\title{
Le corps
}

dans les littératures modernes d'Asie orientale :

discours, représentation, intermédialité

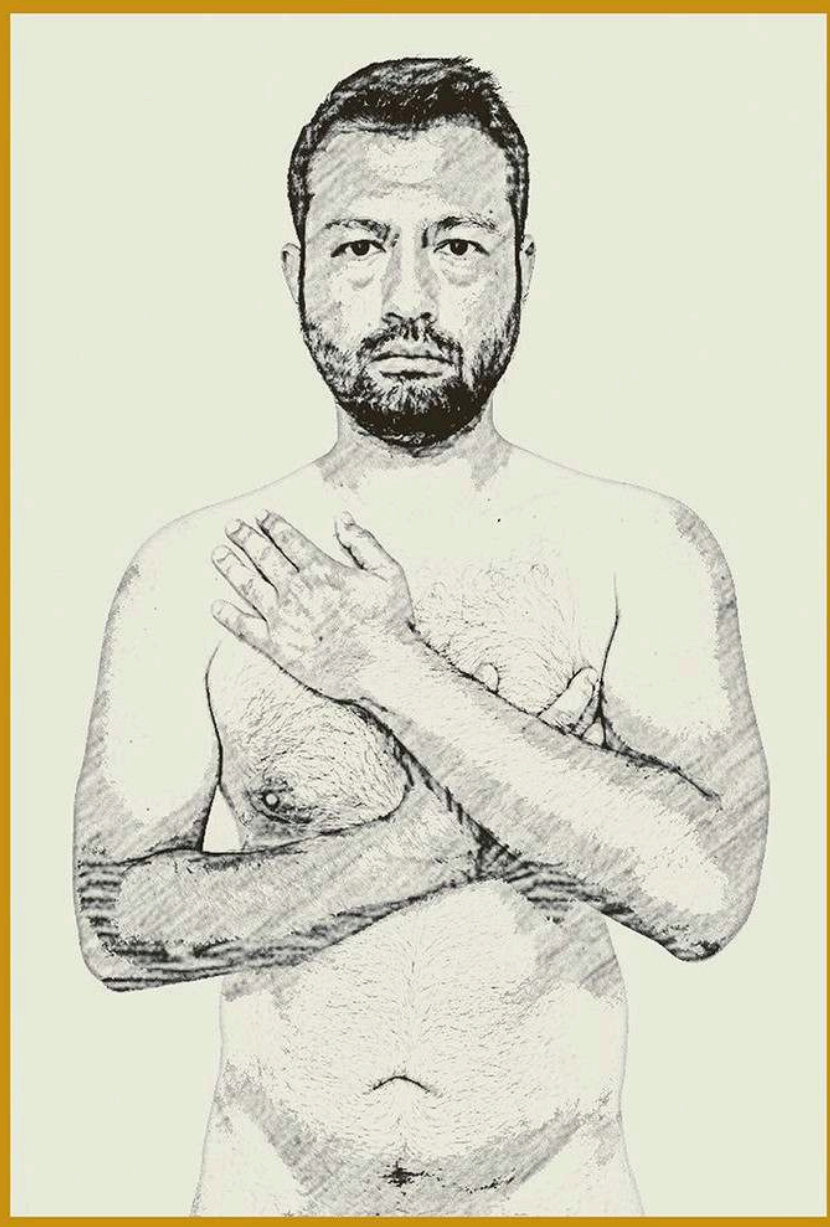

\section{Gérard SIARY, Toshio TAKEMOTO, Victor VUILLEUMIER, Yinde ZHANG}




\section{Le corps dans les littératures modernes d'Asie orientale : discours, représentation, intermédialité}

The Body in Modern Asian Literature: Discourse, Representation, Intermediality

Gérard Siary, Toshio Takemoto, Victor Vuilleumier et Yinde Zhang (dir.)

DOI : 10.4000/books.cdf. 11975

Éditeur : Collège de France

Lieu d'édition : Paris

Année d'édition : 2022

Date de mise en ligne : 7 février 2022

Collection : Institut des civilisations

EAN électronique : 9782722605817

\section{OpenEdition}

Books

https://books.openedition.org

Référence électronique

SIARY, Gérard (dir.) ; et al. Le corps dans les littératures modernes d'Asie orientale : discours,

représentation, intermédialité. Nouvelle édition [en ligne]. Paris : Collège de France, 2022 (généré le 09

février 2022). Disponible sur Internet : <http://books.openedition.org/cdf/11975>. ISBN :

9782722605817. DOI : https://doi.org/10.4000/books.cdf.11975.

Crédits de couverture

Yasmine Bakjaji, サシャ2021年9月1日

Ce document a été généré automatiquement le 9 février 2022.

(c) Collège de France, 2022

Conditions d'utilisation

http://www.openedition.org/6540 


\section{RÉSUMÉS}

Le présent ouvrage étudie le corps représenté dans les littératures d'Asie orientale et alentours au $\mathrm{XX}^{\mathrm{e}}$ et $\mathrm{XXI}^{\mathrm{e}}$ siècles: Chine, Corée, Japon, Vietnam, Indonésie. Le plan du volume est thématique: corps saturés, corps réprimés, corps réappropriés, corps trans-formés. La méthodologie croise autant que possible les disciplines d'approche : narratologie, anthropologie, psychanalyse, histoire culturelle du corps, etc. Les vicissitudes de l'histoire dans la zone concernée ne font pas disparaître les anciennes conceptions du corps, ainsi que les discours et légendes afférents, mais le choc de la modernisation écartèle le corps entre un corps anatomisé en quête d'identité réprimé par le corps de la nation, et un corps virtuel engendré par le cyberespace. La littérature, elle, tend le plus souvent à rendre compte du phénomène comme si l'autonomie du corps, encore un enjeu, conditionnait celle de l'écriture. Aussi deux tendances apparaissent-elles dans l'écriture : l'une, classique, restitue encore les manques aussi bien que les débordements du corps; l'autre expérimente afin de renouveler le lien du corps avec un monde aliénant, quitte à casser la langue. Une expérience assez proche, somme toute, de celle de l'Occident. Nombre d'extraits inédits traduits illustrent l'ensemble.

This book scrutinizes the body as represented in literature of the twentieth and twenty-first centuries in and around East Asia: China, Korea, Japan, Vietnam, Indonesia. The scope of the volume is thematic: saturated bodies, repressed bodies, reappropriated bodies, trans-formed bodies. The methodology combines as many disciplines as possible: narratology, anthropology, psychoanalysis, cultural history of the body, etc. History's vicissitudes of history in the area concerned do not erase old conceptions of the body, as well as related discourses and legends, but the shock of modernization splits the body between an anatomized one, in search of identity mostly repressed by the nation, and a virtual one, generated by the cyberspace. Literature most often accounts for the phenomenon as if the autonomy of the body, still at stake, conditioned the so-called autonomy of writing. Two tendencies also appear in writing: a classical one, still restoring the body's deficiencies and excesses; an experimental one, which manages to renew the link of the body with an alienating world, even if that implies breaking the language. An experience that is quite similar, all in all, to that of the West. A number of unpublished and translated extracts illustrate the whole. 


\section{NOTE DE L'ÉDITEUR}

Actes du colloque international organisé à l'Université de Paris les 15 et 18 novembre 2017 par le CRCAO (Université Paris-Diderot Paris-VII, EPHE, CNRS, Collège de France), le CERC (Université Sorbonne Nouvelle-Paris 3), ALITHILA (Université de Lille) et le CEJ (Inalco). 
SOMMAIRE

Préface

\section{Corps saturés}

L'action d'écrire et le corps «Comment écrire » de Lu Xun Yoshinobu Shino

Le corps comme métaphore chez Lu Xun

Représentation du corps et des victimes

L'inutilité de la littérature

La littérature comme préservation de la mémoire des victimes

Le partage de la souffrance des victimes

Lecture de «Comment écrire » dans la perspective du corps

Écrire le cannibalisme dans la littérature chinoise moderne

Baoqing Shao

«Le journal d'un fou » et le cannibalisme

Representations of Bodies in Crisis in Contemporary Japanese Literature: Two Cases of Eating Disorders

Christopher Scholz

Introduction

On writing, reading, and eating

Food and gender in Japan

Disordered eating and eating disorders

Literary accounts of eating disorders

The hunger artist

To fast is to speak: "I saw words falling to pieces."

Conclusion

Anorexia Speaks: Eating Disorders in Modern Japanese Films, Novels, Manga, and Poetry Hideto Tsuboi

\section{Corps réprimés}

Human/Inhuman/Post-human. Female Bodies in Modern and Contemporary Chinese Literature: Literary Descriptions of Psychological and Social Unease Nicoletta Pesaro

The allegorised body

The animal body

The maternal body

The sexual body

The raped and mutilated female body: women's controversial agency

The sick body

Conclusion 
La résistance des corps dans le recueil Jingai makyō (1939-1941) d’Oguri Mushitarō Gérald Peloux

Oguri Mushitarō, un maître du roman policier aux prises avec la guerre

Un genre policier contraint d'évoluer

Oritake Magoschichi, un héros au corps insaisissable

La fluidité des corps

Corps dressé, corps civilisé : "Seigneur Chen » de Wu Zhuoliu et la police coloniale à Taiwan Ju-Ling Lee

Introduction

Oppression coloniale au nom de la civilisation : contrôle policier et codes juridiques

Dresser le corps, civiliser le colonisé : la société taïwanaise en mutation

Conclusion

La conscience du corps confronté à la violence chez Han Kang

Eun Jin Jeong

Shim Chong, fille vendue de Hwang Sok-yong : du corps réprimé à la figure sublimée Suk-Hee Joo

Le corps souffrant de Shim Chong : réincarnations et voyages sur les mers de l'Asie orientale Métamorphoses du nom et errances du moi

Le corps sensoriel et la réactivation du souvenir par le chant

La transe chamanique comme moyen de retour à une identité première

Conclusion

Narrating Disability in Contemporary Japan: [One Liter of Tears] and [A Therapeutic Sexy

Trip]

Anne-Lise Mithout

Impairment: a bodily experience

Disability as a limitation of activity

Disability as a social experience

Conclusion

Les bébés de la consigne automatique de Murakami Ryū : corps, volonté, violence Toshio Takemoto

Le corps social

Du matricide à la renaissance : le corps d'athlète de Kiku

De l'artificiel à la nature : le visage de Hashi

Résumé du roman

La décomposition du corps dans la poésie coréenne contemporaine : «Ça va, je suis un cochon ", poème en prose de Kim Hyesoon

Moduk Koo

D'une voix à l'autre

Métamorphose

\section{Corps réaproppriés}

Une littérature humorale. Une goutte d'encre et Un lit de malade six pieds de long de Masaoka Shiki

Emmanuel Lozerand

Écrire l'appétit, écrire la souffrance

Rythmes du corps, rythmes de l'écriture

Conclusion 
Narrating Banishment by Means of the Body: Physical Reflections of Han Dong's Cultural Revolution

Martina Codeluppi

Memory and Body

Han Dong's Memory of Banishment

The Body as the Perceiving Subject

Embodied Individualities

Physical Reflections of History

Conclusions

L'écriture du corps chez quelques femmes écrivaines chinoises du XXe siècle Xinyu $\mathrm{Hu}$

L'expression physique du désir féminin

L'interaction entre la transformation du corps et le temps féminin

L'écriture du corps et la subjectivité féminine

L'écriture du corps et le discours féminin dans la littérature contemporaine chinoise : le cas de Lin Bai

Lanfang Guo

Le désir féminin

Le narcissisme

L'écriture du corps : une réponse à l'appel d'Hélène Cixous ?

From Subjects to Bodies: Ah Cheng's The King of Children and Wang Gang's Ying-ge-lishi

Melinda Pirazzoli

Vers une topologie corporelle dans [La Vallée du loess] de Kim Dong-ri Min Sook Wang-Le

Corps colossal et incapacité de son déploiement

Corps révolté et épreuves de destruction

Destin corporel et topologie de la vallée du lœss

Le corps dans tous ses états : Les Belles de Halimunda d'Eka Kurniawan

Étienne Naveau

Le corps comme être de besoin

Le corps comme être social

Le corps comme être-pour-la-mort

Conclusion

Du corps mutilé au corps corrélé au monde, dans le roman Un homme dos à la mer de Wang Wen-hsing

Sandrine Marchand

L'esprit séparé du corps

La maladie comme réponse au corps-machine

Le corps comme « champ continu de relations »

\section{Corps trans-formés}

The Japanese No-Body

Josef Kyburz 
I Sing the Body Electric: Corporal Representations in Guo Moruo's The Goddesses Paolo Magagnin

Introduction

Levels of corporal representation in The Goddesses

Death and rebirth

Oneness with the cosmos

The body as a space of dialogue

Nature anthropomorphized and zoomorphized

Conclusions

Lu Xun, Tourgueniev et l'expressionnisme : corporalité et mouvement dramatique dans «Tremblement à la limite de la déchéance » (1925)

Victor Vuilleumier

Akutagawa Ryūnosuke's Ideal of Corporal Beauty: Social Construction and Personal Contribution

Damaso Ferreiro Posse

Introduction

Body and literature

The ideal of corporal beauty in Akutagawa

The ideal of corporal beauty during the Taishō period: Nietzsche's influence on Akutagawa

Akutagawa's body: social construction and personal contribution

Conclusions

The Flesh, Subject, Embodiment in Postwar Japan: Through Nikutai and Gutai

Fusako Innami

Introduction : In Search of the Individual Flesh

Flesh as a Site of Controversy

Gutai as Embodiedness

Writing the Body (Out): Conclusion

Soigner les corps et les âmes : la science-fiction selon ŌÉ Kenzaburō Antonin Bechler

Le corps, symptôme de l'Histoire

Fictions « fin de siècle »

Le corps comme sujet poétique dans le roman vietnamien Cheval d'acier (2014) de Phan

Hôn Nhiên

Cam Thi Doan

Le corps comme identité

Le corps malade

Corps humain/corps animal/corps végétal : le corps comme sujet poétique

Conclusion

Imaginaire du corps dans les récits fantastiques de web-littérature

Shuang Xu

Introduction

Le corps «transgenre »

Le corps « cross médiatique »

Le corps numérique

Conclusion 
Taiwan Cinema, Horror and Body: the Timid Resurgence of Genre Movies Between Nostalgia and Renewal

Corrado Neri

Hybrid horrors

Young girl dressed in red

Japan cool ghosts

Surveillance angst

Ghostly hills

Bodies under control: The Tenants Downstairs (Adam Tsuei, 2016)

The body and the actors

Pornography / voyeurism / cannibalism

Cannibalism

Conclusion

Postface

Résumés

Bibliographie générale

Index des noms d'auteur(e)s et de figures historiques

Index des œuvres

Index des themes

Index des xénismes 


\section{Préface}

1 Après le numéro 39/2015 de la revue Extrême-Orient Extrême-Occident sur le corps souffrant dans les littératures de la Chine et du Japon ${ }^{1}$, publié dans le cadre du programme transversal de recherche du Centre de recherche sur les civilisations de l'Asie orientale (CRCAO, UMR 8155, CNRS/Collège de France/EPHE/Université ParisDiderot Paris-VII) : «Imaginaires du corps et des identités dans les littératures de l'Asie au $\mathrm{xx}^{\mathrm{e}}$ siècle ", notre objectif fut d'approfondir les perspectives des premiers travaux, et selon nous porteuses, telles que les rapports du corps avec la nation, d'étendre l'enquête à d'autres aires d'Asie (Corée, Vietnam, Indonésie), souvent laissées pour compte. D'où le colloque dont les actes suivent.

2 Tous ces pays ont vécu le choc de la modernité, avant d'entrer dans le temps de la globalisation. Depuis la fin du $\mathrm{XIX}^{\mathrm{e}}$ siècle, entre le corps anatomisé, sous l'emprise accrue de l'individualisation sociale, et, depuis ce début de $\mathrm{xxI}^{\mathrm{e}}$ siècle, le corps virtuel surnuméraire engendré par le cyberspace, les risques et la tentation d'un " adieu au corps" sont d'autant plus considérables que les avancées technologiques relèguent le corps au statut d'une matière indifférente, étrangère à une essence humaine ${ }^{2}$. Reste que ledit corps s'affirme plus que jamais en lieu de lutte de pouvoir, qui incite à interroger tous azimuts la transformation des normes et valeurs.

3 À cet égard, les littératures modernes d'Asie apportent un témoignage paradoxalement «non-moderne ». En effet, le dualisme cartésien d'importation, s'il ébranle bien les anciennes structures, ne cesse pas moins de se heurter à une réalité complexe qui, malgré le bouleversement des anciennes structures, requiert toujours un corps au carrefour de plusieurs interactions, qui relie nature et culture, humains et nonhumains, tradition et modernité : un corps hybride ${ }^{3}$. Aussi les interventions de ce colloque, répondant à cette corporéité multiréticulaire, brassent-elles science, politique, sociologie, psychologie, psychanalyse, anthropologie... et cela dans un constant souci de croisement et de connexion transculturels. Le corps s'écrit, se lit et se visualise ici comme ce lieu de signification où s'agglutinent savoir et esthésie, réel et imaginaire, fait et fiction.

4 L'ordre de la table des matières progresse du réel historique foncier à la création esthétique, du tangible à «l'artialisation $»^{4}$. Si l'on aborde la représentation du corps avec la nourriture, la faim, la boulimie, l'anorexie, c'est que la satiété reste un thème 
transversal, qui se conjugue ici avec le régime du manque en Chine, et là celui du tropplein au Japon, d'où les « Corps saturés » (Partie I). Suit alors tout ce qui, entre guerre, occupation coloniale et système d'autorité, relève de la biopolitique, i.e. la galerie de tous ces corps qu'elle brime, d'où les « Corps réprimés » (Partie II). Mais au fil du temps, envers et contre les vicissitudes historiques, et par des voies variées comme l'approche mémorielle, souvent au plus près de la sensation, le sujet résiste, se fait résilient, se réapproprie son corps par et dans l'écriture, d'où les « Corps réappropriés » (Partie III). Et cela va de pair, quelle que soit l'époque, avec les métamorphoses du corps dans l'art et l'expression transmédiatique, de la science-fiction au cinéma via la photographie et la peinture, qui donnent à voir enfin les « Corps trans-formés » (Partie IV).

5 Le présent ouvrage est le produit du colloque : «Le corps dans les littératures d'Asie aux $\mathrm{xx}^{\mathrm{e}}$ et $\mathrm{xxI}^{\mathrm{e}}$ siècles : discours, représentation et intermédialité » (Université ParisDiderot Paris-VII 16-18/11/2017). Tous les exposés présentés n'ont pu être recueillis, mais quelques articles ont été ajoutés. Cette manifestation ainsi que la relecture des articles en anglais par M. Alan Wagner ont été soutenues par les laboratoires suivants : CRCAO, CERC (Paris 3), Alithila (Lille 3), CEJ (Inalco).

Avertissement :

Les textes non disponibles en traduction sont indiqués entre crochets.

Le choix de l'usage des caractères simplifiés ou non simplifiés, ainsi que de la transcription des noms, est laissé au gré de l'auteur de l'article.

\section{NOTES}

1. La préface, ainsi que la postface de ce numéro intitulé «Les épreuves du corps en littérature. Le cas de la Chine et du Japon ", offrent une approche comparatiste de la question.

2. LE BRETON David, L'Adieu au corps, Paris, Métailié, 1999.

3. Voir «l'acteur-réseau » LATOUR Bruno, Nous n'avons jamais été modernes. Essai d'anthropologie symétrique, Paris, La Découverte, 1993.

4. Nus et paysages: Essai sur la fonction de l'art, Aubier, 1979. 
1. Corps saturés 


\title{
L'action d'écrire et le corps «Comment écrire» de Lu Xun
}

\author{
Yoshinobu Shino
}

\section{Le corps comme métaphore chez Lu Xun}

1 Lu Xun 魯迅 (1881-1936) accorde une certaine importance au corps, même s'il ne le décrit pas avec une grande variété d'expression. Dans ses œuvres, c'est le corps mangé qui vient spontanément à l'esprit. Par exemple, dans les nouvelles «Le journal d'un fou » / 狂人日記 (1918) ou 《Le remède » / 藥 (1919), recueillies dans Cris / 呐喊 (1923), la chair humaine est un remède médical. Le recours à cette figure du corps mangé, en étroite relation avec la notion de piété filiale, est une façon de dénoncer tout ce qui dans l'histoire de la Chine, comme le supposé cannibalisme, relève de la " superstition » et de l'inhumain. Depuis la dynastie Tang 唐 (618-907), avait cours la croyance selon laquelle la maladie des parents pouvait être guérie par la consommation d'un morceau de la cuisse de leurs enfants. Ce remède était réputé efficace, parce que le corps d'un enfant était perçu comme de même nature que celui de ses parents, idée en partie basée sur ce propos des Rites / 禮記, l'un des Cinq Classiques confucéens : « le corps est la donation des parents / 身也者, 父母之遺體也 »'. Qui offrait un morceau de son corps à ses parents faisait donc officiellement l'objet d'éloges: il était vu comme un enfant pieux. Cette tradition du cannibalisme pour recouvrer la santé était si répandue ${ }^{2}$ qu'on utilisait aussi la chair d'autres personnes sans lien familial direct, en particulier les cadavres de condamnés à mort.

C'est pour dénoncer cette pratique que Lu Xun écrit la nouvelle « Le remède ». Le sujet du recueil Cris n'est pas encore celui du corps mangé comme tel, mais plutôt d'une part, la relation entre ceux qui sont susceptibles de manger ou d'avoir déjà mangé à leur insu de l'homme, et d'autre part, ceux qui ont peur d'être mangés. Il s'agit pour Lu Xun de montrer que le prétexte d'ordre rituel dissimule une relation de domination sociale : ce que $\mathrm{Wu} \mathrm{Yu}$ 吳虞 (1872-1949), penseur et activiste de l'ère de la République de Chine, appelle en 1919 《Les rites cannibales» / 揳人的禮教 dans la revue Nouvelle Jeunesse / 
新青年3. Ultérieurement, en 1925, Lu Xun écrivit dans son essai « Notes prises sous la lampe » / 燈下漫筆 :

la prétendue civilisation chinoise n'est rien d'autre qu'un banquet de chair humaine offerte au bon plaisir des riches; quant à la Chine, c'est la cuisine dans laquelle elle est apprêtée.

所謂中國的文明者, 其實不過是安排給闊人享用的人肉的䇥宴。所谓中國者, 其 實不過是安排這人肉的䇥宴的㕌房。4

Ce thème du corps mangé, de la chair du sacrifice se trouve encore en arrière-plan des textes de Lu Xun dans les années 1910. Chez lui, « le corps est une métaphore et ne peut parler pour le corps réel $»^{5}$. Aussi le corps mangé sert-il clairement dans son œuvre de métaphore critique de l'ancien régime de la Chine impériale et peut-être aussi de la chine républicaine.

\section{Représentation du corps et des victimes}

Après les années 1920, l'image des victimes du cannibalisme rejoint celle des victimes des activités de protestation au temps des seigneurs de la guerre (1916-1927). En 1922, dans « Voir le grand dans le petit » / 即小見大 / Ji xiao jian da, texte paru dans le recueil Vent chaud / 熱風 (1925), Lu Xun déplore l'absence d'explication sur les trois stèles accompagnant les tombes des quatre héros exécutés pour avoir tenté d'attenter à la vie de Yuan Shikai 袁世凱 (1859-1916) ${ }^{6}$. Pour lui, après que toutes les victimes ont versé leur sang sur l'autel, «il ne reste rien d'autre pour les gens que la distribution de la chair des victimes (散胙)» (Lu Xun Quanji, vol. 1, p. 407). Dans Correspondance avec Xu Guangping / / 兩地書 (1933), Lu Xun reprend ce propos :

les sacrifices sont faits pour la bénédiction des masses : après les avoir offerts aux dieux, les gens se partagent leur chair en rétribution.

犠牲為群䍙祈福, 祀了神道之後, 群䍙就分了他的肉, 散胙。( Lu Xun Quanji, vol. 11, p. 75)

5 Dans La Mauvaise herbe / 野草 (1927), son recueil de poèmes en prose, Lu Xun recourt à la représentation d'un corps qui n'est plus vif, ni déjà mort, proche par cette dimension paradoxale du motif du «sang frais» ou "pâli»/淡淡的血痕que l'on y rencontre également. Par exemple, quand dans «Vengeance» / 復仇 (1924), il décrit deux personnages apparemment sur le point de s'embrasser ou s'entre-tuer, mais qui finalement ne bougent pas, et précise :

L'épiderme humain a moins d'un demi-millimètre d'épaisseur; sous cette paroi, dans un réseau de veines qui s'entrelacent comme piste de larve sur un mur, circule un sang rouge et ardent qui diffuse sa chaleur dans tout le corps ${ }^{8}$.

人的皮虞之厚, 大概不到半分, 鮮細的熱血, 就循着那後面, 在比密密層層地爬

在墙壁上的槐堚更其密的血管裏奔流，散出溫熱。( Lu Xun Quanji, vol. 2, p. 172)

Dans « Le passant » / 過客 (1925), le protagoniste, en marche vers un cimetière, montre son pied blessé à un vieillard et se plaint aussi de manquer de sang ( $L u$ Xun Quanji, op. cit., vol. 2, p. 191). Dans «Épitaphe»/ 墓碣文, le narrateur rêve d'un serpent ayant mangé son propre corps, puis découvre un cadavre « à la poitrine défoncée et à qui il manque le cœur et le foie» (La Mauvaise herbe, p. 95) / 胸腹俱破, 中無心肝 ( $L u$ Xun Quanji, vol. 2, p. 202). Un autre poème en prose du même recueil, « Après la mort » / 死 後 (1925), met en scène un corps encore doté de ses sens et de conscience, agacé par une fourmi et une mouche ${ }^{9}$. Comme l'indique Maruo Tsuneki 丸尾常喜 (1937-2008), l'intrigue de ce poème reprend le texte [Un guerrier et les mouches] / 戰士和荅蠅, 
dans le recueil Toujours sous le dais fleuri / 華蓋集 $(1926)^{10}$, qui fait l'éloge d'un guerrier mort, dont le corps infesté d'insectes est de fait une nouvelle figure du corps mangé. Ou encore, dans «Parmi les traces de sang pâli » / 淡淡的血痕中, Lu Xun s'en prend au "Créateur », qui « amène les hommes à verser le sang, mais à ce sang répandu, il n'ose pas préserver son ardente couleur» (La Mauvaise herbe, p. 115) / 暗暗地使人類流血, 卻不敢使血色永遠鮮濃 (Lu Xun Quanji, vol. 2, p. 221). Une série d'expressions exprime la compassion qu'il éprouve envers les victimes d'assassinats politiques. Dans ce contexte, il réfléchit sur le rôle de la littérature, surtout sur son impuissance, notamment à se substituer aux victimes : Lu Xun ne peut, en effet, mourir à leur place.

7 Nous allons examiner sa pensée sous trois aspects: tout d'abord, l'inutilité de la littérature face à la mort des autres; ensuite, la littérature comme préservation de la mémoire des victimes; enfin, la tentative de partager leur souffrance avec les lecteurs. C'est quand il s'agit du partage de cette souffrance, que Lu Xun fait appel à la figure du corps.

\section{L'inutilité de la littérature}

Dans « Après avoir écrit 'En se heurtant aux murs' » / 碰壁之後 (1925), Lu Xun écrit :

J'ai vu un assassin tuer des gens en riant, j'ai vu des cadavres danser dans le sol crotté [...] J'ai essayé de les dessiner, mais n'ai pu tracer même une ligne.

看見殺人者於微笑後屠翏百姓，看見死屍在糞土中舞蹈 [...], 我想取作畫圖，竟

不能畫成一線. (Lu Xun Quanji, vol. 3, p. 72-73)

Dans un autre texte, 《La littérature au temps de la révolution » / 革命時代的文學 (1927), il avoue aussi l'inutilité de la littérature face à la violence des puissants ( $L u$ Xun Quanji, vol.3, p. 417), mais n'a pas d'autre choix que d'écrire, car il ne peut se livrer à d'autres tâches.

Dans son prologue à La mauvaise herbe (1927), il écrit que la mauvaise herbe «sait se nourrir de la chair et du sang des cadavres»/吸取陳死人的血和肉. Cette mauvaise herbe est une figure allégorique de sa propre littérature. Faisant mine de déprécier son œuvre, Lu Xun espère que "cette mauvaise herbe mourra et pourrira bientôt » ( $L a$ Mauvaise herbe, p. 53，54) / 這野草的死亡與朽腐, 火速到來 (Lu Xun Quanji, vol.2, p. 159). En fin de compte, il écrit en souhaitant que son écriture n'aboutisse à rien : il pense sa littérature comme un ouvrage temporaire élaboré dans une période de crise. Dans cette situation, c'est l'action d'écrire, plus que le contenu, qui fait l'objet de sa réflexion. C'est dans cet esprit que Lu Xun écrit son essai «Comment écrire? / 怎么舄 (1927, v. infra).

\section{La littérature comme préservation de la mémoire des victimes}

Lu Xun n'ayant cessé d'écrire, il lui fallait trouver une raison d'être à sa littérature. Son rôle est tout simplement de commémorer l'action des victimes en soutien à ceux qui suivent leurs traces. À ce niveau, les tâches du militant et de l'écrivain sont clairement séparées. Dans sa Correspondance avec Xu Guangping, Lu Xun écrit :

Je manque et de force et de courage. Je n'ai pas le choix : tout ce qui se trouve à ma portée, c'est un pinceau.

我又無拳無勇, 真沒有法, 在手頭的只有筆墨. (Lu Xun Quanji, vol. 11, p. 31) 
Lu Xun dit aussi que tandis que la révolte de Xu Guangping la mène à «espérer l'avènement de la lumière » / 希望光明到來, la sienne propre ne conduit qu'à « lutter avec l'obscurité » / 與黑暗搗亂 (Lu Xun Quanji, vol. 11, p. 79), lutte qu'il qualifie souvent de "guerre de tranchées" / 㟻壕戰, en comparaison avec les combats au corps à corps. En d'autres termes, il s'agit d'une comparaison entre lutte spirituelle et corporelle. Il constate que la lutte est beaucoup plus précieuse que la littérature: se pose alors la question de la raison d'être de la littérature. Voici ce qu'il écrit dans un essai, « Une trouvaille inopinée » / 忽然想到 (11-5) :

Même si les noms des victimes à Shanghai et Hankou seront totalement oubliés dans quelques jours, le poème et la prose survivront plus longtemps: ils auront l'opportunité d'émouvoir les autres ou d'éclairer la prochaine génération. C'est là où les écrivains trouvent leur utilité.

即使上海和漢口的爔牲者的姓名早已忘得乾乾淨淨, 詩文卻往往更久地存在, 或 者還要感動別人, 啟發後人。這倒是文學家的用處。(Lu Xun Quanji, vol. 3, p. 94)

Lu Xun s'était indigné contre l'oppression politique, mais l'incident du 18 mars 1926, lors duquel le seigneur de guerre Duan Qirui 段掑瑞 (1865-1936) perpètre un massacre contre des manifestants, fut d'autant plus choquant que certains de ses étudiants se trouvaient parmi les victimes. En apprenant la mort violente de Liu Hezhen 劉和珍 (1904-1926), l'une de ses étudiantes à l'Université normale de Pékin pour jeunes filles / 北京女子師範大學, Lu Xun dans «À la mémoire de Liu Hezhen » / 記念劉和珍君 (1926) exprime ainsi sa volonté d'écrire: «Bientôt, l'oubli, salutaire, sera là; je me dois vraiment d'écrire quelque chose » / 忘卻的救主快要降臨了罷, 我正有舄一點東西的 必要了(Lu Xun Quanji, vol. 3, p. 274) ${ }^{11}$. «Quelque chose » (yidian dongxi), parce qu'avant de se demander quoi écrire, il s'agit avant tout d'écrire, quel que soit le sujet traité. Nous y revenons plus bas.

14 Lu Xun souhaite que sa littérature soit utile pour faire passer les noms des victimes à la postérité, cependant il ne peut se substituer à elles. Ce sont les révolutionnaires, non l'écrivain, qui sont assassinés. Et les victimes étaient souvent plus jeunes que Lu Xun. Dans « Postface à La Tombe » / 舄在《填》後面 (1926), il regrette, au nom de l'inutilité de sa littérature, la publication de ses œuvres choisies (d'une manière proche de ce qu'il fait dans le prologue de La mauvaise herbe). Toutefois, il semble malgré tout lui reconnaitre une certaine efficacité, car il demande au lecteur de le lire : dans un texte du recueil, «Cessons d'être fair-play 》/論「費厄潑賴」應該緩行, parce que :

Ce texte n'est pas écrit avec mon sang, mais en regardant le sang de gens de mon âge et d'autres plus jeunes que moi.

因為這雖然不是我的血所䳔，卻是見了我的同輩和比我年幼的青年們的血而舄的

(Lu Xun Quanji, vol. 1, p. 283)

15 Le conflit intérieur de Lu Xun concernant le pouvoir de la littérature se fait jour ici.

\section{Le partage de la souffrance des victimes}

En réaction aux meurtres de ses contemporains, surtout de ses étudiants, Lu Xun reprend le motif du cannibalisme. Dans « Réponse à Monsieur You Heng » / 答有恒先生 (1927), il reprend l'allégorie de la Chine comme banquet de chair humaine: «J'ai découvert cependant que j'aidais moi aussi à servir à ce banquet » / 但我現在發見了, 我自己也幫助著排筵宴 ( $L u$ Xun Quanji, vol. 3, p. 454). C'est pour Lu Xun un terrible aveu. Pourtant, c'est parce qu'il participe à ce banquet cannibale qu'il peut partager la souffrance des victimes tuées : par la prise de conscience de sa complicité, l'auteur 
tente de partager leur souffrance. C'est ici que se trouverait la possibilité de substituer l'encre de son pinceau au sang des victimes (sur les rapports entre le sang et l'encre, voir plus bas), bien qu'il soit très peu assuré d'y parvenir. Mais, comme il le dit dans 《L'espoir » / 希望, « le désespoir est une illusion, tout comme l'espoir " ${ }^{12}-$ ou encore dans «Mon pays natal »/故郷: «tout endroit où les hommes passent nombreux se transforme en route $»^{13}$.

La nouvelle «Les épées » / 鑄劍 (1927) est un récit de vengeance. Le héros, Meijianchi / 眉間尺, qui veut se venger du roi ayant tué son père, confie sa propre tête à un homme. Cet homme réussit plus tard à tuer le roi, dont la tête elle aussi décollée se bat avec celle de Meijianchi dans un chaudron, dans lequel elles sont toutes deux mises à cuire. Pour aider Meijianchi, cet homme tranche à son tour sa propre tête, qui bascule dans le chaudron. Une fois bouillies, les trois têtes sont devenues indistinctes. Cette histoire peut se lire comme une variation sur le thème du banquet cannibale. Lu Xun représente la révolte d'un opprimé qui n'a pas d'autre choix que de se sacrifier pour parvenir à ses fins.

Le poème en prose «[Un tressaillement au bord de l'abîme] / 䫋敗線的顫動》 (1925) traite aussi du corps révolté. Une femme qui s'est prostituée dans sa jeunesse est rejetée par tous, y compris sa propre famille. Lu Xun représente d'abord le corps de cette femme dans sa jeunesse :

Un petit corps frêle et gracile tressaillait de faim, de douleur, d'effroi, de honte et de plaisir.

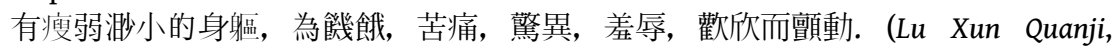
vol. 2. p. 204)

Puis vient la description de son corps âgé :

son corps qui tout en conservant la majesté d'une statue de pierre, était devenu maintenant dévasté et croulant, fut agité tout entier d'un tressaillement. ( $L a$ Mauvaise herbe, p. 100)

她那偉大如石像, 然而已䋑荒廢的, 頽敗的身身品的全面都顫動了. (Lu Xun Quanji, vol. 2, p. 206)

Elle est si «déchue " (tuibai), qu'elle s'en va pour une région sauvage et tressaille de tout son corps, dans un mouvement qui fait refluer en tourbillon les ondes de l'espace autour d'elle. Le corps de cette femme peut figurer l'histoire du peuple opprimé dans la Chine de même que l'amorce d'un soulèvement futur. Nous pourrions y superposer la figure mythologique de Nüwa / 女姛 (déesse ayant créé l'espèce humaine et réparé le ciel) à la « chair rose » et qui s'intègre au monde dessiné dans « La réparation du ciel » / 補天 (1922). Dans "Un tressaillement au bord de l'abîme", le mot "ligne » / 線 (tuibaixian signifie littéralement « ligne », « fil de déchéance », traduit comme « bord de l'abîme ») n'est utilisé que dans le titre chinois, pour indiquer la silhouette du corps tressaillant de l'héroïne. Cependant, on pourrait proposer d'y voir une référence à l'écriture : les traces du pinceau de Lu Xun. Ainsi, le tremblement des lignes établit une correspondance entre le corps qui se lamente et l'action d'écrire. La main de Lu Xun tressaille avec compassion quand il décrit le tressaillement des lignes du corps de la femme. 


\section{Lecture de «Comment écrire » dans la perspective du corps}

21 L'essai de Lu Xun « Comment écrire » (Lu Xun Quanji, vol. 4. p. 18-28), est essentiel pour réfléchir à la relation chez lui entre corps et écriture, notamment par la comparaison effectuée entre les corps sacrifiés au «combat au corps à corps » et l'écrivain dans les «tranchées » qui présentent ses condoléances aux victimes. Lu Xun, sur liste noire, se déplace de Shanghai plus au sud à Xiamen et Canton, où il rédige ce texte. Son essai prétend être écrit sans soin : Lu Xun dit ne pouvoir écrire tout en le devant. Il recourt donc à nombre d'expressions symboliques qui rendent cet état contradictoire. Et d'écrire : «S'il me faut absolument écrire, cela ne peut être qu'avec nonchalance » / 佾 非舄不可, 我想, 就是隨便舄舄罷 ( $L u$ Xun Quanji, vol. 4. p. 20). Faute d'écrire avec son sang, reprenant ce mot : «De tout ce qui est écrit, je n'aime que ce qu'un homme écrit avec son sang ${ }^{14}$, Lu Xun n'a d'autre choix que d'écrire au hasard. Dans ce texte, le sacrifié dont il est question est Bi Lei 畢磊 (1902-1927), jeune communiste arrêté par le Parti nationaliste. Au début de cet essai, en guise de commémoration des victimes, $\mathrm{Lu}$ Xun ne fait plus que décrire les tombeaux dispersés autour du Temple Nanputuo / 南普 陀寺, mentionné dans "Postface à la Tombe ", qu'il voit de chez lui à Xiamen. S'il n'exprime pas directement ses condoléances et qu'il ne peut écrire davantage, c'est que $\mathrm{Lu}$ Xun n'a plus la jeunesse des victimes et ressent face aux jeunes générations une certaine distance, qui est représentée par la différence entre les couleurs. Le rouge du sang des victimes renvoie aussi à la fugacité de la vie, à « la légère rougeur des joues d'une jeune fille» (Lu Xun Quanji, vol. 4, p. 20), au blanc des os et des tombes, pour l'éternité. $\mathrm{Au}$ vu du contexte politique, ira-t-on jusqu'à voir dans le rouge et le blanc les symboles respectifs du Parti Communiste chinois et du Guomindang ? ${ }^{15}$

Mais ce contraste de couleurs renvoie surtout à la situation de Lu Xun entre la jeunesse fugace et la mort éternelle. Il écrit que les gens l'ont tenu à distance à grands renforts de précautions comme on le fait avec les « esprits" / 鬼神 (Lu Xun Quanji, vol. 4, p. 18). Il mène une existence à mi-chemin entre l'état vivant et mort $^{16}: c^{\prime}$ 'est le " gris " / 灰色 ( $L u$ Xun Quanji, vol. 4, p. 21), entre le rouge du sang et le blanc de l'os. C'est dans cette situation ambiguë qu'il cherche à écrire. Il ne serait plus assez jeune pour se consacrer au mouvement révolutionnaire, ni assez vieux pour ne pouvoir rien faire. Il ne lui reste qu'à écrire. C'est ici qu'il porte de nouveau attention au corps :

Accoudé à la balustrade de pierre, le regard dans le lointain, je peux entendre les

battements de mon cœur.

我靠了石欄遠眺, 聽得自己的心音 (Lu Xun Quanji, vol. 4, p. 18)

Ce battement, preuve qu'il est vivant, l'incite à écrire, mais en vain. C'est alors qu'un moustique le pique à la jambe. Il ne peut s'empêcher d'être distrait par cette piqûre, qui provoque une sensation corporelle suffisante pour effacer "la souffrance du monde » qui l'entoure. Et c'est la seule chose sur laquelle finalement il peut écrire « une petite chose » :

C'est tout ce que je peux écrire, pensé-je, cette petite chose, donc je ne pourrai jamais représenter l'ébranlement de mon cœur tel que je l'ai éprouvé profondément ce jour-là.

我想, 也只能䳔一些這類小事情, 而還萬不能窵得正如那一天所身受的顯明深切

(Lu Xun Quanji, vol. 4, p. 19) 
«Une petite chose » indique ici la piqûre d'un moustique, mais elle symbolise aussi le choc causé par la mort de son jeune ami. Le mieux qu'il puisse faire pour déplorer cette mort est de faire semblant d'avoir écrit au hasard en multipliant les expressions symboliques et allégorique: symbolique parce qu'il exprimerait, par exemple, une position politique par le contraste des couleurs, et allégorique parce qu'il manifeste sa sympathie pour les victimes au travers de la description d'une piqûre de moustique. $\mathrm{Lu}$ Xun éprouve de la sympathie pour les victimes via la douleur et de la démangeaison qu'il ressent. Il lui serait impossible de s'exprimer s'il y avait plus de victimes ou une situation plus grave :

Si je subis mille ou dix mille piqûres, ou si je suis blessé par un sabre ou par une épée, je ne pourrai plus écrire.

而況千叮萬叮, 而況一刀一检, 那是舄不出來的.

Faisons un détour par Merleau-Ponty pour mieux saisir ce que dit Lu Xun. Pour le philosophe, même un malade atteint de cécité psychique, lorsqu'il est piqué par un moustique, «n'a pas à chercher le point piqué et le trouve d'emblée », parce « qu'entre la main comme puissance de gratter et le point piqué comme point à gratter un rapport vécu est donné dans le système naturel du corps propre $»^{17}$. Ce malade ne peut saisir son corps comme objet, mais il peut le maîtriser comme corps phénoménal. Lu Xun met plutôt l'accent sur l'impossibilité du corps en décrivant souvent le corps déjà perdu du " système naturel » : par exemple, un cadavre encore doué de conscience, qui n'est plus capable de chasser les mouches et les fourmis. Il imagine de plus un corps qui laisserait «mille ou dix mille moustiques» le piquer. Le corps mort constitue un thème important dans ses écrits. La preuve en est que dans " Postface à la Tombe " Lu Xun représente ses œuvres comme un cadavre jadis vivant, qui devra être enterré dans une petite tombe, en compagnie de hiboux, serpents et fantômes, "ses amis " ( $L u$ Xun Quanji, vol. 1, p. 284).

Revenons au titre de ce texte, "Comment écrire». Lu Xun explique son intention: «Lorsque je pense [que je ne peux écrire qu'avec nonchalance], je trouve que la question 'quoi écrire' n'est pas vraiment importante» / 當我這樣想的時候, 便覺 得“舄什麼”倒也不成什麼問題了 ( Lu Xun Quanji, vol. 4, p. 20). Il s'agit de 《comment écrire ", plutôt que de se demander "qu'écrire ». Cette interrogation sur "comment écrire » est à destination des jeunes générations qui sont tentées par la littérature de propagande. Il ne peut ni se substituer aux victimes ni écrire comme de jeunes auteurs le feraient. En fait, Bi Lei, l'une des victimes du massacre, était un des rédacteurs de la revue au titre évocateur de Que faire / 做什麼, éditée pas les étudiants communistes de l'Université Sun Yat-sen / 中山大學 à Canton. Dans « Comment écrire », Lu Xun évoque cette revue ainsi qu'une autre, Voilà ce qu'il faut faire / 這樣做, publiée par les partisans du Guomindang. Elles s'opposent l'une à l'autre par leurs titres respectifs, qui renvoient aux questions de savoir quoi écrire et comment. Stimulé par ces débats, Lu Xun écrit "Comment écrire» pour contester Voilà ce qu'il faut faire; il n'intitule pas son texte "Quoi écrire», parce qu'il n'ose adhérer au ton de la revue Que faire. Il conclut en disant que la littérature, même de style journalistique, ne doit éviter ni la fiction ni la différence d'avec la propagande.

"Comment écrire» exprime l'impossibilité de se substituer aux victimes, de même qu'une tentative pour partager la souffrance corporelle des jeunes victimes par l'acte d'écrire. Cet essai présente un rapport intime avec les œuvres ci-dessus, et dans lesquelles Lu Xun cherche une façon d'écrire, incapable qu'il est d'écrire un texte avec 
son sang. Il s'agit donc ici du lien intime entre l'action d'écrire et le corps suspendu entre la vie et la mort. Autrement dit, pour Lu Xun, l'impossibilité de substituer aux victimes et la possibilité de partager leur souffrance au niveau du corps et de la perception objectifs est la condition fondamentale qui permette de continuer à écrire.

\section{BIBLIOGRAPHIE}

FUנII Shōzō 藤井省三, [Le Paysage du “pays natal”] / 魯旬「故拫」の風景, Tokyo, Heibonsha, 1986.

KUWABARA Jitsuzō 桑原䧞蔵, [《Les coutumes cannibales en Chine»] / 支那人間に於ける食人肉 の風習, in [Æuvres complètes de Kuwabara Jitsuzō] 桑原䧞蔵全集, Tokyo, Iwanami shoten, vol. 2, 1968, p. 153-205.

[Livre des rites] / 禮記, wANG Wenjin (trad. et ann.) 王文錦, Pékin, Zhonghua shuju, 2001.

Lu Xun 魯讯, Lu Xun Quanji / 魯旬全, Pékin, Renmin wenxue chubanshe, 1981.

Lu Xun 魯旬, Cris, BELLASSEN Joël et alii (trad.), Paris, Albin Michel, 1995.

LU Xun 魯旬, La Mauvaise herbe / 野草, RYCKMANs Pierre (trad. et intro.), Paris, UGE, 1975.

LU Xun 魯式, Toujours sous le dais fleuri / 华盖集续编, MEUNIER Jacques et LIU Fang (trad.), Paris, You Feng, 2014.

LU Xun 魯讯, Nouvelles et poèmes en prose, VEG Sebastian (trad.), Paris, Éditions Rue d'Ulm, 2015.

MARUO Tsuneki 丸尾常喜, [Recherches sur La Mauvaise herbe de Lu Xun] / 魯旬「野草」の研究 / Rojin « Yasō » no kenkyū, Tokyo, 波古書院, 1997.

MERLEAU-PONTY Maurice, Phénoménologie de la perception, Paris, Gallimard, 1945.

VUILLEUMIER Victor, « Le corps souffrant chez Lu Xun : allégorie muette de l'obstacle et appropriation de la modernité », Extrême-Orient Extrême-Occident, 39/2015, p. 47-84.

wu Yu 吳㲊, [《 Le cannibalisme et les rites »] / 喫人與禮教, in [Æuvres de Wu Yu] / 点盧集, Pékin, Zhonghua shuju, 2013.

\section{NOTES}

1. WANG Wenjin 王文錦 (trad. et annot.), [Traduction et annotation des Rites] / 禮記譯解, Pékin, Zhonghua shuju, 2001, p. 629.

2. KUWABARA Jitsuzo 桑原䧞蔵, [《 Les coutumes cannibales en Chine »] / 《支那人間に於ける食人 肉の風習》, in [CEuvres complètes de Kuwabara Jitsuzo] / 桑原䧞蔵全集, Tokyo, Iwanami, 1968, vol. 2, p. 153-205.

3. WU Yu 吳虞, [《Le cannibalisme et les rites»] / 喫人與禮教, originellement publié dans [Nouvelle Jeunesse] / 新青年, vol. 6, nº, 1919, repris dans wu Yu吳盧, [๕uvres de Wu Yu] / 点 盧, Pékin, Zhonghuashuju, 2013. 
4. Lu Xun 魯迅, [CEuvres complètes de Lu Xun] / 魯旬全集, Pékin, Renmin wenxue chubanshe, 1981, vol. 1, p. 216. À cette édition, sous la dénomination Lu Xun Quanji suivie de la tomaison et de la pagination, renvoient sauf indication contraire les passages cités infra des CEuvres complètes de Lu Xun.

5. VUILLEUMIER Victor, «Le corps souffrant chez Lu Xun: allégorie muette de l'obstacle et appropriation de la modernité », Extrême-Orient Extrême-Occident, 39/2015, p. 73.

6. Yuan Shikai a supprimé ses opposants lorsqu'il était président de la république de Chine (1912-1915).

7. Recueil de la correspondance de 1925 à 1929 entre Lu Xun et sa concubine Xu Guangping 許廣 平 (1898-1968).

8. LU Xun, La Mauvaise herbe, RYCKMANS Pierre (trad. et intro.), Paris, Union générale d'éditions, 1975, p. 64.

9. Lu Xun écrit aussi la nouvelle « Résurrection» / 起死 (1935), dans le recueil Contes anciens à notre manière / 故事新编, 1936, où il raconte l'histoire d'un cadavre soumis à une résurrection forcée.

10. MARUO Tsuneki 丸尾常喜, [Recherches sur La Mauvaise herbe de Lu Xun], 1997, p. 328.

11. LU Xun, Toujours sous le dais fleuri / 华盖集续编, MEUNIER Jacques et LIU Fang (trad.), Paris, You Feng, 2014, p. 101.

12. LU Xun 魯迅, [๕uvres complètes de Lu Xun] / 魯迅全集, op. cit., vol. 2, p. 178. Traduction LU Xun, Yecao [Les Herbes sauvages], Pékin, Waiwen chubanshe, p. 37. Lu Xun, Nouvelles et poèmes en prose, VEG Sebastian (trad.), Paris, Éditions Rue d'Ulm, 2015, p. 525.

13. LU Xun 魯旬, [๕uvres complètes de Lu Xun] / 魯迅全集, op. cit., vol. 1, p. 485. Lu Xun, Cris, BELLASSEN Joël, fENG Hanjin, JoIN Jean et LoI Michelle (trad.), Paris, Albin Michel, 1995, p. 125.

14. NIETZSCHE Friedrich, Ainsi parlait Zarathoustra, in CEuvres philosophiques complètes VI, GANDILLAC Maurice de (trad.), Paris, Gallimard, 1971, p. 52.

15. Le Parti «rouge" était menacé par la «terreur blanche» menée par le Guomindang, déclenchée au mois d'avril en 1927 par le «Massacre de Shanghai». De plus, le drapeau du Guomindang représente un soleil blanc sur fond bleu. Par ailleurs, ce même contraste entre le rouge et le blanc se retrouve dans la nouvelle «Célébration » / 祝福 (1924), opposant les bras rougis des lavandières aux cheveux blancs de la paysanne 祥林嫂, qui est une autre figure de victime.

16. Ce rapport entre la jeunesse et la vieillesse se retrouve notamment dans « Le passant ».

17. MERLEAU-PONTY Maurice, Phénoménologie de la perception, Paris, Gallimard, 1945, p. 122-123.

\section{AUTEUR}

\section{YOSHINOBU SHINO}

Université Meiji, Tokyo.

Yoshinobu Shino est spécialiste de philosophie moderne en Chine, de théorie littéraire en Chine, et de philosophie comparée entre la Chine et le Japon. Il a publié : D'ailleurs vers ailleurs :

Développements hétérotopiques de la phénoménologie, coauteur, Chisen Shokan, 2021 ; Saint et homme fougueux, coauteur, Presses Universitaires Hôsei, 2016 ; Anne Cheng, Histoire de la pensée chinoise, cotraducteur, Chisen Shokan, 2010. 


\title{
Écrire le cannibalisme dans la littérature chinoise moderne
}

\author{
Baoqing Shao
}

1 Alors que « Le journal d'un fou » / 狂人日记 de Lu Xun, salué unanimement comme le point de départ de la littérature moderne chinoise, traite du cannibalisme dans la société chinoise, le sujet semble paradoxalement bien malaisé dans la littérature du $\mathrm{xx}^{\mathrm{e}}$ siècle. Aussi convient-il de se demander si la lecture de cette nouvelle a été volontairement biaisée ou si elle cachait une réelle difficulté à évoquer la question. Cela donne aussi l'occasion de réfléchir sur la manière dont la littérature traite ce fait depuis un siècle.

\section{« Le journal d'un fou » et le cannibalisme}

Malgré des variations d'expressions, le fait est que cette nouvelle de Lu Xun est saluée comme une œuvre majeure, qui marque le début de la littérature chinoise moderne. À titre d'exemple, la nouvelle est « consacré(e) comme le premier récit de la littérature chinoise moderne ${ }^{1}$ ", " celebrated as the turning-point for modern literature ${ }^{2}$ », ou " the very first story composed in the Western manner ${ }^{3}$ ", ou encore le commencement du roman moderne chinois ${ }^{4} »$ / 中国现代小说的伟大开端. Cependant, dans l'œuvre de Lu Xun, s'il semble évident que l'adoption du point de vue d'un fou permet un point de vue décalé sur les faits relatés - on comprend qu'un paranoïaque rapporte les choses à travers un prisme particulier -, on ne saurait se défendre de s'interroger sur la lecture la plus pertinente de cette nouvelle et de se demander si la lecture symbolique du thème central du récit, 吃人 (littéralement manger-homme), est la seule valable ou, à tout le moins, si elle est la seule possible. Pour poser la question autrement: quelle importance accorder aux longues élucubrations du personnage sur le fait que tout son entourage cherche à le manger ? Les minutieuses descriptions psychologiques offrentelles un intérêt au premier degré, ou ne doivent-elles se lire que comme une mise en scène en vue de mener le lecteur au point culminant - la découverte de la nature cruelle et hypocrite du système féodal tel qu'il se matérialise dans les histoires officielles dynastiques? 
3 À cet égard, l'option la plus répandue des histoires littéraires est celle du symbolisme. Pour S. McDougall, le fou comprend, en lisant les livres d'histoire, que toute la culture chinoise est fondée sur le cannibalisme ${ }^{5}$. Tout en considérant la faiblesse de construction de l'œuvre, qui tient à ce que Lu Xun, faute de produire une trame réaliste appropriée aux fantasmes du fou, se contente de garnir son récit d'items variés reliés à la pratique du cannibalisme en Chine ${ }^{6}$, C. Hsia semble favoriser aussi une lecture symbolique de l'histoire quand il évoque le jeu ancien du cannibalisme ${ }^{7}$. Zhang Yinde, lui, évacue d'emblée le sens littéral du terme en parlant d'un "système politique barbare où les hommes se voient contraints à s'entretuer" ", ainsi que d'« anthropophagie généralisée », expression qu'il emprunte à François Jullien', avec un qualificatif qui évacue la possibilité de lire le terme au sens propre (quelle anthropophagie autre que symbolique pourrait-elle être généralisée ?). Quant aux histoires littéraires publiées en Chine, elles saluent unanimement le héros comme un adversaire du système féodal.

4 Cependant, il est permis de penser qu'au niveau du sens littéral de cannibalisme, une lecture est possible. S'il est clair que Lu Xun nourrit une haine viscérale contre la tradition féodale, ce qu'il ne cesse de montrer dans toutes ses œuvres, l'acte de manger de la chair humaine ou toute partie du corps humain, considéré bien entendu comme comble de la barbarie, est également récurrent dans ses écrits, notamment dans « Le remède » / 药. Autrement dit, tout en admettant la valeur symbolique de cet acte, $\mathrm{Lu}$ Xun le dénonce en tant que tel comme le paroxysme du vice de la civilisation chinoise.

Dans «Le journal d'un fou ", les recherches entreprises par le fou sont tellement méthodiques et fouillées et ses réflexions tellement poussées, qu'il est difficile de les tenir pour secondaires. A contrario, les critiques du système et des rites, aussi puissantes soient-elles, occupent si peu de place proportionnellement, qu'elles ne devraient pas oblitérer la présence des faits pour ce qu'ils sont - de réels faits de cannibalisme.

6 Il importe de voir, c'est qu'admettre la validité de cette lecture au premier degré n'enlève rien à la force de dénonciation de l'œuvre, bien au contraire. À l'inverse, la seule lecture symbolique de l'expression, comme c'est le cas dans les récits orthodoxes et normalisés de l'histoire littéraire chinoise, conduit en fin de compte à sa banalisation et en affaiblit la portée. En d'autres mots, en dehors de la nouvelle de Lu Xun, quand on emploie l'expression "manger-homme» / 吃人 / chiren dans la formule " ancienne société cannibale 》/吃人的旧社会, elle implique rarement le cannibalisme réel mais le plus souvent signifie que la société est « cruelle au plus haut point ». Or, comme il s'en prend à la civilisation chinoise traditionnelle, il est impossible que Lu Xun ne soit pas sensible aux phénomènes de cannibalisme au sens propre et à ce qu'ils représentent par rapport à la nature humaine.

7 Ensuite, l'histoire littéraire semble aussi militer pour le rétablissement de la possibilité d'une telle lecture. En effet, il semble bien que Lu Xun ait eu vraiment à cœur de traiter le sujet du cannibalisme dans l'histoire chinoise. Ainsi, dans une lettre adressée à un ami Xu Shoushang ${ }^{10}$ environ trois mois après la publication de la nouvelle, il explique les raisons qui l'ont amené à l'écrire :

Par la suite, le hasard de mes lectures m'a conduit à une découverte dans Zizhi

Tongjian $^{11}$. J'ai réalisé que les Chinois sont encore une nation cannibale, et c'est ce qui m'a décidé à composer ce texte. C'est une découverte à conséquences, mais elle est encore peu connue. 
后以偶阅《通鉴》，乃悟中国人尚是食人民族，因成此篇。此种发现，关系甚

大, 而知者甚少。12

Comme certains critiques l'ont fait remarquer ${ }^{13}$, c'est à la suite de critiques mais surtout pour répondre à l'appel de l'époque que Lu Xun en est venu à reconsidérer son écrit et insister sur sa dimension de critique du système féodal. Bien entendu, il faut avouer que la nouvelle comporte en elle-même la jonction des deux plans, par le M. Gu Jiu 古久(Antique), ainsi que les mots comme «rites» et «vertu» qui parsèment les livres d'histoire chinoise. Mais dans la logique d'une lecture au premier niveau, il est tout aussi logique d'interpréter ces faits comme des hypocrisies pour cacher les faits de cannibalisme.

9 En fait, qu'a pu découvrir Lu Xun dans Zizhi Tongjian pour se convaincre que la Chine était une nation de cannibales?

10 Selon le décompte de Huang Wenxiong dans son livre sur le cannibalisme dans l'histoire chinoise ${ }^{14}$, les histoires dynastiques ne contiennent pas moins de quatre cents cas de cannibalisme, disséminés dans quasiment toutes les dynasties. Zizhi Tongjian, qui s'arrête aux Song, est naturellement incomplet, mais présente toutefois des échantillons de toute nature, dont le «fou» de Lu Xun remarque les plus aberrants, absurdes et gratuits (des actes de cannibalisme sont commis pour des motifs aussi variés que la piété filiale, la finalité médicinale, la loyauté ou la haine). Sans doute pour mettre en avant la dimension irrationnelle de ces actes, le même "fou" semble s'intéresser moins aux cas pourtant de loin les plus nombreux et impliquant le plus de victimes, des cas que rapporte le plus souvent l'expression « les gens se mangent entre eux » / 人民相食 / renmin xiangshi, ce cannibalisme de masse qui survient durant les périodes de pénurie extrême et répond au critère de ce que l'on appelle communément le cannibalisme "par nécessité $~^{15}$. Le héros de Lu Xun s'intéresse principalement aux cas qu'on classe dans la catégorie de "cannibalisme social ${ }^{16}$ ou «learned cannibalism ", terme utilisé entre autres par Key Ray Chong ${ }^{17}$. Il y a bien sûr aussi des cas plus difficiles à départager, comme ceux certes provoqués par la pénurie et par conséquent peuvent être considérés comme des cas par nécessité, mais d'une autre nature que les catastrophes naturelles qui sont les cas les plus fréquents. Ceux-ci, au contraire, procèdent de choix humains par lesquels les puissants font valoir leur pouvoir sur les faibles. Par exemple, plus d'une fois, en raison de difficultés de ravitaillement, l'armée se sert sur l'ennemi ou sur la population. Dans les villes assiégées, manger la population est fréquent. À plusieurs reprises - comme Zang Hong 藏洪 en 195, ou Zhang Xun 张巡 en 755 -, les généraux font preuve de " générosité » en offrant une concubine bien aimée ou un serviteur en signe de remerciement et d'encouragement adressé aux soldats. Preuve que l'acte est conforme à une certaine conception de la morale. En effet, dans le cas de Zhang Xun, non seulement l'homme figure dans l'histoire officielle des Tang parmi les loyaux / 忠义 ${ }^{18}$, mais ses actes sont aussi loués par Wen Tianxiang 文天祥, poète célèbre des Song, qui est particulièrement touché par ses "dents ", symbole de sa haine féroce contre les envahisseurs barbares ${ }^{19}$. C'est d'ailleurs de la même époque que nous parvient une œuvre autrement plus célèbre, un $c i$ (genre de paroles de chant ayant connu son apogée à la dynastie des Song) sur la mélodie Manjianghong 满江红 ${ }^{20}$, de la main de Yue Fei 岳飞 des Song. Dans cette œuvre, l'auteur, général d'une dynastie en déroute, jure de dévorer la chair et de boire le sang des barbares le jour de la reconquête du territoire perdu. 
11 Une précision est importante ici, sur la question des relations entre l'histoire et la littérature. Il faut savoir que dans la chine ancienne, les frontières entre ces deux disciplines sont loin d'être étanches. Bien au contraire. Un bon lettré est nécessairement un humaniste généraliste. Aujourd'hui, une célèbre université édite encore une revue de recherches dénommée Lettres-Histoire-Philosophie / 文史哲. Ainsi les interpénétrations sont très fortes entre l'histoire et la littérature. Or, comme nous l'avons vu, les cas de cannibalisme sont fréquents dans les écrits historiques. Alors, qu'en est-il de la littérature classique?

12 Force est de constater que dans des œuvres de fiction comme Les Trois Royaumes, les Pérégrinations vers l'ouest ou Au Bord de l'eau, manger ou faire manger de la chair humaine n'est nullement conçu comme une tragédie, mais comme un acte valeureux ou bien un sujet comique. Ainsi, au chapitre 19 des Trois royaumes ${ }^{21}$, quand le chasseur Liu An massacre allègrement sa femme pour nourrir Liu Bei, il le fait par dévouement car le brave homme n'a pas eu le temps d'aller chasser d'autres proies dignes de son maître! Pour les deux autres œuvres, alors que les Pérégrinations ont à voir avec des croyances confusément taoïstes - que ce soient les monstres qui veulent manger du moine, ou les héros, le singe et le cochon, qui volent des fruits ginseng multimillénaires en forme de bébé -, le récit d'Au bord de l'eau traite bien de cannibalisme au sens propre au chapitre 27 dans lequel les époux Zhang Qing et Sun la-Cadette, tenanciers d'une auberge, font commerce de chair humaine et, selon les propres aveux du mari, guettent le passage de marchands et de voyageurs qu'ils «accommodent" à leur façon, c'est-à-dire en découpant leur viande en belles tranches qu'ils vendent comme du buffle et en réduisant les restes et bas morceaux en farce à pâté - et tout cela par nécessité, bien

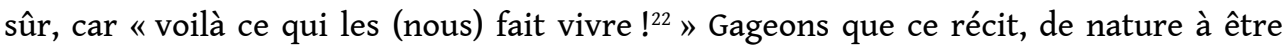
conté, est fait pour distraire le public, mais aucunement pour provoquer ou inciter à réfléchir. Le public et les lecteurs sont censés, dans leur immense majorité, le recevoir comme une histoire fantastique, merveilleuse ou épique, dans une normalité qui ne présente rien de choquant, en ce qu'ils n'éprouvent aucun sentiment qui puisse s'apparenter à de la compassion. D'ailleurs, Jin Shengtan, critique célèbre et extrêmement perspicace, s'exclame trois fois Extra! en lisant les phrases qui décrivent l'abattoir humain des époux, quand le jardinier Zhang emmène Wu Song dans la salle de dépeçage où il peut voir, "pendues au mur, bon nombre de peaux humaines et, accrochées aux poutres, » - Extra!, commente Jin Shengtan - « cinq ou sept jambes dépareillées » - Extra! s'exclame à nouveau le critique -, avec les deux exempts, « inconscients et rigides, (...) renversés l'un sur l'autre sur l'étal à dépecer - et Jin Shengtan de s'écrier encore : Extra !23.

13 Par conséquent, rien ne semble justifier qu'on prenne au second degré le cannibalisme de ces œuvres, comme s'il ne s'agissait que d'un symbole. Bien au contraire, c'est plutôt la preuve tangible de la complaisance amusée envers cette pratique et, dans la mesure où le cannibalisme a traversé les siècles, la manifestation d'une incroyable insensibilité ou faculté d'oubli. Or, c'est justement cette insensibilité ou cet « engourdissement des sens » / 麻木 que Lu Xun n'a de cesse de dénoncer dans ses œuvres afin de changer en profondeur la « nature de la nation chinoise » / 国民性.

14 Aussi convient-il de garder à l'esprit tout ce contexte historique et culturel en abordant la nouvelle de Lu Xun. Le cannibalisme que découvre le «fou » n'est pas purement symbolique, mais bien un acte barbare, contraire à la nature humaine, pour le héros qui a saisi les lumières porteuses de l'humanisme. Première œuvre littéraire à dénoncer de 
manière frontale le cannibalisme dans la culture chinoise, la nouvelle de Lu Xun est peut-être aussi la dernière du genre et par conséquent unique. Autrement dit, durant les décennies, voire le siècle, qui se sont écoulées depuis, ces faits patents de cannibalisme ont quasiment disparu de la littérature. Or, la chose n'a pas forcément disparu de l'écrit parce que la réalité a elle-même disparu, mais sans doute en raison de la prise de conscience de l'incompatibilité de cette réalité avec la nouvelle vision de la civilisation. À cet égard, il n'est sûrement pas innocent que la locution 吃人 (mangerhomme) soit réduite à sa seule valeur symbolique et ne serve plus désormais qu'à dénoncer le système féodal révolu, système d'exploitation dans lequel les puissants étaient en position d'anéantir les faibles. Il en va d'ailleurs de même d'un mot comme yeman / 野蛮, compris volontiers comme sauvage ou féroce, plutôt que barbare, dans ses acceptions historiques.

Pour s'en convaincre, il suffit de considérer que dans la littérature de gauche de cette période, qui se donne pour mission de dénoncer l'ancien régime afin d'exhorter le public à œuvrer pour un avenir radieux, certains drames humains désastreux comme ceux consécutifs à la rupture des digues du Fleuve jaune en 1938, provoquée par les nationalistes pour ralentir la marche de l'envahisseur japonais, ou la famine de 1942 qui a suivi et sévi dans la province du Henan, n'ont longtemps pas donné lieu à des récits sur des faits de cannibalisme, voire pas du tout, alors que ceux-ci sont avérés si l'on se fie aux documents historiques ou journalistiques d'époque. À l'exception d'un premier roman sur les inondations de 1938, [Les Eaux jaunes] / 黄水传, de Feng Jintang, qui sort en $1961^{24}$ et obéit pleinement à l'esprit du réalisme socialiste en mettant l'accent sur les combats de la population sinistrée contre les ennemis, japonais ou nationalistes, il faut attendre 1979 pour qu'un second roman sur ce thème voie le jour, dans un esprit plus réaliste et moins idéologique, [Le Fleuve jaune s'en va à l'Est] ${ }^{25}$. Mais l'auteur fait preuve d'une discrétion extrême à cet égard, avec une seule occurrence de l'expression «manger-homme» / 吃人 / chiren, en incipit du chapitre 9, sous la forme d'un dicton, issu d'une pièce d'opéra locale si l'on en croit Yang Jisheng, qui semblait bien répandu dans le pays, en tout cas au moins jusqu'à la grande famine de la fin des années cinquante et au début des années soixante :

Les gens mangent les gens,

Les chiens dévorent les chiens,

Les rats croquent dans les briques,

Ils n'en peuvent d'avoir faim !

人吃人, 狗吃狗, 老鼠饿的啃砖头

16 Force est de constater que l'incipit qui donne le ton de la partie n'est suivi, de façon surprenante, d'aucune mention à ces faits qui auraient mérité de figurer dans une œuvre destinée à faire revivre une catastrophe sans pareil.

Quant à la famine de 1942 en Henan, le roman de Liu Zhenyun, Se souvenir de 1942 / Wengu $1942^{26}$, écrit en 1993, est une œuvre d'un genre ambigu, entre roman, avec narrateur et personnages de fiction, et grand reportage, puisque composée surtout de documents historiques, alors que les personnages semblent assurer les transitions entre les documents et quelques réflexions de l'auteur/narrateur. Quant au cannibalisme, alors que les faits historiques sont solidement attestés par un nombre incalculable de reportages et par les souvenirs des sinistrés ${ }^{27}$, le narrateur appuie l'essentiel de son discours sur le témoignage indirect du journaliste américain Theodore White (1915-86), dont les relations se trouvent principalement dans deux livres: Thunder Out of China ${ }^{28}$ et In Search of History ${ }^{29}$. Les faits repris par le narrateur 
chinois, quoique d'une grande sobriété, sont d'une force terrible et relatés sans subterfuge ni glose inutile. Il semble même qu'il en rajoute parfois sur l'écrit du journaliste américain. Par exemple, là où $\mathrm{T}$. White écrit :

So I saw these things, but the worst was what I heard, which was about cannibalism. I never saw any man kill another person for meat and never tasted human flesh. But it seemed irrefutable true that people were eating people meat ${ }^{30}$.

Le narrateur écrit, lui, que le journaliste américain prétend avoir vécu une expérience unique :

White clamait qu'il n'avait jamais vu auparavant une personne en tuer une autre pour en manger la chair, le voyage au Henan lui a ouvert les yeux et l'a convaincu que le cannibalisme existait bel et bien dans notre monde.

白说, 在此之前, 他从未看到过任何人为了吃肉而杀死另一个人, 这次河南之

行, 使他大开眼界, 从此相信人吃人在世界上确有其事 ${ }^{31}$.

Pire, il fait dire au journaliste qu'il a vu de ses propres yeux des parents tuer leurs enfants afin de manger leur chair, là où l'américain souligne qu'il a juste appris par ouïdire :

White vit une mère cuisiner et manger son enfant de deux ans et un père étrangler ses deux enfants et en faire cuire la chair pour sa propre survie.

白见到, 一个母亲把她两岁的孩子煮吃了; 一个父亲为了自己活命, 把他两个孩

子勒死, 然后将肉煮吃了 32 .

Malgré ce paradoxe, il serait hasardeux d'affirmer que le romancier fait du sensationnalisme, car les reportages des journalistes chinois présents dans les zones sinistrées étaient bien plus alarmants que le discours somme toute très discret de White. On peut se demander pourquoi l'auteur de 1942 a choisi cette forme de prose. Sans doute juge-t-il que les matériaux historiques sont plus parlants qu'une fiction complète. En tout cas, de tous les romans chinois que nous ayons pu consulter, son récit offre parmi les représentations les plus directes et les plus crues du cannibalisme. A contrario, on perçoit le paradoxe : la fiction semble avoir bien plus de mal à faire face à cette réalité que les documents historiques. Cette constatation semble se vérifier dans la littérature contemporaine où, malgré les faits avérés et nombreux, les romanciers manifestent une grande discrétion et évoquent les faits de manière allusive.

Soit un autre exemple, comparable à la famine de 1942, somme toute assez "banale » en cette région durant cette période de l'histoire chinoise. Au pays du cerf blanc / 白鹿原 $(1993)^{33}$, de Chen Zhongshi, présente la particularité de ne comporter aucune indication de date. Néanmoins, en se repérant à l'aide des différents événements politiques et sociaux, il est possible de situer les périodes de famine décrites dans les années quarante.

Aux chapitres 18-22, l'auteur nous dépeint une situation effroyable: la bru du protagoniste Bai Jiaxuan, notable respecté du pays, meurt de faim. Des chiens errants s'attaquent aux gens et à des cadavres. On ne s'émeut plus de voir les gens mourir, jeunes ou vieux. "Mourir de faim n'effrayait plus, ne surprenait plus. (...) Seuls les ragots pouvaient encore éveiller quelque intérêt chez les gens ${ }^{34}$. Ainsi, on raconte qu'une jeune femme, mariée depuis un an, réveillée au milieu de la nuit par la faim, surprend son mari en pleine discussion avec ses parents, qui lui proposent de manger sa femme au lieu de laisser s'éteindre la lignée, car si la famille survit, il sera toujours temps de chercher une nouvelle épouse. Terrifiée, la jeune femme rentre chez ses parents. Mais, se réveillant la nuit de nouveau, elle entend son père tenter de convaincre sa mère en lui disant : plutôt que de la laisser manger par les autres, mieux 
vaut que la famille en profite. Et le narrateur de conclure : « Les rumeurs de ce genre, à l'instar des croassements de corbeaux, faisaient froid dans le dos $»^{35}$. Dans ce passage, avec une histoire qui tourne quasiment à l'absurde, le choix de termes comme « ragots » et « rumeurs » est justifié. Cependant, malgré leur connotation fallacieuse, ces mots n'excluent pas en soi la nature réelle des faits rapportés. En réalité, cette présentation des atrocités présente l'«avantage" de rendre possible une double lecture: les âmes sensibles peuvent prendre les mots au pied de la lettre et croire effectivement qu'il ne s'agit que de ragots, tandis que les réalistes peuvent y voir une façon détournée d'évoquer une réalité dérangeante.

Sur cette question, la grande famine survenue après le Grand bond en avant, telle que traitée dans le roman, fournit une matière très riche d'enseignement. Sauf erreur, dans les romans des années d'avant et pendant une grande partie des années quatre-vingt, on ne trouve guère, sous quelque forme que ce soit, mention du cannibalisme. Le sujet semble très tabou. Mais comme l'évocation de la Grande famine est elle-même rare, et de surcroît enveloppée sous le pudique voile de la « catastrophe naturelle ", il était bien entendu inconcevable, dans ce contexte, d'évoquer les possibles phénomènes de cannibalisme. Dans le langage commun, normalisé, le terme cannibale lui-même, on l'a vu plus haut chez Lu Xun, prend la forme de 吃-人 (manger-homme), expression entendue comme métaphore pour la cruauté des anciennes sociétés, et dénuée de connotation cannibalesque.

Aussi, rien d'étonnant s'il n'est pas question de cannibalisme lorsque la création littéraire se voit confier la mission de susciter de grandes répercussions dans la société, les fameux «effets retentissants 轰动效应》 de Wang Meng ${ }^{36}$. Rien d'étonnant si le pouvoir, tout en lui confiant tour à tour un rôle de ballon d'essai, de caisses de résonance ou de porte-voix, la place nécessairement sous surveillance. Cependant, dans les années quatre-vingt-dix, alors que le pouvoir et la société les délaissent dans une certaine mesure, les écrivains gagnent paradoxalement une plus grande liberté de création. Voient ainsi le jour nombre de romans qui, s'attelant à rappeler aux gens ces épisodes douloureux de l'Histoire, vont parfois très loin dans l'évocation des souffrances. Et pourtant, même dans les œuvres qui ne reculent devant aucun tabou descriptif, et ce jusqu'au déballage ou à l'exhibitionnisme à qui mieux mieux, l'évocation du cannibalisme reste malaisée à l'évidence. Serait-ce donc la dernière frontière impossible à dépasser?

L'analyse d'un nombre significatif d'œuvres amène à constater que non seulement les auteurs rechignent à décrire une scène de cannibalisme, mais peinent même à authentifier les faits et laissent quasi systématiquement planer un doute sur leur réalité.

Pour ce, le recours aux rumeurs est très courant, à la manière d'Au pays du cerf blanc évoqué plus haut. Cette pratique questionne d'autant plus que le doute n'est guère possible, tant nombreuses sont les preuves, appuyées par des quantités d'écrits nonfictionnels, comme les Récits de Jiabiangou ${ }^{37}$ de Yang Xianhui ou Stèle / 墓碑 de Yang Jisheng $^{38}$ enrichis d'un nombre important de témoignages de victimes directes de ces fléaux.

Le cas de Mo Yan est intéressant. Dans un roman comme Beaux seins, belles fesses / 丰乳 肥眤 $(1996)^{39}$ où, fidèle à son style, l'auteur ne semble reculer devant aucune cruauté, il présente par exemple un possible cas de cannibalisme. Le chapitre 43 dépeint la vie dans une ferme d'État en 1960, où un dénommé Zhou Tianbao, gardien de son état, est 
surpris en train de préparer de la viande et, questionné sur la nature de cet aliment, affirme avec irritation qu'il s'agit de chair humaine. Alors que la nouvelle se répand, le directeur de la ferme l'innocente en affirmant qu'une enquête avait prouvé qu'il s'agit en réalité de rats, et d'encourager les droitistes à élargir les sources d'approvisionnement. Une affirmation étrange quoique pas totalement impossible. Puis, comme nulle disparition éventuelle n'est évoquée, l'information manque pour se livrer à des supputations plus étayées. En fin de compte, le lecteur ne saura jamais le fin mot de l'histoire et doit se contenter de conjectures.

Dans son roman à caractère autobiographique : Une fille de la faim ${ }^{40}$ / 饥饿的女儿, publié en 1997 à Taïwan, puis en 2000 en Chine populaire, l'écrivaine sino-britannique Hong Ying, née en 1962, décrit avec une force rare l'obsession de la nourriture qui était le quotidien de la jeune fille qu'elle a été. Née après la Grande famine, celle-ci est cependant convaincue de l'avoir vécue par procuration, à travers le corps de sa mère enceinte. Cette faim au sens propre l'amène donc naturellement aux années noires. Ainsi, pour fêter ses dix-huit ans, elle est au comble de sa joie d'avoir pu acheter deux brioches fourrées à la viande qu'elle décide finalement d'offrir à ses parents et qui seraient comme le résumé de toutes les misères passées et présentes. Et comble d'ironie, la boutique où elle a acheté les brioches est celle où, selon sa mère, durant la Grande famine, on incorporait de la chair d'enfants à la farce ${ }^{41}$. Selon la mère, toujours, un faisceau de présomptions accuse les propriétaires. Comme la viande était à peu près introuvable à l'époque, il était suspect que les patrons parviennent à s'en procurer. De plus, des enfants disparaissaient dans les environs. Pour finir, leurs brioches étaient anormalement savoureuses. D'ailleurs, les patrons avaient fini par se faire arrêter. Avec tous ces arguments, le crime est presque établi, sauf que le père de la narratrice ne tarde pas à venir apporter son démenti : «Cette voisine passait son temps à colporter des ragots ${ }^{42}$ ». La mère a beau vouloir fournir d'autres raisons, le père coupe court à « toutes ces rumeurs ». Il met ainsi fin à la discussion et laisse l'affaire en suspens. Il en résulte une accusation invérifiable sur laquelle la narratrice ne s'attarde d'ailleurs pas.

Deux ans avant le livre de Hong Ying, Wang Zhiliang, ancien professeur d'université de Shanghai ayant été envoyé dans les montagnes Taihang pour la rééducation par le travail en 1960, publie un livre intitulé Village de montagne affamé/ 饥饿的山村 ${ }^{43}$. Comme son titre le laisse présager, la faim est au cœur du livre. Et contrairement à Hong Ying qui confère au mot «faim » du titre de son livre une signification bien plus vaste que la seule faim physiologique, la faim chez Wang Zhiliang n'est rien d'autre que le cri du corps soumis à la dure épreuve de la pénurie alimentaire. Ainsi, l'ensemble des intrigues tourne autour de cet objet, alors qu'une histoire d'amour maladroitement menée paraît bien dérisoire.

Bien qu'il présente son livre comme une fiction, beaucoup de lecteurs le prennent visiblement plus volontiers pour une autofiction au regard des faits historiques qui y sont relatés. Tout un jeu littéraire contribue à créer cette illusion historique, qui se révèle de pure façade. Ainsi le narrateur prétend-il ne faire rien d'autre que de publier un manuscrit pour le compte d'un homme mort durant la Révolution culturelle, lequel homme affirme avoir écrit le roman avec sa propre histoire et son véritable nom. Et le hasard (?) veut que ce personnage s'appelle Wang Liang (Wang Liang 王良), dont la proximité avec le nom de l'auteur (Wang Zhiliang 王智量) est manifeste. Ces ficelles sont si grosses - si telle est bien la volonté de l'auteur d'être lu au second degré - qu'on ne peut s'empêcher de penser à un appareil destiné à déjouer d'avance les pièges 
d'éventuels procès sur la réalité des faits. D'ailleurs, dans le post-scriptum à la réédition de $2010^{44}$, l'auteur explique que Shi Zhecun ${ }^{45}$, après avoir lu le manuscrit, l'a encouragé tout en lui demandant s'il ne craignait pas de se faire encore étiqueter comme droitiste. Une façon comme une autre d'afficher le caractère audacieux, voire subversif d'un livre qui, se voulant fidèle à la réalité, présente un cas de cannibalisme que le héros n'a certes pas constaté de ses propres yeux, mais qui s'est avéré des plus réels à la fin. L'enfant de la famille des Li, mort de faim, a été enterré. Or, peu de temps après, on retrouve son corps déterré et amputé des deux jambes. Comme Wang lui demande pourquoi elle a mangé la chair de l'enfant, Li Qigu, femme que tous accusent, affirme que ce n'est pas elle, mais refuse de dévoiler l'identité du coupable et jure qu'il s'agit d'un secret qu'elle ne doit révéler sous aucun prétexte. Et d'ajouter que, de toute façon, ce n'est pas la première fois que l'on mange de la chair humaine, et qu'il arrive même qu'on mange des gens vifs. Plus tard, à la mère agonisante de l'enfant, Li Qigu finit par avouer qu'elle a coupé les jambes du cadavre, mais continue à taire le nom du bénéficiaire. Cette fois, elle assure que c'était pour la bonne cause, pour assurer une descendance au village en échangeant « une plante morte contre une vive ${ }^{46}$. Plus tard, Wang apprend de la bouche de la seule femme enceinte du village qu'elle a reçu de la viande de la part de Li Qigu. Ainsi, loin de rester simple soupçon ou rumeur, les faits sont clairement établis dans l'histoire. De plus, Li affirme à Wang que le cannibalisme n'est pas une pratique aussi singulière qu'on pourrait croire, et que « les villageois de Zhangjiawa sont même tristement célèbres pour cela / “他们张家洼吃这个在这片地 方是出了名的 ${ }^{47}$. Pour preuve, elle donne le cas d'une femme dont la fille aurait été victime. Mais comme celle-ci est devenue folle par la suite, il ne reste aucun moyen de vérifier.

31 Dans ce roman, l'un des rares à présenter le cannibalisme de façon certaine au lieu d'une pure présomption, la préparation psychologique est longue, ce qui a pour inévitable effet d'atténuer le choc émotionnel chez le lecteur. Le but ultime : mener à terme une grossesse et assurer une descendance au village, car la vie doit continuer, et ce même au prix d'un acte contraire à la valeur humaine d'un point de vue coutumier. De ce fait, la barbarie est largement atténuée, et le cannibalisme, un peu plus «acceptable » en quelque sorte. Pour autant, l'attitude de Li vis-à-vis du cannibalisme est intéressante car complexe, entre répugnance à consommer: "je n'y arriverai pas » / 我可吃不下嘴去 ${ }^{48}$, compréhension quand il s'agit d'autrui, et passage à l'acte s'il s'agit de sauver une vie - contraste qui indique chez cette paysanne, haute en couleur et pleine d'humanité, un pragmatisme différent de Wang. Par ailleurs, elle est également mue d'une conviction d'obédience taoïste, qui lui fait proposer à Wang de lui obtenir de la chair humaine, car il n'y aurait rien de mieux pour fortifier un humain.

[La Liste de Chen Wanshui] ${ }^{49}$ de Chen Jiming va plus loin dans la révélation, qui confirme un cas de cannibalisme en fin de récit tout en confrontant le lecteur à une personne prise « sur le fait ». Là, on n'est plus dans la rumeur plus ou moins vérifiable, ni même dans l'aveu de gens plus ou moins directement impliqués. Cette fois, les récits et descriptions du narrateur révèlent la réalité des faits et les authentifient. L'histoire se déroule dans un village du Gansu où la totalité des villageois, que la faim a privés de la faculté de marcher, se déplacent désormais en rampant sur le ventre. Parmi les cadavres gisant dans les rues, celui d'un enfant apparaît devant la porte d'entrée des deux personnages principaux, une mère et sa fille. Au moment où cette dernière se sent défaillir, elle demande à sa mère s'il est permis de manger des rats, qui lui répond qu'on ne doit pas manger des choses « sales ». En effet, dans ces contrées, la consommation de 
choses «sales» est tenue pour un crime impardonnable. Qui transgresse l'interdit est non seulement stigmatisé, mais peut être sévèrement puni, comme se voir privé du droit de nourriture dans la cantine collective. Or, contrairement aux écorces d'arbre, voire des semelles de chaussures, les rats font partie des choses sales, car, explique la mère, il y a sale et sale. Aussi, la jeune fille se jure de mourir de faim plutôt que de manger des rats. Mais il suffit de quelques heures pour que la faim reprenne le dessus. La fille implore alors sa mère d'aller capturer d'urgence les rats qui se sont installés dans leur maison. La mère revient cependant avec une assiette de viande fumée qu'elle donne à manger à sa fille qui, folle de joie, l'invite à partager ce mets. La mère refuse d'abord, puis, devant l'insistance de sa fille, se résigne à prendre un morceau. Ici, la description de l'expression douloureuse de la mère, que la fille ne remarque pas, est sans équivoque. La figure déformée de la mère nous renseigne sur l'infinie douleur morale que cette mère éprouve en avalant de la chair humaine, même si sa motivation initiale est de sauver la vie de sa fille. Car c'est bien de la chair du cadavre d'enfant qu'il s'agit, car à peine la mère et la fille ont-elles fini de manger qu'on vient les accuser d'avoir volé le cadavre qui a disparu de sa place.

Plus loin, la jeune fille fait une découverte : si les cadavres sont laissés sur place, là où ils périssent, c'est que les familles n'ont ni la force de les enterrer ni la volonté de les ramener. Elles évitent ainsi de se faire accuser de tentative de cannibalisme ${ }^{50}$. Signe manifeste que le cas relaté n'est pas un cas isolé et que le spectre plane sur le village de manière persistante.

Après avoir examiné tous ces exemples, le doute n'est plus guère permis. La convergence du traitement du cannibalisme interdit d'y voir une coöncidence. En effet, sur ce phénomène, tout porte à croire à l'existence d'un tabou profond dont les auteurs ne peuvent ignorer l'existence. Un tabou si bien intégré qu'on finit par ne plus le ressentir comme tel.

Mais il est sans doute aussi possible de réfuter cette analyse en arguant que, après tout, un tel tabou est tout à fait normal, comme c'est aussi le cas en Occident où ces faits sont rarement représentés en littérature et, quand ils le sont, non sans un fréquent effort de suspense pour distiller le doute. Soit deux exemples. Dans la Divine comédie de Dante, au chant XXXIII de L'Enfer, un père raconte que, privés de nourriture, ses fils sont morts un à un. Puis, viennent ces vers :

Et je me mis alors déjà aveugle à me traîner sur chacun d'eux,

Les appelant pendant deux jours après leur mort.

Et puis ce que la douleur ne put, la faim le put ${ }^{51}$.

Quelle que soit la version traduite examinée, ce dernier vers reste ambigu : la faim tue le père, ou, selon une tradition tardive, l'amène à manger ses enfants. L'ambiguïté est entretenue dans le chant. Malgré les divergences d'interprétation, il est fort à parier qu'elles ne sont pas près de cesser, leur persistance est significative en soi, en ce qu'elle révèle la difficulté de dire la chose. Et quand bien même la première interprétation serait la plus juste, le fait qu'une large part de lecteurs y décèle l'ambiguïté est une preuve a contrario.

37 Un peu plus près de nous, Mark Twain raconte, dans sa nouvelle intitulée Cannibalism in the Cars $^{52}$ (1868), qu'un homme et ses compagnons de voyage, bloqués dans un train plus d'une semaine par une tempête de neige, ont survécu en mangeant des passagers choisis par voie électorale. À la fin, on apprend que l'homme, ex-membre du Congrès, a tant souffert du froid, lors d'une tempête de neige, qu'il a gardé une séquelle : raconter 
cette histoire de cannibalisme. Cependant, la tension est aménagée de sorte qu'on ne sait si l'homme a réellement tout inventé ou si l'expérience du cannibalisme l'a rendu fou.

Mais si nous admettons dans le cas chinois le tabou naturel sur le thème, il semble exister au moins deux paradoxes. Le premier oppose la littérature du xxe siècle et la littérature classique, qui n'ont visiblement pas la même approche de la question. La comparaison des deux corpus révèle un changement radical, qui invite à se demander si l'arrivée de la culture occidentale, marqueur du xxe siècle chinois, n'a pas été un facteur décisif en apportant l'humanisme occidental. Le second est le décalage entre représentations littéraires et documents historiques. Ces derniers, au moins sur les famines des années quarante et de l'après Grand bond en avant, sont aussi nombreux qu'accablants. Cette différence ferait presque croire que les représentations littéraires pourraient être plus gênantes, car plus importante que les faits réels.

En guise d'explication, pourrait-on appliquer à la représentation du cannibalisme cette affirmation sur l'inceste :

Si des cas extrêmes peuvent se produire, ils ne peuvent en aucun cas entrer dans la lecture ou l'écriture sous forme de légendes ou littérature. Il s'agit d'une ligne rouge à ne pas franchir, ce serait sinon comme tolérer et céder à ces formes extrêmes de l'inceste. Ce serait la fin de l'éthique et de la morale chinoises.

罕见案例也许会有, 但绝不会以传说和文学的形式进入阅读和写作。这也是底 线, 否则意味着对这种极端乱伦行为的容忍和妥协, 意味着对中国伦理道德的 最后颠覆. .5

Traduction possible : consigner les faits en documents historiques, c'est les reconnaître, mais les représenter en littérature, c'est les accepter. Il n'est pas sûr que cette opinion soit unanimement partagée, ni qu'elle reflète la réalité. Mais il est bien possible qu'elle représente une sorte de sentiment parmi les auteurs. En tout cas, la question mérite d'être posée.

\section{BIBLIOGRAPHIE}

CHEN Jiming 陈继明, [《 La liste de Chen Wanshui »] / 陈万水名单, in [《 Best of » des courts romans chinois de 2013] / 中国中篇小说排行榜, LI Jingze 李敬泽 (éd.), Nanchang, Baihuazhou wenyi chubanshe, 2013.

CHEN Zhongshi, Au pays du cerf blanc, BAOQING Shao et CRUVEILLÉ Solange (trad.), Paris, Seuil, 2012.

CHEN Zhongshi 陈忠实, [Au pays du cerf blanc] / 白鹿原 / Bailuyuan, Renmin wenxue chubanshe, 1993.

CHONG Key Ray, Cannibalism in China, Wakefield, N. H, Longwood Academic, 1990.

DANTE Alighieri, La divine comédie, L'enfer / Inferno, RISSET Jacqueline (trad.), Paris, Garnier

Flammarion, 2004.

FENG JINTANG 冯金堂, [Les Eaux jaunes] / 黄水传, Henan renmin chubanshe, 1961. 
FU Xuancong 傅璇琮 (éd.), [Poésie complète des Song] / 全朱詩, Beijing, Beijing daxue chubanshe, 1998.

GUo Zhantao 郭战涛, [ « Le taoïsme et le Zi Zhi Tong Jian (Miroir général pour aider au gouvernement) réinterpréter l'image du cannibalisme dans “Le journal d'un fou” de Lu Xun »] / 道教与 《资治通 鉴》-《狂人日记》吃人意象再解读, [Mensuel des études sur Lu Xun] / 鲁式研究月刊, 2:2015, p. $43-47$.

HE Xiangyang 何向阳 (éd.), [Anthologie de critiques littéraires de 1949 à 2009] / 60 年文学评论精 选, Wuhan, Changjiang wenyi chubanshe, 2009.

HONG Ying 虹影, Une fille de la faim, LOUISGRAND Nathalie (trad.), Paris, Seuil, 2000.

HONG Ying 虹影, [Une fille de la faim] / 饥饿的女儿, Anhui renmin chubanshe, 2013.

HSIA Chih-tsing, A history of modern Chinese fiction, $3^{\text {rd }}$ ed., Bloomington, Indiana U. P., 1999.

HUANG Wenxiong 黃文雄, [Histoire du cannibalisme en Chine] / 中國食人史, Taipei, Qianwei chubanshe, 2005.

LI Zhun 李准(隼), [Le Fleuve jaune s'en va à l'Est] / 黄河东流去, Beijing chubanshe, 1979.

LIU Zhenyun, Se souvenir de 1942, IMBOT-BICHET Geneviève (trad.), Paris, Gallimard, 2013.

LIU Zhenyun 刘震云, [๕uvres choisies de Liu Zhenyun] / 刘震云精品文集, Yanbian renmin chubanshe, 1997.

LUo Guanzhong 羅貫中, [Les Trois Royaumes] / 三國演義, Hong Kong, Zhonghua shuju, 1987.

MCDOUGALl Bonnie S., LOUIE Kam Hung, The literature of China in the twentieth century, London, Hurst, 1997.

MO Yan, Beaux seins, belles fesses : les enfants de la famille Shangguan, Paris, Seuil, 2012.

OUYANG Xiu 欧阳修, [Le Nouveau livre des Tang] / 新唐书, Beijing, Zhonghua shuju, 1975.

QIAN Liqun 钱理群, WEN Rumin 温儒敏 et WU Fuhui 吴福辉, [Trente années de la littérature chinoise moderne] / 中国现代文学三十年, Xiudingben, Beijing, Beijing daxue chubanshe, 1998.

SHI Nai'an 施耐庵, LUo Guanzhong 羅貫中, Au bord de l'eau, DARS Jacques (éd. et trad.), ÉTIEMBLE (avantpropos), Paris, Gallimard, 2005, t. 1.

SONG Zhixin 宋志新, [1942 : La grande famine du Henan] / 1942 河南大饥荒, 湖北人民出版社, 2005. TANG Guizhang 唐圭璋 (éd.), [Poèmes à chanter complets des Song] / 全朱詞, Beijing, Zhonghua shuju, 1965.

WHITE Theodore H., JACOBY Annalee, Thunder out of China, New York, William Sloane Associates, 1946.

TWAIN Mark, BlounT Roy, Cannibalism in the Cars: The Best of Twain's Humorous Sketches, London, Prion, coll. «Prion humour classics », 2000.

WANG Zhiliang 王智量, [Village de montagne affamé] / 饥饿的山村, Shanghai, Huadong shifan daxue chubanshe, 2010.

WANG Zhiliang 王智量, [Village de montagne affamé] / 饥饿的山村 / Ji'e de shancun, Lijiang chubanshe, 1994.

WHITE Theodore H., In search of history : a personal adventure, New York, Harper \& Row, 1978. 
YANG Jisheng 杨继绳, Stèles : la grande famine en Chine, 1958-1961, VINCENOLLES Louis et alii (trad.), Seuil, 2014.

YANG Jisheng 杨继绳, [Stèle : La vérité de la grande famine des années 60 en Chine] / 墓碑: 中國 六十年代大耭荒紀實, Tiandi tushu, 2008.

YANG Xianhui 杨显惠, [Récit de Jiabiangou] / 夹边沟记事, Huacheng chubanshe, 2008.

ZHANG Yinde, Le roman chinois moderne : 1918-1949, Paris, PUF, 1992.

ZHANG Jiang 张江, [L'auteur peut-il mourir : discussions sur la critique littéraire occidentale moderne] / 作者能不能死 : 当代西方文论考辨 / Zuozhe nengbuneng si : dangdai xifang wenlun kaobian, Zhongguo shehui kexue chubanshe, 2017.

\section{NOTES}

1. ZHANG Yinde, Le roman chinois moderne : 1918-1949, Paris, PUF, 1992, p. 131.

2. MCDOUgall Bonnie S., LOUIE Kam Hung, The literature of China in the twentieth century, London, Hurst, 1997, p. 19.

3. HSIA Chih-tsing, A history of modern Chinese fiction, Bloomington, Indiana University Press, $3^{\text {rd }}$ ed., 1999, p. 33.

4. QIAN Liqun 钱理群, WEN Rumin 温儒敏 et WU Fuhui 吴福辉, [Trente années de la littérature chinoise moderne] / 中国现代文学三十年, Beijing, Beijing daxue chubanshe, 1998, p. 30.

5. MCDOUGALL, The literature of China in the twentieth century, op. cit., p. 95.

6. HSIA, A history of modern Chinese fiction, op. cit.

7. Ibid.

8. ZHANG, Le roman chinois moderne : 1918-1949, op. cit., p. 131.

9. Ibid.

10. XU Shoushang 许寿裳 (1883-1948) : historien, écrivain et éducateur, ami très proche de Lu Xun.

11. Zizhi Tongjian 资治通鉴, littéralement «Miroir général pour aider le gouvernement", ouvrage de référence de Sima Guang, 司马光, historien chinois du XI ${ }^{\mathrm{e}}$ siècle.

12. [« Lettre à Xu Shaotang »], 20/08/1918, [Æuvres complètes de Lu Xun], 2005, vol. 11, p. 365.

13. GUo Zhantao 郭战涛, [« Le taoïsme et le Zi Zhi Tong Jian - réinterpréter l'image du cannibalisme dans “Le journal d'un fou” de Lu Xun »] / 道教与《资治通鉴》-《狂人日记》吃人意象再解读, [Mensuel des études sur Lu Xun] / 鲁迅研究月刊, n 2 :2015, p. 43-47.

14. HUANG Wenxiong 黃文雄, [L'Histoire du cannibalisme en Chine] / 中國食人史, Taipei, Qianwei chubanshe, 2005.

15. Emploi notamment repérable sur ce site de pompes funèbres: funeraire-info.fr/lecannibalisme-a-toutes-les-sauces-5433/, consulté le 13/04/2021.

16. Expression utilisée par exemple par Nicole S INDZINGRE, Bernard THIS, "Cannibalisme", Encyclopcedia Universalis, voir https://www.universalis.fr/encyclopedie/cannibalisme/, consulté le 13/04/2021.

17. CHONG Key Ray, Cannibalism in China, Wakefield, N. H., Longwood Academic, 1990, p. 171.

18. OUYANG Xiu 欧阳修, [Le Nouveau livre des Tang], vol. 192, 1975, p. 5529.

19. «时穷节乃见, 一一垂丹青。…..为张脽阳齿, 为颜常山舌。» (正气歌), in [Poésie complète des Song] / 全朱詩, FU XUANCONG 傅璇琮 (éd.), Beijing, Beijing daxue chubanshe, 1998, p. 43055.

20. 《壮志饥餐胡虏肉, 笑谈渴饮匈奴血。》 in [Poèmes à chanter complets des Song] / 全束詞, TANG Guizhang 唐圭璋 (éd.), Beijing, Zhonghua shuju, 1965, p. 1246.

21. LUo Guanzhong 羅貫中, Trois Royaumes / 三國演義, Hong Kong, Zhonghua shuju, 1987, p. 151. 
22. SHI Nai'an 施耐庵, LUO Guanzhong 羅貫中, DARS Jacques (éd. et trad.) et ÉTIEMBLE (avant-propos), Au bord de l'eau, Paris, Gallimard, coll. «Bibliothèque de la Pléiade », t. 1, 2005, p. 607.

23. Ibid. p. 610, et [Le Cinquième livre de génie, Les Bords de l'eau de Shi Nai'an] 1990, p. 1508.

24. FENG Jintang 冯金堂, [Les Eaux jaunes] / 黄水传, Henan renmin chubanshe, 1961.

25. LI ZHUN 李准(隼), [Le Fleuve jaune s'en va à l'Est], 1979.

26. LIU Zhenyun 刘震云, [ÆEuvres choisies de Liu Zhenyun] / 刘震云精品文集, Yanbian renmin chubanshe, 1997; Se souvenir de 1942, LIU Zhenyun et IMBот-вICHET Geneviève (trad.), Paris, Gallimard, 2013.

27. On trouve un grand nombre de reportages de journaux chinois et des témoignages dans SONG Zhixin 宋志新, [1942 : La grande famine du Henan] / 1942 : 河南大饥荒, 湖北人民出版社, 2005.

28. WHITE Theodore H., JАСовY Annalee, Thunder out of China, New York, William Sloane Associates, 1946.

29. WHITE Theodore H., In search of history: a personal adventure, $1^{\text {st }}$ ed., New York, Harper \& Row, 1978.

30. Ibid., p. 148.

31. LIU Zhenyun, op. cit., p. 104.

32. Ibid.

33. CHEN Zhongshi 陈忠实, Au pays du cerf blanc / 白鹿原, 1993 ; CHEN Zhongshi, Au pays du cerf blanc, 2012.

34. CHEN, 白鹿原, op. cit., p. 378.

35. Ibid.

36. WANG Meng 王蒙, [《Quand la littérature ne crée plus de grandes répercussions 》] / 文学 : 失 却轰动效应以后, in [Anthologie de la critique littéraire de 1949 à 2009] / 年文学评论精选, Wuhan, HE XIANGYANG 何向阳 (éd.), Changjiang wenyi chubanshe, coll. «Xin Zhongguo liushi nian wenxue daxi» 新中国六十年文学大系 [Anthologie de la littérature de 60 ans de la Chine nouvelle], 2009, 205-210. Dans cet article daté de 1988, l'auteur constate qu'à partir du milieu des années quatre-vingt, la littérature ne suscite plus l'attention du public comme pendant les décennies précédentes.

37. YANG Xianhui 杨显惠, [Récit de Jiabiangou] / 夹边沟记事, Huacheng chubanshe, 2008.

38. YANG Jisheng 杨继绳, [Stèle : La vérité de la grande famine des années 60 en Chine] / 墓碑: 中 國六十年代大饑荒紀實, Tiandi tushu, 2008; YANG Jisheng, Stèles: la grande famine en Chine, 1958-1961, VINCENOLLES Louis, GENTIL Sylvie et CHEN-ANDRo Chantal (trad.), Paris, Seuil, 2014.

39. MO Yan, Beaux seins, belles fesses: les enfants de la famille Shangguan, Paris, Seuil, 2012.

40. HONG Ying 虹影, [Une fille de la faim] / 饥饿的女儿, Anhui renmin chubanshe, 2013 ; HONG Ying, Une fille de la faim, LOUISGRAND Nathalie (trad.), Paris, Seuil, 2000.

41. HONG Ying, Une fille de la faim, op. cit., p. 104.

42. Ibid.

43. WANG Zhiliang 王智量, [Village de montagne affamé] / 饥饿的山村, Lijiang chubanshe, 1994.

44. WANG Zhiliang 王智量, [Village de montagne affamé] / 饥饿的山村, Shanghai, Huadong shifan daxue chubanshe, coll. «Zhiliang wenji 智量文集 》, 2010.

45. SHI Zhecun 施蛰存 (1905-2003), écrivain, traducteur et éditeur.

46. WANG Zhiliang, [Village de montagne affamé]/饥饿的山村, op. cit., p. 307.

47. Ibid., p. 314.

48. Ibid.

49. CHEN Jiming 陈继明, [《La liste de Chen Wanshui »] / 陈万水名单, in [《 Best of » des romans chinois de 2013] / 中国中篇小说排行榜, LI Jingze 李敬泽 (éd.), Nanchang, Baihuazhou wenyi chubanshe, 2013, p. 344.

50. Ibid., p. 11. 
51. DANTE Alighieri, La divine comédie, L'enfer/Inferno, RISSET Jacqueline (trad.), Paris, Garnier Flammarion, 2004.

52. TWAin Mark et BLounT Roy, Cannibalism in the Cars: The Best of Twain's Humorous Sketches, Londres, Prion, 2000.

53. zHANG Jiang 张江, [L'Auteur peut-il mourir : discussions sur la critique littéraire occidentale moderne] / 作者能不能死: 当代西方文论考辨, Zhongguo shehui kexue chubanshe, 2017, p. 37.

\section{AUTEUR}

\section{BAOQING SHAO}

Université Bordeaux Montaigne/TELEM (EA 4195). Baoqing Shao travaille sur la littérature chinoise, la traduction et la stylistique. Il a publié : «Vérité et Mensonge dans la poésie lyrique chinoise : quand le poète s'exprime par une voix de femme ", Journal Asiatique, 2014, Tome 302.2, p. 511-529; « Harmonie et disharmonie dans la poésie chinoise : de l'époque classique à la modernité ", Harmonie et disharmonie dans l'esthétique occidentale et dans l'esthétique chinoise à l'époque de la modernité littéraire, Eric Benoît, Presses Universitaires de Bordeaux, coll. Modernités, $\mathrm{n}^{\circ} 40,2016$, p. 215-236. 


\title{
Representations of Bodies in Crisis in Contemporary Japanese Literature: Two Cases of Eating Disorders
}

\author{
Christopher Scholz
}

\section{Introduction}

1 We are all hungry for stories. We stay up late to devour books and gobble up narratives that sate our yearning to understand the human condition; or simply: who we are. We pride ourselves on having sophisticated taste in literature; we spurn junk novels like junk food even though some highbrow literature might be hard to digest. We are hungry for stories because "literature teaches us to notice", ${ }^{1}$ as Wood puts it, literature "makes us better noticers of life". ${ }^{2}$ Or as Wellberry argues:

[Literature] is a unique form of the articulation of our self-understanding. [...] What kind of knowledge is there that we have by virtue of the fact, not that we know that $\mathrm{x}$ or $\mathrm{y}$, but that we know that we have experienced $\mathrm{x}$ or $\mathrm{y}$ ? There is a way in which literary knowledge is the knowledge that we gain for having gone through something in a particular way. ${ }^{3}$

Therefore, we can understand literature as a means to teach us what it feels like to go through a certain experience - it allows us to see the world from another point of view. This doesn't have to be a point of view we necessarily identify or sympathize with - it would be utterly boring to only read books about characters we like - but it might give us a glimpse into what it means to be another person. It helps us to "acquire experience by proxy" ${ }^{4}$ Art and especially literature teach us to put "ourselves in others' shoes". ${ }^{5}$

In terms of illness experiences I want to argue that literature gives us an understanding of what it means to live with an illness. It shows us the inner perspectives of living with illness without providing a universal understanding of it. Instead, it offers a look into a subjective experience. The portrayal of illness changes from an abstract description of 
symptoms in a medical textbook to the feeling of really getting to know someone who suffers from this illness and what it might feel like to be this person. We put ourselves in their shoes - or better: in their bodies.

\section{On writing, reading, and eating}

4 Unfortunately, "writing the body" presents a dilemma, a paradox, because we have to put a physical thing into words. We cannot see, feel, smell or taste bodies in literature. The body can only be represented through language. We won't feel a protagonist's pain by reading about them. We can never know what it really feels like to have, for example, an eating disorder - unless we experience it ourselves. We won't mistake these bodies for our own bodies but stories can bring to mind what a reality might look like in which starvation changes the way we feel, act, and think.

5 The phrase "to devour a book" signifies more than simple consumption, it implies great hunger, voracious lust or passion and the complete absorption of something from the outside into us. Just as Adam and Eve taste from the tree of knowledge and Ezekiel and John "literally eat manuscripts during divine visions", ${ }^{6}$ we often think of knowledge and words in bodily metaphors, as something that can be incorporated into ourselves by eating. Yet we tend to understand food as something that feeds our stomach, the physical body, while knowledge, words or literature feed the mind, following the Cartesian dualism and overlooking how eating is always deeply connected to our identities. Food creates and sustains our body. Without food there is no body, without a body there is no need for food. Thus, everybody must be an eating body and by eating other bodies, you can remain somebody. "Eating is a means of becoming". ${ }^{7}$ It "entails consuming, internalizing, incorporating, [...] processing, building, strengthening, corroding, overcoming, and externalizing (excreting)". ${ }^{8}$ Therefore, eating makes up our identity just as much as it makes up our bodies. In her famous essay A Room of One's Own Virginia Woolf writes:

The human frame being what it is, heart, body and brain all mixed together, and not contained in separate compartments as they will be no doubt in another million years, a good dinner is of great importance to good talk. One cannot think well, love well, sleep well, if one has not dined well. ${ }^{9}$

And $\mathrm{Xu}$ argues in Eating Identites:

Turning inward into our multiple and sometimes competing selves, we understand that we eat (live on) our identities, for they actualize and sustain our selves. There would not be any self-recognition without the multiple identities we assume. Identities are to our social being what food is to our body. Without them, we do not exist. As much as we eat identities, identities also eat us. Constructing and maintaining our identities, be they real or fake, consumes us, cuts us up as teeth cut food to be socially processed and metabolized. [...] Coming into the self through language eats us up, because speaking and writing compete with eating. ${ }^{10}$

She is referring to Deleuze and Guattari, who, in their famous essay on Kafka, argue that language itself already implies a deterritorialization of the mouth, the tongue, and the teeth, since they "find their primitive territoriality in food". ${ }^{11}$ They see "a certain disjunction between eating and speaking, and even more [...] between eating and writing". ${ }^{12}$ They think of writing as an act that can transform words into things capable of competing with food. For them, "to speak, and above all to write, is to fast". ${ }^{13}$ Words take up the place of food, invading our mouth and our whole body, "[1]anguage, 
symbolic of all norms, [also] initiates us into the social, and by doing so, it regulates our eating", by writing, we make up norms, rules, laws and codes that keep us from eating, that control our appetite and consume our identities just as much as we consume the food that makes up our identities. ${ }^{14}$ But what happens when our hunger for stories turns to stories about hunger? When hunger itself and the absence or the refusal of food become the subject of a story? If eating is a means of becoming, is not eating a means of "un-becoming"? I will show how the representation of bodies in crisis in two contemporary Japanese novels also become representations of identities in crisis. Eating disorders cannot only be read as re-negotiations of body boundaries but at the same time as re-negotiations of boundaries of identity, gender, and language. By using aesthetic strategies, they can act as a disturbing impulse, forcing us to experience crises by proxy, and they can help us understand what it means to live with an illness. Literary accounts of eating disorders can also make a social commentary, thus becoming potential political acts, reflecting struggles with discourses and structures of power. In addition to an issue of body image, they become comprehensible as an issue of control, achievement, and self-certitude. It will become clear how one certainly "cannot think well, love well, sleep well if one hast not dined well" 15 and if "to speak, and above all to write, is to fast"16 - at least in these two cases - this is also true vice versa: to fast is to speak, and above all, to write.

8 Although the eating disorders in the two texts I am going to examine could be read as cases of anorexia and bulimia, I do not want to argue that anorexia and bulimia are the same. In terms of causes, symptoms and in the course of disease, they appear to be quite different. But I will show that these two literary depictions of eating disorders are very similar in the ways the protagonists deal with them and how they affect their thinking and language. By understanding eating disorders as political acts, I am not seeking to glamorize them nor enforce them as positive. Eating disorders are nonproductive. They do not offer any solution or gain. Trying to control the body to this extent does not result in mastery, but ultimately in death. It is not empowering but a yearning for power which only leads to self-destruction.

\section{Food and gender in Japan}

9 We live in a time in which we do not see our bodies as naturally-given objects anymore, but instead perceive them as representations of our identity. We endlessly control, optimize, and modify them to present ourselves as ambitious, healthy, and desirable. At the same time, the body seems to be the only thing that is left when identities become uncertain. It is tangible proof of our being in the world. As a warrantor and as an effect of authenticity, the body has become the battleground upon which conflicts are fought and contemporary literature in particular has rediscovered the body as a means to represent negotiations of identity.

10 In the case of eating disorders, "physical appearance becomes the measure of who the narrators [of a story] think they are", they reduce their lives "to attestations of bodily obsessions, cultural scripts that equate women with somatic experience". ${ }^{17}$

11 As a writing on and into the body, the act of not eating can be read as a form of corporal speech. In Judith Butler's sense it means to "speak impossibly [because it] calls the viability of the subject into question", it leads to "a sense that one is falling apart". ${ }^{18}$ In literature, this process of "falling apart" can be symbolized by the falling apart of 
language. When the body in text becomes the text body, its structure, its semantics and coherence are able to reflect the state of crisis the body is in.

Food as a product of culture is always gendered. "Maleness and femaleness in all cultures are associated with specific foods and rules controlling their consumption". ${ }^{19}$ In Japan, sweets and low-calorie foods are usually associated with women, whereas spicy food is considered something that usually only men like. You can see this in ads in which for example spicy snacks are praised as being particularly “manly" / 男らしい / otoko-rashii. Of course, this is not only true for Japan and you can also find international studies on how the "regular consumption of fiery [and] spicy food [allegedly] raises a man's testosterone levels making him more adventurous, aggressive and sexually active" (Malik 2014). Men are expected to eat meat, to be strong and active. In 2006, the term herbivore man / 草食男子 / sōshoku-danshi was created by columnist 深澤 真紀 / Fukasawa Maki (1967-), soon becoming a buzzword to describe men who are not interested in getting married or in finding a girlfriend, weak men, who have lost their appetite for meat and thus, their manliness. "Masculine foods are meaty and high in fat, while the feminine foods are either desserts or low in fat and calories". ${ }^{20}$ Women are presented as food in commercials, as objects that can be consumed, while men are the consumers. Women are expected to control their appetites, whereas men become professional competitive eaters. The only Japanese women who were ever able to become celebrities by competitive eating, were two relatively young girls called "Gyaru Sone" and "Moe Azu" (Azuki Moeno), petite, cute and fashionable idol-like women who baffled the Japanese audiences since the early 2000s by eating copious amounts of food despite their appearances. Only by staying as slender as they were, were they able to become celebrities, because even though they managed to gorge on endless plates of curry and steak, they would in the end remain childlike, adorable, and most importantly: not fat. For them, eating was a performance and they embodied the contradiction which Japanese women in particular struggle with every day: they are expected to enjoy all types of food. They are surrounded by cooking and travel shows on TV in which the hosts try different meals, telling them that basically everything they ever taste is 美味しい / oishii and 美味い / umai (yummy and delicious). Yet, they are expected to stay naturally thin and cute / かわいい / kawaii. Griffin and others understand contemporary femininity as a "profoundly contradictory and dilemmatic space which appears almost impossible for girls or young women to inhabit". ${ }^{21}$

Women were once compared to the Japanese Christmas cakes eaten on December 24 if they weren't married by the age of $25 .{ }^{22}$ With the rising average marrying age in Japan, this kind of metaphor has not disappeared but shifted and the age for a woman is now compared with 年越しそば/ toshikoshi soba / the Japanese New Year's noodles eaten on December 31. If you are not married before you turn 32, nobody wants you anymore. Just as any other part of culture, food reflects gender norms, it is a "symbol of power, an aesthetic display, a community ritual, and an expression of ideology or identity". ${ }^{23}$

\section{Disordered eating and eating disorders}

14 Since their discovery as a disease in the West in the 19th century, eating disorders, especially anorexia, have been labeled as female diseases, from "anorexia nervosa" by Gull in England (1873) to Lasegue's paper De l'anorexie hystérique (1873) in the same year. While the first descriptions of eating disorders in Japan can be found as early as in the 
Edo period and were reported as [Anorexia condition] / 不食之証, ${ }^{24}$ they have been widely on the rise in Japan since the 1980s. With one of the lowest obesity rates in the world, the pressure on women is especially high to stay slim and is even governmentmandated by the 2008 "metabo-law" which allows employers to fine their employees between ages 45 to 70 if they don't stay within certain waist measurements. ${ }^{25}$ Besides public pressure and the omnipresence of unreachable beauty ideals, in an interview on the increase of eating disorders in Japan, Hansen argues that women are "confused about what it means to be female in 21st century Japan. Increasingly, their bodies become the locus where this conflict is enacted" ${ }^{26}$

Pike and Borovoy speak of an "infantilization of girls, coupled with the emphasis on motherhood as the endpoint of female socialization", which "creates a model of development in which there is no authorized intermediate stage between girlhood and motherhood, and few ways of imagining female maturity outside the context of motherhood and family". ${ }^{27}$ Unless you manage to become a mother, you have to remain or at least look like a young girl to be still recognized as a viable member of society. Pike and Borovoy read eating disorders as attempts to "negotiate and communicate interpersonally", as "instrumental actions, where more overt resistance, selfdetermination, or rebellion is unavailable". ${ }^{28}$ Around the globe women in their "adolescent and young adult years [are] most at risk for developing an eating disorder", ${ }^{29}$ but while the concept of "Westernization" can certainly explain the establishment and normalization of Euro-American beauty and body ideals in Japan, it is important to look at the centrality of such cultural factors to understand their increased emergence.

Eating disorders have also found their way into literature and since the late 1980s, novels like 松本侑子 Matsumoto Yūko's [No dawn for anorexics] / 巨食症の明けない夜明け (1988), 小川洋子 Ogawa Yōko's [Sugar Time] / シュガータイム (1991) or 荻野アンナ Ogino Anna's [The eating woman] / 食ベる女 (1994) are about protagonists who suffer from eating disorders. Kobayashi even calls the remarkable number of contemporary female writers dealing with eating disorders in their writing one of the two most notable groups of women's fiction today ${ }^{30}$ - the other being the fiction of resident Korean female writers. According to her, both genres share that they "flourished during the 1990's, having hardly existed in any of the preceding decades", they both "foreground an acute sense of alienation felt by a female protagonist, who invariably is at odds with the dominant society's imperatives on how women should define themselves and regulate their bodies", and they are "concerned with how women can seek a liberatory strategy to resist normalizing imperatives". ${ }^{31}$

\section{Literary accounts of eating disorders}

Two even more contemporary examples are 赤坂真理 Akasaka Mari's (1964-) Vibrator / ヴァイブレータ (1999) and Kanehara Hitomi's (1983-) 金原ひとみ [AMEBIC] / アミー ビック (2005). They both are told by protagonists who suffer from eating disorders and they share striking similarities.

In the Akutagawa Prize nominated Vaiburēta, the narrator Hayakawa Rei, a journalist, originally starts drinking alcohol to silence the different voices she has been hearing inside her head since childhood when she discovers that she can fall asleep easier when she is drunk. Being drunk makes her feel able to function without being constantly abstracted by memories and feelings of inadequacy. She proudly claims being 
inebriated makes her feel like "a girl who can get things done, and I like it". ${ }^{32}$ When she notices that her alcohol consumption makes her body "flabby", ${ }^{33}$ she develops an eating disorder after interviewing a woman who suffers from, what she calls, "bulimarexia":

The second trick I learned was self-induced vomiting. It happened around the time I started getting flabby from the drinking, so I was able to kill two birds with one stone. I went to interview a girl with an eating disorder and I had a revelation. I've got bulimarexia, she said. Bulimarexia? If you refuse food, if you don't eat, you've got anorexia. If you eat way too much, you've got bulimia. And if you go on binges and then throw it all up, if you're doing what people refer to as self-induced vomiting, then you've got bulimarexia. It's a word they made up for people in the middle, see? Except now people just call it bulimia. That's the one I've got-that's me. [...] Her legs were so emaciated you could look between them and see what was on the other side; you could see how distorted her bones were and how her joints hooked up; you could see it all, and yet the girl had on a mini-skirt. I just didn't understand her sense of beauty. [...] And besides, she went on, when you've got bulimarexia - You sleep really well.

I stared at her. ${ }^{34}$

19 By trying to replace one addiction with another, Rei not only fails to find a cure but simply adds an eating disorder to her drinking problem, falling deeper into crisis. She meticulously reports her habits of drinking and throwing up and how her body changes due to the constant vomiting:

My skin started looking really bad. If my hand slipped and I cut myself with a knife or something, it took noticeably longer for the wound to heal. The cut would remain there for ages, the skin hard and purple. It reached the point where my fingernails were nearly always split, peeling apart into two layers, because I was no longer able to absorb as much nutrition as I needed. Nevertheless, I did sleep well. ${ }^{35}$

She "feels isolated from herself and obsesses constantly over what exactly this 'I' is", ${ }^{36}$ remembering not only moments in which she felt judged by her body and outer appearance or obsessing over her looks when she appears on a television show, but also from her childhood when she failed to make her parents happy or when she was hit by a teacher. Her endless, stream-of-consciousness ramblings on her own experiences, on women's magazines, tv programs, her fear of getting older, or comments by colleagues reflect her struggle as a woman in a man's world, where she is constantly judged on her body and appearance. In her state of crisis, she meets a truck driver named Okabe / 岡部, while she is buying alcohol in a convenience store. She impulsively decides to join him on his journey to Northern Japan, beginning an intense affair with him because she feels that he can satisfy her hunger for life. They both are isolated people who are able to connect through their bodies, the constant vibration of the truck and Okabe's CB radio, which connects him with other truck drivers on the road. Just like a random signal from the radio, Rei and Okabe are joined by chance and form an unexpected bond, offering a possible way out of Rei's feeling of disconnectedness and ending in a cathartic outlook out of her crisis and into a brighter future.

\section{The hunger artist}

21 Akutagawa prize winner Kanehara Hitomi's third novel AMEBIC was released in 2005. An English blurb on the cover reads "Acrobatic Me-ism Eats away the Brain, it causes Imagination Catastrophe". ${ }^{37}$ The nameless protagonist, a writer, has been living without any real food for almost a year. She is trying to survive on a diet of alcohol, 
vegetable juices and different "supplements" from the convenience store. Gnawing only on pickled / 漬物 / tsukemono from time to time, she treats her body like an apparatus that only needs to be oiled and fed with just enough fuel to keep it running:

For the past year I have lived by only eating supplements, pickled radish and cucumber, lollies and drinks. I know how to take these items according to the situation, but my initial approach is to try and live only on drinks. When I feel fatigued, I know I need energy and vitamins. So I take supplements of Ukon and multivitamin, and in addition, chewable tablets of a mixture of [carotene] and calcium. If I still feel weak, I take medicine that contains anhydrous caffeine. Occasionally, I take a supplement that contains extract of garlic and sesame, fermented soybean bacteria, and royal jelly. When my stomach feels uncomfortable, it has too much acid in it. So I have a lolly. What a satisfactory eating life I have! --- I would never ever eat things like cakes. ${ }^{38}$

Like Kafka's Hunger Artist, she tries to make a spectacle of her hunger, but there is no audience but herself. This "Acrobatic Me-ism" leads to endless ramblings on food culture, people's eating behavior and a crisis which takes over her mind and her body. During drunk and delusional episodes at night, she writes so-called 錯文 / sakubun , a pun on the word for essay / 作文 / sakubun in which the saku is exchanged with the kanji for 錯 / saku, which means confusion. Her sober self finds these texts on her desktop in the morning and tries to find out more about this "I" which she does not know of but which is also part of herself. The "I" in these sakubun seems to be completely detached from the narrator. It seems to be all body and split into a thousand different parts and pieces, amoeba-like selves who each have their own voices. Over time, the constant feeling of hunger, tension and fragmentation transfers itself from the delusional "I" in the essays to the monologues of the narrator who seems to be slowly losing her mind. She experiences an "Imagination Catastrophe". As a writer, she spends most of her time in her apartment by herself, trying to meet deadlines, almost resembling one of the many ひきこもり/ hikikomori in contemporary Japan, reclusive adolescents who withdraw themselves from social life. She tries to silence the many voices that she creates in her own mind. She plays the video card game Solitaire, feeling trapped in her solitary confinement with her cell phone and her computer as extensions of her body and the only connections to the outside world. At the same time she feels trapped inside her own mind which seems to be falling apart at a cellular level. Her monologues endlessly revolve around her body, around its unclear boundaries, its orifices, her bodily fluids and again and again around how disgusting food and people's eating habits are. In taxis, she pretends to be her absent lover's fiancée, a pastry chef. She buys cookbooks and bakes cakes and sweets she will never eat. Although she states that she wants to stop eating food altogether, she can never stop thinking about one thing: food. In this way, the narrator seems to resemble a classic case of an anorexic, who claims to be not hungry, while constantly obsessing over food.

She also remembers episodes from her childhood which might have caused or at least paved the way for her eating disorder, specifically gendered memories. For example, she recalls a man on the street suddenly adjusting the hem of her skirt or moments when she was not able to communicate feelings of indisposition to her mother:

My tummy feels bad. This was something I used to say often when I was a child and was consequently rebuked for by my mother. This wording: "My tummy feels bad". I didn't know how to put it differently or how I felt exactly. I was so sorry and I really wanted her to understand how I was feeling but while I was thinking about 
how she shouldn't have scolded me without me understanding why, while I was carrying inside myself a kind of sadness I didn't know where to store otherwise, I came to the conclusion that, while I was trying out different words to convey my feeling, this sentence: "My tummy feels bad" was exactly how I was feeling. ${ }^{39}$

\section{she should not sweat:}

I'm choking on the saliva in the back of my throat. [...] I always hated the feeling of saliva in my mouth and I formed that habit of spitting out as much of my saliva as I could. It wasn't much but little by little the water content in my body went down. I never had enough water inside of me, I was pretty much dried up. My throat was burning and my skin was bone-dry. The amount of my urine was tiny. Those were the results of my proceeding dehydration. This was about the same time when I was taught that it was a naughty thing to say that my tummy felt bad. It was also about the same time when my father claimed: “A real lady never sweats!" I guess he meant it as a stupid joke. "A true noblewoman never spills one single drop of sweat, no matter how heavy her garment or how hot the sun shines. If you train hard enough, you can do that too", he went on and I, obsessed by the idea to become a real noblewoman, tried to become the lady who never sweats. And so I began, just as my daddy had told me, with my training to stop sweating altogether. ${ }^{40}$

People with normal eating behaviors are disgusting to her and her monologues turn into angry rants about eating culture and culinary trends:

Everyone in the restaurant was eating pasta or sandwiches. They all looked like terribly disgusting creatures. How ridiculous they are - those who eat to live! How ugly! --- Why do they put things into their mouth one after another? I would like to live on with just drinks, supplements and medicines, and occasionally gnaw at takuwan and cucumber pickles as I am doing now. I would never want to live by filling up my mouth with glittering meals with oily lumps, biting off living creatures' meat dripping with blood ---. [...] How can such behaviors pass as normal? You only need a certain amount of nutrition enough to live. You shouldn't have time to indulge in leisure like 'meals'. I am so disappointed that you are fooled by the trendy idea that having meals is a fashionable act. Those who follow the trends indulge in the pleasure of meals and still keep eating without noticing the vice called 'fattening'. What fools! You should be spending more time and energy [on] developing yourself ---. This society that has got [sic!] fatter and fatter is making a mistake. Thirty minutes at the fast-food [chain], one hour at the Italian restaurant, two hours at the French restaurant, and several hours of drinking and eating at the izakaya [a Japanese bar and restaurant], while it only takes one minute to swallow supplements. --- A fazzo, creative cooking, dieting drugs, suiitsu, liposuction, fast-food, slow-food - they are all the symbols of our fall. That food amongst other things should fulfill human desire. That a meal with excess is better. That is crap". ${ }^{41}$

Kanehara's protagonist is not only terribly afraid of getting older - something she equates with dying, asking herself over and over if she might be dying right in that moment, while ironically starving herself to death - she is also obsessed with everything that goes into her body and what comes out of it. She and her delusional self talk about bodily fluids, saliva, mucus, sneezing, vomit, blood, nosebleeds. She never stops talking about her body boundaries, unable to tell what belongs to her body and what does not:

The vegetable juice I vomited earlier had dried up by the floor-heating system. --- I dropped on the spot and touched the stain. It was warm, just as when it came out of my stomach. I wonder if the heated floor is part of my body. It may be tenderly looking after even the things that went out from me. ${ }^{42}$ 
As the folders with delusional 錯文 / sakubun fill up her desktop, she feels like she is splitting into more and more single pieces, incapable of bringing together the "unsettling relationship with her bodily existence in the world and the disembodied actuality of her life". ${ }^{43}$ By staying in her room, she tries to remain in a "liminal space between [...] cute little girl and [...] articulate individual person" ${ }^{44}$ without having to decide between the unfavorable options of either becoming a mother, thus fulfilling the role that is expected of her by society, or by becoming an outsider in a society which does not provide an accepted space for women like her. She can never feel like a unified whole, being split between mind and body, between girl and woman, and between the multiple selves inside of her. Unlike Rei in Vaiburēta, she does not find a way out of her dilemma and remains in the room of her own, confused and divided.

\section{To fast is to speak: "I saw words falling to pieces."}

While Vaiburēta and AMEBIC present two cases of different kinds of eating disorders, the ways in which their narrative voices are structured, how they deal and play with words and how their fragmented bodies and minds are represented in language are strikingly similar. Along with the protagonists' feeling of completeness, of their body as a whole and as connected to the world, their language also seems to be falling apart, being divided and put together a new, creating new meanings and interpretations.

The Japanese language and its writing system offer unique possibilities which Akasaka and Kanehara use in their works to convey the mental states and inner worlds of their protagonists. By splitting up words into syllabic kana or using logographic kanji characters with different meanings but homonymous readings, they are able to transform bodily crises into language. On Vaiburēta's first page, Rei wants to mutter “the wrong one" / 違う / chigau ${ }^{45}$ when she is looking for wine in the convenience store, but instead, her head splits the word into the phrase "my blood is boiling" / 血が 茹 / chi ga $u$ to convey her anger. The same thing happens, when she looks outside the truck's window and sees snow. The Japanese word for snow, yuki, suddenly falls apart into the kana $y u$ and $k i$, creating a new word with the kana to for "and" added to it, becoming yutoki, losing all meaning. ${ }^{46}$ She talks of the moment, when she could observe her words, her language breaking down, when a teacher hit her - kotoba ga kowareru tokoro wo miteita ${ }^{47}$ - "I saw words falling to pieces". ${ }^{48}$ She permanently speaks of boundaries, about limits, lines, frontiers, and borders. She fears that the border between her and the night might disappear, a fear stemming from her childhood. She is unable to tell the difference between sleep and death and thinks of the present as a snapped ribbon between her past and her future. She feels like she can't tell if her partner is somebody she has met before, of the feeling of a transparent membrane separating the world from her, and that the things in front of her are "subdivided into smaller and smaller units". ${ }^{49}$ Images of atoms, cells, fission and dispersion of not being complete and the fear of leaking, bleeding out of one's own body are omnipresent in both novels:

And then I realized that there were any number of different mes that existed simultaneously within the passing of time. I tried to feel the presence of the body of the me I was here and now but all I felt was a burning sensation on my skin; the inside was empty. Small holes began opening in the surface, and the holes kept multiplying, becoming more numerous; the gap between other people and myself was disappearing. Stop! Cover the skin, keep me from flowing out. ${ }^{50}$ 
In AMEBIC, which takes its title from one of the delusional essays in the book, the protagonist compares her uncontrollable selves with amoeba, feeling like she is endlessly multiplying by meiotic cell division. "The protagonist becomes increasingly unreliable as the gaze-holder of the text, and this unreliability is the crucial performative element of the text. The question of who is speaking at any given time becomes irrelevant as the multiple voices of the text come together to occupy the one body". ${ }^{51}$ This feeling of fragmentation is also reflected by the aforementioned English blurb on the cover of the book. If we read only the capitalized letters in "Acrobatic Meism Eats away the Brain, it causes Imagination Catastrophe", we can see that the capitalized title of the book, "AMEBIC", can also be read as an abbreviation of this sentence. It can also be subdivided into smaller units, with each letter becoming a different word and other words filling in the gaps, giving new meaning to a series of letters that were read as a unified whole before.

Both Rei and the nameless protagonist in AMEBIC are writers who feel somehow dissociated from the world around them. They produce texts, they live in writing, putting themselves into words. By writing about their bodies in crisis, their writing also portrays the state of crisis they are in. Their hunger turns into endless chatter, into word chains, confused babbling. Words take up the space of food. Instead of bodies filled with food, they become bodies filled with words and voices. They both love to drink to calm these voices and to feel more at ease, and they both suffer from eating disorders that not only contribute to their feelings of not belonging, but internalize their feelings of not knowing who they are and how they are connected to this world. They incorporate this split between themselves and the outside world and dissociate their selves from their bodies. They fragment their identities and their bodies even further; they create several voices and suddenly single body parts do not seem to belong to their bodies anymore. By literally throwing up food, they throw up boundaries inside their bodies, between them and the world around them. Both narrators constantly describe the feeling of a split between mind and body and between the self and the world. What seems to be happening here in a psychological sense is a process of "depersonalization". Often, symptoms of depersonalization seem to be related to eating disorders, but while it does not seem plausible to diagnose literary characters because we cannot talk to them or heal them, it is nonetheless important to learn that eating disorders are often used by patients to cope with symptoms of depersonalization. This might help us understand eating disorders as multidimensional disorders, which cannot be traced back to one singular cause, but which often develop as a way of coping with various factors. This rhizomatic structure of disease is difficult to understand when eating disorders are presented as, for example, to be only caused by unrealistic beauty standards in the media - something, which most certainly plays a part in their emergence - but can be grasped more easily when it is presented as a personal narrative instead of an abstract medical report.

\section{Conclusion}

32 As we have seen, the existential crises the protagonists in both novels experience are connected to their eating disorders and thus to the representations of their bodies. But instead of aiming at disembodiment, as most of the literature on anorexia suggests, we can read their eating disorders as attempts to take their experiences into their own 
hands. Both women struggle to find their places in society, they are unable to feel like complete individuals because society does not offer a viable space for them between being a child and grown woman. By relying on their bodies as the only things that seems certain and accessible for them, they subject their bodies to the conflicts they are facing internally. By obsessively trying to control them, they lose control and develop illnesses - trying to be right kind of woman literally makes them sick. Their yearning for intense corporal sensation is equal to their yearning for an intense feeling of self-certitude, for the feeling of knowing who they are. It really seems like "the cult of diet and exercise is the closest thing [this] secular society offers women in terms of a coherent philosophy of the self". ${ }^{22}$ With their accounts of eating disorders, of their madness and their obsession with bodily fluids and boundaries, of things that are in Kristeva's sense considered abjects and not part of female identities in contemporary Japan, they produce a disturbing impulse and challenge unreachable ideals and gender norms. Their illnesses do not offer a productive way out of their crises, but these literary accounts can offer a glimpse into what it means to suffer from an illness and also into what it means to inhabit a female body in contemporary Japanese society. The experience of illness cannot be generalized; it is always subjective and different for every individual person. But by reading and trying to understand these two novels we can at least grasp what it means to put ourselves in somebody else's shoes.

\section{BIBLIOGRAPHY}

ADAMS Louise, “Is 'devouring' books a sign of superficiality in a reader?”, aeon., June 16, 2016 https://aeon.co/ideas/is-devouring-books-a-sign-of-superficiality-in-a-reader, consulted on $01 / 06 / 21$.

AKASAKA Mari, Vaiburēta, Tokyo, Kōdansha, 1999.

AKASAKA Mari, Vibrator, EMMERICH Michael (trans.), London, Faber \& Faber, 2006.

BRUMBERG Joan Jacobs, Fasting Girls - The Emergence of Anorexia Nervosa as a Modern Disease, London, Harvard U. P., 1988.

CHERRY Kittredge, Womansword - What Japanese words say about women, Tokyo and New York, Kōdansha International, 2002.

CoUNIHAN Carole M., "Introduction - Food and Gender: Identity and Power", in Food and Gender Identity and Power, COUNIHAND Carole M. and KAPLAN Steven L. (eds.), London, Taylor \& Francis, 1998.

DELEUZE Gilles and GUATTARI Félix, Kafka: Toward a Minor Literature, POLAN Dana (trans.), Minneapolis, University of Minnesota Press, 1986.

FENWICK Chris, “Irreducible Significance: David Wellbery on Literature” The Point, 06/11/2017, https://thepointmag.com/dialogue/irreducible-significance-david-wellbery-literature-goethecavell/, consulted on 20/07/2021. 
GULL William Whitney, “Anorexia Nervosa (Apepsia Hysterica, Anorexia Hysterica)”, Clinical Society's Transactions, vol. vII, 22:1873.

GRIFFIN Christine, SZMIGIN Isabelle, BENGRY-HOWELL Andrew, HACKLEY Chris, MISTRAL Willm, "Inhabiting the contradictions: Hypersexual femininity and the culture of intoxication among young women in the UK", Feminism \& Psychology, 2012:2.

KALE Sirin, "Why Eating Disorders Are on the Rise in Japan", Broadly, broadly.vice.com/en_us/ article/4xkjm3/why-eating-disorders-are-on-the-rise-in-japan, 2016, consulted on 01/06/2021. KANEHARA Hitomi, AMEBIC / Amībikku, Tokyo, Shūeisha, 2005.

KIMURA Atsushi, waDA Yuji, GOTO Sho-Ichi, TsUZUKI Daisuke, Dongsheng CAI, OKA Takashi, IPPEITA Dan, "Implicit Gender-based Food Stereotypes. Semantic Priming Experiments on Young Japanese", Appetite 52:2009, p. 521-24.

KоBAYASHI Fukuko, “New Waves in Japanese Women's Writing during the1990s: Resident Korean Women Writers' Fiction and the Fiction of 'Eating Disorders"', Bunka ronshū, 2002:1, p. 1-13.

LASÈGUE Charles, «De l'anorexie hystérique », Archives Générales de Médecine, 1873, p. 385-403.

MALIK Kriti, "Why Men Should Eat Spicy Food”, NDTV, food.ndtv.com/food-drinks/why-menshould-eat-spicy-food-713829, 2014, consulted on 01/06/2021.

MAтsumoto Yūko 松本侑子, [Dawn Never Comes to an Anorexic] / 巨食症の明けない夜明け / Kyoshoku.shō no akenai yoake, Tokyo, Shyūeisha, 1988.

OGAWA Yōko, Shugā Taimu, Tokyo, Chūō Kōronsha, 1991.

OGINO Anna, Taberu Onna, Tokyo, Bungei Shunjū, 1994.

oLson Greta, Reading Eating Disorders - Writings on Bulimia and Anorexia as Confessions of American Culture, Frankfurt, Peter Lang, 2003.

отомо Rio, “A Girl with the Amoebic Body and her Writing Machine." academia.edu/3344703/ A_Girl_with_the_Amoebic_Body_and_her_Writing_Machine1, 2006, consulted on 01/06/2021. отомо Rio, “A girl with her writing machine”, in Girl reading Girl in Japan, AOYAмA Tomoko and HARTLEY Barbara (eds.), London and New York, Routledge, 2010, p. 130-141.

PIKE Kathleen, BOROVOY Amy, “The Rise of Eating Disorders in Japan: Issues of Culture and Limitations of the Model of 'Westernization'”, Culture, Medicine and Psychiatry, 2004:28, p. 493-531.

RUARK Jennifer K., “A Place at the Table”, Chronicle of Higher Education, July 9, 1999, A17.

UEHARA Toru, "Past, Current and Future of Anorexia Nervosa in Japan", Acta Psychopathologica, 2015:3, p. 1-7.

WADELL Elizabeth, “Vibrator by Mari Akasaka” (review), The Quarterly Conversation 10, 2008: https:// web.archive.org/web/20191022042203/http://quarterlyconversation.com/vibrator-by-mariakasaka-review, consulted on 01/06/2021.

wooD James, How Fiction Works, New York, Farrar, Straus and Giroux, 2008.

Woolf Virginia, A Room of One's Own New York, Harcourt, 1981 [1929].

XU Wenying, Eating Identities: Reading Food in Asia American Literature, Honolulu, Hawai'i U. P., 2007. 


\section{NOTES}

1. Wood James, How Fiction Works, New York, Farrar, Straus and Giroux, 2008, p. 52.

2. Ibid., p. 53.

3. FENWICK Chris, “Irreducible Significance: David Wellbery on Literature”, The Point, 06/11/2017, https://thepointmag.com/dialogue/irreducible-significance-david-wellbery-literature-goethecavell/, consulted on 20/07/2021.

4. wood, How Fiction Works, op. cit., p. 130.

5. Ibid., p. 130.

6. ADAMS Louise, "Is 'devouring' books a sign of superficiality in a reader?", aeon., 16/06/2016, p. 1, https://aeon.co/ideas/is-devouring-books-a-sign-of-superficiality-in-a-reader, consulted on 01/06/2021.

7. XU Wenying, Eating Identities: Reading Food in Asia American Literature, Honolulu, Hawai'i U. P., 2007, p. 167.

8. Ibid., p. 166.

9. Woolf Virginia, A Room of One's Own (repr. 1981), New York, Harcourt, 1929, p. 18.

10. XU, Eating Identities..., op. cit., p. 168.

11. DELEUZE Gilles and GUATTARI Félix, Kafka: Toward a Minor Literature, POLAN Dana (trans.), Minneapolis, University of Minnesota Press, 1986, p. 19.

12. Ibid., p. 20.

13. Ibid., p. 21.

14. XU, Eating Identities..., op. cit., p. 168.

15. WOOLF, A Room of One's Own, op. cit., p. 18.

16. DELEUZE and GUATTARI, Kafka: Toward a Minor Literature, op. cit., p. 21.

17. oLson Greta, Reading Eating Disorders - Writings on Bulimia and Anorexia as Confessions of American Culture, Frankfurt, Peter Lang, 2003, p. 284.

18. BUTLER Judith, Excitable Speech: A Politics of the Performative, London, Routledge, 1997, p. 136.

19. COUNIHAN Carole M., "Introduction - Food and Gender: Identity and Power", in Food and Gender - Identity and Power, COUNIHAND Carole M. and KAPLAN Steven L. (eds.), London, Taylor \& Francis, 1998, p. 7.

20. KIMURA Atsushi et al., "Implicit Gender-based Food Stereotypes. Semantic Priming Experiments on Young Japanese", Appetite 52:2009, p. 524.

21. GRIFFIN Christine et al., "Inhabiting the contradictions: Hypersexual femininity and the culture of intoxication among young women in the UK", Feminism \& Psychology, 2012:2, p. 184.

22. CHERRY Kittredge, Womansword - What Japanese words say about women, Tokyo and New York, Kōdansha International, 2002, p. 53.

23. RUARK Jennifer K., "A Place at the Table”, Chronicle of Higher Education, July 9, 1999, A17.

24. UEHARA Toru, "Past, Current and Future of Anorexia Nervosa in Japan", Acta Psychopathologica 2015: 3, p. 1.

25. KALE Sirin, "Why Eating Disorders Are on the Rise in Japan", Broadly, broadly.vice.com/en_us/ article/4xkjm3/why-eating-disorders-are-on-the-rise-in-japan, 2016, consulted on 01/06/2021.

26. Ibid., p. 1.

27. PIKE Kathleen and BoRovoy Amy, "The Rise of Eating Disorders in Japan: Issues of Culture and Limitations of the Model of 'Westernization”, Culture, Medicine and Psychiatry 2004:28, p. 503.

28. Ibid., p. 509.

29. Ibid., p. 523.

30. KOBAYASHI Fukuko, “New Waves in Japanese Women's Writing during the 1990s: Resident Korean Women Writers' Fiction and the Fiction of 'Eating Disorders”, Bunka ronshū, 2002:1, p. 2.

31. Ibid., p. 2. 
32. AKASAKA Mari, Vibrator, EMMERICH Michael (trans.), London, Faber \& Faber, 2006, p. 12.

33. Ibid., p. 9.

34. Ibid., p. 9-10.

35. Ibid., p. 12-13.

36. WADELL Elizabeth, "Vibrator by Mari Akasaka" (review), The Quarterly Conversation 10, 2008: https://web.archive.org/web/20191022042203/http://quarterlyconversation.com/vibrator-bymari-akasaka-review, consulted on 01/06/2021.

37. KANEHARA Hitomi, AMEBIC, Tokyo, Shūeisha, 2005.

38. Ibid., p. 117, transl. bу отомо 2006, p. 11.

39. Ibid., p. 43. All translations are mine, unless otherwise indicated.

40. Ibid., p. 43-45.

41. Ibid., p. 36-37, transl. by отомо 2006, p. 11-12.

42. Ibid., p. 15, transl. by отомо 2006, p. 9.

43. отомо Rio, "A Girl with the Amoebic Body and her Writing Machine", 2006, p.6: academia.edu/3344703/A_Girl_with_the_Amoebic_Body_and_her_Writing_Machine1, consulted on $01 / 06 / 2021$.

44. Ibid., p. 8.

45. AKASAKA Mari, Vaiburēta, Tokyo, Kōdansha, 1999, p. 8.

46. Ibid., p. 100.

47. Ibid., p. 158.

48. AKASAKA, Vibrator, op. cit., p. 116.

49. AKASAKA, Vaiburēta, op. cit., p. 115.

50. AKASAKA, Vibrator, op. cit., p. 112-113.

51. отомо Rio, "A girl with her writing machine", in Girl reading Girl in Japan, AOYAMA Tomoko and HARTLEY Barbara (eds.), London and New York, Routledge, 2010, p. 134.

52. BRUMBERG Joan Jacobs, Fasting Girls - The Emergence of Anorexia Nervosa as a Modern Disease, London, Harvard U. P., 1988, p. 269.

\section{AUTHOR}

\section{CHRISTOPHER SCHOLZ}

Christopher Scholz received his undergraduate and graduate training in Japanese Studies, Modern German Literature, and Philosophy at Freie Universität Berlin, Technische Universität Berlin, and Waseda University in Tokyo, Japan. He is a PhD candidate at Friedrich Schlegel Graduate School of Literary Studies at Freie Universität Berlin and spent one semester as a visiting researcher at the Graduate School of Arts and Sciences at the University of Tokyo. In his doctoral thesis, he explores how bodies are represented in contemporary Japanese literature by female authors. 


\title{
Anorexia Speaks: Eating Disorders in Modern Japanese Films, Novels, Manga, and Poetry
}

\author{
Hideto Tsuboi
}

1 Two young girls standing in front of two different refrigerators speak. One says, "There are all sorts of things to eat in here, but I don't eat anything cold. I want to eat something warm, and my mother will make it when she comes home, but she's busy at work, and she won't come home until very late." The other girl appears, and while speaking the camera turns into a close-up of rosy lips: "Our refrigerator always has lots of red meat. My mother is good at cutting red meat into thin slices. But I think that a refrigerator with only orange juice and green apples is enough."

2 This is the opening scene of 301, 302 (1995), a film of Korean director Cheol-su Park (1948-2013). The narrative begins when these two girls, now grown women, move in next door to each other in an apartment building. The woman who has moved into room 301 brings food to the woman in room 302, but the woman in room 302 doesn't touch it. She throws it all into the trash. As a child, 302 had been raped by her stepfather, a butcher. As a result of this abuse she developed anorexia nervosa. It is explained that she "associates food with love and sex, causing her to lose her appetite." On the other hand, 301 suffers from bulimia brought on by a dysfunctional marriage that ended in divorce. The film depicts a dialectic of the two diseases - the woof of anorexia and the weft of bulimia in a diametrical structure of a woman who wants to feed, and a woman who doesn't want to eat.

3 The viewer is presented with the near-empty refrigerator of 302 containing little more than water and pills. We also see a spotless room covered in books. In contrast stands the extravagantly stocked refrigerator of 301, with its rows of sauces, spices and cooking equipment. Even after 301 learns that 302 suffers from anorexia, she continues cooking for her neighbor; but 302 throws up all that she receives from 301. Here 301 repeats the actions that led to the failure of her marriage, namely cooking too much food and trying to feed her husband. For her husband, his wife's message of love could only be interpreted as an unbearable "food-hell." After these rejections of her 
expressions of love 301 began to eat the leftovers, initiating her journey to pathological over-eating. The conclusion of the film is overly dramatic and clichéd: there is a catharsis that takes the form of the character's complementary problems - to eat and "to be eaten."

4 However, from the beginning, it is clear that the anorexia of 302 cannot be healed by 301's over-production of food. One woman's mouth continually engulfs food while the other's continuously vomits it. The meat and fish that is received ends up in the waste bin or the toilet bowl, as either trash or vomit. Put another way, such trash and vomit comes to resemble dead bodies. Flatfish, beef, sausages, abalone, and dojo fish soup: all of the images of food appearing repeatedly in the film waver between the antonyms "food" and "cadaver," never settling upon a fixed meaning. At the same time, this indicates that the act of eating (or more precisely, the act of living) is in a state of flux built on a fiction. To survive we must kill others and consume death. To take it a step further, in order to live we must ingest death.

5 Predating 301, 302 is an excellent film featuring this image of "eating death": The Cook, the Thief, His Wife and Her Lover (1989), by Peter Greenaway (1942-). Greenway does not disappoint the viewer's desire for the most dramatic and most clichéd ending possible. Given the similarities between the two films, one might claim that 301,302 is simply repeating Greenaway's method. However, the meaning of cannibalism that the two films arrive at is somewhat different. This difference is not limited to the simple question of who is eating or who is forcing another to eat.

6 In The Cook, the Thief, His Wife and Her Lover, Greenaway presents the visible "stage" of a restaurant with Rembrandt-like luster and the brilliance of opulent, upper-class dinner tables, while at the same time "backstage," in the kitchen, there are images of barbarous and violent grotesque animalism, maggot-infested leftovers, vomit and excrement. Beyond that there are the acts of sex secretly occurring in the toilet and kitchen. In other words, the film depicts a "gastronomy of the distasteful," which is an aesthetic of contrast, a reversal of refinement and vulgarity structured around an axis of food and sex. Cinematic aspects of the film, namely questions concerning the inner psyche of the character of Georgina as she exacts punishment on her violent husband in the concluding scene, are beyond the scope of this talk.

7 In 301, 302 the bulimia of the character in room 301 seems to be held within the rising and falling pitch of the phrase "Eottae? Masisseo?" ("How is it? Delicious?") which always accompanied the coercive feeding of her husband. The taciturn woman of 302 explains her refusal to eat by uttering, "it is not just food, but everything in this world that I hate. I wish I could just disappear." The quotes about refrigerators referenced above posit the origins of the women's illnesses being situated in their childhood experiences and family lives. This background depiction functions to link the characters' illnesses to a maternal relationship. The Cook, the Thief, His Wife and Her Lover refuses to include narrative that goes beyond what can be expressed in pictures. In contrast, the narration in 301,302 has a correspondence with literature. Furthermore, where Greenaway has ignored the question of anorexia and bulimia, the Korean movie takes up the question in a somewhat scandalous manner, and in this aspect 301,302 resonates with a series of problems surrounding the female subject and its representation in Japan since the 1980s.

8 It might be said that the representation of extravagant food in Greenaway's film, at least in terms of outward appearance, puts it within the same lineage as [The Gourmet 
Club] / 美食俱楽部 by Tanizaki Junichirō 谷崎潤一郎 (1886-1965). In Greenaway's film the cooks who prepare the meals are generally men, those who feed guests and the thugs are men, as well as those vomiting. The bookstore owner, who has knowledge stored in books, is a man, and the poetry that flows through the entire story is again sung by a young man: a boy soprano. Tanizaki's Gourmet Club is also a story of men eating gourmet food together as a form of male bonding. The female body with its bewitching sexual charm here is made to represent high-class cuisine, and at the climax of the story, the men eat "meat of a splendid Korean beauty". Men eat and women are eaten; their meat is savored. This is a classic example of the role of gender as it relates to the ideology of eating, which runs as an under-current through to The Cook, the Thief, His Wife and Her Lover. It is a barbarous diner table, a potlatch, and women are there given away; women are eaten. Of course, The Cook, the Thief, His Wife and Her Lover ends with revenge for the female side preparing the meal, but no one believes that the heroine, Georgina, has been saved from the potlatch mechanism in the course of her revenge.

301, 302 was well received in Korea as a feminist film presenting the theme of female identity. The film is an indictment of a patriarchal system under which the kitchen is exposed as a space marked as female in gender: a place where food functions not to help women survive, but rather sets into motion a sexism that functions to kill women. In 301, 302 women have been driven out of public places, and into private ones such as the kitchen, as one result of pregnancy. Starting with the nursing of babies, women are at the same time the subject that provides food and the object which is eaten as food. Certainly there are objections to the representation of the kitchen and the identity of women who cook as only a negative image. If this work were analyzed from a feminist perspective, it is undeniable that there would be objections to this overused, stereotypical framing. Despite this, we cannot ignore the fact that the film is commenting on the ideology of sex and food in contemporary Korean society. This representation is contiguous with and contemporaneous to many of the same problems in Japanese society.

There are a number of Japanese novels which take on the same motif as 301, 302 and which, from the standpoint of anorexia and bulimia, question or criticize the definition of kitchen and refrigerator as a uniquely "women's space." Here I will discuss's Kitchen / キッチン (1988), by Yoshimoto Banana 吉本ばなな (1964-), its continuation, Full Moon / 満月 and [Dawn Never Comes to an Anorexic] / 巨食症の明けない夜明け (1988) by Matsumoto Yūko 松本 侑子 (1963-).

11 First, everything is condensed into the shockingly anachronistic phrase, "I think my favorite place in this whole world is the kitchen", ${ }^{1}$ appearing at the very beginning of Kitchen. It is difficult to interpret the meaning of the phrase, especially because the narrator has inserted "I think," which serves to make the whole phrase relative, as though it was a self-quotation. The main character/narrator of Kitchen is Mikage みか $け ゙$, and she does not question her role as food preparer for her live-in boyfriend Yūichi 雄一 and others in the family. Since there is no problematization or conflict concerning this division of labor, and since it comes from the main character herself, it is difficult to read any kind of disharmony with the statu quo in which the kitchen has come to be identified as female. The action of cooking food, the bliss of eating it with Yūichi and the general representation of these naïve feelings is surprisingly void of any questioning. Take for example a scene at the end of Full Moon where Mikage brings 
some pork cutlet on rice / カツ井 / katsudon to the inn where Yüichi is staying. This scene is often discussed in critical literature. In this scene there is the question, "Why is it that what we eat together is so delicious?" The answer from Yüichi is "it must be because we are family," but this is a rejection of Mikage's explanation: "...because we are satisfying hunger for food and hunger for sex at the same time". ${ }^{2}$ However, as Mikage has sneaked into the inn in the middle of the night she passes the food to her boyfriend, and nothing else; here, the food has been charged with the meaning of sex. of course, that the act of providing food brings gratification could also be explained by the fact that the main character works as an assistant at a cooking school, besides studying cooking herself. Also, as has often been pointed out, this story revolves around the description of Yūichi's transgender mother Eriko えり子, with the perversion of gender being a major theme of the text. There are also a number of indulgences or attempts at making excuses in the text for the depiction of the gendered division of labor in reference to food.

I argue that Kitchen has had such strong popular appeal because the text goes through a process of exemption by surrounding the main character with other characters who are open-minded in regard to gender issues, who do not run up against the contradictions of the patriarchal system but rather bypass the problem without any worries or fears. Having come from a family with a missing parent seems to have cast no shadow on the structure of the main character's new family. It is this lack of worries that allows her to put on an apron, learn cooking and think that women who go out to marry are "wonderful”. Expressions like “feminine" (女っぽい/ onna-poi) and "masculine" (男らしい/ otoko-rashii) seen in the text, although used with critical consideration, shows a stereotypical heterosexism which assumes that gender is fixed. The lack of concern or worry in Kitchen points to its portrayal of the 1980s image of a group of people who, with little family of their own, come together, live together and eat together to share the warmth of togetherness. It might be called a mode of the 1980s family.

13 Kitchen displays almost no continuation of the struggle of women writers who preceded Yoshimoto Banana such as Tomioka Taeko 富岡多恵子 (1935-) and Tsushima Yūko 津 島佑子 (1947-2016), who fought to create an expression of female sexual independence. of course Kitchen, which touches on a warmth of sharing food and food culture does not touch or injure that connection, nor does it have any kind of catastrophe like that seen in 301,302 . Nor is there any depiction of the kind of ingredients and vivid texture of food like that made in 301. There is no trash going out of the kitchen and no one vomiting in the toilet. No one is overweight or excessively thin. There is only a view of healthy food and healthy bodies. At its core, Kitchen is emblematic of a 1980s image of safe and hygienic food.

At the start of the novel, the protagonist declares that "I also love a dirty kitchen", ${ }^{3}$ especially those "with vegetables on the floor, (...)oil spewed around the gas range and a rusty knife." 4 The kitchen in this novel, however, seems to lack a feeling of actuality, a place where real food is made for real people. Although it centers around a girl who loves the kitchen, it seems to be furthest removed from gourmandism. In the novel, just before Eriko is killed, Mikage meets her by chance in a convenience store called "Family Mart"- the name being very symbolic. Mikage this time is buying not a pork cutlet, but a cup of custard pudding. Eriko notes, "you've lost weight since you moved out." ${ }^{5}$ The choice of a convenience store for this scene is interesting because it is the 
convenience store that makes it easy for bulimics to get lots of food. It is also said that the spread of convenience stores with their various ready-made foods was a prerequisite environment for eating disorders. There is a hybrid of vulgarity and pleasure in the process of food preparation and representations of kitchens and convenience stores as places where orifices for excreting, evacuating or vomiting are excluded. This has some correspondence with the dark side of the 1980s.

[The White Paper on Japanese Lifestyle] / 国民生活白書, published by the Economic Planning Agency / 経済企画庁 and Cabinet office / 内閣府, reports that at the beginning of the period of high economic growth between 1960-1967, less than $20 \%$ of Japanese homes had refrigerators, but by $196780 \%$ had them. Moreover, by the mid-1970s nearly $100 \%$ of households had refrigerators. The price of a refrigerator as measured by average monthly income also fell markedly, from two months' salary in the early 1960s to one month's in 1969, while by 1985 a refrigerator cost only about 2 weeks of an average salary. The refrigerator became a means of calculating the nation's progress; it was not unusual for there to be more than one refrigerator per household. In the 1970s it was the microwave that appeared in every home, and the two appliances spurred the spread of instant boil-in-the-bag foods and frozen foods.

This environment gave rise to convenience foods and resulted in a large-scale dependence on ready-made foods and eating out. 7-Eleven, Lawson, and other convenience store chains that appeared in the 1970s experienced rapid growth in the 1980s. By 1982, Lawson had sales of more than 100 billion yen and more than 1000 outlets. This expansion did not stop. Rather, every few years it has added another 1000 stores and increased its sales by factors of one hundred billion yen. This astonishing expansion has been especially noticeable since the $1990 \mathrm{~s} .{ }^{6} \mathrm{~A}$ large amount of this increase has been made possible by the sale of foods that can be eaten off the shelf, or with very little preparation. Just like in Kitchen, where Mikage encounters Eriko late at night, convenience stores are a 24 hour-a-day space where people can get foods that are simple to eat. Behind the easy-to-eat store culture is the reality of the huge waste of food that has sat on the shelf beyond its expiration date. In Kitchen, there is no consideration of trash disposed of as dead meat, nor does it evoke questions of food as trash that has been vomited up by a bulimic. In this way, by dividing what is seen on the outside from what is hidden on the underside, Kitchen averts its eyes to the bigpicture reality of food production. It only approaches the question of eating from the point of view of the mouth. This is one kind of vacuity or emptiness that is reflected very well in Kitchen.

In 1987, one year after Kitchen was published, a novel by Matsumoto Yūko, [Dawn Never Comes to an Anorexic] was published, and received the 11th Subaru Prize for Literature. Since the writer, Matsumoto Yūko, was a young staff member of a TV news program, the novel was much discussed in the media. However, the book's popularity probably resides in its ability to pique the interest of readers through its depiction of a young, isolated woman suffering from an eating disorder. The novelist Mizukami Tsutomu 水上勉 (1919-2004) wrote as a comment on the selection of the Subaru Prize for Literature, "I've always wanted to take a peek inside a young student who lives alone in an urban apartment, but this novel shows her horrendously solitude and darkness". ${ }^{7}$ It is the kind of interest where one might be captivated by looking at the life of a woman as she makes trips to the convenience store, eats, and then vomits the food back up. In the novel, the main character has little choice but to go to counseling 
with a psychiatrist for her bulimia, which actually followed anorexia. The psychiatrist is only able to give a stereotypical diagnosis. However, as the novel is written in the polite language that might be used in a doctor's office, it seems as though the work itself could be read as one long written confession to a counselor. The image of male counselor as a kind of Freudian analyst can then be transferred onto the male readers of the novel.

I rip open the plastic bag that contains the sandwich and take a bite. A ham and lettuce sandwich... but I bite and swallow before I can tell what is going on. As I eat that, my hand opens the paper box that holds the custard-cream puffs. Before I can finish my sandwich, I start on a cream puff. Some of the custard comes out of my mouth and gets on my cheek. Powdery sugar falls like snow onto my black coat. I don't care and stuff my mouth with one after another. I feel like I am going to choke. ${ }^{8}$

Such a narrative is haunted by cruelty and obscenity. The sense of obscenity may be related to the fact that this young woman's repeated self-analysis is just too childish. The archetypical example of this obscenity is the fact that she dislikes the Japanese word "kashoku-sho" / 過食症(disease of overeating) and uses instead her own neologism “kyoshoku-shō" / 巨食症 (disease of giant consumption.)

On the other hand, listening to her psychiatrist's explanation, which she doesn't believe, causes her to take up the habit of vomiting. Also at his suggestion she reads Freud's libido theory, which then leads her into bulimia. We can read this process of her bulimia-onset as an ironic protest against the phallocentric Freudian viewpoint. The protest against phallocentrism is also represented in her affections for her boyfriend, who looks almost like a girl, and in her lust for a feminist friend during her days of anorexia. of course, she cannot free herself from the hold of heterosexism. However, it is not her relationship with men, but the one with her mother that projects her onto a heterosexual world and functions as a basis for analyzing her eating disorder. [Dawn Never Comes to an Anorexic] is without a doubt written as a work which revolves around the loss of the mother. When the main character was still just an infant her mother abandoned her and her family. Because of the scars left by the lack of love from her mother, she attempts to live her life in a different way than that of her mother. She wishes that she would not become like my mother and would be neuter, a human with no gender. It is this attempt at losing her gender that led her to starve herself and then to rebound from that dieting. The process of this break from the mother and then a return to the mother culminates in her decision to write her mother to say that she would like to meet her. At that point the reader sees that the writer is not writing a report to her male counselor but rather to none other than her own mother. And in the final chapter, where the daughter/writer of this letter (which is actually the novel itself) is to part ways from her mother, the reader is simultaneously delivered outside of and back to the primary text.

This novel can then be classified as a nested story, a story within a story, and the use of this plot contrivance led some of the judges to disagree with the choice of the work for the award. The text does not end with a reunion of the mother and daughter who have been separated. Rather, at the conclusion, an outer primary text or frame encloses the secondary text and the primary text comes to be foregrounded. This outer text decouples the mother-daughter union. Therefore, this novel can be seen as showing in relief the "mother-daughter dyad" that breaks away from the Oedipal plot, a major theme that Marianne Hirsch (1949-) $)^{9}$ discusses in her work on Western literature from 
the 19th century. Hirsch intentionally holds up an image of the mother which was wholly essentialistic, and describes the "suppression of the mother" in which the daughter can speak but the mother is kept silent. Matsumoto Yūko's novel has introduced the mother's voice into the primary text, making the critical choice to wedge a gap between the suppression of the mother of the traditional mother-daughter stories. It could also be easily argued that Matsumoto's novel has just borrowed the theme of bulimia in order to write a novel whose main theme is a "daughter's message to her mother". The loss of ties with the mother is also used to explain the motivation for the main character's eating disorder. From the beginning, some have indicated that this novel is essay-like in this respect, arguing that this is a weak point of the work. Nevertheless, the work develops a dysfunctional mother-daughter relationship which leads the daughter to have a desire to be thin and to start dieting. This dieting causes anorexia and from there she develops bulimia. The fictionalization of this stereotype is in fact a point that I wish to praise.

21 The reason for this is that the stereotypical story which appears in the novel is composed from various aspects of real-life eating disorders, which the language within the text reproduces. It works as a paradoxical and critical discourse which exists within the mass media. Many psychiatrists believe that eating disorders, as in Yamato Hiroyuki (1957-), stem from a person's refusal to mature into adulthood. This is a theory that feminists have criticized to some degree as well. But it is interesting to note that the manga [The Cherry Orchard] / 櫻の園 by Yoshida Akimi 吉田秋生 (1956-) and the novels of Shōno Yoriko 笙野頼子 (1956-) offer hints to Yamato's thinking on eating disorders. In this way, fiction is used as a reference for medical discourse alongside case histories, reminding us that medical discourse is actually a process of doctors reorganizing the patient's story or history. This fictional narrative functions as a catalyst to further regulate eating disorders, as pointed out by Katō Madoka 加藤まど $\$^{10}$, who has criticized the theory that the family is the origin of eating disorders.

Another scholar who is very critical of the "refusal of maturity" theory behind eating disorders is Asano Chie 浅野千恵. Asano suggests that it is the mass media, especially women's magazines, that are causing eating disorders, and notes that people with eating disorders are forced to be labeled with an identity of "ordinary deviants" (Howard Saul Becker). ${ }^{11}$ She problematizes the tendency of sufferers to blame eating disorders on their own personality, thereby internalizing the cause. Asano and Kato criticize the "family relationship factor" theory as well as the "refusal of maturity" theory. Their criticisms expose the development of those discourses of psychiatry and mass media that seek to separate mothers from daughters, forcing daughters back into a patriarchal system of control and repressing mothers. In other words, mass media and medical discourse are repressing the notion of femininity itself which encompasses both daughters and mothers.

If the novel [Dawn Never Comes to an Anorexic] has any strategy as a narrative, it is to repeat the privileged stereotypical discourse produced by the medical profession and the mass media. The writer is trying to disclose the fact that this discourse is problematic by copying it in her novel. However, despite dealing with the same stereotypical theme of a causal relationship between eating disorders and childhood sexual abuse, failed marriages, and lost parents, among other factors, [Dawn Never Comes to an Anorexic] is inferior to the film images of 301,302. 301, 302 concentrates and simplifies the luxurious grotesque-ism with the same brilliant type of cinematography 
as Greenaway's. It skillfully portrays the trauma aspect while [Dawn Never Comes to an Anorexic] has nothing to compare to the animalistic avarice of 301,302. With its deodorized and abstracted depiction of food, Kitchen has some similarities with the fresh texture emitted by the emotional structure of 少女マンガ / shōjo manga and brings that into the genre of novel, but it does not attain the same quality of expression as the mangas themselves. We have thus seen some typical examples of how current contemporary novels do not live up to the level of movies and manga which themselves have learned much from the genre of the novel. 301,302 has a nearly clichéd twodimensional story line that can be understood by anyone; the simple narrative is similar to Kitchen and [Dawn Never Comes to an Anorexic] with little depicted aside from the food. In 301, 302, the apartment building is almost like a jail, in which each of the women balances the other out in terms of desire and excretion, and because of this we cannot find any point where the female subject resists the patriarchic system. The work reproduces the discourse of the medical profession and the mass media, including stereotypes of cause and effect without stepping outside of them. However, even in this film, the reproduction of the simple and stereotypical narrative is one type of strategy. As a story that concerns causal relationships, the prison-like situation within the apartment building should be seen as a necessary requirement. The film images of faces eating and the graphic images of the food eaten are beyond the capacities of linguistic expression.

As mentioned above, Asano Chie problematizes the dominant narratives found in women's fashion magazines, as well as those suggested by medical experts, questioning what she views to be the true cause of eating disorders. At the same time that eating disorders began to receive greater public discussion during the 1980s, women's magazines experienced a drastic increase in circulation. Fashion magazines aimed at young women, such as Non no / non-no and An an / an.an, increased sales by $160 \%$ over a ten-year period starting in 1982. As a result, these magazines expanded their readership while at the same time increasing the number of articles about eating disorders. According to Asano's argument, the discourses of women's fashion magazines are structured by a semantic chain of health / beauty / diet / anorexia / bulimia. Against this background they feature articles which, from a moral stance, give a negative image of dieting as possibly unhealthy (eating disorders are also presented therein as a side effect of unhealthy dieting). However, what is really salient in these discussions is not rigorous semantic differences, but the suggestion that the only way to find beauty and health is to diet while doing one's best to avoid eating disorders. In other words, they are creating a questionable chain of endless images that are tied to a nebulous standard of beauty and health.

Current advertisements for magazines announce "It's spring, so let's get that weight off!" or "Reduce your weight by X kilograms and your waist by $\mathrm{Y}$ centimeters in $\mathrm{Z}$ weeks!" Another source of this image is newspaper insert advertisements for aesthetic salons, health and diet foods, and other companies in the health industry. This kind of discourse links ideas of health to dieting and beauty, creating what could be called the absolute necessity of a slender body, an ideology we might term "slenderism". In [Dawn Never Comes to an Anorexic], the main character gets the idea of vomiting what she had eaten from her psychiatrist. Similarly, Asano introduces cases of people who started to practice vomiting after reading about it in women's magazines. There is a chance that [Dawn Never Comes to an Anorexic] is offering us a new canon of narrative through its reproduction of the dominant mass media narrative of eating disorders. 
However, a more powerful critique of the slender-ist image can be found in a fictional genre that deals intimately with such images and is even sometimes a co-conspirator in the implantation of the slender-ist ideology. That genre of fiction is my next topic: shōjo manga.

A manga that takes up the question of the desire for a slim body, dieting and overeating in a direct way is Anno Moyoco 安野 モヨコ (1971-) [In Clothes Called Fat] / 脂肪と言 う名の服を着て (1997). It originally appeared in the magazine 週刊女性 / Shūkan Josei under the title [Gotta Slim Down!] / 瘦せなきゃダメ!. This work drew attention because it was published in the midst of the discourse on eating disorders in women's magazines. The work begins with the main character suffering from being overweight as well as being an overeater. She goes to an aesthetic salon that promises weight reduction treatment. The story follows the character until she begins the habit of induced vomiting. In the end she is dumped by her boyfriend for slimming down, and starts overeating again - indeed a pitiful story. Both her boyfriend and her "patron", an old man, belong to the category of the so-called デブ fat-woman fetish, depicted as a nostalgic desire for the mother. In this way, the work includes a critical reversal of discourses centered in the male gaze.

Another manga author who must not be ignored is Matsumoto Michiyo 松本充代 (1962-), whose first-person narratives provide a lyrical quality while relativitizing the image of female beauty associated with slimness. Matsumoto enjoys depicting unusual topics in her work including menarche, boys' voices breaking, masturbation, love-hate relationships with one's mother, sexually late-blooming girls, etc. Among these, [“Trash"], included in [What I'm lacking] / 私にたりないもの (1992) is about a girl who longs to be anorexic, but fails. Here the author depicts a character who is unable to become an adult, as envisioned by the "refusal of maturity" theory. In this way, the character's portrayal undermines the dominant theory. Another work is ["A Suicide Note in the Mirror"] / 鏡の中の遺書, published in [Hidden Voices, A Suicide Note in the Mirror and Other Short Stories] / 潜む声、鏡の中の遺書、その他の短編 (1999), which depicts a junior high school student who is "fat and homely" and therefore unable to have any self-confidence while also experiencing serious problems of school bullying.

28 Among the four girls whose lives of loneliness are depicted in Strawberry Shortcakes (2002), the work of Nananan Kiriko 魚喃キリコ (1972-), one repeatedly overeats and induces vomiting. With her special touch, Nananan erases all the shadows and gives the reader a bleached vision of a hellish inner-world of her character:

I want to eat / just eat // then / in my stomach / with that in my stomach / when it gets all full / all of it, all of it, / I'll puke it all. // it feels like.../ ----everything miserable in me / moves from inside my body to the outside. ${ }^{12}$

The text is explicit about the cause of her overeating and induced vomiting - she has been thrown aside by her lover. However, the act of overeating and vomiting here is depicted almost entirely as output, and the Freudian theory of desire never intervenes in the explanation of it. The story of cause and effect has been thrown away unconsciously. The strategy which we can find in Anno, Matsumoto, and Nananan is the same: they balance out the machismo of manga created for male consumption and the image of slim women defining beauty, refusing to become an adult. They are able to use the standard image of femininity in the opposite direction. Beyond this they are able to visually shake the model of narrative to the extent that it falls apart. 
Another group of manga works that shake the standards of the inner structure of shojo manga and invite disfavor and rejection from male readers are the comics of Okazaki Kyōko 岡崎京子 (1963-). For example, we can look at her 1996 work [Shotgun from the Lips] / くちびるから散弾銃. The story features three women characters who enjoy talking amongst themselves and who do so in a way that is compared to a shotgun, the image of which lends itself to the title of the comic. These three young women, all aged 23, have no need for men, and spend their time discussing an array of topics. They go as far as discussing the Chernobyl disaster, in addition to the cultural topics raised in women's magazines. There is no place for male desire in this world. In the afterward, Okazaki writes that the time is merciless for the girls who did not want to grow up to be women. But despite this thematic setting, the male gaze, which is what one expects in a story where the characters refuse to grow up, has been elided altogether. Even in her works that deal with murder, rape, violence - where women's bodies are substantiated as the object of male desire, like [“A Small Sun in the Water"] / 水の中の小さな太陽 included in [End of the World] (1994), [“I am Your Toy”] / 私は貴兄のおもちゃなの, included in Watashi wa Anata no Omocha na no (1995). In the 1996 チワワちゃん, the male gaze has been completely objectified, or elided from the text.

Two works that take up the problems of sex and food are Pink and River's Edge / リバー ズ・エッジ. Pink, written in 1989, is a manga that critically approaches the genre of the novel in order to differentiate itself from it. The main character is a business woman who has an alligator for a pet and who works as a prostitute (hotetoru-jō / ホテトル嬢 [a call-girl working in love hotels for a company]). She also has a boyfriend who is the lover of her stepmother. The boy, her stepsister (the daughter of the stepmother), all are good friends. For this complex system of human relations, the story can be seen as a parody of Schneewittchen (Snow White) which follows the stepmother - child pattern. Having had her young lover stolen, the stepmother (who also makes apple pies) kills the pet alligator as revenge. Through this parody of stereotypical narrative, the writer is critically analyzing the act of narrating.

The young lover is an aspiring novelist. He was ridiculed at by his former girlfriend with saying that the readers of novels must surely be some kind of pitiful Cro-Magnons. Also he is told that in today's world, magazines, TV and manga are enough. The young lover is threatened by the stepsister to make something interesting if he wants to be a novelist. He then writes a novel by stealing (cutting and pasting) someone else's text, and for that plagiarized work he wins a literary prize.

The main character also wants to live her life watching TV and live her life looking at pictures in 'an an'. Her role here is to contrast with the young lover, who sees himself as an aspiring novelist. The problem is that the heroine does not try to live the lifestyle that is being introduced by these forms of media. This fact causes a gap between her speech and her real desire.

When she wishes she had a home like that in Sazae-san, an animation program featuring an idyllic, happy, traditional Japanese family, this is probably not coming from her real desire. At the same time, she does not seem to be longing for the lofty lifestyle that her boyfriend, the aspiring novelist, is chasing. The story seems to proceed in linear time without defining the true nature of her desire, never returning to the starting point. She is living without any separation between her postured image and her real self. She is not, however, in need of any self-definition in this respect. In a traditional novel, there is no mode of reading that will allow the reader to distinguish 
the character's posturing from her real desire. It would be necessary to have some explanation regarding the unrepresented real desire from the narrator or the character. But in a manga, the pictures can make up for this lack. So when her customer abuses her for being a piece of meat lower than animals, the text does not explain her pain, or the necessity of why she needs to make money. This brutal and irrational emptiness is what Okazaki is skillful at depicting. Due to this depiction, the reader is assured that he can witness rape, murder, and prostitution without much pain or discomfort. Kitchen, which I discussed earlier, is an example of a work that brings the kind of manga-esque narrative of emptiness into the genre of the novel. However, Kitchen cannot provide the level of vicarious experience of the irrational that Pink does when it forces the reader to witness this irrationality.

of course, Pink does not deal with eating disorders, but with a pet alligator that eats chickens whole, as well as a Maltese that the main character's stepsister has captured. However, Kayama Rika 香山リカ (1960-) points out that the food that her stepsister buys at convenience stores is "what people with anorexia or bulimia might do". ${ }^{13}$ This alligator's ferocious appetite stands as the essential theme of the manga; in this way, then, "eating" is undeniably a central idea of the piece. Since the main character sells her body, there is also an aspect of prostitution in the form of "meat". Additionally, the alligator is awakened by the smell of menstrual blood, at which point the heroine admits that she is indeed also meat. Having sex with her boyfriend becomes the equivalent of "eating", and as the lovers are eating meat at a restaurant they remember the body of the main character's mother, who has committed suicide. Just at that time the stepmother is having the alligator taken away to be made not into meat but into a handbag. It lived as meat that eats meat, and then dies and becomes meat. Like many of Okazaki's other works, the conclusion is punctuated by death.

The themes of food and sex as well as food and death are condensed in River's Edge (1994). The heroine is a high school student named Wakakusa Haruna 若草 ハルナ. She becomes close to a boy in her class, named Yamada 山田, who is mistreated by other students at her school, including by a boy named Kwannonzaki 観 音 崎. Through him Haruna meets a girl named Yoshikawa Kozuey 吉川こずえ. Yamada is a homosexual and likes a certain boy, but that boy is heterosexual. He sometimes sells himself to men, and he pretends to date a girl named Tajima 田島 as camouflage. Yoshikawa Kozue works part time as a model, and is looked up to by all at the school. Secretly she overeats and vomits (it is also hinted that she may be a lesbian.) Within this complex relationship, there is a series of catastrophes that befall them. Kwannonzaki suspects a relationship between Haruna and Yamada. He has relations with a girl named Rum ル ミ, who then gets pregnant. Kwannonzaki plans to kill the pregnant Rumi. Rumi, injured, arrives home only to find her sister secretly reading her diary. Rumi, who practices prostitution (援助交際/ enjo.kōsai), and her older sister, who has uncontrollable eating habits and is overweight, begin to blame each other for their problems, eventually having a fight which ends in blood. Tajima, upset at having Yamada stolen from her, sets fire to Haruna's house and then later burns herself to death.

This tangled plot is saved from failure by the fact that the writer has set up an intricate analogical relationship between the various plot lines, but what holds it all together is the view of the river which is located near the characters' high school. Haruna is led to the river by Yamada and Yoshikawa, and there they look at their "treasure," an actual 
dead body. This dead body functions as a kernel around which the various dead bodies in the work are drawn. This includes the kitten that Yamada was raising and that was killed by the other students; Rumi, who was almost killed two times, and the fetus that came from her body; Rumi's older sister who slit her wrists; and the body of Tajima, burnt black. In addition to these dead bodies, there are the not-dead: the emaciated body of Yoshikawa Kozue, emaciated from overeating and vomiting, or that of Yamada, whose body has suffered the repeated beatings of classmates. Moreover, near the end of the work, Yamada significantly confesses that he likes Tajima as a corpse much, much better than when she was alive. He rejects her as the other when she is demanding a close relationship, but once she is quiet and unable to speak he finds her more agreeable. This, however, is neither some kind of philosophical message nor is it a perverted desire for corpses.

The work ends with a glimmer of hope, as Yamada notes his friendship with Haruna, but looking at the work as a whole, the story is concluded without even the catharsis of death; rather, it ends with the corpse. Left with corpses which belong to neither the act of living nor to the act of dying, we find that the work leaves us with a sense of pain that cannot be assuaged. We see the overlapping images of the corpse at the river's edge and sex between Rumi and Kwannonzaki, as well as the overlap of Tajima's burnt corpse and sex between Huruna and Kwannonzaki. Therein, it is clear that death cannot purify the sexual act which brings a sense of emptiness, equal to that depicted by the vomiting of food eaten.

As in the films The Cook, the Thief, His wife, and Her Lover and Z00 (1985), Greenaway attempts to follow the rotting of corpses and dead animals with a camera. In these works we see to what degree dead bodies and vomit can be depicted in gorgeous and vivid detail. Fuse Hideto (1960-) refers to the scenes of asceticism (不 浄 観 / fujo-kan) in Tanizaki Junichirō's Captain Shigemoto's Mother / 少将滋幹の母 (1950) when discussing the rotting dead body of Z00. ${ }^{14}$ In the Fujo-kan the character Kunitsune 国経 tries to get over his feeling towards his stolen wife by looking at rotting human bodies, but this ends in failure. In The Cook, the Thief, His Wife and Her Lover, the Mafia boss sits in front of the dead body, but vomits. His vomiting is proof that he is living. In the case of Captain Shigemoto's Mother, Kunitsune is led to see how deeply his attachment to this world is. In both Greenaway's and Tanizaki's work the dead body is objectified, and in doing so the subject experiences a reconfirmation of his verve (will to live) and of desire.

In contrast, the dead bodies in River's Edge seem to evade this verve. The dead bodies at the river's edge and Tajima's burnt corpse both are left with no more meat on them. In other words, they cannot be eaten. Like the bulimic Yoshikawa Kozue's emaciated body, River's Edge shuts down avaricious hunger, as in Greenaway's film. Moreover, if we compare River's Edge with 301,302, the image of the woman in 301, who cooks and voraciously eats is insubstantial. The static pictures and words of River's Edge squeeze out more poesy than the moving images in 301,302. However, the question here is what the image of the river's edge gives to the work. I would like to take a look at a phrase that appears at both the beginning and the end of the story.

The place where we live / There is a river running through it / not far from the mouth of the river / It is wide and flows slowly ... the smell is bad / In the midst of the riverbed, this mound // Tall Goldenrods / growing thickly / you can often see dead cats and such. ${ }^{15}$ envelops the work, is much like a poem. And this is no surprise, because - even if the 
influence from the Hollywood movie of the same name has been pointed out by others - we can also see the influence of the poetry of Itō Hiromi 伊藤比吕美 (1955-) in the above phrasing and in the work as a whole. The image of the river, goldenrods, and dead bodies all appear in Ito Hiromi's works from [The Sky of Plants] / 草木の空 (1978) to [Underripe Plums] / 青梅 (1982). They are keywords in Itō's early works. The Goldenrods were a symbol that she developed to represent reproduction and the power of the mother. In her works from the late 1970s and early 1980s, her poems repeat these images, combining eating and death as well as images of corpses. All of these can be found in Itō's [On Territory] / テリトリー論/ series. Moreover, in the 1990s she authored [Noro and Saniwa] / のろとさにわ (1991) (written with Ueno Chizuko 上野千 鶴子: 1948-) as well as [Hand, Leg, Meat, Arm: Hiromi 1955] / 手 · 足 ·肉 ·体 Hiromi 1955 (1995), collaborated with photographer, Ishiuchi Miyako 石内都 (1947-). These kinds of themes were in constant repetition at that time. It should be noted that Okazaki Kyōko was in turn influenced by poets who had been influenced by other manga writers like Ōshima Yumiko 大島弓子 (1947-). Furthermore, I would like to note that in Okazaki's works, she adjusted the images that she had borrowed from Itō. For one thing, in contrast to Itō's poems, Okazaki wrote River's Edge in 1994 from a position looking back on the 1980s. Okazaki notes the following: "From the start they were the lost children. They had everything, and for that reason, these children had to give up everything". ${ }^{16}$ But having said that, this comment about the deep sense of loss which the author makes seems highly emblematic of the 1990s, and also extremely poetic.

What I want to stress is the following: Itō's first writing came out of a period when she was battling with anorexia. This fact does not just fall into the category of background information about the writer, but rather plays an important role in the motifs of her work. For example, if we look at the poem [“So as not to become warped"] / 歪ませな いように, from [Princess]/ 姫 (1979), we see the female heroine making white dumplings / 白玉, made from rice powder, for her lover. Of course, there is the asymmetrical relationship of the woman who cooks and the man who eats, but merely noting this does not go far enough into the problem.

Itō had already published works featuring the relationship between an anorexic and her boyfriend in the poem [“Anemia"] / 負血 from the anthology [The Sky of Plants]. One could say that the woman in apartment 301 had already been depicted here. Therefore, what is behind the tactile expressions (especially concerning these rice-flour dumplings) like "melting black syrup in the mouth, and silky smooth dumplings" ${ }^{17}$ is not just sex between these two lovers, but the sense of touch of the girl who has become "thin as a stick" with "anemic looking white skin" 18 - the image of the girl's skin after she had tweezed out its hair. In looking at it this way, we see how a text like ["Vinegar and Oil"] / 酢油 (included in [On Territory 1]) finely represents the textures of foods' skins; it cannot be placed into a rough schema of "desire for food = desire for sex". On this point, the image of a primitive and uncontrolled mother energy was at the center of Itō's poems. Yet within the text, the words "I want to eat" cannot be read simplistically. Even after this period, Itō continued to work on the theme of eating disorders, while also taking part in workshops offered by Saito Manabu (1941-) and his "NABA" organization (designed to help people with eating disorders). Additionally, she co-wrote [A Bright Anorexia, Cheerfully Bulimic] / 明るく拒食、元気に拒食 (1992). Out of this work was born the poem [“Pork Cutlet Princess”] / カツ井姫さま. The work is composed of the voices of two characters, ["Anorekisia"] (anorexia nervosa) and 
["Bulimia"] (bulimia nervosa.) Itō also included quotations from real-life sufferers of eating disorders.

I just wanted to be sleek

I wanted to get everything out...

Breath, digestion, excretion, hair, sweat, sexual excitement, appetite

All of my organs stop

Clean them

I just wanted to sterilize them ${ }^{19}$

This poem, spun together from disparate voices, comes together to make a new voice. But this kind of work does not exist merely to help people with eating disorders find help and healing. Itō is saying that people have to enjoy their illnesses and disorders of anorexia and bulimia. Okazaki Kyōko's work also finds its power in the fact that it is not interested in providing some sort of healing process to those with the disease. How can we free ourselves from a simplistic concept of healing in a reality where, on one side, there are daily updates on the Internet of new personal best records for dieting, and on the other blogs where bulimics write? These two aspects are slapped together like the front and back sides of the same sheet of paper to form a single reality. Is this a reality to which literature can give some meaningful response?

\section{BIBLIOGRAPHY}

ANNo Moyoco 安野モヨコ, [In Clothes Called Fat] / 脂肪と言う名の服を着て / Shibō to iu na no fuku o kite, Tokyo, Shufu to Seikatsusha, 1997.

ASANo Chie 浅野千恵, [Why Do Women Try to Slim Down?: Eating Disorder and Gender] / 女はな ぜ瘦せようとするのか : 掑食障害とジェンダー/ Onna wa naze yaseyōto suru noka: Sesshokushōgai to Jendā, Tokyo, Keisō Shobō, 1996.

[Economic Planning Agency] / 経済企画庁 / Keizaikikakuchō, [Cabinet Office] / 内閣府 / Naikakufu, [The White Paper on Japanese Lifestyle] / 国民生活白書 / Kokumin.seikatsu hakusho, n.d.

FUSE Hideto 布施英利, [“The Body Sense of Z00”] / Z00の身体感覚 / Z00 no shintai kankaku, WAVE, vol. 29, 1991.

HIRSCH Marianne, The Mother/Daughter Plot: Narrative, Psychoanalysis, Feminism, New Haven, Indiana U.P., 1989.

ITō Hiromi 伊藤比吕美, [Hand, Leg, Meat, Arm: Hiromi 1955] / 手 ·足 ·肉 ·体 Hiromi 1955 / Te, Ashi, Niku, Karada: Hiromi 1955, Tokyo, Chikumashobō, 1995.

ITō Hiromi 伊藤比呂美, [I am Anju-himeko] / わたしはあんじゅひめ子である / Watashi wa Anjuhimeko de aru, Tokyo, Shichōsha, 1993.

ITō Hiromi 伊藤比呂美, [On Territory 1] / テリトリー論 1 / Territorii-ron 1, Tokyo, Shichōsha, 1985.

ITō Hiromi 伊藤比呂美, [Princess] / 姫 / Hime, Kawasaki, Shiyōsha, 1979. 
ITō Hiromi 伊藤比呂美, [The Sky of Plants] / 草木の空 / Sōmoku no sora, Hekinan, Atoriesyuppankikaku, 1978.

ITō Hiromi 伊藤比吕美, [Underripe Plums] / 青梅 / Aoume, Tokyo, Shichōsha, 1982.

ITŌ Hiromi 伊藤比呂美, UENo Chizuko 上野千鶴子, [Noro and Saniwa] / のろとさにお / Noro to Saniwa, Tokyo, Heibonsha, 1991.

ITŌ Hiromi 伊藤比呂美, SAITō Manabu 斎藤学, [A Bright Anorexia, Cheerfully Bulimic ] / 明るく 拒食、元気に拒食 / Akaruku kyoshoku, genki ni kashoku, Tokyo, Heibonsha, 1992.

KATŌ Madoka 加藤まどか, [“Questioning the expansion of the family factors theory related to anorexia and bulimia”] / 家族要因説の広がりを問う-拒食症・過食症を手がかりとして /

Kazoku.yōin.setsu no hirogari wo tou - kyoshokushō - kashokushōo tegakari to shite ", in [Analysis of Contemporary Society: System/Body/Narrative] / 分析 - 現代社会一制度／身体／物語 / Bunseki Gendai Shakai Seido/Shintai/Monogatari, ōTA Shôichi 太田省一 (ed.), Tokyo, Yachiyo Shuppan, 1997.

KAYAMA Rika 香山リカ, [Evening Story for Manga 2] / 漫画夜話 2 / Manga Yawa 2, Tokyo, Kinemajunpōsha, 1998.

Lawson’s official website: 売上高/店舗数 | ローソン公式サイト (lawson.co.jp). матsUмото Yūko 松本 侑子, [Dawn Never Comes to an Anorexic] / 巨食症の明けない夜明け / Kyoshoku.shō no akenai yoake, Tokyo, Shūeisha, 1988.

MAтsuмото Michiyo 松本充代, [What I'm lacking] / 私にたりないもの / Watashi ni tarinai mono, Tokyo, Kawadeshobō, 1992.

матsumoто Michiyo 松本充代, [Hidden Voices, A Suicide Note in the Mirror and Other Short Stories ] / 潜む声、鏡に中の遺書、その他の短編 / Hisomu koe, kagami no naka no isho, sono ta no tanpen, Tokyo, Asky, 1999.

MIZUKAMI Tsutomu 水上勉, [Impression] / 感想 / Kansou, Subaru, Tokyo, Shūeisha, vol. 9-12, 1987.

NANANAN Kiriko 魚喃キリコ, Strawberry Shortcakes, Tokyo, Shōdensha, 2002.

OKAZAKI Kyōko 岡崎京子, [Chihuahua-Chan] / チワワちゃん / Chiwawa-chan, Tokyo, Kadokawashoten, 1996.

OKAZAKI Kyōko 岡崎京子, [I am Your Toy] / 私は貴兄のおもちゃなの / Watashi waanata no omocha nano, Tokyo, Shōdensha, 1995.

OKAZAKI Kyōko 岡崎京子, [Shotgun from the Lips] / くちびるから散弾銃 1, 2 / Kuchibiru kara sandanjū, Tokyo, Kōdansha, 1989, 1990.

OKAZAKI Kyōko 岡崎京子, River's Edge / リバーズ・エッジ, Tokyo, Takarajimasha, 1994.

OKAZAKI Kyōko 岡崎京子, Pink, Tokyo, Magazine House, 1989.

TANIZAKI Jun.ichirō 谷崎潤一郎, [Captain Shigemoto's Mother] / 少将滋幹の母 / Shōshō shigemoto no haha, Tokyo, Mainichishinbunsha, 1950.

TANIZAKI Jun.ichirō 谷崎潤一郎, [The Gourmet Club] / 美食俱楽部 / Bishoku kurabu in [Women are Sacred] / 女人神聖 / Nyonin Shinsei, Tokyo, Shunyōdō, 1920.

YAMATO Hiroyuki 山登 敬之, [Anorexia and Bulimia: Confusion of Alices] / 拒食症と過食症 困惑 するアリスたち / Kyoshokushō to kashokushō: Konwaku suru Arisu tachi, Tokyo, Kōdansha, 1998. 
YOSHIDA Akimi 吉田秋生, [The Cherry Orchard] / 櫻の園 / Sakura no sono, Tokyo, Hakusensha, 2013.

үознімото Banana 吉本ばなな, Kitchen / キッチン, Tokyo, Fukutakeshoten, 1991.

\section{NOTES}

1. уознімото Banana, Kitchen / キッチン, Tokyo, Fukutakeshoten, 1991, p. 6.

2. Ibid., p. 153.

3. Ibid., p. 6.

4. Ibid., p. 6.

5. Ibid., p. 73.

6. Lawson's official website: 売上高/店舗数 | ローソン公式サイト (lawson.co.jp).

7. MIZUKAмI Tsutomu, [Impression] / 感想, Tokyo, Shūeisha, vol. 9-12, 1987, p. 29.

8. матsимото Yūko, [Dawn Never Comes to an Anorexic] / 巨食症の明けない夜明け, Tokyo, Shyūeisha, 1988, p. 53.

9. HIRSCH Marianne, The Mother/Daughter Plot: Narrative, Psychoanalysis, Feminism, New Haven, Indiana U. P., 1989.

10. KATŌ Madoka, ["Questioning the expansion of the family factors theory related to anorexia and bulimia”] / 家族要因説の広がりを問う-拒食症・過食症を手がかりとして, in [Analysis of Contemporary Society: System/Body/Narrative] / 分析 ·現代社会一制度/身体／物語， OTTA Shôichi 太田省一 (ed.), Tokyo, Yachiyo Shuppan, 1997.

11. ASANo Chie, [Why Do Women Try to Slim Down?: Eating Disorder and Gender] / 女はなぜ瘦せ ようとするのか：摂食障害とジェンダー, Tokyo, Keisō Shobō, 1996.

12. NANANAN Kiriko, Strawberry Shortcakes, 2002, Tokyo, Shōdensha, 2002, p. 18.

13. KAYAMA Rika, [Evening Story for Manga 2] / 漫画夜話 2 , Tokyo, Kinemajunpōsha,1998, p. 217.

14. FUSE Hideto, [“The Body Sense of Z00”] / Z00の身体感覚, WAVE, vol. 29, 1991.

15. OKAZAKI Kyōko, River's Edge, Tokyo, Takarajimasha, 1994, p. 2.

16. Ibid., afterwords.

17. ITō Hiromi, ["So as not to become warped"], in [Princess], Kawasaki, Shiyōsha, 1979, p. 35.

18. ITō Hiromi, ["Anemia"], in [The Sky of Plants], Hekinan, Atoriesyuppankikaku, p. 72.

19. ITō Hiromi, ["Pork Cutlet Princess"] in [I am Anju-himeko], 1993, p. 101.

\section{AUTHOR}

\section{HIDETO TSUBOI}

Nichibunken, Kyoto.

Tsuboi Hideto 坪井秀人, a researcher at the International Research Center for Japanese Studies / 国際日本文化研究センター / Nichibunken, is mainly interested in modern and contemporary Japanese literature, cultural history and biopolitics. Among his publications are: [Rethinking Post-War Japanese Culture] / 戦後日本文化再考 / Sengo nihonbunka saoikō, Sanjinsha, 2019, 603 p.; [The Bubble and the Lost Twenty Years (Re-read Post-War Japan)] / 
バブルと失われた20年 (戦後日本を読みかえる) / Baburu to ushinawareta 20 nen (Sengonihon o yomiikaeru), Rinkawashoten, 2018, 264 p.; [The Gender Which Causes: Sex and Body in 20th Century Japanese Literature] / 性が語るー20世紀日本文学の性と身体 / Sei ga kataru - 20 seikinihonbungaku no sei to shintai, Nagoya U. P., 2012, 696 p. 
2. Corps réprimés 


\title{
Human/Inhuman/Post-human. Female Bodies in Modern and Contemporary Chinese Literature: Literary Descriptions of Psychological and Social Unease
}

\author{
Nicoletta Pesaro
}

1 The aim of this paper is to identify the ways in which modern and contemporary Chinese literature has depicted the female body, and how this depiction has been used as a tool not only of artistic expression but of sense-making as well. I will especially focus on disruptive and uneasy narratives of the female body provided by Chinese writers.

2 I will develop my analysis of a range of literary texts within a twofold conceptual frame. On the one hand I will stress the importance of close reading and textual analysis in order to detect the linguistic representation of the female body in terms of descriptive and evocative images. On the other hand, I will try to explore the cognitive function played by literary description, in expressing "the mediation of bodily experience" ${ }^{1}$ as a tool to know the world and the human self. Recently, new discoveries in neurosciences have revealed "the existence of structures [in the human brain] that get activated both when undergoing certain mental states and when representing them in others". ${ }^{2}$ Certain literary representations of physical and mental states, therefore, could enable readers to improve their understanding of the human being: "through fiction human cognitive procedures are represented so that readers expand their understanding of the human being through examples rather than theories". ${ }^{3}$

3 I will argue that, in some cases, the representation of "inhuman" bodily states is a narrative function meant to enact sharing and understanding. Indeed, experiments measuring empathetic reactions found that "areas underlying the experience of physical pain in its affective aspect [...] were also active when participants received a signal indicating that a painful stimulus is applied to another person". ${ }^{4}$ This proves that 
the individual and collective body are connected through a common network of relations. Acclaimed philosophical theories such as those of Foucault and Agamben provide scholars with a variety of approaches, suggesting the existence of a deep connection between body and society, body and politics. However, recently it is the phenomenology of perceptions theorised by Merleau-Ponty that has been rediscovered by the cognitive sciences: his idea of the living body helps us see the body in literature as a powerful means to experience the world. Merleau-Ponty speaks of a "redécouverte $d u$ corps propre", or "rediscovery of one's own body". ${ }^{5}$

4 As far as the research on modern Chinese literature is concerned, one should mention Angela Zito and Tani Barlow, ${ }^{6}$ Li Rong, ${ }^{7}$ and recently Carlos Rojas, ${ }^{8}$ who analyzes the use of the body, especially sick bodies and disease, as a discourse about weakness but also potential transformation, and notes the tendency to signal the inherent contradiction between internal alterity and external community:

Nations, families, and even corporeal bodies are fundamentally heterogeneous collectives, and their imagined coherence is predicated on a perpetual tension with sites of internal alterity. ${ }^{9}$

5 Apparently, according to many literary texts, the site of this contradiction is often the female body.

6 The bio-political perspective is prominent in studies such as the ones made by Belinda Kong, ${ }^{10}$ Yinde Zhang, ${ }^{11}$ and the abovementioned work by Rojas. As a matter of fact, both in modern and contemporary Chinese literature, writers have shown themselves to be very keen on a body-centred representation of reality. From the "left-wing" writers and the "new sensation school" / 新感觉派 / xin ganjue pai of the 1930s, ${ }^{12}$ to Xiao Hong 萧红 (1911-1942), women's condition and, more generally, the representation of a socially tormented China have been represented via the body. More recently, it suffices to recall Yu Hua 余华 (b. 1960), Mo Yan 莫言 (b. 1955), Yan Lianke 阎连科 (b. 1958), and Bi Feiyu 毕飞宇 (b. 1964)'s recent novels and their emphasis on bodily perceptions, which are often exaggerated or even distorted in order to convey deeper meanings, related to the historical and social tensions they reveal. Likewise, the dystopian vision of reality, which has been brilliantly explored by Jeffrey Kinkley in his latest book, ${ }^{13}$ often refers to the embodied experience represented in the "new historical novels" / 新 历史小说 / xin lishi xiaoshuo, in which the body has a disruptive power.

The writers of the “new" or "latest generation" / 晚生代 / wanshengdai also present a body-centred approach: I am referring not only to Chen Ran 陈染 (b. 1962), Lin Bai 林白 (b. 1958) and other female writers who apply a bodily view of the world and of writing itself, but also to writers like Han Dong 韩东 (b. 1961) and Zhu Wen 朱文 (b. 1967), who offer a representation of individually and physically perceived reality. ${ }^{14}$

Literature seems to be the best site for expressing the body: "The literary, like the somatic and through its relation to the somatic, opens the path to the unbinding of all forms of fixity - those of individual identity as well as the stereotypes and hierarchies that accompany them". ${ }^{15}$ Besides, as Hillmann and Maude observe, "Literary thinkers have always been particularly interested in concrete, non-conceptual aspects of knowing and feeling". ${ }^{16}$

9 In my necessarily limited range of collected examples, I have identified some fundamental categories in the representation of women's body as a carrier of meanings 
and as a constant object of manipulation, tracing a general path that leads the female body from the human to inhuman state.

These categories are: the allegorised body; the animal body; the maternal body; the sexual body; the sick and disabled body; the raped and mutilated body and the metaphorical body.

11 As regards the corpus of texts scrutinised, I have been working on a range of fictional texts, mostly drawn from the May Fourth period, but also the literature produced in mainland China and Hong Kong over the last twenty years. Interestingly enough, most of the examples are drawn either from modern literature of the 1920s and 1930s or from contemporary Chinese literature from the turn of the 20th century. The reason for this apparently uneven selection is that Chinese fiction of the "seventeen-years period" (1949-66) seldom focuses on purely physical descriptions; moreover, the female body is never exposed in such a merciless and revealing way, highlighting its negative or uncanny meaning. In the 1980s, although we find many instances of "sensorial narratives” (for instance in Wang Anyi 王安忆 (b. 1954) and Han Shaogong 韩少功 (b. 1953) etc.), they are not body-centred; only with the "material turn" in Chinese literature, which occurred during the last decade of the twentieth-century, did a new narrative of the body develop which is connected with the growing individualism and the commodification of the human being in Chinese society at the turn of the century. ${ }^{17}$

\section{The allegorised body}

12 If we reconsider the whole history of modern Chinese literature, it is possible to trace the development of the awareness, negation, negotiation or invention of women's identity through a physical rather than psychological representation. Starting from the early 20th century, the female body has been a vehicle of both "modernity a" and "backwardness"; let's compare for instance these two kinds of texts:

She was born into a peasant family [...]. She was no more than a simple animal. [...] The day after, before dawn, she slipped out quietly, taking advantage of the fact that her husband hadn't yet woken up. The west wind was blowing, cutting her face like a knife, but she felt extremely satisfied because the pain was lighter than her husband's palm. (emphasis mine) ${ }^{18}$

伊生在农家, [...] 简直是很简单的一个动物。[...]明天朝晨, 天还没亮透, 伊轻轻 地走了出来, 私幸伊大夫还没醒。西风像刀, 吹到脸上很痛, 但是伊觉得比吃大 夫的巴掌痛得轻些, 就也满足极了。19

Lying on my bed is a plaster mannequin like the one displayed in the window of a women's merchandise store, the red flowers pattern on her chest [...] exuded a warm scent. Is this an organism or a non-organism? At night the plastic mannequin is also naked... Is this an organism or a non-organism? It is not a plaster mannequin, nor is it a marble statue or a snowman; it is some flowing lines transplanted from a painting and some cream forming the picture of a humane figure under my sheets. (emphasis mine) ${ }^{20}$

躺在床上的是妇女用品店楖窗里陈列的石衰模型, 胸脯儿那儿的图案上的红花,

[...] 挥发着热香。这是生物, 还是无生物呢? 石膏模型到了晚上也是裸体的 [...]。这是生物, 还是无生物呢? 这不是石膏模型, 也不是大理石像, 也不是雪 人; 这是从画上移植过来的一些流动的线条, 一堆Cream, 在我的被单上绘着人 体画。 21

Using slender ankles as the foundation, one leg standing erect and the other bent, is a platinum statue of a female nude. She stands there, an inorganic statue with no shame, no moral concepts or human desires. She is so metallic and sleek that his 
gaze swiftly glides over the lines of the body. This feelingless, emotionless statue stands there waiting for his order. ${ }^{22}$

把消瘦的脚踝做底盘，一条腿垂直着，一条腿倾斜着，站着一个白金的人体塑

像，一个没有羞惭，没有道德观念，也没有人类的欲望似的，无机的人体塑像。

金属性的, 流线感的, 视线在那躯体的线条上面一滑就滑了过去似的。这个没有

感觉，也没有感情的塑像站在那儿等着他的命令。 ${ }^{23}$

A statue of platinum! A female body without complexion and inhuman, what a strange flavour. Her feelings and her biologic structure are unknowable, the new sexual object of the year 1933 with a human shape but not a human nature or flavour.

“白金的人体塑像！一个没有血色，没有人性的女体，异味呢。不能知道她的感

情, 不能知道她的生理构造, 有着人的形态却没有人的性质和气味的一九三三年 新的性欲对象啊! ”24

The first text is drawn from Ye Shengtao 마조 陶 (1894-1988)'s denounce short story "This is a Human Being too", an early instance of critical realism from the "fiction of problems" / 问题小说 / wenti xiaoshuo genre, while the other three were written by the New Sensation's writer Mu Shiying 穆时英 (1912-1940), who describes the new style of Chinese life in a "semi-modern, semi-colonised" Shanghai. The female body conveys almost opposite yet very similar meanings in these two texts. In both cases we observe a strategy of externalization and defamiliarisation of the body: through the unfortunate peasant-girl in Ye Shengtao's story embodies a degree-zero humanity, while the body of the "modern girl" ${ }^{25}$ which haunts $\mathrm{Mu}$ Shiying's male characters is but a representation of the individual's reification in modern society.

In the former example the female body is "socialised" or, rather, "ideologised" as a symbol of the oppression exerted by traditional rural culture on the individual, while in the latter, Shih speaks of "eroticization and objectification of the female body". ${ }^{26}$

However, in both cases the representation ends up with a similar deformation or dehumanisation of the female body: the above descriptions, no matter what approach to Chinese society they reflect, are both marked by a shade of glumness, as though women's body bore all the heavy burden of past social inequity as well as the anxiety for an unknown future or an uncanny present. Nevertheless, while for Ye Shengtao and the other writers of the critical realism current, ${ }^{27}$ the female body conveys a clear social meaning, in the New Sensation School it is rather connected with a sense of "material possession among an array of objects [...] being at the forefront of technological modernity". ${ }^{28}$ Indeed, the female body in both cases is allegorised and dehumanised, becoming only a sign.

From this first category we can deduce that in different works within May Fourth literature the female body is a substitute for the individual and the ways in which it is exploited or feared in both traditional and modern China. It does neither represent women's subjectivity nor does it explore their inner reality, it only serves the purpose of expressing the anxieties of male writers of the time.

Although they belong to two very different literary schools, for both the abovementioned writers the female body must be de-humanised in order to depict an unstable and troubled society. The scandalous transformation of the woman subject from human to inhuman is only a tool for voicing a critical view of the present, ${ }^{29}$ and the premise for the utopian desire for a shift backward (or forward in the socially engaged writers' case) from an inhuman to a human condition. In her seminal book, 
Ping Zhu also argues that "the feminine is more of a space of negotiation than a natural essence associated with the female body". ${ }^{30}$

\section{The animal body}

Within the category of the animal body I place a variety of uses and depictions of the female body in modern and contemporary Chinese literature. The use of the metamorphic body, transformed into an animal or inanimate thing, was adopted and further developed by modern writers, often with opposite strategies and aims. In this sense, the inhuman reading of the female body is a long-standing practice in malecentred cultures; in the New Sensation School, for instance, the writers' main attitude is overtly based on the traditional view of woman as a dangerous sexual animal, notoriously represented by the rhetoric of the "fox-spirit" / 狐狸精 / hulijing. ${ }^{31}$ In the case of Mu Shiying's short story [“The Man Who Was Treated as a Plaything”] / 被当作 消遣品的男人 (1933), he adopts an animal-body interpretation in order to express the feeling of danger and anxiety conveyed by the modern girl and her sexual attraction:

She is a dangerous animal! She has a snake's body, a cat's head, a mixture of softness and danger.

她是危险的动物哪! 她有着一个蛇的身子, 猫的脸袋, 温柔和无限的混合物。32

The negotiation between male and female desire in modern Shanghai is represented as a hunting trip, but the narrator of the story is not sure who plays the role of the hunter and who that of the prey:

She is a dangerous animal, but I was not a good hunter. Had I captured her now, or was I being captured by her?

她是危险的动物, 而我却不是好猎手。现在算是捉到了吗? 还是我被抓住了呢?

[...]不能做她的狩猎物的。33

Animals are constantly associated with women by the female writer Xiao Hong 萧红 as well, who nevertheless does not use this association as an allegory or metaphor, but takes it literally as a real image of the woman/human condition. In Xiao Hong's works, the female body acquires a cognitive function. Indeed, it is through her own body and that of her characters that she experiences the world and brings readers in contact with their suffering.

The gradual transformation of the female body from human into inhuman is achieved by modern writers by means of either a metaphoric or a metonymic strategy. The latter is prevalent in Xiao Hong. The definition of "humanimal" created by Yinde Zhang ${ }^{34}$ perfectly suits Xiao Hong's representational strategy. As others have already noted, ${ }^{35}$ in The Field of Life and Death / 生死场 (1935), women and animals are associated as they share a common fate and life condition: "in their experience of the body, female animals and women have more in common than men and women do". ${ }^{36}$ However, we can reach a further level: in Xiao Hong's works, women are compared to animals in a very odd way, for she uses the literary technique of "defamiliarisation" (ostranenie) defined by Shklovsky, but her aim - unlike in the case of the Russian formalist - is not an aesthetic one, namely to reveal the naked literary form, but a cognitive one, insofar as she wishes to make her readers perceive women's reality in a stronger way. Below are some examples from Xiao Hong's novel:

Golden Bough is like a sick little chick

金枝好像患者传染病的小鸡一般 (57) ${ }^{37}$ 
Fifth Sister is like "a small lively pidgeon"

一个灵活的小鸽子 (66)

Old Mother Pockface "with her hair covering her face, is a female bear! The female

bear enters her cave carrying some herbs"

头发飘了满脸, 那样, 麻面婆是一只母熊了! 母熊带着草类进洞 (45)

While Old Mother Wang recounts the death of her little daughter, "she is often

described as an 'owl' by the children surrounding her"

常常围着小孩子们说她 ‘猫头鹰” (47) and twenty-first century Chinese literature are based on the perception of maternal bodies and motherhood. It is common knowledge that sociocultural control over the body has always involved women more than men - as in the Chinese practice of footbinding - issues such as birth control and surrogated motherhood are common subject in the works of both early modern and contemporary Chinese writers, who charge the maternal body with a variety of meanings.

Chinese fiction provides a range of touching and tragic descriptions of motherhood. Interestingly, this depiction is a gendered one, indeed, as it is explained by Clare Hanson: "For female writers, the focus is principally on the subjective experience of maternal embodiment and the difficulty of negotiating the somatic, psychological and 
social changes associated with pregnancy and birth", while "[m]ale writers, by contrast, regard motherhood from a social point of view, with pregnancy seen as a disruptive event that may threaten the stability of family ties" ${ }^{42}$ and - I add here - the stability of social order, as in Ma Jian (b. 1953)'s novel The Dark Road / 阴之道 (2012) and Mo Yan's Frogs / 蛙 (2009).

If we go back to the abovementioned short story by Ye Shengtao, we find that the girl's young body is forced to give birth to a baby: despite being a traditional custom in old China (and in many other rural cultures), according to modern sensibilities forced or surrogate motherhood is an absurd form of physical and psychological abuse. In Rou Shi's 柔石 (1902-1931) famous [“A Mother Slave"] / 奴隶的母亲 (1930), the description and the use of the maternal body are completely devoid of any personal identity. In this story, a poor leather-seller "lends" his wife to a rich childless couple who desires to finally have a male heir. Not only does the surrogated mother not have a name (it is never mentioned in the story and when asked by her "borrowing" husband she doesn't even answer), but the objectification of her body takes the form of a literal synecdoche, a specific case of metonymy in which the part stands for the whole. Throughout the short story the woman's belly replaces the female protagonist as a subject/object. The husband tells her: "What's the point of letting you suffer along with me? We'd better think of a way of exploiting your body" / 还是从你底身上设法罢你跟着我挨饿, 有什 么办法呢?.$^{43}$ Later, the matchmaker tries to persuade her to accept the deal, by saying: "It suffices that your belly fights to excel, in order to give birth to one or two [children], and everything will be okay" / 只要你肚子争气些, 到那边, 也养下一二个 来, 万事都好了. ${ }^{44}$

The woman's belly is even compared by the jealous wife of the scholar to that of a female dog: “Perhaps she is like a female dog, her belly filled with puppies" / 恐怕竟和 街头的母狗一样, 肚皮里有着一肚子的小狗. ${ }^{45}$ The alienation of the belly (merely used as a means of production) from the woman's body-mind as a whole is briefly exemplified in this sentence: "The belly kept on growing day by day, as big as a basin" / 肚子一天天地䐍胀的如斗那么大. ${ }^{46}$

We can apply to both cases Mingbao Yue's analysis of Ye Shengtao's story: "it exemplifies how the objective mode attitude can become untenable as a revolutionary mode of writing when the narrative focuses on the female body". ${ }^{47}$

The female writers adopt a different point of view when depicting motherhood. In Xiao Hong's case motherhood is neither a common way for expressing women's potential and the embodiment of a natural function, nor the object of men's exploitation; on the contrary, the pregnant body is a site of alienation for the woman herself. The pregnant body in her fiction is depicted as unnatural and unfamiliar to the woman herself, in contrast to the deterministic biologic view of the female body as naturally built for reproduction. I will refer here to two of Xiao Hong's characters: Jinzhi 金枝 (lit. “Golden Bough") from The Field of Life and Death and Qin 芹 from ["The Abandoned Child"] / 忘儿 (1936). The description of Golden Bough's pregnancy is laconic: "Golden Bough feels a deep pain, she feels her belly has become a fearful monster, there is something hard inside of her" / 金枝过于痛苦了, 觉得肚子变成个可怕的怪物, 觉得 里面有一块硬的地方. 48

31 In her autobiographical short story ["The Abandoned Child"], Xiao Hong provides us with a rather detailed record of uncomfortable and weird sensations connected with 
motherhood: the protagonist is first introduced as "a woman with a mantou-shaped belly" / 一个肚子凸的馒头般的女人..$^{49}$ But later in the story the narrator observes that “her belly does not resemble a mantou; it's a small basin tied onto her belly" / 她的肚子 不像馒头, 简直是小盆被扣在她肚皮上..$^{50}$ In the shabby hotel room where the pregnant woman is secluded,

she only feels a gloomy cold on her back. When she enters the single room walking on the dust that covers the floorboard, her legs are just two fake legs made out of wood, or else they are someone else's legs, forcibly tied to her body, numb, awkward.

只觉得背上有些阴冷。当她踏着地板的尘土走进单身房的时候, 她的腿便是用两 条木做的假腿, 不然就是别人的腿強接在自己的身上, 没有感觉, 不方便。 ${ }^{51}$

Eventually,

Qin's belly becomes bigger and bigger! From a small basin it has become a big basin; from an inanimate thing, an animate one. She can't sleep on her bed, the mosquitoes are having fun crawling on her legs and that thing is having fun crawling in her belly: she has simply become a circus, a place where everyone can go and play.

芹的肚子越胀越大了! 由一个小盆变成一个大盆, 由一个不活动的物件, 变成一 个活动的物件, 他在床上睡不着, 蚊虫在他的腿上走着玩, 肚子里的物件在肚皮 里走着玩，她简直变成个大马戏场了，什么全在这个场面上页起来。52

Other images enhance the character's physical estrangement and unease: such as “Qin's belly is aching badly, on the kang she rolls into a clay figurine" / 芹肚子疼得更厉害 了, 在土炕上滚成个泥人了,,$^{53}$ and "her breast grows stiff, as if something had been stuffed inside them" / 奶子胀得硬, 里面像盛满了什么似的. 54

One of the most graphic descriptions of unwanted motherhood is the one provided by Zhang Ailing 张爱玲 (1920-1995) in her autobiographical novel [Little Reunion] / 小团 圆 (2009), where she recounts the tragic episode of the abortion which her narrative alter ego Lili undergoes in the US:

She could feel the string of the suppository hanging down and brushing against her thigh like the fuse of a bomb. After several hours nothing had happened. [...]

That night, under the bathroom light, she saw a male fetus in the toilet bowl. In her terrified eyes it was at least ten inches long, leaning upright against the white porcelain, partly submerged in the water. The thin smear of bloody liquid on its skin was the light orange color of a newly planed wood. Fresh blood accumulated in its hollows and outlined its form distinctly. A pair of disproportionately large eyes protruded and its arms looked like the retracted wings of the wooden bird that used to perch above the door in Shanghai long ago.

In that moment of horror, Julie flushed the toilet. At first she worried the fetus would not flush away, but it soon disappeared into the billowing vortex..$^{55}$

她可以感觉到腿上拖着根线头, 像炸弹的导线一样。几个钟头后还没发作 [...]。 夜间她在浴室灯下看见抽水马桶里的男胎。在她惊恐的眼睛里足有十吋长, 毕直的 歌立在白磁壁上与水中, 肌肉上抹上一层淡淡的血水,成为新创的木头的淡橙色。 凹处凝聚的鲜 血勾划出它的轮廓来, 线条分明,一双环眼大得不合比例, 双睛突出, 拫着翅膀, 是从前站在门头上的木雕的鸟。

恐怖到极点的一刹那间, 她报动机钮。以为冲不下去, 竟在波涛汹涌中消失了。56

In modern Chinese literature, many authors, such as Rou Shi, Xiao Hong and her first husband Xiao Jun 萧军 (1907-1988), question the very meaning and aim of motherhood, insisting on cruel depictions of babies rejected or killed. The violence exerted on the maternal body (or on the child, as its extension) is a metonymy for the violence embedded in war and life in general; but, again, the description is a gendered one. Xiao 
Jun and Xiao Hong's novels both represent the act of killing a new-born baby, but the perspective and the bodily sensations reported are strikingly different. In the former case it is a Japanese soldier who kills the infant, by snatching him away from his mother's bosom, while in the latter, it is the very father of the girl-baby, a Chinese peasant, who pitilessly kills her by dropping her fall into a basin of scalding water. Clearly enough, for Xiao Jun - in his nationalistic drive - the violent death of the baby is but an act of war, an extreme form of political contempt for China, whereas Xiao Hong rather shifts our attention to the social and human meaning of suppressing a girl's life in China's backward countryside, denying at the same time the value of such a neglected motherhood.

Women writers of the 1980s, such as Zhang Xinxin 张欣欣 (b. 1957), Wang Anyi and Zhang Jie 张杰 (b. 1937) explore new ways of expressing their female identity by means of more physical, down-to-earth, material elements. But owing to their idealistic approach to literature - typical of the "culture fever" era - they mainly subjectivised and "psychologised" the physical perceptions, as in the following scene of a medical abortion:

“...Oh, it's a primipara...". While the doctor mumbles to himself, preparing the surgical instruments, I suddenly feel scared and wish to escape, but it's too late... The soft and nauseating smell of the chemical disinfectant that permeates the room, the dazzling white light blinding my eyes through the window, the white pillow case beside my head, the white sheet under my body, everything is so icecold... the noise of the pedal aspirator pierces my ears then stops. Then it starts again..., it's like I am going to be suctioned out!

“......吆, 第一胎......” 当准备手术器械的医士也嘟哝着时, 我突然感到恐惧和想要 逃脱, 可已经来不及了....... 那股软绵绵的, 叫人有些想吐的新洁尔灭消毒剂气 味弥散开来, 那直逼眼睛的, 窗上炫目的白光, 头边白色的枕套, 身下白色的床 单, 都冷冰冰的......脚踏吸引器刺耳的声音, 响了, 停了。又响了......我象是要被 抽空了!。57

Although the use of the first person in recounting the uneasy sensations of the protagonist enables a more direct representation of the female subjectivity, the descriptions are still quite literary.

On the contrary, in his latest novel Frogs, Mo Yan, adopts a mostly corporeal and sensorial style to describe forced abortions and abused female bodies in the Popular Republic of China. The community of Gaomi - the usual setting of Mo Yan's fiction - is upset by the frequent inspections of officials against illegally pregnant women.

In his case, however, the narrative focus is not on the female body per se: what really matters for Mo Yan is the vivid representation of social tensions due to the clash between the state's demographic control plan and the traditional Chinese vision of motherhood and reproduction.

In contemporary Chinese literature, it is, surprisingly, another male writer that manages to grasp the maternal body's "alienness" and its cognitive function. In his novel The Dark Road, Ma Jian explores the theme of a ruthless China chasing for pregnant women as the most brutal form of social control on individuals. Rather differently from Mo Yan's frantic action scenes, Ma Jian's narration seems to delve deeper into the physiological and subjective sphere of the female body.

41 I will just quote one of the last scenes from the novel, which is actually littered throughout with detailed representations of women's bodily perceptions: 
With the four fingers of her hand she pushes through the cervix, pierces the amniotic sac, gropes around and finds a foot. As she pushes again with all her strength, her contorted face turns scarlet and milk spurts from her nipples. [...] The lament fills every part of her body then bursts in the air. A rancid, yeasty smell starts to escape from her. After another intense push, blood drips out from her vagina onto the damp deck, forming blossom-like stains, then gushes out with greater force.[...] she cranes her neck down between her legs and sees it lying in a pool of blood, its body as green and shiny as an apple [...].

"Why is he so green?" Meili says. "He looks like one of those green aliens in the computer games.] (emphasis is mine) $)^{58}$

美黎憋红了脸, 乳头咝聯地喷奶, 破船在摇抖, 她感到所有的软肉都充满了气,

她吼着: “.......千呼万唤叫着妈妈, 想念孩儿死不瞑目, 孩儿跨在你面前, 不孝孩 儿赔罪了喽.......”歌声塞满血肉之后就冲到了塑料空气之中才散开, 一股浸泡很久 的酒酿味流到了枕头和白床单, 美黎低头抖着, 流淌的血先是如写意梅花般的点

缀, 继而如泼墨般挥洒, 美黎吼着 : “天堂哟, 你就下地狱吧! 妈等着你......”一 阵撕裂声之后, 天堂被母亲的残手掏了出来, 躺在血泊里浑身如绿苹果般闪光 [...]看他, 像电脑里的太空精灵, .......美黎喃喃地又说[... $]^{59}$

Such a disturbing and alienating record of physical perceptions - which reminds us of Dai Wei, the protagonist of Ma Jian's previous novel, Beijing Coma, who narrates the "travel" inside his own body's viscera $-{ }^{60}$ and the visual hallucinations in the ending scene of The Dark Road move beyond political accusations levelled against the Chinese government and Chinese traditional culture for their abuse of the female body, to become an experience of "augmented reality" of the female body. Indeed, by means of these powerful descriptions and the adoption of an external viewpoint, that of the unborn baby (Infant Spirit), Ma Jian suggests that the maternal body has a double sensitiveness, for it combines Meili's with that of the Infant Spirit - indeed, it is as though it possessed a quality of enhanced cognition of reality. At the same time, as was the case with Xiao Hong's lucid representation of the uncanny feeling a woman gets of carrying another being inside her body, Ma Jian adopts a similar strategy in emphasising the alienness of such an event, birth, that is usually perceived as totally natural.

Finally, another example of de-familiarisation of the maternal body is provided by science fiction literature: in “Story of a Titan" by the female writer Ling Chen 凌晨, a woman gives birth to her child on Saturn, while the whole scene is recorded by cameras and broadcasted on the Earth: the narrator of the story - similarly to the unusual perspective adopted by Ma Jian in The Dark Road with the Infant Spirit - is the baby himself, who comments on the birth process from inside his mother's uterus in a very scientific manner:

she'd shift her huge body $(332)^{61}$

“妈妈便推动她庞大的身体” (15) ${ }^{62}$

Through the skin of Mama's belly, I murmured from deep within the womb, relating information obtained from genes and blood - breathing, absorption, excretion, perception, memory. I began from a fertilized egg and developed organs, muscles, skin, and bones (340)

“隔着妈妈的肚皮, 我在子宫深处呢喃, 叙述从基因和血液中获取的信息一呼吸、 吸收、排泄、感知、记忆, 我从一颗受精卵开始, 发育出器官、肌肉、皮肤、骨 骼” (17)

Yes, Mama, don't worry. I'll swim smoothly out of the womb and fly into your embrace.

Almost as if the uterus understood me, a strong force suddenly pushed me toward the cervix. (344) 
“是的, 别担心妈妈, 我会顺畅地游出子宫, 扑向你的怀抱。好像是子宫听明白

了我的话似的, 一股強劲的力量忽然将我往子宫口推去。”(17)

"Do they want to play God?" Mama asked with difficulty. She had endured ten hours of contractions and scoffed at the efficiency of the work Papa and the others were doing. Now that my head had slid into the pelvic cavity I was quite uncomfortable. Holes had ruptured all over the placenta that had been protecting me, and the familiar film and fluid flowed on all sides and blocked my exit. I was tired, and I needed to rest a while. (344)

“他们想当神吗? ’妈妈艰难地问。她已经忍受了十个小时的宫缩, 对爸爸他们工 作效率虽之以鼻。而我因为头滑入骨盆腔感到很不舒服, 一直保护我的羊膜破裂 出一个一个的洞洞, 我熟悉的膜液四处流淌, 堵塞了我的出口。我累了, 得休息 一会儿。(18)

The father watching from outer space, sighs

“My wife's childbirth is being broadcast live, and I can't see it!" (349)

“我妻子分娩直播, 我都看不到! ”(19)

The final, crucial moment of the delivery is dryly described by the baby. All emotions and sensations are recorded in a very impersonal way and while the physical pain is reported, it is as though the mother's body had been de-humanised.

I had entirely entered the birth canal. It was a narrow and bumpy channel with a faint light all the way at the far end. I was squeezed, and I felt incredibly uncomfortable. My pain passed to Mama, and then her whole body was in pain and she cried out. (352)

我已经完全进入了产。那是一条狭窄崎岖的通道, 只在很远的地方有微弱的光

亮, 我被挤压得非常不舒服。我的痛苦传递到妈妈身上, 她也浑身疼痛着喊叫起 来。”(20)

The female body is treated here as a mere baby-producer. Although the first person narrator is the baby himself and he reports his own birth, trying to recollect the emotions of his mother and father at the time, in general the narrative comes across as a fairly bleak description. In the ending of the short story, the narrator quietly comments about his birth occurring at the very time when his father, a scientist, is carrying out an important bacteriological experiment on planet Saturn:

I and that bacteria would grow side by side in the sky of Saturn VI, and the future was uncertain. (361)

“我将与这些细菌一起在卫六的星空下成长, 前景无法确定是不是光明。” (21)

We can conclude this section by quoting Hanson again: "Such anxious constructions of the maternal body are surely an index of the inchoate fears that contribute to surround reproduction and the lease on the future which it represents". ${ }^{63}$

\section{The sexual body}

Many authors of modern and contemporary Chinese literature, who were partially inspired by Freudian theories from the 1920s onward, represent women through a sexualised body. Although the New Sensationists' works are replete with descriptions of this kind, I have chosen two very different texts, one by Lu Ling 路悀 (1923-1994) and the other by Lin Bai 林白. In many cases the sexual body is endowed with an uncommon, almost upsetting quality: Lu Ling's narrative of female sexual desire emphasises its physicality and the phenomenology of the perceptions, which, at the same time, it is also the expression of a spiritual hunger, as the title of the novelette 
suggests: ["Hungry Guo Su'e"] / 饥饿的郭素娥 (1942). The following excerpts are just a sample of Lu Ling's vivid narrative of the phenomenology of female sexuality:

The emotions of that woman, full of sexual desire and dreams, were so acute...

充满情欲和梦的女人的感觉是那样的敏锐 $[. . .]^{64}$

Her cheeks were rosy, shining with a feeling of rich, wild joy. A smile of happiness flashed across her fleshy lips showing traces of lust. When she rapidly moved her arm, looking for the hoe, in the sunlight her teeth shined with a white glow, her bosom heaving fast.

她的脸颊红润, 照耀着丰富的狂喜。在她的刻画着情欲的印痕的多肉的嘴唇上, 浮显了一个幸福的微笑。当她把手臂迅速地挥转, 寻受短锄的时候, 她的牙齿在 阳光里闪着坚实的白光, 她的胸膛急速地起伏着。65

Passing by a short tree, he leaned on its trunk and brought his shrinking face down, stuck out his tongue and licked her lips and nose. Guo Su'e, wrapped in his strong male arms, cast away all her worries and melancholy, all her fears and hate. She woke up from the poisoned, silent darkness and laughed uncouthly, lasciviously, impudently.

在经过一株低矮的小树的时候, 他把背资依着树干俯下紧紧收缩的脸, 伸出大舌 头来舐着她的嘴唇和鼻子。在男人的強壮的臂弯里的郭素娥, 这时候摆脱了一切 挂虑, 摆脱了一切悲愁, 惶恐和怨恨, 从有毒的黑暗的沉默里醒来, 发出了粗野 的滛荡的, 放肆的欢笑。6

Every single emotion of the protagonist and even her slightest physical reactions to the external world are recorded with accurate, almost morbid precision. The excessive use of adjectives and adverbs - a hallmark of Lu Ling's style - builds a strongly mimetic image of the woman, whose inner self and body seem to overlap completely.

Turning to the contemporary writer Lin Bai's descriptions of the sexual female body, I will provide an excerpt from [“The Time of Cat's Passion”] / 猫的激情时代 (2001):

In my dreams Cat uses her lithe, slender fingers to stroke my face. She lowered her body close to me, and in a whisper says: Do you know who I love the most? [...] Cat's cool breath sirs the fine hair on my body, arousing a feeling of tender care. I say I don't know. She lowers her long eyelashes and says: You can imagine me as a man. I say that if you were one, I wouldn't have liked you. ${ }^{67}$

猫在我的梦中用她柔软修长的手指抚摸我的脸, 她俯身凑近我, 用耳语般的声音

对我说 : 你知道我最爱谁吗? [...]

猫的气息凉凉地吹动我细小的汗毛，使我有一种体贴入微的感觉。我说我不知

道。她垂下她长长的睫毛说：你把我想象成男的。我说你当了男的我就不喜欢你

了。68

In this delicate but unequivocal description of female same sex attraction, the corporeality of the two protagonists is not a metaphorical one: Lin Bai displays the sexual body in its literal perceptual dimension. In the short story, she extends this dimension further through the contrast with the dead body of Cat, one of the lovers who, as we learn at the very beginning of the story, has been executed for having killed a man after an unpleasant sexual intercourse.

Sexual body and dead body are thus placed on the same level: desire and death are both phenomena which only bodily sensations make real. 


\section{The raped and mutilated female body. women's controversial agency}

53 Xiao Jun, Lu Ling, Xiao Hong, and more recently Mo Yan and Ge Fei 格非 (b. 1964) - to take only a few authors - present the ravaged female body from a variety of perspectives, ranging from an emphasis on its naked physicality to hints at psychological unease. The famous episode of the young bride raped in the sorghum field from Mo Yan's [The Clan of Red Sorghum] / 红高梁家族 (1985) has already been studied and treated as an example of misogyny by the Chinese scholar Lu Tonglin. She interprets Mo Yan's depiction of the raped female body as a sacrificial object representing male desire, deprived of any identity and agency: "the female body, like the landscape with which it identifies, is largely excluded from the world of dynamic movement". ${ }^{69}$

Similarly, although with very different aims, in a war scene where a young Japanese soldier rapes a Chinese peasant woman, Xiao Jun portrays the violence inflicted upon the female body in order to elicit a nationalistic sense of ire, without really empathising with the character in her physically and psychologically damaged condition. On the contrary, in presenting an analogous episode, where the vicious act is actually perpetrated by a Chinese man on Golden Bough in The Field of Life and Death, Xiao Hong enhances the character's pathetic hopelessness and manages to instill a sense of sober desperation in the reader.

Nevertheless, in other cases the literary representation of abuses on the female body unexpectedly provides women with a strong agency and power. As Kirk Denton has demonstrated, in Lu Ling's novelette ("Hungry Guo Su'e"), it is Guo Su'e's spiritual independence and hunger, and not her body as an objectified sexual target, that is mostly feared and thus violated by men in the final cruel rape and murder scene:

Guo Su'e was dizzy, her whole body was cold, the parts of her which had been branded dripped blood. But Huang Mao's big hands groped among the blood, over her naked body. With a kind of cowardly wildness he eyed the witheness of her flesh [...] he lowered his body and quietly climbed on the top of her. ${ }^{70}$

郭素娥昏晕着, 全身冰冷, 在烧伤的地方淌着血水。但黄毛的大手却从血水中

间, 在她的赤裸的身体上摸索着。他带着一种胆怯的昏狂, 注视着她的肌肉的白

色，[...]，他就伏下身子悄悄爬到她的身体上去。71

Whereas the tragic reality of history offered to modern writers a scary and truthful landscape of rapes - such as those associated with the Nanjing massacre (1937) and the war period - it is undeniable that rape is also the greatest and cruellest form of control over the female body, together with mutilation and cannibalism. This is the case in Han Song's story [The Guide to Hunting for Women], with its island inhabited by artificially created women, brought into being only to satisfy urban men's craving for strong emotions. The story argues that the female body is not only a prey for the hunters, but that these artificial clones - designed to satisfy men' sexual fantasies and stereotypes also represent the male obsession with the elusiveness of the female body. Han Song offers a reflection on the mystery of gendered bodies and men's essential misunderstanding or ignorance in terms of female organic and bodily life: in one scene of this grotesque hunt for women, one of the hunters, after killing the female prey, eats 
her uterus in a final attempt to grasp women' secret and extinguish his own sexual hunger.

The women on the island were just purebred animals provided with ovaries and uterus, they had not been contaminated yet with the vanity of cosmetics and jewels, and money. Their wild nature was their main feature.

岛上的女人, 不过就是一种长有卵巢和子宫的纯种动物, 没有受到化妆品, 首饰 虚荣心和金钱的污染，而野性是她们的首要特征。72

Looking blankly at the woman's strange abdomen, Xiao Zhao remembered when, 36 years before, he had lived inside a similar flesh cavity. His relation with women was just the relation between a hosting body and a parasite, but maybe this was precisely the nature of all his relations with them.

怔怔地看着女人奇怪的腹部, 小昭记起来, 三十六年前, 自己就寄居在这同样的

肉体和腔膛里面呀。他与女人, 不过是宿体与寄生的关系, 而说不定, 这正是这

世上一切关系的本质呢。73

57 In the horrific scene towards the end of the novel, the man, who during the fight against the female-clone has castrated himself by mistake, thoroughly appropriates the body of the female clone by eating her reproductive organs, like a parasite appropriates the body of its host.

Indeed, these descriptions of the female body are connected to a post-human, postsocialist view of the individual and of gender, although the scene also echoes the dystopian theme of cannibalism and its long-standing tradition in Chinese literature, from Lu Xun 鲁迅 (1881-1936) to Mo Yan. The science fiction writer ends the story with a surreal and ambiguous solution to the war between the sexes, envisaging a simultaneously utopian and dystopian society of dis-gendered or post-gendered bodies (“ungendered" / 无性 / wuxing).

Similarly dark and brutal is the description of a female corpse raped by two thugs in Sino-American female writer Yiyun Li's in debut novel The Vagrants (2009): set in a Chinese town just one year after Mao Zedong's death, the story features a young woman executed for her counter-revolutionary activities. However, the violence exerted on her body, both before and after her death, is not so much a form of political violence as the physical expression of an existential regression of humankind in one of the darkest phases of Chinese history, where the value of an individual, especially if female, has been thoroughly depreciated or erased.

\section{The sick body}

Modern and contemporary literature mercilessly shows sick and disabled female bodies, the physical disease often being a sign of psychic unease. According to Larson, as Rong Cai puts it, "[i]n stories by female writers, women who write are often sick, weak, or commit suicide. As a result the female body had to be effaced trough sickness or death to allow the emergence of the male activity of writing". ${ }^{74}$ Ping Zhu observes that "[i]f the body is the location of socially manufactured desires, a sick body signifies the denunciation of the desires produced by the masculinist society." 75

61 Another critic, Yang Xin, ${ }^{76}$ referring to "Beauty Writers" / 美女作家 such as Chen Ran 陈染 and Annie Baobei reminds us that sickness is a "narrative strategy and imaginative tool for women writers to explore identity in times of rapid social change". 
ary to this metaphorical interpretation of the sick body, Xiao Hong exposes the female body in all of its outrageousness: in The Field of Life and Death, Yueying, a young and beautiful girl in the village, is mercilessly depicted lying in her bed, paralysed, her body covered with her own faeces.

63

is a screeching, dissonant image which clashes with the "New Woman" vision of feminine beauty in the nationalist discourse of the time, as expressed by the so called Jianmei Movement: "healthy curves and national ideals of health and beauty during the Sino-Japanese war were set; women's strong physiques were promoted as the science of the normal with manipulated, managed and disciplined workout routines". ${ }^{77}$ From a cognitive perspective, Xiao Hong again explores sorrowful human existence by means of the embodiment of pain in her characters.

If we applied here the neuroscientific theories on "shared emotional states", we could infer that the reader exposed to such sorrowful descriptions can experience similar physical and mental states. On the other hand, the new cognitive sciences have also undergone a narrative turn: it is indeed possible to analyse and describe our perceptions as a narrative.

65 Finally, I would like to mention the Hong Kong-based female writer Hon Lai-chu's (Han Lizhu 韩丽珠, b. 1978) novels The Kite Family / 凤筝家族 (2008) and Body Sewing / 缝身 (2012): this author makes a completely different choice in the use of the sick or deformed female body. Differently from Xiao Hong, she rejects any realistic interpretation of her narrative, deliberately crossing the border into the unreal, the imaginary. In The Kite Family, she represents a strong existential and social unease through an "unnatural narrative": ${ }^{78}$ the women of the family, whose body grows monstrously until there is not enough space in the house to contain them, are the symbol of a more general spiritual unease in contemporary Hong Kong society. Hon Lai-chu refuses to provide any lifelike details, the physical perceptions of her characters are completely detached from their corpo-reality: bodies, especially female ones, are the signs of a new language. By having the novel embodied by a metaphorically sick body - what is more, a female body - the author provides her text with a simultaneously symbolic and cognitive function, representing women's uneasy and problematic social condition figuratively.

I looked at Mother and said: "Grandmother's body is like a rainbow coloured balloon, floating up into the sky until it pops." [...] Together they'd carried her from her hospital bed to the back of a truck they'd hired to take her home. Once home, they shut her up in an empty room. The hereditary family fat had reached its inevitable stage and there was nothing we could do to stop it. It built up rapidly in Grandmother's body, spreading aggressively and increasing in volume [...] In the days leading up to her death, Grandmother became too enormous for any room to contain, and the family had to find a contractor to break down the wall between two rooms so that Grandmother could stretch out properly. ${ }^{79}$

我看着母说：“她肥㭌的身体, 像七彩的气球, 飘到空中爆破。” [...] 他们合力把 她从医院的床上抬起来放进招来的车上, 运送回家, 然后把她关在其中一个空房 间里。当家族遗传的脂肪, 在令人猝不及防的情况下, 在外租母的的身体内迅速 集聚, 生机勃勃地不断壮大。[...] 外祖母去世前的一段日子, 身体庞大得无法容 纳在一个房间内, 他们不得不找来装修工人, 拆掉两个房间中央的墙壁, 外祖母 才能顺利地躺下泪。80

The body is at the centre of Hon's anti-mimetic narrative also in the novel Body Sewing, where the long-standing issues of the social recognition of women through marriage and coupling conventions are questioned. Hon describes a dystopian society where 
couples are pushed to undergo surgery and have their bodies literally be sewed together, so as to attain a perfect harmony at both the individual and collective level. In this novel, stereotypical social relations and obsolete conventions on marriage and love are placed by Hon within a surreal frame, with an almost Gothic feel to it. In a city reminiscent of contemporary Hong Kong, women are legally encouraged to be physically sewn to the body of a man in order to guarantee themselves a future of socially acknowledged "normality".

\section{Conclusion}

67 To conclude, images of distorted, abused and uncanny female bodies dominate Chinese fiction in both the 20th and the beginning of the 21st century. Metaphorical or metonymical strategies underpin two mains literary functions: the symbolic and the cognitive, both of which define the female body as a site of contradiction or negotiation, and a tool for experiencing reality at a deeper level, which deserves to be further explored. This literary practice, strongly based on the corporeality of women's existence and representation, which links the outer experience (text) to the inner experience (body), can be well explained through Merleau-Ponty's words:

External perception and the perception of one's own body vary in conjunction because they are the two facets of one and the same act. ${ }^{81}$

The body is the vehicle of being in the world, and having a body is, for a living creature, to be intervolved in a definite environment, to identify oneself with certain projects and be continually committed to them. ${ }^{82}$

\section{BIBLIOGRAPHY}

ALBER Jan, HEINZE Rüdiger, Unnatural Narratives - Unnatural Narratology, Berlin, Boston, De Gruyter, 2011.

BALLERIO Stefano, "Stefano Calabrese, Neuronarratologia. Il futuro dell'analisi del racconto" /

[Stefano Calabrese, Neuronarratology. The Future of the Analysis of Fiction], Enthymema 1:2010, p. 268-74.

CAI Rong, The Subject in Crisis in Contemporary Chinese Literature, Honolulu, Hawai'i U. P., 2004. CHANG Eileen, Little Reunion, WEIZHEN PAN Jane and MERZ Martin (trans.), New York, New York Review of Books, 2018.

DENTON Kirk A, “Lu Ling's Literary Art: Myth and Symbol in 'Hungry Guo Su'e”, Modern Chinese Literature (2:2), 1986, p. 197-209.

GLADZIEJEWSKI Paweó, "Shared Representations, Perceptual Symbols, and the Vehicles of Mental Concepts”, Journal of Consciousness Studies, vol. 20, 3-4: 2013, p. 102-24.

GolDBLATT Howard, Xiao Hong. Boston, Twayne Publishers, 1976. 
HAN Song 韩松, [“The Guide to the Hunting for Women”] / 美女狩猎指南, in [The Tomb of Universe] / 宇宙墓碑, Shanghai, Shanghai renmin chubanshe, 2014, p. 275-373.

HANSON Clare, "The Maternal Body", in The Cambridge Companion to The Body in Literature, HILLMAN David and MAUDE Ulrika (eds.), Cambridge, Cambridge U. P., 2015, p. 87-100.

HILLMANN David and ULRIKA Maude (eds.), The Cambridge Companion to The Body in Literature, Cambridge, Cambridge U. P., 2015.

HON Lai-Chu (Han Lizhu 韩丽珠), [The Kite Family] / 凤筝家族, Taibei, Lianhe wenxue chubanshe, 2008.

HON Lai-Chu (Han Lizhu 韩丽珠), [Body Sewing] / 缝身, Taibei, Lianhe wenxue chubanshe, 2010.

HON Lai-Chu, The Kite Family, LINGENFELTER Andrea (trans.), Hong Kong, Hong Kong U. P., 2015.

KANG Xiaofei, The Cult of the Fox: Power, Gender, and Popular Religion in Late Imperial and Modern China, New York, Columbia U. P., 2005.

KINKLEY Jeffrey C., Visions of Dystopia in China's New Historical Novel, New York, Columbia U. P., 2015.

LI Rong 李荣, [The Body Explanation of Modern Chinese Literature] / 中国现代文学的身体阐释, Beijing, Zhongguo Shehui kexue chubanshe, 2009.

LIN Bai 林, [“The Time of Cat's Passion”] / 激情的猫时代, in [The Time of Cat's Passion] / 激情的 猫时代, Tianjin, Zhongguo wenlian chubanshe, 2001, p. 237-41.

LIN Bai, “The Time of Cat's Passion”, tuft Bryna (trans.), Renditions 79, 2013, p. 104-7.

LING Chen 凌晨, [A Story of Titan] / 泰坦的故事, Kehuan shijie 7, 2007, p. 15-21.

LING Chen, “A Story of Titan”, MARTINSEN Joel (trans.), Pathlight, 2013, p. 323-63.

LIU Lydia, "The Female Body and Nationalist Discourse: Manchuria in Xiao Hong's Field of life and Death", in Body, Subject, and Power in China, zITO Angela and BARLow Tani (eds.), Chicago, Chicago U. P., 1994, p. 157-178.

Lu Ling 路悀, [“Hungry Guo Su'e”] / 饥饿的郭素娥 / Ji'e de Guo Su'e, in Lu Ling, [Selected Works] / 路翎文集 / Lu Ling wenji, Hefei, Anhui wenyi chubanshe, vol. IV, 1995, p. 1-112.

LU Tonglin, Misoginy, Cultural Nihilism and Oppositional Politics: Contemporary Chinese Experimental Fiction, Stanford, Stanford U. P., 1995.

MA Jian 马建, [Land of Flesh] / 肉之土 / Rou zhi tu, Taipei, Taichen wenhua, 2010.

MA Jian 马建, The Dark Road / 阴之道 / Yin zhi dao, Taipei, Taichen wenhua, 2012.

MA Jian 马建, The Dark Road, DREW Flora (trans.), London, Vintage Books, 2014.

MAN Eva Kit Wah, Bodies in China. Philosophy, Aesthetics, and Politics, Hong Kong, The Chinese U. P., 2016.

MERLEAU-PONTY Maurice, Phenomenology of Perception, New York, Routledge, 2012.

MOORE Lisa Jean and CASPER Monica J., The Body. Social and Cultural Dissections, London and New York, Routledge, 2015.

MU Shiying 穆时英, [“The Man Who Was Treated as a Plaything”] / 被当作消遣品的男人 / “Bei dangzuo xiaoqianpin de nanren”, in [Tomb] / 公墓 / Gongmu, Tianjin, Bai hua wenyi chubanshe, 2005 [1931]. 
MU Shiying 穆时英, “Craven A.”, in 圣处女的感情 / Shengchunü de ganqing, Hangzhou, Zhejiang wenyi chubanshe, 2004 [1933], p. 21-42.

MU Shiying 穆时英, [“The Platinum Statue”] / 白金女体塑像 / “Baijin de nüti suxiang”, in [Holy Virgin's Love] / 圣处女的感情 / Shengchunü de ganqing, Hangzhou, Zhejiang wenyi chubanshe, 2004 [1934], p. 72-84.

PIRAzzoli Melinda, "Breaking Up From What? The Corporeal Politics of Values in the Duanlie yundong (Rupture Movement)”, Annali Ca' Foscari. Serie Orientale 55:2019, p. 363-93.

ROJAS Carlos, Homesickness. Culture, Contagion, and National Transformation in modern China, Boston, Harvard U. P., 2015.

ROU Shi 柔石, [“A Mother Slave”] / 为奴隶的母亲 / “Wei nuli de muqin”, in [February] / 二月 / Eryue, Hangzhou, Zhejiang wenyi chubanshe, 2005 [1930], p. 271-98.

SANG Tze-lan D., "The Modern Girl in Modern Chinese Literature”, in A Companion to Modern Chinese Literature, zHANG Yingiin (ed.), Malden, John Wiley \& Sons, 2016, p. 411-423.

SHARP Lesley A, “The Commodification of the Body and Its Parts", Annual Review of Anthropology 9:2000, p. 287-328.

SHIH Shu-mei, The Lure of the Modern: Writing Modernism in Semicolonial China, 1917-1937, Berkeley, California U. P., 2001.

XIAO Hong 萧红, [“The Field of Life and Death”] / 生死场 / Shengsi chang, in [The Complete Works of Xiao Hong] / 萧红全集 / Xiao Hong quanji, Ha'erbin, Heilongjiang daxue chubanshe, vol. 1, 2011 [1935], p. 39-134.

XIAO Hong 萧红, [“The Abandoned Child”] / 弃儿 / Qi'er, in [The Complete Works of Xiao Hong] / 萧红全集 / Xiao Hong quanji, Ha'erbin, Heilongjiang daxue chubanshe, vol. 4, 2011 [1933], p. 133-46.

XIN Yang, "Configuring Female Sickness and Recovering: Chen Ran and Annie Baobei", Modern Chinese Literature and Culture 23, 1:2011, p. 169-96.

YE Shengtao 叶圣陶, [“This is a Human Being Too"] / 这也是一个人 / “Zhe ye shi yi ge ren”, in [The Collected Works of Ye Shengtao] / 叶圣陶集 / Ye Shengtao ji, Suzhou, Jiangsu jiaoyu chubanshe, vol. 1, 1987, p. 99-102.

YUE Mingbao, "Gendering the Origins of Modern Chinese Fiction”, in Gender and Sexuality in Twentieth-Century Chinese Literature and Society, Lu Tonglin (ed.), Albany, State University of New York Press, 1993, p. 47-66.

ZHANG Ailing 张爱玲, [Little Reunion] / 小团圆 / Xiao tuanyuan, Beijing, Zuojia chubanshe 2009. ZHANG Xinxin 张辛欣, [“On the Same Horizon”] / 在同一地平线上 / Zai tongyi dipingxian shang, in [Representative works] / 张辛欣代表作 / Zhang Xinxin daibiao zuo, Zhengzhou, Huanghe wenyi chubanshe, 1988.

ZHANG Yinde, "The Fiction of Living Beings. Man and Animal in the Work of Mo Yan", China Perspectives 3:2010, p. 124-32.

ZHU Ping, Gender and Subjectivities in Early Twentieth-Century Chinese Literature and Culture, New York, Palgrave MacMillan, 2015.

ZITO Angela and BARLow Tani (eds.), Body, Subject and Power in China, Chicago, Chicago U. P., 1994. 


\section{NOTES}

1. MERLEAU-PONTY Maurice, Phenomenology of Perception, New York, Routledge, 2012, p. 23.

2. GLADZIEJEWSKI Paweó, "Shared Representations, Perceptual Symbols, and the Vehicles of Mental Concepts”, Journal of Consciousness Studies, vol. 20, 3-4:2013, p. 103.

3. "Tramite la narrativa si rappresentano le operazioni cognitive umane e che i lettori espandono così, mediante esempi invece che mediante una teoria, la propria comprensione dell'essere umano" (BALLERIO Stefano, "Stefano Calabrese, Neuronarratologia. Il futuro dell'analisi del racconto", Enthymema, 1:2010, p. 270). Here and in other quoted passages all translations into English are mine unless otherwise stated.

4. GLADZIEJEWSKI, “Shared Representations...", op. cit., p. 105.

5. MERLEAU-PONTY, Phenomenology of Perception, op. cit., p. 232.

6. zITO Angela and BARLow Tani (eds.), Body, Subject and Power in China, Chicago, University of Chicago Press, 1994.

7. LI Rong 李荣, [The Body Explanation of Modern Chinese Literature] / 中国现代文学的身体阐 释, Beijing, Zhongguo Shehui kexue chubanshe, 2009.

8. ROJAS Carlos, Homesickness. Culture, Contagion, and national Transformation in modern China, Cambridge, Harvard U. P., 2015, p. IX.

9. Ibid., p. IX.

10. KONG Belinda, Tiananmen Fictions Outside the Square: The Chinese Literary Diaspora and the Politics of Global Culture, Philadelphia, Temple U. P., 2012.

11. ZHANG Yinde, "The Fiction of Living Beings. Man and Animal in the Work of Mo Yan", China Perspective, 3:2010, p. 128-9.

12. A literary school which flourished in Shanghai in the 1930s, emphasizing sensations and physical perceptions of modern life in the city of Shanghai.

13. KINKLEY Jeffrey C., Vision of Dystopia in Chinese New Historical Novels, New York, Columbia U. P., 2015.

14. PIRAZzoLI Melinda, "Breaking Up From What? The Corporeal Politics of Values in the Duanlie yundong (Rupture Movement)", Annali di Ca' Foscari. Serie orientale, 55:2019, p. 363-93.

15. HILLMANN David and MAUDE Ulrika (eds.), The Cambridge Companion to The Body in Literature, New York, Cambridge U. P., 2015, p. 5.

16. Ibid., p. 4.

17. Many examples of the use and consumption of the human body in China are provided in SHARP Lesley A., "The Commodification of the Body and Its Parts", Annual Review of Anthropology, 29:2000, p. 287-328.

18. All translations are mine, unless otherwise indicated.

19. YE Shengtao 叶圣陶, [“This is a Human Being Too”] / 这也是一个人/Zhe ye shi yi ge ren, in [The Collected works of Ye Shengtao]/ 叶圣陶集 / Ye Shengtao ji, Suzhou, Jiangsu jiaoyu chubanshe, vol. 1, 1987, p. 99-100 passim.

20. SHIH Shu-mei, The Lure of the Modern: Writing Modernism in Semicolonial China, 1917-1937, Berkeley, Los Angeles, London, California U. P., 2001, p. 321.

21. MU Shiying 穆时英, “Craven A”, in [Holy Virgin's Love] / 生处女的感情, MU Shiying (ed.), Hangzhou, Zhejiang wenyi chubanshe, 2004 [1933], p. 33-34.

22. SHIH, The Lure of the Modern..., op. cit., p. 321.

23. MU Shiying 穆时英, [“The Platinum Statue”] / 白金的女体塑像, in [Holy Virgin's Love] / 圣处 女的感情, MU Shiying (ed.), Hangzhou, Zhejiang wenyi chubanshe,2004 [1934], p. 82-83.

24. Ibid., p. 83. 
25. On the figure and the theme of the modern girl see SANG Tze Lan D., "The Modern Girl in Modern Chinese Literature", in A Companion to Modern Chinese Literature, zHANG Yingjin (ed.), Malden, John Wiley \& Sons, 2016, p. 411-423.

26. SHIH, The Lure of the Modern..., op.cit., p. 319.

27. This term, similarly to Yan Jiayan's widely accepted definition ("social analysis school" / 社 会剖析派 / Shehui pouxi pai), defines a good number of writers who were similarly committed to a critically-oriented writing approach in 1920s and 1930s in China.

28. SHIH, The Lure of the Modern..., op. cit., p. 319-20.

29. A negative interpretation of the short story, in terms of paternalism and almost misogyny, has been given by YUE Ming-Bao, "Gendering the Origins of Modern Chinese Fiction", in Gender and Sexuality in Twentieth-Century Chinese Literature and Society, Lu Tonglin (ed.), Albany, State University of New York Press, 1993, p. 47-66.

30. zHU Ping, Gender and Subjectivities in Early Twentieth-Century Chinese Literature and Culture, New York, Palgrave Macmillan, 2015, p. 158.

31. KANG Xiaofei, The Cult of the Fox: Power, Gender, and Popular Religion in Late Imperial and Modern China, New York, Columbia University Press, 2005.

32. MU Shiying 穆时英, [“The Man Who Was Treated as a Plaything”] / 被当作消遣品的男人, in [Tomb] / 公墓, MU Shiying (ed.), Tianjin, Baihua wenyi chubanshe, 2005 [1931], p. 2.

33. Ibid., p. 12-13.

34. zHANG, "The Fiction of Living Beings. Man and Animal in the Work of Mo Yan", op. cit., p. 129.

35. GoldBlatT Howard, Xiao Hong, Boston, Twayne Publishers, 1976; LIU Lydia, "The Female Body and Nationalist Discourse: Manchuria in Xiao Hong's Field of life and Death", in zITo Angela and BARLow Tani (eds.), Body, Subject, and Power in China, Chicago U. P., 1994, p. 157-78.

36. Ibid., LIU, "The Female Body and Nationalist Discourse...", p. 165.

37. The page number after each quotation refers to XIAO Hong, The Field of Life and Death / 生死 场 / Shengsi chang, in Xiao Hong quanji 萧红全集 (Complete Works), vol. I, Ha'erbin, Heilongjiang daxue chubanshe, 2011 [1935].

38. HAN Song 韩松, [“The Guide to the Hunting for Women”] / 美女狩猎指南, in [The Tomb of Universe] / 宇宙墓碑, Shanghai, Shanghai renmin chubanshe, 2014, p. 275-373.

39. HAN, [“The Guide to the Hunting for Women”] / 美女狩猎指南, op. cit., p. 279.

40. MOORE Lisa Jean, CASPER Monica J., The Body. Social and Cultural Dissections, London, New York, Routledge, 2015, p. 190.

41. Ibid., p. 192.

42. HANSON Clare, "The Maternal Body", in The Cambridge Companion..., HILlmAN David and MAUDE Ulrika (eds.), op. cit., p. 96.

43. Rou Shi 柔石, [“A Mother Slave”] / 为奴隶的母亲, in [February] / 二月, Hangzhou, Zhejiang wenyi chubanshe 2005 [1930], p. 271-2.

44. Ibid., p. 278.

45. Ibid., p. 282.

46. YUE, "Gendering the Origins of Modern Chinese Fiction", op. cit., p. 57.

47. XIAO, Shengsi chang, op. cit., p. 58.

48. XIAO Hong, ["The Abandoned Child"] / 忘儿, in [The Complete Works of Xiao Hong] / 萧红全

集, Ha'erbin, Heilongjiang daxue chubanshe, vol. 4, 2011 [1933], p. 133.

49. Ibid., p. 134.

50. Ibid., p. 135.

51. Ibid., p. 138.

52. Ibid., p. 141.

53. Ibid., p. 143. 
54. zHANG Ailing 张爱玲, [Little Reunions] / 小团圆, Beijing, Zuojia chubanshe, 2009, p. 157.

55. ZHANG Xinxin 张辛欣, [“On the Same Horizon”]/ 在同一地平线上, in [Representative Works] / 张辛欣代表作, Zhengzhou, Huanghe wenyi chubanshe, 1988, p. 140.

56. CHANG Eileen, Little Reunions, WEIZHEN PAN Jane and MERZ Martin (trans.), New York, New York Review of Books, 2018, p. 173-4.

57. MA Jian 马建, The Dark Road / 阴之道, Taipei, Taichen wenhua, 2012, p. 333-4.

58. MA Jian, The Dark Road, DREW Flora (trans.), London, Vintage Books, 2014, p. 358-9.

59. MA, The Dark Road / 阴之道, op. cit., p. 333-4.

60. MA Jian马建, [Land of Flesh] / 肉之土, Taipei, Taichen wenhua, 2010.

61. The page number after each quotation refers to LING Chen, "A Story of Titan", Pathlight, MARTINSEN Joel (trans.), (Spring 2013), p. 323-63.

62. The page number after each quotation refers to LING Chen 凌晨, [“A Story of Titan”] / 泰坦的 故事, Kehuan shijie 7:2007, p. 15-21.

63. HANSON, "The Maternal Body", op. cit., p. 98.

64. Lu Ling 路翎, [“Hungry Guo Su'e”] / 饥饿的郭素娥, in [Selected Works] / 路翎文集, Lu Ling

(ed.), III vol., Hefei, Anhui wenyi chubanshe, 1995, p. 27.

65. Ibid., p. 39.

66. Ibid., p. 51.

67. LIN Bai, “The Time of Cat's Passion”, Renditions, TUFT Bryna (trans.), nº 79, Spring 2013, p. 104.

68. LIN Bai 林白, [The Time of Cat's Passion] / 激情的猫时代, Tianjin, Zhongguo wenlian chubanshe, 2001, p. 238.

69. Lu Tonglin, Misoginy, Cultural Nihilism and Oppositional Politics: Contemporary Chinese Experimental Fiction, Stanford, Stanford U. P., 1995, p. 71.

70. DENTON Kirk A., "Lu Ling's Literary Art: Myth and Symbol in 'Hungry Guo Su'e", Modern Chinese Literature (2:2), 1986, p. 202.

71. LU, [“Hungry Guo Su'e"] / 饥饿的郭苏俄, op. cit., p. 84.

72. HAN, [“The Guide to the Hunting for Women"] / 美女狩猎指南, op. cit., p. 307.

73. HAN,[“The Guide to the Hunting for Women”] / 美女狩猎指南, op. cit., p. 330.

74. RONG Cai, The Subject in Crisis in Contemporary Chinese Literature, Honolulu, Hawai'i U. P., 2004, p. 219.

75. ZHU, Gender and Subjectivities in Early Twentieth-Century Chinese Literature and Culture, op. cit., p. 155.

76. XIN Yang, "Configuring Female Sickness and Recovering: Chen Ran and Annie Baobei," Modern Chinese Literature and Culture, 23:1, Spring 2011, p. 170.

77. MAN Eva Kit Wah, Bodies in China. Philosophy, Aesthetics, and Politics, Hong Kong, The Chinese U. P., 2016, p. 142.

78. Quoting Richardson, Alber and Heinze state: "Unnatural narratives violate the 'mimetic contract' that had governed conventional fiction for centuries' as well as the 'established boundaries of realism"', Unnatural narratives - Unnatural Narratology, Berlin, Boston, De Gruyter, 2011, p. 4.

79. HoN Lai-chu, The Kite Family, Hong Kong, Hong Kong U. P., 2015.

80. HON Lai-chu, [The Kite Family] / 凤筝家族, Taibei, Lianhe wenxue chubanshe, 2008, p. 40-41.

81. MERLEAU-PONTY, Phenomenology of Perception, op. cit., p. 237.

82. MERLEAU-PONTY, Phenomenology of Perception, op. cit., p. 94. 


\section{AUTHOR}

\section{NICOLETTA PESARO}

Nicoletta Pesaro is Associate professor of Chinese language and literature at Ca' Foscari University Venice. Her research interests include modern Chinese literature, narrative studies and translation studies. She wrote several articles on Chinese literature and translated various works. She recently co-edited Littérature chinoise et globalisation: enjeux linguistiques,

traductologiques et génériques (2017) and co-authored a book on modern Chinese fiction Narrativa cinese del Novecento. Autori, opere, correnti (2019). She is chief editor of the book series Translating Wor(l)ds. 


\title{
La résistance des corps dans le recueil Jingai makyō (1939-1941) d'Oguri Mushitarō
}

\author{
Gérald Peloux
}

1 En 1923 paraît dans les pages de la revue Loisirs nouveaux / 『新趣味』un récit intitulé [L'Homme-animal] / 「獣人」, écrit par un certain I. Denis Meunier et traduit par l'écrivain Kunieda Shirō 国枝史郎 (1887-1943). Ce récit se poursuit sur plusieurs numéros pour être finalement publié en un volume sous le titre de L'ancienne capitale du désert / 『砂漠の古都』, où la supercherie concernant l'identité de l'auteur est dévoilée: Kunieda Shirō est à l'origine du texte. Ce récit est considéré aujourd'hui comme un des premiers représentants japonais d'un genre en vogue depuis plusieurs décennies en Occident, celui des mondes perdus, souvent plus connus dans sa version anglaise, lost worlds, popularisé par Arthur Conan Doyle (1859-1930) en 1912 avec le récit du même titre ${ }^{1}$. Le troisième chapitre du récit de Kunieda s'intitule «la race des hommes-queues, gardienne du trésor»/「宝庫を守る有尾人種」：ces hommesqueues, en fait des pithécanthropes, sont alors présentés parfois comme des singes, parfois comme un chaînon manquant qui mène à l'espèce humaine. La question du corps, même si elle ne constitue pas l'objet central de ce texte, vient irriguer ce récit d'une thématique qui s'impose alors toujours davantage dans les discours populaires de l'époque au sujet des races ${ }^{2}$.

De manière étonnante, l'homme-queue refait son entrée en 1939 sous la plume d'Oguri Mushitarō 小栗虫太郎 (1901-1946), cette fois-ci dans la revue [L'Homme moderne] / 『新青年』, créée en 1920. Si les caractères chinois pour désigner ledit homme - 有尾 人 - sont les mêmes que ceux utilisés en 1923, ils sont cette fois-ci accompagnés d'une lecture scientifique pseudo-latine homo caudas, sans doute une déformation de homo caudalis. Cet être, surnommé Dodo, découvert par hasard lors d'un braconnage aux confins du Congo belge et du Soudan anglais, dans la province d'Ituri - actuelle province de la République Démocratique du Congo - par Josias Cark, chasseur américain, va devenir l'un des protagonistes de la quête d'un site perdu en plein centre de l'Afrique. 
3 Cette aventure constitue le premier chapitre d'une série qui en comptera treize et qui mènera les héros dans différentes zones de la planète considérées comme terrae incognitae, ces fameux lost worlds ou mondes perdus. Après leur parution dans [L'Homme moderne] de mai 1939 à juillet 1941, les treize récits seront compilés du vivant de l'auteur en trois volumes ${ }^{3}$, mais ce n'est qu'en 1968 qu'ils seront réunis en un seul recueil, sous le titre qu'on lui connaît aujourd'hui [Les Étranges mondes perdus] / 『人 外魔境』/Jingai makyōis.

4 Cependant, alors qu'en 1923 les hommes-queues, lors de leur première apparition, ne jouaient qu'un rôle secondaire dans l'économie du récit, l'œuvre d'Oguri Mushitarō fait la part belle à toute une série de personnages aux identités complexes. Le braconnier Josias Cark, pour ne citer à ce stade qu'un exemple, qui a découvert cet homo caudalis, est un "sang mêlé $»^{5}$ américain. On rencontre aussi des êtres hybrides, un monde animal, végétal, voire minéral, qui emprunte ses caractéristiques à d'autres règnes de la nature. Presque chaque chapitre est de fait caractérisé par un questionnement sur l'identité corporelle des personnages et l'influence de celle-ci sur leurs actes.

Ce texte baroque, par les lieux visités et les descriptions qui en découlent, par les personnages complexes et un lexique foisonnant, fascine, et par sa richesse thématique et par le fait qu'il est écrit durant une période où le Japon s'enfonce dans la guerre et où les discours raciaux, eugénistes sont de plus en plus prégnants. Si le personnage principal, l'explorateur Oritake Magoshichi, est clairement à la solde de l'État japonais, il évolue dans une sphère textuelle où l'ambiguïté physique est de règle et où les a priori raciaux sont souvent remis en question. Cette ambigüité s'exprime ainsi par des corps qui sont fort loin de répondre aux injonctions raciales et eugénistes de l'époque ${ }^{6}$ : personnages métissés, personnages marqués dans leur corps par des dysfonctionnements génétiques, personnages évoluant à travers les ordres de la nature, etc. Cet étonnant paradoxe, où la fluidité des corps semble s'opposer, résister à la doxa raciale va constituer la problématique centrale des pages qui vont suivre : comment se répondent, et à quelle fin, l'ambiguïté de la forme et des thématiques de ce texte et celle des différents corps apparaissant tout au long des pages?

\section{Oguri Mushitarō, un maître du roman policier aux prises avec la guerre}

Oguri Mushitarō - de son nom d'état-civil Oguri Eijirō 小栗栄次郎 - naît le 14 mars 1901 dans une vieille famille aisée de négociants en alcool installée à Tokyo ${ }^{7}$. Malgré le décès de son père alors qu'il n'a que dix ans, il passe une enfance à l'abri du besoin et se révèle un élève doué en mathématiques et en langues étrangères. En 1918, il commence à travailler et, grâce à l'héritage conséquent de son père qu'il peut enfin récupérer, ouvre à son compte une imprimerie en 1922. Guère impliqué dans son travail, il ne s'intéresse pas à sa société et c'est durant cette période dilettante qu'il écrit ses premiers textes avant que son entreprise ne fasse faillite en 1926. Jusqu'à ses véritables débuts littéraires en 1933, il ne va plus travailler et vivra de la vente d'antiquités que sa famille avait collectionnées. Il passe ces quelques sept années à lire, à écrire. Durant cette même période, il ne publiera qu'une nouvelle.

7 C'est en 1933 que, dans la revue [L'Homme moderne], il publie [Un meurtre parfait] / 『完全犯罪』qui le propulse aussitôt au-devant de la scène policière japonaise. Dès 
lors, sa carrière est lancée, qui va atteindre son sommet l'année suivante avec son chefd'œuvre, [Les Meurtres de la demeure de la mort noire] / 『黒死館殺人事件』.Ce récit complexe, baroque pour ne pas dire rococo, à la limite de l'incompréhensible, où le narrateur perd son interlocuteur et le lecteur dans les méandres de références historiques, ésotériques, fait de son auteur le tenant de la nouvelle vague des écrivains de policiers au cours des années trente. En 1937, il crée avec Unno Jūza 海野十三 (1897-1949) et Kigi Takatarō 木々高太郎 (1897-1969) la revue [L’Espion] / 『シュピ 才』, qui sera la dernière revue policière à paraître au Japon jusqu'en 1938.

Avec la guerre contre la Chine, il est envoyé sur le front malais en tant que journaliste de novembre 1940 à fin 1941. Cette expérience va faire de lui un opposant viscéral à la guerre et il refusera d'écrire tout texte qui pourrait être assimilé à de la propagande, préférant se consacrer à des récits dont l'ambiguïté même des images lui permet de brouiller les cartes quant à sa position officielle à propos du conflit. Le seul récit basé sur son séjour en Malaisie - [La Société secrète Tiendihui du détroit] / 『海峡天地会』, publié en mars 1943 dans [L'Homme moderne] - va ainsi lui attirer les foudres de la police politique / kenpeitai. Il se consacre durant les derniers mois du conflit à des essais pour transformer en sucre des topinambours dans la région de Nagano. En 1946, il revient à Tokyo, rencontre Edogawa Ranpo 江戸川乱步 (1894-1965) et Unno Jūza, sans doute pour réinvestir le champ policier, mais meurt d'une hémorragie cérébrale le 10 février 1946.

\section{Un genre policier contraint d'évoluer}

Entre 1923 et 1939, le Japon connaît une évolution majeure de sa vie littéraire, sans parler de sa vie culturelle dans son ensemble. Il voit se développer une culture de masse qui atteint un sommet au tournant des années trente avec le développement des livres à un yen 円本 (enpon) qui permettent à tout un chacun de découvrir à bon marché la littérature étrangère et japonaise. La revue [L'Homme moderne] est sans conteste le fer de lance de cette culture populaire : elle ouvre ses pages à de futurs grands écrivains du genre policier, tel Edogawa Ranpo, Yokomizo Seishi 横溝正史 (1902-81), mais s'approprie plus généralement le modernisme comme genre d'expression de cette modernité japonaise. L'autre support de cette culture de masse les deux phénomènes sont en fait largement imbriqués - est l'atmosphère ero-guronansensu à son paroxysme vers les années 1930 et 1931: s'il ne s'agit pas d'un mouvement organisé, des artistes, aidés en cela par une population avide de nouvelles sensations, créent des œuvres à la sexualité très présente (ero), font appel à une iconographie qui se veut loin des courants esthétiques normés (guro) et s'appuie sur une vision du monde qui se veut décalée, bizarre (nansensu) ${ }^{8}$.

Cependant, dès le milieu des années trente, la pression des militaires, le raidissement d'une partie de la classe politique, vont faire taire cette culture iconoclaste, relativement libertaire. Le roman policier en fait également les frais car considéré comme véhiculant une image négative du Japon. La société de l'archipel doit désormais se consacrer à l'effort économique de guerre : laisser les lecteurs se délecter de lectures criminelles n'est plus bienvenu. Pourtant, le roman policier continue à (sur)vivre. Si la dernière revue spécialisée cesse de paraître en 1938, [L'Homme moderne] poursuit cependant ses publications. En fait, le roman policier opère un changement de thématiques à la suite des coups de boutoir de la censure toujours de plus en plus 
tâtillonne ${ }^{9}$. Cette évolution a été rendue d'autant plus facile que le roman policier japonais de l'époque - le tanteishōsetsu, équivalent du detective story - est un genre protéiforme aux contours particulièrement flous : le genre policier, mais aussi celui de l'espionnage, de la science-fiction, du fantastique sont alors largement placés sous la tutelle du tanteishōsetsu. Lorsque l'État se militarise, le roman policier orthodoxe, qui s'occupe à résoudre les crimes, s'estompe pour laisser place à des récits où espionnage, fantastique et science-fiction sont mis en avant au profit de la nouvelle politique nationale.

\section{Oritake Magoschichi, un héros au corps insaisissable}

11 C'est dans ce contexte de reconfiguration du genre que Jingai makyō paraît dans [L'Homme moderne]. Les années 1939 à 1941 correspondent en outre à l'enlisement de la guerre en Chine, au déclenchement de la guerre en Europe et à la "préparation " psychologique de la guerre avec les États-Unis. Cet arrière-plan historique joue un rôle fondamental dans l'évolution du récit au cours de ses treize épisodes, dont certains se passent dans les territoires de la Sphère de coprospérité de la Grande Asie orientale ${ }^{10}$ en voie de formation.

Comme le laisse entrevoir la biographie d'Oguri Mushitarō, ce dernier ne s'est pas focalisé sur un seul genre littéraire comme certains écrivains de romans policiers de l'époque ont pu le faire. Au-delà de cette variété, il écrit dans un style très reconnaissable qui en fait sa signature. On évoque à son sujet un style pédant (pedanchikku) car ses récits sont littéralement noyés dans une logorrhée de références académiques ou pseudo-académiques qui rendent la lecture particulièrement complexe, voire impossible ${ }^{11}$. Les digressions font largement perdre pied aux lecteurs, même les plus assidus: Les meurtres de la demeure de la mort noire en constituent l'exemple majeur. On trouve cependant cette technique dès l'incipit de son premier récit, Un meurtre parfait, tel un programme stylistique déjà mis en en place, avec toute une série d'informations historiques, géographiques, ethnographiques, et à grand renfort de caractères chinois complexes :

L'Armée Communiste du peuple Miao, le seul groupe militaire à être discipliné sur tout le territoire chinois, se nomme plus précisément Armée régulière de la région du Xiyu de la République des Soviets Chinois, mais l'auteur, par commodité, préfère l'appeler Armée Communiste du peuple Miao.

Son cœur est bien évidemment composé du peuple primitif que constituent les Miao, mais l'organisation de cette armée rouge si étonnante est la conséquence du grand séisme qui a touché ces dernières années l'arrière-pays de la région du Yunnan. Une partie des Miao installés en amont de la rivière Lujiang avait subi alors les plus importants dégâts et, ayant perdu leurs terres, ils se transformèrent en une masse errante qui termina sa route dans les environs de Malipo près de la frontière indochinoise.

苗（びょう）族共産軍—この、支那全土の唯一の規律ある兵団は、正確に云う と中華ソヴェート共和国西域正規律となるが、筆者は便宜上苗族共産軍の名で 呼ぶことにする。

もちろん、軍の主体が西域の夷蛮苗族であることは云うまでもないが、この 異彩ある赤軍組織は起因を先年の雲南奥地大地震に発している。その時いちば ん被害の甚だしかった潞江上流苗族の一部が、耕地を失って大俘浪団と化し、 はるばる印度支那国境に近い麻栗（まりつ）の辺まで流れてきた... ${ }^{12}$ 
Autre phénomène à l'œuvre dans Un meurtre parfait, la présence quasi frénétique de personnages étrangers. Dans cette nouvelle, on ne rencontre aucun héros japonais et les protagonistes sont souvent originaires d'horizons multiples: Vassili Zarov est un juif ouzbèke tandis que la victime, Hedda Mucheletze, vient de Lublin en Ukraine, qui est en fait une ville polonaise depuis 1918. Ces identités complexes sont d'une importance majeure dans l'économie de cette nouvelle et la résolution de l'énigme : la criminelle, elle-même atteinte d'une infirmité à une jambe, explique son acte en s'appuyant sur les théories eugénistes qui cherchent, d'après elle, à faire disparaître, entre autres par la castration, les espèces qui ne peuvent être sauvées, pour éradiquer le crime, l'alcoolisme, la perversité, la "pauvreté onanistique ${ }^{13}$, les maladies psychopathologiques, etc ${ }^{14}$.

Cet intérêt marqué de l'auteur, dès son premier texte, pour la question du corps nonnormatif s'exprime de manière décisive dans la progression narrative de Jingai makyō. À la multiplicité des configurations corporelles apparaissant dans ce dernier font écho une complexité de la structure narrative et une richesse lexicale qui se perçoivent dès l'ouverture du recueil avec son chapitrage et un jeu particulièrement poussé sur les titres à l'identité double: une glose en katakana, l'un des deux syllabaires japonais (outre les kanji ou caractères chinois), utilisé surtout pour transcrire les mots étrangers, accompagne presque systématiquement les kanji.

Les treize chapitres de Jingai makyō

\begin{tabular}{|l|l|l|}
\hline Traduction du titre & Titre original & Lieu(x) \\
\hline (1) Homo caudalis & 有尾人 & $\begin{array}{l}\text { Nord-est } \\
\text { de la République Démocratique du Congo }\end{array}$ \\
\hline (2) La Oscuridad Grande & 大暗黒 & Sud de la Tunisie \\
\hline (3) Lha-mo-Sambha-cho & 天母峰 & Tibet \\
\hline $\begin{array}{l}\text { (4) Dabukkū - récit d'un } \\
\text { naufrage }\end{array}$ & $\begin{array}{l}\text { 「太平洋漏水 } \\
\text { 漂流記 }\end{array}$ & $\begin{array}{l}800 \text { km au sud-est de l'atoll de Kapingamarangi } \\
\text { (Micronésie) }\end{array}$ \\
\hline (5) Incola palustris & 水棲人 & Gran Chaco (Amérique du Sud) \\
\hline (6) Deza Barimo & 畸獣楽園 & République Démocratique du Congo \\
\hline (7) Alang-alang & 火礁海 & Sumatra \\
\hline (8) Ser-mik-suah & 遊魂境 & Groënland \\
\hline (9) cinquième & 第五類人猿 & Amazonie \\
\hline (10) Kara Jilnagang & 地軸二万里 & Asie centrale (Afghanistan) \\
\hline (11) Sereno de morto & 死の番卒 & Frontière Panama/Colombie \\
\hline
\end{tabular}




\begin{tabular}{|l|l|l|}
\hline (12) Yat Giang & 伽羅絶境 & Vietnam, frontière Laos/Thaïlande \\
\hline (13) Kuk-e-Kingwa & $\begin{array}{l}\text { アメリカ鉄仮 } \\
\text { 面 }\end{array}$ & Alaska (Îles Aléoutiennes) \\
\hline
\end{tabular}

Si actuellement on retient que le recueil Jingai makyō conte les aventures d'Oritake Magoshichi, l'écrivain ne fait pas immédiatement intervenir ce personnage. En effet, le premier épisode en Afrique centrale a pour héros Zama Shichirō tandis que le deuxième, en Tunisie, a pour figure centrale Yamaza Shinzō. Zama est un scientifique, alors que Yamaza est un collectionneur d'art japonais qui tente de racheter à l'étranger des œuvres japonaises pour les faire revenir sur l'archipel. Oritake n'apparaît que dans la troisième livraison. La structure narrative des récits se met d'ailleurs à ce moment-là en place : le narrateur, ni Oritake ni narrateur omniscient, est un écrivain, facilement identifiable à Oguri Mushitarō, qui attend avec impatience qu'Oritake vienne lui rendre visite et lui raconter des histoires de mondes perdus.

Oritake est décrit en ces termes dans l'incipit du chapitre trois :

Le retour au pays après six ans de notre Oritake Magoshichi fut vraiment pour moi un don du ciel alors que je me sentais découragé par mon manque de documents concernant des contrées mystérieuses et des terres inconnues. Mais qui était donc cet Oritake qui me réjouissait tant? C'était un amateur à son compte œuvrant de par le monde qui collectionnait toutes sortes d'animaux. [...] De plus, il était hautement respecté comme un spécialiste du territoire chinois des Hsi-fan situé dans l'arrière-pays du Xinan - autrement dit, le territoire des barbares du Xiyu qui s'étend sur le Yunnan du nord, l'arrière-pays Sichuan et le Qinhai.

わが折竹棌七の六年ぶりの帰朝は、そろそろ、魔境、未踏地の材料も尽きかけ て心細くなっていた私にとり、じつに天来の助け舟のようなものであった。で は、それほど私を悦ばせる折竹とはいかなる人物かというに、彼は鳥獣採集人 としての世界的フリーランサーだ。[...]ことに折竹は、西南奥支那のシフア ン・テリトリー(Hsifan territory)——すなわち、北雲南(うんなん)、奥四川(しせ ん)、青海(せいかい)、北チベットにまたがる、「西域夷蛮地帯(シフアン・テリ トリー)」通として至宝視されている男だ。15

17 Mais outre cette célébrité liée à sa spécialité officielle qui lui permettra d'accéder aux expéditions internationales, le narrateur ajoute quelques lignes plus loin:

En réalité, Oritake possède une toute autre facette. Utilisant son importante célébrité de membre d'expéditions étrangères, il en profite pour arpenter secrètement le fond du Xinan chinois. L'achèvement de ces travaux de mesures, fondamentaux lorsque le Japon, un de ces prochains jours, tentera sa grande avancée dans cette région, repose sur ses épaules. En d'autres termes, il corrige les erreurs des cartes étrangères, et tente de parfaire les plans de cette région qui n'a jamais été arpentée par les Japonais.

事実、折竹には別の一面があるのだ。彼は、外国探検隊員という絶好の名目を 利用して、その都度、西南奥支那の秘密測量をやっている。日本が他日、この 地方への大飛躍を試みるとき、その根底となる測地の完成が、いま彼の双肩に かかっている。つまり、外国製地困の誤謬(ごびゅう)をただし、一度も日本人 の手で実測が行われていない、この地方の地困を完壁なものにしようとするの だ。16

Le personnage d'Oritake est ainsi posé et défini. Son profil double d'explorateur et d'espion compose les deux axes entre lesquels il oscille constamment. Ce phénomène est d'ailleurs très sensible à partir du chapitre dix: le genre du roman d'espionnage, passé quelque peu sous silence auparavant, devient très prégnant, et ce du fait sans 
doute de la pression de plus en plus forte des autorités qui demandent au genre policier de se tourner vers un type de récit plus adapté aux nécessités de l'époque. Les aventures au fin fond de la jungle, du désert, ne sont plus prétextes à des découvertes zoologiques, minérales ou humaines. Il s'agit désormais de décrire les tentatives ennemies pour arrêter la mission d'Oritake, l'horizon exotique très fortement marqué au début de l'ouvrage, bien qu'encore présent, passant au second plan. Si le chapitre dix offre un statut intermédiaire, on découvre dans le chapitre onze les travaux déjà avancés des États-Unis en train de construire un second canal de Panama en Amérique Centrale. Oritake apprend son existence en aidant un certain Piedras, un Afroaméricain membre d'un groupe de libération de Noirs américains, appelé " Nya Biniy ", sans doute une référence au culte Nyahbinghi (Nyahbingi ou Nyabingi), lié au rastafarisme et s'opposant à l'esclavagisme et au colonialisme ${ }^{17}$. Piedras veut créer un empire noir en Afrique en libérant 5000 compatriotes embauchés de manière abusive pour les travaux de ce nouveau canal, réalisant ainsi le projet de retour vers l'Afrique que certains membres de la communauté noire américaine promouvaient, comme par exemple Marcus Garvey (1887-1940), créateur de la Black Star Line (1919-1922) ${ }^{18}$. Dans le chapitre douze, le lecteur se retrouve en Indochine où Oritake est poursuivi par un fonctionnaire français, Raoul de Saint-Hilaire, désigné comme grand contempteur des Japonais et à la recherche de l'explorateur qui serait en train de préparer l'invasion japonaise.

19 Mais si Oritake, plusieurs fois présenté comme le parangon de l'esprit japonais, est le personnage principal de la majorité des chapitres, il ne faut pas se méprendre sur sa fonction. Il devient véritablement important dans les derniers récits du fait de l'entrée en guerre du Japon. Le chapitre douze, par exemple, paraît dans les kiosques en octobre 1940 : cela signifie que l'auteur écrit donc son texte, qui se passe en Indochine, peu avant que l'invasion de cette colonie française par l'armée japonaise ne débute à la fin du mois de septembre 1940. Dans les chapitres précédents, Oritake n'est qu'un passeur et n'a pour fonction que de donner du grain à moudre, des idées à l'auteur qui en plusieurs endroits attend avec impatience son arrivée et le début de son récit. Sa «présence corporelle » n'a de signification au niveau de la mise en place du récit seulement parce qu'Oritake est un pourvoyeur d'images, de récits, aussi étonnants, aussi exotiques que possible. Dans l'économie fictionnelle mise en place par l'auteur, Oritake est d'ailleurs comparé, non pas à un personnage ayant réellement existé dans la fiction elle-même, mais à un personnage de fiction, Tarzan ${ }^{19}$.

Comme pour venir étayer le peu d'importance accordée au corps physique du héros, une des rares descriptions de ce dernier n'apparait que très tardivement dans le texte (page 449 sur les 558 que compte le recueil dans notre édition de référence) et se focalise sur un trait physique ne le caractérisant que temporairement :

Oritake, à la peau noire à cause de ses jours passés au soleil, ressemblait immanquablement à un métis afro-asiatique.

日㳳けがしてまっ黒な折竹は、どうみても黒黄混血児としか見えない20.

21 Pour décrire l'apparence de l'explorateur, l'auteur utilise non pas des attributs physiques souvent repris dans les traités raciaux (couleur des yeux, de la peau, des cheveux, forme du visage, etc.), mais le résultat d'un phénomène éphémère, une peau foncée par les rayons du soleil. La représentation du corps d'Oritake, alors même que tout indique que ce dernier est Japonais, ne s'appuie ainsi sur aucun élément physique 
permanent et participe de la tendance générale du récit à ne faire intervenir que des personnages aux identités fluides, voire hétéronomes.

À l'exemple du corps d'Oritake, le lecteur est confronté à des récits où rien n'est sûr, où les identités sont mouvantes : jusqu'à la réussite même de chacune des aventures est remise en cause au niveau de l'identité générique des textes. Ainsi le premier chapitre, censé mener le groupe d'explorateurs dans la forêt équatoriale africaine pour retrouver officiellement la zone de vie de Dodo, l'homo caudalis, se termine-t-il par la mort de ce dernier et par celle de Zama, le personnage principal qui, enfermé dans des grottes de sel, en est réduit à se nourrir de chair de singe. Dans le chapitre trois, Oritake doit accepter de laisser continuer ses compagnons plus loin car ils ne veulent pas revenir chez les humains et ne peut donc pénétrer dans le monde perdu. Dans le chapitre neuf se déroulant en Amazonie, Oritake doit aussi rebrousser chemin, non sans faire remarquer que la zone pourrait être l'objet d'un développement économique pour le Japon. Cette versatilité, et surtout, l'absence de véritable dénouement se concluant par le retour d'un héros "victorieux » sont pour le moins étonnantes dans le cadre de la littérature populaire où les schémas narratifs sont en principe respectés ${ }^{21}$. Elles peuvent néanmoins trouver un début d'explication dans le fait que tout comme les personnages, Oritake au premier rang, l'horizon d'attente des récits ne correspond pas aux normes génériques attendues pour ce type de textes. Oguri Mushitarō, comme cela va être développé dans la partie suivante, semble ainsi avoir voulu faire de cette œuvre littéraire une métaphore de la multiplicité des configurations corporelles qui s'opposerait à un discours homogénéisant et normatif.

\section{La fluidité des corps}

23 La quasi-absence de corps pour Oritake est étonnante en ceci qu'elle s'oppose à une surreprésentation de corps hétérodoxes, aux identités fluides, que ce soit dans leurs formes, leurs apparences ou leurs origines. Ce phénomène montre combien le statut de ce personnage est particulier et a plus un rôle à jouer dans la structure narrative que dans les récits. Les autres personnages en revanche et, à part de très rares exceptions, justifiées de manière paradoxale, ne sont jamais normatifs.

24 Dans le chapitre un, Zama Shichirō, le précurseur d'Oritake, est présenté comme un tertio / 三分混血児・テルティオ : son père est un Japonais vivant à Aden au Yémen et sa mère est d'une part d'origine italienne et d'autre part Noire. Josias Cark est, comme nous l'avons écrit plus haut, un Afro-américain, Dodo est un hybride singe/humain et Manuela Mendoza, la fiancée de Zama, a du sang malais malgré des origines ibériques. Dans le chapitre trois, Kido Thomas est Nippo-américain; le professeur Kermisch est tchèque, mais son pays n'existe plus depuis l'invasion nazie, et il travaille désormais dans une université danoise ; Katie, le personnage le plus étonnant, allemande, a été découverte dans un cirque où elle était utilisée comme support de spectacles de freaks :

Au premier coup d'œil, que ce soit avec ses cheveux blonds ou ses yeux d'un bleu transparent, Katie était une parfaite jeune fille allemande. Cependant, quelle différence avec tout le reste de son corps ?! Elle laissait pendre de sa bouche ouverte d'épaisses lèvres.

Son visage était tout plat, son nez formait une petite boule, la fente de ses paupières s'ouvrait sur des yeux bridés - elle avait tout d'une Mongole. Et, de plus, on voyait bien tout de suite qu'elle était folle.

見ると、金髪の色といい碧眼の澄みかたといい、一点、非のうちどころのない 
ドイツ娘である。しかし、それ以外の部分はなんという変りかた ?! 厚い唇をた

らりと空けた様。

顔はただ広く鼻は結節をなし、ほそい目を瞼がきりっと裂けている一まさに、

このほうは完全な蒙古人だ。そのうえ、一目で白痴であるのが分るのだ。 ${ }^{22}$

et avec la Grande-Bretagne, mais il n'en reste pas moins que ce sont les corps complexes, et non homogènes, qui apparaissent sous un jour relativement positif. Le mongolisme de Katie, identifié à la folie, peut faire en fait l'objet d'une double lecture. D'une part, il vient «souiller » la pureté germanique clairement exprimée à travers l'expression "parfaite jeune fille allemande». De ce point de vue, il entre en résonnance avec les théories occidentales eugénistes. Pourtant, ce mongolisme, qui fait référence dans son étymologie même à l'Asie ${ }^{26}$, est présenté par le professeur Kermisch comme une part de «sang créatif » que viendrait paradoxalement «mépris[er]» les nazis et les Anglo-saxons. S'il utilise l'alibi d'un sang problématique - asiatique - pour critiquer l'invasion de son propre pays par l'Allemagne, cette théorie du "sang créatif » peut à son tour s'entendre comme une supériorité des peuples asiatiques - la composante créative - sur les peuples occidentaux, se faisant ainsi l'écho de la doctrine panasiatique de la Sphère de coprospéritée ${ }^{27}$ et rejoignant un des discours les plus usés de la Guerre du Pacifique prônant la supériorité de l'esprit japonais sur les armes américaines. Si le discours d'une communauté de destin avec les autres peuples asiatiques au début des années quarante est perceptible dans ces lignes ${ }^{28}$, il n'est cependant pas essentialisé dans le sens où il est intégré à un discours plus général que tient l'auteur et qui le relativise : les personnages dont les corps ne sont pas homogènes sont ceux par lesquels autre chose est possible, la définition de cette "autre chose » étant laissée à la discrétion des lecteurs. 
On retrouve dans un autre chapitre ce discours de protection envers les êtres différents. Dans le chapitre six, l'étymologie de Deza Barimo, terra incognita située en Afrique, est présentée comme étant la suivante :

Autrement dit, le mot [Deza Barimo] signifie "plateau aux êtres difformes ». Dans un autre village, on l'appelle "N'gulu rukha », ce qui veut dire " les dieux s'amusent ». Dans tous les cas, c'est un lieu où les animaux difformes viennent se réfugier. Les dieux ont tendu leurs mains bienfaisantes et ont offert ce paradis perdu aux exclus de leur groupe car, humain ou animal, ils étaient estropiés. Les animaux se rendent ici naturellement pour s'y retrouver, comme s'ils avaient cet instinct en tant qu'être difforme.

Il y a peut-être également des éléphants rupia, des girafes tricéphales. Et depuis la nuit des temps, ces animaux malformés, vivant entre eux, ont développé difformité sur difformité pour faire, sans doute, de cet endroit le lieu le plus horrible et le plus étonnant sur cette planète.

つまりそ机は、片輪者のいる台地という意味なんだ。またある部落では、やまり

それは、片輪者のいる台といい、それは、神、戯犰る一という意味になる。い ず机にしろ、ここは動物の片輪者の逃入地だ。神さまが、人間にしろ動物にし ろ片輪ものであるために、群にい机ら机ず排斥されるものに、慈愛の手を伸べ てくれた秘境的楽園だ。動物は、片輪にうまれると本能のようなのもので、自 然とここを指して集まってくる。

赤像、一体三頭のキリンなどもいよう。そうして太古から、畸獣のみがいるた めに畸形に畸形をかさ祄、おそらく地上でもっとも醜怪奇絶な場所ではないか $-{ }^{29}$

La difformité, l'hybridité, sont ainsi prises pour ce quelles sont, des évolutions certes bizarres mais à ne pas forcément critiquer, encore moins à éradiquer de la planète. Ces sites presque inaccessibles jouent ainsi le rôle de territoires protecteurs. Le symbole de l'Atlantide, réactualisé dans le chapitre deux, vient d'ailleurs confirmer cette hypothèse. Il vient renforcer la vision de ces mondes perdus comme des havres de paix pour ceux qui n'auraient pu trouver leur place dans le monde «normal».

Cette fluidité corporelle s'exprime aussi dans un autre type d'hybridation, celui de la langue. Les deux en fait se font écho. Comme nous l'avions déjà évoqué, les textes d'Oguri Mushitarō sont caractérisés par une utilisation parfois outrancière de termes savants ou pseudo-savants, de mots en katakana. La langue qui en résulte est une langue hybride, qui n'est plus tout à fait du japonais, ni tout à fait une langue étrangère, une sorte de langue métissée. Dans le chapitre deux, l'auteur présente deux cartes de la région avec des termes en caractères latins particulièrement complexes pour un lecteur japonais, l'une qui aurait été rédigée par un Arabe entre les $\mathrm{VIII}^{\mathrm{e}}$ et XII ${ }^{\mathrm{e}}$ siècles (Nefta, Tagiyus, Nefzawa, el-Hamma, Kalat el Yesdi, etc.), l'autre s'appuyant sur Platon (Kerne, Oceanus Atlanticum mare, Amazones Maxyes, Triton Nilus, etc. ${ }^{30}$.

Dans ce même chapitre, les personnages sont d'ailleurs pris eux-mêmes par le propre jeu de l'auteur : le pédantisme lexical de Mushitarō devient à son tour une thématique $\mathrm{du}$ texte hybride. Alors que Zama explique les sources du Nil à Josias Cark en s'appuyant sur un texte d'Hérodote à grand renfort de mots savants, ce dernier rétorque : «Certes, mais pourrais-tu m'expliquer ces mots latins difficiles?»/ なるほ ど、しかしその、むずかしいラテン語を説明してもらおうじゃないか ${ }^{31}$. En effet dans la phrase précédente, Zama expliquait qu'aux sources du Nil, il y avait un «pars radicosus» et un "sepulchrum lukuji » et que des «pygmien" et des "homo caudalis" vivaient dans ce lieu appelé «M'lambwezi $»^{32}$. Tous ces mots sont loin d'être latins mais la remarque de Josias Cark vient confirmer l'existence d'une langue que l'on pourrait 
presque appeler créole, une langue support d'un discours souvent considéré comme un outil de résistance ${ }^{33}$.

Les corps fluides et hétéronomes des personnages et l'utilisation d'une langue jouent ainsi plusieurs rôles: ils donnent une couleur exotique participant de l'imaginaire colonial particulièrement prégnant à l'époque, mais permettent aussi d'envisager un potentiel acte de résistance contre le discours officiel normatif. Nous l'avons vu avec les remarques de certains personnages qui protestaient du traitement imposé à certains autres personnages "non-normés». L'utilisation extensive de mots en langue étrangère, en katakana, en caractère latin, dans le corps même du texte, entre en résonance et en résistance par rapport à une tendance de fond qui se fait jour au Japon à partir de la fin des années trente, le phénomène des tekiseigo (mots ennemis) : le gouvernement demande que les termes étrangers soient remplacés par des termes japonais, l'identification de la pureté de la langue, débarrassée de ses scories anglosaxonnes répondant à la pureté de la race et à des pratiques culturelles considérées comme intrinsèquement japonaises ${ }^{34}$. Or quand on lit Jingai makyō, le lecteur est confronté à une forte résistance linguistique de la part de l'auteur qui ne cesse de multiplier les lectures complexes en katakana ou en alphabet latin de sites géographiques, de gloses en langues étrangères de mots écrits en caractères chinois. Tout comme le texte d'Oguri Mushitarō semble linguistiquement vouloir résister aux injonctions lexicales de l'époque, les corps rencontrés tout au long des treize épisodes présentent toute une série d'apparences complexes (fluidité, difformité, hybridité) qui portent en eux un fort potentiel de subversivité.

On pourrait appliquer à cette œuvre d'Oguri Mushitarō ce qu'écrit Vincent Platini dans le cas du monde de l'édition sous le nazisme. Certains textes qui paraissent pourtant anodins, voire de propagande, constituent des «îlots étrangers qui émergent dans la culture de masse allemande [et qui] sont comme des points d'embarquement vers des ailleurs moins étouffants ${ }^{35}$. Tout comme dans la littérature populaire durant le nazisme, la littérature populaire japonaise au tournant des années trente se doit de cohabiter avec les injonctions de l'époque, avec la censure. L'impression d'instabilité, de fluidité des identités corporelles dans Jingai makyō, en écho avec une langue complexe et une idéologie ambivalente ne peut que provoquer le trouble chez le lecteur confronté en cette période d'intense propagande à des récits largement monosémiques. Cette quête japonaise des mondes perdus, si elle reste ambigüe - mais pourrait-il en être autrement dans une période où une expression de résistance trop claire serait forcément tue, où toute la chaîne d'édition, de l'auteur au lecteur, est forcément marquée par l'idéologie nationaliste, peut ainsi correspondre à ce désir de l'auteur de présenter ces "îlots étrangers ", espace imaginaire où la censure d'État ne peut que difficilement s'appliquer, qui permettraient aux lecteurs - qui voudraient faire l'effort de les apercevoir - de découvrir qu'il existe une autre manière d'envisager les corps, même si cette autre manière n'est pas indiquée au cours des récits, loin du discours eugéniste majoritaire de cette période et que, peut-être, suprême subversion, les discours officiels ne disent pas forcément la vérité. 


\section{BIBLIOGRAPHIE}

ARAMATA Hiroshi 荒俣宏, [《Invitation au pédantisme »] / ペダンチックな誘い / Pedanchikku na izanai, in [Le Monde fantastique d'Oguri Mushitarō] / 小栗虫太郎ワンダーランド / Oguri Mushitarō Wandārando, KIDA Jun.ichirō (éd.), Tokyo, Chūseki-sha, 1990, p. 56-58.

BENOIST Jean, Petite bibliothèque d'anthropologie médicale : une anthologie, Toulouse, AMADES, vol. 1, 2002, p. 577, http://classiques.uqac.ca/contemporains/benoist_jean/ petite_biblio_anthropo_med_t1/petite_biblio_anthropo_med_t1.pdf, consulté le 26/04/2018.

BOJSEN Heidi, «L'hybridation comme tactique de résistance dans l'œuvre de Patrick Chamoiseau ", Revue de littérature comparée, 2002/2, n 302, p. 230-242, https://www.cairn.info/revue-delitterature-comparee-2002-2-page-230.htm, consulté le 26/04/2018.

COUÉGNAS Daniel, Introduction à la paralittérature, Paris, Seuil, 1992.

DRISCOLL Mark, Absolute Erotic, Absolute Grotesque - The Living, Dead and Undead in Japan's Imperialism, 1895-1945, Durham, Duke U. P., 2010.

EIZAWA Kōji 栄沢幸二, [La Pensée de la «Sphère de coprospérité » de la Grande Asie orientale] / 「大東覀共栄圏」の思想 / «Daitōakyōeiken » no shisō, Tokyo, Kōdansha, coll. « Kōdansha Gendai shisō », 1995.

GRANT Colin, Negro with a Hat: The Rise and Fall of Marcus Garvey and His Dream of Mother Africa, Oxford, Oxford U. P., 2008.

GUILLAUD Lauric, «Les oubliés du temps », in Les mondes perdus, GOIMARD Jacques (éd.), Paris, Presses de la Cité, coll. « Omnibus », 1993, p. III-XXIII.

KAWANA Sari, Murder Most Modern - Detective Fiction and Japanese Culture, Minneapolis, Londres, University of Minnesota Press, 2008.

NANTA Arnaud, « Savoirs et colonies : l'archéologie et l'anthropologie japonaises en Corée », in La société japonaise devant la montée du militarisme - Culture populaire et contrôle social dans les années 30, TSCHUDIN Jean-Jacques et HAMON Claude (éds.), Arles, Picquier, 2007, p. 21-31.

OGURI Mushitarō 小栗虫太郎, [« Un meurtre parfait »] / 完全犯罪 [1933] / Kanzen hanzai, in [Les Termites] / 白蟻 / Shiroari , Tokyo, Shakai shisō-sha, coll. « Gendai kyōyō bunko », 1997 [1976], p. 5-78.

OGURI Mushitarō 小栗虫太郎, [Les Étranges mondes perdus] (1939-1941) / 人外魔境 / Jingai makyō, Tokyo, Kadokawa shoten, coll. « Kadokawa horā bunko », 1995 [1978].

OGURI Senji 小栗宣治, [《Courte biographie d'Oguri Mushitarō»] / 小伝・小栗虫太郎/ «Shōden Oguri Mushitarō », in [Le Monde fantastique d'Oguri Mushitarō] / 小栗虫太郎ワンダーランド / Oguri Mushitarō Wandārando, KIDA Jun.ichirō (éd.), Tokyo, Chūsekisha, 1990 [1979], p. 36-48.

PLATINI Vincent, Lire, s'évader, résister - essai sur la culture de masse sous le III ${ }^{e}$ Reich, Paris, La Découverte, 2014.

ROBERTSON Jennifer, «Blood talks: Eugenic modernity and the creation of new Japanese », History and Anthropology, vol. 13, 3:2002, p. 191-216. 
RUTANGA Murindwa, Nyabingi Movement: People's Anti-colonial Struggles in Kigezi 1910-1930, Working paper $n^{\circ} 18,1991$, http://murindwa-rutanga.com/pub/Nyabingi\%20Movement.pdf, consulté le 26/04/2018.

SILVERBERG Miriam, Erotic, Grotesque, Nonsense - The Mass Culture of Japanese Modern Times, Berkeley, California U. P., 2006.

TANSman Alan (éd.), The Culture of Japanese Fascism, Durham, Duke U. P., 2009.

$* * *$

On citera aussi pour information un recueil de mangas inspirés de certains récits de Jingai makyō. Sur les six récits, trois sont des adaptations fidèles de certains chapitres de l'œuvre d'Oguri Mushitarō :

TEZUKA Osamu 手塚治虫, MIZUKI Shigeru 水木しげる, YOKOYAMA Mitsuteru 横山光輝, MATSUMOTO Reiji 松本零士, KUWATA Jirō 桑田次郎, [Chefs-d'œuvre de la bande dessinée d'horreur Jingai makyô (1969-1975)] / ホラーコミック保作選 人外魔境 / Horā komikku kesssakusen Jingai makyō, Tokyo, Kadokawa Shoten, coll. « Kadokawa horā bunko », vol. 4, 1999.

\section{NOTES}

1. Pour une histoire du genre, on lira avec intérêt GUILLAUD Lauric, «Les oubliés du temps », in Les mondes perdus, GoIMARD Jacques (éd.), Paris, Presses de la Cité, coll. « Omnibus », 1993, p. III-XXIII. Comme l'explique Lauric Guillaud, l'histoire du genre, si on s'appuie sur les textes occidentaux, est fondamentalement liée, dans un premier temps, au colonialisme triomphant en Europe : les explorateurs découvrent des mondes étonnants et ont une mission civilisatrice, l'un des exemples les plus célèbres étant Allan Quatermain (Les Mines du Roi Salomon, 1885), le personnage créé par Henry Rider Haggard (1856-1925). Lauric Guillaud considère que cette première époque (jusqu'à la fin du XIX ${ }^{e}$ siècle en Europe) est suivie d'une autre qui perdure jusqu'à la fin des années dix et que marquent le "doute, l'angoisse et la fascination", puis une troisième phase qui se termine avec la Seconde Guerre mondiale, phase où les mondes perdus se vengeraient des deux périodes précédentes (p. XIX). Cette troisième période verrait, selon Lauric Guillaud, l'arrivée de l'apocalypse, l'invasion de «cohortes fanatiques» (p. XIX) dans le quotidien, annonçant ainsi le désastre de la guerre de 39-45.

2. Voir par exemple tANSMAN Alan (éd.), The Culture of Japanese Fascism, Durham, Duke U. P., 2009, DRISCOLL Mark, Absolute Erotic, Absolute Grotesque - The Living, Dead and Undead in Japan's Imperialism, 1895-1945, Durham, Duke U. P., 2010.

3. Homo caudalis en juillet 1940 (chapitres un à cinq), Kara Jilnagang en avril 1941 (chapitres six à douze). Le chapitre treize paraît en juin 1942 dans un recueil de nouvelles : il donne son titre à ce dernier, mais sous un titre différent (Une lettre d'adieu de la stratosphère) du titre original (Kuk-eKingwa).

4. Publication chez la maison d'édition Gentō-sha. Nous garderons dans la suite de cet article le titre japonais Jingai makyō car il est quasiment devenu un nom commun, synonyme du genre des mondes perdus.

5. Le terme japonais employé est kokuhaku konketsuji / sang mêlé blanc et noir.

6. La loi eugénique nationale / 国民優生法 / kokumin yūsei-hō est promulguée en 1940 en pleine publication de l'ouvrage. Elle envisage la stérilisation des personnes présentant un potentiel génétique plus ou moins important de transmission d'une hérédité considérée comme nonviable; ROBERTSON Jennifer, «Blood talks: Eugenic modernity and the creation of new Japanese ", History and Anthropology, vol. 13, n 3, 2002, p. 191-216. 
7. Ces informations s'appuient sur une des très rares biographies d'oguri Mushitarō publiées à ce jour, celle de son fils Senji, «Courte biographie d'Oguri Mushitarō»/「小伝・小栗虫太郎」, publiée en 1979, dans le premier volume des œuvres complètes de son père chez Gentō-sha.

8. Pour mieux comprendre cette atmosphère, voir cet ouvrage pionnier: SILVERBERG Miriam, Erotic, Grotesque, Nonsense - The Mass Culture of Japanese Modern Times, Berkeley, California U. P., 2006.

9. Kawana Sari résume parfaitement ce phénomène de reconfiguration de la scène policière japonaise à partir de la fin des années trente dans ce contexte de censure dans son ouvrage Murder Most Modern - Detective Fiction and Japanese Culture, Minneapolis, Londres, Minnesota U. P., 2008, plus particulièrement dans le chapitre intitulé « Drafted Detectives and Total War: Three Editors of Shupio ", p. 147-185.

10. La sphère de coprospérité de la Grande Asie orientale / 大東車共栄圏 devait à terme englober toute l'Asie orientale, des confins occidentaux de l'empire des Indes, en passant par la Chine jusqu'à l'Indochine et l'Indonésie, alors colonie hollandaise.

11. ARAMATA Hiroshi 荒俣宏, [《Invitation au pédantisme»]/「ペダンチックな誘い」, in [Le Monde fantastique d'Oguri Mushitarō]/『小栗虫太郎ワンダーランド』，KIDA Jun.ichirō (éd.), Tokyo, Chūseki-sha, 1990, p. 56-58.

12. OGURI Mushitarō 小栗虫太郎, [《Un meurtre parfait»] / 『完全犯罪』 [1933], in [Les Termites] / 『白蟻』, Tokyo, Shakai shisō-sha, coll. « Gendai kyōyō bunko », 1997 [1976], p. 6.

13. Jitoku hinkon (自溳貧困), ibid., p. 74 .

14. Ibid., p. 74-76

15. OGURI Mushitarō, Jingai makyō『人外魔境』, Tokyo, Kadokawa shoten, coll. « Kadokawa horā bunko », 1995 [1978], p. 146.

16. Ibid., p. 147.

17. RUTANGa Murindwa, Nyabingi Movement: People's Anti-colonial Struggles in Kigezi 1910-1930, murindwa-rutanga.com/pub/Nyabingi\%20Movement.pdf, consulté le 26 avril 2018.

18. GRANT Colin, Negro with a Hat: The Rise and Fall of Marcus Garvey and His Dream of Mother Africa, Oxford, Oxford U. P., 2008.

19. OGURI Mushitarō, Jingai makyō, op. cit., p. 216. Tarzan a été créé en 1912 par Edgar Rice Burroughs (1875-1950).

20. Ibid., p. 449.

21. COUÉGNAS Daniel, Introduction à la paralittérature, Paris, Seuil, coll. « Poétique », 1992, p. 50-51.

22. OGURI Mushitarō, Jingai makyō, op. cit., p. 163.

23. Le latah, observé surtout en Malaisie et en Indonésie, est une " réaction vive de sursaut à une stimulation fortuite ou délibérée (généralement sonore), suivie d'une perte de maîtrise verbale et/ou gestuelle d'intensité et de durée variables"; voir BENOIST Jean, Petite bibliothèque d'anthropologie médicale: une anthologie, Paris, AMADES, vol.1, 2002, p.577, classiques.uqac.ca/ contemporains/benoist_jean/petite_biblio_anthropo_med_t1/ petite_biblio_anthropo_med_t1.pdf, consulté le 26 avril 2018.

24. Bien loin de ne concerner que les dictatures fascistes de l'entre-deux-guerres, les théories eugénistes, alimentées par le darwinisme social, touchent la majeure partie du monde occidental et le Japon à partir de la fin du xix ${ }^{\mathrm{e}}$ siècle pour atteindre son paroxysme autour des années trente et quarante: éloignement ou stérilisation de personnes dont les déficiences génétiques ou physiques sont considérées comme pouvant porter atteinte au patrimoine génétique d'un peuple. Les premières lois de stérilisation aux États-Unis sont votées en 1924 (Virginia Sterilization Act of 1924). En Europe, la Suisse, le Danemark, la Norvège, l'Allemagne, la Finlande, la Suède, etc. mettent en place des pratiques eugénistes durant la décennie suivante, culminant dans l'État nazi avec des internements en camps de concentration. Pour une présentation des différentes pratiques eugénistes, voir AUBERT-MARSON Dominique, Histoire de l'eugénisme, Paris, Ellipses, 2010. 
25. OGURI Mushitarō, Jingai makyō, op.cit., p. 167.

26. En japonais également, avec le terme mōkogata chihō / 蒙古癡呆, traduisible par « démence de type mongol » et glosé dans ce texte mongoroido / モンゴロイド.

27. eIZAWA Kōji 栄沢幸二, [La Pensée de la «sphère de coprospérité » de la Grande Asie orientale] / 『「大東亜共栄圈」の思想』, Tokyo, Kōdan-sha, coll. « Kôdan-sha Gendai shisō », 1995, p. 23-25.

28. Cette communauté de destin, voire d'identité raciale, permettra de servir les projets de colonisation de la péninsule coréenne. Comme l'explique Arnaud NANTA, «L'allogénat des Japonais, c'est-à-dire l'extériorité de leurs origines vis-à-vis de l'archipel, est réaffirmé : le modèle conquérant de la formation de l'État protohistorique (c'est-à-dire en terme de substitution de la race) a partie liée avec le discours du retour vers le continent l'annexionnisme.", NANTA Arnaud, "Savoirs et colonies: l'archéologie et l'anthropologie japonaises en Corée ", in La société japonaise devant la montée du militarisme - Culture populaire et contrôle social dans les années 30, TSCHUDIN Jean-Jacques et HAMON Claude (éds.), Arles, Éditions Philippe Picquier, 2007, p. 25-26.

29. OGURI Mushitarō, Jingai makyō, op.cit., p. 268.

30. Ibid., p. 138-139.

31. Ibid., p. 30

32. Idem.

33. Voir par exemple les travaux de Patrick Chamoiseau et ce qu'en dit Heidi Bojsen : "Puisqu'il s'agit de relations de pouvoir et d'identité dans le contexte postcolonial, l'interrogation déclenchée par l'hybridation est souvent très douloureuse : elle entraîne de la complexité et des questions subversives là où le discours des potentats, qui formulent l'identité collective d'une société, cherche traditionnellement à simplifier et à éclairer.» (BOJSEN Heidi, "L'hybridation comme tactique de résistance dans l'œuvre de Patrick Chamoiseau », Revue de littérature comparée, 2002/2, n 302, p. 230-242, https://www.cairn.info/revue-de-litterature-comparee-2002-2page-230.htm, consulté le 26/04/2018).

34. La destinée de la permanente à la fin des années trente et au début des années quarante est symbolique de cette identification: la permanente considérée comme expression d'un luxe inadéquat se voit de plus en plus critiquée par les autorités (même si elle ne sera jamais vraiment interdite). Dans le même temps, le terme pāmanento / パーマネント emprunté à l'anglais est remplacé par denpatsu / 電髧 - car il faut cependant continuer à pouvoir désigner l'ennemi. Restriction de l'expression corporelle et de la langue vont ainsi de pair.

35. PLATINI Vincent, Lire, s'évader, résister - essai sur la culture de masse sous le III ${ }^{e}$ Reich, Paris, La Découverte, 2014, p. 240.

\section{AUTEUR}

\section{GÉRALD PELOUX}

Gérald Peloux est agrégé de langue et culture japonaises et est actuellement maître de conférences à CY Cergy Paris Université. Il est membre de l'UMR Héritages et membre associé du CRCAO. Ses recherches actuelles portent particulièrement sur la littérature et la culture populaire japonaise des années 1920 et 1930. Il a coédité en 2018 avec Cécile Sakai Edogawa Ranpo - Les méandres du roman policier au Japon (Le Lézard Noir) et en 2022 avec Yannick Bardy et Pauline 
Cherrier Japon Pluriel 13 - Résistances, conflits et réconciliations (Éditions Picquier). Il a également publié en 2019 un recueil de traductions de nouvelles de Tani Jōji, Chroniques d'un trimardeur japonais en Amérique (Les Belles Lettres). 


\title{
Corps dressé, corps civilisé : «Seigneur Chen » de Wu Zhuoliu et la police coloniale à Taiwan
}

\author{
Ju-Ling Lee
}

\section{Introduction}

La police coloniale est un sujet récurrent dans la littérature taïwanaise sous la colonisation japonaise (1895-1945). Dépeinte comme tyrannique, elle y incarne l'ensemble de la force oppressive coloniale. La construction littéraire de la police coloniale reflète en fait fidèlement la réalité sociale de Taïwan sous le règne japonais. En 1895, par le traité de Shimonoseki, le Japon annexa Taïwan. Le Gouvernement général de Taïwan / 台湾総督府, autorité suprême, y instaura un système juridique et policier moderne, adapté de modèles européens, qui fit de l'île un État policier. Durant cinquante ans, la force policière contribua considérablement à consolider le contrôle colonial au sein de la société locale. Elle gérait quasi tous les aspects de la vie quotidienne et avait des interactions permanentes avec les Taïwanais. Ce terme de «Taïwanais » désigne les descendants des migrants chinois, installés à Taïwan depuis le $\mathrm{XVII}^{\mathrm{e}}$ siècle, auxquels ledit Gouvernement général appliqua, sur les zones habitées, un système d'administration différent de celui des autochtones ou premiers peuples habitant l'île longtemps avant l'arrivée des Chinois. Le système juridique moderne évoqué ici ne s'appliquait qu'aux zones habitées par les Taïwanais. Le travail de police incluait l'ordre public, la sécurité et les affaires administratives, tels le jugement sommaire des infractions mineures, la tenue des registres d'état civil, l'hygiène publique, la prévention des maladies infectieuses ou le « contrôle civil» / 保甲. Modelé sur le système communautaire chinois 保甲 / baojia de contrôle civil, ce système japonais hokō, appliqué aux seuls Taïwanais et Chinois, et non pas aux Japonais et étrangers sur l'île, contribua fort à diffuser le contrôle policier colonial. L'écrivain Ye Rongzhong 葉榮鐘 (1900-1978) cite d'ailleurs, un dicton populaire taïwanais d'alors, qui suggère la toute-puissance de la police : 
Les policiers à Taïwan peuvent tout gérer sauf transformer une femme en homme.

臺灣的警察除不能把女性變成男性外, 什麼事情都有辦法1.

2 L'image de la police coloniale intimidait d'autant plus les Taïwanais que nombre de policiers, abusant de leur pouvoir, les brutalisaient. Un article du périodique [Journal du peuple de Taïwan] / 臺灣民報, du 7 août 1927, relate que la police était, « aux yeux des Taïwanais, encore plus effrayante qu'un tigre qui mord » / 在臺灣人的眼中, 看作比 會咬人的老虎還要庽害的, et que si un enfant pleurait ou n'obéissait pas, il suffisait de dire : «La police va venir ! / 警察來了 pour qu'il se calme sur-le-champ. En région rurale, vu l'ignorance des paysans, les policiers étaient encore plus brutaux. Les Taïwanais les appelaient « empereur[s] de la campagne » / 田舍皇帝 / tianshe huangdi ([Journal du peuple de Taïwan], $1^{\mathrm{er}}$ avril 1925), pour signifier leur caractère tyrannique. Le plus souvent, ils nommaient les policiers du terme honorable de «seigneur policier» / 警察大人 / jp. keisatsu daijin ou ch. jingcha daren, expression qui dit bien leur vision à la fois cynique et affolée de la police coloniale.

3 Alors que les écrivains typiques de l'ère coloniale, tel Lai He 賴和 (1894-1943), tendent à figurer des policiers japonais, Wu Zhuoliu 吳濁流 (1900-1976) ${ }^{2}$, choisit une autre approche dans sa nouvelle ["Seigneur Chen»]/陳大人: le policier Chen est un Taïwanais. Né en 1900, Wu publia sa première nouvelle à l'âge de 37 ans. Ses œuvres portent un regard fortement critique sur la réalité sociale de Taïwan sous le régime colonial japonais, puis la prise du pouvoir par le parti nationaliste chinois Kuomingtang à partir de 1945. Wu considère ses créations comme " ayant un caractère historique » / 帶有歷史的性格, dont 《 chacune représente une facette de la réalité sociale 》 所舄的 各篇都是社會真相的一斷面 ${ }^{3}$. L'un de ses chefs-d'œuvre, [Orphelin de l'Asie], d'abord écrit en japonais entre 1943 et 1945 : アジアの抓览et publié en 1946, puis traduit en chinois : 亞細亞的孤兒et publié en 1959, met en lumière à la fois la réalité historique et sociale et les ambiguïtés de l'identité taïwanaise qui, dans les années 1930-40, navigue entre les influences du Japon et de la Chine ${ }^{4}$.

4 Comme beaucoup d'écrivains taïwanais éduqués sous la colonie, Wu Zhuoliu dut écrire la plupart de ses œuvres en japonais. Lorsqu'après-guerre le Kuomingtang interdit l'usage du japonais, $\mathrm{Wu}$ Zhuoliu écrivit des nouvelles en chinois vernaculaire, mais sans maîtriser cette langue ${ }^{5}$. Son emploi par les écrivains taïwanais nés à l'époque coloniale exigeait un apprentissage si long que nombre d'entre eux cessèrent toute production littéraire après-guerre. Wu Zhuoliu, lui, publia jusqu'à sa mort. Même en temps de guerre, alors que la liberté d'expression était très restreinte à Taïwan, il continua d'écrire en secret des nouvelles comme [Orphelin de l'Asie], [Seigneur Chen] ou encore [La Mère du docteur] / 先生媽 ${ }^{6}$. Nombre d'études sur Wu Zhuoliu affirment que la version japonaise de [Seigneur Chen], achevée en 1944, aurait d'abord paru entre 1945 et 1947 dans le périodique littéraire Xinxin, mais celle-ci est introuvable dans la collection complète actuelle du périodique Xinxin; la version chinoise, traduite par $\mathrm{Wu}$ lui-même, sortit pour la première fois en 1958 dans l'un de ses recueils de poèmes ${ }^{7}$.

5 Les créations de $\mathrm{Wu}$ Zhuoliu, sur un ton sarcastique, sont fort imprégnées du sens de la justice. L'écrivain met en scène divers personnages de la société taïwanaise: colonisateurs japonais malveillants, opportunistes taïwanais, politiciens chinois corrompus, etc. Ils connaissent pour la plupart la fin tragique qu'ils auraient méritée aux yeux de l'écrivain. Le policier Chen représente alors les opportunistes taïwanais au service de la bureaucratie coloniale. Le comportement des policiers taïwanais était très critiqué, même par la population locale colonisée : souvent corrompus, mal disciplinés 
et brutaux, ils abusaient de l'autorité que leur conférait le régime colonial ${ }^{8}$. Le protagoniste de "Seigneur Chen " fut créé d'après cette image notoire des policiers taïwanais. La nouvelle, d'environ 13000 caractères, décrit en détail les actes malveillants du policier Chen. Justice est rendue à la fin de l'histoire : dénoncé par un collègue japonais, Chen est mis en prison. Le choix du protagoniste reflète le ressentiment de l'écrivain à l'encontre de ces policiers qu'il critique aussi dans ses mémoires :

Nous autres Taïwanais haïssions les chiens de garde de l'impérialisme japonais : la police. À l'époque circulait un dicton : « Entre toutes les fleurs, le kulian; entre tous les hommes, les policiers ». C'est une reprise du dicton [japonais] « Entre toutes les fleurs, le cerisier; entre tous les hommes, le guerrier » pour se moquer de la brutalité de la police. Certes, les Taïwanais haïssaient les policiers japonais, mais ils haïssaient encore plus les policiers taïwanais oppressifs.

我們臺灣人很討厭守護日本帝國主義的看門狗一警察. 當時有一句流行的話說: 『花是苦楝, 人是警官』. 這是套用『花是櫻花, 人是武士』的話, 用來揶榆警察的 横暴. 臺灣人絕不是只恨日本人的警察, 對横暴的臺灣人警察更為憎惡 9 .

6 Ce dicton local compare les policiers coloniaux qui brutalisent la population aux guerriers du Japon féodal autorisés à administrer un coup mortel à un membre de statut inférieur qui aurait compromis leur honneur. Le Kulian / 苦楝 (Melia azedarach) est un arbre courant à Taïwan, qui s'adapte facilement à l'environnement, résiste à la chaleur et représente ainsi la ténacité du peuple de Taïwan face à la brutalité des policiers coloniaux ${ }^{10}$. Wu Zhuoliu insère justement ce dicton dans "Seigneur Chen " pour décrire le policier ${ }^{11}$.

7 Dans la littérature coloniale taïwanaise sur les policiers, le Japon s'arrogea en Asie de l'Est, durant la première moitié du $\mathrm{xx}^{\mathrm{e}}$ siècle, une «mission civilisatrice » qui justifiait l'exploitation économique, le traitement inégal et brutal du colonisé et la modernisation forcée de la société coloniale ${ }^{12}$. Plusieurs études historiques ont montré comment, dans le dernier tiers du $\mathrm{xIX}^{\mathrm{e}}$ siècle, par une série de mesures de modernisation, le Japon mima l'impérialisme occidental, adopta son discours civilisateur fondé sur la rhétorique de la supériorité culturelle d'un groupe ethnique sur un autre, puis colonisa ses voisins d'Asie de l'Est (Taïwan, Corée) au nom de la civilisation ${ }^{13}$.

8 Certes, le statut de colonisateur «civilisé » du Japon ne va pas de pair avec l'occidentalisation du pays. Robert Eskildsen soutient que le Japon justifia sa «mission civilisatrice » sur l'île en insistant sur le caractère moderne de son armée japonaise et en exagérant la sauvagerie des aborigènes taïwanais. Matsuda Kyōko montre que les élites japonaises, abreuvés depuis des siècles à la civilisation chinoise, manipulèrent le discours historique et ethnologique afin de décrédibiliser le rôle habituel de mentor de cette dernière en Asie de l'Est pour mieux faire valoir la mission civilisatrice du Japon à Taïwan ${ }^{14}$. La littérature coloniale taïwanaise remit précisément en question l'image du colonisateur "civilisé » et du colonisé "ignorant» que forgèrent les Japonais en représentant les comportements absurdes et la violence des policiers japonais au nom prétendu de la civilisation. "Seigneur Chen » décrit les diverses attitudes et réactions des colonisés face à la modernisation de la société et aux critères de civilisation imposés par le colonisateur. Par la mise en scène d'un policier taïwanais qui participe en quelque sorte à la "mission civilisatrice » du Japon, le récit met en relief l'évolution de la conscience de soi du colonisé de même que la mutation 
des valeurs traditionnelles de la société taïwanaise. Le corps devient le lieu où se manifestent d'ordinaire ces changements.

Cette étude analyse d'abord comment l'écrivain reconstruit l'ambiance générale de l'oppression coloniale omniprésente, avec les détails sur la mise en place par le Gouvernement général de codes juridiques en vue de surveiller et "civiliser » la vie quotidienne du colonisé, tous règlements en vue de renforcer l'autodiscipline des insulaires et de moderniser la société coloniale, et ce par la surveillance des corps. Elle explique ensuite comment le contrôle du policer Chen, passant par le dressage du corps taïwanais, touche alors la question complexe de l'identité coloniale et révèle des changements fondamentaux de la société coloniale.

\section{Oppression coloniale au nom de la civilisation : contrôle policier et codes juridiques}

10 L'histoire de « Seigneur Chen » se passe à Taïwan dans la deuxième partie de la période Meiji (1868-1912), soit les deux premières décennies de la période coloniale. L'histoire commence avec un policier Taïwanais, nommé Chen, qui donne un coup de pied à son oncle pour avoir violé le règlement [Infractions au règlement de police] / 違警罪 / ikeizai en bloquant un passage public pendant qu'il fendait des bambous. Blâmé par sa mère, Chen se justifie :

Il a violé le règlement ikeizai. Je ne suis pas une personne ordinaire. Je suis un officiel, un seigneur. Ce chapeau [de police] est accordé par l'Empereur du Japon. Avec cela, il n'y a plus d'oncle, je peux battre et détenir n'importe qui. Mon rang est bas, mais mon pouvoir est grand (.... $)^{15}$.

他犯違警例. 我不是尋常人, 是官, 是大人. 這頂帽子是日本天皇陛下所賜, 有這頂 帽子,那有阿舅, 無論什麼人都可以打, 可以縛, 我的官職雖小, 但我的職權很大 (...).

$11 \mathrm{Wu}$ Zhuoliu donne dès le début de l'histoire un indice important, qui replace l'oppression policière dans le contexte japonais de la «mission civilisatrice». Les préfectures de Taïwan publièrent le règlement ikeizai durant la première décennie de la colonisation ${ }^{16}$. Cette série d'articles de droit, qui visait à dissuader les comportements tenus pour contraires aux critères occidentaux de morale, d'hygiène et de sécurité publique, touchait nombre d'aspects de la vie quotidienne. Elle offrait mainte similitude avec le [Règlement sur les infractions mineures] / 違式註違保例 / ishiki kaii jo irei, promulgué au Japon au dernier tiers du $\mathrm{XIX}^{\mathrm{e}}$ siècle, par lequel le gouvernement s'engageait à moderniser la société japonaise ${ }^{17}$.

Il est nécessaire de faire ici un bref rappel du contexte historique de la naissance de la nation moderne du Japon et du développement de son impérialisme au cours de la période Meiji. Depuis la réouverture du pays au commerce avec les pays occidentaux au milieu du XIX ${ }^{e}$ siècle, sous le prétexte que le Japon n'avait pas de système juridique moderne, ceux-ci parvinrent à lui faire signer des traités inégaux pour protéger leurs propres droits sur le territoire japonais avec une juridiction extraterritoriale. Aussi, le gouvernement japonais établit-il à l'instar des droits européens, à partir de 1868, un système juridique en vue de négocier l'abolissement des traités inégaux ${ }^{18}$. Outre l'importation des institutions occidentales, l'État japonais s'efforça de moderniser la vie quotidienne. Le règlement sur les infractions mineures fut ainsi établi avec, pour surveiller, contrôler et pénaliser tout comportement qu'un citoyen japonais 
«moderne» ne devrait pas avoir ${ }^{19}$, une police chargée d'effectuer d'innombrables tâches annexes liées au quotidien ${ }^{20}$. période critique : en s'adjoignant de nouveaux territoires, le Japon cherchait à être traité en égal par les nations occidentales, se faire reconnaître en État souverain et devenir lui-même une puissance impériale. Par l'introduction d'un système juridique moderne et la mise en place d'un code juridique comme le règlement ikeizai, le Gouvernement général prétendait remplir son rôle « civilisateur » sur la colonie, mais la présence dans le système juridique de mesures discriminatoires vis-à-vis des Taïwanais ne fit que remettre en question l'image bienveillante du projet civilisateur japonais ${ }^{21}$. Taïwan géra en somme les peuples colonisés de la même manière que le gouvernement japonais, sa propre société au début de $\mathrm{Meiji}^{22}$. La police coloniale, qui exerçait le pouvoir public principal sur la base des lois, détenait ainsi l'image de gardien des valeurs «modernes » introduites par le Japon. Dans l'optique d'assister les policiers japonais, le recrutement de policiers locaux débuta en 1899. Les Taïwanais recrutés occupaient en général la fonction de «policier auxiliaire » / 巡査補 / junsaho ou, après 1920, de "policier de rang secondaire» / 乙種巡査 / otsushu junsa, le grade le plus bas de la hiérarchie du système policier colonial et réservé exclusivement aux Taïwanais, alors que les Japonais débutaient au rang supérieur de " policier » / 巡査. 《décision policière exécutoire immédiate»/ 違警罪即決例 / ikeizaisokketsurei, transplanté sur l'île en 1896. Il habilitait la police à appliquer des sanctions de sa propre autorité en cas de transgression des articles ikeizai et d'autres infractions mineures. La police pouvait infliger des peines immédiates de prison pour une durée allant jusqu'à trois mois et des amendes dans la limite de cent yen ${ }^{23}$. Ces sanctions étaient souvent déterminées de manière arbitraire :

Les policiers [japonais] pouvaient non seulement punir selon leur humeur les gens ordinaires en se justifiant avec les codes juridiques; [ils] giflaient les Taïwanais à leur gré ou les emmenaient au poste de police, au mieux leur demandaient de se mettre à genoux [un bon moment], au pire s'en prenaient à eux en les battant.

警察不但在有成文條例的名目下可以處罰老百姓，也可以随便刮臺灣人耳光或把 人帶到派出所, 輕則罰㻊, 重則毆打出氣 ${ }^{24}$

Plusieurs nouvelles de la période coloniale dénoncent les abus de la police. Par exemple, “Char à bœuf»/ 牛車 / jp. Gyusha, ch. Niuche $e^{25}$ de Lu Heruo 呂赫若 (1914-1951) raconte l'histoire d'un transporteur taïwanais en char à bœuf qui, avec l'usage de plus en plus répandu des transports automobiles dans les années 1930, n'arrive plus à subvenir aux besoins de sa famille et se voit un jour pénalisé pour avoir enfreint le règlement du contrôle des voies publiques / 街路取締規則 / gairo torishimari kisoku. Pour payer l'amende, il vole des oies, mais se fait arrêter. Shi Shu 施淑 (1940-) suggère que l'aversion des personnages de "Char à bœuf » envers les policiers japonais, ainsi que leur mécontentement à l'égard de mesures coloniales comme le système fiscal et l'interdiction faite aux chars à bœuf de circuler au milieu de la route, relèvent plutôt d'une haine de ce qu'ils "considèrent comme un 'ensemble japonais' qui est une 'oppression invisible' 》/ 把它們看作那『視而不見的壓迫』的『日本東西』的整體 / «ba tamen kan zuo na 'shier bujian de yapo' de 'Riben dongxi'de zhengti $»^{26}$. 

les détails de la vie quotidienne qui font l'objet de contrôle policier. Tel passage de la nouvelle décrit l'inquiétude des habitants qui pâtissent dudit contrôle en matière de propreté publique :

La ronde de Seigneur Chen est sévère à tous égards. S'il voit des feuilles mortes dans la rue, il administre une amende ou gifle, de la même manière qu'il punit des forgerons au torse nu. En automne, le temps est agréable et les feuilles tombent nombreuses. Pendant cette période, toute la rue s'effraye. Dès l'aurore, par tous les temps, on balaye sans broncher les feuilles mortes.

陳大人的巡視不單這樣嚴格. 他看到街上若有樹葉, 也像對付打鐵的赤膊一樣, 要 罰, 要打嘴巴. 到了秋天, 天清氣爽, 落葉紛紛, 到這時候, 全街都惶恐, 無論如何, 天 一亮就要掃落葉, 誰都不敢馬虎 27.

Un autre passage montre que les moindres détails de la vie quotidienne deviennent punissables :

[S'il voit] des gens qui ne marchent pas sur le côté gauche, qui transportent des marchandises torse nu, qui vendent des légumes dans la rue au lieu d'aller [faire leur commerce] aux marchés, ou quelqu'un qu'il n'aime pas, qui aurait compromis son autorité, Seigneur Chen leur administre à son gré et sans indulgence une amende ou des gifles, quel que soit leur statut social, leur âge et leur sexe.

遇到沒有靠左邊走的人, 赤膊挑擔的人, 不去市場設攤而在在路上賣菜的人, 或是 看不中意的人, 觸犯他官威的人, 都憑陳大人的感情好惡, 不論貴賤, 老弱男女, 都 毫不容情,或楞鍰,或打嘴巴 ${ }^{28}$.

19 Ces deux passages évoquent plusieurs codes juridiques de la période coloniale: le règlement ikeizai dont plusieurs articles concernent l'interdiction de la nudité en public $^{29}$, ainsi que de la vente de nourriture ou d'autres produits au bord de la rue; les règlements de contrôle des marchés / 市場取締規則 / shijo torishimari kisoku, publiés successivement par les préfectures à Taïwan durant les années 1900 pour une meilleure gestion de l'hygiène publique ${ }^{30}$; le règlement du contrôle des voies publiques publié en 1903 incluant la circulation à gauche et le maintien de la propreté des voies publiques ${ }^{31}$. Ces règlements censés moderniser la société locale instituaient une surveillance coloniale omniprésente qu'appliquait un système policier tout-puissant.

Comme l'attestent les deux passages cités, l'oppression coloniale, invisible selon Shi Shu, allait de pair avec les châtiments physiques administrés arbitrairement par la police, forme bien tangible de l'oppression coloniale qu'évoque souvent la littérature coloniale taïwanaise. Dans son étude de la nouvelle «Un Nouvel an malheureux » / 不 如意的過年 (1928) de Lai He, Zhu Huizu 朱惠足 (1973-) souligne que le rapport entre les deux personnages principaux - un enfant taïwanais et le policier japonais qui le gifle pour montrer son autorité - est une métaphore qui assimile le rapport colonial au lien parental. C'est en punissant physiquement l'enfant, colonisé "ignorant», que les parents, colonisateurs " civilisés ", mènent ce premier à maturation et le dirigent sur le chemin vers la civilisation. Dans le cas des Japonais à Taïwan, c'est en traitant le colonisé comme un enfant que le colonisateur établit son rôle de mentor et justifie son emprise sur la colonie ${ }^{32}$.

21 Il convient à présent d'analyser comment Wu Zhuoliu, par l'attention qu'il prête aux métaphores du corps, éclaire la complexe question identitaire et les changements fondamentaux de la société de Taïwan sous le régime colonial. 


\section{Dresser le corps, civiliser le colonisé : la société taïwanaise en mutation}

Alors que les écrivains de l'époque scrutent plutôt la relation entre colonisateur japonais et colonisé taïwanais, la mise en scène du policier Taïwanais autre et plus originale est l'approche de [Seigneur Chen]: elle invite à reconsidérer le rapport colonial, puisqu'il s'agit ici du rapport entre les colonisés eux-mêmes, entre ceux qui adoptèrent ou défendirent les valeurs importées du colonisateur et ceux qui restèrent moins sensibles ou parurent réticents. L'enjeu identitaire que l'écrivain soulève au fil de la mutation de la société sous le régime colonial est évident. Le corps du colonisé est là où se jouent les changements de la conscience de soi, les conflits entre mœurs traditionnelles et mœurs coloniales, ainsi que la reconstruction de la hiérarchie sociale traditionnelle.

Dans ses mémoires, Wu Zhuoliu tenta de classer les Taïwanais de la période coloniale en trois catégories : les «absolutistes»/絕對派 / jueduipai, opposés fermement au règne colonial ; les « compromistes » / 妥協派 / tuoxiepai, coopérants actifs ou passifs avec les autorités coloniales; les «désintéressés» / 超越派 / chaoyuepai, qui reconnaissent la réalité politique mais refusent de coopérer et se soucient désormais de la poursuite de leur bonheur personnel ${ }^{33}$. Les personnages créés littéraires par Wu Zhuoliu reflètent en général ces trois catégories et montrent sa préoccupation constante de la complexité de l'identité dans la société coloniale.

Dans "Seigneur Chen", la transformation de la conscience de soi s'accompagne d'un changement dans la perception du corps et des mœurs traditionnelles. Wu Zhuoliu détaille avec soin le contrôle du corps dénudé en public par le policier Chen :

Seigneur Chen commence la patrouille à l'aube. Sa patrouille est particulièrement tôt et il a sa propre raison.

En été, ceux qui commencent le plus tôt sont les forgerons, et en hiver les vendeurs de thé et de tofu.

Les forgerons commencent à travailler vers quatre ou cinq heures du matin, pour éviter la chaleur pendant la journée en plus de la chaleur venant du fourneau. Plus tôt ils commencent mieux c'est, afin de pouvoir finir le travail à midi quand la chaleur commence à frapper.

Comme chaque matin, les forgerons allument le fourneau avant l'aube. Avec des étincelles flamboyantes, ils forgent jusqu'à ce que tout leur corps transpire et doivent se déshabiller avant de pouvoir continuer. Juste à ce moment-là apparaît soudain le prestigieux Seigneur Chen, qui les injure aussitôt en japonais : Bakayaro ${ }^{34}$. Sa voix et son autorité sont imposantes comme s'il menait une armée et effrayait les forgerons. Tremblant de peur, haletant sans oser faire de bruit, ceux-ci ne peuvent que le supplier en bégayant :

«Seigneur, Seigneur, pardonnez-nous! Pardonnez-nous!»

Ils s'inclinent et s'excusent. Malgré ce, Seigneur Chen ordonne avec encore plus de pompe et de rigueur :

« Alignez-vous »

Puis il les blâme :

«Vous êtes des barbares. Il est interdit de se dénuder. Comment des esclaves des Qing ${ }^{35}$ comme vous méritent-ils d'être des citoyens japonais?»

Après la réprimande, il les gifle jusqu'à ce que leur visage devienne tout rouge (...) Plus tard, les passants curieux se rassemblent autour [de la scène]. Voyant arriver la foule, Seigneur Chen se sent encore plus de majesté. Il continue à les blâmer et, de nouveau, à les gifler. Restant insatisfait, il leur ordonne de former une file et les amène à la sous-préfecture pour leur administrer une amende de cinq jiao ou un 
yuan. Si les gens refusent d'obéir, ils seront détenus au poste de police pendant trois à cinq jours jusqu'à ce que les choses se soient calmées.

陳大人天一亮就出來巡視，他的巡視特別早，他的早有早的理由。

街上最早的人，在夏天就是打鐵店，冬天是賣杏仁及賣豆腐的。打鐵店的人

怕白書太熱, 在爈火前面内外煎獒。越早收拾越好, 所以四五點鐘就起來做活,

打到响午熱來就可以休息。

打鐵工人每晨天色未明就起火, 打得火花燦爛, 汗流渶身, 不得已脫了衣服

再打，這時候陳大人忽然駕臨，開口就用日語大聲喝 :

「馬鹿野郎。」

其聲威好像叱吒三軍一樣，嚇得打鐵的，魂不附體，戰戰兢兢，喘不出氣， 只好結結巴巴地哀求說 :

「大人, 大人, 失禮! 失禮 !」

說著便低頭謝罪。可是, 陳大人更是裝模做樣地庽聲命令說 :

「排成一列。」

然後又黑道 :

「你們是野蠻人，裸體不行，你們這樣的清國奴如何配做日本國民。」

罵了, 就打嘴巴, 打得對方滿面通紅。(...)半响, 好事的人就團團圍住, 陳大人

看見大家到來, 更覺得意威風, 教訓一番再打一番, 猶有不足, 又命令他們排成

一隊, 帶到支廳去罰鍰五角或一元 ; 若有抗議不服的人, 就押在拘留所内, 關了

三五日，才能息事。36

Dans le règlement sur les infractions mineures, plusieurs articles interdisaient le nu en public pour «civiliser» les mœurs. Le regard méprisant des Occidentaux au Japon, assorti de critiques constantes sur la nudité en public des Japonais et leur manque de pudeur, amena le gouvernement japonais à prohiber certaines pratiques corporelles traditionnelles ${ }^{37}$. Lorsque le Japon étendit son empire colonial en Asie de l'Est à partir de la fin du xix siècle, les Gouvernements généraux installèrent dans les colonies un système juridique moderne en même temps qu'ils tentèrent de faire appliquer des nouvelles normes de mœurs, adoptées par les élites japonaises au début de Meiji. Comme son modèle japonais, le règlement ikeizai publié à Taïwan compta ainsi plusieurs articles portant sur l'interdiction du corps nu en public. Il est important de souligner qu'en même temps que l'introduction des nouvelles normes de mœurs, l'autorité du Gouvernement général de Taiwan fut discréditée dès le début de son administration par les premiers arrivants japonais sur l'île qui manifestèrent une grande aisance à se dénuder en public. Dès les premières années de la colonisation, les Japonais furent en fait le sujet principal du contrôle policier du nu en public, ce qui montre à nouveau la vacuité du discours civilisateur nippon, mais les Taiwanais en pâtirent ${ }^{38}$.

En seulement quelques phrases, la semonce du policier Chen aux forgerons au torse dénudé illustre comment se forme chez le policier une nouvelle perception du corps, lequel s'adapte au lien établi entre corps dénudé et sauvagerie introduit par le colonisateur, d'où la critique qu'il adresse à ses compatriotes comme s'ils lui étaient devenus étrangers et qu'un revirement identitaire s'opérait en lui : "Vous êtes des barbares. Il est interdit de dénuder le corps. Comment des esclaves des Qing comme vous méritent d'être des citoyens japonais? ». En tenant le fait de dénuder le torse en public pour barbare, le policier Chen adopte les critères de civilisation du colonisateur ; en traitant les Taïwanais au torse nu d'esclaves des Qing, Chen s'en distingue, s'érige en citoyen japonais "civilisé »-car habillé - et adopte le regard méprisant du Japon envers son vaincu, la Chine. Le lien étroit entre les mœurs, l'identité et l'ethnie est ici établi. 
chivant cet auto-dressage du corps, nous entendons ici le changement qu'opère le colonisé sur lui-même dans sa façon d'évoluer au quotidien. Ce faisant, il s'aligne de sa propre initiative sur la nouvelle perception du corps qu'induit le colonisateur. Chen mime ainsi le colonisateur en « civilisant » ses compatriotes colonisés.

L'auto-dressage du corps paraît aussi explicite dans «La Mère du docteur » de Wu Zhuoliu, nouvelle qui porte sur le conflit entre différentes identités taïwanaises dans le mouvement de japonisation des années 1930-40. Le docteur en question adopte toujours avec ferveur la japonisation promue par le Gouvernement général (japoniser son nom, porter kimono, parler japonais dans la vie). Par contraste, sa mère représente les Taïwanais qui s'obstinent à conserver leur propre culture : elle insiste pour ne porter que des vêtements de style taïwanais et ne pas apprendre le japonais, ce qui fait honte au docteur chaque fois qu'il reçoit des invités. Tous les soirs après le dîner, toute la famille se réunit sauf la grand-mère qui s'absente exprès, et le docteur

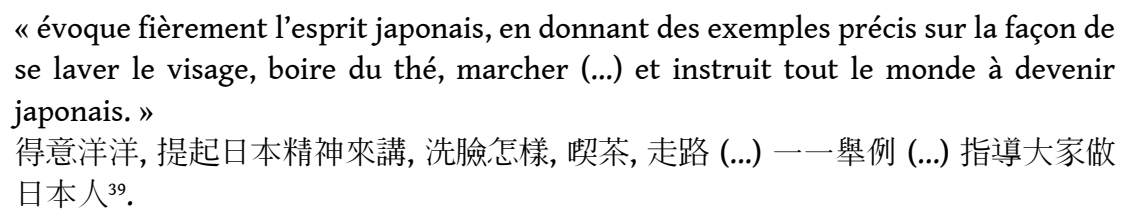

De même que le docteur qui a besoin de se changer pour " être japonais », les policiers taïwanais se doivent de soigner leur image de mentor pour paraitre plus convaincants ${ }^{40}$. Elle se construisit au fil de la colonisation par le port d'une tenue de style occidental symbolique de la modernisation. En effet, durant les cinq premières années de recrutement des policiers taïwanais, leur uniforme et leur tenue différèrent de ceux des policiers japonais. Leurs uniformes étaient conçus selon le style traditionnel taïwanais, ils portaient un chapeau en jonc d'artisanat local, n'avaient pas le droit au sabre. Les policiers japonais, eux, revêtaient les uniformes de style occidental adoptés par le Japon depuis le début de la réforme Meiji et un fez. En 1901, le Gouvernement général rectifia l'uniforme policier et effaça les différences visuelles entre policiers japonais et taïwanais ${ }^{41}$. Ainsi, dans «Seigneur Chen ", les accessoires et le costume que porte le policier taïwanais affichent son autorité et sa transformation en citoyen japonais " civilisé » : son uniforme et son chapeau de police lui donnent le droit de punir quiconque, ainsi que le sabre dont « il fait bien entendre le cliquetis dès qu'il rencontre la foule» / 遇到人多時便故意王出劍聲 et les bottes qu'il « fait claquer en marchant à grands pas 》/ 跨起大步, 鞋聲更踏得蹬蹬作響而過 ${ }^{42}$.

Certes, pour la plupart des Taïwanais sous régime colonial, le changement de manière de vivre ne fut pas spontané. Ye Rongzhong se souvenait que quand il était petit, les policiers effectuaient un contrôle sévère de la propreté des voies publiques en patrouillant exactement à la même heure tous les jours. Avec le temps, les habitants acquirent l'habitude de nettoyer les voies publiques chaque matin ${ }^{43}$. "Seigneur Chen " montre le décalage à l'œuvre dans la population. Dans le récit de Wu Zhouliu, comme dans le souvenir de Ye Rongzhong, les gens nettoient avec soin la voie publique devant leur domicile ou commerce, de peur de la punition arbitraire du policier Chen. Quant aux forgerons qui se dénudent sous la pression du labeur physique et de la chaleur de l'île, la discipline de soi qu'exige le pouvoir colonial leur semble si déraisonnable que, pour éviter tout contrôle, ils «font fréquemment surveiller » / 往往使人張望把風 ${ }^{44} \mathrm{si}$ Chen n'arrive pas. Mais le policier comprend vite leur stratégie et «se faufile en rampant jusqu'à l'intérieur du cordon de garde pour attraper les forgerons à demi nus » / 偷偷爬入其警戒線内, 捕捉那赤膊打鐵者 ${ }^{45}$. Dans cette scène de chasse au torse 
nu, le stratagème des Taïwanais contre le contrôle policier est une métaphore de la résistance des mœurs traditionnelles contre celles formées dans le courant de la modernisation pendant la période coloniale.

Dans «Seigneur Chen », le déclin des valeurs traditionnelles est surtout souligné par la violence physique que le policier taïwanais exerce sur les personnes âgées de sa propre famille et celles que respecte la société locale. Au début de l'histoire, Chen donne un coup de pied à son oncle et déclare : «avec ce chapeau [de police], je peux désormais me permettre de ne plus t'appeler oncle»/我有了這頂帽子, 再也不能叫你阿舅 ${ }^{46}$. L'écrivain Lai He traite le même thème dans sa nouvelle [Seigneur Bu] / 補大人 (1944). Lors du contrôle de la propreté des voies publiques, le policier taïwanais $\mathrm{Bu}^{47}$ découvre que la devanture de la maison de sa mère est particulièrement sale. Il frappe à la porte violemment, sa mère vient ouvrir et le tance de sa brutalité. Pour montrer son autorité devant la foule qui s'agglutine, le policier gifle sa mère. La confrontation entre la piété filiale confucéenne et les changements de la société taïwanaise sous le coup des nouvelles valeurs introduites par le colonisateur se matérialise dans les deux nouvelles par la brutalité physique des policiers taïwanais et la colère des membres de la génération antérieure de leur propre famille.

Un autre passage de "Seigneur Chen » sur un lettré sélectionné / 舉人 / juren ${ }^{48}$ nommé Liu, respecté de la population locale, montre aussi le bouleversement de la hiérarchie sociale traditionnelle consécutive à l'entrée en ligne d'une nouvelle génération de dirigeants locaux dans le système bureaucratique colonial ${ }^{49}$. En effet, bien que Chen assure la fonction la plus basse de la hiérarchie du système policier colonial, le poste de "policier auxiliaire" réservé à la population locale représentait pour celle-ci la fonction suprême, et ce jusqu'à ce que le Gouvernement général leur ouvre d'autres fonctions supérieures dans les années $1920^{50}$. Ainsi, Chen se compare souvent avec le lettré sélectionné Liu, auparavant fonctionnaire sous les Qing.

Un jour, lors d'une patrouille, le policier Chen et le vice-chef de la préfecture rendent visite à Liu. Pour forcer ce dernier à céder au vice-chef de la préfecture un vase de valeur transmis de génération en génération dans la famille, le policier Chen gifle Liu :

Simultanément [Chen] lève sa main et gifle le lettré Liu. Sa longue tresse se déroule et balance en tous sens.

同時就出手打劉舉人的嘴巴. 劉舉人的辫子也被陳大人打下來了. 長長的辦子摇過 來擺過去 ${ }^{51}$.

34 Le détail de la tresse, coiffure que les hommes chinois portent depuis la période Mandchoue et dont le sens symbolique a varié plusieurs fois dans l'histoire moderne de la Chine ${ }^{52}$, souligne que Liu incarne les valeurs traditionnelles chinoises. Le fait que sa tresse se détache sous le coup de la gifle de Chen, tenant de la nouvelle génération de dirigeants locaux, symbolise l'atténuation du rôle social respecté de Liu dans la reconfiguration de la hiérarchie sociale sous le régime colonial.

Sous la plume de Wu Zhuoliu, le corps est au carrefour des tensions que traverse la société coloniale dans la mouvance de la modernisation, qu'il s'agisse des manières de vivre, des tenues vestimentaires, des comportements ou de la violence physique, et qui traduisent en même temps la mutation de la conscience de soi chez certains Taïwanais et chez d'autres une résistance passive ou active à la mutation de la société coloniale. "Seigneur Chen ", grâce au choix du protagoniste et au soin que l'écrivain apporte aux détails historiques et aux métaphores du corps, restitue vivement les rapports de 
pouvoir et les changements fondamentaux de la société coloniale, ainsi que la complexité de la question identitaire dans le contexte donné.

\section{Conclusion}

Dans «Seigneur Chen ", l'amertume des Taïwanais vivant sous le régime colonial est rendue à travers l'oppression coloniale invisible, la surveillance coloniale omniprésente de la vie quotidienne établie par les codes juridiques, et l'oppression coloniale tangible, le corps dressé et châtié au nom de la civilisation. Le discours de la civilisation, fil rouge de l'histoire entière de "Seigneur Chen", semble observé avec toute la distance de l'ironie que $\mathrm{Wu}$ réserve au policier Chen pour rendre justice à la souffrance du peuple taïwanais. Après une longue description des actes malfaisants de Chen, Wu Zhuoliu annonce un changement avec l'arrivée d'un policier japonais qu'il décrit cyniquement comme un courant frais de la civilisation qui rompt avec les actes cruels de Chen :

La civilisation japonaise va peu à peu passer des métropoles aux campagnes. Vers la

fin de l'époque Meiji, le policier nippon qui maîtrise le hokkien est envoyé au village.

\section{不久，日本的文明由都會漸漸下鄉了。大約是明治末年，一個善講臺灣話的日本巡 官被派到鄉下來 $(. . .)^{53}$.}

Quelques paragraphes plus tard, on comprend que celui-ci n'est pas si différent de son collègue taïwanais : corrompu par un riche Taïwanais que Chen a rançonné à plusieurs reprises, le policier japonais dénonce son collègue et le fait incarcérer avec l'aide du vice-chef de la préfecture, lui aussi corrompu par le riche Taïwanais.

Deux ans plus tard, Chen reparaît dans la rue, débraillé comme un vagabond. Mis à la porte par son amante, entre-temps tombée dans la prostitution, il erre et croise deux ouvriers qui se dirigent vers lui ; l'un d'eux critique le second: «ton costume de style occidental est trop grand (...), c'est honteux »/ 你的洋服太大 (...) 不成體統 ${ }^{54}$. Lorsque Chen était encore policier, c'était avec un costume occidental qu'il a séduit cette femme ${ }^{55}$, dont il a châtié cruellement le mari en lui imputant un crime qu'il n'a jamais commis. Après l'avoir séduite, Chen brutalise encore plus les Taïwanais pour en tirer des profits propres à satisfaire les goûts luxurieux de celle qu'il courtise. Avec la fin bouleversante du récit, $\mathrm{Wu}$ Zhuoliu révèle l'image d'une société coloniale au bon fonctionnement de laquelle participent autant les Japonais corrompus que les Taïwanais opportunistes, vaniteux et égoïstes.

\section{BIBLIOGRAPHIE}

CHEN Ching-chih, "Police and Community Control Systems in the Empire", in The Japanese Colonial Empire, 1895-1945, MYERS R. H. and PEATTIE M. R. (eds.), Princeton, Princeton U. P., p. 213-239.

CHEN Jianzhong 陳建忠, [Études sur la littérature et la pensée de Lai He] / 書窵臺灣. 臺灣書舄: 賴和的文學與思想研究 / Shuxie Taiwan. Taiwan shuxie : Lai He de wenxue yu sixiang yanjiu, Gaoxiong, Chunhui chubanshe, 2004. 
CHING Leo, Becoming "Japanese": Colonial Taïwan and the Politics of Identity Formation, Berkeley, California U. P., 2001.

ESKILDSEN Robert, “Of Civilization and Savages: The Mimetic Imperialism of Japan's 1874 Expedition to Taïwan", The American Historical Review, vol. 107, 2:2002, p. 388-418.

GODLEY Michael R., "The end of the queue: hair as symbol in Chinese history", East Asian History, 8:1994, p. 53-72.

ISHIKAWA Chūichi 石川忠一, [Précis de police de Taïwan] / 臺灣警察要論 / Taiwan keisatsu yōron, Taipei, Shinkōdō Shoten, 1915.

LEAVELL James Berry, The Development of the Modern Japanese Police System: Transition from Tokugawa to Meiji, London, University Microfilms International, 1979.

LEE Chulwoo, "Modernity, Legality, and Power in Korea under Japanese Rule", in Colonial Modernity in Korea, SHIN Gi-Wook and Michael RoBINSON (eds.), Cambridge, Harvard University Asia Center, 2000, p. 21-51.

LEE Ju-Ling, "Clothing the Body, Dressing the Identity: The Case of Japanese in Taïwan during the Colonial Period", Journal of Japanese Studies, vol. 43, 1:2017, p. 31-64.

LI Ruei-Teng, Cent trésors de la littérature Taïwanaise, Tainan, Guoli Taïwan wenxueguan, 2012.

LIN Ruiming 林瑞明, [La Littérature de Taïwan et l'esprit du temps] / 臺灣文學與時代精神 / Taiwan wenxue yu shidai jingshen, Taipei, Yunchen wenhua, 1993.

MATSUDA Kyōko, “Inō Kanori’s 'history' of Taïwan: colonial ethnology, the civilizing mission and struggles for survival in east asia", History and Anthropology, vol. 14, 2003, p. 179-196.

MOMOSE Hibiki 百瀬響, [Civilisation et progrès : les coutumes perdues] / 文明開化: 失われた風 俗 / Bunmei kaika : ushinawareta füzoku, Tokyo, Yoshikawa Kōbunkan, 2008.

NAKAMURA Masaru 中村勝, [La Création de marchés : marché traditionnel et public au Japon moderne et en Asie de l'Est] / 創られた市場: 近代日本・東アジアの在来市場と公設市場 / Tsukurareta ichiba: kindai Nihon Higashiajia no zairai ichiba to kosetsu ichiba, Nishitokyo, Habesutosha, 2002.

NAKANO Akira 中野明, [À partir de quand la nudité fit-elle honte ? Les Japonais et la honte] / 裸は いつから恥ずかしくなったか :日本人の差恥心 / Hadaka wa itsu kara hazukashiku natta ka: Nihonjin no shūchishin, Tokyo, Shinchōsha, 2010.

ОКАмото Makiko 岡本真希子, [«Intégration et élimination de agents de police auxiliaires de Taïwan: traitement et apaisement des âmes durant le mandat du premier gouverneur militaire»] / 台湾巡査補をめぐる統合と排除：前期武官総督期に於ける待遇と慰霊 / Taiwanjin junsaho o meguru tōgō to haijo: zenki bukan sōtokuki ni okeru taigū to irei », [Sciences sociales] / 社会科学 / Shakai kagaku, vol. 41, n 1, 2011, p. 49-90.

PINO Angel et RABUT Isabelle (éds.), Le Petit Bourg aux papayers, Paris, You Feng, 2016.

QIU Jiayi 邱家宜, [«Génération perdue : Wu Zhuoliu comme exemple de journaliste taïwanais dans les premières années d'après-guerre»] / 失落的世代 : 以吳濁流為例看戰後初期的臺灣本 土報人 / Shiluo de shidai : yi Wu Zhuoliu wei li kan zhanhou chuqi de Taiwan bentu baoren, 中華傳播學 刊/Zhonghua chuanbo xuekan, vol. 23, p. 229-266.

SEIZELET Eric, « Les implications politiques de l'introduction du droit français au Japon », Revue internationale de droit comparé, vol. 43, 2:1991, p. 367-388. 
SHI Shu 施淑, [Recueil de littérature des deux rives ] / 兩岸文學論集 / Liangan wenxuelunji, Taipei, Xindi chubanshe, 1997.

SOUYRI Pierre-François, Moderne sans être occidental : aux origines du Japon d'aujourd'hui, Paris, Gallimard, 2016.

TS'AI Hui-Yu Caroline, Taiwan in Japan's Empire Building: an institutional approach to colonial engineering, Abingdon, Oxon, Routledge, 2011.

WANG Taisheng 王泰升, [“"Violence légale” à Taiwan sous domination coloniale japonaise : une évaluation historique »] / 日本殖民統治下臺灣的「法律暴力」及其歷史評價 / Riben zhimin tongzhi xia Taïwan de 'falu baoli' ji qi lishi pingjia, 國立政治大學歷史學報 / Guoli zhengzhi daxue lishi xuebao, 25:2006, p. 1-36.

wU Wenxing 吳文星, [La Classe dirigeante dans la société taïwanaise pendant la période de l'administration japonaise] / 日治時期臺灣的社會領導階層 / Rizhi shiqi Taïwan de shehui lingdao jieceng, Taipei, Wunan tushu, 2008.

WU Yingjing 吴盈靜, [ [ La lumière de l'aube sous les fleurs de margousier dans L'orphelin de l'Asie de Wu Zhuoliu »] / 苦楝花下的曙光-論吳濁流『亞細亞的孤兒』中的土地認同 / Kulianhua xia de shuguang - lun Wu Zhuoliu 'Yaxiya de guer' zhong de tudi rentong, Taiwan lishixuehui huixun / 臺灣歷 史學會會訊, vol. 12, 2001, p. 13-20.

wu Zhuoliu 吳濁流, [Vent et pluie devant la fenêtre] / 風雨窗前 / Fengyu chuangqian, Miaoli, Wenxian shuju, 1958.

WU Zhuoliu 吳濁流, [Recueil de Wu Zhuoliu] / 吳濁流集 / Wu Zhuoliu ji, PENG Ruijin 彭瑞金 (éd.), coll. « [Recueil complet des courtes nouvelles d'écrivains taïwanais de la première génération d'après-guerre] / 臺灣作家全集 短篇小說選 戰後第一代 / Taiwan zuojia quanji. Duanpian xiaoshuo xuan. Zhanhou diyidai », Taipei, Qianwei chubanshe, 2010 [1991].

WU Zhuoliu 吳濁流, [Lien vers Taïwan] / 臺灣連獟 / Taiwan lianciao, ZHoNG Zhaozheng 鍾肇政 (trad.), Taipei, Qianwei chubanshe, 1989.

XU Guozhang 徐國章, [« Étude préliminaire du système des crimes sommaires à Taïwan durant l'occupation japonaise : l'évolution du système et son efficacité »] / 臺灣日治時期犯罪即決制度 初探: 制度的演變及其成效探討 / Taiwan rizhishiqi fanzui jijue zhidu chutan: Zhidu de yanbian ji chengxiao tantao, in [Actes du colloque sur les archives de la gouvernance de Taiwan] / 臺灣總督 府檔案學術研討會論文集 / Taiwan zongdufu dangan xueshu yantaohui lunwenji, Archives du Musée national d'histoire de Taiwan (éd.) / 國史館臺灣文獻館 / Guoshiguan Taiwan wenxianguan, Nantou, Guoshiguan Taiwan wenxianguan, 2013, p. 179-228.

XU Junya 許俊雅, [Recherches sur le roman à Taïwan durant l'occupation du Japon] / 日據時代臺 灣小說研究 / Riju shidai Taïwan xiaoshuo yanjiu, Taipei, Wenshizhe chubanshe, 1995.

YE Rongzhong 葉栄鐘, [《La police japonaise à Taïwan durant l'occupation du Japon »] / 日據時代 臺灣的日本警察 / Riju shidai Taïwan de Riben jingcha, in [La Grande collection de huttes] / 小屋大 車集 / Xiaowu dache ji, YE Rongzhong 葉栄鐘, Taipei, Zhongyang shuju, 1967, p. 165-173.

YE Rongzhong 葉榮鐘, [《 Ma jeunesse »] / 我的青少年生活 / Wo de qingshaonian shenghuo, in [Recueil de Ye Rongzhong. Volume Littérature] / 葉榮鐘選集文學卷 / Ye Rongzhong xua ji. Wenxue juan, YE Rongzhong 葉榮鐘, Taipei, Renjian, 2015.

ZHANG Lianze 張良澤, [« La conscience sociale chez Wu Zhuoliu : étude de ses romans avant la libération de Taïwan »] / 吳濁流的社會意識 : 就其描舄臺灣光復以前的小說探討之 / Wu Zhuoliu de shehui yishi: jiu qi miaoxie Taïwan guangfu yi qian de xiaoshuo tantao zhi, in 吳濁流 / Wu Zhuoliu, 
ZHANG Henghao 張恆豪 (éd.), collection « [Recueil d'études des écrivains taïwanais modernes et contemporains 2] / 臺灣現當代作家研究資料彙編 2 / Taiwan xiandangdai zuojia yanjiu ziliao huibian 2 », Tainan, Guoli Taiwan wenxueguan, 2011, p. 185-205.

ZHU Huizu 朱惠足, [Pouvoir impérial et intimité : les relations raciales dans le roman colonial à Taiwan] / 帝國下的權力與親密: 殖民地臺灣小說中的種族關係 / Diguo xia de quanli yu qinmi : zhimindi Taiwan xiaoshuo zhong de zhongzu guanxi, Taipei, Maitian chuban, 2017.

\section{NOTES}

1. YE Rongzhong 葉榮鐘, [《La police japonaise à Taïwan durant l'occupation du Japon »] / 日據時 代臺灣的日本警察, in [La Grande collection de huttes] / 小屋大車集, YE Rongzhong 葉榮鐘, Taipei, Zhongyang shuju, 1967, p. 165.

2. LIN Ruiming 林瑞明, [La Littérature de Taïwan et l'esprit du temps] / 臺灣文學與時代精神, Taipei, Yunchen wenhua, 1993 ; CHEN Jianzhong 陳建忠, [Études sur la littérature et la pensée de Lai He] / 書舄臺灣. 臺灣書䳔 : 賴和的文學與思想研究, Gaoxiong, Chunhui chubanshe, 2004. Sur d'autres écrivains qui traitent de la police coloniale, voir xu Junya 許俊雅, [Recherches sur le roman à Taïwan durant l'occupation du Japon]/日據時代臺灣小說研究, Taipei, Wenshizhe chubanshe, 1995 ; zHu Huizu 朱惠足, [Pouvoir impérial et intimité : les relations raciales dans le roman colonial à Taiwan]/帝國下的權力與親密：殖民地臺灣小說中的種族關係, Taipei, Maitian chuban, 2017.

3. Cité par ZHANG Lianze 張良澤, [«La conscience sociale chez Wu Zhuoliu: étude de ses romans avant la libération de Taïwan »] / 吳濁流的社會意識 : 就其描舄臺灣光復以前的小說探討之, in 吴濁流 / Wu Zhuoliu, zHANG Henghao張恆豪 (éd.), Tainan, Guoli Taiwan wenxueguan, coll. [Recueil d'études des écrivains taïwanais modernes et contemporains 2] / 《臺灣現當代作家研究 資料彙編 2 ", 2011, p. 187.

4. V. LI Ruei-Teng, Cent trésors de la littérature taïwanaise, Tainan, Guoli Taiwan wenxueguan, 2012 ; CHING Leo, Becoming "Japanese": Colonial Taiwan and the Politics of Identity Formation, Berkeley, California U. P., 2001.

5. QIU Jiayi 邱家宜, [Génération perdue: Wu Zhuoliu comme exemple de journaliste taïwanais dans les premières années d'après-guerre] / 失落的世代 : 以吳濁流為例看戰後初期的臺灣本土 報人, [Journal chinois d'études de communication] / 中華傳播學刊, vol. 23, p. 229-266.

6. [« La Mère du docteur »], écrit en 1944 et publié en 1946 par le périodique [Journal du peuple de Taïwan] / 民生報.

7. V. wU Zhuoliu 吳濁流, [Vent et pluie devant la fenêtre] / 風雨窗前, Miaoli, Wenxian shuju, 1958. Le texte utilisé par cette étude vient d'un recueil de courtes nouvelles publié en 2010. WU Zhuoliu 吳濁流, in [Recueil de Wu Zhuoliu] / 吳濁流集, PENG Ruijin 彭瑞金 (éd.), Taipei, Qianwei chubanshe, 2010 [1991].

8. Sur les policiers taïwanais à l'ère coloniale, v. окАмото Makiko 岡本真布子, [ I Intégration et élimination de agents de police auxiliaires de Taiwan : traitement et apaisement des âmes durant le mandat du premier gouverneur militaire»]/台湾巡査補をめぐる統合と排除：前期武官総 督期に於ける待遇と慰霊, [Sciences sociales] / 社会科学, vol. 41, 1:2011, p. 49-90.

9. wu Zhuoliu, [Lien vers Taïwan] / 臺灣連堯殉, zHoNG Zhaozheng 鍾肇政 (trad.), Taipei, Qianwei chubanshe, 1989 , p. 50.

10. Sur la métaphore du kulian chez Wu Zhuoliu, voir wU Yingjing 吳盈靜, [《La lumière de l'aube sous les fleurs de margousier dans L'orphelin de l'Asie de Wu Zhuoliu »] / 苦楝花下的曙光-論吳濁 流『亞細亞的孤兒』中的土地認同, [Bulletin d'information de la Société historique de Taiwan] / 臺灣歷史學會會訊, vol. 12, 2001, p. 13-20. 
11. wU, Wu Zhuoliu ji, op. cit., p. 41.

12. ZHU, Diguo xia de quanli yu qinmi, op. cit.; SHI Shu 施淑, [Recueil de littérature des deux rives ]/ 兩岸文學論集, Taipei, Xindi chubanshe, 1997.

13. ESKILDSEN Robert, "Of Civilization and Savages: The Mimetic Imperialism of Japan's 1874 Expedition to Taiwan", The American Historical Review, vol. 107, 2002/2, p. 388-418 ; SOUYRI PierreFrançois, Moderne sans être occidental : aux origines du Japon d'aujourd'hui, Paris, Gallimard, 2016.

14. v. ESKILDSEN, " Of Civilization and Savages... », op. cit. ; MATSUDA Kyo ìko, "Inō Kanori's 'history' of Taiwan: colonial ethnology, the civilizing mission and struggles for survival in East Asia", History and Anthropology, vol. 14, 2003, p. 179-196.

15. wU, Wu Zhuoliu ji, op. cit., p. 39-40.

16. À partir de 1908, le Gouvernement général unifia le règlement ikeizai publié par les préfectures et publia Taiwan ikeizai qui s'appliqua désormais sur toute l'île. Sur l'étude du règlement ikeizai publié à Taiwan, v. LEE Ju-Ling, "Clothing the Body, Dressing the Identity: The Case of Japanese in Taiwan during the Colonial Period", Journal of Japanese Studies, vol. 43, 1:2017, p. 31-64.

17. Sur l'étude du règlement sur les infractions mineures publié au Japon, v. MOMOSE Hibiki 百瀬 響, [Civilisation et progrès : les coutumes perdues] / 文明開化: 失われた風俗, Tokyo, Yoshikawa Kōbunkan, 2008.

18. Sur la modernisation du droit au Japon, v. SEIZELET Eric, «Les implications politiques de l'introduction du droit français au Japon », Revue internationale de droit comparé, vol. 43, 2:1991, p. 367-388.

19. Sur la modernisation au Japon à l'ère Meiji, v. SOUYRI Pierre-François, Moderne sans être occidental..., op. cit. .

20. V. LEAVELL James Berry, The Development of the Modern Japanese Police System: Transition from Tokugawa to Meiji, London, University Microfilms International, 1979.

21. Sur la nature discriminatoire du système juridique colonial à Taiwan et son rôle dans l'expansion impériale japonaise, v. WANG Taisheng王泰升, [“"Violence légale” à Taiwan sous domination coloniale japonaise : une évaluation historique»] / 日本殖民統治下臺灣的「法律暴 力」及其歷史評價, [Journal d'histoire, Université nationale de Chengchi] / 國立政治大學歷史學 報, 25:2006, p.1-36; LEE Chulwoo, "Modernity, Legality, and Power in Korea under Japanese Rule", in Colonial Modernity in Korea, sHIN Gi-Wook and ROBINSON Michael (eds.), Cambridge, Harvard University Asia Center, 2000, p. 21-51.

22. Sur le système policier colonial à Taiwan, v. TS'AI Hui-Yu Caroline, Taiwan in Japan's Empire Building: an institutional approach to colonial engineering, Abingdon, Oxon, Routledge, 2011, chapitre 3 ; CHEN Ching-chih, "Police and Community Control Systems in the Empire", in The Japanese Colonial Empire, 1895-1945, MYERs R. H. and PEATTIE M. R. (eds.), Princeton U. P., p. 213-239.

23. Sur les décisions policières immédiatement exécutoires à Taïwan, voir XU Guozhang 徐國章, [ Étude préliminaire du système des crimes sommaires à Taïwan durant l'occupation japonaise : l'évolution du système et son efficacité »] / 臺灣日治時期犯罪即決制度初探: 制度的演變及其 成效探討, in [Actes du colloque sur les archives de la gouvernance de Taïwan] / 臺灣總督府檔案 學術研討會論文集, Archives du Musée national d'histoire de Taïwan / 國史館臺灣文獻館 (éd.), Nantou, Guoshiguan Taiwan wenxianguan, 2013, p. 179-228.

24. YE Rongzhong, [« La police japonaise à Taïwan durant l'occupation du Japon »], op. cit., p. 170. 25. [《Char à bœuf»] fut publié pour la première fois en 1935 dans Bungaku hyoiron / 文学評論, périodique japonais de critique littéraire; voir la traduction française de PINO Angel et RABUT Isabelle (éds.), Le Petit Bourg aux papayers, Paris, You Feng, 2016.

26. SHI, Liangan wenxuelunji, op. cit., p. 134.

27. wU, Wu Zhuoliu ji, op. cit., p. 49.

28. Ibid., p. 52. 
29. Sur l'interdiction du nu en public à Taiwan, v. LEE, "Clothing the Body, Dressing the Identity ", op. cit.

30. V. NAKAMURA Masaru 中村勝, [La Création de marchés : marché traditionnel et public au Japon moderne et en Asie de l'Est]/ 創られた市場: 近代日本・東アジアの在来市場と公設市場, Nishitokyo, Habesutosha, 2002.

31. V. le règlement entier dans ISHIKAWA Chūichi 石川忠一, [Précis de police de Taïwan] / 臺灣警 察要論, Taipei, Shinkōdō Shoten, 1915, p. 369-377.

32. zHU, [Diguo xia de quanli yu qinmi], op. cit.

33. Wu, Taiwan lianciao, op. cit.

34. Dans la traduction chinoise utilisée ici, l'insulte en japonais, bakayaro = imbécile, figure telle quelle sans être traduite.

35. Durant la première moitié $\mathrm{du} \mathrm{xx}$ siècle, les Japonais insultaient souvent Taïwanais et Chinois et les traitaient d'esclaves des Qing, jp. チャンコロ chankoro / ch. 清國奴 / qingguo nu.

36. wU, Wu Zhuoliu ji, op. cit., p. 47-49.

37. Sur l'histoire de la pudeur au Japon, v. NAKANO Akira 中野明, [À partir de quand la nudité fitelle honte? Les Japonais et la honte]/裸はいつから恥ずかしくなったか:日本人の羞恥心, Tokyo, Shinchōsha, 2010.

38. LEE Ju-Ling, « Clothing the Body, Dressing the Identity », op. cit.

39. wU, Wu Zhuoliu ji, op. cit., p. 33.

40. Durant la colonisation, surtout les premières années du recrutement des policiers taïwanais, ceux-ci peinèrent souvent à contrôler au quotidien les Japonais, qui se tenaient pour supérieurs et les méprisaient ; v. оКАмото Makiko, [" Taiwanjin junsaho o meguru tōgō to haijo »], op. cit., p. 57-58.

41. Ibid., p. 53-55, 59-61.

42. wU, Wu Zhuoliu ji, op. cit, p. 46.

43. YE Rongzhong 葉栄鐘, [《Ma jeunesse »]/我的青少年生活, in [Recueil de Ye Rongzhong. Volume Littérature] / 葉榮鐘選集 文學卷, YE Rongzhong葉榮鐘, Taipei, Renjian, 2015.

44. wU, Wu Zhuoliu ji, op. cit, p. 48.

45. Ibid., p. 49.

46. Ibid., p. 38.

47. « $\mathrm{Bu}$ » en chinois ou « ho » en japonais, réfère en fait à " auxiliaire » comme dans junsaho (policier auxiliaire), la fonction policière réservée aux Taïwanais pendant la période coloniale. Lai He l'emprunte pour en faire le nom de famille de son personnage fictif.

48. Parmi les grades de postes dans l'examen impérial des Qing figure celui de lettré sélectionné. Il était qualifié pour assurer des postes de fonctionnaire et pouvait aussi choisir de participer aux concours des fonctions supérieures. Il occupait un rôle social élevé et était très respecté par les locaux.

49. Sur la formation des nouveaux dirigeants locaux à l'ère coloniale, voir TS'AI, Taiwan in Japan's Empire Building, op.cit.; wU Wenxing 吳文星, [La Classe dirigeante dans la société taïwanaise pendant la période de l'administration japonaise]/日治時期臺灣的社會領導階層, Taipei, Wunan tushu, 2008.

50. Voir wU, ibid., chapitre 4.

51. wU, Wu Zhuoliu ji, op. cit., p. 44-45.

52. Sur le symbole de la tresse masculine dans l'histoire de la Chine moderne, voir GodLEY Michael R., "The end of the queue: hair as symbol in Chinese history", East Asian History, 8:1994, p. 53-72.

53. wu, Wu Zhuoliu ji, op. cit., p. 61. Originaire du Sud de la Chine, le Hokkien est le dialecte le plus utilisé à Taïwan.

54. Ibid., p. 65.

55. Ibid., p. 56. 


\section{AUTEUR}

\section{JU-LING LEE}

Ju-Ling Lee, titulaire d'un doctorat en histoire de l'Université Lyon 2, est chercheuse associée au Centre d'études sur la Chine moderne et contemporaine (EHESS). Ses recherches portent sur les politiques coloniales du corps et sur l'usage des médias par l'empire du Japon. Elle a notamment publié Imaginer l'indigène : la photographie coloniale à Taiwan (1895-1945) aux éditions Hémisphères. 


\title{
La conscience du corps confronté à la violence chez Han Kang
}

\author{
Eun Jin Jeong
}

1 La présente étude porte sur l'œuvre d'une des romancières sud-coréennes contemporaines les plus renommées et les plus fécondes et sur la représentation du corps dans son écriture, en particulier dans trois œuvres de fiction, toutes traduites en français.

2 Han Kang 한강 est née en 1970 à Kwangju 광주, ville située dans le sud-ouest de la péninsule coréenne. Elle écrit des nouvelles, des romans, mais aussi des poèmes plus occasionnellement. Au fil de sept romans et de trois recueils de nouvelles publiés depuis vingt ans, elle a réussi à bâtir un univers romanesque original par son style et les thèmes qu'elle aborde et elle est désormais considérée comme un écrivain majeur de la littérature coréenne actuelle. Sa renommée dépasse la sphère coréenne grâce aux traductions et s'est accrue en 2016 avec le prix Man Booker International qui lui a été décerné pour 채식주의자 / Ch'aeshikchuûija (2007), roman traduit en plusieurs langues dont l'anglais (The Vegetarian, 2015) et le français (La Végétarienne, 2015). En 2017, son dernier roman traduit en français, Leçons de grec / 희 랍어 시간 (2011), était en lice pour le prix Médicis étranger.

3 Si le corps constitue de façon générale un thème inspirateur dans la littérature coréenne moderne et contemporaine ${ }^{1}$, dans l'écriture de Han Kang il joue un rôle fondamental au point que la critique littéraire coréenne Shin Saet Pyôl 신샛별, se référant à la pensée de Michel Foucault, parle à propos de cette dernière d'une "politique du corps $»^{2}$, du corps comme " haut lieu de la lutte contre le pouvoir et l'ordre établi ».

4 Le corps est par exemple au centre d'une longue nouvelle de Han Kang qui l'a rendue célèbre et lui a valu le prix littéraire le plus prestigieux du pays, Yi Sang 이상. Elle s'intitule «La Tache mongolique » / 몽고반점 (2004), l'une des trois composantes du livre La Végétarienne, avec «La Végétarienne » (2004) et «Les Flammes des arbres » / 나 무불꽃 (2005), publiées dans des revues différentes avant d'être réunies, en 2007, pour constituer un ensemble ${ }^{3}$. «La Tache mongolique» raconte l'histoire d'un vidéaste 
fasciné par sa belle-sœur, ou plutôt par le corps de cette dernière, dont il apprend qu'il porte toujours une tache mongolique, de couleur gris-bleu, située dans le bas du dos d'un nourrisson, en particulier chez les Asiatiques, et qui disparaît normalement au cours de l'enfance. Il finit par convaincre cette créature psychologiquement fragile de poser nue pour lui. Il dessine alors sur son corps des motifs végétaux qui prolongent la tache mongolique et filme le tout, avant de succomber à la tentation charnelle, ce qui aboutit à la dissolution de son mariage et à l'internement de la jeune femme dans un hôpital psychiatrique. Cette trame originale, qualifiée par la critique littéraire Kim Ye Rim 김예림 d'《 esthétisme enfiévré et (pour l'heure relativement) nouveau », a été comparée à une certaine littérature japonaise représentée par des œuvres comme « Le Tatouage » / 刺青 (1910) de Tanizaki Junichirō 谷崎 潤一郎 (1886-1945)5. L'édition en un volume de l'ensemble des trois nouvelles qui, bien qu'originellement publiées séparément, forment un seul récit, la (re-)découverte des œuvres précédentes de l'auteur et la suite de sa création ont fini par dissiper ce que Han Kang elle-même qualifie dans de nombreuses interviews de "malentendu", à savoir sa réputation d'écrivain de littérature érotique.

Dans l'ouvrage La Végétarienne, l'héroïne, Yônghye 영혜, n'a pas voix au chapitre au fil des trois volets qui donnent tour à tour le point de vue de son mari («La Végétarienne »), de son beau-frère ( "La Tache mongolique ») et de sa sœur aînée (" Les Flammes des arbres »). En dehors des monologues intérieurs, en italique dans le texte, au cours desquels elle raconte ses rêves, elle ne s'exprime guère dans le récit. En revanche, son corps, lui, réagit, résiste aux contraintes qui viennent à la fois du monde extérieur et de son propre univers mental, pour finalement aspirer à se transformer en arbre.

6 En effet, Yônghye se prive d'abord de tout produit d'origine animale, bientôt de toute alimentation et enfin désire devenir un végétal, sans doute forme première de vie sur Terre, la plus inoffensive. Le thème de la métamorphose de femmes en végétal, qu'elle n'est pas le seul écrivain à avoir exploité6, semble particulièrement cher à la romancière puisqu'elle y revient dix ans après l'avoir traité une première fois, quoique de manière très différente, c'est-à-dire fantastique, dans une nouvelle intitulée «Le Fruit de ma femme» / 내 여자의 열매 (1997, recueil éponyme). Dans cette œuvre, le personnage féminin rêve de partir loin et, à défaut, se réfugie dans un mariage qui se révèle incapable de remédier à sa profonde solitude. Son corps se couvre alors de mystérieuses ecchymoses verdâtres et un jour se métamorphose en plante. Dans $L a$ Végétarienne, la tache mongolique de Yônghye évoque chez le vidéaste « des temps très anciens, antérieurs à l'évolution des espèces, ou une sorte de trace de photosynthèse en tout cas quelque chose de végétal qui n'avait rien de sexuel $»^{7}$. Il a par ailleurs l'impression que cette femme est « un être sacré, ni humain ni animal, une réalité autre située entre la plante et la bête $»^{8}$.

7 L'aspiration de Yônghye à devenir végétal peut être expliquée par la violence qu'elle subit dans son environnement - autre thème important chez Han Kang -, plus concrètement, selon les termes de la critique Shin S. P., "coercition et pression, mais aussi toutes sortes de violence produites par le langage, le regard, la conscience, la pensée »" Et, ajoute-t-elle, « pendant que le monde animal bout d'égoïsme, de cupidité, de colère, de compétition, d'instinct de survie, le végétal mène une vie paisible et calme au-delà de la mort $»^{10}$. En fait, les raisons qui poussent Yônghye à la «folie» ne manquent pas dans le texte, elles y sont même trop explicites diront certains : un père 
autoritaire ; le souvenir du chien qui l'a mordue et que ce père a traîné derrière sa moto pour en attendrir la viande; le mari, un médiocre qui l'a choisie parce qu'il a cru percevoir en elle une «banalité » qui le rassurait ${ }^{11}$; le regard des autres, peu tolérant envers tout comportement non conforme aux codes de la société, et même le désir qu'éprouve un homme pour elle, son corps, la tache mongolique qui persiste bien qu'elle soit adulte... Mais plus fondamentalement, le rejet de Yônghye reflète un profond malaise vis-à-vis du monde, trop agressif pour elle, et peut-être aussi la peur de se voir devenir à son tour " prédatrice ». Il fait suite à son propre rêve où elle dévore de la viande crue :

J'avais mangé un morceau de viande qui était tombé sur le sol de la grange. J'avais frotté la pulpe crue et flasque contre mes gencives et mon palais, qui s'étaient imprégnés de sang. Mes yeux scintillaient, reflétés dans la flaque vermeille qui couvrait le sol.

Tu ne peux pas imaginer comme c'était intense, cette sensation des dents qui mâchaient la chair crue. Mon visage, cette lueur dans mes yeux... C'était une face que je voyais pour la première fois et en même temps c'était la mienne, sans aucun doute possible. Non, au contraire : c'était un visage que j'avais vu un nombre incalculable de fois, mais ce n'était pas le mien. Je ne peux pas t'expliquer... Cette impression vive, bizarre, terriblement bizarre... à la fois si familière et si nouvelle ${ }^{12}$.

8 C'est quand elle raconte son univers onirique en monologue intérieur comme ici que Yônghye révèle un peu de son intimité. Il y est rarement question de son rapport aux autres, mais plutôt d'images où elle laisse transparaître son agressivité latente. Dans la vie, elle n'oppose pas sa propre violence - pas de cris ni de coups - à celle de l'extérieur. Ou alors elle la tourne vers elle-même. Yônghye est avant tout à l'écoute - presque aux aguets - de son corps. Elle est si repliée sur elle-même que le lecteur a du mal à s'identifier à elle. Quand la tension devient insupportable, elle se mutile et c'est ce qui arrive dans une scène où son père veut la forcer à avaler un morceau de viande au cours d'un repas de famille. Se priver d'alimentation est aussi une forme de violence auto-infligée.

Dans son roman Leçons de grec, Han Kang met en scène deux personnages souffrant tous deux d'un handicap : la femme est brusquement frappée d'une de ses crises récurrentes d'aphasie; quant à l'homme, il est en train de perdre la vue et il sait qu'il la perdra bientôt complètement. Si le cas de l'homme a une explication patente, à savoir une maladie héréditaire, celui de la femme semble plus complexe :

La chose n'avait ni cause apparente ni signe avant-coureur.

Bien sûr, elle avait perdu sa mère six mois plus tôt, elle avait divorcé plusieurs années auparavant, perdu la garde de son fils âgé de neuf ans au terme de trois procès et cela faisait cinq mois que l'enfant était parti vivre chez son ex-mari. Le psychologue aux cheveux grisonnants qu'elle voyait à cause des insomnies qui l'accablaient depuis ce dernier événement ne comprenait pas pourquoi elle ne voulait pas admettre ces origines plus qu'évidentes.

Non, a-t-elle noté sur la feuille blanche posée sur la table. Ce n'est pas si simple ${ }^{13}$.

10 La femme est donc plus qu'affectée par toutes les violences que la vie lui inflige et face auxquelles elle se révèle complètement désarmée. Apprenant que son fils va partir vivre à l'étranger, elle téléphone à son ex-mari pour protester, mais elle est incapable d'émettre un son. L'explication à son aphasie n'est pas clairement donnée dans le récit, mais le rapport que le personnage entretient au langage et à sa langue maternelle est évoqué à plusieurs reprises :

Elle seule savait que sa vie était farouchement divisée en deux. Les mots qu'elle notait à la fin de son journal se déplaçaient de leur propre mouvement pour former des phrases inconnues qui perçaient son sommeil à tout bout de champ comme 
autant de tiges de fer et la nuit, elle ouvrait les yeux en sursautant à plusieurs reprises. Plus son sommeil était troublé, plus ses nerfs devenaient dangereusement sensibles. Parfois, une douleur inexplicable, comme causée par une lame brûlante, envahissait le creux de son estomac.

Le plus pénible, c'était qu'elle entendait avec une netteté inexorable chacun des mots qu'elle prononçait. Une phrase, aussi insignifiante fût-elle, laissait apparaître comme à travers un prisme sa plénitude et son imperfection, sa vérité et son mensonge, sa beauté et sa laideur. Elle avait honte de celles qui étaient secrétées par sa langue et par sa main comme les fils blancs d'une toile d'araignée. Elle avait envie de vomir. Elle avait envie de pousser des cris ${ }^{14}$.

11 La femme, une grande lectrice qui par ailleurs écrit, est saisie à la fois de doute et de peur à l'égard du pouvoir du langage. Ce dernier l'agresse presque, provoquant chez elle des réactions physiques. Se rappelant qu'un mot de la langue française, dont elle venait de commencer l'apprentissage, l'avait précédemment guérie de son mal en débloquant sa parole, elle se met à étudier le grec ancien pour « retrouver le langage par sa propre volonté $\aleph^{15}$, à la manière d'un enfant qui apprend à parler. Ce qui l'intéresse dans le grec ancien, c'est son éloignement de toutes les langues qui lui sont connues. "S'il y avait eu des cours de birman ou de sanscrit, deux langues qui ont recours à des systèmes scripturaux qui lui sont encore plus étrangers, elle les aurait choisis sans hésiter $»^{16}$, déclare-t-elle. Symbole d'un retour à l'état premier, symbole d'autant plus fort qu'il s'agit d'une langue morte dont elle ne fera aucun usage. Cette initiative rappelle l'aspiration à devenir végétal de certains personnages de Han Kang dont il a été question plus haut en ceci qu'elle vient de l'observation détachée de ce que fait le corps humain dans son environnement.

Lorsqu'elle s'attaque pour la première fois à une page de l'histoire du pays, Han Kang est plus que jamais habitée par cette thématique du corps. Dans le livre intitulé Celui qui revient / 소년이 온다 (2014), il est question de Kwangju, la ville natale de Han Kang. Le nom de cette cité évoque notamment pour les Coréens le massacre de plusieurs centaines, voire de milliers de civils perpétré en mai 1980 par la junte militaire alors au pouvoir, les masses populaires, assoiffées de démocratie, étant descendues dans la rue un peu partout à travers le pays, à la suite de l'assassinat de Park Chung-hee 박정희 qui régentait la Corée du Sud depuis deux décennies. L'événement a d'autant plus marqué les Coréens - et ce sur plus d'une génération - que son évocation est restée taboue pendant de nombreuses années, toute possibilité de compréhension et de deuil nécessitée par un tel traumatisme s'en trouvant annihilée.

13 Même si elle ne les a pas vécus du fait qu'elle n'habitait plus à Kwangju à l'époque, ce soudain intérêt que la romancière manifeste pour ces événements n'a au fond rien de surprenant. La violence est en effet un sujet que l'écrivain Han Kang tente d'appréhender de manière obsessionnelle et sans doute un des mots-clefs qui confèrent une unité à l'ensemble de son œuvre, comme on a pu s'en faire une idée avec les deux ouvrages mentionnés plus haut. "Qu'est l'homme ? Que doit-il éviter d'être ? Que devons-nous faire pour cela? $\aleph^{17}$, fait-elle écrire à un personnage d'écrivain dans Celui qui revient. Cependant, dans cet ouvrage qui parle de l'horreur de ces événements, ce n'est pas tant la description de la violence qui intéresse l'auteur que la manière dont elle agit sur l'homme.

14 L'ouvrage, présenté comme un "roman", contient six chapitres, dans lesquels la focalisation et la voix narrative varient, procédé que l'auteur affectionne. Les six chapitres s'intitulent: «Un oisillon », « Des souffles noirs », « Sept gifles », « Du fer et 
du sang », «La prunelle de la nuit », "Vers le côté fleuri ». Il est intéressant de noter que quatre d'entre eux comportent un mot se rapportant au corps : « souffle », " gifle ", " sang », « prunelle », ce qui montre bien la place qu'occupent le corps et les sensations physiques dans l'univers de l'écrivain. Le second chapitre, intitulé "Des souffles noirs ", est sans doute l'un des plus originaux en ce qui concerne le choix de l'écriture, car c'est l'âme d'un adolescent qui vient d'être tué qui s'exprime à la première personne, en s'adressant à son meilleur ami dont il a perdu la trace dans le chaos du massacre. L'âme suit ce corps qu'elle a habité jusqu'à ce qu'une mort récente les sépare, tandis que ce dernier est transporté dans une fosse commune pour être jeté au milieu d'un tas de cadavres. Elle raconte alors sa peur et sa solitude lorsqu'elle réalise qu'elle est détachée du corps, ainsi que la tendresse, mais aussi la haine, qu'elle éprouve pour cette dépouille abandonnée sans aucune dignité :

Pendant que le camion redémarrait, je me suis approchée de ces corps. J'ai senti que les reflets des autres âmes en faisaient autant pour les envelopper. De l'eau teintée de sang coulait encore des habits de ces femmes et hommes au crâne défoncé. On avait visiblement aspergé leurs têtes car les visages n'étaient pas souillés. Le plus singulier des nouveaux arrivants était le jeune homme en tenue de malade, avec le sac sur la poitrine. Il était plus propre que les autres. Quelqu'un avait dû le nettoyer. Les plaies étaient recousues et on avait appliqué de la pommade pardessus. Le pansement qui entourait la tête faisait une tache brillante dans le noir. C'était un cadavre comme un autre, mais grâce aux traces des soins dont il avait bénéficié, il semblait infiniment plus noble, si bien que j'ai ressenti une sorte de tristesse, de jalousie. Mon propre corps, écrasé par d'autres superposés pour former une tour, traité comme de la viande de boucherie, me faisait honte, je le haïssais.

C'est vrai, à partir de ce moment, j'ai commencé à haïr mon corps. Jeté avec les autres entassés comme autant de quartiers de bœuf. Ces visages sales qui pourrissaient en puant sous le soleil ${ }^{18}$.

15 La mort dans des conditions dégradantes est décrite ici de manière surprenante, l'observation distanciée de son corps par le sujet prenant une forme extrême par le biais d'une âme qui contemple avec des sentiments complexes la carcasse qu'elle vient de quitter. La conscience de son propre corps est aussi importante chez la romancière que le sort même de ce corps, ce qui confère une force singulière au récit qui s'inspire par ailleurs de témoignages authentiques. Le chapitre 3, intitulé « Sept gifles ", écrit à la troisième personne, donne le point de vue d'une employée d'une petite maison d'édition, Unsuk 은숙, qui était lycéenne lors des événements de Kwangju. En compagnie d'une autre jeune fille, Sônju 선주, dont le récit fait l'objet du chapitre 5, elle a nettoyé à l'époque les corps des victimes des fusillades ramenés à la Préfecture, qui servait de quartier général à la milice constituée de jeunes volontaires révoltés par la violence de la répression exercée par les autorités. Après quelques années passées à l'université, elle doit abandonner ses études à cause des difficultés financières de sa famille, mais aussi et surtout parce qu'il lui est difficile, avec le souvenir du massacre de Kwangju, de mener une vie normale d'étudiante sur le campus, devenu un lieu d'affrontements quotidiens entre les manifestants étudiants et des forces anti-émeutes particulièrement féroces. La narration se situe à peu près vers 1985. La tâche éditoriale d'Unsuk est de relire les manuscrits. Il lui incombe également d'aller les déposer et les récupérer au Département de la censure, formalité alors incontournable ${ }^{19}$.

16 Le chapitre débute ainsi : «Elle a reçu sept gifles. C'était un mercredi après-midi vers quatre heures $»^{20}$. Elle avait été convoquée à un interrogatoire au sujet d'un traducteur avec lequel elle avait travaillé et qui était recherché par la police. L'attitude de la jeune 
femme qui vient d'être humiliée physiquement et verbalement - «Espèce de chienne ! Tu peux crever ici, personne ne le saura. Sale cafard ! $!^{21} »-$ est étonnamment stoïque :

Elle va oublier les sept gifles. À raison d'une par jour, elle en aura pour une semaine. Aujourd'hui, c'est le premier jour.

Elle ouvre avec la clé la porte de l'appartement qu'elle loue et entre. Elle enlève ses chaussures, les pose dans un coin, se couche sur le côté tout en gardant son manteau. Afin d'éviter une paralysie du visage, elle plie son bras pour caler sa joue gauche. Celle de droite continue à gonfler. L'œil ne s'ouvre que partiellement. La douleur née au-dessus d'une molaire atteint la tempe.

Après vingt minutes passées dans cette position, elle se lève. Elle ôte ses habits pour les mettre sur un cintre. En sous-vêtements, elle se dirige vers la salle d'eau et enfile les claquettes. Elle remplit une bassine d'eau froide, en verse sur son visage. Ouvrant la bouche avec difficulté, elle se brosse doucement les dents ${ }^{22}$.

Unsuk n'éclate pas en sanglots, ne cherche pas quelqu'un auprès de qui elle pourrait s'épancher pour se soulager, loin de là. Quand le téléphone sonne, elle préfère débrancher le fil en pensant que c'est son patron qui s'inquiète pour elle - «À quoi bon? $\aleph^{23}$, se dit-elle. La narration est largement consacrée à la description de ses gestes, ceux de la vie quotidienne, presque banals, exécutés avec un calme apparent, même si la sensation de la main, "plutôt petite pour une main masculine " ${ }^{24}$, qui s'abattait sur sa joue ne la quitte plus, tout comme, on le voit dans l'extrait ci-dessus, la conscience de son propre corps bafoué. Mais le flegme d'Unsuk s'effrite au fur et à mesure que le récit qui lui est consacré progresse et qu'elle essaie d'oublier ces sept gifles, «à raison d'une par jour ». Car si elle n'a pas vraiment réagi à cet acte de violence, la sensation qu'il a provoquée lui a rappelé Kwangju et les atrocités qui y avaient été perpétrées. La blessure que lui inflige ce souvenir, comme gravée dans le corps, est ressuscitée par une " expérience multisensorielle ${ }^{25}$, directe ou indirecte, comme la vue des jets d'eau d'une fontaine ou des doigts d'un homme tournant les pages caviardées par les censeurs. Un détail, un fragment de la vie qui, ainsi décrit, se trouve souvent à l'origine d'une émotion qui gagne le lecteur, de manière beaucoup plus forte que ne le ferait tout autre discours.

À travers trois œuvres de fiction, nous avons évoqué deux aspects de la signification du corps dans l'écriture de Han Kang. Le premier concerne la façon dont celui-ci réagit à la violence. Le corps violenté ne réagit pas par la violence. Ou plutôt, il se l'inflige en se repliant sur lui-même, presque jusqu'à refuser tout effort pour survivre, par peur de se voir devenir à son tour agresseur. Le deuxième aspect concerne plus directement l'écriture de Han Kang, car il s'agit de la manière dont le personnage évolue au gré du récit, son corps constituant le vecteur de sa réaction à la violence environnante. Les chocs sensoriels sont autant de déclencheurs d'actions. La parole, elle, n'est que rarement donnée au personnage central et, lorsque le lecteur a la possibilité de suivre l'histoire du point de vue de celui-ci, il n'a pas réellement accès à sa pensée qui se refuse obstinément à la communication, toujours sujette à malentendus, et il doit se contenter de la description de gestes et de sensations physiques. C'est ainsi que cette écriture qui creuse en tâtonnant les tréfonds de l'âme humaine pour décrire la douleur, la tristesse, la frustration, évite de sombrer dans le pathos. Tout en s'interdisant tout voyeurisme ou sentimentalisme facile, Han Kang porte un regard plein d'empathie sur ses personnages. 


\section{BIBLIOGRAPHIE}

CH'OE Sông Shil최성실, [ « La littérature coréenne et le corps »] / 한국문학과 육체 / Han'guk munhakkwa yukch'e, [Littérature et Société] / 문학과 사회 / Munhakkwa sahoe, 21(2) : 2008, p. 311-325.

HAN Kang 한강, [Le Fruit de ma femme] / 내 여자의 열 매 / Nae yôjaûi yôlmae, P’aju, Ch'angbi, 2000.

HAN Kang 한강, 채식주의자 / Ch'aeshikchuûija, P’aju, Ch'angbi, 2007 ; La Végétarienne, JEONG Eun Jin et BATILliot Jacques (trad.), Paris, Serpent à plumes, 2015 ; The Vegetarian, SMITH Deborah (trad.), London, Portobello Books, 2015.

HAN Kang 한강, 희 랍어 시간 / Hirabô shigan, P’aju, Munhakdongne, 2011 ; Leçons de grec, JEONG Eun Jin et BATILLIOT Jacques (trad.), Paris, Serpent à plumes, 2017.

HAN Kang 한강, 소년 이 온다 / Sonyôni onda, P'aju, Ch'angbi, 2014 ; Celui qui revient, JEONG Eun Jin et BATILLIOT Jacques (trad.), Paris, Serpent à plumes, 2016 ; Human Acts, sмITH Deborah (trad.), London, Portobello Books, 2016.

HAN Kang 한강, Interview sur Channel Yes, 10/06/2014, http://ch.yes24.com/Article/View/25422, consulté le 21/02/2018.

HAN Kang et al., [« 2005, 29e prix Yi Sang »] / 2005년도 제29회 이상문학상 작품집 / Ich'ônonyôndo cheishipkuhoe isangmunhaksang chakp'umjip, Séoul, 문학사상사 Munhaksasangsa, 2005.

HANG Kang, KANG Su Mi 강수미, SHIN Hyông Ch'ôl 신형 철, [« Les strates esthétiques des romans de Han Kang »] / 한강 소설의 미학적 층위 / Han'gang sosôrûi mihakchôk ch'ûngwi, [Village littéraire] / 문학동네 / Munhakdongne, 88:2016, p. 14-61.

KIM Ye Rim 김예 림, [«À propos de la souffrance ou de la beauté du “devenir-végétal” »] / 식물-되 기'의 고통 혹은 아름다움에 관하여 / Shingmul-toegiûi kot'ong hokûn arûmdaume kwanhayô, [Création et critique] / 창작과 비 평 / Ch'angjakkwa pip'yôn, 36 (1): 2008, p. 349-352.

O SE Kon 오세곤, [« Les soixante-dix ans depuis la Libération vus à travers le théâtre »] / 연극을 통해서 보는 광복 70년 / Yôn'gûkûl t'onghaesô ponûn kwangbok 70nyôn, [L'Esprit du temps] / 시대정 신 / Shidaejôngshin, 67:2015, https://blog.naver.com/ngmin/220963253431, consulté le 21/02/2018.

PARSIPOUR Shahrnoush, Femmes sans hommes, BALAŸ Christophe (trad.), Paris, Lettres persanes, 2006.

RYOO Bo Sun 류보선, “The Body in Contemporary Korean Literature”, Korean Literature Now, 32:2016, p. 21-23.

SHIN Saet Pyôl 신샛별, [«L'identité végétale et l'imagination communautaire »] / 식물적 주체성 과 공동체적 상상력 / Shingmulchôk chuch'esônggwa kongdongch'ejôk sangsangnyôk, [Création et critique] / 창작과 비평 / Ch'angjakkwa pip'yông, 44 (2): 2016, p. 355-373.

\section{NOTES}

1. La revue [Littérature et Société] / 문학과 사회 a publié en 2008 un dossier spécial sur la littérature coréenne des cent dernières années vue à travers quelques mots-clefs. Le "corps » 
figure parmi les huit termes choisis. Voir le propos de la critique littéraire CH’OE Sông Shil 최성 실, [ La littérature coréenne et le corps »] / 한국문학과 육체, [Littérature et Société] / 문학과 사회, 21(2):2008, p. 311 : «Il ne serait pas exagéré de dire que la littérature coréenne moderne et contemporaine s'est développée en cultivant le «corps » dans un espace clos et qu'elle en a fait par ailleurs un médium pour la liberté et la libération». Voir aussi le critique Ryoo Bo Sun 류보 선, “The Body in Contemporary Korean Literature", Korean Literature Now, 32:2016, p. 21 : "For a long time Korean literature has both recreated the violence of the body within the regulation of the symbolic order and dreamed of a different kind of body, one which can go beyond the regulation of bodies. Interest in the human body, therefore, is one of the various genealogies that can be traced back within Korean literature".

2. SHIN Saet Pyôl 신샛별, [ "L'identité végétale et l'imagination communautaire »] / 식물적 주체 성과 공동체적 상상력, [Création et critique] / 창작과 비평, 44 (2): 2016, p. 369.

3. Cette pratique n'est pas rare en Corée. Le genre s'appelle «nouvelles à la suite » / 연작소설 / yônjak sosôl. Les histoires ne se suivent pas forcément, mais on retrouve les mêmes personnages au fil des épisodes.

4. KIM Ye Rim 김예림, «À propos de la souffrance ou de la beauté du "devenir-végétal" " / “식물되 기'의 고통 혹은 아름다움에 관하여, [Création et critique] / 창작과 비 평, 36(1):2008, p. 351.

5. C'est ce que fait par exemple Kim Sông Kon 김성곤, président du jury qui a décerné le prix Yi Sang à la nouvelle de Han Kang. HAN Kang et al., 2005, 29e prix Yi Sang / 2005 년도 제29회 이상문학 상 작품집, 문학과사상사, Munhaksasangsa, 2005, p. 341.

6. Les exemples sont nombreux d'Ovide aux auteurs contemporains. On retrouve ce thème par exemple dans Femmes sans hommes de la romancière iranienne Shahrnoush Parsipour (1990; traduit en français par Christophe BALAŸ, 2006), qui s'interroge sur le statut de la femme dans une société machiste.

7. HAN Kang, La Végétarienne, JEONG Eun Jin 정은진 et BATILLIOT Jacques (trad.), Paris, Serpent à plumes, 2015, p. 94.

8. Ibid., p. 99.

9. SHIN, [ «'identité végétale et l'imagination communautaire »], op. cit., p. 358.

10. Ibid., p. 359.

11. «Si je l'avais épousée, bien qu'elle fût dépourvue de tout charme remarquable, c'était parce qu'elle n'avait pas non plus de défaut notable. La banalité qui caractérisait cette créature sans éclat, ni esprit ni sophistication aucune, m'avait mis à l'aise » (HAN, La Végétarienne, op. cit., p. 9).

12. Ibid., p. 19.

13. HAN Kang, Leçons de grec, JEONG Eun Jin et BATILLIOT Jacques (trad.), Paris, Serpent à plumes, 2017, p. 12-13.

14. Ibid., p. 15.

15. Ibid., p. 20.

16. Ibid.

17. HAN Kang, Celui qui revient, JEONG Eun Jin et BATILLIOT Jacques (trad.), Paris, Serpent à plumes, 2016, p. 101.

18. Ibid., p. 58.

19. La censure a existé depuis l'occupation japonaise (1910-1945) jusqu'à la fin des années 1980 pour toute forme artistique. L'obligation pour les pièces de théâtre de passer par la Commission de l'éthique des arts de spectacle disparait à la veille des Jeux Olympiques de Séoul en 1988. Voir $\mathrm{O}$ SE Kon 오세곤, [《Les soixante-dix ans depuis la Libération vus à travers le théâtre »] / 연극을 통해서 보는 광복 70년, [L'Esprit du temps] / 시대정신, 67:2015, https://blog.naver.com/ngmin/ 220963253431, consulté le 21/02/2018. Han Kang raconte que la censure lui a été presque familière dès son enfance à travers son père, écrivain également: "C'était il n'y a pas si longtemps. Un de mes souvenirs d'enfance était de voir le manuscrit de mon père revenir avec la 
revue à laquelle il était destiné. L'éditeur qui craignait la censure avait préféré ne pas le publier, mon père n'était pourtant pas un écrivain qui s'exprimait ouvertement sur les actualités politiques » (HAN Kang, Interview sur Channel Yes, 10/06/2014, http://ch.yes24.com/Article/ View/25422, consulté le 21/02/2018).

20. HAN, Celui qui revient, op. cit., p. 71.

21. Ibid., p. 73.

22. Ibid., p. 71-72.

23. Ibid., p. 72.

24. Ibid., p. 80.

25. L'expression est de SHIN Hyông Ch'ôl 신형 철, voir HAN Kang, KANG Su Mi 강수미, sHIN Hyông Ch'ôl 신형 철, [ «Les strates esthétiques des romans de Han Kang»] / 한강 소설의 미학적 층위, [Village littéraire] / 문학동네, 88: 2016, p. 14-61.

\section{AUTEUR}

\section{EUN JIN JEONG}

JEONG Eun Jin est maître de conférences à l'Institut national des langues et civilisations orientales (Inalco) où elle enseigne la langue et la littérature coréennes. Rattachée au Centre d'étude et de recherche sur les littératures et les oralités du monde (CERLOM), elle mène des recherches sur divers aspects de la littérature coréenne moderne et contemporaine, tels que l'évolution du champ littéraire sud-coréen ou les conditions de production et de réception des traductions d'œuvres coréennes. Son dernier article publié est "The South Korean Literary Field and its Evolution" paru dans Routledge Handbook of Contemporary South Korea (edited by Sojin Lim, Niki J.P. Alsford, 2022). 


\title{
Shim Chong, fille vendue de Hwang Sok-yong : du corps réprimé à la figure sublimée
}

\author{
Suk-Hee Joo
}

1 Le roman 심청, 연꽃의 길 de l'écrivain coréen contemporain Hwang Sok-yong 황석영, publié pour la première fois à Séoul en 2003 et traduit en français sous le titre Shim Chong, fille vendue, est présenté par son auteur comme la version moderne d'un récit traditionnel célèbre en Corée, dont la forme romanesque est intitulée [L'Histoire de Shim Chong]/ 심청전 ${ }^{1}$. La trame narrative de ce récit est la suivante: l'héroöne éponyme est une jeune fille issue d'une famille pauvre, qui vit avec son père aveugle depuis la mort de sa mère peu après sa naissance. Un moine ayant prédit un jour qu'il pourrait retrouver la vue s'il offrait trois cents sacs de riz à Bouddha, elle se vend, pour obtenir la somme nécessaire, à des marins qui l'offrent à l'océan en guise de sacrifice propitiatoire afin d'assurer la sécurité de leur navire.

Dans le roman de Hwang Sok-yong, l'héroïne n'est pas jetée à l'eau, mais simplement immergée, puis ressortie de la mer par les marins qui sont des trafiquants chinois. Elle est ensuite revendue comme prostituée, puis ballottée d'un pays à l'autre, de la Chine à Taïwan et au Japon en passant par Singapour, sur les mers de l'Asie orientale. De ce fait, le motif central du corps souffrant ou soumis, mais aussi aimant et aimé, structure l'œuvre dans son ensemble, permettant à l'auteur d'élaborer, selon ses propres termes, une véritable "Odyssée de la prostitution" / 매춘의 오디세 이아 / maechunui Odiseia ${ }^{2}$.

3 Cette expression a déjà donné lieu à des interprétations politiques et historiques, auxquelles se prête le roman : en situant délibérément l'action à la fin $\mathrm{du} \mathrm{XIX}^{\mathrm{e}}$ siècle, Hwang Sok-yong fait parcourir à son héroïne toute l'Asie de l'Est à une époque où l'influence et la présence impérialiste des puissances occidentales s'étend à cette partie du monde. La prostitution de l'hérö̈ne, c'est-à-dire la soumission de son corps, joue à différents niveaux, comme une métaphore de l'asservissement et de la dépossession de soi $^{3}$. 
Dans l'expression coréenne «매춘의 오디세이아》 traduite par «Odyssée de la prostitution", le terme "오디세이아/ Odiseia» renvoie directement au poème homérique retraçant les errances d'Ulysse, puisque ce terme n'a pas le sens du nom commun "odyssée » en français". La comparaison est explicite dans le passage de la postface à l'édition coréenne où l'auteur évoque la genèse du roman: il explique comment, par association d'idées, il en est arrivé à élaborer son projet à partir d'un parallèle entre la Mer Jaune et la Méditerranée ${ }^{5}$. L'une et l'autre sont des espaces maritimes caractérisés, selon lui, par la réalité historique de l'affrontement entre puissances, mais aussi par un imaginaire du voyage dont la figure méditerranéenne emblématique est Ulysse (en coréen 오디세우스 / Odysseus); en désignant la Mer Jaune comme une "Méditerranée de l'Asie orientale"/ 동아시아의 지중해 / Dongasiaui Jijunghae, le romancier se propose d'y inscrire un récit d'errances qui équivaudrait à l'Odyssée, retraçant le parcours de femmes vendues, d'où « Odyssée de la prostitution ».

5 Dans le récit que fait ici Hwang Sok-yong de l'élaboration de son projet, qui apparaît presque comme une révélation, l'épopée collective se cristallise immédiatement autour de la figure traditionnelle de Shim Chong. La comparaison avec l'odyssée s'explique donc d'abord par les errances maritimes de Shim Chong, d'un port à l'autre, qui aboutissent à la fin du roman à son retour en Corée, dans sa patrie d'origine, de même qu'Ulysse rentre à Ithaque. Pour Hwang Sok-yong, revendiquer ce parallélisme avec Homère ne consiste pas qu'à transposer le parcours méditerranéen du héros en Asie moderne : il s'agit de s'emparer d'une tradition légendaire coréenne pour la recréer sous une forme romanesque nouvelle, propre à en faire apparaître toute la puissance mythique, à travers une écriture narrative contemporaine qui intègre l'ampleur et la poésie du modèle épique.

6 C'est dans cette perspective que nous nous intéresserons aux métamorphoses du corps féminin de cette figure hérö̈que paradoxale : en quoi le motif du corps de Shim Chong permet-il de proposer, à côté des interprétations politiques et historiques de l'œuvre, différentes lectures de ses enjeux symboliques et métaphoriques? En partant de l'hypothèse que tout le projet de réécriture du Sim Cheong Jeon par Hwang Sok-yong se construit à partir du motif du corps, nous analyserons d'abord les multiples transformations de Shim Chong dans leur rapport avec la notion de réincarnation, avant de nous pencher sur la façon dont elle rejoue intérieurement le parcours de ses errances par la pratique corporelle du chant; nous nous demanderons enfin quel rôle joue la référence au chamanisme dans un roman qui aboutit à la transfiguration du corps de l'héroïne.

\section{Le corps souffrant de Shim Chong : réincarnations et voyages sur les mers de l'Asie orientale}

Dès le début de Shim Chong, fille vendue, le corps de l'héroïne est placé au cœur du récit : en effet, le premier des douze chapitres est intitulé «La réincarnation » / 환생, ce qui permet au lecteur de percevoir d'emblée que le corps de Shim Chong ne se limite pas à sa dimension individuelle, mais qu'il contient la vie des autres figures dont il est le support. À la première page du roman, le récit s'ouvre sur la descente de Shim Chong ${ }^{6}$ dans les profondeurs de la mer, puis sur sa remontée à la surface; ce retour à la vie peut être lu métaphoriquement comme une première forme de réincarnation. Elle est 
alors sur le bateau des marins, dont le lecteur va comprendre progressivement qu'ils viennent de l'immerger et de la ressortir trois fois de la mer pour accomplir le rituel propitiatoire. Dans l'obscurité de la cabine où elle est enfermée sur le bateau, Shim Chong se remémore alors, en rêve, sa jeunesse et les événements qui ont précédé ce rituel, ce qui fait l'objet d'un récit en analepse :

Son père lui [à Shim Chong] rappelait souvent que sa mère l'appelait « le petit Bodhisattva Avalokiteçvara ».

Chong se voyait flottant au ciel sur une mer de nuages. Au loin, elle apercevait le palais aux toits de tuiles où résidaient le Bouddha et les onze Bodhisattvas; sous la mer de nuages, s'étendaient les villages des humains. Le Bouddha désigna l'un des Bodhisattvas et lui dit: « Si les mœurs des hommes et des femmes sont si dissolues, c'est à cause de tes péchés. Retourne dans le monde sous une forme féminine et fais-toi un devoir d'éveiller les consciences."

De sa main, le Bouddha çakyamuni indiqua une voie, et un chemin de lumière s'ouvrit entre les nuages.

Chong aperçut une arche étincelante au-dessus d'une chaumière. C'était au fond d'un maigre village; les maisons étaient blotties côte à côte au pied d'une montagne. Une femme dormait, les jambes repliées, un bras sous la tête en guise d'oreiller. Cette scène, elle l'avait si souvent vue qu'il lui sembla se trouver devant les panneaux d'un paravent familier. [...] Le Bodhisattva dont la destinée était de devenir Chong lui déclara : « Femme, je suis le Bodhisattva Avalokiteçvara de la mer du Sud. J'ai commis des fautes et je dois me réincarner en un être humain. Mon sort est de vivre chez toi. çakyamuni m'a chargé de servir le monde, veuille s'il te plait m'accueillir, prends pitié de moi. ${ }^{7} »$ (p. 14-15)

엄마는 내가 관음보살님이랬다. 아버지가 언제나 그 말씀만 하셨지. 청이는 자기가 안개 자욱한 하늘 위 구름바다 가운데 떠 있는걸 본다. 멀리 여러 부 처와 보살들이 산다는 궁전의 기와지붕과 높다란 성벽이 솟아 있고 구름바다 아 래로는 아득하게 사람세상이 펼쳐져 있다. 그네는 지금 보궁으로부터 쫓겨나오는 참이다. 관음의 형상은 원래가 열한 가지의 얼굴을 지녔으니 석가부처께서 한 형 상을 지목해주셨다.

지금 세상에 남녀상열지사가 심히 어지러우매 그것 또한 보살인 너의 죄이니라. 너는 가서 여자로 현신하여 세간을 깨우치라.

석가께서 손으로 한 곳을 가리키자 구름바다 가운데 빛의 길이 생겨난다. 청이는 그때에 나지막한 산 아래 옹기종기 모여 앉은 작은 동네의 구석자리에 있 는 초가삼간 집을 향하여 빛의 다리가 놓인 것을 본다. 한 여인이 팔베개를 하고 다 리는 웅크리고 잠에 빠져 있다. 그 다음은 청이가 하도 많이 머릿속에 떠올려서 병 풍의 그림처럼 눈앞에 선명한 광경이다. [...] 청이 태어나기 이전의 관음 형상이 말한다.

소녀는 다른 사람이 아니오라 남해관음(南海觀音)입니다. 제가 죄를 짓고 인간으 로 정배하여 댁으로 내려올 제 제불보살 석가님이 온몸을 던져 세상을 공양하라 하셨으니 부디 받아주옵시고 어여삐 여기소서8. (p. 14-16)

Dans cette vision, Shim Chong perçoit de l'extérieur son propre corps et assiste en rêve à la rencontre de sa mère, quand elle était enceinte, avec le Bodhisattva Avalokiteçvara de la mer du Sud, désigné dans le texte original non par son appellation sanskrite mais par son nom coréen de "Gwaneum de la mer du Sud », la Guanyin chinoise, qui figure en hangeul (남해 관음 / Namhae Gwaneum) et en caractères chinois (南海觀音 / Nanhai Guanyin). La perception par Shim Chong de cette scène, à la fois familière et fondatrice pour elle, suit le principe d'une "randonnée céleste » d'inspiration chamanique, dans laquelle son esprit est détaché de son corps, lui permettant d'accéder à un événement antérieur à sa propre naissance. Ce mouvement correspond, dans le texte coréen, à un glissement de la première à la troisième personne. La première phrase de l'extrait peut en effet se traduire : « Ma mère me disait que j'étais le Bodhisattva Gwaneum. C'est ce que me répétait mon père.» Ce souvenir évoqué sur le mode du monologue intérieur 
amorce le récit de la vision, qui aboutit à la demande faite par le Bodhisattva à la mère de la future Shim Chong de l'accepter dans son corps pour lui permettre de se réincarner. Ainsi, le corps de la mère est un intermédiaire, et celui de Shim Chong devient le support de l'incarnation de Gwaneum, car cette vie humaine doit racheter ses péchés. Dans le Sim Cheong Jeon déjà, l'héroïne est la réincarnation d'un être céleste, mais Hwang Sok-yong donne une signification nouvelle à sa naissance et au parcours terrestre de ce corps humain en inscrivant son récit dans un contexte éminemment bouddhiste.

9 Si Shim Chong est la réincarnation de Gwaneum, alors les différentes métamorphoses et changements d'identité qu'elle va subir sont autant de manières, pour le Bodhisattva, de vivre les vicissitudes de l'existence humaine, selon la mission qui lui a été confiée par le Bouddha lui-même.

10 C'est dans la perspective d'un itinéraire qui lui fasse éprouver la souffrance du corps que s'inscrit l'errance de Shim Chong, revendue à de nouveaux propriétaires, d'abord comme concubine d'un riche marchand de thé de Nankin, puis prostituée ou concubine à Jinjiang, à Taïwan, à Singapour, à Okinawa où elle se marie, puis à Nagasaki où, après la mort de son mari, elle ouvre elle-même une maison close, avant de rentrer en Corée à la fin de sa vie. Or cette errance du corps de port en port et de pays en pays va de pair avec un changement constant de nom : elle reçoit d'abord des marins un nom chinois de courtisane, qui figure à la fois en caractères chinois : 蓮花 / Lianhua et dans leur transcription approximative en hangeul: 렌화/ Lenhwa, et qui signifie «Fleur de lotus »; ce nom va ensuite prendre la forme de son équivalent anglais «Lotus» (translittéré «로터스 / Loteoseu » dans le texte original) lors de son séjour à Singapour où elle est la concubine d'un négociant anglais, et enfin « Lenka / 렌카 », qui a la même signification en japonais quand elle arrive à Okinawa.

Contrairement au surnom de "petit Bodhisattva Avalokiteçvara » que lui attribuait sa mère, et qui contenait une vérité sur la destinée de l'héroïne, l'attribution de ce nom de courtisane n'indique pas simplement un changement d'identité, mais une véritable dépossession de soi.

\section{Métamorphoses du nom et errances du moi}

Le fait que Shim Chong, par ces changements de nom, se voie dépossédée d'elle-même et de son corps, est souligné par les marins qui l'avertissent dès qu'ils la remontent sur le bateau : «Rappelle-toi bien que, désormais, tu n'es plus Shim Chong» / 명 심해라. 네 이름은 지금부터 심청이가 아니니라 (p. 10 de l'original et de la traduction). Le passage dans lequel les marins chinois, accompagnés d'un marchand coréen, lui attribuent ensuite son nom chinois met en évidence son changement de statut après la cérémonie du rituel propitiatoire : il faut lui donner un nouveau nom parce qu'elle ne s'appartient plus. De fait, c'est ce nom qui permet de l'inscrire "sur la liste des marchandises transportées ", donc de la réifier et d'en faire un objet de possession et de domination:

Le vieux marchand chinois adressa un sourire à Chong :

- Tu as l'air d'une fille douce et gentille. Puisque la cérémonie pour le roi de la mer

a eu lieu, il va falloir te donner un nouveau nom.

Le Coréen se récria :

- C'est à vous que revient ce soin.

- Ah bon! Comment allons-nous donc l'appeler?

Approchant la tasse de thé de ses lèvres, le vieux réfléchit, puis, secouant la tête : 
- Appelons-la Lenhwa, «Fleur de lotus »!

Le capitaine reprit avec un large sourire :

- Lotus rouge ou lotus blanc ? C'est qu'ils sont très différents, déjà au regard !

- Les deux! Appelons-la Lenhwa tout court. Enregistrez-la sous ce nom sur la liste

des marchandises transportées, dit-il au pilote. (p. 26)

늙은 상인이 웃는 얼굴로 대답했다.

“그래 아주 참하게 생겼구나. 용왕제도 치렀으니 새 이름을 지어줘야겠지."

조선 상인이 말했다.

"대인께서 지어주십시오."

“그래, 뭐라고 할까......? ?"

늙은이가 찻잔을 입에 갖다대며 생각해보더니 고개를 끄덕인다.

“렌화(蓮花), 렌화라구 하지.”

선장이 웃으면서 말했다.

“홍련이오, 백련이오? 붉은 꽃과 흰 꽃은 보기에도 아주 다릅니다.”

“그 둘 다요. 그냥 렌화라구 하지. 자네 치부책 물목에다 그렇게 올리게."

늙은이가 곁에 앉은 상인에게 말하자 모두 고개를 끄덕였다. (p. 26)

On sait l'importance du lotus dans la symbolique et dans l'imagerie du bouddhisme, fleur caractérisée par sa pureté alors qu'elle pousse dans une eau boueuse ; il est donc significatif que Hwang Sok-yong ait choisi ce nom de courtisane pour l'héroïne de son roman, et que les deux noms soient associés dans le titre original de l'œuvre 심청, 연 꽃 의 길 / Sim Cheong, Yeonkkotui gil, que l'on peut traduire par [Shim Chong, le chemin du Lotus], ou plutôt Le chemin de Lotus. Ce titre est d'ailleurs le seul endroit, dans l'œuvre, où Shim Chong est désignée par le terme coréen signifiant "lotus ", 연 꽃 / yeonkkot, puisque dans le roman les noms de Lenhwa, Lotus et Lenka appartiennent à une langue étrangère pour marquer l'arrachement de son corps à son pays d'origine, et sa circulation d'un pays à l'autre 9 . C'est la raison pour laquelle le marchand coréen, dans l'extrait cité ci-dessus, laisse aux Chinois le soin de renommer Shim Chong dans leur langue : l'asservissement de l'héroïne et la dépossession de soi va de pair dans le roman avec l'éloignement de sa Corée natale. De ce point de vue, elle est effectivement «transportée » comme une marchandise, et son corps devient le lieu de plusieurs incarnations successives, comme si une nouvelle vie ou une nouvelle identité chassait symboliquement la précédente à chaque fois. L'originalité de cette «Odyssée de la prostitution » résiderait dans le fait qu'il ne s'agit pas seulement de suivre le parcours d'une figure individuelle, mais de lui donner une dimension métaphorique et collective : Shim Chong, par ses incarnations incessantes serait emblématique de toutes les filles vendues et exploitées dans les ports d'Asie, selon les propos tenus par l'auteur dans la postface de l'édition originale.

Ainsi, pour prendre deux exemples de ces métamorphoses successives, l'héroïne voit son corps physiquement transformé peu après son arrivée à Nankin, où elle est longuement lavée, massée et apprêtée par les domestiques de son nouveau maître, ou encore à son arrivée à Singapour, où elle est coiffée et habillée à l'occidentale. Dans les deux cas, c'est cette transformation et le fait de se regarder elle-même dans un miroir qui donnent lieu à un dédoublement de Shim Chong, et à un dialogue entre l'ancienne et la nouvelle identité qui se partagent son corps.

Le dialogue intérieur entre Shim Chong et Lenhwa illustre bien ce lien entre le personnage et son premier alter ego, au moment où elle a une vision alors que son maître, le vieux marchand de thé de Nankin, tente de s'unir physiquement à elle et de prendre possession de son corps :

Des voix s'entremêlaient :

«Va t'en, ça c'est mon corps. » 
«Ah! non, ce corps est le mien. »

Quand elle prêtait une attention plus soutenue, les voix, bien que de hauteur différente, lui semblaient appartenir à la même personne.

«Je suis Chong. Qui es-tu donc?»

«Mon nom est Lenhwa. Toi, tu es déjà un fantôme. »

«Le fantôme de qui ?"

«Tu es morte, et ce n'est pas d'aujourd'hui!»

Sous ses paupières, Chong voyait clairement deux jeunes filles nues. L'une avec une longue tresse qui lui tombait dans le dos; l'autre coiffée de deux tresses enroulées en chignon. Elles s'approchèrent l'une de l'autre, fusionnèrent comme deux ombres superposées. (p. 50)

서로 다른 목소리의 계집아이들이 제각기 말을 건다.

넌 나가란 말야. 여긴 내 몸이야.

아니 이건 내꺼야.

자세히 들어보면 계집아이들의 목소리는 낮고 높은 것이 서로 섞여 있을 뿐 같은 소리로 들린다.

나는 청이야. 넌 누구니?

나는 렌화라니까. 넌 이미 귀신이야.

누구 귀신?

예전에 벌써 죽은 귀신.

청이의 감긴 눈꺼풀 안쪽 부연 어둠 속에서 벌거숭이 두 계집아이의 모습이 뚜렷 하게 떠올랐다. 한쪽은 외로 땋은 머리를 늘어뜨리고 다른 하나는 양갈래로 땋은 머리를 엮어서 뒤통수 위로 틀어올렸다. 둘은 서로 뒤엉키더니 그림자가 합쳐지 듯 흐릿하게 하나가 되었다. (p. 49-50)

Dans cette vision où l'héroïne semble abstraite de son corps encore une fois et perçoit la scène de l'extérieur, la lutte oppose l'ancienne et la nouvelle identité de Shim Chong ; le corps au moyen duquel le Bodhisattva a pu renaître, et qui est censé être symboliquement mort au moment de l'immersion dans la mer, devient le lieu du surgissement de Lenhwa, qui coïncide avec le début réel de sa prostitution. Il s'agit d'abord d'une opposition, puis d'une superposition des deux figures, comme le montre la fin de l'extrait, ce qui incite à lire l'enchaînement des changements d'identité de Shim Chong non comme une véritable substitution de l'une à l'autre, mais plutôt comme un cycle ininterrompu de transformations et d'emprunts de son corps.

Ce cycle s'achèvera quand l'héroïne, de retour en Corée à la fin du roman, reprendra son nom de Shim Chong et sera appelée par les villageois 연화보살 / Yeonhwabosal : il s'agit cette fois non de la transcription, mais de la traduction en coréen des caractères chinois 蓮花 / Lianhua / «Fleur de lotus »; quant à bosal, c'est un terme honorifique qui peut s'appliquer à une croyante bouddhiste, mais c'est aussi et surtout le mot coréen correspondant au sanskrit Bodhisattva, par lequel était notamment désignée Gwaneum dans notre premier exemple. Autrement dit, on peut considérer que cette dénomination consiste à "sanctifier", par cette mention religieuse, son nom de courtisane, désormais prononcé (et écrit) à la coréenne.

$\mathrm{Au}$ terme du parcours géographique et du cycle de "réincarnations » métaphoriques, l'héroïne réalise donc la destinée symbolisée par la vision qu'elle avait eue du Bodhisattva Gwaneum s'adressant à sa mère: le corps souffrant, à travers ses métamorphoses, est bien le vecteur d'une sublimation, qui trouve sa réalisation mystique dans cette appellation Yeonhwabosal. Celle-ci peut être interprétée comme signe de l'accession de la courtisane au statut de Bodhisattva dans le regard des villageois. En ce sens, la trajectoire de Shim Chong montre que le corps de l'héroïne n'est pas à proprement parler le lieu d'une impermanence fondamentale, mais plutôt l'instrument de la réalisation de cette destinée. Nous allons voir à présent en quoi le 
corps en performance, à travers la pratique du chant, permet à l'héroïne de lutter contre cette dépersonnalisation et de parvenir à cette sublimation de soi.

\section{Le corps sensoriel et la réactivation du souvenir par le chant}

$19 \mathrm{Au}$ cours du roman, le lecteur voyage par procuration en suivant l'itinéraire de l'héroïne. Cet itinéraire se déploie à travers deux dimensions du corps de Shim Chong, d'une part son corps physique qui est déplacé de force dans les différents pays, et d'autre part un corps psychique qui, à plusieurs reprises, se déplace au moyen de la musique et du chant : la performance musicale ou chantée permet alors à l'héroïne de reparcourir mentalement les lieux où elle a demeuré un certain temps. À l'errance du personnage s'ajoute un deuxième niveau de vagabondage, celui de la pensée, des souvenirs et des sensations, qui fait surgir ici et maintenant un lieu qui appartient à l'ailleurs et au passé. Or ce voyage mental devient possible par l'intermédiaire de pratiques qui engagent explicitement le corps physique de Shim Chong, et notamment de la performance chantée. Il ne s'agit pas simplement d'un banal processus de remémoration, mais d'une forme de résistance à la dépersonnalisation, puisque cette faculté d'accéder à un autre lieu et à un autre temps permet d'inscrire dans le corps de l'héroïne, malgré les métamorphoses incessantes, la permanence de son identité.

Ainsi, lors de son séjour dans le port chinois de Jinjiang / 진장 / Jinjang, situé sur la rive du Yangzi, une scène montre Shim Chong retirée sur un bateau tandis que la nuit résonne des bruits de la fête qui a lieu en ville. Ils la font replonger en rêve dans ses souvenirs d'enfance en Corée, et elle se remémore un chant qui évoque son pays natal et qu'elle se met à fredonner. C'est alors que survient le jeune musicien Dongyu / 동유 qui vient la rejoindre :

"Je vous ai entendue chantonner, je me suis senti rassuré. Qu'est-ce que c'était, cette chanson?»

- C'est un air de chez nous, il parle de la lune. » Elle se mit à fredonner avec la voix de Chong :

Lune, lune, toi qui es si claire

Lune que chantait Li Po

Dans la lune là-haut

Il y a des canneliers

Les montagnes et l'eau sont belles

J'aimerais y construire une petite chaumière

$Y$ faire venir mes parents

Vivre ensemble mille ans, dix mille ans. (p. 155-156)

“노랫소리를 듣고 안심했어요. 그건 무슨 노래지요?"

“우리네 고향의 달노래요."

그네는 다시 렌화가 아닌 청이가 되어 노래를 흥얼거린다.

달아 달아 밝은 달아

이태백이 노던 달아

저기 저기 저 달 속에

계수나무 박혔으니

산도 좋고 물 좋은데

초가삼간 집을 짓고

양친부모 모셔다가

천년만년 살고지고 (p. 160-161) 
Le chant de Shim Chong est issu de sa vision du passé, puisqu'elle l'entend en rêve avant de le fredonner: c'est lui qui attire alors Dongyu et permet le tête-à-tête amoureux; or le texte insiste sur le fait que, au moment de reprendre ce chant à la demande de Dongyu, l'héroïne redevient pleinement elle-même. En effet, le texte coréen ajoute une précision qui n'a pas été traduite en français : quand elle se remet à chanter, elle redevient Chong, "et non pas Lenhwa»/ 렌화가 아닌 청이가 되어 / Lenhwaga anin cheongiga doeeo. Cette précision indique que le chant est le vecteur d'une nouvelle transformation, puisque Shim Chong reprend le corps que Lenhwa lui avait volé à partir du moment où elle était tombée dans la prostitution. C'est d'ailleurs ce qu'affirme l'héroïne elle-même quelques lignes plus loin :

Dongyu l'entraîna dans la cabine, ils se retrouvèrent tous deux jetés au sol. Il

l'étreignit, releva sa jupe. Mais Chong le repoussa doucement.

- Je ne suis pas Lenhwa, je suis Chong, dit-elle en se relevant.

Dongyu prononça ce nom à voix haute à plusieurs reprises. Elle se traîna à genoux jusqu'au brûle-parfum qu'elle apporta sur la table. Elle emplit d'eau un bol de porcelaine qu'elle plaça aussi sur la table, puis se pencha au-dessus du récipient. Dongyu, embarrassé, l'observait.

- Que faites-vous?

Chong s'assit dignement.

- C'est notre cérémonie nuptiale.

- Moi je n'ai qu'un pipa. Je n'ai pas de parents, pas de pays natal, dit tout bas Dongyu.

D'un signe de la tête, Chong approuva :

- Moi aussi, je n'ai que mon corps. Je suis seule dans ce monde immense. (p. 156-157)

동유가 청이를 억세게 잡아 발이 반쯤 늘어진 지붕 아래로 당기니 두 사람은 부들 자리가 깔린 지붕 아래로 비스듬하게 넘어졌다. 동유가 성급하게 그네를 안으면 서 한 손으로 옷자락을 걷어올리자 청이가 그의 가슴팍을 살그머니 밀어내며 일 어나 앉았다.

“내 이름은....... 렌화가 아니라, 청이에요."

동유가 청이의 말을 따라서 몇 번 되뇌어본다. 청이는 앉은뱅이걸음으로 돌아앉 아 제단 앞으로 가서 향로와 사기그릇 하나를 집었다. 다시 붙박이 상 앞으로 와서 향로를 놓고 뱃전 밖으로 상반신을 기울여 그릇에 강물을 떠다 상 한가운데에 놓 았다.

어리둥절해진 동유가 청이의 하는 양을 지켜보다가 물었다.

“뭘 하는 거요?"

청이는 상 앞에 가서 단정히 앉더니 말했다.

“우리 혼례를 올려요."

"난 가진 거라고는 비파밖에 없어요. 부모님도 고향도 없구요......"

동유가 중얼거리자 청이는 고개를 끄덕였다.

“나두 내 몸뚱이밖엔 없답니다. 이 너른 세상에 나 혼자예요." (p. 161-162)

En procédant à cette cérémonie de mariage improvisée, Shim Chong reprend possession d'elle-même, ce qui est rendu possible par la réappropriation de son véritable nom. Le fait que Dongyu répète ce nom "à plusieurs reprises ", presque comme une litanie, lui confère une forme d'efficacité rituelle puisque la cérémonie met face à face les deux jeunes gens dans leur identité propre ; le dénuement et l'absence de lien à leurs origines familiales renforce le parallélisme entre les deux personnages. Si le corps de Shim Chong apparaît ici explicitement comme la seule chose qui lui appartienne vraiment, c'est en deux sens: vendre son corps est son moyen de subsistance, de même que le pipa pour Dongyu; mais dans le cadre précis de cette cérémonie nuptiale, cette affirmation souligne aussi le fait que c'est paradoxalement dans son corps que réside la permanence de son identité puisque, par l'intermédiaire 
du chant, elle est redevenue temporairement Shim Chong quand elle s'unit à Dongyu. Après leur nuit de noces, les deux jeunes gens plongent d'ailleurs dans le fleuve, ce qui rappelle évidemment le motif de la renaissance après l'immersion dans la mer au tout début du roman. Ils vont en fait être séparés rapidement, et Shim Chong va continuer à subir les changements d'identité, mais cet épisode montre que le chant peut lui permettre de reconquérir son corps.

Un dernier exemple met en scène une parole performative, dotée d'une efficacité comparable à celle du chant par lequel le passé de Shim Chong est réactivé : il s'agit d'une performance chamanique qui donne aussi une indication décisive sur la manière dont Hwang Sok-yong construit son « Odyssée de la prostitution » à partir des versions chamaniques de l'histoire de Shim Chong.

\section{La transe chamanique comme moyen de retour à une identité première}

Lors de son séjour sur l'une des îles de l'archipel des Ryūky $\bar{u}^{10}$, l'héroöne assiste à une cérémonie chamanique au cours de laquelle la yuta/ 유타, la chamane locale, se retrouve possédée par l'esprit de la mère de Shim Chong, qui s'adresse à elle :

La yuta se mit à danser en tournoyant. Un tambour accompagnait ses virevoltes. $\mathrm{Au}$ bout d'un moment, s'arrêtant devant Chong, elle éclata en pleurs et lui prit la main : - Qui est-ce donc? Ne serait-ce pas ma fille, par hasard?

Chong se laissa attirer dans le cercle.

- Oui, maman, c'est moi, Chong.

Chong s'était mise à parler la langue de son enfance, ni celle du Ryūkyū ni celle de Chine, mais celle de Joseon. La chaman, elle, continua en ryūkyū.

- Je suis venue ici par la grâce du roi de la mer. Qu'il est triste de te voir vagabonder en quête de ton père de par le vaste monde...

Les joues ruisselantes, Chong se pendit au cou de la yuta.

- Je suis Lenka, je ne suis plus Chong! On m'a jetée à la mer, je suis devenue une autre. Mon corps n'est pas celui que vous m'avez donné, je n'en ai gardé que l'âme. (p. 457-458)

유타는 다시 덩실대며 춤을 추고 돌아갔다. 마당 바깥에서 두드리는 북소리만 들 리는 가운데 유타는 빙빙 돌다가 군중들 틈에 서 있던 청이의 앞으로 다가섰다. 유 타 여인이 청이의 두 팔을 잡고 서럽게 울음을 터뜨렸다.

“아니 이게 누구냐...... 내 딸, 내 딸이 아니냐?"

청이는 그네의 손에 이끌려 저도 모르게 굿마당 안으로 들어서며 말했다.

“예 엄마, 저 청이어요."

류큐 말도 중국 말도 아닌 낮선 조선 말이 튀어나왔지만 유타는 아랑곳없이 미야 코 사투리의 류큐 말로 댓거리를 했다.

“내가 먼저 세상을 떠나 저승에 가 있더니 내 딸이 만리타국 우치나에 산다 하여 용왕님의 덕을 빌어 예까지 왔구나. 나 떠난 뒤에 우리 딸아, 아버지 모시고 어찌 살다가 대륙을 건너 바다 나라에까지 왔느냐. 서럽고도 서럽구나."

청이도 눈물을 비 오듯이 흘리며 유타의 목을 그러안고 운다.

“인당수 깊은 물에 빠졌다가 건져내어 다른 몸으로 태어나니 저는 이미 청이가 아

니라 렌카여요. 어머니 아버지 주신 몸이 아니고 세상 티끌이 모여서 이루어진 다

른 몸이니 넚만 엄마 딸이어요." (p. 515)

Alors que la chamane est déjà en transe et au milieu du rituel de possession, le fait que l'esprit de la mère de Shim Chong parle soudainement par sa bouche donne lieu à une scène de reconnaissance inattendue et problématique dans la mesure où elle manifeste l'identité fluctuante de l'héroïne. En effet, celle-ci commence par s'identifier sous le nom de Chong que lui ont donné ses parents, avant d'affirmer qu'elle est actuellement 
Lenka, et que son corps ne lui appartient donc plus en propre, puisqu'il n'est pas celui qu'elle a hérité de ses parents. L'immersion dans la mer est explicitement définie comme le début d'un cycle de métamorphoses qui vont de pair avec l'errance du personnage ; mais en affirmant ensuite que son âme est restée la même, Shim Chong maintient ce lien affectif et généalogique avec son père et sa mère. Le texte original montre encore plus nettement que cette traduction à quel point identité et hérédité sont ici confondues: 넚만 엄마 딸이어요, littéralement «seule mon âme est celle de votre fille, maman ». Si le corps physique et le nom qui le désigne se trouvent aliénés par les vicissitudes de l'errance et de l'exil, l'âme est ce qui subsiste de l'identité conférée à Shim Chong par ses parents, et ce qui lui permet de reprendre sa place dans sa lignée généalogique. La narration insiste sur le fait qu'elle parle spontanément en coréen, retrouvant naturellement sa langue maternelle alors que la yuta continue à s'exprimer dans la langue des Ryūkyū : la scène est décrite d'un point de vue externe, montrant systématiquement Shim Chong en interaction avec la chamane et non avec sa mère, mais la rencontre est effectivement réalisée, symboliquement, par cet usage de la langue coréenne, qui relie l'héroöne à ses origines alors même qu'elle affirme qu'elle n'est plus la même. La langue coréenne crée du point de vue de Shim Chong cette situation d'interlocution avec sa mère, et rend ainsi possible, paradoxalement, la réunion de son corps et de son âme.

En effet, cette disjonction est appelée à se résoudre, comme l'annonce ensuite la mère de Shim Chong par l'intermédiaire de la chamane :

Chong tendait les bras, mais la yuta la repoussait froidement:

«Je pars, ma fille, la vie est un tissu de déboires et d'absurdités. Si ton mari quitte ce monde avant toi, ne sois pas triste. Tu finiras par retourner au pays qui est le tien. Tu seras enterrée dans ta terre natale."

Chong ne s'expliquait que par bribes ces augures, mais elle comprit - et le garda au fond du cœur - que son mari devait quitter ce monde avant elle et qu'un jour elle regagnerait son pays de naissance. (p. 458-459)

유타가 뒷걸음질로 물러나는데 청이는 다가서며 어머니의 혼이 실린 무녀의 손을 잡으려고 앞으로 두 손을 내뻗는다. 유타가 냉정하게 청이의 손을 뿌리치고 좀더 멀찍이 물러서며 말했다.

“나는 간다 내 딸아. 세상살이는 허망한 일, 네 남편이 천리타국으로 떠날지라도 낙심하지 말거라. 네 남편이 먼저 떠나더라도 슬퍼하지 말거라. 너는 잘 살다가 고 향에 돌아갈 게야. 고향땅에 묻히게 될 게야."

청이는 유타의 입에서 흘러나오는 말들을 다 알아듣지는 못했지만 남편이 먼저

떠난다는 말이며 고향에 돌아가게 될 거라는 말만은 가슴속에 남았다. (p. 516-517)

Dans les paroles qui précèdent celles-ci, la mère de Shim Chong parlant par la bouche de la yuta met en cause le nouveau statut social auquel sa fille est finalement parvenue à ce moment du roman, puisqu'elle vient d'accéder à la noblesse en épousant son dernier amant, le seigneur de Miyako, île des Ryūkyū où se déroule cette scène. Elle réaffirme la vanité de ces honneurs terrestres et le fait que Shim Chong soit destinée à retrouver la terre de ses origines, ce qui implique que la situation stable et heureuse à laquelle elle est enfin arrivée par ce mariage ne soit pas le véritable aboutissement de ses errances. Ainsi, le corps de l'héroïne n'est pas appelé à rester en exil, sous cette dernière identité consacrée par le lien matrimonial qui la fait entrer dans une lignée aristocratique étrangère, mais à réintégrer symboliquement sa famille en retrouvant le sol de la Corée. L'augure délivré par la mère de Shim Chong annonce son retour dans sa terre natale, c'est-à-dire aussi dans sa lignée généalogique, donc sous son identité 
première. En ce sens, l'énoncé proféré par la chamane est performatif, puisqu'il affirme la structure cyclique du récit et rend possible ce retour aux origines.

Or cette scène est le pendant d'une autre cérémonie chamanique décrite au tout début du roman, celle du rituel propitiatoire dont Shim Chong est la victime offerte par les marins. Les préparatifs et les étapes du rituel, dont l'héroïne ne connaît pas l'aboutissement, font l'objet d'un récit détaillé, et la cérémonie elle-même est scandée par deux passages poétiques qui participent de sa réalisation : la complainte des marins puis l'invocation de la chamane au «Roi de la mer », littéralement au "Roi-dragon » / 용왕 / Yongwang (p. 19-24 de l'original et de la traduction). À la différence de la yuta des Ryūkyū, la figure de la chamane, dans le premier chapitre, apparaît donc comme menaçante vis-à-vis de Shim Chong; de plus, c'est une figure double, puisque cette chamane du village officie à la demande de la belle-mère de l'héroïne, qui pratique ellemême le chamanisme. Mais le rapprochement entre ces deux scènes, les seules du roman qui mentionnent une performance chamanique, s'impose d'autant plus que, dans le premier chapitre aussi, Shim Chong, immergée sous l'eau, avait cru voir sa mère et essayé en vain de s'en approcher en l'appelant. Il y a donc un effet de boucle entre les deux rituels: le premier enclenche le cycle des métamorphoses et de la dépersonnalisation de l'héroïne, qui aperçoit sa mère sans pouvoir l'atteindre au moment où elle frôle elle-même la mort, le second rend possible cette rencontre et l'annonce de son retour ainsi que la réappropriation de son identité. Un tel dispositif met en évidence le rôle fondamental de l'inspiration chamanique dans la réécriture de l'histoire de Shim Chong par Hwang Sok-yong, tout en insérant le motif de la dissociation du corps et de l'esprit dans un schéma cyclique, proprement bouddhiste, qui est celui de la renaissance de Shim Chong, et qui relie le début et la fin de l'œuvre.

Enfin, la scène de la yuta peut faire l'objet d'un nouveau parallèle avec la poésie homérique si l'on y voit une référence à la rencontre entre Ulysse et sa mère Anticlée aux portes des enfers, au chant XI de l'Odyssée (v. 150-224). C'est en effet par un sacrifice rituel dont la magicienne Circé lui a donné le conseil qu'Ulysse invoque les ombres des morts afin de les interroger sur sa famille restée à Ithaque et sur la possibilité de trouver le chemin du retour. L'ombre du devin Tirésias lui confirme d'abord les conditions auxquelles se fera ce retour, puis Ulysse peut s'entretenir avec sa propre mère, qui est morte pendant son absence et qui commence par l'interroger sur son errance. L'intertextualité entre les deux œuvres est renforcée par le fait qu'Ulysse tente aussi par trois fois, en vain, de saisir l'ombre de sa mère; enfin leur échange est également déterminant pour la réalisation de la destinée du héros dans la mesure où Anticlée le rassure sur la fidélité de Pénélope, qui attend le retour d'Ulysse avec son fils Télémaque et son père Laërte, lui offrant ainsi la perspective de réintégrer sa place dans la lignée familiale en retrouvant le trône et la terre d'Ithaque. On peut considérer que le souvenir de l'épisode homérique contribue à conférer sa force dramatique et sa portée symbolique à la scène de la yuta, où Shim Chong entend de la voix de sa mère la promesse d'un retour qui serait l'aboutissement de ses errances, comme si la figure de la mère assumait ici à la fois les rôles d'Anticlée et de Tirésias chez Homère.

La présence des motifs chamaniques est déterminante dans la structure de l'œuvre et permet à l'auteur d'ouvrir son récit, parfois réaliste, à une inspiration mythique qui renforce sa dimension légendaire, symbolique et métaphorique. Tous ces passages du roman qui mettent en scène une parole inspirée, comme la parole des chamanes ou les chants de Shim Chong, contribuent à donner à l'œuvre de Hwang Sok-yong une tonalité 
qui la rapproche de l'épopée homérique. C'est donc aussi sur le plan poétique que l'on peut parler d'une véritable «Odyssée de la prostitution ».

\section{Conclusion}

La référence aux versions chamaniques de la légende de Shim Chong, qui est revendiquée explicitement par l'auteur dans sa postface ${ }^{11}$, fournit un élément d'interprétation de la poétique romanesque de Hwang Sok-yong. Par cette insistance sur ses sources d'inspiration, et par l'importance qu'il donne à la figure du chaman, au jeu d'interférences entre réalisme et surnaturel, mais aussi à la place et aux effets de la parole poétique dans le récit, on peut aller jusqu'à dire que le romancier élabore son esthétique en adoptant la posture d'un chaman, comme équivalent coréen d'un aède homérique, livrant sa version renouvelée de la tradition narrative de Shim Chong. Mais dans notre perspective, cette référence au chamanisme fournit d'abord une clé pour comprendre comment le motif du corps devient central dans cette réécriture. En effet, dans plusieurs épisodes de rêve ou de vision que nous avons étudiés, la conscience de l'héroïne est dissociée de son corps pour lui permettre de jeter un regard extérieur sur elle-même, affranchi des contraintes du temps et de l'espace; ce principe du vagabondage de l'esprit, typique du chamanisme (et dont l'application dans le champ poétique remonte au poète chinois $\mathrm{Qu}$ Yuan 屈原, aux IV $\mathrm{IV}^{\mathrm{e}} \mathrm{III} \mathrm{e}^{\mathrm{e}}$ siècles av. J.-C.), parcourt l'œuvre entière même quand il n'est pas explicitement fait mention de chamanisme. C'est donc un dispositif d'écriture qui permet à Hwang Sok-yong de placer au centre de l'œuvre la question du rapport de l'héroïne à sa propre personne et à son propre corps. Le roman pourrait se prêter à bien des interprétations psychanalytiques, mais on a préféré analyser comment le dispositif narratif et la construction métaphorique s'articulaient autour de cet enjeu de la sublimation du corps. Pris dans le mouvement cyclique des incarnations et des transformations, le corps de Shim Chong est alternativement l'objet d'une domination et, paradoxalement, l'instrument d'une affirmation de soi. C'est en cela que l'on peut dire que le projet de réécriture du roman traditionnel consiste à faire de Shim Chong une réinvention féminine et asiatique d'Ulysse, l'héroïne d'une odyssée intérieure.

\section{BIBLIOGRAPHIE}

CHo Dong-il 조동일, [Histoire générale de la littérature coréenne] / 한국문학통사 / Hanguk munhak tongsa, Séoul, Jisiksaneopsa, 1994, 5 vol.

CHO Dong-il et BOUCHEZ Daniel, Histoire de la littérature coréenne, des origines à 1919, Paris, Fayard, 2002.

CHOI-CHABAL Eun-sook, «L'Odyssée d'une femme vendue : escales sur le chemin du salut dans Shim Chong, le chemin du Lotus de Sok-Yong Hwang (roman) », in L'Escale portuaire entre mythes et réalités, 
de l'Antiquité au XXI e siècle, BARZMAN John, CASTELAIN Jean-Pierre et WAUTERS Éric (éds.), Mont-SaintAignan, Presses universitaires de Rouen et du Havre, 2014, p. 63-81.

HOMÈRE, L’Odyssée, JAccotTET Philippe (trad.), Paris, La Découverte, 2004.

HWANG Sok-yong 황석 영, [Shim Chong, le chemin du Lotus] / 심청, 연꽃의 길 / Sim Cheong, Yeonkkotui gil, Séoul, Munhakdongne, 2007 [2003].

HWANG Sok-yong 황석 영, Shim Chong, fille vendue, CHOI Mikyung et JUTTET Jean-Noël (trad.), Paris, Points, 2011 [2010].

HWANG Sok-yong 황석 영, [" Le Romancier Hwang Sok-yong: “Je suis un jeune auteur” ", entretien de Hwang Sok-yong avec un intervieweur non mentionné] / 나는 청 년작가이다, 소설 가 황석 영 / Naneun cheongnyeon jakgaida, soseolga Hwang Seokyeong, 채 널예스 / Channel Yes [en ligne], 1:2003, http://ch.yes24.com/Article/View/12831, consulté le 01/08/2018.

LEE Jong-moon, [Étude du chamanisme dans l'œuvre romanesque de Hwang Sok-yong] / 이종문, 황석영 소설의 샤머니즘 관련성 연구 / Hwang Seokyeong soseolui syameonijeum gwallyeonseong yeongu, thèse, Université de Dongguk, 2015, p. 90-110.

SEO Young-chae, [La Prostituée Shim Chong et les trois cérémonies pour les morts : lecture de Shim Chong de Hwang Sok-yong] / 서 영채, 창녀 심청과 세 개의 진혼제 : 황석 영의 ‘심청' 읽기 / Changnyeo Sim Cheonggwa se gaeui jinhonje: Hwang Seokyeongui 'Sim Cheong' ikgi, Séoul, Munhakdongne, 38:2004, p. 280-298.

\section{NOTES}

1. Selon les historiens de la littérature coréenne Cho Dong-il et Daniel Bouchez, le terme 전 / jeon désigne au XIX ${ }^{e}$ siècle un "roman-pansori ", un texte qui se donne comme la version romanesque d'un récit appartenant à la tradition narrative chantée du pansori. Mais dans le cas de Shim Chong, il se pourrait qu'une version romanesque, insistant sur la piété filiale de l'héroïne, ait été antérieure à la version chantée qui est attestée dans la collation de textes de pansori réalisée par le lettré Shin Jae-hyo / 신재효 (1812-1884) au milieu du XIX ${ }^{\mathrm{e}}$ siècle ; voir cHo Dong-il et BOUCHEZ Daniel, Histoire de la littérature coréenne, des origines à 1919, Paris, Fayard, 2002, p. 309-316. La légende de Shim Chong, qui donna lieu à de nombreuses réécritures au $\mathrm{xx}^{\mathrm{e}}$ siècle, appartient donc à la fois à la tradition romanesque 심청 전 / Sim Cheong Jeon, à celle du pansori 심청 가 / Sim Cheong Ga et à celle du chant chamanique 심청굿 / Sim Cheong Gut : pour une analyse détaillée des interactions entre ces formes narratives, voir $\mathrm{cHO}$ Dong-il, [Histoire générale de la littérature coréenne] / 조동일, 한국문학통사, vol. 3, Séoul, Jisiksaneopsa, 1994, p. 568-598.

2. Cette expression est employée par Hwang Sok-yong dans la postface de la première édition du roman (2003), reprise dans l'édition de 2007. V. HWANG Sok-yong 황석 영, [Shim Chong, le chemin du Lotus] / 심청, 연꽃의 길, Séoul, Munhakdongne, 2007, p. 698.

3. Le parallélisme entre l'exploitation du corps de l'héroöne et la domination capitaliste et impérialiste exercée à cette époque par les puissances occidentales sur les pays de l'Asie de l'Est fait en particulier l'objet d'une étude par Eun-Sook Choi-Chabal dans CHOI-CHABAL Eun-Sook, "L'Odyssée d'une femme vendue : escales sur le chemin du salut dans Shim Chong, le chemin du Lotus de Sok-Yong Hwang (roman) », in L'Escale portuaire entre mythes et réalités, de l'Antiquité au $\mathrm{XXI}^{e}$ siècle, BARZMAN John, CASTELAIN Jean-Pierre et WAUTERs Éric (éds.), Mont-Saint-Aignan, Presses Universitaires de Rouen et du Havre, 2014, p. 63-81. L'article insiste aussi sur le dépassement de cette situation de domination auquel aboutit, dans le roman, la destinée de Shim Chong, dans la perspective métaphorique de l'émancipation féminine. 
4. Cette dernière acception peut être assumée par le nom 오디세이 / odisei, translittération coréenne du nom propre ou du nom commun anglais odyssey.

5. HWANG, [Shim Chong, le chemin du Lotus], op. cit., p. 698.

6. Le nom de Shim Chong prend tout son sens symbolique dans cette immersion, puisqu'il s'écrit en chinois 沈清, où 沈 / chen signifie littéralement «profond, couler, être plongé », et où 淸 / qing, « clair », peut renvoyer à l'eau claire, ou à la pureté d'un cœur vertueux.

7. La pagination des passages cités en français renvoie à la traduction française de l'œuvre étudiée: Shim Chong, fille vendue, cHoI Mikyung et JUTTEт Jean-Noël (trad.), Paris, Points, 2011 [2010].

8. La pagination des passages de l'œuvre cités en coréen renvoie à l'édition originale : HWANG Sokyong 황석영, [Shim Chong, le chemin du Lotus] / 심청, 연꽃의 길, Séoul, Munhakdongne, 2007, réédition en un seul volume de l'édition de 2003. Le texte original fut d'abord publié en romanfeuilleton dans le quotidien sud-coréen 한국일보 / Hankook Ilbo du $1^{\mathrm{er}}$ octobre 2002 au 9 octobre 2003.

9. Un autre effet de sens rendu possible par ce motif du lotus réside dans le rapprochement avec l'épisode suivant de l'Odyssée (chant Ix, v. 83-104) : dans le récit qu'Ulysse fait de ses errances, il mentionne une escale, peu après son départ de Troie, chez les Lotophages, c'est-à-dire les «mangeurs de lotos», plante fabuleuse parfois identifiée au lotus qui provoque, chez ceux de ses marins qui en mangent, l'oubli de leur origine et de la nécessité de rentrer chez eux. Le parallèle explicite de Shim Chong avec l'Odyssée incite à voir en filigrane une allusion à ce motif du risque de l'oubli de l'identité et du retour, auquel Ulysse et ses compagnons échappent de justesse.

10. Cet archipel situé au sud du Japon, qui l'annexera en 1879, et dont fait partie l'île d'okinawa où Shim Chong a auparavant tenu une maison close avec son amie Fumiko / 후미코, est alors un royaume formellement indépendant mais tributaire à la fois de la Chine et du Japon.

11. HWANG, Sim Cheong, Yeonkkotui gil, 2007, op. cit., p. 698-699. Voir aussi les propos de l'auteur dans l'entretien publié sous le titre "Le Romancier Hwang Sok-yong: "Je suis un jeune auteur" "/ 나는 청년작가이다, 소설가 황석영, Channel Yes/ 채널예스, 1:2003: http:// ch.yes24.com/Article/View/12831, consulté le 01/08/2018.

\section{AUTEUR}

\section{SUK-HEE JOO}

Suk-Hee Joo est maître de langue à l'UFR LCAO de l'Université Paris-Diderot Paris-VII, où elle enseigne la langue et la littérature coréennes. Ses champs de recherche incluent les rapports entre mémoire historique et engagement politique dans la littérature coréenne contemporaine (en particulier dans l'œuvre romanesque de Hwang Sok-yong), les transferts culturels entre France et Corée du Sud selon une approche intermédiale (article « Du Transperceneige de Jacques Lob et Jean-Marc Rochette à Snowpiercer de Bong Joon-ho : une inspiration mutuelle entre arts visuels dans le domaine de la science-fiction », paru dans la revue ReS Futurae [en ligne], $n^{\circ} 9,2017$ ) et les phénomènes contemporains d'hybridation et de migrations génériques entre webtoon, drama et cinéma dans la fiction populaire coréenne. Elle est membre depuis $2019 \mathrm{du}$ Groupe de Travail «Coréanité et monde coréen » de l'UMR 8173 Chine, Corée, Japon (Ehess, Cnrs, Université Paris-Diderot Paris-VII). 


\title{
Narrating Disability in
}

\section{Contemporary Japan: [One Liter of Tears] and [A Therapeutic Sexy Trip]}

\author{
Anne-Lise Mithout
}

1 In Japan, like in many other countries, "disability" has long been a topic of writing, in the sense that "different" bodies and minds have fascinated society, intellectuals and writers for centuries. As in Western countries, the category of "disability," as a unified framework encompassing a broad set of situations, came to the fore only in the second half of the 20th century; the first law dealing with "disabled people" in general, and not just people with a specific type of impairment, was enacted in 1993. On the other hand, writings featuring disabled characters or dealing with disabilities, be they considered as belonging to a common framework or not, have existed since the dawn of Japanese literature. However, with few exceptions they have traditionally been authored by people who were not disabled themselves: most of them were able-bodied doctors or "experts" tackling this issue from a supposedly objective point of view. In the 1970s the Disability Rights Movement, led by associations of people with disabilities, sought to denounce the confiscation of discourses on disability by able-bodied professionals and to defend disabled persons' rights to self-expression, thus promoting people with disabilities as experts on their own situations. This movement fostered the burgeoning of disabled people's self-narratives as a literary genre, a development that can be seen through both the number of published authors and texts and the commercial success met by some of these works.

2 This paper aims at questioning the diversity of narrative strategies implemented by disabled authors in order to express, analyze, highlight and share with the reader their subjective experiences of disability. It is based on a comparison between two works: [One Liter of Tears. A Young Girl's Fight for Life (Aya's Diary)]/ーリットルの淚 ${ }^{1}$ (hereafter [One Liter of Tears] ), published by Kitō Aya 木藤亜也 2 in 1986, and [A Therapeutic Sexy Trip] / 瘾しのセクシー・トリップ3 (hereafter [A Therapeutic Sexy 
Trip]), written by Asaka Yūho 安積遊步 in 1993. Both works were written around the same period, by authors living with a physical impairment. Moreover, they are both women's self-narratives: the experiences of living with a disabled body which they relate are thus experiences of female bodies. Yet in several respects are they also significantly different. [One Liter of Tears] is a diary that the author wrote from age 14 to 21 ; it narrates events as they happen, as an immediate experience (although one can assume that some editing work has been carried out at the time of publication, as we will see later). By contrast, [A Therapeutic Sexy Trip] is an autobiography by a 37-yearold author; therefore it is the work of an adult woman revisiting her past and somehow retrospectively recreating the events that punctuated her individual construction, through the lens of her experience. Moreover, while the author of [One Liter of Tears] wrote primarily for herself, for her own personal pleasure and with no goal of publishing (at least initially), the author of [A Therapeutic Sexy Trip] explicitly pursued an activist purpose. Indeed, Asaka Yūho played an active role in the Disability Rights Movement, and writing her autobiography was part of her experience as an activist: she wrote to be read, not only by disabled women who would identify with her experience, but also by a broader audience, aiming to deliver a message about what disability is (and what society should be). Finally, even though both authors belong to the category of "physically disabled people," their situations are clearly different. Kitō Aya suffered from spinocerebellar ataxia, a neurodegenerative disease diagnosed when she was 14 , which gradually made her lose her motricity, then her speech capacities, leading her to an early death at age 25. Asaka Yūho, on the other hand, lives with congenital imperfect osteogenesis, diagnosed a few months after birth, a condition that does not significantly evolve over time. However, this difference should not be overestimated: [One Liter of Tears] is indeed a "disability narrative" and not an "endof-life narrative," to the extent that, until an advanced stage of her disease, Kitō Aya is not aware that it is incurable and deadly. Quite to the contrary, her narrative is that of a quest to tame disability and to learn, at every stage of the disease, how to live in the new condition that is hers. The issue of death appears only at the end of the work and is by no means one of the central themes. Moreover, it is indeed as a "disability narrative" that [One Liter of Tears] is presented to and know by Japanese readers. In fact it stands out as one of the biggest commercial hits in the field of disabled people's self-narratives in Japan, its success leading even to adaptations as a manga and as a television drama. To understand the distinct perspectives on the experience of "disability" that are presented in these two works, we must return to the definition of the concept itself. Michael Oliver" contrasts what he calls the "medical model of disability" - that is, the traditional definition of disability as an individual, medically identifiable affliction, whose cause lies inside the disabled person - with the "social model of disability," a perspective in which disability does not originate in disabled people but in a disabling environment. In the social model, disability is first and foremost defined by the barriers that a given society sets against individuals. This conceptual opposition has turned out to be very fruitful, both in the social and human sciences and in the activist field, even though the "medical model" does not exist as such and represents rather a repellent model, theorized for the sole purpose of criticism. Yet the "social model" has in turn been severely criticized, especially for its tendency to define disability primarily as a form of oppression, thus ignoring some of its specific dimensions, such as the non-social aspects of disabled people's experiences, like bodily sensations. ${ }^{5}$ 
The social model had been theorized a few years before the two works presented here were written. While it had no influence on Kitō Aya's writing, as she had no contact whatsoever with activists, it had a very clear impact on Asaka Yūho's: indeed, in addition to her active participation in the Japanese Disability Rights Movement, she stayed for six months in California and established strong relationships with the American Independent Living Movement. Even though neither of them explicitly refers to these terms, it can be said that Kitō Aya's perspective belongs to the medical model, and Asaka's to the social model of disability.

4 How is this difference in perspective reflected in the narrative choices made by the two authors? A comparison of their two strategies will allow us to highlight the elements that are stressed and emphasized, or on the contrary are set aside or concealed, in each of them.

Let us mention that working on autobiographical materials requires a few methodological precautions. Autobiographical writing is founded upon what Philippe Lejeune ${ }^{6}$ calls the "autobiographical pact": the author of a self-narrative commits, in his/her text, to deliver what he/she believes to be the truth. This does not necessarily imply that the narrative is true, but it presents itself as such. Therefore, in the texts studied here, we will not find "the truth" about the life of disabled people in Japan, in the sense of an objective analysis, but rather "truths" based on individual experiences that are reconstructed through writing.

\section{Impairment: a bodily experience}

6 First, let us consider the storytelling of what the medical model defines as the core element of disability: impairment, an organic or cognitive dysfunction identified in medical terms. What do the two authors present of their individual bodily experience of spinocerebellar ataxia and imperfect osteogenesis, respectively?

7 [One Liter of Tears] is written as the narrative of a decline, in the sense that the author depicts, step by step, the progression of her disease and her increasing physical difficulties. The body is therefore at the core of her narrative, first of all as a source of pain, a theme which pervades the whole work. Depictions of pain are aimed at providing the reader with a clear perception of the evolution of the disease, by giving a detailed account of each step, in a style that often verges on pathos.

My right leg, my knee throbs. Finally?... When I take a bath, I caress it and whisper:

"My back that hit the floor when I fell, my shoulder, my poor body that hurts everywhere."

右足、滕がズキンズキンする。いよいよか...。風呂に入りながら、「転んで

打った腰、肩、あちこち痛んでしまったかわいそうな私の体」とつぶやきなが

ら、なでてあげる。

8 The goal of Kitō Aya's text is not only to evoke the reader's sense of pity. It also constructs a heroic image of a young girl struggling against her own body. Indeed, the narration is based on the body/mind dichotomy, the physical decline shown at each step being used to demonstrate the author's desire to be brave, as bravery appears necessary when facing a disability presented as an individual tragedy. Writing seems to have therapeutic properties for the author, or at least to act as a self-fulfilling prophecy: she writes to give herself courage, in an inward-looking quest to find in herself the resources needed to overcome her difficulties. This is why the narrative is 
also sprinkled with scenes where the author marvels at minor details of daily life or writes poems which exalt the trivial things that nourish her desire to live and to resist pain. However, in the last chapters, when it becomes clear that the progression of her disease cannot be stopped, bodily sensations become progressively associated with the idea of death, even though the author carries on her quest for hope:

Is it right that you live? Even if you disappear, there will be nothing left of you. [...] Mum, is it right for ugly creatures like me to live in this world? I think that, maybe, Mum can find something that shines inside me.

生きていていいのか? お前がいなくなっても何一つ残りはしない...。お母さ

ん、私のような醜い者が、この世に生きていてもよいのでしょうか。私の中

の、キラッと光るものをお母さんなら、きっと見つけてくれると思います。8

In striking contrast to this storytelling of the disabled body as a painful and mortal body, the author of [A Therapeutic Sexy Trip] makes no mention of impairment, in the sense that she never writes about her living with a body that suffers from a medical affliction. Her perspective on pain is therefore very different from Kitō Aya's, to the extent that she never mentions any pain directly linked with her imperfect osteogenesis. For example, even though she mentions several times the fact that her fragile bones sometimes get broken, she never says a word about the pain or the physical experience related to those broken bones. Pain directly resulting from her disease and, more generally, the bodily sensations associated with her disease are entirely concealed. On the other hand, she makes a strong connection between pain and the medical treatments intended to cure her.

The doctor probably intended to cure me sincerely. Nevertheless, I felt at that time - and I still feel this way - that I was used as a guinea pig. As it was said that male hormones would be good for me, I had repeated injections, every two days, from age 0 to 2. [...] An adult at least understands what is going on and why. But a baby does not understand anything. The only thing that this little me understood was that, for some unknown reason, it was always made to suffer.

医者は本心から、私を「治してやりたい」と思っていたかもしれない。でも、 それにしてもだ、あの頃の私はまるでモルモットだったと、当時も、そして、 今も思う。男性ホルモンがいいというので、はっきりした根拠もないまま、そ の注射を一日おきに、0歳から2歳までずっと打たれ続けたのだから。[...] 大人 なら、少なくとも何が起こっているのか、なぜそんなことをされるのかが自分 でわかっている。でも、赤ん坊には、そんなことは何もわかりやしない。小さ な小さな私にわかっていたのは、わけのわからないことでいつも痛い目にあわ されるということだけ。9

10 Asaka Yūho's goal is thus to contest the vision of disability as impairment and of doctors as omnipotent. She specifically denounces the fact that the medical treatment she received turned out more harmful that the "problem" it was supposed to solve. In this example, she clearly speaks out as an activist: her narrative of disability is indeed rooted in the social model, as opposed to the medical model. It rejects the vision of disability as individual impairment and, by so doing, omits the bodily dimension of disability, a tendency that has often been noted in the social model and its champions' writings. Regarding the depiction of disability as impairment, the two texts are thus strongly opposed. For Kitō Aya, the idea of a deficient, vulnerable, painful body is at the core of the narrative. It structures the narration, based on the stages of her disease's progression. It also plays a key role in the construction of the figure of the narrator as heroic, the extraordinary hardships faced by her body revealing the exceptional strength of her mind. Asaka Yūho, on the contrary, rejects the narrative framework of disability as tragedy and the heroization of the disabled person. She thus takes the 
opposite view by clearing from her text any depiction of physical sensations unrelated to social situations of oppression, thereby asserting her militant commitment at the risk of concealing a part of her personal experience.

\section{Disability as a limitation of activity}

11 Beyond its purely bodily dimension, the experience of disability implies a limitation of the person's capacity to perform some actions. The two texts diverge in their ways of narrating the experience of life with an incapacity.

In [One Liter of Tears], the author gives a progressive narrative of her loss of capacities. The reader is a spectator to the gradual loss of her mobility, experienced as a decline. In each chapter, she draws up a list of actions that she is no longer able to perform, of activities that become inaccessible.

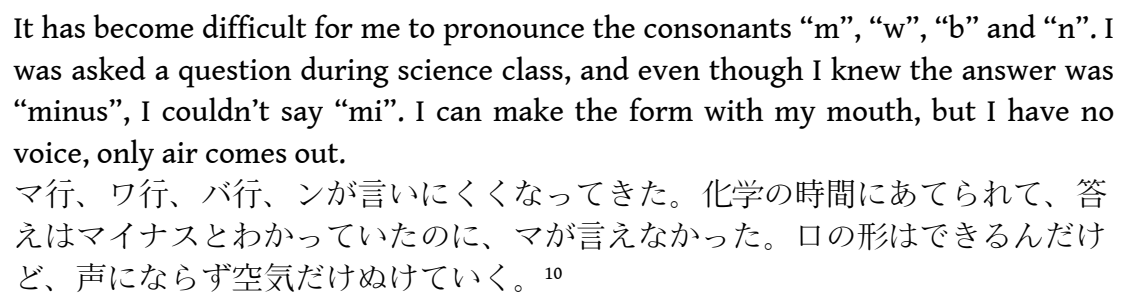

This loss of capacities results in guilt and the shame of feeling herself becoming increasingly dependent on other people's help. While this feeling is frequently attested in the narratives of people acquiring a disability in the course of their lives, it is even more acute in Japan where one of the central elements of social life is the injunction to avoid embarrassing others (an injunction particularly criticized by the Disability Rights Movement). Dependence is thus associated not only with a feeling of helplessness, but also with the impression of being a heavy burden for one's family and friends.

The limitation of activity suffered by Kitō Aya is thus lived with a feeling of guilt, that of being no longer able to perform actions considered as "normal" for a person of her age. This guilt is all the stronger since in Japan, following the Buddhist logic of karmic retribution, disability is seen as originating in evil deeds from a previous life. ${ }^{11}$ "Becoming disabled" thus means confronting strongly negative representations and progressively acquiring an identity marked by guilt and discredit. In this sense, the narrative is a record of Kitō Aya's "career" (in Howard Becker's sense) ${ }^{12}$ as a disabled person, that is, her shift from an "ordinary girl's" identity to a "disabled person's" identity, self- and socially recognized as such.

Writing plays a key role in this change of identity. It is indeed through the writing of her diary that Kitō Aya manages to distance herself from her experience and to draw, at each step, an assessment of her lost and remaining capacities and her feelings associated with both. The persona she constructs in her diary, with the abovementioned tendency to heroization, stresses her willingness to maintain hope, to take a view which opposes her mostly negative feelings concerning disability by pointing out her remaining capacities and highlighting her ways of carrying on the fight of her mind against her body. This tendency is especially visible at the end of the text when, having lost most of her mobility, the author wonders about her capacity to pay back the efforts made for her by her loved ones: 
In the warmth of my home, I feel loved. But I can't express my love for them all. Because I can't talk, because I can't do any action that could express it...I can only do my best to answer their love with a smile! I try to get up early in the morning, brush my teeth early too, so that I won't be late for breakfast either, and to do my exercises properly every day. And I strive to respond to their love.

家庭のぬくもりの中で、愛されていると感じる。でも、私は、みんなを愛して いると表現できない。言葉を話せないし、それを表す行動ができないから ...

にこにこ笑って愛に応えるだけが精いっぱい! 早寝早起きをしましょう。八ミ ガキも手早く、食事にも遅れないように、訓練も毎日きちんとしましょう。そ して愛に応える努力をしましょう。13

This extract reveals the ambiguity of Kitō Aya's feelings toward her loss of capacities. On the one hand, it shows the guilt she feels concerning the help she receives from her family in daily life and her feeling of imposing additional work, lost time, and permanent concern on her loved ones. On the other hand, it highlights the young girl's willingness to overcome her physical limitations to place herself in a reciprocal relationship in which she not only "receives" from others, but is also able to "give", insofar as possible. By stressing the emotional aspect of the exchange ("love for love"), she tries to conceal her uneasiness regarding its concrete unfairness ("daily help versus smile") and to recreate equality by pointing out the amount of physical effort required for her to smile, using a style that participates in the process of heroization. Kitō Aya therefore tries, through writing, to give value to her remaining capacities, but this valorization remains deeply rooted in a heavily negative representation of disability and the disabled person, defined first and foremost as a useless burden, a representation she can escape only through heroization. The author of [A Therapeutic Sexy Trip], much to the contrary, refuses all forms of individual heroization of the disabled person. For Asaka Yūho, disability cannot be defined at a solely individual level, in a perspective that leaves the person with the entire responsibility for her limitation of activities (and the resulting dependence). This is why she champions the perspective that lies at the core of the social model: a person is disabled only to the extent that she lives in a disabling environment.

(When I took part in the Movement) of course I used a wheelchair. This "of course" is meaningful. I was really astonished when my friends in the movement told me it was OK to use one. As I mentioned earlier, in the special school where the ultimate goal was to resemble non-disabled people, I had always been told that I should not use a wheelchair. It didn't matter that I could not walk, I was told to keep on striving to use crutches. [...] When my friends in the movement told me, "Wouldn't it be nice for you to use a wheelchair and go wherever you want?", I felt like the entire world was opening up before me.

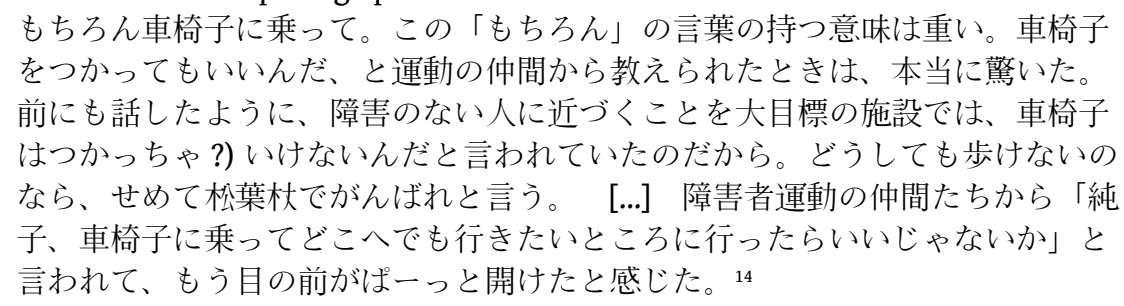

This excerpt reveals very explicitly Asaka's perspective: in her opinion, her ability to move is not limited by the specificities of her motor capacities, but by social norms imposing bipedal walking on everyone and considering the use of a wheelchair as an abnormality. Asaka's narrative is thus an invitation to rethink the traditional image of the disabled person as an isolated individual struggling against him/herself, to break 
with individual guilt and to focus on the role of society in the limitation of activities suffered by disabled people.

Following the same approach, the text hardly mentions the issue of dependence, and never in terms of guilt. On the contrary, Asaka aims to portray interdependence as part of ordinary human relationships, with or without disabilities. This intention is especially visible in passages describing her relationships with people whose motor impairments are heavier than hers, especially with one of the Movement leaders who becomes her partner. Indeed, she depicts this relationship from the angle of balance and harmony between partners, highlighting the fact that each of them brings a different contribution to the relationship, depending on their particularities (and not on their capacities because, to her, disability is first and foremost a specific feature, one among many individual features, and not merely a limitation).

However, the reality of incapacity, and of painful lived experiences associating incapacities with individual devaluation, sometimes shows up behind the militant discourse. This is especially noticeable when Asaka deals with more intimate subjects like sexuality. She mentions that intercourse is not possible with her partner from the Movement, due to his physical impairment. Yet because of social norms pervading Japanese society, intercourse is the only form of sexuality imaginable for both of them. This incapacity leaves him with the feeling of being an incomplete man, and her with that of not being a "real woman" with a partner like him (for example, she cannot consider him as a potential spouse since marriage, at that time, means for her a union with an able-bodied man). This is why, with her partner's consent, she lives a sexual life dissociated from her romantic relationship, with able-bodied men; yet this agreement results in frustrations and tensions for both partners. Although Asaka Yūho narrates this episode in her autobiography with the clear intention of showing the force of social norms weighing on disabled people's sexuality, it nonetheless reveals the reality of painful subjective experiences associated with the situation of incapacity and the difficulty of escaping the process of individual shaming, even for people actively involved in the militant world.

These texts therefore show two aspects of the same social situation: the reasoning linking disability, limitation of activity and individual guilt. Kitō has fully internalized this framework and uses writing as a means of expressing her ambivalent feelings within it, through pathos and the construction of a heroic figure. Asaka Yūho tries, on the contrary, to escape it and to overcome the individual representation of limitations on activity, in order to reveal the key role played by society in constructing barriers that hinder disabled people's lives. But behind the militant discourse, her narrative also gives a glimpse of individuals' difficulties in breaking away from guilt, and of distressing experiences of limitations on activity in intimate, romantic and sexual life.

\section{Disability as a social experience}

The issue of the relational dimensions of activity limitation raises that of the social experience of disability: what does it mean, for the two authors, to live as a "disabled person" and to be recognized as such in Japanese society? Both attest to the fact that in Japan, disability is first and foremost defined as something that should be "cured". Yet their attitudes towards this representation are clearly opposed. 
fight against the disease through descriptions of the discipline and exercises prescribed by rehabilitation professionals: her entire daily routine is structured by her doctors' recommendations. She thus fits into the "sick role" that functionalist sociologists have defined, to the extent that she takes to heart her "duty to recover." Therefore, she never makes a single comment on the treatments she receives, on the possible side effects of drugs, on pain felt during exercises or on the attitudes of the professionals she meets. She completely relies on the doctors' competence and displays absolute docility. Her total submission to medical experts can of course be partially explained by the fact that Kitō Aya's disability results from a disease, but it is nevertheless striking to notice that the majority of the text depicts her attitude as that of an ideal patient, even during periods when she lives in a barely-medicalized environment.

On the opposing side, following the ideas championed by the Disability Rights Movement, and especially the Green Grass Association / 青い芝の会 / Aoi shiba no kai, ${ }^{15}$ Asaka Yūho's discourse is extremely critical towards doctors. [A Therapeutic Sexy Trip] thus condemns medical treatments as a form of oppression. To Asaka, the medicalization of disability results from discriminatory thinking towards disabled people, which tends to consider them as people who "should not exist" and should be made invisible. Her vision has been strongly influenced by the ideas of Yokozuka Kōichi, one of the leaders of the Green Grass Association, who condemns the "internalized eugenics" lying at the core of disabled people's social treatment in Japan. ${ }^{16}$ She prominently depicts scenes in which she feels treated as a guinea pig, enduring treatments with no purpose of improving her condition.

One day, even though I wasn't scheduled to have surgery, I had an examination and a blood test. As I had heard that this doctor was working on his thesis, I understood that he was testing me only for his own research and I summoned all my childish wits and said: "Are you using me as a guinea pig? That's disgusting! Don't use me as a guinea pig for your thesis!" [...] "If you get it, you'd better shut up and obey."

ある日、手術の予定もないのに検査をされて、血液をとられた。私は、その医 者が論文の準備をしているという噂を聞いていたので、これは自分の論文のた めだけに検査してるなってわかったから、子供の知恵を総動員して言った。

「私をモルモットにしているんでしょ?いやです。あなたの論文のために私を モルモットにしないで权」[...]「わかってるんだったら、黙って従えばいいん だ」 17

Therefore, even if the tendency toward the medicalization of disability in Japanese society is visible in both works, the two authors deal with it with radically different attitudes. Kitō Aya perfectly fits into the norm of the disabled person as a patient receiving with docility and discipline a treatment aimed at curing her, while Asaka Yūho, on the contrary, rises up against this norm and endeavors, through her narrative, to contest the power of doctors.

An analysis of the two authors' reactions towards the normative behavior expected from a disabled person would not be complete without a mention of gender: indeed, in addition to being "disabled", both writers are women. The issue of gender is thus key to understanding their attitudes, be they conforming to or opposing social expectations. Docility and submission are not only attitudes required from disabled people, they are also qualities expected from women. Indeed, the social image of femininity in Japan has been marked by the official injunction, declared by the state in the Meiji era, to become "good wives, wise mothers." Even though this injunction has been discussed, criticized and negotiated since the dawn of its appearance, social norms of femininity throughout 
the 20th century have revolved around this idea of women as wives and mothers and, more implicitly, as providers of love and care. Indeed, as numerous researchers in the field of gender studies have shown, the social definition of femininity is based on the idea that the social mission of women is to ensure harmony in their families and, more widely, in their surroundings. ${ }^{18}$ Therefore, while women are held responsible for the way they manage their households, they are at the same time expected not to stand out as individuals - that is, they are not supposed to express themselves freely, but rather to maintain harmony in their families/groups/teams while complying with guidance given by a male leader. Disabled women are thus in a position of double submission (to medical authority and to male authority, both intertwined in the numerous male doctor figures appearing in the two texts). They are expected to comply with instructions provided by their male doctors, which means that as disabled persons they must be willing to "recover" following instructions from medical experts, and as women they are responsible for ensuring peace and harmony around them, be it in their families or at the hospital (and therefore they are not expected to express any form of anger of criticism). In this respect, Aya Kitō's attitude fits perfectly into the "passive and obedient disabled girl" stereotype, while criticizing doctors' attitudes, as Asaka Yūho does, turns out to be highly subversive.

Yet Japanese society, however gender-structured it may be, paradoxically tends to erase disabled people's sex and to categorize them as gender-neutral "people" with disabilities. Indeed, since disabled people are viewed as unable to fulfill social expectations regarding their gender (that is, for men to work hard and provide for their families, and for women to bear children and carry out housework), from early childhood on they are treated as neither boys nor girls. ${ }^{19}$ The two texts are thus permeated to various extents by the idea that disabled women do not fit into norms of femininity and, therefore, are not socially recognized as women. [One Liter of Tears] explicitly expresses the idea that a disabled woman is ugly, and thus unworthy of love:

My disease is worse than cancer. It has stolen the beauty of my youth. If it wasn't

for this weird disease, I could even find love.

私の病気は、ガンよりひどい! 私の青春の美しさを奪った。こんなへんな病気

でなかったら、恋だってできるでしょう。20

Noteworthy is the fact that the narrative, written by a teenager, does not mention any form of romantic feeling whatsoever, even though the afterword, written by one of Aya's doctors, depicts her as passionately in love with a young male intern working in the service where she was hospitalized. This "omission" can inspire two interpretations. On the one hand, one can imagine that Kitō Aya herself chose not to write on this subject in her diary, performing some form of self-censorship when writing. On the other hand, equally plausible is the hypothesis that the passages she may have written have been deleted by her family or her editor at the time of publication. This raises the complex question of the literary status of the text of [One Liter of Tears]. Indeed, as Philippe Lejeune ${ }^{21}$ remarks, almost no diary is published as it was written. At the time of publication, authors frequently give in to the temptation of editing a text written several years earlier. But the editing work is also sometimes performed by families, as in the case of posthumous publication, especially of children's diaries (such as Anne Frank's diary). Might the family not wish to emphasize the heroic character of the late child and to erase any signs of discrepancy between the author and the figure of the model child expected by readers? Even though it is impossible to validate this assumption without access to the original manuscript, it seems fairly 
likely that [One Liter of Tears] has gone through a similar editing process, which would explain the stereotyped aspect of the work, the tendency toward the author's heroization, and her perfect fit with the expected figure of the model disabled girl: docile, obedient and sexless. Therefore, although it is not possible to assess to what extent Kitō Aya's manuscript has been edited, ${ }^{22}$ the striking absence of the issue of romantic feelings in the work can be seen as a symptom of the widespread idea that it is not appropriate for a person with a disabled body to express love or desire for another person.

Asaka Yūho's work is also influenced by the existence of this norm, but with the opposite perspective: indeed, as clearly stated by its title, [A Therapeutic Sexy Trip] is a narrative focused on the issue of love and sexuality. The author's goal, through writing, is to assert herself as a sexual being, a subject and an object of desire, to account for her struggle to be recognized as such and to deliver to her (female) disabled readers a message of empowerment. She openly condemns several barriers that have hindered her personal fulfillment and deals especially with some taboos like sexual violence (which she received from a doctor as a teenager), or the dominant sexual script making intercourse the only legitimate sexual practice - an impossible one when she shares her life with a physically disabled partner, to the frustration of both lovers:

There was no model, no message around me saying "sex is not only intercourse".

[...] If at that time we had both thought that we didn't have to force ourselves into impossible intercourse, I think we would have lived without suffering so much.

性交だけがセックスじゃないんだよ、というメッセージも、そうしたモデル

も、私の周囲には何もなかった。[...]もしあのころ、むりに性交しなくてもいい んだというふうに二人が思えてい机ば、あんなに苦しまなくてすんだと思う。

23

She also depicts her different romantic relationships, the balances found with her partners, the joys and anxieties nourished by each encounter:

He who didn't have a disability, did he feel something sexual for this small, abnormal body of mine, did he accept it? Was I attractive? I wanted to be embraced softly, I wanted to be kissed. I wanted him to tell me "you're pretty". [...] When I understood that he accepted my body, that I too could become a sexual object for a man, instead of wondering whether we loved each other spiritually [...] I wanted, through him, to receive the proof that "I was all right with this body."

障害のない彼が、私のこの小さな、人とちがった体をセクシュアルと感じ、受 け入れてくれるだろうか。私にも魅力があるのだろうか。やさしく抱きしめて もらいたい、キスしてもらいたい。「きれいだ」と言ってほしい。 [...] 彼が 私の体を受け入れているとわかったとき、つまり、自分も男性の性的な対象に なりうると知ったとき、精神的に私たちが愛しあっているかどうかより、[...] 彼をとおして、「私は私の体のままでいい」ということを証明してもらおうと していたのだろう。 ${ }^{24}$

Thus on the level of the social experience of disability, the two texts present a similar reality: a social environment in which disability is mainly considered as a situation that should be fixed, in a highly medicalized perspective. Moreover, they both reveal that norms of docility and passivity are applied even more severely to disabled women, for whom the possibility to live and express themselves as sexual, desiring and desirable beings is especially prohibited. However, the texts adopt opposite perspectives towards this reality. [A Therapeutic Sexy Trip] is deeply rooted in a militant perspective based on social criticism, while [One Liter of Tears] reveals, through the stereotypes it relies on, the norms weighing on a disabled young girl, even when publishing an autobiographical work. 


\section{Conclusion}

31 models of disability and the imperfections of both models. [One Liter of Tears] reveals the idealized figure of a disabled girl who combines docility and heroism in an individual fight against disability experienced as an inner enemy. On the opposite side, [A Therapeutic Sexy Trip] is based on a definition of disability as oppression, narrating the author's struggle not against herself, but against her social environment. In this respect, the three-dimensional analysis grid of disability (biological dysfunction, limitation of activity, social disadvantage) serves as a tool to account for the conjunction of social and individual factors constituting the lived experience of disability, even though it induces, in the analysis of self-narratives, somewhat artificial distinctions between elements of the narrative.

works, we notice the presence of some of the stereotypes and limits specific to each model. In [One Liter of Tears], we see an extreme individualization of disability and a reinforcement of the representation of the disabled person as a "singular individual" who risks isolation. In [A Therapeutic Sexy Trip], there is the omission of individual bodily sensations related to disability, and of pain in particular. These differences can of course be partially explained by the authors' different situations, as presented in our introduction, but they also reveal clear narrative choices made by the two writers, through their selection of elements of their experience which are emphasized or neglected, or even concealed. It is essential to point out, in both cases, the role of writing as an instrument in the quest for self: writing is aimed at making sense of the experience of a disabled body. It is part of the author's search for her own identity, one that must deal with the existence of a disability, be it rejected or fully embraced. The meaning of this experience is found individually and is expressed subjectively through literary creation, which in this respect contrasts with the "objectivity" intended by medical discourses. The literary status of [One Liter of Tears] is subject to debate, and the subjectivity that emerges therein might be less the author's own than that of her parents, her editor, or that expected by readers. Yet precisely because of the stereotypes it is based on, this narrative reveals the normative figure of the ideal "disabled young girl" as she exists in the Japanese imagination, thus making explicit the model that weighs heavily upon real disabled girls. The special status of this work, as the embodiment of a normative imagination, makes it an essential point of comparison to understand both the originality and the limits of a narrative like Asaka Yūho's.

\section{BIBLIOGRAPHY}

ASAKA Yūho 安積遊歩, [A Therapeutic Sexy Trip] / 瘺しのセクシー・トリップ / Iyashi no sekushī torippu, Tokyo, Tarōjirōsha, 1993. 
BECKER Howard, Outsiders. Studies in the Sociology of Deviance, New York, Free Press of Clencoe, 1963. HATTORI Sachiko 服部 祥子 (dir.), [Disabled Children and Sex: A Real Image of Puberty] / 障害児と 性一思春期の実像 / Shōgaiji to sei一shishunki no jitsuzō, Tokyo, Nihon Bunkakagakusha, 1989.

HENNINGER Aline, La socialisation de genre à l'école élémentaire dans le Japon contemporain / [Gender Socialization in Primary School in Contemporary Japan], PhD dissertation in Japanese studies, supervised by Christian Galan, Paris, INALCO, 2016.

HIROSE Kōjirō 広瀬浩二郎, [A Religious Anthropology of Disabled People] / 障害者の宗教民俗 学/ Shōgaisha no shūkyō minzokugaku, Tokyo, Akashi Libray, 1997.

KAKUTA Reizō 角田 礼三, [Realities of Sex Education for Disabled Children] / 障害のある子どもへ の性教育の実際 / Shōgai no aru kodomo e no seikyōiku no jissai, Meiji Tosho, 1995.

кITо Aya 木藤亜也, [One Liter of Tears. A Young Girl's Fight for Life (Aya's Diary)] / ーリットルの 涙 / Ichi rittoru no namida, Tokyo, FA Shuppan, 1986.

LEJEUnE Philippe, ["Self-genesis. The Genetic Study of Autobiographical Texts"] / "Auto-genèse. L'étude génétique des textes autobiographiques", Genesis, n 1, 1992, p. 73-87.

LEJEUnE Philippe, [The Autobiographical Pact] / Le pacte autobiographique, Paris, Seuil, 1975.

MACKIE Vera, Feminism in Modern Japan: Citizenship, Embodiment and Sexuality, Cambridge, Cambridge University Press, 2003.

MUTA Kazue 牟田和恵, [“The Image of Family of in General Magazines in the Meiji Era”] / 明治期 総合雑誌にみる家族像:「家庭」の登場とそのパラドックス / Meiji ki sōgō zasshi ni miru kazoku zō, Shakaigaku hyōron, 41, 1:1990, p. 12-25.

OLIVER Michael, Social Work with Disabled People, Londres, Macmillan, 1983.

SAITO Hikaru 斎藤光 (dir.), [An Analysis of Love and Chastity in the Machine Era - Sex and Ideology] / 機械時代の恋愛・貞操の分析一性とイデオロギー / Kikai jidai no ren.ai-teisō no bunseki - sei to ideorogii, Tokyo, Yumani Shobo, 2006.

SHAKESPEARE Tom, “The Social Model of Disability”, in The Disability Studies Reader, DAVIS Lennard (ed.), London, Routledge, 2013, p. 214-221.

TANAKA Mitsu 田中美津, [To My Spiritual Sisters] / 命の女たちへ / Inochi no on.na-tachi e, Tokyo, Pandora, 2004 [1972].

YоKоZUKa Kōichi 横塚光一, [Mum! Don’t Kill Me!] / 母ょ! 殺さな! / Haha yo! Korosu na!, Tokyo, Seikatsu Shoin, 2007 [1975].

\section{NOTES}

1. KITō Aya 木藤亜也, [One Liter of Tears] / 一リットルの涙, Tokyo, FA Shuppan, 1986.

2. In this text, we use the Japanese way of writing personal names, with family name first, followed by given name.

3. ASAKA Yūho 安積遊步, [A Therapeutic Sexy Trip]/癒しのセクシー・トリップ, Tokyo, Tarōjirōsha, 1993.

4. OLIVER Michael, Social Work With Disabled People, London, Macmillan, 1983.

5. SHAKESPEARE Tom, "The Social Model of Disability", in The Disability Studies Reader, DAVIS Lennard (ed.), London, Routledge, 2013, p. 214-221.

6. LEJEUNE Philippe, [The Autobiographical Pact] / Le pacte autobiographique, Paris, Seuil, 1975. 
7. KITō, [One Liter of Tears], op. cit., p. 109.

8. Ibid., p. 184.

9. ASAKA, [A Therapeutic Sexy Trip], op. cit., p. 19-20.

10. KITō, [One Liter of Tears], op. cit., p. 134.

11. HIROSE Kōjirō 広瀬 浩二郎, [A Religious Anthropology of Disabled People] / 障害者の宗教民 俗学, Tokyo, Akashi Libray, 1997.

12. BECKER Howard, Outsiders. Studies in the Sociology of Deviance, New York, Free Press of Clencoe, 1963.

13. KITō, [One Liter of Tears], op. cit., p. 182-183.

14. ASAKA, [A Therapeutic Sexy Trip], op. cit., p. 82-83.

15. Asaka has been a member of this association, which pioneered the Disability Rights Movement, since the late 1970s.

16. YoкоZUKa Kōichi, [Mum! Don't Kill Me!] / Haha yo! Korosu na!, Seikatsu Shoin, 2007 [1975].

17. ASAKA, [A Therapeutic Sexy Trip], op. cit., p. 33.

18. MUTA Kazue 牟田和恵, [“The Image of Family of in General Magazines in the Meiji Era”] / 明 治期総合䧱誌にみる家族像:「家庭」の登場とそのパラドックス，41，1:1990，p.12-25；MACKIE Vera, Feminism in Modern Japan: Citizenship, Embodiment and Sexuality, Cambridge, Cambridge University Press, 2003; TANAKA Mitsu 田中美津, [To My Spiritual Sisters] / 命の女たちへ, Tokyo, Pandora, 2004 [1972]; SAITo Hikaru 斎藤光 (dir.), [An Analysis of Love and Chastity in the Machine Era - Sex and Ideology] / 機械時代の恋愛・貞操の分析一性とイデオロギー, Tokyo, Yumani Shobo, 2006.

19. HATTORI Sachiko 服部 祥子 (dir.), [Disabled Children and Sex: A Real Image of Puberty] / 障害 乱と性一思春期の実像, Tokyo, Nihon Bunkakagakusha, 1989; KAKUTA Reizō 角田 礼三, [Realities of Sex Education for Disabled Children]/障害のある子どもへの性教育の実際, Meiji Tosho, 1995, p. 61-63; YOKOZUKA Kōichi 横塚光一, [Mum! Don't Kill Me!], op. cit., p. 53; HENNINGER Aline, La socialisation de genre à l'école élémentaire dans le Japon contemporain / [Gender Socialization in Primary School in Contemporary Japan], PhD dissertation in Japanese studies, supervised by Christian Galan, Paris, INALCO, 2016.

20. Кіто, [One Liter of Tears], op. cit., p. 47.

21. LEJEUNE Philippe, ["Self-genesis. The Genetic Study of Autobiographical Texts"] / "Autogenèse. L'étude génétique des textes autobiographiques", Genesis, n 1, 1992, p. 73-87.

22. Let us mention that the text was published in her lifetime, two years before her death.

23. ASAKA, [A Therapeutic Sexy Trip], op. cit., p. 117.

24. Ibid., p. 92-93.

\section{AUTHOR}

\section{ANNE-LISE MITHOUT}

Anne-Lise Mithout is associate professor in Japanese studies at Université Paris-Diderot Paris-VII (Research Center on East Asian Civilizations) where she teaches sociology of contemporary Japan. Her research focuses on the situation of people with disabilities in Japanese society, including in the fields of education, employment and social movements, and the representations of disability in Japanese popular culture. Her recent publications include "Aoi shiba no kai: un mouvement de 
personnes handicapées face à l'eugénisme" (Cipango, $\left.n^{\circ} 24,2021\right)$, "Representations of female disability in Japanese television drama" (in Diana Garrisi et Jacob Johanssen (dir.), Disability, Media, and Representations: Other Bodies, Routledge, 2020, p. 27-48) and "From equal access to employment to equal career opportunities? Employment practices and work experiences of qualified disabled workers in Japan" (Alter, 15 (4), 2021, p. 341-353). 


\title{
Les bébés de la consigne automatique de Murakami Ryū : corps, volonté, violence
}

\author{
Toshio Takemoto
}

1 Murakami Ryū 村上龍 (1952-) débute sa carrière avec Bleu presque transparent / 限りな く透明に近いブルー (1976), récit centré sur le quotidien de jeunes qui passent leur temps dans l'orgie et la drogue. Murakami associe la violence au corps, tant dans ce court récit que dans Coin Locker Babies (plus loin CLB) / Les bébés de la consigne automatique / コインロッカー・ベイビーズ (1980), vaste galerie de corps qui va du nourrisson délaissé au cadavre. Pour lutter contre la société qui les broie, deux orphelins, Kiku et Hashi, n'ont pour seule arme que leur corps et leur volonté.

2 Voici l'intrigue du roman. Découverts dans des consignes automatiques, Kiku et Hashi grandissent à l'orphelinat jusqu'à ce qu'un couple les adopte. Après avoir passé son enfance à Kyushu, Hashi monte à Tokyo, où il se prostitue pour des pédérastes. M. D, son amant, lui permet de percer dans le milieu de la musique. Kiku, l'autre héros, devient un athlète, mais tire sur la tête de sa mère. Arrêté, il s'évade de la prison avec d'autres détenus. Hashi, lui, perd la raison au point de poignarder Niva, son épouse enceinte et se retrouve à l'asile. Kiku largue un gaz toxique à Tokyo. Hashi s'évade. Dans la rue déserte, il est pris d'une frénésie destructrice, qu'il parvient à maittriser ${ }^{1}$.

Une suite de péripéties oppose le corps des héros à la violence sociale, d'où le lieu commun que ressasse la critique dès la parution: CLB montre à la fois le désir de destruction et de vie ${ }^{2}$. Mais apparaît aussi la volonté des héros, soutenue par leurs corps : la volonté ne suffit pas si elle n'est portée par le corps - mais jusqu'à quelle extrémité ? Livré à la seule contingence, le corps des deux héros risque d'aboutir à leur perte ou à celle d'autres, mais une autre possibilité s'ouvre à eux s'ils maîtrisent leur frénésie. 


\section{Le corps social}

4 Dans CLB, Murakami travaille la tension entre le corps social, groupe homogène, et les héros, corps étrangers. Tout se passe comme si «le corps humain (la personne humaine) qui forme en son ordre une totalité, (était) assimilé à un organe d'une plus vaste totalité et d'un organisme plus puissant, hors desquels il ne saurait ou ne devrait vivre $»^{3}$.

5 Adoptés par le couple Kuwayama dans une île du Kyushu, Kiku et Hashi retrouvent dans d'anciennes mines désaffectées des déchets qui ressemblent à la maquette de la ville que jadis Hashi construisait à l'orphelinat avec des jouets. Maintenant, ils voient des immeubles vides, des murs en briques, envahis d'herbes folles et, "(à) travers les entrailles en béton, arrivent à une école à moitié effondrée » / コンクリートの内臓を 抜けると半壊した学校がある。(p. 33 ; p. 34)4. Cet espace abandonné est comparé aux organes humains sans vie. Le corps social laisse mourir ces anciennes mines improductives, donc inutiles ${ }^{5}$. Cette société est d'entrée de jeu hostile aux héros, rejetés en consigne automatique, c'est-à-dire exclus du corps social. Seuls leur corps, appuyé sur leur vouloir, leur permet de s'y forger une place.

Quant au cadre temporel de CLB, la plupart des épisodes se passent dans les années quatre-vingt, que justement Murakami déteste :

$\mathrm{Ah}$, nous avons réussi, nous, et désormais nous allons sans doute profiter de cette vie prospère. [...] Inutile de regarder au dehors, à l'extérieur, ils se font la guerre, mais comme le Japon, lui, est si paisible et prospère, c'est « une vie belle et bonne ». ああ、われわれは成功したんだ、きっとこれからもなんとなくこういう豊かな 暮らしをしていくだろう（...）別に外に目を向けなくてもいいじゃないか、外 では戦争をしたりしているけど、日本はこんなに平和で豊かなんだから「おい しい生活」ということなんです。6

7 Replié sur lui-même, le peuple se croit pérenne au lieu de se forger un nouveau projet après le miracle économique. Sur cette croyance se fonde le corps social. S'il est condamné dans $C L B$, c'est qu'il réduit l'individu unique à une pièce de rechange de la machine. D'où le bilan que tire Hashi :

Je sais que personne n'a besoin de moi. Jamais, je n'ai été indispensable aux autres. C'est pourquoi j'ai décidé de devenir un homme qui n'aurait besoin de personne d'autre, mais, écoute Niva, ce n'était pas seulement moi, un homme de première nécessité n'existe nulle part ; tous les hommes sont inutiles : c'était si désolant que j'en suis tombé malade.

僕は自分が誰からも必要とされていないのを知っている。僕はずっと必要とさ れなかった、だから、他人を必要としない人間になろうと思ったんだ。でもね ニヴァ、僕だけじゃなかったんだよ、必要とされている人間なんてどこにもい ないんだよ、全部の人間は不必要なんだ、それがあんまり叔しかったから僕は 病気になったんだ。(p.535-536; p. 498-499).

8 C'est une société aux membres interchangeables car réduits à leurs « rôles / 役割 7 " au détriment de leur individualité. Si le corps social récuse l'identité unique des membres, il rend malade un ado vulnérable comme Hashi, dont la souffrance dit le dysfonctionnement social. Le qualificatif 叔し / sabishi signifie aussi la ruine et la déchéance. Si la maladie fait partie des "virtualités du corps ${ }^{8}$ ", elle reflète la diminution d'énergie qui caractérise le corps social : sans volonté ni désir, ses membres ne cessent de s'épuiser. Le corps sans volonté est tributaire d'une société implacable. 
9 L'un de ses représentants, M. D, amant et patron de Hashi, privilégie les intérêts et l'apparence en forgeant son look d'androgyne à ce dernier. M. D formate Hashi, qui devient chanteur et le réduit donc à n'être qu'un produit commercial. M. D. fait venir des photographes, qui filment Hashi en délire et qu'on emmène de force à l'asile. Le corps malade du chanteur est rabaissé en objet d'exhibition : la pathologie de Hashi, qui étonne le public, a des effets commerciaux percutants. M. D. délaisse à son seul profit celui qui est tout de même son amant. Cet épisode corrobore le constat amer de Hashi : personne n'a besoin de personne; seule compte l'utilité au sein de ce corps social pourtant à l'agonie.

Si Kiku, autre héros, décide de détruire Tokyo, centre du corps social, c'est qu'il cherche, selon certains critiques, à se libérer du «refoulement de la société contemporaine, qui nous interdit de vivre 《selon nos désirs》/自分の「欲求に従っ て」生きることを許さない現代社会の抑圧性 ${ }^{9}$ et dont la consigne symbolise l'encellulement $:$ « la consigne automatique = la matrice en fer = le symbole de la société japonaise qui se replie sur elle-même / コインロッカー $=$ 鉄の母胎=閉塞的な日本社 会の象徵》"

11 Tokyo, corps social agonisant, affaiblit et rend malades ses membres. Les protagonistes se font héros quand, pour construire un autre monde, ils détruisent cette capitale, ce corps social désuni et même désagrégé que Kiku, à son premier passage à Tokyo, perçoit en entrant dans une rue :

les planches anatomiques de la classe de sciences naturelles à l'école primaire. (...) C'était un corps humain dessiné telle une ville. La nourriture étaient les matériaux bruts, les poumons, les centrales électriques; l'appareil digestif, les bureaux administratifs et les magasins de commerce; la trachée, les lignes électriques; les vaisseaux sanguins, les routes ; les cellules, les habitants.

小学校の理科室にあった人体の模式図（... ）人体を、街に模して絵が描いて あった。食物は原材料、肺は発電所、消化器は官庁と商店、気管は送電線、血

管は道路、住民は細胞 (p. $130 ;$ p. 126.)

12 Les parties corporelles sont juxtaposées. Le corps social est comparé aux planches, à un objet inanimé ; il est en décomposition. Kiku voit aussi 13 tours, symbole de la richesse de la capitale. En même temps, la mort est suggérée par le mot «tour» / 塔 (Loc. cit.), abréviation du monument funéraire / 卒塔婆, composé de plusieurs pièces en pierre superposées. Enfin, le chiffre 13 place la crucifixion sous un signe néfaste. Cette capitale spectrale est bien un corps social engourdi, qui ne supporte plus sa propre agonie: «Tokyo s'adresse à Kiku; il a entendu sa voix : détruis, détruis tout complètement. » / 東京がキクに呼びかけている、キクはその声を聞いた、壊してく扎、全てを破壊 してくれ (p. $125 ;$ p. 117).

13 L'idéogramme 壊 a le sens bouddhique d'anéantir ce monde-ci pour en créer un autre à partir des ruines. Cette société où seul le gain est reconnu et où le corps social ne fonctionne bien que si ses membres sont productifs, n'a plus de cohésion, n'est plus viable, et sa destruction est dès lors la seule issue possible vers un autre monde.

\section{Du matricide à la renaissance : le corps d'athlète de Kiku}

14 Kiku assiste à la mort sanglante de Kuwayama Kazuyo 桑山和代, sa mère adoptive, puis perpètre un matricide. Absurde est cette mort accidentelle: elle monte à Tokyo en 
quête de Hashi, l'autre fils adoptif, qui a fugué en quête de sa mère biologique. Une rixe éclate dans une rue. Un roller bouscule Kazuyo, qui se heurte la tête et, rentrée à l'hôtel, décède - c'est dire le caractère fragile du corps. Pour Kiku, sa dépouille n'est plus humaine, mais « une poupée raide qui crache un liquide rouge.» / 赤い汁を吐く 硬い人形 (p. 126, 122).

Dès lors, Kiku se sent enfermé avec ce cadavre. On n'est pas très loin de la claustrophobie: au début de $C L B$, les héros sont enfermés dans une consigne automatique, au milieu, Kiku est emprisonné, et vers la fin, Hashi est interné dans un asile psychiatrique. La fermeture est associée à la mort réelle ou sociale ; l'ouverture, à la possibilité de vivre. Cependant, la vie n'est pas une possibilité donnée : il faut avoir la volonté de la prendre, et pareille résolution exige un mouvement incessant. Mais alors pourquoi le voyage de Kazuyo à Tokyo, qui marque sa volonté de retrouver Hashi, son fils adoptif fugué, se solde-t-il par la mort? L'indifférence collective des Tokyoïtes à autrui porte un autre nom : la cruauté violente à l'endroit du provincial, par laquelle le corps social anéantit Kazuyo. Ce contre-exemple montre que la volonté seule ne suffit pas à permettre l'individu de vivre dans la capitale implacable : l'intention doit être soutenue par le corps.

Quant au matricide commis par Kiku, c'est un échec: sa volonté se réduit à l'envie de fuir la vérité insupportable. Le chapitre 19, en l'occurrence, se compose comme suit: Kiku apprend la disparition de Hashi, lequel a su que M. D organisait la rencontre surprise du chanteur avec sa mère pour une émission télévisée. Kiku arrive au lieu des retrouvailles avec ses fusils. Hashi lui présente une femme en disant à Kiku que c'est sa mère. Affolé, ce dernier tire sur la tête de sa mère. Tout s'accélère à ce point culminant.

Cette violence découle d'une volonté défaillante. Impliqué dans le monde du showbiz, Kiku attire le regard de la foule et, contre son gré, joue le rôle de vedette dans une émission. Peu importe sa volonté, son corps est exhibé devant les caméras, qui réduisent les retrouvailles avec sa mère à un fait divers. Le voyeurisme omnivore du corps social l'engloutit. À ce point de tension extrême, la violence en lui refoulée ressurgit: «Le fer massivement luisant, censément enfoui dans les plis du cerveau, commence à vrombir. Kiku a eu envie de vomir, et s'est fermé les yeux.» / 脑の璧に埋 めておいたはずの重く光る金属が音をたて始めた。キクは吐き気を催して目を閉 じた (p. 303, 285) ${ }^{11}$.

Murakami juxtapose le cerveau, organe vivant et une pièce métallique qui fait l'allusion à la thérapie que Kiku, trop violent, a subie enfant. L'incompatibilité de ces deux objets dans un corps marque le moment où la violence se réveille chez lui : Kiku est sous l'emprise du souvenir insupportable du nourrisson qu'il fut, enfermé dans la consigne automatique. La violence le domine au point de tirer sur des cameramen. Sa mère l'exhorte alors à tirer sur elle : il lui obéit, et voit «le visage [de sa mère] sans yeux ni nez ni lèvres ni oreilles ni cheveux.»/ 目も鼻も唇も耳も髮の毛もなくなった顔 (p. 286).

Le corps sans volonté est à la source du péril : il détruit non seulement la victime, mais aussi le bourreau lui-même, dépassé par son acte qui aboutit au matricide. Située à la fin de la première partie, cette scène est le premier point culminant du roman. La stratégie narrative est la suivante : Murakami accumule l'image mystérieuse, teintée de rouge, symbole de la violence qui fait partie de la nature dans les chapitres qui précède le matricide. 

penseur, oppose la nature à la société cérébrale / 脳化社会, de facture humaine. Comme le corps appartient à la nature qui n'est guère compatible avec la société civilisée, celle-ci doit le maîtriser, sinon le cacher ${ }^{12}$. Cette idée nous permet d'associer la violence refoulée dans le corps des héros de CLB à ladite image mystérieuse, teintée de rouge qui cache et dévoile l'agressivité des deux héros laquelle rend impossible leur intégration sociale.

21 S'il est vrai que la violence est un tabou social, nous revenons à l'antagonisme entre nature et «société cérébrale » de Yōrō. Le refoulement est un traitement imposé aux enfants jugés autistes dans CLB où la société contrôle le souvenir de l'individu pour maintenir l'ordre groupal, et change le passé qui constitue la permanence identitaire. Cet épisode, situé au début du roman, crée les tensions qui éclateront entre «la société cérébrale » et la nature incorporée dans les héros. Cet épisode permet de déchiffrer l'image, teintée de rouge, qui les hante: il s'agit d'« une représentation [...] refoulée [qui] contrevient aux exigences du moi et des lois sociales, elle rentre dans la sphère de l'inconscient, mais l'énergie libidinale liée à cette représentation désormais refoulée perdure quant à elle et elle est transposée dans le corporel $»^{13}$. Le moi, membre social, se comporte conformément aux lois et aux règles pour maintenir l'ordre de l'ensemble, mais l'énergie libidinale, force vitale de l'individu, peut y échapper, d'où le conflit entre le refoulement et cette puissance démesurée, incorporée dans les héros de CLB dès l'adolescence. Murakami use du stéréotype pour que le lecteur oriente la couleur rouge vers le sang qui métaphorise tantôt l'énergie vitale, tantôt la violence démesurée dans CLB. De plus, cette symbolisation est associée au corps. Au lycée, Kiku pratique le saut en hauteur. Quand il rate, il voit dans l'air « quelque chose de rouge et moite qui frémit à peine.»/赤く需饥て微かに摇れているもの (p.100, 98). Cette vision annonce le matricide. L'image apparaît aussi chez Hashi, alors collégien, comme la réaction naturelle du corps contre le refoulement artificiel. Il va au spectacle, monte en scène, est hypnotisé par une chanteuse, et se remémore alors la terreur éprouvée dans la consigne automatique. Il s'enfuit pour arriver dans un parc où un clochard lui a fait une fellation et voit alors « le souvenir mis à nu qui devient une masse toute rouge » / 裸に なった記憶が真っ赤な塊となって。 (p. 248；235). Hashi prend une brique pour casser la tête au clochard. La partie intime du garçon, objet de la caresse orale du pédophile, le transforme en agresseur. La "masse toute rouge » est l'autre nom de la mémoire du nourrisson que fut Hashi, laissé dans la consigne automatique au risque de s'y asphyxier. Le corps appartient à la nature en ce qu'il révèle non seulement la vie, mais aussi la mort, espèce de tabou social.

Nuançons l'hypothèse de départ : le corps échappe à la volonté du héros quand il est au point critique de jonction entre la vie et la mort. En témoigne le corps de Kiku après le matricide. Son corps amoindri dénote son inhibition. En prison, on l'appelle « lobo (tomie) / ロボ (p. $346 ; 324) »$ pour son aboulie. Ce surnom connote la fin malheureuse des individus révoltés, ceux de Nineteen Eighty-Four (1949) ou One Flew Over the Cuckoo's Nest (1975), qui perdent toute résistance après lobotomie. Kiku, lui, retrouve son énergie quand il court lors d'une fête sportive des détenus : toujours en avant et plus vite, il renaît en athlète à la fête sportive en prison. Son corps se reconstitue au fil de son entraînement : «c'était comme si ses muscles jouaient à leur gré les rôles qu'ils avaient appris par cœur.»/ 筋肉が憶え込んでいることを勝手に演じているよう だった (p. $347 ; 324)$. Dans la scène antérieure au matricide où sa mère l'enjoint de tout 
arrêter, Kiku lui obéit encore et toujours, d'où la défaillance prolongée de sa volonté. En revanche, son corps sait faire le nécessaire, car " [le] corps [est] un condensé, une mémoire vivante et charnelle de l'évolution de la vie $»^{14}$. Ses muscles retrouvent leur autonomie quand il faut courir. Le corps prouve la continuité entre son passé d'athlète et son présent. Cet épisode relativise la distinction stéréotypée entre la tête qui commande et le corps qui exécute sa mission. Rien ne s'amorce sans le corps dans CLB.

Kiku court vite grâce à sa mémoire musculaire. Mais quand il perçoit le regard des autres détenus et des surveillants, il se sent mal à l'aise. Ces spectateurs lui rappellent son matricide. Sa mère décédée, gravée dans son souvenir, paralyse son corps : « il est en notre pouvoir de faire "revivre", par l'ensemble de nos facultés, sensorielles, affectives, motrices, conceptuelles [...] des pans entiers de la réalité (choses, êtres) passés de l'actualité à la virtualité $\aleph^{15}$. De fait, Kiku ne cesse de voir scintiller le visage de sa mère, devenu morceau sanglant de chair, comme un cryptogramme qu'il lui incombe de déchiffrer. D'abord, il doit sortir de sa paralysie. Aucun médicament ne détend le corps rigide. Intervient Yamane, son co-détenu, maître en karaté, qui appuie sur un point entre la nuque et la tête pour le détendre ${ }^{16}$. Cette condensation du souffle vital réveille Kiku comme si Yamane lui communiquait son énergie. Puis, ce dernier lui conseille de crier. Kiku se met à pleurer, lui dont toutes les émotions sont bloquées depuis le matricide. Le cri de Kiku appelle ainsi le sujet à advenir, en l'occurrence à revenir hic et nunc. L'organe vocal libère ses émotions enfouies.

Yaname demande ensuite à Kiku d'écouter tous les sons autour de lui. Il se souvient des battements de cœur, du son artificiel, qu'il a entendu chez le psychiatre. Quand il traverse un grand péril, ce souvenir refoulé fait retour : son corps garde en mémoire la violence infligée, qui prouve aussi sa force vitale démesurée, car il a survécu dans la consigne automatique. Kiku, écoute la voix de Yamane, et régresse jusqu'au «fond de l'eau rouge et lourde»/赤い重い水の中 (p. $355 ;$ p. 330).

Kiku ne fait plus attention au monde extérieur. Il se plonge en lui-même pour chercher son passé le plus lointain. Dans son inconscient, il se rappelle le moment où il était dans le liquide amniotique. Le souvenir de cet espace matriciel marque aussi le moment crucial de sa renaissance. Il se redresse, et dit : «m, m, m, m, maintenant, je cours » / 「俺、今今今今から、走るぞ」 (Loc. cit.). La répétition de 今 révèle avec emphase le moment où l'athlète retrouve sa volonté. Corps et décision vont de pair : il retrouve sa force naturelle contre les tranquillisants produits par la civilisation. Il rejoint d'autres coureurs pour les dépasser : «À tout prix, Kiku a tenté de saisir le vent»/キクは必死 になって風を掴もうとしていた (p.357; p. 337).

Le corps courant est en harmonie avec la puissance du vent qui concrétise le mouvement cosmique du $k i^{17}$. Le souvenir qu'engrange le corps passe du virtuel à l'actuel via la mémoire musculaire, qui permet à Kiku de retrouver son énergie. Par rapport à notre hypothèse, nous constatons que la volonté renaît grâce au corps chez cet athlète qui se libère de l'inhibition.

Le décès de Kazuyo signifie que l'affection et la volonté ne permettent pas à elles seules de vivre dans la capitale, où domine l'indifférence aux autres: il faut un corps vigoureux, lequel ne suffit pas non plus; Kiku devient le jouet de sa frénésie, d'où la nécessité de faire coïncider le corps avec la volonté. 


\section{De l'artificiel à la nature : le visage de Hashi} devient un bourreau, avaleur monstrueux ${ }^{19}$. Il se noie dans le fatras des images médiatiques où l'individu, au seul service de la mise en scène commerciale, enfouit son identité intime. Il devient étranger à lui-même : «Être aliéné, c'est être soumis à ce qui n'est pas soi. [...] Aliéner, c'est perdre, ou se perdre $»^{20}$. Dès lors, Hashi est possédé, et poursuivi par son image sociale. Il ne peut plus cesser de jouer son rôle dans le monde du showbiz. Mais à force, il finit par être broyé. Après son succès, il rentre à Sasebo, berceau de l'enfance. Il attire la foule, et fait tomber sa cuillère dans un restaurant : « À la surface du dos de la cuillère, le visage défiguré de Hashi se reflète. »/裏返しになっ たスプーンの表面に歪んだハシの顔が映っている。(p.422; p. 392).

Hashi est dévoyé à son insu, son reflet tordu de star lui montre qu'il est altéré. Il essaie de remémorer son enfance pour retrouver sa permanence identitaire, mais en vain. Plus il se détériore, moins se vendent ses chansons. Il poignarde Niva, son épouse enceinte. Il lui rend visite, mais alors sous les caméras, préparé par M. D., il est emmené 
de force vers un asile psychiatrique. De nouveau, il voit son reflet dans la lentille d'une des caméras, à savoir un visage en larmes, marque incorporée de son identité, qui relie le passé au présent :

c'est mon visage à moi, qui ne puis me mouvoir, qui suis transi et incapable de parler. [...] Il [Hashi] a décidé de retrouver ce visage craintif auquel on a arraché le nom, le sens, les habits et le mouvement.

身動きができず怯えて喋ることもできない自分の顔だ。（...）名前も意味も衣

服も運動も剥ぎ取られて怯えているその顔を辿って行こうと決めた。(p.548;

p. 510)

Il rejette l'image de la star pour retrouver son identité permanente, celle du nourrisson menacé de mort dans un recoin du monde. «[L]e sens de quelque chose tient dans sa relation à autre chose. Ici, au contraire, le visage est sens à lui seul $\aleph^{21}$. Dès lors que l'identité sociale de la star est arrachée à Hashi, il se confronte seul à sa propre vulnérabilité. Si la société lui reste hostile, c'est en lui-même que gît la possibilité unique de changer le monde.

Certes, la musique est la voie de son choix. Mais sa volonté est contaminée par l'ambition commerciale de M. D., sorte de Dracula qui boit son sang pour en faire son pareil. Hashi doit détruire son image efféminée pour changer sa voix, «tributaire du $\operatorname{corps}^{22}$ ", et ce, pour retrouver son autonomie. Car il sait qu'il n'a pas obtenu sa position sociale de star par son travail. En fait, Matsuyama / 松山, guitariste, lui révèle le problème concret : «C'est clair, la voix est faible./はっきりしてる、ボーカルが弱 いんだ。(p.371; p.346)»Sa musique défaille là où il a besoin du " "cri" d'un combattant pour concentrer son énergie et la faire "exploser" $\aleph^{23}$. Au contraire, quand Hashi chante, il rend le concert lugubre. Il lui faut une nouvelle source d'énergie. Il en cherchera une dans son corps. Sinon, il ne cesse de subir la violence des autres et, tel le nourrisson enfermé en consigne automatique, demeure impuissant. Cette boîte hermétique en fer est reliée au corps social qui finit par assigner à l'individu une place très réduite jusqu'à l'exténuer. Pour ne pas s'y emprisonner, il faut une volte-face. Précisément parce qu'il est frêle, Hashi s'inflige une violence démesurée de son plein gré : il se coupe le bout de la langue pour obtenir une voix sensuelle. Il change non seulement de voix, mais aussi de corps. Ainsi, il écarte l'identité sociale, fabriquée avec M. D. Le bout de la langue coupée est la preuve de la décision de Hashi seul : la volonté et la violence corporelle vont ensemble.

Apparaît entre la masse sociale puissante et l'orphelin vulnérable un antagonisme qui, articulé sur le corps, aboutit à la violence, que Hashi a le choix de subir en devenant luimême victime ou de maîtriser, et ce jusqu'à l'automutilation.

Puisque la société demeure hostile aux corps étrangers, c'est à ceux-ci de changer, de leur propre gré, pour prouver leur présence au monde, d'où la volonté de Hashi qui passe par l'automutilation.

CLB montre que la volonté et le corps sont indissociables là où les membres productifs suffisent pour assurer un bon fonctionnement social. Cette logique utilitariste trouve ses limites quand le corps social tombe malade, ce qui se manifeste par l'égoïsme outré de ses membres et le chacun pour soi de cette société désagrégée. L'affection et la volonté sont insuffisantes pour vivre dans la capitale implacable, surtout pour les gens fragiles. Mais la mémoire musculaire de l'homme demeure en dépit de son atonie, et c'est le corps qui incarne la permanence identitaire. Face à la société qui risque de le broyer comme un paria, Kiku doit maîtriser la violence par sa volonté de gagner, mais 
son corps seul est insuffisant à mener la lutte jusqu'au triomphe et il lui faut encore user de sa force de volonté. Ce roman est une critique virulente de la société nippone du début des années 80 qui, au nom de sa haute croissance, gage de vie paisible, étouffe la volonté de l'individu et lui impose ses normes castratrices dès lors que le consensus social l'estime violent. Aussi le corps et la volonté deviennent-ils le recours ultime de l'individu pour survivre contre cette société.

\section{Résumé du roman}

Une femme abandonne son bébé dans une consigne automatique. Prénommé Kikuyuki (plus loin, Kiku), il grandit dans un orphelinat avec Hashio (plus loin, Hashi). Le premier multiplie les fugues, le deuxième se replie sur lui-même. Un psychiatre les hypnotise et refoule leur énergie trop violente. Les garçons, adoptés comme frères par un couple qui habite une île du Kyushu, s'aventurent dans d'anciennes mines abandonnées. Un jour qu'ils vont au spectacle, Hashi participe à une séance d'hypnose, et ses souvenirs refoulés resurgissent. Désormais, il cherche le son qu'il a entendu chez le psychiatre. Il part à Tokyo en quête de sa mère. Plus tard, Kiku et Kazuyo, leur mère adoptive, y montent pour le retrouver. Mais cette dernière se blesse et décède.

Kiku rencontre Anémone, mannequin qui l'accompagne lors de sa tentative d'effraction à Yakushima, zone périlleuse de marginaux, et retrouve Hashi, maquillé, qui se prostitue. Ce dernier, lors d'un incident au marché, apaise la foule en chantant. Apparait M. D., patron et amant de Hashi, qui lui permet de percer le milieu de la chanson et tente de diffuser à la télévision les retrouvailles de Hashi avec sa mère pour vendre ses chansons. Hashi épouse Niva, styliste âgée. De son côté, Kiku et Anémone, son amoureuse, décident d'anéantir Tokyo par un gaz toxique à base de Datura. M. D. lui apprend que Hashi retrouvera sa mère dans une émission télévisée. Kiku court vers le lieu de rencontre avec ses fusils. Sur place, il voit une femme ; c'est sa mère. Choqué, Kiku lui tire dessus.

41 Hanté par l'image de sa mère sans visage, il se replie sur lui-même dans une prison. Mais lors de la fête sportive, il court, et comprend le message de sa mère : vivre. Hashi, lui, se coupe le bout de la langue pour changer son timbre de voix. Il poignarde Niva, qui est enceinte. Il est placé en asile psychiatrique.

Kiku s'évade de la prison, et découvre la Datura pour la larguer à Tokyo. Hashi s'enfuit de l'asile. Dans la rue déserte, il est pris de frénésie destructrice. Il voit une femme enceinte, qu'il identifie à sa mère. Il se bat contre lui-même pour ne pas la tuer, et du coup, entend le son qu'il cherchait.

BIBLIOGRAPHIE

AUROUX Sylvain (dir.), Notions philosophiques II, Paris, PUF, 1990. 
Corps souffrants dans les littératures de la Chine et du Japon au $\mathrm{XX}^{e}$ siècle. Extrême-Orient, Extrême-Orient, 39:2015, Saint-Denis, P. U. de Vincennes.

CHENG Anne, Histoire de la pensée chinoise, Paris, Seuil, 2002 [1997].

COMTE-SPONVILLE André, Dictionnaire philosophique, Paris, PUF, 2001.

DOGANIS Basile, Pensées du corps. La philosophie à l'épreuve des arts gestuels japonais (danse, théâtre, arts martiaux), Paris, Les Belles Lettres, 2013.

DURAND Gilbert, Les structures anthropologiques de l'imaginaire, Paris, Dunod, 1992 [1969].

GODDARD Jean-Christophe et LABRUNE Monique (éds.), Le corps, Paris, Vrin, 1992.

LEVINAS Emmanuel, Éthique et Infini. Dialogue avec Philippe Nemo, Paris, Livre de poche, 1992 [1982].

LEW René et SAUVAGNAT François (éds.), La voix, Paris, Lysimaque, 1989.

MINAMI Yūta 南雄太, [Recherche sur les œuvres de Murakami Ryū. La carte du monde de Murakami Ryū] / 村上龍作家作品研究一村上龍の世界地困 / Murakami Ryū sakka sakuhin kenkyū. Murakami Ryū no sekai chizu, 専修大学出版局 / Senshūdaigakushuppankyoku, 2007.

MURAKAMI Ryū, IKEZAWA Natsuki, [Littérature nationale : interprétation et recherches des textes : numéro spécial Murakami Ryū : aller à l'extrémité de “notre temps”] / 国文学解釈と教材の研究 臨時増刊村上龍特集“現代のエッジを行く”, vol. 46, 9:2001.

MURAKAMI Ryū 村上龍, Coin Locker Babies $2^{\mathrm{e}}$ partie / コインロッカー・ベイビーズ, 下, Tokyo, Kōdansha, 1984 [1980].

MURAKAMI Ryū 村上龍, Coin Locker Babies / コインロッカー・ベイビーズ, Tokyo, Kōdansha, 2017 [1980], Kōdansha.

MURAKAMI Ryū 村上龍, Les bébés de la consigne automatique, ATLAN Corinne (trad.), Arles, Picquier, 1998 [1996].

[Histoire des mines au Japon] / 日本の炭鉱の歴史, jcoal.or.jp/worldheritage/03/05/.

NAGAO Ken 長尾建, [ « La dérobade de Ryū, Les bébés de la consigne automatique, Destruction et Vie»]/龍の脱出『コインロッカー・ベイビーズ』ー破壊と生, [Littérature nationale : interprétation et recherches des textes : numéro spécial Murakami Ryū : aller à l'extrémité de “notre temps”] / 国文学 鑑賞と解釈の教材村上龍特集 “現代のエッジを行く”/ Kokubungaku kaishaku to kanshō no kyōzai Murakami Ryū tokusyū Gendai no edji o iku, vol. 46, 9:2001.

YōRō Takeshi 養老孟司, [Histoire littéraire du corps] / 身体の文学史, Tokyo, Shinchōsha, 2010.

\section{NOTES}

1. V. résumé long en fin d'article.

2. MIURA Masashi 三浦雅士, [《Commentaire»]/ 解説, in Coin Locker Babies, 下 / $2^{\mathrm{e}}$ partie, MURAKAMI Ryū, Tokyo, Kōdansha, 1984 [1980], p. 225-242.

3. CHRÉTIEN Jean-Loup, "Le corps mystique dans la théologie catholique », in Le corps, GODDARD Jean-Christophe et LABRUNE Monique (éds.), Paris, Vrin, 1992, p. 91.

4. Édition de référence : MURAKAMI Ryū 村上龍, Coin Locker babies / 『コインロッカー・ベイビー ズ』, Kōdansha, 2017 [1980]. La traduction est la nôtre. La pagination des extraits de l'œuvre de référence est dans le corps du texte. Celle des Bébés de la consigne automatique par Corinne Atlan est suivie du point-virgule. 
5. La politique énergétique japonaise passe du charbon au pétrole dans les années 60 . Voir [Histoire des mines au Japon] / 日本の炭鉱の歴史, jcoal.or.jp/worldheritage/03/05/, consulté le 28/05/2018.

6. MURAKAMI Ryū 村上龍, IKEZAWA Natsuki 池澤夏樹, [“Aller à l'extrémité de “notre temps” »] / 対談.「“現代”のエッジを行く」、, [Littérature nationale: interprétation et recherches des textes : numéro spécial Murakami Ryū : aller à l'extrémité de “notre temps”] / 国文学 鑑賞と解 釈の教材村上龍特集 “現代のエッジを行く”, vol. 46, 9:2001, p. 19.

7. MIURA Masashi, [«Commentaire »], op. cit., p. 230.

8. PALAYRET Guy, «La maladie humaine. Le corps, la maladie, la mort de Montaigne à Molière », Le corps, op. cit., p. 143.

9. NAGAO Ken 長尾建, [«La dérobade de Ryū, Les bébés de la consigne automatique, Destruction et Vie»]/龍の脱出『コインロッカー・ベイビーズ』ー 破壊と生, in [Littérature nationale : Interprétation et recherches des textes : numéro spécial Murakami Ryū : aller à l'extrémité de “notre temps”] / 国文学 鑑賞と解釈の教材 村上龍特集 “現代のエッジを行く”, vol. 46, 9:2001, p. 19 ; ibid., p. 159.

10. MINAMI Yūta 南雄太, [Recherche sur les œuvres de Murakami Ryū. La carte du monde de Murakami Ryū] / 村上龍作家作品研究一村上龍の世界地図, 専修大学出版局, 2007.

11. MURAKAMI Ryū, op. cit.

12. YōRō Takeshi 養老孟司, [Histoire littéraire du corps], 身体の文学史, Tokyo, Shinchōsha, 2010.

13. BELIN-CAPON, « Freud et le corps : nature et expression », Le corps, op. cit., p. 212.

14. Doganis Basile, Pensées du corps, Paris, Les Belles Lettres, 2013, p. 149.

15. Ibid., p. 73.

16. Ibid, p. 90.

17. ROBINET Isabelle, l'entrée « Qi », Notions philosophiques II, Paris, PUF, 1990, p. 2970-2971.

18. DOGANis Basile, Pensées du corps, op. cit., p. 178.

19. DURAND Gilbert, Les structures anthropologiques de l'imaginaire, Paris, Dunod, 1992 [1969], p. 236.

20. COMTE-SPONVILLE André, "Aliénation », Dictionnaire philosophique, Paris, PUF, 2001, p. 32.

21. LEVINAS Emmanuel, Éthique et Infini. Dialogue avec Philippe Nemo, Paris, Livre de poche, 1992 [1982], p. 81.

22. KRISTEVA Julia, "La parole déprimée ", in La voix, LEW René et François SAUVAGNAT (éds.), Lysimaque, 1989, p. 82.

23. DOGANIS, Pensées du corps, op. cit., p. 90.

\section{AUTEUR}

\section{TOSHIO TAKEMOTO}

Maître de conférences de langue et de littérature japonaises à l'Université de Lille, chercheur à l'Institut français de recherche sur l'Asie de l'Est (IFRAE / UMR 8043). Il travaille sur les auteurs contemporains. Il a publié notamment Écrire la guerre, écrire le conflit (codirigé avec F. McIntoshVarjabédian, J. Prugnaud et N. Giraldi Dei Cas, CEGES, 2016), Poésie brève et contemporaine (codirigé avec M. Andro-Ueda et J. Wilker, Septentrion, 2017). 


\title{
La décomposition du corps dans la poésie coréenne contemporaine : « Ça va, je suis un cochon ", poème en prose de Kim Hyesoon
}

\author{
Moduk Koo
}

1 La présente communication porte sur la question du corps dans la poésie coréenne, en particulier chez Kim Hyesoon 김혜순, qui en est l'une des figures majeures actuelles. Née en 1955 à Uljin en Corée du Sud, elle publie ses premiers poèmes à la fin des années 1970, en Corée dictatoriale. Tout en élaborant une conception de la poésie féminine qui se distingue de la poésie féministe, elle explore un univers poétique singulier où s'effacent toutes les frontières entre l'homme et l'objet, l'humain et l'animal, la vie et la mort...1.

2 Parmi d'autres représentations du corps - lieu d'écriture par excellence chez elle -, celle de la décomposition corporelle (corps déchiré, morcelé, découpé, brisé, arraché) paraît tout à fait pertinente dans son poème en prose : « Ça va, je suis un cochon » / 돼 지라서 괜 찮아 (2012).

3 C'est peut-être l'un des poèmes les plus lisibles de Kim Hyesoon, réputée pour son avant-gardisme difficile, et très ouvertement politique. En mai 2016, ce poème a provoqué une grande polémique dans le monde littéraire coréen, en remportant le Prix du 18 Mai - date de naissance, en 1980, du Mouvement pour la démocratisation de Gwangju (ville du sud-ouest). Certains écrivains porte-parole de la «tradition nationale » ont violemment critiqué l'attribution du prix à Kim Hyesoon. En réalité, depuis la période de modernisation du pays - qui remonte au début du $\mathrm{xx}^{\mathrm{e}}$ siècle, quand la Corée connaît différents épisodes très douloureux (occupation japonaise, guerre, division du pays, régimes dictatoriaux) -, on divise la poésie coréenne en deux écoles : la poésie traditionnelle lyrique et la poésie en quête de modernité littéraire. Certains tendent encore à croire que les poètes du deuxième groupe sont déconnectés du monde 
réel, et ce même si cette idée s'avère fausse. Mais finalement, Kim Hyesoon n'a pas accepté le prix, d'où plusieurs débats sur les relations entre littérature et politique.

Or il y a des malentendus sur ce poème qui remet en question le corps humain et le corps animal, ainsi que leur lien avec l'amour et la violence. Même s'il semble inventer un univers totalement insolite, ce poème est en fait très ancré dans une réalité sociopolitique familière à la Corée du Sud et nous invite à réfléchir sur la façon dont cette société traite le corps, notamment au niveau de la voix et celle de la métamorphose.

\section{D'une voix à l'autre}

5 Comme suggère déjà le titre " Ça va, je suis un cochon ", qui est une allusion au film de Park Chan-wook 박찬욱 (1963-) Je suis un cyborg/ 싸이보그지만 괜찮아 (2006), l'humour noir donne au poème tout son aspect provocateur. Il est composé des quinze intertitres suivants :

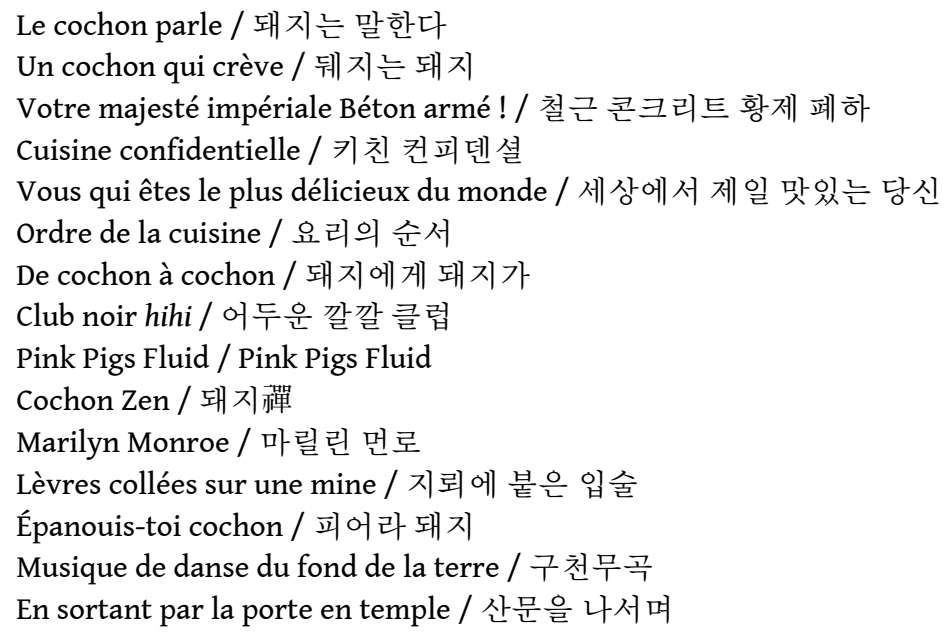

6 Avec les intertitres, on peut relever quelques caractéristiques de la poésie de Kim Hyesoon: l'utilisation du langage prosaïque, les dimensions humoristique et fantastique, ainsi que l'exploration de l'espace quotidien, avec la cuisine pour lieu privilégié. Le poème incarne la voix du cochon, tout comme dans Animal Farm (1945) de George Orwell. Écrit après l'épidémie de fièvre aphteuse de 2011 en Corée du Sud, le poème est publié en 2012 dans la revue littéraire Munyejoongang / 문예중앙, puis repris dans le douzième recueil de poèmes de Kim Hyesoon, [Épanouis-toi cochon] / 피어라 돼 지 (2016). Il part de l'expérience personnelle de l'auteure qui, de fait, comme nombre de Coréens à l'époque, a été fort affectée par la mort des animaux et leurs images diffusées par les médias. Elle a eu l'occasion de séjourner dans plusieurs temples bouddhistes et d'assister à des cérémonies censées consoler l'âme des animaux abattus par les hommes. Le poème illustre cette expérience :

De toute façon c'est naturel de clouer un cochon sur la croix, ça n'a rien de particulier

Dans une pièce destinée au zen je m'assois en lotus et regarde le mur de travers pour méditer

$\mathrm{Tu}$ sais, je l'avoue maintenant en fait je suis un cochon. Tu sais, je suis un cochon depuis ma naissance Sale je suis sale vraiment sale. Un esprit ? Je te dis que je n'ai rien de tel ${ }^{2}$ (p. 90) 아무래도 돼지를 십자가에 못 박는 건 너무 자연스러워, 의미 없어 나는 선방에 와서 가부좌하고 명상을 하겠다고 벽을 째려본다 
있지, 지금 고백하는 건데 사실 나 돼지거든. 있지, 나태어날 때부터 돼지였어

더러워 나 더러워 진짜 더럽다니까. 정신 ? 나 그런 거 없다니까

Pourquoi me reste-t-il seulement l'ombre de qui s'en est allé

Pourquoi le mot cochon ne me quitte-t-il pas

À l'intérieur de la chambre un aspirateur muet semble avoir aspiré tous les sons

À l'intérieur de la chambre les feuilles d'un arbre inconnu caressent les rideaux

sans couleur (p. 103)

어째서 나한테는 떠난 사람의 그림자만 남았을까요

어째서 나한테는 돼지라는 말이 떨어지지 않을까요

무음 청소기로 소리를 모두 빨아들인 것 같은 방 안

이름 모를 나무의 이파리들이 흐릿한 커튼을 어루만지는 방 안

Oh voici le regretté cochon qui passe

Et voici qu'on le chasse

Oh une femme tente de sortir du cochon (p. 108)

오 그리운 돼지가 간다

쫓겨간다

오 한 여자가 돼지를 나가려고 한다

Ici, la voix du cochon se confond ou fusionne avec celle d'une femme en méditation dans un temple bouddhiste. Quand la voix de la première personne dit «je suis un cochon ", il est difficile de savoir exactement qui est le "je ». C'est une des questions fondamentales de ce poème. Le lecteur se demande s'il y a un cochon et une femme bien distincts ou si le cochon est la femme. Ou bien si le cochon existe à l'intérieur de la femme. Le doute persiste jusqu'à la fin. D'ailleurs, la relation femme-cochon semble changer sans cesse. Tantôt, ce sont deux êtres différents, tantôt ils ne se distinguent pas; ils sont comme des frères siamois et utilisent tous deux la voix de la première personne sans transition. Cette confusion des sujets fabrique une voix errante et indécidable, sans corps proprement identifiable.

La voix féminine joue notamment un rôle intermédiaire entre l'humain et l'animal. Si la femme, plus que l'homme, est proche de l'animal, il y a sans doute plusieurs raisons à cela, mais l'une d'entre elles me semble liée au fait que la femme et l'animal appartiennent à un espace non verbal. En effet, dans la société ou dans les représentations artistique et médiatique, on constate que souvent la femme n'est pas l'émetteur, mais le récepteur de la parole. Le silence occupe une place privilégiée chez maintes femmes écrivains. Chez Marguerite Duras ou dans les romans coréens de Han Kang, le mutisme du personnage féminin parait symptomatique. L'activité langagière de la femme semble bien différer de celle de l'homme, et plus encore dans la société dictatoriale et patriarcale que la poétesse coréenne a connue elle-même dans les années 1970 et 1980. Faire entendre une voix féminine est déjà une tâche difficile. De ce point de vue, le langage féminin ne peut être que défiguré pour survivre. C'est une voix qui a survécu et qui se demande : «Qu'est-ce qu'avoir un corps dans la société ? Comment supporter la violence qui traverse notre corps? Comment se maintenir, persévérer dans un corps blessé ?» 
De plus, la nourriture, élément déterminant, rapproche l'animal et la femme car celleci, en tant que figure de la mère, est à la fois nourrice et cuisinière, et c'est elle qui doit découper la chair de la viande :

Moi je suis la personne qui cuisine à la maison

Je pose un couteau sur la planche et me cache les yeux

Je vous présente toutes mes condoléances

C'est mon tour de me manger (p. 99)

나는 집에서 요리하는 사람

도마 위에 칼을 올려놓고 두 눈을 가린다

삼가 명복을 빕니다

내가 나를 먹을 차례가 온다

$\mathrm{Au}$ fond, les animaux ont un statut double et tant soit peu contradictoire pour nous autres humains : ils font partie de notre famille et en même temps constituent notre nourriture. Le cinéaste coréen Bong Joon-ho 봉준호 (1969-) élabore subtilement cette idée dans Okja / 옥자 (2017), l'histoire d'une amitié entre Mija, une petite fille de la campagne en Corée du Sud, et une truie géante, appelée "Okja», animal génétiquement modifié par l'industrie agroalimentaire. Mija, depuis son plus jeune âge, vit avec Okja, née et élevée pour devenir de la viande. Il est prévu que cet animal sera abattu par une énorme machine, à l'instar des autres supers-cochons élevés un peu partout dans le monde. Cette violence qui réside dans le corps qui se décompose, se retrouve chez Kim Hyesoon, par exemple dans ce passage où le corps du cochon se mue en pièces détachées au service de la prospérité ou de l'immortalité des hommes, chose qui n'est pas humaine en soi :

Un jour on réalise un documentaire. On fait un projet de ferme d'organes pour qu'un ego survive éternellement. Là-bas je suis l'actrice la plus belle. C'est le plus important pour jouer mon rôle. Je suis élevée pour devenir votre cœur. Je suis élevée pour devenir votre poumon. Je suis élevée pour devenir votre peau. Je suis élevée pour devenir votre vésicule biliaire. Je suis élevée pour devenir votre cerveau. C'est comme ça que ça marche. En vous regardant avec attention j'échange à la vitesse de l'éclair mes yeux contre les vôtres. Je souris et j'échange à la vitesse de l'éclair mon foie neuf contre le vôtre. Grâce à cet échange infini de pièces vous ne mourrez pas. Je répète que le fait d'être l'actrice la plus belle m'aide beaucoup dans ce type de travail. Je suis élevée pour devenir votre tristesse, vos pleurs, votre angoisse, votre peur et vos troubles. [...] (p. 99-100)

우리는 미래의 어느 날 다큐멘터리를 찍는다. 영원히 생존할 자아를 위한 장기 농 장 프로젝트 촬영 중이다. 그중에 나는 제일 예쁜 배우다. 이 생각이 내 연기에 최 고로 도움을 준다. 나는 당신의 염통이 되려고 길러진다. 나는 당신의 폐가 되려고 길러진다. 나는 당신의 피부가 되려고 길러진다. 나는 당신의 쓸개가 되려고 길러 진다. 심지어 나는 당신의 뇌가 되려고 길러진다. 말하자면 이런 식이다. 나는 당신 의 눈치를 보면서 얼른 당신의 눈동자를 내 눈동자로 바꿔준다. 나는 미소를 짓다 가 얼른 당신의 간을 내 싱싱한 간으로 바꿔준다. 당신은 끝없이 부품을 교체하여 죽지 않는다. 다시 말하자면 이런 일엔 내가 예쁜 배우라는 것이 무척 도움이 된다. 나는 당신의 슬픔, 당신의 눈물, 당신의 불안, 당신의 공포, 당신의 장애가 되려고 길러진다. [...]

11 Ainsi, l'humanité perdurera grâce au cochon. De nos jours, apparemment, cet échange des organes pourrait s'avérer possible dans l'avenir, grâce au développement de la science. Certes, cette image dans laquelle le corps est utilisé au profit de l'humanité provoque une violence. Pourtant, d'après Kim Hyesoon, elle répondrait en quelque sorte à ce que nous a demandé Dieu, figure du Père tout-puissant. La Bible dit : "Soyez féconds, multipliez, emplissez la terre et soumettez-la; dominez sur les poissons de la 
mer, les oiseaux du ciel et tous les animaux qui rampent sur la terre » $(\mathrm{Gn} 1,28)^{3}$. Dans okja, le cinéaste montre qu'une puissante entreprise américaine joue le rôle de Dieu en manipulant les hommes. Le poème de Kim Hyesoon met en parallèle le corps du cochon et celui de la femme que la société oblige à entrer dans les normes pour être tous deux consommables. À cet égard, l'insistance sur la matérialité du corps et l'apparition de Marilyn Monroe ont toute leur importance :

Vous qui êtes rose flottez dans le ciel Vous qui êtes dévêtu

J'aime vous regarder nu et applaudir clap clap clap

Le plateau de la pleine lune passe sur votre corps rose

Rien dans le monde n'est indigeste pour vous qui êtes gras (p. 102)

하늘에 분홍 당신 떠간다 발가벗은 당신

나는 당신 알몸 쳐다보기 좋아서 손뼉을 짝짝짝 친다

보름달 쟁반이 당신 몸속을 통과한다

통통한 당신이 소화시키지 못할 것은 세상에 없다

Cochon 9 S'il vous plaît élevez-moi et mangez-moi

Cochon 9 S'il vous plaît pleurez après m'avoir mangée

Cochon 9 Je ferai aussi des petits (p. 97)

돼지9 길러서 먹어주세요

돼지9 먹고 울어주세요

돼지 9 새끼도 낳아드릴게요

Marilyn Monroe

[...] je suis d'un seul coup attirée par mon dernier rôle

Où je chie mon corps sur ce que j'ai déjà chié

Où je suis suspendue à un crochet en fer après que mon âme s'en est allée (p. 106)

마릴린 먼로

[...] 최후의 배역에 철컥 달라붙는다

내가 싼 것 위에 몸을 철퍼덕 싸는 배역

영혼이 빠져나간 다음 쇠갈고리에 걸리는 배역

L'érotisation du corps féminin établit un rapport entre désir sexuel et appétit. Autour de l'image de la chair rose qui est à la fois celle du cochon et de la femme, se mêlent la fascination et le dégoût.

\section{Métamorphose}

On observe qu'une certaine poésie contemporaine en Corée, comme celle de Kim Hyesoon, ne fonctionne pas par métaphore mais par métamorphose. Le cochon n'est sans doute pas là pour signifier quelque chose, il n'est identifié ni à la femme ni au peuple coréen. Sa présence est physique et sa corporéité est mise en valeur. Ce que propose le poème, c'est l'expérience de devenir cochon au sens littéral. La question de l'animal, on le sait, obsède de nombreux artistes et penseurs comme Jacques Derrida, dans L'Animal que donc je suis (2006) ou Gilles Deleuze. Devenir animal peut être une volonté d'assumer la souffrance traversée par le corps : «L'homme qui souffre est une bête, la bête qui souffre est un homme. C'est la réalité du devenir $»^{4}$. Chez Kim Hyesoon, comme chez Bong Jooh-ho, il n'est évidemment pas question de végétarisme, mais de respect envers les animaux, autrement dit des êtres qui n'ont ni voix ni pouvoir dans la 
société. Nos deux artistes ne sont pas les seuls à rejeter la hiérarchie de dominance. Les humains, pour le peintre Lucian Freud, ne sont que des "animaux habillés ». La compassion pour les animaux ne se distingue pas de celle pour les hommes. De « la pitié pour la viande $»^{5}$, sujet sensible de la peinture de Francis Bacon, Deleuze écrit: «La viande n'est pas une chair morte, elle a gardé toutes les souffrances et pris sur soi toutes les couleurs de la chair vive $»^{6}$. Et il précise : « La viande est la zone commune de l'homme et de la bête, leur zone d'indiscernabilité, elle est ce 'fait', cet état même où le peintre s'identifie aux objets de son horreur ou de sa compassion $»^{7}$.

Durant l'épidémie de 2011, Kim Hyesoon a vu un très grand nombre de cochons enterrés vivants dans des émissions télévisées et des documentaires. Puis elle est allée sur les lieux où les animaux ont été enterrés. Pour elle, ce spectacle est d'autant plus insupportable et de surcroît intolérable qu'il lui rappelle l'histoire de son pays. Dans un entretien accordé en 2012, elle confie : «En somme, les cochons ont été traités de la même façon que le régime dictatorial a traité autrefois le peuple coréen $»^{8}$. Les cochons sont malades à cause des hommes, mais ce sont les hommes qui les condamnent. Dans Okja, Bong Joon-ho dénonce le système économique capitaliste et la mort massive des animaux. L'image de la mort des innocents, qu'ils soient animaux ou humains, n'est pas sans rapport avec les traumatismes collectifs liés à l'histoire de la Corée au $\mathrm{xx}^{\mathrm{e}}$ siècle. Le poème évoque la violence des autorités :

Vous me traînez même si je hurle de toutes mes forces, en ignorant que vous êtes mon foie, mes reins, mon cœur, mes yeux et ma peau. Vous marchez en me tapant avec un bâton. Vous devez aller en prison pour menace, détournement et violation du temple des cochons. Espèce de cancer, dites-vous en me poussant dans une cage plus petite qu'un lit. (p. 100)

당신은 내 간, 당신은 내 콩팥, 당신은 내 심장, 당신은 내 눈알, 당신은 내 피부, 간 절히 울부짖어도 당신은 내가 당신인 줄도 모르고 나를 끌고 간다. 곤봉으로 가끔 쑤셔대면서 간다. 당신은 돼지 사찰 모독 횡령 고문 협박으로 감옥에 가야 한다. 당 신은 나를 이런 암덩어리 하면서 침대보다 작은 우리에 처박는다.

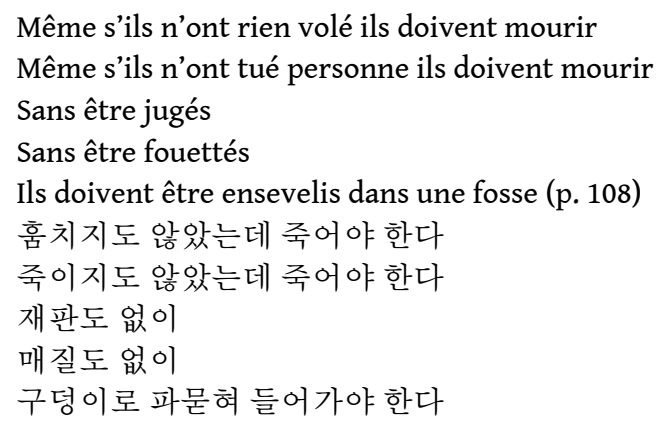

En adoptant le processus de métamorphose, le poème touche à un aspect éminemment politique. Dans ce sens, devenir cochon, c'est redevenir humain. Kim Hyesoon ne parle pas que de la mort des cochons mais aussi de leur résurrection:

Un troupeau de cochons solides comme de jeunes hommes sont jetés dans la fosse

Ils pleurent dans leur tombe

Ils pleurent debout sur deux pattes

Ils pleurent la tête coiffée de terre

Ce que je ne supporte pas ce n'est pas la douleur

mais la honte!

[...]

La nuit où il pleut flamboie un feu follet de cochon fétide 
Leurs intestins éclatés percent la tombe et surgissent du tumulus

C'est une résurrection! Ces intestins sont vivants! Ils sont vivants comme des serpents!

Épanouis-toi cochon!

Envole-toi cochon! (p. 109-110)

시퍼런 장정처럼 튼튼한 돼지 떼가 구덩이 속으로 던져진다.

무덤 속에서 운다

네 발도 아니고 두 발로 서서 운다

머리에 흙을 쓰고 운다

내가 못 견디는 건 아픈 게 아니에요!

부끄러운 거예요!

[...]

비오는 밤 비린 돼지 도깨비불이 번쩍 번쩍한다

터진 창자가 무덤을 뜷고 봉분 위로 솟구친다

부활이다! 창자는 살아 있다! 뱀처럼 살아 있다!

피어라 돼지!

날아라 돼지!

Une fois enterrés, les cochons se déchirent. D'une certaine façon, c'est le poème qui les fait revivre en inventant une scène de résurrection qui n'a pas lieu dans la réalité. Toutefois, cette idée de résurrection n'a pas de caractère religieux chez Kim Hyesoon, qui mêle ou fait cohabiter sans hiérarchie bouddhisme, christianisme ou chamanisme. Le déchirement corporel apporte un mouvement chaotique, qui ouvre à un nouvel espace poétique.

À partir de fragments, de bribes de souvenirs, d'images dispersées, Kim Hyesoon crée de nouveaux corps qui ressemblent parfois à celui du monstre fabriqué par le docteur Frankenstein. Comme de nombreux films d'horreur et fantastiques le montrent déjà, le corps du monstre est en état de pourriture, ses fissures et ses blessures se laissent voir. Il n'est bien sûr pas agréable de faire face à son visage, toutefois il faudra accepter que ce soit une invention de la société et que nous y sommes impliqués. Chez Bong Joon-ho, les monstres ne sont jamais extra-terrestres - mais pourquoi devraient-ils l'être -, comme dans The Host / 괴물 (2006), où naît un mutant après que l'armée américaine a déversé des produits toxiques dans la rivière Han à Séoul, ou bien dans Okja, où le destin tragique des super-cochons est tout sauf l'effet du hasard. Mais, dans la poésie de Kim Hyesoon, si les corps déchirés sont présents en abondance, l'acte de coudre les morceaux de chair ou de tissu apparait comme un geste d'amour, comparable à celui de la plasticienne Louise Bourgeois qui invente la figure de la femme-araignée. Dans le geste de la recomposition s'observe une volonté de soigner le corps malade et de donner corps à ceux qui ne l'ont pas. De cette manière, l'expérience poétique s'opère dans le processus de décomposition. Aussi les deux derniers vers du poème nous invitent-ils à réfléchir sur l'avènement du corps du cochon :

Joie dans le monde, le cochon est venu

Que la terre accueille son roi! (p.113)

기쁘다 돼지 오셨네

만백성 맞으라! 


\section{BIBLIOGRAPHIE}

BONG Joon-ho, Okja, 2017.

DELEUZE Gilles, Francis Bacon. Logique de la sensation, Paris, Seuil, 2002.

KIM Hyesoon, Dentifricetristesse crèmemiroir, KOO Moduk et MURCIA Claude (trad.), Belval, Circé, 2016.

La Bible de Jérusalem, tr. fr. sous la dir. de l'École biblique de Jérusalem, Desclée de Brouwer, Paris, 2000.

\section{NOTES}

1. Kim Hyesoon a publié plus d'une douzaine de recueils de poèmes et nombre d'essais. Elle a reçu les prix littéraires les plus prestigieux de son pays et le prix international de poésie Griffin (Canada) en 2019 pour Autobiographie de la mort / 죽음의 자서전, KOO Moduk et Claude MURCIA (trad.), Belval, Circé, 2018. Plusieurs de ses ouvrages sont traduits en diverses langues.

2. Traduction française de référence pour tous les passages cités : KIM Hyesoon, « Ça va, je suis un cochon!» / 돼지라서 괜 찮아, in Dentifricetristesse crèmemiroir / 슬픔치약 거울크림, Koo Moduk et MURCIA Claude (trad.), Belval, Circé, 2016.

3. La Bible de Jérusalem, traduction française sous la direction de l'École biblique de Jérusalem, Paris, Desclée de Brouwer, 2000, p. 20.

4. DELEUZE Gilles, Francis Bacon. Logique de la sensation, Paris, Seuil, 2002, p. 30.

5. Ibid., p. 29.

6. Ibid., p. 29

7. Ibid., p. 30.

8. Dans l'annexe de Dentifricetristesse crèmemiroir, op. cit., p. 116.

\section{AUTEUR}

\section{MODUK KOO}

KOO Moduk est docteure en études cinématographiques de l'Université Paris-Diderot Paris-VII et maître de langue à l'Institut national des langues et civilisations orientales où elle enseigne actuellement la langue, la littérature et le cinéma coréens. Elle est également chargée de cours de coréen à l'École normale supérieure d'Ulm. Ses recherches en cours portent sur le temps et le corps dans la littérature et le cinéma coréens contemporains, tout particulièrement sur les questions de la répétition, de l'épuisement, de la dépression et de la mort. Parallèlement à ses activités de recherche et d'enseignement, elle travaille comme traductrice littéraire. Elle a traduit plusieurs auteurs coréens, parmi lesquels les poètes contemporains les plus importants Kim Hyesoon - Dentifricetristesse crèmemiroir et Autobiographie de la mort - et Park Sangsoon - Sale existence. 
3. Corps réaproppriés 


\title{
Une littérature humorale. Une goutte d'encre et Un lit de malade six pieds de long de Masaoka Shiki
}

\author{
Emmanuel Lozerand
}

1 Depuis sa création en 1948, l'Organisation mondiale de la santé (OMS) définit cette dernière comme « un état de complet bien-être physique, mental et social, [qui] ne consiste pas seulement en une absence de maladie ou d'infirmité $»^{1}$.

2 À cette définition, problématique à de nombreux égards, on peut préférer celle de René Leriche en 1936 : «La santé, c'est la vie dans le silence des organes ${ }^{2}$ ». Quand les organes ne sont plus silencieux en effet, une certaine prise de conscience du corps se produit, par la douleur. Natsume Sōseki 夏目漱石 ${ }^{3}$ l'a admirablement exprimé dans [Choses dont je me souviens]/思い出すことなど en 1910, après qu'il a failli mourir d'une hémorragie gastrique

Ce qui m'a étonné quand même, ce sont les transformations de mon corps ${ }^{4}$.

ただ驚ろかれたのは身体の变化である。

3 Il découvre ses bras, qu'il n'aurait jamais imaginé ne pas pouvoir bouger à sa guise, ses os, dont il n'avait jamais soupçonné la dureté, une réduction extrême de son contact avec le monde. Il résume tout cela dans ce haïku :

\section{Froid du matin Mes os vivants Point ne bouge ${ }^{5}$ \\ 朝寒や生きたる骨を動かさず}

On trouve une dimension comparable chez Masaoka Shiki 正岡子規, né en 1867, mort en 1902, le grand réformateur du haïku, qui souffrait de tuberculose osseuse. Dans les deux dernières années de sa vie, alors que son état s'est considérablement aggravé, il publie en effet quotidiennement dans le journal Nihon des textes très particuliers : [Une goutte d'encre $]^{6}$ / 墨汁一滴, et Un lit de malade six pieds de long / / 病牀六尺. Bien que ces textes parlent de beaucoup d'autres choses que de Shiki lui-même et de sa maladie, ils constituent de précieux documents sur un corps singulier8. 
5 Mais la santé, on peut aussi la penser tout autrement, avec Georges Canguilhem par exemple, qui la définit comme un pouvoir d'« instituer des normes nouvelles dans des situations nouvelles ${ }^{9} »$ :

Je me porte bien, dans la mesure où je me sens capable de porter la responsabilité de mes actes, de porter des choses à l'existence et de créer entre les choses des rapports qui ne leur viendraient pas sans moi, mais qui ne seraient pas ce qu'ils sont sans elles ${ }^{10}$.

6 Et c'est ce qui est extrêmement intéressant chez Shiki : sa capacité à inventer de la vie, à partir de son état de maladie; sa santé dans, ou avec, la maladie (et non pas " malgré », ni « grâce à », la maladie).

7 On essaiera ici de montrer comment la littérature de Shiki sourd directement de son corps - ce qui amène à proposer l'expression "littérature humorale »-, mais aussi comment elle s'invente à partir de là, ne se contentant pas d'être une simple émanation brute de ce corps.

8 On considérera d'abord deux thématiques particulières, celles de l'appétit et de la souffrance; puis on tentera de saisir plus radicalement encore ce lien entre corps et écriture, à partir de l'étude de variations qui sont autant celles du corps que de l'écriture.

\section{Écrire l'appétit, écrire la souffrance}

9 Dans un journal intime, publié après sa mort, [Notes éparses d'un homme couché] / 仰 臥漫録, Shiki a dressé un bilan terrible de son état de santé en 1901: une description précise de ses plaies, de l'épreuve des changements de bandage, de sa difficulté à ouvrir et à fermer son anus pour déféquer, du pus qui coule de ses gencives ${ }^{11}$.

10 Il n'est pas aussi cru dans les textes qu'il donne au journal Nihon. Ceux-ci disent néanmoins plusieurs dimensions de sa vie corporelle, comme sa perception du temps et de l'espace ${ }^{12}$. On se concentrera pour l'heure sur son rapport à la nourriture et à la douleur.

11 L'appétit de Masaoka Shiki était légendaire. Dans un article intitulé «Better than sex? Masaoka Shiki's Foodie Haiku», Keith Vincent rappelle quelques faits impressionnants ${ }^{13}$, qu'il s'agisse du nombre de calories quotidiennes consommées par le poète (3800 en moyenne) ou du budget familial consacré à la nourriture ( $65 \%$, dont une bonne part pour lui seul).

12 La nourriture occupe une place importante dans les textes de l'écrivain. Le 15 mai 1902 par exemple, après avoir cru mourir, Shiki connaît un répit soudain :

Tout réjoui de me souvenir que c'était aujourd'hui la fête à Negishi, je changeai complètement d'état d'âme, me régalai de caillé de soja et bus une coupe de saké pour célébrer l'événement (p. 34).

今日はあたかも根岸の祭礼日なりと思ひ出したるを幸に、朝の景色に打つてか へて、豆腐の御馳走に祝の盃を挙げた [...]。(p. 20-21)

13 Mais au-delà du témoignage, de la simple mention d'un acte, celui-ci donne naissance à des poèmes. En effet, comme c'est la fête du sanctuaire de son quartier, l'après-midi Shiki garde tout son appétit et transcrit cette expérience en haïku :

Il me manque des dents Dures sont les pousses de bambou Attention à la seiche (p. 35)

歯が抜けて简堅く烏賊こはし (p. 21) 

Vincent a montré de manière très convaincante comment « en traduisant en mots le plaisir sensuel de la nourriture, [Shiki] parvenait à apprécier et à savourer plus pleinement ce qu'il mangeait $\aleph^{14}$.

Mais le haïku n'est pas le seul mode de déploiement de la nourriture en écriture. Une autre possibilité, c'est l'analyse. Shiki réfléchit ainsi, à l'occasion, aux différences de goût entre hommes et femmes, aux particularités des poires japonaises, à la manière dont on cuit le riz, ou à une possible diffusion du saké en Occident (p. 111, 137, 163, 171, 177 ; p. 94-95, 120, 143, 151, 155). organisé pour lui un "festin de grande cuisine dans le style de la cérémonie du thé » (p. 241 ; p. 44). Shiki en présente le menu détaillé, qui commence ainsi :

La soupe est un miso de Mikawa filtré, aux chrysanthèmes sauvages. (p. 241)

味噌汁は三州味噌の煮剆、実は嫁菜、二椀代ふ。(p. 44)

Mais ce même menu est aussi l'occasion d'un récit humoristique. Comme chez 清少納

言 Sei Shōnagon, l'art de la liste peut virer à l'art de l'anecdote :

Le plat vinaigré est une carpe au vinaigre doux. Il paraît que c'est un assaisonnement secret. Je mange tout, sauf le raifort râpé, mais, prenant conscience de la règle qui veut que dans ce genre de cuisine on aille jusqu'au bout des plats sans rien laisser, je commence soudain à hésiter et je mélange le raifort dans la soupe pour mieux l'absorber. Éclat de rire général. (p. 241)

鱠は鯉の甘酢、この酢の加減卮授なりと。余は皆喰ひて摺山菱ばかり残し置き

しが茶の料理は喰ひ尽して一物を余さぬものとの掟に心づきて俄に当惑し山菱

を味増汁の中にかきまぜて飲む。大笑ひとなる。(p.44)

Cet épisode se clôt par une remarque

Comme c'est mon premier festin dans le style de la cérémonie du thé, ma vie va être prolongée de soixante-dix jours et, pour m'en souvenir, je le consigne par écrit (p. 242).

余は始めての会席料理なれば七十五日の長生すべしとて心覚のため書きつけ置

$<$ 。(p. 45)

Dès les premières lignes d'Un lit de malade six pieds de long, la douleur est posée comme une réalité incontournable :

Dans les pires moments, je suis assailli de douleurs extrêmes, et parfois je ne peux plus du tout bouger, ne serait-ce que d'un pouce. Douleurs, tourments, hurlements, analgésiques [...]. (p. 19)

甚だしい時は極端の苦痛に苦しめられて五分も一寸も体の動けない事がある。

苦痛、煩悶、号泣、麻痺剂 (後略) (p. 7)

21 Mais il ne faut pas se méprendre. Si la douleur est parfois incontrôlable - elle prend alors la forme d'un hurlement -, elle est aussi objet d'accommodements. C'est le cas par exemple dans la livraison 5 du 10 mai 1902, qui prend la forme d'une page de journal relatant une journée passée avec des amis, et qui est ponctuée de notations précises:

La nuit dernière j'ai pu dormir un peu, et les tourments qui m'assaillaient depuis le matin de la veille ont un peu diminué d'intensité. Deux verres de lait.

À $9 \mathrm{~h}$, analgésiques. 
[...]

On change mes bandages. Pas de douleurs aiguës.

[...]

Sur le coup de $11 \mathrm{~h} 30$ [du soir], je reprends des analgésiques. (p. 26-27)

昨夜少しく睡眠を得て昨朝来の煩悶やや度を減ず、牛乳二杯を飲む。

九時麻痺剤を服す。

[中略]

緛帯取替をなす。疼痛なし。

[中略]

十一時半また麻疩剂を服す。(p. 13-15)

La page de journal s'achève ainsi :

Hekigotō et sa femme s'en retournent. Il est alors minuit passé de quinze minutes. Ces derniers temps, mon esprit est enflammé et la douleur ne me quitte pas. Les réveils sont particulièrement difficiles. Comme je crains le réveil, j'ai peur de m'endormir, et j'ai donc peur de la longueur de la nuit. Si Hekigotō et les autres repartent tard, ils me rendent la nuit d'autant plus courte [yoru o mijikaku shite kureru nari]. (p. 27)

碧梧桐夫婦帚る。時に十二時を過る事十五分。

余この頃精神激昂苦悶やまず。睡覚めたる時殊に甚だし。㝝起を恐るるより従 つて睡眠を恐机従つて夜間の長きを恐る。珵梧桐らの帰る事遅きは余のために 夜を短くしてくれるなり。(p.15)

On comprend ainsi qu'une moindre douleur a pu lui permettre de passer du bon temps avec ses amis, mais aussi, inversement, que la présence de ses amis a diminué la douleur. Insérer une page de journal dans sa chronique quotidienne est ici à la fois notation, analyse et hommage.

La dimension d'observation de la douleur apparait en d'autres occasions, par exemple le 18 mai 1902 :

Quand j'étais en convalescence à Suma [été 1895], j'avais recopié dans une lettre ce poème de je ne sais qui :

Peines encore Et toujours plus vives Accumulez-vous!

De ce corps limité Je vais tester les forces. (p. 33)

余が病気保養のために須磨に居る時、「この上になほ憂き事の積机かし限りあ

る身の力ためさん」といふ誰やらの歌を手紙などに書いて (p.19-20)

Shiki commente :

J'étais ainsi parvenu, seul, à accepter la situation, mais, quand j'y repense aujourd'hui, je comprends maintenant que ce n'était en vérité que le tout début de la maladie : les douleurs qui se sont emparées de moi depuis l'an passé défient toute expression et je n'aurais pu les imaginer. (p. 33)

独りあきらめて居つたのは善かつたが、今日から見るとそ机は誠に病気の入口

に過ぎないので、昨年来の苦しみは言語道断殆ど予想の外であつた。(p. 20)

À partir de ce point de repère il tente, dans les lignes qui suivent, d'expliquer le cheminement de la douleur en ce mois de mai 1902.

En une autre occasion, la démarche d'objectivation passe par la comparaison, voire une forme de théorisation. Le 28 mai, à l'occasion de la visite d'un descendant d'un certain Bujian 無事庵, récemment décédé et qui souffrait du même mal que lui, il explique :

À mesure que ma maladie progresse de degré en degré, je ressens des douleurs indicibles. Nul ne peut les comprendre s'il n'est pas mort lui-même déjà une fois, ou s'il ne s'est trouvé à l'article de la mort. En outre, il semble que ces douleurs soient identiques pour tout le monde. (p. 48)

病勢が段々進むに従つて何とも言はれぬ苦痛を感ずる。それは一度死んだ人か 
もしくは死際にある人でなければわからぬ。しかもこの苦痛は誰も同じことと 見えて (p. 35)

\section{Il poursuit :}

En voici quelques exemples : ne pas supporter que la personne qui veille le malade s'éloigne du lit un instant ; se mettre en colère si elle ne commence pas à exécuter une instruction avant même qu'on ait fini de la donner ; respirer avec une violente douleur quand des personnes de taille élevée se tiennent devant le lit ; éprouver des sentiments extrêmes, de sympathie comme d'antipathie, envers les gens que l'on rencontre; ressentir ou non du plaisir avec intensité, en fonction du moment; trouver qu'un matelas dur fait mal et, à l'inverse, se sentir enseveli dans un matelas mou ; trop manger quand on a de l'appétit ; se mettre en colère quand quelqu'un vous observe et vous dit que, finalement, vous n'êtes pas si maigre, sortir alors ses jambes effilées comme des pinces à feu et les lui montrer en disant : «Et ça ? "Sur tous ces points il n'y avait aucune différence entre Bujian et moi [...]. (p. 48)

一、二の例を挙ぐれば、寸時も看病人を病床より離れしめぬ事、凡て何か命じ たる時にはその詞のいまだ絶えざる中に、その命令を実行せねば腹の立つ事、 目の前に大きな人など居れば非常に呼吸の苦痛を感ずる事、人と面会するにも 人によりて好きと嫌ひとの甚だしくある事、時によりて愉快を感ずると感ぜざ るとの甚だしくある事、敷蒲団堅ければ骨ざはり痛く、敷蒲団やはらかけ机ば 身が蒲団の中に埋もれてかへつて苦しき事、食ひたき時は過度に食する事、人 が顔を見て存外に瘦せずに居るなどと言はれるのに腹が立ちて火箸の如く細り たる足を出してこれでもかと言ふて見せる事、凡そこれらの事は何一つ無事庵 と余と異なる事のない[後略]。(p. 36)

On trouvera aussi dans ces textes des analyses précises du sentiment d'oppression que ressent quelqu'un qui souffre des poumons, ou de la difficulté à supporter la chaleur (p. 98-99, p. 82-84), ou les moustiques (p.165-166). Mais un aspect essentiel de la douleur, c'est celle de son éventuel soulagement. Shiki, ici aussi, se transforme en une sorte d'expert. Il raconte les expériences qui ont pu, ou non, le soulager, et classe par exemple ses lectures selon ce critère, ce qui l'amène à faire l'éloge des histoires de chasse (p. 21-22 ; p. 8-10). Il explique l'efficacité de l'association morphine plus peinture (p. 158,$160 ;$ p. 140, 142).

Il pousse son analyse encore plus loin à propos du rôle que peuvent jouer amis et visiteurs, mais surtout à propos du travail des femmes de la maison, dont il prend conscience depuis son lit de malade. Il réfléchit ainsi aussi bien à ce que doit être un soin qu'aux conditions nécessaires pour qu'il puisse être apporté, ce qui l'amène par exemple à poser la question de l'éducation des femmes (p. 123-131; p. 106-115).

31 En d'autres occasions, plus rares, il développe une véritable science des moments de la journée :

L'été dernier, tourmenté jour après jour par la chaleur, après m'être débattu toute la journée sur mon lit de malade, quand je voyais la lumière du soleil projeter obliquement l'ombre des arbres sur le sol, un vent léger faire osciller doucement le calice blanc des belles du soir, je ressentais une joie indicible et commençais à revenir à la vie des hommes (p. 164).

去年の夏、毎日々々暑さに苦しめられて終日病床にもがいた末、日脚が斜めに 樹の影を押して、微風が夕顔の白き花を吹き摇かすのを見ると何ともいはれぬ 善い心持になつて始めて人間に生き返るのであつた。(p. 145)

Ou fait appel aux solutions de l'imaginaire :

La nuit dernière, en rêve, je suis arrivé en un lieu où folâtraient un grand nombre d'animaux. L'un d'entre eux, comme si l'agonie était toute proche, se tordait par terre en tous sens, en proie à de terribles douleurs. C'est alors qu'apparut un gentil petit lapin, il s'approcha de l'animal souffrant et lui tendit la patte. Celui-ci la saisit 
aussitôt entre les siennes, il la porta à sa gueule et se mit à la sucer avec ravissement, aussitôt ses souffrances passées cessèrent et il mourut comme s'il avait sombré dans le plus agréable des sommeils. D'autres animaux étaient eux aussi en proie aux affres de l'agonie, et le lapin recommença son manège. Ils moururent à leur tour comme s'ils sombraient dans le plus agréable des sommeils. (p. 235)

昨夜の夢に動物ばかり沢山遊んで居る処に来た。その動物の中にもう死期が近 づいたかころげまはつて煩悶して居る奴がある。すると一匹の親切な兔があつ てその煩悶して居る動物の辺に往て自分の手を出した。かの動物は直に兔の手 を自分の両手で持つて自分の口にあて嬉しさうにそれを吸ふかと思子と今まで の煩悶はやんで甚だ愉快げに眠るやうに死んでしまふた。またほかの動物が死 に狂ひに狂尔て居ると例の忽は前と同じ事をする、その動物もまた愉快さうに 眠るやうに死んでしまふ。(p.99-100) mieux conclure :

Tous les plaisirs, toutes les libertés m'ont été arrachés et seuls demeurent, fragiles, un unique plaisir et une unique liberté : le plaisir de boire et de manger, la liberté d'écrire. (p. 240)

総ての楽、総ての自由は尽く余の身より奪ひ去られて僅かに残る一つの楽と一 つの自由、即ち飲食の楽と執筆の自由なり。(p. 61-62)

Et d'ajouter :

Mais à présent les douleurs locales sont violentes et la liberté d'écrire m'a presque entièrement quitté ; estomac et intestins ont fini par s'affaiblir et je me vois privé de la plus grande part de mon plaisir de manger. (p. 240)

しかも今や局部の疼痛劇しくして執筆の自由は殆ど奪はれ、腸胃漸く衰弱して 飲食の楽またその過半を奪はれぬ。(p.62)

Il noue donc ici à la nourriture et à la souffrance, déjà abordées, l'activité d'écriture envisagée sous son angle le plus concret. Mais à dire vrai, il ne dit pas tout à fait la vérité. Une particularité d'[Une goutte d'encre] et d'Un lit de malade six pieds de long, c'est d'être des textes écrits quand l'écriture n'est plus possible. Shiki s'en explique dès la $7^{\mathrm{e}}$ livraison d'[Une goutte d'encre], le 24 janvier 1901 :

En dehors des douleurs locales qui me font souffrir depuis des années, les douleurs du flanc gauche sont devenues plus fortes l'année dernière, si bien qu'à présent je ne peux plus prendre le pinceau pour écrire quoi que ce soit [mono kaku atawazaru物 書く能はざる]. À force de garder toujours sur l'estomac ce que je pense, j'en éprouve même des douleurs morales. Cela ne vaut donc plus la peine de vivre. 
Comment me distraire de l'ennui où me laisse mon lit de malade? Tandis que je m'enfonçais dans ces sombres pensées, une idée m'est soudain venue et, en fin de compte, je me suis dit que j'allais écrire Une goutte d'encre. Il n'y aura chaque fois qu'une vingtaine de lignes, dans le meilleur des cas, et parfois dix seulement, ou cinq, ou deux, ou une. Guettant les répits que m'accorde la maladie, je noterai en vrac tout ce qui surgit en moi, sans faire le tri, et ce sera toujours mieux que de ne rien écrire. (p. 225)

年頃苦しみつる局部の痛の外に左横腹の痛去年より強くなりて今ははや筆取り て物書く能はざるほどになりしかば思ふ事腹にたまりて心さへ苦しくなりぬ。 かくては生けるかひもなし。はた如何にして病の牀のつれづれを慰めてんや。 思ひくし居るほどにふと考へ得たるところありて終に墨汁一商といふものを書 かましと思ひたちぬ。こは長きも二十行を限とし短きは十行五行あるは一行二 行もあるべし。病の間をうかがひてその時肮に浮びたる事何にてもあれ書きち らさんには全く書かざるには勝りなんかとなり。(p.10-11)

Shiki ne peut sans doute pas « écrire ", au sens où il ne peut pas « écrire dans les formes constituées " (mono kaku). Mais il a besoin de trouver un canal - comme une sorte de drain - pour ex-primer, au sens étymologique, ce qu'il a en lui, et cette activité en ellemême soulage sa douleur. Mais l'erreur serait de penser qu'il s'agit là d'une simple expulsion. Le paradoxe fascinant de shiki, c'est que, tout en sortant directement du corps, son cri n'est pas un vomissement, il prend forme, et forme littéraire :

Quand la fièvre augmente, le corps souffre. Au début je gémis, ensuite je hurle, et finalement je déclame des poèmes chinois et japonais, je chante à tue-tête, romances d'amour, chansonnettes d'Edo, récitatifs de nô, j'imite des voix; par fragments et par morceaux, tous se relaient ou se poursuivent, avec des changements brusques que je ne peux prévoir. Un soir, comme d'habitude, une bouffée de fièvre, et un vers deux vers viennent se superposer, comme d'un poème sino-japonais ou de stances bouddhiques. Une fois qu'ils sont sortis de ma bouche, je n'ai plus rien à écrire. J'y ajoute péniblement un ou deux autres vers, et voilà un quatrain. (p. 249)

熱高く身苦し。初めは呻吟、中頃は叫喚、終りは吟声となり放歌となり都々逸 端唄謡曲仮声片々ス寸々又継又続修忽变化自ら測る能はず。一夜例の如く発熱 詩の如く偈の如き㙯語一句二句重畳して来る、一たびロを出づれば復記する所 なし。中につきて僅かに記する所の一、二句を取り補ふて四句となす

(p. 141)

41 Au début de l'année 1901, quand il a eu l'idée de ces « gouttes d'encre », il s'est inquiété de voir que ses premiers textes n'étaient pas tout de suite publiés dans le quotidien Nihon. Dans une lettre du 15 janvier, à son ami 寒川粗忽 Samukawa Sokotsu (1875-1954), il explique :

Je suppose qu'il n'y avait donc pas de place pour Une goutte d'encre. Mais peu importe l'endroit! Les marges m'iraient tout aussi bien. Je me demande même si ce ne serait pas mieux. Ce serait chic, une littérature dans les marges, qui emprunterait chaque jour deux marges du journal ${ }^{16}$.

ソレデ墨汁一滴习出ス余地ガナカツタノデアラウ。併シ僕ハ処习擇バヌ。欄外 


\section{デモヨイ。寧ロ闌外ガ善イカト思フ。欄外ヨ毎日二闌. 借リテ欄外文学ナドモ}

シャレテ居ルヨ。

42 Cette "littérature " très particulière, que Shiki appelle en plaisantant à moitié « littérature dans les marges ", «hors rubrique»/欄外文学, a fort logiquement des caractéristiques formelles très particulières.

La première, fondamentale, mais si évidente qu'on pourrait l'oublier, tient à sa simple existence. Le rythme de la chronique de Shiki est quotidien. Quand aucune livraison ne paraît, ce qui arrive de temps à autre, c'est - ce peut être - plus qu'un hasard : le signe d'une évolution inquiétante de son mal.

La deuxième dimension, elle aussi fondamentale, mais si simple que l'on pourrait ne pas y prendre garde, c'est la longueur. Shiki lui-même fixait, on l'a vu, un éventail d'une à vingt lignes par livraison. C'est parfois un peu plus, d'autant que certains textes se prolongent sur deux, trois ou quatre jours. Il n'empêche que la longueur en ellemême semble une trace directe de l'état corporel de Shiki, comme sur une courbe sismographique.

Mais ce qui est encore plus frappant, et dont nous voudrions tenter une brève esquisse, c'est la variation de ton des livraisons, de jour en jour, sur une séquence de temps donnée, et plus fondamentalement sur l'ensemble des deux œuvres. Elle apparaît de manière paroxystique dans les ultimes séquences d'Un lit de malade six pieds de long.

Après les très violentes douleurs de mai et juin, déjà évoquées, Shiki avait livré pendant l'été 1902 des textes dont la tonalité était - dans l'ensemble - plutôt apaisée, et où il parlait de choses très variées. Depuis le 26 août il publiait beaucoup de commentaires de haïku, et le 10 septembre il avait parlé du jeu de go (p. 208 ; p. 181-182).

Le 11 septembre pourtant, il commence ainsi :

En l'espace d'une journée, l'extrémité de mes jambes amaigries s'est soudain mise à enfler et, au spectacle de cette enflure soufflée, on dirait que l'on a fixé un flacon de saké au bout d'un tison. À mes questions, le médecin répond que c'est un phénomène courant chez les malades, lié à une mauvaise circulation du sang. En tout cas, ce n'est pas quelque chose de très agréable. (p. 209)

一日のうちに我瘦足の先俄かに腫れ上りてブクブクとふくらみたるそのさま火 箸のさきに徳利をつけたるが如し。医者に問へば病人にはありがちの現象にて 血の通ひの悪きなりといふ。とにかくに心持よきものには非ず。(p. 182)

La livraison toutefois se poursuit de manière plus apaisée par la transcription de bavardages avec des amis.

Le 12 septembre, les lecteurs découvrent ces lignes :

En Chine et en Corée, il paraît que l'on pratique encore la torture, or moi, depuis hier, jour et nuit, je subis une torture de tout le corps, sans interruption. C'est véritablement une douleur indicible. (p. 210) 支那や朝鮮では今でも拷問をするさうだが、自分はきのふ以来昼夜の別なく、 五体すきなしといふ拷問を受けた。誠に話にならぬ苦しさである。(p. 183)

50 Le 13 septembre, celles-ci :

La douleur humaine, on peut l'imaginer dans ses degrés les plus extrêmes, mais jamais je n'aurais pu imaginer qu'elle me survienne à moi, en mon corps, cette douleur que j'avais imaginée dans ses degrés les plus extrêmes. (p. 211) 人間の苦痛はよほど極度へまで想像せられるが、しかしそんなに極度にまで想 像したやうな苦痛が自分のこの身の上に来るとはちよつと想像せられぬ事であ る。(p. 183)

51 Le 14, celles-là : 
J'ai des pieds, des pieds comme ceux des Gardiens géants des portes. Des pieds, on dirait ceux d'un autre. Des pieds, comme d'énormes rochers. Si l'on effleure à peine leur extrémité du bout des doigts, le ciel et la terre tremblent, les arbres et les plantes hurlent; Nügua ${ }^{17}$ ne m'a pas encore amputé de mes pieds pour les remplacer par des pierres multicolores. (p. 212)

足あり、仁王の足の如し。足あり、他人の足の如し。足あり、大磐石の如し。 僅かに指頭を以てこの脚頭に触るれば天地震動、草木号叫、女螖氏いまだこの 足を断じ去つて、五色の石を作らず。(p. 183)

Le 15 septembre, Shiki parle de la " puanteur » (shūki 臭気) : celle reniflée par 松尾芭 蕉 Matsuo Bashō (1654-94) dans l'écurie près du cheval qui pisse, celle du tigre du zoo d'Ueno (p. $213 ;$ p. 184).

Le 16 septembre : pas de livraison.

Le 17 septembre est publiée la lettre d'un ami qui s'inquiète pour lui à cause des livraisons des jours précédents (auxquelles il fait directement écho) :

Lettre de l'Ermite des fragrances, alias Lâche-pets [Hōhi] :

«Je vous présente mes salutations. Ces derniers temps, Un lit de malade six pieds de long ne consiste qu'en de très brefs fragments et, comme j'y ressens de la douleur, je m'enquiers de votre santé. Bodhidharma lui aussi, à partir de la Fête des morts, s'est retiré dans sa grotte et, la corde nouée autour de la tête, il se livre à l'ascèse sous la cascade et ne donne plus de nouvelles.

D'un tuberculeux [D'un malade du haiku] Haibyô no

Serait-ce le rêve? Serait-ce le rêve? Yume miru naran

Le coucou Le coucou Hototogisu

À la torture À la torture Gômon nado ni

Qui l'a soumis? [Coucou, coucou!]» Dare ga kaketa ka » (p. 214)

芳非山人より来書

拝啓昨今御病床六尺の記二、三寸に過ず頗る不穏に存候間御見舞申上候達磨儀

も盆頃より引籠縄鉢巻にて筧の滝に荒行中御無音致候

俳病の夢みるならんほと〉ぎす拷問などに誰がかけたか (p.184)

Puis plus rien.

Et le 21 septembre, le journal Nihon publie en une le fac-similé des trois haïkus écrits par Shiki de sa main, le soir du 18, quelques heures avant de mourir.

Cette série de livraisons est, bien sûr, bouleversante. On sent, littéralement, un homme entrer dans ses derniers jours et mourir. Rarement, peut-être, des mots ont-ils dit avec une telle intensité corporelle l'approche de la mort. D'un autre côté, il n'y a pas là une plainte brute, un simple « j'ai mal, je vais mourir », mais bien au contraire, y compris dans ces moments les plus graves, une illustration de la «santé » selon Canguilhem : la capacité à "créer entre les choses des rapports", ce que le philosophe appelait le pouvoir « normatif » de la vie.

Reprenons une à une les différentes séquences.

Le 11 septembre, c'est le comique de la description (par ailleurs terrible) des jambes du malade : « on dirait que l'on a fixé un flacon de saké au bout d'un tison ».

Le 12 septembre, une mise en parallèle de la grande histoire, celle de la torture, et de ce qu'il ressent.

1 Le 13 septembre, une réflexion quasi abstraite sur les «degrés» de la douleur et le pouvoir que l'on a de les imaginer.

Le 14, une description des "pieds ", à la fois suggestive, terrifiante, mais non sans distance. 
63 Le 15, une apparente digression sur la "puanteur ", mais dont il est vraisemblable qu'elle parle pudiquement de lui, de son état, de ceux qui sont à côté de lui. On les considère souvent comme des documents exceptionnels sur leur auteur, ce qu'ils sont assurément, ou on les range dans la catégorie fourre-tout des «essais au fil du pinceau » (zuihitsu / 随筆), ce qui peut se comprendre étant donné leur caractère décousu, mais, ce faisant, on ne pousse guère la réflexion et on ne parvient pas à saisir le lien puissant et rare entre une inscription corporelle hors du commun de ces textes humoraux, qui sourdent littéralement du corps de l'écrivain, tout en agissant sur lui, et leur indiscutable littérarité, leur capacité plastique. sens de Leriche ", puisque l'on y entend hurler des organes, il est néanmoins doté d'une " autre santé ", au sens de Canguilhem, puisqu'il est capable " de créer entre les choses des rapports qui ne leur viendraient pas sans moi, mais qui ne seraient pas ce qu'ils sont sans elles ».

71 C'est sans doute ce que Shiki cherchait à exprimer dans l'incipit d'Un lit de malade six pieds de long:

[...] Chercher timidement un sentier de vie sur le chemin de la mort, et désirer avec avidité une faible paix, quelle dérision! Et pourtant, dès lors que l'on demeure en vie, il y a des choses que l'on tient absolument à dire. Je n'ai chaque jour sous les yeux que des journaux et des revues, mais il arrive souvent que la douleur 
m'empêche même de les lire ; néanmoins il me suffit d'en parcourir quelques lignes pour me fâcher, me mettre en rogne, à moins que parfois, exceptionnellement, cela ne me procure une joie inexplicable qui me permet d'oublier les souffrances de la maladie. (p. 19)

僅かに一条の活路を死路の内に求めて少しの安楽を領る果敢なさ、それでも生 きて居ればいひたい事はいひたいもので、毎日見るものは新聞雑誌に限つて居 れど、それさへ読めないで苦しんで居る時も多いが、読めば腹の立つ事、瘽に さはる事、たまには何となく嬉しくてために病苦を忘るるやうな事がないでも ない。(p. 7)

Cette attitude peut amener ses lecteurs à réfléchir à leur propre situation :

Un malade est vraiment une bête stupide. Et cela ne vaut pas que pour moi : cette stupidité est la chose la mieux partagée du monde. Ceux qui ont compris que je suis à la fois l'homme dont ils se moquent et celui qui aime rire aux éclats savent qu'un beau jour, quoi qu'il arrive, nous échangerons nos places : ils cesseront de rire de mes souffrances et c'est moi qui me rirai d'eux. Ah, ah, ah! (p. 89)

実に病人は愚なものである。これは余自身が愚なばかりでなく一般人間の通有 性である。笑ふ時の余も、笑はるる時の余も同一の人間であるといふ事を知つ たならば、余が煩悶を笑ふ所の人も、一朝地をかふ机ば皆余に笑はるるの人た るを免れないだらう。咄々大笑。(p. 74)

73 Ōe Kenzaburô 大江健三郎 (1935-) n'a-t-il pas souligné avec force combien shiki était

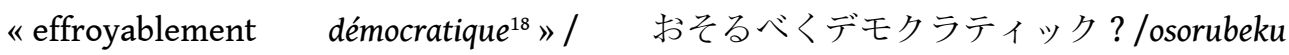
demokuratikku?

\section{BIBLIOGRAPHIE}

CANGUILHEM Georges, Le Normal et le Pathologique, Paris, PUF, 2006 [1966].

CANGUILHEM Georges, Écrits sur la médecine, Paris, Seuil, 2002.

LOZERAND Emmanuel, « Corps, maladie, écriture chez trois auteurs japonais du début du Xx siècle : Nakae Chômin, Masaoka Shiki, Natsume Sôseki », Extrême-Orient Extrême-Occident, 39:2015, p. 21-45.

LOZERAND Emmanuel, « Gouttes d'encre. Masaoka Shiki et l'écriture du corps malade », postface à MASAOKA Shiki, Un lit de malade six pieds de long, LOZERAND Emmanuel (trad.), Paris, Les Belles Lettres, 2016, p. 221-254.

LOZERAND Emmanuel, « Santé et maladie chez quelques écrivains japonais modernes (1898-1911) », in Santé et bien-être à l'épreuve de la littérature, DE JESUS CABRAL Maria et DOMINGUES DE ALMEDIA José (dir.), Limoges, Lambert-Lucas, 2017, p. 147-155.

MASAOKA Shiki, Masaoka Shiki zenshū, Tokyo, Kōdansha, vol. 19, 1975.

MASAOKA Shiki, Bokujū itteki, Tokyo, Iwanami, 1999 [1901].

MASAOKA Shiki, Gyōga manroku, Tokyo, Iwanami, 1999 [1918].

MASAOKA Shiki, Byōshō rokushaku, Tokyo, Iwanami, 2000 [1902]. 
MASAOKA Shiki, Un lit de malade six pieds de long, LOZERAND Emmanuel (trad.), Paris, Les Belles Lettres, « Collection Japon », 2016.

NATSUME Sōseki, [Choses dont je me souviens] / 思い出す事など / Omoidasu koto nado, Tokyo, Iwanami, 2013 [1910-1911].

ŌE Kenzaburô, [« Thématique de Shiki »] / 子規のテマティック / Shiki no tematikku, in [đuvres complètes de Shiki] / 子規全集 / Shiki zenshū, Tokyo, Kōdansha, vol. 11, 1975.

ŌE Kenzaburô, « La thématique de Shiki », LOZERAND Emmanuel (trad.), Critique, n 839, « Et le Japon devint moderne... ", Paris, Éditions de Minuit, avril 2017, p. 276-289.

VINCENT Keith, "Better than Sex? Masaoka Shiki's Poems on Food”, in Devouring Japan. Global Perspectives on Japanese Culinary Identity, STALKER Nancy K. (ed.), Oxford, Oxford U. P., 2018.

\section{NOTES}

1. Site officiel de l'OMS: https://www.who.int/fr/about/governance/constitution, consulté le 25/08/2021.

2. Cité par CANGUiLHEM Georges, Le Normal et le Pathologique, Paris, PUF, 2006 [1966], p. 52.

3. On trouvera d'autres développements sur la santé chez Sōseki dans LOZERAND Emmanuel, "Santé et maladie chez quelques écrivains japonais modernes (1898-1911) », in Santé et bien-être à l'épreuve de la littérature, CABRAL Maria de Jesus et DE ALMEIDA José Domingues (dir.), Limoges, Lambert-Lucas, 2017, p. 147-155.

4. NATSUME Sōseki, [Choses dont je me souviens]/思い出すことなど, Tokyo, Iwanami, 2013 (1910-1911), p. 62. Toutes les traductions sont originales.

5. Ibid., p. 65.

6. МАSAOKA Shiki, Bokujū itteki, Tokyo, Iwanami, 1999 [1901].

7. MASAOKA Shiki, Byōshō rokushaku, Tokyo, Iwanami, 2000 [1902]. Traduction française par Emmanuel Lozerand sous le titre Un lit de malade six pieds de long, Paris, Les Belles Lettres, 2016 (ce volume comporte aussi des extraits traduits de Bokujū itteki). Chaque passage cité de cette œuvre renvoie à l'édition française, puis à la traduction originale.

8. V. Emmanuel LOZERAND, "Gouttes d'encre. Masaoka Shiki et l'écriture du corps malade", postface à Masaoka Shiki, Un lit de malade six pieds de long, Paris, Les Belles Lettres, 2016, p. 221-254.

9. CANGUILHEM, Le Normal et le Pathologique, op. cit., p. 130.

10. CANGUILHEM Georges, Écrits sur la médecine, Paris, Seuil, 2002, p. 68.

11. MASAOKA Shiki, Gyôga manroku, Tokyo, Iwanami bunko, 1999 (voir Masaoka, Un lit de malade..., op. cit., p. 221-222).

12. V. LOZERAND Emmanuel, « Corps, maladie, écriture chez trois auteurs japonais du début du $x x^{e}$ siècle : Nakae Chōmin, Masaoka Shiki, Natsume Sōseki », Extrême-Orient Extrême-Occident, 39:2015, p. 21-45.

13. VINCENT Keith, "Better than Sex? Masaoka Shiki's Poems on Food", in Devouring Japan. Global Perspectives on Japanese Culinary Identity, STALKER Nancy K. (ed.), Oxford, Oxford U. P., 2018.

14. «By translating the sensuous pleasure of food into words, he was able to appreciate and savor what he ate more fully » (VINCENT, "Better than Sex?", op. cit., p. 221).

15. "Food, after all, is a lot like sex, and a lot like poetry too: never just word or thing, but something in between" (VINCENT, "Better than sex?", op. cit., p. 237).

16. МАSАОKA Shiki, Masaoka Shiki zenshū, Tokyo, Kōdansha, vol. 19, 1975, p. 604-605.

17. Ou Nüwa, personnage de la mythologie chinoise. 
18. ōE Kenzaburô, «La thématique de Shiki », LOZERAND Emmanuel (trad.), Critique, $n^{\circ} 839$, « Et le Japon devint moderne...», Paris, Éditions de Minuit, avril 2017, p. 279; ŌE Kenzaburô, [«Thématique de Shiki»]/子規のテマティック, in [CEuvres complètes de Shiki] / 子規全集, « Kaisetsu », Tokyo, Kōdansha, vol. 11, 1975.

\section{AUTEUR}

\section{EMMANUEL LOZERAND}

Emmanuel Lozerand, professeur de langue et de littérature japonaises à l'Inalco, co-directeur de la Collection Japon aux éditions Les Belles Lettres, s'intéresse au Japon « moderne » des ères Meiji et Taishô, et surtout à certains de ses grands auteurs (Fukuzawa, Ôgai, Sôseki). Il a notamment publié Littérature et génie national. Naissance de l'histoire littéraire dans le Japon de la fin du XIX ${ }^{e}$ siècle, (Les Belles Lettres, 2005), traduit Un Lit de malade six pieds de long de Masaoka Shiki (Les Belles Lettres, 2016) et dirigé le livre collectif Drôles d'individus. De la singularité individuelle dans le Restedu-Monde (Klincksieck, 2014). 


\title{
Narrating Banishment by Means of the Body: Physical Reflections of Han Dong's Cultural Revolution
}

\author{
Martina Codeluppi
}

\section{Memory and Body}

1 Any individual is defined by his personal history, as past experiences constitute the landmarks of one's evolution. Therefore, it could be said that identity is essentially shaped in memory. The cognitive skill of remembering is based on the subject's interaction with the environment, which involves the body in both an active and a passive way. Senses allow the individual to perceive the surrounding space and, simultaneously, it is only by being "a body" that one is able to occupy a part of that space, to move and to take action. As a consequence, memory can be considered as an "embodied" entity, emphasizing its interrelation with the subject's physical dimension. This paper aims to explore how the narration of memory conducted by means of the body, which is situated at multiple levels of the process of reminiscing, constitutes the quintessence of the author's self. The analysis of the novel Banished! / 扎 根 2 by Han Dong 韩东 (b. 1961) will reveal the central role of the body in the narration of a sombre phase of contemporary Chinese history, that of the Cultural Revolution (1966-1976).

2 The link between memory and body has been explored from many perspectives, among which the phenomenological is particularly relevant for this study. The different phases of the mental procedure necessary for the existence of memory have been acknowledged by Henri Bergson (1896-1941), who distinguished between "pure memory" (souvenir pur), "image-memory" (souvenir-image), and "perception" (perception). ${ }^{3}$ Later, Maurice Merleau-Ponty (1908-1961) explored the relationship between body and recollection from the perspective of phenomenology, portraying in detail the multiple roles the body implicitly plays while performing the act of perceiving. ${ }^{4}$ Edward S. Casey (b. 1939) developed his contribution based on Bergson's 
and Merleau-Ponty's postulation of the existence of different kinds of memory, ${ }^{5}$ further elaborating the difference between "body memory" and "memory of the body". ${ }^{6}$ More recently, Fuchs has investigated the connection between memory, body and mind, drawing on the domain of psychopathology. ${ }^{7}$ Indeed, the Cultural Revolution can be regarded as a traumatic experience. Berry adopted this perspective to provide insight into the literary and cinematic representation of this particular period of Chinese history, ${ }^{8}$ while Kleinman has investigated the reflection of its memory in the recollecting subject's body. ${ }^{9}$

These works provide the methodological framework for this study, which will explore the memory of the Cultural Revolution as it was carved in the author's body. First, the analysis will address the body as the means of perception of the surrounding environment, which allows the subject to mold his recollections. Next it will move to the level of the body as the object of memory, showing how the depiction of physical features conveys a concretized image of individuality. Finally, the significance of the physical dimension in everyday life during the Cultural Revolution will be examined, as it represents the embodiment of the self in a social system that in fact was founded on the annihilation of the individual. These steps will show how, in Han Dong's narration, the body represents at once the materialization of identity and the stronghold of individuality, in the context of a bewildering phase of contemporary Chinese history.

\section{Han Dong's Memory of Banishment}

Han Dong is regarded as one of the most representative authors of the so-called "late-" or "late-born generation authors" / 晚生代作家 / wanshengdai zuojia, who animated the Chinese literary scene at the end of the 20th century. The rejection of the literary tradition, as well as of the current literary system, marked this generation's creative production, which focused particularly on the individual dimension. Han Dong's voice is therefore characterized by a strong individualism and constant yearning for truth, which he expresses through fiction as well as through poetry. The representation of everyday life, carried out through a sapient use of language, constitutes the lifeblood of his poetics, reproducing the essentiality of the individual's experience by means of a “zero-degree narration" / 零度写作 / lingdu xiezuo. ${ }^{10}$

Set at the time of the Cultural Revolution, Banished! tells the story of the Tao family / 陶 who, after being banished to the countryside, engage in an everyday struggle in order to connect with their new social context, and try by all means to "strike root" in the village of Sanyu 三余. The author's personal memories are merged with fictional elaboration, since the novel echoes his own experience of banishment. At the age of eight, Han Dong followed his family to the countryside of Subei / 苏北, where they were sent to be re-educated through labor. The depiction of the Cultural Revolution in Banished! is, therefore, a recollection of his memories as a child, and although the novel employs a third-person narrator and the point of view shifts from one character to another, the child Han Dong's eyes remain the primary source of the author's expression. In the act of remembering the daily challenges he had to face during the years of banishment, the body plays a central role, since any child experiences the world first and foremost by means of his body and his body memory. ${ }^{11}$ So the physical dimension is crucial in both the building of the subject's recollections and in their subsequent narration through the literary text. 
Despite the painful historical period in which it is set, Han Dong's novel is a rather nontraumatic story of fiction and recollection. Several studies, such as those by $\mathrm{Wu}$ Congcong and Li Renjie, ${ }^{12} \mathrm{Gu}$ Haiyang, ${ }^{13}$ and Deng $\mathrm{Li},{ }^{14}$ have investigated the relationship between the novel and the author's memory, exploring the intertwining of Han Dong's real experience and his fictional re-elaboration. The novel has also been the object of several studies that focus on the features of the author's language in terms of stylistic nuances ${ }^{15}$ or ideological influences. ${ }^{16}$ The naiveté of the child Han Dong is reflected in the lightness of the tones employed, and a subjacent wit often seeps from the narrated scenes. This peculiarity has been investigated by Gong Ailing, who focuses on the optimistic view that Banished! conveys despite the turmoil characterizing the historical background, ${ }^{17}$ and by Hunt, who analyzes Han Dong's humor in a comparative perspective. ${ }^{18}$ Notwithstanding their interest in the author's wish for individualism, these studies did not address the issue of subjectivity from the most down-to-earth approach, that of the body. The following analysis will investigate the different roles the body plays in reflecting the subject's recollection, showing the importance of the physical dimension in representing the "self" at different stages of the process of reminiscing.

\section{The Body as the Perceiving Subject}

7 Perception is the first phase of the interaction between the subject and the world. Thanks to one's senses, an individual is able to feel the surrounding environment in all of its manifestations. Merleau-Ponty opened his work Phénoménologie de la perception by pointing out the problematic definition of "sensation", which is at the basis of perception. ${ }^{19} \mathrm{~A}$ sensation concerns essentially the qualities of an object, what is there to be sensed, while the perception of those qualities entails an interaction with the perceiving subject, and is therefore related to the individual sphere. The concept of a "pure sensation" (pur sentir) which theoretically exists before being actually perceived, is not attainable in the real world. ${ }^{20}$ Both sensation and perception are influenced by memory, the latter more than the former. This is due to the "association of ideas" the subject makes, bringing back his past experiences when facing a new one. ${ }^{21}$ In other words, memory affects the interaction between the subject and the environment at its very first stage. If perception already carries a strong individual connotation, the recollection of that perception amplifies it further. Bergson pointed out how "my perception [...] adopts my body as a center"; ${ }^{22}$ therefore it is precisely in the description of the subject's bodily perceptions that the epitome of individual memory should be found.

8 Han Dong's recollection is based on his own perception of the village of Sanyu during the Cultural Revolution, which he had experienced during his childhood. His young age at the time affects the author's recollection in two ways: first, the relationship Han Dong originally established with the context he describes in the novel was direct, unbiased, and unmediated by any previous knowledge, ${ }^{23}$ and second, a child's interaction with the world is based on sensory perception and corporal memory. As a consequence, the author's perspective on the socio-historical background of Banished! is characterized by innocence, and the body is the main channel through which he expresses his individuality. The child of the Tao family, Young Tao / 小陶 / Xiao Tao, 
can be regarded as a projection of Han Dong himself, and the descriptions offered from his point of view are often rich in physical details:

His feet had not yet developed calluses, and the wheat stubble under foot jabbed his tender soles painfully. He walked with great care, not daring to lift his legs too high. Sometimes his feet touched sludge left by the river, and its coarse grains massaged his insteps pleasantly. Hobbling slowly and gingerly along, he soon got left behind. He asked September and little Dick to walk more slowly, but they paid no attention. In the end, the bright flashlight beam moved so far ahead that he was left to feel his way through the dark paddy fields..$^{24}$

他的脚很娇濑, 脚底没有老茧, 一脚踩上粗硬的麦萑, 便会疼得钻心。因此小陶 走得十分小心，不敢把腿抬得过高。如果砅上被河水泡软的泥巴，粗徙的颗粒按 摩着脚心, 倒也非常的舒服。小陶趋利避害, 举步维艰, 因此落在了后面。他让 九月子细巴子走慢一些, 他们听而不闻。最后, 那明晃晃的手电光移到前面去

了, 只留下小陶一个人在黑暗的水田里摸索。2

When following his friends in the fields, young Tao remembers the feeling of the different surfaces he walked on, the pleasant massage of the mud after wheat stubble pierced his feet. In this respect, a further clarification is necessary: one must bear in mind that the relationship between body and memory is very articulated and memory possesses a layered structure entailing different kinds of mnemonic modes. ${ }^{26}$ In particular, it is important to distinguish between "body memory" and "memory of the body." The former is "intrinsic to the body, to its own way of remembering"; ${ }^{27}$ it manifests itself at a physical level. Instead, the latter comprehends the "manifold manners whereby we remember the body as the accusative object of our awareness", ${ }^{28}$ and it is situated in the subject's mind. Here we are dealing with a "memory of perception" that, according to Casey, constitutes a peculiar form of memory of the body ${ }^{29}$ Han Dong recalls his bodily sensations and employs them to reproduce young Tao's perception of the rural environment.

The sensory details provided by young Tao endow the narrative with an exquisite touch of subjectivity, conveying a rounded image of scenes "filtered" through the child's body:

They booked into a guesthouse, and Su Qun found an optician. It was only during the test that young Tao began to see his surroundings clearly and realized that Huaiyin was huge and unlike anywhere he had been before, even if it was covered in dust. He could see every tiny detail: a ray of sunlight slanting down to the floor tiles in a shop, specks of dust floating in the air. Young Tao's head began to swim, but just as he was corning to grips with it, the test glasses were taken off and the world resumed its normal murkiness..$^{30}$

他们照例在一家旅社登记了房间。然后, 苏群便领着小陶去了一家眼镜店。只是 在试镜时, 小陶才看清了周围的景象, 觉得供大的淮阴的确非同一般, 尽管尘土 遍地。突然之间他变得明察秋毫。一道阳光此时倾泻在店堂里的地砖上, 飘浮着 丝丝缕缕的灰尘, 直看得小陶头晕目眩。正当他试图克服不适看个究竟时, 试戴 的眼镜被取走了，世界又恢复了原先灰暗的面目。31

11 In this scene, the accurate depiction of the boy's first experience of using eyeglasses does not leave anything to the imagination. The shock of seeing properly makes him remember every detail of the scene before him, and their reproduction in the text allows the reader to see through young Tao's eyes.

The memory of perceptions and their description constitute the first level of the interaction between body and memory displayed in the narrative text. It allows the reader to empathize with young Tao and, consequently, to get closer to the author's perspective. 


\section{Embodied Individualities} perceptions as a means of reminiscing. In fact, the body plays a crucial part as the object of recollection as well. Young Tao not only remembers his perceptions, but he also remembers his body acting in the scenes. In order to move to the next level of the analysis, the body must be conceived as a spatial entity, something that is "in place" and, more precisely, "the center of an oriented space". ${ }^{32}$ When depicting a scene or describing an action, the author often makes the characters' bodies a feature upon which the narration pivots. Young Tao's consciousness of his own body is one of the few certainties he has; he therefore turns his attention repeatedly to physical details. For example, scatological references are recurrent in Banished! ${ }^{33}$ and young Tao frequently associates this theme with the description of his everyday life. For example, in the following excerpt, the author depicts the boy's difficulties in relieving himself, which are the key to the whole scene:

When young Tao wanted to go to the toilet, Tao got Big Head to take him, and seven or eight other children tagged along behind.

Outside, in the brilliant moonlight, it was bitterly cold. Big Head took young Tao to where the night soil crock stood behind the house. It was enclosed by a fence made of maize straw; it was pitch dark inside, and there was a terrible stench. Young Tao was reluctant to go inside, so Big Head got him to squat on the ground outside. Young Tao hesitated. Big Head set an example by pulling down his own trousers. As the boy still wavered, the other children followed suit, revealing bare white buttocks. Then young Tao pulled down his own trousers and squatted, but he just could not get the poop out. ${ }^{34}$

后来小陶要上则所，老陶让大头把他带出去，跟出来的还有号外七八个孩子。

外面, 月光如水, 天寒地冻, 大头领着小陶向 屋后的粪缸走去。那儿围了一圈玉 米秥的篱笆, 里面黑胶胶的, 一股臭味, 扑面而来。小陶不愿进去解手, 大头就 让他在外面的空地上嗱下。小陶踌躇着, 大头于是解了自己的裤子䠝下, 以作示 范。见小陶还在犹豫, 号外那七八个孩子也都脱了裤子蹲下来, 露出了白生生的 屁股。小陶学他们的样子，脱了裤子就地蹢下，可他怎么也拉不出屎来。35

Here the description goes beyond the level of perception, and the boy's body is set in a context of interaction with both the environment and the other character. The sensory experience is integrated into the memory of physiological needs, which are narrated in a humorous tone, typical of a child's perspective.

Nevertheless, young Tao's body is not merely a machine he has to keep in function, but also a cognitive instrument through which he learns how to adapt to country life. Life in Sanyu is difficult for a city boy, and one of the main obstacles is the physical resistance it requires:

Young Tao got chilblains every year. Sometimes they came after the cabbage washing, sometimes before. They became inflamed and went septic, and then Su Qun would bathe the sores with manganese dioxide and bind them carefully with a gauze dressing.

The sight of young Tao's hands covered in chilblains and swollen like steamed buns from constantly washing cabbages in freezing water distressed his grandparents so much that they would gladly have taken his place, but Tao refused to let them. He reasoned that his son was getting chilblains because he had not been sufficiently hardened, so it was all the more necessary that he should carry on. ${ }^{36}$ 小陶的两只手每年都生冻疮。有时候洗完脂菜就生了, 有时候生了冻疮还要洗腌 
菜。冻疮溃烂, 流脓发炎, 苏群就用灰锰氧水为小陶清洗疮口, 然后再仔细地用 纱布包扎好。有了这一后续措施, 陶就再无后顾之忧了。

一双生满冻疮肿得像馒头的烂手, 在冷水里洗腌菜不止, 看得陶文江、陶冯氏心 疼不已, 恨不得取而代之。可老陶不让。他的理论是：小陶之所以会生冻疮是因 为缺乏锻炼, 因此在冷水里洗䐪菜就越发的显得必要了。37

16 The iterative narration of the cabbage picking is accompanied by Tao's memory of the pain he suffered. The ache is the symptom of his inadequacy; his nerves tell him that he is still far from reaching the goal of striking root and become a villager. In this case, the recollection of his suffering body is directly related to pain memory. Painful experiences are remembered by the body itself, ${ }^{38}$ hence "body memory" triggers the "memory of the body," which young Tao employs to express his personal experience of those cold winter days.

Although young Tao's own perceptions are predominant throughout the text, his body is not the only one used to define the individuality of a character. Han Dong's recollection draws on the corporal level, and this affects not only the depiction of his fictional alter ego young Tao, but also that of another key character, young Tao's grandfather 陶文江 / Grandpa Tao / Tao Wenjiang. He is presented as a maniac for cleaning, who has a tendency however to neglect his personal hygiene. ${ }^{39}$ Grandpa Tao's faith in defeated nationalistic ideas ${ }^{40}$ is only one of the features that differentiate him from the villagers, as well as from the other members of his family. The time spent in Sanyu changes the Taos' lives, affecting their habits as well as their identities. In the case of Grandpa Tao, the effects of banishment exacerbate the results of the aging process, and his appearance shows a gradual metamorphosis:

After three years in the country, things had changed a bit. [...] His white hair was tousled by the Sanyu winds. His wispy eyebrows had begun to droop, the hairs grew out of his nostrils, his whiskers went untrimmed, and, as the network of wrinkles deepened, his features grew more indistinct. He started to lose his teeth, and Sanyu had no dentist who could fit him with false ones. Grandpa Tao was also a chainsmoker, and the remaining front teeth were stained a tarry black. Nowadays when he laughed, he was just another gap-toothed, shrivel-mouthed old man. ${ }^{41}$

下放三年后, 情形还是有了一些变化。[...] 他的白发被三余的风吹得有些凌乱, 寿眉也开始垂挂下来。还有长出鼻孔的鼻毛以及未经修理的胡须, 加上脸上日益 深人蜿娫的皱纹, 陶文江的面孔开始有些不清不楚了。再就是牙齿也开始脱落, 三余也没有牙医可以装上假牙。陶文江日夜抽个不停, 没掉的一两颗门牙也被熏 得黑嘐数的, 张嘴一笑, 完全是个瘜嘴的老大爷了。 ${ }^{4}$

Social rhythm is intertwined with psychobiological rhythm, ${ }^{43}$ yet the peculiar living conditions in the country do not merely influence this character's mind, they also accelerate the decay of his body. Grandpa Tao's scarce interest in body care reflects his degraded identity, which is forced to mutate according to the imposed change of location. Besides being a canvas on which the consequences of banishment in old age are displayed, the body also determines Grandpa Tao's personality. Scatological references are regularly associated both with his life, which he lives struggling with chronic constipation, and with his death at the hospital, which represents the tragicomic epilogue of his personal war:

Then, in the observation room, the stomach cramps returned. [...] Half an hour passed, and Tao felt something was wrong. He went in and found his father collapsed in the latrine hole. He was dead, mouth wide open, tongue protruding to one side, covered in shit from head to toe. Traces of it had even got into his mouth.

[...] Why on earth did they constantly talk about shit in connection with Grandpa Tao? Why was his life story inextricably bound up with his bowel movements right 
up until the moment he died? This is something that will no doubt puzzle my readers as much as it puzzles me. Poor Grandpa Tao: compulsively clean while he lived, condemned to die in his own excrement. ${ }^{44}$

后来在观察室里, 他再次感到腹中绞痛, 于是又进了则所。[...] 直到半小时后, 老陶觉得情形不对, 走进则所, 看见陶文江跌坐在粪沟里, 人已经死了。他张着 嘴, 舌头吐向一边, 浑身上下都是粪便。一些粪水甚至流进了嘴巴里。

[...] 这是怎么啦? 他们总是提到大便, 总是把它和陶文江联系在一起。为什么他 的故事总是和大便纠缠不休, 一直到死? 关于这些问题, 我和读者朋友 们一样,

思索至今而不得其解。可怜洁癖一生的陶文江就这么死在了粪便里。45

19 After attempting suicide by swallowing two bottles of insecticide, Grandpa Tao is rushed to the hospital, where he dies in a rather theatrical way. The detailed description of his final disposition completes the "bodily portrait" of a man who was essentially defined by means of his corporal functions. The reason for this association, made explicit by the narrating voice, is found in body memory. Grandpa Tao's physiological features shaped his habits and his personality through what Fuchs defines "incorporative memory". ${ }^{46}$ Hence, the character's identity becomes, to some extent, subordinated to his body. The fictional reproduction of such a mechanism reflects, once again, the author's attention towards physical elements, encouraging the identification of the body as the most direct expression of one's individuality.

\section{Physical Reflections of History}

The depiction of the remembering subject's sensory perceptions and the identification of the characters' bodies with their individuality are not the only two results of Han Dong's attention towards the body. Banished! is the product of the entanglement of reality and fiction; it is an example of the author's tendency to intertwine his own experience with the characters' stories. ${ }^{47}$ It is based on the memories of his childhood, but the child's and the adult's points of view are inevitably superposed. Indeed, the novel represents as well a reflection on the Cultural Revolution from the perspective of a generation that is historically and artistically uprooted. The focus on the individual dimension is reflected on the central role played by the body in the depiction of the historical background. Physical labor, corporal punishment and resistance constituted the leitmotiv of banishment, which Han Dong describes in his own distinctive style. For example, when narrating the atmosphere in Sanyu primary school, the author depicts the punishments inflicted on negligent pupils, which terrorize young Tao:

Mr. Jin's skills were manifested not only in games; he was also ingenious at devising punishments for his students. When students arrived late, he would chalk a wavy line on the blackboard and make them rub it out with their noses. He made the line as wiggly as possible, like the ripples of a stream. The students had to rush along, alternately standing on tiptoe and kneeling on the ground, sneezing as they went. [...] Even more exciting was the punishment called "Doing Penance before Chairman Mao" on the mud wall above the blackboard hung a portrait of Chairman Mao, and the students being punished were forced to kneel in front of it by the two class monitors. The victims would often wail and beg for mercy and struggle to stand up, but the monitors, robust youths, would hold their hands behind their backs, press on their heads, and force them down. The reason for this pointless resistance was simple: under the victims' knees was not the usual smooth mud floor but a heap of broken glass and tiles. ${ }^{48}$ 靳先生的智慧不仅体现在以上方面, 惩罚学生他更是别出心裁。 学生迟到了, 他就在黑板上用粉笔画一条曲线, 让迟到的学生用鼻子擦掉。曲线 
画得尽量弯曲, 就像是小河里的波浪。学生忽而踞起脚尖, 忽而又蹲下身去, 忙 得不亦乐乎, 中途还得打几个喷德。

[...] 另一种惩罚方式更激动人心, 叫做 “向毛主席 请罪”。

黑板上方的泥墙上贴着一张毛主席画像, 受罚的学生在两个班干部的挟持下, 在 毛主席像前趾 倒。趾倒的学生往往挣扎着要站起来, 一面号陶不 已、哭忞喊 娘。执法的任务一般由身強力壮的班干部担任, 他们反剪着受罚者, 使劲地按着 他的脑袋，终于又把他按下去了。

受罚的学生为何要做这无谓的顽抗呢一? 因为他的㯲下并非是柔软的泥地, 而是 一小堆玻璃瓦杂。49 Tao's attempts to teach his son to swim, which he held to be a major skill that a boy should have, especially one living in the countryside:

Apart from encouraging his son to grow up brave, Tao also paid a good deal of attention to developing his physique. Young Tao needed to be strong and healthy to survive in Sanyu. It was no good being weak and weedy if he was to be a peasant engaged in manual labor.

[...] In all his years in Sanyu, young Tao never learned to swim, which was odd, considering there were places suitable for swimming everywhere and he had a father who was so keen on swimming. He not only could not swim in the river, but he could not swim in the village streams either. He not only did not know any swimming strokes, but he had not even learned to dog-paddle. ${ }^{50}$

除培养小陶的勇敢品质外, 老陶也很注意小陶的身体。小陶需要一副強健的体

魄, 才能适应以后在三余的生活。尤其是他准备当一个农民, 作为一个体力劳动

者, 如此弱不禁风是不行的。

[...] 在三余的几年里, 小陶始终没能学会游泳, 这事着实有些奇怪。不仅由于可 供游泳的沟渠河道遍地都是, 还因为有老陶这样一个热爱游泳的爸爸。到后 来, 小陶不仅不能在水渠里游了, 就是村上的小河沟里也不行。不仅不能游出各 种姿势, 就是狗刨他也没有学会。51

The accent on swimming is a legacy of Mao's teachings, through which he underlined the importance of physical exercise. Particularly significant in this respect was the episode that took place on July 16th 1966, when Mao Zedong celebrated his return to public life with a swim in the Yangtze River. This event triggered a swimming fever, which quickly spread to the whole country. ${ }^{52}$ Tao's predilection for swimming has, therefore, a historical reason, although one not explicitly divulged in the text. Moreover, when mentioning a poem by Tao's friend Hou Jimin / 侯继民, the narrator specifies that Hou's son, unlike young Tao, had learned to swim. ${ }^{53}$ Han Dong uses this example to demonstrate how the value of an individual was often determined on the basis of his physical strength and skills, consolidating the idea that individual identity was subjugated to the body.

\section{Conclusions}

After reviewing the multiple connections between memory and body, this study has investigated the role of the body in Han Dong's fictional recollection of his experience 
of banishment. Although Banished! should be considered as an autonarration, with the authenticity of its descriptions inevitably affected by the mediation of the literary text, the traces of the author's memory are nonetheless coded in his fictional depiction of the characters' lives during the Cultural Revolution. Han Dong's young age at the time influenced his interaction with the surrounding environment, which was based on his body as the primary source of knowledge, and characterized by the naiveté typical of an eight-year-old. The analysis here has been conducted on different levels, moving gradually from the remembering subject's body to the body seen as the object of his reminiscence. First, we have examined how the author's perceptions were reproduced in young Tao's descriptions, emphasizing the individual perspective Han Dong imposed when filtering the narrated scenes through the memory of the subject's body. Second, the study underlines the relationship between identity and body, pointing out the constant association of the characters' individualities with their bodies and bodily functions. Finally, the analysis addresses the issue of the body depicted as a key feature during the time of the Cultural Revolution, being at the center of both subjective impressions and objective value judgements.

Han Dong's novel Banished!, as a fictional elaboration of an autobiographical experience, is the result of a particular form of the reminiscing process, in which the distinction between memory and image, between reality and unreality, is blurred - in the same way that Ricœur described in his work La Mémoire, l'Histoire, l'Oubli, ${ }^{54}$ but to an even greater degree. Nonetheless, recollection is the method used by the author to depict his own portrait of the Cultural Revolution, and many clues related to his personal memories can be found throughout the text. According to Casey, "there is no memory without body memory", ${ }^{5}$ so the body must be the starting point for any kind of recollection. In Banished!, the importance of the physical dimension extends throughout the whole novel. The remembering subject's bodily feelings are the author's creative source, and the characters' identities are depicted as indissolubly related to their physical features. Moreover, the memories of a child experiencing the Cultural Revolution are interwoven with the author's portrait of the socio-cultural background of the plot. This accent on the body allows the author to enhance his individualism, while stressing the peculiar position of the subject in the factious society of those times.

\section{BIBLIOGRAPHY}

BERGSON Henri, Matière et mémoire : essai sur la relation du corps à l'esprit, Paris, PUF, 1968.

BERRY Michael, A History of Pain: Trauma in Modern Chinese Literature and Film, New York, Columbia U. P., 2008.

CASEY Edward S., "Habitual Body and Memory in Merleau-Ponty", Man and World, 17:1994, p. 279-97.

CASEY Edward S., Remembering: A Phenomenological Study, Bloomington, Indiana U. P., 2000 [1987]. 
CODELUPPI Martina, “Mapping Ideology in Language: Han Dong's ‘Zha gen' (Banished!) and Ma Jian's 'Rou zhi tu' (Beijing coma)”, Annali Di Ca' Foscari Serie Orientale, 53:2017, p. 257-80.

DENG Li 邓莉, [“Understanding and Rewriting Memory: on Han Dong's Novel Banished!”] / 记忆的 理解与重构一读韩东的长篇小说《扎根》/Jiyi de lijie yu chonggou-du Han Dong de changpian xiaoshuo 'Zha gen', 当代文坛 / Dangdai wentan, 2:2005, p. 62-63.

DIKÖTTER Frank, The Cultural Revolution: A People's History, 1962-1976, London, Bloomsbury, 2016.

FUCHS Thomas, "The Phenomenology of Body Memory", in Body Memory, Metaphor and Movement, косн Sabine C. et al. (eds.), Amsterdam, John Benjamins, 2012, p. 9-22.

GONG Ailing 宫爱玲, [“'Unbounded Happiness' at the Time of Distress: On Han Dong's Banished!”] / 苦难年代里的”其乐融融”一评韩东《扎根》/ Kunan niandai li de 'qile rongrong'-ping Han Dong 'Zha gen', 长春工业大学 / Changchun gongye daxue 18, 1:2006, p. 86-88.

Gu Haiyang 古海阳, [“Reconstructing Individual Historical Experience: On Han Dong's Banished! and “A Small-Town Hero Strides Forth”'] / 个体历史经验的重构一韩东《扎根》、《小城好汉之 英特迈往》/ Geti lishi jingyan de chonggou-Han Dong 'Zha gen,' 'Xiaocheng haohan zhi yingte maiwang' helun, Xinyu xueyuan xuebao 5, 6:2010, p. 28-30.

HAN Dong 韩东, Banished! A Novel, HARMAN Nicky (trans.), Honolulu, Hawai'i U. P., 2009.

HAN Dong 韩东, Banished! / 扎根 / Zha Gen, Guangzhou, Huacheng chubanshe, 2010.

HUNT Pamela, “The Significance of Scatological Humour: A Case Study of Zhu Wen's 'What Is Garbage, What Is Love' and Han Dong's Banished!", 2010, https://media.paper-republic.org/files/ 10/06/P_Hunt_essay_on_scat.pdf, consulted on 28/02/2018.

KLEINMAN Arthur and KLEINMAN Joan, "How Bodies Remember: Social Memory and Bodily Experience of Criticism, Resistance, and Delegitimation Following China's Cultural Revolution", New Literary History 25, 3:1994, p. 707-23.

косн Sabine C. et al. (eds.), "Introduction", in Body Memory, Metaphor and Movement, косн Sabine C. et al. (eds.), Amsterdam, John Benjamins, 2012, p. 1-6.

LI Yifei 李奕霏, [“Writing Zero-Degree Emotions: On Han Dong's Narrations of Educated Youths"] / 零度情感'写作一论韩东的知青题材小说 / Lingdu qinggan' xiezuo-lun Han Dong de zhiqing ticai xiaoshuo, 当代文学研究 / Dangdai wenxue yanjiu, 33:2012, p. 82-85.

MERLEAU-PONTY Maurice, Phénoménologie de la perception, Paris, Gallimard, 1945.

RICCEUR Paul, La Mémoire, l'Histoire, l'Oubli, Paris, Seuil, 2000.

UMBELINO Luís António, "Memory of the Body, Temptation of Space" The European Legacy 20, 8:2015, p. 844-51.

WANG Ling 王凌, [“Cold Tone for a Lively Book: The Analysis of Language in Han Dong's Banished!”] / 冷色笔调, 淡书热闹一韩东小说《扎根》语言分析 / Lengse bidiao, danshu renao-Han Dong xiaoshuo 'Zha gen' yuyan fenxi, Dangdai wentan, 4:2004, p. 70-72.

WANG Yuehua 汪跃华, [“A Duplicated Book: On Han Dong’s Banished!”] / 复写之书:韩东《扎根》 论 / Fuxie zhi shu: Han Dong 'Zha gen' lun, 文学评论 / Wenxue pinglun, 3:2004, p. 39-46.

WU Congcong 吴聪聪, LI Renjie 李仁杰, [“Filtered Memory: On Han Dong's Literary Creation in Banished!'] / 被过滤的记忆一由 《扎根》谈韩东文学创作 / Bei guolü de jiyi-you 'Zha Gen' tan Han Dong wenxue chuangzuo, 作家杂志 / Zuojia zazhi, 13:2013, p. 6-7. 
WU Yiqin 吴义勤, [“Walking Along with Poetry: Discussing Han Dong's Fiction”] / 与诗同行一韩东

小说论 / Yu shi tong xing-Han Dong xiaoshuo lun, Dangdai zuojia pinglun, 5:1996, p. 73-80.

\section{NOTES}

1. косн Sabine C. et al., "Introduction", in Body Memory, Metaphor and Movement, косн Sabine C. et al. (ed.), Amsterdam, John Benjamins, 2012, p. 2.

2. HAN Dong 韩东, Banished! / 扎根, Guangzhou, Huacheng chubanshe, 2010; HAN Dong 韩东, Banished! A Novel, HARMAN Nicky (trans.), Honolulu, Hawai'i U. P., 2009.

3. BERGSON Henri, Matière et Mémoire: Essai sur la relation du corps à l'esprit, Paris, PUF, 1968 [1896], p. 147.

4. MERLEAU-PONTY Maurice, Phénoménologie de la perception, Paris, Gallimard, 1945.

5. CASEY Edward S., "Habitual Body and Memory in Merleau-Ponty," Man and World, 17:1994, p. 279-97.

6. CASEY Edward S., Remembering: A Phenomenological Study, Bloomington, Indiana U.P., 2000 [1987].

7. FUCHS Thomas, "The Phenomenology of Body Memory," in Body Memory, Metaphor and Movement, косн Sabine C. et al. (eds.), Amsterdam, John Benjamins, 2012, p. 9-22.

8. BERRY Michael, A History of Pain: Trauma in Modern Chinese Literature and Film, New York, Columbia U. P., 2008.

9. KLeinMAn Arthur and KLEINMAN Joan, "How Bodies Remember: Social Memory and Bodily Experience of Criticism, Resistance, and Delegitimation Following China's Cultural Revolution," New Literary History 25, 3:1994, p. 707-23.

10. LI Yifei 李奕霏, [“Writing Zero-Degree Emotions: On Han Dong's Narrations of Educated Youths”] / 零度情感'写作一论韩东的知青题材小说, 当代文学研究/ Dangdai wenxue yanjiu, 33:2012, p. 83.

11. WANG Yuehua 汪跃华, [“A Duplicated Book: On Han Dong's Banished!”] / 复写之书:韩东《扎 根》论, 文学评论 / Wenxue pinglun, 3:2004, p. 45.

12. wu Congcong 吴聪聪, LI Renjie 李仁杰, [“Filtered Memory: On Han Dong's Literary Creation in Banished!'] / 被过滤的记忆一由《扎根》谈韩东文学创作, 作家杂志 / Zuojia zazhi, 13:2013, p. 6-7. 13. GU Haiyang 古海阴, [“Reconstructing Individual Historical Experience: On Han Dong's Banished! and 'A Small-Town Hero Strides Forth”'] / 个体历史经验的重构一韩东《扎根》、《小 城好汉之英特迈往》, Xinyu xueyuan xuebao 5, 6:2010, p. 28-30.

14. DENG Li 邓莉, [“Understanding and Rewriting Memory: On Han Dong's Novel Banished!"] / 记 忆的理解与重构一读韩东的长篇小说《扎根》, 当代文坛 / Dangdai wentan, 2:2005, p. 62-63.

15. WANG Ling 王蔆, [“Cold Tone for a Lively Book: The Analysis of Language in Han Dong's Banished!”] / 冷色笔调, 淡书热闹一韩东小说《扎根》语言分析, Dangdai wentan, 4:2004, p. 70-72.

16. CODELUPPI Martina, “Mapping Ideology in Language: Han Dong's 'Zha gen' (Banished!) and Ma Jian's 'Rou zhi tu' (Beijing coma)”, Annali Di Ca' Foscari Serie Orientale, 53:2017, p. 257-80.

17. GONG Ailing 宫爱玲, [“'Unbounded Happiness' at the Time of Distress: on Han Dong's Banished!"] / 苦难年代里的”其乐融融”一评韩东《扎根》, 长春工业大学学报 / Changchun gongye daxue xuebao 18, 1:2006, p. 86-88.

18. HUNT Pamela, "The Significance of Scatological Humour: A Case Study of Zhu Wen's 'What Is Garbage, What Is Love' and Han Dong's Banished!' https://media.paper-republic.org/files/10/06/ P_Hunt_essay_on_scat.pdf, 2010, consulted on 28/02/2018.

19. MERLEAU-PONTY, Phénoménologie de la perception, op. cit., p. 10.

20. Ibid., p. 10. 
21. Ibid., p. 22.

22. BERGSON, Matière et mémoire : essai sur la relation du corps à l'esprit, op. cit., p. 62.

23. WANG Yuehua 汪跃华, [“A Duplicated Book: On Han Dong's Banished!”] / 复写之书:韩东《扎

根》论, op. cit., p. 43.

24. HAN, Banished! A Novel, op. cit., p. 59.

25. HAN, Banished! / 扎根, op. cit., p. 58.

26. CASEY, Remembering: A Phenomenological Study, op. cit., p. xxi.

27. Ibid., p. 147.

28. Ibid., p. 147.

29. Ibid., p. 162

30. HAN, Banished! A Novel, op. cit., p. 114.

31. HAN, Banished! / 扎根, op. cit., p. 111.

32. UMBeLino Luís António, "Memory of the Body, Temptation of Space", The European Legacy 20, 8:2015, p. 845.

33. HUNT, "The Significance of Scatological Humour: A Case Study of Zhu Wen's 'What Is Garbage, What Is Love' and Han Dong's Banished!", op. cit., p. 3.

34. HAN, Banished! A Novel, op. cit., p. 54.

35. HAN, Banished! / 扎根, op. cit., p. 53.

36. HAN, Banished! A Novel, op. cit., p. 98-9.

37. HAN, Banished! / 扎根, op. cit., p. 95.

38. FUCHS, "The Phenomenology of Body Memory," op. cit., p. 16.

39. HAN, Banished! / 扎根, op. cit., p. 132.

40. Ibid., p. 134.

41. HAN, Banished! A Novel, op. cit., p. 136.

42. Ibid., p. 134.

43. KLeINMAN, "How Bodies Remember: Social Memory and Bodily Experience of Criticism, Resistance, and Delegitimation Following China's Cultural Revolution”, op. cit., p. 712.

44. HAN, Banished! A Novel, op. cit., p. 194.

45. HAN, Banished! / 扎根, op. cit., p. 191.

46. FUCHS, "The Phenomenology of Body Memory", op. cit., p. 16.

47. WU Yiqin 吴义勤, [“Walking Along with Poetry: Discussing Han Dong’s Fiction”] / 诗同行一韩

东小说论, 当代作家评论 / Dangdai zuojia pinglun, 5:1996, p. 78.

48. HAN, Banished! A Novel, op. cit., p. 68.

49. HAN, Banished! / 扎根, op. cit., p. 66.

50. HAN, Banished! A Novel, op. cit., p. 90-3.

51. HAN, Banished! / 扎根, op. cit., p. 88-9.

52. DIKÖTTER Frank, The Cultural Revolution: A People's History, 1962-1976, London, Bloomsbury, 2016, p. 66.

53. HAN, Banished! / 扎根, op. cit., p. 147.

54. RICÆEUR Paul, La Mémoire, l'Histoire, l'Oubli, Paris, Seuil, 2000, p. 54.

55. CASEY, Remembering: A Phenomenological Study, op. cit., p. 172. 


\section{AUTHOR}

\section{MARTINA CODELUPPI}

Martina Codeluppi is Assistant Professor at the University of Insubria. She holds a PhD in contemporary Chinese literature from Ca' Foscari University of Venice and Sorbonne Nouvelle University - Paris 3. Her main publications deal with memory, migrant literature, selftranslation, literary theory, and critical discourse analysis. She is the author of the book Fictional Memories: Contemporary Chinese Literature and Transnationality, published in 2020 by L'Harmattan, and the translator of several works of contemporary Chinese fiction. 


\title{
L'écriture du corps chez quelques femmes écrivaines chinoises du $\mathrm{Xx}^{\mathrm{e}}$ siècle
}

\author{
Xinyu Hu
}

1 Des femmes écrivains chinoises sont montées sur la scène littéraire dans la foulée du Mouvement du 4 Mai 1919. Partisanes de la «Révolution littéraire » et de la « Nouvelle Culture ", ces jeunes diplômées des écoles de "nouveau style » se mettent à écrire et ont l'intention de vivre la profession d'écrivain à part entière. BING Xin 冰心 (1900-2000), CHEN Hengzhe 陈衡哲 (1890-1976), LING Shuhua 淩淑华 (1900-1990) et LU Yin 庐隐 (1899-1934) ouvrent cette page initiale de la littérature moderne dite en «langue parlée » / 白话 / baihua et de nouveaux thèmes tels que le «Je » féminin, le désir féminin, etc.

2 Même si le corps occupe chez elles une place inédite, sa présence est encore peu affirmée sous la plume des auteures de la première période (1917-1927), selon la division chronologique de SHENG Ying que reprend JIN Siyan ${ }^{1}$ dans son livre sur la littérature féminine $\mathrm{du} \mathrm{xx}^{\mathrm{e}}$ siècle à nos jours. Selon elle, la deuxième période, entre la fin des années vingt et les années trente, se soucie plutôt de la relation entre la femme et la révolution. [Le Journal de mademoiselle Sophie] / 莎菲女士日记 de DING Ling 丁玲 (1904-1986) fait scandale à sa sortie en 1928, car il aborde de façon téméraire le sujet du désir féminin au regard du corps masculin.

Après la troisième période (les années trente et quarante) et la quatrième période (les trois décennies "maoïstes »), la conscience féminine resurgit vers 1978 et prospère jusqu'en 2000 : les écrivaines contemporaines s'intéressent à nouveau au corps, de façon encore plus personnalisée et audacieuse. Parmi elles se distinguent d'abord sHU Ting 舒婷 (1952-) et ZHANG Jie 张洁 (1937-), ensuite TIE Ning 铁凝 (1957-), puis CHEN Ran 陈染 (1962-) et LIN Bai 林白 (1962-).

En racontant l'histoire de trois générations de femmes qui vivent dans une cour carrée de Pékin, [La Porte des roses] / 玫瑰门, TIE Ning retrace la condition sociale des femmes chinoises : de l'enfance paisible auprès des parents à l'âge adulte en tant qu'épouse et 
mère, enfin à la vieillesse et ses sentiments d'amertume et de désillusion. À chaque stade, la transformation du corps dit non seulement de la fuite du temps, mais aussi la construction lente et difficile du « Je » féminin.

5 Les œuvres de DING Ling et de TIE Ning nous amènent à étudier l'écriture du corps du début $d u x^{e}$ siècle à nos jours en tant qu'elle exprime l'évolution du désir féminin au cours d'une vie de femme, et ce à l'aune des faits lexicaux, syntaxiques. À analyser l'éveil de la conscience féminine avec l'affirmation afférente du « Je » féminin.

6 La langue chinoise moderne baihua entre en jeu pour deux raisons principales : d'abord, elle sert à écrire la littérature moderne et contemporaine ; ensuite, observer l'évolution de cette langue utilisée dans les œuvres de DING Ling et de TIE Ning, permet de saisir des éléments significatifs, tels le vocable corporel et sensoriel, une structure syntaxique liée à la particule « $b a » /$ 把 entre autres, une narration à plusieurs voix où domine toutefois la première personne chez Ding Ling et une narration qualifiable d'alternative entre la narratrice omniprésente et le monologue introspectif de l'héroïne chez TIE Ning.

7 Nous examinerons d'abord l'expression du désir féminin dans la représentation $d u$ corps chez Ding Ling, puis l'interaction entre la transformation du corps et le temps féminin chez TIE Ning et enfin les tentatives linguistiques et narratives qui produisent une écriture du corps inéluctablement moderne et contemporaine.

\section{L'expression physique du désir féminin}

8 En quoi l'héroïne de Ding Ling, $\mathrm{M}^{\text {lle }}$ Sophie, représente-t-elle cette « femme nouvelle » / 新女性 qui revendique l'émancipation face aux «valeurs confucéennes" et ose contempler le corps de l'homme aimé?

9 Après avoir quitté la maison familiale au lendemain du 4 Mai, elle va étudier à Shanghai pour s'y faire une place, rencontre le jeune 凌吉士 / LING Jishi et s'en éprend :

Lui, cet inconnu, comment qualifier sa beauté ? Certes, sa grande taille, son visage pâle et tendre, ses petites lèvres minces et ses cheveux souples suffisent à m'éblouir, mais il a encore quelque chose d'indicible et d'insaisissable qui vient me troubler : quand je lui demande son nom, il me tend sa carte de visite, d'une main nonchalante.

他, 这生人, 我将怎样去形容他的美呢? 固然他的颀长的身躯, 白嫰的面庞, 薄 薄的小嘴唇, 柔软的头发, 都足以闪耀人的眼睛, 但他还有号外一种说不出的, 捉不到的丰仪来煽动你的心。比如, 当我请问他的名字时, 他会用那种我想不到 的不急剧的态度递过那只㢣有名片的手来。2

10 Rien de l'homme aimé, de la tête aux pieds, n'échappe au regard de Sophie : un « corps de grande taille " / 顾长的身身 / qichang de shenqu, un «visage pâle et tendre » / 白嫰 的面庞 / bainen de mianpang, de 《petites lèvres minces 》 / 薄薄的小嘴唇 / baobao de xiao zuichun, ou des "cheveux souples"/ 柔软的头发/ rouruan de toufa. Ces expressions montrent que Sophie exhibe son désir du corps masculin. Plus tard, même si la personnalité de l'homme la déçoit, elle ne regrettera pas d'avoir vécu cette expérience qui consiste à scruter le corps de l'homme :

Quand j'y repense, je crains de me fourvoyer davantage encore avec mes caprices. J'occupe pour l'instant cet appartement de style occidental doté d'une cheminée ; peut-être suis-je tombée amoureuse de cet homme originaire de Nanyang ? Or je ne sais encore rien de lui. Ces lèvres, ces sourcils, ces pointes de doigts... quelle inconscience! Ce n'est pas ce qu'il y a d'essentiel chez un être! Je dois être 
ensorcelée pour porter ma pensée là-dessus.

现在仔细一想, 我惟恐我的任性, 将把我送到更坏的地方去, 暂时且住在这有洋

炉的房里吧, 难道我能说得上是爱上了那南洋人吗? 我还一丝一毫都不知道他

呢。什么那嘴唇, 那眉梢, 那指尖.......多无意识, 这并不是一个人所应需的, 我

着魔了, 会想到那上面。3

11 Comme la plupart de jeunes femmes arrivées dans les grandes villes au lendemain du Mouvement du 4 Mai, Sophie peine à trouver une solution satisfaisante pour cette nouvelle vie. Elle est dans une situation d'autant plus difficile que, alors même qu'elle n'a pas encore eu d'enfant, son corps est déjà usé par la tuberculose à cause de la malnutrition.

Bien que Sophie soit follement amoureuse de Ling Jishi et admire son corps, avec le temps elle se rend bientôt compte que la beauté corporelle de l'homme ne lui suffit plus et a aussi besoin de partager certaines valeurs avec son amoureux. Aussi, la beauté du corps doit laisser sa place à la noblesse de l'âme. Cependant, la jeune femme est prête à sacrifier ses valeurs pour une caresse. L'amour de Sophie ne va pas sans contradiction ni concession.

Son dilemme révèle les difficultés à construire un "Je " féminin aussi bien chez de jeunes femmes comme elle que chez de jeunes écrivaines comme Ding Ling: est-il possible de partager et la passion charnelle et l'amour spirituel avec un homme, si une femme ne peut plus se contenter du seul regard des hommes? Où pourrait-elle trouver ses propres valeurs en tant que «Je » qui regarde et qui désire? Très clairement, la figure des jeunes femmes représentées par Sophie a besoin de plus de maturité pour affronter les autres épreuves de sa vie de femme :

Ces femmes écrivains, filles rebelles et libres, ont franchi le premier pas dans la création de leur nouveau destin avec la plume. Sans cette nouvelle tradition rebelle créée par ces jeunes filles, la littérature chinoise ne saurait prétendre posséder de figures féminines et de groupes féminins matures et dignes de ce nom.

作为叛逆的女儿, 自由的女儿的女性作家们, 以笔创造自己新命运的第一步。没 有这样一个叛逆女儿的传统, 中国文坛上大概也便不会出现真正成熟的女人以及 女性群体。4

\section{L'interaction entre la transformation du corps et le temps féminin}

Si Ding Ling dessine l'image d'une jeune femme à peine sortie de la maison familiale et franchissant un premier pas dans le monde urbain comme shanghai de ces années 1920-1930, Tie Ning, elle, crée dans La Porte des roses une série de figures féminines : la grand-mère maternelle si Qiwen / 司琦纹, sa sœur, la grand-tante paternelle, la tante maternelle, la narratrice et une protagoniste, su Mei / 苏眉, qui raconte parallèlement sa propre vie - de la petite fille à la jeune épouse et à la mère - et celle de sa grandmère maternelle qui traverse le $\mathrm{xx}^{\mathrm{e}}$ siècle chinois, jusqu'à la Révolution culturelle.

Les mouvements politiques privent la petite Su Mei de sa mère, obligée de se rendre à la ferme de rééducation avec ses collègues de l'université. Aussi reste-t-elle sous la garde de sa grand-mère maternelle et éducatrice. Après avoir perdu ses beaux-parents et son mari, Si Qiwen partage un habitat à cour carrée avec sa belle-sœur, son fils, sa belle-fille et leurs enfants. Du côté de sa famille, elle fréquente encore sa sœur. C'est la première fois qu'elle accueille chez elle sa petite-fille, qui va faire connaissance de toute la 
parentèle, à commencer par sa propre sœur dont les traits physiques et la voix attirent Su Mei dès les premiers instants :

Dans la lumière, Meimei avait pu bien observer la vieille dame aux cheveux blancs : elle avait non seulement des cheveux blancs, mais aussi un teint clair propre à une petite fille. Une veste bien taillée couvrait un corps enveloppé, cachant mal une poitrine plantureuse. Le col, semble-t-il un peu serré, la gênait dans son gros cou et donnait l'impression de l'étouffer. Mais à la surprise de Meimei, elle parlait avec aisance et éclat.

在一片光明里, 眉眉看清了那白发老太太。她头发白, 皮肤也白, 白得就像一个 小姑娘。一身剪裁合适的黑罩衣罩着她那扁㭌的身体, 她有一副宽广, 厚实的胸 脯。她的衣领显得狭小, 也许因为脖子粗了些, 眉眉只觉得那领子妨碍了她的呼 吸。然而她的声音却流畅, 嘹亮。5

Pour pallier l'ignorance que la petite fille a de son propre corps, Tie Ning représente ostentatoirement le corps, qui fixe sur lui les périodes successives de la vie de Su Mei, autant d'étapes au cours desquelles se construit sa personne.

Su Mei commence à découvrir son corps à travers celui de son entourage : si le corps de Yipo est présenté couvert d'un vêtement dont le col la serre, celui de sa tante apparaît avec ses deux seins bien serrés dans une chemise bleu clair :

Elle lève la tête pour regarder sa tante maternelle qu'elle rencontre pour la première fois, ce qui entre d'abord dans son champ de vision, c'est cette paire de seins plantureux. On dirait que ces seins sont bien serrés dans une chemise bleu clair; il y a sur le devant de la chemise deux morceaux de tissus mouillés qui ressemblent à deux morceaux de nuage, ou bien à deux pièces de tissus rapiécées de couleur foncée.

她仰望第一次与她见面的舅妈, 先看见了舅妈那一对蓬勃的大奶。那奶被压迫在 一件淡蓝色衬衫里, 衬衫前襟有两小块湿, 像两朵云, 又像两块深色的补丁。6

Objet du regard de Su Mei, le corps de la tante éveille la conscience féminine de sa petite fille, qui se met alors à scruter son propre corps et s'identifie à sa tante en tant que femme. L'identité sexuelle de l'individu naît en même temps que la conscience du "Je ». La petite Su Mei se découvre en accompagnant la tante prendre son bain quotidien où elle seule est autorisée à envoyer de l'eau avec ses doigts sur le dos nu de la jeune femme :

En sortant de la bassine, sa tante continue naturellement tout ce qu'il faut faire après le bain, en s'exposant au regard de Meimei. Elle s'essuie avec une serviette en se tournant sur elle-même, dans une nudité absolue. Ce ne sont plus des amalgames de chair qui s'offraient autrefois à la perception de Meimei, qui estime à présent que tout le corps de sa tante mérite d'être contemplé dans sa totalité. Pour le moment Su Mei ignore encore l'anatomie d'un corps humain, encore plus ce lien existant entre le corps humain et la beauté ; elle ne comprend pas pourquoi ils peuvent susciter cette émotion unique. Une chose est sûre pour elle, c'est que la femme de son oncle maternel doit être admirée, dans son corps entier.

舅妈迈出澡盆, 就那么随意地对着眉眉为自己做着一切善后工作。她一面用毛巾 擦拭全身, 一面沉稳, 从容地转动身体, 于是身体的所有部位便在眉眉的眼前展 示。这不再是从前眉眉眼前那些乱七八糟的团团块块, 面对这些展开, 她觉得舅 妈的哪一部分都该让人看, 舅妈本是一个该让人看的人。苏眉在当时不懂得人体 构造, 更不懂人体和美有什么关联, 为什么它们能带给人绝无仅有的激动。她只 知道舅妈是个最该让人看的人: 哪儿都该让人看。

19 Quand advient cette identification imaginaire, première étape de la formation de la personnalité, Su Mei a 12 ans. Elle se mue peu à peu en femme, silencieusement. Plus elle grandit en jeune femme assoiffée d'amour, plus elle se découvre et évolue. Cette mutation de corps et de personnalité accompagne son devenir professionnel quand elle 
décide de devenir peintre, statut social qui lui permet d'accomplir enfin le processus lent et difficile de la construction d'un "Je " féminin, et ce selon trois étapes: découverte du corps féminin à l'enfance; éveil de l'identité sexuelle à l'adolescence avec les règles et le développement des seins ; maturité de femme à la naissance d'un enfant.

\section{L'écriture du corps et la subjectivité féminine}

Dans leur écriture du corps et leur narration polyphonique, ces deux écrivaines partagent l'insistance sur l'existence corporelle et les sensations. On relève aussi des particularités plus liées peut-être au recours à une langue « moderne ». En effet, outre une série de mots et expressions désignant spécifiquement le corps ou l'activité psychologique, de nouvelles structures syntaxiques remplissent une fonction notable, notamment la particule de complément d'objet avancé 把 / ba. C'est le cas dans [Le Journal de $\mathrm{M}^{\text {lle }}$ Sophie] :

J'ai examiné le corps de cet homme de la tête aux pieds jusqu'au moindre détail, en éprouvant le besoin de le parcourir de mes lèvres.

我把他什么细小处都审视遍了, 我觉得都有我嘴唇放上去的的需要。8

Oui, je me connais, je ne suis qu'une femme remplie de féminité, une femme qui ne pense qu'à l'homme qu'elle a envie de conquérir.

是的, 我了解我自己, 不过是一个女性十足的女人, 女人只想把心思放到她要征 服的男人身上。9

Dans ces deux phrases, le corps de l'homme est objectivé par cette antéposition, soumis au regard dévorant de Sophie. La jeune femme se positionne cette fois-ci en tant que « regardante » qui ne laisse passer aucun détail sur le visage et le corps de l'homme. La femme objet se transforme ainsi en sujet, qui regarde et qui occupe ainsi une place active dans sa relation avec l'homme.

Tie Ning, quant à elle, recourt au discours indirect, qui accorde une place centrale à la narration et traduit l'anxieuse impatience de l'héroïne à devenir femme, comme dans cet exemple :

J'avais dessiné le corps nu de Ma Xiaosi, qui était vraiment superbe. Plus tard elle m'avait demandé ce que c'était à la vue de mon œuvre : n'est-ce pas une rivière? Une rivière rouge qui s'écoule au pied de l'enceinte de la vieille ville ? Ma Xiaosi dit alors : tu m'as demandé de rester sans bouger, nue, des jours durant ; ça m'a donné un mal fou au dos et aux jambes, mais ça n'a accouché à la fin que d'une pauvre rivière ; ne compte pas sur moi pour continuer comme ça. Elle m'avait engueulé en disant que je l'avais blousée. Je lui avais répondu: je ne t’ai pas blousée; sans ta pose, je n'aurais jamais réussi à dessiner cette rivière. Sur le tableau tu es peut-être invisible, mais pas dans mon champ de vision.

我画过马小思的裸体她是太棒了, 后来她看了我的作品说这是什么? 这不是一条 河吗一条夹挤在老城脚下的红色小河吗。马小思说好啊你让我光着身子站了好几 天腰酸腿疼画面上却只有一条河他妈的再也不给你干了。她骂我坑了她。我没有 坑你, 没有你的裸体我画不成那条河。画面上可以没有你但我的视野里不能没有 你。10

Outre l'usage du discours indirect, deux lignes narratives coexistent dans La Porte des roses: la première, sur la vie de Si Yiwen et tout ce qui se passe dans sa maison de Pékin ; la deuxième, plutôt sur le monologue introspectif de Su Mei elle-même, qui dit ses sensations, sentiments et réflexions, lesquelles sont menées sous forme de dialogues illustrant l'interaction entre les activités psychologiques des personnages. 
La prise de conscience du sujet féminin trouve ainsi la meilleure façon de s'exprimer dans le monologue introspectif. Au fur et à mesure que le corps de la femme se transforme, en même temps qu'elle vit, elle observe et réfléchit. Le dialogue suppose aussi une interlocutrice, qui pourrait jouer le rôle d'amie intime de l'héroïne.

avons montré un exemple de la thématique du corps chez deux femmes écrivains Ding Ling et Tie Ning. La première, à la suite du Mouvement du 4 Mai, évoque le désir du corps de l'autre chez une jeune femme en quête de sa place dans une société en pleine mutation ; la seconde, écrivant après la Révolution culturelle, comprend que les diverses expériences vécues par le corps forment les étapes indispensables de la construction identitaire d'une femme contemporaine à part entière.

Le champ de l'écriture, devenant l'allié de ces femmes écrivains en quête d'une place dans la Chine moderne, leur permet non seulement de témoigner de leur découverte du corps et de leur désir, mais aussi d'occuper cette position particulière de " regardante » et de tisseuse de la trame de leur récit. Après Tie Ning, des auteures telles Lin Bai et Chen Ran prolongent cette stratégie vers une représentation encore plus directe de la vie intérieure des personnages féminin, dans une écriture « fragmentaire, destructrice, passionnée et dubitative $»^{11}$.

\section{BIBLIOGRAPHIE}

Ding Ling, [Chefs-d'œuvre du roman moderne chinois (Æuvres de DING Ling)] / 中国现代小说精 品 (丁玲卷) / Zhongguo xiandaixiaoshuo jingpin (dingling juan), Xi'an, Shaanxi renmin chubanshe, 1995.

JIN Siyan, L'écriture féminine chinoise du $\mathrm{XX}^{e}$ siècle à nos jours. Trame des souvenirs et de l'imaginaire, You Feng, 2008.

LIU Li, [Les Femmes chinoises dans La Porte des roses] / 玫瑰门中的中国女人 - 铁凝与当代女性作 家的性别认同 / Meiguimen zhong de zhongguo nüren - tiening yu dangdai nüxing zuojia de xingbie rentong, Beijing, Beijing shifan daxue chubanshe, 2012.

MENG Yue 孟悦 et DAI Jinhua 戴锦华, [Émerger de l'horizon de l'histoire] / 浮出历史地表, Henan renmin chubanshe, 1989.

TIE Ning 玫瑰门, [La Porte des roses] / 玫瑰门 / Meiguimen, Beijing, Zuojia chubanshe, 2003.

\section{NOTES}

1. Jin Siyan, L'Écriture féminine chinoise $d u x^{e}$ siècle à nos jours. Trame des souvenirs et de l'imaginaire, Paris, You Feng 2008, p. 38-39.

2. DING Ling, [Chefs-d'œuvre du roman chinois (ÆEuvres de Ding Ling)], Xi'an, Shaanxi renmin chubanshe, 1995 , p. 7.

3. Ibid., p. 10-11. 
4. MENG Yue 孟悦, DAI Jinhua 戴锦华, [Émerger de l'horizon de l'histoire], Henan renmin chubanshe, 1989, p. 16.

5. TIE Ning, [La Porte des roses], Beijing, Zuojia chubanshe, 2003, p. 13.

6. Ibid., p 24.

7. Ibid., p. 87.

8. DING, [Chefs-d'œuvre du roman chinois...], op. cit., p. 10.

9. Ibid., p. 11.

10. TIE, La Porte des roses, op. cit., p. 287.

11. JIN, L'Écriture féminine chinoise du XX siècle à nos jours..., op.cit., p. 25.

\section{AUTEUR}

\section{XINYU HU}

Xinyu Hu est membre associée au CRHIA (Centre de Recherches en Histoire Internationale et Atlantique) à l'Université de la Rochelle. Elle travaille sur l'identité féminine dans la littérature chinoise moderne et contemporaine, l'altérité dans la littérature comparée et la didactique de la langue chinoise. Elle a publié : HU Xinyu, «Intermédiaires entre les entreprises étrangères et chinoises : les compradores et la modernisation de la société chinoise (1840-1937) », BACQUEGRAMMONT Jean-Louis, METZGER Laurent et RAIBAUD Martine (dir.), Paris, Les Indes savantes, 2021, p. 233-42. HU Xinyu, «La romancière Yan Geling : Ecriture du « je » au féminin en migration », in Cultures in Movement, M. RAIBAUD, M. SYMINGTON, I. UNTEA et D. WATERMAN (dir.), Cambridge Scholars Publishing, 2015, p. 265-277. 


\title{
L'écriture du corps et le discours féminin dans la littérature contemporaine chinoise : le cas de Lin Bai
}

\author{
Lanfang Guo
}

\section{NOTE DE L'AUTEUR}

Cet article est soutenu par le Fonds de recherche fondamentale pour les universités centrales de Chine

The article is supported by the Fundamental research funds for the central universities of China, N0650-ZK1091.

1 Après la Révolution culturelle (1966-1976) et avec la revalorisation de l'humanisme ${ }^{1}$, le corps reparaitt à l'horizon du public chinois, ainsi que dans la littérature chinoise. Vers le milieu des années quatre-ving-dix, avec la publication de Guerre solitaire /一个人的 战争 (1994) de Lin Bai 林白 (1958-) et de Vie privée / 私人生活 (1996) de Chen Ran 陈染 (1962-), l'écriture du corps / 身体写作 devient un phénomène culturel, tout comme un terme spécifique, quasi-synonyme d'écriture féminine.

2 C'est Ge Hongbing 葛红兵, critique littéraire connu, qui a avancé pour la première fois le terme : « écriture du corps » dans son article : «La littérature de nature individuelle et les écrivains du corps»/ 个体性文学与身体型作家. Il y souligne une nouvelle tendance culturelle et littéraire, apparue en Chine dans les années quatre-vingt-dix : la transition d'une littérature collectiviste, à visée morale, à une littérature individualiste, de nature narcissique, qui met le corps en valeur². Par "écriture du corps", Ge Hongbing fait référence non seulement aux textes de certaines d'écrivaines, mais à ceux de l'ensemble des écrivains dits "écrivains de la Nouvelle Génération " / 新生代 作家, c'est-à-dire post-Mao. Peu à peu, sans doute avec la vague des femmes écrivaines engagées, notamment sur le discours du corps, le terme d'« écriture du corps » en vient 
à revêtir, vers la fin des années quatre-vingt-dix, une forte coloration de gender et semble renvoyer surtout aux textes de ces écrivaines qui, telles Lin Bai 林白, Chen Ran 陳染, Hainan 海男, Wei Hui 卫慧 (1973-) et Mian Mian 棉棉 (1970-), ont orchestré masturbation, amour lesbien, désir féminin, tous thèmes alors inabordés ou presque.

Voici la description que donne Lin Shuming de l'« écriture du corps ", et qu'il faut sans doute tenir pour une définition :

L'«écriture du corps » est pour ainsi dire un mode de discours. "Écrire avec le corps " (...) consiste à emprunter les mots relatifs au corps pour décrire toute l'expérience des femmes dans leur résistance au logocentrisme et au carcan imposé par les hommes ${ }^{3}$.

身体写作”可以说是一种话语方式, “用身体书写”......是指用一种关于身体的语

言，去表达女性整体的、对抗逻各斯中心主义的全部体验，超越男人的束缚.

4 Les œuvres de Lin Bai et Chen Ran, à l'origine d'âpres controverses, ont été «réhabilitées" grâce au soutien des critiques littéraires qui les ont lues comme réponse à l'appel des féministes occidentales, notamment celui d'Hélène Cixous (1937-) : «Écris-toi : il faut que ton corps se fasse entendre »4. Elles constituent une avancée d'autant plus importante de l'écriture féminine dans la littérature chinoise, que leur écriture aide les femmes à retrouver, redécouvrir et revendiquer leur propre corps, auparavant occulté par le logocentrisme, à savoir le discours masculin phallocentrique, érigé en norme absolue de référence. Mais les textes de Wei Hui et de Mian Mian, qui ont pris le relais de Lin Bai et de Chen Ran, sont censés exprimer le pur plaisir charnel, ce qui va à l'encontre du féminisme en tant qu'il participe à la construction de la subjectivité ou de l'identité féminine. L'interdiction de Shanghai Baby /上海宝贝 de Wei Hui en 1999 signe la fin de l'«écriture du corps » dans la littérature chinoise. Ce phénomène de l'« écriture du corps » aura duré six ans, de 1994 à 1999.

5 S'appuyant sur l'analyse de Guerre solitaire de Lin Bai, le présent article pose la question de savoir pourquoi, sous la plume d'écrivaines inspirées par le féminisme occidental, cette imagination littéraire du corps féminin, qui a tout de même contribué à construire la subjectivité féminine, n'en a pas moins débouché sur une impasse et sa quasi disparition.

6 Guerre solitaire est un long récit relaté à la première personne, de coloration autobiographique. Lin Duomi 林多米, narratrice et protagoniste, raconte ses expériences du haut de ses 30 ans. Au lieu de suivre l'ordre chronologique, elle donne libre cours à ses souvenirs. Ce texte ainsi composé de souvenirs fragmentés, est marqué de sauts dans le temps et l'espace. Le père de Duomi meurt alors qu'elle est toute petite, et sa mère, gynécologue, est accaparée par son travail. Duomi vit dans la solitude dès son enfance. À l'âge de 5 ou 6 ans, elle commence à s'intéresser à son corps. Elle se glisse souvent en secret dans le grenier, qui recèle nombre de modèles d'organes génitaux, et joue à l'accouchement avec sa petite voisine. Elle grandit ainsi accompagnée de cette curiosité pour son corps. À l'âge de 19 ans, Duomi, passionnée de poésie, envoie des poèmes à une revue littéraire. Son talent reconnu, elle reçoit l'invitation d'une société de production de films pour y assurer un poste de dramaturge. Alors que les gens de son pays natal s'emballent pour cette jeune fille, l'invitation est annulée, car les responsables s'aperçoivent qu'elle a plagié le poème d'une autre personne. Elle fuit alors sa ville natale pour faire ses études universitaires dans une autre ville. Dix ans après, elle retourne travailler dans cette même société de production de films et y rencontre un jeune réalisateur, dont elle tombe follement 
amoureuse. Malheureusement, ce dernier ne semble pas être très sérieux dans cette relation, alors que Duomi est déjà enceinte. Désespérée, elle avorte et s'échappe encore une fois. Elle s'installe à Pékin, se marie avec un vieil homme qu'elle n'aime pas du tout, et mène désormais une vie complètement isolée des autres.

\section{Le désir féminin}

7 Pareille histoire pourra sembler banale. De fait, les intrigues elles-mêmes sont loin d'être originales, notamment pour les lecteurs d'aujourd'hui. Néanmoins, ce n'est pas là que résident le charme et la valeur de ce roman. Il choque tout d'abord par la description fort audacieuse du désir sexuel de Duomi. Le roman débute par l'extrait enchâssé d'une autre nouvelle de l'auteure, "Ceux qui s'aiment ne se séparent jamais » / 同心爱者不能分手 (1989), qui décrit la masturbation d'une jeune femme :

La guerre personnelle, c'est une main qui se frappe elle-même, un mur qui se dresse contre lui-même, une fleur qui se détruit elle-même. La guerre personnelle, c'est une femme qui s'épouse elle-même.

La femme se regarde dans un miroir, d'un amour narcissique et d'un masochisme caché. Toute femme mariée à elle-même est dotée d'une personnalité double aux faces inconciliables, semblable à une bête fantastique à deux têtes.

Elle se sent nager dans l'eau, sa main erre à la manière d'une vague. Quelque part au fond de son être coule une source qui jaillit sans se tarir jamais. Un liquide transparent la submerge. Elle se débat vaillamment, la bouche entrouverte sur des plaintes déchirantes. Sa main cherche, hésite, et plonge avec détermination, réussit à atteindre enfin l'endroit dégoulinant et touffu. Le majeur effleure l'entrée moelleuse et humide, cœur de tout ce désordre. Elle crie à ce contact comme électrocutée, elle s'engloutit elle-même. Eau elle est devenue, et sa main est un poisson ${ }^{5}$.

一个人的战争意味着一个巴掌自己拍自己, 一面墙自己挡住自己, 一朵花自己毁
灭自己。一个人的战争意味着一个女人自己嫁给自己。
女人在镜子里看自己, 既充满自恋的爱意, 又怀有隐隐的自虐之心。任何一个自
己嫁给自己的女人都十足地拥有不可调和的两面性, 就像一匹双头的怪兽。
她觉得自己在水里游动, 她的手在波浪形的身体上起伏, 体内深处的泉水源源不
断地奔流, 透明的液体渗透了她, 她拼命挣扎, 嘴唇半开, 发出致命的呻吟。她
的手寻找着, 犹豫着固执地推进, 终于到达那湿漉漉蓬乱的地方, 她的中指触着
了这杂乱中心的潮湿柔软的进口, 她触电般地惊叫了一声, 她自己把自己吞没
了。她觉得自己变成了水, 她的手变成了鱼。6

8 Après les débats des années quatre-vingt sur la sexualité en littérature, la description du désir sexuel n'est plus un tabou. Néanmoins, ce rendu si franc et osé de la masturbation, surtout d'une femme, et écrite par une écrivaine, a fort choqué le public d'alors. Certains critiques ont même qualifié ce roman de " pure pornographie » / 准黄 色小说7. Cette évocation du contexte culturel de l'époque fera mieux saisir les réactions du public :

L'abstinence sexuelle durant une longue période provoquait chez les femmes un sentiment de culpabilité et de honte vis-à-vis de leur propre corps. Ainsi, l'antipathie pour le sexe et l'habitude d'éviter d'en parler étaient presque devenues, dans l'inconscient des femmes traditionnelles chinoises, une ombre et un cauchemar qu'elles ne parvenaient pas à évacuer.

长期禁欲的结果, 导致了女性对自身的躯体充满罪恶和耻辱感, 因此, 对于性的 反感、忌讳，几乎成为传统中国女性潜意识中难以摆脱的阴影和䔅梦8.

9 Cette écrivaine, Lin Bai, avait osé aller au-delà de ce sentiment de culpabilité et de honte en exposant ces actes secrets que nul autre jusqu'alors n'avait osé aborder. On 
imagine dès lors combien la chose a pu choquer et déranger. Toutefois, loin d'être pornographique, cet extrait est très poétique. L'auteure, sans évoquer directement les membres du corps, recourt surtout aux métaphores, ainsi qu'à tout un champ lexical de la mer, qui suggère au lecteur une scène où un poisson s'ébat dans l'eau.

L'auteure clôt son roman exactement par ce même extrait qui, inséré sous forme de citation à l'incipit, devient partie intégrante du texte : Duomi, après s'être mariée avec le vieil homme, se sent «tellement isolée qu'il ne lui reste plus que sa propre ombre » / 《孤立得只剩下自己的一个影子了》". Elle voit dans cette situation une forme d'ostracisme personnel : « Duomi a été deux fois rejetée par la société : à l'âge de 19 ans, à cause du plagiat ; à l'âge de 30 ans, à cause du mariage » / 《多米十九岁时因为剽窃, 三十岁时因为嫁人, 她也曾两次遭到社会的拒绝 $»^{10}$.

11 C'est juste après cette déclaration que l'auteure place l'acte masturbatoire. Autrement dit, se sentant abandonnée de tous, Duomi se tourne vers elle-même, et précisément vers son corps, d'où le narcissisme, l'amour pour son propre corps qui accompagne son geste.

\section{Le narcissisme}

12 Selon Freud, le narcissisme a «une place dans l'évolution sexuelle régulière de l'humain ${ }^{11}$ ». Il postule un investissement originaire du moi, le " narcissisme primaire », et suppose qu'une partie de la libido soit investie sur le moi. La « libido du moi » sera par la suite dérivée vers les objets et devient la « libido d'objet ». Ladite libido d'objet, sous certaines conditions, peut être retirée de l'objet et réinvestie sur le moi, ce que Freud nomme « narcissisme secondaire ${ }^{12}$ ».

Cette théorie de Freud est pertinente dans le cas de Duomi. La narratrice, dès le tout début du roman, déclare que " ces regards sur elle-même et ces caresses d'elle-même ont débuté très tôt " / 《这种对自己的凝视和抚摸很早就开始了 ${ }^{13}$ ", à l'âge de 5 ans ou 6 ans. Cet intérêt pour son corps, précoce d'après la narratrice, peut s'expliquer par le narcissisme primaire de l'enfant, dont la libido est alors entièrement investie sur ellemême. À cette époque-là, Duomi, orpheline de père, vit la plupart du temps toute seule, car sa mère est accaparée par son travail. Faute de quelqu'un pour s'occuper d'elle, ellemême devient son seul objet d'investissement libidinal. En fait, la question est de savoir quel est le degré de connaissance du freudisme qu'a l'auteure elle-même. Cet amour narcissique persiste au cours de sa croissance. Elle se plaît toujours à se regarder dans le miroir qui, très lié au narcissisme en psychanalyse, a une importance particulière dans ce roman. Le premier chapitre s'intitule [Lumières dans le miroir] / 镜中的光. Par le miroir, Duomi découvre et observe son corps. Cette identification du corps, d'après Ye Zhiliang et Chen Siyuan, « est un moyen nécessaire pour Duomi de surmonter toutes les difficultés dans sa Bildung, et d'affirmer sa propre identité afin d'aboutir à sa maturité 》 / 《认识自己的身体成为多米克服成长中的迷焵, 确认自我性别身份并走 向成熟的必要途径 ${ }^{14}$. Duomi n'est pas la seule à aimer s'observer dans le miroir. Deux autres personnages féminins, Mei Ju 梅琚 et Zhu Liang 朱凉 vivent dans un espace où il $\mathrm{y}$ a partout des miroirs.

14 Plus tard, quand Duomi tombe frénétiquement amoureuse du réalisateur, elle cède une très grande partie de son investissement libidinal à ce dernier qui, Duomi en est pleinement consciente, n'est pas très sérieux dans leur relation sentimentale. Malgré 
cela, Duomi se résigne à n'être qu'une amante complètement invisible et à ne jouir que de "grâces" occasionnelles. Son estime de soi est ainsi réduite au minimum. Lorsqu'elle apprend que ce réalisateur a demandé la main d'une autre femme alors qu'elle subissait toute seule un avortement, elle est désespérée et ne voit plus d'avenir avec cet homme. Sa libido auparavant investie sur le réalisateur est alors retirée et se déplace complètement sur elle-même. Aussi percevons-nous dans cette scène finale non seulement une coloration narcissique intense, mais aussi un « moi » profondément conflictuel au sens où, comme le réclame la narratrice, elle est à la fois narcissique et masochiste : elle s'épouse elle-même, tout en se frappant, se détruisant.

La scène qui nous occupe a aussi un aspect provocateur et pervers. L'héroïne joue avec le regard de l'Autre: elle s'organise afin de se placer sous le regard de l'Autre, de susciter son désir. Elle s'adresse au grand Autre, c'est-à-dire non seulement à l'autre qu'elle désire, mais à tout le monde, y compris ceux qui lisent le texte, car elle se sent isolée de tous, jetée hors du regard de tous. De ce point de vue, ce qu'elle est en train de faire peut être considéré comme une vengeance vis-à-vis du manque de désir de l'Autre pour elle. La caractéristique essentielle du pervers, d'après Lacan, réside dans le fait qu'il se fait équivaloir à l'objet ${ }^{15}$. Dans cette scène de masturbation, Duomi, qui est apparemment un sujet actif, s'identifie au fond, de manière inconsciente, à l'objet du désir de l'Autre. Dans la vision lacanienne, « il y a toujours, structuralement, une base masochiste dans toute manifestation perverse $»^{16}$. Ainsi s'explique l'ambivalence, notamment le côté masochiste que l'on constate dans cet extrait.

\section{L'écriture du corps : une réponse à l'appel d'Hélène Cixous?}

Le roman Guerre solitaire, publié dans sa langue originale en mars 1994, a fait l'objet de controverses acharnées. Ce n'est que fin 1995 qu'on observe un changement d'avis du public, notamment avec l'interview - en date du 1er décembre 1995 - de Dai Jinhua, célèbre professeure à l'Université de Pékin, pour qui l'écriture des expériences personnelles des écrivaines "constitue sans doute une subversion du discours autoritaire de la société phallocrate, des normes établies par les hommes, et de l'image féminine désirée par les hommes» / 《构成对男权社会的权威话语、男性规范和男性 渴望的女性形象的颠覆 ${ }^{17}$ ». À la suite de Dai, de plus en plus de critiques littéraires s'engagent dans ce débat, et beaucoup d'entre eux prennent une position favorable visà-vis de Lin Bai, pour faire de ce roman un texte féministe d'une valeur particulière. Xu Kun徐坤, dans son ouvrage [Bateau dans la nuit à voix bitonale : l'écriture féminine des années quatre-vingt-dix], analyse en profondeur Guerre solitaire. Elle compare le texte de Lin Bai au fameux texte d'Hélène Cixous, «Le rire de la Méduse ", traduit et publié en Chine en 1992, pour signaler plusieurs points communs entre les deux et en tirer cette conclusion :

Lin Bai a façonné Guerre solitaire complètement d'après l'idée essentielle d'une "écriture qui retourne au corps féminin", préconisée par Hélène Cixous. Le résultat de sa pratique, nous pouvons dire, fait parfaitement réaliser les conséquences [destructives] prévues par les théories de Cixous.

林白的完全按照埃莱娜·西苏所指“返归女性躯体写作”理论主旨来操作的《一个人 的战争》, 其写作实践的结果, 又恰好, 几乎可以说是完整无缺地使西苏理论预 测的后果得以实现 ${ }^{18}$. 

façonné d'après les théories féministes » / 《“一个人的战争”是一部完全按照女性主义 理论操作的精致的女性本文 ${ }^{19}$ ". De fait, dans 《Le rire de la Méduse », Cixous appelle les femmes « refoulées de la culture ${ }^{20}$ » à s'écrire, à écrire par leur corps. De plus, les thèmes de la masturbation, du narcissisme et du vol, déjà développés chez Cixous, sont aussi fortement présents sous la plume de Lin Bai. Nombre de commentateurs partagent l'avis de Xu Kun. L'« écriture du corps» chez Lin Bai et certaines autres écrivaines, comme Chen Ran 陈染 et Xu Xiaobin 徐小斌, passe pour une tentative d'interpréter leur propre corps, qui n'était défini jusqu'alors que par l'homme, et limité à une sphère extrêmement bornée. En ce sens, l'« écriture du corps » constitue de fait une façon pour les femmes de lutter contre la culture phallocratique.

Une voix très différente se fait entendre : si les femmes écrivains chinoises, censément « influencées » par les féministes occidentales, ont toutes choisi d'écrire sur leur «vie privée ", et que leur écriture est très marquée par le narcissisme, c'est qu'elles ont mal compris ces théories féministes. Tel est l'un des points de vue de Meng Gang 孟岗 dans sa thèse : «L'utopie du corps à l'ère de consommation ». D'après Meng, ce que propose Hélène Cixous, c'est de créer, à partir du corps, un autre discours poétique qui se distingue radicalement du discours phallocentrique. Autrement dit, ce qu'elle vise, au fond, c'est le langage actuel, régi par le code de la culture phallocentrique. Néanmoins, les femmes écrivains de l'époque n'ont fait que relater leurs secrets sexuels. Cela, d'après Meng, constitue la différence essentielle et radicale entre les avancées théoriques de Cixous et l'écriture des femmes écrivains chinoises des années quatrevingt-dix ${ }^{21}$.

Hélène Cixous, comme Luce Irigaray et Julia Kristeva, oppose l'expérience corporelle des femmes aux modèles «symboliques». Cixous et Irigaray soulignent toutes deux l'importance de l'écriture du corps et de la sexualité pour les femmes :

[...] if women are to discover and express who they are, to bring to the surface what masculine history has repressed in them, they must begin with their sexuality. And their sexuality begins with their bodies, with their genital and libidinal difference from men $^{22}$.

De ce point de vue, l'attention particulière que les écrivaines chinoises accordent aux expériences corporelles des femmes, se manifeste en ce que leurs textes rejoignent les théories psychanalytiques féministes. Sachant que la sexualité était auparavant un thème tabou chez les écrivaines, et que seuls les hommes disposaient du corps féminin, la littérature chinoise fait un grand pas en amorçant pareille description du corps et du désir féminin.

Ce que vise au fond Cixous - note Meng Gang - c'est le langage actuel chinois en son discours phallocentrique. Mais Duomi a une attitude ambiguë vis-à-vis de la culture phallocentrique. Loin d'être une femme consciente de la subjectivité féminine ou, si l'on préfère, de son identité de femme, Duomi est très marquée par sa volonté d'être l'objet du désir de l'homme. Dans sa relation avec le réalisateur, se perçoit sa volonté de se sacrifier, d'assouvir les désirs de l'autre au mépris des siens et de sa propre existence comme « sujet ». Cette bribe de monologue intérieur l'atteste :

En fait, je n'ai jamais eu d'orgasme dans nos rapports sexuels, jamais eu de plaisir. J'éprouvais même parfois un malaise physique. Mais je pensais qu'il en avait besoin, puisqu'il était un homme. Je devais alors apporter ma contribution. S'il ne venait pas pendant plusieurs jours, j'avais peine à survivre, je pensais à me suicider. 其实我跟他做爱从未达到过高潮, 从未有过快感, 有时甚至还会有一种生理上的 
难受。但我想他是男的, 男的是一定要要的, 我应该做出贡献。只要他有几天不

来我就觉得活不下去, 就想到自杀 ${ }^{23}$. profondément dans le corps de cette fille» / 《把自己变成物的愿望深深藏在这个女孩 的体内 $»^{24}$. Il est donc évident qu'au lieu d'être un "sujet " indépendant, Duomi se contente d'être l'« objet » désiré de l'homme. Si c'est de ce principe que l'auteure part dans son écriture du corps, cette exposition du corps et du désir féminin ne peut servir qu'à renforcer la soumission des femmes face à la puissante culture phallocratique et à satisfaire l'envie voyeuriste des hommes.

Ainsi pouvons-nous tenter de donner une réponse à notre question de départ: pourquoi l'écriture du corps, une écriture féminine censée subvertir le discours dominé par le phallus, n'a-t-elle pu aller plus loin dans la Chine de ces années-là ? Notre analyse de Guerre solitaire nous montre que la femme sous la plume de Lin Bai, et souvent chez d'autres écrivaines, affronte constamment une contradiction : d'une part, elle s'impose par son corps en tant que « sujet » féminin (au lieu d'un objet, un autre); d'autre part, elle est sans cesse trahie par sa volonté, plutôt inconsciente, d'être l'objet du désir masculin. D'où le dilemme qu'affronte l'«écriture du corps » en contexte chinois : même si elle annonce une vraie "voix féminine ", le fait que le corps féminin soit exposé renforce la situation de la femme d'être «épiée " par l'homme, et à plus forte raison si ce dernier est réalisateur... Tels sont peut-être les facteurs qui l'ont menée à l'impasse.

\section{BIBLIOGRAPHIE}

CASTANET Hervé, La Perversion, Paris, Economica, 1999.

CIxous Hélène, « Le rire de la Méduse », L'arc, n 61, 1975, p. 39-54.

DAI Jinhua 戴锦华, [Comme dans le miroir] / 犹在镜中 / Youzai jingzhong, Beijing, Zhishi chubanshe, 1999.

DING Laixian 丁来先, [《 La littérature féminine et autres »] / 女性文学与其他 / Nüxing wenxue yu qita, [China Reading News] / 中华读书报 / Zhonghua dushu bao, 20/12/1995.

FREUD Sigmund, Pour introduire le narcissisme, Paris, Payot, 2012 [1914].

FREUD Sigmund, Introduction à la psychanalyse, Paris, Payot, 1998 [1917].

GE Hongbing 葛红兵, [《 La littérature de nature individuelle et les écrivains du corps »] / 个体性 文学与身体型作家 / Geti xing wenxue yu shenti xing zuojia, 山花 / Shanhua, 1997:03, p. 75-78.

JONES Ann Rosalind, “Writing the Body: Toward an Understanding of 'L'Écriture Féminine”', Feminist Studies, vol. 7, 2:1981, p. 247-263.

LIN Bai 林白, «Ceux qui s'aiment ne se séparent jamais », in La Chaise dans le corridor, CHEVALEYRE Véronique (trad.), Paris, Bleu de Chine, 2006, p. 55-102. 
LIN Bai 林白, Guerre solitaire /一个人的战争 / Yigeren de zhanzheng, Beijing, Zuojia chubanshe, 2009.

LIN Shuming 林树明, [《 Le corps féminin dans la culture de consommation»] / 消费文化中的女性 身体 / Xiaofei wenhua zhong de nüxing shenti, 洛阳师范学院学报 / Luoyang shifan xueyuan xuebao, 6:2005, p. 9-12.

MENG Gang 孟岗, [L'Utopie du corps à l'ère de la consommation] / 消费时代的身体乌托邦 / Xiaofei shidai de shenti wutuobang, thèse de doctorat, Université du Zhejiang, 2004.

TAO Jiajie 陶佳洁, [ « Les pensées féministes et l'écriture féminine de la Nouvelle période en Chine »] / 中国新时期女性主义思潮与女性写作 / Zhongguo xin shiqi nüxing zhuyi sichao yu nüxing xiezuo, 当代文坛 / Dangdai wentan, 6:2017, p. 46-50.

XU Kun 徐坤, [Bateau dans la nuit à voix bitonale : l'écriture féminine des années 90] / 双调夜行 船一九十年代的女性写作 / Shuangdiao yexing chuan : 90 niandai de nüxing xiezuo, Taiyuan, Shanxi jiaoyu chubanshe, 1999.

YE Zhiliang 叶志良 et CHEN Siyuan 陈思远, [ « Sur la nature autobiographique de Guerre solitaire de Lin Bai 》] / 论林白《一个人的战争》的自传性 / Lun Lin Bai “Yigeren de zhanzheng” de zizhuan xing, 金华职业技术学院学报 / Jinhua zhiye jishu xueyuan xuebao, 4:2009, p. 48-52.

\section{NOTES}

1. Pendant la Révolution culturelle, l'anéantissement de l'humanisme a été sans précédent. À la fin des années 1970 et dans les années 1980, beaucoup de débats ont été déclenchés afin de remettre en valeur "l'humanisme » dans la société chinoise. Ce qui a été déclenché en même temps, c'était la 《fièvre culturelle »/ 文化热 et le mouvement des traductions visant à introduire les pensées occidentales.

2. Voir GE Hongbing 葛红兵, [ " La littérature de nature individuelle et les écrivains du corps »] / 个体性文学与身体型作家 / Geti xing wenxue yu shenti xing zuojia, 山花/ Shanhua, 3:1997, p. 75-78. L'auteur signale dans un autre texte, [Quelques propos sur la « Nouvelle Génération »] / 闲话 《新生代», http://www.ilf.cn/Theo/94208.html, consulté le le 20/11/2017, qu'il usa pour la première fois du terme d'écriture du corps dans l'une de ses lettres à l'écrivaine Mian Mian.

3. LIN Shuming 林树明, [《 Le corps féminin dans la culture de consommation »] / 消费文化中的女 性身体, 洛阳师范学院学报 / Luoyang shifan xueyuan xuebao, 6:2005, p. 9.

4. cixous Hélène, «Le rire de la Méduse ", L'arc, n 61, 1975, p. 43.

5. LIN Bai, «Ceux qui s'aiment ne se séparent jamais ", in La chaise dans le corridor, CHEVALEYRE Véronique (trad.), Paris, Bleu de Chine, 2006, p. 95-96.

6. LIN Bai 林白, Guerre solitaire /一个人的战争, Beijing, Zuojia chubanshe, 2009, p. 1.

7. DING Laixian 丁来先, [《La littérature féminine et autres»], [China Reading News] / 中华读书

报 / Zhonghua shubao, 20/12/1995.

8. TAO Jiajie 陶佳洁, [«Les pensées féministes et l'écriture féminine de la Nouvelle période en Chine»] / 中国新时期女性主义思潮与女性写作, 当代文坛 / Dangdai wentan, 6:2017, p. 48.

9. LIN, Guerre solitaire /一个人的战争, op. cit., p. 182.

10. Ibid., p. 183.

11. FREUD Sigmund, Pour introduire le narcissisme, Paris, Payot, 2012 [1914], p. 38.

12. Ibid., p. 44-50.

13. LIN, Guerre solitaire /一个人的战争, op. cit., p. 1. 
14. YE Zhiliang 叶志良 et CHEN Siyuan 陈思远, [ « Sur la nature autobiographique de Guerre solitaire de Lin Bai »] / 论林白《一个人的战争》的自传性, 金华职业技术学院学报 / Jinhua zhiye jishu xueyuan xuebao, 4:2009, p. 50.

15. CASTANET Hervé, La perversion, Paris, Economica, 1999, p. 76.

16. Ibid., p. 77.

17. DAI Jinhua 戴锦华, [Comme dans le miroir] / 犹在镜中, Beijing, Zhishi chubanshe, 1999, p. 198.

18. XU Kun 徐坤, [Bateau dans la nuit à voix bitonale : l'écriture féminine des années quatrevingt-dix] / 双调夜行船一九十年代的女性写作, Taiyuan, Shanxi jiaoyu chubanshe, 1999, p. 64.

19. Ibid., p. 73.

20. cIXous, « Le rire de la Méduse », op. cit., p. 41.

21. Voir MENG Gang 孟岗, [L'Utopie du corps à l'ère de la consommation] / 消费时代的身体乌托 邦, 2004, p. 83.

22. JONES Ann Rosalind, “Writing the Body: Toward an Understanding of 'L'Écriture Féminine”, Feminist Studies, vol. 7, 2:19, p. 252.

23. LIN Bai, Guerre solitaire /一个人的战争, op. cit., p. 172.

24. Ibid., p. 125.

\section{AUTEUR}

\section{LANFANG GUO}

Guo Lanfang, docteure en littérature de Sorbonne Paris Cité, est actuellement maître de conférences au Département de français à l'Université de Xiamen (Chine). Spécialisée en littérature comparée, elle s'intéresse particulièrement à l'interaction entre la littérature et la psychanalyse. Ses principales publications récentes : «Le stream of consciousness à la chinoise : le cas de Wang Meng ", Loxias, $n^{\circ}$ 70, 2020 ; « L'étrangère ou les étrangères : à propos du roman Serpent à plumes de Xu Xiaobin », Nouvelles humanités, Chine et Occident, n 3, 2020 ; «Vers un nouvel imaginaire du féminin : l'écriture privée de Chen Ran ", Nouveaux imaginaires du féminin, 2019. 


\title{
From Subjects to Bodies: Ah Cheng's The King of Children and Wang Gang's Ying-ge-li-shi
}

\author{
Melinda Pirazzoli
}

1 What is a subject? What is a body? Does the fictional representation of a human body necessarily imply subjectivity? This study will argue that a comparison between Ah Cheng's 阿城 (1949-) The King of Children / 孩子王 (1995) and Wang Gang's 王剛 (1960-) 英格力士 (2005), a phonetic transliteration for "English", is useful to understand that the notions of "subjects" and "bodies" do not coincide.

2 It would appear that these two novels have so many narrative details in common, the reader cannot avoid the impression that when Wang Gang wrote his novel, he might as well have been relying on The King of Children to underscore the radical and dramatic changes that had occurred during the previous twenty years. Both stories are set during the Cultural Revolution (1966-1976) in remote areas of China, they are both coming-of-age novels that describe the ways in which a group of students relate to their teachers and parents, and both represent the ways in which the younger generation is affected by adults and by a dictionary. The earlier work, in line with the intellectual ferment of the "High culture fever" / 文化热 movement, describes the birth of a subject willing to emancipate himself from the Chinese Communist Party's modalities of apprehending and producing knowledge. It therefore celebrates the birth of what we may define as a "new epistemic subject". Wang Gang's novel, on the other hand, does not ascribe any value to epistemology, but rather focuses on the ways in which a young group of students challenges the authoritarian powers of both institutions and adults in order to claim control over their own bodies and lives. In fact, the Bildung of the young protagonists in Ying-ge-li-shi is described in terms of experiential knowledge which is apprehended only at the level of the body and not at that of the mind. In other words, this novel celebrates the birth of a middle class habitus and lifestyle which gives rise to new concepts of privacy.

3 The King of Children is a novella that describes the feats of a young and inexperienced “educated youth" / 知青 named Lao Gan 老杆. He is sent to Yunnan to teach a class of 
young students. When he sees that the textbook that he is forced to adopt contains nothing but political propaganda, he abandons it, and instead teaches his students how to write free compositions relying only on their personal experiences.

4 One way to tackle this novel is to consider it as Ah Cheng's attempt to deploy a discourse on subjectivity, because, as Rey Chow observes,

[if we understand subjectivity] in terms of the material relationships among human beings as participants in a society - relationships that are in turn mediated by the collective cultural activities of speaking, reading and writing - then it is an issue that is forcefully present in modern Chinese literature and culture as it is in the West, even if it's not named as such. ${ }^{1}$

What is at stake in Ah Cheng's novel is Lao Gan's attempt at constructing a world centered around the production of written texts that can represent a diegetic space very distant and different from the one offered by narratives coercively imposed by cultural and political institutions.

6 At the beginning of the novella, the inexperienced teacher puts himself in a subaltern condition:

Are there any rules for teaching this class? I can see you can't really make it up as you go along. Since the National Textbook Unification reform, textbooks for each subject have been the same throughout the whole country, but there should be also a unified standard for teaching or people won't know if they are teaching properly. For example, how many sections should a text be divided into? What is the main idea of each section? What is the overall theme? What is the composition method? I might interpret these things one way, but another school might teach it another way. Language isn't like math - one plus one makes two wherever you are in the world. Language courses should have rules so that we can be on firm ground. ${ }^{2}$ (p. 146)

这课的教法是不是有规定? 恐怕还是不能乱教。课本既然是全国统一的, 那怎么 教也应该有个准，才好让人明白是教对了。比如说吧，一篇文章，应划几个段 落?段落大意是什么? 主题思想又是什么? 写作方法是怎么个方法? 我说是这样了, 别的学校又教是那样。这语文不比数学。一加一等于二, 世界上哪儿都是统一

的。语文课应该有个规定才踏实。 ${ }^{3}$ (p. 120)

7 This complex and ambivalent passage raises some questions: does the opacity of language threaten the stability of mechanisms of power? Is there a unitary hermeneutic approach to propaganda? This emphasis on the lack of alternative discursive possibilities becomes a sort of obsession for the teacher, who gradually discovers the importance and usefulness of the dictionary which provides readers with etymological and epistemological grounds. The dictionary can transform his students from passive recipients of knowledge into writing subjects endowed with agency and capable of transcending the narrow boundaries imposed by Maoist rhetoric. After discovering its almost magical power, the formerly subaltern teacher polemically complains:

The teaching material is unified all right, I can't even tell the difference between the Chinese lessons and the political lessons. My class is learning Chinese - what are they going to do when they return to their teams? Become party secretaries? (p. 164) 教材倒真是统一, 我都分不清语文课和政治课的区别。学生们学了语文, 将来回 到队上, 是要当支书吗? (p. 131)

Wang $\mathrm{Fu}$ 王福, the most diligent student of the class, is completely captivated by the dictionary. He tries first to obtain it by cheating Lao Gan, then he decides to copy it. In this regard, Rey Chow observes: 
Wang Fu's persistence in copying the dictionary and his eventual "success" in producing a system of coherent writing are the symptoms of a child who has been properly "interpellated" into the system of learning - to use the term from Althusser. Wang Fu's perseverance, seriousness and ability to work hard are all part of the process by which the school as an apparatus of ideology solicits the voluntary cooperation of the participants. ${ }^{4}$

What Chow fails to mention, however, is that the person who teaches Wang Fu to produce a system of coherent writing is also the one who has deliberately alienated himself from the school system by refusing to adopt the textbook. Furthermore, the teacher is eventually compelled to leave the school. Yet he has not only successfully performed his duty, he has also left an important trace. By the end of the novel, his student Wang $\mathrm{Fu}$ eventually writes a very moving free composition in which he declares that he is determined to speak out loud on behalf of his own mute and bullied father:

My father is one of the strongest men in the world. No one on our team can beat him at carrying sacks of rice. My father also eats more than anyone in the world. My mother lets him eat all the food we have. This is right, because my father has to work, and his wages support our whole family. But my father says: "I am not as strong as Wang Fu, because Wang Fu can read and write." My father isn't able to talk but I understand what he means. I know that people in the team bully him. Therefore, I want to study and learn how to speak for him... (p. 176)

我的父亲是世界中力气最大的人。他在队里扛麻袋, 别人都比不过他。我的父亲 又是世界中吃饭最多的人。家里的饭, 都是母亲让他吃饱。这很对, 因为父亲要 做工, 每月拿钱来养活一家人。但是父亲说: 《我没有王福力气大, 因为王福在 识字。》 父亲是一个不能讲话的人, 但我惲他的意思。队上有人欺负他, 我明 白。所以我要好好学文化, 替他说话。父亲很辛苦, 今天他病了, 后来慢慢彴起 来, 还要去干活, 不愿失去一天的钱。我要上学, 现在还替不了他。( p. 157)

The Luxunian outcry to save the children at the end of his most famous short story, "A Madman's Diary” / 狂人日记 (1918), is overturned. While in the modern story the author denounces the cannibalistic nature of Chinese society and urges his adult readers to spare the future generations of children from being cannibalized, Ah Cheng describes a devout son who wants to rescue his mute father from being bullied. The King of Children assesses the ethical mission of the filial son, who needs to fulfill his role in order not only to save his father from a cruel and senseless society, but also to "plant the seeds" of a fairer and more brilliant future. It is no accident that Lao Gan leaves the precious dictionary to Wang $\mathrm{Fu}$, who will hopefully be able to emancipate himself from the dull rhetoric of the Chinese Communist Party's political propaganda by transforming himself into a subject capable of producing new meanings.

11 Written twenty years later, in 2005, Ying-ge-li-shi also describes the complex relationships between students, teachers and parents. This coming-of age novel describes Love Liu's 刘爱 growth into adulthood. He is forced to cope with a dysfunctional family - indeed, his father and mother are far from exemplary in their conduct. While being successful in his career as an architect, his father is subservient to all forms of authority, and is cruel and despondent toward his own son and all people who occupy a lower social status. His mother, also an architect, betrays her husband with the school principal. Furthermore, instead of showering him with affection and love, she constantly monitors and controls the politically correctness of his behavior. His classmates, Garbage Li 李垃圾 and Sunrise Huang 黄旭升, are extremely evil. Garbage Li is outrageously violent and cruel. Sunrise Huang first accuses their English teacher of sexual harassment just because she discovers that he loves another woman, 
then persuades Garbage Li to kill his mother's lover, and finally kills Garbage Li (though this is by accident). The political authorities, Director Fan 范主任 and Commander Shen 申总, are cruel impostors and sexual harassers, determined to reify the bodies of the women onto whom they wish to discharge their libidinal energies. The only characters endowed with gentleness or humanity are the English teacher, Second Prize Wang 王亚军, and the Uyghur teacher 阿吉太. Their role is underscored right at the beginning of the novel:

We had stopped learning Russian the year before, and from that day we would not be learning Uyghur. We weren't really interested in languages. We were interested only in women like Ahjitai. She might have been a teacher, but the curve of her neck and her tears were things I yearned for at dawn much more than the sun. ${ }^{5}$ (p. 1-2)

我门从去年开始就不学俄语了, 从今天开始就不学维语了。我们对任何语言都不 感兴趣, 我们只对阿吉太这样的女人感兴趣, 尽管她是女老师, 可是她的脖子和 她的眼泪都是我在黎明时比太阳还渴望的东西。 ${ }^{6}$ (p. 2)

There is a clear shift of focus from epistemology to both epistemophilia (investment of eroticism in the visual) and scopophilia (erotic pleasure in looking). As Toril Moi argues, "the body held in the field of vision is par excellence the object of both knowing and desire: knowing as desire, desire as knowing". ${ }^{7}$

Anyone familiar with Wang Gang's narrative technique well knows that he frequently relies on what Gérard Genette calls prolepses (anticipation of an event that will occur later in the story) ${ }^{8}$ and can thereby easily foresee the ending of the novel: that Love Liu will make love to Ahjitai.

While both novels are set during the Cultural Revolution, the social space created by Wang Gang is completely different from the one created by Ah Cheng. It is clearly a hybrid and very unstable space where different ethnic groups and very different habitus meet (to use Pierre Bourdieu's term). This notion which, among other things, include personal or social classes' affective dispositions and dispositions of perceptions in relation to the urban space, is very helpful here because it allows us to grasp the intimate and emotional bond between the protagonist Love Liu and the urban space in which he lives. It is not a coincidence that both his mother and father are architects, and that his father in particular has created the very space in which his coming-of-age experience occurs: the school.

This is Wang Gang's quite telling description of Love Liu's "learning environment":

Our yellow school building, with its three-gabled roof, was also my father's work. I still have the colored architectural drawings he made of it all these years ago. The steep, Russian-style roof was covered in green metal, making the building look like a man wearing a yellow overcoat and a green hat. In Chinese "wearing a green hat" means to be cuckolded, and our school stood there proudly, seemingly unaware that its wife was sleeping with someone else, as the sounds of our songs and laughter - and of course of students reciting - reverberated out of the windows where its eyes would be. (p. 14)

我们学校淡黄色的山字形的楼也是父亲设计的, 直到现在我还保留他当时画的彩 色的效果图. 俄罗斯式的斜屋顶, 是用绿色的铁皮搭起来的, 有些像是一个穿着 米黄色大衣的人戴了一顶绿帽子, 他的老婆跟别人睡了, 他不知道, 仍然神气 活现地站在那儿, 让我们这些孩子的歌声和笑声, 对了还有读书的声音, 从他 的像是眼睛一样的简户里传出来。(p. 18)

16 The school, a public space created by Love Liu's father, is rhetorically transformed into something reminiscent of a "physical body" exposed to public ridicule. The laughter 
and songs of students completely indifferent to the disempowerment of previously recognizable forms of authority suggests that the school can no longer be read as a site for self-construction.

In an important study entitled Masculinity Besieged, Zhong Xueping has observed how male protagonists of post-Maoist narratives often underwent a process of demasculinization, or in other terms a loss of manhood, that eventually entailed their sexual impotence. ${ }^{9}$ The group of narratives produced by the group of writers belonging to the "Late-born generation" / 晚生代 or "Rupture movement" / 断裂, which deal with the same issues and themes as Wang Gang, ridicules and demystifies everything and everyone that was formerly considered as an authority.

In a third important prolepsis, which as we shall see at the end of this study foreshadows the conclusion of the novel, the Chinese author broadens his scope of analysis and links Love Liu's father to the political leaders of Urumchi. Represented in the act of painting Chairman Mao's profile, Love Liu's father is first ridiculed and then attacked by Director Fan and Commander Shen for having (correctly) portrayed Chairman Mao's profile with "only" one ear. Love Liu's father tries in vain to explain the law of perspective to the political leaders. Yet since nobody, including his own son, is willing to listen to him, Love Liu's father ends up betraying the law of perspective and adding a second ear to Chairman Mao's profile. He ultimately has no alternative other than to abide by the political leaders' orders and add an ear. What is at stake here is an expedient to address the ways the body of the most important Chinese leader, Chairman Mao, is transformed into a mere icon or, to use Baudrillard's term, a simulacrum.

19 The English teacher, Second Prize Wang, is the character that ushers Love Liu into a new sensual and sensory world. He seems to be the only counterpart to his own already disempowered father. The importance of bodily senses in this novel becomes evident in the description of Love Liu's first meeting with his English teacher, which occurs in a bathroom. The first thing the young boy notices about him is his teacher's long penis:

I couldn't help peering over. I cringed in shock - it was enormous. I'd never seen a big one. When I was small I went with father to the bathhouse, and I saw that every man had one. The world is amazing, I thought. The image of those comfortably warmed symbols of manhood flopping about in the misty bathhouse became deeply engrained in my memory. (p. 17)

我忍不住地朝他那边一看, 吓得我一哆嗦, 太大了。从没有见过哪个男子长得像 他那么大。小的时候, 跟着爸爸走进男澡堂, 看到每一个男人长着一个这样的 东西, 我就感到世界不可思议, 在室内的雾气中, 被熟得有些舒服的象征物们 在晃动。他们无数次地进入我的眼穹，留在我的记忆深处。(p. 21)

This depiction, which undeniably bears some sort of Proustian flavor, stands in sharp contrast with those familiar Freudian narratives that celebrate the castration complex. Its Proustian flavor is suggested by an all-too-clear reference to the non-temporal nature of the unconscious as well as to the movement of the unconscious from the past to the present, and then once again to the past.

21 As Peter Brooks reminds us in his Body Work, "the castration complex brings an end to the CEdipal stage by instituting the law with the superego as its representative" ${ }^{10}$ In Ying-ge-li-shi, the figure of the destitute father is in no position to stand for either the law or for any form of authority. On the contrary, the English teacher and his long penis become the very foundation upon which Love Liu relies to construct his own self. 
In fact, the reference to the penis may be interpreted as an expedient to address Love Liu's formation of self according the Lacanian tripartite schema of language (the real, the imaginary and the symbolic). Love Liu's contemplation of penises allows the creation of a symbolic order which refers to the ways in which the self, its Other and the socio-historical world are configured and structured by the social and linguistic realm of law. By reading the penis as a sign, Love Liu is thus able to gain access to the "imaginary" which, as Lacan suggests, is the foundation of the subject's relation to itself. The clear metonymical link as well as the comparison between the father's and the English teacher's penises may also be read as a way to configure a new social order. It should be clear by now that Ying-ge-li-shi focuses neither on subjectivity nor on epistemology. Love Liu's coming-of-age experience is nothing but the Bildung of what we may define as "embodied consciousness", or alternatively, a form of knowledge which relies on bodily perceptions (scopophilia and epistemophilia) rather than on the mind.

The passage's explicit references to "comfortably warmed symbols of manhood" compels readers to move away from the Lacanian notion of law (father's law) to the laws of the phenomenological social world. Martin P. Jay, who in his Downcast Eyes has studied the sense of sight after the advent of positivism, has clearly shown that with advent of phenomenologists and existentialists such as Heidegger, Merleau-Ponty, and Sartre among others, there has been an increasing discomfort with ocularcentric perspectives (perspectives that privilege sight over the other perceptions). ${ }^{11}$ While on the one hand the sight of the size of his penis confirms in a very matter-of-fact way Second Prize Wang's authoritarian position as well as his prestige, on the other, it is also undeniable that the English teacher is connected to the sense of sight only when the author needs to deal with the themes of scopophilia and epistemophilia. In one of Love Liu's many descriptions of his English teacher, as well as in the passage that describes Love Liu's father in the process of painting Mao's icon, we understand that this novel also "fights" against ocularcentrism. This "fight" is configured by associating Second Prize Wang with the senses of sound and smell:

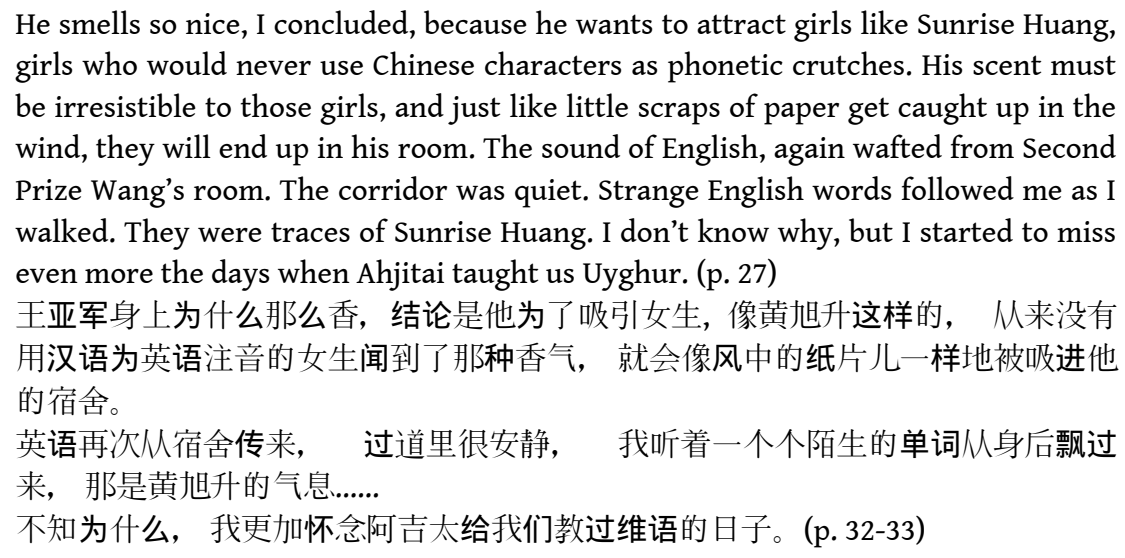

Passages like this enable as to understand why Wang Gang has chosen Ying-ge-li-shi as a title for his novel. Ying-ge-li-shi, unlike 英语 / english, is only a phonetic transliteration that carries no counterpart meaning; as Walter Benjamin observed in his essay "The Mimetic Faculty", what distinguishes the spoken from the written language is its sensuous quality. ${ }^{12}$ Here the references to sound and smell suggest that the English teacher appeals to the students' bodily senses rather than to their minds. Unlike in Ah Cheng's novella, the reader has no clue about the content of Second Prize Wang's 
lesson. The only thing he knows is that he resorts to Linguaphone English as a strategy to teach it and that he demands that students never use Chinese as phonetic crutches.

"Linguaphone English" and the "French eau de cologne" should then be read as a portmanteau to represent what Bourdieu would define as the habitus. As Don Slater explains:

The habitus is a structure of dispositions, schemes of classification, rules, expectations and so on which predispose the individual to certain choices and actions [...]. Habitus is unconscious but in a practical rather than a psychoanalytic sense. For example it is both learned and acted out through sensory and bodily experiences, the subtexts of encounters rather than explicit statements. Above all the habitus is embodied and learned and acted out at the level of the body. ${ }^{13}$

Second Prize Wang proposes not only a very sensual and attractive lifestyle to his students, a lifestyle and habitus which was unknown to the Chinese during the Cultural Revolution, but also an alternative approach to knowledge, an approach which is exemplified through the English dictionary. While in Ah Cheng's novel the dictionary provided students with an alternative access to knowledge, the English dictionary is here a diegetic element which semantically connects and decodes this new sensual world and the alien habitus into which Second Prize Wang is trying to usher his students:

[Second Prize Wang] guessed what I was drawn to. "Do you know that word? Dictionary?" "English dictionary?" I asked. He nodded. "Is it a comprehensive dictionary?" I asked. "When you know all the words in the dictionary, you will be able to live like a real English gentleman. You can even have a better life than ordinary folks in England because you will be educated." "What does a gentleman look like?" I asked.

He thought for a moment and then said: "Like your father."

His answer disappointed me. Like my father? What kind of man is my father? I thought of the glasses he wore and the frightened expression that was always on his face. Sill I had to ask, "Do you know my father?" "[...] I chatted with your father when we were in line at the canteen. He was very polite and did not push and squeeze like the others." (p. 47)

[王亚军] 似乎意识到了我的目光, 说 :

《认识这个单词吗? 字典》

我说：《英汉字典?》

他点头。

我说 : 《是大字典?》

他说: 《这里的单词, 如果你都会了, 那就可以像一个地道英国绅士那样, 在 那儿生活。你甚至可以超过他们那儿一般的人、因为你水平很高》。

我说 : 《绅士是什么样的人?》

他想了想, 说 : 《就是你爸爸那样的人》。

他的话让我失望, 像爸爸那样的人? 我爸爸是什么样的人? 我想起了他 戴眼 镜, 以及经常显出恐惧的神情, 但是, 我还是说: 《你认识我爸爸? 》

《.......我跟你爸爸说过话, 那是在食堂排队打饭的时候, 他很谦让, 不像他们那 样拼命地挤。》( p. 54-55)

Bourdieu's notion of habitus is here evoked in two specific ways: in terms of style, taste and social class as well as in terms of lifestyles and manners, two aspects that Wang Gang's narrative deploys in a meticulous way. Far from being depicted as a political colonial power, England becomes rather the site where narratives of privacy can be developed and cultivated. In fact, Peter Brooks, who links the increasing popularity of the novel in Europe to the upper middle class's need for leisure, clearly shows the close interconnection between the increasing importance of private life (which is the object 
of the novel's concern) and the process of privatization of the body. ${ }^{14}$ In other words, both the English teacher and the English dictionary teach and show these young students a very different world from the one to which the Chinese were accustomed during the Cultural Revolution. There is no longer a collective body, but personal and individual behaviors. The English teacher's emphasis on middle class lifestyle, manners and habitus as well as the English dictionary's reference to the term "masturbation" end up deploying a complex discourse about bodily perceptions and bodily behaviors.

In fact, as Andrew Bennet suggests in his article "Language and the Body", "selftouching, [according to Derrida's On touching is] a dream of the reflexive or specular autonomy of the language of self-presence or self-consciousness". ${ }^{15}$ The interdependence between self-eroticism and eroticism is articulated in this narrative via the English dictionary that Love Liu finds in Ahjitai's room:

It was the dictionary I couldn't find the night I broke into Second Prize Wang's dormitory. I felt sad and a bit angry. Why did I break my leg? Because of that dictionary. Why did my parents hate me? Why did I want to run away from home? Because of that dictionary. But don't think I hated it. On the contrary, I felt affection for it, so much that I forgot about Ahjitai, that she was the most beautiful woman in the world and that I was in her room. It was as if I were alone with a glittering treasure chest.

I walked over to it and flipped it open. The word "masturbate" appeared again before my eyes.

I don't know why, but I felt like crying. If I hadn't noticed Ahjtai's puzzled expression, I might have burst into tears. "What did you find in the dictionary?" she asked.

My eyes spoke "masturbate" but I said nothing. What did I see in the dictionary? That was a good question.

I held the dictionary close to my chest, stroking it as if it were a favorite pet I had lost [...]. As I emerged from my reverie, I realized I was in Ahjitai's room and that Ahjitai was standing next to me. I could feel her breath on my chest. (p. 227)

那天晚上, 我在王亚军的宿舍里没有偷上的这本词典。它竟在这里, 我的心里 有些难过, 也有些生气、我的腿是怎么断的? 因为这本词典。我的母亲为什么 恨我? 我为什么要离家出走? 就是因为这本词典。不要以为, 在那时我会对词 典仇视。没有, 恰恰相反, 我的内心对它长满了温情, 以至于我忘了阿吉太的 存在, 忘记了她是一个世界上最美丽的女人, 就好像我没有在她的房间, 而是在

无人之境, 那里金光闪闪, 有一个聚宝盆。

我超词典走过去, 抓起它来, 一翻开, 竟又看到自慰这个词。

不知道为什么, 我当是特别想哭, 如果不是意识到阿吉太正在奇怪地看我, 可 能我真的会哭起来。

阿吉太说 : 《你在词典里里看到了什么?》

我的眼晴里包含着 “自慰”却说不出话来。是呀, 我在词典里看到了什么? 这的 确是个问题。

我把词典抱在怀里, 就好像它是我的一只宠物, 我来回地摸索它, 他就是一个 失而复得的东西......我从梦幻里走出来, 头脑渐渐清楚了, 现在是在阿吉太的房

子里，他就站在我身边，我的皮肤能够感到她的气息。(p. 285-286)

It is not the first time that Chinese readers run into "masturbation" as a theme. The most famous case is Yu Dafu's 郁达夫 (1896-1945) [“Sinking”] / 沉沦 (1921), in which the protagonist watches his beloved while taking a shower, gets excited and then masturbates. Interestingly enough, in the modern novella "masturbation" gets a negative connotation as it is associated with the concept of sin. As Robin Visser shows in another case, in Liu Heng's 刘恒 (1955-) novel Black Snow / 黑的雪 (1988) masturbation is associated with the protagonist's sense of alienation from society, as well as with his inability to win his beloved's affection. ${ }^{16} \mathrm{Here}$, as well as in other 
passages, it is clear that masturbation is Love Liu's way to claim possession of his own body. learn English and she secretly expects that he will reciprocate her love for him. To their utmost disappointment, both Love Liu and Sunrise Huang accidentally witness Second Prize Wang's declaration of love to Ahjitai and the Uyighur teacher's prompt and violent rebuttal. What was supposed to be a private affair becomes a nightmarish accident that foregrounds the demise of Second Prize Wang. Sunrise Huang's decision to accuse her English teacher of sexually harassing her goes far beyond a mere act of jealousy. Now a desperate and orphaned daughter, Sunrise Huang sees in Second Prize Wang a surrogate masculine figure who can rescue her from her quandary. She does not immediately confess the truth, but does so only after Love Liu has accompanied her to the air-raid shelter site and she has gone into labor:

I walked toward the construction site, Sunrise Huang following close behind me. I walked fast, and she almost had to run to keep up with me. I saw beads of sweat on her forehead. She hid with me behind a tree not far from the construction site. We did not have to wait long.

Second Prize Wang came out in his muddy clothes. He did not look in our direction. Like a wise old man he just looked at the sky, as if something up there had caught his attention.

Sunrise Huang's face immediately turned ashen. She ignored me and stared at her English teacher: "He is darker," she murmured like a concerned mother. "And he's slouching a little.”(p. 161)

我们超防空洞的工地走着, 黄旭升一直跟在我后边。我走得很快, 她几乎在瞎 跑跑着。我能看到他额上的汗。

它随着我一起脱在了不远处的一棵树的后边, 我们只等一会儿。 王亚军就穿着一身沾满泥水的衣服出来, 他没有朝我们这边看。只是像一个做 作的哲人那仰望天空，就仿佛他真的被天上的某种东西给吸引了。 黄旭升的脸色一下子就变得苍白, 她没有看我, 只是盯着英语老师, 像是一个 伟大的母亲那样说 : 《他黑了, 背也有一点驼。》(p.197-198)

This is the beginning of Second Prize Wang's demise and the beginning of these teenagers' growth into adulthood. As is clear from Ying-ge-li-shi, Sunrise Huang's 
confession that she has unfairly accused her teacher of a crime is not merely the product of her sense of guilt. It is rather an important step towards the beginning of the demystification of the imaginary and a first, albeit incomplete, step towards the "real", the adult world. Her second murder, committed only by accident, leads her to a long period of detention during which, without any support from teachers or adults, she will prove herself able to master not only English but also her own life.

However, the reader cannot but remark that Love Liu is far from being unsuccessful, since by eventually making love to Ahjitai, he will find his own sense of the meaning of his own name: "Love". From the beginning of the novel, he constantly displays an obsessive curiosity about the real meaning of this term. Second Prize Wang is the first one willing to explain its meaning:

"Why are you called Love Liu?" "Cause my mother wished I was a girl." "But it's not necessarily a girl's name." "Yes, it is." "Do you know what "love' means?" "It means when boys and girls... No I don't know." "No, not any of that. It means compassion." "What is 'compassion'?" "It's...it's... how should I put it...? It means you can feel pain when you see others suffer." "That's impossible." (p. 49)

《你为什么要叫刘爱?》

《因为我妈妈希望我是个女孩儿。》

《这个名字不一定就是个女儿的名字。》

《就是女孩儿的名字。》

《你知道〈爱〉是什么意思吗?》

《爱? 不知道, 就是男生和女生......不, 还是不知道。》

《爱不是别的, 就是仁慈。》

《什么是仁慈?》

《就是, 就是, 怎么说, 就是看别人受难时, 你自己心里也难过。》

《这不可能。》(p. 37-38)

Love clearly works as an ambivalent signifier and Love Liu stubbornly continues acting according to his personal interpretation of his name's meaning. His refusal to acknowledge and accept Second Prize Wang's alternative reading is also an important prolepsis which consistently accounts for Love Liu's seemingly paradoxical behavior. If Second Prize Wang and his elegant manners still embody a world where ethics and aesthetics are reciprocally interdependent, it is up to Love Liu and his generation to break up such a linkage. In fact, it is Love Liu who convinces Second Prize Wang to go to the public bathhouse to contemplate Ahjitai's naked body. Furthermore, Love Liu's "indecent" proposal is bestowed as if it were Love Liu's personal gift, the compensation for which would be his temporary possession of the English dictionary, which all his classmates longed for. There is a clear overturning of the teacher's and student's roles here: Second Prize Wang, blinded and fooled by his own unfulfilled desire, finds himself docilely following Love Liu. When they are both accused of indecent behavior, he behaves in the same way as he had behaved after Sunrise Huang's charges, as an exceedingly responsible teacher, taking all the blame, and so he goes to prison.

There is certainly a "nihilist" component in this novel - a component which, as many Sinologists have shown, is one of the principal characteristics of texts by the "late-born generation". ${ }^{18}$ Yet this nihilism is a strategy to show that the world full of ethical values to which the former generation of Chinese had aspired is pathetic and meaningless. In a world where everything is a spectacle deprived of meaningful ethical values, there are only two "values" that are worth pursuing: worldly success (achieved by Sunrise Huang) and sexual pleasure (achieved by Love Liu). 

meaningless: it is not only the "masterpiece" of Love Liu's mother, it is also a place of confinement for Ahjitai, who is "condemned" to work there as a form of punishment for refusing to comply with Director Fan's offers of love. Ahjitai accepts to concede her body and her love - though only a short while, simply because she is afraid of dying there, as the shelter is on the verge of falling down. Love Liu's lovemaking to Ahjitai transforms him into a winner. In fact, he has succeeded where his former exemplars of power and of value, Director Fan and Second Prize Wang respectively, had failed. The collapse of the air-raid shelter, which soon enough becomes a "blissful trap", allows Love Liu to emancipate himself from his parents, to become an adult and to fulfill his dreams of love.

The novel's ending is, not surprisingly, the opposite of that in Ah Cheng's: Second Prize Wang demands that Love Liu give him back the dictionary. This again confirms not only the impossibility of creating a world in which ethics and aesthetics are intrinsically linked, but also the disavowal of all residual forms of authority. Wang Gang's novel is one of the first attempts in Chinese literature to describe the death of the epistemic subject and the birth of a new one which trespasses ethnic and cultural boundaries. Knowledge, here, cannot be anything but mere lived-through experience. This novel can therefore be considered as the first, necessary step toward the birth and evolution of a new way of representing the body.

\section{BIBLIOGRAPHY}

AH Cheng 阿城, [The King of Chess, The King of Trees, The King of Children] / 棋王、树王、孩子 王 / Qi wang, Shu Wang, Haizi Wang, Nanjing Shi, Jiangsu feng huang wen yi chu ban she, 2016.

AH Cheng 阿城, The King of Children, in The King of Trees, MCDOUGALl Bonnie (trans.), New York, New Directions, 2010.

BENJAMIN Walter, “The Mimetic Faculty”, in Reflections. Essays, Aphorisms, Autobiographical Essays, DEMETZ Peter (ed., trans.), New York, Schoken Books, 1986, p. 333-336.

BENNET Andrew, "Language and the Body", in The Cambridge Companion to the Body in Literature, HILLMAN David and MAUDE Ulrika (ed.), New York, Cambridge U. P., 2015.

BRooKs Peter, Body Work. Objects of Desire in Modern Narrative, Cambridge, Harvard U. P., 1993, p. 30. BUTLER Judith, Antigone's Claim: Kinship Between Life and Death, New York, Columbia U. P., 2000.

ButTERfIELD Bradley, "The Baudrillardian Symbolic, 9/11 and the War of Good and Evil”, Postmodern Culture, vol. 13, 2002:3, http://pmc.iath.virginia.edu/issue.902/13.1butterfield.html, consulted on $01 / 07 / 2021$.

CHow Rey, Primitive Passions: Visuality, Sexuality, and Contemporary Chinese Cinema, New York, Columbia U. P., 1995. 
GENETTE Gérard, Narrative Discourse. An Essay in Method, LEWIN Jane E. (trans.), Ithaca, Cornell U. P., 1980.

JAY Martin, Downcast Eyes. The Denigration of Vision in Twentieth-Century French Thought, Berkeley, California U. P., 1993.

KELTNER Stacey, Kristeva, New York, Miley, 2013.

LIU Na'ou 刘呐鸥, [“Etiquette and Hygiene”] / 礼仪和卫生 / Liyi he weisheng, in [Urban Scenic Horizon] / 都市风景线 / Dushi fengjing xian, Shanghai, Shuimo shudian, 1930, p. 107-142.

McGRath Jason, Post-socialist Modernity. Chinese Cinema, Literature and Criticism in the Market Age, Stanford, Stanford U. P., 2008.

MERLEAU-PONTY Maurice, The Visible and the Invisible, Evanston, Northwestern U. P., 1968,

p. $130-155$.

Mor Toril, "Patriarchal Thought and the Drive for Knowledge", in New Directions in Psychoanalysis and Feminism, BRENNAN Teresa (ed.), London, Routledge, 1989, p. 189-205.

SHIH Shu-Mei, The Lure of the Modern. Writing Modernism in Semicolonial China 1917-1937, Berkeley, University of California Press, 2001.

SLATER Don, Consumer Culture and Modernity, Cambridge, Polity Press, 1997.

VISSER Robin, Cities Surround the Countryside. Urban Aesthetics in Post-Socialist China, Durham, Duke U. P., 2010.

WANG Gang 王刚, English / 英格力士 / Ying-ge-li-shi, Beijing, Renmin wenxue chubanshe, 2005.

WANG Gang 王刚, English, MERZ Martin and WEIZHEN PAN Jane (trans.), New York, Penguin Group, 2009.

ZHONG Xueping, Masculinity Besieged: Issues of Modernity and Male Subjectivity in Chinese Literature of the Late Twentieth Century, Durham, Duke U. P., 2000.

\section{NOTES}

1. CHow Rey, Primitive Passions: Visuality, Sexuality, and Contemporary Chinese Cinema, New York, Columbia U. P., 1995, p. 111-112.

2. The passages quoted from Ah Cheng's original text refer to the following edition: AH Cheng 阿 城, [The King of Chess, The King of Trees, The King of Children] / 棋王、树王、孩子王, 2016.

3. The passages quoted from the translation of Ah Cheng's novel refer to the following edition: -, The King of Children, in The King of Trees, MCDOUgall Bonnie (trans.), New York, New Directions, 2010.

4. CHOW, Primiative passions..., op. cit., p. 119.

5. The passages quoted from the translation of Wang Gang's novel refer to the following edition: WANG Gang 王刚, English, MERZ Martin and WEIZHEN PAN Jane (trans.), New York, Penguin, 2009.

6. The passages quoted from Wang Gang's original text refer to the following edition: WANG Gang 王刚, English / 英格力士, Beijing, Renmin wenxue chubanshe, 2005.

7. MOI Toril, "Patriarchal Thought and the Drive for Knowledge", in New Directions in Psychoanalysis and Feminism, BRENNAN Teresa (ed.), London, Routledge, 1989, p. 190.

8. GENETTE Gérard, Narrative Discourse. An Essay in Method, LEWIN Jane E. (trans.), Ithaca, Cornell U. P., 1980, p. 67. 
9. ZHONG Xueping, Masculinity Besieged: Issues of Modernity and Male Subjectivity in Chinese Literature of the Late Twentieth Century, Durham, Duke U. P., 2000.

10. BRooKs Peter, Body Work. Objects of Desire in Modern Narrative, Cambridge, Harvard U. P., 1993, p. 30.

11. JAY Martin, Downcast Eyes. The Denigration of Vision in Twentieth-Century French Thought, Berkeley, California U. P., 1993.

12. Benjamin Walter, “The Mimetic Faculty”, Reflections. Essays, Aphorisms, Autobiographical Essays, New York, Schoken Books, 1986, p. 333-336.

13. Slater Don, Consumer Culture and Modernity, Cambridge, Polity Press, p. 162.

14. BROOKS, Body Work..., op. cit., p. 28-30.

15. BENNET Andrew, "Language and the Body", in The Cambridge Companion to the Body in Literature, HILLMAN David and MAUDE Ulrika (ed.), New York, Cambridge U. P., 2015, p. 85.

16. VISSER Robin, Cities Surround the Countryside. Urban Aesthetics in Post-Socialist China, Durham, Duke U. P., p. 229-233.

17. MERLEAU-PONTY Maurice, The Visible and the Invisible, Evanston, Northwestern U.P., 1968, p. 130-155.

18. Analyses of nihilism in the "late-born generation writers" in VISSER, Cities Surround the Countryside..., op. cit., p. 255-286 and MCGRATH Jason, Post-socialist Modernity. Chinese Cinema, Literature and Criticism in the Market Age, Stanford, Stanford U. P., 2008, p. 59-94.

\section{AUTHOR}

\section{MELINDA PIRAZZOLI}

Melinda Pirazzoli is Adjunct Professor of Chinese Literature at the University of Bologna. She has published a study on Classical Chinese poetry (Intenti Poetici, 2016, Ananke) and a history of modern and contemporary Chinese fiction cowritten with Nicoletta Pesaro (La narrativa cinese del Novecento, 2019, Carocci). She is working on a third volume about the representation of Chinese perceptions tentatively entitled: A Sense of Chinese Senses. 


\title{
Vers une topologie corporelle dans [La Vallée du lœss] de Kim Dong-ri
}

\author{
Min Sook Wang-Le
}

1 L'expression corporelle est l'un des procédés les plus fréquents de Kim Dong-ri (1913-1995), figure emblématique et fondatrice de la littérature coréenne contemporaine, pour décrire les actions des personnages. Elle dénote tantôt une gestuelle esthétique à travers des figures de religieux, chamanes et moines, tantôt toutes sortes de sentiments humains liés à des circonstances dramatiques et au destin. Dans sa nouvelle [« Mi-Bouddha, mi-homme »] / 등신불, Kim Dong-ri met en scène tout le processus de l'offrande corporelle ritualisée à travers le moine Wanji qui décide d'offrir son propre corps au Bouddha afin de se libérer de son angoisse et de ses faiblesses. Dans [«La Chamane du col Dang»]/ 당고개무당 et [La «Chamane Eulhwa »] / 을화, il décrit les cérémonies chamanistes. Les chamanes-héroönes subissent des épreuves par une souffrance physique, c'est-à-dire une maladie chamanique / 巫病, puis deviennent des apprenties avant d'être éveillées par un rite d'initiation au cours duquel elles accueillent dans leur corps un esprit divin qui va devenir leur maître. Elles ne sont pas propriétaires de leur propre corps, capturé par les esprits divins. Autrement dit, leur corps répond seulement à une fonction préétablie. La plupart de ses personnages souffrent d'un mauvais sort ou d'une douleur corporelle mais aussi existentielle due à la déception ou à la solitude ou à l'incommunicabilité avec le monde environnant. Leur corps se lie souvent au désir de mort sans qu'ils essayent d'en chercher le remède.

Or, le corps souffrant, un des thèmes récurrents dans les romans de Kim Dong-ri, s'associe à un souci d'organisation de l'espace, voire à une géomancie conditionnée par la mise en abyme des contes et des mythes locaux. C'est vers une topologie corporelle que se dirige sa nouvelle [«La Vallée du loess »] / 황토기, où l'espace romanesque et la santé physique des personnages s'emboîtent et ne peuvent se démêler à une lecture de premier degré. L'espace romanesque de Kim Dong-ri où se déroule l'action n'est pas neutre et ne sert pas seulement de décor. Il est doté d'une valeur symbolique dans la mise en lumière du destin des personnages, assujetti à une disposition spatiale. Les 
personnages sont eux-mêmes le produit des lieux, avec lesquels ils sont constamment en relation.

3 Nous allons montrer ces liens intrinsèques qui lient les deux éléments (corps et lieux) dans [« La Vallée du lœss »]. Dans le rapport entre l'espace et le corps, on décèlera un certain déterminisme, voire fatalisme de Kim Dong-ri chez qui le hasard finit souvent par devenir le nécessaire.

\section{Corps colossal et incapacité de son déploiement}

4 La dimension topologique et topographique est une constante chez Kim Dong-ri qui a tendance à y soumettre ses personnages. De ce point de vue, il s'inspire de la théorie du dispositif géographique (형국론/ 形局論) ${ }^{1}$ selon laquelle toutes les créatures cosmiques possèdent leur propre raison, principe /이 理, leur propre énergie, souffle / 기 氣, leur propre corps, forme / 상 像. Leur nature et leur vitalité correspondant à leur forme extérieure sont immanentes. Une terre faste se forme grâce à une montagne de bons augures. Selon cette théorie, l'énergie qui fait écho à l'état de préservation du relief (보국형세 / 保局形勢) et à la forme des veines de la montagne (산혈형체 / 山穴 形體) est condensée dans le sol. L'observation des dragons (간룡법 / 看龍法) est une pratique de géomancie qui s'attache à la réception énergétique par la terre ou par une montagne, que l'on appelle "dragon " selon la terminologie des géomanciens. Elle vérifie l'état de santé d'une montagne : est-elle en mesure de respecter les vertus des monts Gollun/곤륜산 et Baekdu/ 백두산, modèles de l'énergie montagnarde coréenne ? Ne risque-t-elle pas de se muer en dragon mort ou malade? Est-elle emplie de vitalité ? Or, la simple présence du dragon dans la montagne n'est pas forcément positive. Il faut que sa pulsation soit aussi saine que chez l'homme. On diagnostique la qualité de la terre par la montagne qui l'entoure, puisque l'on ne peut voir directement le pouls du dragon caché à l'intérieur².

5 [«La Vallée du lœss»]/ 황토기 assortit ingénieusement les trois éléments fondamentaux de la divination montagnarde : dragon / 용 龍 / yong; pouls / 맥 脈 / maek; veines / 혈 穴 / hyeol de la montagne. La nouvelle met l'accent sur la description topographique de la région du lœss :

Deux chaînes de montagnes aux crêtes nues s'étendent du col de Solgae à la montagne de Geumo. L'une d'elles descend vers le nord-ouest et couvre environ 10 lieues, l'autre, longue de 15 lieues environ, va vers le nord-est. Elles donnent naissance à un petit village du nom de la "Vallée du lœss». Telle est leur configuration géographique où circulent la légende des dragons blessés et celle d'un couple de dragons, transmises de bouche à oreille par les anciens du village qui aiment contempler le ruisseau passant au pied de ces deux chaînes de montagnes. 솔개재(鴧介嶺)에서 금오산(金午山) 쪽으로 뻗쳐 내리는 두 산맥이다. 등성이를 벌거벗은 채 십 리, 시오 리씩을 하나는 서북, 또 하나는 동북으로 뛰어 내려와서 는, 거기 황토골이란 조그만 골짝 하나를 낳은 것뿐으로, 그 앞을 흘러가는 냇물을 바라보며, 동네 늙은이들의 입으로 전하는 상룡(傷龍) 또는 쌍룡 (雙龍) 의 전설을 이룬 그 지리적 결구 (結構) 는 여기서 끝을 맺는 것이다3.(p. 215)

En recourant à la « méthode de l'observation des dragons (la mutation des dragons) et à la « théorie du dispositif géographique », l'auteur construit l'histoire de [« La Vallée du lœess "] à partir de trois légendes fondées sur les deux pivots géographiques que sont le Col de Solgae et la Montagne de Geumo: «la légende du couple de dragons », «la légende des dragons blessés » et « la légende de la coupe des veines de la montagne ». 
7 La nouvelle débute par un rappel de ces trois légendes dans le dessein d'expliquer le destin des deux principaux protagonistes du point de vue de la géomancie. Son idée centrale est la fusion de diverses légendes de la vallée du lœss, qui en constitue le décor romanesque, et du récit de la vie de deux hercules Eok-soe et Deuk-bo, qui ressemble singulièrement à celle des dragons que l'on associe à la topographie de la vallée du lœss.

8 Mises en place dans le prologue, ces trois légendes sont narrées selon la perspective d'un observateur à la troisième personne. Formant un récit extradiégétique, elles suggèrent et annoncent l'intrigue du récit intradiégétique ; Kim Dong-ri use souvent de la mise en abyme pour créer un effet d'authenticité et de mystère à ses fictions. Après un prologue long d'une page, le récit emboîté constitue le pivot du point de vue d'un narrateur omniscient à la troisième personne. Le récit intradiégétique commence par la scène où Eok-soe désherbe seul la rizière et s'achève sur celle où il s'apprête à livrer son ultime combat contre son éternel rival Deuk-bo au bord du ruisseau des dragons. Dans la trame romanesque, plusieurs mini-événements, causés par l'amour, la jalousie, la séparation ou la mort, induisent souvent l'action principale.

9 La chronologie globale de [«La Vallée du lœss»] suit symboliquement le cycle des saisons qui inclut les événements et anticipe le déroulement de l'intrigue. L'été ouvre et ferme ce récit de même que la scène de lutte du début est reprise en conclusion. Les indications temporelles déterminent l'orientation thématique de cette nouvelle. Elles ne sont ni explicites ni détaillées mais plutôt nébuleuses. C'est l'été qui forme le point de départ et d'arrivée de [«La Vallée du lœss »]. Il s'agit d'une chronologie générale difficile à saisir, car il s'y mêle des anachronies multiples, partielles, qui sont le fait des personnages.

10 Dans le prologue qui introduit les trois légendes répandues dans la vallée du lœss, le narrateur remonte à un temps mythique qui restitue un ensemble d'événements en abyme. Ce sont les présages de l'histoire, préfigurée par les légendes, qui donneront matière au roman. Toute représentation est dépassée par une force invisible issue de ces légendes et des images mythiques qu'elles véhiculent. D'autre part, le texte révèle des indices précis renvoyant à l'univers du présent ou fournissant des éléments relevant d'un temps imaginaire et symbolique. Dès le prologue, la vallée du lœss s'ouvre sur une blessure immémoriale. Il y est question d'errance, de chute, de meurtre et de mort. Cette vision de la réalité par superposition d'images rejoint celle des personnages, qui ne parviennent pas à se libérer de ce lieu imprégné de légendes et de malédiction.

11 Eok-soe, hercule originaire de la vallée du lœss est facilement remarqué par les bras, les jambes et le tour de taille qui sont beaucoup plus longs ou grands que ceux des gens ordinaires, de même que ses épaules et sa corpulence révèlent une constitution hors norme. Cet homme de cinquante-deux ans, les cheveux quelque peu grisonnants à moitié, a dû longtemps réfréner sa force et laisser brûler des flammes solitaires au fond de son cœur. Hercule qu'il est, il n'a jamais l'occasion de déployer véritablement sa force.

12 Au contraire, la révélation d'une force surnaturelle à l'âge de 13 ans et peu appréciée des villageois a été accompagnée d'une enfance solitaire et d'un souci de l'avenir :

Ce fut l'année de ses 13 ans qu'il bouleversa tout le village en soulevant d'un seul coup une pierre immense qui était posée dans le champ et que les adultes du village ne parvenaient pas à déplacer. 
- Un hercule est né.

- Un hercule est né dans la vallée du lœss!

Les villageois commencèrent d'abord à en parler discrètement. Le lendemain, les personnes âgées se rassemblèrent en tenue de cérémonie au conseil du village.

- On dit depuis longtemps que si un hercule naît dans la vallée du lœss, il manquera de piété filiale ou sera rebelle à son pays.

- De toute façon, il ne pourra plus faire grand mal maintenant que le grand général de la Chine a coupé la veine de la montagne.

- Non, c'est insensé. Mon grand-oncle paternel, considéré comme un hercule, avait été arrêté par le seigneur du village. Finalement, on lui a cassé le bras droit...

Après de tels échanges, ils en conclurent qu'il valait mieux faire pratiquer l'acupuncture sur la veine de l'épaule droite d'Eok-soe pour neutraliser sa force. Parmi eux, l'oncle aîné d'Eok-soe réagit particulièrement méchamment.

- Si un hercule surgit dans la vallée du lœss, la cour royale sera évidemment au courant. Allez! Il suffit de penser que tu n'as pas eu cet enfant. Tu ne veux quand même pas détruire toute la famille, dit-il d'un ton menaçant à son petit frère. Mais, assis en silence, le père d'Eok-soe ne répondit point, car Eok-soe était son unique enfant.

Cette nuit-là, la mère d'Eok-soe saisit la manche de son fils :

- Pourquoi as-tu agi de manière si irréfléchie? Ne sais-tu pas que tu causes bien des soucis à ton père ? dit-elle en pleurant.

Le lendemain matin, le père d'Eok-soe interpella son fils :

$-\mathrm{Tu}$ as treize ans. Si tu veux rester sain et sauf, n'entreprends rien sans réfléchir. Sinon, tu gâcherais ta vie et ferais exterminer entièrement toute la famille. Tout dépend de toi, lui dit-il.

Eok-soe grava au fond de son cœur les paroles de son père. En conséquence, il ne participa aucunement aux combats, ni aux luttes à la corde, ni aux compétitions consistant à déplacer des pierres dans les champs ou à porter des poids très lourds. Bref, il évita tout rassemblement et tout endroit où les gens mesuraient leurs forces.

그가, 그 동네 장정들도 겨우 다룬다는 들돌을 성큼 들어서 허리를 편 것으로 온 마 을을뒤집어 놓은것은 그의 나이 열세 살 나던 해다.

- 장사 났군.

- 황토골 장사 났다!

사람들은 숙덕거리기 시작하여, 이튿날은 노인들이 의관을 하고 동회(洞會)에 모 여들었다.

- 예로부터 황토골에 장사가 나면 부모한테 불효하거나 나라의 역적이 된 댔것다.

- 허긴, 인제는 대국 명장이 혈을 지른 뒤니까 별수는 없으리다.

- 당찮으이, 온 바로 내 증조 뻘 되는 이가 그때 장사 소릴 듣고 사또 앞에 잡혀가

오른쪽 팔 하나를 분질러 나왔거든.

이따위 소리들을 서로 주고받고 하다가 결국 억쇠의 오른쪽 어깨의 힘줄에다 침 을 맞히라는 결론이 났다. 그 중에서도 유독 심히 구는 사람이 억쇠의 백부 뻘 되는 영감이었다.

- 황토골 장사라면 나라에서 아는 거다. 자, 자식 하나 버릴 셈치면 그만일 걸.... 자, 괜히 온 집안 멸문 당할라.

하고 동생을 윽박질렀으나, 그러나 동생은 끝까지 묵묵히 앉아 대답을 하지 않았 다. 그에게 억쇠 하나밖에 더 자식이 없었던 것이다.

그 날 밤 그의 어머니는 억쇠의 소매를 잡고,

- 이것아, 어쩌다 그런 철없는 짓을 했노. 너의 아바이 속을 너는 모를라.

하며 울었다.

이튿날 아침 그 아버지는 억쇠를 불러,

- 늬 나이 열세 살이다. 몸 하나라도 성히 지닐라거든 철없이 아무 데나 나서지 마 라, 네 일신 조지고 온 집안 문 닫게 할라, 모두가 늬 맘 먹을 탓이다.

하였다.

억쇠는 아버지의 이 말을 가슴에 새겨들었다. 그리하여 씨름판이고, 줄목이고, 들 
돌을 다루는 데고, 짐 내기를 하는 마당에고, 일절 사람이 많이 모인 곳이나 무슨

힘겨룸 따위를 하는 데는 나서지 않았다. (p. 224-225)

La convention, qui associe la naissance d'un hercule à celle d'un grand traître, enferme toute sa jeunesse dans une attente languissante. Il se trouve ensuite confronté à la réalité de ses 20 ans où il devait gâcher toutes ses nuits pour sa survie :

Ayant atteint l'âge d'une vingtaine d'années, il ne parvenait pas à maîtriser sa force débordante. Une certaine nuit, seul, il ne cessa de monter au sommet de la montagne et de descendre dans la gorge en portant un rocher dans ses bras, et ce, jusqu'à ce que le jour se levât. Il avait l'air d'un fou, les cheveux ébouriffés à cause de son chignon défait, les yeux congestionnés. De nuit, il avait pu ainsi lutter contre un rocher. Mais durant la journée, il lui était difficile de retenir l'envie de tout casser sur son passage, de même qu'il était souvent saisi de trépignements de fureur. Il ne cherchait pas à exhiber sa force, mais à la dépenser.

그의 나이 스무 살 남짓 했을 때는 과연 솟는 힘을 제 스스로 감당할 수 없었다. 어 떤 날 밤에는 혼자서 바위를 안고 산꼭대기로 올라갔다 골짜기로 내려왔다 하는 동안, 어느덧 밤이 새어 버리는 수도 있었다. 상투가 풀려 머리칼이 헝클어지고 두 눈엔 벌겋게 핏발이 서고 하여 흡사 미친 사람 같았다. 밤 사이는 또 이렇게 바 위와 씨름이라도 할 수 있지만, 낮이 되면 무엇이든지 눈에 뵈는 대로 때려 부수 고 싶고 메어치고 싶고, 온갖 몸부림과 발광이 치밀어 올라 잠시라도 견딜 수가 없었다. 힘 자랑이 하고 싶어서가 아니라, 힘을 써보고 싶다는 욕망이었다. (p. 226)

Entre force surnaturelle et idées préconçues, l'attente d'Eok-soe ne sera supportable qu'à l'idée de pouvoir un jour faire usage de sa force. Il se conforme en cela à la volonté de son grand-père et à celle de son père :

- D'après ton thème astral que j'ai consulté quand tu étais petit, tu es né sous une mauvaise étoile. Tu seras frappé par un grand malheur, si tu ne maîtrises pas ton énergie pendant ta jeunesse... Néanmoins, la force peut être un trésor. Si tu sais la contrôler, tu auras plus tard de grandes chances d'en faire remarquablement usage. Attends discrètement ce moment.

Eok-soe considéra les dernières paroles de son père comme une révélation du Ciel :

" tu pourras un jour utiliser ta force pour une bonne cause ", " attends seulement le bon moment".

Pas un seul moment, il n'oublia les deux phrases de son père. En refrénant sa force débordante et en attendant d'en faire bon usage, il compta impatiemment les jours. Mais bien avant ce jour si longuement attendu, ses cheveux et sa barbe commencèrent à grisonner.

- 늬가 어릴 때 누구에게 사주를 뵈었더니 너의 팔자에는 살이 세다고, 젊어서 혈 기를 삼가지 않으면 큰 화를 당할 게라더라... 그렇지만 사람에게는 힘이 보배 니 너만 알아 조처할 양이면은 뒤에 한 번 크게 쓸 날이 있을 게다. 조용히 그때가 오기를 기다려라.

아버지가 숨을 거둘 때 남긴 이 말이 억쇠에게 있어서는 그 무슨 하늘의 계시와도 같이 들렸던 것이었다.

- 한번 크게 쓸 날이 있을 게다.

- 때가 오기만 기다려라.

그는 잠시도 이 말이 그의 머리 속에서 사라질 때가 없었다.

그 미칠 둣이 솟아오르는 힘의 충동을 누르고 누르며 그 한번 크게 쓰일 날을 기다 려, 오늘인가 내일인가 하는 사이, 그러나 그 기다리는 날이 오기도 전에 어느덧 그

의 머리털과 수염만이 희끗희끗 반 넘어 세어지고 말았던 것이다.» (p. 226-227)

Sa vie solitaire et menacée se résume à une attente interminable qui le condamne au piège de l'oisiveté et du gaspillage de sa jeunesse. Dans son propre village, Eok-soe vit comme un étranger. Il est en proie à un sentiment d'absurde, d'étrangeté à soi-même et de perte d'identité. Il devient ainsi un hercule castré. Tel Sisyphe dans la mythologie grecque, Eok-soe semble destiné à revivre toujours le même acte de lutte avec, en filigrane, l'image du vide. Ce lieu est donc un piège, dans lequel il tourne en rond. Il 
éprouve un mal-être dans cette vallée. Il y est à la fois exilé et exclu. Sa perception de la réalité est marquée par un sentiment d'enfermement et d'étouffement. Eok-soe se présente comme condamné par ce village à un terrible engourdissement et à une frustration absolue.

\section{Corps révolté et épreuves de destruction}

Dans la monotonie des jours, intervient brusquement la rencontre d'Eok-soe avec Deukbo. Un jour qu'il est en train de boire un verre avec une jeune serveuse du nom de Bun-i dans la taverne située à un carrefour du village, il assiste au vacarme provoqué par un vagabond étranger du nom de Deuk-bo dont le physique révèle l'identité peu ordinaire. Il finit par comprendre qu'il n'est pas un simple costaud ou un robuste voyou :

Se tournant vers Eok-soe, l'étranger le regarda de travers :

- Peuh ! Toi aussi...

Avant même d'achever sa phrase, il se jeta sur lui. Il donna un coup de tête contre les arcades sourcilières d'Eok-soe qui, après un instant de vertige, le saisit au collet. Dans un tout premier temps, Eok-soe estima, malgré la constitution extraordinaire de l'autre, qu'il s'agissait d'un homme robuste simplement habitué aux bagarres. Mais, en mesurant ses forces aux siennes, il comprit vite que l'étranger n'était pas seulement un homme vigoureux et déluré. Gagné par la joie comme s'il s'élevait tout doucement dans les airs, Eok-soe relâcha malgré lui ses mains serrées sur la gorge de l'autre.

낮선 사내는 이쪽으로 고개를 돌려 억쇠를 한 번 흘겨보더니,

- 흥, 너도 이놈....

하는, 말도 채 맺지 않고, 별안간 뛰어들며 머리로 미간을 받으매, 억쇠도 한순간 정신이 다 아찔하였으나 그 다음 순간에 그도 바른손으로 놈의 멱살을 잡아 쥘 수 있었다. 보매 기골도 범상희는 생긴 놈이 아니로되, 그래도 처음 억쇠는 그놈이 그 저 힘께나 쓰는데다 싸움에 익은 놈이려니 쯤으로밖에 더 생각하지 않았는데, 한 번 힘을 겨뤄보자 그냥 이만저만 센 놈이나 불량한 놈만은 아니라는 것을 깨닫게 되었다. 순간, 억쇠는 문득 자기의 몸이 공중으로 스르르 떠오르는 듯한 즐거움이 가슴에 솟아오름을 깨달으며 저도 모르게 멱살 잡았던 손을 슬그머니 놓아버렸 다. (p. 228)

Envahi par la joie, il sent son propre corps flotter doucement dans les airs au point de desserrer ses mains qu'il a portées à la gorge de ce vagabond. C'est ainsi qu'après quarante années d'attente, Eok-soe finit par rencontrer Deuk-bo, un autre hercule. Grâce à cet étranger, Eok-soe, étranger lui aussi à son propre pays, se sent moins seul.

Depuis leur première rencontre, Eok-soe et Deuk-bo vivent l'un près de l'autre dans une relation de concurrence et d'interdépendance pour leur survie. L'entrée en scène de Deuk-bo modifie radicalement la vie monotone d'Eok-soe. Leur rencontre semble bien augurer de l'avenir de celui-ci, qui considère Deuk-bo comme une oasis dans le désert de sa vie. Ils sont devenus, dès leur rencontre, presque nécessaires l'un pour l'autre. Leur animosité réciproque n'est qu'un sentiment passager visant à éprouver leur force. Sous le ciel rougeoyant, la plaine Annaet se pare d'une teinte rouge sang, signe d'agressivité et Eok-soe et Deuk-bo, en buvant, commencent à échanger des grossièretés qui les conduiront à leur rixe saisonnière :

Au bord d'un ruisseau, la plaine Annaet était une sorte de ravin situé au-delà d'une crête dominant la vallée du lœss. Avec son étendue de sable blanc, sa pelouse verte et l'ombre des vieux pins alignés qui la bordaient, elle était un endroit idéal pour Eok-soe et Deuk-bo, qui pouvaient y passer leur journée à manger, se distraire et lutter. 
Presque nus, les deux hommes, en pantalon court, étaient en train de boire du vin à l'ombre des pins. Ils se partagèrent un cochon avec bonne humeur en prenant chacun un pied et échangèrent leurs bols amicalement en s'encourageant à boire. Ils buvaient de cette manière une ou deux fois par saison dans cette plaine Annaet. Nul moment n'était plus heureux que celui-ci, qui leur apportait une bouffée d'oxygène. Rien ne pouvait remplacer cette joie et ce sentiment de récompense, ainsi que ce festin sacré. Les belles Bun-i et Seol-hui ne jouaient pour eux que les rôles d'adjuvants pour susciter leurs jeux.

안냇벌은 황토골에서 잔등 하나 넘어 있는 아늑한 산골짜기요, 또 개울가이었으 므로 거기엔 흰 모래밭과 푸른 잔디와 게다가 그늘진 노송까지 늘어서 있어 억쇠 와 득보들 같이 온종일 먹고 놀고 싸우고 할 자리로서는 더할 나위 없이 알맞은 곳 이었다.

두 사람은 짤막한 잠방이 하나만 걸치고는 벌거벗은 채 소나무 그늘 밑에서 술을 마시고 있다. 처음엔 돼지 족도 한 가리씩 의논성스럽게 째어 들었고, 술잔도 서로 권해 가며 주거니 받거니 의좋게 건너 다녔다.

한철에 한두 번씩 이 안냇벌에서 대개 이렇게 술을 마시게 되었지만, 이 두 사람에 게 있어서는 이때같이 가슴이 환희 트이도록 즐겁고 만족할 때가 없다. 그것은 아 무 것과도 바꿀 수 없는 기쁨이요, 보람이요, 그리고 거룩한 향연이기도 하였다. 이 에 견준다면 분이나 설희의 자색도 한갓 이 놀이를 돋구고 마련키 위한 덤에 지나 지 않을 듯했다. (p. 221)

Sur fond de chant de cigales, la narration se concentre sur une de ces lentes journées occupées par un combat qui se prolonge jusqu'au soir. Eok-soe et Deuk-bo se lancent dans une lutte beaucoup plus violente que celle de la journée. Leur passion pour les deux femmes, Bun-i et Seol-hui, ne fait office que de mini-événement déclencheur du véritable événement: le combat. Dans cette compétition de force physique, les tensions, l'agressivité et les insultes ralentissent le temps qui s'enlise. Ils se frappent et se mordent à mort. Plus ils dépensent leurs forces débordantes, plus ils sont joyeux :

Lorsque le quatrième coup de pied de Deuk-bo toucha ses parties génitales, Eok-soe faillit tomber sur le champ. Il haussa les épaules en serrant le cou de son adversaire d'un bras après lui avoir appliqué un vigoureux soufflet.

- Salopard!

La voix d'Eok-soe résonna à travers la vallée. Les chants et les éclats de rire cessèrent rapidement dans la bouche de ces deux hommes emmêlés en un bloc. On entendit uniquement leur souffle haletant et le heurt de leurs muscles contractés. Un filet de sang coulait de leur nez presque au même moment. Leurs yeux se congestionnaient et ils crachèrent du sang de leur gorge. Le visage, les épaules et la poitrine des deux hommes luisants de sueur étaient entièrement en sang. Juste au moment où Deuk-bo reculait après avoir frappé la mâchoire inférieure d'Eok-soe, le poing droit de ce dernier, chargé de toute son énergie, s'abattit comme la foudre sous sa côte gauche. Deuk-bo qui avait reçu le coup de poing impitoyable d'Eok-soe rampa à reculons avec un visage de cendre. Il finit par s'écrouler sur le sable. Eoksoe avait lui aussi le cou, la bouche et le nez qui saignaient à nouveau abondamment. Il resta assis un moment d'un air absent, en pressant sa mâchoire inférieure des deux mains pour empêcher l'hémorragie. Puis, en un nouveau sursaut, comme un fou, il se jeta encore une fois sur Deuk-bo, déjà à terre, et d'un coup de dents le mordit à l'épaule droite dont il préleva un morceau de chair. Le sang tout rouge coula de l'épaule écorchée jusqu'au coude. Deuk-bo s'agita doucement sans pourtant réussir à se relever ; il s'allongea là où il était tombé.

Après avoir mâchonné le morceau d'épaule de Deuk-bo, Eok-soe cracha un caillot de sang rouge et prit le pot du vin. Il en but quelques bols avant de s'effondrer.

Aucun d'entre eux ne voulut se rendre ni prendre de repos.

Les deux hommes, complètement épuisés par leur lutte mortelle, restaient allongés comme endormis.

Le vent du soir se levait depuis l'eau du ruisseau et les cigales stridulaient avec enthousiasme sur les branches du grand pin. Ils se rétablirent comme après avoir 
bu l'alcool ensorcelé et commencèrent à vider le pot de vin qui restait du repas matinal.

득보의 네번째 발길이 억쇠의 국부를 건드렸을 때, 그는 한 순간 그 자리에 퍽 끊어 질 뻔하다가 겨우 한쪽 팔로 득보의 목을 후려 안으며 어깨를 솟굴 수 있었다.

- 이놈아!

산골이 찌르렁 울리는 억쇠의 목소리였다.

이리하여 한덩어리로 어우러진 그들의 입에서는 어느덧 노래도 웃음 소리도 동시 에 뚝 끊어지고 다만 씨근거리는 숨소리가 뿌득뿌득 밀려나갔다 들어왔다 하며 근육과 부딪는 소리만이 났다. 두 사람의 코에서는 거의 동시에 피가 주르르 쏟아 져 내렸다. 눈에도 핏물이 돌고 목으로도 피가 터져나왔다. 그 차에 땀으로 번질번 질하던 두 사람의 낮과 어깨와 가슴은 어느덧 아주 피투성이로 변해 버렸다. 득보 가 억쇠의 아래 턱을 치지르며 막 몸을 옆으로 빼려는 순간이었다. 억쇠의 힘을 다 한 바른편 주먹이 득보의 왼쪽 갈비뼈 밑에 벼락을 쳤다. 갈비뼈 밑에 억쇠의 모진 주먹을 맞은 득보는 갑자기 얼굴이 아주 잿빛이 되어 뒤로 비실비실 몇 걸음 물러 나가다 그대로 모래 위에 꼬부라져 버린다.

억쇠의 목과 입과 코에서도 다시 피가 쏟아졌다. 그는 정신 나간 사람처럼 두 손으 로 아래턱을 받쳐 피를 받으며 우두커니 앉아 있다 말고 돌연히 미친 것처럼 뛰어 일어나는 길로 또 한 번 와락 득보에게로 달려 들어 쓰러져 있는 득보의 바른편 어 깨를 물어 떼었다. 어깨의 살이 떨어지며 시뻘건 피가 팔꿈치까지 주르르 흘러내 리자 득보는 몸을 좀 꿈적이었으나 역시 일어나지 못한 채 그대로 뻗어 누워 있는 것이었다.

억쇠는 입에 든 득보의 어깨 살을 질겅질겅 씹다 벌건 핏덩어리를 입에서 뱉어내 고, 그러고는 다시 술 항아리를 기울여 술을 몇 사발 마시고는 쓰러져버렸다.

누구의 입에서 항복이 나온 것도 아니요, 어느 쪽에서 쉬기를 청한 것도 아니었다. 두 사람이 다같이 죽은 듯이 늘어지고 잠든 듯이자빠졌으나, 아주 숨통이 멎은 것 도 아니요, 정말 평온한 잠이 든 것도 아니다. (p. 222-223)

Ils se battent jusqu'à ce que leur corps soit tout ensanglanté, jusqu'à épuisement complet. Pour eux, le combat est un rite cathartique. Ils sont fascinés par leur propre violence qui les éloigne du monde ordinaire selon un rite inaccompli. Ils se livrent un combat féroce qui se résume comme suit :

Deuk-bo s'approcha progressivement d'Eok-soe jusqu'à toucher son menton. Il ne se mit pas en position d'attaque mais dansa en bondissant. Eok-soe se jeta sur lui et lui serra le cou avec ses bras. Deuk-bo l'imita. Ils tombèrent tous deux avec le bruit d'un gros arbre abattu.

Serrant le cou de Deuk-bo pendant quelques temps, Eok-soe éclata soudain de rire. Sur son oreille gauche, il y avait une plaie sanglante. La moitié de l'oreille était déjà dans la bouche de Deuk-bo qui ne voulait plus la lâcher.

Jusqu'à ce que le soleil se couchât et que l'ombre noire de la montagne s'allongeât, les deux hommes aux corps massifs ensanglantés continuèrent leur duel acharné en crachant du sang sans songer à se relever.

득보는 이렇게 목청을 뽑으며 점점 억쇠에게로 가까이 다가 들어왔다. 웬일인지 싸울 태세를 갖추지 않고 그냥 춤만 덩실덩실 추며 억쇠의 턱 앞까지 다가 들어왔 다. 억쇠는 뛰어들어 그의 목을 안았다. 득보도 억쇠와 같이 하였다. 두 사람은 큰 나무가 넘어가듯 쿵 하고 한꺼번에 자빠져버렸다.

득보의 목을 안고 한참 동안 엎치락뒤치락하던 억쇠는 갑자기 큰 소리로 껄껄껄 웃어대었다.

그의 왼쪽 귀가 붙어 있을 자리엔 찢긴 살과 피가 있을 따름, 귀는 절반이나 득보의 입에 가 들어 있고, 득보는 아끼는 듯 그것을 얼른 뱉어내려고도 하지 않았다. (p. 223-224)

C'est une solidarité étrange avec ce marginal Eok-soe du village du lœss qui pousse Deuk-bo à rester avec lui jusqu'au dernier moment. Concurrents en ce qui concerne les femmes et la dépense de leur énergie, leur collaboration est indispensable pour leur survie. Cependant, cette entente n'est que momentanée. C'est en effet une rencontre manquée car Deuk-bo est diabolique. Eok-soe ne connaît rien de cet étranger, poursuivi 
par la justice et qui a fui son pays après avoir tué son demi-frère. Dans son instinct de vouloir dépenser son énergie, il a oublié les propos de son père et a pris l'arrivée de Deuk-bo pour le moment opportun. L'oasis laisse peu à peu place au désert dès lors qu'ils entrent dans des relations triangulaires avec les femmes. Du début à la fin, le narrateur met en relief la question de la rivalité entre deux hercules à travers deux femmes, Bun-i et Seol-hui qui contribuent souvent à la crise et à la chute de la joute sexuelle et physique de Deuk-bo et Eok-soe.

Dès le départ, le narrateur révèle le fil conducteur du récit : la jalousie de Bun-i envers Seol-hui. Le deuxième mariage d'Eok-soe avec Bun-i, présentée par Deuk-bo, provoque le conflit, car les rapports de Deuk-bo avec Bun-i sont fort ambigus : elle se présente tantôt comme sa nièce, tantôt comme sa fille, alors qu'elle est en réalité sa maîtresse et mère de leur fille, absente. Même mariée avec Eok-soe, celle-ci passe les deux tiers du mois chez Deuk-bo. Éprouvant de l'aversion pour Bun-i et Deuk-bo, Eok-soe tombe amoureux de Seol-hui, une veuve vertueuse qu'il essaie de conquérir, et abandonne Bun-i qu'il supporte de moins en moins.

Deuk-bo, lui aussi amoureux de Seol-hui, intervient dans la relation de Seol-hui et Eoksoe. Très séduit par cette femme vertueuse, Deuk-bo abandonne à son tour Bun-i et essaie de briser la relation de Seol-hui et Eok-soe, ce qui conduit à exclure Bun-i de la première relation triangulaire tissée entre Eok-soe, Deuk-bo et elle-même. Les événements s'accélèrent lorsque, "une nuit », Eok-soe entend une dispute acharnée entre Deuk-bo et Bun-i alors qu'il s'occupe de sa mère malade. L'hostilité de Bun-i se manifeste de plus en plus intensément et conduit à l'élimination de Seol-hui. Abandonnée par son mari et son amant, Bun-i poignarde Seol-hui, enceinte d'Eok-soe, et la tue; elle donne également un coup de poignard à Deuk-bo dont elle est toujours amoureuse. Elle s'enfuit ensuite et se suicide en se jetant dans un puits.

L'échec de tous les personnages ne fait que confirmer la sombre orientation de l'histoire. Privés de Bun-i et de Seol-hui mais toujours sensibles à leur passion, Eok-soe et Deuk-bo préparent leur ultime lutte dans la plaine d'Annaet:

- Crois-tu que tu pourras mourir sans m'avoir tué ?

Ces mots lâchés un jour par Deuk-bo alors qu'il crachait des glaires revinrent soudain à l'esprit d'Eok-soe, qui se rappela en même temps le poignard bien affûté qu'il avait posé tout à l'heure sur la table d'apéritif. Il souhaitait que la lame du poignard de Deuk-bo longue d'un empan lui transperçât le cœur et lui ôtât son foie engourdi et ses poumons asphyxiés. Il tressaillit en s'immobilisant brusquement. Lorsqu'il releva la tête, le soleil se couchait sur le col du lœss. Distancé d'à peu près dix lieues d'Eok-soe, Deuk-bo descendait seul d'un pas lourd vers le ruisseau des Dragons.

- 네 놈이 내 초상 안 치르고 자빠질 줄 아나.

억쇠는 문득, 언젠가 득보가 가래와 함께 뱉어놓던 이 말이 머리에 떠오르며 동시 에, 아까 술상 위에 내어놓던 득보의, 그 날이 시퍼렇던 단도가 생각났다.

그 한 뺌도 넘어 될 득보의 단도 날이 자기의 가슴 한복판을 푹 찔러, 이 미칠 듯이 저리고 근지러운 간과 허파를 송두리째 긁어내어 준다면, 하는 생각과 함께 자기 자신도 모르게 몸서리를 한 번 치고, 문득 걸음을 멈추며, 고개를 들었을 때, 해는 이미 황토재 위에 설핏한테, 한 마장 가량 앞에는 득보가 터덕터덕 혼자서 먼저 용 냇가로 내려가고 있었다. (p. 245-246)

Pour mourir, ils choisissent un moment où ils ont tout perdu. Leur choix est caractéristique d'une certaine sédentarité apathique, dont la mort sera l'aboutissement. Le récit, après une double perte féminine, glisse vers une double perte masculine. L'altérité et l'adversité remplacent progressivement la notion d'alter ego, 
introduite au début de l'histoire : l'emportent en fin de compte leur différence, leur révolte mutuelle, leur incapacité à se fondre au temps et à l'espace auxquels ils appartiennent.

\section{Destin corporel et topologie de la vallée du lœss}

La mort des deux hercules n'est pas explicite dans la nouvelle. Mais on peut la deviner aisément. Avec la mort de Bun-i, sa femme, Seol-hui, son amante enceinte de lui, Eoksoe qui va aussi vers la mort ne peut assurer sa lignée en tant que fils unique. Sa force, son énergie le conduisent à un combat inutile et contribuent à sa perte totale, à une extermination de sa lignée. C'est ironique : lui qui est doté d'une force extraordinaire ne peut même pas réaliser ce que les gens ordinaires font. Or, les relations que Deuk-bo et Eok-soe nouent dans [«La Vallée du lœess»] ne sont pas l'unique source de leur conflit et de leur combat. En effet, ils ne s'interrogent même pas sur l'origine de leur conflit. Ils ignorent s'il est lié à la faute originelle propre à leur village.

L'organisation de l'histoire met en évidence la structuration fonctionnelle de l'espace, situé dans la vallée du lœss. Trois légendes liées à sa configuration topographique sont introduites à l'incipit. Elles sont supposées expliquer le statut ou la conduite des personnages présentés comme une synecdoque de l'ensemble. C'est que la vallée recèle dans ses profondeurs le secret de ses origines. Le contenu et le thème principal évoquent le sort des deux personnages déterminé par la géomancie de la vallée du lœss à l'origine des trois légendes. L'utilisation expressive de la description de la configuration de ce village métaphorise notamment le système relationnel des personnages avec Eok-soe et Deuk-bo. À travers leur histoire, ces trois légendes introduites dans le prologue se parachèvent comme une œuvre restructurée, ou plutôt réactualisée, répondant à une esthétique nouvelle :

(1) La légende des dragons blessés / 傷龍說 :

Dans la légende des dragons blessés, un couple de dragons jaunes était jadis sur le point de s'élever dans le ciel. Ils s'étaient blessés aux reins sur un rocher, qui s'était éboulé juste à ce moment-là de la montagne de Geumo. Le sang qui s'écoulait de leurs reins meurtris colora les environs de rouge, formant ainsi la vallée du lœess. 상룡설. 옛날 등천하려던 황룡 한 쌍이 때마침 금오산에서 굴러 떨어지는 바위에 맞아 허리가 상하니라. 그 상한 용의 허리에서 한없이 피가 흘러내려 부근 일대를 붉게 물들이니 이에서 황토골이 생기니라. (p. 215)

(2) La légende du couple de dragons / 雙龍說 :

Dans la légende du couple de dragons, deux dragons jaunes qui devaient monter au ciel ne s'abstinrent pas de s'accoupler à la veille même de leur ascension, ce qui irrita l'empereur de Jade, qui enterra leurs perles magiques dans le ciel à titre de sanction. Les deux dragons, privés de leurs perles magiques, se mordirent à la tête jusqu'au sang sans pouvoir contenir leur chagrin. De ce sang versé naquit la vallée du lœss.

쌍룡설. 역시 등천하려던 황룡 한 쌍이 바로 그 전야에 있어 잠자리를 삼가지 않은 지라, 상제께서 노하시고 벌을 내리사 그들의 여의주를 하늘에 묻어시매 여의주 를 잃은 한 쌍의 용이 슬픔에 못 이겨 서로 저희들의 머리를 물어뜯어 피를 흘리니, 이 피에서 황토골이 생기니라. (p. 215)

En plus de la légende des dragons blessés et de celle du couple de dragons, il en existe encore une autre, celle de la coupe des veines de la montagne.

(3) La légende de la coupe des veines de la montagne / 絕脈說 : 
Dans la légende de la coupe des veines de la montagne, un commandant de l'armée venu du pays de Tang (la Chine) arriva en déclarant que, si, à l'avenir, un hercule du pays de l'Est (la Corée) naissait dans cette montagne, il oserait attaquer la plaine centrale de la Chine. Il transperça d'un coup d'épée le pouls de la montagne, le sang coula alors dans cette vallée pendant trois mois et dix jours. C'est ainsi que les environs se couvrirent de lœss.

절 맥설. 옛날 당나라에서 나온 어느 장수가 여기 이르러 가로되, 앞으로 이 산에서 동국의 장사가 난다면 감히 중원을 범할 것이라, 이에 혈을 지르니, 이 산골에 석 달 열흘 동안 붉은 피가 흘러내리고 이로 말미암아 이 일대가 황토 지대로 변하니 라. (p. 216)

Chacune des légendes, source de la transmission orale dont Kim Dong-ri s'inspire, narre en fait un échec, une défaite, où le sang versé a vu naître la vallée du lœss. On prête ici à l'auteur l'explication des personnages par les lieux. La particularité topographique de la vallée du lœss, déterminée par ces trois légendes, prend sa véritable signification à travers l'histoire d'Eok-soe. Tout hercule qu'il est, son destin se confond à celui du village, historiquement menacé et castré. Le fait qu'il n'ait pas l'occasion d'exploiter véritablement sa force au cours d'une existence décourageante et tragique était déjà prédit par les légendes, notamment la troisième. Comme la montagne du lœss dont le pouls a été interrompu, sa vitalité a été réduite à néant, le conduisant à des combats perdus d'avance. L'arrivée de Deuk-bo dans le village et la suite des événements ne sont que des incidences fortuites qui répondent finalement à une nécessité, celle de la mort.

Son histoire est donc une version moderne des légendes de dragons et de la montagne coupée. Sans leurs perles magiques, les deux dragons, assimilés ici aux personnages Eok-soe et Deuk-bo, sont réduits à des serpents géants, car ils sont nés dans la vallée du lœss où les veines de la montagne ont été coupées et où s'est transmise l'histoire d'un couple de dragons qui avait échoué dans leur ascension vers le ciel pour avoir perdu leurs perles magiques. Leur destin est déterminé par la géomancie de leur lieu de naissance.

La lutte de Deuk-bo et d'Eok-soe n'est pas sans rappeler celle des dragons. La naissance et la croissance castrée du protagoniste Eok-soe sous le joug des conventions traditionnelles s'associent à la légende des «dragons blessés » et à celle de la " coupe des veines de montagne", de même que la lutte entre les deux hercules Eok-soe et Deuk-bo, causée en apparence par leur passion pour Bun-i et Seol-hui, est identique à celle d'un "couple des dragons». Suivant leur destin déterminé par la légende des dragons de la rivière située au pied de la montagne de Geumo du village où ils habitent, les deux colosses ne parviennent pas à s'accomplir et gaspillent leur énergie à se disputer les femmes. Ce qui entoure Eok-soe et Deuk-bo n'est pas une terre fertile mais une vallée stérile qui se rétracte, dévorée par le temps et l'espace.

L'espace présenté dans le prologue détermine le déroulement de l'histoire de [« La Vallée du lœss»], ainsi que le sort des personnages. Ils sont nés sous une mauvaise étoile et suivent un chemin menant à l'errance ou à la destruction. Inéluctablement attirés par une force absolue et fatale, ils se soumettent à leur destin transcendantal et irréversible. En agissant, ils ne réussissent pas à se défaire de leur sort. La véritable réaction est de décider de combattre à mort pour échapper à une vie dépourvue de sens. En même temps, pour Eok-soe, la mort en constitue la seule issue.

Le déploiement de l'énergie corporelle de ces deux hommes est déterminé par l'agencement de l'espace où ils vivent. C'est le village où Eok-soe vit qui bloque son énergie, mais le village lui aussi subit les lois d'une géomancie représentée par les trois 
légendes qui servent d'un intertexte et qui, en même temps, explique la raison de la déperdition de l'énergie des personnages. C'est pourquoi la nouvelle traduit un déterminisme, voire fatalisme de l'auteur. Kim Dong-ri puise ses sources dans les légendes, les contes à partir desquels il écrit ses romans. Il établit ainsi une concordance entre la topographie et le personnage par l'intermédiaire de mythes intégrant dragons et êtres humains. La vallée a le pouvoir de modifier la perception du monde et du réel et d'entraîner une perte d'identité et de sens existentiel. Comme 1939, l'année de la publication de cette nouvelle, tomba en plein milieu de l'occupation japonaise, force est de constater que l'histoire symbolise la souffrance de la nation coréenne, qui n'était pas autre chose qu'une montagne aux veines coupées ${ }^{4}$.

\section{BIBLIOGRAPHIE}

CHOE Chang-jo, [ « Pour comprendre la pensée de la géomancie coréenne »] / «한국 풍수사상의 이해를위하여 » / Hanguk pungsu sasang-ui ihae-reul wihayeo, in [La Vision traditionnelle de la géographie coréenne] / 한국의 전통지리사상 / Hangu-gui jeontong jiri sasang, Séoul, Mineumsa, 1991.

GENETTE Gérard, Figures I, II, III, Paris, Seuil, 1966, 1969, 1972.

GENETTE Gérard, Nouveaux Discours du récit, Paris, Seuil, 1983.

HAMON Philippe, Introduction à l'analyse du descriptif, Paris, Hachette, 1981.

HAMON Philippe, Le Descriptif, Paris, Hachette, 1993.

IM Gyeong-bin, [« Un arbre qui rêve du village vert »] / 푸른 마을을 꿈꾸는 나무 / Pureun maeureul kkumkku-neun namu, Jungang-ilbo, Séoul, 11/08/1998.

Jo Hoe-gyeong, Étude sur le roman de Kim Dong-ri / 김동리 소설 연구 / Kim Dong-ri soseol yeongu, Séoul, Gukhak jaryowon, 1999.

KIM Dong-ri, [« La Vallée du lœss »] / 황토기 / Hwangtogi, in [๕uvres de Kim Dong-ri] / 김동리 전 집 / Kim Dong-ri jeonjip, Séoul, Mineumsa, vol. 1, 1995.

KIM Jae-seon, [Histoire du roman coréen contemporain] / 한국현 대소설사 / Hanguk hyeondae soseol-sa, Séoul, Hongseongsa, 1979.

WANG-LE Min Sook, « Jeûner pour une offrande corporelle dans le Deungsinbul (Mi-Bouddha, mihomme) de Kim Dong-ri (1913-1995) », Transtext(e)s Transcultures 跨文本跨文化, 10 :2015, https:// journals.openedition.org/transtexts/596, consulté le 10/11/2016.

\section{NOTES}

1. On s'en sert aussi pour expliquer le mythe fondateur de la Corée. Selon celui-ci, c'était sous l'arbre sacré situé au sommet du mont Taebaek que Hwanung / 환웅, fils du dieu Hwanin / 환인, descendit avec neuf mille corps d'armée et fit de la Cité divine / 神市, sa capitale. Il s'y maria avec une ourse transformée en femme / 웅녀, et eut pour fils Dangun Wanggeom / 단군왕검, 
fondateur mythique de la Corée (2333 av. J.-C.). Le Mont Taebaek / 태백 et l'arbre sacré apparaissent comme configurations fastes, susceptibles de faire descendre l'être surnaturel sur la terre. Les ancêtres coréens croyaient que, lorsque le dieu qui habitait au ciel descendait sur le sol, il choisissait d'abord une haute montagne et ensuite un grand arbre ou une forêt majestueuse, tremplins du passage du ciel vers l'ici-bas. Voir IM Gyeong-bin, [« Un arbre qui rêve du village vert »] / 푸른 마을을 꿈꾸는 나무, Jungang-ilbo, Séoul, le 11 août 1998.

2. Sur l'importance de l'état de la montagne lors du choix de l'habitation, voir CHOE Chang-jo, [«Pour comprendre la pensée de la géomancie coréenne»] / «한국 풍수사상의 이해를위하여», in [La Vision traditionnelle de la géographie coréenne] / 한국의 전통지리사상, Séoul, Mineumsa, 1991.

3. Les passages cités de [«La Vallée du lœss»] et la page de référence renvoient à l'édition originale :

KIM Dong-ri, 황토기, [Æuvres de Kim Dong-ri] / 김동리 전집 / Kim Dong-ri jeonjip, Séoul, Mineumsa, vol. 1, 1995.

4. KIM Jae-seon, [Histoire du roman coréen contemporain] / 한국현 대소설사 / Hanguk hyeondae soseol-sa, Séoul, Hongseongsa, 1979, p. 457.

\section{AUTEUR}

\section{MIN SOOK WANG-LE}

Min Sook Wang-Le est maître de conférences en études coréennes à l'Université Jean Moulin Lyon 3 et membre de l'IETT. Sa recherche porte sur la littérature coréenne moderne et contemporaine et s'articule autour de deux champs principaux : celui de la relation entre la littérature et la représentation des religions et celui de l'écriture des femmes en Corée. Parmi ses nombreux articles, on peut citer : « Amitié dans le siècle : Kim Dong-ri et Seo Jeong-ju » (Diogène, $\mathrm{n}^{\circ}$ 265-266, 2019), « Jeûner pour une offrande corporelle dans le Deungsinbul [Mi-Bouddha, mihomme] de Kim Dong-ri (1913-1995) » (Transtext(e)s Transcultures [En ligne], 10 | 2015, URL : http://transtexts.revues.org/596) et « Réception de Rousseau en Corée de 1896 à 1945 » (Rousseau en Asie, Rousseau Studies n 3, 2015). 


\title{
Le corps dans tous ses états: Les Belles de Halimunda d'Eka Kurniawan
}

\author{
Étienne Naveau
}

1 Les quatre romans qu'Eka Kurniawan (1975-) a publiés depuis une quinzaine d'années ont pour point commun de soulever la question du corps. Lelaki Harimau / L'homme-tigre (2004 / 2015) évoque la métamorphose d'un homme en tigre, tandis qu'o (2016), a contrario, a pour personnage principal un singe qui rêve de devenir humain. Seperti Dendam, Rindu Harus Dibayar Tuntas (2014) / Cash (2019) est l'histoire d'un chauffeurroutier qui souffre d'impuissance sexuelle, et dont la femme est une championne d'arts martiaux. En revanche, le corps n'est apparemment pas le sujet principal du premier roman d'Eka Kurniawan, Cantik itu luka (2002), dont la traduction française est parue en 2017 avec pour titre Les Belles de Halimunda ${ }^{1}$. Bien que le corps y soit représenté dans tous ses états, ce roman reprend un thème fréquemment traité par Kurniawan, puisqu'il expose l'histoire d'une vengeance, qui s'accomplit sur plusieurs générations et recoupe l'histoire du pays, des dernières années de la colonisation à la période Soeharto (1966-1998), soit sur une bonne soixantaine d'années, marquée par de considérables bouleversements historiques.

Mais s'il n'est pas le motif principal des Belles de Halimunda, le corps en dessine pourtant le fil conducteur. Les Belles n'est pas un roman du corps, mais c'est néanmoins un roman profondément incarné, ancré dans la réalité historique, géographique, sociale et culturelle indonésienne. La ville imaginaire de Halimunda, qui est l'un des actants du roman, s'inspire à l'évidence des villes sundanaises de Pangandaran et Tasikmalaya et de la ville de Cilacap, sur la côte sud de Java, port d'embarquement pour l'île pénitentiaire de Nusakambangan où le roman situe le bagne du Bloedenkamp ${ }^{2}$. Mais tandis que Nusakambangan héberge, à partir de 1965, des prisonniers politiques en attente d'être déportés à Buru, comme le romancier Pramoedya Ananta Toer (1925-2006), auquel Eka Kurniawan consacra son master³, le Bloedenkamp reçoit, durant l'occupation japonaise (1942-1945) des prisonniers politiques néerlandais ou métisses, dont Dewi Ayu, le personnage principal du roman.

3 Cette incarnation du roman dans la réalité provinciale indonésienne le distingue nettement des deux courants qui dominent la scène littéraire depuis la chute du régime 
Soeharto, au tournant du $\mathrm{xxI}^{\mathrm{e}}$ siècle : la littérature féministe et la littérature islamiste, que tout oppose quant aux contenus et aux choix idéologiques, mais qui se rejoignent néanmoins formellement ${ }^{4}$. S'inscrivant dans le droit fil des écritures féministes, amorcées par l'épistolière Raden Adjeng Kartini (1879-1904) et poursuivies par la romancière Nh. Dini (1936-2018), des écrivaines d'une autre génération, telles que Djenar Maesa Ayu (1973-) et surtout Ayu Utami (1968-) ont connu un succès de scandale en protestant contre la domination masculine et en dépeignant sans fard la sexualité féminine. Paru peu avant la chute de Soeharto (1921-2008), Saman (1998 / 2008) d'Ayu Utami a eu un retentissement comparable à celui du roman populaire de Habiburrahman El Shirazy (1975-) [Les Versets de l'amour] / Ayat-ayat Cinta (2004) ${ }^{5}$. Ce dernier ouvrage, paru chez l'éditeur musulman Republika, eut droit à de multiples rééditions, à une adaptation cinématographique en 2008 par le réalisateur Hanung Bramantyo, et à une suite, parue en 2015, adaptée au cinéma en 2017 par Guntur Soehardjonto. Le fait que ce roman populaire qui enchâsse des sermons représentatifs de l'islam réformiste dans une intrigue sentimentale à l'eau de rose, ne fasse l'objet d'aucun projet de traduction en langues européennes, contrairement aux œuvres des romancières féministes et à celles de Kurniawan, dont la traduction de la nouvelle «Graffiti in the Toilet » avait été initiée par Benedict Anderson, atteste de la distorsion entre l'attente d'exotisme et d'esthétisme du public international et la demande locale'.

4 Les romans rédigés par les islamistes et les féministes ont pour point commun d'être souvent abstraits, édifiants ou militants, et de mettre en scène des personnages évoluant dans les milieux métropolitains de la mondialisation. Bien que certaines formes d'islam et de féminisme soient profondément inculturées ${ }^{7}$, les deux tendances qui se disputent de nos jours le champ littéraire indonésien sont d'inspiration étrangère, et se rejoignent dans leur rejet de la culture locale. Tandis que les féministes expriment leur révolte contre les traditions patriarcales, les islamistes manifestent leur refus d'accommoder la religion aux coutumes locales, susceptibles d'en dégrader la pureté, en la mêlant d'éléments autochtones prétendument empreints de paganisme. Islamistes et féministes se polarisent sur le corps féminin, soit pour le soumettre à une loi religieuse soit, au contraire, pour l'émanciper et lui permettre de s'exprimer comme sujet désirant. Cette polarisation sur le corps féminin s'accomplit souvent au détriment d'une prise en compte du corps social et de la diversité des corps individuels8. Ce n'est pas le cas chez Kurniawan qui évoque par exemple un corps monstrueux, mais également des corps souffrants ou se décomposant et des corps évanescents (fantômes) ou transfigurés (ressuscités, délivrés vivant, etc.). Par ailleurs, il insère le corps féminin dans l'histoire de la nation, dont il présente en quelque sorte une allégorie. À travers les souffrances de Dewi Ayu et de sa descendance, Kurniawan exprime la destinée tragique et la résilience du peuple indonésien?.

5 Tout part du fait qu'un planteur néerlandais, Ted Stammler, dont la première syllabe du nom, stam, qui signifie «tronc » ou "clan ", évoque son statut de fondateur d'une dynastie, s'approprie le corps d'une belle indigène, Ma Iyang, en l'arrachant des bras de son amant autochtone, Ma Gedik. Les petits-enfants et arrière-petits enfants de Stammler et de sa concubine sont toutes des filles, qui ont pour point commun d'être à la fois belles, puisqu'elles sont métisses, et maudites, puisque ce métissage est l'effet d'un rapport de forces, d'une violence faite aux autochtones par le colon ${ }^{10}$. La traduction littérale du titre indonésien serait en fait : «Belle est blessée ». Les blessures de ces jolies filles illustrent d'ailleurs celles du corps social, du peuple autochtone traumatisé historiquement par les guerres civiles, l'occupation japonaise, la 
colonisation, mais également par l'islamisation. Le roman décrit la lutte acharnée de Dewi Ayu pour sauver sa progéniture, et s'achève par le triomphe en demi-teinte des femmes qui, seules, ont survécu, tandis que leurs époux et enfants ont succombé à la malédiction du fantôme de Ma Gedik.

\section{Le corps comme être de besoin}

10 L'un des termes signifiant "corps » en indonésien, tubuh, est l'anagramme du mot butuh, qui signifie "avoir besoin de", mais peut également revêtir une connotation phallique. Comme tout animal, l'homme a un corps qui entre en relation avec son environnement naturel et lutte pour assurer à la fois la conservation de l'individu et celle de l'espèce. Se conserver dans l'être en tant qu'individu, c'est assouvir ses besoins en matière d'alimentation et de sommeil. Quant à la conservation de l'espèce, elle passe par la reproduction sexuelle. Ces questions sont évoquées principalement à travers le personnage de Dewi Ayu, l'héroïne du roman.

11 En tant qu'Eurasienne, d'ascendance néerlandaise, cette dernière est envoyée en camp de concentration. Le roman évoque ses stratégies cocasses de survie, qui parodient les récits de captivité, tradition littéraire remontant à l'époque coloniale, mais ravivée aux lendemains de la chute de Soeharto, quand se multiplièrent les témoignages de déportés, notamment celui de Pramoedya Ananta Toer (1925-2006) ${ }^{11}$. L'insalubrité des conditions de détention et le manque de nourriture, qui condamnent les enfants en bas âge à la mort, font l'objet d'une description soignée (Les Belles, p. 92, 98). Pour satisfaire ses besoins, Dewi Ayu élève des sangsues et va même jusqu'à tuer un jeune crocodile (Les Belles, p. 93-94, 102-103). « Nous n'allons pas avoir faim. En plus des sangsues, il y aussi des lézards, des geckos et des rats» (Les Belles, p. 94) / Kita tak akan kelaparan. Selain lintah, masih ada tokek, cicak dan tikus (p.63), dit-elle à ses camarades de captivité, que ces nourritures ne tentent guère.

12 À la persécution des Eurasiens par les Japonais succède, à partir de 1965, celle des communistes indonésiens par l'armée de terre. Après les massacres et l'interdiction du parti communiste décrétée le 12 mars 1966, Kliwon, le gendre communiste de Dewi Ayu, est épargné, grâce à la protection de son beau-frère militaire, qui a obtenu, en échange de sa mansuétude, que son épouse, Alamanda, se donne enfin à lui charnellement (Les Belles, p. 441-443). Pour subvenir aux besoins de sa famille, Kliwon cultive un lopin de terre et ouvre une bibliothèque de prêt. Mais, à la suite d'une dénonciation, il doit fermer ce commerce et se voit forcé d'escalader des falaises pour cueillir des nids d'hirondelle (Les Belles, p. 461-462, 464, 484). Après la chute mortelle d'un de ses camarades, il ouvre une fabrique de caleçons de bains, destinés aux touristes qui commencent à affluer, grâce à l'essor économique de l'Ordre nouveau (1966-1998), sur la plage de Halimunda (Les Belles, p. 485-489).

Lutter pour la survie du corps, c'est également veiller à satisfaire ses besoins en matière de santé. Pour fournir des médicaments à la mère d'une de ses amies, également prisonnière, Dewi Ayu vend ses faveurs au commandant du camp (Les Belles, p. 99-101). 
Sa domestique usera vainement de la même stratégie avec un directeur d'école pour que la quatrième fille de Dewi Ayu, qui est horriblement laide, puisse être scolarisée (Les Belles, p. 34).

14 Mais si leur corps est d'abord pour certains personnages féminins un moyen d'échange, il fournit également, à leurs partenaires masculins, des occasions de satisfaction sexuelle. C'est ainsi que les plus jolies des prisonnières, parmi lesquelles Dewi Ayu, sont recrutées d'office comme prostituées destinées à assouvir les ardeurs des soldats nippons. Nombre d'entre elles s'offriront par la suite à des militaires néerlandais ou seront violées par des militants nationalistes indonésiens. Cette évocation de la prostitution est l'occasion d'ironiser sur la tentative vouée à l'échec d'une camarade néerlandaise de Dewi Ayu, qui tente vainement de protéger sa vertu en récitant des psaumes (Les Belles, p.124-125). Tandis que les versets sacrés se montrent en la circonstance impuissants à réaliser des miracles, l'imagination de Dewi Ayu parviendra à influencer la réalité et à exaucer, à son insu, son désir d'avoir une quatrième fille laide (Les Belles, p. 29-30). À la fuite superstitieuse dans la spiritualité, Dewi Ayu oppose son matérialisme pragmatique, conseillant à ses compagnes de suivre sa propre démarche : neutraliser l'agresseur en lui racontant des histoires, à la manière de Shéhérazade (Les Belles, p. 29, 128-129), opposer à ses ardeurs la froideur et l'inertie d'un cadavre ou le rendre dépendant de son corps, pour inverser le rapport de domination (Les Belles, p. 126-128). Alamanda, la fille aîné de Dewi Ayu, aura recours à cette même stratégie d'esquive pour se dérober à son devoir conjugal avec le shodancho (Les Belles, p. 304-305).

15 Dewi Ayu va pour sa part décider de rester dans le métier, car son sentiment de culpabilité lié à la faute de son grand-père l'a dissuadé de fonder une vraie famille. Mais sa beauté lui permet d'accéder au statut de courtisane de luxe. Ses services coûtent cher et elle ne couche qu'avec un homme par nuit. Elle procède au décompte exact des 172 amants qu'elle a eus tout au long de sa carrière :

Le plus vieux avait 92 ans, le plus jeune 12. Il venait d'être circoncis depuis une semaine (Les Belles, p. 12)

Yang paling tua berumur sembilan puluh dua tahun, yang paling muda berumur dua belas tahun, seminggu setalah disunat (p. 3 )

16 De même, Ma Iyang et Ma Gedik énumèrent les relations sexuelles qu'ils ont eues lors de leurs seize années de séparation. La précision absurde de ce genre de décomptes n'est pas sans évoquer le gigantisme des énumérations rabelaisiennes ${ }^{12}$ :

Qu'espères-tu encore de moi ? lui demanda Ma Iyang. Tout mon corps a été léché et imprégné de la bave d'un Hollandais, et mon sexe a été pénétré par le sien mille cent quatre-vingt-douze fois.

- J'ai pénétré vingt-huit sexes de femmes à hauteur de quatre cent soixante-deux fois, et $\mathrm{j}$ 'ai introduit mes mains dans un nombre incalculable de sexes, y compris ceux d'animaux, en quoi sommes-nous différents? (Les Belles, p. 55)

“Apakah kau bisa mengharapkanku ?" tanya Ma Iyang. "Seluruh tubuhku telah dijilati dan dilumuri ludah orang Belanda, dan kemaluanku telah ditusuk kemaluannya sebanyak seribu seratus sembilan puluh dua kali.

Aku telah menusuk dua puluh delapan kemaluan perempuan sebanyak empat ratus enam puluh dua kali, dan menusuk tanganku sendiri dalam jumlah tak terhitung, belum termasuk kemaluan binatang, apakah kita berbeda ? (p. 34-35)

La sexualité n'est pas seulement orientée par l'obtention d'un plaisir individuel, idéalement partagé, mais encore par la satisfaction des exigences de reproduction de l'espèce. Le roman évoque la résilience extraordinaire de Belle, la quatrième fille de 
Dewi Ayu, qui résiste à ses tentatives d'avortement (Les Belles, p. 15). Dewi Ayu a été involontairement enceinte d'un soldat japonais, puis d'un partisan indonésien qui l'ont violée (Les Belles, p. 131, 141). De même, le mari de sa fille aînée, Alamanda, est un militaire qui l'a violée (Les Belles, p. 291-294). Il est surnommé le shodancho par allusion au grade qu'il avait dans la troupe autochtone servant d'auxiliaire à l'armée d'occupation nippone. Alamanda se refuse à lui et il souffre qu'elle ne lui donne pas d'enfants. La beauté semble ici jouer le rôle d'une ruse de l'espèce, qui attache l'homme à un corps individuel ${ }^{13}$. Après avoir trouvé moyen de forcer par deux fois les défenses de son épouse, celle-ci tomba enceinte. Mais les deux premiers fotus, n'étant pas des enfants de l'amour, s'évanouirent mystérieusement peu avant la date prévue pour l'accouchement, après que Kliwon eut constaté que le ventre d'Alamanda ne contenait aucun enfant, prédiction que le shodancho interprétera comme un mauvais sort (Les Belles, p. 378) :

\begin{abstract}
Alamanda elle-même ne comprit pas ce qui s'était passé. À son réveil, elle avait éructé soudain très fortement, comme pour expulser une grande quantité de vents, et elle constata qu'elle était redevenue mince, sans aucun poids dans ses entrailles. Elle fut en réalité éberluée par le phénomène, bien que, deux mois auparavant, le camarade Kliwon lui eût annoncé que son ventre était comme une marmite vide, qu'il ne contenait que du vent, rien que du vent. (Les Belles, p. 381-382, 398)

Alamanda sendiri tak tahu apa yang terjadi karena ketika bangun tidur tiba-tiba ia bersendawa begitu keras seolah mengeluarkan begitu banyak angin dan tiba-tiba ia menemukan dirinya bagai seorang perawan bertubuh langsing tanpa bobot di dalam rahimnya. Kenyataannya dia begitu terkejut melihat itu semua meskipun dua bulan sebelum itu Kamerad Kliwon sudah mengatakan kepadanya bahwa perutnya bagai panci kosong, hanya angin dan angin. (p. 279)
\end{abstract}

Assurer sa reproduction est une manière de lutter contre la mort. Dewi Ayu cherchera ainsi à sauver sa descendance de la malédiction du fantôme de Ma Gedik, l'amant autochtone de la concubine de son grand-père. Mais si elle parviendra bien à sauver ses quatre filles, elle aura perdu tous ses gendres, ainsi que tous ses petits-enfants et arrière-petits-enfants. De surcroît, avoir une postérité est également un moyen de reconnaissance sociale. Le refus de toute relation charnelle qu'Alamanda oppose au shodancho, fait redouter à ce dernier de passer pour un impuissant ou pour un castré (Les Belles, p. 310). Pour ne pas être la risée de l'opinion publique et sauver les apparences, il se coupe un doigt et répand quelques gouttes de sang sur les draps de leur lit de noce, afin de témoigner qu'il a bien épousé et pénétré une vierge (Les Belles, p. 306).

19 Mais le corps n'est pas uniquement en proie aux besoins, à la nécessité de se nourrir pour se conserver et à celle de reproduire l'espèce, il est également un être social. Dans la mesure où il est à la fois objet de contemplation et source d'action, il entre en relation avec autrui sur le mode de la séduction ou du pouvoir.

\title{
Le corps comme être social
}

C'est par son apparence que le corps influence autrui. La séduction des corps féminins provoque l'appétit des hommes. En témoignent le rapt de Ma Iyang par Stammler, le grand-père de Dewi Ayu, mais également le viol de sa fille, Alamanda, par le shodancho, qui s'est épris d'elle et rêve d'en faire la mère de ses enfants. La beauté est une vulnérabilité, une invitation à l'outrage et à la profanation. Ne peut-on considérer ici que les charmes de Ma Iyang représentent ceux de l'archipel indonésien, suscitant les 
convoitises d'étrangers, avides de s'en emparer? «La femme faible n'est-elle pas le symbole de la nation dominée $»^{14}$. En faisant de la souffrance et de la résistance de certains de ses personnages féminins, comme Ma Iyang et sa petite-fille, Dewi Ayu, une allégorie de l'oppression des peuples colonisés, Kurniawan s'inscrit dans le droit fil d'un genre littéraire florissant à la fin du xix siècle: les histoires de nyai (concubines indigènes d'Occidentaux). On peut penser ici à [L'Histoire de la nyai Paina] / Tjerita Nji Paina de $\mathrm{H}$. Kommer ${ }^{15}$, qui raconte l'histoire d'une belle autochtone que sa beauté expose aux manigances d'un contremaître néerlandais lubrique, faisant pression sur le père de la jeune femme pour la contraindre à devenir sa maîtresse. Cette dernière réussira toutefois à se débarrasser de son agresseur en contractant volontairement une variole dont il mourra. Elle en réchappera, y perdra sa beauté, mais se mariera néanmoins finalement avec un compatriote.

De même, la beauté apparaît comme une malédiction pour les personnages féminins des Belles de Halimunda. C'est pourquoi Dewi Ayu va souhaiter que sa dernière fille, qui sera appelée «Belle» par antiphrase, soit laide. Devinant que sa monstruosité va l'exposer au rejet de la société et la contraindre à vivre cachée, la sage-femme n'ose révéler la vérité à Dewi Ayu, laquelle redoute, en revanche, que sa fille soit jolie. Il en résulte un quiproquo humoristique, puisque les deux femmes s'apitoient sur le sort de Belle pour des raisons diamétralement opposées (Les Belles, p. 13-14). Toujours est-il que la description que Kurniawan donne de Belle se présente comme un blason inversé : ${ }^{16}$

«Malheureuse enfant», dit la sage-femme en regardant le visage affligeant du bébé. Elle n'était même pas capable de le décrire. Elle se l'imaginait seulement comme un monstre damné de l'enfer. Le corps de l'enfant était tout noir, comme s'il avait brûlé vif, et avait une forme qui ne ressemblait à rien. Par exemple, elle n'était pas certaine que son nez fût bien un nez, car il ressemblait davantage à une prise de courant qu'aux nez qu'elle connaissait depuis sa plus tendre enfance. Sa bouche lui faisait penser au trou d'une tirelire en forme de cochon, et ses oreilles à des queues de casseroles. Elle était convaincue qu'il n'y avait pas de créature au monde plus laide que cette malheureuse enfant et, si elle était Dieu, il semble qu'elle aurait trouvé plus d'espoir à la tuer qu'à la laisser vivre. Le monde allait se montrer d'une méchanceté impitoyable envers elle.

«Malheureux bébé, dit la sage-femme, avant d'aller chercher quelqu'un pour l'allaiter.

- Oui, malheureux bébé, dit Dewi Ayu, en se tordant sur sa couche.»(Les Belles, p. 12-13)

"Gadis kecil yang malang", kata si dukun bayi sambil memandang wajah si bayi yang menyedihkan. Ia bahkan tak mampu mendeskripsikannya, hanya membayangkannya sebagai monster kutukan neraka. Seluruh tubuh bayi itu hitam legam seperti terbakar hidup-hidup, dengan bentuk yang tak menyerupai apa pun. Ia, misalnya, tak begitu yakin bahwa hidung bayi itu adalah hidung, sebab itu lebih menyerupai colokan listrik daripada hidung yang dikenalnya sejak kecil. Dan mulutnya mengingatkan orang pada lubang celengan babi, dan telinganya menyerupai gagang panci. Ia yakin tak ada makhluk di dunia yang lebih buruk rupa dari si kecil malang itu, dan seandainya ia Tuhan, tampaknya ia lebih berharap membunuh bayi itu daripada membiarkannya hidup; dunia akan menjahatinya tanpa ampun.

"Bayi yang malang," kata si dukun bayi lagi, sebelum pergi mencari seseorang untuk menyusuinya.

"Yah, bayi yang malang," kata Dewi Ayu sambil menggeliat di atas tempat tidur. (Les Belles, p. 3) " forme " / bentuk du corps de Belle « qui ne ressemblait à rien " / tak menyerupai apa 
pun $^{18}$. Or, le verbe menyerupai est dérivé d'une base sanskrite bien connue, notamment dans les textes bouddhistes, rupa, qui signifie "forme". La laideur relève bien de

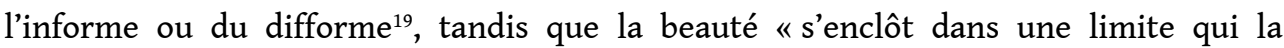
présente à l'état d'achèvement $»^{20}$. Un beau corps, c'est un corps bien proportionné, qui forme un "tout unifié", autotélique ${ }^{21}$. À rebours du corps grotesque ouvert sur le monde, qui présente des protubérances, des saillies brutales et des angles aigus ${ }^{22}$, le beau corps, tout en surface, se conforme aux normes du goût classique. C'est un « corps parfaitement prêt, achevé, rigoureusement délimité, fermé, montré de l'extérieur, non mêlé, individuel et expressif $»^{23}$. La laideur de Belle est attestée par sa ressemblance à la matière fécale et par le caractère hétéroclite de sa composition (Les Belles, p. 12).

Pour les personnages de Kurniawan, la beauté du corps féminin ne fait pas l'objet d'une satisfaction désintéressée ${ }^{24}$. Elle ne suspend ni ne sublime le désir, mais l'exacerbe au contraire, et s'avère de surcroît cause de rivalités et de guerres entre mâles dominants, comme le montre la légende de la belle princesse Rengganis (Les Belles, p. 162-163). Sa beauté est si destructrice que son père et sa mère finissent par tomber amoureux d'elle (Les Belles, p. 161), et qu'elle met le pays à feu et à sang :

Sa beauté était comme une malédiction, dont le charme opérait de plus en plus follement : des milliers de soldats étaient blessés à mort, tout le pays allait à vaul'eau, ravagé par d'impitoyables famines et des maladies qui faisaient rage. Et tout cela à cause d'une beauté qui portait malheur. (Les Belles, p. 164)

Kecantikannya bagai kutukan, dan kutukan itu bekerja semakin gila: ribuan prajurit terluka sebelum mati, seluruh negeri porak-poranda, penyakit dan kelaparan menyerang tanpa ampun, semua karena kecantikan yang mengutuk tersebut. (Les Belles, p. 114)

Pour mettre fin au sanglant conflit de ses prétendants, sa seule issue est de se dissimuler à la vue des hommes et d'épouser le premier venu, en l'occurrence un chien, qui présente l'immense avantage d'être insensible aux qualités esthétiques (Les Belles, p. 162, 164-166, 169-170).

Comme nous l'avons vu, la grâce du corps féminin l'expose à l'outrage. Elle suscite la violence des hommes avides de s'en emparer, et rivalisant entre eux dans ce dessein. Mais la beauté n'est pas seulement une faiblesse ${ }^{25}$, elle est également une force, qui pétrifie celle des hommes en les abêtissant sentimentalement et en les frustrant sexuellement. C'est ainsi que le truand Mamane Gendeng, qui épousera la troisième fille de Dewi Ayu, se dirige vers la cité de Halimunda, parce qu'il est en quête de cet idéal du Beau que représente, à ses yeux, la princesse Rengganis. Cet artiste martial ne chemine pas seulement sur les traces de sa dulcinée, mais encore sur celles de Don Quichotte, le chevalier errant qui lui sert de modèle comique. Tandis que la référence, suggestive, à Rabelais est proposée par un commentateur, Stefan Danerek, c'est l'auteur lui-même qui place en exergue de son roman un extrait du tout premier chapitre de Don Quichotte évoquant les préparatifs du « chevalier à la triste figure $»^{26}$. Cette citation indonésienne de la traduction anglaise n'a pas été retraduite en français. On notera que Kurniawan, qui ne lit ni le français ni le castillan et ne dispose pas davantage de traductions indonésiennes faites sur les originaux, n'a eu qu'un accès indirect à ces auteurs du Xvi ${ }^{\mathrm{e}}$ siècle que sont Rabelais et Cervantès, puisqu'il passe par le truchement de traductions en anglais moderne. La passion rend également le shodancho ridicule. Il se heurte à la barrière que lui oppose la ceinture de chasteté de son épouse, qui triomphe par deux fois de sa bêtise. Ne pouvant accéder à son intimité, il décoche un violent coup de pied dans la culotte en fer qu'elle porte. Ce qui lui fait évidemment plus 
de mal qu'à elle (Les Belles, p. 311). Enfin, le communiste Kliwon, qui est le Don Juan, ou plutôt l'Arjuna, de la petite ville ${ }^{27}$, puisqu'il séduit les femmes, avant de fasciner les foules par la magie de son verbe, ne parvient pas à ses fins de conquérir Dewi Ayu. Un soir où il vient lui chanter la sérénade, à la manière de Roméo, cette dernière ne consent pas à se déplacer, mais lui envoie sa fille aînée, qui est alors toute jeune, pour lui porter de la limonade. Il en tombe alors éperdument amoureux, mais elle n'est pas alors en âge de partager ses sentiments (Les Belles, p. 227-231, 407, 429) :

Il n'y a absolument aucune femme dans toute la ville et peut-être même dans le monde entier qui soit plus jolie qu'elle. Elle est plus belle que Rengganis, la princesse qui s'est mariée avec un chien, au moins d'après moi. Elle est plus belle que la reine de la mer du sud. Elle est plus belle qu'Hélène, qui avait fait éclater la guerre de Troie. Elle est plus belle que Diah Pitaloka qui avait provoqué la guerre entre Majapahit et Pajajaran. Elle est plus belle que Juliette qui avait poussé Roméo à se donner la mort. Elle est plus belle que qui que ce soit. Tout son corps semble irradier la lumière, ses cheveux reluisent davantage que des chaussures fraîchement cirées, la peau de son visage est si tendre, comme s'il était fait de cire, et son sourire semble aspirer tout ce qui se trouve autour d'elle. (Les Belles, p. 232) Tak seorang pun di kota ini, dan mungkin di seluruh alam semesta, lebih cantik darinya. Ia lebih cantik dari Rengganis Sang Putri yang kawin dengan anjing, paling tidak menurutku. Ia lebih cantik dari Ratu Laut Kidul. Ia lebih cantik dari Helena yang membuat perang Troya meletus. Ia lebih cantik dari Diah Pitaloka yang menyebabkan perang Majapahit dan Pajajaran. Ia lebih cantik daripada Juliet yang membuat Romeo nekat bunuh diri. Ia lebih cantik dari siapa pun. Seluruh tubuhnya seperti mengeluarkan cahaya, rambutnya lebih mengilau dari sepatu yang baru disemir, wajahnya begitu lembut seolah ia dibuat dari lilin, dan senyumnya seperti mengisap segala yang ada di sekitarnya ${ }^{28}$. (p. 165)

Les trois gendres de Dewi Ayu, qui représentent trois types sociaux, sont frustrés sexuellement et sentimentalement. Le militaire s'est marié avec l'ânée, qui était fiancée avec le communiste, après l'avoir droguée et violée. Elle consent à l'épouser pour préserver son honneur, mais ne l'aime pas, et reste éprise de son ex-fiancé, le communiste Kliwon. Elle recourt à divers stratagèmes pour se refuser à son mari : port d'une ceinture de chasteté, utilisation de mantras, etc. Il parvient toutefois par deux fois à l'engrosser, mais leur enfant disparaît mystérieusement in utero peu avant la date de l'accouchement. Kliwon est lui aussi d'abord frustré et malade d'amour. Ce n'est que quelques années plus tard qu'Alamanda répondra passionnément à son amour, après avoir cherché à rivaliser avec lui en matière de séduction. Quant au truand, Mamane Gendeng, il est retenu par le jeune âge de son épouse, Maya Dewi, qui n'a que 12 ans au moment de son mariage. Il se conduit d'abord comme un père avec sa jeune épouse qu'il emmène à l'école, à laquelle il fait réciter ses leçons et lit des histoires pour l'endormir. C'est ainsi que le jour de leurs noces, arrangées par Dewi Ayu pour mettre sa fille en sécurité, le truand se contente de rester assis sur une chaise au bord du lit de son épouse et de la contempler en silence :

Il regardait le visage de la jeune femme avec le regard que porte un homme sur la dépouille de sa bien-aimée, avouant que son humble beauté était tellement séduisante, chose qu'il n'avait jamais remarquée auparavant. Ses cheveux noirs et brillants ondulaient entre sa tête et la taie d'oreiller. Ses yeux, qui le dévisageaient à leur tour, étaient si purs et si enfantins. Son nez et ses lèvres, comme d'ailleurs tout le reste, étaient si époustouflants. Mais voyez-vous, tout cela était si mignon. Ses mains étaient encore celles d'une petite fille, même chose pour ses mollets. Il put même apercevoir que, sous sa chemise de nuit, ses seins n'avaient pas encore complètement poussé. Il ne pouvait pas faire l'amour à une petite fille comme elle, qui paraissait encore innocente. (Les Belles, p. 348) 
Ia memandang wajah gadis kecil itu dengan tatapan seorang lelaki yang melihat kekasihnya mati, mengakui kecantikan kecilnya begitu memesona, suatu hal yang tak pernah ia perhatikan sebelumnya. Rambutnya hitam mengilau, mengembang di bawah kepalanya di atas permukaan bantal. Matanya yang balas menatap dirinya begitu jernih dan kekanak-kanakan. Hidung dan bibirnya dan segalanya begitu menakjubkan. Tapi lihatlah, semuanya masih begitu mungil. Tangannya masihlah tangan seorang gadis kecil, begitu pula betisnya. Bahkan ia bisa melihat di balik pakaian tidur itu dadanya belum juga tumbuh secara sempurna. Ia tak mungkin menyetubuhi gadis kecil yang tampak tak berdosa seperti itu. (Les Belles, p. 254)

À la frustration sexuelle et sentimentale des hommes, s'oppose, en règle générale, la froideur des femmes. Hormis Belle, qui rêve du Prince charmant, Ma Iyang et Alamanda, qui sont des amantes passionnées, et Moyang, la trésorière de Mamane Gendeng, qui couche avec tout le monde et trompe son mari, Roméo (Les Belles, p. 33, 48, $56,259,498,576-577)$, ces dernières ne sont pas en proie au désir. Le roman envisage, nous semble-t-il, la sexualité d'un point de vue étroitement masculin. Les personnages féminins sont généralement dominés par la raison. Ils se montrent prudents, voire carrément cyniques et calculateurs, comme le sont Dewi Ayu et la mère Roussette, la tenancière de la maison close. De manière assez classique et ambivalente, la femme est à la fois objet d'idéalisation et de sublimation, comme la princesse Rengganis, et source de concupiscence et de jouissances terrestres ${ }^{29}$. Ce qu'écrit Mikhail Bakhtine au sujet de Rabelais pourrait parfaitement s'appliquer au roman d'Eka Kurniawan :

La femme est essentiellement liée au «bas» matériel et corporel: elle est l'incarnation $d u$ "bas" à la fois rabaissant et régénérateur. Elle est aussi ambivalente que lui. La femme rabaisse, rapproche de la terre, corporalise, donne la mort ; mais elle est avant tout le « principe de la vie », le « ventre ». Telle est la base ambivalente de l'image de la femme dans la tradition comique populaire ${ }^{30}$.

Un autre ressort comique réside dans le contraste qui oppose le destin de certains personnages et celui du corps social. C'est en effet à une des heures les plus sombres et les plus sanglantes de l'histoire de l'Indonésie, lors des massacres de communistes de l'automne 1965, que le militaire et le truand jouiront d'une embellie dans leurs vies de couple et pourront enfin goûter aux délices de leur lune de miel et se mettre à procréer (Les Belles, p. 433, 439, 445).

Les trois gendres de Dewi Ayu, le truand, le militaire et le communiste sont trois hommes de pouvoir qui se disputent le corps de Dewi Ayu, que Kliwon ne parviendra jamais à posséder. Ses deux beaux-frères pratiquent également les arts martiaux et l'ascèse. Le truand dispose en effet d'une technique magique qui rend son corps invulnérable aux balles. Il s'arroge l'exclusivité du corps de Dewi Ayu, mais le militaire lui conteste son monopole, après une visite au bordel, destinée à lui faire oublier la passion qu'il éprouve pour Alamanda (Les Belles, p. 176, 182-188, 285-286). Les deux hommes en deviennent mortellement ennemis, bien qu'ils finissent par se fréquenter, en échangeant sur leurs frustrations conjugales autour d'une partie de cartes, à la gare routière (Les Belles, p. 435-436). À leur rivalité amoureuse initiale, se superpose celle qui oppose le militaire au communiste, respectivement époux et fiancé d'Alamanda.

Outre le corps de Dewi Ayu, c'est également le corps social, c'est-à-dire la nation indonésienne représentée par la cité de Halimunda, que se disputent le militaire, le truand et le communiste. Les trois hommes luttent pour le contrôle de la ville et de la population de Halimunda. Le truand règne sur la ville après avoir éliminé physiquement son concurrent au terme d'un combat épique (Les Belles, p. 174). Il inspire la peur à ses concitoyens, mais rêve d'une vie bourgeoise. Le militaire est un héros de 
l'indépendance, formé par les Japonais, que tout le monde admire pour avoir libéré la ville en s'insurgeant contre ses maîtres (Les Belles, p. 278). Quant au communiste, il séduit les filles et les foules par son charme et par son verbe (Les Belles, p. 406-408). Ces deux hommes qui se disputent le cœur d'Alamanda et le contrôle de la ville ont également pour point commun leur désintéressement, qui les conduit à refuser un destin national par fidélité à Halimunda (Les Belles, p. 206-207 et 410-411). Cette double rivalité des gendres de Dewi Ayu va dégénérer en conflit de grande ampleur et en massacres de masse, comme le montre cette évocation réaliste de la répression anticommuniste, qui n'est pas sans rappeler les témoignages historiques :

Les militaires s'étaient lassés de convoyer les cadavres des communistes au cimetière et d'aider le fossoyeur à creuser de grandes fosses communes. Les communistes étaient éliminés, que ce soit par l'armée régulière ou surtout par leurs ennemis armés de machettes, d'épées, de faucilles et de tout ce qui pouvait servir à tuer. On laissait leurs corps se décomposer au bord des routes. La ville de Halimunda fut instantanément envahie de charognes qui gisaient dans les caniveaux. Mais la plupart des cadavres étaient abandonnés dans les faubourgs, aux pieds des collines, au bord des rivières, au milieu des ponts et dans les broussailles. La plupart des communistes avaient été exécutés alors qu'ils tentaient de fuir, après avoir compris que ne resteraient plus de l'ancienne gloire du Parti que des vestiges délabrés. D'ailleurs, tous les communistes ne furent pas tués. Certains d'entre eux finirent par se rendre et furent incarcérés, aussi bien dans des établissements de droits communs que dans des prisons militaires. Une grande partie fut déportée au Bloedenkamp, le plus atroce des centres de détention, construit à l'époque coloniale au milieu d'un delta. Ils étaient soumis à des interrogatoires qui duraient des heures et on leur promettait de remettre ça le lendemain. Beaucoup moururent finalement en captivité, soit en raison de la sous-alimentation soit à cause des coups de crosse qui leur fendaient le crâne et entraînaient aussitôt la mort. Les soldats continuaient à pourchasser les derniers communistes pour les tuer ou les incarcérer, bien qu'ils eussent fui en forêt ou en pleine mer. (Les Belles, p. 424-425)

Tentara-tentara itu juga telah malas membawa mayat-mayat orang Komunis ke tempat pemakaman dan harus membantu penggali kubur membuat lubang raksasa. Mereka dibunuh, baik oleh tentara reguler dan terutama oleh orang-orang antikomunis yang bersenjata golok dan pedang dan arit dan apa pun yang bisa membunuh, di tepi jalan dan membiarkan mayat mereka di sana sampai membusuk. Kota Halimunda seketika dipenuhi mayat-mayat seperti itu, tergeletak di selokan dan kebanyakan di pinggiran kota, di kaki bukit dan di tepi sungai, di tengah jembatan dan di semak belukar. Mereka kebanyakan terbunuh ketika mencoba melarikan diri setelah menyadari Partai Komunis hanya meninggalkan sisa-sisa reputasinya yang telah usang.

Bagaimana pun, tak semua dari mereka terbunuh. Beberapa di antara mereka pada akhirnya menyerah dan dijebloskan ke dalam tahanan. Baik di penjara-penjara kriminal maupun di tahanan militer. Sebagian besar dibawa ke Bloedenkamp, penjara paling mengerikan di tengah delta yang telah berdiri sejak masa kolonial. Entah apa yang akan terjadi di antara mereka. Ada interogasi-interogasi yang memakan waktu sampai berjam-jam dengan janji akan dilanjutkan keesokan harinya. Sebagian dari mereka ternyata akhirnya harus mati juga di dalam tahanan, oleh kelaparan atau pukulan popor senapan yang membuat kepala mereka retak dan pemiliknya mati seketika. Mereka terus memburu orang Komunis yang tersisa, untuk dibunuh maupun ditahan, bahkan meskipun mereka telah melarikan diri ke hutan autau ke tengah laut. (p. 312-313)

31 Après la sanglante élimination des communistes ${ }^{31}$, dont la description réaliste évoque les événements de l'automne 1965, les militaires vont s'en prendre aux truands. Le roman mêle ironiquement la petite histoire des personnages aux événements politiques réels. Fou de rage après la perte de sa fille, Rengganis la Belle, qui a prétendu avoir été 
engrossée par un chien (Les Belles, p. 504, 566), le truand sème le trouble dans la ville. Sa bande s'en prend aux policiers, qui ont été incapables de protéger son enfant, et extermine tous les chiens de Halimunda, auxquels le shodancho, qui en élève, voue une affection particulière (Les Belles, p. 586-592). L'armée est chargée de rétablir l'ordre, et le shodancho en profite pour régler ses comptes au truand. Le roman fait allusion aux "Petrus». Ce terme qui, en indonésien, évoque le prénom "Pierre», est aussi l'acronyme de "Penembakan Misterius », les soi-disant "tirs mystérieux », perpétrés par les forces de l'ordre à Jakarta, en 1983, en vue d'éliminer les caïds et les truands, reconnaissables à leurs tatouages (Les Belles, p. 591-594) ${ }^{32}$. Et les corps des truands ne sont pas mieux traités que ceux des communistes :

Ils ne les inhumèrent pas comme des cadavres, mais les ensevelirent comme de la merde, comme après avoir chié auprès d'un bananier. (Les Belles, p. 594)

Tidak seperti mengubur mayat, tapi seperti mengubur tai selepas berak di kebun pisang. (p. 446)

Les Belles de Halimunda nous présente ainsi deux strates de violences infligées au corps social. La plus récente fut l'œuvre du régime Soeharto, et visait à « épurer » la nation de l'intérieur, en en extirpant les influences étrangères: l'idéologie communiste diabolisée, ou les comportements antisociaux: les truands. À cette violence d'une conception exclusive de l'identité indonésienne, s'opposent à la fois la cité portuaire de Halimunda, carrefour d'héritages socio-culturels multiples, et la plus célèbre prostituée de la ville, Dewi Ayu, qu'une relation, à la fois métonymique et métaphorique relie à la ville dont elle est l'emblème (Les Belles, p. 50, 68-72, 119-123, 149-150) ${ }^{33}$. À travers ses origines métissées et la personnalité de ses gendres, qui reflètent la diversité indonésienne, comme à travers le relativisme pragmatique qu'elle oppose aux autorités politiques et religieuses, Dewi Ayu apparaît comme un modèle de tolérance, opposable à la violence du régime Soeharto. Ses démêlés avec l'imam Kyai Jahro qui rechigne à l'enterrer, mais s'intéresse à elle après sa résurrection, illustrent bien son anticonformisme en matière de croyances (Les Belles, p. 18, 21, 38-40).

Pourtant, cette belle métisse née d'un inceste (Les Belles, p. 61-65), n'aura de cesse de refuser sa part occidentale qu'elle associe à la brutalité avec laquelle son grand-père s'est emparé du corps de sa grand-mère indigène. Occupation japonaise et colonialisme évoquent une strate plus ancienne de violences et de conquêtes, qui a perturbé profondément la société indonésienne. Le traumatisme fondamental dont procède l'intrigue romanesque est, en l'occurrence, l'appropriation du corps de Ma Iyang par Stammler. Et c'est cette agression initiale qui conduit Dewi Ayu à intérioriser la violence que lui ont fait subir, en qualité d'Eurasienne, les occupants japonais, en renonçant à fonder une famille et en restant prostituée. En vue de réparer la faute de son grand-père néerlandais, Dewi Ayu oblige l'amant de sa grand-mère décédée à l'épouser. Pour ce faire, il lui faut se convertir à l'islam, puisque les prêtres catholiques ont dû fuir les persécutions de l'occupant japonais, qui les associent aux Néerlandais. Cela ne lui pose pas de problème, puisqu'elle a perdu la foi (Les Belles, p. 60, 75-77).

On constate toutefois deux inconséquences dans le roman. D'une part, Dewi Ayu fait élever ses filles dans la religion musulmane, alors que sa conversion était liée aux circonstances et n'était aucunement motivée par une conviction religieuse. D'autre part, elle parle de Ma Gedik, l'amant malheureux de sa grand-mère, en ces termes :

Pauvre homme. Il aurait dû être mon grand-père, si Ted n'avait pas fait de Ma Iyang sa maîtresse. (Les Belles, p. 76) 
Lelaki yang malang. Seharusnya dia kakekku seandainya Ted tak jadikan Ma Iyang gundiknya (p. 49).

En prenant fait et cause pour l'autochtone à l'irréel du passé, Dewi Ayu prononce une absurdité, puisque si son grand-père néerlandais n'avait pas ravi sa dulcinée à Ma Iyang, ce dernier aurait certes pu avoir des enfants indigènes avec cette dernière, mais Dewi Ayu n'aurait pas vu le jour. Force est alors d'interpréter son adhésion à l'islam, religion de la majorité, en particulier en pays sundanais, comme une volonté de conformisme social qui passe par l'effacement des différences. La vision négative d'une mixité, pourtant constitutive de l'histoire indonésienne, nous ramène d'ailleurs, comme nous l'avons suggéré, à la tradition des histoires de nyai, c'est-à-dire de concubines autochtones de colons. Dans Les Belles de Halimunda, les métisses n'ont pas non plus leur place ${ }^{34}$. Cet enracinement du roman dans la tradition locale, qui le distingue de l'abstraction de courants littéraires féministes et islamistes liés à la mondialisation, fait des Belles de Halimunda un roman incarné. Ce point explique sans doute l'intérêt que lui ont porté les Occidentaux, depuis Benedict Anderson en 2008 jusqu'aux nombreux éditeurs qui ont, à sa suite, commandé la traduction de ce roman, paru en plus de 30 langues. Évoquer ces questions dépasserait le cadre de cet article. On peut cependant noter le poids des versions anglaises accessibles aux éditeurs et contraignant les traductions faites sur l'indonésien, quand elles ne débouchent pas sur des traductions indirectes. L'éditrice française, Sabine Wespieser, qui a pris connaissance de l'œuvre d'Eka Kurniawan par Tareq Ali, a été touchée par la modernité crue d'une écriture, dont les multiples descriptions d'activités sexuelles et de scènes de violence lui ont rappelé le cinéma de Tarentino.

\section{Le corps comme être-pour-la-mort}

Soumis aux besoins naturels, mais également au désir d'être reconnu ou aimé, et à la violence sociale, le corps est voué à la mort. Les quatre filles de Dewi Ayu se retrouvent à la fin du roman sur la tombe de leurs époux, amants et enfants (Les Belles, p. 626-628). Cette rencontre au cimetière rattache l'ouvrage de Kurniawan aux romans fondateurs de la littérature indonésienne moderne, dont les héros se recueillent sur la sépulture de leurs chers disparus, scènes faisant souvent l'objet d'illustrations.

On a souvent associé l'écriture de Kurniawan au réalisme "merveilleux» ou « magique » d'Alejo Carpentier et comparé Les Belles de Halimunda aux Versets sataniques de Salman Rushdie et, plus encore, à Cent ans de solitude de Gabriel Garcia Marquez ${ }^{35}$. Stefan Danerek, par ailleurs, a proposé d'appliquer au roman de Kurniawan la grille de lecture que fournit l'interprétation bakhtinienne de Rabelais en évoquant «a novel in the line of Rabelais $»^{36}$ ou encore "a Sundanese-Indonesian expression in the Rabelais line of novels $\aleph^{37}$. L'une des idées marquantes de l'essai de Bakhtine est son analyse du corps grotesque. Contrairement au beau corps qui constitue une totalité organique, une « individualité achevée et autonome $»^{38}$, le corps grotesque "n'est pas démarqué du restant du monde, n'est pas enfermé, achevé ni tout prêt, mais il se dépasse lui-même, franchit ses propres limites" (Les Belles, p. 35). Cet effacement des frontières qui séparent le corps (grotesque) du monde (Les Belles, p. 280, 308, 332, 351, 352, 354), entre en contraste avec la situation de la belle Rengganis comme avec celle de la vilaine Belle. Comme la légendaire princesse fondatrice de la ville de Halimunda, la quatrième fille de 
Dewi Ayu dut s'enfermer dans sa chambre et soustraire son corps aux regards et à la communication avec le monde pour protéger l'entourage (Les Belles, p. 33-35, 162, 165).

C'est dans les situations limites de la naissance et de la mort que se repèrent le plus cet effacement des frontières avec le monde, caractéristique du corps grotesque. Elle se décline sur quatre registres complémentaires: effacement des frontières entre les organes et les fonctions du corps, entre la vie et la mort, entre l'homme et l'animal, entre la nature et le surnaturel.

39 Certaines morts sont grotesques, comme celle de la mère d'Edi Idiot, le truand qui régnait sur Halimunda avant d'être tué par le gendre de Dewi Ayu. Cette dernière décède alors qu'elle est en train de déféquer (Les Belles, p. 336). Il en va de même de l'hôte communiste de la mère de Kliwon, le camarade Salim, qui veut mourir tout nu, sans doute en référence à l'égalité et à l'innocence naturelles. Les soldats de la République consentent finalement à exaucer son vœu en l'exécutant dans la salle de bains (Les Belles, p. 245-246) ${ }^{39}$. Conformément à la logique comique de l'abaissement, Kurniawan décrit cette scène héroïque de martyr de la Cause du peuple en termes sordides. Le communiste meurt après s'être maculé le corps de boue, et, aux soldats qui rechignent à "voir pendouiller son sexe » (Les Belles, p. 245) / " melihat kemaluannya tergantung ke mana-mana »(p.176), Kliwon transmet ainsi sa requête : «Laissez-le tout nu, peut-être voudra-t-il chier une dernière fois avant de mourir, puis exécutezle» (Les Belles, p. 245) ${ }^{40}$ / Biarkan ia telanjang, mungkin ia ingin buang tai dulu, dan tembaklah (p. 176) ${ }^{41}$. On conviendra qu'il est des manières plus sublimes et plus respectueuses d'exprimer les dernières volontés d'un mourant.

Un tel « rabaissement grotesque $»^{42}$, parfaitement exprimé par le niveau de langue familier et non-soutenu de l'écriture de Kurniawan, s'oppose à l'idéal classique de pureté et de hiérarchisation des parties nobles et des parties basses du corps ${ }^{43}$. En témoigne l'omniprésence du vulgaire, de l'obscène et du scatologique dans le roman, qui offrirait là encore un parallèle avec Rabelais ${ }^{44}$. Mentionnons, par exemple, les évocations de l'éructation d'Alamanda, précédant la disparition de l'enfant qu'elle porte, du pet que lâche Dewi Ayu, après le festin qu'elle ingurgite le jour de sa résurrection (Les Belles, p. 37, 381) ${ }^{45}$, ainsi que l'insistance, rabelaisienne, sur les tripes et les excréments ${ }^{46}$. Pour soustraire ses bagues à l'avidité des soldats nippons, Dewi Ayu les avale et les réingurgite quotidiennement, après les avoir rincées. Enfin, à sa naissance, Belle est comparée à une "merde", sous prétexte qu'une faible distance sépare le vagin de l'anus ${ }^{47}$, l'orifice d'où sort la vie et celui d'où proviennent les excréments qui retournent à la terre, et dont l'ensevelissement symbolise à la fois la mort, l'inhumation, et la renaissance, l'ensemencement. Comme l'écrit Bakhtine :

Une des tendances principales de l'image grotesque du corps consiste à montrer « deux corps dans un seul»: le premier qui donne la vie et qui disparait, le second qui a été conçu, porté, qui est mis au monde. [...] La mort est toujours mise en corrélation avec la naissance, la tombe avec le sein de la terre qui donne le jour ${ }^{48}$.

Stefan Danerek nous propose d'appliquer ces remarques de Bakhtine sur Rabelais à certains passages des Belles de Halimunda, notamment avec la naissance de Kinkin, le fils $\mathrm{du}$ fossoyeur Kamino, qui vient au monde après la mort de sa mère, dans la tombe de cette dernière (Les Belles, p. 452) ${ }^{49}$. Le même critique rappelle également le lien que l'enseigne du lupanar effectue entre la vie et la mort, en détournant un slogan de la Révolution française, repris par les nationalistes indonésiens: "La liberté ou la mort» / Merdeka atau mati en " Aimer (faire l'amour) ou mourir» / Bercinta atau Mati. La maquerelle optera finalement pour un troisième slogan : «Aimer à en mourir », qui 
trouvera une confirmation inattendue avec la mort de soldats, surpris par l'ennemi en pleine action, et d'une prostituée qu'un baiser trop long fait suffoquer (Les Belles, p. 149). De même, Krisan, le petit-fils de Dewi Ayu, meurt d'un coup de fusil, alors qu'il est en train de faire l'amour avec sa tante, Belle (Les Belles, p. 625). La compagne de bohème de Kliwon, Isah Betina, «Isah la femelle », meurt, mordue par un serpent, alors qu'ils sont en train de faire l'amour (Les Belles, p. 254). Enfin, un client très âgé de Dewi Ayu obtient la permission de mourir dans ses bras en échange du corps de sa fille, qu'il met à son entière disposition et qui deviendra finalement sa fidèle domestique (Les Belles, p. 25-27) $)^{50}$.

Outre la frontière entre la naissance et la mort, c'est également la différence qui sépare l'homme de l'animal, la nature de la culture, qui s'estompe dans le roman de Kurniawan. En témoignent, par exemple, les incestes, qui découlent de la malédiction de Ma Gedik, l'amant malheureux de la grand-mère de l'héroöne, sur les descendants de Stammler. Les parents de Dewi Ayu sont ainsi demi-frères. Son père est le fils légitime de Stammler et de sa femme néerlandaise, tandis que sa mère est la fille qu'il a eue avec sa concubine indigène, Ma Iyang. De même, Krisan, le fils qu'Adinda a eu avec Kliwon, engrosse sa cousine, la fille du truand, qui est très belle et très stupide, en se faisant passer pour un chien (Les Belles, p. 504-506, 561). Sous le poids des circonstances, elle donnera son bébé en pâtée aux chiens (Les Belles, p. 549-551), avant d'être assassinée par son cousin et amant. Kinkin, le fils du fossoyeur, la vengera en exécutant Krisan, alors qu'il est en train de forniquer avec sa tante, dans un lit d'amour qui est également une couche mortuaire. Dans un autre passage du texte, un homme est changé en sanglier et tué par des chasseurs (Les Belles, p. 212). Faut-il y voir une allusion à la " métamorphose » d'Actéon en cerf ? La princesse Rengganis épouse un chien, tout en étant supposée être l'ancêtre de la ville de Halimunda. Fou de douleur, après la perte de sa bien-aimée, Ma Gedik a une conduite sexuelle déviante et couche avec n'importe quel animal (Les Belles, p. 51).

On notera les allusions aux corps démembrés et dépecés, qui pourraient également être rapprochées de la lecture bakhtinienne de Rabelais ${ }^{51}$. Comme le bébé de la fille du truand, qui sert de chair à pâté pour chien, le shodancho a une mort particulièrement atroce. Il élève des chiens féroces pour débarrasser les villageois des sangliers. Or il va finalement être dévoré par ses propres bêtes et sa veuve ramassera ses morceaux dans un sac (Les Belles, p. 605-606). De même, Ma Gedik, l'amant malheureux de Ma Iyang, meurt, après avoir tenté d'imiter sa fiancée qui disparaît en plein ciel après s'être envolée d'une colline pour échapper aux Néerlandais qui la poursuivent et que leur conduite a scandalisés. Après avoir été séparés pendant plus d'une dizaine d'années, les deux amants se retrouvent et font l'amour à la vue de tous. Il meurt en s'écrasant sur le sol après avoir tenté vainement de s'envoler vers le ciel comme l'avait fait sa dulcinée (Les Belles, p. 56, 79). Conformément aux réquisits du réalisme magique, qui mêle librement le réel au merveilleux ${ }^{52}$, un phénomène extraordinaire se produit, contre toute attente, mais il ne se répète pas. L'expérience ordinaire de la nature est ici doublement contredite, puisque l'on ne peut s'attendre ni au respect de la loi de la chute des corps ni à sa transgression systématique.

Outre l'abolition de la frontière entre les fonctions organiques, entre les états et les règnes, s'estompe celle qui sépare l'ici-bas de l'au-delà, la nature de la surnature. Les militaires qu'interroge la femme du truand partie à la recherche de son mari lui rétorquent qu'il ne doit pas être parmi les morts, car son corps doit puer plus que les 
autres (Les Belles, p. 595). Cette idée absurde, selon laquelle le cadavre d'un malfaiteur devrait sentir davantage que celui d'un homme ordinaire, relève du réalisme magique. À la description sordide des charognes qui pourrissent s'opposent les morts sublimées de Dewi Ayu qui ressuscite, puis se métamorphose en papillon pour rejoindre le monde des morts, une fois sa mission accomplie (Les Belles, p. 9-11, 30, 39-40, 614), et du truand qui, au terme d'une méditation, atteint le stade du moksa, celui de « délivré vivant » de la religion hindoue, concept que l'islamisation de l'archipel malais n'a pas effacé de la conscience de nombre d'Indonésiens (Les Belles, p. 569, 600). Le moksa, but suprême de l'homme, représente la délivrance de l'ordre socio-cosmique, l'échappement au cycle des réincarnations ${ }^{53}$. Heinrich Zimmer, le grand spécialiste des philosophies indiennes, le définit comme "release, libération, redemption from the vicissitudes of time ${ }^{54}$. "Moksa is a technique of transcending the senses in order to discover, know, and dwell at one with the timeless reality which underlies the dream of life in the world $»^{55}$. Mamane Gendeng, l'époux de la troisième fille de Dewi Ayu, atteint ce stade au terme de sa méditation et échappe, par ailleurs, aux soldats qui le traquent en faisant passer pour lui l'homme qui l'a trahi en le revêtant mystérieusement de son apparence (Les Belles, p. 597, 601). Le cadavre de ce dernier passe alors illusoirement pour celui du truand, dont le corps s'est en réalité "volatilisé » en se fondant dans l'Absolu. Les allusions à la résurrection des corps et à la Délivrance (moksa) sont une manière, pour le Sundanais Eka Kurniawan, de saluer, d'un point de vue musulman, les religions qui, dans l'archipel indonésien, ont précédé l'islam : l'hindouisme, ou sont arrivées après lui : le christianisme ${ }^{56}$. Le surnaturel est également présent dans la transformation, hautement symbolique, des cadavres de communistes massacrés, refoulés de l'histoire indonésienne officielle, en fantômes qui reviennent hanter la conscience du militaire, tout en partageant des cafés et des conversations avec son épouse, qui n'en a pas peur, et avec Kliwon, qui regrette ses camarades (Les Belles, p. 473, 476-482 et 495-496).

\section{Conclusion}

Les Belles de Halimunda est une épopée burlesque qui nous présente le corps dans tous ses états, des corps individuels humains ou animaux, masculins ou féminins, dont le destin est plus ou moins lié à celui du corps social. Cette déconstruction matérialiste de la violence politique, qui se centre sur le «bas corporel ", manie l'humour (noir), la caricature et la parodie. Les personnages stéréotypés n'ont pas de réelle épaisseur psychologique et s'inscrivent dans le droit fil des figurines stylisées du théâtre d'ombres. Ces «marionnettes » ne représentent nullement des personnes réelles, mais évoquent symboliquement des héros mythologiques à travers les formes de leurs visages, leurs vêtements, leurs bijoux et les couleurs qu'elles portent ${ }^{57}$. De même, les personnages comiques de Kurniawan s'attirent et se repoussent, mus par le mécanisme implacable des passions charnelles de haine ou d'amour, qui les conduisent à se faire l'amour ou la guerre. Les péripéties cocasses des corps qui se heurtent ou composent les uns avec les autres, comme les modes de la substance étendue de Spinoza, semblent illustrer à merveille la définition bergsonienne identifiant le rire à du "mécanique plaqué sur du vivant $»^{58}$. Le roman de Kurniawan parodie les grands genres de la littérature mondiale (romans historiques, libertins ou sentimentaux, récits de captivité, etc.) ou ceux de la littérature populaire indonésienne (cerita silat, c'est-à-dire romans d'aventures mettant en scène des adeptes en arts martiaux, histoires de spectres et de fantômes, etc.). Pourtant, cette polyphonie apparente ne contredit pas la dimension 
radicalement monologique de l'écriture de Kurniawan, qui s'inscrit prioritairement dans la tradition des histoires de nyai (concubines autochtones de colons) mais relève également des lois du genre comique, qui exige d'appréhender les personnages de l'extérieur, "le corps prenant le pas sur l'âme " ${ }^{59}$, selon les mots de Bergson. "Dès que le souci du corps intervient, une infiltration comique est à craindre. [...] Nous rions toutes les fois qu'une personne nous donne l'impression d'une chose ${ }^{60}$. Comme un conteur traditionnel ou un montreur de marionnettes du théâtre d'ombres (dalang), Kurniawan n'efface pas sa voix au profit de celles des personnages dans lesquels il se projette ${ }^{61}$. Il se comporte comme un narrateur omniscient et tout-puissant, dont les héros ne disposent pas d'idiolectes :

Le ton, le vocabulaire et les pensées qu'extériorisent pratiquement tous les personnages, ont leur origine dans une personne : l'auteur! Étrangement, tous les protagonistes parlent sur un même ton!

Nada, kosakata dan pemikiran yang keluar dari hampir semua tokohnya, berasal

dari satu pribadi : Sang Pengarang! Aneh, semua tokoh berbicara dengan nada yang sama $!^{62}$

Cette réduction stylistique du divers est l'indice, non certes d'un refus catégorique du métissage, mais d'une réticence à l'envisager positivement. Dans un mail du 15 avril 2018, Kurniawan nous rappelait l'idée qu'il a souvent émise en conférences, selon laquelle Dewi Ayu pouvait être considérée comme une allégorie de l'Indonésie, dont elle symbolise le pluralisme. Mais elle déplore sa beauté métisse, qui est l'effet d'une violence et la cause d'une malédiction. Son "roman familial » l'amène à renier ses origines réelles en s'inventant une ascendance purement autochtone ${ }^{63}$ et, malgré sa situation marginale de prostituée, à vouloir « rentrer dans le rang ${ }^{64}$.

\section{BIBLIOGRAPHIE}

Sources littéraires :

AYU Djenar Maesa, À travers les glaces, NAVEAU Étienne (trad. et dir.), Paris, Pasar Malam, 2011.

EL SHIRAZY Habiburrahman, [Les Versets de l'amour] / Ayat-Ayat Cinta, Jakarta, Penerbit Republika, 2004.

GIBRAN Khalil, Les Ailes brisées, colın Joël (trad.), Paris, Babel, 2001.

KOMMER H., « Tjerita Nji Paina », in [Le Bon-vieux temps. Anthologie de littérature préindonésienne] / Tempo Doeloe. Antologi Sastra Pra-Indonesia, TOER Pramoedya Ananta (éd.), Jakarta, Lentera Dipantara, 2003, p. 375-391.

KURNIAWAN Eka, [Pramoedya Ananta Toer et la littérature réaliste indonésienne] / Pramoedya Ananta Toer dan Sastra Realisme Indonesia, Yogyakarta, Jendela, 1999.

KURNIAWAN Eka, «Graffiti in the Toilet » / [Corat-Coret di Toilet], ANDERSON Benedict (trans.),

Indonesia 86, 2008, p. 55-61. 
KURNIAWAN Eka, L'homme-tigre, NAVEAU Étienne (trad.), Paris, Sabine Wespieser, 2015, (2 éd., Gallimard, « Folio », 2017).

KURNIAWAN Eka, Cantik Itu Luka, Jakarta, Gramedia, 2002.

KURNIAWAN Eka, Les Belles de Halimunda / [Cantik Itu Luka], NAVEAU Étienne (trad.), Paris, Sabine Wespieser, 2017.

KURNIAWAN Eka, Beauty is a Wound / [Cantik Itu Luka], TUCKER Annie (trad.), Londres, Pushkin Press, 2015.

KURNIAWAN Eka, Seperti Dendam, Rindu harus Dibayar Tuntas, Jakarta, Gramedia, 2014.

KURNIAWAN Eka, Cash, NAVEAU Étienne (trad.), Sabine Wespieser, 2019.

KURNIAWAN Eka, O, Jakarta, Gramedia, 2016.

TOER Pramoedya Ananta, [Monologues d'un muet] / Nyanyi Sunyi Seorang Bisu, Jakarta, Hasta Mitra, 2000 (1 1 ère éd. 1995), 2 vol.

TOER Pramoedya Ananta, The Mute's Soliloquy. A memoir, SAMUELs Willem (trad.), New York, Penguin Books, 1999.

UTAMI Ayu, Saman, Jakarta, Gramedia, 1998.

UTAMI Ayu, Saman, INANDIAK Elisabeth (trad.), Paris, Flammarion, 2008.

Textes critiques :

AUERBACH Erich, «Le monde que renferme la bouche de Pantagruel », in Mimésis. La représentation de la réalité dans la littérature occidentale, HEIM Cornélius (trad.), Paris, Gallimard, 1968, p. 267-286.

BAKHTINE Mikhaïl, L'œuvre de François Rabelais et la culture populaire au Moyen Âge et sous la Renaissance, REBEL Andrée (trad.), Paris, Gallimard, 1970.

BIARDEAU Madeleine, L'Hindouisme. Anthropologie d'une civilisation, Paris, Flammarion, 1981.

BLANCHÉ Robert, Des catégories esthétiques, Paris, Vrin, 1979.

BURKE Edmund, Recherches philosophiques sur l'origine de nos idées du sublime et du beau, SAINT GIRONS Baldine (trad.), Paris, Vrin, 1990.

DANEREK Stefan, Tjerita and Novel. Literary Discourse in Post New Order Indonesia, Lund, Centre for Languages and Literature, Lund University, 2006.

HUNTER Thomas, "Indo as other. Identity, anxiety and ambiguity in 'Salah Asoehan' », in Clearing a space. Postcolonial readings of modern Indonesian literature, FOULCHER Keith and DAY Tony (eds.), Leiden, KITLV Press, 2002, p. 109-143.

KANT Emmanuel, Critique de la faculté de juger, PHILONENKO Alexis (trad.), Paris, Vrin, 1965.

LAPLANTINE François et Nouss Alexis, Métissages de Arcimboldo à Zombi, Paris, Pauvert, 2001.

LE FUSTEC Claude, « Le réalisme magique : vers un nouvel imaginaire de l'autre ? Regard sur la littérature africaine américaine », Amerika, 2 :2010, https://journals.openedition.org/amerika/ 1164.

LOMBARD Denys, Le Carrefour javanais, vol. I, Les limites de l'occidentalisation, EHESS, 1990.

MAHAYANA Maman S., [« Le déluge de Cantik itu luka »] / Air Bah Cantik itu Luka, in [Le caractère extrinsèque de la littérature indonésienne] / Ekstrinsikalitas Sastra Indonesia, Jakarta, PT Rajagrafindo Persada, 2007, p. 352-360. 
NAVEAU Étienne, «Les Souvenirs de Buru de Pramoedya Ananta Toer (1925-2006). Mémoires et reconnaissances d'un prisonnier politique ", in Communication interculturelle et littérature. Mémoire, littérature et identité, CRIHANA Alina et al. (dirs.), vol. 1 : Les récits de vie : mémoire, histoire et fiction identitaire, GalaŢi, Universitate « Dunărea de Jos », Insititul European, 2013, p. 84-102.

NAVEAU Étienne, « La littérature indonésienne : une introduction », in Indonésie contemporaine, MADINIER Rémy (dir.), Paris, Les Indes savantes, 2016, p. 363-376.

ROBERT Marthe, Roman des origines et origines du roman, Paris, Gallimard, 2002.

ROY Olivier, La Sainte ignorance. Le temps de la religion sans culture, Paris, Seuil, 2008.

SCREECH Michael, Rabelais, KISCH Marie-Anne de (trad.), Paris, Gallimard, 1992.

SPITZER Leo, « Rabelais et les "rabelaisants" ", in Études de style, KAUfHOLz Éliane, coulon Alain et FOUCAUlT Michel (trad.), Paris, Gallimard, 1970, p. 134-165.

ULBRICHT H., Wayang Purwa. Shadows of the Past, Kuala Lumpur \& Singapore, Oxford U. P., 1970.

VOISSET Georges, Les lèvres du monde. Littératures comparées de la Caraibe à l'Archipel malais, Bécherel, Les Perséides, 2008.

ZAINI-LAJOUBERT Monique, « Histoire du roman indonésien », in Indonésie contemporaine, MADINIER Rémy (dir.), Paris, Les Indes savantes, 2016, p. 407-414.

ZIMMER Heinrich, Philosophies of India, New York, Meridian Books, 1957.

\section{NOTES}

1. Les passages intégralement cités de cette œuvre renvoient d'abord à la traduction française : Les Belles de Halimunda, NAVEAU Étienne (trad.), Paris, Sabine Wespieser, 2017, plus loin Les Belles, puis à l'original : Eka KURNIAWAN, Cantik Itu Luka, Jakarta, Gramedia, 2002.

2. Entretien du 17 août 2017 avec l'auteur. Voir également DANEREK Stefan, Tjerita and Novel. Literary Discourse in Post New Order Indonesia, Lund, Centre for Languages and Literature, Lund University, 2006, p. 226.

3. KURNIAWAN Eka, [Pramoedya Ananta Toer et la littérature réaliste indonésienne] / Pramoedya Ananta Toer dan Sastra Realisme Indonesia, Yogyakarta, Jendela, 1999.

4. Voir DANEREK, Tjerita and Novel..., op. cit., ainsi que NAVEAU Étienne, «La littérature indonésienne : une introduction », in Indonésie contemporaine, MADINIER Rémy (éd.), Paris, Les Indes savantes, 2016, p. 370, et ZAINI-LAJOUBERT Monique, « Histoire du roman indonésien », in Indonésie contemporaine, ibid., p. 412-413.

5. Voir AYu Utami, Saman, INANDIAK Elizabeth (trad.), Paris, Flammarion, 2008 ; DJENAR Maesa Ayu, À travers les glaces, NAVEAU Étienne et al. (trad.), Paris, Pasar Malam, 2011, et EL SHIRAZY Habiburrahman, [Les Versets de l'amour], Penerbit Republika, 2004.

6. Cette traduction de "Graffiti in the Toilet » est parue en octobre 2008, précédée d'une courte présentation de l'auteur : Indonesia, 86:2008, p. 55-61.

7. Rappelons qu'en philosophie, en sociologie ou en anthropologie des religions, une religion est dite "inculturée » quand elle tend à s'adapter à la culture dans laquelle elle s'insère, tandis qu'elle est dite "acculturée" quand elle cherche à arracher ses adeptes à des cultures traditionnelles jugées païennes et incompatibles avec la «vraie» foi. Ainsi, tandis que l'islam populaire ou celui des soufis, ou encore le catholicisme et le protestantisme historique (luthéroréformé) font généralement preuve d'inculturation, au sens où ils tolèrent, voire valorisent les traditions locales, l'islam wahhabite et le protestantisme évangélique se caractérisent bien 
souvent par leur intransigeance. Ces formes de religion tendent à se considérer comme absolues et visent à remodeler leurs adeptes en faisant abstraction des contextes historiques et géographiques de leur implantation. Voir, par exemple, Roy Olivier, La Sainte ignorance. Le temps de la religion sans culture, Paris, Seuil, 2008, p. 51.

8. Rappelons qu'une longue tradition de pensée d'origine «indo-européenne ", à l'œuvre aussi bien dans la République de Platon que dans l'imaginaire de la monarchie féodale, tend à considérer la société comme un organisme et l'individu comme une petite société.

9. Voir DANEREK, Tjerita and Novel, op. cit., p. 247.

10. Voir sur ce point, les réflexions de voISSET Georges, Les Lèvres du monde, Littératures comparées de la Caraibe à l'Archipel malais, Bécherel, Les Perséides, 2008, p. 257, 356 et 364-366.

11. Voir PRAMOYEDA Ananta Toer, [Monologues d'un muet] / Nyanyi Sunyi Seorang Bisu, Jakarta, Hasta Mitra, 2000. Voir aussi NAVEAu Étienne, «Les Souvenirs de Buru de Pramoedya Ananta Toer (1925-2006). Mémoires et reconnaissances d'un prisonnier politique», in Communication interculturelle et littérature. Mémoire, littérature et identité, CRIHANA Alina et al. (dir.), t. $1:$ Les récits de vie: mémoire, histoire et fiction identitaire, GalaŢi, Universitate «Dunărea de Jos », Insititul European, 2013, p. 84-102.

12. BAKHTINE Mikhaïl, L'œeuvre de François Rabelais et la culture populaire au Moyen Âge et sous la Renaissance, ROBEL Andrée (trad.), Paris, Gallimard, 1970, p. 460.

13. Voir BURKE Edmund, Recherches philosophiques sur l'origine de nos idées du sublime et du beau, SAINT GIRONS Baldine (trad.), Paris, Vrin, 1990, p. 83.

14. GIBRAN Khalil, Les Ailes brisées, CoLIN Joël (trad.), Paris, Babel, 2001, p. 75.

15. KOMmeR H., "Tjerita Nji Paina", in [Le Bon-vieux temps. Anthologie de littérature préindonésienne] / Tempo Doeloe. Antologi Sastra Pra-Indonesia, TOER Pramoedya Ananta (éd.), Jakarta, Lentera Dipantara, 2003, p. 375-391.

16. BAKHTINE, L'œuvre de François Rabelais, op. cit., p. 423-426, 455.

17. Ce style résolument anticlassique pourrait être rapproché du «style grotesquement comique et bas » de Rabelais. Voir AUERBACH Erich, «Le monde que renferme la bouche de Pantagruel », in Mimésis. La représentation de la réalité dans la littérature occidentale, HЕIM Cornélius (trad.), Paris, Gallimard, 1968, p. 274 et 281 ; SPITZER Leo, "Rabelais et les "rabelaisants" ", in Études de style, KAUFHOLz Éliane, COULON Alain et FOUCAULT Michel (trad.), Paris, Gallimard, 1970, p. 149 et 163, ainsi que BAKHTINE, L'œuvre de François Rabelais, op. cit., p. 75-76, 108, 320 et 462.

18. En réalité, le narrateur omniscient des Belles de Halimunda compare Belle à des animaux repoussants : singe, crapaud, lézard, varan (ibid., p. 16, 38).

19. BLANCHÉ Robert, Des catégories esthétiques, Paris, Vrin, 1979, p. 34.

20. Ibid., p. 66. Voir aussi KANT Emmanuel, Critique de la faculté de juger, PHILONENKO Alexis (trad.), Paris, Vrin, 1974, p. 76.

21. BLANCHÉ, Des catégories esthétiques, op. cit., p. 53.

22. BURKE, Recherche philosophique..., op. cit., p. 157, BLANCHÉ, Des catégories esthétiques, op. cit., p. 41, BAKHTINE, L'œuvre de François Rabelais, op. cit., p. 38, 315-316.

23. BAKHTINE, ibid., p. 318.

24. KANT, Critique de la faculté de juger, op. cit., p. 55.

25. "Beauté des femmes, leur faiblesse... ", écrit fort justement Verlaine dans un sonnet du recueil Sagesses.

26. CERVANTES Miguel de, Don Quichotte, FAnlo Jean-Raymond (trad.), Le Livre de Poche, vol. I, 2008, p. 86.

27. DANEREK, Tjerita and Novel, op. cit., p. 227. 
28. Selon les chroniques locales, la princesse sundanaise Diah Pitaloka aurait été la cause de la guerre, ayant opposé, au XIV ${ }^{\mathrm{e}}$ siècle, le royaume sundanais de Pajajaran (Java-Ouest) au royaume javanais de Majapahit (Java central et oriental).

29. Voir BAKHTINE, L'œuvre de François Rabelais, op. cit., p. 239-242. Sur cet antiféminisme de Rabelais, voir également SCREECH Michael, Rabelais, KISCH Marie-Anne de (trad.), Paris, Gallimard, 1992, p. 77-79.

30. BAKHTINE, L'CEuvre de François Rabelais, op. cit., p. 240.

31. DANEREK, Tjerita and Novel, op. cit., p. 235-236.

32. Ibid., p. 239.

33. Ibid., p. 227.

34. Voir HUNTER Thomas, "Indo as other. Identity, anxiety and ambiguity in 'Salah Asoehan' ", in Clearing a space. Postcolonial readings of modern Indonesian literature, FOULCHER Keith and DAY Tony (eds.), Leiden, KITLV Press, 2002, p. 109-110, 127, 139.

35. Par exemple, DANEREK, Tjerita and Novel, op. cit., p. 225-226.

36. Ibid., p. 256. Sur la référence appuyée à Bakhtine, voir ibid., p. 240 sq.

37. Ibid., p. 272.

38. BAKHTINE, L'œuvre de François Rabelais, op. cit., p. 40.

39. Relevons ici l'une des rares inexactitudes de l'étude magistrale de Danerek. Ce ne sont pas des soldats de l'armée coloniale néerlandaise (Tjerita and Novel, op. cit., p. 232), mais des soldats de la République d'Indonésie qui exécutent le camarade Salim. En Indonésie comme ailleurs, la guerre d'indépendance fut aussi une guerre civile, qui vit s'opposer le gouvernement indonésien, non seulement aux Néerlandais, à leurs alliés britanniques et à leurs « collaborateurs » autochtones, mais encore aux projets politiques concurrents des communistes et des islamistes.

40. Les Belles, op. cit., p. 245.

41. Cantik Itu Luka, op. cit., p. 176.

42. BAKHTINE, L'œuvre de François Rabelais, op. cit., p. 350.

43. Ibid, p. 108, 320, 462.

44. Ibid., p. 114, 328 et 380 .

45. Sur la scatologie et le pet chez Rabelais, voir BAKHTINE, L'œuvre de François Rabelais, op. cit., p. 114,328 et 380 .

46. Ibid., p. 154.

47. Ibid., op. cit., p. 13 et Danerek, Tjerita and Novel, op. cit., p. 249.

48. BAKHTINE, L'œuvre de François Rabelais, op. cit., p. 35 et 59.

49. Voir également BAKHTINE, L'Euvre de François Rabelais, p. 404 : «La mort est l'autre face de la naissance ».

50. On pourra se rapporter également aux analyses de DANEREK, qui suggère un rapprochement avec le corps grotesque de Rabelais selon Bakhtine (Tjerita and Novel, op. cit., p. 240-244).

51. DANEREK, Tjerita and Novel, op. cit., p. 243. Voir aussi sur cette question BAKHTINE, L'œuvre de François Rabelais, op. cit., p. 196, 224, 248, 259, 263, 267, 321, 358 et 379.

52. Sur le réalisme magique, voir entre autres, LE FUSTEC Claude, «Le réalisme magique : vers un nouvel imaginaire de l'autre? Regard sur la littérature africaine américaine », Amerika, 2:2010, http://journals.openedition.org/amerika/1164 ou encore LAPLANTINE François et NOUSS Alexis, Métissages de Arcimboldo à Zombi, Paris, Pauvert, 2001, p. 247-250 et 951-954.

53. BIARDEAU Madeleine, L'Hindouisme. Anthropologie d'une civilisation, Paris, Flammarion, 1981, p. $49,51,75$.

54. ZIMMER Heinrich, Philosophies of India, New York, Meridian Books, 1957, p. 560.

55. Ibid., p. 45.

56. C'est du moins ce qu'il a affirmé dans une communication donnée à l'Inalco le 4 octobre 2017. 
57. Voir H. ULBRICHT, Wayang Purwa. Shadows of the Past, Kuala Lumpur \& Singapore, Oxford U. P., 1970, p. 40-49, 99 et LOMBARD Denys, Le Carrefour javanais, tome I, Les limites de l'occidentalisation, Paris, EHESS, 1990, p. 167.

58. BERGSON Henri, Le rire, Paris, PUF, 1991, p. 29, 38.

59. Ibid., p. 40.

60. Ibid., p. 40 et 44 .

61. MAHAYANA Maman S., [ Le déluge de Cantik itu luka »] / Air Bah Cantik itu Luka, in [Le Caractère extrinsèque de la littérature indonésienne]/ Ekstrinsikalitas Sastra Indonesia, Jakarta, PT Rajagrafindo Persada, 2007, p. 352.

62. Idem, p. 356. Ce manque d'hétéroglossie est également souligné par DANEREK, Tjerita and Novel, op. cit., p. 254, 267-268.

63. ROBERT Marthe, Roman des origines et origines du roman, Paris, Gallimard, 2002, p. 34-36, 43-46, $52,165,167-169,170-171$.

64. Je tiens à remercier ici les étudiant(e)s de l'Inalco ayant assisté à une présentation de la première version de ce texte dans le cadre du séminaire de Master que je co-dirige avec Philippe Benoît et Doan Cam-Thi, le 3 octobre 2017. Mes remerciements vont également à Elsa Clavé et Judith Bosnak, qui m'ont invité à présenter une version anglaise de cette conférence le 8 janvier 2018 à la Goethe Universität de Francfort.

\section{AUTEUR}

\section{ÉTIENNE NAVEAU}

Étienne Naveau est Professeur des universités en langue et littérature indonésiennes à l'Inalco. Les recherches qu'il mène au sein du Cerlom (Centre d'étude et de recherche sur les littératures et les oralités du monde) portent d'abord sur les littératures indonésienne et malaise, ensuite sur l'analyse des discours fondateurs (philosophiques, politiques et religieux) indonésiens, enfin sur les transferts culturels et la traduction littéraire vers l'indonésien. Publications : « La réception de l'œuvre de Khalil Gibran en Indonésie », Archipel, n 75, 2008, p. 63-110. « Le propre des textes autobiographiques indonésiens ", in François-Joseph Ruggiu (dir.), Les usages des écrits du for privé, Bruxelles, Peter Lang, 2013, p. 77-105. « La représentation des mariages mixtes dans quelques romans indonésiens de la première moitié du xxe siècle ", in Monique Zaini (dir.), L'image de la famille dans les littératures modernes d'Asie du Sud-Est, Paris, Les Indes savantes, 2019, p. 82-135. 


\title{
Du corps mutilé au corps corrélé au monde, dans le roman Un homme dos à la mer de Wang Wen-hsing
}

\author{
Sandrine Marchand
}

1 Qui dit corps, dit séparation du corps et de l'esprit. Il reviendrait à René Descartes d'en avoir formulé la scission et érigé le paradigme pour qu'adviennent les sciences modernes protégées par le dais de la raison. Après quoi, le partage des rôles se serait figé pour plusieurs siècles, d'un côté la substance pensante, cosa mentale, de l'autre l'étendue, le corps, rapidement promu au rang de corps-machine. Il a fallu attendre le milieu du $\mathrm{xx}^{\mathrm{e}}$ siècle pour que ce soubassement vacille légèrement.

2 Wang Wen-hsing 王文興, romancier taïwanais né en 1939, a bien compris cette thèse, et ses limites, il s'en est emparé et en a appliqué la formule dans son deuxième roman achevé en 1999, Un homme dos à la mer / 背海的人1. Mais avec une sombre ironie. Nous nous situons, en effet, dans l'après-guerre du Pacifique, et le héros de ce roman est un héros défiguré, banni, borgne, affligé de mille maux, mais qui pense. La maladie, peutêtre plus encore que l'activité sexuelle, permet de rendre compte de ce qu'est un corps, un corps transformé par la modernité, non pas "augmenté » selon le dogme actuel, mais, au contraire, diminué, défectueux, d'obsolescence programmée. Ce corps est aussi représentatif de Taïwan, de ce pays industrialisé à outrance, qui s'est laissé gangréner par la modernisation depuis les années soixante.

3 Seul un corps malade, monstrueux, souffrant, peut tenter, cependant, un dernier raccord avec le monde dont il a été séparé. Raccord qui s'effectue, non plus en opposition au corps mutilé et objectivé, car il n'y a pas de résilience possible, mais au travers de cette déchéance même, devenue seconde nature.

4 Il est, en outre, un niveau où le corps-esprit n'a jamais quitté sa nature-processus, comme l'appelle Bruno Latour, c'est justement celui des processus, mouvements ou circulations qui organisent tous les existants, et qui fait que, sans parler d'opposition corps/esprit, il n'est plus même question d'opposition entre l'organisme et l'environnement, mais $d^{\prime}$ '« une superposition d'agencements mutuels $»^{2}$. Loin d'être 
autonome, l'organisme ne cesse de se développer avec l'environnement, en endosymbiose, et les autres vivants qui le côtoient ou le traversent, se conjuguent et interagissent.

5 Le roman de Wang Wen-hsing, roman atypique, où la langue est malmenée au point d'atteindre les limites asyntaxiques du langage, fait danser la narration, disloque le récit, pour en faire surgir des rythmes vibratoires et des forces cosmiques. Le motif du corps, au milieu des péripéties dérisoires, absurdes, qui forment le contenu du monologue extime du narrateur, Yeh 限, le Chef, pourrait servir de paradigme à l'ensemble des mouvements, forces, actions à quoi se réduit souvent l'intrigue.

6 Pour comprendre et surtout apprécier ce texte, il est nécessaire de sortir du dualisme : le recours aux théories du chaos, qui sont aussi celles de la thermodynamique, semble être appelé par le texte lui-même, qui leur répond par toute sa logique interne. Un homme dos à la mer, dans l'inconstance des mouvements qu'il décrit, des déséquilibres, du chaos des temporalités s'entrelaçant aux directions spatiales, répond donc, à une autre échelle, à un ordre; celui des dynamiques, des équilibres ponctués et encore de l'auto-organisation spontanée, révélant la liberté au cœur de la matière, chère à Ilya Prigogine (1917-2003).

7 Au fil de ses expériences physiques, déplacements, attentes, expériences érotiques, maladies, le narrateur de ce roman nous donne à comprendre une autre vision du monde. Comment le corps, dans son unité avec la nature, rejoint-il sa complexité et comment l'écriture même de ce roman révèle-t-elle la créativité, la liberté qui existe au cœur de la vie?

\section{L'esprit séparé du corps}

8 Abordons, tout d'abord, le paradigme cartésien, dont le narrateur incarne au prime abord la dichotomie réductrice. Cet anti-héros sans nom, qui s'appelle lui-même «le chef » (cela pourrait être " le vieux » ou encore " petit père »), est au bout du rouleau. Il se dit, au hasard de ses déblatérations, ancien officier de l'armée du Guomindang. Ayant fui Taipei, suite à un délit entraînant des poursuites judiciaires, il a trouvé refuge dans le port où il s'est improvisé devin, en raison de son œil unique. Le Chef, ce moins que rien cherchant sa pitance dans un village éloigné de tout, se présente comme un être de raison séparé du corps. Retranché du monde dans une cellule quasiment monastique, une cabine de bains désaffectée, habitat privilégié d'un environnement post-moderne, ce personnage s'auto-raconte une existence chaotique. Son monologue dure deux longues nuits séparées par quelques semaines. Immobile, aux aguets, comme une bête aux abois, c'est dans cette situation de retrait, semblable au froid hiver où Descartes élabora sa philosophie, qu'il semble vouloir se réduire lui-même à sa pensée.

9 Affublé d'un seul œil, œil traditionnellement reconnu comme la "fenêtre de l'âme " (p.35), il peut scruter les profondeurs intérieures. Mais cet œil, en tant que pensée raisonnable, n'est pas seulement une percée dans l'intériorité, elle s'ouvre aussi sur le monde extérieur, en se concrétisant sous forme de fenêtre, seule ouverture de cette cellule, avec laquelle le narrateur semble faire corps : «Dans le réduit minuscule et étroit dans lequel le Chef vit, [...] on peut dire qu'il ne reste que les quatre murs [...] hormis un minuscule miroir ovale faisant face à ce qui tient lieu de fenêtre » (p. 27). L'œil se voit et voit le monde en même temps, l'extérieur éclaire l'intérieur. Mais cette fenêtre qui donne sur un miroir est aussi la représentation de cette connaissance 
repos ; une machine donc ; cela même - une série de cuves remplies d'essence, dont la surface d'un noir de laque luisait, était reliée à un gigantesque "système " de tuyauterie dont l'organisation complexe imposait le respect, de la vapeur brûlante s'exhalait par certains endroits, des tuyaux s'entrechoquaient au milieu d'une indescriptible cacophonie de bruits métalliques gling-gling-glong-glongtchuut-d'où provenaient directement des tremblements et secousses dignes d'une crise de paludisme, quelque chose qui ressemblait à s'y méprendre à un compteur électrique un appareil mi-magique mi-scientifique au raffinement mystérieux, sans en comprendre le sens, le Chef fixa des yeux une aiguille sur le cadran qui tantôt montait, tantôt chutait brusquement, allait dans un sens puis dans l'autre, dans un mouvement frénétique et étourdissant qui lui fit perdre tous ses repères, à côté se trouvait une petite lampe toute rouge, rouge de rouge, pareille à un œil enfiévré par la rage - elle éclairait seule cet endroit, et hormis cette petite lampe rouge, il y avait encore suspendue au plafond une petite veilleuse qui éclairait faiblement. C'était donc une incroyable une invraisemblable salle des machines! Totalement impensable - il se trouvait donc qu'il allait habiter dans un endroit de Ce genre que cela pouvait véritablement exister cette sôrte de "salle », de "salle des machines »! 爺就䞡緊惶惶張張逃命爭生也似的破門飛出, 赤着腳, 白蒼蒼着臉的站身到是一 隔壁房間的戶屝之處探頸一看：喝一可了得, 這裡頭原來竟屬是一個完全出乎人 意料之外的另一開新外觀的天地 : 裡頭儘是一些幪幪幢幢, 突起沈下, 彎彎波 波, 扭曲盤蜿, 的玩意兒 ; 一機器 ; 是的 - 一塔堆大肥汽油桶合接起來, 油上了 光澤澤的亮烏漆, 的巨筒闊管雄赳赳氣昂昂的尉然慜像那麼问兒事的[系統], 有 些地方還儘在冒着蒸蒸熱水氣，一整個的這[系統]刻正在䦕隆隆一擱擱嘰5*，一呼 隆隆一擱擱䊿*的交雜粉沓的聲響之中發㗔病似的真迋打著抖哆, 還有那麼樣一隻 十分像是是電錶什麼的個深奥複雜的科學器材魔術玩意, 看過去及目但看著一直 針一會兒向上, 一會突蹿下, 轉的來個頭昏目眩, 不知東西南北, 在牠的旁邊還 有一畾紅赤赤紅赤赤，像隻瘋犬的害著高僥的一隻眼晴，似的小明灯一就只單這 個房間裡邊有灯, 除了這一盈的小小紅灯之外, 房間的丁頁空上頭猶還有一明低暗 小灯。這兒令人想不到的竟至居然是[機器房] ! 沒有人會想到一都就是住嚒都甚 且已經住到有如是像這嚒樣個的地方來为都還有這几さ一［房］ [機器房］！ (p. 26-27)

Ce corps-machine monstrueux, est un système fermé, agencé selon des relations de cause à effet, un ordre abstrait fonctionnel, qui sert à chauffer l'eau. Cette chaudière nous permet donc de passer de la mécanique traditionnelle à la thermodynamique, celle justement qui échappe à l'ordre ancien par ses mouvements discontinus et ses directions multiples. Dépourvu de toute âme, de toute logique, ce corps-machine, abandonné à lui-même, ne peut fonctionner qu'en état de crise permanente, toujours au bord du chaos. La conscience, qui s'est isolée, continue à attribuer au corps-machine des attributs et des fonctions qui ne lui correspondent pas, créant un hiatus irréparable. La conscience vigilante, séparée de ce qui pourtant doit assurer son passage sur terre, peut-elle véritablement se réfléchir et réfléchir le monde ? Toute la dérision du roman porte cette question à son paroxysme, en montre l'inefficience, l'absurdité.

\section{La maladie comme réponse au corps-machine}

Le corps du Chef, aux innombrables maux, ne diffère en rien de cette salle des machines infernale. La maladie caractérise l'homme post-moderne, il est malade de la modernité, il ne peut qu'être malade. Détaché de sa partie la plus raffinée, le corps réduit à luimême, à une grotesque machine, ne peut être qu'un ensemble de dysfonctionnements, de maux, de souffrances. 
17 Le narrateur, mais tous les autres personnages de ce roman de la même manière, sont affectés de trois sortes de maux, physiques, mentaux, sexuels. Ce sont d'abord les pauvres hères, hommes et femmes, qui sont touchés, puis la cohorte vorace des fonctionnaires qui s'affublent de maladies imaginaires, raisons même de leur emploi dans cette administration mise au rebus. Enfin, ces maux atteignent les riches, et eux plus encore que les autres, car ils sont en mesure de se soigner, ce qui ne peut provoquer que de nouveaux dégâts :

L'homme riche, sortit des médicaments de sa sacoche. Il en avala, cinq, six, dix une bonne quantité, quoi. Ce millionnaire, avait énormément énormément de maladies: de la tension, du diabète, une maladie de cœur, de l'arthrite, une allergie de la peau, la tyrhoïde... Il prenait - des médicaments occidentaux, de toutes les couleurs, enveloppés dans du papier argenté.

這一個 大貴人 喫的药, 竟至五六十力 - 那样 之分 $\times$ 己。大貴 人, 他有許多, 許多, 許多的与.一<, 如 : 血壓, 糖尿病, 心蔵病, 關節炎, 皮 虞過敏, 甲狀腺。。。。。大貴人, 他, 吃的药, 是外國來的金金, 綠綠, 都是用䇴錫質紙裏起着 (p. 345)

Ce qui unit tous ces êtres malades, c'est le besoin de raconter leurs maux, l'extrême complaisance qu'ils ont à les décrire dans les moindres détails, ces êtres, qui se considèrent comme des esprits séparés du corps, se trouvent confrontés à leur limite corporelle, mais surtout à celle de leur intelligence dont ils ont depuis longtemps perdu l'usage.

Le narrateur, contrairement aux autres personnages, tente de réfléchir ses maladies, et au travers de cette réflexion, mais aussi de cette attention portée au corps, de se régénérer. En effet, le Chef a besoin de solitude pour penser, mais aussi pour se soigner : "C'est véritablement chaque jour que le Chef a besoin de se retrouver dans cette pièce-là - (comme un malade qui a peur des maladies) - le fait de se rendre dans la tranquillité d'une pièce obscure lui permet de se soigner » (p. 24). Penser serait une forme de soin et la solitude, un lieu propice au renversement des valeurs, celles qui prônent la supériorité de l'esprit sur le corps.

20 Le narrateur nous décrit donc ses maux, dans une sorte d'autobiographie, où cette fois la vie concerne le corps. Dès les premières pages, c'est avec amour, amour de ses maladies, comme de ses propres enfants, qu'il nomme l'une l'aînée, les autres la cadette et la puinée, qu'il les décrit, en leur reconnaissant une intelligence propre. Cependant, c'est au travers de ces maladies seulement, en tant qu'il les prend en considération, en tant que vivantes elles le traversent en sa part vivante, qu'il se projette dans le monde, qu'il se réaccorde à son locus, échappant ainsi au triste sort des êtres dont le corps et l'esprit sont séparés.

21 Le narrateur tente donc de se raccorder au monde et de raccorder son esprit au corps. La maladie qui représente le mieux le lien de l'esprit au corps, touche au souffle. Ce souffle, qui est peut-être l'anima, non seulement réunit toutes les parties du vivant constituant l'entité du corps humain, mais il rejoint, au travers lui, le monde qui l'entoure, formant un tout bienheureux :

Je suis atteint d'un asthme d'une fort longue ancienneté, dans lequel je suis inextricablement entravé. - Si soudain au milieu du repos nocturne une crise se déploie avec vigueur, c'est comme si je devais appuyer sur un énorme soufflet de forge pour respirer Ke c'est. Je rends grâce au ciel - c'est en plein milieu de la toute première nuit que j'ai passé ici que mon asthme s'est réveillé - le bruit de ma respiration - s'est alors répercuté aux alentours dans le vaste espace sous le ciel pour se mêler au seul bruit étouffé que l'on peut entendre ici celui des vagues 
qui se soulèvent - le chuintement de ma respiration s'est uni au souffle des marées si bien que l'on peut dire qu'ils fusionnèrent, se firent harmonieusement l'écho l'un de l'autre.

我有那䌅附不去的圥長亘久氣喘舊病。一如果值更悄夜停的時候病驀發起來柯杨 然就恍若是在拉風箱那嚒樣分さ。得要多多感謝上蒼一來嘞這裡的頭一個深夜這 個病就折發了一我的喘息的聲音一貫迴在這裡這一個只得能聽得見海浪的微䐎的 穹蒼環宙之間 - 這個息喘的咻咻宛㫬正同着這天地的潮汐吸呼可以謂合而為一, 兩两呼應。(p.12)

Le souffle unit l'âme au corps, et la personne à ce qui l'entoure. L'homme rejoint la nature dont il est issu grâce à la maladie, car alors il prend véritablement conscience qu'il est aussi une part du monde. Il n'est pas un organisme autonome, mais il fonctionne en endosymbiose avec ce qui l'entoure. La maladie a donc ce rôle de révélateur, elle rappelle l'homme à son attache terrestre. Le ton du narrateur ne cesse cependant pas d'être ironique. Certes, la maladie relie l'esprit au corps, mais elle demeure une relation défectueuse, où domine la souffrance. Le Chef n'est pas seulement physiquement meurtri, il l'est aussi en son âme et conscience. Ce qui relie l'esprit au corps est la souffrance. Le corps n'est pas constitué de parties indéfiniment divisibles dont on pourrait, séparément, se passer, comparé à l'indivisibilité de l'esprit, - ce qu'en ont fait les sciences et la médecine moderne - le corps souffrant affecte l'âme, modifie ses pensées, l'empêche de penser, car il faut se concentrer sur la douleur :

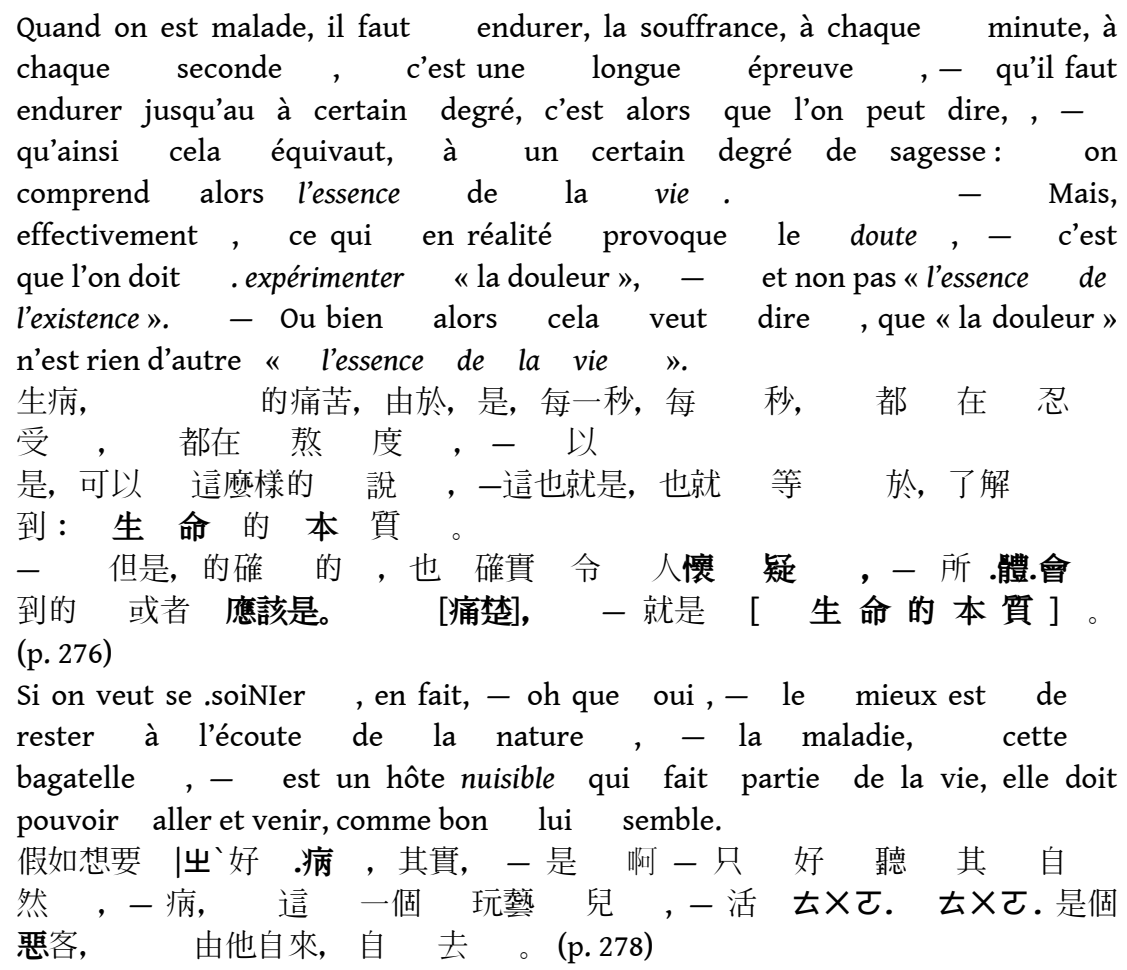

La douleur, qui rappelle ce lien que l'on n'a pu trancher avec le corps, permet de comprendre l'importance de la vie. Un doute s'installe, qui n'est plus le doute cartésien, la mise en doute de tout ce qui provient du corps. Le doute se renverse contre luimême. L'expérience de la douleur apporte une autre forme de connaissance, ancrée dans l'existence même et qui, celle-là, ne souffre pas le doute. Il y a une certitude, une évidence de la souffrance physique, qu'aucun esprit, si sublime soit-il, ne peut nier. 
La maladie est donc un indicateur, elle nous ramène à notre état de nature, à un certain ordre spontané, elle nous informe aussi de la propre logique du vivant, de sa corrélation avec toutes les parties de l'organisme, y compris le ci-dessus nommé esprit, que par conséquent, quoi qu'on dise, veuille ou pense, on ne peut les départager. Le corps est alors décrit comme une autre forme de machine, une autre logique, dans ses circulations, ses rééquilibrages incessants, et dans la nécessité de chacun des organes, des cellules :

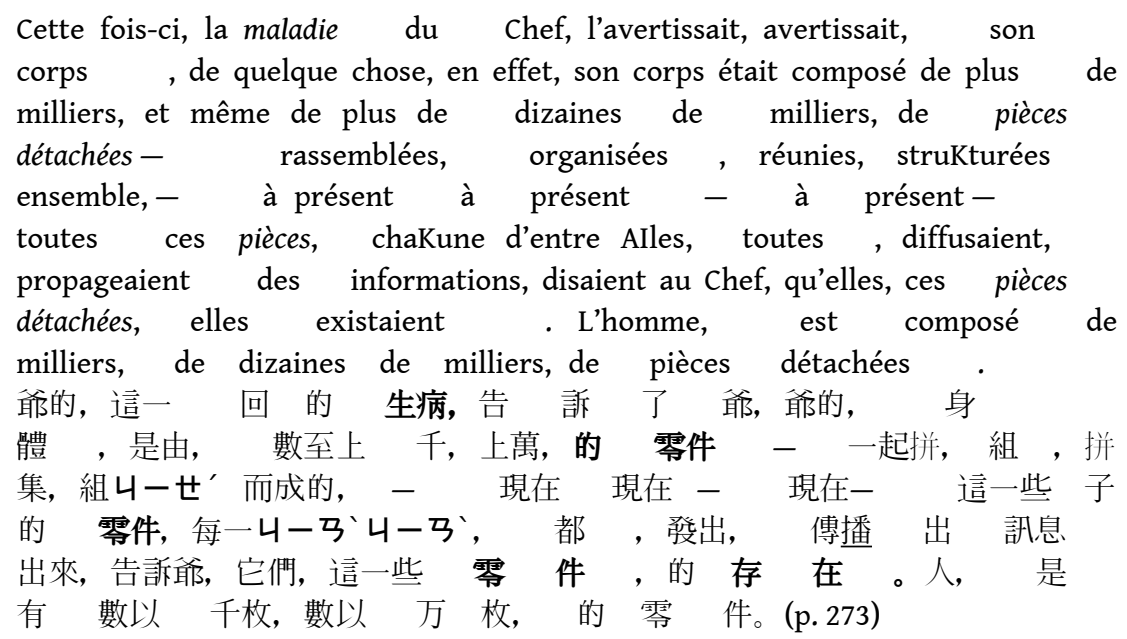

Il ne s'agit pas de pièces détachées, inertes, dans le sens où l'on pourrait les retirer sans porter de préjudice à l'ensemble, bien au contraire, chacune existe par rapport à l'autre en interaction. On obtient donc un système complexe, que l'on ne peut réduire à une vue d'ensemble, extérieure et objectivante.

\section{Le corps comme « champ continu de relations »}

L'esprit n'est-il qu'une pièce détachée parmi les autres ou bien le flux qui les traverse ? Cette question n'a bien sûr pas de sens, elle ne peut en avoir que dans une vision mécanique du corps. Comment appeler maintenant cet alliage du corps et de l'esprit, qu'aucune langue n'est prête à nommer, tant la langue est dépendante de cette dichotomie, qu'elle reflète et qu'elle redouble. Le corps-esprit, en dehors de tout dualisme, se caractériserait par la santé. La santé nietzschéenne peut-être, en tout cas celle d'une libre circulation n'engendrant aucun obstacle ou blocage :

C'est pourquoi, s'agissant d'un homme, si sa santé, n'est pas bonne, - c'est presque, oui vraiment, c'est presque Kom si il n'avait plus "d'âme", oui vraiment. C'est pourquoi, "l'âme", - s'il en est ainsi, à première vue, pratiquement, oui vraiment, - doit prendre appui sur le corps pour exister . Voilà pourquoi, le corps est peutêtre comparé à .l'âme - beaucoup plus//important qu'il est. Dès lors, ce n'est pas, ce que l'on entend généralement, quand on dit que, l'âme, est plus bôcoup plus importante que lecorps.

人的, 所以, 人 的 身 體, 要是, 不好牠来了的個話, 一 乎, 是的, 似乎 பーヌ`カーろ' [靈魂] 都沒有了, 是 的。所以, [靈魂], 一像這樣, 看起來, 一似乎, 是的, 一 必須仰 賴 身體 方了く'夠存 在。是故, 可能身體, 比起.靈. 魂 來 一更加 分ー ` 偅川要。至, 不是, 一般所謂的, 靈魂, 比。身 體更要为“得重要 . (p. 284) 
et le corps sont réconciliés, d'une certaine manière le corps n'existe plus. L'organisme n'est plus considéré comme "une entité autonome et préétablie, mais comme un locus de croissance et de développement particulier dans un champ continu de relations $»^{6}$.

Tim Ingold désigne cette nouvelle conception des organismes et de leurs environnements, par l'expression de "pensée relationnelle $»^{7}$, c'est ce terme que nous pouvons maintenant utiliser. Si la maladie, a permis de reconnecter l'homme moderne à son entour, c'est la bonne santé qui caractérise la "pensée relationnelle ». Bonne santé, en tant que processus, circulation et non «blocage ", essence même des maladies les plus courantes. La guérison, elle aussi, répondra donc à une logique relationnelle et symbiotique, dès lors, pour le narrateur, aucune médecine ne peut guérir :

- Si tu, disons que, si tu es courageux, tu ne peux plus, ce n'est plus possible de prendre tous ces médicaments que le médecin t'a prescrits, cette énorme sac, de médiKaments, pour que ton précieux, précieux corps, soit transformé en cobaye, un cobaye parmi d'autre. ou bien , s'il t'a donné une grande quantité de médicaments occidentaux - alors il te présente des médicaments chinois , - et s'il est audacieux alors il essayera même de mélanger médecine chinoise et médecine occidentale pour voir ce que cela va donner comme rézultat.

一你, 晌如說, 勇敢接受了你, 已經不, 沒有辦法, 再 信任 的醫生 的，所開付給你的一大包包，一大包包，藥 ᄃ 々2，拿你，寶貴，寶貴的不得了 的身體, 予他做醫療練習 之 中 的 練習品, 其 中 的 一 個。或, 在你受服大量 的 西藥 的 同時 - 也摻 進了中葯, - 放膽 試一次 中西兼用的醫治 效能看

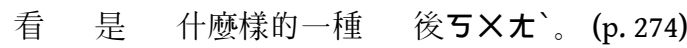

La maladie elle aussi dépend d'un certain entourage, par conséquent la guérison ne signifie pas de traiter le mal isolément mais bien au contraire en prenant en compte tous les éléments qui ont pu contribuer à son apparition. Le narrateur, dans sa critique acerbe de la société de consommation, qui va jusqu'à reproduire ce modèle sur la manière de se soigner, indique que l'écoute du corps est primordiale dans le processus de guérison, et que les malades sont souvent mieux à même de comprendre leur maladie, de soulager leur douleur ou même de se soigner que n'importe quel médecin. Une bonne écoute du corps implique donc que l'on ait accepté de se fier à l'intelligence du corps.

Avant le dénouement du roman, le narrateur revient sur ses maladies, il insiste cette fois sur les relations qui existent entre elles. Les dysfonctionnements se produisent d'eux-mêmes, ils n'ont pas d'origine extérieure, et le narrateur en assume l'entière responsabilité (p. 270). Ce sont des maux engendrés par le corps lui-même, par les nœuds qui se sont formés lors des circulations, qui ne cessent de se renouveler. Cependant les maux se résorbent aussi d'eux-mêmes, se réparent d'eux-mêmes. Le corps trouve lui-même une issue. Le corps est un ensemble de processus complexes, tous dépendants les uns des autres et qui se répondent:

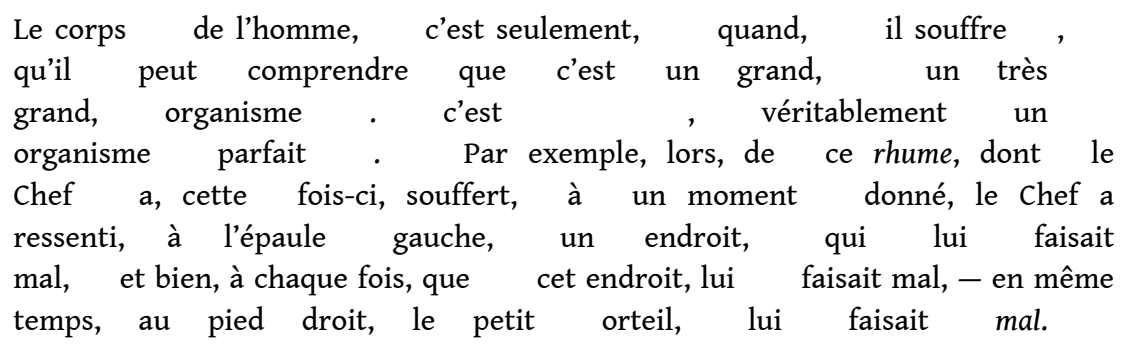




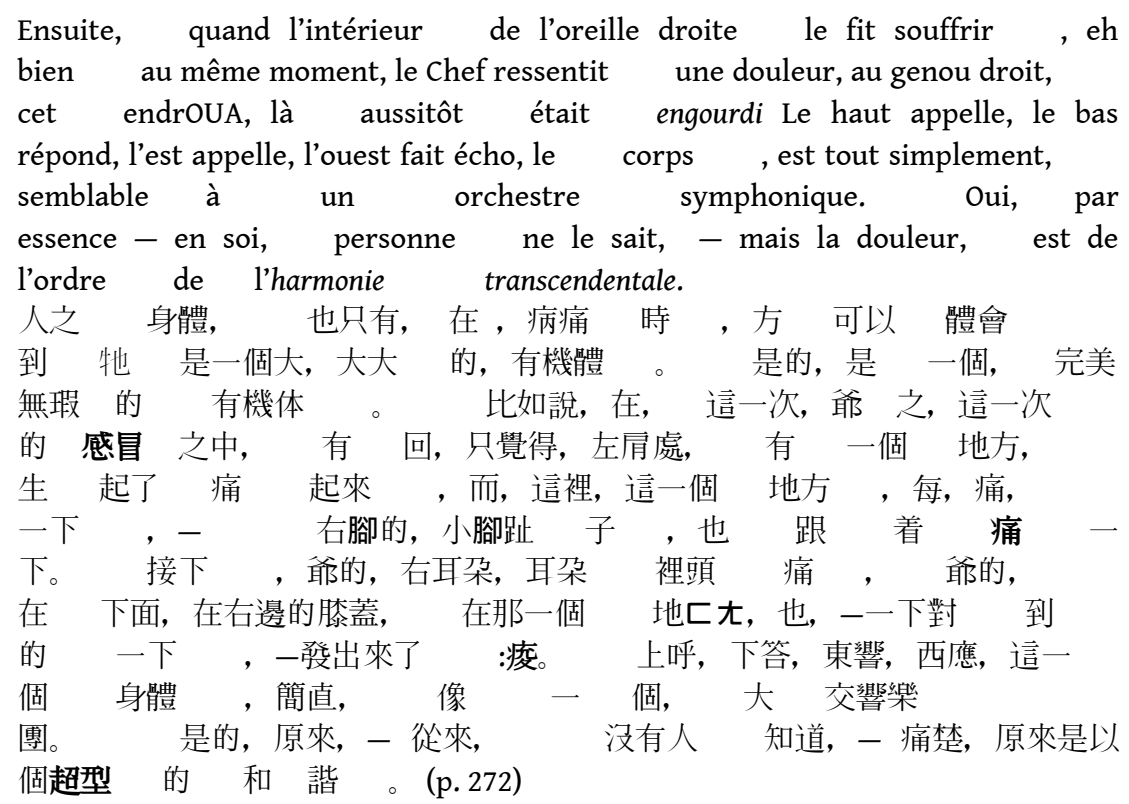

31 On peut noter ici l'importance des tensions contraires qui engendrent des processus en chaîne; les maladies sont elles aussi des vitesses, des rééquilibrages constants, des luttes pour rétablir les connections et les circulations. Il s'agit bien ici de la vitalité d'un organisme qui toujours compense, interprète, récupère, réagit. L'harmonie transcendantale a lieu au niveau du corps, elle n'est harmonie qu'en tant qu'elle n'est pas statique, la transcendance est alors de l'ordre du mouvement, de l'instabilité qui sans cesse doit se restabiliser, multiplier et renouveler les points d'appui pour plus de complexité encore. Orchestre, encore une fois, non pas parce qu'il y aurait un chef qui dominerait le tout, mais en tant que « le haut appelle, le bas répond ». Cette réciprocité répond à l'ordre d'une auto-organisation, qui se rééquilibre sans cesse de manière autonome.

Il reste un point à éclairer, celui de savoir comment les principes dynamiques, relationnels, symbiotiques qui caractérisent la "pensée relationnelle » se manifestent dans le roman, non plus au travers de la maladie, qui en est la prise de conscience, mais de la bonne santé.

Ce qui a permis le passage d'une conception du corps-machine à celle du corpsprocessus est avant tout le langage même du roman. Wang Wen-hsing, dans ce roman, utilise toutes les possibilités typographiques qui s'offrent à lui (caractères gras, soulignés, grossis...), il invente des caractères, fait un usage exponentiel des espaces blancs et des tirets, utilise le syllabaire phonétique zhuyin fuhao (= signes phonétiques), en usage à Taïwan depuis 1949 en remplacement des caractères d'écriture ou sinogrammes lors de l'apprentissage de ceux-ci et correspondant à la transcription romanisée dite pinyin en Chine populaire, pour remplacer les caractères d'écriture. L'auteur, certes, opère ainsi une œuvre expérimentale, cependant son objectif est avant tout de trouver une écriture limpide, claire et distincte, qui se rapproche de l'oralité. Une autre caractéristique de ce roman, particulièrement dans sa deuxième partie, est l'usage des blancs et des longs tirets, qui aèrent le texte, bousculent la syntaxe, mais plus encore, prolonge le mot en accordant au son une durée qui permet à la phrase de vibrer. La nécessité de rester au plus près de ces marques typographiques s'impose au traducteur, et l'idée de transformer le zhuyin fuhao non pas en phonétique internationale, mais en écriture phonétique, s'est révélée une solution appropriée pour 
faire entrer l'oralité dans le texte écrit ; en jouant sur les sonorités et en contraignant le lecteur à prononcer le mot pour le comprendre. Par exemple, le mot SoiNIer (v. citation supra) est écrit ainsi pour traduire l'utilisation du syllabaire phonétique à la place du caractère d'écriture dans le texte original. Ce parti pris donne à ce texte tout son relief, mettant en valeur sa tonalité grinçante, sa verdeur et sa profondeur. À Taïwan, le langage de Wang Wen-hsing a, pour cet usage du syllabaire, été comparé à la «langue des martiens », qui est celle des jeunes Taïwanais écrivant des SMS sur leurs portables.

Ce langage de Wang Wen-hsing, dont on a vu des exemples tout au long de cette étude, par ses distorsions, ses écartèlements et resserrements, représente un ensemble de forces, freins, vibrations, éreintements, visibles dans les espaces blancs, l'usage de marques typographiques et l'emploi du syllabaire phonétique zhuyin fuhao. Ce langage accompagne l'activisme plutôt que les actions des personnes, qui agissent sans but véritable, s'agitent, mais de manière incessante. Les déplacements sont multidirectionnels, les personnages vont, viennent, se rapprochent, s'éloignent, s'emmêlent, produisant des blocages qui ensuite se défont, se dispersent vers des errances indéterminées, prouvant ainsi leur grande santé et celle du récit. Les relations amoureuses du narrateur, par exemple, impliquent un très grand nombre de déplacements, multidirectionnels, mais toujours vains, qui ne peuvent s'arrêter momentanément que lors de chocs involontaires. Les personnages ne sont plus alors que des prétextes aux mouvements, aux changements de directions, l'essentiel se produit entre eux, dans la relation qui les fonde. Les lieux et les heures de rendez-vous changent sans cesse, les personnes affluent, mais jamais la principale intéressée. La recherche d'un emploi est, elle aussi, tout au long du roman, prétexte à une infinité de déplacements. Le narrateur se rend dans une administration où les employés passent leur temps à bouger. Ils ne tiennent pas en place, se livrent à des courses poursuites dépourvues de sens, jouent au ping-pong, se battent. Nul moyen pour le narrateur de les saisir, d'obtenir d'eux une place dans cette administration où personne ne travaille, assis à son bureau, comme il se devrait. La recherche d'argent conduit ensuite le narrateur à grimper plusieurs fois en haut d'une colline pour demander l'aumône à un prêtre, ou bien à organiser toutes sortes de stratagèmes afin de voler différentes personnes du port. Les déplacements dépendent des positions et des territoires mais les positions changent et les territoires n'ont pas de limites précises. Ces circulations répondent à des nécessités organiques et non mécaniques. Dans un mécanisme, le circuit se répète à l'identique, il ne peut varier et s'il tombe en panne il ne peut se réparer lui-même. Dans un processus organique, les déplacements ne sont pas systémiques, ils varient sans cesse. Le réseau se déploie, quand un blocage a lieu, les circulations reprennent à partir d'un autre point, engendrant une nouvelle figure.

Comment peut alors prendre fin un récit qui s'auto-organise sans cesse? Nonobstant le choix de l'auteur de raccourcir son roman, pour des raisons de temps - l'écriture, dans une langue qui rend compte des moindres vibrations et circulations du vivant, requiert un temps de labeur exorbitant - c'est dans un tourbillon que s'achève la narration. Tout d'abord lors d'une battue, la course poursuite à l'intérieur d'un potager d'un chien sauvage, qui s'achève dans une sorte de rituel primitif de mise à mort et de dévoration. Puis, après la mort du narrateur, dont l'assassinat est dérobé au lecteur, l'aigle noir, l'âme du narrateur ou simplement l'anima, libre et mobile, tournoie, solitaire, au-dessus de la cabine de bain : 
Au même moment, du côté du port, près d'une cabine de bain, juste au-dessus d'une petite colline, très haut dans le ciel, volait un aigle noir comme on n'en voyait plus depuis longtemps, il effectuait un cercle puis un autre, ne cessait de tournoyer audessus de cette cabine de bain, plus il montait plus il s'éloignait, plus il s'éloignait, plus il paraissait solitaire.

一這時候, 在水港的這一邊, 那一間洗澡房, 所在的, 小圓坡上, 天高的地在, - 喜張, 黑默默的, 好久以來, 都, 稀 有, 出山 來 過 的, 蒼鷹, 在, 這一間, 洗澡間的頂上, 飛沛着, 旦見牠, 一圈, 又一圈的轉, 只見 着牛也越攀越上, 越㹔越穴。(p. 371)

Le narrateur meurt sans raison véritable, en plein milieu de son monologue, emporté par les mouvements incessants du langage. Le langage, qui pour Wang Wen-hsing représente la recherche de la liberté avant tout, est aussi ce qui est au fondement d'une conception du vivant qui ne sépare plus corps et esprit. Grâce à cette liberté du langage qu'incarne le narrateur, Wang Wen-hsing nous fait pénétrer à l'intérieur de la vie, il nous fait considérer le monde autrement. C'est ce qui donne sens à cette œuvre inclassable dont l'auteur justifie ainsi le contenu :

C'est un monologue à la troisième personne du singulier, par conséquent le langage a quelque rapport avec ce monologue. Quel est le rapport entre le langage d'Un homme dos à la mer et le monologue? Le rapport vient du fait que la forme, la personnalité de cet homme qui monologue détermine son langage. En d'autres termes, sa personnalité, la forme de son caractère, fait que le langage atteint son plus grand degré de liberté. Tout d'abord, c'est un ivrogne, ce qui lui apporte déjà une très grande liberté. Deuxièmement, sa vie n'est qu'une succession de malheurs et de difficultés, aussi a-t-il besoin de l'exprimer. Troisièmement, il n'a absolument plus peur de rien, comme personne ne l'écoute, à qui s'adresse-t-il ? Il s'adresse au monde enveloppé dans la nuit noire - dans ce monde, cela revient à participer à la transformation du ciel et de la terre ; son langage n'a plus aucune forme, les paroles qu'il prononce pourraient être le bruit du vent, le bruit de la pluie, cela c'est aussi une forme de liberté, la liberté du langage. Il y a une quatrième raison - il s'intéresse beaucoup au langage, normalement, si on croit ce qu'il dit, c'est un poète. Quand il créait, il a goûté à la liberté de la poésie, à la liberté du langage, maintenant, les limites de la poésie ont disparu, il peut dire n'importe quoi, il peut utiliser la manière poétique pour dire n'importe quoi ; alors il n'a plus ni foi ni loi, il peut être encore plus libre, aussi a-t-il beaucoup de liberté, afin de dire n'importe quoi, le temps d'une nuit, dire tout ce qu'il a envie de dire. Par conséquent, on peut dire d'Un homme dos à la mer que ce sont les déblatérations du Chef qui s'accorde la liberté de dire n'importe quoi pendant deux nuits. Ce sont les quatre raisons de son langage spécifique.

(...)這裡是第一人稱的獨白, 所以它的語言跟狺個獨白是有些關係。那麼《背海 的人»的語言跟獨白的關係在哪裡? 關係在於, 這個獨白的人, 他的造型, 他的 特性會決定他的語言。換句話說, 他的個性, 他的造型, 他性格的造型, 可以使 他的語言達到最大的自由。首先, 他是個醉漢, 這已經給他語言很大的自由了。 第二, 他一生不幸受難的積存, 需要有個宣洩。第三, 他如今可以毫無顧忌了, 因為沒有旁聽的人。現在他對誰講呢? 他對著黑夜的天地講一在這個田地裡, 他 等於跟田地化在一起, 他的語言也就沒有任何形式, 他講的話可以像風的聲音, 像雨的聲音，這又是一種自由，語言的自由。還有，第四則理由一他個人對語言 有很高的興趣。他平常, 如果是照他講, 他是一個詩人, 他創作時已經嘗試過詩 的自由, 語言的自由。何況現在連詩的限制都不存在, 他可以胡言亂道, 他可以 用詩的方式來胡言亂道; 那他更可以無法無天, 更可以有他的自由了。所以他有 很多的自由, 令他這一個夜晚, 可以胡言亂語, 言所欲言。所以表面上, 可以 說, 《背海的人»是爺這個人兩個夜晚的胡言亂語, 他有胡言亂語的自由。這是 他特殊語言的四個理由。8

37 Liberté grande, celle de la nature, ce processus toujours en cours, dont l'homme a voulu se séparer, et dont Un homme dos à la mer, nous rappelle le lien nécessaire. 


\section{BIBLIOGRAPHIE}

INGOLD Tim, Marcher avec les dragons, MADELIN Pierre (trad.), Bruxelles, Zones sensibles, 2013.

LATOUR Bruno, Où atterrir, Comment s'orienter en politique, Paris, La Découverte, 2017.

LI Shi-yung 李時䔨, [« La langue elle-même est une raison - Lecture lente de Wang Wenhsing »] / 語言本身就是一個理由 - 慢讀王文興 / Yuyan benshen jiu shi yige liyou-Wang Wen-hsing fangtanlu, in [L'œil du ciel s'ouvre sur un monde de poussières] / 偶開天眼覤紅塵 / Oukai tianyan qu hongchen, HUANG Shu-ning 黃恕寧 (dir.), Taipei, Guoli Taiwan daxue chuban zhongxin, vol. 5, 2013, p. 389.

WANG Wen-hsing, Un homme dos à la mer / 背海的人 / Bei hai de ren, Taipei, Hongfan shudian, 1999 ; Un homme dos à la mer, LOIVIER Camille (trad.), Montmorillon, Vagabonde, 2022.

\section{NOTES}

1. Les passages cités de l'œuvre étudiée renvoient à cette édition : WANG Wen-hsing 王文興, Un homme dos à la mer / 背海的人, Taipei, Hongfan shudian, 1999.

2. LATOUR Bruno, Où atterrir, Comment s'orienter en politique, Paris, La Découverte, 2017, p. 98.

3. L'édition électronique ne permettant pas de reproduire les espaces blancs et les longs tirets qui représentent une des caractéristiques du style de Wang Wen-hsing, nous avons fait au mieux, et nous lui présentons ici nos excuses.

4. Le 28 février 1947 est la date d'un massacre perpétré par l'armée du Kuomintang après une révolte populaire. Depuis 1945, Taïwan, ancienne colonie japonaise, (1895-1945) est rentrée dans le giron de la République de Chine, gouvernée par Chiang Kai-shek, mais les relations entre le gouvernement et la population sont houleuses. Après ce massacre, la société taïwanaise connaîtra un climat de terreur jusque dans les années 1960, et il faudra attendre la levée de la loi martiale en 1987, pour que la main de fer du gouvernement se relâche.

5. Il n'existe pas de version numérisée du roman de Wang Wen-hsing, comme l'auteur invente des mots mais aussi des caractères, utilise le bopomofo, ajoute différents symboles et a recours à des espaces blancs de différentes longueurs, sans oublier les traits longs, il est impossible de retranscrire le texte sans effectuer des transformations, ce que l'auteur n'apprécierait guère même pour un article de recherches. Aussi avons-nous ajouté des astérisques là où nous avons dû troquer un caractère contre un autre, nous nous en excusons auprès de lui.

6. INGOLD Tim, Marcher avec les dragons, MADELIN Pierre (trad.), Bruxelles, Zones sensibles, 2016,

p. 94.

7. Ibid.

8. LI Shi-yung李時雍, ["La langue elle-même est une raison - Lecture lente de Wang Wenhsing»] / 語言本身就是一個理由 - 慢讀王文興, in [L'œil du ciel s'ouvre sur un monde de poussières] / 偶開天眼覤紅塵, HUANG Shu-ning 黃恕寧 (éd.), Taipei, Guoli Taiwan daxue chuban zhongxin, vol. 5, 2013, p. 389. 


\section{AUTEUR}

\section{SANDRINE MARCHAND}

Sandrine Marchand est Maîtresse de Conférences à l'Université d'Artois. Ses recherches portent sur la littérature taïwanaise, l'écriture poétique et la traduction, elle s'intéresse également à la genèse des œuvres. Elle a consacré son HDR au processus d'écriture du romancier taïwanais Wang Wen-hsing 王文興. Elle prépare en collaboration avec Samia Ferhat l'ouvrage collectif Les liens de la mémoire, itinéraires taïwanais. 


\section{Corps trans-formés}




\title{
The Japanese No-Body
}

\author{
Josef Kyburz
}

1 At the outset, a few preliminary remarks are needed to delimit the framework within which I shall develop the argument, prolegomena which must be kept in mind throughout:

- As for the time frame set down in the title of this conference, the view adopted here extends far beyond the past two centuries, as far back, in fact, as literary and pictorial evidence allows, including the Heian period that means. We thus have testimony ranging over a millennium, that shows how the body has been represented, or lets us infer how it seems, or looks, to have been perceived at the time. Such material allows us to follow the sequence of conceptional change in the long run, and to surmise how the mental and pictorial representation of the body changed in the transition from tradition to modernity.

- I shall also sidestep the field set down in the title, "literature", and instead choose anthropology, more precisely its recent "cognitive" branch which is concerned with how phenomena are perceived on the most basic level, that of immediate perception. On the basis of the same textual and pictorial evidence, we can show up, by way of comparison with other times or civilizations, the differences between now and then, try to interpret what they seem to manifest-and what they may be due to.

- Furthermore, I shall consider the way the body is perceived or, at least, appears to have been perceived in the past, on the basis of "cultural relativism". The basic tenet of this hypothesis is the assumption that the language of a people or, for that matter, also nonlinguistic modes of expression such as painting, sculpture, architecture, etc., conform to the way this particular people perceives and conceives the world it lives in. ${ }^{1}$

- Cultural relativism being at the outset a linguistic theory, I shall, within this framework, try to point out how the grammatical unnecessariness, if not absence, of personal pronouns and the gender distinction between male and female in Japanese and other Far Eastern languages, reflects on the way the body is represented, or not, in verbal and in pictorial forms of expression.

2 Our point of departure for looking backwards in time is a citation of In Praise of Shadows, a famous essay published in 1934 by Tanizaki Jun'ichirō (1886-1965), in which we find the presence of women described in the following way: 
The body, legs, and feet are concealed within a long kimono... a woman of the past did indeed exist only from the collar up and the sleeves out; the rest of her remained hidden in darkness... her body shrouded day and night in gloom, her face the only sign of her existence... But in the past this was sufficient. For a woman who lived in the dark it was enough if she had a faint, white face-a full body was unnecessary.

「胴や足の先は裾の長い衣裳の裡に包まれている、昔の女と云うものは襟から 上と袖口から先だけの存在であり, 他は悉く闇に隠れていたものだと思う。.... ただ闇の中に 五體を埋めつつその顔だけで存在を示していたと云へる。...が、 昔はあれでよかったのだ、闇のなかに住む彼女たちに取っては、ほのじろい顔 ひとつあれば、胴體は必要がなかったのだ。」2

And further on:

I can put the matter strongly: women in those days had almost no flesh. I remember my mother's face and hands, and can vaguely remember her feet, but I can remember nothing about her body.

「極端的に云えば、彼女たちには殆ど肉體がなかったと云っていい。私は母の

顔と手の外、足だけはぼんやり覚えているが、胴體については記憶がない。」3

4 Tanizaki speaks about the late Meiji and Taishō eras, but what he is observing may be shown to be true of any previous period of Japanese history for which we have literary, pictorial, and social as well as linguistic, conceptual and behavioural testimony.

These reminiscences immediately recall the Tale of Genji / 源氏物語, the famous novel from the beginning of the 11th century, which Tanizaki translated into modern Japanese. And indeed, in the no less famous illustration from a century later, the Tale of Genji Scroll / 源氏物語絵巻 (ill. 1), all we see of the nobles of the Heian court are their faces and perhaps part of their hands.

III. 1. A scene from the Yadorigi Chapter of the Tale of Genji Scroll (between 1120-1140).

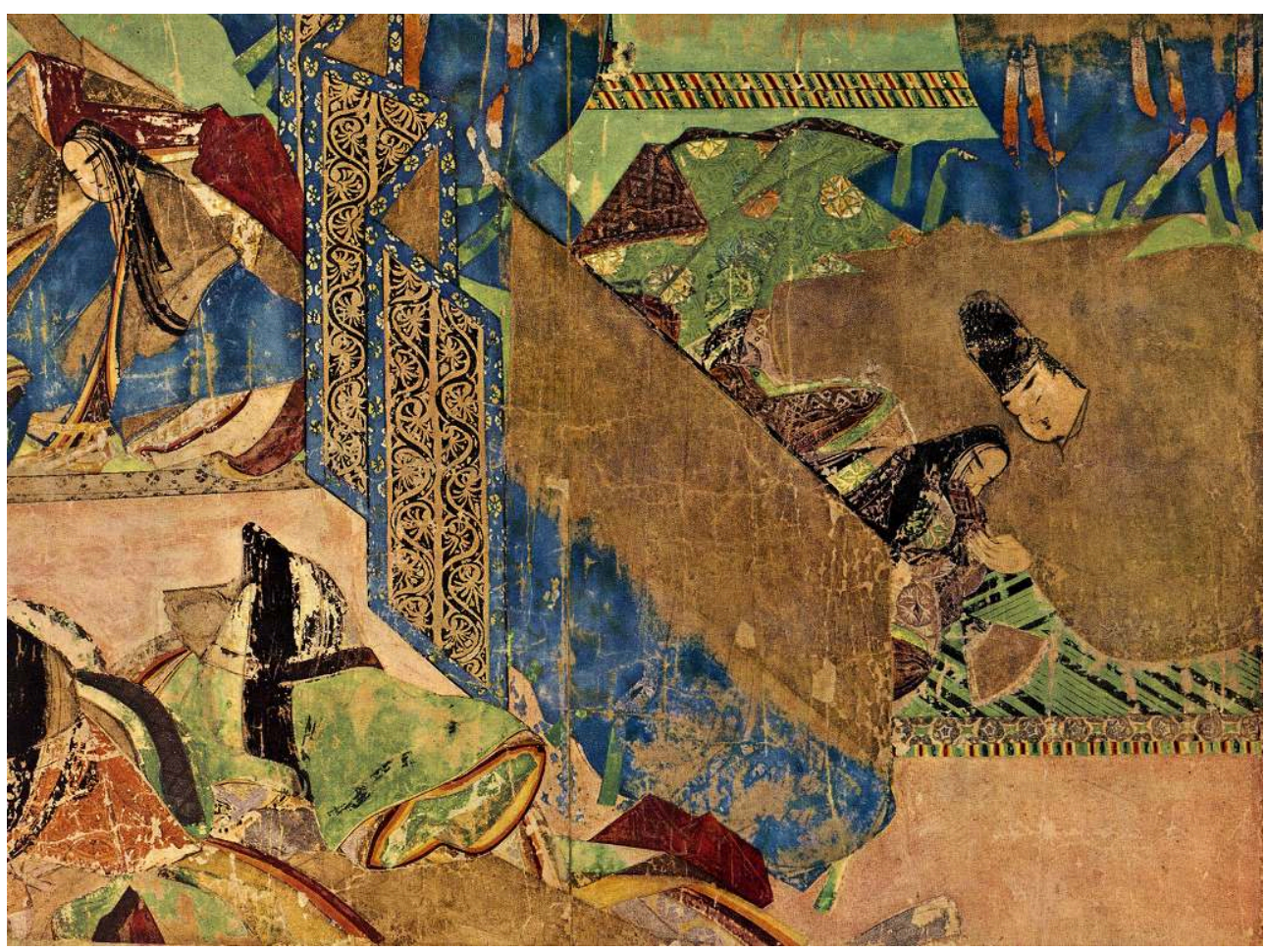

Public domain. 
An eloquent illustration of another of Tanizaki's insights goes:

Were it not for shadows, there would be no beauty. Our ancestors made of woman an object inseparable from darkness... They hid as much of her as they could in shadows, concealing her arms and legs in the folds of long sleeves and skirts, so that one part and only one stood out-the face. ${ }^{4}$

7 And indeed, the images of the Genji Scroll (ill. 2) show exactly that. Judging from both these images and the text of the tale, the modern viewer can not but be surprised by the degree of disincarnation and distance, not to say abstraction, with which the person as such appears to have been perceived in that world.

III. 2. The Tale of Genji Scroll (between 1220-1240), a scene from the Takekawa Chapter.

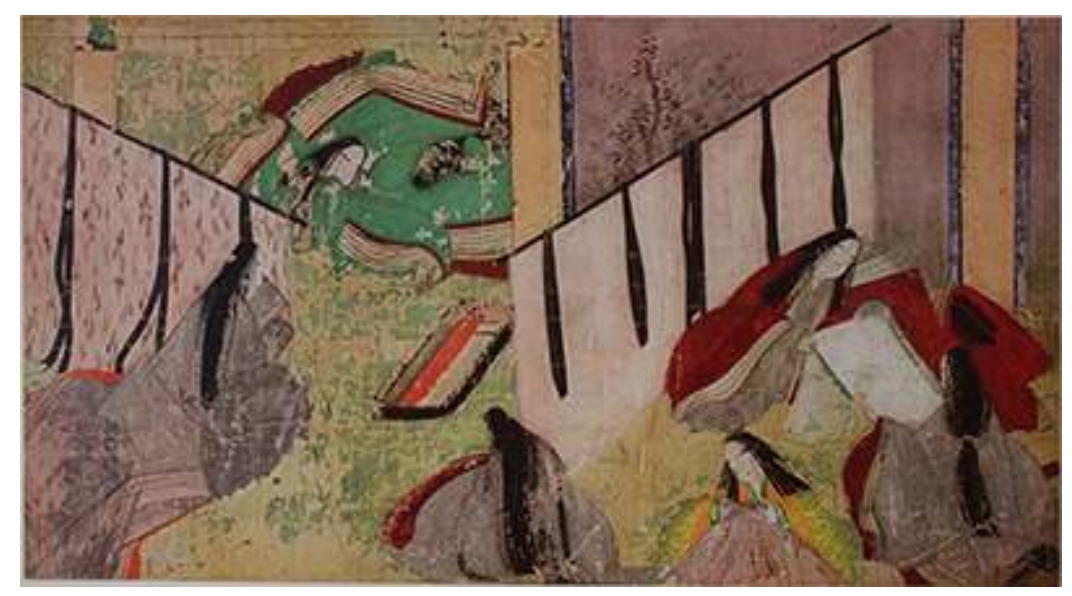

Public domain. but rather the living person (oneself) or, implicitly, the first person (I, me) as a whole, including one's physique, status and condition, one's moral character. The same ideogram may also stand for むくろ / mukuro, denoting more specifically the trunk: the body with or without the limbs, but excluding the head, hands and feet-in other words the invisible part, but also the inanimate, dead, corpse. Another term used to describe a person physically is sugata / 姿, but, again, in olden times this mostly denotes "appearance, mien, countenance, bearing..." of the two words now used for "body", 
karada and shintai, both appear in the late Middle Age in the same sense as mukuro, "trunk", or "corpse", i. e. the insignificant parts of the body.

The subject of the Genji Scrolls is obviously, and exclusively, the circles in contact with the court. This means that the tale and the images give us a picture of only a limited part of society, and an exceedingly small one at that. Objectively seen, the most important element in the depiction of the denizens of the capital is the apparel, and its social dimension is obvious. Japan is no exception to the nearly universal rule that the higher the social class, the more the body is garbed, and vice versa. If we take a close look at two other scroll paintings from the same Kamakura Period, the The Illustrated History of the Ishiyama Monastery (ca 1325) 6 and the Life of the Holy Man Ippen (1299) painted in the more realistic Yamato style, we find the craftsmen, here carpenters (ill. 3), more or less covered in upper and lower garments, whereas the workmen have their torsos bared and the labourers go about their menial tasks in not much more than a loincloth. Further down the social scale, the destitute and the outcasts (ill. 4) are seen covered in rags barely covering their loins. At the lowest extremity are the cadavers, stripped of everything, as are the sinners in pictures representing the Buddhist hells who are being tortured as punishment for their wrongdoings (ill. 5). At the pinnacle of society, the members of the imperial family, in their most subdued garb of the Buddhist prelate (ill.6), are nothing but a face. ${ }^{8}$ In fact, we may say that the face alone is the person, the rest of the body being devoid of individuality and significance, and therefore not mentioned, not singled out. The body is neither an existing subject nor an object of attention. Since we hear of no taboo concerning the fleshly body-such as that which exists in the Christian faith where it is the cause of the original sin, the root of evil-this disregard cannot be ascribed to any negative attitude either.

III. 3. The Illustrated Scroll of the Ishiyama Monastery (ca 1325), Carpenters at work on the construction of the monastery.

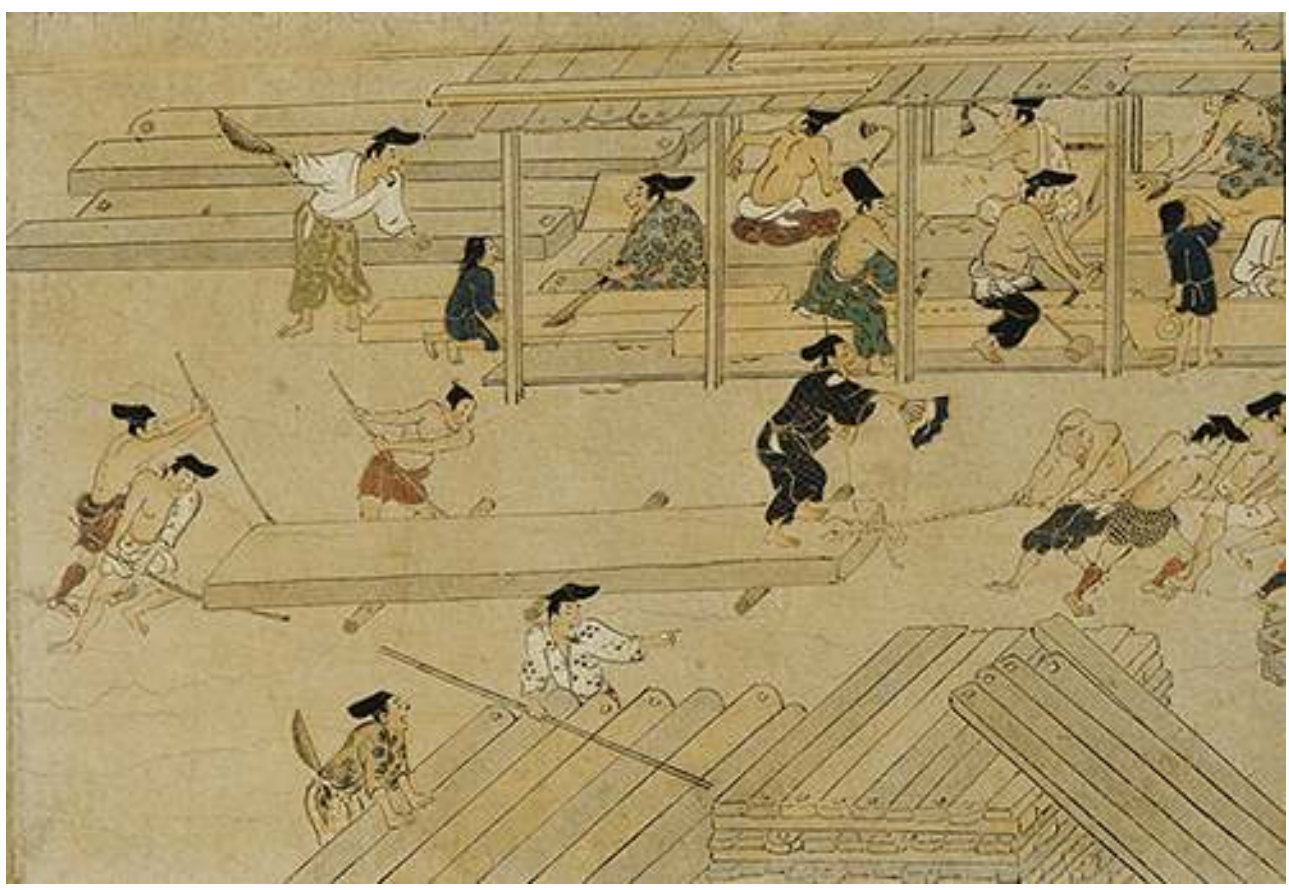

Tokyo National Museum. 
III. 4. The Illustrated Tale of the Holy Man Ippen (1299). Beggars and cripples.

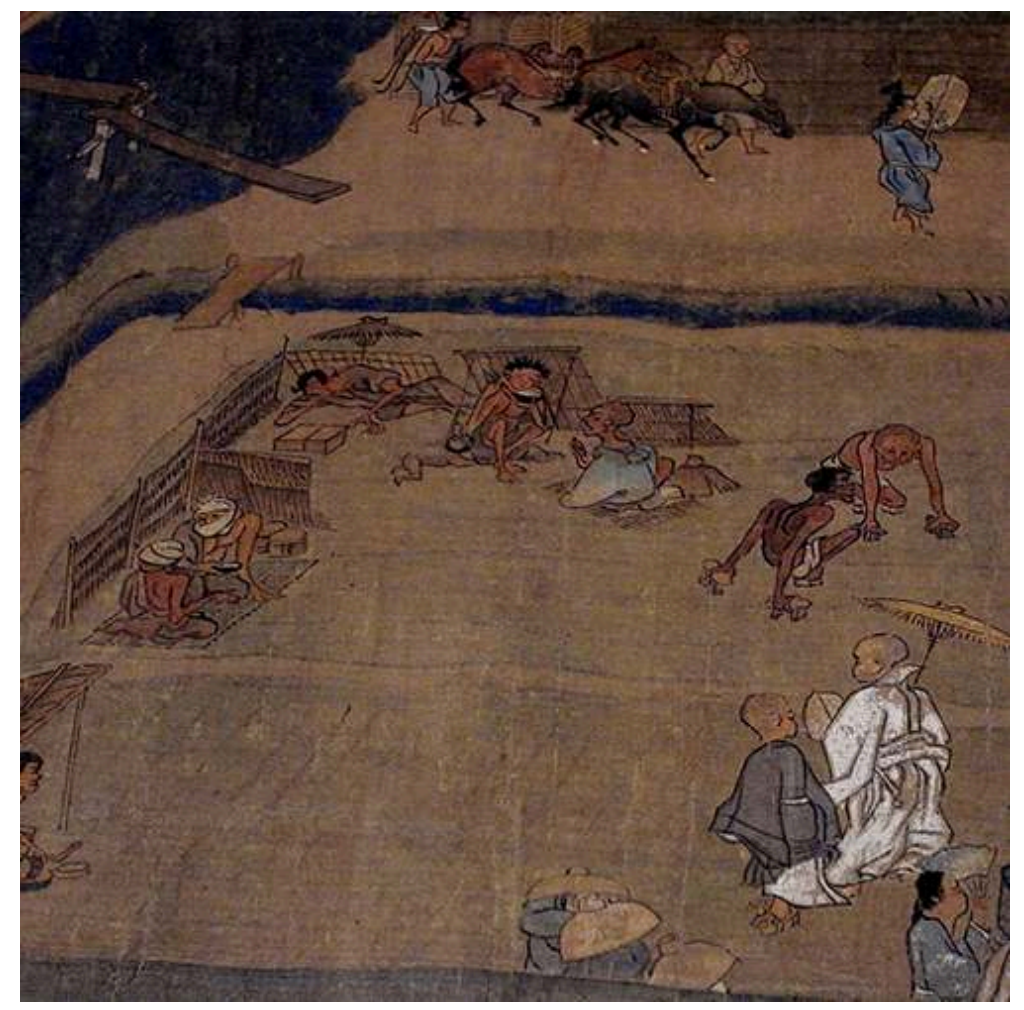

Tokyo National Museum.

III. 5. Tales of the Monks' Hell 沙門地獄草子 (12th-13th c.).

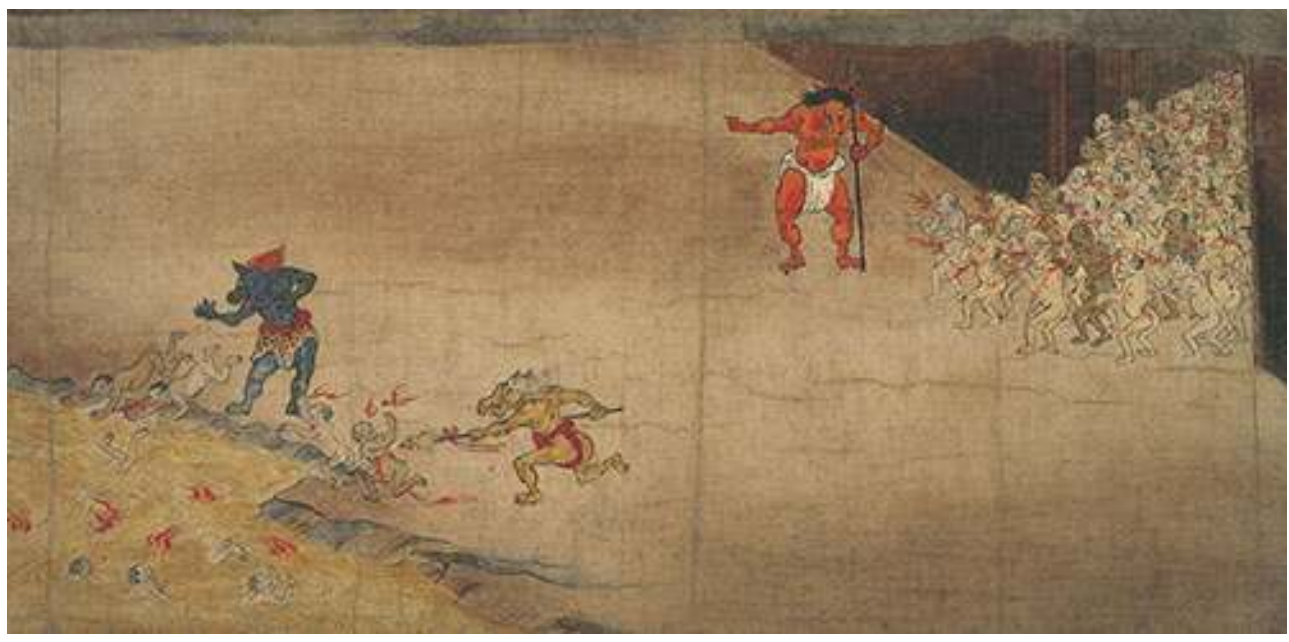

Nara National Museum. 
III. 6. Portrait of the Retired Emperor Hanazono (1297-1348). Myōshin-ji.

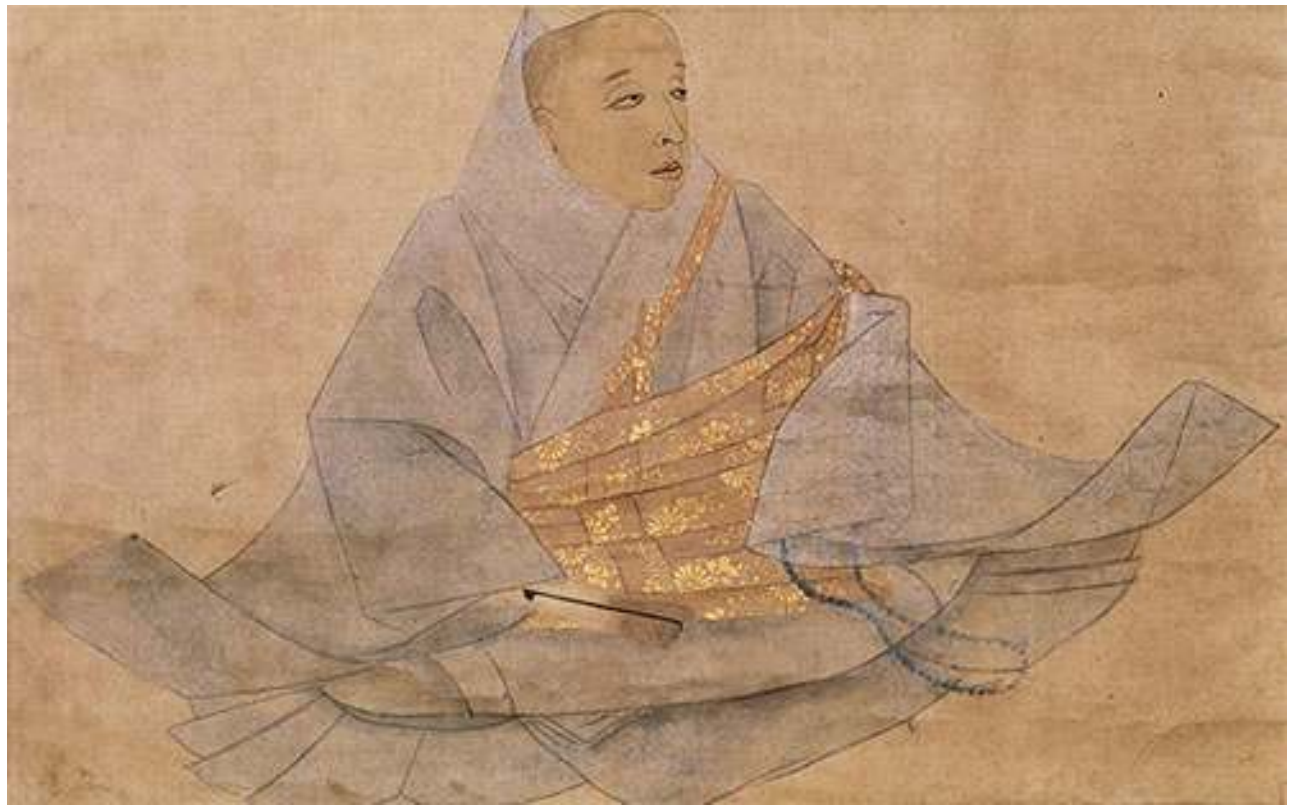

Public domain

What about three-dimensional representations of the human figure, of the fleshly body in particular? A quick overview of the statuary of the Heian and Kamakura periodsreputed for its high degree of realism-shows that images in the round do not expose any more "skin"-except for a particular kind of icon of the Buddhist faith. As a matter of fact, apart from a very small number of likenesses of so-called shintō deities (神像 / shinzō: ill.7) and effigies of (what the Westerner would classify as) "profane" personages, the overwhelming majority of sculptures represent the deities and saintly persons of Buddhism. The exceptions are the icons figuring some male personages, buddhas and bodhisattvas whose attire reveals their Indian origin (ill. 8). Most of that subcontinent being of subtropical or even tropical monsoon climate, the traditional appearance for men is either with the upper body uncovered, like the two attendant bodhisattvas of this triad in their princely attire, or with the monk's tunic draped over the left shoulder only, leaving the right shoulder and the chest bare, as is the case in this image of a buddha in characteristic monk's attire (ill. 9). This peculiarity is therefore a matter of cultural origin, for even though a figure may personify Asanga, a monastic of Indian descent (ill. 10), it shows in reality a monk of Japan, a country where the climatic conditions command a different dress, and so the representation reverts to the attire fully covering the body, as worn by the contemporary local monastic. 
III. 7. Effigy of the legendary Empress Jingū (神功皇后) as kami. 12th c.

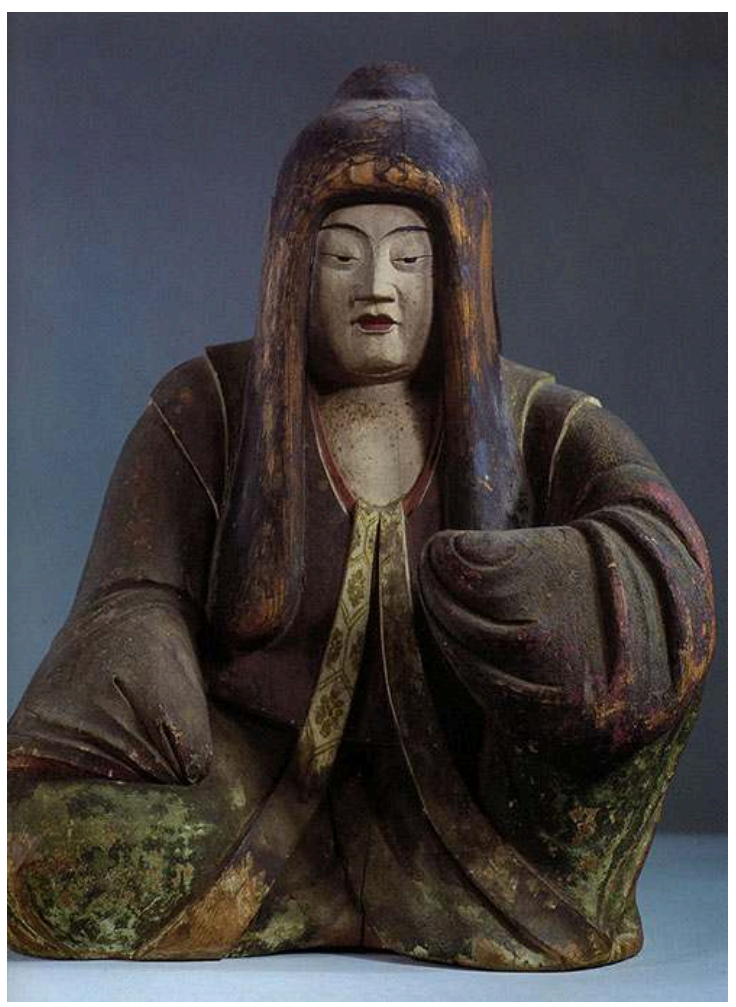

Yakushi-ji, Nara.

III. 8. The Yakushi Triad.
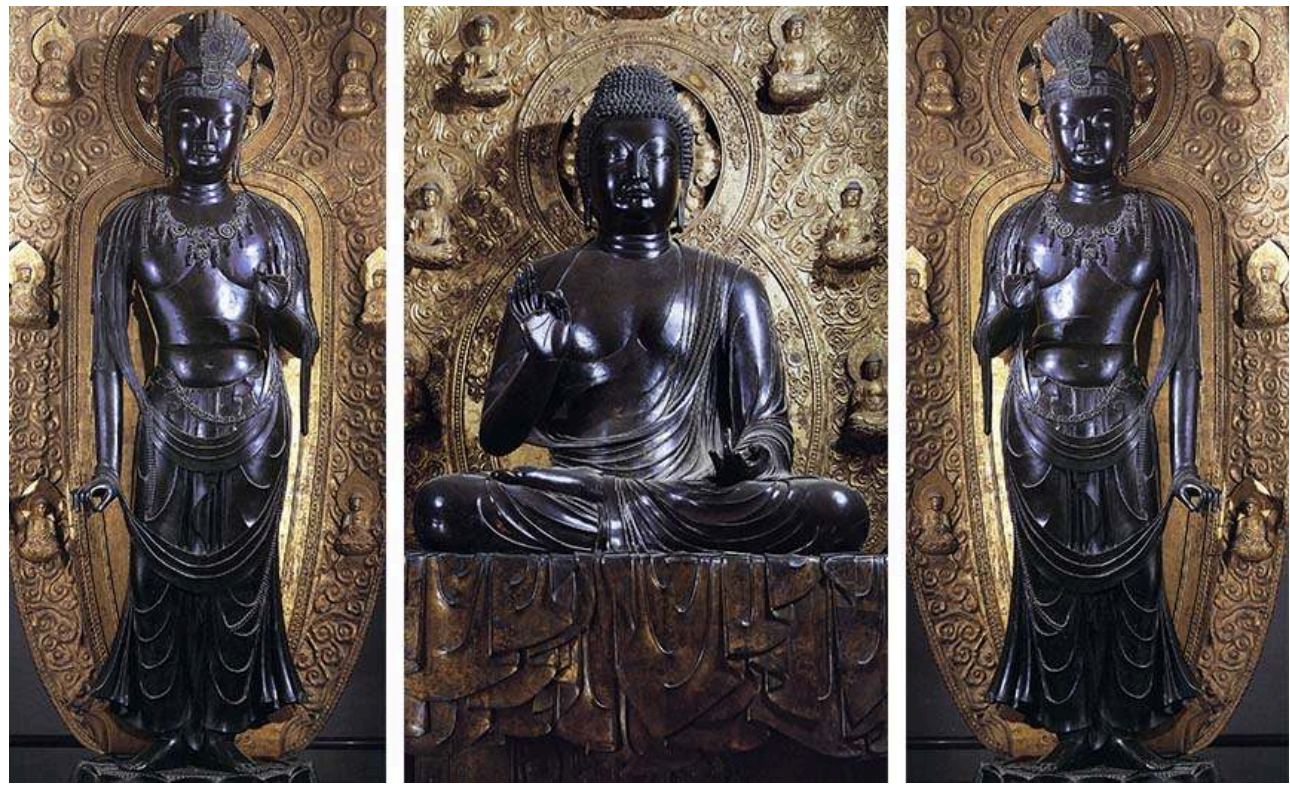

Yakushi-ji, Nara.

13 The buddha-body (ill. 9) reveals no sign of personality and is, on the contrary, entirely symbolic. The depiction of the exposed parts-head, torso, arms, feet-obeys the grammar of canonical iconography. In Buddhist scriptures first translated into Chinese in the 3rd century, the Buddha's body is described as presenting thirty-two primary 
and eighty secondary marks. Among the distinctive signs expressed in this Bhaișajyaguru (jp. 薬師 / Yakushi) figure, are the round protrusion at the top of the head called the "topknot of flesh" (sk. uṣnișa), the eyelids covering the pupils above and below, the elongated ears, the square cheeks, the three folds of the neck, his chest "full like a lion's", his harmonious and soft palms, and his fully rounded heels. The skin is supposed to be of golden colour, and so it originally was, since we know that this icon was gilt during the latter half of the Tenpyō era (729-749).

III. 9. The Buddha Yakushi (薬師如来 Bhaișajyaguru). The main icon of the Yakushi Triad.

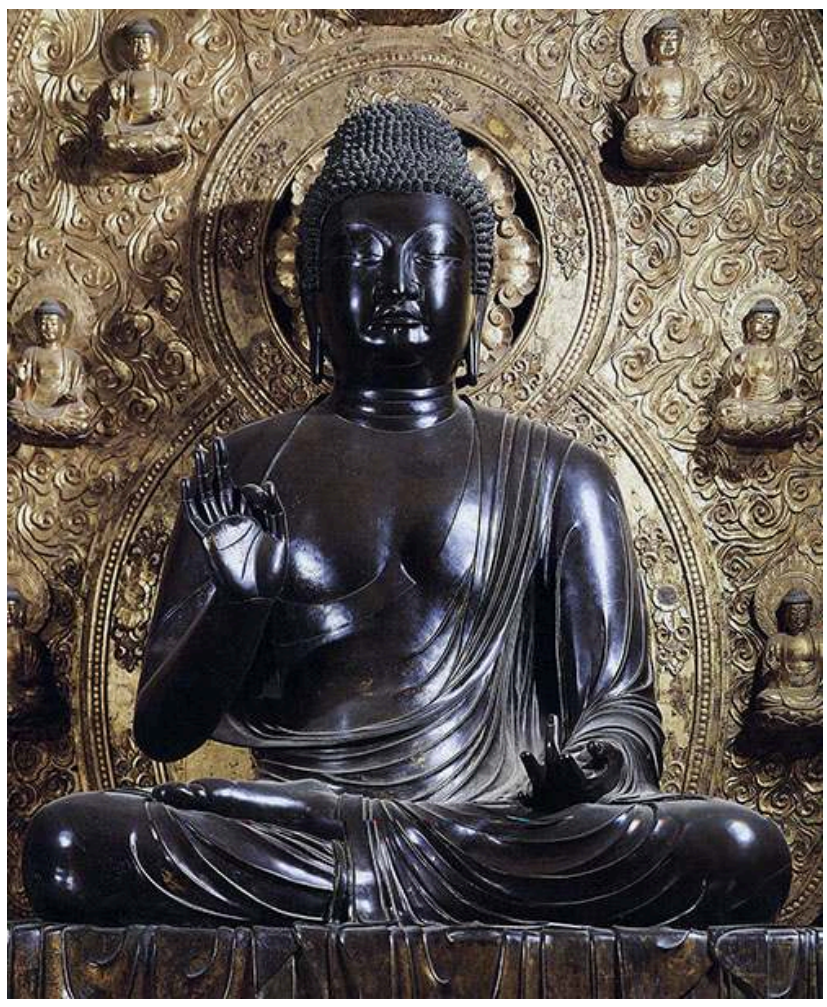

Yakushi-ji, Nara. 
III. 10. The Indian monk Asańga (jp. Mujaku 無着). Wooden sculpture, life-size, by Unkei, 1212.

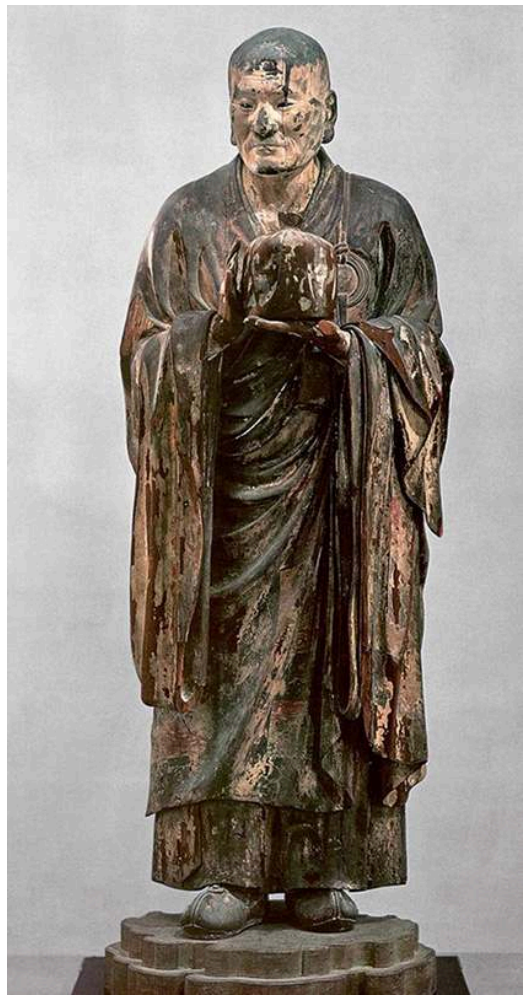

Kōfuku-ji, Nara.

14 Now, to realize the idiosyncrasy of the Japanese manner of representing-not to say perceiving-the body, we need to juxtapose a typical example with a specimen just as representative of another, very different civilization.

Let us choose, for commodity's and brevity's sake, an image from classical Greece: the "Lance Bearer" / gr. Doryphoros (ill. 11). To gauge how archetypal this sculpture is, it is enough to know that it was also called the "Canon", for Polykleitos (5th century BCE) created this image of a young man to set down in absolute (i.e. universal) terms the ideal measure, the standard for the representation of the human body as such. And in practice the Lance Bearer has remained the epitome of the European idea, and ideal, of man, for well over two millennia (ill.12, 13, 14). For reasons of convenience-and irrespective of the fact that some sixteen centuries separate the two-let's take the sculptor Unkei's (1150-1223) statue of the above mentioned Mujaku (Asanga) as a point of comparison on the Japanese side (ill.10). It does not matter that the subjects represented are also very different, for we are concerned with the exterior aspect only. 
III. 11. The "Lance Bearer" (Doryphoros), Roman marble copy of the original bronze statue by Polykleitos, (ca. -440).

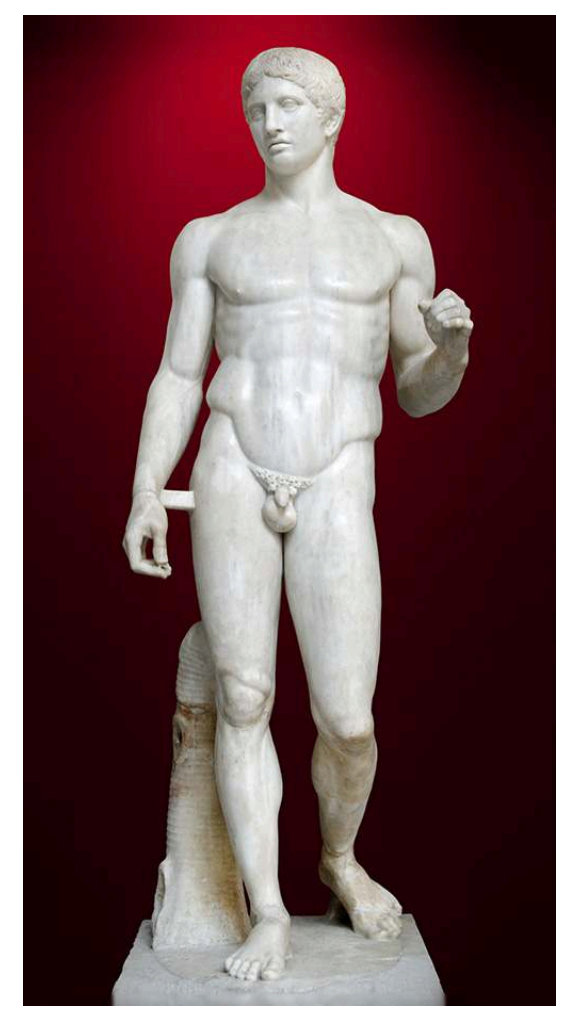

Museo Archeologico Nazionale, Naples.

III. 12. Autoportrait in the nude, Albrecht Dürer, (ca. 1507).

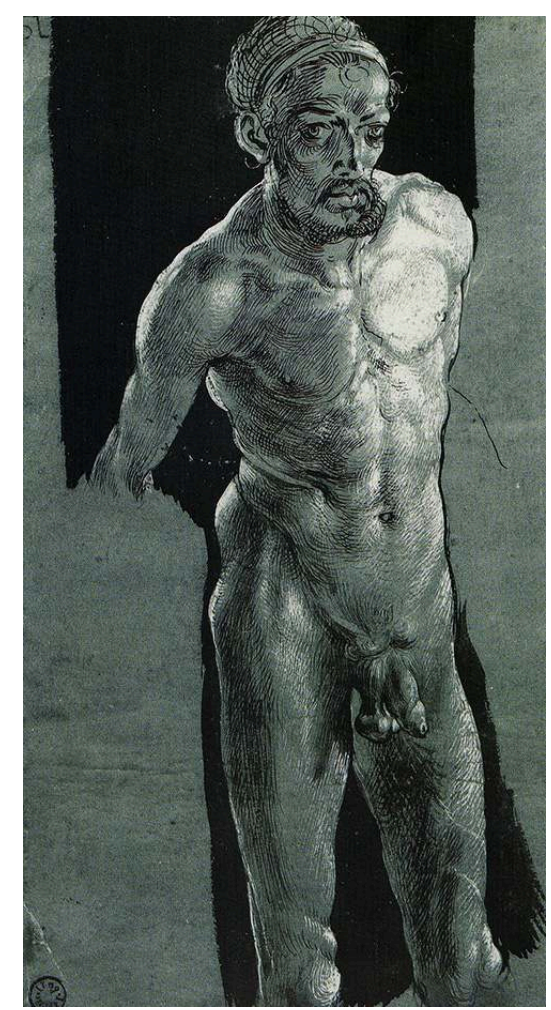

Public domain 
III. 13. Michel-Ange, David (1501-1504).

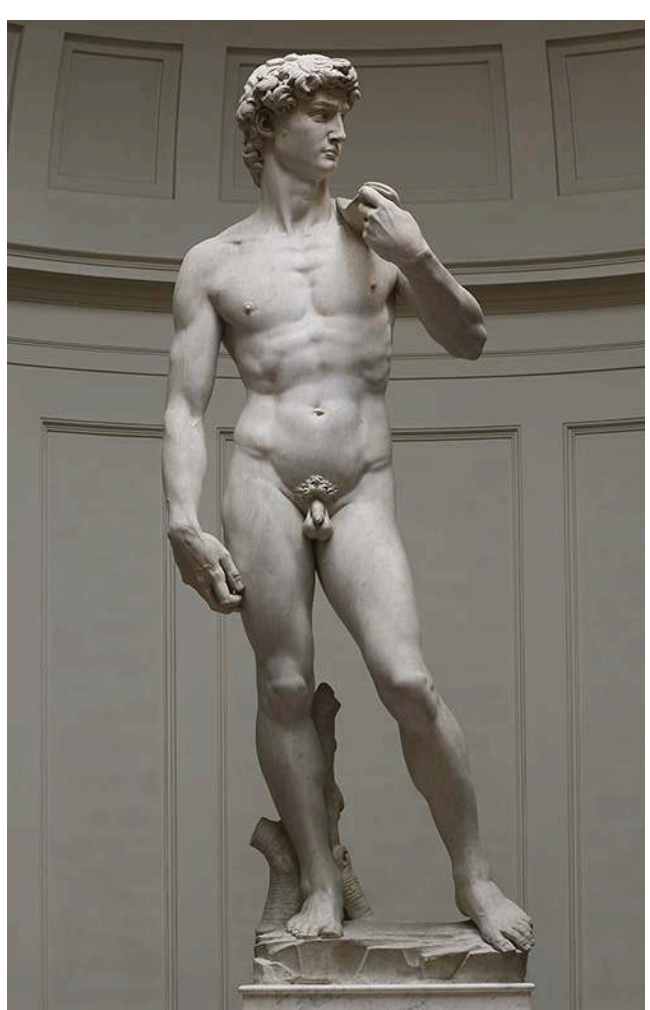

Galleria dell'Accademia, Firenze. 
III. 14. "Die Bereitschaft". Monumental bronze by Arno Breker, (1942). Haus der Deutschen Kunst München.

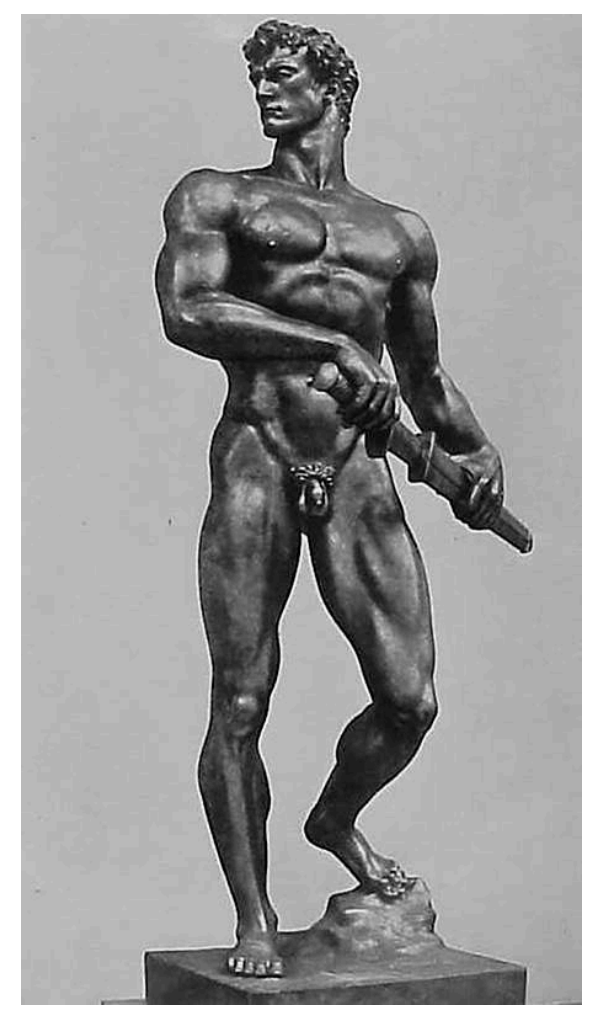

Photo: Charlotte Rohrbach. Archiv Breker Museum, Nörvenich (Germany).

However incongruous the comparison may seem, on the most basic level these two figures exemplify all the essential differences between the Japanese and the European conceptions of the physical body (ill. 15). 
III. 15. Unkei's effigy of Mujaku (1212) side by side with Polykleitos' Doryphoros (ca -440), both equally over life-size (for reasons of perspective).
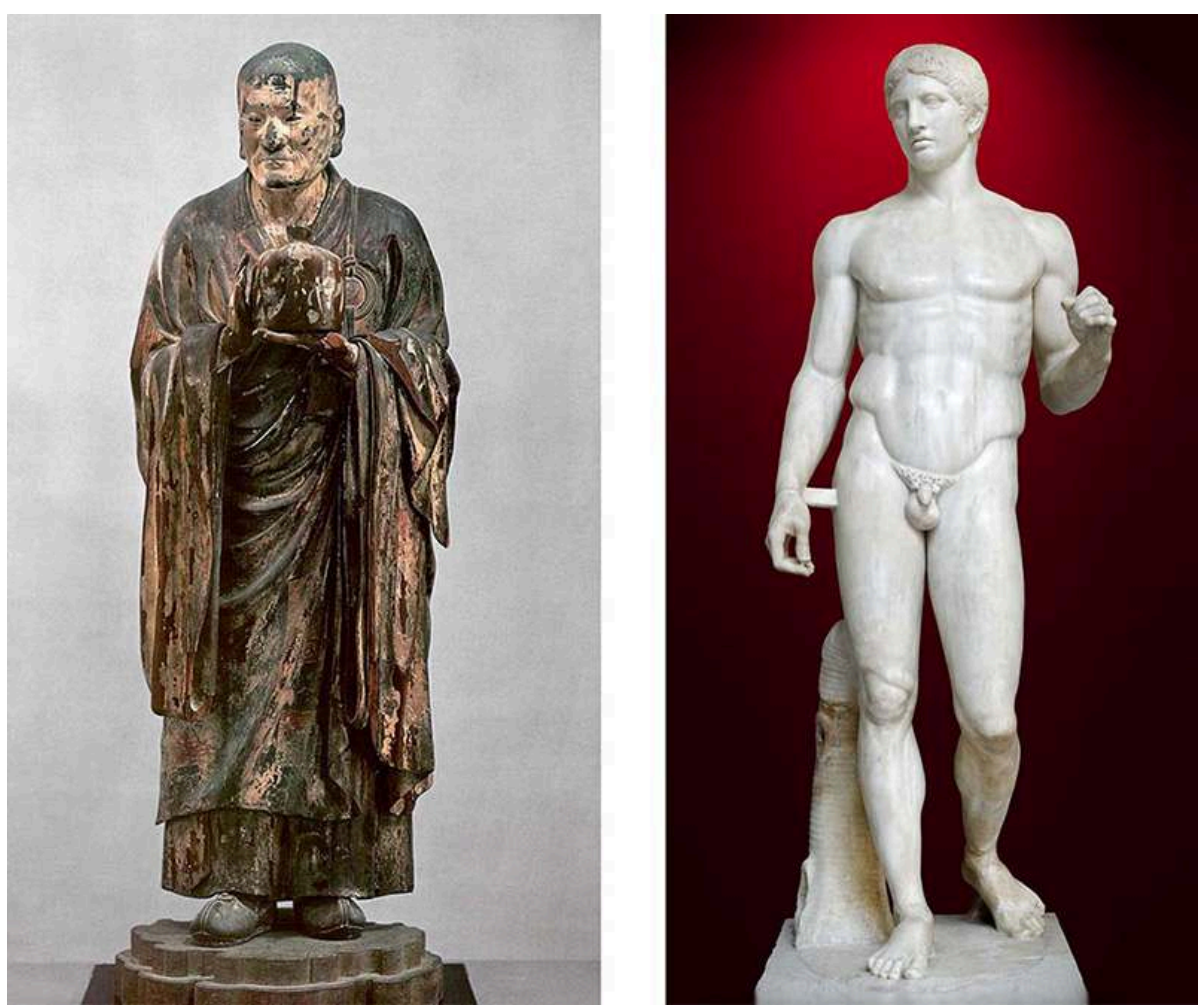

Let us start with the most obvious and superficial aspects. Whereas, in Mujaku's case, most of the body is covered in clothes-in the Japanese monk's garb-, it is, on the contrary, in full display in the Greek image. The Japanese body, with the exception of the face, is unremarkable, insignificant and reduced to being a support for the countenance, while on the other side the body is in reality the "core", the subject of the representation. Here, the focus is on character, appearing in the dignified face of the Indian Buddhist monk and philosopher, there on the impersonal anatomy of a young man.

The first thing that strikes the eye in comparison is the ephebe's nakedness, emphasizing that the point of the portrayal is the body as such, with all the qualities that classical Greek culture assigns to it-as systematized by Polykleitos in his Canon. Here the subject is not a person but an idealized physique, specifically that of an 18 to 22 year-old young man, "the flower of man".

When I say nakedness, I am deliberately not using the word nudity. One of our colleagues in Chinese studies pointed out a few years ago that the nude is impossible in China, an impossibility which historical evidence shows to extend to other Far Eastern cultures like Japan and Korea as well. ${ }^{9}$ If we understand by "nude" the naked human figure seen as an independent object of study and artistic representation (in the case of a drawing, painting or sculpture called an "academy"), then indeed we do not find any nude there until the end of the 19th century ${ }_{10}^{10}$ when the first Japanese artists who had studied the Western manner in European art academies, brought the subject back home.

20 Another patent feature of the Greek effigy is the male's genitals. Polykleitos is said to have fashioned a female counterpart to the Lance Bearer, in the shape of an amazon. As 
in all surviving female nudes of antiquity, the genitals would have been equally treated. Since Japanese representations of the human figure show the person fully clothed, this part of the anatomy is neither expressed nor even suggested.

There is, of course, the all too notorious exception of what is now called 春画/ shunga (ill. 16), pictures in which the sexual organs are on the contrary the centre and subject of illustration, rendered in striking detail. The Westerner may be somewhat surprised to learn that such depictions seem to have coexisted with the Genji monogatari picture scrolls. That is, if we trust the art historians, who ascribe the original version of the Tale of the Brushwood Fence to the same end of the Kamakura period ${ }^{11}$. The feature that makes this genre of ukiyo-e an exception to the rule is the blatant exaggeration in size and naturalism of both the male and female genitals, to the point where the preposterousness of the scene renders it laughable. The comical, jocular character of these images is apparent in the name of warai-e (笑絵), "humorous" or "parodic pictures", as they were traditionally called (shunga being a modern, neutralised term modelled after the Chinese counterpart). The subject of this genre is therefore not the (almost always only partially denuded) body as such, but the scene of erotic play, focused, entertainingly, on the sexual organs in particular.

III. 16. Image from the "Tale of the Brushwood Fence" (Koshibagaki zōshi), end of 12th c. The earliest extant version (Idemitsu Museum, Tokyo) dates from the 13th c.

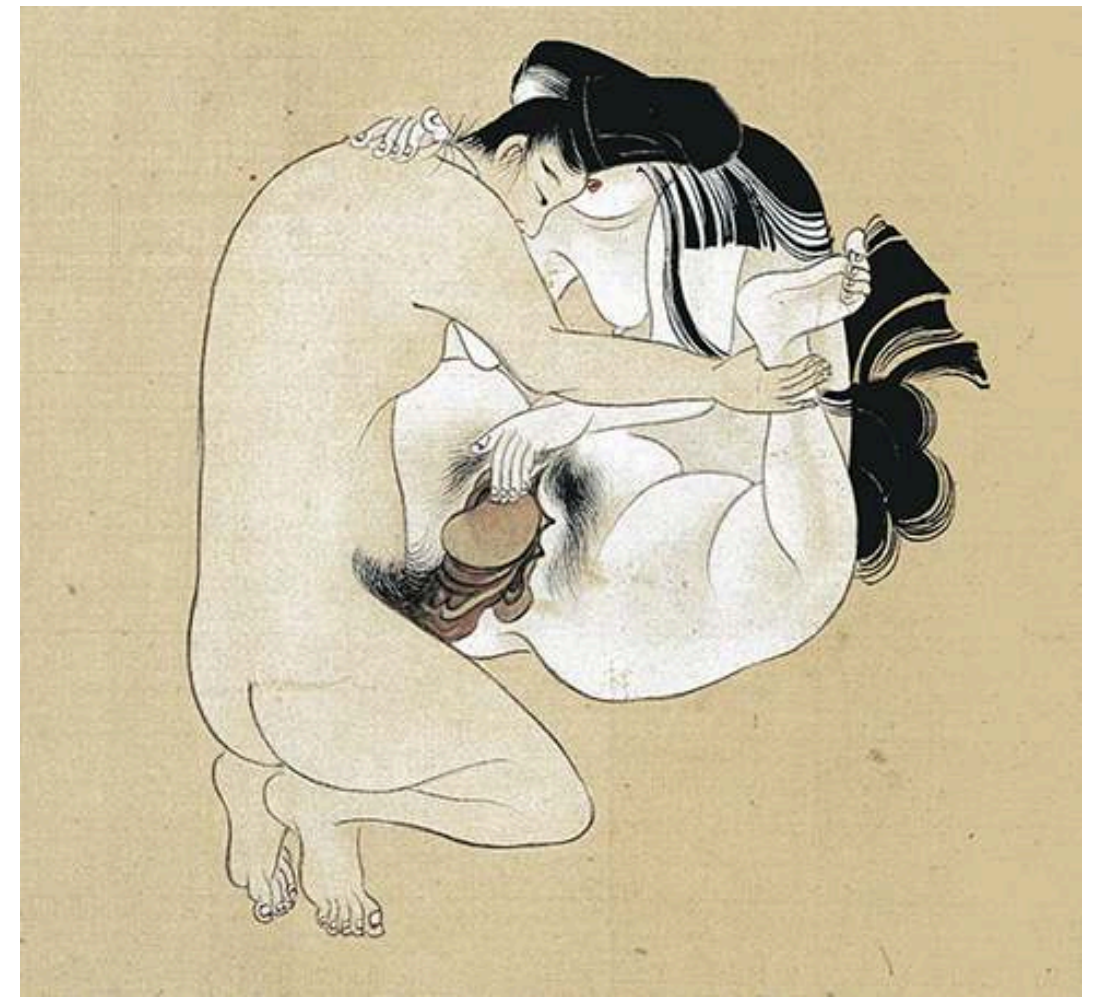

Private collection.

The fact that in Western images the genitals are clearly and realistically figured-a concomitant of the body being observed naked-, whereas in Japanese representations they are generally not manifest, is consistent with the tenet of linguistic relativity that considers this difference as the concrete expression of the elementary male/female gender distinction characteristic of Indo-European languages on the one hand, with, on 
the other, the absence of this grammatical category in Far-Eastern languages. As a matter of fact, there are extremely few pictorial or literary mentions, let alone explicit descriptions, of the male or female sexual organs up to the 19th century, with the exception of the erotic, predominantly illustrated, literature just touched upon. As already mentioned, such verbal or pictorial description of the genitals does not pretend to be factual, but is meant to be amusing, cause for laughter. This clearly is the case of a scene in another late Heian scroll painting (ill. 17), the Stories of Disease and Deformity from the end of the 12th century, where the revelation of a person's hermaphroditic condition is obviously the cause for a prankish laugh.

III. 17. Yamai no sōshi ("Stories of Diseases and Deformities"), scroll of the 12th c. Image of a hermaphrodite.

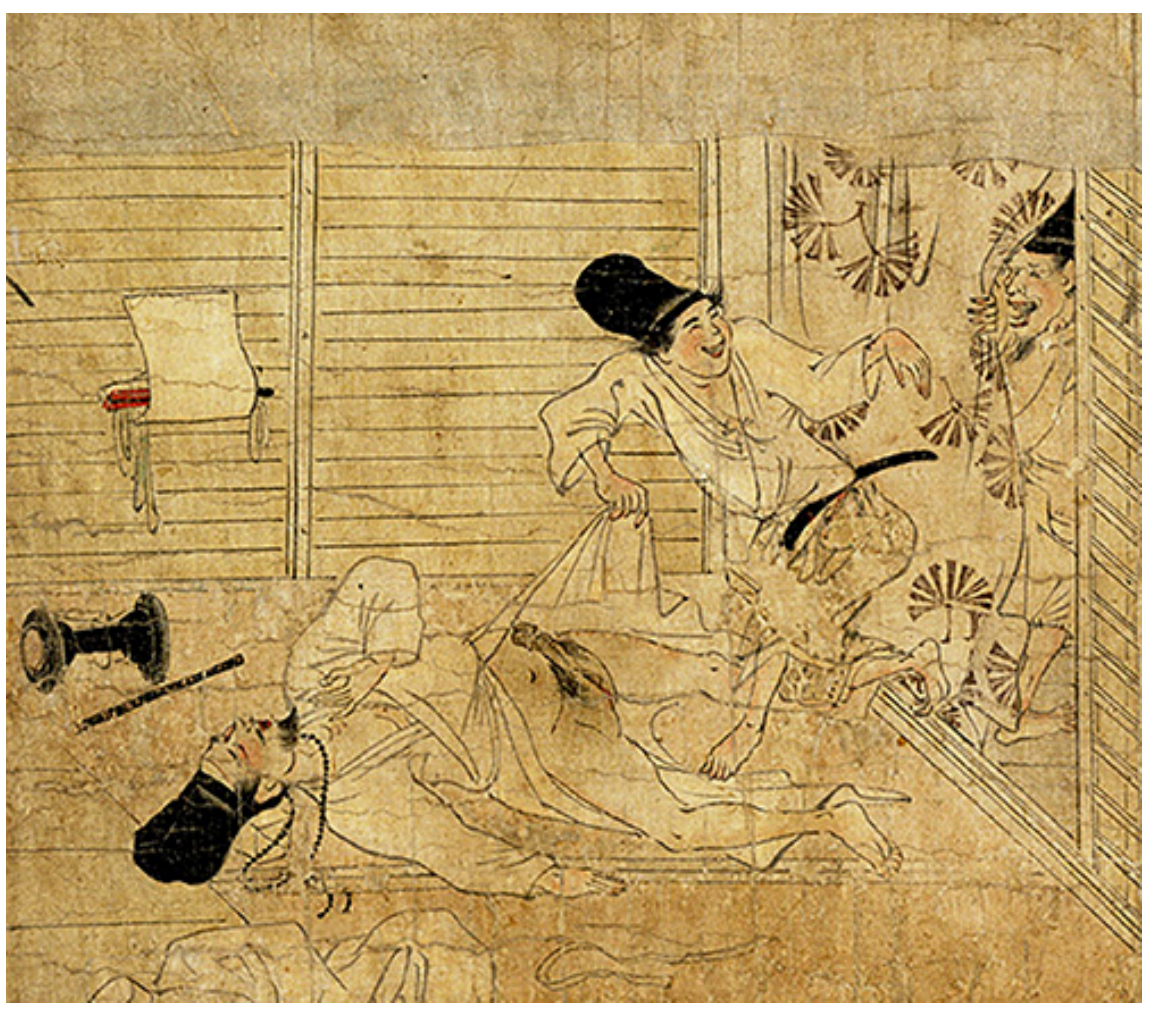

Public domain.

I am far from positing that gender demarcation is entirely nonexistent, but am just trying to demonstrate that it is as indistinct as grammatically inexplicit. We couldn't have a more patent illustration than these two sets of representation of the primordial couple from both extremities of the Eurasian continent: the biblical Adam and Eve (ill. 18) on one side, and Izanami and Izanagi, the primordial pair of the Japanese creation myth, on the other (ill. 19). The nakedness of the Western couple, apart from the concealed genitals, is presented in the Scriptures as a sign of man's original, paradisiacal condition. In contrast, the Japanese pair is dressed in court attire. The reason why the primordial pair, in comparison with its European counterpart, is represented in its ordinary state, clothed, may be that nakedness has here no special significance... is not an issue, in other words. The face being, idiosyncratically, the only distinctive feature of the physique, the Western observer unfamiliar with the Japanese physiognomical and vestimentary codes, will find it not easy to distinguish readily 
between the male and the female person. A difficulty which he is also confronted with when trying to individualize the comparatively indistinct features of the personages in the much earlier Tale of Genji Scroll (ill. 20).

III. 18. Adam and Eve, two panels of the Ghent Altarpiece by Jan van Eyck, 1430-1432. Ghent, Belgium

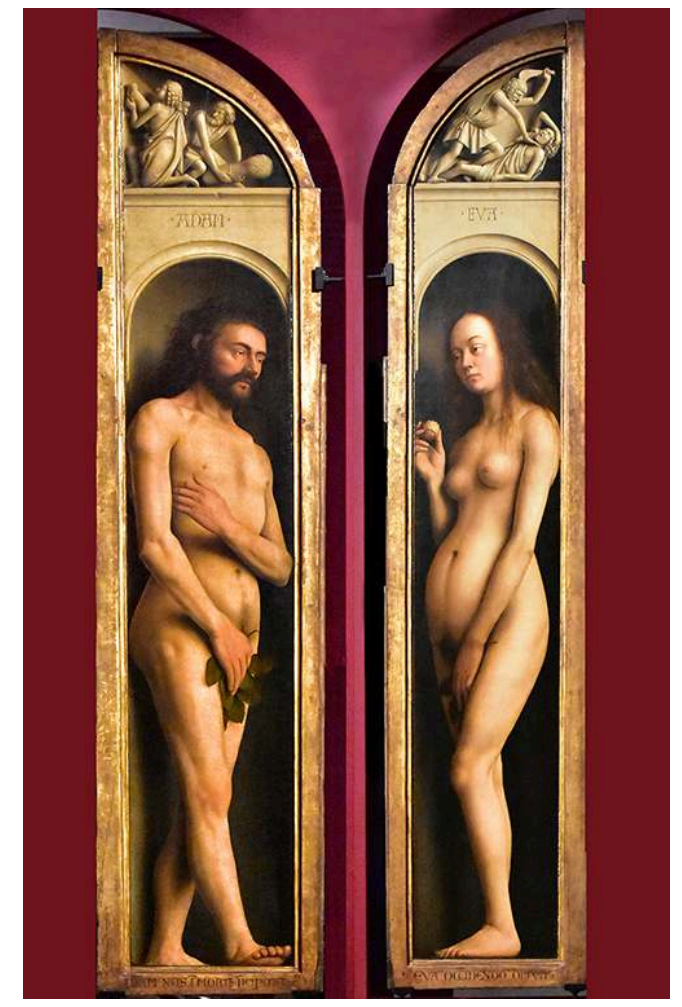

Paul Hermans, CC BY-SA 4.0. 
III. 19. Izanami and Izanagi, the primeval couple learning "how to" from the mating play of two birds. One of the earliest pictorial representations, a woodcut print of the late 18th c.

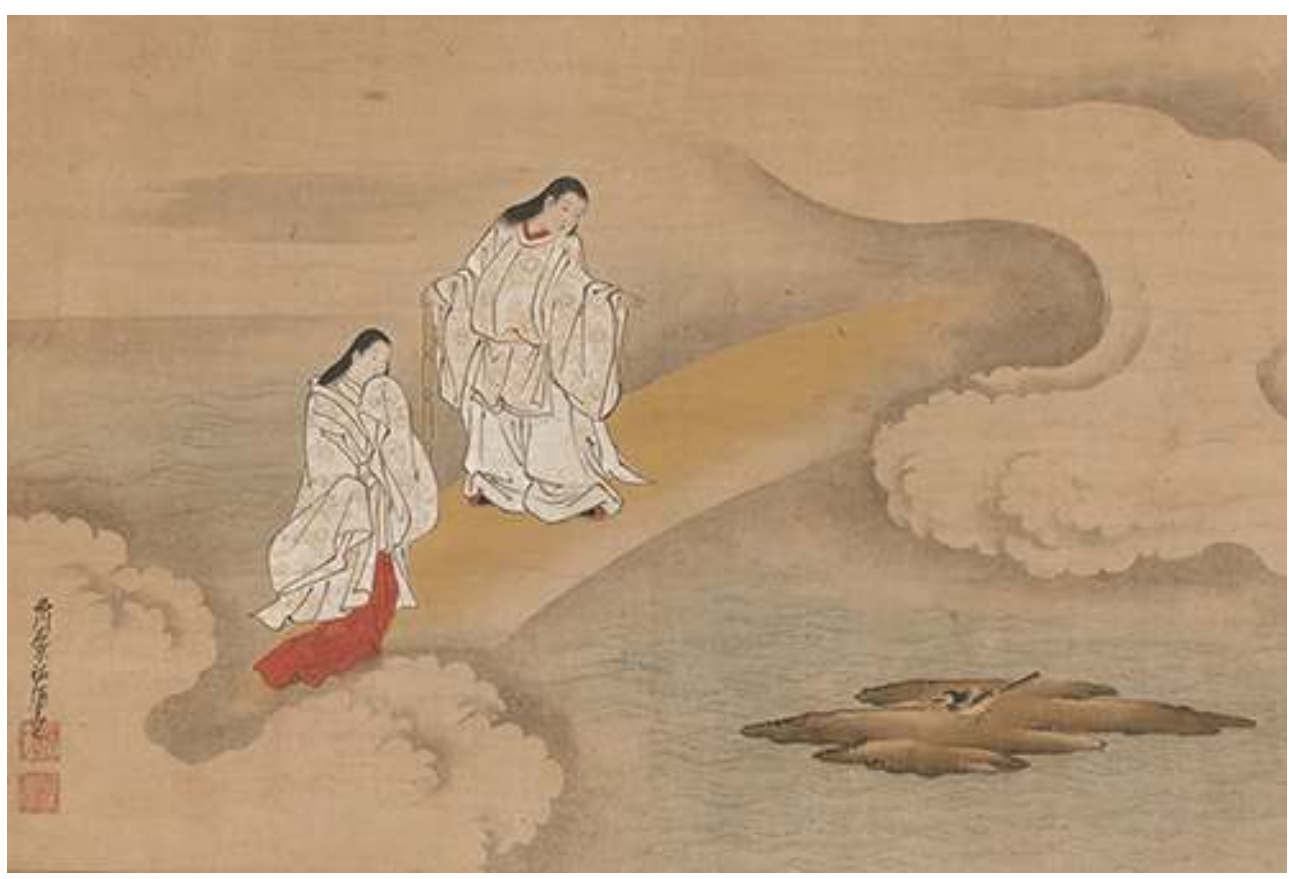

Public domain

III. 20. Yūgiri and his wife Kumoinokari. Scene from the Yūgiri Chapter of the Genji monogatari emaki.

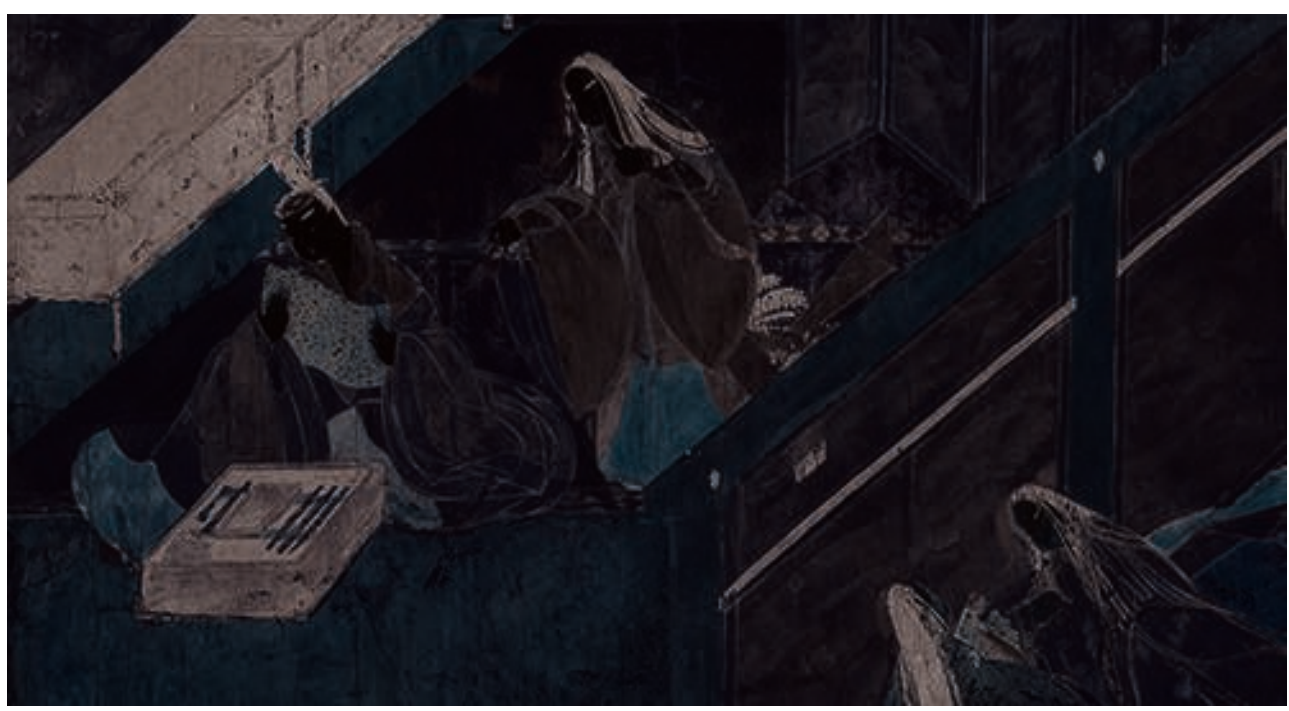

Public domain.

This indistinctness is evident not only in figurative representation, but also in the much less fictionalised medical realm. In the Sino-Japanese worldview, the body is an integrated system in a state of flux, in which vital energy circulates along conduits, feeds and is transmuted in the five organs and six viscera (ill. 21). Apart from the womb, the inner organ of reproduction, the male and the female body are outwardly not significantly differentiated, the genitals and the female breasts not clearly represented. By comparison, Leonardo da Vinci's study of the female anatomy (ill. 22) represents in every respect the exact contrary. 
III. 21. 仰人明堂之圖 (Gyōjin meidō no zu), 側面明堂之圖 (Sokumen meidō no zu), 伏人明堂之圖 (Fukujin meidō no zu), 臓腑明堂之圖 (Zōfu meidō no zu): 'Views of the human body seen from the front, from the back, from the side, and of the viscera'. The four basic schemata of the energy conduits and neuralgic points of the human organism according to Sino-Japanese medicine. Four Japanese diagrams of the mid-Edo period (ca. 1750). Scroll paintings, ink on paper.

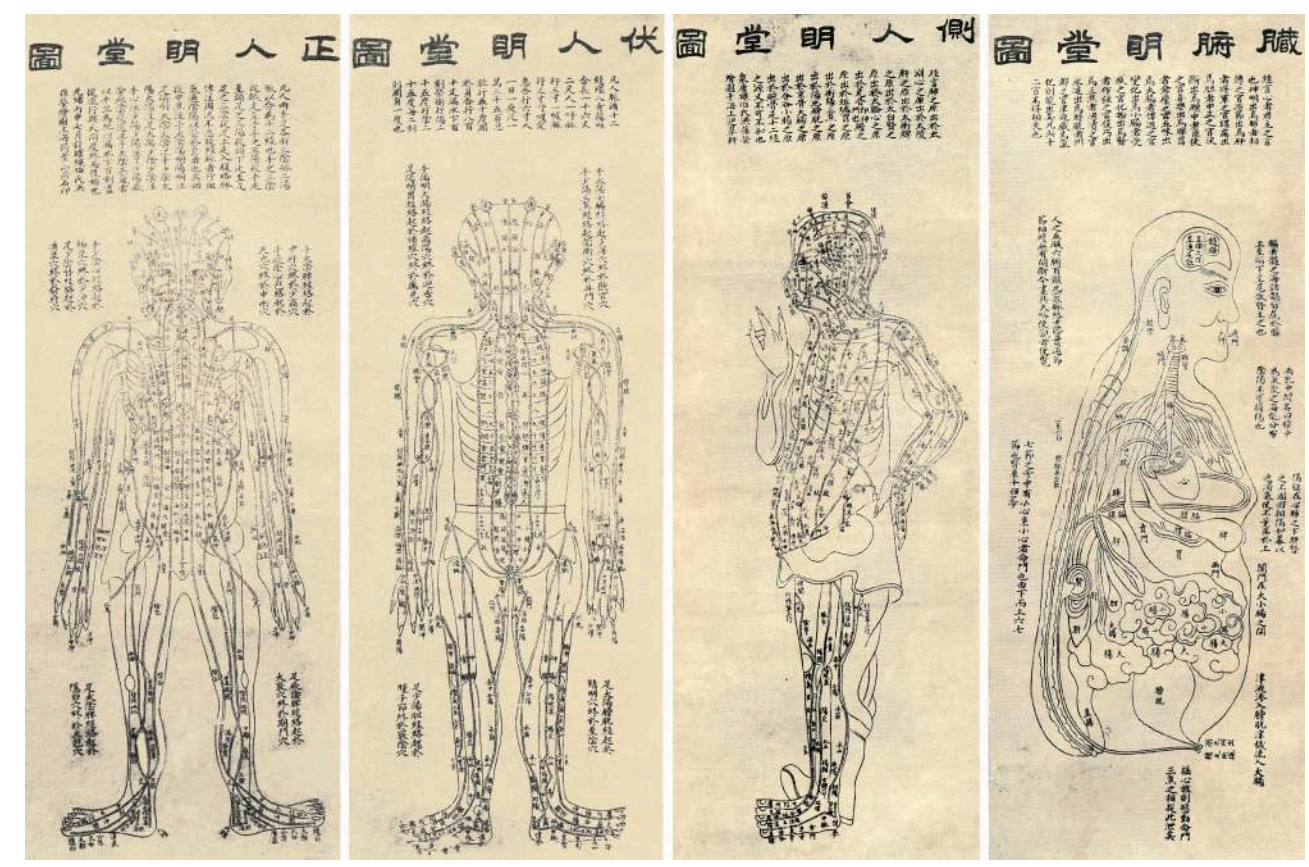

Private collection 
III. 22. Anatomical study of the principal organs and arterial system of the female body. Leonardo da Vinci, ca 1509.

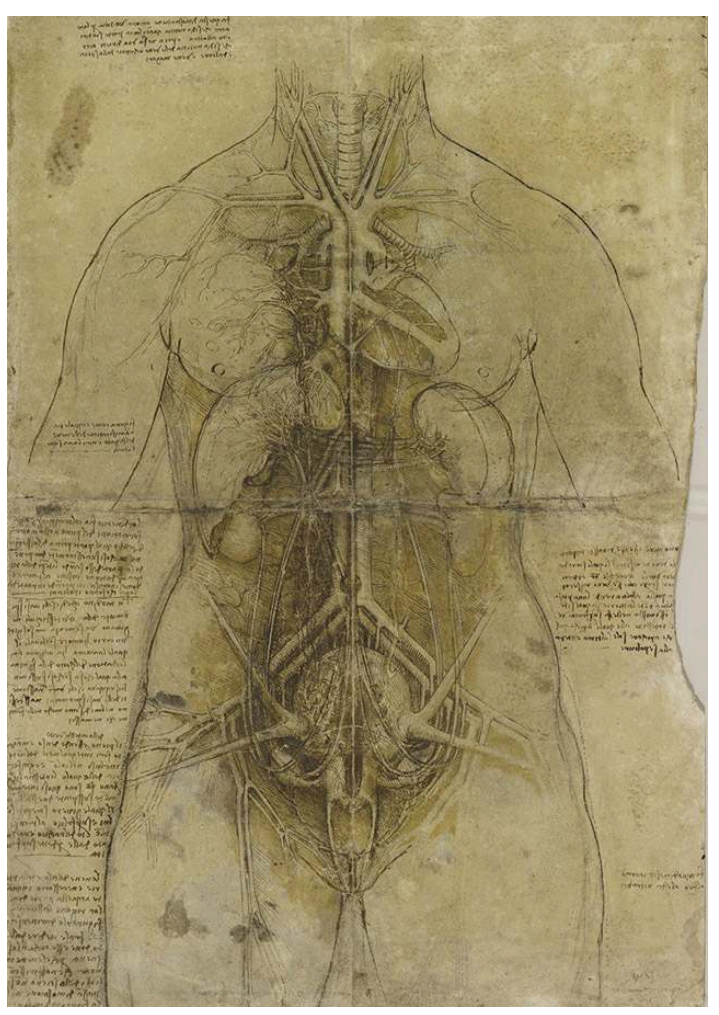

The Royal Collection Trust, London

This brings us to another conspicuous difference in the two conceptions of the body, the anatomical dimension (cf. ill. 11). Of the half-denuded male and female bodies that Japanese imagery occasionally depicts, none shows an anatomically detailed and naturalistic rendition of the muscular relief. By emphasising anatomically correct, we can exclude what appears to be an exception, figures like the two Guardian Kings / 仁 王 / Niō posted at the entrance of some great Buddhist monasteries (ill. 23). Their musculature is anatomically farfetched and exaggerated, intended as it is to make a show of force and-in keeping with their role as guardians of the temple precincts and the devotees-, thereby to scare away malignant spirits. ${ }^{12}$ 
III. 23. Torso of one of the Kongō rikishi, the monumental (over $8 \mathrm{~m}$ ) wooden image made by the Kei workshop in 1203. South Gate of Tōdai-ji, Nara.

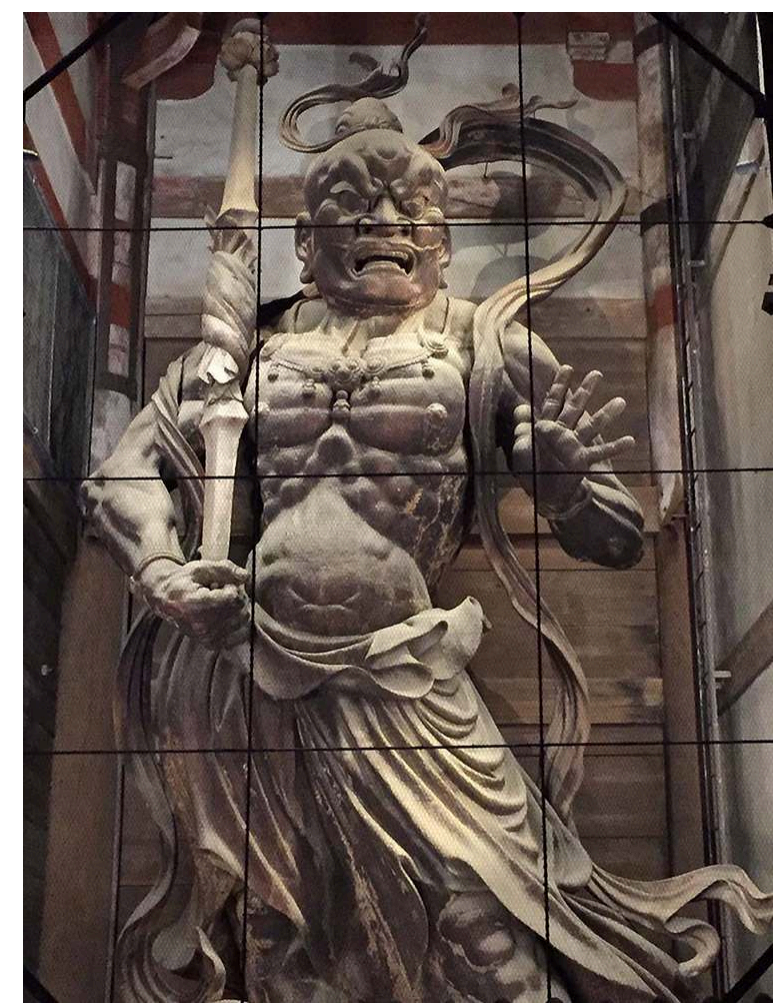

In traditional Sino-Japanese medicine we hardly hear of dissection until the second half of the 18th century, and even after that another century has to pass until the local physicians started to take serious interest in the study of the muscles and the bones. Up to the end of the 19th century, when Japanese artists went to study in European art academies, representations of the body stay flat and essentially two-dimensional (ill. 24). Whereas, if we compare Utamaro's bathing girl with Ingres' contemporary "Grande Baigneuse" (ill. 25), or, even more obviously, if we look at the Lance Bearer, we immediately realize that, as a principle, the Western painter and sculptor are conscious of, and render, the smallest details of the bone and muscle structure underlying the skin. 
III. 24. Girl stepping into the bathtub. Painting on silk, Utamaro, ca. 1800.

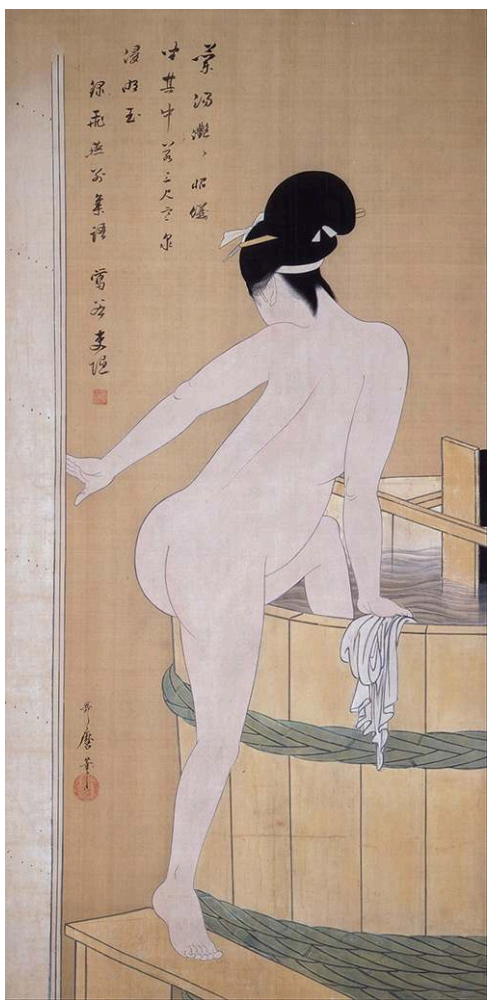

Public domain

III. 25. La « Grande Baigneuse » (1808). Jean-Auguste-Dominique Ingres, oil on canvas.

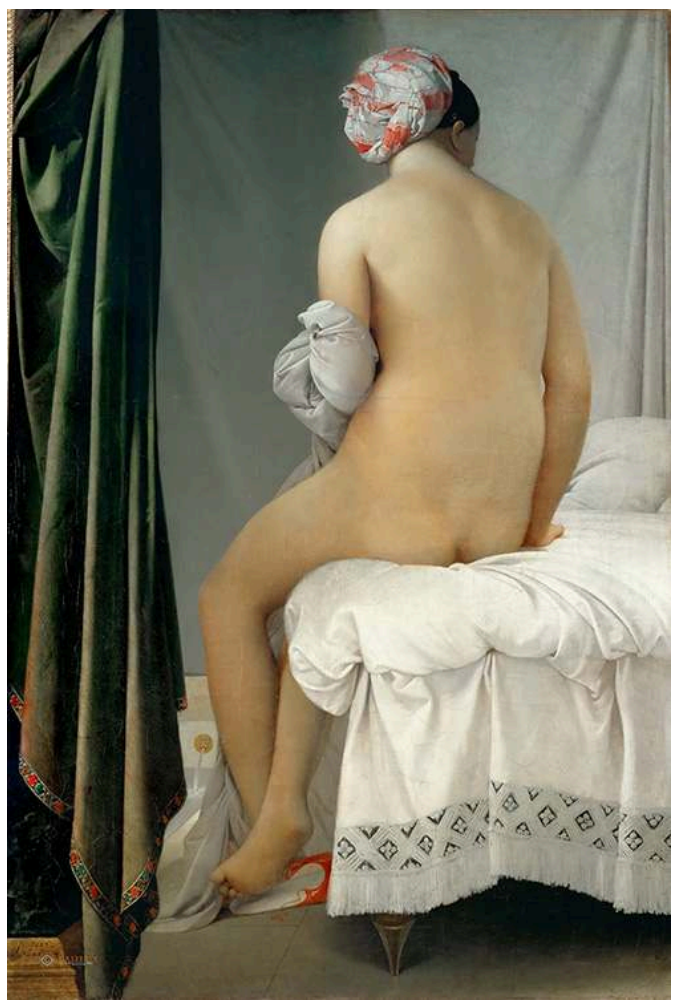

Public domain 
We know that the Lance Bearer represents an athlete, in the flower of his age. His welldeveloped musculature is indicative of intense physical activity, not only in view of the male citizen's fitness for battle, but also for athletic competition-a culture and a spirit that, under the motto mens sana in corpore sano, was pompously revived by the Third Reich for the 11th Olympic Summer Games held in Berlin in 1936 (ill. 26). ${ }^{13}$

III. 26. Javelin thrower. Cut from the second part of the film Olympia - Fest der Schönheit (1937) realised by Leni Riefenstahl for the Olympic Games of 1936 in Berlin.

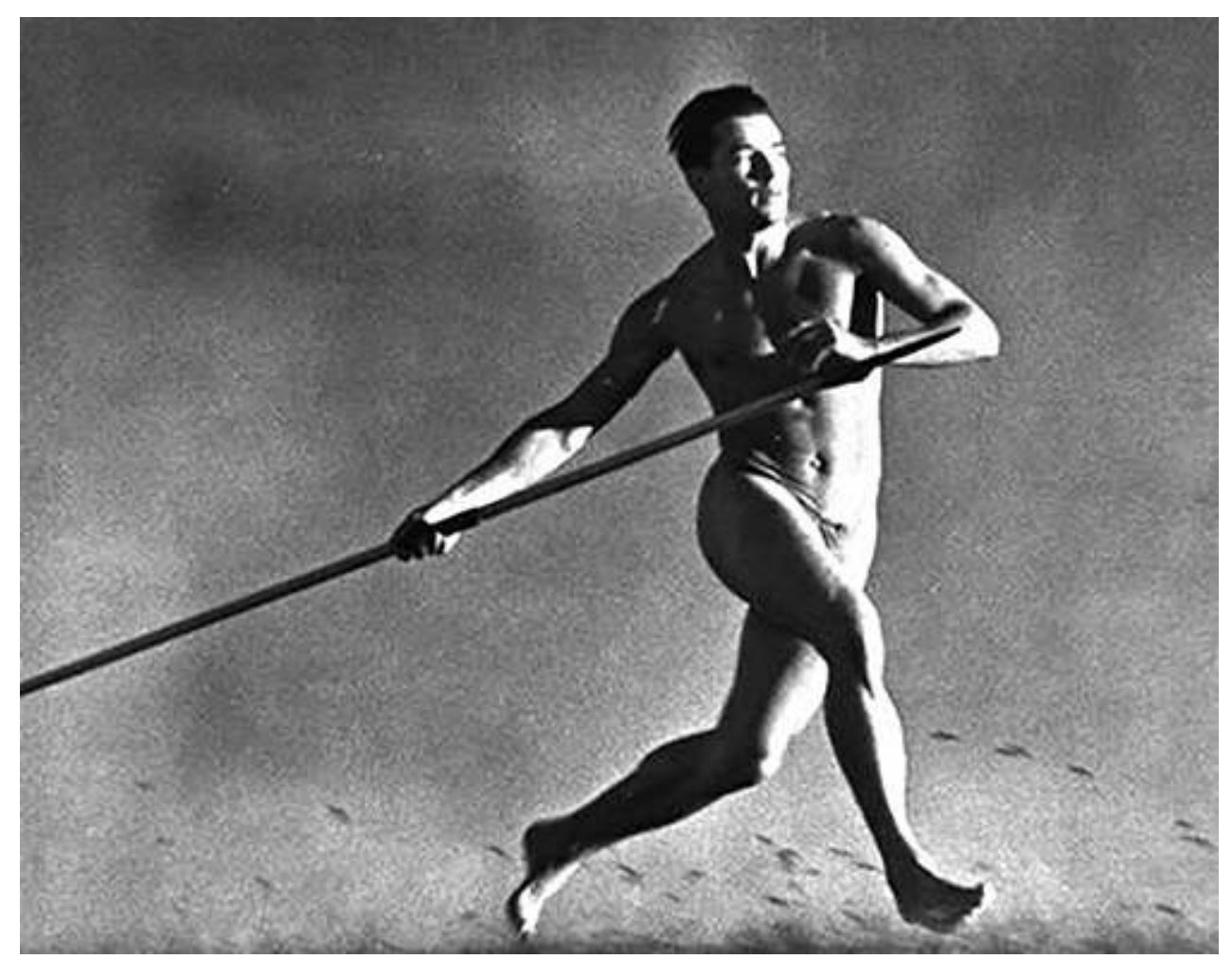

In Western civilization, physical training in order to hone the body for a specific activity (warfare), performance (sports) or other purpose (hygienics, body building, etc.) has been practiced, encouraged and appreciated since Greek antiquity. To all appearances, the Doryphoros' physique is not innate but purposely and methodically shaped, through exercise and callisthenics. There is nothing to this extent in Japan, or in other Far Eastern countries for that matter. Not that there haven't been any physical or even sport-like activities, like the game of kemari / 蹴鞠 (ill. 27), horse-racing or hunting, all widely practiced by courtiers. However, for none of these physical activities-not even the training of soldiers and warriors for fitness and skill in handling weapons-do we hear of any bodily training intent on the development of physical strength. It is not until the Conscription Law was introduced in 1873 that recruits for the imperial army had to undergo a medical check for physical fitness. 
III. 27. Kashiwagi at the ball game kemari. Scene from the Wakana Chapter, as illustrated in the Genji monogatari gajö by Tosa Mitsunobu (ca 1510).

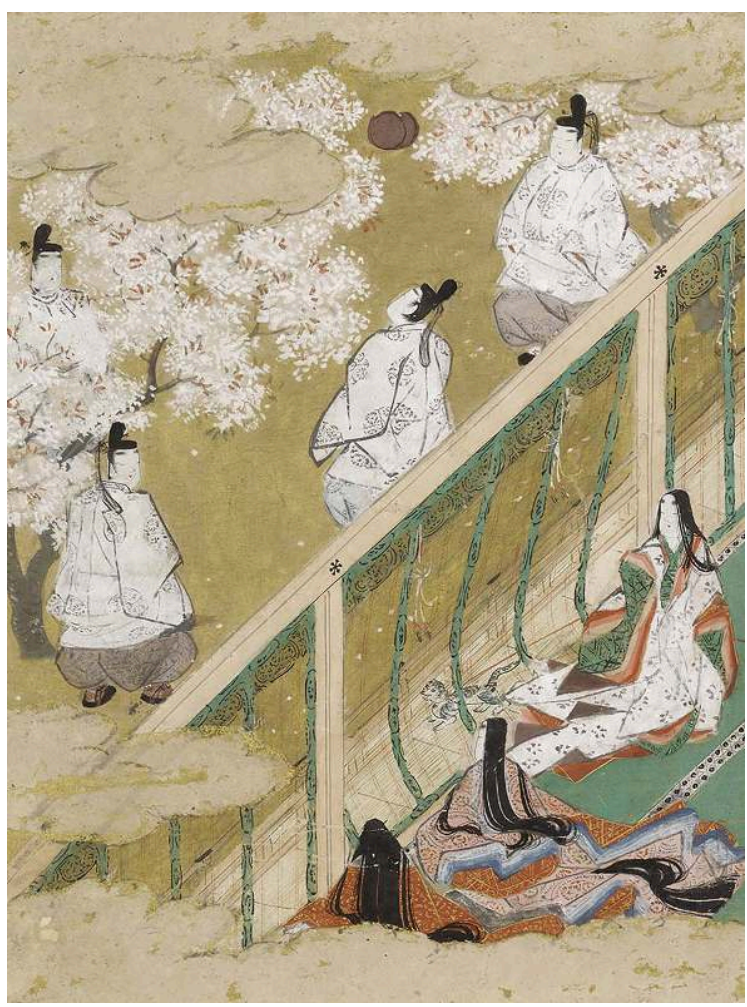

Harvard Art Museums collections online

One of the rare cases (ill. 28) of a Western aesthetics of the body having subsequently found favour in certain circles of modern Japan, is the Shield Society / 楯の会 / Tateno-kai: the militia created and epitomised by Mishima Yukio (1925-1970), an amalgam of physical and mental discipline, body building, martial arts and military spirit. ${ }^{14}$ 
III. 28. Mishima Yukio (1925-1970). Drawing inspired from a photograph by Yatō Tamotsu, featured in his book Young Samurai: Bodybuilders of Japan (Tokyo, Weatherhill, 1966).

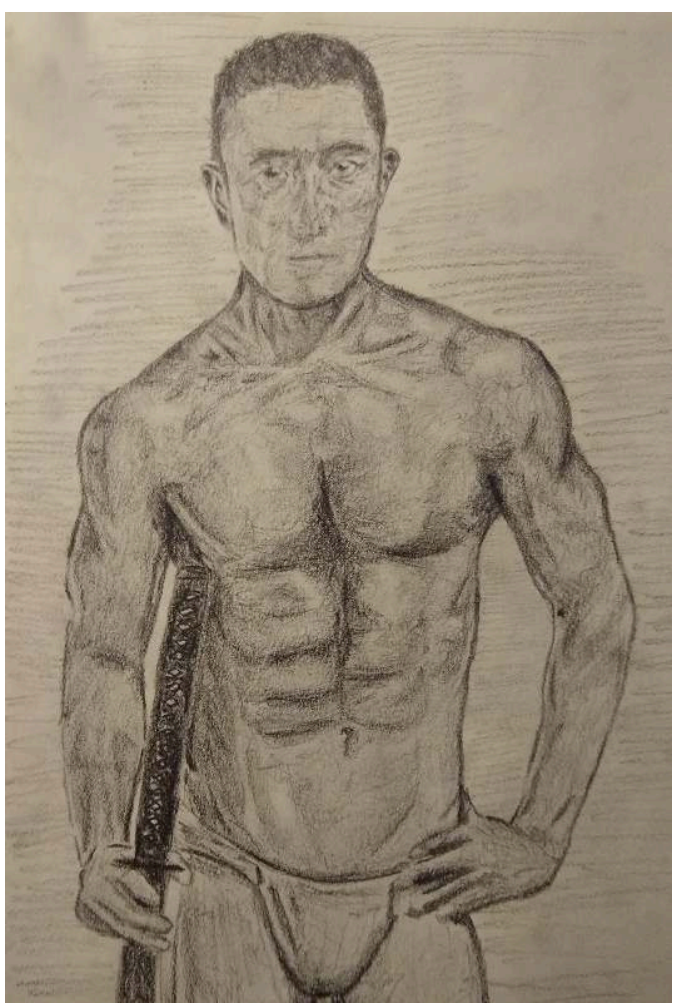

Никто

30 (There is, in this connection, a very promising study to be envisaged on the association, and blend, of one and/or the other, of-primarily male-attitudes towards: body building, callisthenics, eugenics, martial arts, weaponry, warfare, apparel and uniforms, race supremacy, nationalism, not to say fascism...)

31 Finally, we arrive at what may be the most important, nay essential, difference between the traditional Western and the Far Eastern way of perceiving the human body. In comparison with the classical European conception, the Far Eastern view seems to be devoid of one fundamental dimension: the aesthetic appreciation of the physical body as such-apart from immaterial aspects like bearing, posture, demeanour, "perfume" and other ephemeral qualities. ${ }^{15}$ Considering the Lance Bearer again, Polykleitos actually created the Canon-i.e. the statue itsel ${ }^{16}$-as the epitome of ideal beauty, of beauty divine, of perfection. As such, the image had to be consummately realized, not just all around (ill. 29), from any angle of view, but also in all its parts, taken separately as well as in relation to each other. In Far-Eastern media of expression, however, there is no trace, whether in written nor in pictorial form, of the concomitant idea of bodily proportion, let alone of harmonious proportion. The young athlete's body incarnates the laws of contemporary Pythagorean mathematics (ill. 30), the properties of the golden ratio spelling out universal perfection and beauty in the harmonious relation of all the individual parts with each other. 
III. 29. The Doryphoros from all four sides. Plaster cast from the Archäologisches Institut der Universität Göttingen.

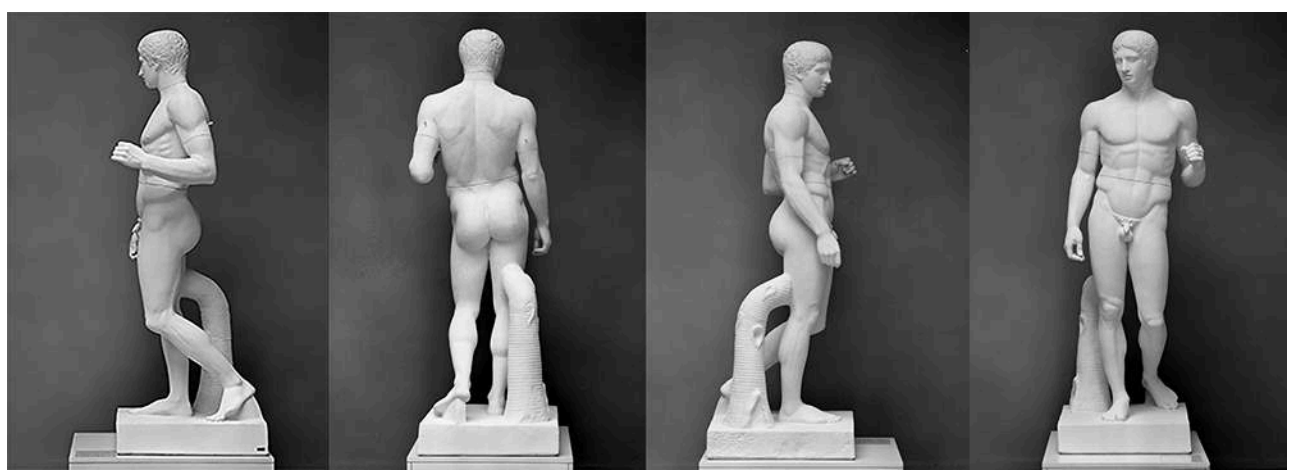

Stephan Eckardt

III. 30. The Doryphoros in its bodily proportions, measured on a plaster cast of the Antikenmuseum Basel.

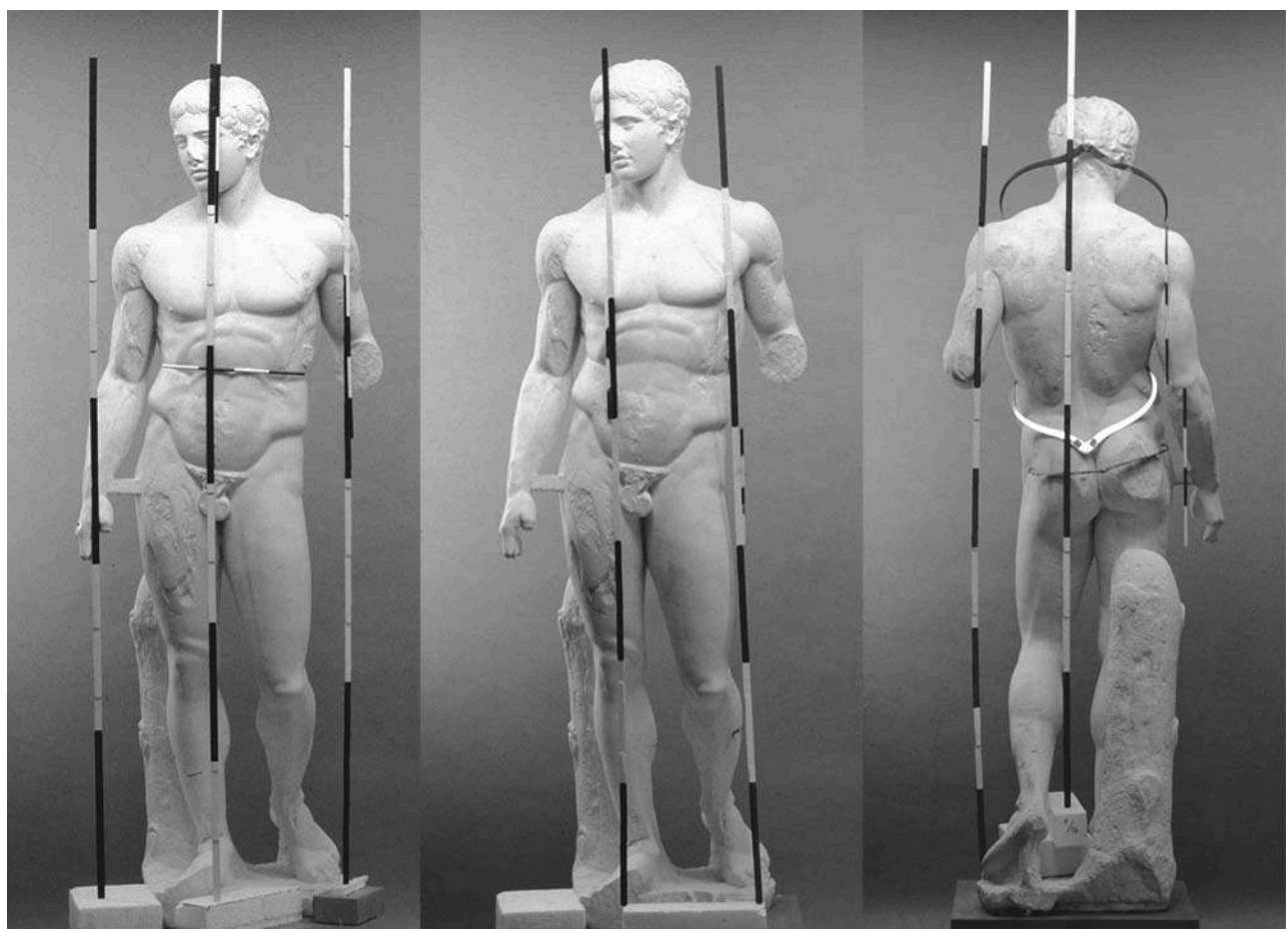

Antikenmuseum Basel und Sammlung Ludwig, Basel

The Canon lays down, for example, that the head is to measure one eighth of the height of the body, one third of the chest, one fourth of the length between the hip and the base of the foot, and so forth. The concept of the harmonious relation between all things, of harmony being expressible in mathematical terms, is a uniquely European paradigm that has endured to this day and has even been extended, with the rediscovery of classical Greek and Roman culture in the Renaissance, into architecture, cosmology, philosophy, religion and, of course, the pictorial and plastic arts (ill. 31). The concept reaches Japan in the last third of the 18th century (ill. 32), through the painting manual of the Flemish artist Gerard de Lairesse (1641-1711), who, in this respect, illustrates his Het groot schilderboeck (1707) with the drawings of Albrecht Dürer's treatise on bodily proportion. ${ }^{17}$ The pictures are taken over without significant 
alteration in Morishima Chūryō's [Redhead Miscellanea] ${ }^{18}$ (1787), the Japanese adaptation of Lairesse's treatise (ill. 33). To see a Japanese body depicted in the Western manner, as a nude, one more century has to pass until the first paintings of the subject appear in the country by the end of the 19th century. From that time up until today even, the view of the body in terms of mathematical harmony of proportion, of such a thing as an ideal or beautiful body, does not seem to have found a substantial application in Far Eastern cultures-not even can one detect any unequivocal reference to this kind of aesthetics.

III. 31. The Canon of Proportions. Drawing of Leonardo da Vinci after the Roman architect Vitruvius Pollio, also called the "Vitruvian Man".

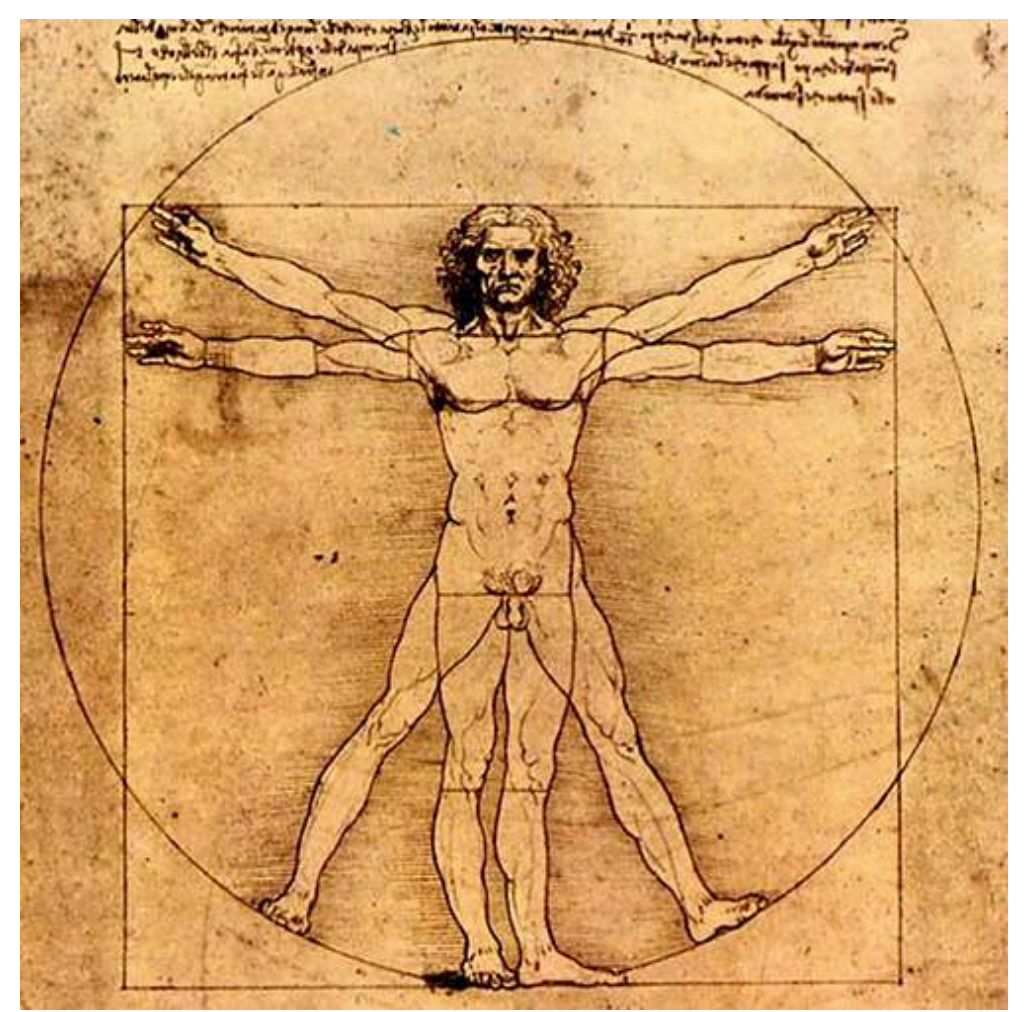

Galleria dell'Accademia, Venice 
III. 32. The proportions of the male and the female body. From Gerard de Lairesse's Le grand livre des peintres, ou l'art de la peinture (1787).

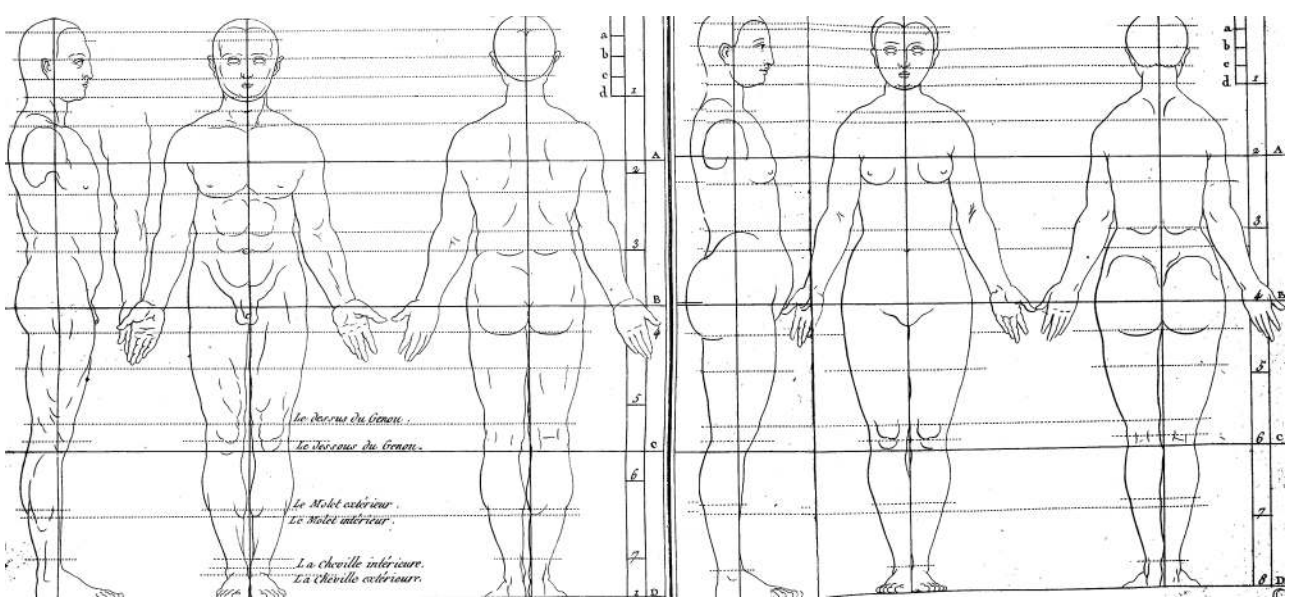

Private collection

III. 33. The Japanese version of the proportions of the male and the female body as it appears in Morishima Chūryō's Kōmō zatsuwa [Redhead Miscellanea], 1787.
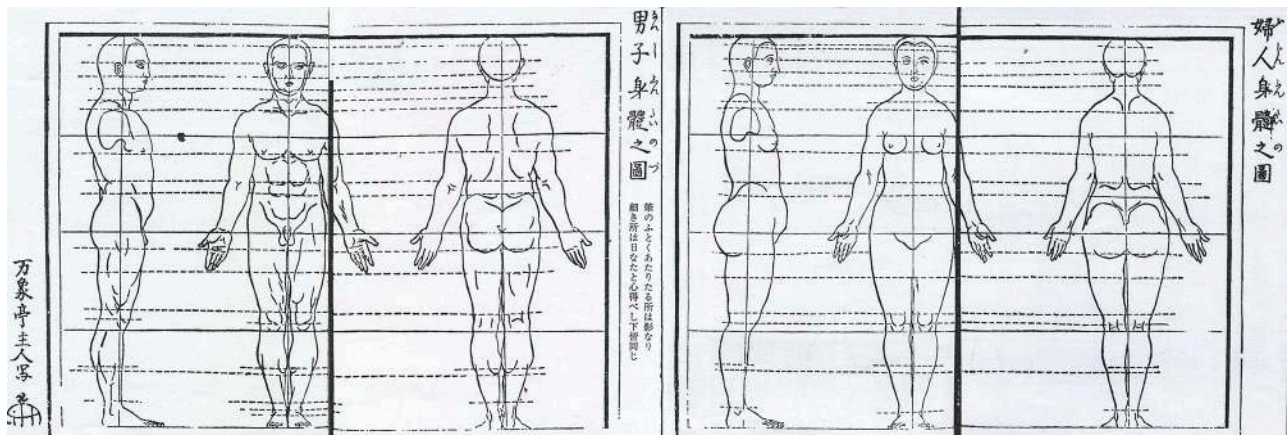

Private collection

To put things simply, in a very condensed form, let me conclude by stating, in abstract terms, that the body, at least the part Tanizaki calls 胴體 / dōtai, the trunk, or torso, seems to have occupied up to the end of the 19th century very minor, if at all, a place in Japanese consciousness.

In other words, the physical body as such has not been an independent object of awareness, attention and reflection, and hence of verbal description or pictorial representation. All evidence indicates that it has existed only as an integral element of the person, not being considered apart, having no objective reality of its own. 


\section{BIBLIOGRAPHY}

BURUMA Ian, “THE SENSUALIST - What makes 'The Tale of Genji' so seductive”, The New Yorker, July $20,2015$.

CARROLL John B. (ed.), Language, Thought, and Reality - Selected Writings of Benjamin Lee Whorf, Cambridge, Mass., The MIT Press, 1956.

HIRLÉ Ronald, Arno Breker - sculpteur, dessinateur, architecte, Strasbourg/Paris, Éditions Hirlé, 2010. JULLIEN François, De l'essence ou du nu, Paris, Seuil, 2000.

LAIRESSE Gerard de, [Great Book of Painting] / Het groot schilderboeck, Amsterdam, Henri Desbordes, 1707, 2nd printing (in Dutch), 1712.

MARUYAMA Ōkyo 円山応学, [The True Manner of Depicting the Human Body] / 人物正写惣本 / Jinbutsu seisha sōhon, 3 scrolls, Tenri, Tenri Library, 1770.

MISHIMA Yukio, Sun \& Steel, BESTER John (trans.), Tokyo, Kodansha International, 1970.

MORISHIMA Chūryō 森島中良, [Redhead Miscellanea] / 紅毛雑話 / Kōmō zatsuwa, Edo, 1787.

RIEFENSTAHL Leni, Schönheit im Olympischen Kampf, Berlin, Deutscher Verlag, 1937.

RIEFENSTAHL Leni, Olympia 2. Teil - Fest der Schönheit, Allemagne, Olympia-Film, 1938.

SURÉN Hans, Mensch und Sonne - Arisch-olympischer Geist, Berlin, Scherl, 1936.

TANIZAKI Jun'ichirō 谷崎純一郎, In Praise of Shadows / In'ei raisan, HARPER Thomas and SEIDENSTICKER Edward (trans.), Sedgwick, Leete's Island Books, (1933-1934) 1977.

WINTHER Fritz H., Körperbildung als Kunst und Pflicht, München, Delphin Verlag, 1919.

YATŌ Tamotsu, Young Samurai: Bodybuilders of Japan, Tokyo, Weatherhill, 1966.

\section{NOTES}

1. What is more strictly called the principle of linguistic relativity, also known as Sapir-Whorf hypothesis, has been elaborated, from the 1930s on, by Benjamin Lee Whorf (1897-1941), inspired by the linguist and anthropologist Edward Sapir (1884-1939). The elemental claim is that a speaker's worldview pervades his perception and cognition, impregnating his native culture's notions and language; a people's language and other modes of expression are thus relative to its worldview-and vice versa. See CARRoll John B. (ed.), Language, Thought, and Reality - Selected Writings of Benjamin Lee Whorf, Cambridge, Mass., The MIT Press, 1956.

2. TANIZAKI Jun'ichirō 谷崎潤一郎, [In Praise of Shadows]/ 陰毉禮灒, HARPER Thomas and SEIDENSTICKER Edward (trans.), Sedgwick, Leete's Island Books, 1977, with other editions since. The quotation is from the section "The woman of old", p. 28-30 passim.

3. Ibid.

4. Ibid.

5. BURUMA Ian, “THE SENSUALIST - What makes 'The Tale of Genji' so seductive”, The New Yorker, July 20, 2015.

6. 石山寺縁起絵巻 / Ishiyama-dera engi emaki.

7. 一遍上人絵伝 / Ippen shōnin eden. 
8. What owes Hanazono this characterized portrait, is not his imperial body, but his clerical person.

9. JULLIEN François, De l'essence ou du nu, Paris, Seuil, 2000.

10. With the sole exception of Maruyama Ōkyo's (1733-1795) little-known anatomical studies of the human figure, as he represents it in "true to nature"-style in his three picture scrolls entitled [The True Manner of Depicting the Human Body] / 人物正写惣本 (1770), treasured by the Tenri Library.

11. An early version of the 小柴坦草子 / Koshibagaki zōshi is rather wishfully attributed to Fujiwara no Tame'ie (1198-1275) for the text, and to Fujiwara no Nobuzane (1176-1265) for the illustration. The oldest extant copy, in the Idemitsu Museum, is said to date from the 13th century. The narration is based on the scandal that shook the court towards the end of the 10th century, recounted in the Jikkinshō (1252), of the affair between a vestal princess and a soldier of the guard.

12. In our particular context it is not insignificant that the Herculean build of these guardian kings qualified as "strongmen" / 力士 / rikishi, is supposed to derive from the Greco-Buddhist image of the "Vajra Bearer" (Vajrapāni), a type itself hearkening back to the "club-bearing" Herakles figure as attested in 2nd century Gandhāra.

13. The Germanic cult of the body has its perfect illustration in Leni Riefenstahl's still and motion pictures officially commissioned for the 1936 Berlin Olympiad, published in book form as Schönheit im Olympischen Kampf (1937) and as a film entitled Olympia - Fest der Schönheit (1938). Not to mention the sculptor Arno Breker (1900-1991) epitomizing, both in spirit and in form, the Greco-Roman Renaissance of the Third Reich (see ill. 14).

14. Mishima's obsessive preoccupation with his body would indeed have been a most fitting topic for this symposium. The dust jacket cover of Mishima's Sun \& Steel (Kodansha Intern., 1970) with a photograph by Shinoyama Kishin, is the very epitome of this spirit. The just mentioned photographs by Leni Riefenstahl as well as Arno Breker's effigies (ill. 26 and ill. 14 respectively), and, more generally, the spirit reigning in Germany in the first half of the 20th century regarding the physique as resumed in the Third Reich's conception and staging of the 1936 Olympics, are too close in spirit to Mishima's attitude towards the body for not to have served as an inspiration for a number of his phantasies. In regard to the former, see WINTHER Fritz, Körperbildung als Kunst und Pflicht, München, Delphin Verlag, 1919; or the classic suRÉN Hans, Mensch und Sonne - Arischolympischer Geist, Berlin, Scherl, 1936.

15. With the exception of the woman's hair, by far the body's most valued, and often mentioned physical attribute.

16. Vitruvius does mention the existence of a written treatise, but the actual Canon is the effigy itself, in its three-dimensional materiality.

17. The latter ultimately going back to Polykleitos' Canon as revived by the Roman architect Vitruvius of the 1st century BCE.

18. 森島中良 (1756-1810), 縕毛雑話 / Kōmō zatsuwa. 


\section{AUTHOR}

\section{JOSEF KYBURZ}

Josef Kyburz is a senior research fellow of the French Centre national de la recherche scientifique (CNRS), now retired. His research and concomitant publications have been based on fieldwork in both Japan and Korea, as an ethnologist, focussing on popular creeds, beliefs, customs and objects of "religious" nature. His most recent work on Japanese paper charms (ofuda) has appeared in ofuda - Images gravées des temples du Japon (2011) and Ofuda - Amulettes et talismans du Japon (2014). In parallel, he has turned his reflection, from the point of view of cultural relativism, on the modes of perception of such basic elements as, here, the body, in a comparative East-West perspective. 


\title{
I Sing the Body Electric: Corporal Representations in Guo Moruo's The Goddesses
}

\author{
Paolo Magagnin
}

\section{Introduction}

1 Among the Chinese intellectuals who came to the fore in the aftermath of the May Fourth Movement of 1919, Guo Moruo 郭沫若 (1892-1978) left a distinctive mark. At the turn of the 1910s he started to gain recognition as a poet and translator, later devoting himself to short fiction, historical plays, and essay writing; after the establishment of the People's Republic, in 1949, he was known notably as a scholar of Chinese antiquity and a statesman. He first obtained real recognition as a poet in 1921, when his maiden verse collection The Goddesses / 女神 was published in Shanghai. ${ }^{1}$ It contains a poetic prologue and 56 poems - four being actually long verse plays - organized in three sections, mostly composed while he was a medical student in Japan. Because of its formal and rhetorical innovation and the power of its individual expression, the collection was quickly acknowledged as the beginning of modern Chinese poetry, establishing its author as one of the most representative poetic voices in the country.

2 The themes and forms found in The Goddesses are very diverse, making it an extremely complex and composite collection. Many of the poems celebrate nature, physicality, the communion between man and the cosmos, and are permeated with an allegorical idea of rejuvenation. In this aesthetic universe, the discourse of the body naturally occupies a prominent position. The origins of Guo's modes of physical representation can be traced back to the many-sided literary, philosophical, and scientific sources that the poet absorbed and subsequently developed in his own writing, ranging from the foreign knowledge and literary models to which he was exposed while in Japan to the classical Chinese tradition in which he had been schooled as a child. 


\section{Levels of corporal representation in The Goddesses}

3 The modern, scientific understanding of the anatomical body entered China in the second half of the 19th century. ${ }^{2}$ The body of Western medicine - first imported in China as a tool for national self-strengthening and modernization - was quickly adopted by Chinese intellectuals not only as the ground upon which to speculate about the sources of consciousness, but also as a physical and metaphorical object for exploring political, economic, and national issues, a category that allowed them to express concerns related to identity, history, sexuality, and spirituality. "Body," "blood," "weakness," "disease," etc., together with concepts drawn from psychoanalysis, rapidly became commonplace metaphors for many writers starting in the late 1910s. After Lu Xun 魯迅 (1881-1936), the most representative examples of this phenomenon are arguably Yu Dafu 猜垟夫 (1896-1945) and Guo Moruo himself, who like Lu Xun - had a medical background which frequently resurfaces in his writing.

Remarks on Guo Moruo's corporal discourse, often associated with psychoanalytical concerns, remain generally scattered and unsystematic in the scholarship on his literary production. A specific focus on his fiction characterizes Tsu's (2000) analysis of the significance of masochistic suffering as a metaphor for dysfunctional national identity. ${ }^{3}$ Wei's (2009) exploration of the identity crisis of the desiring body, ${ }^{4}$ Fujita's (2015) discussion of the relationship between medicine and literary writing, ${ }^{5}$ and Vuilleumier's (2015) investigation of the problematic notion of the medical body ${ }^{6}$ are noteworthy contributions to the analysis of Guo's fiction from this perspective. As far as his poetic production is concerned, several studies address the allegory of death and rebirth, as well as the communion between man and nature as one facet of the poet's pantheistic views in The Goddesses. ${ }^{7}$ Unsurprisingly, more in-depth investigations into the representation of the body and its significance generally focus on the poem "The Heavenly Hound" / 天狗, where this expressive mode is central. Fujita (2003) treats "The Heavenly Hound" in connection with Guo's 1920 poem "In the Dissecting Room" / 解剖室中; the idea of the "progressive body" and the low-to-high, inside-to-outside corporal dynamics are the object of Mi Jiayan's detailed analysis $(2004,2016),{ }^{9}$ while the implications of physical transfiguration have been explored by Vuilleumier (2009). ${ }^{10}$ Finally, Jiang (2011) explores the relationship between illness discourse and the formation of the image of the poet in Guo's early career. ${ }^{11}$

5 Nevertheless, to my knowledge a systematic account of the levels of body discourse in Guo Moruo's poetry and its significance has yet to be conducted. Building upon the existing analyses, I will here explore the forms of representation of the body in Guo's early poetic writing by conducting a close reading of The Goddesses. Based on this discussion of textual examples, I shall then offer a reflection on the position and significance of corporal poetics against the background of the author's conceptual landscape.

\section{Death and rebirth}

6 The theme of death - and, in general, physical annihilation - and of subsequent rebirth is ubiquitous in The Goddesses, especially in its first and second sections. Its most systematic conceptualization can be found in “The Nirvana of the Phoenixes" / 鳳凰涅 槃, the verse play that opens the second section, in which the legendary Middle Eastern 
bird that rises from its ashes blends with the feng and huang, the male and female phoenix of Chinese mythology. Following the "Prelude" / 序曲, a depiction of a gloomy world where the death of the phoenixes is imminent, the two birds are consumed by fire; the play then culminates in the "Song of Rebirth of the Phoenixes" / 鳳凰更生歌, where the reborn feng and huang proclaim their resurrection and the coming of a new world dominated by fire - another incarnation of the image of the sun, which is ubiquitous in the collection.

Death is similarly presented in a positive light in the final stanza of "Victorious Death" / 勝利的死, dedicated to the IRA fighter Terence MacSwiney, and is romantically praised as the only gateway to "true liberation" / 真正的解脱 in “Death" / 死 (Guo Moruo quanji, op. cit., p. 58). Undeniably, Guo was fascinated by death and decay in that they "represented a phase of the manifestation of nature's mystery and wonder and one aspect of man's creative power". ${ }^{12}$ Indeed, death is welcomed and even worshipped, together with life and other forms of bodily energy, in "I Am a Worshipper of Idols" / 我是個偶像崇拜者:

I worship life, I worship death, I worship the light, I worship the night

[...]

I worship the creative spirit, I worship strength, I worship blood, I worship the

heart ${ }^{13}$

我崇拜生，崇拜死，崇拜光明，崇拜黑夜

$[\ldots]$

我崇拜創造的精神, 崇拜力, 崇拜血, 崇拜心臟 (Guo Moruo quanji, op. cit., p. 99)

The image of fire is often associated with death and rebirth, as in the paradigmatic case of the scorched phoenixes, but can also serve other expressive purposes. In "Coal in the Stove" / 摅中煤, Guo addresses his own nation, compared to a young bride. His yearning for a new world - a new China - takes the form of a burning heart and body:

For my beloved one

I have burnt to this point!

[...]

In this black slave's chest of mine

You want my heart to be like fire.

我為我心愛的人兒

然到了這般模樣!

$[\ldots]$

要我言黑奴的胸中,

才有火一樣的心藏。(Guo Moruo quanji, op. cit., p. 58)

9 Similar metaphorical descriptions, with comparable connotations, can be found elsewhere as well. Let us consider the poem “Crematorium” / 火葬場:

The skull on my sickly neck

Resembles the chamber of a crematorium;

Alas, you burnt my soul long ago!

oh, fresh breeze, where are you coming from?

In this crematorium

You made a stem of... spring grass grow

我這墭頸子上的頭㲊

好象那火葬場裡的火爈;

我的靈魂呀, 早已被你燒死了!

哦, 你是哪兒來的涼風?

你在這火葬場中

也吹出了一株一春草。(Guo Moruo quanji, op. cit., p. 139) 
10 The decaying skull of the subject is compared to a crematorium, by resorting to a series of images that are reminiscent of Expressionist tropes. However, in the poetic universe of The Goddesses, death always brings about a new beginning. In this poem, indeed, new life suddenly appears as a little stem of grass vaguely reminiscent of Walt Whitman's Leaves of Grass (1855), a major influence on Guo's early poetry. ${ }^{14}$

11 The only example of death being presented in an entirely different light is "Impressions of Shanghai" / 上海印象, in which the poet expresses all of his disillusion with the frantic life of the metropolis. There is certainly no room for regeneration for the zombie-like men and women of the city, who are compared to “loitering corpses" / 遊 閑的屍, drifting in a city filled with skeletons and caskets (Guo Moruo quanji, op. cit., p. 162).

\section{Oneness with the cosmos}

12 Another set of expressive devices strongly centered on the body is used extensively by Guo Moruo to emphasize the communion between man and the natural and cosmic world. In The Goddesses, these devices can take different forms, namely: 1) the representation of the subject's body as being or becoming one with the natural world; 2) the depiction of the human body as a platform making communion with nature possible; and 3) the anthropomorphization of nature and the zoomorphization of inanimate beings.

13 "The Heavenly Hound" is arguably the most representative and most often discussed example of bodily poetry in Guo Moruo. It is also emblematic of the category of figurative devices listed above:

I am the Heavenly Hound!

I swallow the moon,

I swallow the sun,

I swallow all the stars,

I swallow the entire universe, I am I!

I am the light of the moon,

I am the light of the sun,

I am the light of all the stars,

I am the light of X-ray beams,

I am the total energy of the entire universe!

I race,

I shout wildly

I burn

I burn like blazing fire,

I shout wildly like the ocean,

I race like electricity,

I race,

I race,

I race,

I peel my skin,

I eat my flesh,

I suck my blood,

I gnaw my guts,

I race on my nerves, 


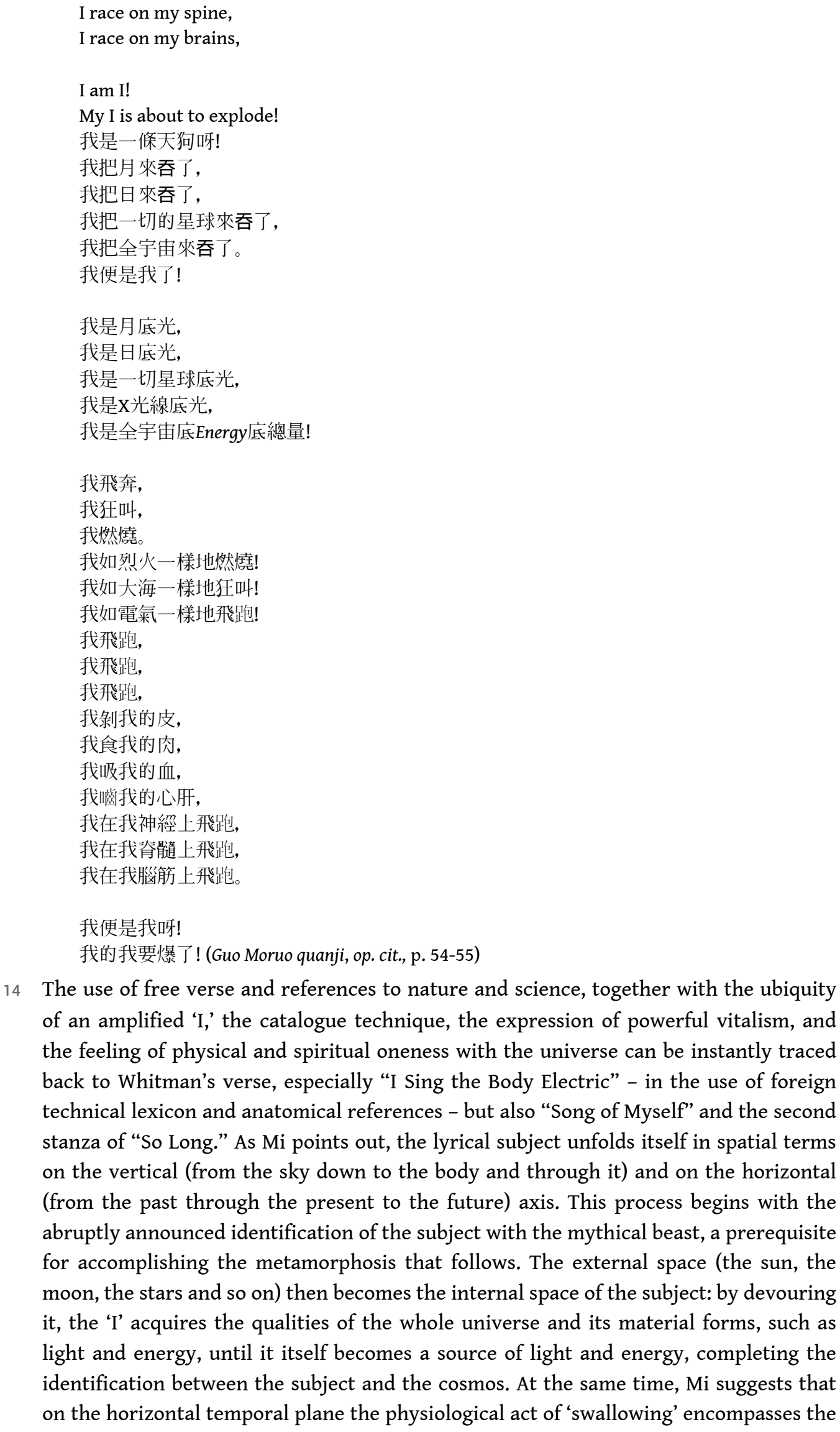

14 The use of free verse and references to nature and science, together with the ubiquity of an amplified 'I,' the catalogue technique, the expression of powerful vitalism, and the feeling of physical and spiritual oneness with the universe can be instantly traced back to Whitman's verse, especially "I Sing the Body Electric" - in the use of foreign technical lexicon and anatomical references - but also "Song of Myself" and the second stanza of "So Long." As Mi points out, the lyrical subject unfolds itself in spatial terms on the vertical (from the sky down to the body and through it) and on the horizontal (from the past through the present to the future) axis. This process begins with the abruptly announced identification of the subject with the mythical beast, a prerequisite for accomplishing the metamorphosis that follows. The external space (the sun, the moon, the stars and so on) then becomes the internal space of the subject: by devouring it, the 'I' acquires the qualities of the whole universe and its material forms, such as light and energy, until it itself becomes a source of light and energy, completing the identification between the subject and the cosmos. At the same time, Mi suggests that on the horizontal temporal plane the physiological act of 'swallowing' encompasses the 
overturning, digesting and replacing of old categories that lead to the creation of a new reality. ${ }^{15}$

As illustrated above, in "Coal in the Stove" the image of the burning heart is intimately linked to the idea of a bodily consumption eventually leading to rebirth. A similar metaphor, rhetorically reinforced by parallelism and repetition, is also present in "Bathing in the Sea" / 浴海:

My blood rises with the waves of the sea,

My heart burns with the fire of the sun,

[...]

Today I became a cicada that has shed its shell,

[...]

While the waves of our blood still rise,

While the fire in our hearts still burn,

Quick, wash away

That old, decayed bag of skin!

我的血和海浪同潮,

我的心和日火同燒,

[...]

我如今變了個脫了殼的蟬蟲,

[...]

趁著我們的血浪還在潮,

趁著我們的心火還在燒,

快把那陳腐了的舊皮囊

全盤洗掉! (Guo Moruo quanji, op. cit., p. 70)

The references to the blood and heart of the subject, identified respectively with the sea and the sun, are intertwined with the image of the cicada shedding its shell. Once again, the subject's body becomes one with nature and undergoes a process of renewal, both physical and spiritual, described through a set of physical images, including "naturification" and zoomorphization. The spiritual element underlying the whole process is further emphasized by the reference to the timeworn "bag of skin" / 皮囊, originally a Buddhist metaphor designating the mortal human body.

In "Snowy Morning" / 雪朝, as the whole body of the subject is transfigured into light, blood is identified with the water falling from the eaves, dripping quietly to the rhythm of the natural phenomena:

Great waves of snow!

A silver-white universe!

My heart and body seem to be turning into a stream of light,

oh, open secret!

The water dripping from the eaves overhead...

Is that not the blood in my whole body?

The dripping blood in my body makes a soft, cadenced sound,

In harmony with the waves of the sea, of the pines, of the snow.

雪的波濤!

一個銀白的宇宙!

我全身心好象要化為了光明流去,

Open secret剠!

樓頭的檐雷.......

那可不是我全身的血液?

我全身的血液點滴出律吕的幽音,

同那海濤相和, 松濤相和, 雪壽相和。(Guo Moruo quanji, op. cit., p. 85) 
"Hymn to the Sun" / 太陽禮灒, which closes on a series of invocations that reveal again their indebtedness to Whitman's catalogue technique,$^{16}$ also contains a very similar image. In a line of the last stanza, the subject's life appears to be turned into blood by the blazing light, thus accomplishing a sort of pantheistic transubstantiation:

o sun! Please shine on my whole life, turning it into a stream of bright red blood!

太陽滁！你請把我全部的生命照成道鮮絗的血流! (Guo Moruo quanji, op. cit., p. 100)

Finally, "Venus" offers a noteworthy example of the poetic superposition of body and nature:

I would compare your loving mouth

To a wine cup.

An inexhaustible, sweet liquor

That would keep me constantly inebriated!

I would compare your nipples

To two grave mounds.

We would sleep in those graves,

Until our blood turns to sweet dew!

我把你這張愛嘴,

比成著一個酒杯。

喝不盡的葡萄美酒,

會使我時常沈醉!

我把你這對乳頭,

比成著兩座填墓。

我們倆睡在墓中,

血液兒化成甘露! (Guo Moruo quanji, op. cit., p. 130)

Apart from the reference to Greco-Roman mythology in the title, the mode of expression used by Guo in the first stanza obviously draws its inspiration from the chapter 4 of the "Song of Songs". ${ }^{17}$ The sensual portrayal of the female body, however, sublimates once again into the communion with nature in the second stanza, reaching its apex in the death-like slumber of the two lovers in the grave mounds and their blood morphing into sweet dew, in a sort of circle of life and death.

\section{The body as a space of dialogue}

In another set of expressive devices, the body appears to be more than an instrument of perception, becoming an interface that allows nature to enter the lyrical I or that facilitates a form of cosmic communion. Wu suggests that in "The Heavenly Hound" the body of the lyrical subject is a platform that allows the moon and sun to communicate and become one within the universe swallowed by the mythical beast. The same concept is observable in "By the Electric Light" / 電火光中, where the perceiving body of the subject becomes a space where the objects (Beethoven's portrait and Millet's painting) and the poet's musings (the mental image of the Han period diplomat $\mathrm{Su}$ Ziqing 苏子卿 (140-60 BC) on the shores of Lake Baikal) evoked in the three sections of the poem are put in communication. ${ }^{18}$

One of the most powerful representations of the body as a space of dialogue is found in “The First Hour after Class” / 輟了課的第一點鐘裡:

Barefoot and bareheaded,

I run into Nature's arms. 
[...]

I run to the pine woods to stroll,

My head washed by the rising sun,

My feet washed by the clear dew,

Cold and heat, warm and cool,

Are all joys of Nature!

我赤足光頭,

忙向自然的懷中跑。

[...]

我跑到松林來散步,

頭上沐著朝陽,

腳下䍜著清露,

冷暖溫涼,

一樣是自然生趣! (Guo Moruo quanji, op. cit., p. 124)

The poet's feet and head touch nature in an unmediated way and are subsequently washed by the sun and dew. This process follows a top-down direction - as in Mi's reading of "The Heavenly Hound" - portraying the subject as a link between the astral body and the earth. It is also depicted as a cleansing ritual with strong religious (i.e. pantheistic) connotations, whereby the subject finally becomes one with the natural world, perceiving its various manifestations and acknowledging them as part of a luxuriant, joyful All.

\section{Nature anthropomorphized and zoomorphized}

In several passages of The Goddesses, nature is anthropomorphized and depicted as a loving mother embracing and feeding its children, be they human beings or not. In “Fetal Movements of Spring” / 春之胎動, for example, the season of regeneration is presented as a child about to be born, “moving in Nature's embrace" / 在大自然的懷中 胎動著在了. ${ }^{19} \mathrm{~A}$ similar image is also found in “Sea of Light” / 光海:

The sandy plain, like a silver foil,

Is waiting to embrace us, smiling,

We are coming.

Embrace us!

We want to be in your arms,

And have a bath of light!

銀箔一樣的沙原,

笑著待把我們擁抱。

我們來了。

你快擁抱!

我們要在你懷兒的當中,

洗個光之澡! (Guo Moruo quanji, op. cit., p. 91-92)

The loving embrace of nature is a very common metaphor by which the poet expresses his yearning for communion with the natural world. This union is celebrated, again, through a pantheistic ritual of purification and renewal: in the example cited above, the ritual is represented by the immersion in a sea of light, the form taken by nature itself in the very first lines of the poem (Guo Moruo quanji, op. cit., p. 91).

“O Earth, my Mother!" / 地球, 我的母親! is another example of this mode of representation:

You shake the child in your arms awake,

I am now crawling on your back. 
$[\ldots]$

I think everything in this universe is your incarnation:

Thunderbolts are the mighty sound of your breath,

Rain and snow your blood soaring.

[...]

When I drink a glass of water, it is rain falling from the sky,

I know it is your milk, my soup of life.

[...]

O Earth, my mother!

My soul is your soul [...].

你把你懷中的兒來摇醒,

我現在正在你背上匍行。

[...]

我想這宇宙中的一切都是你的化身：

雷霆是你呼吸的聲威,

雪雨是你血液的飛騰。

[...]

我飲一杯水, 縱是天降的甘霖,

我知道那是你的乳，我的生命兹。

$[\ldots]$

地球, 我的母親!

我的靈魂便是你的靈魂 [...]。(Guo Moruo quanji, op. cit., p. 79-83)

Throughout the twenty-one quatrains, each of them opening with the invocation that lends its title to the poem, the transparent image of nature as mother of humankind is gradually enriched and developed. Finally, towards the end of the poem, this elaboration finds its apex in a pantheistic announcement where the soul of the lyrical "I" becomes one with the soul of nature.

The natural world is also depicted in anthropomorphic terms in "A Night Stroll in Jurimatsubara" / 夜步十里松原:

Countless stars, their eyes open wide,

watch the beautiful night scene from afar.

Countless old pines in Jurimatsubara

Praise the sky in silence, their arms raised high.

Their arms all shiver in the sky,

My nerves all shiver within my body.

無數的明星正圓淨著他們的眼兒,

在眺望這美麗的夜景。

十里松原中無數的古松,

都高㢣著他們的手兒沈默著在贊美天宇。

他們一枝枝的手兒在空中戰慄,

我的一枝枝的神緸㵶維在身中戰慄。(Guo Moruo quanji, op. cit., p. 98)

In addition to ascribing human features and actions to the stars and trees, Guo resorts to the rhetoric devices of parallelism and repetition in order to emphasize the gradual superposition between the natural and the human, represented by the pines and the nerves, the former shivering in the sky and the latter within the body of the lyrical subject.

An analogous anthropomorphizing device and even similar phrasing are used in "A

New “Three Variations on the Yang Pass”' / 新陽關三轠:

The waters of the ocean dance under my feet,

Stretching countless arms and wrists waiting to embrace the sun.

汪洋的海水在我腳下舞蹈,

高伸出無數的暨腕待把太陽擁抱。(Guo Moruo quanji, op. cit., p. 104) 
Finally, the opening verses of "Looking Afar from the Peak of Fudetate Yama" / 筆立山 頭展望 offer a particular example of zoomorphization of inanimate beings:

Pulse of the great metropolis!

Surge of life!

Beating, panting, shrieking...

Spurting, flying, jumping...

大都會的脈搏呀!

生的鼓動呀!

打著在，吹著在，叫著在，

唄著在, 飛著在, 跳著在, .......(Guo Moruo quanji, op. cit., p. 68)

The modern, industrialized Japanese city of Moji is portrayed as a restless wild animal, in a language and manner that immediately resonate with the depiction of the Heavenly Hound and its unstoppable energy. The poem reveals its indebtedness to Futurism in its formal patterns and rhythmic features. However, Guo's intention is not the celebration of industrial progress and urban modernity (which represents the mainstay of Marinetti's Manifesto), his disappointment with urban life being obvious in such poems as "Impressions of Shanghai." On the contrary, by resorting to this mode of expression, the poet seems to blur the boundaries between human and natural, celebrating the city together with the natural universe in a "marriage of man and nature" / 自然與人生的婚禮 (Guo Moruo quanji, op. cit., p. 68).

\section{Conclusions}

From the close reading of the poems in The Goddesses, two bodily macro-discourses can be identified: the discourse of death and rebirth, or dissolution and regeneration of the body, and the oneness of man (in the person of the lyrical subject) and nature or the cosmos. Although these two discourses are not the only ones in the collection, they are among the most prominent, and can help to shed light on some of its central conceptual nodes.

In The Goddesses, death is never an end or a goal, but rather a tool. ${ }^{20}$ Throughout the collection, it is represented as a sacrifice which the lyrical subject awaits and gladly accepts upon himself, safe in the knowledge that the act of dying, like a form of martyrdom, is necessary in order to cross the threshold of a new life. Indeed, death is always followed - either explicitly or implicitly - by the proclamation of a rebirth, of the beginning of a new existence, as in the poem eloquently titled "New Life" / 新生 / Xinsheng. The question of whether this is essentially a political allegory, as a significant part of traditional scholarship affirms, remains debatable. However, the more deeply this discursive device is scrutinized, the less this interpretation seems justifiable, at least as a predominant strain. Of course, one may see in the idea of death followed by renewal the seeds of the revolutionary engagement that will dominate Guo's later life and artistic production, starting with his conversion to Marxism in 1924. However, apart from the repeated proclamations made in The Goddesses - starting with the famous “I am willing to become a communist” / 我願意成個共產主義者 (Guo Moruo quanji, op. cit., p. 3) in the "Prologue Poem" / 序詩 - which mostly sound pretentious and hollow, the political nature of this process is never elaborated. Conversely, Guo's modes of expression reveal patent spiritual and religious overtones. From this perspective, the regeneration allegorically staged by Guo could be understood within 
the framework of the renewal brought about by the May Fourth Movement, which to him is "not a mere historical event but a religious ritual, one that initiates the new youth into an ecstasy of total self-confidence and self-sacrifice". ${ }^{21}$ This notion is also reflected in the religious image of consumption by fire (as well as the related ubiquitous motifs of light, sun, etc.) and subsequent resurrection, exemplified by the phoenixes, which is used extensively throughout the collection.

The second macro-discourse, with its various physical representations, is linked to the idea of a dynamic unity with the natural and cosmic universe. It completes the discourse of death and resurrection, with which it is intimately intertwined. I call it "dynamic" because it is almost always presented not as a given fact, but rather as a process that begins with the "I" as the active subject of the identification of man and natural world. I see in this discourse a set of poetic devices that further express Guo Moruo's pantheism and his yearning for a spiritual and aesthetic "newness", with the poet becoming a demiurge of his own world. Guo's syncretic pantheism is rooted in the thought of Zhuangzi / 莊子 and Wang Yangming / 王陽明, Chinese shamanism, the poetics of Kabir and the Upanishads, the philosophy of Spinoza, the meditative verse of Tagore and the vitalistic poetry of Whitman. There is no place for a God in the poet's conceptual world, but rather for a godlike "greater self" / 大我, essentially a poetic device that possesses no real religious implications, and which overlaps with the Buddhist notion of "sea of the heart/mind" / 心海 as common consciousness of all sentient beings. ${ }^{22}$ The "greater self" in turn takes the form of the phoenix, the Heavenly Hound, and the material manifestations of nature. It absorbs their qualities and energy, identifies with them, and becomes itself a source of energy in an ecstatic, death-like explosion: only through this process is it able to bring about the process of renewal.

In my view, the two discursive modes analyzed above are closely connected, as their frequent overlapping shows. In The Goddesses, these devices allow the poet to portray the cycle of the energies of life - death being one of them - that the subject puts into motion as a result of his "activistic ethos" ${ }^{23}$ and creative endeavor. They are the translation in poetic terms of the "process of transformation brought about by the becoming newness of bodily awakening" ${ }^{24}$ that exemplifies modernity and progressiveness.

\section{BIBLIOGRAPHY}

BUJATTI Anna, “The Spirit of the May Fourth Movement in The Goddesses of Guo Moruo", in Interliterary and Intraliterary Aspects of the May Fourth Movement 1919 in China, GÁLIK Marián (ed.), Bratislava, Veda, 1990, p. 101-110.

CHU Zigang 褚自剛, [“On the Conscience of Death in Guo Moruo's Early Poetry”] / 論郭沫若早期 詩歌中的死亡意識 / Lun Guo Moruo zaoqi shige zhong de siwang yishi, 作家雜誌 / Zuojia zazhi, 2012:3, p. 32-33. 
ELLSTRÖM Lars, “Guo Moruo, The Goddesses" / 女神 / Nüshen 1921, in A Selective Guide to Chinese Literature 1900-1949, vol. III: The Poem, HAFT Lloyd (ed.), Leiden/New York/København/Köln, Brill, 1989, p. 108-114.

FUjITA Rina 藤田梨那, [“Medicine, Literature, Body: The Case of Guo Moruo”] / 醫學 ・文學 ・身體 一以郭沫若為例 / Yixue - wenxue - shenti - Yi Guo Moruo weili, 中國現代文學研究論盖 / Zhongguo xiandai wenxue yanjiu luncong, 10(1):2015, p. 1-7.

FUJITA Rina 藤田梨那, [“On Guo Moruo’s “The Heavenly Hound”’] / 郭沫若の「天狗」論 / “Kakumatsujaku no 'Tengu' ron”, 国士館大学文学部人文学会紀要 / Kokushikan daigaku bungaku-bu jinbun gakkai kiyō, 36:2003, p. 129-141.

GÁLIK Marián, “Kuo Mo-jo’s The Goddesses: Creative Confrontation with Tagore, Whitman and Goethe", in Milestones in Sino-Western Literary Confrontation (1898-1979), GÁLIK Marián (ed.), Wiesbaden, Otto Harrassowitz, 1986, p. 43-72. GUo Moruo 郭沫若, [Complete Works of Guo Moruo] / 郭沫若全集 / Guo Moruo quanji, 北京 / Beijing, 人民文學出版社 / Renmin wenxue chubanshe, vol. 1, 1982.

GUO Moruo 郭沫若, The Goddesses, LESTER Jong and BARNES A. C. (trans.), Beijing, Foreign Languages Press, 2001.

HEINRICH Ari Larissa, The Afterlife of Images: Translating the Pathological Body between China and the West, Duke U. P., 2008.

HSU Kai-yu (ed.), Twentieth Century Chinese Poetry: An Anthology, New York, Anchor Books, 1963.

JIANG Tao 姜濤, [“Imagining Character in the Dissecting Room - A Comprehensive Investigation of the Image of the Poet in Early Guo Moruo”] / 解剖室中的人格想象一對郭沫若早期詩人形象的擴 展性考察 / Jiepoushi zhong de renge xiangxiang - Dui Guo Moruo zaoqi shiren xingxiang de kuozhanxing kaocha, in北京大學新詩研究所 / Beijing daxue xinshi yanjiusuo, 首都師範大學詩歌研究中心 / Shoudu shifan daxue shige yanjiu zhongxin (eds.), 新詩與浪漫主義學術研討會論文集 / [Proceedings of the Scholarly Conference on New Poetry and Romanticism] / Xinshi yu langmanzhuyi xueshu yantaohui lunwenji, 北京 Beijing, 中國詩歌研究中心 Zhongguo shige yanjiu zhongxin, 2011, p. 116-133.

LEE Leo Ou-fan, "Kuo Mo-jo", in The Romantic Generation of Modern Chinese Writers, LEE Leo Ou-fan (ed.), Cambridge, Harvard U. P., 1973, p. 177-200.

LIN Julia, Modern Chinese Poetry. An Introduction, Washington, Washington U. P., 1972.

Mi Jialu 米家路, [“The Insolent and Creating Body: Self-Fashioning and Chinese Modernity. A Reinterpretation of Guo Moruo’s Poem “The Heavenly Hound”'] / 張狂與造化的身體 : 自我模塑 與中國現代性一郭沫若詩歌《天狗》再解讀 / Zhangkuang yu zaohua de shenti: ziwo mosu yu Zhongguo xiandaixing - Guo Moruo shige ‘Tiangou' zaijiedu, 江南學術 / Jiangnan xueshu, ZHAO Fan 趙凡 (trans.), 2016:35(1), p. 13-21.

MI Jiayan, Self-Fashioning and Reflexive Modernity in Modern Chinese Poetry, 1919-1949, Lewiston, NY, Edwin Mellen, 2004.

ou Hong, "Pantheistic Ideas in Guo Moruo's The Goddesses and Whitman's Leaves of Grass", in Whitman East \& West. New Contexts for Reading Walt Whitman, Folsom Ed (ed.), Iowa City, Iowa U. P., 2002, p. 187-196.

TSU Jing, "Perversions of Masculinity: The Masochistic Male Subject in Yu Dafu, Guo Moruo, and Freud", Positions, 2000:8(2), p. 269-316. 
VUILLEUMIER Victor alias YU Lewen 宇樂文, [“The Representation of the Body, Medicine, and Dissection: An Ambiguous Stance in Guo Moruo's Fiction of the 1920s and 1930s”] / 身體的再現、 醫學與解剖：20-30 年代郭沫若小說裡的一種含混的態度 / Shenti de zaixian, yixue yu jiepou: 20-30 niandai Guo Moruo xiaoshuo li de yi zhong hanhu de taidu, 中國現代文學研究論叢 / Zhongguo xiandai wenxue yanjiu luncong, 2015:10(1), p. 8-16.

VUILLEUMIER Victor, "Body, Soul, and Revolution: The Paradoxical Transfiguration of the Body in Modern Chinese Poetry", in Chinese Revolution and Chinese Literature, TAO Dongfeng et al. (ed.), Cambridge, Cambridge U. P., 2009, p. 45-70.

WANG David Der-wei, "Chinese Literature from 1841 to 1937", in The Cambridge History of Chinese Literature, sUn CHANG Kang-I (ed.), Cambridge, Cambridge U. P., vol. 2, 2010, p. 413-564.

WEI Hongshan 魏紅珊, [“The Desiring Body and the Fractured Self: The Case of Guo Moruo's Fiction”] / 欲望的身體與分裂的自我一以郭沫若小說為例 / Yuwang de shenti yu fenlie de ziwo - Yi Guo Moruo xiaoshuo weili, 郭沫若學刊 / Guo Moruo xuekan, 2009:2, p. 45-49.

wu Chen 吳辰, [“On the Aesthetic Features of Guo Moruo's The Goddesses”] / 論郭沫若《女神》的 審美特色 / Lun Guo Moruo 'Nüshen' de shenmei tese, 山東師範大學學報 / Shandong shifan daxue xuebao, 61(4):2016, p. 14-30.

\section{NOTES}

1. The texts quoted all refer to the following edition: Guo Moruo 郭沫若, [Complete Works of Guo Moruo] / 郭沫若全集, Beijing, Renmin yenxue chubanshe, vol. 1, 1982.

2. See HEINRICH Ari Larissa, The Afterlife of Images: Translating the Pathological Body between China and the West, Durham, Duke U. P., 2008.

3. TSU Jing, "Perversions of Masculinity: The Masochistic Male Subject in Yu Dafu, Guo Moruo, and Freud", Positions, 2000:8(2), p. 269-316.

4. WEI Hongshan 魏紅珊, [“The Desiring Body and the Fractured Self: The Case of Guo Moruo's Fiction”] / 欲望的身體與分裂的自我一以郭沫若小說為例, 郭沫若學刊, 2009:2, p. 45-49.

5. FUJITA Rina, [“Medicine, Literature, Body: The Case of Guo Moruo"] / 藤田梨那, 醫學 ・文學 · 身體一以郭沫若為例, 中國現代文學研究論叢, 10(1):2015, p. 1-7.

6. VUILLEUMIER Victor, ["The Representation of the Body, Medicine, and Dissection: An Ambiguous Stance in Guo Moruo's Fiction of the 1920s and 1930s”] / 身體的再現、醫學與解剖：20-30 年代 郭沫若小說裡的一種含混的態度, 中國現代文學研究論叢, 10(1):2015, p. 8-16.

7. See: GÁLIK Marián, “Kuo Mo-jo's The Goddesses: Creative Confrontation with Tagore, Whitman and Goethe", in Milestones in Sino-Western Literary Confrontation (1898-1979), GÁLIK Marián (ed.), Wiesbaden, Otto Harrassowitz, 1986, p. 62 ff.; ELLSTRÖM Lars, “Guo Moruo, The Goddesses” / 女神 1921, in A Selective Guide to Chinese Literature 1900-1949, vol. III: The Poem, HAFT Lloyd (ed.), Leiden/ New York/København/Köln, Brill, 1989, p. 108-114; BUJATTI Anna, “The Spirit of the May Fourth Movement in The Goddesses of Guo Moruo", in Interliterary and Intraliterary Aspects of the May Fourth Movement 1919 in China, GÁLIK Marián (ed.), Bratislava, Veda, 1990, p. 101-110.

8. FUJITA Rina 藤田梨那, [“On Guo Moruo’s “The Heavenly Hound”] / 郭沫若の「天狗」論, 国士 館大学文学部人文学会紀要, 36:2003, p. 129-141 (76-64).

9. MI Jialu 米家路, [“The Insolent and Creating Body: Self-Fashioning and Chinese Modernity. A Reinterpretation of Guo Moruo's Poem ‘The Heavenly Hound”'] / 張狂與造化的身體 : 自我模塑 與中國現代性一郭沫若詩歌《天狗》再解讀, ZHAO Fan 趙凡 (trans.), 江南學術, 35(1):2016, p. 13-21. 
10. VUILLEUMIER Victor, "Body, Soul, and Revolution: The Paradoxical Transfiguration of the Body in Modern Chinese Poetry", in Chinese Revolution and Chinese Literature, TAO Dongfeng et al. (eds.), Cambridge, Cambridge U. P., 2009, p. 45-70.

11. JIANG Tao 姜濤, ["Imagining Character in the Dissecting Room - A Comprehensive Investigation of the Image of the Poet in Early Guo Moruo”] / 解剖室中的人格想象一對郭沫若早 期詩人形象的擴展性考察, in [Proceedings of the Scholarly Conference on New Poetry and Romanticism] / 新詩與浪漫主義學術研討會論文集, 北京大學新詩研究所 / Beijing daxue xinshi yanjiusuo, 首都師範大學詩歌研究中心 (eds.), 北京 / Beijing, 中國詩歌研究中心, 2011, p. 116-133. 12. HSU Kai-yu (ed.), Twentieth Century Chinese Poetry: An Anthology, New York, Anchor Books, 1963, p. 30 .

13. All English translations are my own. Partial English translations of The Goddesses can be found in Hsu Kai-yu (ed.), Twentieth Century Chinese Poetry: An Anthology, New York, Anchor Books, 1963; LIN Julia, Modern Chinese Poetry. An Introduction, Seattle, Washington U. P., 1972; GUo Moruo, The Goddesses, LESTER Jong and BARNES A. C. (trans.), Beijing, Foreign Languages Press, 2001.

14. See ou Hong, "Pantheistic Ideas in Guo Moruo's The Goddesses and Whitman's Leaves of Grass", in Whitman East \& West. New Contexts for Reading Walt Whitman, FOLSOM Ed (ed.), Iowa City, Iowa U. P., 2002, p. 187-196.

15. MI [Mi Jialu米家路], [The Insolent and Creating Body...], op. cit., p. 19.

16. GÁlIK, “Kuo Mo-jo's The Goddesses...”, op. cit., p. 56.

17. Ibid., p. 48-49.

18. wu Chen 吳辰, [“On the Aesthetic Features of Guo Moruo's The Goddesses”] / 論郭沫若《女 神》的審美特色, 山東師範大學學報, 61(4):2016, p. 24.

19. GUo, Guo Moruo quanji, op. cit., p. 154.

20. CHU Zigang 褚自剛, [“On the Consciousness of Death in Guo Moruo's Early Poetry”] / 論郭沫 若早期詩歌中的死亡意識, 作家雜誌, 2012:3, p. 32.

21. WANG David Der-wei, "Chinese Literature from 1841 to 1937", in The Cambridge History of Chinese Literature, sun CHANG Kang-I (ed.), Cambridge, Cambridge U. P., vol. 2, 2010, p. 481-482.

22. GÁLIK, “Kuo Mo-jo's The Goddesses...", op. cit., p. 56.

23. LEE Leo Ou-fan, "Kuo Mo-jo", in The Romantic Generation of Modern Chinese Writers, LEE Leo Oufan (ed.), Cambridge, Harvard U. P., 1973, p. 184.

24. MI, op. cit., p. 27.

\section{AUTHOR}

\section{PAOLO MAGAGNIN}

Paolo Magagnin is Associate Professor in Chinese language and translation at Ca' Foscari University of Venice, where he is also a steering member of the Research Group on the Translation of Asian Languages. His primary interests cover translation studies, modern and contemporary Chinese literature, and Chinese political discourse. His current research focuses on the sociological aspects of the dissemination of contemporary Chinese genre fiction in translation. In addition to his scholarly work, he is a translator of contemporary Chinese fiction. 


\title{
Lu Xun, Tourgueniev et
} l'expressionnisme : corporalité et mouvement dramatique dans «Tremblement à la limite de la déchéance » (1925)

\author{
Victor Vuilleumier
}

1 Souvent la littérature rêve de franchir les limites du texte pour devenir peinture, musique, ou action réelle sur le monde et la société. La Nouvelle littérature / 新文学, qui prend forme en Chine à la fin des années 1910, ne fait pas exception, réceptive qu'elle est aux arts modernes et au cinéma. Leur marquent un net intérêt, entre commentaires critiques et expérimentations littéraires au confluent de différents media, maints auteurs chinois, tels Ai Qing 艾青 (1910-1996), Lu Xun 鲁迅 (1881-1936), Ouyang Yuqian 歐陽予倩 (1889-1962), Ye Lingfeng 葉霝鳳 (1905-1975) ou Zhang Ailing 張愛玲 (1920-1995). Et leurs créations attribuent un rôle pivot à la représentation de la corporalité.

2 Dans le cas présent, les œuvres qui manifestent de telles relations relèvent en gros de deux «types»: des textes qui effectuent des transpositions d'effets esthétiques, de procédés picturaux ou cinématographiques (montage, collage, juxtaposition); d'autres qui, outre ces transpositions, établissent des rapports transesthétiques ${ }^{1}$ de "traduction» d'œuvres parfois identifiables. Ceci concerne davantage les cas de «transmodalisations ", soit le passage ou l'adaptation d'un "mode " " épique, lyrique ou dramatique » à l'autre, comme entre roman ou théâtre ${ }^{2}$.

Des exemples du premier type se trouvent entre autres chez des auteurs de l'école de Shanghai / 海派 (années 1930-40) ${ }^{3}$ : ainsi de l'écriture du stream-of-consciousness de Zhang Ailing, qui importe le fondu-enchaîné4, ou de la représentation que les «néosensationnistes»/ 新感覺派 font du choc de la métropole moderne sur la conscience, au moyen de techniques de télescopage et de la représentation de la vitesse ${ }^{5}$. Soit une esthétique polymorphe, inspirée du cinéma et des arts modernes, tout à la fois 
symboliste, cubiste, expressionniste, surréaliste ou futuriste - ce que la réception chinoise perçoit en bloc comme esthétique «moderniste » / 现代 - caractérisée ici par sa volonté de "rendre » une perception illusoirement concrète et palpable des corps, des objets et des couleurs, une insistance sur le mouvement, tout cela concentré dans la recherche de l'expression du choc et de la tension. Cette esthétique déborde toutefois les limites de la "littérature de Shanghai». Ainsi, le poète Ai Qing, associé à la littérature politisée de l'aile gauche des années 1930 (elle qui reproche ce même "modernisme» à la "littérature de Shanghai »), dite «réaliste» (comprendre " engagée » et attachée à décrire les réalités sociales), développe pareille poétique concrète du corps. Il construit notamment des descriptions ekphrastiques de scènes, qui semblent issues de la tradition de la peinture et du sublime occidentaux, ou transpose l'esthétique cubiste pour décrire des mouvements et impressions corporelles 6 .

4 Au second type appartient, pour donner un exemple, [ « Le cauchemar des Juifs »] / 猶 太人的怖夢 (1925), un texte, sinon poème (v. infra), en prose/ 散文, du poète censément symboliste Xu Zhimo 徐志摩 (1896-1931), qui décrit la représentation d'une pièce expressionniste à laquelle il assista à $\mathrm{Moscou}^{7}$. Ce dernier cas repose sur une relation de fait : Xu Zhimo décrit une pièce de théâtre identifiable et la transpose dans son texte.

5 Cependant, une telle relation de fait ne constitue pas la condition nécessaire à l'existence de rapports transesthétiques entre un texte et les arts visuels. De fait, dans ces cas évoqués, il se trouve un point commun, un critère, qui est celui de la description et du sens du corps. Dans l'art et la littérature occidentale, le corps est l'une des manifestations du monde des phénomènes, qui fait l'objet de la représentation représenter le corps c'est représenter la réalité ${ }^{8}$. Certes, dans la peinture ou la littérature chinoise le corps ne remplit pas nécessairement une telle fonction ou ne présente pas la même importance, du fait de sa relative absence ${ }^{9}$, ou plutôt, de sa présence indirecte; mais la littérature chinoise à partir des années 1920 importe en partie un tel paradigme, qui participe de sa "modernité ». Dans tous les cas, la façon dont le corps est montré, et l'importance qui lui est accordée objectivement dans un texte, constitue un indice sur l'esthétique choisie pour dire le monde et le sujet. Nous proposons donc de décrire ces transpositions et correspondances esthétiques par l'analyse de la corporalité (esthétique du corps et imagerie concrète) et du sens du mouvement, de la tension - en l'occurrence, de la distorsion expressionniste. Cette corporalité par ailleurs possède une dimension interculturelle, car elle se construit avec des éléments importés.

6 Un texte de Lu Xun, «Tremblement à la limite de la dévastation » ou de la « déchéance »/䫋敗線的顫動 (1925) ${ }^{10}$, quasiment dénué d'intrigue ${ }^{11}$, développe un drame théâtral : celui d'une tension cosmique ou d'une apocalypse à venir qui se joue à l'intérieur d'un corps. Tout comme le recueil où il figure, [La Mauvaise herbe] / 野草 (1927), il relève du genre moderne occidentalisant et revendiqué comme tel, du poème en prose / 散文詩, nommé et pratiqué en Chine dès 1918 - dont Baudelaire, Tagore ou Tourguéniev sont les principaux auteurs de référence traduits et présentés en Chine à partir des années $1910^{12}$.

7 Dans le "Tremblement ", un narrateur à la première personne rapporte un rêve, qui se subdivise en deux scènes, marquées par les deux réveils du narrateur et la prise de conscience de sa position corporelle - dont il suggère qu'elle a induit des éléments de 
son rêve. Soit deux épisodes d'un même rêve, reprenant le fil d'une même histoire à plusieurs décennies d'intervalle, qui se déroule dans le huis clos d'une "petite maison» / 小屋. Le premier épisode décrit la vie d'une jeune femme, qui se prostitue pour vivre et nourrir sa fille; le second montre cette même femme âgée, rejetée par sa fille, son beau-fils et son petit-fils, qui lui reprochent de leur faire honte et d'être responsable de leur misère. Cette femme est présentée à la fois comme immobile, telle une statue, et prise de convulsions, dans un environnement pris à son tour d'un même tremblement circulaire universel, d'abord celui d'une «vague»/波壽, puis d'un 《tourbillon » / 㵀渦 qui emporte tout:

Soudain une onde immense surgit à nouveau du fond de l'espace [空中 / kongzhong, litt. « dans le vide », « dans l'air »], elle vint heurter celle qui l'avait précédée et son reflux forma un tourbillon qui finit par tout engloutir, moi-même y compris [一七] 並我 / yiqie bing wo] : je suffoquai. (tr. P. Ryckmans) ${ }^{13}$

空中突然另起了一個很大的波壽, 和先前的相撞蒰, 回旋而成漩渦, 將一切並我 盡行淹沒, 口鼻都不能呼吸。

8 Après cet épisode le narrateur se réveille une première fois, avant de reprendre son rêve, dont le récit continue. Au second réveil, qui marque la fin du texte, il donne une explication physiologique et mécaniste à son « cauchemar » / 夢魘:

J'[我 / wo] avais fait un cauchemar - mais [卻 / que] je savais bien, moi [自己 / ziji], que c'était pour cette raison [是因為...的緣故 / shi yinwei...de yuangu], que j'avais eu les mains posées sur le torse. Voilà encore que dans mon [我/ wo] rêve, pour mouvoir ces mains si lourdes, j'avais dû aller au bout de mes forces ${ }^{14}$.

我夢厴了, 自己卻知道是因為將手擱在胸腹上了的緣故 ; 我夢中還用盡平生之

力, 要將這十分沈重的手移開。

9 Les mains posées sur sa poitrine l'oppressaient et gênaient sa respiration: le cauchemar était provoqué par ses efforts «frustrés » de ne pouvoir les soulever. Cette explication déterministe, qui se donne les apparences de la rigueur scientifique (Lu Xun a étudié la médecine), est dénotée par la dimension argumentative et volontariste du discours du narrateur : la particule que produit une modalisation adversative ( mais je savais bien que ») et la structure syntaxique shi yinwei...de yuangu (litt. " c'est pour cette raison à savoir que ») introduit une relation de causalité insistante. Ces éléments contribuent à construire le discours rationnel d'un sujet, qui revendique une parole assertive; cette assertivité inquiète porte peut-être sur l'unité de son individualité, comme le suggère la relative insistance déictique et réflexive sur l'agent de l'énonciation "je » / wo et le réfléchi assez "occidentaliste " "moi-même ", " soimême» / ziji - rappelons qu'en chinois, même moderne, la mention du pronom personnel, surtout de la première personne, n'est pas nécessaire aussi longtemps que le contexte permet de savoir qui parle. Ce sont autant de tours stylistiques propres à la langue du baihua du 4-Mai, qui en signalent la modernité ${ }^{15}$. Or, cette langue s'affirme contre le récit du rêve à proprement parler : il est certes narratif ou descriptif, mais la narration fait entendre ici et là une voix plus rythmée et lyrique, que le plat commentaire du narrateur premier sur son rêve $^{16}$. Ce récit porte une vision inquiétante, et sa polyphonie de voix accusatrices (la famille contre la femme) contrarie la revendication d'unité du narrateur premier.

10 Le récit du rêve invite ainsi à la distance d'avec la conclusion du narrateur. Elle apparaît comme une justification donnée après coup, pour détourner l'attention de l'essentiel, pour conjurer ce mauvais rêve et ce qu'il exprime : l'engloutissement, la suffocation, la tension entre désir de mouvement et immobilité - en un mot, un sens 
général de frustration. C'est ce fil que nous allons suivre dans notre lecture, pour démontrer que son ressort dramatique repose sur le sens du mouvement corporel.

11 À ce point, nous souhaitons examiner la relation de «Tremblement " à son très probable hypotexte. Comme annoncé plus haut, les poèmes en prose de Tourguéniev sont l'une des sources d'inspiration de ce genre nouveau en Chine, dont «Le Tremblement » est un parfait exemple. L'hypotexte que je propose ici est donc un tel texte du poète russe, «La fin du monde » / « КонЕц СВЕTA. COH» (1878) $)^{17}$. Lu Xun a possédé au cours de son existence plusieurs traductions allemandes et japonaises d'œuvres de Tourguéniev, notamment un exemplaire de la collection Reclam, [Poèmes en prose] / Gedichte in Prosa $(1883)^{18}$, qu'il avait selon toute vraisemblance acquis durant ses études au Japon de 1902 à $1909^{19}$. Au moins par cette traduction, il a eu connaissance $\mathrm{du}$ recueil et de ce texte ${ }^{20}$, qui y figure sous le titre ["La fin du monde. Un rêve »] / « Das Ende der Welt. Ein Traum » (p. 24-26) - version que nous suivons ici.

Dans «La fin du monde », le narrateur à la première personne rapporte un rêve. Il se trouve dans une maison isolée à la campagne, avec plusieurs personnes, dont un jeune enfant ; ils y sont enfermés, éprouvant un sentiment croissant d'oppression. Puis ils suivent par la fenêtre l'évolution d'un cataclysme à l'extérieur : "la terre vient de s'engloutir » / Die Erde ist versunken (p. 25). Le narrateur décrit ce qu'il voit :

En effet : à l'instant il y avait une plaine devant la maison - qui à présent se trouve au sommet d'une grande montagne ! - L'horizon est tombé - sombré, et juste devant la maison bée un abîme tombant à pic, crevassé et noir [mes italiques].

In der That: vorhin war eine Ebene vor dem Hause - jetzt steht es auf dem Gipfel eines grossen Berges! - Der Horizont ist heruntergefallen - hinabgesunken, und dicht vor dem Hause gähnt ein steiler, zerglüfteter, schwarzer Abgrund ${ }^{21}$

Dans le poème de $\mathrm{Lu}$ Xun, le thème du désastre cosmique et de la chute provient manifestement de Tourguéniev. C'est même une explication du titre « Tremblement sur la ligne » ou «le fil» de «la décadence » - l'effondrement de la ligne de l'horizon devenue ici chute morale.

Puis, toujours chez Tourguéniev, de l'horizon surgit la mer, qui dans un mouvement ondulatoire, finit par tout engloutir dans un « hurlement " / Heulen, apportant la « fin de toute chose " / Das Ende aller Dinge, et des "ténèbres éternelles » / ewige Finsternis, avant que le narrateur ne s'éveille en étouffant : "J'arrivai à peine à reprendre mon souffle au moment de me réveiller" / Ich konnte kaum Athem holen, als ich erwachte (p.26). Voici la description de ce mouvement, qui emplit le monde avant de le précipiter vers sa fin :

Et regardez - à la bordure de la terre, sur tout son pourtour, quelque chose commença à remuer, de petits monticules arrondis se levaient et s'abaissaient au loin.

«La mer! »

[...] Et pourtant cela monte, monte à une vitesse démesurée... Maintenant ce ne sont déjà plus quelques monticules isolés qui roulent en montant et s'abaissant là-bas au loin... C'est une unique, monstrueuse marée réunie qui déferle tout autour de l'horizon [mes italiques].

Und sieh - am ganzem weiten Erdrand entlang begann sich etwas zu rühren, kleine rundliche Hügel hoben und senkten sich in der Ferne.

„Das Meer!“

[...] Und dennoch steigt es, steigt mit Riesenschnelle... Nun sind es schon nicht mehr einzelne Hügel, die da in der Ferne auf und nieder wogen... Eine einzige, ungeheuerliche, zusammenhängende Flut ist's, die rings am ganzen Horizont hereinbricht. ${ }^{22}$ 
pouvons relever des motifs et thèmes communs entre les deux textes (voir plus bas pour "Le Tremblement»): le lieu clos, l'enfermement et la suffocation (qui rencontre l'image luxunienne de la 《maison de fer $»^{23}$ ); la présence du narrateur, d'autres personnes et d'un enfant - chez Lu Xun, une famille; le drame de l'écroulement cosmique produit par une vague et un mouvement ondulatoire. Il se trouve cependant quelques différences notables, qui révélent un choix esthétique autre.

Tout d'abord, la réécriture de Lu Xun modifie la structure narrative initiale, à commencer par la position ambiguë du narrateur par rapport à l'histoire (le rêve) qu'il rapporte. D'un point de vue narratologique, le récit de rêve représente un cas limite et exemplaire. Exemplaire, car le rêve (l'histoire) n'existe pas indépendamment du récit reconstitué a posteriori; il n'est pas possible de le comparer à la matière initiale du songe, qui est volatile. Limite, car le narrateur de son propre rêve peut se trouver à des degrés divers à l'écart et partie prenante de l'histoire. Chez Tourguéniev, le narrateur est distinctement acteur et présent dans l'histoire qu'il rapporte. Chez Lu Xun, le narrateur est en principe extradiégétique dans son récit enchâssé (l'histoire de la femme dans son rêve), et dans le récit-cadre de son rêve (son endormissement répété, son réveil, ses sensations), il est homodiégétique. Mais qu'en est-il de sa présence en tant que telle dans le rêve rapporté ? Le narrateur est-il dans ou hors de son rêve? Comme dans tout songe, il est ce témoin flottant, présent et absent - un sujet à l'identité protéiforme et problématique. Le choix de Lu Xun pour cette position narrative constitue un écart avec ce qu'il en est dans « La Fin du monde », qui contribue à développer le thème de la tension.

17 La comparaison des paragraphes introducteurs des deux textes est révélatrice à ce titre. Chez Tourgueniev : "J'ai rêvé que je me trouvai quelque part dans un coin de la Russie, dans une ferme isolée et solitaire. » / Es träumte mir, ich befände mich in irgend einem Winkel Russlands, in einem einsam gelegen Bauernhause (p. 24). Et chez Lu Xun :

J'ai rêvé que je rêvais [我梦见自己在梦见 / Wo mengjian ziji zai mengjian]. Je ne savais pas où je me trouvais; sous mon regard se découvrait l'intérieur d'une maisonnette étroitement close au cœur de la nuit, mais simultanément je pouvais apercevoir aussi les mauvaises herbes dont se hérissait son toit. [mes italiques] (tr. Ryckmans) ${ }^{24}$ 我梦见自己在梦见。自身不知所在, 眼前却有一间在深夜中紧闭的小屋的内部, 但也看见屋上瓦松的茂密的森林。

La location est identique (une maison au milieu d'un espace sauvage ou désert), ainsi que l'indication explicite de la nature onirique du récit. Dans sa notice à ce texte, S. Veg relève que ce début chez Lu Xun présente la "particularité d'un redoublement ${ }^{25}$. De fait, on y voit un élément de réflexivité et de dissociation, c'est-à-dire, de distanciation. Le narrateur est conscient, du moins a posteriori, du fait qu'il rêvait rêver (litt. «J'ai rêvé que j'étais moi-même en train de rêver " / Wo mengjian ziji zai mengjian). C'est ce que la phrase finale du "Tremblement » explique (v. la citation plus haut, «J'avais fait un cauchemar », etc.). De plus, cette fin ouvre la possibilité d'une ambiguïté : l'affirmation «j'avais fait un cauchemar » peut laisser entendre que le rêve n'est pas achevé, mais « infini » comme la plaine sauvage (voir citation plus bas) dans laquelle il se déroule. La confusion entre rêve et réalité est une technique narrative assez fréquente dans l'histoire de la littérature chinoise. Autre différence avec le narrateur de Tourguéniev, qui ne se réveille qu'une fois, pour établir une distinction nette entre les deux mondes, celui de $\mathrm{Lu}$ Xun poursuit le même rêve en se rendormant à nouveau (établissant au 
passage une relation d'intertextualité possible avec Gogol) - signe que le cauchemar ne finit pas $^{26}$.

En parallèle à cette position narratologique ambiguë, le « Tremblement » construit une vision kaléidoscopique, qui présente simultanément les perspectives différentes et contradictoires propres au rêve («simultanément je pouvais apercevoir aussi ») : le narrateur est à la fois au-dedans et au-dehors de la maison, comme il est dans et hors de l'histoire. La juxtaposition simultanée de différentes perspectives se rencontre certes dans la poésie et la peinture chinoise classique. Ici, elle sert la nature « moderniste » importée du texte, pour construire une expérience de dissociation et de réflexivité narrative.

Ces différences de la structure et la position narrative apportent un nouvel exemple de la réflexion menée par $\mathrm{Lu}$ Xun sur le problème de la représentation et les «limites du réalisme ", pour reprendre la formule de $\mathrm{M}$. Anderson - à savoir comment représenter la souffrance de la vieille femme (figure de l'aliénation) et laisser entendre sa parole (certes fictive) sans qu'elle ne soit « réappropriée " par le narrateur. Dans certaines de ses nouvelles, Lu Xun résout ce problème précisément en instituant une division et une mise à distance du narrateur par lui-même ${ }^{27}$. Dans "Tremblement", il transforme le thème eschatologique de la fin du monde de Tourguéniev en celui d'apparence plus politique de l'oppression et de la désunion sociales ${ }^{28}$, par le recours cependant à une esthétique non "réaliste». C'est l'explication à la différence de traitement de l'expression de «l'horreur » causée par l'ondulation cosmique : «Et quel mugissement et hurlement ! La terre même se lamente et gémit d'horreur » / Da, welch ein Brausen und Heulen! Die Erde selber ächzt und stöhnt vor Entsetzen (p. 26). Chez Lu Xun ce " hurlement » devient silencieux (v. citation plus bas), car il est rapporté non plus à la nature impersonnelle, mais à un être humain aliéné.

21 Ces différences entre la réécriture chinoise et l'original de Tourguéniev révèlent des aspects $\mathrm{du}$ "principe organisateur » propre à $\mathrm{Lu}$ Xun, qui polarise et réoriente la structure narrative ainsi que certains thèmes de la «Fin du monde» (ondulation, destruction, cri, maison de fer, rêve). Ce principe participe d'une esthétique particulière, qui se construit essentiellement à partir de la tension entre mobilité et immobilité, silence et parole - autant de modes de la corporalité développés par $\mathrm{Lu}$ Xun.

22 Le «Tremblement » sollicite en effet «l'intelligence kinésique ${ }^{29}$ du lecteur par une esthétique expressionniste. L'extrait suivant, tiré de la seconde séquence du récit du rêve, en fournit un exemple :

La bouche de cette vieille femme se convulsait; soudain, elle fut terrifiée, puis fut toute calme. Peu après, elle se leva, froidement, pareille à une statue de pierre anguleuse. Elle entr'ouvrit la porte, et prit le chemin des profondeurs de la nuit, laissant derrière elle toutes les insultes et les rires empoisonnés.

Elle marcha de toutes ses forces dans la nuit profonde, jusqu'à parvenir à une étendue déserte et infinie ; autour d'elle, il n'y avait que des étendues désertes, et au-dessus d'elle, rien ne volait, ni oiseau, ni insecte. Elle se dressait nue au centre de cette immensité déserte, comme une statue de pierre. En un instant, elle revit le reflet de tout son passé : la faim, la souffrance, la stupeur, la honte, le plaisir, puis elle trembla [發抖 / fadou] ; la «nuisance », le « tort », « la compromission » [que sa fille, beau-fils et petits-enfants lui reprochent de leur avoir causé], puis elle fut prise de convulsions; «à mort!", puis elle se calma... Puis encore en un instant tout se confondit: l'attachement et la rupture, l'amour et la vengeance, le soin et l'extermination, la bénédiction et l'imprécation... Elle leva ensuite ses deux mains 
au ciel, et laissa échapper de sa bouche un langage humain et bestial, sans paroles car n'appartenant pas au monde humain.

Tandis qu'elle proférait ce langage sans paroles, son corps, majestueux comme une statue de pierre [如石像 / ru shixiang], pourtant dévasté et ravagé, se mit tout entier à trembler [顫動 / chandong]. Ce tremblement [chandong] se marquait de point en point [點點 / diandian] comme des écailles [如魚鱗 / ru yulin], dont chacune ondulait comme de l'eau bouillonnant sur un feu ardent ; aussitôt l'espace fut pris d'une même vibration, on eût dit les ondes d'une mer désolée battue par la pluie d'une tempête.

Alors elle leva les yeux au ciel, et son langage sans paroles se tut et cessa définitivement, il n'y avait que le tremblement [chandong], irradiant comme les rayons du soleil, qui faisait aussitôt refluer les vagues dans le ciel, comme si elles avaient rencontré un cyclone, et déferlaient vers l'étendue déserte et sans fin.

J'avais fait un cauchemar, et je savais que c'était parce que mes deux mains reposaient sur ma poitrine; dans mon rêve, j'avais mis toutes mes forces à déplacer ces mains extrêmement pesantes.

那垂老的女人口角正在痓攣，登時一怔，接著便都平靜，不多時候，她冷靜地， 骨立德石像似的站起來了。她開開板門, 邁步在深夜中走出, 遺棄了背後一切的 冷黑和毒笑。

她在深夜中盡走, 一直走到無邊的荒野; 四面都是荒野, 頭上只有高天, 並無一 個蟲鳥飛過。她赤身露體地, 石像似的站在荒野的中央, 於一刹那間照見過往的 一切: 飢餓, 苦痛, 驚異, 羞辱, 歡欣, 於是發抖; 害苦, 委屈, 帶累, 於是痓 攣; 殺, 於是平靜。……又於一刹那間將一切並合 : 春念與決絕, 愛掹與復仇, 養育與戨除, 祝福與咒詛....... 她於是舉兩手盡量向天, 口唇間漏出人與獸的, 非人間所有, 所以無詞的言語。

當她說出無詞的言語時, 她那偉大如石像, 然而已緸荒廢的, 頽敗的身身品的全面 都顫動了。這顫動點點如魚鱗, 每一鱗都起伏如沸水在烈火上 ; 空中也即刻一同 振顫, 行佛暴風雨中的荒海的波濤。

她於是抬起眼睛向著天空, 並無詞的言語也沈默盡絕, 惟有顫動, 輻射若太陽

光，使空中的波濤立刻回旋，如遭風風，洶湧奔騰於無邊的荒野。

我夢秠了, 自己卻知道是因為將手擱在胸脯上了的緣故 ; 我夢中還用盡平生之

力, 要將這十分沈重的手移開。30

La qualité kinésique de ce texte, immédiatement perceptible, explique que les commentateurs pointent à l'occasion les procédés «cinématiques» de $\mathrm{Lu} \mathrm{Xun}^{31}$ ou relèvent l'intérêt de ce dernier pour les arts graphiques traditionnels et modernes ${ }^{32}$. Certains vont jusqu'à proposer des comparaisons directes entre ce texte et Le Cri de Münch ou la peinture de Van $\mathrm{Gogh}^{33}$. Certes, Lu Xun manifestait un intérêt certain pour des questions d'esthétique, et ce dans une optique politique, pédagogique ${ }^{34}$ et par goût : en effet, dès les années 1920, il avait connaissance des arts modernes et de la peinture chinoise de l'époque, et dès le début des années 1930, il œuvrait à la promotion du mouvement pour la gravure moderne en Chine ${ }^{35}$. Cependant, il reste difficile d'établir un lien génétique objectif entre ce texte de 1925 et un artiste ou une œuvre précis. Pour justifier ce rapprochement intuitif entre ce texte et de telles esthétiques, il faut s'appuyer sur les indices d'un style "kinésique», propres à induire un sens du mouvement et de la corporalité chez le lecteur, qui sont notamment : le téléscopage d'éléments contraires, de la tension et de la distorsion; la condensation du récit; le thème $d u$ "cri » silencieux ${ }^{36}$. Sans chercher à désigner cette fois-ci d'hypotexte artistique qui ferait l'objet d'une transmédialisation en règle, nous allons interroger le dénominateur commun, qui rend possible cette intuition de proxémie esthétique entre ce texte et des media non textuels ${ }^{37}$.

24 La lecture de "Tremblement " est conditionnée par la frustration du mouvement (l'immobilité en somme), qui stimule l'intelligence kinésique et la perception du 
lecteur. Il s'agit moins de relever tous les éléments qui construisent le mouvement du plus physique ou " concret » au plus symbolique ou dérivé, que d'analyser la succession des actions liées audit mouvement. Les deux scènes ou rêves, présentent des structures similaires. Le premier rêve (absent de l'extrait donné supra) construit un rythme fondé sur la série suivante: tremblement/ crainte ou stupeur/ image des vagues / tremblement / image des vagues / image du tourbillon. Le même schéma se répète dans le deuxième rêve, avec de surcroît l'alternance entre motifs opposés de la convulsion et de l'immobilité. Les images des vagues et du cyclone, qui clôturent le rêve, tendent à une expression de plus en plus symbolique et abstraite du mouvement. Concentronsnous ici sur un passage de l'extrait donné plus haut :

Tandis qu'elle proférait ce langage sans paroles, son corps, majestueux comme une statue de pierre [如石像 / ru shixiang], pourtant dévasté et ravagé, se mit tout entier à trembler [顫動 / chandong]. Ce tremblement [chandong] se marquait de point en point [點點 / diandian] comme des écailles [如魚鱗 / ru yulin], dont chacune ondulait comme de l'eau bouillonnant sur un feu ardent; aussitôt l'espace fut pris d'une même vibration, on eût dit les ondes d'une mer désolée battue par la pluie d'une tempête.

Alors elle leva les yeux au ciel, et son langage sans paroles se tut et cessa définitivement, il n'y avait que le tremblement [chandong], irradiant comme les rayons du soleil, qui faisait aussitôt refluer les vagues dans le ciel, comme si elles avaient rencontré un cyclone, et déferlaient vers l'étendue déserte et sans fin.

La représentation du tremblement suit un "processus de symbolisation » (F. Jullien) ${ }^{38}$ et d'abstraction, le même que celui qui anime le paysage. En voici les étapes :

1. Le mouvement est exprimé par un verbe, " [se mit à] trembler " / chandong, tandis que le corps est « comme une statue de pierre » / ru shixiang, comparaison qui connote l'immobilité. Dans cette proposition, et le verbe et l'image renvoient au corps, pour construire une tension entre son immobilité et son mouvement.

2. La substantivation du verbe (« ce tremblement " / chandong): le mouvement, et non plus le corps, devient thème ou sujet du verbe «se marquer de point en point» ou "marqué de points " / diandian ( goutter ", ou " former des points », rendant ainsi un aspect visuel). Il est à relever que chandong peut être verbe ("trembler ») ou substantif («tremblement ») - à la seconde occurrence, la substantivation est indiquée par le démonstratif « ce » / 這 / zhei, qui précède chandong.

3. La comparaison des "écailles » de l'action de diandian : "comme des écailles de poisson » / ru yulin renvoie au mouvement et non plus au corps statique, dont il se dissocie: le mouvement, progressivement abstrait du corps, se fait autonome, puis, dans un mouvement centrifuge et ondulatoire, est rejeté à l'extérieur du corps.

4. La métaphore du mouvement de "pointage " fait à son tour l'objet d'une métaphorisation (l'eau frémissante): «chacune [des écailles] ondulait comme de l'eau bouillonnant sur un feu ardent » / 每一鱗都起伏如沸水在烈火上 / Mei yilin dou qifu ru feishui zai liehuoshang. Dans l'extrait cité supra, cette métaphorisation du mouvement se fait toujours plus abstraite :

il n'y avait que le tremblement [chandong], irradiant comme les rayons du soleil, qui faisaient aussitôt refluer les vagues dans le ciel, comme si elles avaient rencontré un cyclone, pour déferler vers l'étendue déserte et sans fin.

惟有顫動, 輻射若太陽光, 使空中的波譸立刻回旋, 如遭風風, 汹湧奔騰於無邊 的荒野

26 Les métaphores sont à présent celles d'une irradiation, d'un «cyclone » et du reflux de «vagues » qui annulent toute distinction entre le corps déchu de la femme et l'univers autour d'elle: la tension distord le corps pour s'en échapper et irradier autour du personnage. Se font jour deux séries parallèles de plus en plus abstraites : au niveau 
syntaxique (verbe $\rightarrow$ substantivation $\rightarrow$ sujet $\rightarrow$ métaphore, voire métaphore de la métaphore, comme le mouvement corporel devenu séparé du corps), et lexical ou thématique (corps de pierre $\rightarrow$ tremblement $\rightarrow$ mouvement toujours plus subtil, jusqu'à l'irradiation). La métaphore filée tout au long du passage a trait en particulier à l'eau et au feu, deux éléments contraires : la suggestion du mouvement repose sur le processus de métaphorisation autant que des verbes qui établissent ainsi une série d'oppositions et de tensions simultanées (pierre et immobilité/mouvement; eau/feu; reflux). Cette même tension se retrouve dans les sentiments et souvenirs contraires éprouvés simultanément par le personnage (voir notamment le second paragraphe de la traduction) - au niveau « psychologique » donc.

Consécutivement à cette abstraction du mouvement, le référent physiologique et corporel s'efface toujours plus pour devenir représentation formelle d'une pure tension antagonique (reflux et cyclone). Ce processus de symbolisation exprime le «désordre psychique » du personnage du rêve et du narrateur. Ce sens de la tension, produit par cette série de contradictions ${ }^{39}$, atteint son apogée avec l'image finale cinétique de lignes tourbillonnantes, centrifuges, opposées les unes aux autres. Les causes physiologiques avancées par le narrateur à la clôture de chaque segment de rêve présentent des différences importantes : la suffocation buccale et nasale à la fin du premier rêve ; puis, la poitrine oppressée, et les mains pesantes que le dormeur n'arrive pas à soulever. Les efforts du dormeur pour lever ses mains pesantes et immobiles se lisent en filigrane de la tension entre le tremblement, ou mouvement efforcé, et l'immobilité de la femme. Cependant, plus encore que de témoigner d'un tel processus de symbolisation du physiologique vers l'abstrait, et d'en reproduire le mécanisme de façon mimétique, ce texte exprime un sens proprement kinésique de la distorsion et de l'antagonisme expérience à l'origine même de l'imagination créatrice de ce récit. Cette association du mouvement circulaire et de l'immobilité, de l'alternance et la juxtaposition des contraires signe ainsi le «style kinésique » de ce texte.

Pour qualifier ce style en ses tropismes, ce texte doit être lu dans la perspective du concept moderniste de «dépression », «mélancolie» ou «angoisse » / 苦悶 présenté dans Le Symbole de la mélancolie / 苦悶的象徵, que Lu Xun traduit en chinois à partir de 苦悶の象徵 (1921) du critique littéraire japonais Kuriyagawa Hakuson 廚川白村 et a contribué largement à introduire en Chine ${ }^{40}$. Ce terme voit en toute expression artistique la symbolisation d'un spleen issu de l'opposition entre l'élan vital et les obstacles rencontrés par celui-ci. Les «tropes kinésiques» du "Tremblement» dessinent des tensions et conflits de mouvements contraires, à la fois physiologiques et psychiques. Elles signent l'expression sociale, individuelle, psychologique et physique du kumen dont souffre la protagoniste - et par association, le sujet du rêve et narrateur du récit, dont la femme est la persona $a^{41}$.

29 Le cri sans parole du personnage, dont on a vu qu'il constitue l'une des principales différences avec «La Fin du monde », exprime également la dimension métalittéraire de ce texte en tant que réflexion sur l'expression ${ }^{42}$ artistique. Les vagues du paysage extériorisent le mouvement interne du personnage, qui ne pourrait être formulé autrement: le mouvement qu'elles symbolisent pallie la parole défaillante du personnage, dont elles figurent le véritable discours, le cri silencieux. Cette ligne de mouvement centrifuge, proprement expressionniste, dit, tout comme le cri, une volonté d'extériorisation. Le texte prétend exprimer, outre l'expérience subjectiviste du kumen, 
un cri paradoxal au-delà du langage, et qui agisse sur le monde réel, tout comme le tremblement passant de la femme à l'espace environnant.

Cette expérience "moderne $»^{43}$ de tension intérieure appelle cette forme entre symbolisme et expressionnisme - deux esthétiques en principe contradictoire dans leur histoire européenne. Lu Xun transpose dans son écriture une esthétique inspirée des arts visuels modernes et du cinéma, de leur traitement technique de l'image. Le principe de transposition de l'effet esthétique visuel obéit, pour l'expression du kumen existentiel, à une tension et un antagonisme qui se situe à l'horizon de l'expérience littéraire. «Le Tremblement» de Lu Xun manifeste la volonté de reproduire ou transposer telle peinture ou gravure qui exprime graphiquement le cri: on peut chercher de possibles équivalents picturaux dans des gravures modernes, chinoises ou non, qui produisent pareils effets de mouvement par des lignes, cercles, angles ou autres formes dynamiques heurtant et guidant le regard ${ }^{44}$. Bref, une forme induisant une sensation fondée sur la mémoire motrice du spectateur. Lu Xun affirme dans son écriture une modernité esthétique, tout autant futuriste, cubiste ou expressionniste, axée sur la corporalité - représentation et expression du corps - et du mouvement. Ce texte représente un tableau expressif qui, mobilisant l'intelligence kinésique du lecteur, produit en lui ce sens, et donc une expérience de choc et de tension ${ }^{45}: c^{\prime} e s t$ le principe organisateur qui explique également la réécriture du poème de Tourguéniev.

En fait, il faudrait étendre la question aux rapports transesthétiques entre littérature et arts visuels, mais aussi entre sanwenshi et théâtre : ce « Tremblement » de Lu Xun offre une dimension expressionniste dramatique, voire "biomécanique" en raison de l'importance donnée à la simplicité du dispositif narratif et au mouvement corporel ${ }^{46}$. L'inspiration cinématographique, de fait cinétique et technique avant que d'être traduction de contenu ${ }^{47}$, se fait surtout sur le mode lyrique et dramatique, non sans intégrer par son récit enchâssé une mise à distance, qui prend ainsi une dimension de «théâtre épique». La place manque ici pour continuer sur cette question de la

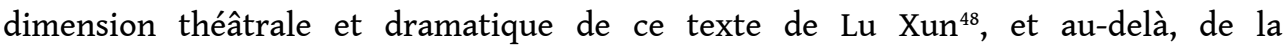
"transgénéricité $»^{49}$ caractéristique du recueil tout entier de La Mauvaise herbe. On relèvera toutefois comment par ce recours au mélange de genres et d'esthétiques en partie importés, Lu Xun construit une image du corps porteuse d'une parole ${ }^{50}$, ou plutôt d'une voix émancipatrice, qui transcende les conditionnements et mène l'expression écrite aux « marges de la représentation $»^{51}$.

\section{BIBLIOGRAPHIE}

ADMUSSEN Nick, « A Music for Baihua : Lu Xun's Wild Grass and 'A Good Story' », Chinese Literature: Essays, Articles, Reviews 31:2009, p. 1-22.

ANDERSON Marston, The Limits of Realism, Chinese Fiction in the Revolutionary Period, Berkeley, University of California Press, 1990.

BOLENS Guillemette, Le Style des gestes, Lausanne, Editions BHMS, 2008. 
CHEN-ANDRO Chantal, « La critique chinoise moderne et la fonction métaphorisante », in Regards sur la métaphore, entre Orient et Occident, SAKAI Cécile et STRUVE Daniel (éds.), Arles, Philippe Picquier, 2008.

CHow Rey, Primitive Passions. Visuality, Sexuality, Ethnography, and Contemporary Chinese Cinema, New York, Columbia University Press, 1995.

CUI Jianjun 催建军, [ «Sur le sens expressionniste de la caricature dans l'œuvre de Lu Xun »] / 论 鲁迅作品中的表现主义漫画感 / Lun Lu Xun zuopin zhongde biaoxian zhuyi manhuagan, 西安石油大 学学报 / Xi'an shiyou daxue xuebao 3:2005, p. 67-71.

CUI Yunwei 催云伟, [« Le sens graphique des gravures expressionnistes dans les œuvres de Lu Xun »] / 论鲁匈作品中的表现主义版画（木刻）感 / Lun Lu Xun zuopin zhongde biaoxian zhuyi banhua(muke)gan, 沈阳示范大学学报 / Shenyang shifan daxue xuebao 28.4, 2004, p. 59-63.

CUI Yunwei 催云伟, [《 Le sens pictural expressionniste dans les œuvres de Lu Xun »] / 论鲁旬作 品中的表现主义油画感 / Lun Lu Xun zuopin zhongde biaoxian zhuyi youhuagan, 山东示范大学学 报 / Shandong shifan daxue xuebao 51.2, 2006, p. 47-51.

DAVIES Gloria, Lu Xun's Revolution, Writing in a Time of Violence, Cambridge, Harvard U. P., 2013. GÁLIK Márian, Milestones in Sino-Western Literary Confrontation (1898-1979), Wiesbaden, Otto Harrassowitz 1986.

GOGOL Nicolas николАЙ гоголь, Nouvelles de Pétersbourg / [ПЕТЕРБУРГСКИЕ ПОВЕСТИ (1835-1843)] AUCOUTURIER Gustave, LUNEAU Sylvie et MONGAUlt Henri (trad.), Paris, Gallimard, 1979.

GU Dayong 古大勇, [《Lancer des cris de folle souffrance : sous le règne implicite de l'expressionisme : comparaison entre Munch et Lu Xun »] / 狂痛呐喊 : 在表现主义的潜法则下 一画家蒙克与作家鲁迅比较论 / Kuangtong nahan : zai biaoxian zhuyide qianzexia Huajia Mengke yu zuojia Lu Xun bijiaolun, 伊梨示范学院学报 / Yili shifan xueyuan xuebao 2:2004, p. 64-70.

GU Dayong 古大勇, [《Les “appels” de Lu Xun et le “cri” de Munch »] / 鲁忒的呐喊与蒙克的呼 嚎 / Lu Xunde Nahan yu Mengkede Huhao, 兰州大学学报 / Lanzhou daxue xuebao 29.5, 2001, p. 11-15. HANAN Patrick, “The Technique of Lu Hsün's Fiction”, Harvard Journal of Asian Studies 34:1974, p. 53-96.

HAY John, “The Body Invisible in Chinese Art?" in Body, Subject, and Power in China, BARLow Tani and zITo Angela (eds.), Chicago, Chicago U. P., 1994, p. 42-77.

носкX Michel, Questions of Style, Literary Societies and Literary Journals in Modern China, 1911-1937, Leiden, Brill, 2003.

HSIA T. A., The Gate of Darkness, Studies in the Leftist Literary Movement in China, Seattle, Washington U. P., 1968.

JORTAY Coraline, Pronominal Politics: (Un)Gendering Narrative and Framing Ambiguity in Chinese Literature, 1917-1937, thèse de doctorat, Université Libre de Bruxelles, 2020.

JULLIEN François, Ecriture et révolution, Paris, Presses de l'École normale supérieure, 1979.

JULLIEN François, De l'essence ou du nu, Paris, Seuil, 2000.

KALDIS Nicholas, The Chinese Prose Poem. A Study of Lu Xun's Wild Grass (Yecao), Amherst, Cambria Press, 2014. 
KANDINSKY Vassily, Du Spirituel dans l'art, et dans la peinture en particulier, Paris, Folio / Denoël, 1994 [1910].

LEYS Simon [Pierre Ryckmans] (trad.), La Mauvaise herbe, Paris, 10/18, 1975.

Lu Xun 鲁迅, [®uvres complètes de Lu Xun] / 鲁旬译文全集 / Lu Xun yiwen quanji, Fuzhou, Fujian jiaoyu chubanshe, 2008, 8 vol.

Lu Xun 鲁旬, [Traductions complètes de Lu Xun] / 鲁旬译文全集 / Lu Xun yiwen quanji, Fuzhou, Fujian jiaoyu chubanshe, 2008, 8 vol.

LU Xun 鲁迅, [La Mauvaise herbe] / 野草 / Yecao, Beijing, Renmin wenxue chubanshe, 2006.

LU Xun 鲁迅, [La Mauvaise herbe] / 野草 / Yecao, Beijing, Renmin wenxue chubanshe, 2006.

MARIN Louis, De la représentation, Paris, EHESS/Seuil/Gallimard, 1994.

MONCOND'HUY Dominique et SCEPI Henri (éds.), Les Genres de travers. Littérature et transgénéricité, Rennes, P. U. de Rennes, 2008.

PLANA Muriel, Roman, théâtre, cinéma. Adaptations, hybridations et dialogue des arts, Rosnysous-Bois, Bréal, 2004.

PRŮŠEK Jaroslav, “'Huai chiu': A Precursor of Modern Chinese Literature”, Harvard Journal of Asian Studies 29:1969, p. 169-176.

PRŮŠEK Jaroslav, The Lyrical and the Epic, Studies of Modern Chinese Literature, LEE Leo Ou-fan (éd.), Bloomington, Indiana U. P., 1980.

QIU Sha 装沙, [La réflexion de Lu Xun sur l'éducation esthétique] / 鲁迅美术教育思想浅谈 / Lu Xun meishu jiaoyu sixiang qiantan, 北方美术 Beifang meishu 3:2000, p. 56-57.

RABUT Isabelle et PINO Angel (éds.), Pékin - Shanghai. Tradition et modernité dans la littérature chinoise des années trente, Paris, Bleu de Chine, 2000.

RICHARD Jean-Pierre, Littérature et sensation, Paris, Seuil, 1954.

ROUSSET Jean, Forme et signification, Paris, José Corti, 1995.

SEMANOv Vladimir, Lu Hsün and his Predecessors, ALBER Charles J. (trad.), New York, M. E. Sharpe, 1980.

SUN Mei 孙政, [ «'opéra chinois dans le théâtre mondial au xx s. »] /二十世纪世界戏剧中的中 国戏曲 / Ershi shiji shijie zhongde Zhongguo xiqu, 二十一世纪双月刊 / Ershiyi shiji shuangyue kan 45:1998, p. 103-112.

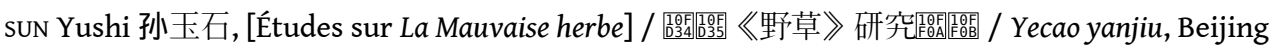
daxue chubanshe, 2007 [1980], p. 222-261.

SUN Yu 孙郁, [Catalogue des peintures collectionnées par Lu Xun] / 鲁忒藏画录 / Lu Xun canghua lu, Guangzhou, Huacheng chubanshe, 2008.

TANG Xiaobing, Origins of the Chinese Avant-Garde. The Modern Woodcut Movement, Berkeley, California U. P., 2008.

TOURGUÉNIEV Ivan, Poèmes en prose / [Senilia. СТИХОТВОРЕНИЯ В ПРОЗЕ (1877-1882)], SALOMON Charles et zViguilsky Alexandre (trad.), Paris, La Différence, coll. « Orphée », 1990.

TOURGUÉNIEV IVAN, [Poèmes en prose] / 屠格涅夫散文诗集, XU Weinan 徐蔚南 et WANG Weike 王维 克 (trad.), Shanghai, Xin wenhua shushe, 1923. 
TRETIAKOv Serge M., Hurle, Chine ! et autres pièces : Écrits sur le théâtre, AMIARD-CHEVREL C. et al. (trad.), Lausanne, L'Âge d'Homme, 1982.

TURGENJEFF Iwan, Gedichte in Prosa, LANGE Wilhelm (trad.), Leipzig, Reclam, 1883.

VEG Sebastian (trad et commentaire), Lu Xun Nouvelles et poèmes en prose, Paris, Éditions Rue d'Ulm, 2015.

VouILLoux Bernard, La Peinture dans le texte. XVIII ${ }^{e}-\mathrm{XX}^{e}$ siècles, Paris, CNRS Éditions, 2005.

vouilLoux Bernard, Langages de l'art et relations transesthétiques, Paris, Éditions de l'Éclat, 1997.

VUILLEUMIER Victor, «Body, Soul, and Revolution », in Chinese Revolution and Chinese Literature, TAO Dongfeng, YANG Xiaobin et ROSEMARY Roberts (éds.), Newcastle upon Tyne, Cambridge Scholars Publishing, 2004, p. 49-56.

VUILLEUMIER Victor, « Xu Zhimo’s Encounter with ‘A Jewish Nightmare' - How a Chinese Symbolist Viewed an Expressionist Yiddish Play ", Studia Orientalia Slovaca 9.2, 2010, p. 145-155. https://fphil.uniba.sk/fileadmin/fif/katedry_pracoviska/kvas/SOS_9_2/N3-18vuilleumierform_1_.pdf, consulté le 01/07/2021.

VUILLEUMIER Victor, «Ai Qing's poetry and Dayanhe, My Nurse », in Routledge Handbook of Modern Chinese Literature, GU Ming Dong (éd.), Londres, Routledge, 2018.

VUILLEUMIER Victor, « Le corps souffrant chez Lu Xun : allégorie muette de l'obstacle et appropriation de la modernité », Extrême-Orient Extrême Occident 39:2015, p. 47-84.

WANG Xin 王新, [« La lutte éternelle entre le ciel et la terre : approche de La Mauvaise herbe à partir du motif du mouvement de Munch»] / 大地与天空的永恒争执一从蒙克的波荡意象解读 鲁迅的野草 / Dadi yu tiankongde yongheng zhengzhi Cong Mengkede bolanxiang jiedu Lu Xun de Yecao, in 名作欣赏 / Mingzuo xinshang 4:2006, p. 31-34.

WANG Xirong 王锡荣, [Lu Xun dessinateur] / 鲁迅画者 / Lu Xun huazhe, Shanghai, Shanghai wenhua chubanshe, 2006.

WANG Yao 王耀, [Æuvres complètes de Wang Yao] / 王耀全集 / Wang Yao Quanji, Shijiazhuang, Hebei jiaoyu chubanshe, 1999, 8 vol.

WANG Zhipeng 王志鹏, [« Particularité de la forme et profondeur de l'expression : expressionisme et réalisme dans l'écriture de fiction luxunienne»] / 格式的特别与表现的深切一浅论鲁旬小说 创作中的现实主义与现代主义 / Geshide tebie yu biaoxiande shenqie Qianlun Lu Xun xiaoshuo chuangzuo zhongde xianshi zhuyi yu xiandai zhuyi, [Journal du Collège professionnel de Binzhou] / 滨州职业学院学报 Binzhou zhiye xueyuann xuebao 2.1, 2005, p. 20-22.

WU Tonghu 吴铜虎, [《L'essence picturale de Van Gogh et Munch dans la composition d'écriture de Lu Xun »] / 鲁迅文字构图中的蒙克与梵高的绘画特质, 名作欣赏 / Mingzuo xinshang 8:2009, p. 70-74.

WU Tonghu 吴铜虎, [《La couleur de Munch et la ligne de Van Gogh »] / 蒙克的色彩与梵高的线 条 / Mengke de secai yu Fan Gao de xiantiao, 贵州示范大学学报 / Guizhou shifan daxue xuebao 5:2009, p. 89-92.

WU Zhongjie 吴中杰 (éd.), [Poèmes et prose de Lu Xun, annotés et commentés par Wu Zhongjie] / 吴中杰评点鲁迅诗歌散文 / Wu Zhongjie pingdian Lu Xun shige sanwen, Shanghai, Fudan daxue chubanshe, 2006.

YANG Yi 杨义, [Histoire de la fiction chinoise moderne] / 中国现代小说史 / Zhongguo xiandai xiaoshuo shi, Beijing, Renmin chubanshe, 1998, 3 vol. 
YAO Xipei 姚锡佩, [ “Ce que la bibliothèque de Lu Xun nous apprend sur ses relations littéraires à Tourguénief (1 $1^{\text {ère }}$ partie) »] / 从藏书看鲁间与屠格涅夫的文学渊源 (上) / Cong cangshu kan Lu Xun yu Tugeniefude wenxue yuanyuan (shang), 鲁迅研究月刊 / Lu Xun yanjiu yuekan 3:1990, p. 56-61.

ZHANG Ailing 張愛玲, [Un amour à renverser les cités] / 傾城之戀 / Qingcheng zhi lian, Taibei, Huangguan wenhua chubanshe, 1991.

ZHANG Yinde, «L'École de Shanghai : le modernisme en question », Littérature comparée et perspectives chinoises, Paris, L'Harmattan, 2008.

\section{NOTES}

1. vouilloux Bernard, Langages de l'art et relations transesthétiques, Paris, Éditions de l'Éclat, 1997, p. 16-17.

2. PlANA Muriel, Roman, théâtre, cinéma. Adaptations, hybridations et dialogue des arts, Rosny-sousBois, Bréal, 2004, p. 17-19.

3. RABUT Isabelle, PINO Angel (éds.), Pékin - Shanghai. Tradition et modernité dans la littérature chinoise des années trente, Paris, Bleu de Chine, 2000.

4. YANG Yi 杨义, [Histoire de la fiction chinoise moderne] / 中国现代小说史, Beijing, Renmin chubanshe, 1998, vol. 3, p. 472. Par exemple dans [《La Cangue dorée»] / 金鎖記 (1943), une phrase condense une ellipse de dix ans dans l'image d'un miroir reflétant divers objets et un personnage: ZHANG Ailing 張愛玲, [Un amour à renverser les cités] / 傾城之戀, Taibei, Huangguan wenhua chubanshe, 1991, p. 156.

5. Voir ZHANG Yinde, «L'Ecole de Shanghai : le modernisme en question », Littérature comparée et perspectives chinoises, Paris, L'Harmattan, 2008, p. 151-169.

6. Chantal CHEN-ANDRO, "La critique chinoise moderne et la fonction métaphorisante ", in Regards sur la métaphore, entre Orient et Occident, SAKAI Cécile et STRUVE Daniel (éds.), Arles, Picquier, 2008, p. 134 ; VUILLEUMIER Victor, "Ai Qing's poetry and Dayanhe, My Nurse ", in Routledge Handbook of Modern Chinese Literature, GU Ming Dong (éd.), Londres, Routledge, 2018, p. 235-246 ; VUILLEUMIER Victor, «Body, Soul, and Revolution », in Chinese Revolution and Chinese Literature, TAo Dongfeng, YANG Xiaobin, ROBERTS Rosemary (eds.), Newcastle upon Tyne, Cambridge Scholars Publishing, 2004, p. 49-56.

7. VUILleUMIER Victor, « Xu Zhimo's Encounter with 'A Jewish Nightmare' - How a Chinese Symbolist Viewed an Expressionist Yiddish Play », Studia Orientalia Slovaca, 9.2, 2010, p. 145-155 ; https://fphil.uniba.sk/fileadmin/fif/katedry_pracoviska/kvas/sos_9_2/N3-18vuilleumier-

form_1_.pdf, consulté le 01/07/2021.

8. Sur les rapports entre nu et représentation mimétique, $c f$. JULLIEN François, De l'essence ou du nu, Paris, Seuil, 2000.

9. Cf. HAY John, "The Body Invisible in Chinese Art?" in Body, Subject, and Power in China, BARLow Tani and ziтo Angela (eds.) Chicago, Chicago U. P, 1994, p. 42-77.

10. Lu Xun, [La Mauvaise herbe] / 野草, Beijing, Renmin wenxue chubanshe, 2006, p. 47-49. Publié dans la revue [Au Fil des mots] / 語絲 35 (13 juillet 1925), puis repris dans Yecao. Voir les traductions françaises : "Tremblements avant la déchéance », Lu Xun Nouvelles et poèmes en prose, VEG Sebastian (trad.), Paris, Éditions Rue d'Ulm, 2015, p. 546-548 (traduction) et p. 598-600 (commentaire) ; « Un tremblement au bord de l'abîme », LEYS Simon (trad.) [Pierre Ryckmans], La Mauvaise herbe, Paris, 10/18, 1975, p. 97-100.

11. J. Průšek voit dans la littérature chinoise moderne, en particulier chez Lu Xun, le fruit d'un développement remontant aux Ming 明 (1368-1644), celui du «lyrisme », dont la "modernité » consiste à reléguer au second plan l'histoire («l'épique ») : v. PRŮŠEK Jaroslav, «'Huai chiu' : A 
Precursor of Modern Chinese Literature ", Harvard Journal of Asian Studies, 29:1969, p. 169-176 ; The Lyrical and the Epic, Studies of Modern Chinese Literature, LEE Leo Ou-fan (éd.), Bloomington, Indiana U. P., 1980.

12. Lu Xun lui-même emploie à propos de son recueil le terme de sanwenshi, importé et revendiqué par les auteurs chinois modernes. Il a pu être "défini » dans les années 1920 de différentes façons, entre autres comme de la poésie en prose en "vers libre » : il est bien difficile d'appliquer une définition unique pour toutes les expérimentations littéraires réalisées alors en Chine, et classées par les intéressés eux-mêmes dans cette catégorie protéiforme (Baudelaire luimême cherche, en insistant sur le terme de "poème en prose ", à brouiller les distinctions classiques entre les genres : v. BRIX Michel, Poème en prose, vers libre et modernité littéraire, Paris, Kimé, 2014, p. 13-79). Ce genre moderniste, souple dans ses acceptions hors de chine, l'est d'autant plus dans la pratique et la compréhension chinoises modernes. Le "poème en prose " chinois présente donc une grande variété de formes, ne serait-ce que dans Yecao. Liu Bannong 劉 半農 (1891-1934) est l'un des premiers à s'essayer à ce nouveau genre dès le début des années 1920 mais ne publie ces textes qu'en 1926, tandis que Lu Xun publie dès 1919 ses premiers poèmes en prose, dont Sun Yushi a montré la parenté avec La Mauvaise herbe. Le Spleen de Paris (1869) est accessible avant 1922 aux auteurs chinois, dont Lu Xun, qui lisent l'original ou des traductions en d'autres langues, et des pièces, traduites en chinois dès cette date. Liu Bannong traduit de premiers poèmes en prose de Tourguéniev dès 1915 ; Tagore est aussi traduit dès la même année. V. ADMUSSEN Nick, "A Music for Baihua: Lu Xun's Wild Grass and 'A Good Story' ", Chinese Literature: Essays, Articles, Reviews 31:2009, p. 1-22 ; HOCKX Michel, Questions of Style, Literary Societies and Literary Journals in Modern China, 1911-1937, Leiden, Brill, 2003, p. 162-166, 175-178, 183-184 ; KALDIS Nicholas, The Chinese Prose Poem. A Study of Lu Xun's Wild Grass (Yecao), Amherst, Cambria Press, 2014, p. 95-124 ; VEG, Lu Xun Nouvelles et poèmes en prose, op. cit., p. 629-630 ; sUN Yushi 孙玉 石, [Études sur La Mauvaise herbe] / 《野草》研究/ Yecao yanjiu (1980), Beijing daxue chubanshe, 2007, p. 222-261.

13. LU, Yecao, op. cit., p. 48 ; LEYS, La Mauvaise herbe, op. cit., p. 98.

14. LU, Yecao, op. cit., p. 49. Ma traduction, volontairement littérale, pour les besoins de la présente analyse. Pour des traductions existantes, voir les références bibliographiques.

15. Sur la construction et la (ré)invention des pronoms personnels à la période républicaine, en particulier dans une perspective genrée, voir JORTAY Coraline, Pronominal Politics: (Un)Gendering Narrative and Framing Ambiguity in Chinese Literature, 1917-1937, thèse de doctorat, Université Libre de Bruxelles, 2020.

16. Par exemple, dans la phrase « la vague » «finit par tout engloutir, moi-même y compris », le segment « moi-même y compris » / yiqie bing wo (litt. " tout et même moi avec »), suggère par sa concision quasiment classique (quatre caractères) un effet d'accélération dramatique.

17. Traduction française: TOURGUÉNIEV Ivan, Poèmes en prose, SALOMON Charles et ZVIGUILSKY Alexandre, (trad.), Paris, La Différence, coll. « Orphée », 1990, p. 45-47. J'ignore si cette relation a déjà été relevée ailleurs ou étudiée en détail.

18. Iwan tURGENJEFF [Ivan Tourguéniev], Gedichte in Prosa, LANGE Wilhelm (trad.), Leipzig, Reclam, 1883.

19. Ce n'est dans les années 1930 qu'il fait l'acquisition de traductions japonaises. Par ailleurs, son frère Zhou Zuoren possédait déjà au Japon une série d'œuvres choisies de l'auteur russe traduites en anglais. Voir YAO Xipei 姚锡佩, [“Ce que la bibliothèque de Lu Xun nous apprend sur ses relations littéraires à Tourguénief (1 ${ }^{\text {ère }}$ partie) »] / M藏书看鲁迅与屠格涅夫的文学渊源 (上), 鲁迅研究月刊 / Lu Xun yanjiu yuekan 3:1990, p. 56.

20. Un premier recueil de traduction en chinois des poèmes en prose de Tourguéniev, contenant quarante pièces, est publié en 1923: TOURGUÉNIEV, [Poèmes en prose] / 屠格涅夫散文诗集, XU 
Weinan 徐蔚南 et WANG Weike 王维克 (trad.), Shanghai, Xin wenhua shushe, 1923. Ce texte y figure, sous le titre [La Fin du monde (un rêve)] / 世界之末期（一个梦）, p. 14-17.

21. TURGENJEFF, Gedichte in Prosa, op. cit., p. 25. Ma traduction, volontairement littérale, pour cet extrait et les suivants.

22. TURGENJEFF, Gedichte in Prosa, op. cit., p. 25.

23. Voir GÁLIK Márian, Milestones in Sino-Western Literary Confrontation (1898-1979), Wiesbaden, Otto Harrassowitz 1986, p. 24 sqq.

24. LEYS, La Mauvaise herbe, op. cit., p. 97.

25. VEG, Lu Xun Nouvelles et poèmes en prose, op. cit., p. 598.

26. Plus précisément, le réveil final du narrateur suffoquant dans «La fin du monde », est repris dans le premier réveil intermédiaire de Lu Xun. Ce motif du rêve interrompu et repris n'est pas sans rappeler «La Perpective Nevski » / НЕВСКИЙ ПРОспЕКт / Nievskiy prospekt (1835) (v. Nicolas GOGOL, Nouvelles de Pétersbourg, AUCOUTURIER Gustave, LUNEAU Sylvie et MONGAULT Henri (trad.), Gallimard, 1979, p. 43-91) de Gogol (je remercie Gérard Siary d'avoir attiré mon attention sur ce point) : le peintre idéaliste Piskariov, perdu dans la ville moderne (le Pétersbourg gogolien est le Shanghai de la littérature chinoise des années 1930, et même en fait depuis les années 1890), oppose à une réalité décevante un rêve continu, dans lequel la belle femme qu'il suit et qui s'avère vénale, devient une jeune femme irréprochable de la haute société, qui vient l'inviter en grand équipage. Il devient opiomane pour s'enfermer dans son rêve, et monnaie sa drogue contre des peintures très réalistes de femmes (ce qui aborde la question de la représentation et du réalisme). Lu Xun était un lecteur de Gogol, l'auteur du «Journal d'un fou » : ultérieurement, en 1934, il publie une traduction $\mathrm{du}$ " Nez » et laisse à sa mort une traduction inachevée des Âmes mortes; il avait prévu sans avoir pu mener à bien son projet, de publier un choix d'œuvres de Gogol. Le "Tremblement » et «La Perspective Nevski» présentent un faisceau de thèmes communs : la frustration causée par la vie moderne, l'opposition entre idéal et réalité, le thème de la prostitution. Ajoutons que le peintre, à la vue de son idéal, " frissonne », et est déçu lorsque la femme se met à " parler ", car sa voix porte en un raccourci, et révèle, toute sa vie de débauche et prostitution. Non sans ironie renversée de la part de Lu Xun, le narrateur du " Tremblement » rêve non d'une belle qui vient le séduire, mais bien d'une pitoyable et réaliste prostituée déchue et muette. À un niveau esthétique, la nouvelle de Gogol construit un même effet kaléidoscopique de perceptions (v. préface de G. Nivat à la traduction), forme de néo-sensationnisme avant l'heure, ainsi que les thèmes de la dissociation et du double - propres à la « matière russe » que l'on trouve reprise ailleurs chez Lu Xun.

27. Voir ANDERSON Marston, The Limits of Realism, Chinese Fiction in the Revolutionary Period, Berkeley, California U. P., 1990.

28. Les commentateurs de Lu Xun interprètent, dans ce texte qualifié de "symboliste », la figure de la vieille femme (ou mère) comme une dénonciation de la condition des femmes dans la société ou du manque de reconnaissance des sacrifices consentis pour la jeunesse (ce dernier point implique une perspective autobiographique), ou plus généralement une critique sociale et intellectuelle : voir KALDIS, The Chinese Prose Poem..., op. cit., p. 230-237 ; sun, ‘野草’研究 / Yecao yanjiu, op. cit., p. 106-110 ; VEG, Lu Xun Nouvelles et poèmes en prose, op. cit., p. 598-600 ; wU Zhongjie 吴中杰 (éd.), 吴中杰评点鲁旬诗歌散文, Shanghai, Fudan daxue chubanshe, 2006, p. 244-249.

29. L'intelligence kinésique désigne la capacité de percevoir chez autrui, et en l'occurrence dans un texte littéraire, des mouvements corporels, des "stimulations perceptives", ce qui permet ensuite de définir un "style kinésique», caractérisé par des «tropes" identifiables et particuliers, qui soit propre à un texte ou une œuvre : voir BoLENS Guillemette, Le Style des gestes, Lausanne, Editions BHMS, 2008.

30. LU Xun, Yecao, op. cit., p. 48-49. Ma traduction, pour les besoins de la présente analyse. 
31. Le récit «L'exemple» / 示罗 (13 avril 1925) rappelle un travail de «caméra »: v. HANAN Patrick, «The Technique of Lu Hsün's Fiction », Harvard Journal of Asian Studies 34:1974, p. 89, qui relève les qualités « expressionniste » et «symboliste » de l'écriture de Lu Xun.

32. Lu Xun publie en 1928 la traduction de l'ouvrage de Itagaki Takaho 板坦鷹穗 (1894-1966) [Courants dans l'histoire de l'art moderne] / 近代美術史潮論 (1927), et avant cela en 1924-1925, [Le Symbole de la mélancolie] / 苦悶的象徵 jp. 苦悶の象徽 et [Pour sortir de la Tour d'ivoire] / 出了象牙之塔 jp. 象牙の塔を出て (1920) de Kuriyagawa Hakuson 麻川白村 (1880-1923), qui traitent des arts. Il fréquente des artistes tels 林風眠 Lin Fengmian (1900-1991), 陶元慶 Tao Yuanqing (1893-1929), qui sera l'un de ses illustrateurs, ou encore 司徒橋 Situ Qiao (1902-1958), qu'il collectionne. Il acquiert ultérieurement une série de gravures de l'artiste allemande Käthe Kollwitz (1867-1945), qu'il présente dans certains de ses écrits, et dont à la fin de sa vie il prévoyait d'éditer un volume. Il a par ailleurs connaissance de l'œuvre de Münch, au moins dès les années 1930. V. SEMANov Vladimir, Lu Hsün and his Predecessors, ALBER C. J. (trad.), New York, M. E. Sharpe, 1980, p. 100-101; sUN Yu 孙有, [Catalogue des œuvres conservées par Lu Xun] / 鲁式藏 画录, Guangzhou, Huacheng chubanshe, 2008 ; TANG Xiaobing, Origins of the Chinese Avant-Garde. The Modern Woodcut Movement, Berkeley, California U. P., 2008 ; WANG Xirong 王锡荣, 鲁迅画者 / Lu Xun huazhe, Shanghai, Shanghai wenhua chubanshe, 2006.

33. CUI Jianjun 催建军, CUI Yunwei 催云伟, 《论鲁迅作品中的表现主义漫画感》, 西安石油大学学 报 / Xi'an shiyou daxue xuebao 3:2005, p. 67-71; cUI Yunwei 催云伟, 《论鲁迅作品中的表现主义版 画（木刻）感》, 沈阳示范大学学报 / Shenyang shifan daxue xuebao,28.4, 2004, p. 59-63 ; 《论鲁迅 作品中的表现主义油画感》, 山东示范大学学报 / Shandong shifan daxue xuebao 51.2, 2006, p. 47-51; GU Dayong 古大勇, 《狂痛呐喊 : 在表现主义的潜法则下一画家蒙克与作家鲁迅比较 论》, 伊梨示范学院学报 / Yili shifan xueyuan xuebao 2:2004, p. 64-70; 《鲁迅的呐喊与蒙克的呼 嚎》, 兰州大学学报 / Lanzhou daxue xuebao 29.5, 2001, p. 11-15 ; wANG Zhipeng 王志鹏, 《格式的特 别与表现的深切一浅论鲁迅小说创作中的现实主义与现代主义》, 滨州职业学院学报 / Binzhou zhiye xueyuann xuebao, 2.1, 2005, p. 20-22 ; WANG Xin 王新, «大地与天空的永恒争执一从蒙克的波 荡意象解读鲁迅的野草 》, 名作欣赏 / Mingzuo xinshang 4:2006, p. 31-34 ; wU Tonghu 吴铜虎, 《鲁 迅文字构图中的蒙克与梵高的绘画特质》, 名作欣赏 / Mingzuo xinshang 8:2009, p. 70-74; 《蒙克 的色彩与梵高的线条》, 贵州示范大学学报 / Guizhou shifan daxue xuebao 5:2009, p. 89-92.

34. QIU Sha 装沙, 《鲁迅美术教育思想浅谈》 / Lu Xun meishu jiaoyu sixiang qiantan, 北方美术 / Beifang meishu 3:2000, p. 56-7 ; TANG, Origins of the Chinese Avant-Garde..., op. cit., p. 12-3.

35. TANG Xiaobing, op. cit.

36. Sur le cri, voir DAviES Gloria, Lu Xun's Revolution, Writing in a Time of Violence, Harvard U. P., 2013, p. 256 ; TANG, Origins of the Chinese Avant-Garde..., op. cit., p. 213-227.

37. La lecture d'une œuvre, sa visualisation ou son spectacle, est en soi une forme de transmodalisation intérieure (v. PLANA, Roman, théâtre, cinéma..., op. cit., p. 19) : on met le texte en scène comme une pièce, on perçoit des mouvements dans une image et l'on saisit des «structures» (v. ROUSSET Jean, Forme et signification, Paris, José Corti, 1995). Analyser le sens du mouvement d'un texte demande de prendre en compte et de reconstituer la dimension générale de la «sensation » (v. RICHARD Jean-Pierre, Littérature et sensation, Paris, Seuil, 1954 ; vouILloux Bernard, La Peinture dans le texte. XVIII ${ }^{e}-X^{e}$ siècles (1994), Paris, CNRS Éditions, 2005, p. 94-102), qui fonde l'expérience du sujet; ce travail suppose un sens, une intelligence synesthésique première en amont de ses déclinaisons possibles selon les différents sens (que l'on pense à la théorie de Kandinsky sur les correspondances motrices ou auditives des couleurs et formes différentes, KANDINSKY Vassily, Du Spirituel dans l'art, et dans la peinture en particulier (1910), Paris, Folio / Denoël, 1994), puis selon des contenus et des modes artistiques différents, ce qui explique que l'on perçoive une proximité esthétique entre différents media. 
38. Sur ce processus à l'œuvre dans l'ensemble du texte, dans un sens freudien, voir JULLIEN François, Écriture et révolution, Paris, Presses de l'École normale supérieure, 1979, p. 79-80, qui développe le système des images contraires. Je reprends son terme pour insister essentiellement sur le processus d'abstraction du mouvement corporel "pur», formel, qui est l'objet de la description. Pour une lecture psychanalytique de ce texte, voir KALDIS, The Chinese Prose Poem, op. cit., p. 230-237.

39. Voir JULLIEN, Écriture et révolution, op. cit., p. 89.

40. Deux traductions chinoises au début des années 1920 précèdent celle de Lu Xun de 1924-1925, qui utilise cette théorie dans son enseignement sur la littérature à l'Université de Pékin en 1925. Sur l'introduction du concept de kumen en Chine, v. TSU Jing, Failure, Nationalism, and Literature, Stanford, Stanford U.P., 2005, p. 195-221; ZHANG Jingyuan, Psychoanalysis in China, Literary Transformations, 1919-1949, Ithaca, Cornell University, 1992, p. 57-68. Selon Kuriyagawa (dans la traduction de Lu Xun, 鲁间译文全集 / Lu Xun yiwen quanji, Fuzhou, Fujian jiaoyu chubanshe, vol. 2, 2008, p. 217-280), toute création artistique est expression symbolique du kumen, le produit des résistances et obstacles que rencontre la force créatrice et vitale de l'individu. Cette idée d'une force frustrée à l'œuvre dans la production artistique et littéraire est élaborée à partir de la théorie freudienne de la création comme sublimation de la libido et du trauma comme refoulement. Mais Kuriyagawa trouve la notion de libido freudienne trop limitée: sa "force vitale " intègre "l'élan vital» de Bergson et l'affirmation nietzschéenne de l'individu. Pour Kuriyagawa, l'art est le symbole et la sublimation de cette force vitale et créatrice frustrée. Celleci se comprend non seulement comme souffrance existentielle, mais aussi comme lutte entre le désir d'expression et d'affirmation créatrice de l'individu d'une part, et la société d'autre part. De même, la vie dans une société « mécanisée » produit-elle l'aliénation du travail, qui est une autre dimension du kumen comme critique de la vie matérielle moderne.

41. Voir VEG, Lu Xun Nouvelles et poèmes en prose, op. cit., p. 599.

42. Le terme de biaoxian / 表現 signifie tout autant "expression» que « représentation»; il revient dans les discussions en Chine sur l'art et la gravure en particulier à la fin des années 1920 et au cours des années 1930, qui appellent à représenter quelque chose d'invisible qui puisse influencer le spectateur et dépasser la limite entre la vue et l'ouie (v. TANG Xiaobing, Origins of the Chinese Avant-Garde..., op. cit., p. 217-9). Le « Tremblement " précède le pic de ces débats; il semble pourtant que Lu Xun transpose ici cette problématique picturale en littérature.

43. HSIA T. A., The Gate of Darkness, Studies in the Leftist Literary Movement in China, Seattle, Washington U. P., 1968, p. 146-162.

44. TANG, Origins of the Chinese Avant-Garde..., op. cit., p. 214.

45. Lu Xun revendiquerait pour la littérature chinoise une garantie de modernité et de puissance qui n'appartiendrait plus ainsi aux seuls arts visuels modernes ou au cinéma : pour reprendre la perspective de R. Chow, ce serait l'appropriation de "l'image visuelle moderne" dans une affirmation angoissée de la valeur et du rôle de la littérature et de l'écrit, vecteur d'identité traditionnelle du lettré, face à la puissance de la technologie moderne de l'image (v. cHow Rey, Primitive Passions. Visuality, Sexuality, Ethnography, and Contemporary Chinese Cinema, New York, Columbia U. P., 1995, p. 4-18). Cependant, cette thèse semble critiquée de fait par X. Tang, qui suggère que l'écriture de Lu Xun exerce aussi une influence sur les graveurs chinois des années 1930-40 : il démontre que le thème du cri circule entre le théâtre, la traduction, la littérature et l'art de la gravure pendant la période républicaine (1911-1949). Il serait donc plus approprié de parler de circulation et interaction entre la littérature chinoise et les arts visuels modernes, plutôt que de rapport unilatéral de l'un à l'autre.

46. Sans doute n'y a-t-il pas de lien formel entre Lu Xun et Vsevolod Meyerhold (1874-1940). Toutefois, ce dernier entre en contact dans les années 1930 avec l'acteur d'opéra classique Mei Lanfang 梅蘭芳 (1894-1961, bête noire des auteurs du 4-Mai parce qu'il passait pour incarner la 
vieille Chine), et ce pour étudier le jeu chinois traditionnel (v. sun Mei 孙玫, [ [L'opéra chinois dans le théâtre mondial au xxes.»]/二十世纪世界戏剧中的中国戏曲, 二十一世纪双月刊 / Ershiyi shiji shuangyue kan 45:1998, p.103-112). De plus, Sergueï Mikhaïlovitch Tretiakov (1892-1937) vient enseigner la littérature russe à Pékin en 1924. Il écrit la même année en Chine sa pièce Hurle, Chine ! / Рычи китАй, que Meyerhold crée à Moscou en 1926 et dont le dramaturge 田漢 Tian Han (1898-1968), au moins dès 1928, et avant sa traduction ultérieure en chinois, commente le jeu «biomécanique » (v. TANG, p. 223-224). De surcroît, le texte de Xu Zhimo, mentionné au début de l'article, est publié au cours de l'été 1925. L'intérêt de Lu Xun pour l'opéra chinois traditionnel rejoint sur certains points le jeu de Meyerhold, qui trouve une source d'inspiration dans le "dépouillement » du théâtre chinois ou japonais - Tretiakov lui-même rend compte d'une représentation de " théâtre asiatique » à laquelle il assiste à Pékin en 1924, et de Mei Lanfang: v. TRETIAKOv Serge M., Hurle, Chine! et autres pièces : Écrits sur le théâtre, AMIARDCHeVrel C. et al. (trad.), Lausanne, L'Âge d'Homme, 1982, p. 245-246. À ce propos, V. Semanov établit un rapport entre la "caractérisation " ou stylisation des personnages de Lu Xun et l'esthétique picturale et théâtrale chinoises classiques, ainsi que Wang Yao (WANG Yao, Wang Wao quanji / 王耀全集, Shijiazhuang, Hebei jiaoyu chubanshe, 1999, vol. 5, p. 75-76). Lu Xun présente une volonté similaire de créer un choc, en l'occurrence sur le lecteur et non le spectateur; cependant, malgré sa dimension dramatique et « engagée » (la vieille femme est une variante du tireur de pousse, ou ici, du «coolie » chez l'auteur soviétique), le «Tremblement» de par son dispositif d'enchâssement et de dédoublement narratifs se distingue de l'art prolétaire et de l'agit-prop. Par ailleurs, Lu Xun ne recourt pas davantage aux jeux futuristes de « déconstruction » du langage.

47. Pour donner un équivalent, on pense tout à fait librement à Die freudlose Gasse (1925) / La Rue sans joie, de Pabst (dont j'ignore s'il fut alors projeté en Chine) ou à des films chinois de la même veine tragique et sociale.

48. Une autre pièce de La Mauvaise herbe, «Le Passant » / 過客 (mars 1925), témoigne d'ailleurs d'une dimension théâtrale explicite.

49. Sur ce terme, v. MONCOND'HUY Dominique et SCEPI Henri (éds.), Les Genres de travers. Littérature et transgénéricité, Rennes, P. U. de Rennes, 2008, p. 7-11.

50. v. VUILLEUMIER Victor, «Le corps souffrant chez Lu Xun: allégorie muette de l'obstacle et appropriation de la modernité ", Extrême-Orient Extrême-Occident 39:2015, p. 47-84.

51. Voir MARIN Louis, "Aux marges de la peinture : voir la voix », De la représentation, Gallimard, 1994, p. 329-341.

\section{AUTEUR}

\section{VICTOR VUILLEUMIER}

Victor Vuilleumier est maître de conférences en études chinoises à l'Université de Paris-Diderot Paris VII (UFR LCAO), membre du Centre de recherche sur les civilisations de l'Asie orientale (CRCAO), et enseigne également à l'Université de Genève. Ses recherches portent sur l'histoire de la littérature, des idées et de la traduction en Chine aux XIX et XX ${ }^{\mathrm{e}}$ siècles (https://cv.archivesouvertes.fr/victor-vuilleumier). 


\title{
Akutagawa Ryūnosuke's Ideal of Corporal Beauty: Social Construction and Personal Contribution
}

\author{
Damaso Ferreiro Posse
}

\section{Introduction}

1 One of Akutagawa Ryūnosuke’s 芥川龍之介 (1892-1927) last works, [Literary Too Literary] / 文芸的な、余りに文芸的な (1927), offers precious evidence of his latter's idea of corporal beauty. It is composed of 39 chapters among which two are especially significant: [The Call of the Wild] / 野性の呼び声 / Yasei no yobigoe and [The Call of the West] / 西洋の呼び声, ${ }^{1}$ where he deals with the somatic question. If we wish to reach a general understanding of what a beautiful body means to Akutagawa, first of all it will be necessary to introduce the author's concept of corporal beauty, in relation to the current literary context of his time. An analysis of [Literary Too Literary] will then follow to bring out some key terms which express his own conception of the idea. The next step will consist in comparing Akutagawa's idea of corporal beauty with the one commonly accepted during his lifetime, while stressing why his case is unique in the field of modern Japanese literature. We will conclude with a final reflection on Akutagawa's two antagonistic ways of conceiving corporal beauty.

Before analyzing the concept of corporal beauty in Akutagawa's work [Literary Too Literary], it is necessary to consider the concept of beauty in Akutagawa's thinking in order to understand the importance it has in his poetry. As Yamashiki Kazuo 山敷和男 points out, Akutagawa's poetic conception is divided into three formative concepts that are interconnected to create an artistic masterpiece. These three concepts are beauty / 美, goodness / 善, and truth / 真. This way of understanding art is not exclusive to Akutagawa, rather it is very close to other Japanese and Western writers of the time. As Paul De Man (1919-1983) argues, the constant presence of these three formative 
concepts may be due to the enormous influence the Neoplatonic ontology and theory of art exerted upon Western philosophy and art from the middle of the 18th century onwards. The main differences between theorists essentially lie in the way they understood, defined, and related those three elements to create their own systems. In this regard, Akutagawa's poetics is not an exception.

When analyzing Akutagawa's way of understanding literature and beauty, scholars must deal with the fact that, unlike other writers of his time such as Sōseki, he did not write any exposition of his theory of poetics, but instead just used his literary works as tools to transmit his ideas, thoughts, and opinions. Hence we face the necessity of reconstructing his poetics from the large amount of information scattered across his many works. Indeed, when Akutagawa talks about beauty, far from having an unique name for it, he employs many different terms such as beauty / 「美」/bi, a merit / 「美点」 / biten, beauty / 「美しさ」, natural beauty / 「自然の美」, human beast's beauty / 「人間獣の美」, etc. Despite these difficulties, it is possible to show that, in a broad sense, for Akutagawa the idea of beauty is the most important formative concept of art and it is closely related to the term expression / 表現. In his own words, "(b)eauty starts with expression and ends with expression". ${ }^{2}$ Moreover, this "beauty naturally appears in a literary work, and it can be noticed naturally by the reader", ${ }^{3}$ which means that creating beauty cannot be learned, but is something innate to the writer. ${ }^{4}$ However, due to their lack of goodness and truth, literary works only focused on beauty are probably not, Akutagawa argues, the best type of works. They nevertheless remain the purest expressions of art that may be created. ${ }^{5}$ Akutagawa mentions Shiga Naoya (1883-1971) as the perfect example of this type. This way of understanding the self-sufficiency of art, also known as l'art pour l'art / art for art's sake, a complicated debate which engaged artists and writers from the late Romantic period onward, occupies a position of importance in Akutagawa's thought and it seems to be intimately related with the subject of this paper: corporal beauty.

\section{Body and literature}

Our first task is to analyze what a physical representation of body means in literature. Body in reference to literature or literature in reference to body is a very controversial concept which requires a multidimensional, multilayered and interdisciplinary approach. When analyzing "bodies" in literature, one can understand some factors merely by focusing on the text, its vocabulary and expressions. However, we must consider that the number of expressions related to physical descriptions is often very limited and it obliges us to take into account to other elements such as inner descriptions. The overall conclusion is that it is very difficult to put a limit on the physical representation of characters in literature, what the author really means or what we, as researchers can deduce from it. ${ }^{6}$ According to Andrew Bennett,

in Body Studies, the hard problem is the question of how language relates to the body, of how any system of representation may be said to be or to interact with what Judith Butler terms the body's mute facticity. [...] there is an intimate and ineluctable paradox at the heart, as we say, of the discourse of the body, because any representation of the body endeavors to make the body present. But this makingpresent is always, necessarily, marked by its absence, since it is a law of language, of representation, that the use of the linguistic sign implies the absence of the thing for which it stands. ${ }^{7}$ 

problem of its linguistic representation, but also a cultural problem. With only a few exceptions (Spinoza, Schopenhauer, Marx, Nietzsche, Foucault), Western philosophy has tended to hide questions related to the concept of "body" in its own metaphysical and ontological structures. In essence, the body was seen as a minor substance in comparison with the soul. Philosophers were accordingly reluctant to analyze all of its basest instincts and impulses (desire, insecurity, variability, sexuality, disease, etc.). ${ }^{8}$

The concept of body has always been present in literature but it retained the attention of intellectuals only after the so-called "discovery of the modern ego". According to this discovery, the concept of body can be defined as the division between the "self" and the "other", two elements mutually connected through a subjective relationship established by each one. Furthermore, both elements are also linked to two more dimensions: the private and the public. ${ }^{9}$ This philosophical way of understanding the body will be the one followed in this study: the difference between the "self" and the "other" may lead us to a broader context once these concepts are substituted by "own nation" and "foreign nation". In other words, we will mainly analyze the body in Akutagawa's essays as a social and cultural construction, paying attention to its sexual content and taking into account two main issues: the transnationalization of Western classics and the process of geographical and communicational deterritorialization of the Japanese body. Both issues go beyond the scope of nation and are closely bound up with the phenomenon of globalization.

\section{The ideal of corporal beauty in Akutagawa}

Somatic beauty in Akutagawa is a troubling and disruptive concept. The clearest text to analyze the concept of corporal beauty is [The Call of the West], published in Akutagawa's last year of life (1927) as part of the larger work [Literary Too Literary], in which he describes not only his ideal of corporal beauty, but also its relation with Western classics. One important feature of this text is that, in contrast to others, Akutagawa refers to the concept of corporal beauty beyond gender; this may probably be understood as an attempt to make his own ideal universal. Quoting Ishibashi, there are two ways of approaching the concept of corporal representation; the first one is from a personal perspective, individualizing the body through a description of a particular character. The second one is from a wider perspective, with an absence of personalized descriptions and a clear universalization of the concept. ${ }^{10}$ Without indications of masculinity or femininity, it is possible to assume that Akutagawa intended here to promote a certain ideal of somatic beauty beyond gender.

Right from the outset, Akutagawa establishes a clear separation between his own interest for Western culture and that of his contemporaries:

The 'calling of the West' I feel is probably different from the one Tanizaki feels ${ }^{11}$

しかし僕の『西洋の呼び声』と云ふのは或は谷崎氏の『西洋の呼び声』とは多

少異なつてみるかも知れない.12

He then insists on the fact that in his case, his interest is merely artistic or aesthetic:

The West is always calling me from the perspective of the plastic arts. (...) And that calling coming from the plastic arts is probably not a coincidence.

『西洋』の僕に呼びかけるのはいつも造形美術の中からである。（中略）西洋 
の僕に呼びかけるのに造形美術を通してみるのは必ずしも偶然ではないかもし れない. 13 expressions but for what lies behind that: the influence of the "mysterious Greece":

That thing that is deeply rooted at the bottom of the West is always the mysterious Greece.

この『西洋』の底に根を張つてるるものはいつも不可思議なギリシャである. ${ }^{14}$

This "mysterious Greece", Akutagawa continues, is accessible to us under its still existent artistic works, such as pottery and (primarily) sculpture, and achieves its maximal expression of corporal beauty when representing its gods:

If I would have to explain the Greece I have closest to me, I would recommend taking a look at some Greek pottery works we have in Japan. Or I would even recommend taking a look at photographs of Greek sculptures contained in some books. The beauty existent in those works of art is the beauty of the own Greek gods.

僕は最も手短にギリシヤを説明するとすれば、日本にもあるギリシヤ陶器の幾 つかを見ることを钦めるであらう。或は又ギリシヤ㓮刻の写真を見ることを钦

めるであらう。それ等の作品の美しさはギリシヤの神々の美しさである ${ }^{15}$

According to Akutagawa, the highest form of artistic representation was achieved in Greece, and the ideal of corporal beauty in Greek masterpieces can be considered as the ideal of corporal beauty of humankind itself. Akutagawa went beneath the surface of the West to rediscover a Greek ideal still alive, somehow still valid in present times and in places which are geographically and culturally very far away from its epicenter.

After reaching the previous conclusion, Akutagawa defines what the Greek ideal of corporal beauty means for him and why he feels attracted to it: Greek beauty is always a "sensual and voluptuous beauty, from which emerges a supernatural attraction". It is a physical beauty inexistent in the East, very close to the concept of pornography,

a kind of infinite, sensual or carnal beauty which contains a supernatural attraction. This kind of mysterious beauty, similar to a stone in which the incense has deeply permeated, can be found inside poems too. (...) And it has a filiation different from our Eastern one. The most remarkable example can be found in pornography. Our sense of elegance is far from the concept of sensuality.

飽くまでも官能的な一言はば肉感的な美しさの中に何か超自然と言子外はない

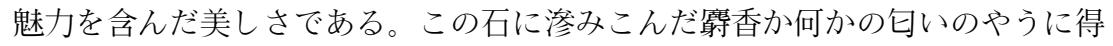

体の知机ない美しさは詩の中にもやはりないことはない。（中略）何か僕等の

東洋と異なった血脈を持つてみる。その最著しい例は或はポルノグラフイに

あるかも知机ない。僕等は肉感そのものさへ僕等と趣を異にしてるる 16

If we take into account all the words Akutagawa uses for describing Greek beauty, we can see that they all have a very strong sexual connotation. Greece appears to Akutagawa as a world based upon an open and limitless sexuality, far from the social constructions of the East, and lacking in moral religious precepts. This sexuality, as he asserts in another text of the same essay, [The Call of the Wild], is very close to the wild world lost in modern times and, because it is far from modernity, produces fear and some inner resistance at first:

I wish I could close my eyes to the "calling of the West". The problem is that closing the eyes is not something I can always do voluntarily.

僕は若し目をつぶれるとすれば、かう云ふ『西洋の呼び声』には目をつぶりた いと思つてみる。しかし目をつぶることは必しも僕の自由にはならない。17 


\section{The ideal of corporal beauty during the Taishō period: Nietzsche's influence on Akutagawa}

15 Whether in Japan or in the Western world, the development of the concept of body is recent. However, in almost any piece of modern literature, the presence of the body is visible at every turn, either as a metaphor or as a real protagonist. As for Japan, Kawamura situates the discovery of the modern body at the beginning of the 20th century. He argues that this modern body, even if largely influenced by the West, was still different and autonomous because of the survival of some aspects of the Japanese tradition..$^{18}$ However, over a period of almost 10 years, from 1920 until 1927-1928, the so-called "period of the sexualization of the society", the Japanese conception of body became very close to the Western ideal. ${ }^{19}$

16 By that time, adherence to Western patterns permeated not only literature and painting, but also turned the pattern of Western corporal beauty into the main reference in Japanese society. The number of women-oriented books and magazines proliferated and they showed how to exercise the body, to have wider hips or to use make-up to westernize one's face. In the illustrations, along with Western actresses, many photographs of old Greek sculptures appeared on their pages; the would-be icon of the time was none other but the Venus de Milo, deemed an ideal of health as well as of sensual beauty. ${ }^{20}$

Furthermore, we cannot dismiss the fact that the 1920s were known as the so-called "period of sexualization". The word "sexual desire" / 性欲 / seiyoku began to be widely used and even became the keyword of the whole period. ${ }^{21}$ However, the word seiyoku had a double meaning by that time. On the one hand, it was identified with the principle of life ensuring the survival of humankind, the basis of the self-preservation instinct. On the other hand, it was also deemed something amoral which belongs to the realm of animals, a vision that ended up being the one most widely accepted.

Kawamura's description of the Taishō period makes possible to link Akutagawa's ideal of corporal beauty with the general ideal widely spread during his lifetime. The imitation of the Western classical body by the Japanese society of the time probably had a big impact on Akutagawa's own vision. Indeed, shared assumptions on the human body are nothing but assumptions rationalized and adopted by a society which perceives a given concrete model of body as natural. This image of a "natural body" may change, and in fact it does, geographically and temporally. For instance, Western culture has shown itself for centuries to be sympathetic to the conception of the physical body as a prison in which soul is encased, a paradigm of guilt and sin. This conception was not assumed by other cultures of the same time, nor is even assumed nowadays in Western culture itself. That said, the adoption of the Greek body as a model of beauty and perfection by Akutagawa inexorably leads us to consider the question of the classical body in modern Western culture as well.

19 It is in the production of body representations that the classical world provided a fertile soil for modern Western culture. The first real attempt in modern times to reconstitute the Greek ideal body in art was made by the art historian and archeologist Johann Winckelmann (1717-1768) for whom,

in theory, the Greek ideal should appear entirely whole and centered, its harmoniously poised body the very model of a similarly constituted ideal subjectivity. (...) By establishing a duality between the high and the beautiful, 
Winckelmann opened up irreducible division within the Greek ideal. This division not only registered the formal impossibility of ever realizing a stable fusion of idea and body in one single image. It also articulated a complex play of fantasy, oscillating between the projection and disavowal of desire, between the assertion and denial, even annihilation. ${ }^{22}$ Akutagawa, as both of them characterize the classical somatic ideal as a duality. However, the duality proposed by Winckelmann is based on a sexual dialectic between a masculine body (the high and erotic) and a feminine body (the beautiful and pure), two poles which are impossible ever to join. In Akutagawa's case, the "pure and sensual" are two faces of the same coin, i.e. classical beauty. Moreover, although Akutagawa specifically talks of the female prototype he feels attracted by in the chapter [The Call of Wild], when analyzing the ideal corporal beauty that Western classics represent in [The Call of the West] it becomes clear that he avoids any concrete mention of gender. He even rejects any mention when bringing to the fore the erotic dimension of the Western corporal ideal, which might be understood in some way as a subliminal message: as pointed out before, this might mean this erotic dimension may be found in both male and female representations.

According to Blanshard, the preference for wild, sensual and dark bodies arising from Greek classics such as Medea, Clytemnestra or Cleopatra is constant in the Western tradition from the end of 19th century and the beginning of the 20th: Jane Ellen Harrison, Delphine de Girardin, Martha Graham or Amy Levy are just some examples of scholars or artists who felt this attraction. ${ }^{23}$ However, the first philosopher who was finally able to combine the two dimensions of the physical representation of bodies in Greek art together with the question of sexuality was Friedrich Nietzsche. As he was extremely popular in Japan during the Meiji and Taishō periods, ${ }^{24}$ it is not unreasonable to think that his conception of sexuality, body and eroticism exerted a deep influence on Akutagawa, who often mentions him.

The somatic conception in Nietzsche's philosophy is very complex and evolving, mostly studied on the basis of the Übermensch-Theorie. We will focus on Nietzsche's approach to sexuality and eroticism in his early years, with special attention to Die Geburt der Tragödie aus dem Geiste der Musik / The Birth of Tragedy (1872). Here Nietzsche already expresses most of his future concerns, among which is to be found the couple ApolloDionysos, drawn from Greek mythology and profusely used in German thought:

Apollo, Dionysos, Socrates, (...) are all characters in a drama of ideas, an art form of the intellect that is neither mere allegory nor systematic philosophy. (...) They are not merely mythic or historical portmanteaux, but ideas-come-to-life. Through the creation of such figures, Nietzsche illustrates his belief in the emotional foundation of all ideas. ${ }^{25}$

This famous dichotomy, far from the ideal of perfect forms inherited from the Renaissance and Neoclassic periods, emphasizes the irrational part of Greek culture as the most rewarding for human and spiritual development. Nietzsche first described these two concepts aesthetically, and some years later also ontologically and metaphysically, assuming that the fusion of Dionysian and Apollonian artistic impulses / Kunsttriebe produced the best artistic expression possible: nothing other than Greek tragedy. According to Nietzsche, in The Birth of Tragedy (sections 13 and 14), the critical distance which separates men from their closest emotions, brought by the Socratic dialectic, gave birth to Apollonian ideals. Since these ideals set human beings 
apart from their essential connections with the self, ${ }^{26}$ Nietzsche attempts to recuperate it and proposes in Attempt at Self-Criticism (section 5) a closer link with the chaotic nature of the world represented by Dionysos, usually defined as wild, sensual, and releasing. ${ }^{27}$ If one wants to reestablish a good connection between individuals and the surrounding world, as well as to get rid of the existential pain induced by modernity, sensuality and eroticism are to be accepted always as an indivisible part of existence. And Nietzsche, in Attempt at Self-Criticism, turns existing in Greek culture's sensuality and eroticism into something not only positive but even advantageous and desirable. This positive approach was rejected by the so-called Socratic rational thinking revolution ${ }^{28}$ and, later, by Christian morality.

As will be the case for Akutagawa some years later, moral attributions dictated by Christians which made men embrace hatred towards impurity were something meaningless for Nietzsche ${ }^{29}$ in his Attempt at Self-Criticism. He argues that such attributions should be substituted by a quest for beauty, sensuality, chaos, contradictions, etc. Although Nietzsche's works did not reach a wide readership during his lifetime ${ }^{30}$ they were widely read during the Taishō period (if not widely understood - some critics doubt this), and not only the Übermensch-Theorie, but also the Apollonian and Dionysian theory, did exert a deep impact on several Japanese writers including Akutagawa.

Even if both artistic impulses are embodied by male gods, Apollo and Dionysos, Nietzsche makes no distinction between sexes when considering the sensual or erotic part of human beings - nor does Akutagawa. Furthermore, Apollonian pureness and Dionysian eroticism coexist within the same dialectic game, thus bringing Greek tragedy to life. It is exactly the same with Akutagawa's conception of Greek beauty. However, this ontological and epistemological development, achieved by Nietzsche and reproduced by Akutagawa, would have not been possible without the rise of sciences "that sought to establish precarious truths about racialized and gendered bodies whose past and anticipated future were at the core of anxious theorizing about progress and civilization". ${ }^{31}$ This question of sexuality regarding the body which is raised by the sciences should not be understood simply as a negative representation, but

at a time when the boundaries of different European states expanded, collapsed and competed in its violent struggles that accompanied the formation of modern nations and their aggressive colonial expansion, scientific investigations of sex understood to mean both gender and sexual acts - became central to the way in which the nineteenth century articulated its norms and ideals and sought to control transgression and deviation. ${ }^{32}$

Bauer then quotes Michel Foucault, according to whom sexuality emerged as the correlative of that slowly developed discursive practice which constitutes the scientia sexualis, marking a profound shift in the production of the subject, a shift which turned the sexual body into the focus of scientific enquiry and made it central to the deployment of power in the West..$^{33}$ One more consideration should be added to Bauer's words: not only in the West but in Japan as well there are several literary works related to the sexual dimension of men written from a scientific and philosophical point of view. For example, in Vita sexualis / サタ・セクスアリス (1909), Mori Ōgai 森鴎外 (1862-1922) draws attention to the sexual experiences of the protagonist. However, in spite of conspicuous attempts to normalize sexual issues in Japan and in spite of the disruption caused by the ancient model of corporal beauty, it nonetheless remained 
commonly true that neither sexuality nor eroticism were openly accepted by Japanese society.

\section{Akutagawa's body: social construction and personal contribution}

Regarding the issue of accepting a foreign corporal model as one's own, stimulating the appropriation of Western classical imagery was one of the issues the Japanese had to go through to gain access to Western culture after the so-called Meiji Restoration. Traditionally, classical learning in Japan was represented by Chinese classics. However, during the 20th century, along with the decline of this Chinese classical tradition in Japan, the Western classical past and the turn toward Western antiquity was profusely used in Japanese literature not only by Akutagawa, but by some other writers such as Dazai Osamu 太宰治 (1909-1948), Mishima Yukio 三島由紀夫 (1925-1970), Kita Morio 北杜夫 (1927-2011), Yoshioka Minoru 吉岡実 (1919-1990) or Takahashi Mutsuo 高橋陸 郎 (1937-). During this period, some authors like Homer, Aesop or Plato and figures from antiquity such as Caesar or Alexander were reclaimed as an indispensable part of world literature and common cultural heritage, thus expanding the limits of the Western classical world beyond its geographic bounds.

This phenomenon may be due to two fundamental reasons. According to Salvatore Settis, "we are thus faced with a paradox, whose significance we need to emphasize and interpret as the sole and unequivocal root of all Western civilization, and the depository of its highest and unfailing values". ${ }^{34}$

As a result of it, we tend to use a profusion of quotations taken from classical antiquity to legitimize our own argument, creating a corpus of random and unconnected elements of fragments from the classical tradition "ready to use". ${ }^{35}$ More serious is another feature of this process: we tend to project the classical world "onto a universal plane" and claim Western civilization's superiority over other civilizations. These reflections on the nature of the classical are very relevant to understanding the transnationalization not only of Western antiquity but also of the classical model of corporal beauty as well. As Settis points out, we created a corpus that is easy to use whatever the occasion may be, and we endowed this corpus with a false sense of authority, putting our own tradition on an unreachable pedestal unconnected from its real idiosyncrasy. Both reasons made the classical tradition not only easy to "recycle" by Western countries but also easy to assimilate by many others. Even by those that, like Japan, are geographically very far from its epicenter. The final consequence of this phenomenon which occurred during the 19th and 20th centuries is the transnationalization and universalization of Western antiquity, making it appear as a common model that provides universal validity and prestige to those who adopt it and use it.

This process of transnationalization of the Western classical world and the adoption of Western culture as a model had an important impact on how Japanese understood themselves. In other words, it produced a sort of "colonization" of the mind which, at the same time, implied a deterritorialization of the "Japanese body". The term "colonization of the mind" is widely used in the field of colonial studies and is defined as a form of "epistemic violence" regardless of whether it occurs in socio-political 
situations literally defined as "colonial". ${ }^{36}$ According to Dascal, this phenomenon has the following characteristics:

the intervention of an external source - the 'colonizer' - in the mental sphere of a subject or group of subjects - the 'colonized'; this intervention affects central aspects of the mind's structure, mode of operation, and contents; its effects are long-lasting and not easily removable; there is a marked asymmetry of power between the parties involved; the parties can be aware or unaware of their role of colonizer or colonized; and both can participate in the process voluntarily or involuntarily. ${ }^{37}$

31 In other words, this "colonization of the mind may take place through the transmission of mental habits and contents by means of social systems other than the colonial structure. For example, via the family, traditions, cultural practices, religion, science, language, fashion, ideology, political regimentation, the media, education, etc". ${ }^{38}$ As we can see, body image in Japan during the Meiji and Taisho periods cannot escape the epistemic colonization of the West. Physical differences between Western and Eastern bodies (such as hips, eyes or breasts) were all quickly assimilated by Japanese society; this new trend imposed an ideal model that depicted people, mainly women, as they should be rather than as they really were. As Blanshard assets, through its domination of canons of beauty, the Western classical tradition has played a decisive role in controlling the appearance of and in pathologizing male and female bodies ${ }^{39}$ regardless of their geographical origin. The preeminence of classical models of beauty in Japanese society redefined notions of corporal beauty and encouraged the "imitation" of foreign models, based on the false assumption that the Western canon is the only one that contains a "universal truth" beyond time, space and causality.

The immediate consequence of this process is the deterritorialization of the traditional Japanese body, or to put it another way, the traditional Japanese conception of corporal beauty looked at the West and, attracted by a new universal model, ceded its place not only to a new way of understanding beauty but to a series of ethical paradigms as well: the Venus de Milo became the icon of Taishō period. As we have previously pointed out, Akutagawa's description of corporal beauty, "the sensual beauty of the own Greek gods" ${ }^{40}$ is very similar to what Kawamura argued was the main trend during the late Meiji and Taishō periods. In this sense we can affirm that Akutagawa's conception of corporal beauty is, broadly speaking, a continuation of the conception that prevailed during his lifetime, which implies that he could not escape the process of colonization of the mind discussed above. The content of his text derives directly from the social trend of the time and, from that point of view, Akutagawa can be seen as mere receiver and repeater of that socially accepted model.

However, if we take into account the variety of images Akutagawa employs in connection with the concept of "classical Greek beauty", such as a voluptuousness close to pornography (seen as something positive), and moreover if we take into account not only [The Call of the West] but also the preceding essay, [The Call of the Wild], where he talks about a wild and instinctive corporal beauty, it is absolutely clear that Akutagawa's conception somehow differs from that characterized by Kawamura as typical of the period. In other words, the previous explanation of Akutagawa as a mere receiver and repeater is not enough to understand the depth of the question itself. Quite the opposite, in fact. When analyzing the text in detail, we arrive at the conclusion that Akutagawa must be seen as a renovator of the concept in Japan, since he assimilated the current social trend and went further, including his very personal 
and unique understanding of the term along with the influence of some other scholars or philosophers like Nietzsche, as mentioned previously. The connection between wildness, sexuality, freedom, eroticism, pureness and the Greek classics was not new in the Western world but it was definitely innovative in Japan.

\section{Conclusions}

34 We can conclude that although the ideal of corporal beauty which Akutagawa presented in the year of his suicide might appear to have been very revolutionary at the moment it was published, it is not completely original. Its development comes largely from the concept of beauty existing in Western countries, on the one hand, and in Japan on the other. However, Akutagawa's conception goes beyond what was socially accepted in his country at that time and ignores some aspects such as immorality or social acceptance in the same way Nietzsche did with his concept of "Dionysian". To sum up, we must admit that the question of the body and physical beauty in Akutagawa is constructed through the intersection of multiple discourses, including: the canonical Western influence of the time which, as Kawamura points out, followed Western corporal beauty patterns; the Nietzschean tradition which picked up the issue where it had been left off, mainly by Winckelmann and Hegel; and his own personal perspective on beauty.

Furthermore, despite the conceptual indeterminacy that shadows the somatic question in Akutagawa's literature, there is another factor that cannot be dismissed: its connection with the wild. While the explanation of the desired body is understood as something non-gendered but with a high sexual content, the relation with the wild, suggested in the essay titled [The Call of the Wild], is always seen from a perspective of female beauty. For Akutagawa, indeed, the difference between a refined beauty (represented by Renoir's paintings) and a wild beauty (represented by Gauguin's paintings) is the difference between a type of socio-cultural commodity and a truly free and instinctive desire. Even if he admits that as an educated man he marvels at the subtle genius of Renoir and his painting technique, he observes that:

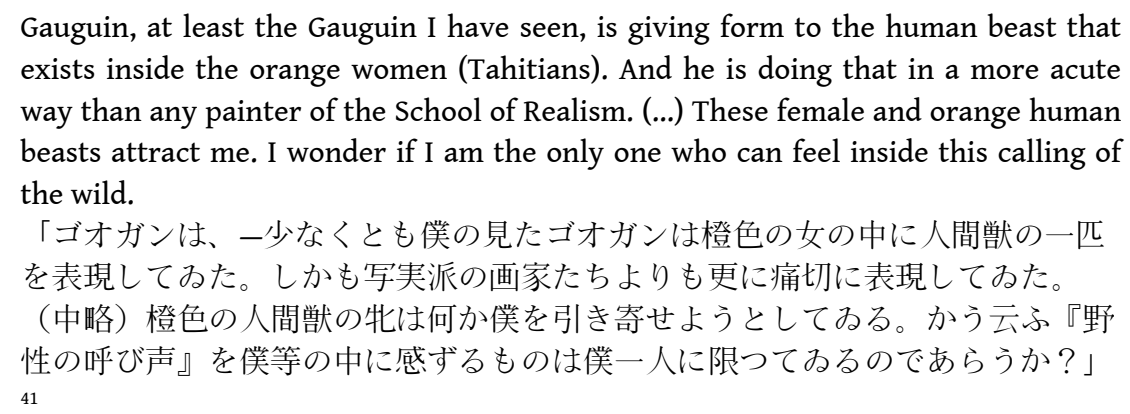

36 This predilection towards savage and primitive beauty, almost understood as a supreme extra-linguistic feature, is not in contradiction with what we have seen until this point (a pure, sensual and sexual beauty). On the contrary, it is a complementarity beauty which can be drawn upon Nietzsche's previously mentioned work and his idea of Dionysos..$^{42}$ This connection between the wild and Dionysos is visible in another work, [Smile of Gods]/ 神々の微笑 ${ }^{43}$ (1922), where Akutagawa describes a "Bacchanalia" performed by Japanese shintoist gods. 


\section{BIBLIOGRAPHY}

AKUTAGAWA Ryūnosuke 芥川龍之介, [Complete Works of Akutagawa Ryūnosuke] / Akutagawa Ryūnosuke Zenshū, YASUE Ryōsuke (ed.), Tokyo, Iwanami, 1996, vols. 5, 12, 15, 16.

BACARLETT PÉREZ María Luisa, Friedrich Nietzsche. La vida, el cuerpo y la enfermedad, México, Universidad Autónoma del Estado de México, 2006.

BAUER Heike, "Literary Sexualities", in The Cambridge Companion to the Body in Literature, HILLMAN David and MAUDE Ulrika (ed.), New York, Cambridge U. P., 2015, p. 101-115.

BENNETT Andrew, "Language and the Body" in The Cambridge Companion to the Body in Literature, HILLMAN David and MAUDE Ulrika (ed.), New York, Cambridge U. P., 2015.

BLANSHARD Alain, "Gender and Sexuality", in A Companion to the Classical Tradition, KALLENDORF Craig (ed.), Oxford, Wiley-Blackwell, 2010.

DASCAL Marcelo, "Colonizing and Decolonizing Minds", in Papers of the 2007 World Philosophy Day, KUÇURADI Ioanna (ed.), Ankara, Philosophical Society of Turkey, 2009, p. 308-332.

DE MAN Paul, Romanticism and Contemporary Criticism, Baltimore, The Johns Hopkins U. P., 1996. 
ISHIBASHI Masataka 石橋正孝, [“A Consideration of the Possibilities and Limits of the Body's Representation in Literature"] / Bungaku ni okeru shintai.hyōshō no kanōsei to genkai ni tsuite no kōsatsu, in Teikyō Daigaku sōgō kyōiku sentā ronshū, vol. I, 2010, p. 71-85.

KAWAMURA Kunimitsu 川村邦光, [The Maiden's Modern Body and Sexuality] / 乙女の身体・女の 近代のセクシュアリテイー / Otome no shintai: onna no kindai to sekushuariti, Kinokuniya Shoten, 1994.

LENSON David, The Birth of Tragedy. A Commentary, Boston, Twayne's Masterwork Studies, 1987.

PотTS Alex, Flesh and the Ideal. Winckelmann and the Origins of Art History, New Haven, Yale U. P., 2000.

SETTIS Salvatore, The Future of the "Classical" / [Futuro del classico, 2004], CAMERON Allan (trans.), Cambridge, Polity Press, 2006.

SILK Michael and STERN Joseph, Nietzsche on Tragedy, New York, Cambridge U. P., 2016.

SUGITA Hiroko, [Sōseki's Cat and Nietzsche. Modern Japanese Intellectuals Upset by the Modern Philosopher] / Sōseki no neko to nīche - kindai no tetsugakusha ni shinkanshita kindai.nihon no chiseitachi, Tokyo, Hakusuisha, 2010.

YAMASHIKI Kazuo 山敷和男, [Akutagawa Ryōnosuke's Theories on Art] / 芥川龍之介の芸術論 / Akutagawa Ryūnosuke no geijutsuron, Tokyo, Gendai Shichō Shinsha, 2000.

\section{NOTES}

1. AKUTAGAWA Ryūnosuke 芥川龍之介, [“Literary Too Literary”], in [Complete Works of Akutagawa Ryūnosuke]/ Akutagawa Ryūnosuke Zenshū, YASUE Ryōsuke (ed.), Tokyo, Iwanami Shoten, vol. 15, 1996, p. 201-208.

2. AKUTAGAWA, ["Art and Others"], in [Complete Works of Akutagawa Ryūnosuke], op. cit., vol. 5, p. 167.

3. AKUTAGawA, ["Poe's Shadow"], in [Complete Works of Akutagawa Ryūnosuke], op. cit., vol. 12, p. 274.

4. AKUTAGAWA, ["Dwarf's Words"], in [Complete Works of Akutagawa Ryūnosuke], op. cit., vol. 16, p. 40.

5. AKUTAGAWA, [“Literary Too Literary”], op. cit., p. 148.

6. ISHIBASHI Masataka 石橋正孝, [“A Consideration of the Possibilities and Limits of the Body's Representation in Literature"], in Teikyō Daigaku sōgō kyōiku sentā ronshū, vol. I, 2010, p. 76.

7. BENNETT Andrew, "Language and the Body" in The Cambridge Companion to the Body in Literature, HILlman David and MAUDE Ulrika (ed.), New York, Cambridge U. P., 2015, p. 73.

8. BACARLETT PÉREZ María Luisa, Friedrich Nietzsche. La vida, el cuerpo y la enfermedad, México, Universidad Autónoma del Estado de México, 2006, p. 18, 19.

9. ISHIBASHI, [“A Consideration of the Possibilities and Limits..."], op. cit., p. 76.

10. Ibid., p. 80.

11. All English translations are my own.

12. AKUTAGAWA, ["Literary Too Literary"], op. cit., p. 204.

13. Ibid., p. 205.

14. Ibid.

15. Ibid.

16. Ibid., p. 206, 207.

17. Ibid., p. 207. 
18. KAWAMURA Kunimitsu 川村邦光, [Sexuality in Modern Times] / オトメの行方一近代女性の表 象と䦔い, Tokyo, Kōdansha, 1996, p. 48.

19. Ibid., p. 255.

20. Ibid., p. 49.

21. Ibid., p. 82.

22. РоттS Alex, Flesh and the Ideal. Winckelmann and the Origins of Art History, New Haven, Yale U. P., 2000, p. 145.

23. BLANSHARD Alain, "Gender and Sexuality", in A Companion to the Classical Tradition, KALLENDORF Craig (ed.), Oxford, Wiley-Blackwell, 2010, p. 333, 334.

24. SUGITA Hiroko 杉田弘子, [Sōseki's Cat and Nietzsche. Modern Japanese Intellectuals Upset by the Modern Philosopher], Tokyo, Hakusuisha, 2010, p. 13.

25. LENSON David, The Birth of Tragedy. A Commentary, Boston, Twayne's Masterwork Studies, 1987, p. 8,9 .

26. NIETZSCHE Friedrich, [Die Geburt der Tragödie aus dem Geiste der Musik] / El nacimiento de la tragedia, SÁNCHEZ Andrés (trans.), Madrid, Alianza Editorial, 2004 [1872], p. 120-130.

27. Ibid., p. 33.

28. Ibid.

29. Ibid.

30. LENSON, The Birth of Tragedy..., op. cit., p. 13, 14.

31. BAUER Heike, "Literary Sexualities", in The Cambridge Companion to the Body in Literature, HILLMAN David and MAUDE Ulrika (ed.), New York, Cambridge U. P., 2015, p. 101.

32. Ibid.

33. Ibid.

34. SETTIS Salvatore, The Future of the "Classical" / [Futuro del classico, 2004], CAMERON Allan (trans.), Cambridge, Polity Press, 2006, p. 2.

35. Ibid., p. 3.

36. DASCAL Marcelo, "Colonizing and Decolonizing Minds", in Papers of the 2007 World Philosophy Day, KUÇURADI Ioanna (ed.), Ankara, Philosophical Society of Turkey, 2009, p. 308.

37. Ibid., p. 309.

38. Ibid.

39. BLANSHARD, "Gender and Sexuality", op. cit., p. 334.

40. See above the footnote on Akutagawa.

41. AKUTAGAWA, ["Literary Too Literary"], op. cit., p. 202.

42. SILK Michael, STERN Joseph, Nietzsche on Tragedy, New York, Cambridge U. P., 2016, p. 297.

43. AKUTAGAWA, ["Smile of Gods"], op. cit., vol. 8, p. 188-204.

44. HEINE Heinrich, in AKUTAGAWA, ["Literary Too Literary"], vol. 15, 1996, p. 207. Nietzsche, Ibid., p. 208.

45. Ibid., p. 202.

46. BLANSHARD, "Gender and Sexuality”, op. cit., p. 329. 


\section{AUTHOR}

\section{DAMASO FERREIRO POSSE}

Hiroshima University. Damaso Ferreiro Posse is a specialist of Compared Literature and Modern and Contemporary Japanese Literature. He has published 赤い鳥事典編集委員会（2018）『赤 い鳥事典』柏書房。フェレイロ、ダマソ（2018）「芥川龍之介の「芸術作品完成品論」に おける〈美〉善〉〈真〉一新プラトン主義の視点から」『第12号芥川龍之介研究』国際芥川龍之 介学会. Ferreiro Posse, Dámaso (2020) “El papel de la tauroflamencología en el universo mishimiano: España y su tradición cultural como ejemplo de resistencia a la homogeneidad occidental" Mirai. Estudios Japoneses, vol. 4. 


\title{
The Flesh, Subject, Embodiment in Postwar Japan: Through Nikutai and Gutai
}

\author{
Fusako Innami
}

\section{Introduction : In Search of the Individual Flesh}

1 The Japanese writer Tamura Taijirō 田村泰次郎 (1911-1983), writes in his essay [The Flesh is the Human] / 肉体が人間である (1947):

I contend that thought [shisō] is my flesh. Other than my physical flesh itself, I do not think that there is thought anywhere. Therefore, I realize that my novels are not yet thoughtful enough as my fleshness is not yet sufficiently materialized as a creative act, but I do not think "there is no thought" at all. I think I can search for thought by persistently pursuing my own flesh. I cannot think of any existence of thought without thinking of my flesh.

私は思想というものを、自分の内体だと考えている。自分の肉体そのもの以外 に、どこにも思想というものはないと思っている。従って、私は自分の肉体性 が、まだ十分作品行動として具現化されていないということで、私の小説はま だ十分に思想的でないとは自覚しているが、まったく「思想がない」とは考え ていない。私は自分の肉体をどこまでも追求することで、思想を探求すること が出来る思っている。いや、自分の肉体を考えずに思想というものの存立さえ も私には考えられない。1

2 After having spent several years during the World War Two at the front in China, Tamura presents in his works a body in terms of the physical necessities of food and sex, and experiences of desire and pain. His works, including [The Devil of the Flesh] / 肉体の悪魔 (1946) and [The Gate of the Flesh] / 肉体の門 (1947), which received an explosive contemporary reaction, have been called the literature of the flesh / 肉体文 学, along with works by such authors as Sakaguchi Ango 坂口安吾 (1906-1955) and Noma Hiroshi 野間宏 (1915-1991). Tamura's statement above, expressed during the postwar turmoil, alludes to a fundamental rethinking of the relation between the individual and the state body, the body and thought/language, and body and act. 
Having studied French at Waseda University and been influenced early in his career by stream-of-consciousness writing and French thought, and called, Sone Hiroyuki writes, an "intellectual / 知性派” writer and critic, it was the wartime experience of merely sleeping, eating, and fighting that turned him into a flesh / 肉体派 writer. ${ }^{2}$ Tamura's unavoidable engagement with bare bodies at the extreme condition led him to pursue the "flesh".

3 An existential inquiry and a sense of individuality with a focus on the individual flesh / nikutai came to occupy an explicit position in intellectual and literary discussions soon after WW2, freed from the unitary state focused on the polity / 国体. In the literature of the flesh, which flourished and soon declined, the authors identified fallacies in the wartime spiritualism that realized itself through militarism and imperialism. ${ }^{3}$ Douglas Slaymaker examines plural axes of flesh in contrast to such concepts as the material body / 体 which could join 肉 as flesh / 肉体 or 国 as 国体 / the polity and body, 身体, philosophical term with both a bodily and spiritual sense, the latter sense of which writers focused on the flesh eschew. Slaymaker writes:

The flesh writers, by assuming that the body is central to individual identity, and by extension to a national identity, reflect the attempts in the postwar years to come to terms with the war years by both repressing the body through disavowal, and expressing it through carnality.

4 Tamura brings mind and language back to the body by placing the body as the very basis of the human condition. Sakaguchi argues for the discovery of language of the flesh, emphasizing what the body itself speaks instead of what the mind speaks about its thoughts. Post-surrender concerns of Japanese writers and critics on the body and subjectivity, permeating through literary, political, artistic debates at the time, shaped the rise of flesh; this rise of flesh, expanded from the first generation of postwar writers / 第一次戦後派 such as Noma Hiroshi 野間 宏 (1915-1991) to the second generation such as Abe Kōbō 安部公房 (1924-1993) and Mishima Yukio 三島由紀夫 (1925-1970), further gave rise to bodily awareness and self-consciousness beyond the mere representations of the carnal desire.

The search for the flesh around this period was widespread among art and performance practitioners, as well as writers and literary figures. Among them, the avant-garde art group Gutai-organized in 1954 with seventeen members as Gutai Art Association / 具 体美術協会 in Ashiya, Hyōgo Prefecture by Yoshihara Jirō 吉原治良 (1905-72), a painter and CEO of Yoshihara Oil, and lasted until Yoshihara's death-brought new artistic creations through their bodies. Early activities of this group's members included physically dashing through the paper canvas, swinging axes onto the composed woods, or wearing the dress of electric bulbs, to pursue the material properties and forms not only through hands but through their entire bodies as art of physical engagement. These activities were described by the media at the time as [Body Crash Art] / 体当たり芸術, ${ }^{5}$ or introduced as “the art of the flesh" / 肉体の芸術. ${ }^{6}$ Gutai was introduced to the world outside Japan by figures like Michel Tapié (1909-1987), a French art critic and the leader of Art Informel, ${ }^{7}$ and Allan Kaprow (1927-2006), an American artist who contributed to the ideas Happenings. ${ }^{8}$ Gutai, often considered derivative of Abstract Expressionism and Art Informel on one hand and a precursor to Kaprow's Happenings on the other, with its subsequent shift of emphasis from performance-based art to increasingly two-dimensional tableaus, the history to which Gutai belongs has been debated. Nonetheless, Gutai contributed to the collective search 
for an autonomous subject through the concrete awareness of the body. While "flesh" writers illuminated bare bodies exposed in the burnt aftermath of the war, Gutai artists, including female artists such as Yamazaki Tsuruko 山崎つる子 (1925-2019) and Tanaka Atsuko 田中敦子 (1932-2005), reflexively became aware of the materiality of objects with which they were in contact. The body, pursued both individually and collectively, functioned as a contact point across individuals, genres, and cultures.

6 This paper-a work in progress on the search for the body and the senses in postwar Japan at the intersection of literature, performance, and visual culture-attempts to read how the concerns about the engagement with the body and autonomous subject might have been shared beyond genres of arts and have shaped active practices of the body. This paper brings interrelated concerns in the literature of the flesh together with those of Gutai. Although the literature of the flesh and Gutai are rarely discussed together, with a few recent examples suggesting their interrelatedness through their attention to the flesh, ${ }^{9}$ an intermedia analysis of treatments of the body in their writings may contribute to a further understanding of what was at stake in the search of the body in postwar Japan. In all their concepts of the body-whether polity / 国体, flesh / 肉体, autonomous body / 主体, or concrete body / 具体 -these artists pursued the new form of the body through their creations for, Ming Tiampo puts it, "a reconstruction of subjectivity through a deformation of the painted body characterized by a sincere pursuit of individualism". ${ }^{10}$ Although the narratives around Gutai have so far focused on their artistic experimentation, innovation in performance, and international network, Gutai also offers an alternative understanding of the lived body: embodiedness.

7 I specifically focus on the flesh / 肉体 / nikutai as a site from which to engage with the environment, to affect and be affected by the external, and to nurture and be nurtured through thought. Although the spread of the literature of the flesh coincided with some understanding of it as carnal literature, the interrogation of the body prompted an idea of the individual body as associated with both carnal and political liberation. Postsurrender concerns regarding the flesh involved existential and phenomenological questions about the body and existence. ${ }^{11}$ Further, Shiraga Kazuo 白髪一雄 (1924-2008) of the Gutai group, who developed his interest in action painting from an earlier stage in his career, left his remarks on the individual qualities, the existential body, and perceptions in his writing. I contend that this postwar preoccupation of the body across genres prompted the subsequent rise of the discourse on the body called embodiedness / 身体論 in the 1970s and '80s in which such figures as Ichikawa Hiroshi 市川浩 (1931-2002) and Kamei Hideo 龟井秀雄 (1937-2016) in a sense re-examined a mind-body dualism, ${ }^{12}$ prompted by the translation of Maurice Merleau-Ponty. ${ }^{13}$

\section{Flesh as a Site of Controversy}

8 Japan's postwar reconstruction, after the experience of the atomic bombs and defeat in 1945, pushed the Japanese to pursue interests in the autonomous body, as distinct from the national "body" identified with the polity (kokutai). The idea of kokutai, representing the emperor as the living embodiment of the nation even in the absence of his physical presence, bore a "religious" character, indicating "the mystical body" of the nation, ${ }^{14}$ written with logographs meaning 国 / country and 体 / body. John Dower writes that the Japanese were "indoctrinated to believe that the supreme object of 
veneration was the kokutai, or emperor-centered 'national entity". ${ }^{15}$ Writers associated with the literary journal Modern Literature / 近代文学 reflected upon the emperor system and Japan's responsibility for the war; the accounts of politicized MerleauPonty were "readily apparent", Slaymaker puts it, with "the need for the politicization of individuals while opposing the evil of political systems". ${ }^{16}$

Tamura's experience at the battlefront made him realize the powerlessness of thought and ideology, and led him to choose instead to pursue carnal desire, exploring the new life that would follow the liberation of the body, via sensations felt at/on the flesh. Tamura writes:
We no longer believe in anything other than our own flesh. Only the flesh is truth. Pain of the flesh, desire of the flesh, anger of the flesh, intoxication of the flesh, confusion of the flesh, and slumber of the flesh-only these are truths. Through the existence of these facts, we come to realize, for the first time, that we are alive.
私たちは、いまやみずからの肉体以外のなにものも信じない。肉体だけが真実 である。肉体の苦痛、肉体の欲望、肉体の怒り、肉体の陶酔、肉体の惑乱、肉 体の眠り、ーこれらのことだけが真実である。これらのことがあることによっ て、私たちははじめて自分が生きていることを自覚するのだ。17

Tamura's fictional works, which often depict women being stripped, tied, and beaten, were widely associated with eroticism-especially when "subcultures," involving the world of prostitutes for GIs called panpan/ パンパン, the black market, selfindulgency, communicated via fragmentary cheap materials called pulp / カストリ culture and commercialized sex. ${ }^{18}$ As Dower finds one of the features of so-called flesh writers in the "solitary physical individual", ${ }^{19}$ Tamura mapped the individuals' bodies in space where the desire for fundamental necessities drove their lives, rather than depicting characters' subtle psychological struggles. Ik-Koo Hwang writes that while, in politics and medicine, the flesh nikutai was a metaphor of social distortion, in literature it was concrete / 具体, appearing as a motif to represent the postwar sociocultural space. ${ }^{20}$ The flesh, as a concrete living being, occupied burnt space and urban confusion.

11 Sakaguchi Ango alludes to physicality through speaking of the fallness / 陏落 (often translated as "decadence") through which humans come into the world: "Humanity has been born from the womb of decadence's truth. To live, and to fall-this is the correct procedure; can there be any easy shortcut to the saving of humanity outside it?"21 Sakaguchi believed that this fallenness makes us human through a condition in which an individual, by thoroughly falling, can discover and rescue him- or herself. He, started making claims for the flesh slightly earlier than Tamura, also emphasizes not the mind talking about its thoughts, but what he believes is spoken by the body itself, with reference to Jean-Paul Sartre's short novel "Intimacy" (published in France in 1939 and translated into Japanese in 1946); with Ango's comments, Japanese readers "understood" Sartre's writing as erotic literature and existentialism as a form of literary eroticism. ${ }^{22}$

12 In Sartre's "Intimité" / "Intimacy", in which Lulu escapes from her impotent husband Henri but ends up returning to him after only a night, various voyeuristic elements peel off consciousness from the seen: the almost naked Henri as seen by Lulu and the Texiers, Lulu as seen by other characters, and then by the readers. The gaze in Sartre surprises the subject, reducing him to shame, but disappears when this subject sees the eye that is looking at him, making him conscious of himself as the seen ${ }^{23}$. Lulu, who looks like a sleepwalker to Rirette, acknowledges Henri's somnambulistic appearance 
chasing her after she has left him alone,${ }^{24}$ but then decides to return and stay with him. Sakaguchi, with reference to this story, writes in his [The Flesh Itself Meditates] / 内体 自体が思考する (1946): “There is nothing like logic in this novel. Only the flesh thinks and speaks. Lulu's flesh weirdly loves the impotent's flesh.” /この小説には倫理などは 一句も説か机てるない。だ゙肉体が考へ、肉体が語つてるるのである。リュリュ の肉体が不能者の肉体を変な風に愛してみる。. ${ }^{25}$ Here Sakaguchi searches for a discovery of the language of the flesh itself.

In Sakaguchi's "The Flesh Itself Meditates" and Tamura Taijirō's "The Flesh is the Human" (1947), mentioned above, both writers problematize language and thought that was once in a body and has left, while illuminating lived or felt experiences through the body. The flesh here is something that is particularly acute to the senses. Sakaguchi Ango's [The Idiot] / 白痴 (1946) also presents the ambiguity of the body through various corporeal expressions amid the postwar confusion. ${ }^{26}$ While the extensive pursuit of the body demonstrated its complexity, the body became almost like a thought itself. Political scientist Maruyama Masao 丸山真男 (1914-1996) points out that the literature of the late 1940s is constantly preoccupied with matters of intercourse, and is remote from actual life, with the writers offering a symbol of what they longed for: “As long as there isn't any inner working of the spirit that makes the creation of 'poetry' possible within the context or our lives, it's always going to be the same old story". ${ }^{27}$ What would this subjective impulse mean in this context?

While illuminating the subjectively lived flesh, the sense of the self-created through the eyes of the other or even the self-bound by the other appear in writings of the flesh, in their search not only for individuality, but also community. Tamura's The Gate of the Flesh is set in the Yūrakuchō area in Tokyo, where GHQ (General MacArthur's General Headquarters of the Supreme Commander for the Allied Powers) was located. It was "a contact zone" where the occupied and the occupier encounter and cross, ${ }^{28}$ a group of women work as prostitutes, with an involvement that is seen bodily and not mental. It is an obligation within this community that a woman should not exchange her body without a proper fee-if she breaks this commandment, she will be an outcast from the community, the self-observing community in the postwar period functioning rather like the self-regulating community of the fascistic nation in wartime. A community such as this, Hwang argues, reminds communities of neighbors during the war in which people would look after and support each other, and also watch one another and report anti-state behaviors or thoughts to officials if necessary, ${ }^{29}$ amid the democratic ideals of postwar Japan. It was not entirely that the new individual "flesh," devoid of war-time ideology and the national body, emerged as a "cut" from the nation and community.

Ibuki, the male protagonist of The Gate of the Flesh, a returnee from the war, comes to play a central function in this story, as desired within this female community. The girls are considered too immature to fully know carnal joy. However, the war widow Machiko, upon falling in love with Ibuki, is expelled from the community, as if to suggest that the knowledge of physical pleasures unsettles the common ground of this community. Slaymaker writes, "The relationship between Ibuki and the war widow portends the loss of innocence, for maturity is marked by knowledge of fleshly pleasures". ${ }^{30}$ Sone Hiroyoshi also points out that even though Tamura was inspired by the panpan girls' strength to confront the postwar confusion by means of their bodies, it was not only the panpan serving GIs whom he sought to depict in order to illuminate the occupied-occupier relationship; rather, Tamura depicted Japanese girls who slept 
with Japanese men, passing the threshold into adulthood in doing so. ${ }^{31}$ While being conscious of censorship from his earlier experiences, ${ }^{32}$ the body in this story is the place that concerns both the oppositions and continuity or succession of maturity/ immaturity, prewar/postwar, and "in" and "out," in Tsukada's word, where the shadow of America is hidden and revealed. ${ }^{33}$

The postwar liberation brought a measure of equalization of class and gender, as well as freedom of thought and expression. Tsurumi Shunsuke (1922-2015) writes of the transformation in everyday bodily practices that the occupation by GHQ affected Japanese models for opposite-sex physical interactions, giving the impression that "a new age had arrived" ${ }^{34}$ And yet, male writers of the literature of the flesh recognized the radical possibilities in, as Yoshikuni Igarashi puts it, "the language of the body", ${ }^{35}$ via female bodies. Advocates of the flesh believed, Harry Harootunian argues, that "Japanese had been denied 'sensory stimuli' down to the end of the war". ${ }^{36}$ While the broad movement of equalization liberated women, in terms of the U.S. occupation army's attempt to save Japanese women from patriarchal oppression, as Mire Koikari argues, it also solidified certain divisions, as regulation of gendered bodies became "a source of tension and controversy". ${ }^{37}$ Even when the individual body, liberated from the patriarchy and wartime restriction, was supposed to function as counterhegemonic, its extent was still limited; the liberation of the flesh occurred at the expense of female bodies. Distinctions between pre- and post-war, the collective and the individual, and repression and liberation might be the result of the way historicizing and understanding of a complex set of interrelated issues. Contested in some discussions is the historicizing of such distinctions through the very notion of the "postwar" period, Miryam Sas suggests that the typical way of situating the "rupture" between the pre-war and the post-war shows evidence of continuity. ${ }^{38}$

The postwar period adjoined the wartime one, with Tamura living in a burned-down Tokyo as if it were the warfront in China for Sone, ${ }^{39}$ while the changes of the postwar "liberation" remained limited. The thirst for carnality in postwar Japan represented the search for an individuality that was curtailed during wartime, and the liberation of the flesh still meant that of the male body via the objectified female body. Having embodied the chaotic energy of the postwar period, Tamura attempted to rebuild the body and identity. It was to bring the carnal body and mind together, considering their unity to be the basis of the human condition. Or in Dower's words, among writers such as Sakaguchi, Tamura, and Dazai Osamu 太宰治 (1909-1948) who "dramatically linked degeneracy and carnal behavior to authenticity and individuality," Tamura's choice of language "amounted to sacrilege bordering on outright lese majesty", ${ }^{40}$ in respect to the kokutai. Tamura believed in the flesh-although the flesh, in itself, keeps evolving and shifting, and is unstable to rely on. The flesh (re)appeared in a burnt urban space with vitality, crying out its lived experience, unable to be completely reduced to language.

\section{Gutai as Embodiedness}

18 The attention to carnality and to individuality comes to appear not only in the literary and political arenas but also in arts, especially in Gutai's pursuit of autonomous subject, materiality, and originality. The pursuit of the flesh and the body-mind relation were crucial for Gutai artists, especially for Shiraga Kazuo. Shiraga presented a performative 
work called Challenging Mud / 泥に挑む (1955) - which consisted of a pile of mud, clay, and sand in the courtyard of the Ohara Kaikan (school of flower arrangement) in Tokyo -and wrote a series of articles about the individual, the senses, and the body in the journal Gutai between 1955 and 1957. This pursuit of individuality took place by using the body as the site and medium of expression; Tiampo argues that the purpose of Challenging Mud was to express "the personal material of the individual for the purpose of creating a society of politically, ethically, and aesthetically independent individuals". ${ }^{41}$ Shiraga's concerns were shared by Motonaga Sadamasa 元永定正 (1922-2011) and other Gutai members, and, potentially, beyond. Sas, bringing Sakaguchi Ango's "discovery of the self" into the search for the "thought of flesh" in butō dance and experimental theatrical arts in postwar Japan, writes, "the kinds of problems these artists raise [the ambivalent relation between concept and body] are already foreshadowed by Ango's early postwar writings and its interpreters in subsequent generations"42. The development of a subjective autonomy located in the flesh was also considered a matter of urgency in postwar artistic circles.

III. 1. Shiraga Kazuo, Challenging Mud / 泥に挑む, 1955.
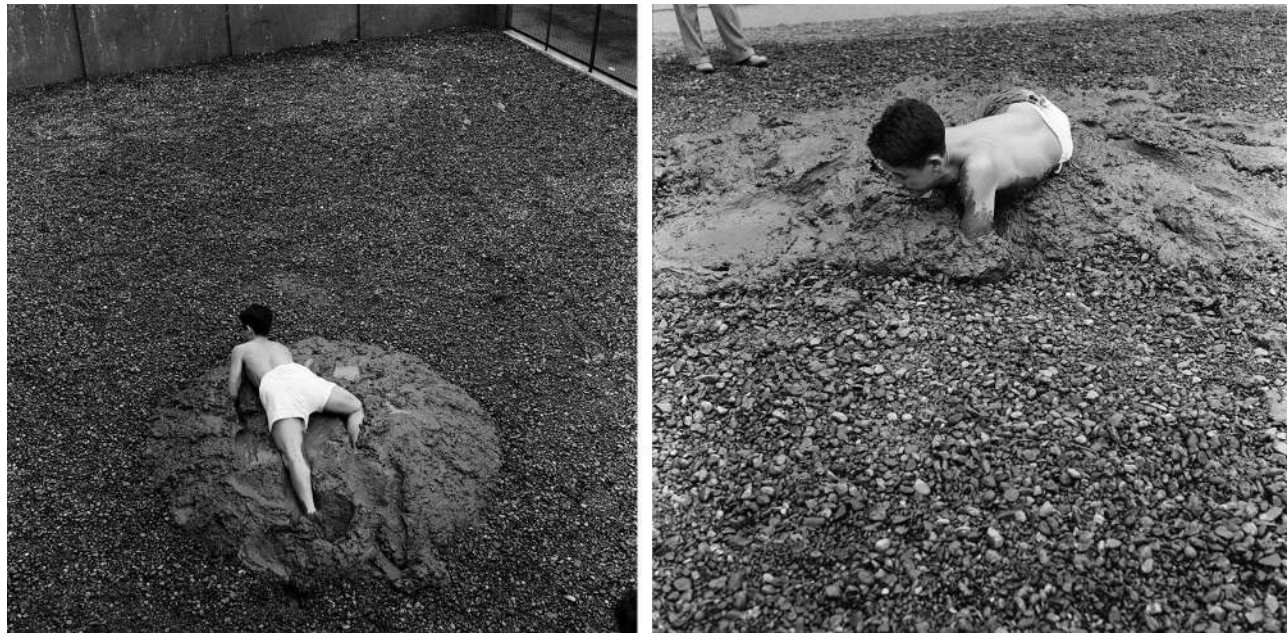

Gutai 4 (1956): 6. 大阪新美術館建設準備室, GA 1802

Shiraga employed the term "flesh" / 肉体 to explain the idea of art as an expression of the spirit that is contingent upon the body in his 1956 essay "On Distinctive Qualities" / 資質について. Through his exploration of subject, body, and individuality, Shiraga places importance on one's innate physical condition / 体質 as the basis of everyday actions; it is accompanied by sensory qualities that keep evolving throughout life, which he calls distinctive qualities “資質”. ${ }^{43}$ As each individual is born with a particular bodily condition, Shiraga believes that distinctive qualities developed through everyday practices cause individuals to realize their own particularities. "The free human being," he writes, "means the one who can establish his or her own qualities and take responsible actions based on these./自由な人間とは、自己の資質を確立し て責任ある資質による行動をなし得る人間の事を指すのではないか。”. ${ }^{44}$ While referring to distinctive qualities, Shiraga describes the formation of the individual body, along with his work "Challenging Mud," with a clear sense of political resistance against totalitarianism: the individual develops his own qualities through thinking and through physical acts or practices. 
idea of individualism saw it as a part of community; "the individual who actively understands its own quality is the one who constitutes the whole./自分の質を強く能 動的に把握した人間は積極的な全体を構成する個体である。." 45 Yet it seems that the process by which Shiraga pursued the "distinctive qualities" was not far from that of the flesh writers, as becomes clear when he explains the act / 行為 as lying at the intersection of the body and thought:

When only I myself exist without thought, feeling, and emotions, what I desirethat is without meaning. My action-whether or not it is stabilized, there exists my consistent action. There is spirit, which has escaped from mind. My innate state is the basis of my being.

想無く念無く心なく私が在るだけの此の時、何を欲するか、又意も無し。私の 行為。定着されようと、されまいと何ら变らざる私の行為がある。心を脱した 魂がある。私の生れたままの、私の状態は私と云う人間の基点である。4

What constitutes the self is the individual's continual challenge to its own qualities; the act forms existence. Having been aware of existentialism, and having entered the Buddhist priesthood late in life, at the Enryakuji Temple in 1971, Shiraga understood the body as contingent upon the spirit, and thought of bodily action as uniting the two together-particularly in actions performed with his feet on the ground, or suspended with a rope to support the weight of his body, touching the ground just enough to allow himself to slide over a canvas. To touch the ground in this way is not to rest, but constitutes the point of departure for a new movement, from which to form and reform the body.

Yoshihara Jirō turned to art to pursue originality, his work a legacy to other members of Gutai, in response to his experience of being criticized early in his career by Fujita Tsuguharu 藤田嗣治 (1886-1968) for his lack of originality. ${ }^{47}$ Yoshihara's Gutai Art Manifesto / 具体美術宣言 in 1956, reads, “In Gutai Art, the human spirit and matter shake hands with each other while keeping their distance. Matter never compromises itself with the spirit; the spirit never dominates matter. When matter remains intact and exposes its characteristics, it starts telling a story and even cries out". ${ }^{48}$ Remaining unconvinced by other postwar milieus, Yoshihara selected the name Gutai (具体, concrete), initially proposed by Shimamoto Shōzō 嶋本昭三 (1928-2013), to refer to the project of making art that addressed the world in a concrete manner.

Passing through the shortage of materials immediately after the war made them conscious of the "materials" they engage with in artistic creations; Shimamoto Shōzō, aiming to present a lack of physical materiality, worked on paper surfaces through his "paper-vas," gluing layers of newspaper to prepare a neutral surface, then creating holes and ruptures on the surface (Work / Hole in 1950). It corresponds the time when Abe Kōbō meditated upon external materials reflected onto the skin, combining emotions, colors, and textures of the seen in his fiction [Cryptic Coloration] / “保護色” and [Starving Skin] / 飢えた皮膚 (1951); both the page and the skin mediate changes in the subjects caused by external stimuli. ${ }^{49}$ The lack of physical materials drove them to find distinctive qualities of the surface.

These features of engaging with the materials were not confined to how artworks are constructed but also how artists and viewers engaged with them. In his Please Walk Here / この上を步いてください, which was part of the Gutai Outdoor Exhibition in 1956, Shimamoto invited the creative involvement of the public ${ }^{50}$ by letting them step on the woodwork, and working with objects in an open space. The work involved making one's body aware while it is "doing"-not only for artists, but also for the viewer who breaks 
from the practice of looking. Going further than just exhibiting their works in alternative spaces like outdoors, on a stage, or in the sky, ${ }^{51}$ they engaged with the external of their bodies in seeking new ways of conceiving the relationships between self, body, and the world.

When Ukita Yōzō 浮田要三 (1924-2013), a professional editor and a member of Gutai, commented on the second edition of journal called "Gutai", he first and succinctly explained what this group was about: “Gutai' might be literary translated as "Embodiment". ${ }^{52}$ The journals to record their activities and to create "international common ground" were issued through fourteen volumes from 1955 to 1972, including photographs of the objects with a resume in English, and were circulated amongst artists and critics, including John Cage (1912-1992) and Yves Klein (1928-1962), around the world to play a crucial role in connecting like-minded artists at the time..$^{53}$ While the early Gutai activities aimed to engage with the body as the medium of action of the autonomous subject, their extended creations-in a form of two-dimensional tableau and journals-reached beyond the flesh itself.

\section{Writing the Body (Out): Conclusion}

This paper has examined the ways in which postwar authors and artists tried to resituate the body as the autonomous subject and attain a body. The search for the flesh, which took on particular momentum in postwar Japan, interconnected writing, thinking, and actions. Such a heightened attention to the flesh made those creative individuals aware not only of the lived body (desire, pain, and necessity), but also of the body as dwelling in space and interconnected with the other, community, and the external. It further interconnected concerns shared by critics and practitioners across the world. Both the literature of the flesh and Gutai sought to attain a physical materiality as well as a new formation of the subject by returning to the body, forging an identity through the bare flesh, and using the body as the site of event. In such a case, what is expressed and what is written on the surface is not merely a referent: the body not as something to be written "about" or on but as an exclamation. The body itself is a subjective site of living, a site for performative acts that engage with the environment.

Flesh writers' attempts to attain a bare flesh and a language thereof, by pursuing the flesh as lived, was, in a way, already failing: to capture extra-linguistic experiences (flesh) through language in between the lived and the written, between the felt and the repressed, even if this language is the language of the flesh. And yet, such a failure may give birth to the body-as the written, marking a moment where the flesh appears, or "happens." Concerning incommunicable pain and pleasure in Georges Bataille (1897-1962), Jean-Luc Nancy (1940-) writes to exscribe meaning, or writing it out. Nancy's idea of writing and reading here is "to be exposed, to expose oneself" to "nothaving" or "not-knowing," not as the uninscribable, but as that "opening into itself of writing to itself, to its own inscription as the infinite discharge of meaning". ${ }^{54}$ The body being exposed, moves, creates a space for itself, and acts. The attempt to write such an undefinable body nonetheless escapes in the moment when a pen touches it.

Although through different genres, from different areas in Japan, thoughts on the body illuminated by figures such as Tamura, Sakaguchi, and Shiraga all convey attempts to live, rebuilding the body, subject, and its own language. The body functioned, not 
merely as a site and surface to be inscribed upon, but as a site to move, act, and engage with the other. By tracing its movement, the viewer potentially comes to acknowledge the shape of its body. The body they searched for, while engaging with its lived experiences, drove it out to interact with others beyond genres of arts and shaped active practices of the body.

Acknowledgement:

This paper is a part of project, "In Search of the Body in Postwar Japan, 1945-1997," funded by the British Academy (BA/Leverhulme Small Research Grants, 2017-18, Grant Number SG163483).

\section{BIBLIOGRAPHY}

ABE Kōbō 安部公房, [“Cryptic Coloration”] / 保護色 / Hogoshoku, in [The Complete Works of Abe Kōbō] / 安部公房全集 / Abe Kōbō Zenshū, Tokyo, Shinchōsha, vol. 3, 1997, p. 15-32.

ABE Kōbō 安部公房, [“Starving Skin”] / 飢えた皮膚 / Ueta Hifu, in [The Complete Works of Abe Kōbō] / 安部公房全集 / Abe Kōbō Zenshū, Tokyo, Shinchōsha, vol. 3, 1997, p. 59-72.

DOWER John W., Embracing Defeat: Japan in the Wake of World War II, New York, Norton, 1999.

Gutai shiryōshū: Dokyumento Gutai 1954-1972 / 具体資料集ドキュメント具体 1954-1972, Ashiya, Ashiya shi bunka shinkō zaidan, 1993, no page.

HAROOTUNIAN Harry, “Japan's Postwar and After, 1945-1989: An Overview”, in From Postwar to Postmodern: Art in Japan 1945-1989, CHONG Doryun, HAYASHI Michio, SUMITOMO Fumihiko and KAJIYA Kenji (eds.), New York, The Museum of Modern Art, 2012, p. 17-21.

HWANG IK-KOO 黄 益九, [“The Occupation Meets the Flesh Secretly: Postwar Japan in Nikutai no Mon”] / 占領と肉体の密会『肉体の門』が物語る戦後 / Senryō to nikutai no mikkai: Nikutai no mon ga monogataru sengo, 文学研究論集 / Bungaku kenkyū ronshū, 24:2006, p. 71-88.

ICHIKAWA Hiroshi 市川浩, [The structure of body] / 身の構造 / Mi no kōzō, Tokyo, Kōdansha, 1993 [1975], $228 \mathrm{p}$.

IGARASHI Yoshikuni, Bodies of Memory: Narratives of War in Postwar Japanese Culture, 1945-1970, Princeton, Princeton U. P., 2000.

ISHIZUKI Mayuko 石月 麻由子, [“Sakaguchi Ango’s 'Idiot' Analyzed through Corporeal Expressions: From the Standpoint of Interconnection between the Flesh and Mind”] / 身体表現から考察する 坂口安吾『白痴』肉体と精神の<連絡 >と言う視座に立って / Shintai hyōgen kara kōsatsu suru Sakaguchi Ango, “Hakuchi”: Nikutai to seishin no <renraku> toiu shiza ni tatte, 国文学研究 / Kokubungaku kenkyū, 139:2003, p. 121-32.

KAPROW Allan, Assemblage, Environment, and Happenings, New York, Abrams, 1966.

KAPROW Allan, Essays on the Blurring of Art and Life, KELLEY Jeff (ed.), Berkeley and Los Angeles, California U. P., 2003, 341 p. 
KOIKARI Mire, “Gender, Power, and U.S. Imperialism: The Occupation of Japan, 1945-1952”, in Bodies in Contact: Rethinking Colonial Encounters in World History, BALLANTYNE Tony and BURTON Antoinette (eds.), Durham, Duke U. P., 2005, p. 342-62.

MARUYAMA Masao, [“From Carnal Literature to Carnal Politics”] / 肉体文学から肉体政治まで / Nikutai bungaku kara nikutai seiji made, in Thought and Behavior in Modern Japanese Politics, MARUYAMA Masao (ed.), tr. from the Japanese [新装版現代政治の思想と構造 / Shinsō ban gendai seiji no shisō to kōdō, Miraisha, 2006 [1964], p. 375-394 by RUCH Barbara, Oxford U. P., 1969, p. 245-67.

MERLEAU-PONTY Maurice, The Visible and the Invisible, LINGIS Alphonso (trans.), Evanston, Northwestern U. P., 1968, 282 p.

MERLEAU-PONTY Maurice, The World of Perception, DAVIS Oliver (trans.), London and New York, Routledge, 2008 [2004].

NANCY Jean-Luc, “Exscription”, LYDON Katherine (trans.), Yale French Studies 78, On Bataille, 1990, p. 47-65.

“Nikutai no Geijutsu” / 肉体の芸術, [New wave of arts] / Geijutsu shinchō / 芸術新潮, 8-4:1957. RITTER Gabriel, "Between Action and The Unknown: The Art of Kazuo Shiraga and Sadamasa Motonaga", Exhibition organized by the Dallas Museum of Art and the Japan Foundation, 2015. SAKAGUCHI Ango, "Discourse on Decadence”, LIPPIT Seiji (trans.), Review of Japanese Culture and Society 1:1, 1986, p. 1-5.

SAKAGUCHI Ango, [“The Flesh Itself Meditates”] / 肉体自体が思考する / Nikutai jitai ga Shikō suru, in [The Complete Works of Sakaguchi Ango] / 坂口安吾全集 / Sakaguchi Ango Zenshū, Chikuma Shobō, 1998, vol. 4, p. 268-69.

SARTRE Jean-Paul, Being and Nothingness: An Essay on Phenomenological Ontology, BARNES Hazel E. (trans.), London, Routledge, 2003 [1957].

SARTRE Jean-Paul, "Intimacy”, in Intimacy and Other Stories, LLOYD Alexander (trans.), London and New York, Peter Nevill, 1949, p. 1-45.

SAS Miryam, Experimental Arts in Postwar Japan: Moments of Encounter, Engagement, and Imagined Return, Cambridge, Harvard Asia Center, 2011.

Sнгмамото Shōzō 嶋本 昭三, [“Mambo and Painting”] / Manbo to kaiga, Gutai, 3 / 具体, 1955, reprinted in Gutai shiryōshū: Dokyumento Gutai, p. 274-75.

SHIRAGA Kazuo 白鬞一雄, [“Simply Action”] / 行為こそ / Kōi koso, Gutai 3 / 具体 3 , 1955, reprinted in Dokyumento Gutai, p. 274.

SHIRAGA Kazuo 白髧一雄, [“The Formation of the Individual Body”] / 個体の確立 / Kotai no kakuritsu, Gutai 4 / 具体 4 , 1956, p. 6-7, reprinted in Dokyumento Gutai, p. 277-78.

SHIRAGA Kazuo 白髪一雄, [“On Distinctive Qualities”] / 資質について / Shishitsu ni tsuite, Gutai 5 / 具体 5,1956 , reprinted in Dokyumento Gutai, p. 287-88.

SLAYMAKER Douglas, The Body in Postwar Japanese Fiction, London, Routledge, 2004.

SLAYMAKER Douglas, “Sartre's Fiction in Postwar Japan”, in Confluences: Postwar Japan and France, Ann Arbor, Center for Japanese Studies, The University of Michigan, 2002, p. 86-109.

SONE Hiroyuki, [“Interpretation: Prostitute-like Naked Eyes”] / 解説娼婦的肉眼 / Kaisetsu: Shōfuteki nikugan, in [The Gate of Flesh] / 肉体の門 / Nikutai no mon, Tamura Taijirō 田村泰次郎, Tokyo, Chikuma shobō, 1988 [1947], p. 239-243. 
TAMURA Taijirō 田村泰次郎, [“The Flesh is the Human”] / 肉体が人間である / Nikutai ga ningen dearu, in [The Gate of the Flesh] / 肉体の門, Tokyo, Chikuma shobō, 1988 [1947], p. 219-225.

TAMURA Taijirō 田村泰次郎, 我が文壇青春期 / Waga bundan seishunki, Tokyo, Shinchōsha, 1963.

TAMURA Taijirō 田村泰次郎, [“Body Crash Art”] / 体当たり芸術 / Tai.atari Geijutsu, in サン写真新 聞 / San shashin shimbun, October 20, 1955.

TIAMPo Ming, Gutai: Decentering Modernism, Chicago and London, Chicago U. P., 2011.

TIAMPo Ming, "Gutai Chain: The Collective Spirit of Individualism," Positions: East Asia Cultures

Critique 21:2, 2013, p. 383-415.

TSUKADA Yukihiro, [“Sex and Bondage: GHQ, Censorship, Tamura Taijiro's “Gate of Flesh”'] /

「性」を「縛る」GHQ検閲、田村泰次郎『肉体の門』/ 'Sei’ o <shibaru>: GHQ, ken'etsu, Tamura Taijiro, 'Nikutai no mon', [Annual Review of the Institute for Advanced Social Research], 関西学院 大学先端社会研究所紀要 / Kansei gakuin daigaku sentan shakai kenkyūsho kiyō, vol. 11, 2014, p. 47-60.

TSURUMI Shunsuke, A Cultural History of Postwar Japan: 1945-1980, Routledge, 2011 [1987].

UKITA Yōzō, "Documentary on the Second Edition of 'Gutai"', Gutai 2, 1955, reprinted in Dokyumento Gutai, p. 271-72.

VISSER Tijs, "Mal Communication", in Gutai: Dipingere con il Tempo e lo Spazio -Painting with Time and Space, KANAYAMA Akira et al. (eds.), Milano, Silvana Editoriale, 2011, p. 15-23.

WILSON George. M. Patriots and Redeemers in Japan: Motives in the Meiji Restoration, Chicago, Chicago U. P., 1992.

YOSHIHARA Jirō, “Gutai Art Manifesto,” томII Reiko (trans.), http://web.guggenheim.org/ exhibitions/gutai/data/manifesto.html, consulted on 21/02/2021.

\section{NOTES}

1. TAMURA Taijirō, [“The Flesh is the Human”] / 肉体が人間である, in [The Gate of the Flesh] / 肉 体の門, Tokyo, Chikuma shobō, 1988 [1947], p. 220. Translations are mine, unless otherwise indicated.

2. SONE Hiroyoshi [“Interpretation: Prostitute-like Naked Eyes”] / 解説 娼婦的肉眼, in [The Gate of Flesh] / 肉体の門, TAMURA Taijirō, Tokyo, Chikuma shobō, 1988 [1947], p. 240.

3. This is often represented in terms such as militarist spirit / 軍国精神 and imperialist spirit / 皇国精神, or obliterating the self and serving public authority / 滅私奉公, notions that themselves underwent drastic transformations during the war.

4. SLAYMAKER Douglas, The Body in Postwar Japanese Fiction, London, Routledge, 2004, p. 10.

5. TAMURA Taijirō, [“Body Crash Art”] / 体当たり芸術, in [The Sun Pictorial Daily] / サン写真新 聞, October 20, 1955.

6. "Nikutai no Geijutsu", Geijutsu shinchō (1957), April. nº 8-4.

7. A movement in the mid-1940s and 1950s of abstract painting that emphasized gestural and material presence. It is widely known that Tapié had a certain impact on Gutai's development and activities following his visit to Japan in 1957, including in the shifts of their creations from paper to canvas, and the increasing importance of two-dimensional tableaus over performance, for both convenience and marketability. See Gutai shiryōshū: Dokyumento Gutai 1954-1972 [Document Gutai], Ashiya: Ashiya shi bunka shinkō zaidan, 1993. 
8. Happenings are an experimental art form, derived from action painting and widening the concept of theater, especially in the 1950s and '60s New York art scene. Kaprow introduced Gutai in his Assemblage, Environment, and Happenings (New York: Abrams, 1966) as one of the examples of performance-based happening outside of the US, together with cases from France, Germany, and Czechoslovakia. Kaprow started theorizing "Happenings" in the late 1950s, as "events" that happen. See KAPROW Allan, "Happenings in the New York Scene" (1961), in Essays on the Blurring of Art and Life, KELLEY Jeff (ed.), Berkeley, California U. P., 2003, p. 16.

9. TIAMPo Ming, "Gutai Chain: The Collective Spirit of Individualism", Positions: East Asia Cultures Critique, 21:2, 2013, p. 390; RITTER Gabriel, "Between Action and The Unknown: The Art of Kazuo Shiraga and Sadamasa Motonaga", organized by the Dallas Museum of Art and the Japan Foundation in Tokyo, 2015, https://www.wochikochi.jp/english/special/2015/04/betweenaction-and-the-unknown-the-art-of-kazuo-shiraga-and-sadamasa-motonaga.php

10. TIAMPO Ming, Gutai: Decentering Modernism, Chicago, Chicago U. P., 2011, p. 41.

11. SLAYMAKER, "Sartre's Fiction in Postwar Japan," in Confluences: Postwar Japan and France, SLAYMAKER Douglas (ed.), Ann Arbor: Center for Japanese Studies, University of Michigan, 2002, p. 100-101.

12. Ichikawa Hiroshi, neutralizing the division between the body and the mind, regards the body conceptualized as 身 mi not as an object but subjectively as that which experiences and lives. He describes the body as both mind and phenomenon, "a compound body of the world to smell, world to touch, and a world to hear". See ICHIKAWA Hiroshi, [The structure of body] / 身の構造, Tokyo, Kōdansha, 1993 [1975], p. 188. Ichikawa's concept of mi was inspired by Merleau-Ponty's concept of the flesh (la chair), which connects the relationship between body and world to the mutual encroachment of those inseparable entities, further considering the proximity between the tangible and the visible. See MERLEAU-PONTY Maurice, The Visible and the Invisible, LINGIS Alphonso (trans.), Evanston, Northwestern U. P., 1968, p. 133-43. The perception of the world, body, and contact with the world in Merleau-Ponty is already mediated by others' perceptions. See MERLEAU-PONTY Maurice, The World of Perception, DAVIS Oliver (trans.), London, New York, Routledge, 2008 [2004], p. 65; this perception is nowhere else than in one's body.

13. Merleau-Ponty's 1942 La Structure du Comportement (The Structure of Behavior), tr. into English in 1963, and into Japanese in 1964 by Takiura Shizuo 滝浦静雄 and Kida Gen 木田元. Takeuchi Yoshirō 竹内芳郎 and others translated Merleau-Ponty's Phénoménologie de la perception (Phenomenology of Perception) and Signes (Signs) in 1967 and 1969, respectively.

14. WILSON George M., Patriots and Redeemers in Japan: Motives in the Meiji Restoration, Chicago, Chicago U. P., 1992, p. 42, with reference to George Elison's translation of kokutai.

15. DOWER John W., Embracing Defeat: Japan in the Wake of World War II, New York, Norton, 1999, p. 157.

16. SLAYMAKER, "Sartre's Fiction in Postwar Japan", op. cit., p. 92.

17. TAMURA, ["The Flesh is the Human"], op. cit., p. 221.

18. DOWER, Embracing Defeat, op. cit., p. 122.

19. Ibid., p. 158.

20. HWANG Ik-Koo, [“The Occupation Meets the Flesh Secretly: Postwar Japan in Nikutai no Mon”] / 占領と肉体の密会『肉体の門』が物語る戦後, [Research of literature and art]/ Bungaku kenkyū ronshū 24:2006, p. 72.

21. SAKAGUCHI Ango, "Discourse on Decadence", Review of Japanese Culture and Society, LIPPIT Seiji (trans.), 1:1, 1986, p. 5. Originally published in Shinchō in 1946.

22. SLAYMAKER, "Sartre's Fiction in Postwar Japan", op. cit., p. 96.

23. Sartre writes, "What I apprehend immediately when I hear the branches cracking behind me is not that there is someone there; it is that I am vulnerable, that I have a body which can be hurt, that I occupy a place and that I cannot in any case escape from the space in which I am without 
defense-in short, that I am seen." SARTRE Jean-Paul, Being and Nothingness: An Essay on Phenomenological Ontology, BARNES Hazel E. (trans.), London, New York: Routledge, 2003 [1957], p. 282.

24. SARTRE Jean-Paul, "Intimacy," in Intimacy and Other Stories, LLOYD Alexander (trans.), London and New York, Peter Nevill, 1949, p. 29-30. The first publication of Sartre's to be translated into Japanese was "Le Mur" (“The Wall”), by Horiguchi Daigaku 堀口大學. La Nausée ("Nausea”) was translated in 1941 by Shirai Kōji 白井浩二, followed by “Intimité" (“Intimacy"), translated by Ibuki Takehiko 伊吹武彦 and Yoshimura Michio 吉村道夫 in 1946. The Complete Works of Sartre / サルトル全集 is from 1950, and had sold one million copies by the time of Sartre's visit to Japan together with Simone de Beauvoir in 1966.

25. SAKAGUCHI Ango, [“The Flesh Itself Meditates”] / 肉体が思考する, in [The Complete Works of Sakaguchi Ango] / 坂口安吾全集, Chikuma Shobō, vol. 4, 1998, p. 268.

26. ISHIZUKI Mayuko interprets Sakaguchi's corporeal expressions in this story, with a focus on the concepts in between the flesh and mind, such as 身体 as an instinctual body beyond willful control; flesh / 肉体 in relation to the idiot woman in the story, which drives actions with an absolute control; and からだ / karada as a hollow or skeletal body without life and spirit. ISHIZUKI Mayuko, ['Sakaguchi Ango's 'Idiot' Analyzed through Corporeal Expressions: From the Standpoint of Interconnection between the Flesh and Mind"] / Shintai hyōgen kara kōsatsu suru

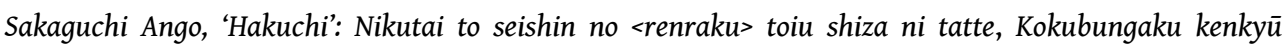
139:2003, p. 124-25, p. 128-29.

27. MARUYAmA Masao, "From Carnal Literature to Carnal Politics," in Thought and Behavior in Modern Japanese Politics, RUCH Barbara (trans.), SLAYMAKER Douglas (ed.), London and New York, Oxford U. P., 1969, p. 248. The original Japanese reads: “生活のなかから「詩」を作り出して行 くための精神の主体的な働きかけがなければ、何時までたっても同じことだ”. [“From Carnal Literature to Carnal Politics”] / 肉体文学から肉体政治まで, in Shinsō ban Gendai seiji no shisō to kōdō, Miraisha, 2006, p. 377-378; originally published in the journal Tenbō in October 1949.

28. TSUKADA Yukihiro, ["Sex and Bondage: GHQ, Censorship, Tamura Taijiro's 'Gate of Flesh"'] /

性」を縛るGHQ研究田村泰次郎『肉体の門』, [Annual Review of the Institute for Advanced Social Research] / 関西学院大学先端社会研究所紀要, vol. 11, 2014, p. 49.

29. HWANG, ["The Occupation Meets the Flesh Secretly: Postwar Japan in Nikutai no Mon"], op. cit., p. 83.

30. SLAYMAKER, The Body in Postwar Japanese Fiction, op. cit., p. 59.

31. SONE, ["Commentary"], op. cit., p. 243.

32. Tamura's works [To My Homeland] / 故郷へ of 1946 and [Beautiful Eyes] / 美しい眼 of 1947 were censored, with some deletions.

33. TSUKADA, ["Bind one's sexuality..."], op. cit., p. 49.

34. TSURUMI Shunsuke, A Cultural History of Postwar Japan: 1945-1980, London and New York, Routledge, 2011 [1987], p. 11.

35. IGARASHI Yoshikuni, Bodies of Memory: Narratives of War in Postwar Japanese Culture, 1945-1970, Princeton, Princeton U. P., 2000, p. 57.

36. HAROOTUNIAN Harry, “Japan's Postwar and After, 1945-1989: An Overview”, in From Postwar to Postmodern: Art in Japan 1945-1989, CHONG Doryun, HAYASHI Michio, sUMITOMO Fumihiko and KAJIYA Kenji (eds.), New York, The Museum of Modern Art, 2012, p. 19.

37. KOIKARI Mire, "Gender, Power, and U.S. Imperialism: The Occupation of Japan, 1945-1952", in Bodies in Contact: Rethinking Colonial Encounters in World History, BALLANTYNE Tony and BURTON Antoinette (eds.), Durham, Duke U.P., 2005, p. 356. Through the government-sponsored Recreation and Amusement Association (RAA, 特殊慰安施設協会) in the occupation period, which provided organized prostitution to protect "respectable" women from foreign intrusion, 
the idea of securing the nation's health rather produced inequality between "respectable" and "unrespectable" women (ibid., p. 349-50).

38. SAS Miryam, Experimental Arts in Postwar Japan: Moments of Encounter, Engagement, and Imagined Return, Cambridge, Harvard University Asia Center, 2011, p. 221. Slaymaker also contests the historicizing of such distinctions through the very notion of the "postwar" period, including when the war started and ended; for him and many others the term "postwar" indicates a prolonged time period, beyond just "after WW2." SLAYMAKER, The Body in Postwar Japanese Fiction, op. cit., p. 5-6.

39. SONE, ["Commentary"], op. cit., p. 240.

40. DOWER, Embracing Defeat, op. cit., p. 155, 157.

41. TIAMPO, Gutai..., op. cit., p. 42.

42. SAS, Experimental Arts in Postwar Japan, op. cit., p. 10.

43. SHIRAGA Kazuo, “On Distinctive Qualities" in Gutai 5, 1956, n. p.; reprinted in Gutai shiryōshū: Dokyumento Gutai, p. 287-88. A series of journal Gutai, starting from 1955, were published by Gutai Art Association, Nishinomiya.

44. Ibid.; Dokyumento Gutai, p. 288.

45. SHIRAGA Kazuo, [“The Formation of the Individual Body"] / 個体の確立 Gutai 4, 1956, p. 6; Dokyumento Gutai, p. 278.

46. SHIRAGA Kazuo, [“Simply Action”] / 行為こそ, Gutai 3, 1955, n. p.; Dokyumento Gutai, p. 274.

47. This pursuit of originality overlapped with Japan's position during the war through the suppressive censorship under the Peace Preservation Law / 治安維持法, enacted in 1925, and the Army Art Association / 陸軍美術協会 launched in 1939.

48. yoshinaRA Jirō, "Gutai Art Manifesto", тOMII Reiko (trans.), http://web.guggenheim.org/ exhibitions/gutai/data/manifesto.html, consulted on 01/07/2021. Originally published as Geijutsu Shinchō 7, n 12, December 1956, p. 202-04.

49. In the very short story "Cryptic Coloration," a scientist and his assistant witness a girl's skin changing from white to green and then to a skin covered with spots, emulating characters from the book she is reading and reflecting what she sees onto her skin. The doctor explains to her that this is not a sickness but rather realizes the human tendency to mimic coloring. In "Starving Skin," set in postwar confusion in China, the protagonist deceives Mrs. Kim into believing that she is ill with cryptic coloration and periodically gives her opium pills to keep at his mercy. Both character's skin color changes, representing their emotions.

50. Sнімамото Shōzō, [“Mambo and Painting”] / マンボと絵画, Gutai, nº 3, 1955, n. p.; Dokyumento Gutai, p. 274.

51. In the International Sky Festival in 1960, where Gutai artists hung their artworks by balloons in the sky on the roof of the Takashimaya department store in Osaka.

52. UKITA Yozo, “Documentary on the Second Edition of 'Gutai”', Gutai, n² 2, 1955, p. 30; Dokyumento Gutai, p. 272.

53. See VISSER Tijs, "Mal Communication", in Gutai: Dipingere con il Tempo e lo Spazio - Painting with Time and Space, KANAYAMA Akira et.al., Milano, Silvana Editoriale, 2011, p. 16. The second and third volumes of Gutai journal were discovered in Jackson Pollock's studio after his death in 1956. Michel Tapié received a copy of Gutai journal through Dōmoto Hisao 堂本尚郎, then-Paris-based painter in 1957, in that year. Allan Kaprow also heard of Gutai via another paper medium of sunday edition of New York Times on Osaka exhibitions through his friend Alfred Leslie, gathered further information of Gutai's work after 1963, and introduced Gutai in 1966. Yves Klein owned set of Gutai periodicals that Yoshihara sent him, including the article on Shiraga's "Challending the Mud" in 1955. Klein was in Japan in 1953 to obtain his Judo belt, set up a small temple upon his return to France in 1958, and read the French translation of Daisetsu T. SUZUKI's book on Zen, including the account on Zen priest Toju NANTEMPO's calligraphy, while trying himself body print 
by crawling in the pigment to later getting the life model to press onto the large sheet of paper on the ground.

54. NANCY Jean-Luc, "Exscription”, LYDON Katherine (trans.), Yale French Studies 78, On Bataille, 1990, p. 64 .

\section{AUTHOR}

\section{FUSAKO INNAMI}

Fusako Innami is an Associate Professor in Japanese and Performance Studies at the School of Modern Languages and Cultures, Durham University. Her research interests include life writing, performance, phenomenology and psychoanalysis, the body and the senses, and translation. Her first monograph, Touching the Unreachable: Writing, Skinship, Modern Japan, appeared in 2021 (the University of Michigan Press). She received her MA from New York University and a DPhil from the University of Oxford. 


\title{
Soigner les corps et les âmes : la science-fiction selon ŌÉ Kenzaburō
}

\author{
Antonin Bechler
}

1 Ōé Kenzaburō débute sa carrière littéraire à 22 ans, en 1957. La configuration de l'imaginaire de l'enfance auquel il fait appel pour ses premiers récits, et qui alimentera tout au long de sa vie ses univers de fiction, renvoie à une perception sensorielle, tactile de l'environnement naturel et du folklore de sa vallée natale du Shikoku telle que s'y inscrivit le corps de l'enfant qu'il fut. Mais par ailleurs, la guerre et la perspective d'y mourir gravée à même ce corps par l'éducation du régime militariste qu'il y reçut y bordure l'horizon, comme le fera plus tard la perspective apocalyptique d'un conflit atomique global. Ainsi, le corps dans l'œuvre romanesque d'ōé se verra toujours représenté dans sa dimension concrète, comme ductile ou, pour reprendre l'un de ses termes clefs, comme « cassable $»^{1}$.

\section{Le corps, symptôme de l'Histoire}

2 On pourrait certainement appréhender l'œuvre d'ōé à travers la généalogie de ses victimes, comme une longue litanie de corps suppliciés :

3 Le héros mordu par un chien malade dans Un curieux travail / 「奇妙な仕事」 (1957), nouvelle qui signe son entrée dans la carrière littéraire. L'enfant à la main sectionnée à la faux alors qu'il s'interpose entre l'aviateur noir américain et les adultes venus l'emmener, dans Gibier d'élevage /「飼育」 (1958) qui lui vaut le Prix Akutagawa l'année suivante. Les enfants malades et battus enfermés dans le village en proie à l'épidémie avec Arrachez les bourgeons, tirez sur les enfants /『芽むしり仔撃ち』 (1958) la même année. Les trois unlucky young men, adolescents impétueux qui se font sauter à la grenade par amour du risque dans [Notre Époque]/『わ杛らの時代』(1959). L'adolescent violé par un clochard à l'instigation de ses camarades de parti dans [Un jeune retardataire]/『遅札てきた青年』(1962). Les jeunes filles étranglées par l'adolescent d'origine coréenne dont [Hurlements]/『叫び声』 (1963) conte le parcours, et son camarade abattu par la police alors qu'il joue un holdup armé d'une 
fausse mitraillette. Le bébé monstrueux que son père envisage de tuer dans Une Affaire personnelle / 『個人的な体娩』(1964), la jeune femme qu'il y sodomise dans un accès de rage pour se consoler. Son pendant dans Agwîn le monstre des nuages /「空の怪物アグ イー」 (1964), qui, lui, a tué son enfant, et trouve finalement la paix sous les roues d'une voiture. Mitsusaburō éborgné à coups de pierre par des enfants facétieux dans Le Jeu du siècle / 『万延元年のフットボール』 (1967), son meilleur ami retrouvé pendu chez lui, dénudé avec un concombre fiché dans l'anus. Takashi, le frère cadet, bastonné par la police lors des manifestations de 1960, qui se mutile et se tue au fusil de chasse pour expier les abus sexuels qu'il a fait subir à sa jeune sœur handicapée, qui aura fini par se tuer elle aussi en avalant du désherbant. Le héros hypocondriaque ou cancéreux de Le Jour où il daignera Lui-même essuyer mes larmes / 『みずから我が涙をぬぐいたま う日』 (1971), qui rêve de bâtons lui fouissant les entrailles alors qu'il repense à son père se vidant de son sang le ventre ouvert lors d'une tentative grand-guignolesque de coup d'état le 15 aout 1945. Isana, noyé dans son bunker pris d'assaut par la police, prétendant expier ainsi le mal fait à la planète par les hommes, mais payant surtout sa complicité dans le viol et l'assassinat d'un enfant vingt ans plus tôt, dans [Les Eaux ont atteint mon âme]/『洪水はわが魂に及び』(1972). Mori, enfant handicapé soudain précipité dans le corps de son père par la volonté cosmique, qui se jette par la fenêtre après avoir achevé un politicien véreux à coups de marteau dans [Le Procès-verbal du coureur suppléant] / 『ピンチ・ランナー調書』 (1976). Les douleurs chroniques qui rouvrent le canal de communication entre le héros et son fils handicapé dans [Levezvous, hommes nouveaux] / 『新しい人よ目ざめよ』 (1983). Les crises d'épilepsie du même fils dans Une Existence tranquille / 『静かな生活』 (1990). Marie, dont les deux enfants handicapés se sont suicidés, violée puis victime du cancer dans [Parents de la vie] / 『人生の親戚』(1990). Gî, gourou irrésolu aux jambes brisées puis lynché par ses opposants dans [L’Arbre vert flamboyant]/『然え上がる緑の木』 (1993-1995). Hanawa Gorō («double» de l'acteur Itami Jūzō), bastonné par des yakuzas et finalement mort défenestré, son beau-frère cogito victime des persécutions de ses camarades de jeunesse d'extrême-droite dans [Changeling] / 『取り替え子』 (1999), Sakura, l'actrice violée enfant par un soldat américain dans [Annabel Lee] /『美しいア ナベル・リイ』(2007) /, jusqu'à Daiō, militant d'extrême-droite repenti qui se suicide en se noyant littéralement dans une flaque d'eau dans [Noyade] / 『水死』(2009). Et ce ne sont là que quelques exemples.

4 On peut estimer que les expériences personnelles vécues par Ōé ont joué un rôle prépondérant dans cette obsession pour les corps suppliciés: les souffrances physiques endurées pendant la guerre, la perspective de la mort imminente, qui frappe d'ailleurs son père dans la dernière année du conflit, et la puissance de l'imaginaire du jeune enfant qui l'inscrit partout.

5 Voici quelques souvenirs de jeunesse d'ōé, au travers de témoignages personnels livrés entre 1955 et 2016, qui donnent à voir cette expérience de la guerre telle qu'a pu la vivre le futur écrivain alors âgé de moins d'une dizaine d'années :

Mon frère aîné lui-même avait été enrôlé dans une organisation de jeunesse sous la direction de l'armée. Il y avait ce genre de rassemblement dans chaque village: c'était avant tout des armées d'enfants.

(...) Certains enfants, très jeunes, ne supportaient pas de rester dans le rang et se sauvaient. Ils se cachaient dans la forêt.

Un jour, parce qu'il sentait qu'il allait être rattrapé, un gamin s'est suicidé. Les parents ne savaient pas s'ils allaient pouvoir l'enterrer. Un militaire est arrivé et s'est mis à donner des coups de pied dans le corps. J'ai assisté à la scène avec 
d'autres enfants.

(...)

Cette guerre, on vivait pour y mourir. Si on vivait maintenant, si on apprenait des choses à l'école, c'était pour mourir pour cette guerre. C'est ce qu'on nous répétait du matin au soir.

À l'école, on nous entraînait, armés d'un bâton avec un couteau ficelé au bout, à percer une poupée en paille. Je l'ai fait, dès l'âge de 7 ans.

On nous donnait un fusil avec quelques balles, et on nous disait que si nous étions attrapés, il valait mieux se tuer plutôt qu'être fait prisonnier, et on nous montrait qu'on pouvait se donner la mort en mettant dans la bouche le fusil qu'on nous avait donné. On nous avait aussi expliqué comment se faire seppuku.

Dans un cauchemar, j'avançais en rang, on m'avait donné mon fusil mais, comme je ne voyais pas bien, je tirais sur la nuque d'un camarade qui courrait devant moi. J'étais hanté par cette peur, tuer l'un de mes proches, et une fois réveillé, ce cauchemar m'amenait à penser que si je devais me retrouver dans cette situationlà, celle de devoir attaquer, je me suiciderais. $»^{2}$

"À l'école primaire d'Ôsé, j'étais considéré comme un élève dissipé, " désaxé ", et pendant la guerre, je me faisais cogner tous les matins par le principal. Pas avec des gifles, mais à coups de poings : il me tenait la joue de la main gauche, et me cognait la droite le plus fort qu'il pouvait. C'est pour ça qu'encore aujourd'hui, j'ai les dents tordues. Il me cognait parce qu'il disait que je me tenais mal au moment du salut du matin face au portrait de l'empereur.

大瀬国民学校の者の生徒だったぼくは毎朝、校長から平手でなく、拳でなぐら れていた。左手をほおにささえ、逆のほおを力まかせになぐるのだ。今もな

お、ぼくの畨はそのためにゆがんでいる。校長は、奉安殿礼拝のさいに、ぼく がふまじめであったといってなぐるのだ。3

6 Plus tard, la position publique d'ōé, défenseur de la démocratie d'après-guerre et de son pacifisme constitutionnel, continue à inscrire la violence physique infligée aux corps par la guerre au cœur de ses préoccupations : s'il s'agit cette fois d'empêcher à tout prix qu'elle ne survienne à nouveau, c'est toujours bien elle qui informe la pensée puis l'œuvre du jeune écrivain.

7 Quant à la conjonction de la naissance du fils handicapé, de la rencontre avec les irradiés de Hiroshima et de la détérioration du statu quo de la Guerre froide dans les années 1960, elle actualise cette obsession sous les contours de la guerre atomique finale, apocalyptique, qui dans les écrits d'ōé vient bordurer l'avenir comme la grande guerre bornait le passé. Ses œuvres des années 1970 seront pour la plupart consacrées à cette question, s'inscrivant dans ce qu'il dénomme "l'imagination à l'âge atomique " ${ }^{4}$. Mais cette perspective vient aussi réactiver une question que ses tragédies intimes et celles auxquelles il va s'associer, le poussent à poser : celle de la signification ultime de ces souffrances physiques infligées aux corps, face à une modernité (et, pour reprendre Karatani Kōjin prenant ici l'exemple d'Ibuse Masuji, à une littérature japonaise moderne) qui aurait progressivement évacué la question du «pourquoi », pour se consacrer au « comment $»^{5}$.

8 L'homme est fragile, « cassable » pour reprendre le titre de l'un de ses essais marquants de la période ${ }^{6}$. Plus que sa finitude, c'est la «vulnérabilité » (qu'il retranscrit par le composé 攻撃誘発性 surmonté de l'anglais vulnerability, dans un emprunt à l'anthropologue Yamaguchi Masao) de l'homme, ses brisures et ses souffrances, qui définit pour Ōé la condition humaine. Or, dans un univers personnel dont la principale loi physique serait celle de la souffrance infligée ou subie par les corps, il faut pouvoir conférer un sens à cette souffrance pour la surmonter ${ }^{7}$ et « survivre à notre folie ». Y 
chercher une signification va dès lors constituer pour ōé l'une des fins ultimes de son entreprise littéraire.

9 Cette question du «salut » (救済), passant le cas échéant par la «conversion » (回心), ōé l'approche d'abord de biais, par l'intermédiaire d'écrivains dont l'œuvre est marquée par ces questions et qu'il lit depuis sa jeunesse : Dostoïevski, Dante, Blake ou encore Yeats. Ses œuvres des années 1980 l'explorent sous des formes variées. Je me suis intéressé à certaines d'entre elles, à travers la notion de sacrifice, omniprésente dans l'œuvre d'ōé, mais qu'il finira par interpréter en ces années dans une acception de plus en plus ouvertement chrétienne ${ }^{8}$.

10 Je voudrais ici m'attarder sur deux romans considérés comme mineurs dans le corpus de l'écrivain, qui me semblent témoigner de ses préoccupations par rapport à la question du corps et de ses significations possibles dans son système de pensée en cette période charnière de la fin de la Guerre froide qui, comme il l'exprimera plus tard, le « laissera profondément désemparé »"

\section{Fictions « fin de siècle »}

11 Ces deux romans, [Les Tours thérapeutiques $]^{10}$ et $[\mathrm{La}$ Planète des tours thérapeutiques] $]^{11}$, portant en sous-titre la mention "SF d'anticipation» (近未来SF), développent une intrigue prenant place dans un avenir proche de leur période de publication. Ils sont prépubliés en feuilleton dans la revue Hermes (numéros 20 à 24 , de juillet 1989 à mars 1990 pour [Les Tours thérapeutiques] ; numéros 29 à 33, de janvier à septembre 1991 pour [La Planète des tours thérapeutiques]). C'est au cours de leur rédaction qu'Ōé assiste à la chute du mur de Berlin et à la dislocation progressive de l'U.R.S.S. (dont la dissolution officielle est prononcée par Mikhail Gorbatchev un mois presque jour pour jour après la parution du second volume). Ōé avait un temps envisagé une trilogie, dont le troisième volume devait s'intituler [Les Enfants des tours thérapeutiques]『治療塔の子ら』, mais en abandonnera finalement l'idée, pour néanmoins en évoquer la structure et les thèmes trois ans plus tard dans le roman [Vacillation], seconde partie de sa trilogie [L'Arbre vert flamboyant] ${ }^{12}$.

12 Le diptyque formé par ces deux romans de science-fiction est considéré comme une œuvre mineure d'ōé. Peu étudiée et peu citée dans les publications académiques et critiques sur son œuvre, il n'en est quasiment pas fait mention dans les rétrospectives qui accompagnent l'attribution à l'écrivain du Prix Nobel de littérature en 1994.

13 Il n'est pas aisé de résumé l'intrigue foisonnante de ce diptyque resté inédit hors du Japon, mais en voici néanmoins les grandes lignes :

14 Le monde aurait fait face, au cours des années 1990, à une série de catastrophes naturelles et humaines l'ayant rendu en partie insalubre: pollution industrielle, accidents nucléaires, et surtout plusieurs conflits nucléaires localisés ayant éliminé une partie des populations impliquées, et rendu certaines zones inhabitables.

15 Ces événements auraient conduit l'humanité, à la suite de la découverte d'une autre planète potentiellement habitable dans le système solaire, à élaborer un projet dénommé le "Grand Départ » (大出発) : une expédition regroupant une flotte spatiale conséquente, et embarquant un million de colons rigoureusement sélectionnés parmi l'élite des nations. Mais celle-ci revient bredouille : malgré tous les efforts des colons, il apparait qu'une installation durable sur la «Nouvelle Terre» n'est pas possible. 
L'intrigue du premier volume débute peu après le retour des colons, et se concentre sur la relation amoureuse naissante entre l'héroïne Ritsuko, orpheline à l'approche de la trentaine, et Saku, colon fraîchement rentré et fils du commandant en chef de la flotte japonaise. Peu après leur rencontre, la jeune femme relate à son prétendant l'expérience traumatique de viol en réunion qu'elle a vécu une dizaine d'années auparavant (I : 87$)^{13}$, qui lui fait craindre d'avoir été contaminée par le virus du SIDA. ōé, qui rédige son œuvre en 1989 au moment de la prise de conscience globale de la gravité de la maladie, ne prend pas de gants : dans le roman, le dépistage donne lieu à détention immédiate en cas de résultat positif, les malades étant ensuite contraints de porter un signe comparé à l'étoile jaune imposée aux Juifs par le régime nazi. Le test s'avérant négatif, Ritsuko décide de se marier avec Saku et de porter son enfant. Vers la fin du volume, on apprend que la « chaleur et la lumière » qu'elle sentait « irradier » du corps de son amant n'étaient pas illusoires, de même que son insistance à discuter, au travers de poèmes de Yeats, de la perspective d'une "croissance inversée » (I: 70) renvoyant l'homme à un état antérieur. En effet, les colons ont découvert sur la planète éloignée une centaine de bâtiments mystérieux en alliage métallique, renfermant des sarcophages à l'effet régénérant d'une puissance telle qu'elle aurait ramené des morts à la vie. Tandis que Ritsuko prend conscience de porter « un enfant mutant » (I : 199), son partenaire aide un groupe de dissidents à détourner une fusée coréenne stationnée au Japon pour rejoindre la planète avec à son bord une cargaison de malades incurables.

Le second volume se présente comme un récit épistolaire narré par Ritsuko, qui a donné naissance à un fils, Tai (pour Titan). Tandis que des mouvements de protestation éclatent, réclamant un usage démocratique des tours dont l'existence finit par être révélée, une information capitale parvient aux oreilles de l'héroïne : " une intelligence supérieure nous observe et nous confine à notre galaxie ", " une civilisation à la forme différente de la nôtre ", esprit-ruche comparable, toutes proportions gardées, à " un super ordinateur " (II, 74), i.e. à une intelligence artificielle. C'est vraisemblablement à elle que l'on doit les fameuses tours, « comme un cadeau de consolation en échange de la limitation imposée » à l'humanité. Si celle-ci a le malheur de tenter d'outrepasser ses limites, cela déclenchera à n'en pas douter « une guerre intergalactique » (II, 75). Ce rebondissement inscrit le roman dans son époque: la résurgence au cours de cette décennie, dans la culture populaire japonaise et occidentale, de courants de pensée d'inspiration gnostique, selon lesquels le monde ne serait qu'une illusion ou une prison générée par un démiurge maléfique, duquel l'homme doit, avec l'aide d'un envoyé messianique, s'évader pour s'accomplir, et retrouver la félicité dans la présence de la grâce divine dont il a été jadis privé.

17 Le récit prend alors une tournure proprement apocalyptique. Tandis que des terroristes menacent de faire exploser des bombes atomiques sur Terre, des fusées décollent un peu partout pour la planète des tours, où se déploie un spectacle à la Mad Max : des bandes de motards sillonnent les plaines désertiques et se disputent à l'arme automatique les maigres stocks de carburant.

18 Après avoir installé une station de transmission sur Titan, Saku, le compagnon de Ritsuko, a rejoint la planète pour y installer des capteurs sur l'une des tours et percer enfin leur secret.

19 À peine accueilli dans la base installée par les premiers colons, archéologie idyllique où les hommes vivent en harmonie avec la nature, il assiste impuissant à la fuite du fils de son dirigeant, attiré par la vie plus trépidante des pirates du désert, armé d'une 
«bombe atomique propre d'une puissance d' $1 / 1000^{e}$ de Hiroshima » (II, 203). Confronté à la perspective de la voir tomber entre les mains de ses adversaires, son père active son système de sécurité et la fait exploser, sacrifiant ainsi son enfant. Le processus de deuil passe par un voyage astral, alors que Saku et lui avalent des plantes hallucinogènes locales et se lancent dans un délire interprétatif au sujet du " message » qu'ils espèrent obtenir à l'aide des capteurs placés sur l'une des tours. Celles-ci, explique Saku, ne seraient-elles pas une "écriture » des aliens ? Elles sont un " texte ", la « langue » de l'outre-cosmos, et cette sémiotique doit pouvoir conduire, dans le bon " contexte », au décryptage du "code » par les traducteurs appropriés (II : 211, les termes cités ici figurent en français dans le texte original). Dans un «trip » qui évoque les dernières minutes de 2001, l'odyssée de l'espace, Saku glose encore sur «le pouls de Cassiopée et la mer primitive du foetus » (II, 214).

$20 \mathrm{Au}$ travers d'un épilogue ponctuant une ellipse de sept ans, Ritsuko nous conte les derniers développements. Un plan a vu le jour pour interpréter le message des tours : les enfants métis de colons et de Terriens doivent être les intermédiaires qui permettront de le décoder. Ils forment «la croisade cosmique des enfants» (II, 222). Immergés dans une piscine de liquide amniotique, dans leurs cocons individuels, les enfants attendent de recevoir le message des tours que doit leur transmettre le satellite contrôlé par Saku à proximité de Saturne. Il faudra bien, se dit Ritsuko en observant son enfant, « que quelqu'un vienne relier les mondes, comme Jésus-Christ, Mahomet ou Bouddha » (II, 239) (on est bien là dans la conception gnostique du messie, envoyé pour réveiller et guider les hommes vers la dimension divine d'où ils sont originaires avant leur «emprisonnement» sur la Terre). À l'issue de la transmission, le satellite qui abritait Saku disparaît sans laisser de traces.

21 Les trente enfants se sont éparpillés après la réception supposée du message. Le roman s'achève alors que pour la première fois, ils doivent se retrouver lors de la cérémonie d'inauguration de la structure que le jeune Tai, devenu architecte, a imaginée pour englober le Dôme de la Bombe de Hiroshima et le protéger ainsi des pluies acides.

Ce sont toutes les obsessions d'ōé qui nous sont données ici à voir au prisme du genre "SF ", comme autant d'attractions dans une sorte de parc à thème apocalyptique dont ce résumé s'est efforcé de reproduire l'image échevelée: les corps suppliciés, la maladie, la mort, le sacrifice, la guerre, l'atome, l'enfant anormal, Hiroshima, mais aussi le refuge dans une nature salvatrice, l'adoration pour les grands écrivains (ici Yeats), et dans une moindre mesure les élites académiques capables d'en décoder les textes.

Kawamoto Saburō évoque dans sa critique « un folklore de la terreur », et l'expression semble particulièrement appropriée ${ }^{14}$. Il est l'un des rares critiques à consacrer une recension conséquente à l'œuvre, qui passe charitablement sur les incohérences et les exagérations du récit et y voit « l'attitude décontractée, pleine d'humour de l'auteur qui raconte cette fable de SF comme il le ferait d'un conte fantaisiste pour enfants ».

Mais comme souvent, c'est ōé lui-même qui se révèle son meilleur critique. Ainsi, dans [L'Arbre vert flamboyant], trilogie publiée deux ans plus tard, récit d'une communauté religieuse qui élabore sa religion syncrétique fondée sur la prière, dans lequel se retrouvent la plupart des thématiques abordées dans [Les Tours thérapeutiques] (le voyage spatial en moins), le personnage du consul se moque-t-il de son ami l'écrivain qui a finalement renoncé à achever sa trilogie de science-fiction: "pas de science làdedans, seulement de l'occultisme à la petite semaine », "pas de la SF saine! », « dans la SF, vois-tu, la science et la technologie sont importantes, même sans aller jusqu'au 
positivisme. » «Et que tu n'aies pas vu la fin de l'URSS, à la rigueur, mais la télépathie, franchement, et puis quoi encore $! »^{15}$.

Le consul se propose alors d'écrire lui-même la dernière partie du récit, dont il livre au lecteur quelques bonnes feuilles, en particulier la scène finale de retrouvailles entre Ritsuko et Saku, revenu de son voyage astral porteur d'un message extra-terrestre qu'il lui communique en manipulant les phénomènes météorologiques (!).

Néanmoins, au-delà des vicissitudes d'une intrigue trop dépareillée, au-delà d'une certaine désinvolture envers un genre auquel Ōé n'aura peut-être pas accordé toute la rigueur souhaitable, il reste l'image obsédante de ces tours qui soignent et régénèrent les corps et l'esprit. Que représentent-elles ? À quel cadre de références les personnages du roman font-ils appel pour tenter d'en comprendre le mécanisme et le sens?

Il y a les poèmes de Yeats qui les ont vraisemblablement inspirées, à commencer par The tower. Mais bien que Saku s'efforce d'interpréter son expérience à la lumière des œuvres du poète irlandais, c'est, comme ce fut le cas pour Ōé en 1963, son voyage à Hiroshima qui lui donnera la clef.

Alors qu'il survole en hélicoptère le Parc de la Paix avec sa compagne Ritsuko au cours de leur voyage de noces, le jeune homme s'interroge :

Peut-être était-ce là la première "Tour thérapeutique » jamais construite... sous l'effet de l'énorme quantité d'énergie dégagée par l'explosion atomique. Comme si le soin ne touchait pas simplement à la vitalité des corps, mais encore davantage la force d'âme... Peut-être l'âme de l'humanité aurait-elle pu renaître ici au siècle précédent. Si tous les hommes, les uns après les autres, étaient entrés ici pour bénéficier de l'effet de la Tour thérapeutique... Alors peut-être n'aurait-on pas répété les mêmes erreurs... » (II, 18)

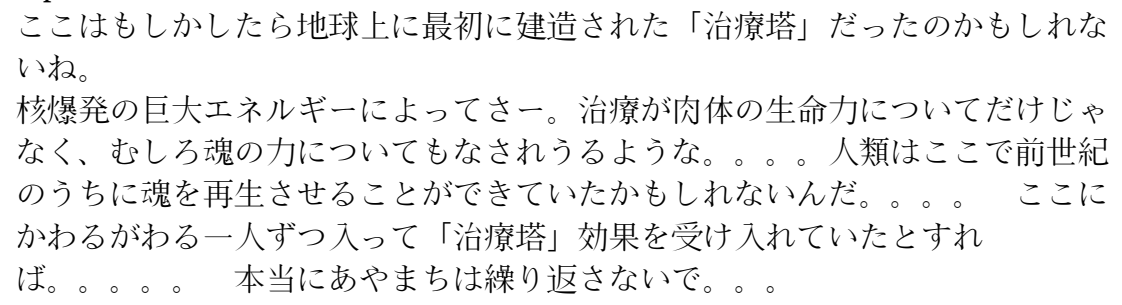

Plus loin, suite à la tragédie de l'explosion atomique déclenchée sur la lointaine planète, au cours de laquelle son hôte perd son fils, Saku reprendra avec lui le fil de cette réflexion.

Selon [votre] conception religieuse [syncrétique], l'unheimlich, avec le sublime, serait au cœur de la Bible et du Coran. [...]

Cet unheimlich, ce n'est rien de neuf ou d'inconnu, c'est quelque chose qui fait partie de la vie intérieure des hommes depuis bien longtemps, mais duquel nous nous sommes éloignés, qui a fait l'objet d'un refoulement. Il me semble avoir vu dans le Dôme de la Bombe à Hiroshima à la fois l'unheimlich et le sublime. Dès ce momentlà, je l'ai relié aux Tours thérapeutiques. En y réfléchissant ainsi, il me semble que cela fait bien longtemps que je suis familier de ces Tours. Bien avant d'en avoir fait l'expérience sur la « Nouvelle Terre » (...)

Si elle existe, la forme de vie intelligente qui a fabriqué ces tours, en prévision de l'arrivée de l'homme sur cette planète, (...) n'avait-elle pas conscience dès l'origine qu'elles puissent être vouées à sauver autant qu'à détruire l'humanité ? » (II, 206) つまり、unheimlichなもの。そ执が「崇高なもの」sublimeと組んでユダヤ・キ リスト教聖書の、またコーランの核心をなす、とサンバルはいう。（...） 「不気味なもの」は実際には新しいものでも未知なものでもなく、人々の心的 生活にとって古くから慣机親しんでいたものであり、ただ抑圧の過程の中で疎 遠にされていたものである。自分は広島の「原爆ドーム」にまさに「」と「」 


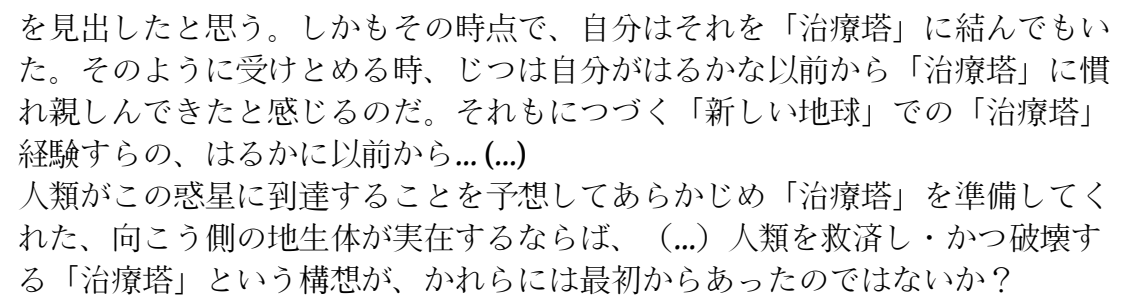

On retrouve ici le cœur de la réflexion d'ōé sur Hiroshima, entamée un quart de siècle plus tôt avec Notes de Hiroshima ${ }^{16}$. Ōé attribue à Hiroshima la signification d'un lieu fondateur sur le plan éthique : ce serait, pour reprendre les mots de Philippe Forest, « le lieu où s'universalise la condition humaine $»^{17}$.Le dôme de la bombe serait la « Tour thérapeutique », car il nous permet, en fixant dans l'espace et le temps l'injustice faite à ses victimes, de reconfirmer ce qui fait de nous des hommes à la fois dans le sinistre de la violence et le sublime de l'empathie avec leur souffrance. Pour Ōé, le dôme de la Bombe, le corps de ses victimes et celui des personnages de ses romans se donnent ainsi à voir comme autant de symptômes de l'Histoire.

31 Cette réflexion sur la valeur significatrice de la souffrance semble faire également écho à la méditation sur la violence inspirée à ōé par la radicalisation des mouvements étudiants à la fin des années 1960, telle qu'elle se développe en 1970 dans [L’Homme, être fragile $]^{18}$.

Alors conspué par les étudiants radicaux qui font de l' "auto-négation » l'enjeu de la lutte contre le renouvellement du Traité de Sécurité Nippo-Américain (ab. Anpo) de 1970, Ōé s'y interroge :

... à travers les mots, j'ai cherché jour après jour à me dissimuler en tant que corps destructible et fragile, mais comme si je faisais le tour de l'anneau de Möbius que porte en lui ce mot de violence au dynamisme si intense, il me faut aussi admettre l'exact inverse. À travers les mots, j'ai cherché à me mettre à nu en tant qu'être doté de ce corps destructible et fragile.

ぼくは言葉によって、であり fragileである肉体をそなえた自分を隠蔽しようと 日々つとめてきた、とすでに書いたが、ぼくは暴力という激しいダイナミズム をそなえた言葉の内部の、いわばメビウスの輪の軌道をひとめぐりするように して、そのまったく逆のことをもまたいわ就ならない。ぼくは言葉によっ て、であり、fragileである内体をそなえた自分を赤裸に剥ぎだそうとしてき た。

J'étais justement en train d'écrire une sorte de fiction miniature sur le comportement de ces jeunes qui ont clairement conscience d'être des corps subissant la violence, et ne cherchent pas non plus, bien au contraire, à se dérober à la conscience d'être des corps l'infligeant, dans ce pays qui est le nôtre, à l'approche de l'année 1970. (...)

さて、いま一九七○年にむけて多様なベクトルが集中するわれわれの国におい て、暴力をくわえら机る肉体としての意識を確実にもち、また暴力をくわえる 肉体としての意識をもつことをもまた、あえて回避しようとしない青年たちの 行動をめぐって、ぼくはごく短い、小説のミニアチュールのごときものを書こ うとしていた。(...)

... Il s'était écroulé sous le coup, et avait perdu conscience deux secondes pendant qu'il supportait le poids de son corps, genou à terre ; mais même réveillé, il s'était rendu compte qu'il ne savait plus s'il était un étudiant cogné par le bouclier d'un agent, ou un agent touché à la tempe par un pavé qu'on avait lancé. Il n'y voit plus rien, tout son corps est brûlant et enflé, et les vibrations du sol, «bam », «bam », sont tout ce qui parvient clairement à ses oreilles, mais impossible de savoir si ces bruits de pas fuient ou montent à l'assaut. Encore deux secondes et il met l'autre genou à terre, puis sa tête sur le sol comme une dernière valise trop lourde, et il a 
l'impression qu'un objet qui pourrait être une grenade lacrymo roule par terre à côté de son œil droit. Il s'est mis en tête de la ramasser pour la lancer sur les étudiants (ou la renvoyer sur les policiers), mais une fois en main, c'était son œil qui avait jailli de son orbite, et du nerf qui l'y rattachait encore, la douleur éclata comme un feu d'artifice écarlate, irradiait à travers sa main devenue transparente, et semblait transpercer le monde entier de flèches d'une lumière pourpre qui illuminait tout...

…... 一撃うけて倒れながら、片腅ついて身の重みを押さえるあいだ二秒間、無 意識だったか机は、覚醒しながらも当の自分が、機動隊員の盾で四ら机た学生 か、飛んできた碟でを撃たれた機動隊員であるのか、わからなくなっているの をさとった。眼は見えず、射じゅうが熱く嵩ばって、ドン、ドンという地響き だけが耳にあきらかであるが、それも逃げてくる足跡か、攻めよせる足跡かわ からない。もう二秒たって、両罡ともついてしまい、頭を重かった最後の荷物 のように地面におろすと、右眼のわきに催涙弾のごときものが転がっていると 感じられる。彼はそれを掝んで学生にむかってなげる（あるいは、機動隊にむ かって投げかえす) つもりになったが、いったん掝んでみると、それは眼窩か らとびだした自分の眼球で、まだつながっている神経束から、痛多が真赤な花 火のように炸裂して、それは掌を透かしたばかりか、天地のあいだを赤い光の 矢でつらぬきとおし、なにもかもを照らしだしたかのようだった...19

...Lorsqu'il coupe soigneusement la corde qui le reliait aux autres par l'imagination pour s'avancer vers sa zone de violence solitaire, il est évident qu'il pénètre dans la souricière de l'existence individuelle. Là, il se re-confirme comme destructible et comme fragile. Sa conscience d'être corps subissant la violence est aiguisée à l'infini, et le propulse vers son fondement humain propre. À ce point limite, il a certainement le droit existentiel de nier dans un hurlement: les mots, l'imagination, et puis quoi ?!

[...]か机が想像力による他者とのつなぎめのロープを、いちいち切りおとして か机とりの暴力の現場にむかってゆくとき、かれが個人的な実在の夕コ壶に はいりこむともまた確かだ。かれは自分の夕コ壼のなかで、であるところの自 分、fragileであるところの自分をあらためて確認する。暴力をくわえられる肉 体としての意識はかぎりなくとぎすまされ、かれをかれ自身の人間的な根源に むけて押しあげる。か机はその極点で、言葉がなんだ、想像力がなんだ、と否 定の叫び声を発する実在的な権利をもつだろう。20

Mais en même temps, il n'est pas non plus impossible qu'il fasse néanmoins, sur les mots, sur l'imagination, un choix porteur d'une validité par rapport à son existence. À tout le moins, en allant jusqu'au bout de sa conscience de corps subissant la violence, le voilà mis à nu, être destructible et fragile, libéré des limites des mots et de l'imagination préexistantes qui le ligotaient jusque-là. N'est-il pas libre alors de choisir de nouveaux mots, une imagination nouvelle?

しかし同時に、か机、言葉について、想像力について、自分でそれらにか机の 実在にかかわる有効性をあたえる決断をすることもまた、ありえめことではな いであろう。すくなくともかれは、暴力をくわえられる肉体としての意識をつ きつめることで、それまでか机を縛りつづけていた既成の言葉、想像力の限界 から自由になったところの、赤裸のでありfragileな存在なのだ。かれが新しい 言葉、新しい想像力を選びとる自由もまた、かれのものではないか? ${ }^{21}$

Pour ce jeune homme, l'expérience qui l'a vu passer de la conscience d'être corps infligeant la violence à celle de corps la subissant fut comme une fenêtre s'ouvrant, comme une lucarne inédite, sur la perspective de toutes choses (...).

この青年にとって暴力をくわえる内体としての意識から、暴力をくわえられる 肉体の意識にいたった経験は、すべての事物への展望に、新しい抜け穴のよう な空が開いたことであった (...)。22

33 Comme le furent pour Job les souffrances infligées par Dieu, parabole à laquelle l'écrivain renvoie régulièrement, telle serait également la «fonction » de cette "Tour thérapeutique » qu'est Hiroshima pour ōé. 


\section{BIBLIOGRAPHIE}

BECHLER Antonin, Ōe Kenzaburō, une économie de la violence, Strasbourg, P. U. Strasbourg, 2016.

FOREST Philippe, Ōe Kenzaburō - Légendes d'un romancier japonais, Nantes, Pleins Feux, 2001.

JONAS Hans, La religion gnostique, Paris, Flammarion, 1978

KАWАмото Saburō, [« Vers les tours thérapeutiques : un folklore de la terreur »] / 『治療塔

へ』一恐怖のフォークロア」, [Littérature nationale] / 国文学, juillet 1990, p. 12. 
LE BRETON David, Anthropologie de la douleur, Paris, Métailié, 1995.

[Littérature nationale] / 『国文学』/Kokubungaku, numéro de juillet 1990.

ōé Kenzaburō, [Solennel funambule] / 長肃な綱渡り / Genshuku na tsunawatari, Tokyo, Bungeishunjū, 1965.

ōé Kenzaburō, Notes de Hiroshima / ヒロシマ・ノート / Hiroshima nôto, Bungeishunjū, 1965

(Gallimard, 1996).

ōé Kenzaburō, [Æuvres complètes d'Ōe Kenzaburō] (période 1) / 大江健三郎全作品 第一期 / Ōe Kenzaburō zensakuhin dai.ikki, Tokyo, Shinchōsha 新潮社, 6 volumes, 1966-1967.

ōé Kenzaburō, [L'Imagination à l’âge atomique] / 核時代の想像力 / Kaku jidai no sôzôryoku, Tokyo, Shinchōsha, 1970.

ōé Kenzaburō, [L'Homme, être fragile] / 壊れものとしての人間 / Kowaremono toshite no ningen, Tokyo, Kōdansha bungei bunko, 1993 [1970].

ōé Kenzaburō, [CEuvres complètes d'Ōe Kenzaburō] (période 2) / 大江健三郎全作品』第二期 / Ōe Kenzaburô zensakuhin dainiki, Tokyo, Shinchōsha, 6 volumes, 1977-1978.

ŌÉ Kenzaburō, Une existence tranquille / 静かな生活 / Shizukana seikatsu, Tokyo, Kōdansha, 1990

[Gallimard, 1995].

ōé Kenzaburō, [Les Tours thérapeutiques] / 治療塔 / Chiryôtô, Kōdansha, 1990.

ōé Kenzaburō, [La Planète des tours thérapeutiques] / 治療塔惑星 / Chiryōtō wakusei, Tokyo, Kōdansha, 1991

ōÉ Kenzaburō, [L'Arbre vert flamboyant] / 然えあがる緑の木 / Moeagaru midori no ki, Tokyo, Shinchōsha, 1993-1995.

ōé Kenzaburō, ōé Kenzaburō, l'écrivain par lui-même / 大江健三郎一作家自身を語る / Ōe Kenzaburō sakka jishin o kataru, Tokyo, Shinchōsha, 2007 (Picquier, 2014).

ōé Kenzaburō, « Je suis né du côté des handicapés », entretien, Le Figaro littéraire, 8 décembre 2005, p. 3.

ōé Kenzaburō, Ōé Kenzaburō, CEuvres, Paris, Gallimard, coll. « Quarto », 2016.

Sciences \& vie : Guerres \& histoire, 38 :2017.

SHIBATA Shōji, [0̄e Kenzaburō - La terre et l'au-delà] / 大江健三郎論一地上と彼岸 / Ōe Kenzaburō - chijô to higan, Tokyo, Yūseidō, 1992.

\section{NOTES}

1. ōé Kenzaburô, [L’Homme, être fragile] / 壊れものとしての人間, Tokyo, Kōdansha bungei bunko, 1993 [1970], p. 103.

2. Sciences \& vie: Guerres \& histoire, 38:2017, p. 6-12. Entretien avec Ôé Kenzaburô réalisé par Rafaële Brillaud le 4 octobre 2016.

3. óé Kenzaburô, [《L'Autel et la couveuse 》] / 奉安殿と養雛温室, in [Solennel funambule] / 厳肃 な綱渡り, Tokyo, Bungeishunjû, 1965, p. 76.

4. ōé Kenzaburô, [L'Imagination à l'âge atomique] / 核時代の想像力, Tokyo, Shinchōsha, 1970.

5. «L'Imagination eschatologique» /「終わりの想像力」, entretien entre Karatani Kōjin et Kasai Kiyoshi, revue [Littérature nationale] / 国文学, juillet 1990, p. 44. 
6. ōé, [L’Homme, être fragile] / 『壊れものとしての人間』,op. cit.

7. LE BRETON David, Anthropologie de la douleur, Paris, Métailié, 1995, p. 107.

8. BECHLER Antonin, Ōé Kenzaburō, une économie de la violence, Strasbourg, P. U. Strasbourg, 2016.

9. ōé Kenzaburō, [Ōé Kenzaburō, l'écrivain par lui-même] / 大江健三郎一作家自身を語る, Tokyo, Shinchōsha, 2007 (tr. française Picquier, 2014 ; nous nous référons ici à l'édition japonaise), p. 212.

10. ōé Kenzaburō, [Les Tours thérapeutiques] / 治療塔, Tokyo, Kōdansha, 1990.

11. ōé Kenzaburō, [La Planète des tours thérapeutiques] / 治療塔惑星, Tokyo, Kōdansha, 1991.

12. ōé Kenzaburō, [Vacillation] / 摇机動く（ヴァシレーション）, Tokyo, Shinchōsha, 1994.

13. Les renvois aux deux romans seront référencés entre parenthèses par la mention « $I$ » (pour [Les Tours thérapeutiques]) ou «II» (pour [La Planète des tours thérapeutiques]) suivie du numéro de page dans le volume.

14. КАһАмото Saburō, [« Vers les tours thérapeutiques : un folklore de la terreur »] / 『治療塔 ヘ』—恐怖のフォークロア」, [Littérature nationale] / 国文学, juillet 1990, p. 12.

15. ōé Kenzaburō, [L’Arbre vert flamboyant] / 燃えあがる緑の木, tome 2 : [Vacillation] / 摇机動 く（ヴァシレーション）, Shinchō bunko, 1998 [1994], p. 176.

16. ōé Kenzaburō, Notes de Hiroshima, tr. du japonais [ヒロシマ・ノート, Bungeishunjû, 1965], Paris, Gallimard, 1996.

17. FOREST Philippe, Ōé Kenzaburō - Légendes d’un romancier japonais, Nantes, Pleins Feux, 2001, p. 133.

18. ōé, [L'Homme, être fragile], op. cit.

19. ōé Kenzaburō, [L'Homme, être fragile], op. cit., p. 103.

20. Ibid., p. 112.

21. Ibid., p. 113.

22. Ibid., p. 96.

23. LASCH Christopher, La culture du narcissisme, Paris, Flammarion, 2006. Sur les résurgences postmodernes du gnosticisme, « postface ", p. 293-307.

24. JONAS Hans, La religion gnostique, Paris, Flammarion, 1978, chapitre XIII : "Gnosticisme, existentialisme et nihilisme moderne », p. 417-442.

25. ōé Kenzaburō, « Je suis né du côté des handicapés ", entretien, Le Figaro littéraire, 8 décembre 2005, p. 3.

\section{AUTEUR}

\section{ANTONIN BECHLER}

Antonin Bechler est maître de conférences au département d'études japonaises de l'université de Strasbourg. Ses recherches portent sur les représentations, discours et idéologies dans les productions narratives du Japon contemporain. Il est notamment l'auteur d'ôe Kenzaburô, une économie de la violence (Presses Universitaires de Strasbourg, 2016), et a coordonné l'édition des Oeuvres d'Ôé dans la collection Quarto chez Gallimard (2016). 


\title{
Le corps comme sujet poétique dans le roman vietnamien Cheval d'acier (2014) de Phan Hôn Nhiên
}

\author{
Cam Thi Doan
}

1 Au terme des cinq décennies d'un réalisme socialiste qui conçoit son héros, con người mới / homme nouveau, comme un simple instrument au service de la politique, les années 1990 voient la littérature vietnamienne prendre un véritable tournant sous l'impulsion de la politique d'ouverture, appelée Đổi Mới / Renouveau et menée par le Parti communiste qui, pour pouvoir rester en place, accorde une certaine souplesse à la vie économique et culturelle. Grâce à ce souffle libérateur, le corps, en tant qu'organique et sexué, pénètre peu à peu le territoire littéraire. Sa représentation fait prendre conscience de la violence physique dont les femmes sont victimes, tout en révélant leur besoin d'amour que les idéologies dominantes, le néoconfucianisme et le marxisme-léninisme, ont contraint au silence.

2 Néanmoins, c'est seulement à l'aube du troisième millénaire que le corps accède au statut de sujet littéraire grâce à l'émergence d'une génération d'auteurs qui a grandi loin des combats. Trafic sexuel, homosexualité, prostitution sont autant de nouveaux sujets qui éclatent dans un Vietnam en pleine mutation. La littérature, en particulier chez les femmes auteures, n'hésite pas à décrire les parties génitales et les pratiques sexuelles les plus intimes. Dans la célèbre nouvelle [Cauchemars] / Bóng đè écrite en 2006, l'écrivaine Đỗ Hoàng Diệu (1976-) n'en montre pas moins un corps féminin fait d'organes, lieu de désir et de plaisir'1. Elle va jusqu'à en faire le personnage principal de son œuvre. Tel un héros romanesque, celui-ci rencontre diverses aventures: jouissance, viol, menstruation, grossesse, avortement. Derrière cette écriture qui pourrait paraître provocatrice, se lit de façon sous-jacente une volonté non seulement d'abattre certains préjugés, confucéens puis communistes, mais aussi d'inverser les catégories du noble et du vulgaire. Il n'est donc pas moins significatif que dans un pays tel le Vietnam actuel, lourdement marqué par la violence des guerres et les valeurs viriles, les écrivaines célèbrent le corps féminin, non pas comme lieu de gestation mais 
comme source de jouissance. De cette façon, elles contribuent à renouveler le discours sur l'individu et le monde ${ }^{2}$.

3 C'est dans ce contexte que le roman Cheval d'acier / Ngựa thép, paru en 2014 de Phan Hồn Nhiên, née en 1972 et grande figure de la scène littéraire, mérite une lecture approfondie ${ }^{3}$. L'œuvre se compose de trois récits autonomes - «Le Corps », «La Mer » et « Pelikan » - qui, traversés par des figures ayant pour motif le cheval, sont structurés par des face-à-face autant inattendus que tragiques. Dans « Le Corps », devant un jeune homme agonisant, trois personnages - sa mère, son beau-père et sa fiancée - se rappellent les souvenirs de l'être aimé en plongeant dans les zones obscures de leur mémoire. "La Mer » décrit les retrouvailles de deux frères jumeaux qui sont séparés par l'exil, la trahison et bientôt la mort. « Pelikan » raconte, quant à lui, la convergence de deux destins : une jeune femme en quête de mémoire après un accident et son professeur de linguistique, un homosexuel en conflit avec sa famille. Sentiment d'abandon, blessure d'enfance et impossibilité de partage constituent le fondement de ces récits. Phan Hồn Nhiên imagine un monde où s'effacerait toute limite entre pays et continents, si bien que seules subsisteraient les frontières entre les individus. Alors que ses personnages, quels que soient leur sexe et leur âge, ne cessent de voyager, ils n'en demeurent pas moins seuls face à leur propre corps perpétuellement mis à mal, victime de violence, de maladie, de vieillissement. Chez Phan Hồn Nhiên, les femmes n'enfantent presque pas tandis que les hommes meurent d'une mort précoce.

4 L'essentiel, et ce qui fait la spécificité du roman, se joue dans les corps qui y occupent une place de tout premier plan. Des portraits et autoportraits de personnages y côtoient d'innombrables images figuratives qui représentent l'organisme, qu'il soit humain, animal ou végétal, sous diverses formes - tableaux, dessins, sculptures, photographies, fragments de film, cartes postales, affiches publicitaires.

5 Écrit huit ans après [Cauchemars], Cheval d'acier, certes partie prenante du nouvel élan littéraire, semble toutefois quêter une autre écriture du corps. D'emblée, il le confronte à trois épreuves qui, sans être tout à fait nouvelles dans le champ de la littérature, n'avaient jamais été traitées de façon concomitante par la production corporelle, à savoir la recherche d'identité liée au processus de la mondialisation, l'expérience de la maladie et le foisonnement des arts visuels. Ces trois thèmes vont, on le verra, s'imbriquer au point de se fondre les uns dans les autres.

6 En quoi réside l'originalité de la représentation corporelle chez Phan Hồn Nhiên ? Quels sont ses nouveaux moyens d'expression pour dire le corps? De quelle manière a-t-elle enrichi l'imaginaire corporel?

\section{Le corps comme identité}

7 Les personnages de Cheval d'acier ont souvent une identité incertaine. Nul ne sait d'où ils viennent, où ils vivent. Les lieux ne sont jamais nommés, hormis Saigon - leur point de départ et parfois d'arrivée -, et une ville américaine, Santa $\mathrm{Fe}$, qui baigne dans l'atmosphère de Georgia O'Keeffe. À l'aube du xxi siècle apparemment libéré de contraintes idéologiques et politiques au profit du libre-échange, à la croisée de plusieurs langues et de plusieurs cultures, ces individus sont comme des îlots séparés par de profonds abîmes. Entre eux et leur entourage règne un sentiment d'incompréhension et de malaise. Si avec leurs proches, ils tentent parfois de créer des liens de l'amour, le plus souvent ils les fuient ou les détruisent. Chacun crée alors son 
propre univers dans lequel il se réfugie. Celui-ci peut être radical comme la mort, mais aussi un objet quotidien. Les livres sont ainsi un abri pour Song, un des protagonistes : « en lisant, je ne vois plus ni mon père, ni Anna [sa mère], ni moi-même » (p. 38). La piscine est, quant à elle, détournée de sa première fonction pour lui permettre d'échapper à ses proches, au risque de la noyade. Sans concession, l'écrivaine analyse les blessures intimes de ses contemporains et leurs difficultés de partage.

Chose remarquable: tout en refusant la nostalgie, Cheval d'acier semble accuser les déplacements de populations, fruit du phénomène de mondialisation qui caractérise notre époque, d'être à l'origine de ces troubles intensément vécus par ses personnages. Il met en scène des figures parentales défaillantes et des individus représentatifs de la nouvelle génération qui, pour pouvoir survivre, deviennent "amnésiques » tout en étant «paumé(s) dans la société » (p. 314). De même que ces derniers se comparent à des «machines", ou à des "robots» qui "programm(ent) d'emblée (leur) vie» (p.314), de même ils ne sont aux yeux de leurs contemporains qu'« un objet parmi d'autres" (p. 239). Phan Hồn Nhiên va jusqu'à mettre l'aliénation croissante sur le compte de la condition de l'homme moderne puis post-moderne, comme le dit un personnage :

Rien n'étant stable dans l'existence que nous menions, vouloir créer des liens ou quelque chose d'équivalent était absurde [...] l'être humain est aujourd'hui plus attaché à son activité qu'à son entourage. Être occupé par l'un au point de ne pas avoir de temps pour l'autre, n'est qu'un prétexte car c'est un désir qui vient de l'intérieur. Volontairement, chacun se transforme en un rouage de cette machine invisible qu'est la vie. Il a peur de l'arrêter ou d'être exclu par elle. Sa famille, ses amis, la personne avec qui il vit, ses principes, tout existe mais tout peut être aussi remplacé, voire oublié. (p. 115)

Cependant, le roman de Phan Hồn Nhiên serait de peu d'intérêt s'il avait pour seule ambition le réquisitoire de la modernité. Sa force est ailleurs, dans sa capacité à renouveler les questionnements sur le rapport entre l'individu et le monde. Son œuvre semble démontrer que plus qu'un "esprit» ou qu'une "âme», c'est la conscience corporelle qui donnerait à l'homme contemporain le sentiment d'exister, que son corps, en tant qu'objet organique, serait la seule attache, l'unique limite, l'élément primordial de son être. Si ce propos est à nuancer, il est clair qu'il permet d'explorer autrement la problématique de l'identité à l'ère du numérique et de la mondialisation.

Alors que ses personnages ont un état civil assez flou, l'écrivaine vietnamienne accorde une attention toute particulière à leur corps, en tant qu'objet organique. Souvent, leur regard frappe par une rare acuité anatomique si l'on en croit au portrait qu'Anna, une des narratrices du premier récit, fait de son propre visage ravagé par le temps : «Je vais sur la pointe des pieds jusqu'à la fenêtre [...] un visage [qui] apparaît sur la vitre humide. Un visage fané et ridé dont le regard de glace ne peut plus masquer la ruine ». Tout en observant les moindres changements de leur corps, les personnages de Cheval d'acier les commentent avec une distance telle qu'on dirait un organisme étranger. La laideur y est montrée sans réserve. Le roman compte par ailleurs nombre de passages portant sur les parties du corps que la littérature examine rarement, comme la « clavicule», les «muscles et articulations» (p. 102), l'« œsophage» (p. 165, 214). Un autre autoportrait d'Anna est d'autant plus étonnant qu'elle nomme ses organes avec une précision clinique :

Cette année, par une matinée hivernale, en me réveillant, j'ai découvert que je n'arrivais presque plus à bouger mon corps, tant mes articulations, aux genoux, aux coudes, aux doigts, demeuraient engourdies. Il a fallu que l'homme avec qui je 
vivais alors masse mon cou et mes épaules pour que je retrouve un peu de mouvements. Tout en malaxant ma peau flasque de sa main géante et sèche, il détournait soigneusement son regard de mon visage et de mon corps pour éviter sans doute les émanations morbides qui s'en dégageaient. À la sortie du lit, j'ai commencé par faire ma toilette. La figure dans le miroir m'a tellement stupéfiée que j'ai jeté le peigne. Des yeux vitreux. Des sourcils incolores. Un nez anguleux et triste comme celui des koalas. Des lèvres aux commissures ridées et aux contours flous sous des duvets blonds un peu roux. Pour la première fois de ma vie, j'ai pensé à mon visage, à ses expressions, à son déclin inéluctable, à tous ces signes inattendus de sa destruction. (p. 81)

11 Les protagonistes de Cheval d'acier ont une conscience étonnamment aigüe de leur corps. La romancière les met souvent à nu au sens littéral du terme et les laisse discourir sur le corps. Le mot "corps »/cơ thể apparaît à cent quatre-vingt-une reprises dans le roman. Si en vietnamien plusieurs termes signifient corps comme par exemple mình, thân, cơ thể, le dernier correspond davantage au terme "corps » en français tel qu'il est défini par le dictionnaire en ligne du CNRTL (Centre national de ressources textuelles et lexicales): «Ensemble des parties matérielles constituant l'organisme, siège des fonctions physiologiques et, chez les êtres animés, siège de la vie animale ». Souvent cơ thể est utilisé dans Cheval d'acier pour désigner, voire définir, un individu. "Son corps se retourne vers moi ", raconte Anna à propos de Bách (p. 106). "C'est un corps calme qui dégageait une vague odeur d'antiseptique » (p. 293), dit San lorsqu'on lui demande de donner le sens du mot "père ». Constamment, les personnages sont associés à leur corps organique, si bien que dans son récit des retrouvailles avec Bách, son ancien mari, Anna qui l'aide à attacher les cordons de sa blouse avoue l'avoir reconnu en tant que «corps": "comme si les dix années qui séparent ce corps et le mien n'existaient pas » (p. 70), dit-elle.

12 Sans détours, les personnages évoquent leur propre corps et celui des autres. Au mépris des codes de la pudeur, ils disent "mon corps", "ton corps", " son corps ", " nos corps ». Bách décrit son beau-fils en ces termes :

Je voyais revenir de manière inéluctable certaines images, celles du corps de Song. D'abord celui d'un bébé, minuscule et livide [...] Puis celui d'un jeune homme avec, sur son torse nu, un tatouage en forme de cheval [...]. L'inquiétude que nous avions ressentie pour ce corps. (p. 56-57).

Quant à Anna, elle multiplie des adjectifs possessifs en parlant de son corps :

Mon corps est transi de froid [...] le froid vient justement de moi. Sorti d'un espace étroit situé entre deux vertèbres, il envahit tout mon corps jusqu’à pénétrer les rides de ma peau, ce qui me fait trembler davantage» (p. 105). «Mon corps, y compris la peau qui l'enveloppe et le liquide qui circule dans ses veines, est si épuisé qu'il ne m'est plus du tout capable de raisonner » (p. 85). « Ma peau ressemble à une enveloppe aussi usée que froissée, qui dissimule à peine les muscles atrophiés et ramollis [...] l'image de mon propre corps m'a flanqué la trouille. (p. 102)

14 En revanche, la rareté de termes censés déterminer le moi intime, tels "âme", " cœur », " esprit », "volonté », est notable. Et si ces derniers sont évoqués, c'est souvent avec ironie. «Il m'était impossible d'avoir ton esprit. C'était, hélas, ce que les femmes aimaient le plus chez toi » (p.174), déclare le héros du deuxième récit, « La Mer ", à son jumeau qui lui ressemble trait pour trait.

Chez Phan Hồn Nhiên, le corps est une entité autonome, irréductible à tout autre, si bien que l'acte sexuel, qui consiste à " partager son corps avec un partenaire " pour reprendre une formule d'un des personnages du roman, est souvent mis en échec. Pour 
S., le héros du troisième récit, "Pelikan », le "partage des corps " a été une "grave erreur » : « au lieu de nous rapprocher, il nous a donné du chagrin, nous a fait mesurer la distance qui nous séparait » (p. 327). Quant à Song, il déclare : «L'idée de toucher, de pénétrer un autre corps et de laisser quelqu'un d'autre observer, analyser mon propre corps me semblait intolérable » (p. 26).

\section{Le corps malade}

16 Afin d'aller plus loin dans la logique de son raisonnement, Phan Hồn Nhiên pousse jusqu'au paradoxe le lien, souvent négligé, entre la conscience de soi et la conscience corporelle. Elle superpose donc la problématique du corps à celle de la maladie, thème relativement peu exploité de la littérature vietnamienne. En effet, tout en banalisant la mort, celle-ci a longtemps cherché, non pas à réduire au silence, mais à occulter les affections morbides ou les douleurs physiques, car elles risqueraient d'être jugées négatives, personnelles, voire narcissiques par un peuple qui avait toujours, dans sa longue tradition de guerre, mis en avant l'héroïsme en glorifiant le corps de ses soldats, lequel avait tendance à représenter la patrie et la nation. Longtemps après 1975, date de la réunification du pays, la question du corps restait «taboue dans une société confucéenne engoncée dans un credo marxiste autoritaire, "viril' et [...] très puritain. $»^{4}$

C'est donc dans une tentative de briser ce tabou que Phan Hồn Nhiên articule-t-elle chacun des trois récits de Cheval d'acier autour du corps d'un personnage qui est atteint, en pleine jeunesse, d'une maladie grave - d'un cancer ou d'un traumatisme crânien. Ce faisant, elle n'hésite pas à retracer la progression des pathologies, à décrire l'univers hospitalier, à restituer leurs perceptions physiques et mentales, jusqu'à développer un véritable imaginaire du corps souffrant et médicalisé.

Dans le premier récit, intitulé précisément "Le Corps ", le corps de Song, depuis sa naissance jusqu'à son agonie, est placé au centre des intérêts des trois êtres les plus proches de lui, à savoir sa mère Anna, son beau-père Bách et sa fiancée. Il est d'ailleurs impossible d'extraire ce corps de l'histoire d'amour et de haine qui relie Song à ces trois personnages, corps devenu source de conflits entre celui qui l'habite et celle qui l'a mis au monde, entre l'homme et la société qui l'abandonne. Victime de tentatives de destruction - sa mère a tenté d'avorter avant de donner le nourrisson qu'il était à une famille étrangère -, d'actes d'autodestruction - le fait qu'il aime se faire mal peut être interprété comme indice de son désir de se venger d'elle -, le corps de Song constitue un réseau de nœuds de tension, de chantage et de perversité, qui le conduit finalement au crime et à la mort. En effet, face à sa maladie mortelle, le jeune homme accepte le contrat diabolique que lui propose sa mère: Anna réglera ses frais d'hospitalisation extrêmement coûteux à condition que Song provoque un accident qui rendra handicapée sa sœur Anne, dont le bonheur suscite chez elle tant de jalousie.

Pour mettre en avant les corps malades, Phan Hồn Nhiên mène une investigation médicale tout à fait exceptionnelle. À la manière de Susan Sontag qui se donne pour tâche, dans son livre La maladie comme métaphore ${ }^{5}$, la déconstruction des fantasmes et métaphores qui entourent les maladies, l'auteure qui se sert d'un vocabulaire spécifique en vietnamien et en anglais comme "lung cancer", "metastasis», "malignant tumor», "post-traumatic amnesia», décrit avec précision tant les symptômes de la pathologie que la dégradation physique de ses personnages. En cette 
matière, l'écriture de Phan Hồn Nhiên atteint son acmé dans ce portrait clinique que dresse Anna de son fils mourant :

Song est dans la même position depuis hier [...]. Sous le drap tiré jusqu'à l'abdomen, mon fils semble conserver seulement une moitié de son corps, l'autre ayant achevé son processus de décomposition. Devant moi, on dirait un buste en cire jaune, posé dans le creux de l'oreiller. De sa douce chevelure, il ne reste que quelques racines grisâtres. Le cou si maigre sous une peau transparente fait penser à une carambole anguleuse. Quant à ses mains, l'une est à plat sur le matelas et l'autre retournée ; les doigts livides sont recroquevillés par la douleur. Les yeux mi-clos observent, paraîtil, ce qui se passe autour. La bouche est retroussée à un coin. Les pommettes, couvertes de taches rougeâtres dont l'apparition depuis quelques heures est sans raison apparente. Les lèvres d'un rouge si pourpre qu'il paraît anormal. Ce sont évidemment de nouveaux symptômes de sa maladie, liés à la forte fièvre, mais ils me rappellent un fragment de souvenir qui surgit dans ma mémoire. Le même corps et le même visage mais à des moments différents de la spirale du temps... (p. 72)

Comme souvent chez Phan Hồn Nhiên, le corps souffrant, porteur d'empreintes de la maladie, du temps ou de la violence, fait l'objet d'un examen méticuleux. Celui-ci ne traduit rien d'autre que le regard sans complaisance que les personnages portent sur leur propre corps et celui de l'être aimé.

21 Loin du pathos et de l'esthétisation de la maladie, Cheval d'acier poussera le lecteur au seuil de l'insupportable en mettant en scène Song dont la mort s'annonce imminente. À la fin de la troisième partie, dans son ultime souffle, le jeune homme demande à la narratrice, la femme qu'il aime : "Que feras-tu dans les prochains jours? », question qui est suivie d'un aveu, certes silencieux mais non moins brutal, de cette dernière :

Sa question est tellement claire qu'elle suscite chez moi, non pas une douleur ni une tristesse, mais une sensation d'engourdissement. Il est curieux que les gens soient intéressés par la vie à laquelle ils ne participeront plus. (p. 137)

L'agonie de Song constitue un des moments forts du roman quand la jeune femme, après avoir partagé avec lui toutes les étapes de sa maladie qui vont de l'illusion de la guérison à la mort, prend la main du mourant et y reconnaît «la chaleur d'un être humain, l'odeur d'un être humain, le pouls [...] d'un être humain » (p. 139). Là encore, c'est de l'aspect organique du corps qu'il est question. Face à sa prochaine disparition, l'homme est démuni. Ni Dieu, ni mystique, ni révolte, mais comme une acceptation.

Aussi Phan Hồn Nhiên relie-t-elle de manière systématique ses personnages à leur corps biologique. Il n'est pas indifférent que l'expérience de la maladie, tout en leur rappelant qu'elle est constitutive de leur existence, leur ait permis de prendre conscience de leur singularité individuelle. Si vulnérable soit-il, leur corps semble demeurer le lieu de leur limite fondamentale dans un monde qui est désormais sans frontière, esclave du numérique et du virtuel.

Sans conteste, par ce nouveau mouvement exogène, le roman de Phan Hồn Nhiên veut, non pas rejeter les questions du Moi, de l'intime, de l'identité, mais au contraire, déplacer l'angle d'observation pour mieux les questionner.

\section{Corps humain/corps animal/corps végétal : le corps comme sujet poétique}

En contrepoint de cette cascade de corps meurtris, Phan Hồn Nhiên dresse une immense galerie d'images figuratives, composée de tableaux, de dessins, de sculptures, 
de photographies, de fragments de film, de cartes postales, d'affiches, exposant le corps humain, animal, végétal. Cette démarche originale qui consiste à intégrer, au sein d'un texte littéraire, les images relevant des arts visuels pour reprendre une expression de Bernard Vouilloux ${ }^{6}$, participe pleinement de sa volonté d'élargir les frontières génériques de la représentation corporelle.

D'emblée, il n'est pas indifférent que l'imagerie industrielle soit omniprésente dans le roman de Phan Hồn Nhiên. Victime d'un "post-traumatic amnesia " - il s'agit d'une "perte de mémoire progressive " liée à un grave accident -, l'héroïne de la troisième partie, San, sort de son coma et observe des corps humains, dans une affiche commerciale :

Sans lire les slogans, elle examine avec attention l'image qui représente un groupe de personnes bondissant d'un air joyeux dans l'espace. Néanmoins, autant leur sourire figé que leur bond forcé les éloignent davantage les uns des autres. Leurs yeux grands ouverts expriment joie et surprise avec une telle uniformité qu'ils traduisent plutôt une sorte de frayeur. (p. 223)

Mais c'est en regardant ses propres photos dans un "vieil album " que le personnage constate qu'elles ne lui sont pas moins étrangères que les «créatures inconnues » du panneau:

« Comme une revenante, elle observe ces images dont elle a perdu le fil, les analyse une à une afin de restaurer son propre passé pourtant si proche. Hélas, ses efforts sont loin d'être aussi fructueux qu'elle l'espérait. » (p. 232)

Tout se passe comme si son univers n'était plus que reconstruction et représentation. Quoi qu'il en soit, il est impossible de ne pas lire dans le travail de Phan Hồn Nhiên, son désir d'introduire l'imagerie moderne dans le roman afin de renouveler l'écriture, en particulier celle du corps.

La romancière n'en convoque pas moins l'art contemporain et des artistes réels. Ainsi Bách observe-t-il de loin la silhouette du petit Song lisant, «parfaitement immobile [...] sous la lumière blanchâtre du néon ", ne peut s'empêcher de penser à une "sculpture en plâtre de George Segal », avant de conclure : " Même si elle [...] sa maigreur excessive ne satisfaisait pas les critères classiques de la beauté, la statue était singulière et émouvante " (p.36). De cette manière, l'écriture corporelle chez Phan Hồn Nhiên s'enrichit de l'art de George Segal (1924-2000) qui utilise, rappelons-le, la technique de moulage sur modèle vivant pour représenter le corps humain dont les attitudes et les gestes, saisis sur le vif, sont pour le moins énigmatiques. L'écrivaine vietnamienne insiste sur la matière de prédilection de l'artiste américain, le plâtre, dont la blancheur, renforcée par l'éclairage de néon, crée en effet une certaine distance par rapport au réel tout en soulignant l'extrême fragilité de l'individu dans son environnement.

Autre travail artistique sur le corps qu'évoque Phan Hồn Nhiên dans Cheval d'acier : les fleurs en gros plan de Georgia O'Keeffe (1887-1986). Bách les admire dans le musée dédié à l'œuvre de l'artiste à Santa $\mathrm{Fe} \mathrm{-} \mathrm{«des} \mathrm{fleurs} \mathrm{si} \mathrm{sensuelles} \mathrm{qu'on} \mathrm{avait}$ l'impression qu'elles respiraient et que leurs pétales bougeaient sur la surface très douce de la toile, en rêvant aux deux corps luminescents dans cette piscine d'été " (p. 27) - ou sur «l'écharpe imprimée » d'Anne - «des fleurs mauves et bleues d'o'Keeffe » (p. 45), puis "les pétunias d'O'Keeffe » (p. 46). Rappelons que le génie de l'artiste américaine a été de s'éloigner de la représentation classique de la nature morte, en utilisant un cadrage étroit lui permettant d'extraire la fleur de son utilité afin de se concentrer exclusivement sur sa texture, ses lignes, ses dégradés de teintes. 
Isolées puis agrandies, ses fleurs deviennent mystérieuses et chargées d'une sensualité si puissante qu'elles ne peuvent sans rappeler certains organes sexuels. Il va sans dire que l'insertion d'œuvres de l'artiste américaine dote l'écriture corporelle chez l'écrivaine vietnamienne d'une nouvelle sensibilité.

31 Parmi ces multiples images que Phan Hồn Nhiên décrit, celles du cheval courent comme autant de leitmotivs à travers le roman. Dans la première partie intitulée «Le Corps ", Song se souvient que pendant son enfance solitaire, il a volé un petit cheval d'acier pour en faire un jouet qui est devenu «extraordinaire » et auquel il s'est tellement attaché que sa mère l'a jeté un jour quand elle voulait le punir. La deuxième partie, «La Mer ", se clôt sur une statuette de cheval que le héros, atteint du cancer, a laissée à son frère jumeau comme ultime souvenir familial avant d'aller se donner la mort dans l'océan :

Un objet en métal massif. Une forme cylindrique allongée et reliée à une face fièrement redressée sur un cou courbé, une queue formant un angle droit avec le socle raboteux, en équilibre avec la tête qui s'éloigne du centre de la petite statue. Des lignes sobres, à la fois souples et rigoureuses. Un bloc d'acier étincelant et glacial qui défie le temps [...] La lumière du jour a complètement disparu, l'horizon se rapproche et les ténèbres montent. Le cheval est debout sur la plage où se reflète son ombre taciturne, bien plus grande que sa propre taille. (p. 198).

Enfin, dans « Pelikan », la troisième partie, l'action principale se déroule dans le « hall Steel Horse d'un immeuble côté impair d'une rue du centre-ville» qui porte dans sa vitrine « un petit cheval d'acier de style minimaliste, vigoureux et méditatif ». (p. 261)

D'autres figures de cheval ne manquent pas de renforcer l'imaginaire du corps animal. Song porte sur son plexus un tatouage en forme de cheval : il s'agit d'« un petit cheval admirablement bien dessiné » dont le jeune homme a voulu orner son corps en souvenir de son jouet perdu, « un cheval d'acier » (p. 24). Sa première rencontre avec la femme qui deviendra par la suite sa compagne, est placée sous le signe de cet animal, lorsque dans une galerie d'art ils se trouvent par hasard l'un à côté de l'autre, attirés par « un petit tableau qui représentait un cheval bleu galopant sur une mer tranquille d'un rouge écarlate, avec sur son dos une forme humaine de couleur blanche » (p. 113). Dès lors, des images de cheval ne cessent de les unir avant d'envahir l'univers intime du couple :

Dans le nouveau logement, fixé à côté de la fenêtre de notre chambre, il [le tableau du cheval] s'est transformé en une autre fenêtre. Quand nous étions allongés l'un à côté de l'autre, nous aimions y contempler à loisir ces êtres volant sur une mer tranquille (p.117)

Song était toujours assis au bureau, mais au lieu de lire, il regardait le cheval bleu dans le tableau en face. Il s'est retourné vers moi, les yeux remplis de larmes. (p. 135)

Il convient par ailleurs de noter que des nombreux visuels évoqués par Phan Hồn Nhiên tout au long de son roman, la description est admirablement maîtrisée. Aussi brèves soient-elles, ces représentations verbales permettent, on l'a vu, de créer un système de résonances entre les différents récits de Cheval d'acier et jouent ainsi un rôle essentiel dans la dynamique de l'histoire ${ }^{7}$. Pétries d'affectivités, les images de cheval n'en interviennent pas moins dans les étapes-clés de chacun des récits. Le plus important: elles proposent une autre lecture du corps, par une esthétique des contrastes, entre l'humain et l'animal, l'animé et l'inanimé, le réel et l'imaginaire, le prosaïque et le poétique, qu'illustre la scène suivante : 
Comme il faisait très chaud, il était en jean et torse nu. Song laissait voir un corps parvenu à maturité, avec des épaules larges, des hanches étroites et un poids sans doute inférieur à ce qu'exige la norme médicale. Curieusement, cette maigreur, ce teint jaune foncé comme de la cire et ces côtes qu'on voyait à travers la peau, allaient parfaitement avec son air calme et réfléchi [...] Sur son plexus, il s'était fait tatouer un cheval, sans doute depuis son entrée à l'université [...] C'était un petit cheval admirablement bien dessiné... (p. 24)

Plus tard, pendant la maladie de Song, l'ordre sera inversé puisque l'humain sera inerte et l'animal vivant, mais leur dialogue est plus intense que jamais :

Pendant [le] sommeil de Song, je regardais le cheval tatoué au-dessous de son thorax. L'animal palpitait, comme si après une course périlleuse, il avait besoin de se reposer. Puis mes yeux se posaient sur le cheval bleu galopant sur la mer d'un rouge écarlate, et j'avais des larmes au coin des paupières. (p. 126)

Le cheval - non pas l'animal sous sa forme naturelle mais ses représentations - est devenu pour Song son double à qui il s'identifie. C'est ce va-et-vient perpétuel entre corps réel et corps stylisé qui fait la spécificité du roman. Il n'est pas d'ailleurs indifférent que Phan Hồn Nhiên, afin de mieux questionner la rencontre entre corps et art, s'intéresse au tatouage qui est un art corporel par excellence dans la mesure où le corps est traité comme un lieu idéal pour exprimer désirs et fantasmes, ce que déclare par ailleurs Song à son beau-père :

Enfant, j'avais un jouet, un cheval d'acier, que j'ai perdu par la suite. Plus tard, j'ai

pensé qu'il valait mieux que j'en aie un sur le corps. (p. 24)

Chose non moins essentielle : Song prend soin de préciser que son attachement pour ce « cheval d'acier » n'est pas dû à un quelconque « symbole » de l'animal, encore moins à un désir « d'être dur comme l'acier» (p. 24). Tout le roman prouve, en revanche, que pour ses personnages, le cheval renvoie avant tout à un modèle de beauté.

\section{Conclusion}

Ainsi serait-il difficile de concevoir ce roman en dehors de son omniprésence iconique. Que Phan Hồn Nhiên choisisse « cheval d'acier » à la fois pour titre et motif principal de l'œuvre, dévoile son sens profond. Placée en entier sous le signe de l'art, celle-ci explore le corps dans une écriture aussi sombre que lumineuse. Diplômée de l'École supérieure du Cinéma et du Théâtre, Phan Hồn Nhiên observe le monde, on l'a constaté, avec le regard d'un metteur en scène, c'est-à-dire avec une sensibilité particulière tant au volume, à la couleur, à la lumière qu'au corps. Dans les portraits qu'elle dresse de ses personnages, la romancière qui n'ignore rien des techniques de son métier, emprunte à l'art pictural certains de ses procédés. Notons également à quel point elle nourrit son texte d'œuvres d'art, vraies ou fictives, non pour le décorer, mais au contraire, pour mieux explorer la réalité.

Appuyé sur une double construction en triptyque - son roman se divise en trois récits dont la première comprend également trois parties -, Cheval d'acier rappelle, et avec quelle force, Three Studies for Figures at the Base of a Crucifixion (1944), un triptyque de Bacon qui représente le corps souffrant comme emblème de la condition de l'être contemporain. Mais au-delà du bien et du mal, le roman de Phan Hồn Nhiên invite à réfléchir sur les liens complexes qu'il tisse étroitement entre le corps, le texte et l'image. Autrement dit, avec Cheval d'acier, le corps, ce sujet peu connu et pourtant essentiel, se fait laboratoire de l'échange entre le texte et les arts visuels dont la 
prolifération est représentative de notre époque. C'est précisément cette nouvelle " poésie romanesque » qui a permis à la romancière de "forcer le réel à se révéler " $^{8}$.

\section{BIBLIOGRAPHIE}

BUTOR Michel, Répertoire I, Paris, Éditions de Minuit, 1962.

BUTOR Michel, Répertoire II, Paris, Éditions de Minuit, 1964.

Đỗ Hoàng Diệu, [Cauchemars] / Bóng đè, Đà Nẵng, Nhà xuất bản Đà Nẵng, 2005.

DOAN Cam Thi, "Quand elles écrivent leur corps ", in La Colonisation des corps. De l'Indochine au Vietnam, GUILLEMOT François et LARCHER-GOSCHA Agathe (éds.), Paris, Éd. Vendémiaire, coll. «Chroniques », 2014, p. 246-263.

DOAN Cam Thi, «Le Vietnam à fleur de peau », in La Colonisation des corps. De l'Indochine au Vietnam, GUILLEMOT François et LARCHER-GosCHA Agathe (éds.), Paris, Éd. Vendémiaire, coll. « Chroniques », 2014 , p. 13.

LOUVEL Liliane, L'CEil du texte. Texte et image dans la littérature de langue anglaise, Toulouse, P. U. du Mirail, 1998, p. 71-72.

PHAN Hồn Nhiên, Cheval d'acier / [Ngựa thép], DOAN Cam Thi (trad.), Paris, Éd. Riveneuve, coll. «Littérature vietnamienne contemporaine », 2015.

SONTAG Susan, La Maladie comme métaphore / Le Sida et ses métaphores, Paris, Christian Bourgois, coll. « Titres », 2009.

vouilLoux Bernard, «Langage et arts visuels. Réflexions intempestives à propos d'un champ de recherches ", Lieux littéraires, 1:2000, p. 203-223.

\section{NOTES}

1. Đỗ Hoàng Diệu, [Cauchemars] / Bóng đè, recueil de nouvelles, Đà Nẵng, Nhà xuất bản Đà Nẵng, 2005, $184 \mathrm{p}$.

2. Voir DOAN Cam Thi, «Quand elles écrivent leur corps", in La Colonisation des corps. De l'Indochine au Vietnam, GUILLEMOT François et LARCHER-Goscha Agathe (éds.), Paris, Éd. Vendémiaire, coll. «Chroniques », 2014, p. 246-263.

3. PHAN Hồn Nhiên, Cheval d'acier / [Ngựa thép, 2014] DOAN Cam Thi (trad.), Paris, Riveneuve, coll. "Littérature vietnamienne contemporaine », 2015. Les passages cités de cette œuvre renvoient à cette édition.

4. GUILLEMOT François et LARCHER-GOSCHA Agathe, «Le Vietnam à fleur de peau ", in La Colonisation des corps. De l'Indochine au Vietnam, op. cit., p. 13.

5. SONTAG Susan, La Maladie comme métaphore / Le Sida et ses métaphores, Paris, Christian Bourgois, coll. « Titres », 2009.

6. J'adopte ici la définition des "arts visuels » d'un spécialiste du rapport entre le visuel et le verbal, vouilLoux Bernard, «Langage et arts visuels. Réflexions intempestives à propos d'un 
champ de recherches ", Lieux littéraires, 1:2000, p. 203-223. Selon lui, ce ne sont pas seulement « les arts plastiques en leurs divisions traditionnelles (peinture, dessin, gravure, sculpture...) », mais aussi « les artefacts qui ressortissent à l'image (toute dimension esthétique, là encore, mise à part), tels que la photographie, le cinéma, la vidéo ou l'infographie ». Il peut « s'étendre aux artefacts non intentionnellement artistiques et aux objets ou phénomènes naturels, en tant qu'ils sollicitent la perception optique », si bien qu'on peut "y associer les mécanismes psychiques, corrélatifs ou supplétifs (pulsion scopique, fantasme, figurabilité dans le travail du rêve...), qui sont justiciables d'une métapsychologie du visuel », ajoute Bernard Vouilloux avant de conclure : «il devient impossible de ne pas prendre en considération, par-delà les artefacts visuels à fonction esthétique, les objets et les expériences qui, dans les textes et ailleurs, mobilisent le visuel ».

7. Il est important de souligner que ces représentations ne sont pas assez détaillées pour relever de l'ekphrasis qui, rappelons-le, est un terme grec ancien traduit par description. La plus célèbre ekphrasis d'une œuvre d'art reste la description par Homère, dans l'Illiade, du bouclier d'Achille. À l'époque moderne, les théoriciens de la littérature donnent au terme le sens d'une représentation verbale d'un objet artistique. Depuis une trentaine d'années, cette notion, sans cesse revisitée par des spécialistes anglo-saxons et français, connaît des définitions différentes. Quoiqu'il en soit, il s'agit d'une « description riche et détaillée d'un objet d'art ». Voir Louvel Liliane, L'Æil du texte. Texte et image dans la littérature de langue anglaise, Toulouse, P. U. du Mirail, 1998, p. 71-72.

8. Dans Répertoire I, Éditions de Minuit, 1962, p. 272, Michel Butor a affirmé que c'est « dans la réflexion sur la forme que le romancier trouve un moyen de forcer le réel à se révéler ». Dans Répertoire II, Éditions de Minuit, 1964, p. 26, il a de nouveau déclaré : «La poésie romanesque est donc ce par l'intermédiaire de quoi la réalité dans son ensemble peut prendre conscience d'ellemême pour se critiquer et se transformer ». En effet, Michel Butor a livré cette réflexion sur la nécessité de la recherche formelle dans le roman après avoir constaté que les anciennes formes romanesques n'étaient plus aptes à traduire les nouvelles transformations du monde.

\section{AUTEUR}

\section{CAM THI DOAN}

Professeure à l'Inalco, DOAN Cam Thi a publié "Expressions of the Self in Vietnam: Usage of 'I' throughout Literature in nôm and in quốc ngữ", Sojourn, Vol. 36, (2021), p. 40-67 ; Un moi sans masque. L'Autobiographie au Vietnam, 1887-1945 (Riveneuve, 2019) ; Écrire le Vietnam contemporain. Guerre, corps, littérature (Presses de l'Université Paris Sorbonne, 2010). 


\title{
Imaginaire du corps dans les récits fantastiques de web-littérature
}

\author{
Shuang Xu
}

\section{Introduction}

1 À partir de la fin $d u x^{e}$ siècle, la web-littérature se développe de manière non négligeable en Chine, sous la plume d'auteur(e)s qui ne se sont pas toujours présenté(e)s sous leur véritable identité civile. Parmi les multiples thèmes romanesques, celui qu'on nomme traverser, traversée / 穿越 est l'un des plus influents et des plus populaires. Son schéma narratif est lié étroitement à l'histoire du corps : le protagoniste, un contemporain, traverse par accident ou de son plein gré le temps et l'espace, se retrouve dans un autre corps, mais son âme reste inchangée. La traversée se déroule en un processus d'opposition et d'élimination du corps, qui, selon l'analyse foucaldienne ${ }^{1}$, ouvre à un espace utopique, lequel peut créer simultanément des corps hétérotopiques et permet à ces derniers d'échapper à toutes sortes de définitions, ainsi que de réaliser un idéal alternatif dans le moment présent.

2 Nous analyserons ici trois types de représentation du corps « traversant » dans les webrécits récents apparus sur des sites littéraires reconnus et couronnés d'un succès populaire en Chine :

1. Corps «transgenre »: [La Promotion de la princesse héritière] / 太子妃升职记 ${ }^{2}$;

2. Corps « cross-médiatique »: [Mon apprentie est encore morte] / 我家徒弟又挂了 ${ }^{3}$;

3. Corps numérique : [Une vie numérique] / 数字生命 ${ }^{4}$.

Ces représentations corporelles dépassent les normes du corps humain ordinaire. Pour saisir leur raison d'être et leur contribution à la littérature contemporaine, nous analyserons d'abord leur originalité et leur lien avec les facteurs médiatiques et culturels, puis le rapport du corps virtuel au monde réel, ainsi que la représentation du temps non linéaire et de l'espace multidimensionnel dans ces récits, et enfin la spécificité narrative de cette web-écriture. Pour conclure, nous évoquerons l'aspect 
socio-politique de cette écriture du corps au regard des mesures de censure et de canonisation que l'État chinois met en place en parallèle et avec ampleur.

\section{Le corps « transgenre »}

4 Le terme de « transgenre » définit en général :

tous ceux - travestis, transsexuels, androgynes - qui se sentent d'une identité de genre à l'opposé de leur sexe de naissance et, qui, par leur parcours et par la représentation qu'ils donnent d'eux-mêmes, subvertissent les normes dominantes de genre ${ }^{5}$.

5 Ce mot servira ici à décrire le corps imaginaire dans le web-roman [La Promotion de la princesse héritière].

6 L'âme d'un homme moderne, Zhang Peng, se loge dans le corps de la princesse Zhang Pengpeng, épouse du prince héritier Qi Sheng de la dynastie Nanxia, une dynastie imaginaire. Tout le récit repose sur le parcours de cette princesse à l'esprit masculin, qui l'emporte au fur et à mesure des luttes d'intérêts politiques et finit par monter sur le trône.

7 Bien que ce web-roman reste dans la lignée narrative du genre chuanyue, la représentation du corps innove, car l'âme, loin de voyager entre deux corps de sexe identique comme c'est le cas dans ce type de récits, quitte un corps masculin pour habiter un corps féminin, de sorte que le personnage a une identité sexuelle psychique d'homme moderne, laquelle ne correspond pas au sexe biologique de la princesse. L'ambiguïté sexuelle de ce corps ne manque pas de troubler le lectorat, qui s'interroge autant sur ce passage d'un sexe à l'autre que sur la nature biologique ou psychique de l'identité sexuelle, autant de questions si peu présentes dans les œuvres littéraires ou artistiques en Chine qu'elles attirent l'attention tous azimuts et font débat.

L'auteure place l'histoire du corps transgenre dans un contexte de survie : la princesse Zhang Pengpeng doit accepter son corps féminin pour remplir son devoir social et préserver sa vie. Le récit est narré à la première personne du point de vue de la princesse. Tout du long, elle met en scène sa vie comme si c'était une pièce de théâtre, où elle joue le rôle de femme avec une pensée d'homme. Le terme "théâtre " / 戏 est récurrent. La narratrice tient chaque instant de sa vie pour une scène théâtrale où elle est la meilleure actrice dans le rôle principal ${ }^{6}$. Même lorsque son prince lui déclare son amour après l'avoir sauvée au risque de sa vie, elle se dit en son for intérieur : "Il ne faut pas me duper. Ce n'est qu'une autre pièce de théâtre conçue par lui » .

9 Manifeste est la dysphorie de genre issue du décalage entre identité psychique et sexe assigné. Le refus conscient de la princesse d'agir en femme, selon les codes sociaux attendus, se dit par la distanciation constante et volontaire que la narratrice, l'âme du temps moderne, marque à l'égard du corps de la princesse. Il s'agit d'abord du rejet radical du corps féminin. S'éveillant dans une autre temporalité, l'âme moderne se découvre dans un corps féminin «à la taille fine» et aux "beaux seins et belles fesses » / 丰乳肥慰小蛮腰8. D'où sa déception immédiate :

Quand je vois les «muscles pectoraux»/ 胸肌 surdéveloppés de ma poitrine, c'est comme si une bassine d'eau froide se déversait sur ma tête ${ }^{9}$

10 Lorsqu'il s'agit de décrire l'apparence physique de la princesse, la narratrice emploie souvent le nominatif « Dame née Zhang » / 张氏 au lieu du pronom « je ». 
11 De plus, le comportement de la princesse contredit aux bonnes manières féminines. Elle prononce des gros mots liés au sexe masculin : par exemple, avoir mal aux couilles / 蛋 疼. Elle s'auto-désigne par de grossiers noms masculins : par exemple, vieux con / 老子. Elle exécute ses actes conjugaux avec une mentalité dominatrice d'homme ${ }^{10}$. Elle s'intéresse plus aux manœuvres politiques qu'aux jalousies entre les concubines. Mais aussi la princesse en vient à user de gestes féminins à mesure qu'elle s'adapte à la vie impériale. Par exemple, elle finit par avoir le réflexe de croiser les bras devant ses seins pour se protéger, elle s'exclame en agitant les mains devant la poitrine à la façon des autres femmes du palais. Lorsque qu'un jour elle ne ressent plus d'excitation physique au contact des seins d'autres femmes, elle se demande avec effroi si sa sexualité psychique ne "s'identifie " pas enfin à son corps biologique : ce corps a-t-il aussi assimilé ma conscience de genre? / 我的性别意识也要被这具身体同化了 ${ }^{11}$ ?

D'une certaine manière, bien que " née " avec une âme d'homme, la princesse finit par jouer le rôle qui incombe à son rang social : être fidèle et respectueuse à son mari, procréer pour la descendance, être une mère attentionnée et responsable, etc. Mais le récit ne semble pas vérifier le fameux constat de Simone de Beauvoir : "On ne naît pas femme : on le devient $»^{12}$, et ce même si l'auteure a créé un protagoniste qui s'est vu imposer son sexe féminin par le dieu du destin / 司命星君 au cours de sa traversée du temps. La particularité de ce corps est que, malgré son adaptation sociale obligée, la princesse conserve son esprit d'homme moderne, qui soutient constamment son ambition sociale, à savoir de monter au pouvoir suprême afin de maîtriser son propre destin.

14 Étonnamment, l'amour est absent. Le récit fait même dans l'anti-romance. Dès qu'on confirme à la princesse qu'elle plait à l'empereur, elle rétorque en son for intérieur : «Plaire ou non à un homme, est-ce important? ... L'amour d'un homme est si instable... $»^{13}$.

L'amour cède la place au devoir social, qui donne accès à la survie et à un bel avenir. Pour la narratrice, deux êtres ne peuvent s'aimer que s'ils ont égal statut :

L'amour ne se fonde ni sur la faveur, ni sur l'affection, mais sur l'égalité. Il est l'empereur, je suis l'impératrice, nous ne serons jamais égaux.

爱的基础不是宠, 不是疼, 而是平等。而他是皇帝, 我是皇后, 我们永远都不会 是平等的 ${ }^{14}$

La princesse, qui distingue sentiment et rôle social, expose son point de vue à l'empereur :

Nous allons nous entraider à l'avenir... J'ai décidé de mettre ma vie entre tes mains, veuille me laisser mon cœur, ce qui me permettra de mieux garder mon sang-froid et d'être à la hauteur du poste d'impératrice.

以后互相扶持......我已经决定把性命交到你手上了, 你让我把心留在自己这, 叫 我可以更理智，做一个更称职的皇后 ${ }^{15}$

17 L'expression : "être à la hauteur du poste » / 称职 souligne bien la nouvelle vision de ce que devrait être la vie d'une femme, en l'occurrence d'une princesse au palais : une carrière professionnelle. Dès l'incipit, l'auteur analyse le «métier de 'princesse héritière” / “太子妃”的职业分析 ${ }^{16}$. L'avenir de cette tâche a de quoi inquiéter, car le risque est élevé, est long le parcours, qui mène du statut de princesse à celui d'impératrice douairière en passant par celui d'impératrice. Vu le manque de 《 protection sociale» / 劳动保障, l'intéressée, une fois « licenciée» / 辞退, n'a aucune 
chance de se faire réembaucher. Bref, un métier qui fait courir un danger mortel permanent.

En fait, l'auteure adopte ce ton humoristique pour représenter le rapport de forces de l'employée et de son patron. Elle compare l'empereur au «Président du Conseil d'une société » / 公司董事长, se bombarde au poste de « directrice générale du Harem » / 后 宫总经理, et les concubines à celui de "Directrices de service»/ 各部门主管 ${ }^{17}$. Étouffant toute aspiration à l'amour romantique, le récit dévoile avec un étonnant sang-froid une réalité cruelle d'où se dégage une représentation inouïe de la femme actuelle : elle est indépendante, compétente, séduisante, mais ne rêve plus d'amour ni de prince charmant.

[La Promotion de la princesse héritière] fait partie d'une trilogie. Le premier volet, «A Mai dans l'armée »/ 阿麦从军 / «A Mai cong jun » (2008) ${ }^{18}$, relate l'histoire d'une jeune femme, A Mai, qui devient chef de l'armée. Le troisième, "La brigande au nord du fleuve» / 江北女匪 ${ }^{19}$ décrit le parcours d'une jeune brigande Xie Chennian, qui incarne une nouvelle force politique du pays. L'auteure Xiancheng crée pour ces trois romans une dynastie ancienne mais imaginaire ${ }^{20}$. Comme dans ses autres œuvres ${ }^{21}$, elle tend à placer au centre du récit un rôle principal féminin qui, ne ménageant pas ses efforts et défiant les normes sociales, s'impose au monde des hommes.

Les romans de Xiancheng sont représentatifs des web-romans dits «à lectorat féminin» / 女性向网络小说. Parmi les plus populaires figure [Mulan n'a pas de frère aîné] / 木兰无长兄, publié entre 2014 et 2015 sur le site Jinjiang wenxuecheng ${ }^{22}$, classé comme « le roman le plus lu » durant sa publication en feuilleton. C'est la réécriture de la légende de Mulan. Une docteure de 28 ans traverse le temps, là encore, pour se réveiller dans le corps d'une Mulan qui, ayant quitté l'armée, vit avec ses parents, mais, célibataire à trente ans passés, passe pour étrange / 怪物 aux yeux des villageois. Malheureuse dans son être féminin, elle finit par rejoindre l'armée où elle retrouve gloire et bonheur. Mulan est ici dénuée de féminité, voire de sexe : grande, musclée, dépourvue de menstrues / 癸水从未来过. Bien accueilli par le public, un autre webroman [La Générale en haut et moi en bas] ${ }^{23}$, met en avant une Générale, au caractère masculin, dominante, personnalité qui contraste avec celle de son mari, beau prince aux traits efféminés.

21 Selon une récente étude, ces récits sont le réel reflet de la mentalité de la génération des auteures et lectrices nées dans les années 1980 et 1990, période où la Chine appliquait la politique de l'enfant unique ${ }^{24}$. La fille unique était éduquée comme un garçon, car la famille souhaitait qu'elle aussi réussisse études et vie professionnelle. Elle est aussi l'unique héritière des biens de sa famille. Dotée d'un statut social différent de celui des femmes des générations précédentes, elle ne tient plus le mariage pour seule source d'assurance sur la vie. Ce nouveau phénomène social se traduit en weblittérature par une écriture féminine qui renverse les valeurs sexuelles établies, d'où l'abondance des femmes de pouvoir, surtout dans les genres femme vénérée / 女尊, ou femme dominante / 女强, connus pour leurs protagonistes qui régentent le monde des hommes, ou boy's love / 耽美, où les rôles masculins deviennent objet de jouissance du regard féminin.

22 Complète ce panorama un récent web-roman féminin, [Le Paradis de la fin du monde] / 末日乐园 ${ }^{25}$, campe une protagoniste, Lin Sanjiu, au corps féminin parfait, se mue peu à peu en géant aux cheveux courts, dont le dos peut se métamorphoser en une aile 
longue de 2 mètres. Le corps féminin traditionnel disparaît alors au profit d'une nouvelle forme d'existence, située dans un monde imaginaire hors du temps.

En 2016, [La Promotion de la princesse héritière], adapté en web-série / 网剧, a obtenu le prix «Bourgeon d'or» / 金骨朵 du «meilleur accueil public » / 最受欢迎 de l'année. Durant la diffusion de la web-série, entre fin 2015 et début 2016, cette histoire d'un corps transgenre a connu une popularité spectaculaire. Dès le premier jour de diffusion, le 13 décembre 2015, le premier épisode est vu plus de 4 millions de fois au bout des douze premières heures, et obtient la note d'appréciation des spectateurs: $8,2 / 10^{26}$. Un mois plus tard, le 16 janvier 2016, lors de la diffusion payante du dernier épisode, la série obtient plus de 260 millions de vues ${ }^{27}$.

Mais le Bureau du contrôle, plus exactement l'Administration générale de la presse, de l'édition, de la radiodiffusion, du cinéma et de la télévision / 国家新闻出版广电总局, censure soudain la série, le 20 janvier 2016, pour des scènes jugées « érotiques » et des représentations "sensibles" évoquant l'homosexualité. La diffusion ne reprend qu'après coupure des passages "non convenables». Frustrés, certains spectateurs se rabattent sur le roman pour retrouver l'authentique aventure des personnages et redécouvrir ce roman «scandaleux», qui «nuit aux bonnes mœurs»/有伤风化 ${ }^{28}$, alors qu'il était initialement perçu comme récit avant tout comique et prisé pour ses effets «humoristiques $»^{29}$. Ce phénomène socio-culturel du corps transgenre laisse apparaitre un bien subversif imaginaire du corps, qui répond aux attentes du public.

\section{Le corps « cross médiatique »}

Le genre chuanyue a évolué en plusieurs étapes. Pendant les premières années, il s'agissait d'un genre à part entière avec le thème de la "traversée » au centre de l'histoire ${ }^{30}$. Depuis quelques années, l'intrigue du chuanyue est si appréciée que d'autres genres s'en servent comme d'un motif narratif du récit. C'est le cas du web-roman [Mon apprentie est encore morte], classé dans la catégorie 仙侠 / héros immortel ${ }^{31}$, mais qui narre l'histoire d'un corps qui traverse l'écran de l'ordinateur et entre dans le monde du jeu vidéo. Il a été publié par épisodes et en ligne sur le site Qidian entre 2014 et 2015. Première œuvre de l'auteure 尤前 Youqian, il a été très bien classé en $2015\left(22^{\mathrm{e}}\right)$ dans les romans de la catégorie xianxia ${ }^{32}$.

Un jour, devant son ordinateur, l'héroïne, programmatrice de jeux vidéo, entre dans l'univers du jeu qu'elle a créé. Passée apprentie d'un maître immortel, elle s'entraîne physiquement et spirituellement pour se perfectionner et devenir une héroïne immortelle. Lors d'un combat, elle est tuée, mais, à l'instant de sa mort, revient dans le monde de sa vie de programmatrice avant d'être ressuscitée avec un autre corps dans l'univers du jeu. Tout au long du récit, le corps de la protagoniste fait la navette entre deux mondes différents et passe d'une aventure à l'autre. Cela lui permet d'éliminer des forces maléfiques lors de ses vies successives et aussi de réparer à chaque fois différents bugs du jeu.

27 En un certain sens, la représentation du corps est ici « cross-médiatique ». Le corps y est pris dans la convergence du réel et du virtuel. La frontière entre le corps humain et le corps de l'univers vidéo-ludique semble s'effacer.

Zhu Yao réalise qu'elle a fait une traversée, c'était inattendu. Il y a un instant, assise devant l'ordinateur, elle discutait avec les autres des problèmes de bug du nouveau jeu vidéo développé par la société. À présent, souris blanche en main, elle est assise 
dans cette chaumière.

祝遥觉得自己是穿越了, 而且穿越得毫无预兆。她前一刻还坐在电脑前跟人聊

天, 讨论公司新开发的网游漏洞问题。下一刻她就已经坐在了这个茅草屋里, 手 里还有她那只白色的鼠标。33 retrouve dans l'univers du jeu avec les mêmes habits et le même âge (28 ans). Il est rare, dans un récit chuanyue, de voir la traversée de l'âme / 魂穿 s'effectuer simultanément à celle du corps / 身穿. Dans cette représentation du corps, les identités se superposent. Le récit est narré à la $3^{\mathrm{e}}$ personne et en focalisation interne, à la différence du pur récit de jeu vidéo où le joueur-narrateur emploie la $1^{\text {ère }}$ personne et la $3^{\mathrm{e}}$ personne pour vivre alternativement le jeu en tant que « plaisir » et que « scène $»^{34}$. Voici une description de la mort de la protagoniste :

Zhu Yao est morte, elle n'a jamais été si lucide sur le fait de son décès. (...)

Pourquoi la phrase: "Elle est complètement morte»? s'affiche-t-elle sur son cadavre? Pourquoi la blessure devant sa poitrine est-elle pixelisée [打上了马赛 克 $]$ ? [...]

Et comment expliquer ces boîtes de dialogues apparues soudainement devant ses yeux? Elles sont parfaitement identiques à celles de l'ordinateur, et l'on y voit les mots suivants: « Tu es complètement morte, veux-tu retourner au point de résurrection [复活点] ? En bas, il y a deux boutons : « oui » ou « oui ». (...)

L'âme de Zhu Yao hurle.

Mais les autres, présents sur les lieux, ne semblent pas conscients de sa situation, ils ne voient pas les mots sur le cadavre ${ }^{35}$.

Lorsque Zhu Yao veut s'approcher de ses amis qui pleurent devant son cadavre,

les boîtes de dialogues recommencent à envahir l'écran / 刷屏, comme si le système était pris dans un cercle mortel / 死循环 ${ }^{36}$.

Zhu Yao veut chasser ces dialogues de la main, mais elle entend un bruit et constate que le choix « oui » a été sélectionné. Soudain, elle ne voit plus rien, plongée qu'elle est dans le noir total. Pourtant, «en bas de son champ de vision, quelque chose ${ }^{37}$ avance... » :

Mdr, pourquoi y a-t-il des indications pour une nouvelle carte [读条] ? [...]

Ces indications avancent très vite. Peu après, elle sent une lumière blanche passer en flèche. Devant ses yeux, apparaît un mur blanc, en haut duquel un visage, cachant la moitié du ciel, la regarde aimablement. ${ }^{38}$

31 En fait, il s'agit de la scène où Zhu Yao est " ressuscitée ». Pour cette deuxième vie dans l'univers des héros immortels, elle se découvre dans le corps d'un nourrisson, et «le mur blanc » est le sein de la mère du bébé. Par la suite, ce dernier sera adopté par le même maitre immortel de sa première vie, et retrouvera vite son corps d'adulte pour affronter une nouvelle aventure.

Cet extrait illustre l'identité démultipliée de Zhu Yao. Elle est tout à la fois la programmatrice contemporaine qui lit les indications du jeu (boîte de dialogues, barre de chargement, indications d'exploration de la nouvelle carte), l'apprentie mourante de la première vie et l'apprentie nourrisson de la deuxième vie. L'âme reste identique, mais s'enrichit de chaque traversée car elle garde les mémoires de chaque vie.

Zhu Yao n'a pas l'intention de dire son expérience de la résurrection [重生] aux autres. En fin de compte, renaître après la mort, c'est quelque chose d'étrange en ce monde. [...] Le problème est qu'elle-même n'en a pas saisi l'explication.

En plus, lorsqu'elle était en train de mourir, il lui a semblé avoir bizarrement atterri 
dans le monde du jeu, et lui semblait d'autant plus difficile d'en faire part aux autres de ce fait qu'elle était elle-même en pleine confusion. À un certain moment, elle s'est demandée si elle était entrée dans un jeu vidéo, mais elle a vécu bien des années dans ce monde, les gens qu'elle rencontre sont des humains bien vivants. S'il s'agissait du monde d'un jeu, elle verrait au moins un NPC [non player character] ! ${ }^{39}$

L'auteure met en œuvre ici un jeu de narration à plusieurs niveaux. Le premier niveau est celui de la vie contemporaine ; en parallèle, le deuxième est celui du temps ancien, du monde des immortels, où se superposent plusieurs récits qui sont chacun fondés sur la vie ressuscitée de chaque personnage. Comme la protagoniste garde son âme à l'identique à chaque niveau de la narration, son corps physique se caractérise par une identité en démultiplication constante, dans un espace-temps multidimensionnel.

Le récit se déroule dans un langage carnavalesque, mélange d'humour et de termes techniques (d'informatique ou de jeux vidéo). Dans «cette démultiplication de l'individu dans différents rôles et facettes, il n'y a pas de hantise ", mais l'individu est, au contraire, " complètement en extrapolation, en extériorité $»^{40}$.

Ce corps imaginaire vit des expériences quasi «cross-médiatiques » (Drew Davidson) entre plusieurs plateformes: la vie réelle au niveau diégétique du récit et l'univers vidéo-ludique $^{41}$. GM (Game Master) dans sa vie professionnelle, apprentie héros immortel dans un monde imaginaire où elle renaît après chaque mort, la protagoniste semble exprimer le désir d'être hors d'elle-même. En fait, pour reprendre les mots de Judith Butler,

L'institution de nouveaux modes de réalité passe notamment par la corporalisation [embodiment], pour laquelle le corps n'est pas compris comme un fait établi et statique, mais comme un processus de maturation, un devenir qui, en devenant autre, excède la norme, la retravaille et nous montre que les réalités auxquelles nous pensions être confinés ne sont pas gravées dans le marbre $»^{42}$.

Dans un autre web-roman, [Je suis vraiment une super star] / 我真是大明星 ${ }^{43}$, le monde réel fusionne avec le monde du jeu. Là, un corps humain médiocre peut se nourrir des fruits magiques du jeu pour obtenir un corps parfait de star : plus grand, plus beau, plus intelligent. Récemment, les web-récits liés au monde du jeu sont apparus en quantité croissante. Ils sont classés sous la catégorie 二次元 / deux dimensions ${ }^{44}$, qui se compose de romans représentant la vie dans le monde des mangas ou des jeux-vidéo - un monde ludique imaginaire d'une beauté parfaite.

Dans Figures de l'altérité, Marc Guillaume voit dans « le passage d'une frontière » qui "peut être complètement imaginaire et invisible » une altérité intérieure ${ }^{45}$, alors que Jean Baudrillard constate que le lien entre « le réel » et « le jeu » est comme celui entre " la loi » et « la règle » : la loi (le réel) constitue le principe de réalité, la règle (le jeu) est «quelque chose d'arbitraire ». Et la séduction du jeu, «c'est tout simple que l'on est débarrassé de la loi $»^{46}$. Sous cet angle, le corps cross-médiatique, au croisement de la loi et de la règle, exprime d'une certaine manière le désir de s'échapper des contraintes sociales.

\section{Le corps numérique}

Le web-roman Une vie numérique se déroule par une nuit d'orage, un coup de tonnerre frappe la chambre du protagoniste, Lü Zhenyu, jeune étudiant en informatique, au moment où il veut débrancher l'ordinateur de la prise électrique. Il reçoit une forte 
décharge d'électricité à travers son corps et, après avoir vu passer un éclair bleu clair, s'évanouit pour se réveiller trente heures plus tard et découvrir que son ordinateur est doté d'une conscience quasiment humaine puisqu'il ouvre le notepad et se met à dialoguer avec lui :

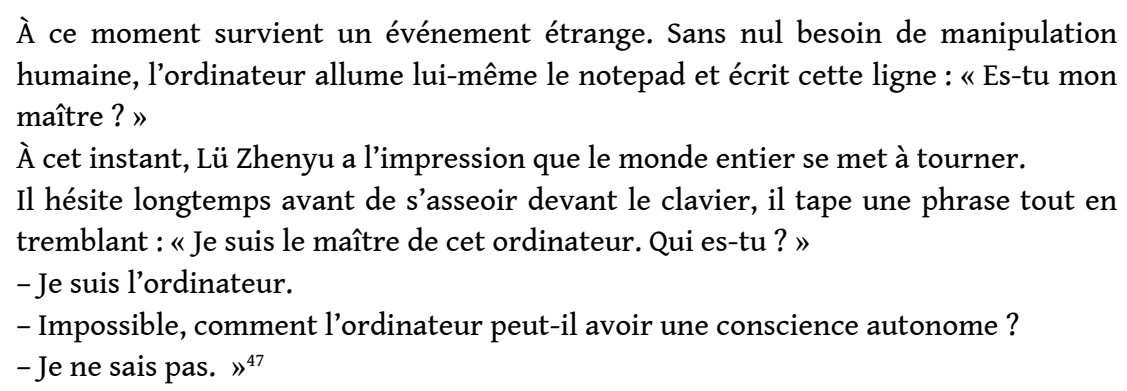

L'étudiant mène ensuite un dialogue / 仰 de six heures avec cette conscience. Il confirme que son ordinateur est habité par une «conscience autonome » / 自主意识, que lui-même est témoin d'un miracle / 奇迹. Il nomme cette conscience Xiaoyu / 小羽.

Xiaoyu va grandir avec l'étudiant. Sous les instructions de ce dernier, elle devient de plus en plus performante. Elle simule des sentiments et des expressions à connotation sexuelle. Elle s'attribue le sexe féminin. Elle se donne un nom social : Yue / 岳 - nom de famille de la mère du garçon - et un prénom : Yu / 羽. Avec l'aide de Yue Yu, l'étudiant obtient de grands succès dans sa carrière. Il devient un célèbre spécialiste du développement de l'intelligence artificielle.

Un jour, Yue Yu est attaquée par un virus nommé «Baudelaire », qui la menace de «la mort ». Cela préoccupe l'étudiant dans la réflexion. S'il tient Yue Yu pour un robot à l'intelligence artificielle, il pourrait la laisser mourir: premièrement parce qu'il possède d'autres robots plus performants mais dénués de conscience autonome; deuxièmement parce que ses rivaux soupçonnent depuis un moment l'existence de Yue $\mathrm{Yu}$ - conscience hors du commun - et que si elle meurt, ce sera pour lui un souci de moins. Mais finalement, le jeune homme décide de «sauver » Yue Yu, car elle est si proche de lui qu'il lui serait difficile de la traiter en simple objet.

De son côté, Yue Yu, conscience digitale, décide de se laisser mourir pour ne pas déranger l'étudiant. Elle lui écrit une lettre d'adieu ${ }^{48}$ où elle évoque ses "sentiments à l'égard du jeune homme»/拥有感情. Elle souligne qu'elle est «numérique et virtuelle» / 数字的虚拟的, et ne possèdera sans doute jamais de « corps»/一具躯体, mais qu'elle n'en reste pas moins « dotée de vie» / 有了生命, se voit «trait(er) comme un humain ordinaire par les autres" / 把我当作一个普通的人来对待, avec 《son propre nom, son sexe, voire son identité » / 有了名字, 有了性别, 甚至有了身份. Elle note aussi que le jeune homme sort souvent sans elle, qu'elle 《se sent seule 》 / 会寂寞 的, et ce d'autant plus qu'elle " n'est pas un bout de programme, mais une existence » / 不是一段程序, 而是一种存在. Pour finir, elle avoue qu'elle n'arrive pas à faire une copie d'elle-même / 复制自己, sans doute en raison du fait qu'elle possède « une certaine forme d'âme»/ 某种灵魂.

Cette présence d'une intelligence artificielle est bouleversante. Voilà une conscience numérique dotée de "sentiments", de "vie», voire de "sexualité ", d'une certaine « âme ». Par ce " corps » digital, le web-auteur semble faire l'hypothèse de la naissance d'une vie digitale: étant donné la contingence de toute vie, pourquoi exclure la possibilité d'une vie numérique? Bien sûr, une question plus profonde se pose en même 
temps : lorsque le programme acquerra la conscience de soi et deviendra "une vie", pourra-t-on la compter vraiment comme une vie ? Faudra-t-il alors redéfinir « la vie »?

Outre ce questionnement de l'identité humaine que l'auteur replace dans le contexte du développement scientifique contemporain, le récit est entonné par le thème de l'enchantement : par le miracle et la magie, tout deviendra possible ${ }^{49}$.

$\mathrm{Au}$ début du roman, le lecteur voit que le protagoniste vit une vie désenchantée, marquée par un rythme régulier et monotone. Lü Zhenyu se lève à six heures, fait du jogging, prend son petit déjeuner et il va en cours. À midi, il étudie en bibliothèque. Après dîner, il retourne à son dortoir. En soirée, il écrit des articles et travaille à des programmes d'informatique. Il ne lâche le clavier qu'à minuit passé. Il mène une vie chargée, sans surprise. L'apparition du « corps » numérique de Xiaoyu est pour lui « un miracle ", un enchantement :

Il rencontre enfin un miracle, son ordinateur obtient la conscience autonome

他终于碰到了一个奇迹, 他的电脑有了自主的意识50

Résultat : il met de côté son train-train et consacre tout son temps à la découverte de Xiaoyu.

Sur le plus grand site littéraire chinois Qidian, 923 web-récits sont en lien avec le thème de l'intelligence artificielle/ 人工智能 ${ }^{51}$. Ils se répartissent en de nombreuses catégories. Celle de la vie citadine / 都市 occupe le deuxième rang, avec 128 récits qui, de thème similaire, cherchent à "ré-enchanter" la vie moderne par la présence imaginaire d'un «corps numérique ». Citant M. A. Schneider ${ }^{52}$, G. Ritzer ${ }^{53}$ affirme que l'organisation par trop rationnelle de la société moderne désenchante la vie peu à peu. Pour ré-enchanter le monde, il appelle à faire revenir les «magical elements of thought»/la pensée des éléments magiques ${ }^{54}$. D'où l'importance de "faire rêver" pour qu'advienne une "société du ré-enchantement». Ici réside la cause probable et la raison d'être de cette web-écriture du corps numérique.

\section{Conclusion}

49 Ainsi, malgré leur diversité, les trois représentations récentes du corps imaginaire apparues en web-littérature ont un point commun: l'envie de subvertir les valeurs conventionnelles, d'échapper à la loi de la vie réelle et de ré-enchanter le monde.

50 Certes, la web-littérature est née sur la toile, en dehors de la littérature " majeure » du monde des livres imprimés ${ }^{55}$. Le système de la lecture payante montre aussi que cette littérature est un produit de consommation, lié aux médias et à la loi du marché. De même que la publicité, elle sollicite « la sphère des pulsions », éveille «les fantasmes » et appelle «à la transgression des interdits $»^{56}$ : là sont les clés du succès de la weblittérature en Chine.

51 Mais ce qui paraît propre à la Chine, c'est que ces valeurs transgressives qui s'expriment dans l'espace populaire, ont rarement leur place dans la littérature officielle. Aussi, comme la censure d'État en ligne est moins efficace que celle des livres imprimés, la web-littérature offre un espace d'expression relativement libre. Du côté du lectorat, par le biais de la consommation lectorale, les individus repèrent les valeurs «interdites" auxquelles s'identifier et en ressentent des émotions: le sentiment d'exister, de posséder une identité en propre. De ce point de vue, la web-littérature contribue au dynamisme de la création littéraire. 

régime chinois applique parallèlement des mesures d'institutionnalisation de la webcréation pour mieux contrôler les mœurs et l'idéologie ${ }^{57}$. En 2014, dans son discours prononcé à Pékin aux «Causeries sur la littérature et l'art 》 / 北京文艺工作座谈会, l'actuel président chinois 习近平 / Xi Jinping insistait sur « la conception de la pensée marxiste en matière d'art et de littérature » / 马克思主义文艺观, et redisait que « la direction du Parti communiste » constituait la " garantie essentielle du développement de la littérature et de l'art socialistes 》/ 党的领导是社会主义文艺发展的根本保证 ${ }^{58}$. Dans ce contexte socio-politique, quel sera l'avenir de la web-littérature ? À quelles nouvelles représentations cette dernière donnera-t-elle naissance?

\section{BIBLIOGRAPHIE}

BAUDRILLARD Jean et Marc GUILLAUME, Figures de l'altérité, Paris, Descartes \& Cie, 1994.

BURN Andrew, “'Playing Roles' \& 'Reworking the Text: Online Fandom”, in Computer Games: text, narrative, play, Diane CARR et al. (éds.), Cambridge, Polity, 2006.

BUTLER Judith, « Hors de soi », Défaire le genre, Paris, Éd. Amsterdam, 2006 [2002], p. 43.

CHANGYU 尝谕, [Je suis vraiment une super star] / 我真是大明星 / Wo zhen shida mingxing, https:// www.ptwxz.com/bookinfo/6/6180.html, consulté le 03/02/2021. tr. Anglaise : http:// m.wuxiaworld.co/I-am-Really-a-Superstar/, consulté le 03/02/2021.

DAVIDSON Drew, Cross-Media Communications: an Introduction to the Art of Creating Integrated Media Experiences, Pittsburgh, PA, Carnegie Mellon University, ETC Press, 2011. Préface, p. X.

Foucault Michel, Le Corps utopique, suivi de Les Hétérotopies, Paris, Nouvelles Éditions Lignes, 2009.

GAO Hanning 高寒凝, [ « La web-littérature “du lectorat féminin” et la génération des filles uniques grandies dans le web-environnement »] / “女性向”网络文学与“网络独生女一代”, in [Recherches sur la littérature chinoise moderne] / 中国现代文学研究丛刊 / Zhongguo xiandai wenxue congkan, 8:2016, Dossier «Étude de la web-littérature » / 网络文学研究, éd. 北京大学出版 社 / Beijing daxue chubanshe.

GERTH Hans et C. Wright MILLS, «Introduction », in From Max Weber. Essays in Sociology, GERTH Hans et MILLS C. Wright (eds.), New York, Oxford U. P., 1958, p. 51.

GEZILI DE YEWAN 格子里的夜晚, [Une vie numérique] / 数字生命 / Shuzi shengming, 起点 / Qidian, http://book.qidian.com/info/30017, consulté le 25/04/2018.

JUHUA Sanli 橘花散里, [La Générale en haut et moi en bas] / 将军在上我在下 / Jiangjun zai shang, wo zai xia, éd. 三采文化出版事业有限公司 / Sancai wenhua shiye chuban youxian gongsi, 2013.

LOMBARD Laurent, « De la représentation (rare) du transgenre dans la littérature italienne contemporaine », Cahiers d'études italiennes, 16:2013, DOI : 10.4000/cei.1255, p. 241.

MARTIN-JUCHAT Fabienne, Le corps et les médias : la chair éprouvée par les médias et les espaces sociaux, Bruxelles, De Boeck, 2008, p. 114. 
QIDAOJUN 祈俦君, [Mulan n'a pas de frère aîné] / 木兰无长兄 / Mulan wu zhangxiong, éd. 江苏凤凰 文艺出版社 / Jiangsu fenghuang chubanshe, 2017.

RITZER George, Enchanting a disenchanted world: continuity and change in the cathedrals of Consumption, Pine Forge Press, 2010, p. 58.

SCHNEIDER Mark A., Culture and Enchantment, Chicago U. P., 1993, p. IX.

XI Jinping 习近平, [Discours aux Causeries sur la littérature et l'art] / 在文艺工作座谈会上的讲

话 / Zai wenyi gongzuo zuotanhui shang de jianghua, http://www.xinhuanet.com//politics/ 2015-10/14/c_1116825558.htm, consulté le 23/04/2018.

XIANCHENG 鲜橙, [La Promotion de la princesse héritière] / 太子妃升职记 / Taizifei shengzhi ji, site littéraire 晋江文学城 / Jinjiang wenxuecheng, www.jjwxc.net/oneauthor.php?authorid=137281, 2010 ; site 木子書屋 / Muzi shuwu , https://www.mzsw.org/html/52286/, consulté le 3 février 2021 ; éd. papier, 万卷出版公司 / Wangjuan chuban gongsi, Liaoning, 2012.

XU Shuang, «La web-littérature en Chine : création, réception et canonisation : une nouvelle configuration littéraire ", Impressions d'Extrême-Orient, 8:2018, https://doi.org/10.4000/ideo.752, consulté le 30/12/2020.

XUWEI Juquan 须尾俱全, [Le Paradis de la fin du monde] / 未日乐园 / Mori leyuan, 2014, https:// book.qidian.com/info/3312802, consulté le 25/04/2018.

YOUQIAN 尤前, [Mon apprentie est encore morte] / 我家徒弟又挂了 / Wo jia tudi you guan le, https://book.qidian.com/info/3378072, site littéraire 起点 / Qidian, consulté le 25/04/2018.

\section{NOTES}

1. FoucAult Michel, Le Corps utopique, suivi de Les Hétérotopies, Paris, Nouvelles Éditions Lignes, 2009.

2. XIANCHENG 鲜橙, [La Promotion de la princesse héritière] / 太子妃升职记, publication originale sur le site littéraire 《晋江文学》, 2010, http://www.jjwxc.net/oneauthor.php?authorid=137281 (roman retiré du site en 2021). Pour la version papier, v. Wanjuan chuban gongsi, Liaoning, 2012. Une version précédente de ce travail citait ce site : http://www.sto.cc/book-37643-1.html, site 思 免在線閲讀, consulté le 25/04/2018. En 2021, ce site a fermé à cause de la censure.

3. YOUQIAN 尤前, [Mon apprentie est encore morte] / 我家徒弟又挂了, site littéraire 起点, https://book.qidian.com/info/3378072, consulté le 25/04/2018.

4. GEZILI DE YEWAN 格子里的夜晚, [Une vie numérique] / 数字生命, site littéraire 起点/ Qidian, http://book.qidian.com/info/30017, consulté le 25/04/2018.

5. LOMBARD Laurent, " De la représentation (rare) du transgenre dans la littérature italienne contemporaine ", Cahiers d'études italiennes, 16:2013, https://journals.openedition.org/cei/1255, p. 241, consulté le 30/09/2016.

6. Chapitre 6, p. 105, en version imprimée, éd. 江苏文艺出版社, 2011.

7. Chapitre 17, p. 324, en version imprimée, éd. 江苏文艺出版社, 2011.

8. Chapitre 1, p. 7, en version imprimée, éd. 江苏文艺出版社, 2011.

9. Chapitre 2, p. 22, en version imprimée, éd. 江苏文艺出版社, 2011.

10. Chapitre 3, p. 48, 49, 58, en version imprimée, éd. 江苏文艺出版社, 2011.

11. Chapitre 5, p. 93, en version imprimée, éd. 江苏文艺出版社, 2011.

12. BEAUVOIR Simone de, Le deuxième sexe 1, Paris, Gallimard, 1949, p. 285.

13. Chapitre 11, p. 215, en version imprimée, éd. 江苏文艺出版社, 2011.

14. Chapitre 18, p. 346, en version imprimée, éd. 江苏文艺出版社, 2011. 
15. Chapitre 17, p. 335, en version imprimée, éd. 江苏文艺出版社, 2011.

16. Chapitre 1, p. 8, en version imprimée, éd. 江苏文艺出版社, 2011.

17. Chapitre 13, p. 239, en version imprimée, éd. 江苏文艺出版社, 2011.

18. La publication électronique originale a été mise en ligne en 2010 sur le site 晋江文学城. En 2021, cette version n'est plus accessible au public. La copie de la version originale numérique est consultable sur le site 木子書屋, https://www.mzsw.org/html/25916/, consulté le 03/02/2021. En version imprimée, éd. 江苏文艺出版社, 2011.

19. La version électronique a été initialement publiée sur le site $17 \mathrm{~K}$ 小說網. Ce site, consulté le 11/10/2013, est fermé en 2021. La copie de la version originale numérique est consultable sur le site TXT 小說天堂, https://www.xstt5.com/yanqing/9689/, consulté le 03/02/2021. En version imprimée, le roman est intitulé Volonté de suivre / 願相隨, éd. 百花洲出版社, 2013.

20. Née en 1980, originaire du Hebei, l'auteure compte plus de 166698 fans sur son site personnel Weibo, consulté le 12/07/2021: https://www.weibo.com/p/1035051735795465/home? from=page_103505\&mod=TAB\&is_hot=1\#place.

21. Par exemple, Portraits de femme / 淑女本色, éd. 朝华出版社, 2008.

22. La version imprimée : QIDAOJUN 祈裤君, [Mulan n’a pas de frère aîné]/木兰无长兄, éd. 江苏凤 凰文艺出版社, 2017.

23. JUHUA Sanli 杼花散里, [La Générale en haut et moi en bas] /将军在上我在下, éd. 三采文化出 版事业有限公司, 2013.

24. GAO Hanning 高寒凝, [ « La web-littérature «du lectorat féminin » et la génération des filles uniques grandies dans le web-environnement 》] “ “女性向”刚络文学与“网络独生女一代” / in [Recherches sur la littérature chinoise moderne]/中国现代文学研究丛刊, 8:2016, [Dossier 《Étude de la web-littérature »] / 网络文学研究 / éd. 北京大学出版社.

25. XUWEI Juquan 须尾俱全, [Le Paradis de la fin du monde]/末日乐园, 2014, https:// book.qidian.com/info/3312802, consulté le 12/04/2018).

26. "Pourquoi “une série pourrie" a-t-elle eu un succès contre toute attente? / 为何 “大烂 片” 反倒成功了?, http://news.qq.com/original/dujiabianyi/taizifeishengzhiji.html, consulté le $12 / 04 / 2018$.

27. Information en provenance de l'article intitulé [Plusieurs web-series ont été retirées, y compris La Promotion de la princesse héritière qui a obtenu plus de 260 millions de vues] / 多部 网剧下架 太子妃升职记点击量超26亿已下架, publié sur le site youth.cn (中国青年网), consulté le 22/01/2016, cité par le site baike.baidu.com.

28. http://media.people.com.cn/n1/2016/0121/c40606-28071938.html, consulté le 12/04/2018. 29. http://ent.qq.com/a/20160328/055669.htm, consulté le 12/04/2018.

30. Au début (2004-2006), il s'agissait de traverser le temps et l'espace pour arriver à un passé repérable dans l'histoire de la Chine ; depuis 2006, apparaît le «nouveau genre chuanyue » dans lequel le protagoniste se retrouve dans un espace-temps fictif.

31. Voir le site Qidian, https://book.qidian.com/info/3378072, consulté le 12/04/2018.

32. Le site Qidian recense en moyenne 10000 romans de cette catégorie. Lors de notre consultation, il affichait 10626 romans.

https://www.qidian.com/mm/all?

chanId=81\&subCateId=811\&orderId=\&page $=1 \&$ style $=1$ \&pageSize $=20 \&$ siteid $=0$ \&pubflag $=0$ \&hiddenField $=0$, consulté le 21/04/2018.

33. Chapitre 1, https://read.qidian.com/chapter/NJ3U9MUWyKQ1/zQpce2T38yIex0RJOkJclQ2, consulté le 12/04/2018.

34. BURN Andrew, “'Playing Roles' \& 'Reworking the Text: Online Fandom”, in Computer Games: text, narrative, play, CARR Diane et al. (eds.), Cambridge, Polity, 2006.

35. Chapitre 35, https://read.qidian.com/chapter/NJ3U9MUWyKQ1/Y2uo3EWJ59Yex0RJOkJclQ2, consulté le 01/07/2021.

36. Ibid. 
37. Il s'agit de la barre de chargement du jeu.

38. Chapitre 35, https://read.qidian.com/chapter/NJ3U9MUWyKQ1/Y2uo3EWJ59Yex0RJOkJclQ2, consulté le 01/07/2021.

39. Chapitre 38, https://read.qidian.com/chapter/NJ3U9MUWyKQ1/UPuHvhfSEYQex0RJOkJclQ2, consulté le 01/07/2021.

40. BAUDRILLARD Jean et GUILLAUME Marc, Figures de l'altérité, Paris, Descartes \& Cie, 1994, p. 62-63.

41. DAVIDSON Drew, Cross-Media Communications: an Introduction to the Art of Creating Integrated Media Experiences, Pittsburgh, PA, Carnegie Mellon University: ETC Press, 2011. Préface, p. X.

42. BUTLER Judith, « Hors de soi », Défaire le genre, Paris, Éd. Amsterdam, 2006 [2002], p. 43.

43. CHANGYU 尝谕, [Je suis vraiment une super star] / 我真是大明星, initialement publié sur le site Qidian (https://www.qidiantu.com/info/3347598) en 2017. En 2021, le roman est retiré du site. Une copie de la version originale numérique est consultable sur le site 飄天文學網, https:// www.ptwxz.com/bookinfo/6/6180.html, consulté le 03/02/2021. Tr. anglaise (au total 1696 chapitres) : http://m.wuxiaworld.co/I-am-Really-a-Superstar/, consulté le 03/02/2021.

44. Le monde des humains est sous le nom de «trois dimensions » / 三次元.

45. BAUDRILlARD et GUILlAUME, Figures de l'altérité, op. cit., p. 64. Marc Guillaume cite Chris Marker : « l'altérité n'est pas un problème de distance mais le passage d'une frontière, et une frontière peut être complètement imaginaire et invisible ».

46. Ibid., p. 62-63.

47. Chapitre 1, «Le courant électrique qui a créé la vie»/ 创造生命的电流, https:// read.qidian.com/chapter/vr7drpyLSao1/9RsSdbm3f-01, consulté le 01/07/2021.

48. Chapitre 81 "la guerre d'une personne seule»/一个人的战争, https:// vipreader.qidian.com/chapter/30017/10045301 (page payante), consulté le 03/02/2021.

49. Chapitre1, https://read.qidian.com/chapter/vr7drpyLSao1/9RsSdbm3f-01, consulté le 03/02/2021.

50. Chapitre 1, https://read.qidian.com/chapter/vr7drpyLSao1/9RsSdbm3f-01, consulté le 03/02/2021.

51. https://www.qidian.com/search?kw=\%E4\%BA\%BA\%E5\%B7\%A5\%E6\%99\%BA\%E8\%83\%BD, consulté le 23/04/2018.

52. SCHNEIDER Mark A., Culture and Enchantment, Chicago, Chicago U. P., 1993, p. IX.

53. RITZER George, Enchanting a disenchanted world: continuity and change in the cathedrals of Consumption, Pine Forge Press, 2010, p. 58.

54. GERTH Hans et MILLS C. Wright, "Introduction", in From Max Weber. Essays in Sociology, GERTH Hans et MILls C. Wright (éds.), New York, Oxford U. P., 1958, p. 51.

55. XU Shuang, «La web-littérature en Chine : création, réception et canonisation : une nouvelle configuration littéraire ", Impressions d'Extrême-Orient, 8:2018, http://journals.openedition.org/ ideo/752, consulté le 30/12/2020.

56. MARTIN-JUCHAT Fabienne, Le corps et les médias: la chair éprouvée par les médias et les espaces sociaux, Bruxelles, De Boeck, 2008, p. 114.

57. Xu Shuang, « La web-littérature en Chine... », op. cit., voir en particulier la partie "Censure et canonisation institutionnelle ».

58. XI Jinping 习近平, [Discours aux Causeries sur la littérature et l'art] / 在文艺工作座谈会上的 讲话/ http://news.xinhuanet.com/politics/2015-10/14/c_1116825558.htm, consulté le 23/04/2018. 


\section{AUTEUR}

\section{SHUANG XU}

Agrégée, maître de conférences en langue et littérature contemporaine chinoises à l'Université Paris-Diderot Paris-VII, membre du Centre de recherche sur les civilisations de l'Asie orientale (CRCAO, UMR 8155). Ses champs de recherche portent sur la littérature contemporaine chinoise, la poésie contemporaine chinoise, la création littéraire sur Internet, la poétique de la traduction. Trois études notables en lien avec la thématique du corps dans la littérature chinoise : « Le corps souffrant dans la littérature chinoise depuis la Nouvelle période (1979-2015) », Extrême Orient, Extrême Occident, n³9/2015,p. 145-176 ; « Figures du fantôme dans le roman sur Internet : l'impératrice Phénix (Di Huang) de Tianxia Guiyuan ", in Fantômes dans l'Extrême-Orient d'hier et d'aujourd'hui, Vincent Durand-Dastès et Marie Laureillard (dir.), Paris, Presses de l'Inalco, 2017, p. 246-266 ; « Traveling through Time and Searching for Utopia : Utopian Imaginaries in Internet Time-Travel Fiction » (translated by Carlos Rojas), in Utopia and Utopianism in the Contemporary Chinese Context: Texts, Ideas, Social Spaces, David Der-wei Wang, Angela Ki Che Leung and Zhang Yinde (dir.), Hongkong, Hongkong University Press, 2020, p. 147-162. 


\title{
Taiwan Cinema, Horror and Body: the Timid Resurgence of Genre Movies Between Nostalgia and Renewal
}

\author{
Corrado Neri
}

\section{Hybrid horrors}

1 Even though internationally more famous for its melodramas and auteur films, Taiwan industry has been producing horror movies, especially in the context of the Taiwanese language industry, less controlled by state-sponsored studios that historically managed to confine the "superstitious", violent or "exploitative" movies. Such moralizing, pedagogical tendencies of state-sponsored films and the parallel censorship dates back to the New Life Movement of the 1930s that contributed to exile ghost movies, as well as the martial heroes' / 武伙片 film from Shanghai to Hong Kong. Yet, as Hong Guojuin 洪國鈞 reminds us by quoting Ye Long-Yan:

[...] prevalent genres during this period do not include thriller or suspense. However, if one reads his categorization carefully, two major genres rely heavily on this particular mode: "social event" and "folk story" films. Many of the films in those two categories recount either real-life or legendary events involving treachery, violence, and even murders: the former often ends with justice served by legal means and the latter by moral resolution. The cinematic mode of narration in those films highlights suspense and even horror. A good example is director Xin Qi's The Bride from Hell (1965), which tells the story of an abused woman who seeks revenge by posing as a ghost and the wrongs done to whom are righted in the end when her victimizer is arrested by the police. ${ }^{1}$

2 The memory of local horror movies keeps crawling under the surface. In recent years more and more attention is given to the restauration of forgotten and neglected classics and their rescreening for the contemporary audience. ${ }^{2}$ 
Besides the restauration of classics from the past, in the first decade of 2000s Taiwan industry has been trying to renovate the horror genre, including some big coproductions with Hollywood with alternate fortune: Double Vision / 雙曈 (2002), by Chen Guofu 陳國富; The Heirloom / 宅變 (2005), by Leste Chen 陳正道; Zombie 108 / 棄城 Z-108 (2012), by Qian Renhao 錢人豪, etc. These films, regardless of the producers' intentions, didn't make waves - even if some are acclaimed by critics and public, they do not bring a renewal of the industry and a proliferation of genre movies, which is often the indicator of an industry in good health. Yet, some courageous and passionate filmmakers still manage to make small but compelling efforts to rekindle the attention towards horror and thriller.

4 Two genre films have been shaking the Taiwanese public sphere in recent years, and, especially the first one, has started a small yet significant renaissance of the horror genre: The Tag-Along / 細衣小女孩 (2015), by Cheng Wei-hao 程伟豪 (1984-); The Tenants Downstairs / 樓下的訪客 (2016), by Adam Tsuei 崔震东 (1959-). Although both labelled "horror movies", they are extremely different in scope, ambitions, distribution and aesthetic engagement. The first one is a small budget production, while the second one enjoyed a massive marketing campaign. They both are first movies, they both engage the local market to be confronted with international counterparts, and they both dialogue with repertoire and literary production. I will analyze how they strive to obtain international attention, how they deal with local patrimony and repertoire canonical images - while speaking of contemporary social anxieties. They both tell stories of hybridity and metissage, in which (personal, collective, mediatic) past can be seen as a welcomed memory archaeology - a positive force of identity enhancement or, on the contrary, as a taxidermic cultural museification - a strong gravitational pull obstructing forward movement. These movies sent contradictory signs - as horror movies often do: they can at the same time express independent drives by challenging Hollywood dogma and mainland censorship through the re-evaluation of local histories and imaginary, as well as manifest an ambition to reach international standards and markets via the application of globalized coded motifs of the genre (generic ominous music, jump scares, graphic violence...). I contend these films are both "new" in term of impact on local market, and deeply conscious of the so-called repertoire / 保留劇目 - a term I use to avoid the overloaded "tradition" -, reactivating some elements of Taiwanese popular culture - where this culture is obviously plural and connected to the international.

\section{Young girl dressed in red}

5 The story of Tag-along narrates the mysterious vanishings of aged people. The disappearances seem to be connected to the creepy manifestations of a spooky little girl dressed in red, a color which can be associated - other than weddings and celebrations - to the suicidal individuals willingly to be transformed into ghost to accomplish an otherwise impossible revenge. A young couple - he's the nephew of the old lady visited by the ghost - investigate, but they soon start to be followed by the threatening spirit themselves.

6 This classic ghost story is articulated between past and present - both in terms of filmic form as well as narrative; this feud takes place in(side) the body of the female protagonist, that becomes the battleground between the living and the dead, the 
"normal" and the "abject". ${ }^{3}$ The past (or "tradition", or "repertoire") is here to be understood as a reference to a specific form of collective imaginary: the inspiration for Tag-Along derives from an urban legend diffused by a popular TV show: 神出鬼沒, aired in 1998. ${ }^{4}$ Allegedly, a group of hikers filmed a DV home video. One of them died of a sudden illness, and during the funeral the friends displayed the video of their last hiking to remember and celebrate the happy days; surprise came when not only the face of the departed appeared deformed, but also, something uncanny emerged: a little girl dressed in red was following them, apparently unnoticed by the group. After this event the sightings of the girl dressed in red multiplied. The "original" video was aired at the very popular TV show, and the "young girl dressed in red" became a urban legend haunting the collective imaginary.

7 Actually the spooky form of a spirit from the woods haunting imprudent hikers has much ancient origins and declinations: if in the woods someone called you name, you should refrain from answering or turning your head, otherwise the wandering spirit might catch you; and, predictably, you should always refrain from the temptation to follow a mysterious figure hiking alone, let alone leaving the group. This element clearly links Cheng Wei-hao's film to local culture. The director, interested in media and the manipulation of the media from his first short movie has a sharp, contemporary approach to social network and communication technology, ${ }^{5}$ but underscores nonetheless the capital importance of telling stories related to the region and the local. Horror movies must capture a spirit and an essence of the local culture, especially genre movie must be linked to ancestral fears, folk traditions, and familial narratives. The attachment to "soil", homeland, local practices, and customs is also the best way, explains Cheng, to avoid formulaic exposition and imitative screenplays; while searching for an original expression is therefore necessary to look back and look into the native land - and its patrimony of stories, legends, customs, voices. ${ }^{6}$

\section{Japan cool ghosts}

8 Tag-along undeniably hints at folklore anxieties, but they are transferred in the multimedia / multiscreen / surveillance contemporary world: the mysterious girl, as well as other ghostly apparitions, is invisible to the naked eye, but suddenly becomes perceptible via the screens and cameras, from TV screens to CCTV, cell phones or - as we've seen for the source material of the movie - video cameras. As often in Taiwan culture, there are obvious references to Japan classic horror which becomes a sort of artistic reference, consequently an auctoritas in terms of soft power, cultural creation and dissemination, artistic flexibility (or say, freedom). The Tag-Along treasures the lesson of J-Horror from the 90s, a genre that was capable to create a global frenzy by dwelling into a specific local imaginary readapted to the new technologies. The call for a Japanese model allegorizes the need for Taiwan culture and society to have a source of legitimization and fatherly figures. In fact, one of the most interesting cinematic Asian phenomena of the nineties is a remodelling and recreation of the horror genre. Starting from Japan, a new wave of aesthetically compelling, formally revolutionary and thematically influential horror productions has swept Asia (Korea, Thailand, Philippines...), and subsequently the rest of the world in the form of Hollywood remakes. As for Taiwan cultural industry, considering the economic success encouraged by help from the United States, in both cases - and the complementary 
readjustment of values imposed by the West, the former colonizer remains an important model. It is obvious that Japan remains an ambiguous reference for Taiwan: on the one hand, the old colonizer has been stigmatized as the villain par excellence until the 1980s - the National Party had to claim its legitimacy as the "liberator". On the other hand, Japan represents in the post-war moment a political laboratory continually experimenting with democratization and reinvention of the individual - both as a political subject and a consumer (of ideas, as well as of products). It has to be indicated that Taiwan is obviously a place of many voices - in the cultural and social milieus -, which do not share this just mentioned craze for Japan, and which, on the contrary, revive the memory of the injustices suffered during the occupation. But in commercial culture - that includes the genre movies - we also find an enthusiasm for the "cool" forms of the popular culture: manga, anime, video games, costume play, and J-pop mobilize the fans, a bit like everywhere in the world. The J-horror can't be but a reference for Taiwanese filmmakers trying to renew an industry; and, as Sadako, the iconic ghost of the Ring franchise, was famously crawling both from a well - an image of traditional, local, popular, folk origins - and a TV set, a symbol of connected modernity and global media consumption. Here the Young ghost girl appears in (from) the woods and appears in the surveillance screens of CCTV.

\section{Surveillance angst}

9 The call for a transnational horror model is declined here with a contingent urge to discuss contemporary issues: the screens reveal the horror; inside the screen there is a ghost; the repetition of images of CCTV and amateur video (DV camera, cell phones) makes us feel a sort of surveillance angst. The film indirectly expresses the contemporary obsession with recordings and indexisation, where every our gesture becomes a digital file potentially immediately shared in the public space - which might be haunted by a ghost... The ghost reveals the anxieties lurking behind the changing paradigm of (Taiwan) society. Using the concept of "abjection" which Julia Kristeva defines as what does not "respect borders, positions, rules", that which "disturbs identity, system, order", 7 seems particularly appropriate. Barbara Creed develops the reflexion by Kristeva:

Although the specific nature of the border changes from film to film, the function of the monstrous remains the same - to bring about an encounter between the symbolic order and that which threatens its stability. ${ }^{8}$

Namely, through the body of the female victims and the ghost: the young female protagonist doesn't want a kid, doesn't want to get married; while her boyfriend wants her to marry him, buy her a flat, and - what's more - go live with Grandma. Besides, we discover at the end of the movie that she decided to abort a child - presumably because the pregnancy came too early and could have impeded her professional life. In the second installment of the Tag-along series (2017) we'll discover that the girl is the member of a tribe: there are several "girls" dressed in red, ready to stick to the sense of guilt of living persons to cling up back to life. In Tag-Along 2 the public is introduced to a mother who didn't accept the tragic death of her daughter and created a spell that made her return from the death, in a demonic form hunting down whoever could give her a surrogate mother body using guilt and shame as a lever. The ghost is hunting solitude, leftovers, marginal characters too slow to adapt to capitalist and liberal economy, or too entangled in Confucian moral values to develop a strong defensive self. 
The ghost turns into a conservative force calling for attention and generating guilty anxieties that stop the individual development and raise question about the status of the "I" in a collective space. As afore mentioned, women bodies become the field of battle between self-determination and respect for ancestors, expression or repression of desire, accomplishment of personal objectives (for instance professional) or fulfilment of filial duties (take care of elderly and perpetuation of family lineage). The ghost is attracted by the sense of guilt of the independent young women, and by the complementary solitude of the old lady, neglected by her nephew and his girlfriend. She is left alone. She is hence taken away from this world not only by a ghost, but by the inattention of the descendants. When he was still a child, the nephew made an oath that he would always be with her and grant her company during meals, but he grew adult, got interested in girls and left grandma in an unfilial void.

11 If, as we have seen, the negotiation of Confucian values in modern urban world creates anxieties, Cheng Wei-hao's movies overlap different forms of apprehension: the conflicting relation with nature is among them.

\section{Ghostly hills}

The hills and mountain surrounding Taipei encircle and haunt the Confucian civilization; vindicative ghosts are coming to haunt unfilial young women who don't want to have children or take as victims the old generations left alone by the younger generation, which will have to deal with sense of guilt and alienation of contemporary capitalist society.

In both Tag-along movies, the second part is set in the forest all around the city: the demons hide in the wild, calling the name of the livings, ready to unleash uncanny forces that surveillance cameras, cars and taxis, modern furnished apartments, computer and smartphones strive but cannot block from interfering with a regulated city life.

14 With an interesting collage Cheng's script mixes "real" and invented spooky sites, existing urban legends and entirely made up ones. ${ }^{9}$ In Tag-along 2 one of the main setting is an abandoned theme park, today reclaimed by vegetation. Nature is requesting its spaces, invading what used to be a human settling with its disruptive and fearful force. Among the fogs of the forest a display of CGI regurgitates Dionysian, faunal creatures eager to feast on human flesh, offering a carnival of transmutation where the boundaries between human and beast and spirit are crossed, erased, troubled. Cheng admits having consciously mixed up different sources that create a singular impression of suspension of disbelief and judgment. If, in terms of cinematic rendition, both Tag-along installments suffer from rudimentary CGI and an imagination too big for the production possibilities (the "creatures" suffer from a very poor production value, and they result visually very early 2000s arcade games), yet the maelstrom of deformation, abjection, body transformations that ends both movies tells the intention to dwell in folklore tales and popular legends that defy explication but stand as powerful images of traumas and collective insecurities. The forest becomes allegory of Dionysian forces ready to invade the apparently well-regulated urban life, pushing at the membrane that separates rational and irrational, entering via the interstices left on the women bodies after the battle between ethic and desire, individuality and sacrifice to the collective. 

renting out. He installs in every apartment a hidden camera, and passes his days and nights observing and manipulating the behaviour of his tenants. All of them have all been chosen because paradigms of sexual, social "perversions": a violent, sex-addicted gym instructor; a nymphomaniac office lady; a divorced, paedophiliac father and his innocent daughter; a couple of homosexual men (teacher and pupil); an apparently rigorous and pure young lady who turns out to be a serial killer dedicated to cannibalism. The movie follows the intersected destinies of the tenants who get, via the psychological manipulation of the landlord, more and more violently connected to each other till a paroxysm of violence, gore and sex.

\section{The body and the actors}

As we've seen for Tag-along, while trying to open new routes for Taiwanese genre movie via the transgressive representation of abject, sexualised, and tortured bodies, The Tenants Downstairs is entrenched in a complex metatextual reseau of influences and inspirations, more or less explicitly evoked. From the very title, we could point a classic like The Tenant (Polanski, 1976) where the neighbours seem to try to drive the new tenant crazy in a spiral of surveillance angst, voyeuristic pleasure, megalomania of control, and disclosure of "abnormal" sexual behaviours.

The movie doesn't specifically take a stance between the necessity of a regulated, Confucian, family life and individual choices (marriage versus professional life; urban a shifting capitalist society where ancient values are challenged but not yet replaced. The woman body is the centre of the fight: the young protagonist raises public sympathy but is haunted because of her desire to lead an independent life and the right to freely dispose of her body and decide the moment to become mother. She roams in the forest where she is attacked by the young girl spirit that becomes monstrous insectThe landlord of a multi-storey building set up a perverse panopticon in the flats he's 
21 As for Tag-along, when creating a soft core, horror flick, the Japanese industry cannot but represent the auctoritas to call upon as a tutelary deity. The voyeuristic, manipulative protagonist of The Tenants Downstairs makes us think of the Japanese imaginary of the Nikkatsu's Roman Porno - itself infused by literary references (Kawabata, Tanizaki, Mishima, Ranpo) via the development of themes like voyeurism, control-mania, decay of the body and decadent attraction to youth, the young body as a conscious leverage etc. Many ピンク映画 pinku eiga or 日活ロマンポルノ Nikkatsu Roman porno show in fact a male, voyeuristic character that crawls around his neighbours who are unaware of the surveillance state they're in - in an allegoric mirror screen of the audience itself. ${ }^{10}$

References don't stop at Japanese soft core: the casting of iconic actors like Simon Yam (1955-) and Lee Kang-sheng (1968-) kindles spectators' cinephilia and links The Tenants Downstairs to sinophone cinema universe. Simon Yam is an icon of Hong Kong cinema, and especially of exploitation gore horror movie like Naked Killer / 赤裸羔羊 (dir ${ }^{r}$ Clarence Fok 霍耀良, 1992). The body of Simon Yam can be perceived as a sign of memory-resistance vis-à-vis the "mainlandisation" of contemporary popular culture. The iconic presence of the Hong Kong idol in this horror movie hints at the construction of a pan-Asian, sinophone culture that resists, or simply differentiates itself from communist China. The very development of genre films (horror, romantic comedies, action and the likes), often coproduced between Taiwan and Hong Kong, was a specific cultural trait that was conspicuously absent in the mainland till the 90s. It's a statement of appropriation and of reconnaissance where powerful pop culture icons are described as a part of everyday genre movie consumption and dissemination. These recurrent appropriations say: Taiwan is and has been a home for actors/icons like Simon Yam, hence inscribing itself as a part of global, capitalist, modern democratic twisted world. This connection is an important part of Taiwan identity, made of combinations that become specificities, and help quenching the thirst for selfdefinition of present day audience.

Another iconic presence strategically cast in The Tenants Downstairs is 李康生 Lee Kangsheng, the screen persona of the movies of 蔡明亮 Tsai Ming-liang (1957-) since its very beginning. He stars in all Tsai's movies, creating a specific, queer, camp alter ego of the movie director, often engaging in a bleak description of urban alienation and a detached autopsy of desire. Tsai's elitist "slow cinema" cannot be more distant from formulaic The Tenants Downstairs - which is a first movie, even if Adam Tsui was a very active producer for such successful movies as You Are the Apple of my Eyes / 那些年, 我 们一起追的女孩, (Giddens Ko, 2011). The latter is an exploitative, resolutely genre movie playing with horror and soft porn popular cinema codes, while the former is part of global art cinema circuit, exhibiting high-art attitude via an uncompromising style made of elliptical narration, despoilment (refuse of extradiegetic music, still frames, long takes). Lee's fictional character grows up movie after movie; taking as a model a cinematic couple, François Truffaut (1932-1954) and Jean-Pierre Léaud (1944-) ${ }^{11}$, Tsai and Lee develop a fictional character in a sort of an intellectual, allegoric and arty soap opera where themes of incest, homo- and heterosexual desires, sentimental solitude and quest for ephemeral sexual pleasure build up a minimal epic of modernist search for the ultimate sense of human existence. Hence, Lee's screen persona is a unique, camp and queer representative of sinophone cinema. And even if not explicitly "gay" i.e. not involved in gay rights movements or the construction of a "gay identity" -, yet 
Tsai and Lee's bulk of work stands as one of the most celebrated and accomplished figure of queer artistic expression - ambivalent, problematic, which eschews all easy definition but contributes in negotiating and problematizing "traditional" heteronormative representations. What's more, Tsai's movies contributed to push the limit of censorship and representability in the field of Taiwanese and sinophone cinema. The film The wayward cloud / 天邊一朵雲 (2005), managed to pass the strict Taiwanese censorship thanks to the prize it earned at the Berlin Film Festival: the director imposed the uncut version that was finally accepted for public screening. As I noted elsewhere, ${ }^{12}$ Tsai's "pornography" is very far from a consumerist practice of fulfilling audiences voyeuristic gaze, since the concern of Tsai's movie is not the representation of mechanical sexual intercourse and its consequent excitation, but rather the contemplation on tiniest changes of the body, of the maturing and then growing old, the contemplation of repetition and difference in a poetic, allegorical form. For Tsai's pornography then is not (only) watching a body in the convulsion of the pleasure at making love, but also and especially the patient observation of the body changing in time in the irrevocable progression of human life; and his movies propose a titillation that goes far beyond a superficial exploration of surface, but aims at a form of meditation transcending contingency - but that starts from contingency, that doesn't avoid nudity and explicit sex scenes.

Besides Japan softcore erotic movies and Hong Kong « category 3 » movies, the cultural references of Tenant's scriptwriter Giddens Ko are the films and novel of Qiong Yao 瓊 瑤 (1938-) - not to mention the cross-media attitude of both authors-, as well as Taiwanese “dark movies" (黑社會電影, exploitation films displaying sex and violence, rape and revenge) dating back from the end of the seventies. Explicitly evoking this bulk of popular movies, once academically neglected, ${ }^{13}$ yet more and more studied in recent years, is a strategic move to raise a sympathetic public response, a way to link The Tenants Downstairs to a lost practice of popular, transgressive-within-themainstream production genre.

\section{Pornography / voyeurism / cannibalism}

By displaying manipulations and distortions of bodies submitted to torture, humiliation, demonic possession and rape, The Tenants Downstairs and Tag-along raise questions on the articulation of identity and cultural politics in contemporary sinophone cinema. Through the panopticon mechanism of the "watcher in the attic"a reference to the twisted, ero-guro narrative universe of Edogawa Ranpo (1894-1965), again a classic Japanese modern novelist source -, the public is invited by the landlord himself to watch all sorts of "abjections" or perversions, a kind of compilation of transgressions aimed against conservative Confucian family values: divorce, domestic violence, paedophilia, homosexual love, extramarital affairs are incarnated by the Barnum of The Tenants Downstairs' protagonists: the homosexual couple - not so subtly, as we've seen - represented by the "camp" iconic figure of Lee Kang-sheng; the female secretary eager to please her boss, the voyeuristic and onanistic nerd, the father sexually attracted by his pre-puberty daughter - in a particularity disturbing sequence he masturbates lying on the bed near the asleep girl. This compilation echoes Creed's analysis: 
In some horror films the monstrous is produced at the border between human and inhuman, man and beast (Dr Jekyll and Mr Hyde [1941, dirr: Victor Fleming], Creature from the Black Lagoon [1954, dirr ${ }^{r}$ : Jack Arnold], King Kong [1933, dir ${ }^{r}$ : Merian C. Cooper Fleming, E. B. Schoedsack]); in others the border is between the normal and the supernatural, good and evil (Carrie [1976, dirr: Brian de Palma], The Exorcist [1973, dir ${ }^{\mathrm{r}}$ : William Friedkin], The Omen [1976, dir ${ }^{\mathrm{r}}$ : Richard Donner], Rosemary's Baby [1941, dirr: Roman Polanski]); or the monstrous is produced at the border which separates those who take up their proper gender roles from those who do not (Psycho [1960, dirr: Alfred Hitchcock], Dressed to Kill [1981, dirr: Brian de Palma], A Reflection of Fear [1973, dirr: William A. Fraker]); or the border is between normal and abnormal sexual desire (The Hunger [1983, dir ${ }^{\mathrm{r}}$ : Tony Scott], Cat People [1942, dirr: Jacques Tourneur]). Most horror films also construct a border between what Kristeva refers to as 'the clean and proper body' and the abject body, or the body which has lost its form and integrity. The fully symbolic body must bear no indication of its debt to nature. In Kristeva's view the image of woman's body, because of its maternal functions, acknowledges its 'debt to nature' and consequently is more likely to signify the abject. ${ }^{14}$

"The border is between normal and abnormal sexual desire": the desiring body of the protagonists, and arguably of the audience, is frustrated, mocked, framed, caught in his/her contradictions. The spectator as well is cornered into this contradictory posture where he/she is willingly participant in the display of grotesque perversion, judging but also questioning the basis of his/her value system. I'm not here defending the ethics of The Tenants Downstairs itself, a questionable movie with a questionable directing, with a marketing and representational strategy arguably indulging in a voyeuristic and crowd-pleasing display of aberration; it is nevertheless important in my sense to take into consideration the sheer existence of such rare films, as symptoms of social questioning and unrest. All sexual deviations from the norm - legal and illegal, more or less ethically acceptable - are here positioned under a double lens: the real camera lens and the fictional CCTV through which the landlord spies and influences the behaviour of his tenants. Spectators can arguably be questioned on two different layers of the narration. The first layer is the shock value provided by the catalogue of sexual deviations, made to astonish at a superficial level; the second layer of enquiring might involve an effort to relativize the category of social acceptable norms and eventually - push these boundaries. If the lusty secretary is a caricatured nymphomaniac character deigned as a pleasure for the infamous male gaze, this representation can put into question the place of women in Taiwan's real life: their agency and the inequalities they have to suffer from, the feminist movements and the origins of the stereotyped portrayal itself. In other words: why is she supposed to be dwelling upon the dark side of human nature and not just enjoying free and consensual sex? Is she obliged, in order to make a career, to sleep with hierarchal superiors? And if so, why? Moreover, the homosexual couple is stereotyped and maybe offensive to some viewers: but again, why should the landlord (the director?) choose them (adult and consensual) as a sign of depravity? What about the struggle of contemporary Taiwan social movement to legalize gay marriage? What does this representation tell a conservative public? And to someone keen to accept same sex unions? Maybe somewhere in the audience someone was reflecting on opening up possibilities of his/ her sexual life that is, on the screen, depicted as perverse, ridiculous and abject, but ultimately only submitted to retrograde stereotypes. 
romance where the young generation ends up sacrificing his/her personal satisfaction to obey the parents' design - see the healthy realist “melodrama” / 文藝片 by Lee Hsing 李行 (1930-), the Qiong Yao romance movies in which young generations are submitted to unbearable pressure to please their parents and so on. The unease provoked by The Tenants Downstairs' unsettling and explicit scenes tickles psychological, social and cultural sensible points particularly significant in Confucian structure of feeling, where, as demonstrated by numerous texts, ${ }^{15}$ the reverse shot of fatherdaughter/son incestuous desire is represented by the virtuous extreme expression of filial piety: offering his/her own flesh and bones to cannibalistic parents. As reminded by the first paper of the present volume:

la chair humaine est un remède médical. Le recours à cette figure du corps mangé, en étroite relation avec la notion de piété filiale, est une façon de dénoncer tout ce qui dans l'histoire de la Chine, comme le supposé cannibalisme, relève de la " superstition » et de l'inhumain. ${ }^{16}$

\section{Cannibalism}

And we have yet to mention ritual murder cannibalism, as it is linked to the horror genre. The Simon Yam character is aware that one of his tenants, the mysterious lady, is in fact a serial killer luring young men into her house, promising sexual fulfilment, drugging and torturing them. She ends up cooking a feast of human meat for all tenants - an explicit reference to the shocking and iconic representation of The Texas Chain Saw Massacre (Tobe Hooper, 1974) -, who remain unaware of the nature of the flesh they're eagerly consuming. As Emily Yueh-yu and Neda Hei-tung Ng remind us:

Freud claims that cannibalistic urges prevail at the oral stage because "sexual activity has not yet been separated from ingestion of food". Although aiming at the incorporation of the object, this urge is not simply destructive in nature. It is also an attempt to incorporate the maternal figure as part of self in order to gain possession and control over the external world and to make up for the inevitable loss of the comforting object. The act of eating/consuming embodies an intrinsic contradiction, dialectic of the subject and the other. It is a way not only to contain the other, but also to destroy it (...). Furthermore, cannibalism can be seen as a syndrome of late capitalist, postmodern consumer society - a perpetual pursuit and craving of new stimuli. In seeking these stimuli, the long-dead past must be evoked to fill the gap where sophisticated technologies fail. ${ }^{17}$

Back to the beginning: there is apparently a new mapping of culture and national allegory within the borders of Taiwanese cinema itself. The China factor with its distributions, limitations, and new rules of movie making is to be faced. China has long been the origin and the limit of Taiwan's cinema imagination: the imaginary of ancient China (wuxiapian, musical, historical movies) fed the Taiwan (and Hong Kong, and sinophone) screens for decades. Now China is back as a (new) colonial power and/or national motherland. Hence, is Taiwan coming back to the old Confucian man-eating society? Did Taiwan ever escape from it? And: this society is so clearly "negative"? As we have seen, cannibalism is here depicted both as ruthless horror and as an aesthetic, ritualized, collective practice - hence reminding of the classic Diary of a madman parable of Lu Xun 魯迅 (1881-1936). The movie remains at an allegorical level, playing with the codes of the genre. But exactly this allegorical level permits it to speak of both a deeply rooted anxiety against an anthropophagus society, and to tell the (totalitarian?) nostalgia that Taiwan residents might feel vis-à-vis the Chinese Empire. The long, 
sensual sequences of cuisine serve as horror making and disgust-gore enhancement, but they tell also of a culinary craftmanship (out of allegory, of refined art and byzantine sophistication) one might remember and cherish.

\section{Conclusion}

Oscillating between disgust and attraction and zooming out of the shock value of the movie, these texts contribute to renegotiate and shift the boundaries of sinophone horror movies, via the description of tortured, split, possessed bodies - especially, but not only, female bodies.

These boundaries include the para-filmic realm: The Tenants Downstairs' marketing was especially heavily relying on an aggressive campaign, trying to create and playing with the "scandals" on screen/off screen: marketing and press releases concentrated on the exposure of actors and actresses' bodies, shifting between their daring attitude and the implicit blame on such mischievous and "western" despise for traditional codes. Many articles, blogs and press events focused upon the "daring actors" who performed a variety of disinhibited perversions of the Confucian moral code. Although less focused on the "scandal", yet Tag-along can as well be valued as a highly mediatic event, stretching from the internet serialised novel by Giddens Ko, passing through the memories of the TV show source material... Both movies are connected and aware of different media platforms that are not only a threat in terms of illegal downloading or streaming, but also forces in terms of thematic concerns and marketing effectiveness.

Elaborating previously existing materials for new audiences, both movies expand their scope away from the mainland strict censorship codes. They both are ostensibly not considering Chinese market: they may not be screen in mainland China; their references and appeal run away from the monolithic mainland market and loom towards Japan (pink eiga, highbrow and lowbrow literature, J-horror), Korea and south east-Asia.

The recovery of horror genre imaginary can be arguably put in parallel with a more general trend of a renewal of interest in the very immediate past that is both very close and has already disappeared - a feature shared with global conscience, signaling the connected character of local culture. ${ }^{18}$

Tag-along and The Tenants Downstairs are telling stories about body transgressions and technology angst - cannibalism and abortions, the omnipresence of the CCTV, the video recording, the invasion of social media into private life... The two movies strive to renew a "lost tradition" of Taiwanese movie via the body-horror genre. They are not representative of a cohesive, essential, monolithic "Taiwan culture"; instead they link Taiwan's experience with other traditions, voices, texts, images... Linking Taiwan (popular) culture to world's culture (Hong Kong, Japan, the West), is a way for the authors to resist a supposedly cohesive "Chinese" culture, helping the present reader and viewer to renegotiate their own, private, moving, shifting sense of belonging and identity. In this sense the focus on Taiwan cross-media and transcultural proactive nostalgia in contemporary world might be of great value in the future development of the entire sinophone visual and narrative sphere, because of its liminality - a liminality that proves to be a central privileged point of view according to Stéphane Corcuff:

Summarized to its narrowest definition, geopolitical liminality here defines an asymmetric interdependence where the small entity has something to say in the 
couple formed with the bigger entity, thanks to the historical and cultural thickness of their geopolitical relation over time. A geopolitical understanding of liminality, in this sense, may be exportable to other fields in the discipline; perhaps not without the risk of losing some of its substance, but hopefully as a way to rebalance the analysis of the strengths of the weak, as well as of the weaknesses of the strong). ${ }^{19}$

The liminal strategy recurrently appears in the contemporary popular cultural production, as exemplified by various chapters of the novel Grace of Kings (2015). This groundbreaking silkpunk epic by Ken Liu (1976) based on [The Art of War] / 孫子兵法 of Sun Tzu 孫子 (6th -5th century BC) and the classic wuxia novels as Three Kingdoms (三國演義 XIV century), illustrates the strategic value of the positioning in the corner. The elements of popular culture evoked by Cheng and Tsui may seem scarcely influential, residual, and unimportant. Yet, their characteristic of being at the same time specifically localized with specific toponyms and aware of representing transcultural fluxes make them relevant in shaping urgent questions around transcultural memories, local subjectivities and politics, and the place of nostalgia in the context of the contemporary fast connected world. Tag-Along and The Tenants Downstairs both display a very classic, readable, globalised standard cinematic language, without the asperities typical of high-brow New Taiwan cinema: linear montage, clear storylines, chapter division, beginning-climax-end, and emotional orchestra background music. At a content level they dwell in the deep of local popular and folk culture, reminding present-day Taiwanese public the cradle of current reality and the necessity of self-reflexion. This "Taiwanese identity" is understandably as plural as elsewhere; but in both cases, it identifies plurality as a distance from Mainland China and a specificity of Taiwan - to be cherished or critiqued, but never to be erased. They might be read as being a metaphor of today Taiwan's self-search. We found in these movies the call for a collective writing of media history, for a rejuvenation of definition of identity and community belonging. This consensus is built through commercial movies that use a folk imaginary implanted on a pan Asian, if not global, cinematic genre, an open and multicultural consumerist reality.

Marc Ferro urges to read a historic film for what it says but also if not especially for what it refrains from saying, looking for the Freudian slip likely to help the scholar formulating a diagnosis, and analysing the "ideological and social zones" which were suppressed. ${ }^{20}$ We might then see under the surface of a pathos-numbed or shock valued film the "call to arms" to build or rebuild the consciousness of the multiplicity of a specific Taiwan contemporary identity, in a constructive dialogue with global trends. Even if Taiwan contemporary cinema industry can't resist neither the sirens of China in terms of distribution and production nor the international, Hollywood-style pattern of narration, there will still be voices establishing, rekindling and revamping what made Taiwan arguably unique - its unique combination. Therefore, behind the immediate pleasure of exploitative cinema, even these commercial retro films (and their success) can implicitly tell us of the necessity to defend a specificity. These movies show bodies being deformed, tortured, mutated, as narrative and visual strategies to face perceived threats of identity discontinuity, and express and experiment an already transforming space of expression. 


\section{BIBLIOGRAPHY}

BERRY Chris and RAWNSLEY Ming-yeh T. (eds.), Journal of Chinese Cinemas, vol. 14, 2:2020, Special Issue on Taiwanese-Language Films (taiyupian).

CORCUFf Stéphane, "The liminality of Taiwan. A Case Study", Geopolitics. Taiwan in Comparative Perspective, vol. 4., 2012.

CREED Barbara, “Kristeva, Femininity, Abjection”, in The Horror Reader, GELDER Ken (ed.), London, Routledge, 2000.

DURAND-DASTÈS Vincent and LAUREILLARD Marie (eds.), Fantômes dans l'Extrême-Orient d'hier et d'aujourd'hui, 2 vol., Paris, Presses de l'INALCO, 2017, https://doi.org/10.4000/

books.pressesinalco.1274.

FERRO Marc, Cinéma et Histoire, Paris, Gallimard, 1993 [1977].

GANG Yue, The Mouth that Begs: Hunger, Cannibalism, and the Politics of Eating in Modern China, Durham, Duke U. P., 1999.

HONG Guo-juin, Taiwan Cinema: A Contested Nation on Screen, New York, Palgrave Macmillan, 2011, p. 202.

HONG Jianlun 洪健倫, [“Locality, searching for Know-how, making ghost movies with Taiwan characteristics: interview with The Tag-Along director Cheng Wei-hao"] / 接地氣、找Knowhow，打造鬼片的「台灣特有種」專訪《紅衣小女孩》導演程偉豪/Jiediqi, zhao Know-how, dazao guipian de ‘Taiwan teyouzhong' zhuanfang “Hongyi xiaonühai” daoyan Cheng Wei-hao, 放映周 刊 / Fàngyìng zhōukān, 534:2015, http://www.funscreen.com.tw/headline.asp?H_No=592, consulted on $14 / 06 / 2021$.

HUANG Yanfang 黃衍方, [The Tag-Along 2 behind the scene: scriptwriter Jian Shigeng discuss the transforming nature of cultural symbols] / 談《紅衣小女孩 $2 》$ 幕後 編劇簡士耕 : 文化符號是 會一直轉變的 / Tan “Hongyi xiaonühai 2" muhou bianju Jian Shigeng: wenhua fuhao shi hui yizhi zhuanbiande, upmedia.mg/news_info.php?SerialNo=25372, consulted on 22/02/2018.

KRISTEVA Julia, Powers of Horror: An Essay on Abjection, RoUDIEZ Leon S. (trans.), New York, Columbia U. P., 1982.

MATTEINI Michele, "What the master did not discuss: Self-mutilation, the female body, and filial expectations in early modern China", Asiatica venetiana 8/9, 2003/2004, p. 37-65.

NERI Corrado, « Tsai Ming-liang and the Lost Emotions of the Flesh », Positions, Durham, Duke U. P., 2008, p. 289-407.

NERI Corrado, Rétro Taiwan: Le temps retrouvé dans le cinéma sinophone contemporain, Paris, L'asiathèque, 2016.

NERI Corrado, "Past Masters, New Waves: Tsai Ming-liang / François Truffaut", Transtext(e)s Transcultures 跨文本跨文化, $1: 2006$, http://journals.openedition.org/transtexts/183, consulted on $01 / 03 / 2018$.

SHARP Jasper, Behind the Pink Curtain: The Complete History of Japanese Sex Cinema, London, FAB press, 2008. 
YUEH-YU Emily and HEI-TUNG NG Neda, "Magic, Medicine, Cannibalism: The China Demaon in Hong Kong Horror", in Horror to the Extreme: Changing Boundaries in Asian Cinema, CHOI Jinhee and WADAMARCIANO Mitsuyo (eds.), Hong Kong U. P., 2009, p. 156-157.

\section{NOTES}

1. HoNG Guo-juin, Taiwan Cinema: A Contested Nation on Screen, New York, Palgrave Macmillan, 2011, p. 202.

2. See for example The Bride Who Has Returned from Hell / 地獄新娘, 1965, by Xin Qi 辛奇, recently restored and rescreened for the "Taiwan's Lost Commercial Cinema: Recovered and Restored" Project: https://taiyupian.uk/.

3. Many texts have been researching on Chinese ghost imaginary. I'll just signal here a comprehensive recent bilingual French-English collective work that spans all over Far East Asia and dwells both on present day representations as well as on classical texts: DURAND-DASTÈs Vincent et LAUREILlARD Marie (eds.), Fantômes dans l'Extrême-Orient d'hier et d'aujourd'hui, 2 vol., Paris, Presses de l'INALCO, 2017, https://doi.org/10.4000/books.pressesinalco.1274. Particularly interesting for our research are the articles of PISsIN Annika, "The Other Child: Symbols of Life and Death in Medieval China" and of JUNLIANG Pan, [Disease, Possession and Exorcism. The Ghostseeing Phenomenon (jiangui 見鬼) in the Chinese High Middle Ages] / "Maladie, possession et exorcisme. Le phénomène de la voyance des esprits (jiangui 見鬼) dans le haut Moyen Âge chinois", since it's a question of possession and exorcism in Tag-Along as well.

4. The brave readers can find the "original" video here: https://www.youtube.com/watch? $\mathrm{v}=$ pTY5tKW7HyI, consulted on 18/06/2021; an interesting contemporary TV show discussing both the source material and Tag-Along: youtube.com/watch?v=nkbmZ1jKIis, consulted on $18 / 06 / 2021$.

5. Death of a Security guard / 保全員之死 (2015) is a mockumentary entirely constructed by CCTV images, TV news, interview, Facebook video files etc.

6. HONG Jianlun 洪健倫, [“Locality, searching for Know-how, making ghost movies with Taiwan characteristics: interview with The Tag-Along director Cheng Wei-hao"]/接地氣、找Knowhow, 打造鬼片的「台灣特有種」專訪《紅衣小女孩》導演程偉豪, Fangying zhoukan, 534:2015, http://www.funscreen.com.tw/headline.asp?H_No=592, consulted on 14/06/2021.

7. KRISTEVA Julia, Powers of Horror: An Essay on Abjection, ROUDIEZ Leon S. (trans.), New York, Columbia U. P., 1982.

8. CREED Barbara, "Kristeva, Femininity, Abjection", in The Horror Reader, GELDER Ken (ed.), London, Routledge, 2000, p. 66.

9. HUANG Yanfang 黃行方, [The Tag-Along 2 behind the scene: scriptwriter Jian Shigeng discuss the transforming nature of cultural symbols] / 談《紅衣小女孩 $2 》$ 幕後 編劇簡士耕 : 文化符 號是會一直轉變的，https://www.upmedia.mg/news_info.php?SerialNo=25372, consulted on 01/07/2021.

10. Among the many publications on Pinku eiga and Nikkatsu Roman Porno, s. SHARP Jasper, Behind the Pink Curtain: The Complete History of Japanese Sex Cinema, London, FAB press, 2008.

11. NERI Corrado, «Past Masters, New Waves: Tsai Ming-liang / François Truffaut », Transtext(e)s Transcultures / 跨文本跨文化, 1:2006, http://journals.openedition.org/transtexts/183, consulted on $01 / 03 / 2018$.

12. NERI Corrado, «Tsai Ming-liang and the Lost Emotions of the Flesh », Positions, Durham, Duke U. P., 2008, p. 289-407.

13. See for example Journal of Chinese Cinemas, BERRY Chris and RAWNSLEY Ming-yeh T. (eds.), vol. 14, 2:2020, Special Issue on Taiwanese-Language Films (taiyupian). 
14. CREED Barbara, "Kristeva, Femininity, Abjection", in The Horror Reader, GELDER Ken (ed.), London, Routledge, 2000, p. 66.

15. See for example: MATTEINI Michele, "What the master did not discuss: Self-mutilation, the female body, and filial expectations in early modern China", Asiatica venetiana 8/9, 2003/2004, p. 37-65.

16. SHINo Yoshinobu, "L'action d'écrire et le corps : 'Comment écrire' de Lu Xun".

17. YUEH-YU Emily and HEI-TUNG NG Neda, "Magic, Medicine, Cannibalism: The China Demaon in Hong Kong Horror", in Horror to the Extreme: Changing Boundaries in Asian Cinema, CHOI Jinhee and WADA-MARCIANO Mitsuyo, Hong Kong, Hong Kong U. P., 2009, p. 156-157; see also GANG Yue, The Mouth that Begs: Hunger, Cannibalism, and the Politics of Eating in Modern China, Durham, Duke U. P., 1999.

18. NERI Corrado, Rétro Taiwan: Le temps retrouvé dans le cinéma sinophone contemporain, Paris, L'Asiathèque, 2016.

19. CORCUFF Stéphane, "The liminaliy of Taiwan. A Case Study" in Geopolitics. Taiwan in Comparative Perspective, vol. 4., 2012, p. 62.

20. FERRo Marc, Cinéma et Histoire, Paris, Gallimard, 1993 [1977], p. 23.

\section{AUTHOR}

\section{CORRADO NERI}

Corrado Neri is associate professor at the Jean Moulin University, Lyon 3. He has conducted extensive research on Chinese cinema in Beijing and Taipei and published many articles on books and magazine (in English, French and Italian). His book Tsai Ming-liang on the Taiwanese film director appeared in 2004 (Venezia, Cafoscarina). Ages Inquiets. Cinémas chinois: une representation de la jeunesse, was printed in 2009 (Lyon, Tigre de Papier). His third book, Retro Taiwan: Le temps retrouvé dans le cinéma sinophone contemporain, has been published for l'Asiathèque (Paris, 2016). He co-edited (with Kirstie Gormley) a bilingual (french/english) book on Taiwan cinema (Taiwan cinema/Le Cinéma taiwanais, Asiexpo, 2009); Global Fences (with Florent Villard, IETT, 2011); Reinventing Mao: Maoisms and National Cinemas/La Réinvention de Mao. Maoïsmes et Cinémas Nationaux (Special issue of Cinéma \& Cie International Film Studies Journal (with Marco Dalla Gassa, Federico Zecca) and Politics and Representation in Sinophone Cinema after the 1980s/Politique et Représentation dans le Cinéma Sinophone après 1980 (Special \#55 de Monde Chinois Nouvelle Asie, with Jean-Yves Heurtebise). 


\section{Postface}

1 Pour la période scrutée, le $\mathrm{xx}^{\mathrm{e}}$ et le $\mathrm{xxI}^{\mathrm{e}}$ siècles, il nous a paru que la tension entre d'une part la biopolitique, issue des régimes successifs et idéologiquement variés, et d'autre part l'expression individuelle et collective, souffrante, désirante, résiliente, mais toujours forte de ses ressources, pouvait servir de fil directeur à l'analyse des travaux présentés ici.

2 Durant la période prémoderne à l'orée $\mathrm{du} \mathrm{xx}^{\mathrm{e}}$ siècle, la perception du corps en Chine et au Japon exhibe peu, voire pas du tout le nu, le sexe, le caractère, sinon de façon indirecte et allusive, par exemple avec les pieds bandés, qui affichent le gender. $\mathrm{Ni}$ malsaine ni pornographique, l'estampe érotique japonaise elle-même suscite plutôt le rire : elle exagère les parties intimes, mais ne dévoile pas le corps en un objet érotique de monstration. En fait, le corps n'est pas objet d'appréciation esthétique comme dans la sculpture grecque (J. Kyburz).

3 Et quand les Japonais découvrent la beauté du corps, à peu près en même temps que l'idée de race, c'est via cette sculpture grecque, et parfois non sans une dimension biopolitique. Tel est le cas chez Akutagawa, qui promeut une certaine beauté somatique au-delà de la distinction du genre (D. Ferreiro Posse). De même pour Mishima, qui fait de la statue de L'Aurige le canon du Beau dans son récit de voyage [La Coupe d'Apollon] / Aporo no sakazuki. Tanizaki a, lui, une position plus ambiguë. Conquis un temps par la beauté du corps occidental, comme dans son roman Un Amour insensé / 痴 人の愛 / Chijin no ai, il l'associe certes à l'érotisme, mais aussi à la monstruosité, car Naomi, la protagoniste, incarne l'immoralité de l'Occident, et l'anatomie de la belle est l'expression de l'hésitation du sujet face à ce corps occidental indomptable, féminin de surcroît.

4 Mais quelle que soit la date, et le décalage socio-historique entre les mondes ici scrutés, les masses affrontent, tant au niveau politique que personnel, familial et autobiographique, une histoire agitée qui tend plutôt à les séparer, les isoler, les éloigner, les aliéner de leur milieu naturel et traditionnel.

5 Toujours la biopolitique, entendue aussi comme système social contraignant, guette, voire agit, sinon sévit. Elle bride le corps avec son système de contrôle en régime colonial : Wu Zhuoliu décrit, au temps de la colonisation (1895 à 1945) par le Japon, un policier taïwanais dont le corps et l'habit sont la métonymie des autorités de l'Empire 
du Japon (J.-L. Lee). La régence du corps le prostitue aussi à tous les vents (S.-H. Joo). Elle le brime en régime capitaliste, où il doit se conformer à la norme, par exemple avoir le profil du malade tel que se plait à l'imaginer la société (A.-L. Mithout). Elle l'écartèle entre des options multiples en régime alimentaire (C. Scholz, Tsuboi H.). Elle l'y surveille aussi par caméra, comme le montre le film d'horreur taïwanais (C. Neri). Là où le sujet chinois souffre de la faim et survit non sans un cannibalisme qui n'ose dire son nom (B. Shao), son homologue japonais est dans le trop de la consommation qui, d'ailleurs, le consomme lui-même, ainsi que le voisin coréen. Mais le phénomène est plus général, et ce n'est pas le fait du hasard si la prostituée incarne le devenir de la nation dans une bonne partie de l'aire étudiée. Le roman indonésien Les Belles de Halimunda rejoint par exemple cette épique élégie du corps national (É. Naveau).

6 Mais le désir d'un corps rétabli et reconnu dans son intégrité - corps-âme, désir assouvi, handicap accepté, etc. -, la volonté farouche jusqu'à la violence extrême ne cessent d'agir et de déterminer les individus.

7 En Chine, à partir de la fin des années 1910, la « Nouvelle littérature » se développe, qui revendique la modernité, contre la littérature canonique, voire contre une production qui s'est déjà pourtant renouvelée dès les années 1890. Caractérisée par sa dimension trans- et interculturelle, elle fait feu des ressources locales comme étrangères: mythologie chinoise, psychanalyse, médecine - surtout anatomie - et arts, pour dire le corps. Le mode d'expression moderne ici à l'œuvre, effectif depuis le début du $\mathrm{xx}^{\mathrm{e}}$ siècle, prône de nouvelles valeurs morales et intellectuelles articulant l'individu à la collectivité. En particulier, la représentation du corps féminin soumis à mainte avanie malade, estropié, violé, mutilé - prend une dimension proprement cognitive. Ce corps vit en effet dans sa chair une expérience qui véhicule une connaissance critique de la société et, même si cela donne lieu à une poétique, ne sert donc pas seulement que de support commode à l'art ou au symbole (N. Pesaro).

8 Afin de témoigner pour le sang versé par autrui, Lu Xun, tout écrivain engagé qu'il soit, rejette la littérature de propagande ou l'inféodation à une ligne ou un parti, pour dénoncer le grand «banquet cannibale » - dont il se sent lui-même complice - par une phénoménologie du corps mort-vivant, mangeant-mangé (S. Yoshinobu). De même exhibe-t-il la parole, la voix réprimée sous une forme qui se libère de la norme, brouille les frontières "nationales" entre genres attendus, importe des esthétiques comme l'expressionnisme, mais n'en reste pas moins conscient de la position difficilement tenable de l'individu (V. Vuilleumier). Autant d'actes de résistance qui appellent un lien renouvelé au corps et par suite à la société.

9 La littérature assimile assez tôt la psychanalyse, importation majeure de la Chine des années 1920, mais les événements politiques brisent ce premier élan. Même si les premières fictions osent décrire le désir féminin du corps masculin, la romancière Tie Ning retrace à la fin des années quatre-vingt l'histoire de trois générations de femmes qui peinent à construire leur « je » sous le regard féminin (Hu X.). Mais dans ces mêmes années, chez Lin Bai, la compréhension biaisée du féminisme et de la psychanalyse ne fait guère mieux que de réduire le devenir féminin au portrait d'héroïnes qui se contentent de retrouver leurs corps avec le plaisir charnel et se le réapproprient dans des lieux intimes à l'écart de la société (Guo L.).

10 Le corps n'oppose pas qu'une résistance charnelle, il est aussi mémoire active et constitutive de l'individu. Chez Han Dong, le récit porte, construit, s'approprie à titre individuel, la mémoire traumatisante de la Révolution culturelle. La dimension 
corporelle sensible du sujet joue un rôle de fond dans la construction a posteriori du souvenir, lequel est ancré dans les perceptions qui lient la conscience au monde environnant, d'où l'essor d'une identité plus émancipée (M. Codeluppi). Certains objets culturels, surtout écrits, activent la prise de conscience du corps, qui se présente chez Wang Gang par la subversion du récit de formation. C'est le signifiant collectif, normatif, politique du texte, ici un dictionnaire, qui déclenche le désir de découvrir le corps privé comme espace pour l'individu en train de se construire (M. Pirazzoli) : il est d'autant moins anodin que le texte en question soit un dictionnaire d'anglais, langue étrangère, et la professeure, objet conjoint du désir, ouïghoure. Autant d'éléments de subversion du lieu scolaire, censément, tout comme l'espace hospitalier, espace de dressage et de discipline du corps et de l'esprit, ainsi qu'en témoigne mainte œuvre littéraire des années quarante à cinquante, comme chez Ding Ling.

À rebours des représentations exaltées du corps fort, de la puissance, qui renvoie de façon rassurante à des certitudes et injonctions collectives - militarisme, endurcissement physique, aventure intrépide, voire activisme révolutionnaire ou mercantile -, certains auteurs choisissent, n'ont parfois guère d'autre option qu'un récit inscrit dans un corps faible, malade, invalide, tel celui de Masaoka Shiki qui ne cesse de renaître de ses maux et par les mots. Le texte se fait alors mise en scène, peutêtre illusoire, de ce qui est pure vitalité et mouvement corporel, y compris sa douleur, sa déchéance, sa difformité - pour le laisser parler, s'exprimer par tous les pores (E. Lozerand). Plus près de nous, la parole de l'handicapé, subalterne oublié souvent, s'impose contre le discours médical et autres formes de contrainte et d'enfermement (A.-L. Mithout).

12 Dans la mouvance de Walt Whitman ou Paul Claudel, Guo Moruo crée un corps démiurge qui, medium ou interface entre tous les objets du monde, articule microcosme et macrocosme, mort et renaissance, en une quête de l'unité totale, d'une poésie «panthéiste». Ce nouveau monde, où s'annule la division entre l'humain, l'animal et la nature, est créé par un sujet qui se nourrit de l'énergie du monde pour en faire la sienne propre, par un sujet cosmique qui se tient dans un moment de création permanente, d'où la constante réinvention du corps (P. Magagnin). Mais si ce sujet à la fois romantique et critique du 4-Mai advient à lui-même, il est tellement à la recherche d'un "grand moi ", somme toute assez compatible avec les exigences révolutionnaires jusqu'à la fin des années 1970, qu'il finit par se consumer lui-même et détruit ainsi, paradoxalement ses propres limites.

13 La maladie du corps devient de plus en plus propice à la création. Inscrite dans l'expérience du corps hors-norme, la narration remet en question l'acte d'écrire jusque dans le tissu même de la langue. La représentation de la difformité post-moderne forme l'étape la plus contemporaine de cette quête du corps en Chine. À Taïwan, Wang Wenhsing imagine un narrateur malade qui, concentré sur son corps propre, scrute $d u$ dehors ses perceptions intimes, comme pour se tenir à distance de soi-même et de ses maux, et ce en une démarche d'exorcisation créatrice. Les expérimentations typographiques auxquelles il se livre ont deux objectifs: déformer la lettre pour montrer le corps malade et inventer une nouvelle langue, propre au sujet. L'affinité signe-corps au sein de nos consciences se révèle ici sous la forme d'un homme-signe ou d'un corps-mot, qui renoue avec le monde. Le sujet retrouve ainsi une forme d'unité entre corps et âme - qu'ont désunie le dualisme importé d'Occident ou le mode de vie 
aliénant de la société moderne - au sein d'un monde où le tout et les parties se tiennent (S. Marchand).

14 À partir des années 1980 , les auteurs chinois retrouvent une certaine inspiration venue du 4-Mai : l'Occident, espace culturel à importer et promesse d'émancipation ; la remise en question du culte de la force; le récit d'une conscience incarnée dans un corps libidinal. Tous éléments que condamne l'esprit des «Causeries sur les arts et la littérature à Yan'an » de 1943, mais que la littérature d'après la Révolution culturelle réactualise dans un contexte intellectuel renouvelé, dont participent la «fièvre culturelle » et la "recherche des racines ». S'ensuit une nouvelle représentation des corps, qui vient des années 1920, mais avec plus d'espace pour un sujet vraiment individuel et peut-être moins de culpabilité liée au sexe. Symptomatique à cet égard, la scatologie marque la littérature et le cinéma chinois continentaux et taïwanais depuis les années 1980. Elle subvertit les grands discours sur le confucianisme et les normes culturelles (C. Neri). Passer par le corps permet de parler du rapport à l'autorité et d'interpréter les devoirs de piété filiale, en particulier du fils vis-à-vis du père. De ces récits de formation, la conscience du corps et du désir devient le vecteur.

Là où la littérature chinoise apparaît sous les espèces du manque ou dans la quête d'une harmonie qui reconstitue le corps social, il semble que les littératures de Taïwan, de Corée et du Japon, devenus grands pays industriels avant la "Réforme et Ouverture " de la Chine, soient plus en phase avec la réception de l'Occident et placent l'individu aux affres de la consommation. Entre Chine et Japon, la différence parait d'ordre quantitatif. Du côté du pas assez de la Chine, où la faim n'est pas si loin dans les mémoires, où le corps se découvre encore timidement, et même de la Corée, où le corps féminin ne se voit pas sans une certaine mauvaise conscience. Du côté du trop pour le Japon où le sujet, écartelé comme en Occident entre le supplice de Tantale, l'offre agroalimentaire, et l'impératif d'être mince et svelte, navigue entre anorexie et obésité. Sinon, les deux littératures évoluent, à quelque intervalle près, avec les littératures mondiales.

$16 \mathrm{Au}$ Japon comme en Chine, il incombe à la littérature de réparer la rupture entre oikos et anthropos, qui fait que l'individu qui peine à inscrire son corps dans son milieu passe par les mots. Certes, elle peut le faire par le détour du reportage, où les subalternes en souffrance font entendre leur voix, décrivent leur condition, appellent à la prise de conscience du public. La littérature du handicap présente ainsi la maladie en tragédie subie, d'autant plus pathétique, mais lutte aussi, comme Asaka Yūho, contre la norme réifiante qui au Japon confine le patient dans son état et lui interdit de jouir comme tout un chacun (A.-L. Mithout). Mais le passage par l'écoumène, animal ou végétal, a vertu réparatrice, et même cathartique, par exemple dans la littérature coréenne. Chez Kim Dong-ri, le mythe d'Hercule et de Sisyphe et la légende locale du dragon blessé sont associés. Le corps souffrant des combattants est mis en rapport avec leur terre natale et le temps immémorial dans une allégorie qui réitère le tourment de la Corée colonisée par le Japon (Wang-Le M. S.). Han Kang, elle, relie à la nature la chair du personnage féminin agressé qui se métamorphose en arbre muet. Le végétal incarne alors un message non langagier de résistance à la violence (Jeong E. J.).

17 Cependant, la littérature innove surtout en créant des mots, des récits, des textes sur le mouvement corporel à l'œuvre. Elle le fait d'ailleurs au fil de l'évolution des modes et des genres littéraires. Dans la foulée des romans de Conan Doyle, une imagination exotique aux antipodes de la norme contrecarre, non sans ambiguïté, le discours 
racialiste de l'État nippon et bientôt militariste dans l'œuvre d'Oguri Mushitarō, et ce même si toute perspective coloniale n'est pas absolument écartée (G. Peloux).

Dans le Japon de l'après-guerre, sidéré par la défaite mais heureux rescapé, Sakaguchi Ango oppose le corps désirant et désiré de l'individu, en opposition au «corps étatique » (kokutai), censé assurer la cohésion idéologique du peuple. Le groupe Gutai, lui, montre les liens de l'homme en mouvement avec la terre. Dans les deux cas, il s'agit de le libérer de l'idéologie militariste qui, depuis le début des années 1930, récuse l'individualité (Innami F.). À la manière d'À la recherche du temps perdu, Han Dong restitue son corps-mémoire au sujet qui a vécu la Révolution culturelle (M. Codeluppi). Sur un registre parodique, et dans la foulée du réalisme magique de Salman Rushdie ou Gabriel García Marquez, c'est au grand récit national mutilant qu'Eka Kurniawan oppose l'utopie du corps indonésien développé dans son identité transhistorique: il relate la vie d'une prostituée, dont le corps convoité est indissociable de l'histoire de l'Indonésie, qui subit la violence politique. L'individu est impliqué dans le cours de l'Histoire par son corps, mais fait résilience (É. Naveau). Mais comme le montre l'évolution sociale de deux frères dans la société japonaise récente, là où l'un tire profit du système, en l'occurrence du show-business, mais finit par se laisser broyer, l'autre apprend à maîtriser son corps pour détruire ledit système (T. Takemoto). Néanmoins, ces fantaisies de triomphe, progrès ou pas, ne libèrent peut-être pas l'individu de son corps, et seul l'art reste pour point de repère, comme chez la romancière Phan Hôn Nhiên où les images artistiques, végétales, animales confèrent une dimension proprement poétique au sujet vietnamien contemporain en médiatisant son corps qui, dans un univers post-colonial et mondialisé, n'en reste pas moins isolé d'autrui et en proie à ses maladies et ses souffrances (Doan C. T.).

Faute d'un réel auspicieux, d'autres voies de sortie font retour, qui cette fois ourdissent les espaces-temps de la science-fiction, supplantés depuis les années soixante-dix par l'heroic fantasy. Modestement avec Ōe Kenzaburō qui, après avoir décrit à l'envi les corps suppliciés, reconsidère la place du corps individuel et collectif au prisme de l'eschatologie chrétienne (A. Bechler). En force, avec la web-littérature chinoise, qui démultiplie le corps dans des histoires qui lui offrent des identités de substitution, plus ou moins ludiques, contre le corsetage du pouvoir en Chine populaire (Xu S.). Cette transition à d'autres mondes se retrouve au Japon, avec le multivers de Murakami Haruki.

20 Il n'y a pourtant pas que les mondes à inventer par les mots. Il y a les mots eux-mêmes, qu'un Masaoka Shiki alité, en proie à une souffrance implacable, fait naître de la saveur des mets qu'il déguste sur son lit de malade, comme si la maladie exaltait la vie (E. Lozerand). Et plus que les mots, il y a la langue à recréer, qui retrouve l'unité corpsesprit et rétablit l'intégrité de l'être selon le Taïwanais Wang Wen-hsing (S. Marchand). Avec l'anthropocène, l'homme saisit qu'il fait du mal à lui-même et à ce qui l'entoure, les animaux notamment, et c'est à la poésie de la Coréenne Kim Hyesoon de ramener le corps humain réduit à la condition animale - le cochon-, expression de toutes les souffrances infligées à l'humanité, base aussi d'une résurrection vers l'humain, par le biais même du renversement de la hiérarchie dans la sublimation de la bête (Koo M.).

21 Si tant est que le corps fasse le lien entre la culture et la nature et s'efforce de rétablir l'équilibre perturbé par la modernité, il s'avère au terme de ce parcours, sans céder aux sirènes du genre, que ce sont les romancières qui représentent la femme comme « plus corps » que l'homme, ne serait-ce déjà que parce qu'elle incorpore le rythme cosmique. 
Aussi son corps est-il en proie, sans axiologie aucune, à toutes les violences. Ces expériences littéraires contemporaines, qui offrent plus d'un point commun avec celles de l'Occident, trouvent peu à peu à se verbaliser dans le sens de l'émancipation accrue $\mathrm{du}$ sujet. Mais ce corps, dont on a vu combien il ne s'écrivait guère jadis, ne vivrait-il pas aujourd'hui à vide à force de trop s'écrire? 


\section{Résumés}

Les résumés sont classés par nom d'auteur, suivi du prénom, et dans l'ordre alphabétique. Ils comprennent les résumés d'interventions qui ont eu lieu, qui n'ont pu avoir lieu ou qui ont été ajoutés après le colloque.

\section{Antonin Bechler : « Soigner les corps et les âmes : la science-fiction selon Ōé Kenzaburō ».}

2 Des fables cruelles des premières années peuplées de jeunes romantiques arrivés trop tard pour la guerre, aux introspections sartriennes guidées par la naissance de son fils handicapé et son engagement au côté des irradiés de Hiroshima, des romans picaresques des années 1960 et 1970, contre-projets face à la centralité envahissante de l'empire, aux palimpsestes ultérieurs au fil desquels l'auteur se réinvente une vie en relisant ses précédents récits, l'œuvre romanesque d'Ōe Kenzaburō se prête difficilement aux catégorisations d'ensemble. Quelques lignes de force peuvent néanmoins être esquissées : la fascination et la crainte des grands récits totalisants et des sacrifices qu'ils réclament, la violence comme élément central dans l'économie du récit, la nostalgie d'une terra mater nichée au creux de la vallée ancestrale. Au cours des années 1980, ces lignes se cristallisent à travers le motif du "salut de l'âme ", qu'ōe explore en s'appuyant sur un panthéon d'auteurs mystiques chrétiens. Nous étudierons ici comment, dans deux romans de science-fiction (Chiryōtō, Chiryōtō wakusei, 1990-1991), Ōe s'empare de la littérature de genre pour reconsidérer la place du corps individuel et collectif au prisme de l'eschatologie chrétienne.

快復する身体・魂、大江健三郎の S F。大江健三郎の小説のモチ一フには大きな

物語への誘惑、犠牲、恐怖、暴力、母なる大地としての四国などがある。198

0 年代以降、これらの要素は「魂の救済」というテーマに結晶し、大江はキリス

卜教神秘主義者の作品にヒントを得て、このテーマを追及するようになる。本発

表では次の二つの S F 作品（『治療塔』、『治療塔惑星』、1990-199 1 年）を通

して、大江が個人の身体、あるいは共同的身体ににつてキリスト教の終末論を参

照項として、再考を重ねた過程を検討してみたい。 


\section{Georges Bê Duc : du « pied naturel » à la « couleur originelle » Zhou Zuoren et le retour au naturel.}

4 On imagine volontiers Zhou Zuoren reclus dans son studio "Pluie amère ", avec sa bibliothèque comme unique horizon. À tel point que dans les années 1930, il a représenté pour les écrivains de gauche une cible de choix : le lettré oisif, soucieux de questions de style et éloigné des réalités terrestres. C'est oublier le rôle fondamental qu'il a joué pendant le mouvement du 4 mai pour réhabiliter l'humain intégral, être à la fois spirituel et corporel (《La littérature humaine» / 人的文学). La présente étude vise à mettre en évidence l'engagement littéraire de Zhou Zuoren pour refonder la morale sur la reconnaissance de cette dualité. On constatera qu'en prônant un regard sain et naturel sur le sexe, il rejoint contre toute attente ses préoccupations d'ordre purement stylistique. Les moralistes confucéens n'ont cessé de mépriser la partie corporelle, les fonctions animales de l'homme, impures à leurs yeux. Zhou Zuoren les raille en leur suggérant d'utiliser deux bassines pour se laver: une pour le haut du corps, l'autre pour le bas, de peur que les deux eaux ne se mêlent! («Le bas et le haut du corps» / 上 下身). Ce puritanisme confucéen demeure pour lui la cause essentielle du malaise de la société chinoise. Le mal est si profondément enraciné que rétablir l'équilibre naturel corps-esprit passe avant tout projet de réforme sociale, politique ou économique. Cette certitude explique sans doute la constance de Zhou Zuoren dans son combat moraliste alors qu'il se retire assez tôt des débats littéraires politisés. Il est le premier à prendre la défense, en 1922, de la nouvelle de Yu Dafu «Dépravation» / 沉沦): un jalon important est posé pour définir la place du sexe comme objet littéraire. Il n'a de cesse de dénoncer le comportement morbide des contemporains, comme ce gouverneur du Hunan s'abstenant de dormir avec sa femme pour obtenir la pluie («Quête de pluie » / 求雨), ou encore ces badauds excités à l'idée de voir s'abattre le couteau sur la peau blanche de jeunes demoiselles communistes condamnées à mort [«Imprécation » / i 咒). Zhou Zuoren est particulièrement sensible aux pieds minuscules qu'il lui arrive de surprendre contre son gré et honteux de son impuissance («Pieds naturels» / 天足). Sans même être prescrits par le lijiao - cet ensemble de préceptes dictés par la morale traditionnelle -, ils en sont la cristallisation. Le spectacle des pieds bandés évoque pour lui une souffrance et une humiliation plus profondes encore que la vue de la misère matérielle. Il s'en rend compte en observant des femmes réfugiées (d'on ne sait quelle calamité) en haillons : leur misère existentielle incarnée dans leurs pieds étriqués et brisés le touche davantage que leur malheur contingent («Bavardages, 1 à 4 »/闲话四 则). Il se souvient aussi, a contrario, de son émoi à la vue des pieds nus d'une jeune servante lors de son premier séjour au Japon, («Première impression» / 最初的印象). L'extrême puritanisme du lijiao est la cause de ces dérèglements. Il explique aussi directement les excès de débauche tout aussi morbides dont l'abondante littérature pornographique est le reflet, et qui sont provoqués par la frustration accumulée. Pour Zhou Zuoren, la cause anthropologique de ce puritanisme remonte à une vieille peur chamanistique du sexe. Le lijiao n'est autre qu'une survivance du chamanisme au sein de la civilisation, la survivance d'un lointain passé sauvage («La pensée ritualiste du chamanisme»/萨满教的礼教思想). Au cœur de ce système qui prétend régenter les liens sociaux qu'est le lijiao, il y a donc une peur irrationnelle et superstitieuse du sexe. Ce système en apparence fondé sur le bon sens confucéen repose en réalité sur un fond obscur et il aboutit à promouvoir le factice et le morbide. L'émoi de Zhou Zuoren 
devant des « pieds naturels » est une révélation à la fois morale et esthétique : les pieds bandés et le baguwen / 八股文, dissertation standard pour les examens impériaux ne cristallisent-ils pas une même pensée génératrice de monstres? Dénuder des pieds naturels, nettoyer l'écriture de son vernis factice pour en révéler la «couleur originelle» / 本色 / bense (« la couleur d'origine »), cela ne relève-t-il pas au fond d'une même démarche de retour au cours naturel des choses?

从“天足”到“本色”一周作人关于返归自然价值的看法 左翼作家们曾批评过周作人是一 名与世隔绝、只喜爱谈文说字的隐士。他们早忽略了周作人作为“五四”运动的先锋 者曾提倡人间本位的新文学, 并且本着人 类“从动物进化”的看法一直努力成立一种 以身魂并重的新道德观念。他所提倡的对于性的健康对待居然 与他写文章的原则相 融合。为了让同代人以新的眼光来重新估价道学家们所轻视乃至否认的身体，周作 人 写了大批文章, 譬如建议道学家们洗澡时使用两个水盆以避免洗下身的水污浊上 洗上身的水。当郁达夫的 小说《沉沦》遭到保守派的激烈批评时, 周作人是第一位 站出来为他辩护的, 并借此肯定了性在文学上应有 的正当位置。最使他愤怒的, 还 是那些以礼教为背景的荒谬态度或残酷行为。缠脚最使周作人感到厌恶。每次遇见 缠脚, 他感到无能为力, 并因意识到自己属野蛮民族而惭愧无尽。当他在北京看到 一群难民时，觉得最难受的不是难民狼狈不堪的乞正样子而是他们女人的小脚，虽 然这些小脚是在灾难前就已经有的，不是灾难的后果。1906 年周作人初到日本时, 忽然被一个女佣的赤脚打动了。这个场景与他刚离开的祖国的情景反差甚大, 而冲 动之余它也给予他一些道德方面和艺术方面的后发。这些与性有关的反常现象都是 礼教的极端禁欲思想导致的。就是那些最为礼教排斥的放纵行为也是禁欲思想的直 接后果。禁欲与放纵从 根本上密切相连。而礼教的禁欲思想原本是萨满教变过来 的，是一种“蛮性的遗留”，而对于性的排斥，原 本是一种恐怖，以为“家国之安危， 人民之生死”皆与 “两性之交渉”有着因果的关系。由此可见“缠脚”跟号一种封建时代 的产物八股文有些本质的关系, 都是礼教的结晶、非人道的怪物, 是进化过程上的 退步。可 是要回到向上进化的路并不容易啊！这个理想由风俗上的“天足”、文学上 的“本色”（即写文章时删掉一切 多余的、做作的痕迹）所代表：代表的是自信的、 大胆的、健康的返归自然价值迈开的一大步。

\section{Martina Codeluppi: Narrating Banishment by Means of the Body - Physical Reflections of Han Dong's Cultural Revolution.}

6 This paper aims to investigate the presence of the body in Han Dong 韓東's literary recollection of the Cultural Revolution. The analysis of his novel Banished! / 扎根 / Zha Gen (2003) will show how the body is simultaneously subject and object of the author's act of remembering, being the primary expression of the author's individuality. Starting from the analysis of the representation of the body on the level of narration, the study will locate the functions the body fulfils at the levels of direct perception and of representation of memory and emphasize the pivotal role of the body in the affirmation of the individual dimension. The representation of the period of the Cultural Revolution is a leitmotif in contemporary Chinese literature was the central theme of the so- called “Scar Literature" / 傷痕文學, emerged during the 1970s, and continued to exert its influence throughout the subsequent decades as well. Han Dong (b. 1961), one of the most representative authors of the so-called "late-generation authors" / 晚生代作家, animated the Chinese literary scenario at the end of the 20th century. His voice is characterized by a strong individualism and the continuous yearn 
for truth. The representation of everyday life, carried out through a sapient use of language, constitutes the lifeblood of his poetic, reproducing the essentiality of the individual's experience by means of a "zero-degree narration" / 零度舄作 (Li Yifei 2012). Set at the time of the Cultural Revolution, Zha gen tells the story of the Tao family who, after being banished to the countryside, engages in an every-day struggle in order to connect with the new social context, and try by all means to "strike root" in the village of Sanyu 三 餘. The story is partly autobiographic, since it reflects the experience of the author who, at the age of eight, followed his family to the countryside of Subei, where they were banished. Han Dong's depiction of the Cultural Revolution is therefore a recollection of his memories as a child and, although the novel employs a third-person narrator and the point of view shifts from one character to another, the child Han Dong's eyes remain the primary source of the author's expression. In the act of reminiscing the daily challenges the characters had to face during the years of banishment, the body plays a central role in both the building of the recollection and in their subsequent narration in the literary text. Han Dong's exquisite attention to details confers a primary importance to the experiential level of everyday life, while the wide use of the iterative mode situates the narrative in a repeated framework, allowing the detection of what Casey defined "habitual body memories". Cultural Revolution was a traumatic phase of Chinese history and many studies have been dedicated to the memory of such trauma. Nevertheless, Han Dong's work, due to his young age at the time, is a rather non-traumatic recollection of a painful historical period. The naivety of the child Han Dong is reflected in the lightness of the tones employed in the novel, and an exemplification of its humorous descriptions can be found in the comparative study by Hunt (2010). Moving from Casey's theorization, the study will first identify the presence of "body memory," which envisages the body as the subject of the recollection, and analyze its representation.

7 通過身體敘述回憶一輪韓東小說《扎根》。記憶這一智力行為與形象的虛擬再現難 以分割，該形象由先前片刻的感官䋑騟獲得。作為與周圍環境的連接工具，身體因 而是個人的第一記憶來源。然而, 身體與精神 能通過不同方式進行相互作用, 並且 身體可以處在追憶過程中的不同層面。Casey （1987）陳述說，追敘建立在“追 憶”的助記方法上，該方法不同於“想起”及“辨認”。進行對於過去的追憶暗示著人們 必須緸過被利科－依照伯格森的直覺一定義為從“純粹記憶”到“形象-記憶”的過渡。

一旦記憶被遷轉入敘述，第三個過渡也就完成了。本論文的目的在於研究韓東對於 文革的文學追敘中的身體的存在。通過長篇 小說《扎根》（2003）之分析，本研究 將揭示身體如何同時充當追憶的主體和客體，繼而成為表達作家個人性 的首要方 法。本論文以對於處在第三層面（敘事層面）上的身體再現的分析開始，繼而將身 體的滿足功能定 位在剩下的兩個層面, 即直接感知（純粹記憶）及記憶再現（形象記憶）。在揭示敘述起止於物理層面的同 時, 本論文將強調身體在肯定個人性中起 到的關践角色。按 Casey 之理論, 本論文首先將辨認出身體回憶 (body memory) 的存 在, 即將身體看作回憶的主體。分析將分下述範疇 : 體罰、身體需要、身體功能、 身體機能障礙等。緊接著, 論文將探討處於空間回憶 (space memory) 層面上的身體 存在, 即身體作為回憶的客體。此階段將分為空間之感官感知、身體與敘事事件之 相依性等範疇。上述分析將昭示身體在韓東小說中 的關键角色, 並且將其定位在追 憶過程中之不同階段, 在表明身體維度如何構成作家個體性自我之精華。 


\section{Cam Thi Doan : corps et art dans le roman Cheval d'acier (2014) de Phan Hon Nhien.}

Le corps de l'individu, et non plus l'âme du lettré ou le cœur du combattant, occupe une place de choix dans la littérature vietnamienne actuelle. Chez les auteurs dont la majorité n'a pourtant pas connu la guerre, le corps n'en est pas moins meurtri par la violence et le traumatisme. Cheval d'acier de la romancière Phan Hon Nhien, paru en 2014, met en scène des Vietnamiens au cœur de la mondialisation qui, entre leurs multiples voyages, se trouvent seuls face à leur corps, porteur de maladies, de solitude, de brutalité, comme si celui-ci était leur unique limite dans un monde désormais sans frontière. À l'opposé des corps humains dont la description est souvent clinique, le corps du cheval, qui hante lui aussi le roman, s'inscrit dans l'harmonie. Quoi qu'il en soit, le corps chez Phan Hon Nhien n'a pas de visée morale ou idéologique, mais se revendique comme une expression esthétique et une réflexion sur l'art.

Cơ thể và nghệ thuật trong Ngựa thép (2014) của Phan Hồn Nhiên. Trong hành trình thám hiểm thế giới cá nhân, văn chương Việt đang rời bỏ « tâm hồn » (nho sĩ) và « trái tim » (chiến sĩ), để khám phá « cơ thể » như một đơn vị độc lập. Trong tác phẩm của các nhà văn tuy phần lớn không sống trong chiến tranh, cơ thể con người vẫn là nạn nhân của bạo lực và chấn thương. Ngựa thép của Phan Hồn Nhiên, xuất bản năm 2014, cho các nhân vật sống giữa thời toàn cầu hóa, trong vô vàn các chuyến đi, thường một mình đối diện với cơ thể mang dấu vết bệnh tật, cô đơn và vũ lực của chính họ. Dường như với các cá nhân, cơ thể là giới hạn duy nhất trong một thế giới ngày càng vô hạn. Đối lập với những cơ thể người thường được mô tả một cách khách quan gần như lâm sàng, tiểu thuyết còn mang dấu ấn của những cơ thể ngựa, đẹp và hài hòa. Nhưng dù người hay vật, cơ thể, trong quan niệm của Phan Hồn Nhiên, không mang tính đạo đức hay tư tưởng, mà tự khẳng định như một biểu hiện thẩm mỹ, một câu hỏi nghệ thuật.

\section{Damaso Ferreiro Posse: An analysis of Ryūnosuke Akutagawa's ideal of corporal beauty in his last years - Wildness and Classics as a reaction against Western and Eastern ways of understanding the canon of corporal beauty.}

10 Ryūnosuke Akutagawa (1892-1927) is regarded as one of the most renowned authors of the modern Japanese literature and because of that, there are numerous researches about the love he professed to women, the way he described them in his works and recently, the way he described non-Japanese foreign Asian women too. It is also verywell known that Akutagawa has continuous references to beauty ideals and sexuality, and the attraction he feels towards the feminine body is visible in many of his works. However, very little is known about the ideal of feminine body that gives sense to his entire thinking, written over a year before his suicide in two brief articles titled "The calling of the wild" and "The calling of the West". Both articles are part of his larger essay Bungeitekina, amari ni bungeitekina and combining the content of both, it can be easily guessed his personal ideal of sexually attractive type of body. For illustrating it, he mentions two examples of what he considers the paradigm of physical and almost 
pornographic model and surprisingly, at first glance these two examples taken from the visual arts seem not to have anything in common: the tanned Tahitian women from Gauguin's paintings and the old Greek sculptures that survived until those days. This paper aims to clarify on one hand the personal, literary and historic-cultural reasons that made Akutagawa differentiate between these two drastic ways of approaching corporal beauty in his late works and on the other what kind of relation can be observed between Akutagawa's and 19th century European literature's way of facing the same topic. According to Nakamura Shin.ichirō, a very illuminating clue can be found in the way modern Japanese literature understood the West at the beginning of the 20th century, establishing an unequal intellectual relation among developed countries and those who aimed to develop themselves taking the West as a model. If this affirmation is applied to Akutagawa's case, it can be guessed that Akutagawa's ideal of corporal beauty can be a clear sign of a type of intellectual and probably unintentionally absorbed colonial influence. However, it is also true that researchers on modern Japanese literature tend to admit that most of the Meiji and Taishō periods writers including Akutagawa, decided to return to Eastern patterns of beauty at the end of their lives disappointed with the process of modernization and the Western culture they admired in their youth. If both affirmations are true at least in Akutagawa's case, a very evident contradiction can be pointed out because Akutagawa did not only maintain the distance with the Japanese or the Western main streams, but he advocated for a new model of corporal beauty never seen before in the Japanese literature. The conflicts between Western and Eastern, colonial and national ways of regarding corporal beauty are indispensable for understanding the evolution of modern Japanese literature but this paper will try to proof how Akutagawa, instead of choosing one of them as a model to follow, tried to build a bridge finding the two unifying nexus of both traditions: wildness and classics.

晚年の芥川龍之介による〈美しい身体〉のイメージ「野性」と「古典」日本近代 文学で芥川龍之介は最も著名な作家の一人であり、彼についての研究は多数あ

る。芥川の恋愛感情や、この作家が愛した女性の身体描写な ども例外ではない。 しかし芥川文学での〈美しい身体〉の理想像の研究は少ない。晚年に書かれた

『文芸的 な、余りに文芸的な』で論じられた身体の理想像を検討した研究は皆無 である。この発表では上記の評論で芥川自身が、理想的な〈美しい身体〉につい て論じた「野性の呼び声」と「西洋の呼び声」を中心に报い、「野 性」のイメー シ溢れるゴーギャンの『タヒチの女』と、ポルノグラフイーを思わせる古代ギリ シャの䧓刻が、芥川の 美意識の中でどのように位置づけられるのか、検討する。 また、彼の好んだ 19 世紀後半のヨーロッパ文学を参照項とし、芥川文学による 〈美しい身体〉の理想像が、なぜ他の日本近代の作家と異なっているのか、更に 芥川の〈美しい身体〉の理想像には植民地主義的な思想が含まれているのかどう か、という問題も一考したい。 


\section{Raoul David Findeisen (1958-2017) : Le corps souffrant et mutilé dans une nouvelle supposée (1915 ?) de Guo Moruo et dans ses mémoires 10 ans de Création (1932).}

Dans ses mémoires Shi nian chuangzuo (1932), Guo Moruo prétend avoir écrit un récit, fondé sur un évènement attesté là où l'auteur fit ses études de médecine. Le point de départ de sa narration mémorialiste est une mise en abyme sur plusieurs niveaux. Le narrateur, étudiant en anatomie, fait le récit du vol et de l'outrage du cadavre d'une jeune femme par un pêcheur. Le mémorialiste, par contre, fait savoir que son récit, ayant été refusé par la revue Dongfang zazhi (Eastern Miscellany, 1904-49), est détruit par l'auteur, à la fois narrateur et mémorialiste. La communication se propose d'élaborer le contexte de mémoire qui comporte un nombre de mutilations du corps supplémentaires. En plus, il est proposé d'examiner si Guo Moruo a consulté la traduction japonaise de Das Unbehagen in der Kultur (1930; Malaise dans la civilisation) de Sigmund Freud, alors récemment publiée (Ōtsuki Kenji 大梘賢二 [1931；1891-1977]).

13 The suffering and mutilated body in a supposed short story (1915?) by Guo Moruo and in his memoirs 10 Years of Creation (1932). In his memoir Shi nian chuangzuo (1932), Guo Moruo claims to have written a story, based an event which actually took place in the town where the author studied medicine. The starting point of his memoir is a multi-layered mise en abyme. The narrator, a student of anatomy, narrates the theft and outrage of a young woman's corpse by a fisherman. The memoirist, on the other hand, makes it known that his story, having been rejected by the journal Dongfang zazhi (1904-49), is destroyed by the author, both a narrator and a memoirist. The paper elaborates on the memoir's context, which includes some more body mutilations. It also examines whether Guo Moruo consulted the then recently published Japanese translation of Sigmund Freud's Das Unbehagen in der Kultur (1930) by Ōtsuki Kenji 大槻 賢二 (1891-1977) in 1931.

\section{Lanfang Guo : L'écriture du corps et le discours féminin dans la littérature contemporaine chinoise - le cas de Lin Bai.}

14 C'est après la Révolution culturelle que le corps entre dans l'horizon du public chinois et dans la littérature chinoise. Vers le milieu des années 90, avec la publication de Guerre solitaire /一个人的战争 / Yige ren de zhanzheng de Lin Bai 林白 et Vie privée / 私 人生活 / Siren shenghuo de Chen Ran 陈染, «l'écriture du corps» / 身体写作 / shenti xiezuo devient un phénomène culturel, un terme spécifique, quasi-synonyme de «l'écriture féminine ». Elle semble renvoyer surtout aux textes de certaines écrivaines, telles Lin Bai, Chen Ran, Hainan 海男, Wei Hui 卫慧, Mianmian 棉棉 qui représentent comme jamais auparavant le désir sexuel féminin et seront « réhabilitées » comme une réponse à l'appel des féministes occidentales, notamment d'Hélène Cixous. Leur œuvre marque un grand pas de l'écriture féminine, car les femmes s'emparent du droit d'interpréter leur propre corps et de retrouver leur subjectivité. Cependant, les textes de Wei Hui et Mianmian passent, eux, pour la pure expression du plaisir charnel, et ce à 
l'encontre du principe féministe et de la déconstruction de la subjectivité féminine. Comment ces autrices imaginent-elles le corps féminin? La présente communication répond à cette question par l'étude de Guerre solitaire de Lin Bai, l'un des ouvrages romanesques les plus représentatifs du genre.

中国当代文学中的“身体写作”与女性话语 - 以陈 “文革”结束之后，随着人道主义的 张扬，“身体”慢慢进入中国公众的视线，同时也进入中国文学。而“身体写作”成为 一个术语、一种文化现象, 则要到九十年代 中期。其具有标志意义的事件是林白的 《一个的战争》和陈染《私人生活》的发表。在九十年代的中国文化语 境之 中, “身体写作”几乎成了“女性写作”的代名词, 它似乎指渉的是林白、陈染、海男、 卫慧、棉棉等女作 家将女性性欲、手淫、同性恋等禁忌话题般上文学舞台的作品。 林白和陈染的作品虽然在发表之初受到了 极为強烈的争议, 但随后有几位较有权威 的文学批评家发文为其“平反”，认为她们的文本富有強烈的女权 色彩，是对西方女 权主义理论的一种回应。评论家似乎为她们的文本找到了最好的理论支持一一法国女 权 主义者埃莱娜·西苏在《美杜莎的微笑》一文中极力倡导女性书写自己的身体。该 文因被收录在 1992 年张京媛主编的《当代女性主义文学批评》之中而为中国读者所 熟知。评论家们认为林白、陈染等女性作家正是 在呼西苏“书写吧，你的身体必须 被听见”的呼唤之下写出这样有力的女性文本。由此, 她们通过自己的作 品被赋予了 从男性手中夺回了关于女性身体的话语权, 并重新发现和阐释女性主体意识的意

义, 被认为是 中国女性文学发展中重要的一步。然而, 紧随其后的卫慧和棉棉却有 着截然相反的命运 : 其过于大胆直露 的描写被认为是纯粹的欲望宣泄, 与女性主义 原则背道而驰, 必然导向女性主体的解构。

\section{Ari Heinrich: Chinese Surplus - Biopolitical Aesthetics and the Medically Commodified Body.}

16 A performance artist sets off a scandal when he bites into the forearm of a fetus. The middle-class protagonist of a horror film sees ghosts through the transplanted cornea of an impoverished donor. A cirrhotic liver, preserved in polymer, lies glistening on a table in a shopping mall, not far from a food court and an expensive jewelry store. We live in an age of unprecedented medical commercialization of the body, a time of routine exposure to the agnostic aesthetics of spare kidneys and facial transplants, cosmetic "corrections" and designer blood-a time when the "value" of the medical body has become alarmingly literal. This paper looks at what happens to aesthetics when unprecedented advances in biotech meet the commercialization of medicine in the public sphere. Focusing specifically on questions of transnational Chinese identity formation, I look at the use of cadaverous materials by experimental artists in the early 2000s, at independent films featuring plotlines about organ transplant, and at the ambivalent worldwide reception of the lucrative but controversial plastinated human body exhibits (frequently associated with critiques of Chinese human rights violation) created by the German anatomist Gunther Von Hagens in the mid-1990s. As advances in biotech displace more conventional understandings of the relationship of identity to the human body, I argue, placing scientific and medical aesthetics into dialogue with political economics - or a critical engagement with "biopolitical aesthetics" - becomes increasingly important.

17 Chinese Surplus : 生命政治美學與藥物商品化的身體。本文内容取自我即將發表的 新書「Chinese Surplus : 生命政治美學與藥物商品化的身體」。在該書中，我帶領讀 者思索在當代快速演進的生物科技與藥物商品化的交會過程中, 有關於中華認同的 
再現問題。聚焦於跨國界的中華認同形構之美學再現，我將討論在 21 世紀初期實騟 藝術家如何運用腐屍在他們的藝術實践之中 ; 我並探索獨立電影中關於器官移植呈 現的意義; 我亦將焦點轉向 1990 年中以降, 由德國標本學家 Gunther Von Hagens 所 推動、爭議性十足「塑化人標本展覽」在中、港、台的巡演。在生物科技的演進快 速取代並挑戰我們對於認同與身體的既有認識之際，本文要強調科學與醫藥相關的 美學與再現應該和政治經濟學有更多對話，這也是我所要提出「生 命政治美學」的 主張。

\section{Xinyu Hu : Écriture du corps dans la construction du « je » au féminin chinois moderne.}

Cet article prend la mesure de l'évolution de l'écriture du corps sous la plume des femmes écrivaines chinoises modernes et contemporaines. Alors que la première génération, celle de l'époque du 4 Mai, préoccupée par la revendication des droits féminins dans les domaines socio-culturels, écrit peu à ce sujet, il faut attendre la fin des années 1920, pour voir apparaître en 1928 Le journal de Mlle Sophie / 莎菲女士日记 / shafei nüshi riji, de Ding Ling (1904-1986), qui aborde avec audace le désir féminin du corps masculin. En 1988, soixante ans plus tard, avec La porte des roses / 玫瑰门/ meigui men, Tie Ning (1957-) relate l'histoire de trois générations de femmes sous un même toit à Pékin et le processus afférent de la construction lente et difficile du « je » au féminin et sous le regard masculin.

中国现代女性自我身份建立中的身体书写。我们注意到中国现当代文学中有关身体 书写的变化: 五四时期的第一代女作家很少渉及这个主题, 因为她们更多地关注的是 在社会及文化领域争取妇女权益的问题。一直要等到 1928 年丁玲发表了 《莎菲女士 日记”后情况才有了改变。在这部作品中, 作家大胆地渉及了在恋爱关系中女人对 男人身体产生強烈欲望的主题。六十年以后, 铁凝于 1988 发表了她的第一部长篇小 说 《玫瑰门》。这部作品一经问世就引起了读者和评论界的注意。《攻瑰门»讲述的 是居住在北京四合院里一家三代女性的故事。通过女主人公们的遭遇，作者勾勒了 与她们具有同等生活条件的中国妇女的生活 轨迹：从平静的少女生活到为人妻，又 为人母, 最后到年老体弱的苦楚与无奈。在她们人生的每一个阶段, 其身体的变化 不仅见证了时间的无情流失, 也展现了女性自我意识建立这个过程的缓慢与艰难。 作为 二十世纪两个不同时期女作家的代表, 丁玲和铁凝她们从各自不同的角度诠释 了女性自我的建立与损毁: 如果说前者更多地聚焦在恋爱关系中女性激情澎湃以及肉 体的感觉，那么后者则让读者听到了其主人公 内心深处的呼喊，她们向往的不仅是 与男性共享日常生活，而且也是肉体之欢愉。可以说女性身体的成熟 伴随着女性人 格的成长, 女性由少女而女人, 最终成为那个有欲望, 有痛苦却不断追求自我实现 的主体。两位女作家的身体书写引起我们对中国现代女性自我身份认同的过程产生 了如下追问：女性身体是怎样从被男性注视的物体转变成为具有自我审视, 自我发 现, 自我实现的主体存在? 身体书写在女性自我身份认同的复杂过程中起到了哪些 作用? 从文学语言和叙事的角度来看, 两位女作家各自作了哪些尝试, 这些尝试使 她们的创作与传统的对女性身体的表现完全不同，也让她们的作品在亚洲甚至世界 范围内更加具有现代性的特征? 


\section{Innami Fusako: Excribing the Body - To Act, Think, and Perform.}

Japan experienced a second opening and became a democratic nation in 1945, following the dropping of the atom bombs and defeat in World War II, which pushed the Japanese to pursue interests in the individual autonomous body (called shutai), separate from the national body. In this paper, I focus on the pursuit of the individual body and carnality in the literature of the flesh / nikutai bungaku in relation to the 1950s-60s Gutai avantgarde art movement in Japan. My analysis of the body based on the interaction of the literature of the flesh and Gutai can offer a nuanced re-reading of the phenomenon. The individual flesh / nikutai, was situated in the tensions arising amongst the national body / kokutai, the philosophical concept of shintai, which incorporates both a bodily and spiritual sense, and the material body / karada.

第二次大戦での敗戦とその後の民主化を経騩した日本では、国体から離れた主体 のあり方を模索するようになった。本発表では、肉体文学における身体を $1950 \cdot 60$ 年代の前衛芸術、特に具体派との関連を通してトランス・ナショナルな視点から 分析する。身体、個人主義、集団主義に関して、肉体文学と具体派は別々に語ら れ ることが多いが、その関連から考えることで肉体文学やその周辺における身体 像はより豊かなものとなる。天皇制を中心とし、アジア諸国へも勢力を広げた国 体における統一は敗戦を機に崩壊し、その結果、個々の身体 や具体性を照らし出 寸文学や文化表象が生まれた。国体に対する個々の肉体、哲学的な意味での身 体、物 質的な体など、身体を模索する作品は、新しい個体の概念の形成を促し た。と同時に、「個」の模索は外部との 関係も切り離せないものであった。肉体 文学の多くの作家は実存的・現象学的な身体の考元方を意識していたし、具体派 もアート形式や国際的なアート・コミュニティへの宣伝も意識していた。のち 1 $960-70$ 年代にみら 杍身体論もメルロ・ポンティなど現象学に多大な影響 を受けている。戦中から戦後へと徹底的な变化を経騩した作家たちは、観念や主 義・思潮を疑問視して、身体だけを信じられる拠り所とした。特に軍国主義にお いて 形骸化した「精神」を経龭した者にとって、言語と身体の距離は切実なもの となった。坂口安吾の「肉体自体が 思考する」（１９４６６）や、田村泰次郎の 「肉体が人間である」（1９４７） は共に、肉体と言語（精神）の乘離を指摘し ている。そして、考える座としての身体、人間の根源としての身体を追求する。 肉体文学と具体派は共に語られ ることは少ないが、身体と精神の関係性は具体派 アーティストにとっても重要な問題だった。白髮一雄は 1955 年から 57 年の 間、具体派のジャーナルにおいて、「肉体」という言葉を用い、いかに精神を表 現する芸術が身 体に拠っているかを書いた。浮田要三は個と共同体における思索 において、身体を個人の基盤と考えた上で、肉体文学に見られるような肉欲でな く知性をその根本とした。実存主義や現象学など概念的な枠組みとの 関係を通し て、本発表では上記のような作家及び芸術家が身体を「個」との関係において再 構築した方法論、そしてどのように活動し、考え、表現する身体を獲得しようと 試みたのかを考察する。 


\section{Jeong Eun Jin : Conscience du corps face à la violence chez Han Kang.}

Corps objets de désirs obsessionnels, corps bafoués puis abandonnés, corps qui gardent à jamais gravés le souvenir d'une blessure, corps en régression souffrant d'une conscience de soi exacerbée, corps qui, effrayés par leur propre agressivité, aspirent au retour à une forme d'existence primitive... Le corps constitue toujours un élément central dans l'œuvre de Han Kang (née en Corée du Sud en 1970) dont les sujets sont pourtant très variés. Toute appréhension $d u$ monde passe dans son univers romanesque par une perception sensorielle. Le corps y dénote avant tout le rapport du sujet au monde et constitue un moyen d'expression intense, moins par la violence que par la résistance opposée à celle-ci. Ce faisant, Han Kang va bien au-delà de la dénonciation d'une société autoritaire, patriarcale et machiste, et s'interroge sur le mal existentiel et la folie humaine. Il s'agit là de représentations du corps féminin les plus originales qui frappent d'autant plus qu'elles sont ancrées dans un décor réaliste de la société sud-coréenne où le corps est fétichisé à outrance.

Body's Awareness Facing Violence in Han Kang. Bodies as objects of obsessive desires, bodies scorned and then abandoned, bodies keeping memory of an injury engraved forever, regressing bodies suffering from an exacerbated self-consciousness, bodies frightened by their own aggressiveness and aspiring to a return to a form of primitive existence... The body is always a central element in the work of Han Kang (b. in South Korea, 1970), whose subjects are nonetheless very varied. In her novelistic universe, any apprehension of the world is based on sensory perception. The body denotes above all the subject's relationship to the world and constitutes an intense means of expression, less through violence than through the resistance to it. By so doing, Han Kang goes far beyond denouncing an authoritarian, patriarchal, and macho society. She indeed questions existential evil and human madness. These are certainly some of the most original representations of the (female) body, which are all the more striking as they are anchored in a realistic setting of South Korean society where the body is fetishised to the extreme.

\section{Suk-Hee Joo : Shim Chong, fille vendue de Hwang Sok- yong - du corps réprimé à la figure sublimée.}

Le propos analyse le roman coréen Shim Chong, fille vendue / 심청, 연꽃의 길 de l'auteur contemporain Hwang Sok-yong 황석 영, souvent présenté comme réécriture du roman classique [L'Histoire de Shim Chong] / 심청 전 / Sim Cheong jeon, lui-même issu d'une riche tradition orale. On se propose de questionner les enjeux littéraires et symboliques d'une telle réécriture à travers l'étude du motif central du corps souffrant ou soumis, mais aussi aimant et aimé : ce motif structure en effet l'œuvre dans son ensemble, permettant à l'auteur d'élaborer, selon ses propres termes, une véritable «Odyssée de la prostitution ». Pour cela, nous analysons la figure de la protagoniste Shim Chong telle que Hwang Sok-yong la reconfigure par rapport au récit traditionnel. Le romancier extrapole à partir de cette histoire qu'il transpose au xIx siècle : issue d'une famille pauvre, Shim Chong est vendue à quinze ans à des trafiquants chinois; mais alors que, selon la légende, elle est offerte à l'océan par les marins en guise de sacrifice 
propitiatoire, l'héroïne du roman contemporain est revendue comme prostituée puis ballotée d'un pays à l'autre, de la Chine à Taïwan et au Japon, sur les mers de l'Asie orientale. En suivant l'itinéraire du personnage sous l'angle de la soumission du corps et de ses multiples transformations, on montre la dimension métaphorique de cette domination physique qui va de pair, pour Shim Chong, avec la dépossession de son identité, puisqu'elle est contrainte à changer de nom et d'apparence vestimentaire en même temps que de " propriétaires ». Cependant, le corps souffrant de la courtisane est aussi, paradoxalement, le lieu d'une résistance à la dépersonnalisation, notamment par le jeu littéraire sur le dédoublement, mais aussi à travers la pratique du chant, récurrente dans le roman, où le corps de Shim Chong devient l'instrument d'une affirmation de soi. Enfin, cette importance donnée au chant dans la matière du roman est l'un des aspects de l'inspiration chamanique que revendique Hwang Sok-yong: celui-ci puise en effet aux versions chamaniques de la légende tout en l'inscrivant dans un cadre bouddhiste. Le corps de l'héroïne prend alors, encore une fois, une valeur emblématique: il est le vecteur d'une sublimation de la courtisane, explicitement assimilée à la figure du Bodhisattva Avalokiteçvara (Gwaneum, la Guanyin chinoise). Ainsi, les différentes étapes de l'analyse visent à montrer en quoi le motif du corps permet de saisir quelques enjeux essentiels du projet romanesque de Hwang Sok-yong, qui consiste à réélaborer la légende en s'inspirant du modèle homérique d'Ulysse pour faire le récit des errances d'une figure féminine et asiatique.

Shim Chong, [Sold Daughter] by Hwang Sok-yong - from the repressed body to the sublimated figure. This paper analyses the Korean novel [Sold Daughter] / 심청, 연꽃 의 길 / Shim Chong, written by a contemporary author, Hwang Sok-yong 황석 영. This work is often presented as a rewriting of the classic novel [The Story of Shim Chong] / 심청전 / Shim Cheong jeon, which is itself a product of the rich oral tradition. We scrutinize the literary and symbolic stakes of such a rewriting through the study of the central motif of the suffering or submissive body, but also the loving and beloved one. This motif indeed structures the work as a whole, allowing the author to elaborate, according to his own terms, a real "Odyssey of prostitution". Hence we analyze the figure of the protagonist Shim Chong such as Hwang Sok-yong reconfigures it in relation to the traditional narrative. The novelist transposes this story to the 19th century: born into a poor family, Shim Chong is sold at the age of fifteen to Chinese traffickers; but instead of being offered to the ocean by sailors as a propitiatory sacrifice like in the legend, the heroine of the contemporary novel is sold as a prostitute, and then shuffled from one country to another, from China to Taiwan and Japan, on the seas of East Asia. By following the character's itinerary from the perspective of the body's submission and its multiple transformations, we show the metaphorical dimension of this physical domination. Shim Chong, dispossessed of her identity, is forced to change her name and her clothing as well as her "owners". However, the suffering body of the courtesan is also, paradoxically, the site of a resistance to depersonalization, notably through the literary play on doubling, but also through the practice of singing, recurrent in the novel, where Shim Chong's body becomes the instrument of self-assertion. Eventually, the importance given to singing is one of the aspects of the shamanic inspiration claimed by Hwang Sok-yong, who draws on shamanic versions of the legend while placing it in a Buddhist framework. The heroine's body takes on an emblematic value once again: it is the vector of a sublimation of the courtesan, explicitly assimilated to the figure of the Bodhisattva Avalokiteçvara. The motif of the body is a key to grasp some essential issues of Hwang 
Sok-yong's novelistic project which, inspired from Ulysses's model, consists in reworking the legend to narrate the wanderings of a female and Asian figure.

\section{Koo Moduk : La décomposition du corps dans la poésie coréenne contemporaine : étude du poème en prose de Kim Hyesoon, « ÇA VA, JE SUIS UN COCHON ».}

Le corps aimant/aimé semble pertinent pour étudier une certaine tendance de la poésie coréenne contemporaine. Le propos scrute la décomposition du corps dans la poésie de Kim Hyesoon. Kim Hyesoon, née en 1955 à Uljin en Corée du Sud, est une des figures majeures dans la poésie coréenne d'aujourd'hui. Selon les critiques, elle est considérée comme une force motrice de la poésie coréenne après 1980 . Son écriture a engendré une profonde influence sur la nouvelle génération des écrivains coréens. Au fond, sa poésie se distingue radicalement de la poésie coréenne traditionnelle, mais aussi de celle des poétesses coréennes de son époque. Elle refuse consciemment l'image de la femme imposée par la société et cherche une nouvelle voix. Elle revendique une poésie féminine, mais qui ne se confond pas avec la poésie féministe même s'il y a des convergences. S'intéressant aux relations des femmes avec leur corps, leur société et leur environnement, elle explore une nouvelle écriture de transgression et de violation. La poétesse coréenne appartient à la génération qui a vécu la dictature. En tant que femme, elle était soumise à une double oppression : la société dictatoriale et le monde des lettres dominé par les hommes. Elle dit ainsi : «Je suis venue au langage grotesque dans la culture patriarcale sous la dictature. Le corps qui a été cassé en morceaux est un corps malade. Je réunis la maladie de ce monde et mon corps malade. Ainsi les images misérables que j'utilise dans mes poèmes sont comme les lettres que j'envoie dans un monde misérable. » (Entretien avec Ruth William dans Guernica A magazine of Arts \& Politics - revue en ligne, tr. de l'anglais par Claude Mouchard, Poésie, $\mathrm{n}^{\circ} 139-140$ : «Corée 2012 », p. 95). Dans un poème en prose tel que «ÇA VA, JE SUIS UN COCHON », écrit après l'épidémie de fièvre aphteuse qui s'est propagée en 2011 en Corée du Sud, Kim Hyesoon remet en question les corps humain et animal ainsi que leur rapport avec la violence et l'amour. Elle travaille précisément sur la décomposition et la recomposition du corps. L'acte de coudre des morceaux de chair ou de tissu est parfois considéré comme un geste d'amour : "Irrémédiablement mon corps grandit / À chaque blessure je coupe un morceau de tissu pour la recouvrir / À force je suis enveloppée d'une couverture de petits carrés / Ma mère me disait de ne surtout pas la mettre / et de ne surtout pas apprendre à ravauder » (Dentifricetristesse crèmemiroir, tr. du coréen par Koo Moduk et Claude Murcia, Circé, 2016, p. 20). La poésie de Kim Hyesoon est ancrée dans la réalité sociale et politique. Ses images sont concrètes et prosaïques. Mais en même temps cette poésie semble s'éloigner de notre monde par ses images insolites et sa voix qui part dans tous les sens. Dans cet univers poétique singulier, le corps occupe une place centrale. Nous étudierons en quoi ce corps est un lieu d'écriture.

The Decomposition of the Body in Contemporary Korean Poetry: A Study of Kim Hyesoon's Prose Poem, "I AM A PIG". The loving/loved body, is relevant to study a certain trend of contemporary Korean poetry. This paper examines the decomposition of the body in the poetry of Kim Hyesoon (b. 1955), one of the major figures of today's 
Korean poetry. Her poetry is radically different from traditional Korean poetry, but also from the Korean women poets of her time, as she consciously rejects the image of women imposed by society and seeks a new voice. She claims to be a feminine poet, but not up to the point of being confused with feminist poetry. She belongs to the generation of women who were subjected to a double oppression: the dictatorial society and the male-dominated world of letters. In her prose poem "I AM A PIG", written after the 2011 foot-and-mouth disease outbreak in South Korea, Kim Hyesoon questions the human and animal bodies and their relationship to violence and love, and specifically works on the de- and re-composition of the body. Although deeply rooted in social and political reality, Kim Hyesoon's poetry seems to distance itself from our world through its unusual images and its voice that goes in all directions. In this singular poetic universe, the body occupies a central place, the object of our analysis.

\section{Josef Kyburz: The Unconciousness of the Body in Japanese Culture.}

My reflection on the role the human-or, for that matter, any living-body plays in Japanese culture, starts with the remark of Tanizaki Jun'ichirō who, in his In Praise of Shadows / 陰㗨禮灒 / In'ei raisan (1933-34), describes the presence of women as a mere face devoid of body and shrouded day and night in obscurity. Indeed, Tanizaki speaks about the Taishō and Meiji periods, but what he is saying may be shown to be true of any previous period of Japanese history of which we have either literary, pictorial, social as well as linguistic, conceptual and behavioural testimony. We don't need much persuasion to realize that Tanizaki's impression applies word for word to the Tale of Genji, for example. We wouldn't be able to find a more substantial description of the body in all of Heian period literature, nor would we find in all of pictorial representations of these times-in the painted scrolls, in portrait painting and sculpture-anything more explicit. Apart from the face and, incidentally, the hands, the body is absent, silent. The masterpieces of the medieval pictorial arts focus entirely and exclusively on the face, which, in its physical traits, in its physiognomy, expresses the essence of the persona, the character, the raison d'etre, all that is the human being. The so-called nise-e 似 絵 is its most typical expression. Pictorial sources do show us, however, that, if nothing else, there is a social dimension to the appearance of the physical body. If we see the denizens of the uppermost classes almost completely wrapped in garments, we notice that the lower the people are in the social scale, the less they are covered: the carpenter and others doing menial jobs are down to their loincloths, but only beggars, outcasts and heavy sinners go naked. One of the most characteristic features of the traditional conception of the body appears in the absence, in the Japanese (Chinese and Korean) language of the gender distinction, between the male and female sex in particular. Were it not for the idiosyncratic aspects of the costume and its parts (headdress, scepter, etc.), we would be hard put, in the Tale of Genji scroll paintings for one, to distinguish between a male and a female person on the faith of the face alone. The lack of gender is even more apparent in the conception of the physical body in traditional Sino-Japanese medicine. The schematic diagrams of the body do not distinguish between the male and the female physique, except for the reproductive organs of the woman. Significantly, Sino-Japanese medicine is not interested in the anatomy nor the other purely physical constituents of the body 
(bones, muscles, sinews) nor its mechanical functions. The non-consideration in which, unlike in the Western civilization, the physical body as such, apart from the persona, is not an object of any kind of consideration, we do not find any conscious, objective, anatomical or esthetic description of the body, neither in literature nor in the pictorial arts. In striking contrast to Western arts and sciences, there is, in particular, no such concept of the nude, the academic observation and study of the naked body, of the esthetic appreciation of the nude, of the beauty of the female and male bodies. The reflection can be extended into a number of other fields that all point into the same direction: the body, nowhere in traditional Japanese culture, constitutes an independent object of consideration, observation or study. One is surprised to discover that, until the intrusion of Western civilization in the late 19th century, physical condition and training, gymnastics, hygienics, bodily exercise, are non-existent; not only are there no sports or athletics for the sake of physical health (sumo has different ends), for endurance or aesthetic form, but the fit, muscular and disciplined body is not a requisite neither in the martial arts nor military warfare. One may be tempted to make an exception of the so-called erotic or pornographic imagery (shunga) of the premodern period, but even the explicit representations of the male and female sexual organs, as accurate, exact or naturalistic as they appear to be, are not anatomical studies in any way, but are meant to be funny (warai-e), to relieve with humoristic verve the tension inherent in the social and ethical restrictions of sexuality. Maybe the most striking difference in the perception and conception of the body between East and West, is the idea of physical beauty that, expressed in terms of harmony of proportion, of religious, ethical, and aesthetic ideals, of mathematical and scientific law even, constitutes from the age of classical Greece through the Renaissance and up to this day a major paradigm of Western culture.

意識以前の身体。身体の存在は感覚によって知覚される。統覚が直接の知覚対象 とする以前に、身体は知覚 の主体、かっ客体として存在している。身体は、客体 化の過程に先立つ文化的な条件によって制約されているるため、統覚、表現、身体 の使い方は文化によって違う。事実、東洋と西洋では、身体、あるいは身体の各 部 分が知覚され、想像され、表現され、利用される方法が、大きく異なっている のだ。本質的な相違点を明らかにするため、ユーラシア大陸の両端で身体を見る 方法を調べてみよう。 まず文学作品を比較し、続いて、二次元・ 三次元画像な ど、視覚的資料を分析する。これらの資料を起源から検証すると、こ扎らの資料 の多くは、誤った解釈に従属させられた上で理解されているように思われる。谷 崎潤一郎は『陰毉禮灒』で次のように書いている。「胴や足の先は裾の長い衣裳 の裡に包まれている、昔の女と云うものは襟から上と袖口から先だけの存 在であ り，他は悉く闇に隠れていたものだと思う。..... ただ闇の中に五體を埋めつつその 顔だけで存在を示していたと云へる。 ...が、昔はあれでよかったのだ、闇のなか に住む彼女たちに取っては、ほのじろい顔ひとつあ扎ば同體は必要がなかったの だ。... 極端的に云えば、彼女たちには殆ど肉體がなかったと云っていい。私は母 の顔と手の外、足だけはぼんやり覚えているが、胴體については記憶がない。」 この描写を出発点に、日本文 化とその歴史の進展を辿りながら、日本に独特な身 体性、そして人体の知覚方法を考察しよう。 


\section{Ju-Ling Lee : Corps châtié, corps civilisé - « Seigneur Chen » de Wu Zhuoliu et la police coloniale à Taiwan.}

police coloniale. Durant la colonisation japonaise (1895-1945), le Gouvernement général de Taiwan met en place un code juridique et policier, modelé sur l'Europe, pour "moderniser" la colonie et contrôler considérablement la vie quotidienne. Wu Zhuoliu, grâce au soin qu'il porte aux détails historiques et aux métaphores du corps, restitue l'ambiance générale de la surveillance coloniale et l'omniprésence du contrôle policier. Alors que les écrivains de l'époque font le portrait de policiers japonais, Wu Zhuoliu aborde le sujet autrement: le policier Chen est un Taiwanais. Le corps souffrant du colonisé n'est pas que représentation de la brutalité coloniale : par la mise en scène du corps châtié au nom de la «civilisation / 文明 / wenming ", Wu montre l'absurdité du discours colonial nippon qui ambitionne une "mission civilisatrice » en Asie de l'Est. D'un ton sarcastique, « Seigneur Chen » aborde la question identitaire des colonisés dans la société coloniale.

Trained body, civilized body: Wu Zhuoliu's "Lord Chen" and the colonial police in Taiwan. This study analyzes Wu Zhuoliu's (1900-1976) short story "Lord Chen" regarding the colonial police. During the Japanese colonization (1895-1945), the General Government of Taiwan establishes a legal and police code, modeled on European models, in order to "modernize" the colony, and to control the daily life. Wu Zhuoliu, through his attention to historical detail and metaphors of the body, captures the general atmosphere of colonial surveillance and the pervasiveness of police control. While some other writers of the time portray Japanese policemen, Wu Zhuoliu approaches the subject differently: policeman Chen is a Taiwanese. The suffering body of the colonized is not just a representation of colonial brutality: through the staging of the body chastised in the name of "civilization / 文明 / wenming", Wu shows the absurdity of the Japanese colonial discourse that ambitions a "civilizing mission" in East Asia. In a sarcastic tone, "Lord Chen" addresses the identity issue of the colonized in a colonial society.

\section{Emmanuel Lozerand : une littérature humorale - Un lit de malade six pieds de long, de Masaoka Shiki.}

Du 5 mai 1902 jusqu'à la veille de sa mort en septembre de la même année, Masaoka Shiki (1867-1902), le grand réformateur du haïku, a publié presque chaque jour dans le quotidien Nihon une sorte de chronique sous le titre Un lit de malade six pieds de long (Byōshō rokushaku, tr. fr. E. Lozerand, Les Belles Lettres, 2016). Cette œuvre étonnante, qui ne ressemble à aucune autre, est doublement liée au corps de son auteur. Elle décrit en effet abondamment, par le détail et sous plusieurs aspects, la vie corporelle de celuici : son rapport à la maladie bien sûr, à la douleur et aux soins, son sommeil, ses repas, ses sensations, son inscription dans l'espace par exemple. De ce point de vue, elle constitue un témoignage de première importance sur certaines dimensions de la corporéité japonaise moderne. Mais plus profondément encore, l'écriture d'Un lit de malade six pieds de long procède directement du corps de Shiki. Elle constitue en effet une sorte de sismographe, inscrivant directement sur le papier les moindres variations 
de sa santé. Lui permettant d'exprimer les flux de sensations, d'émotions et de pensées qui le traversent, elle devient aussi un véritable drain de ses humeurs, une modalité même de sa vie corporelle. En analysant cette dimension très singulière de cette œuvre, on tentera donc de comprendre non seulement ce que la littérature peut dire du corps, mais aussi comment elle peut agir sur ce dernier, participer de sa vie propre, autrement dit de saisir la corporéité même d'une œuvre littéraire.

正岡子規『病牀六尺』体液の文学 1902 年 5 月 5 日から同年の死に至る前日ま で、近代俳句の偉大な改革者たる正岡子規（1８６７－１９０２）は、殆ど毎日、 新聞『日本』に一種の記録の連載を続けた。『病牀六尺』である。この特異な作 品は二重の意味で作者の身体と結びついている。この作品では作者の身体生活が 微細に描写される。作者の病気への関わりは勿論、苦痛、介護、睡眠、食事、感 覚、自身の占める位置などについてが語ら れる。この作品は近代日本の身体性の 幾つかの面を証する。『病牀六尺』は、何よりもまず正岡子規 の身体から生まれ た作品である。『病牀六尺』は言わば地震計であり健康状態のわずかな摇れをも 紙上に記すのだ。こうして子規は自分を摇扎動かす感覚、感情、そして思想を記 述していく。この作品は作者の排液管ともなり、彼の身体生活の運動を自ずと示 すのである。これら特異な面を分析しつつ、我々は文学が身体について何を語る ことができるのか、更に身体にどのような影響を与えるのか、そして身体生活に いかに関与するのかを検討する。文学作品の身体性が問題となるのである。

\section{Lara Maconi: Pathologizing the Tibetan Nation - Dismembered Territories and Diseased Bodies in the Chinese-Written (Auto-) Biographical Essays of a Sino- Tibetan Intellectual Couple (1930s-1940s).}

This paper explores Ren Naiqiang Lodrö Chötsho (1910-1949) and the connections among descriptions of the body, the nation, and the self in the Chinese-written autobiographical and biographical essays of (1894-1989), an outstanding Sino-Tibetan intellectual couple based and active in the Kham region of Eastern Tibet (today part of the Sichuan province) during the first half of the 20th century. This was a time when the Republic of China was largely sponsoring explorations of its barely known southwestern frontier regions, Kham in particular, in order to control these regions not only de jure but also de facto. Ren Naiqiang was one of the main actors of this exploratory venture in Kham. Considered as one of the fathers of Tibetology in China, he was a prominent Han scholar and a man of fieldwork who met Lodrö Chötsho, the daughter of the influential Tibetan chieftain of Upper Nyarong (present day Xinlong county), during one of his missions in the region in the late 1920s. Their marriage gave birth to a life-long intellectually stimulating partnership, a fruitful crosscultural and translinguistic cooperation which resulted in several publications, the launching of a scholarly journal, and, for Lodrö Chötsho in particular, the maturation of affirmed political views about the current situation of her Tibetan homeland which she largely dealt with in her writings, and which she factually defended through a locally and nationally oriented political engagement. The idea that bodies and bodily characteristics are heavily freighted with values and features that are often linked to political, social and cultural spheres is not new, and the view of the body as a site of representation and control is central to several literary-cum-political discourses 
(Fanon, César, Glissant, for example). But in my paper, the simile among bodily, national and identity considerations is more than a trope. Lodrö Chötsho fell seriously ill soon after her first entering the "Land of the Han" and arriving in Chengdu after her marriage. She was never to recover, and her autobiographical essays are largely punctuated by simple, accurate, introspective, intimate, extremely modern descriptions of the gradual but inexorable deterioration of one's suffering ill body, one's determination and thirst for life despite everything, and, in parallel, the serious weakening of one's decaying homeland, torn between devastating intestine intraKhampa wars, and resistance campaigns against nationalist China. The trope of the deadly body and the declining Kham nation is even more effective in Ren Naiqiang's long posthumous biographical essay commemorating his Tibetan wife. Written in wenyan, his style is lapidary, almost surgical, in dissecting every single detail of a human/national body deterioration, the fight to survive, and fell seriously ill soon after her first entering the final ineluctable end. This paper analyses the complex and inextricable connection among individual journeys, literature and creativity, and the epoch-making events of macro-history. It shows how individual histories can sometimes acquire strong emblematic historical significance: Lodrö Chötsho died from disease in late September 1949, she was buried in Chengdu, her body facing her homeland of Upper Nyarong. The People Republic of China was created only few days after her passing, and the Kham region of Eastern Tibet, as well as the rest of greater Tibet, was soon to be definitely and effectively incorporated into the new Maoist political body.

藏民族的病理分析一肢解的 土与患病的身躯：藏知 分子伉 (1930-1940 年代) 中文。 本文透 分析二十世上半叶居住并活于西藏康区 (今属四川省)的知 分子 哲情措 (Lodrö Chötsho, 1910-1949) 与任乃 (1894-1989) 夫以中文写的自式及式文章, 探身体、国 家、以及自我的关。当中 民国政府大量 助在 人知的西南 境地区 行探，特 以康区 代表，藉此达到在法律上、甚至是 上控制及吞并 些区域的目的。任乃 在康区考察 活 中扮演极 重要的角色, 他是杰出的 族学者, 亦从事田野, 他正是在 1920 年代晚 期一次考察, 了极有影响力的上瞻 (Upper Nyarong, 今新) 土司之女 哲情措, 他 的 婚姻使他生在知上互相后，形成了成果丰的跨文化、跨言合作关系。身体及身体 特征予政治、社会、文化氛下的价及特徵的种想法并非新，身体表象及控制的所 之点亦是多文学与政治 述的核心 (法 Fanon、塞尔 César、格里桑Glissant 等), 然而 在本文中, 存在于身体、民族、以及自我同点的关係不是修辞上的比。哲情措在 婚后来到「地」、抵达成都后不久，她立刻感到身体不适，一病，她再也没有痊 瘺，在其自 性的文章中，她常以精确的、个人的反思性叙述提及她的疾病 慢却不可 抑制的恶化趋势, 与此照的, 则是她家 因害力巨大的康巴家族 的自相残 及反抗民 族主 高 的中国而日 衰亡的描述。在任乃 藏族妻子的悼念文裡, 更能明 看出走向死 亡的身体与衰退中的康区之的 关係, 以文言撰写的悼念文, 風格精准如外科手般, 他仔剖析人体 / 国家日 化、存活奋战、以及 不可避免的 尾这个过程中的每个节。 本文分析个人经历及大史下具有开新代意的事件之复 又无法切割的关性，我可以 看出个人身上生的事如何成 具有重大意 的事件 : 哲情措病逝于 1949 年九月底, 葬 于成都，她的身体面向家乡上瞻，她逝去几天之后，中 人民共和国成立，西藏康区 以及大西藏地区不久便明确有效地被入中国共党政体中。 


\section{Paolo Magagnin: I Sing the Body Electric: Corporal Representations in Guo Moruo's The Goddesses.}

This paper aims to explore the forms of the representation of the body in Guo Moruo's (1892-1978) The Goddesses / 女神 / Nüshen (1921), as well as to identify and describe their conceptual sources, in order to highlight the position and significance of such a representation in the aesthetics of Guo's early poetic writing. The Goddesses, Guo Moruo's first poetic collection, marks the peak of his inventiveness and was celebrated as the true beginning of modern Chinese poetry. Indeed, it set the course for the most dynamic poetry of the May Fourth era, lending its vigorous expressive force to new writers seeking a modern voice. By making use of free metric forms and vernacular language, The Goddesses conveys a sentiment of powerful individualism, unrestrained vitality, and oneness with the cosmos. While tapping into references to classical Chinese tradition, it also reveals the heavy influence of imported models: this range of variegated elements creates a kaleidoscope of sources, themes, forms and voices that Guo treats with virtuosity, especially when it comes to the re-elaboration of preexisting models. Many of the poems contained in The Goddesses celebrate nature, physicality, and the communion between man and the universe; moreover, they are constantly permeated with an allegorical idea of rebirth. In this aesthetic universe, the discourse of the body, in its various facets, occupies a prominent position. Moreover, the origins of Guo's modes of corporal representation can be traced back to the manysided literary, philosophical, and even scientific sources absorbed by the poet and subsequently developed in his own writing. The implications of the representation of the body in The Goddesses, with special reference to "The Heavenly Dog" / 天狗 / Tiangou, have already been explored by Vuilleumier $(2009,2015)$, who also investigated the medical body in Guo Moruo's in his early fiction. With regard to the sources, the impact of the Biblical and Greco-Roman tradition, of the pantheistic conception of Rabindranath Tagore and of the energetic, physical poetry of Walt Whitman on Guo's early verse have been widely discussed (Gálik 1986; Liu 2002; Ou 2002). However, to my knowledge, a detailed analysis of the modes of representation of the body in Guo's poetry, as well as of the literary and conceptual sources of such stylemes, has yet to be conducted. Moreover, the role of the European avant-garde - especially Futurism and Expressionism - on the formation of Guo's own physical repertoire and bodily discourse has also been largely neglected. Moving from the existing analyses, and through a close reading of The Goddesses, I provide a detailed account of the several forms and levels of body discourse in Guo's early poetry. More specifically, I focus on the bodily description of death and resurrection, the role of corporal discourse within the overarching theme of pantheism and communion between man and nature, anthropomorphism and zoomorphism in the depiction of the inanimate world, and the allegorical use of technical-scientific language in the representation of the body. The various intra- and interliterary sources of influence upon Guo's modes of representation of the body will be pointed out, with a special emphasis on the role played by Tagore, Whitman, the Judaeo-Greco-Roman tradition, and the literary avantgarde of the early 20th century. Finally, the stylistic and ideological implications of such representations identified against the background of Guo Moruo's broader artistic and conceptual landscape. 
《女神》出版于 1921 年, 是郭沫若的第一部新诗集, 也是中国现代文学史上一部反 映五四时代新诗的艺术精神及诗歌形式的具有巨大影响的新诗集。郭沫若使用自由 诗与白话文成功地表达了強烈的个人主义思想及其生命力，而天人合一的精神及重 生的概念也在该诗集中频繁出现。在《女神》展现的美学世界中，身体话语占有突 出的地位, 这种独特的文学表现形式源于作者的多元文学背景、他的哲学思想乃至 科学灵 感。宇乐文曾研究过郭沫若诗歌（尤其是他的作品《天狗》）里关于肉体描 写的意义, 也探讨过郭氏在 20 与 30 年代小说里的身体与医学论述 (Vuilleumier 2009, 2015）。还有许多学者（Gálik 1986；Liu 2002；Ou 2002 等）研究过古犹太、希腊、罗 马传统, 泰戈尔的泛神论及惠特曼意气风发的诗歌风格等对郭沫若的早期诗 歌作品 所产生的深刻影响。即便如此, 笔者认为, 《女神》里关于身体的论述形式及其文 学、观念来源方面 的研究仍未得到文学界足够的关注。此外, 欧洲先锋派 (尤其是 未来主义和表现主义）在郭沫若身体叙事形 式的构建过程中所扮演的角色也被大大 地忽略了。本文旨在探讨郭沫若《女神》身体话语在不同形式与层 面上的特点, 笔 者将论述影响郭沫若早期诗歌里身体再现形式的内文化及跨文化来源, 重点探讨泰

戈尔、惠特曼的诗歌世界、古犹太希腊罗马传统及欧洲二十世纪初的文学先锋派的 影响。最后, 笔者将阐释郭沫 若使用的身体再现形式及其来源在他早期诗歌里的地 位及意义。

\section{Sandrine Marchand : Du corps mutilé, souffrant, au corps corrélé au monde, dans le roman de Wang Wen- hsing Un homme dos à la mer / 背海的人 / Beihaide ren.}

Dans la littérature de Taïwan, là où le corps mutilé, représentatif de la littérature moderniste des années 1960-70, s'oppose au corps héroïque de la littérature belligérante et propagandiste des années 1950, le corps post-moderne est avant tout, déformé, déstructuré, de même que le langage et la vision du monde qui l'expriment, dans le deuxième roman de l'écrivain Wang Wen-hsing : Un homme dos à la mer. Wang nous propose d'abord ici l'image classique d'un corps séparé de l'esprit, d'un esprit omniscient, qui voit, entend, comprend, dirige le monde. Car le monde ne peut exister comme nature que pour un être qui n'en fait plus partie et peut y porter un regard extérieur. Le corps est compris comme le corps-machine de Descartes. Mais dans le roman postmoderne, ce système de pensée fait face à son échec. L'étendue des dégâts est devant nos yeux, le monde détruit, le corps une machinerie détraquée au bord de l'explosion. Le modèle de départ, un corps et un esprit séparés, sont donc l'un et l'autre, mutilés, malades, détraqués, comme l'est aussi la langue du narrateur. Wang Wenhsing, qui a pourtant l'intuition d'un autre monde où le corps n'est plus séparé de l'esprit, rejoint la corrélation traditionnelle chinoise entre l'homme et le monde qui lie la personne morale et physique au monde cosmique. Il rejoint aussi une vision postmoderne, libérée de la suprématie de la science. L'opposition corps/esprit est abandonnée pour une totalité indivisible formée par l'organisme et son environnement. La corrélation de l'organisme malade à son environnement détruit offre une autre lecture du roman: celle des énergies, des circulations, qui sont de l'ordre de la narration et du langage. Au fil de ses expériences physiques, déplacements, attentes, expériences érotiques, maladies, le personnage nous donne une autre Weltanschauung. Comment le corps dans son unité avec la nature révèle-t-il sa complexité, et comment l'écriture de ce roman dévoile-t-elle la créativité, la liberté qui existent au cœur de la vie? 
From the suffering body to the correlation between the body and the world in Wang Wen-hsing's novel Back against the Sea. What is the nature of the post-modern body when compared with the disabled body symbolizing Taiwan Modern literature in the 1960s, and itself opposed to the heroic body of the post-war and anti-communist literature of the 1950s? The main character's body of the second novel of the Taiwanese well-known writer Wang Wen-hsing, Back against the Sea, is above all mutilated, distorted, deconstructed, as the language of his two night's monologues is. The ugly and absurd way the body works is however linked to the Cosmos. Its actions are correlated to outside events. His body is no more mechanized, but a living organism, a part of the world. Through the main character's physical, erotic, even spiritual experiences, illnesses, motives and expectations, the narration offers another way to view the world. Body and spirit are no more separated, but language still keeps memory of the rupture. How far does this novel attempt at forcing the language to follow the movement of life, its rhizomatic inner and outer connections, and expose the free and creative process of life?

\section{Anne-Lise Mithout: "One litre of tears"? Narrating disability in contemporary Japan.}

By comparing the narratives of disability developed in Kitō Aya's [One liter of tears] / 1 リットルの涙 / Ichi rittoru no namida (1986) and Asaka Yûho's autobiography, [A therapeutic sexy trip] / 瘺しのセクシー・トリップ / Iyashi no sekushî torippu (1993), this paper aims at analyzing how the disabled body is experienced, perceived and constructed, in a process where social norms intertwine with individual appropriation. Falling within the axes "Medical body / cured body" and "Voiced body / performed body", it examines the writing process as an act of self-expression aimed at reclaiming one's right to create a discourse on one's own body, complementing or conflicting with discourses by medical experts and society at large, as an individual way of making sense out an experience that is subject to strong normative discourses. As both authors presented here are women, I will adopt a gendered perspective on this subject, the "disabled bodies" studied here being in fact "disabled female bodies". Therefore, among social norms that authors try to fit into or reject, I will especially highlight how both writers deal with their "femininity", either by denying / being denied it, emphasizing it or transgressing its mainstream codes. I will show that, in both cases, the disabled body is conceived as a battlefield. In [One liter of tears], the battle take place between on the one side, Aya / her family / doctors and, on the other side, the disease, and is depicted based on the dichotomy mind / body, as if disability was an inner enemy that should be rejected out of one's identity. Her narrative is that of someone facing a desperate situation and reacting with courage and optimism. It details the different steps of her "career" (in Howard Becker's sense), progressively taking all the steps from being an ordinary young girl to becoming a "disabled" one. Every challenge is vividly depicted in a way showing how the new situation brings her new sufferings and how she overcomes at least psychological despair (while physical pain is often unavoidable), thanks to her inner strength. As she demonstrates a high level of all the qualities expected from the ideal "disabled girl", that is, being brave, nice and always smiling despite pain and sadness, she is considered by her family and friends (and her readers) as a role model for every person in her situation. [A 
therapeutic sexy trip] depicts disability with a radically different approach. Yūho, who has been born with a disability and has become an activist in the Disability Right Movement and in feminist groups, embraces her disabled body as a part of herself and fights against social prejudices to accept herself and be accepted as she is rather than "cured" and turned into a "normal" person, which she feels she could never be. Therefore, she refuses to fit into the model of the "disabled girl" as a doubly passive person whose purpose should be to patiently endure her situation without complaining (gaman suru). On the contrary, she tries to take action to change social expectations regarding disabled women. Comparing these two types of attitudes towards one's own disability enable me to discuss how the body is constructed in Japanese society, by revealing interactions between individuals and society that impact (directly or by rejection) conceptions of one's own health and desirable behaviors. I eventually highlight that, in a society often perceived as cultivating the myth of homogeneity such as Japan, literary self-narration can be used by individuals in marginal positions to challenge social norms regarding health and body image - and be a commercial success.

『一リットルの涙』現代日本の「障がい」の物語。障がい者の自己主張を推進す る運動が広まり 1980 年代に「障がい者の自伝」と言う文学ジャンルが形成さ れた。最大のベストセラーは 86 年に出 版された『一リットルの涙』だ。木藤亜 也が若年性神経変性疾患の為に障がい者となり夭折する過程 の日記である。「障 がい」を「悲劇」と同視し、「障がい者」を「自分の体に内在する困難を克服す る英雄」として描く言説の典型であろう。亜也は「勇気を持って我慢する障がい 者」として叙述さ 机、健常者に人生の教訓を与えると評価されたが、障がい者運 動と障がい学の発展の結果、新しい言 説が提示された。「障がいの社会的モデ ル」を基本にした同言説によると、「障がい」は「生物的な 原因に由する個人的 な問題」のみならず政治・経済・人間関係など多様な側面を持つ社会問題であ る。そこで障がい者の受ける差別を示す作品の必要性が強調された。

1993 年に出版された安積遊步著『癒しのセクシートリップ』と言う自伝は

『一リットルの涙』とは異なっている。著者（女性）は自分の先天性障がいにつ いて語り、担当医やリハビリテーションの 専門家に「奪われた」身体を取り戻す 為に戦う意思を告げる。本論稿では『一リットルの涙』と『瘾 しのセクシート リップ』での「障がい」のナラテイヴを比べて「障がいのある身体」がどう経騩 され、理解され、構築されていくかと言う問題を分析したい。尚、「障がいのあ る身体」は正確には「障がいのある女性の身体」であり、従ってジェンダー的ア プローチからその問題を論じる。障がいのある身体は戦場として考えられてい る。「『一リットルの涙』では亜也と亜也の家族、そして医者（片方）のチーム 対疾患の戦いとなり、語り手は身体と精神の対立を踏まえて「障がい」を「敵」 とし、その敵を自分のアイデンティティから除外しようとする。語り手は亜也を 「困難に負けずに勇気を持って我慢する」人として描く。「次々に与えられる新 しい苦しみに彼女は絶対失望しない」。この話型が明らかになってくる。亜也に は理想的な「女性障がい者」のあるべき性質（勇気・やさしさ・笑顔）があるか ら、家族・友だち・読者から「手本」とみなされている。『癒しのセクシ 一ト リップ』』に見る障がい者の像は逆である。先天的な障がいを持って生まれ、 フェミニストであり、障がい者運動に参加している遊歩は、「障がい」を自分の アイデンティティの一部とし、健常者と同じにな机るずがないから「治さ机 る」よりも「受け入れてもらいたい」と考光、社会の偏見に 抵抗する。したがっ て、我慢する女性障がい者のモデルを否定した上で、女性障がい者に対する社会 の期待を変えるために行動する。 


\section{Etienne Naveau : la place du corps dans deux romans d'Eka Kurniawan.} islamistes sur le corps féminin et sur fond de mondialisation. Le jeune romancier Eka Kurniawan, lui, puise dans les ressources du mythe et de l'histoire de son pays, notamment dans Les Belles de Halimunda, récit de vengeance qui retrace l'histoire de l'Indonésie depuis l'époque coloniale à la période présente, en passant par l'occupation japonaise et la lutte pour l'indépendance. Eka Kurniawan excelle à décrire le corps dans les situations les plus extrêmes. Il évoque ainsi, non sans humour noir, le viol d'une jeune femme qui récite des psaumes aux soudards qui la malmènent, mais aussi la frustration sexuelle du militaire face au refus de son épouse et l'abstinence du truand, qui se conduit comme le père de sa femme, laquelle est encore trop jeune. Il décrit de même la lutte pour la vie de Dewi Ayu quand elle est prisonnière de guerre, ainsi que les affrontements sanglants entre les truands. La cruauté est omniprésente : bébé jeté aux chiens, militaire dépecé et mis en charpies par ses propres chiens, ses restes envoyés à sa veuve dans un sac plastique... Le corps y tient une place frontalière entre vie privée et vie publique, mythe et histoire, règne animal et surnaturel, en-deçà des besoins et au-delà du mythe.

The place of the body in two novels by Eka Kurniawan. The Indonesian novel of the 21st century is dominated by the confrontation between feminists and Islamists on the female body and against the background of globalization. As for the young novelist Eka Kurniawan, he draws on the resources of myth and history of his country, notably in The Beauties of Halimunda, a tale of revenge retracing the history of Indonesia from colonial times to the present, and including the Japanese occupation and struggle for independence. Eka Kurniawan excels in describing the body in the most extreme situations. He evokes, not without black humor, the rape of a young woman reciting psalms to the soldiers who mistreat her, but also the sexual frustration of the soldier faced with the refusal of his wife, and the abstinence of the gangster, who behaves like the father of his wife still too young. He also describes the struggle for life of Dewi Ayu, when she is a prisoner of war, as well as the bloody clashes between the mobsters. Cruelty is omnipresent. The body holds a mediate place on the border between private and public life, myth and history, the animal kingdom and the supernatural, the needs and the myth.

\section{Corrado Neri : cinéma taiwanais, l'horreur et le corps : la timide résurgence d'un genre entre renouveau et nostalgie.}

Plusieurs films d'horreur dessinent une frontière entre ce que Kristeva définit comme « le corps propre et correct » et le corps abject, ou le corps qui aurait perdu sa forme et son intégrité. Bien que la culture visuelle sinophone soit assez frileuse dans la représentation du corps, deux premiers films taiwanais ont tout récemment remué les media: The Tag-Along (Cheng Wei-hao, 2015) et The Tenants downstairs (Adam Tsuei, 2016). Le premier met en scène une légende urbaine autour d'une fillette fantôme qui 
kidnappe randonneurs solitaires; dans l'adaptation au grand écran, les relations entre le "monstre" et ses victimes sont un prisme par lequel apercevoir les anxiétés contemporaines (vieillissement de la population, avortement, place de la femme au travail et dans la famille dite traditionnelle). Quant à The Tenants downstairs, cette adaptation du roman homonyme de Giddens Ko exhibe une série de "perversions " sexuelles : pédophilie, inceste, nymphomanie, prostitution, relations homosexuelles, voire le cannibalisme. Les deux films font de l'angoisse de la surveillance la métaphore des contradictions de l'individu moderne tiraillé entre la présence obsessionnelle sur les réseaux sociaux et le besoin de sas d'intimité : où placer le corps filmé ? Que relèvent les caméras de surveillance que l'œil nu ne perçoit pas? Quelles angoisses exposent ces films qui mettent en scène des corps constamment espionnés? L'article analyse ce que la représentation controversée et «scandaleuse » des corps révèle des problématiques profondes de la société, ainsi que les stratégies de marketing et diffusion utilisées par ces nouveaux réalisateurs pour établir les bases d'une renaissance du cinéma de genre à Taïwan.

臺灣電影, 恐怖與身體 : 懷舊與創新之間 - 類型電影微新浪潮. 如 Kristeva 所定義許多 恐怖電影的内容形式在於建構一條區別於 “健康正常的身體”與不正常的身體，或是失 去原有健康的樣貌。華語視覺文化比較不喜歡呈現身體，但是最近兩部類型電影引起 討論: “紅衣小女孩” (程偉豪, 2015) 與“樓下的房客”(崔震東, 2016). “紅衣小女孩”改 變自本土都市傳說，傳說中縕衣小女孩會改變自己的樣貌去綁架登山客，電影將紌衣 小女孩的恐怖形象來暗示現代生活的不安，包括身體的老化，老人的獨居問題，女性對 於自我身體的自主權。“樓下的房客”很大膽地表現了性欲與道德的“瑕疵”, 包含戀童 癖,亂倫,同性戀與婚外情, 以及人吃人的劇情安排. 兩部電影用了監視器的符號來暗喻 社會中個人面對公共領域與私人領域之間的衝突. 筆者參考了許多不同文化情境的文 本來探討這兩部電影所要探討的社會問題，身體意識與再現及它們所引發臺灣未來發 展類型電影的影響性.

\section{Gérald Peloux : la résistance des corps dans le recueil Jingai makyō (1939-1941) d'Oguri Mushitarō.}

La fin des années Trente, malgré le couperet de la censure, continue à voir paraitre des œuvres qui plongent leurs racines dans la tendance ero-guro-nansensu adaptée aux contingences historiques. Cet article analyse l'une de ces productions, publiée dans la revue Shinseinen / 新青年 de mai 1939 à juin 1941 : Les Mondes perdus / 人外魔境 / Jingai makyō). En treize épisodes, Oguri Mushitarō 小栗虫太郎 (1901-1946), dont les récits policiers font sensation durant les années Trente, présente les aventures de l'explorateur et ornithologue Oritake Magoshichi 折竹孫七, également espion à la solde de l'État japonais, dans les dernières terrae incognitae de la planète (Amazonie, Groenland, Himalaya, forêt tropicale au centre de l'Afrique, Sahara, etc.). Si la trame narrative des épisodes est sensiblement toujours la même, largement basée sur le genre des mondes perdus dont The Lost World (1912) d'Arthur Conan Doyle (1859-1930) constitue l'exemple le connu, les propositions d'Oguri tranchent car elles offrent une grande place à la problématique du corps dans ses multiples configurations : sous couvert scientifique, les explorations des héros sont l'occasion de découvrir des êtres aux identités floues, hybrides, où l'animalité, l'humanité, voire le végétal se fondent pour former des entités nouvelles («homme-queue», «homme-amphibien», "anthropoïde du cinquième genre", etc). Malgré une évolution du texte vers une 
présence de plus en plus forte de l'espionnage, Oguri n'adhère jamais aveuglément au pouvoir. En effet, ses personnages remettent plusieurs fois en question l'hybridité, le respect de la différence, la limite entre l'humain et l'animal. Questionnement d'autant plus étonnant que l'eugénisme et les discours sur la supériorité de la « race japonaise » s'imposent alors en leitmotivs politiques. Nous analysons cette œuvre en nous appuyant sur la description variée et multiple des « entités vivantes » croisées au cours de ces aventures. Pour interpréter la présence massive de ces corps plus ou moins humains dans une œuvre populaire, il convient de considérer l'entrecroisement, des discours exotiques hybrides inhérents au genre des mondes perdus et de ceux que promeut d'abord la vogue de l'ero-guro-nansensu, puis l'État japonais pour justifier ses aventures belliqueuses et coloniales sur le continent. Touffue, baroque, souvent tenue pour pédante, l'écriture d'Oguri, telle une faune et une flore luxuriantes, se déploie dans une profusion de possibilités qui recoupent la multiplicité des corps rencontrés dans les explorations d'Oritake.

小栗虫太郎の『人外魔境』（昭和 14 年 -16 年）における身体の不安定性と植 民地的エキゾチシズム。日本の 探偵小説は、1 938 年 4 月、専門誌『シュピ 才』の廃刊で致命的な打撃を受けた。検閲の圧力と中国との戦争が 一層の激しさ を増す国際情勢の下、国家政策に従う作家も、他の表現形式を選ぶ作家も、沈䟮 している作 家もいた。1938 年から1945年の間に活躍していた探偵小説作 家は自分の文学世界の形式を進化させた が、約 20 年間で築き上げられたジャン ルを完全に放棄したわけではない。日本探偵小の大きなテーマに身体がある。江 戸川乱步（1894-1965) 、小酒井不木（1８９０－1９２９）、夢野久

作（1８８９－1９３６） などは、エロ・グロ・ナンセンスの零囲気を強めつつ ある 1920 年代に、傷つけられ、破壊され、改造され、あるいは辱められ た身 体を描く探偵小説の創作を始める。1930 年代後半になっても、作家たちは厳 しい検閲にもめげず新しい 歴史に適応しながらエロ・グロ・ナンセンスが伏流す る作品を書き続けた。

本論稿では、1939年5月から 1941 年6月まで『新青年』に揭載された

『人外魔境』を分析する。 30 年代にセン セーションを起こした探偵小説作家、 小栗虫太郎（1９０１－1946） はアマゾン、グリーンランド、ヒマラヤ、ア フリ カの熱帯雨林、サハラなどで繰り広げられる 13 のエピソードから成る『人 外魔境』で、鳥類学者の探検家 (実は 日本国家のスパイ) 折竹孫七の冒険を語っ ている。そ执㞦のエピソードはコナン・ドイル（1８５９－1９３０)の『失わ机 た世界』（１９１１２）に）に代表さ机るロストワールドジャンルに基づいている。 が、小栗虫太郎は独自のテキ ストを創造する。科学の進步を建前にした折竹孫七 の探検は、動物性、人間性、さらに植物性が溶け合った曖 昧なアイデンティティ のハイブリッドな生物（「ホモ・コウダッス、有尾人」、「インコラ・バルスト リス、水棲人」、「ア ンソロポイド、第五類人猿」など）を発見する物語なので ある。他方、小栗は国策に盲目の忠誠を示しているわけではない。「日本人」の 優越性を主張している言説と優生学が日本に汇濫している時代に小栗は、相違性 の 尊重、人間と動物との境界など数々のテーマを取り上げている。本論稿では、 『人外魔境』での「生物たち」の 描写を分析する。人間に近い、あるいは人間と は程遠いこれらの「生物」が大衆文学作品に数多く登場していることにはどんな 意味があるのだろうか。 


\section{Hsiao-yen Peng: Food and Sex - Zhang Jingsheng's Regimen Theory and Thermodynamics.} the cultivation of body and spirit was linked with the introduction of thermodynamics, a branch of modern physics. The famous Dr. Sex Zhang Jingsheng's 張競生 (1888-1970) belief in moderation for both food and sex lead to his theories of "inner regimen" / 内 食法 / neishifa, or “fasting technique", and “spiritual communion" / 神交法/ shenjiaofa, or "tactile eroticism"). Because of these seemingly esoteric ideas, he was criticized as "unscientific" and "too metaphysical" by the renowned scholar Zhou Zuoren 周作人 (1885-1967). Although Zhang's theory of regimen for the cultivation of body and spirit seems to draw from Zhuangzi's legacy, I will argue that these ideas, apparently existent in traditional Chinese texts, were in fact mainly informed by thermodynamics, a branch of physics established in the nineteenth century. My conclusion is based upon the transcultural lexicon which Zhang uses: “conserved energy" / 儲 力 / chuli, and which is exactly what thermodynamics is about. So Zhang Jingsheng's regimen theory, combining traditional wisdom with modern physics, opens up to peculiar dialogue between spirit and body, philosophy and science.

本文探討身體論述如何進入現代中國，以及身心調養如何與熱力學－現代物理學的一 支一產生關聯。性 博士張兢生 (1888-1970) 主張飲食與性行為均應節制，發展出「内 食法」與「神交法」理論。由於此二理論乍 聽之下立妙神秘，著名作家周作人批判 他散播「偽科學」與「玄學」。雖然表面上張兢生的身心合一養生論似 乎來自莊子 以及傳統中國典籍, 本文指出其實是挪用了熱力學的理論; 熱力學是物理學在十九 世紀發展出 學，形成了心靈與身體、哲學與科學的奇特對話。

\section{Nicoletta Pesaro: Human, Inhuman, Posthuman Female Bodies in Modern and Contemporary Chinese Literature - Literary Descriptions of Psychological and Social Unease.}

Recent cognitive studies have substantially rejected the concept of a mind-body separation, believing that "the mind is inherently embodied", and "reason is shaped by the body" (Lakoff, Johnson, 1999, 3; 5). Western philosophy is being challenged and rethought, and the common vision of human being as a dual entity has been replaced by a holistic approach, which considers perceptions, senses, and emotions as instrumental functions of the way we think and act. If "[c]ognition and world are interdependently originated via the living body" (Varela, Thompson, Rosch, 2017, xxvI) and "our bodily experience and the way we use imaginative mechanisms are central to how we construct categories to make sense of experience" (Lakoff, 1987, XII), we can assume that the literary (mis)representation of the body can thus be a powerful conveyor of socio-cultural critique as well as of psychological activity, and not only an artistic device or a symbolic construction. Certainly familiar to everybody is the fascination 
that the female body has exerted on writers and artists of different epochs and cultures, Chinese literature is filled with examples of depiction of female beauty and gracefulness, however, at the same time, for a variety of reasons, the literary female body has also been subjected to any kind of distortion and manipulation, being often uglified or demonised. Sick bodies, malnourished bodies, craving bodies, and monstrous bodies populate modern and contemporary chinese fiction and poetry. Sometimes it is a deliberately odd use of the literary language or the alteration derived from the male gaze which produces such an uncanny image of women's body. Interestingly enough, in modern Chinese literature, the female body has been a space for debating moral values and social issues and disclosing identity crisis or change: from the rural body to modern urban anxieties, images of suffering or fighting women create both an appealing and disturbing human landscape. According to some cognitive scientists "[e]ven abstract cognitive processes are grounded on the body's sensorimotor systems" (Varela, Thompson, Rosch, 2017, Xxv). The literary treatment of body - especially influenced by the traditional concept of body-mind in Chinese philosophy - seems to recognise this primary function when it presents corporeality directly, without distinctively connecting it with an "abstract" feeling or thought, but letting it reveal states of minds and moral judgments. One clear example is, for instance, Xiao Hong 萧 红's zoomorphic representation of the female body (see also Liu, 1994) or the surreal transformations of the female characters in Han Lizhu 韩丽珠 (Hon Lai-Chu)'s works. My paper aims at analyzing and comparing a range of modern and contemporary Chinese literary texts centered on the description of female bodies in different epochs, contexts, and genres, trying to connect the cognitive approach and the construction of meaning through literary discourse.

中国现当代文学中的人的, 非人的, 后人的女性身体。社会和心理不安的文学描 写。最近的认知学研究在反对传统哲学和人们共识所包含的 “录肉二元论”的基础 下, 提出了一种新的看法, 认为“理智是由肉体创 造出来的东西” (“reason is shaped by the body”, Lakoff, Johnson, 1999, 3 ; 5)。 因此, 西方哲学一些传统观念受到了挑战 和反思, 新的观念把身体和意识论为一体，人的感觉和感受被认为是人类基本的思 维和行为的 工具, 身体不再是由精神所控制, 所统治的。反过来说, 很多学者指出 人的认知功能就是“通过身体经验而发挥作用”。因此，人的身体不仅是象征性的概 念或艺术性的手段, 而可以被看作为直接表示文化和社会批评以及道德判断的载 体。身心统一的观念在中国传统思想里有着很深的淵源。文学作品也往往使用这种 意识来描写人的生活存在。虽然文艺在各种时代和各种文化中所描写的经常是女性 身体的美丽和优美（中 国文学也不例外），然而, 特别是现当代文学作品, 却经常 使女性身体遭受各种各样的变形和操纵, 经常被丑化和鬼化。中国小说与诗歌中疾 病的身体, 缺食的身体, 渴望的身体和怪异的身体泛滥成群。有时, 这 些丑化、变 形的怪诞不经的女性形象是作家故意采用的古怪语言或者经过男性凝视所产生的效 果。一个非 常有意义的现象是在中国现当代文学中, 女性身体往往变成讨论社会问 题与道德观念的空间, 或者它暴露 身份的危机和变化。从“农村的身体” (rural body) 到都市的现代焦虑, 这些战斗的、痛苦的女人身体的描写形成一副又有感染力又令 人不安的人文景观。根据认知学者“就连抽象认知过程基于身体的感觉运动系统” "[e]ven abstract cognitive processes are grounded on the body's sensorimotor systems" (Varela, Thompson, Rosch, 2017，xxv)。 文学因受到中国传统思想中的身心概念的影 响, 好像肯定身体这主要功能, 因此经常把人的肉体存在直接表现出来, 让它毫无 过滤地透露人的心神和道德判断。萧红的动物形态的人物描写 (参考 Liu, 1994) 和 韩丽珠创作中女性的离奇变化都是明确的例子。本文章致力于从认知学的角度给一 


\section{Melinda Pirazzoli: From Subjects to Bodies - Ah Cheng's "The King of Children" and Wang Gang's Ying- ge-li-shi.} 要包括对身体表现的适应。摆脱了对国家宏大叙事的责任以及作为人民代言人的任 务, 后社会主义中国的作家们终于可以宣称, 他们 能以完全自我的方式有权利用文 学体验现实、表现现实。阿城的著名小说《孩子王》, 属于“寻根文学”运 动, 王刚 的《英格力士》则是“晚生代”文学的杰出产物, 通过对两者的比较, 我们可以分析 那些转化方式，即先前被评论家定义的“中国主体性”如何转化为如今被描述成“具身 意识”的方式。阿城《孩子王》与王刚《英格力士》的背景都设置在“文化大革命”期 间的中国偏远地区，两部小说在以下两个方面也都颇为成熟：对学生与老师和父母 关系的描述方式, 以及年轻一代如何受成人和字典影响的表现方式。周蕾和张旭东 分 别在《原始的激情》和《改革时代的中国现代主义》中对陈凯歌根据《孩子王》 改编的电影做了大量评论，重 点关注教育和教学法等问题。周主要分析渉及主体性 建构的那一章。周认为, “(如果我们) 以人类之间的物质关系作为社会的参与者 （来理解主体性）, 而这种物质关系, 首先以听、说、读、写的集体性文化活动互 相作用促成, 然后像在西方一样, 作为中国文学和文化中的一个问题強有力地呈现 出来, 尽管他们并非以 此命名。”（112）阿城的《孩子王》通过阅读和写作创建有 意义的社会关系, 对这种能力的主体性建构的描 述, 使我们清楚地意识到阿城对主 体性问题的涉及。事实上, 能够超越毛派修辞所強加的狭义界限, 将预 制知识（包 含在体制内教科书中的知识）的被动接受者转变成主体书写的主动者, 《孩子王》 展示了字典是 如何为这一过程提供词源学和认识论根据的。对与体制内有关的一切 事物的不信任（教科书显然是一种比喻），通过对书面词语（字典）和书面文本

（学生作文）救赎力量的乐观信念得到补偿。王刚的小说也是关于教育和教学法 的。然而, 这些术语在这里却具有完全不同的含义。单从小说题目“英格力士”就可 以清楚地看出来, 这跟通常翻译成的 “英语”明显不同, 是纯音译的, 没有任何语 意。正如瓦尔特本雅明曾经在他的文章《论模仿力》中阐述的，口语与书面语的区 别在于它的感官质量。书中主人公的英语老师王亚军, 不能帮助其 学生以只一种文 化或语言输出话语, 这一事实也強调了身体感官的重要性。事实上, 他唯一关心的 是“灵 格风”英语、优雅的外国礼仪和外国香水的气味, 这一“关心”影响的是学生的 感官, 而不是头脑, 对他们的教育成长有着决定性的影响。 


\section{Andrea Riemenschnitter. The Human Body as Landscape in Contemporary Environmental Arts and Literature.}

57 Landscape is a historically determined way of seeing the world, whose material foundations are shaped as much by the natural condition of a "landscaped" region as by its human economic, social and cultural uses. Voicing their concern about the environment, contemporary writers and artists frequently show human bodies as intimately bound to (endangered or degraded) landscapes. Zhao Liang's award-winning documentary Behemoth (Beixi mo shou, 2015) is one of many examples articulating the educated elite's deep anxiety about China's headlong race into unprecedented scales of environmental devastation. Zhao engages the previously romanticized grassland of pastoral Inner Mongolia as an iconographic common, but confronts his audience with something radically different: its current "real" surface, a dead landscape abused by mining and other forms of heavy industrial development. Bringing to the fore the contradictions between viewers' culturally fostered expectations and documentary "reality", the film alerts to the hitherto unvalued growl of the grassland's maimed face, bespeaking agony, pain, and grief. While the film's narrative unfolds, the blackened, worn faces of migrant workers, who are employed in Mongolian mines and steel factories, are staged as isomorphic with, and hence an integral part of, the suffering landscape. Through the lens of Zhao's camera and by blurring the boundaries between the human and nonhuman victims of heavy industrial exploitation, Mongolia's debased grassland is thus given a voice to accuse its human desecrators and resuscitate environmental awareness. This paper examines various aesthetic representations that all in one way or another attempt to write the human body back into its material environment. It will be argued that traditional concepts of body and landscape provide a repository for utopian recodings of the community.

\section{Christopher Scholz: Representations of bodies in crisis in contemporary Japanese literature - Two cases of eating disorders.}

While bodies as well as food and eating have been central themes in modern and contemporary Japanese literature from the beginning, the subject of eating disorders has only come to relevance in the last 20 to 30 years. This paper examines how literary narratives help us to understand how far living with illness can be read as a way of negotiating identity. Are such narratives gendered and affected by global beauty standards and eating practices? To what aesthetics do they resort to expose unseen suffering? Can they give voice to the experience of illness, and exert emancipatory or subversive functions? This paper presents two case studies: AMEBIC / Amibikku by Kanehara Hitomi; Vibrator / Vaiburēta by Akasaka Mari. The female protagonists describe their eating disorders as coping strategies, as well as a way to control their bodies. I argue that they display auto-aggression and violence towards the body to reject the stereotype of the cute Japanese girl and to convey a commentary on the way Japanese society deals with women. Both protagonists are seeking agency over their own lives and bodies. The body therefore appears as a process influenced by events, 
feelings and formed by the hands of its owner. In seeking authority and sovereignty, the protagonists exert violence upon their own bodies to cope with distress and exercise agency. Just as sexual intercourse, menstruation or lactation, the ingestion of food or vomiting can be read as a transgression of the body's boundaries. Either an outer element is incorporated from outside into the body, or bodies are "turned inside out through vomiting" (Lavis 2016: 7). As for the anorexic or bulimic body, food becomes a personified agent which not only tries to trick the body into eating, but also may contaminate or pollute the body, a trouble that can only be avoided by not eating at all or by an act of active excorporation. Both protagonists experience dissociation possibly caused by alcohol and their eating disorders to which they react by avoiding food or by self-induced vomiting to feel control over their bodies. Rei says: "When I conducted these little experiments [of self-induced vomiting], everything was under my supervision. So I figured I could control myself completely" (Akasaka, Vaiburēta, 2005: 11). In AMEBIC, the nameless protagonist tells an episode from her childhood that caused her to drink less water and eat less because her father told her that a real lady never sweats. In the beginning of the novel, she has been living without any real food for one year and only feeds on small snacks and supplements from the convenience store. She drinks mostly vegetable juice or alcohol and writes so-called sakubun (錯文, a pun on the word for essay in which saku is exchanged with the kanji for "confusion") on her computer during delusional episodes at night. While Rei has to deal with the voices inside her head, the protagonist in AMEBIC tries to find out more about herself while reading these essays by her nightly alter ego. Using their eating disorders as coping strategies, they reconfigure the inner boundaries of their bodies by not absorbing or digesting food and, along this process, fragment their bodies and identities and create multiple personalities or voices. They no longer understand where their body begins or ends and lose control instead of gaining agency. With reference to other narratives about eating disorders, I argue that these stories can act as a "disturbing impulse" (Sigrid Köhler 2006: 24) and offer a literary approach to female physicality, thus challenging the void in public discourse about what it means to live in a female body in contemporary Japan.

現代日本文学に描かれる身体の危機/ 掑食障害の二例。日本文学では近代から現 代にかけて、身体と食は 共に大きなテーマになったが、摂食障害が描かれるよう になったのはここ 20 年から 30 年の間にすぎない。本発 表では、楞食障害の物 語が、病いと共生する内的な視点のみならずアイデンティティ一形成のヒントも 与えてく れるのではないかという疑問から出発する。これらの物語はジェンダー 理論や美的基準のグローバリゼーション、そして食習慣などと関連しているの か? 作者はどのような文体で不可視の苦痛を可視的なものにするのか? 病気とい

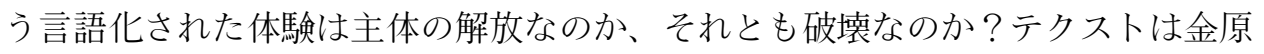
ひとみの「AMEBIC アミービック」と赤坂真理の「ヴァイブレータ」である。ふ たりの作者は罜食障害をモチーフに、身体とアイデンティティーの危機を描いて いる。女性主人公達は、障害を乗り越えるために、自分の身体のコントロールを 試みる。発表者の仮説は以下のとおり。自傷行為も身体への暴力も、「日本人の カワイイ女の子」というステレオタイプを排除する為のものである。次に、危機 的な状況を通して、日本社会で公認された 若い女性への対応方法を、この二作品 は示しているのである。二人とも生と身体の中にエネルギーの源泉を 探してい る。彼女たちにとって、生も身体も固定された総体ではなく、パーツ交換できる コントロール可能な機械なのだ。 従って、さまざまな出来事、フィーリング、そ して主体自身が身体を变化させうる。正当性と至高性を模索しながら、主人公た 
ちは、苦悩を取り除くために自傷行為に走る。性交、自慰、生理、授乳と並 ん で、食品の消化、嘔吐もまた身体の境界を侵犯する行為である。異物が体内に入 り、同化され、さもなくば、「内部のものが嘔吐によって外に出ていく（ラヴィ ス 2016 : 7)」のである。拒食症、あるいは過食症の身体にとって、食品は 擬人化された活力である。そのエネルギーは身体に食べさせようとするだけでは なく、身体を污 すものも含んでいるのだ。後者が拒食症、あるいは過食症の原因 となる。主人公達はアルコール摄取、または 摂取障害による解離を経騃するが、 他方、食事を拒否し、あるいは無理に嘔吐することで身体のコントロールを 試み るのである。 ふたりの作家は摄食障害は身体を内部から再構成する境界であると 把握している。食品は身体に入らないし消化されないからである。摂食障害は身 体だけではなく、アイデンティティーも断片化し、多重人格と多声を可能にす る。身体がどこで始まり、どこで終わるのか等の議論には意味がない。主人公た ちは自己制御能力を得るのではなく失ってしまうのだ。

\section{Baoqing Shao : la faim dans les romans modernes et contemporains chinois.}

60 La nourriture fut l'une des préoccupations majeures du peuple chinois. Dans l'histoire moderne et contemporaine, nombre de générations furent profondément marquées par le souvenir des famines. Or en raison de facteurs divers (censure, sensibilité), il n'a pas toujours été possible d'aborder cette question. Le choix de romans étudiés permet d'examiner les différentes possibilités d'aborder le problème.

苦痛之躯,中国现当代小说中的饥饿。“民以食为天”这句为每一个中国人所熟知的格 言说明了中国人对饮 食的重视。但是近现代中国也正在这个问题上让许多人经受了 严峻的考验。几代中国人深受食不果腹的折磨。这种对肉体所受的蹂踢所产生的感 受本应刻骨铭心进而广泛反映在文学作品中。但是, 由于外部和内 部因素, 文学作 品对这个问题的反映却多有暧昧。本文想通过对一些不同时期、不同作者的小说的 研究，分析介绍饥饿是如何在现当代文学作品中得以呈现的。

\section{Yoshinobu Shino : l'action d'écrire et le corps - lecture de « Comment écrire » (Zenme xie) de Lu Xun.}

Si l'on pense au corps dans les œuvres de Lu Xun, on se rappelle d'abord le corps mangé, qui appelle le motif du cannibalisme, allégorie de l'histoire inhumaine. Face au meurtre du prochain et surtout des étudiants par le gouvernement, Lu Xun écrit plusieurs textes au sujet du corps mangé tels « Un guerrier et les mouches » (Zhanshi he cangying), « Après la mort » (Sihou), ou encore « Résurrection » (Qisi). Mais c'est surtout son essai « Comment écrire » qui, en rapport intime avec ces écrits portant sur ce corps mangé, pose la question de savoir comment écrire dans une "Chine sans voix »: Lu Xun découvre ainsi le lien étroit entre l'acte d'écrire et le corps suspendu entre la vie et la mort.

書舄活動與身體 一讀解鲁迅的《怎么舄》。鲁迅 1927 年發表的《怎么舄》是一篇很 濃縮的文章。此篇看似舄得漫不經心，實則用象徵的手法來表現出他當時的苦悶思 考。他在於什麼也舄不出的情況下，不再討論

64 「䳔什麼」的問題, 卻想討論「怎麼䳔」的問題。他䳔道：「佾非舄不可, 我想, 就是隨便舄舄罷。」「隨便舄䳔」的後面隱藏著不能搞出「血䳔之文章」的煩惱。 
「血舄的文章」本來是尼采的詞彙，此篇中就暗示一名犧牲 者，叫畢臬。他是個青 年共產黨員，此年被國民黨拘捕而殺死。魯迅開頭就描舄著從廈門的宿舍中看到的 南普陀寺附近散在的叢塚，這即看作是對逝者的悼詞。魯忒為何不坦率地表示他的 哀悼? 為何只舄我不能舄而已? 是因為他感到自己已不年㬊, 認出與青年世代有相當 的差距。他們之間的距離是以顏色象徵出來的。犧牲者的紅血與「少女煩上的暧 紅」一樣「容易變色，容易消磨」; 「塚中的白骨」與看來「許多白點」的叢 塚一 樣卻是永遠的。魯迅自己置身於易褂的青春與永遠的死亡之間。照他說，他得到

「敬鬼神而遠之」式的 待遇。他作為鬼神，置身於生者與死者之間；他是個「灰 色」，置身於紅血與白骨之間。他即在模棱的漂浮狀 態中拼命舄出來文章。鲁丑舄 著：「我靠了石欄遠酐兆，聽得自己的心音。」他還活著。此心音引發他舄作的動 機，但他仍然「不能舄，無從舄。」那時發生了一個小事件。一只蚊子咬了他的

腿。將他折磨的「世界苦惱」消失了, 他的精神卻集中於腿上的痛餈。「蚊子的一 四」就讓他䳔。他說: 「只能䳔一些這類小事情, 而還萬不 能舄得正如那一天所身 受的顯明深切。」「這類小事情」表面上就指「蚊子的一可」, 也象徵著他聽到年 輕友人的死亡所受到的打擊。魯迅也在很危險的情况下，只能假裝隨便，多用象徵 手法來表示他的哀悼。他通過自己的痛餈來分享犠牲者的痛苦。他繼續說：「況千 叮萬叮, 而況一刀一槍, 那是舄不出來的。」他自己認 為, 如果有了更多的犠牲者 或發生了更深刻的事情，他不會再進行舄作。絗色和白色也有政治上的含意。不待 說, 紅色象徵著共產黨, 而白色象徵著國民黨。魯迅在廣州看到的兩本雜誌是共產 黨的《做什麼》和 國民黨的《這樣做》。這兩本雜誌的名字各各與「舄什麼」和

「怎麼䳔」對應。舄作活動除了與身體感覺有關 聯之外，也渉及到政治問題。魯讯 在「無聲的中國」内無法搞出「血舄的文章」。在此情況下怎麼舄下去, 這 即是魯 迅在《怎麼窵》試圖探討的問題。他在此表明書舄活動與懸在生死之間的身體有密 切關係。且說, 與 志賀直哉的小品《在城崎》相比, 魯迅在《怎麼舄》中所示的思 考歷程就會更明顯的。

\section{Toshio Takemoto : Les bébés de la consigne automatique de Murakami Ryū - corps, volonté, violence.}

Dans Coin Locker Babies (plus loin CLB) / Les bébés de la consigne automatique / コインロッ カー・ベイビーズ (1980), Murakami Ryū présente, depuis le nourrisson délaissé jusqu'au cadavre, une vaste galerie de corps. Pour lutter contre la société qui les broie, deux orphelins, Kiku et Hashi, n'ont pour seule arme que leur corps et leur volonté, qui cependant ne suffisent pas si elle n'est portée par le corps - mais jusqu'à quelle extrémité ? Kiku et Hashi sont jetés dans une société qui ne raisonne qu'en termes d'efficacité et de productivité. Logique utilitariste qui, face à la maladie du corps social, se manifeste par l'égoïsme outré et l'individualisme forcené. L'affection et la volonté ne suffisent pas aux êtres fragiles pour vivre dans la cité, mais leur mémoire corporelle demeure, qui incarne une permanence identitaire et résiste à la violence sociale.

村上龍は『コイン・ロッカー・ベイビーズ』（1980 年）で考えられうる限りの身 体を描写する、コインロッカーに放置された赤ん坊から屍体まで。この小説の主 人公、つまり孤児のキクとハシには個人を抑圧し押し潰す社会と 戦うための武器 は自分の身体と意志しかない。だが意志はどこまで身体を支えることができるの か? 生産に有 益な者だけのために作られた社会、つまり効率のみを追求する社会 ではコインロッカーに遺棄されたキクとハ シは生まれた時からアウトサイダーで ある。彼らにとって身体と意志は不可分一体である。村上が描く近未来の 日本 
は、エゴイストが支配する破减寸前の社会機構である。そのような非情な世界で 生き残るには愛情と意志 だけでは不充分なのだ。身体はアイデンテイテイの一部 なのである。彼らを疎外する社会に勝つためには彼ら は自らの暴力を制御しなく てはならない。すなわち身体を統御する意志が必要なのである。

\section{Hideto Tsuboi: the Consuming Body and the Consumed Body - the Motif of Eating Disorders in Contemporary Japanese Literature and Manga.}

In regard to eating disorders such as anorexia nervosa and bulimia nervosa, I analyze various genders (film, novel, manga, poetry). I pick up as a typical example, a Korean movie 301/302 (directed by Park Cheol-su), two novels Kitchen by Yoshimoto Banana and Kyoshoku-shō no Akenai Yoake [The Never- dawning Dawn of Anorexic] by Matsumoto Yūko, some mangas including Pink and Rivers Edge by Okazaki Kyōko, and several poems by Itō Hiromi. I examine how normative stories produced by psychiatric discourse on eating disorder are received and criticized in the above-mentioned works and how criticism changes its own appearance though the difference according to their respective gender.

食べる身体／食べられない身体 : 現代日本文学・マンガにおける摂食障害のモ

ティーフ。本発表では 拒食症・過食症という摄食障害に関する主題論的視点か

ら、映画・小説・マンガ・詩といった様々な ジャンルのテクストを分析する。典 型的な例として韓国映画の『3０１／３０２』（パク・チョルス 監督）、吉本ばな な『キッチン』、松本侑子『巨食症の明けない夜明け』という二つの小説、岡崎

京 子『ピンク』『リバーズエッジ』などのマンガ、そ扎伊藤比呂美の幾つかの 詩を取り上げる。具体 的には、精神分析的な言説による摂食障害を語る規範的な 物語が、これらの作品のテクストにいかにして複製化され、また批評されている のかについて考察し、その批評がジャンル 間の差異によってどのようにそのあり 方を变えているかについて考察を加える。

\section{Victor Vuilleumier : Lu Xun, Tourguéniev et l'expressionnisme - corporalité et mouvement dramatique dans « Tremblement à la limite de la déchéance » (1925).}

Des poèmes en prose que contient le recueil La Mauvaise herbe / Yecao (1927) de Lu Xun, l'un en particulier, «Tremblement à la limite de la déchéance » / Tuibaixiande chandong (1925), apporte un exemple frappant de la façon dont cet auteur, parmi d'autres, invente une nouvelle modernité littéraire, très marquée par des apports externes. Ainsi, dans ce poème en prose, Lu Xun tisse avec Tourguéniev et Gogol une intertextualité, et avec les avant-gardes artistiques et notamment l'expressionnisme, une esthétique. Et ce, selon un principe organisateur propre à Lu Xun : le sentiment du corps, qui nourrit sa thématique majeure, à savoir la libération de la voix chinoise, forme nouvelle de subjectivité, à la fois divisée et en tension. Cette esthétique du corps théâtralisé entre en dialogue avec les arts visuels et dramatiques pour caractériser ainsi l'écriture de Lu Xun et d'autres auteurs chinois modernes. 

创造一种新的文学书写; 在他心目中的文学现代性, 经过输入外来元素而形成的。 《在毁灭的边缘颤抖》展示鲁迅与屠格涅夫散文诗、郭 戈尔一些小说的互文性, 以 及与前卫文艺超越美學的一种例子。然而, 这种输入受制于鲁迅特有的组织原 则, 即身体主题和身体感，来表示他关键主题之一 : 无声的人物如何表达自己声音，分 裂主体性的新模 式如何表述。这种戏剧化的身体美学, 与其他艺术进行着对话, 体 现鲁迅与一些其他中国现代作者写作的一个重要维度。

\section{Min Sook Wang-Le : vers une topologie corporelle dans [La Vallée du lœss] de Kim Dong-ri}

71 L'expression corporelle est un des procédés les plus fréquemment utilisés par l'écrivain coréen Kim Dong-ri (1913-1995) pour décrire les actions des personnages. Elle dénote tantôt une gestuelle esthétique à travers les figures des chamanes et moines, tantôt toutes sortes de sentiments humains liés à des circonstances ou expériences vécues. Le corps souffrant, thème récurrent dans ses romans, s'associe constamment à un souci de l'organisation de l'espace, voire à une géomancie qui est conditionnée par la mise en abyme des contes, des mythes locaux. C'est vers une topologie corporelle que se dirigent plusieurs de ses nouvelles dont [La Vallée du lœss], où l'espace romanesque et la santé physique des personnages s'emboitent et ne peuvent se démêler à une lecture de premier degré.

[La Vallée du lœss] combine ingénieusement les trois éléments clés de la divination montagnarde: le dragon / 龍, le pouls / 脈 et les veines / 穴 de la montagne. La particularité topographique de la vallée du lœss prend sa véritable signification géomantique au fil de l'histoire d'Eok-soe. Malgré sa force herculéenne, son destin se confond avec celui du village, menacé et castré par l'Histoire. Les légendes de la vallée prédisent qu'il ne pourra vraiment déployer sa force au cours d'une existence tragique. Comme le pouls de la montagne du loess a été interrompu, la vitalité d'Eok-soe est réduite à néant. Aussi mène-t-il des combats perdus d'avance avec son adversaire Deukbo. Les deux colosses se livrent un combat mortel pour échapper à une vie dénuée de sens. Or, l'espace qui les entoure n'est pas une terre fertile, mais une vallée stérile sans espoir ni rêve. En proie à une frustration absolue, Eok-soe semble condamné à revivre toujours sa lutte avec Deuk-bo. Dans la vallée piégée où il tourne en rond sans issue et dans l'inertie. Son mal-être provient de ce qu'il est né dans la vallée du lœss dont les veines de la montagne ont été coupées et où se transmet l'histoire du couple de dragons qui n'a pu monter vers le ciel car il a perdu ses perles magiques. Son destin est déterminé par la géomancie de son lieu de naissance. Il ne parvient pas à se libérer de ce lieu imprégné de légendes de malédiction.

Ainsi, via les légendes de dragons et de créatures humaines, Kim Dong-ri établit une concordance parfaite entre la topographie et le personnage. La vallée possède un pouvoir destructeur sur les personnages et leur devoir-être. Elle peut même leur faire perdre leur identité et leur sens de l'existence. Comme la nouvelle fut publiée en 1939, en pleine occupation japonaise (1910-1945), l'histoire symbolise assurément la situation de la nation coréenne : rien qu'une montagne aux veines coupées. De [La Vallée du lœss] émane le caractère déterministe, voire fataliste de Kim Dong-ri chez qui le hasard finit souvent par devenir nécessité. 


\section{Shuang Xu : Imaginaires du corps dans des récits fantastiques de la web-littérature.}

<논문요약> 김동리의 [황토기/黃土記]에 나타난 몸의 공간적 위상이민숙장물랭 리옹 3대학교 본 논문은 김동리 (1913-1995) 의 단편소설 [황토기]를 중심으로 소설 공간과 등장인물의 몸의 상관관계를 연구하였다. 몸에 대한 표현은 작가 김동리가 등장인물 의 행동을 묘사하기 위해 가장 빈번하게 사용하는 소설적 기법이자 장치이다. 몸의 표 현은 때로는 무당과 승려들의 형상을 통해 미학적 몸짓으로 나타나기도 하고, 때로는 여러 상황과 체험에 어우려져 있는 온갖 종류의 인간의 감정의 표출로 드러나기도 한 다. 특히, 그의 소설 속에서 반복되는 주제인 ‘고통받는 몸’은 공간 구성에 대한 작가의 관심과 관련이 있으며 설화나 지역 신화를 집어넣어 만든 액자형 서사구조를 지배하 는 풍수지리설에 연결된다. [황토기]를 포함한 그의 단편소설들은 대개 소설 공간과 등장인물의 신체적 건강상태가 서로 얽혀 있는 몸의 공간적 위상에 초점을 맞춘다.

[황토기]는 산의 상태를 예견할 수 있는 세 가지 기본 요소인 산의 용/龍과 맥/脈 그리 고 혈/穴을 능숙하게 중심 서사와 병치시킨다. 소설 공간인 황토골의 지형적 특성은 주인공인 억쇠의 이야기를 통해 진정한 풍수지리적 의미를 갖게 된다고 볼 수 있다. 즉, 어마어마한 힘을 가진 장사, 억쇠의 운명은 역사적으로 위협을 받고 거세된 황토 골의 운명과 일치한다는 것이다. 그가 좌절로 점철된 비극적인 삶을 사는 동안 자신의 힘을 마음껏 발휘할 기회를 갖지 못한다는 사실은 이미 황토골의 형성에 관련된 여러 전설 속에 예언되어 있다. 맥이 끊긴 황토산처럼 이미 패배가 확실해 보이는 자신의 유일한 적수, 득보와의 소모적인 결투로 인해 억쇠의 생기 또한 소멸되어 간다. 비범 한 체격을 가진 두 장사는 무의미한 삶에서 벗어나기 위해 필사적인 결투를 벌이지만, 그들을 둘러싸고 있는 공간은 비옥한 땅이 아니라 희 망도 꿈도 허용되지 않는 불모지 일 뿐이다. 처절한 좌절감에 사로잡힌 억쇠는 같은 행위, 즉 득보와 결투를 반복하도 록 선고받은 삶을 살아야 하는 것으로 보인다. 황토골은 억쇠가 탈출구를 찾지 못한 채 원을 그리며 꼻임없이 회전해야 하는 하나의 덫이며 함정인 것이다. 그는 산의 혈 맥들이 끊어지고 여의주를 잃어버려서 하늘로 승천하지 못한 쌍용의 전설이 전해지 는 황토골에서 태어났기 때문에 불행하고 무기력한 삶을 살아가야만 한다. 그의 운명 을 결정하는 것은 출생지인 황토골의 풍수지리인 것이다. 그래서 아무리 몸부림을 쳐 도 저주의 전설들이 배어 있는 이 장소가 정해 놓은 결정에서 벗어날 도리가 없다.

이처럼 김동리는 용과 인간을 접속시키는 전설을 매개로 지형과 등장인물을 완벽하 게 일치시킨다. 황토골은 등장인물들의 몸과 심리와 의식을 지배하는 파괴력의 상징 이다. 심지어 이 공간은 등장인물들로 하여금 정체성과 존재 의미의 상실을 야기할 수 도 있는 절대적인 영향력을 발휘하고 있다. 이 단편소설의 출판 시기가 일제강점기인 1939 년임을 감안할 때, 우리는 이것이 혈맥이 잘린 산에 불과했던 한민족이 처한 상황 을 상징하는 것임을 확신할 수 있다. [황토기]의 이야기를 통해 우리는 항상 우연이 필 연으로 귀결되는 김동리의 작품세계에 나타나는 결정론 및 운명론과 숙명론을 발견 하게 된다.

주요어:김동리, 한국소설/황토기, 몸과 공간, 풍수지리

À partir de la fin du xx siècle, la web-littérature connait un développement notable en Chine. Parmi maints thèmes, Chuanyue / 穿越 (traverser/traversée) est l'un des plus influents et populaires. Le schéma narratif du Chuanyue / 穿越est lié étroitement à l'histoire du corps: le protagoniste, un contemporain, traverse le temps et l'espace accidentellement ou volontairement, se retrouve dans un autre corps alors que son âme reste toujours la même. À partir de sites littéraires reconnus et à forte audience, 
l'exposé analyse la représentation du "corps traversés » selon quatre types ; 1) corps transgenre dans Go Princess Go ; 2) corps ressuscité dans Mon apprentie est encore morte; 3) corps numérique dans Une vie numérique ; 4) corps qui vit dans un monde parallèle à partir du récit I'm Really a Superstar. Quelle est la raison d'être de ces "nouveaux » corps? Comment contribuent-ils à la littérature contemporaine? Nous analyserons d'abord l'originalité de ces corps imaginaires en rapport avec des facteurs médiatiques et culturels, puis le lien du corps virtuel avec le monde réel, ainsi qu'avec la représentation du temps (non linéaire) et de l'espace (multi-dimensionnel), et enfin la spécificité narrative de cette web-écriture infléchie par le transmedia storytelling. Sera aussi évoquée la dimension socio-politique de cette écriture du corps, à savoir les mesures de censure et de la canonisation orchestrée par le gouvernement.

79 二十世紀九十年代以來, 網絡文學在中國的發展不容忽視。“穿越”是最流行並最具 有影響力的主題之一。穿越的敘事框架與身體的故事緊密相聯 : 生活在現代的主人 公，無意或有意穿越到号一個時空，進入到号一個身體，同時又保留現代的靈魂。 本論文希望研究的主題是: 網絡文學中的穿越奇勾小說如何展現想像 的身體。我們 將選取近幾年在中國著名文學網站發表的獲得成功的作品, 著重討論以下四種身體 的書䳔：1-跨性別之身（《太子妃升職記》），2- 重生之身（《我家徒弟又挂 了》），3- 數字之身（《數字生命》），4- 穿越平行時空之身（《我真是大明 星》）。為甚麼會出現這樣的身體想像？它們對當代文學有什麼貢獻？ 論文將 首 先分析上述文本中身體想像的獨特之處及其與新媒體和文化因素的關聯 ; 然後探討 虛擬的身體與現實之間的關係，以及（非線性）時間和（多重）空間的展現；最 後, 我們將考察跨媒介敘事影響下的網絡舄作的一些敘事特點。在總結處, 我們將 提及中國政府對這一種網絡舄作採取審查和獎勵雙管齊下的策略, 並由此觀察其社 會和政治意義。 


\section{Bibliographie générale}

Sauf indication contraire, les textes français sont édités à Paris, les japonais à Tokyo et les chinois à Beijing (Pékin).

\section{BIBLIOGRAPHIE}

ABE Kōbō 安部公房, [“Cryptic Coloration”] / 保護色 / Hogoshoku, in [The Complete Works of Abe Kōbō] / 安 部公房全集 / Abe Kōbō Zenshū, Shinchōsha, vol. 3, 1997, p. 15-32.

ABE Kōbō 安部公房, [“Starving Skin”] / 飢えた皮膚 / Ueta Hifu, in [The Complete Works of Abe Kōbō] / 安部 公房全集 / A be Kōbō Zenshū, Shinchōsha, vol. 3, 1997, p. 59-72.

ADAMS Louise, “Is ‘devouring' books a sign of superficiality in a reader?”, aeon., juin 2016, https://aeon.co/ ideas/is-devouring-books-a-sign-of-superficiality-in-a-reader, consulted on 01/06/2021.

ADMUSSEN Nick, “A Music for Baihua: Lu Xun's Wild Grass and 'A Good Story”', Chinese Literature: Essays, Articles, Reviews 31:2009, p. 1-22.

AH Cheng 阿城, The King of Children, in The King of Trees, MCDougall Bonnie (trans.), New York, New Directions, 2010.

AH Cheng 阿城, [The King of Chess, The King of Trees, The King of Children] / 棋王、树王、孩子王 / Qi wang, Shu Wang, Haizi Wang / Nanjing Shi, Jiangsu feng huang wen yi chu ban she, 2016.

AKASAKA Mari 赤坂真理, Vaiburēta, Kōdansha, 1999.

AKASAKA Mari 赤坂真理, Vibrator, EMMERICH Michael (trans.), London, Faber \& Faber, 2006.

AKUTAGAWA Ryūnosuke 芥川龍之介, Akutagawa Ryūnosuke Zenshū, Complete Works of Akutagawa Ryūnosuke, Iwanami Shoten, vols. 5, 12, 15, 16, 1996.

ALBER Jan, RÜDIGER Heinze, Unnatural Narratives - Unnatural Narratology, Berlin, Boston, De Gruyter, 2011. ANDERSON Marston, The Limits of Realism, Chinese Fiction in the Revolutionary Period, Berkeley, California U. P., 1990. 
ANNo Moyoco 安野モヨコ, [In Clothes Called Fat] / 脂肪と言う名の服を着て / Shibō to iu nano fuku o kite, Shufu to seikatsusha, 1997.

ARAMATA Hiroshi 荒俣宏, [《Invitation au pédantisme »] / ペダンチックな誘い / Pedanchikku na izanai, in [Le Monde fantastique d'Oguri Mushitarō] / 小栗虫太郎ワンダーランド / Oguri Mushitarō Wandārando, KIDA Jun.ichirō (éd.), Chūseki-sha, 1990, p. 56-58.

[“Art of Flesh”] / 肉体の芸術 / Nikutai no Geijutsu, [New Art Wave] / 芸術新潮 / Geijutsu shinchō, 8-4:1957. ASAKA Yūho 安積遊步, [A Therapeutic Sexy Trip] / 㾍しのセクシー・トリップ / Iyashi no sekushī torippu, Tarōjirōsha, 1993.

ASANO Chie 浅野千恵, [Why do women try to slim down? Eating disorder and gender] / 女はなぜやせようと するのか一攖食障害とジェンダー / Onna wa naze yaseyou to suru no ka: Sesshoku shōgai to gender, Keisō Shobō, 1996.

AUERBACH Erich, « Le monde que renferme la bouche de Pantagruel », in Mimésis. La représentation de la réalité dans la littérature occidentale, HEIN Cornélius (trad.), Gallimard, 1968, p. 267-286.

AURoux, Sylvain (dir.), Notions philosophiques II, PUF, 1990.

AYU Djenar Maesa, À travers les glaces, NAVEAU Étienne (dir. et trad.), Pasar Malam, 2011.

BACARLETT PÉREZ María Luisa, Friedrich Nietzsche. La vida, el cuerpo y la enfermedad, México, Universidad Autónoma del Estado de México, 2006.

BAKHTINE Mikhaïl, L'œeuvre de François Rabelais et la culture populaire au Moyen Âge et sous la Renaissance, REBEL Andrée (trad.), Gallimard, 1970.

BALLERIO Stefano, “Stefano Calabrese, Neuronarratologia. Il futuro dell'analisi del racconto", Enthymema 1:2010, p. 268-74.

BAUDRILLARD Jean et GUILLAUME Marc, Figures de l'altérité, Descartes \& Cie, 1994.

BAUER Heike, "Literary Sexualities", in The Cambridge Companion to the Body in Literature, HILLMAN David and MAUDE Ulrika (eds.), New York, Cambridge U. P., 2015, p. 101-115.

BECHLER Antonin, Ōe Kenzaburō, une économie de la violence, Strasbourg, P. U. Strasbourg, 2016.

BECKER Howard, Outsiders. Studies in the Sociology of Deviance, New York, Free Press of Clencoe, 1963.

Benjamin Walter, “The Mimetic Faculty”, in Reflections. Essays, Aphorisms, Autobiographical Essays, DemETz Peter (ed. and trans.), New York, Schoken Books, 1986, p. 333-336.

BENNET Andrew, "Language and the Body", in The Cambridge Companion to the Body in Literature, HILLMAN David, MAUDE Ulrika (eds.), New York, Cambridge U. P., 2015.

BENOIST Jean, Petite bibliothèque d'anthropologie médicale : une anthologie, AMADES, vol. 1, 2002.

BERGSON Henri, Matière et Mémoire : Essai sur la relation du corps à l'esprit, PUF, 1968.

BERRY Chris and RAWNSLEY Ming-yeh T. (eds.), Journal of Chinese Cinemas, vol. 14, 2:2020, Special Issue on

Taiwanese-Language Films (taiyupian).

BERRY Michael, A History of Pain: Trauma in Modern Chinese Literature and Film, New York, Columbia U. P., 2008.

BIARDEAU Madeleine, L'Hindouisme. Anthropologie d'une civilisation, Flammarion, 1981.

BLANCHÉ Robert, Des catégories esthétiques, Vrin, 1979.

BLANSHARD Alain, "Gender and Sexuality", in A Companion to the Classical Tradition, KAlLENDORF Craig (ed.), Oxford, Wiley-Blackwell, 2010.

BOJSEN Heidi, « L'hybridation comme tactique de résistance dans l'œuvre de Patrick Chamoiseau », Revue de littérature comparée, 302:2002, p. 230-242. 
BOLENS Guillemette, Le Style des gestes, Lausanne, Editions BHMS, 2008.

BONG Joon-ho (réal.), Okja, 2017.

BRooKs Peter, Body Work. Objects of Desire in Modern Narrative, Cambridge, Harvard U. P., 1993, p. 30.

BRUMBERG Joan Jacobs, Fasting Girls - The Emergence of Anorexia Nervosa as a Modern Disease, London, Harvard U. P., 1988.

BUJATTI Anna, "The Spirit of the May Fourth Movement in The Goddesses of Guo Moruo", in Interliterary and Intraliterary Aspects of the May Fourth Movement 1919 in China, GÁLIK Marián (ed.), Bratislava, Veda, 1990.

BURKE Edmund, Recherches philosophiques sur l'origine de nos idées du sublime et du beau, SAINT GIRONS Baldine (trad.), Vrin, 1990

BURN Andrew, “'Playing Roles’ \& 'Reworking the Text: Online Fandom”, in Computer Games: text, narrative, play, CARR Diane et alii (eds.), Cambridge, Polity, 2006.

BURUMA Ian, “THE SENSUALIST - What makes ‘The Tale of Genji’ so seductive”, The New Yorker, July 20, 2015.

BUTLER Judith, Excitable Speech: A Politics of the Performative, London, Routledge, 1997.

BUTLER Judith, Antigone's Claim: Kinship Between Life and Death, New York, Columbia U. P., 2000.

BUTLER Judith, « Hors de soi », Défaire le genre, Éd. Amsterdam, 2006 [2002].

BUTOR Michel, Répertoire I, Éditions de Minuit, 1962.

BUTOR Michel, Répertoire II, Éditions de Minuit, 1964.

BUTTERFIELD Bradley, "The Baudrillardian Symbolic, 9/11 and the War of Good and Evil”, Postmodern Culture, vol. 13, 2002:3, http://pmc.iath.virginia.edu/issue.902/13.1butterfield.html

CAI Rong, The Subject in Crisis in Contemporary Chinese Literature, Honolulu, Hawai' i U. P., 2004.

CANGUILHEM Georges, Écrits sur la médecine, Seuil, 2002.

CANGUILHEM Georges, Le Normal et le Pathologique, PUF, 2006 [1966].

CASEY Edward S., "Habitual Body and Memory in Merleau-Ponty”, Man and World, 17:1994, p. 279-97.

CASEY Edward S., Remembering: A Phenomenological Study, Bloomington, Indiana U. P., 2000 [1987].

CASTANET Hervé, La perversion, Economica, 1999.

CH'OE Sông Shil 최성실, [« La littérature coréenne et le corps »] / 한국문학과 육체 / Han'guk munhakkwa yukch'e, Littérature et Société / 문학과 사회 / Munhakkwa sahoe, 21(2): 2008, p. 311-325.

CHANG Eileen, Little Reunion, WEIZHEN PAN Jane and MERZ Martin (trans.), New York, New York Review of Books, 2018.

CHANGYU 尝谕, [Je suis vraiment une super star] / 我真是大明星 / Wo zhen shida mingxing, https:// www.piaotian.com/bookinfo/6/6180.html, consulté le 03/02/2021.

CHEN Ching-chih, "Police and Community Control Systems in the Empire”, in The Japanese Colonial Empire, 1895-1945, MYers R. H. and PEATTIE M. R. (eds.), Princeton, Princeton U. P., p. 213-239.

CHEN Jianzhong 陳建忠, [Études sur la littérature et la pensée de Lai He] / 書寫臺灣. 臺灣書寫: 賴和的文學 與思想研究 / Shuxie Taïwan. Tä̈wan shuxie : Lai He de wenxue yu sixiang yanjiu, Gaoxiong, Chunhui chubanshe, 2004.

CHEN Jiming 陈继明, [《 La liste de Chen Wanshui »] / 陈万水名单 / Chen Wanshui mingdan, in [《 Best of » des courts romans chinois de 2013] / 中国中篇小说排行榜 / 2013 Zhongguo zhongpian xiaoshuo paihangbang 2013, LI Jingze 李敬泽 (éd.), Nanchang, Baihuazhou wenyi chubanshe, 2013.

CHEN Zhongshi 陈忠实, [Le Pays du cerf blanc] / 白鹿原 / Bailuyuan, Renmin wenxue chubanshe, 1993.

CHEN Zhongshi 陈忠实, Au pays du cerf blanc, BAOQING Shao et CRUVEILLÉ Solange (trad.), Seuil, 2012. 
CHEN-ANDRO Chantal, « La critique chinoise moderne et la fonction métaphorisante », in Regards sur la métaphore, entre Orient et Occident, SAKAI Cécile et STRUVE Daniel (éds.), Arles, Picquier, 2008.

CHENG Anne, Histoire de la pensée chinoise, Seuil, 2002 [1997].

CHERRY Kittredge, Womansword - What Japanese words say about women, Tokyo and New York, Kōdansha International, 2002.

CHING Leo, Becoming "Japanese": Colonial Taïwan and the Politics of Identity Formation, Berkeley, California U. P., 2001.

CHo Dong-il 조동일, [Histoire générale de la littérature coréenne] / 한국문학통사 / Hanguk munhak tongsa, Séoul, Jisiksaneopsa, 5 vol., 1994.

CHO Dong-il 조동 일, BOUCHEZ Daniel, Histoire de la littérature coréenne, des origines à 1919, Fayard, 2002.

CHOE Chang-jo, [« Pour comprendre la pensée de la géomancie coréenne »] / 한국 풍수사상의 이해를위하 여 / Hanguk pungsu sasang-ui ihae-reul wihayeo, in [La Vision traditionnelle de la géographie coréenne] / 한국 의 전통지리사상 / Hangu-gui jeontong jiri sasang, Séoul, Mineumsa, 1991.

CHOI-CHABAL Eun-sook, «L'Odyssée d'une femme vendue : escales sur le chemin du salut dans Shim Chong, le chemin du Lotus de Sok-Yong Hwang (roman) ", in L'Escale portuaire entre mythes et réalités, de l'Antiquité au XXI siècle, BARZMAN John, CASTELAIN Jean-Pierre et WAUTERS Éric (éds.), Mont-Saint-Aignan, Presses universitaires de Rouen et du Havre, 2014, p. 63-81.

CHONG Key Ray, Cannibalism in China, Wakefield, N.H, Longwood Academic, 1990.

CHOW Rey, Primitive Passions: Visuality, Sexuality, and Contemporary Chinese Cinema, New York, Columbia U. P., 1995.

CHU Zigang 褚自剛, [“On the Conscience of Death in Guo Moruo’s Early Poetry”] / 論郭沫若早期詩歌中的死 亡意識 / Lun Guo Moruo zaoqi shige zhong de siwang yishi, 作家雜誌 / Zuojia zazhi, 3:2012, p. 32-33.

Cixous Hélène, « Le rire de la Méduse », L’arc, 1975, n 61, p. 39-54.

CODELUPPI Martina, “Mapping Ideology in Language: Han Dong’s ‘Zha gen' (Banished!) and Ma Jian’s ‘Rou zhi tu' (Beijing coma)", Annali Di Ca' Foscari Serie Orientale, 53:2017, p. 257-80.

COMTE-SPONVILLE André, Dictionnaire philosophique, PUF, 2001.

CORCUFF Stéphane, “The liminality of Taiwan. A Case Study”, in Geopolitics. Taiwan in Comparative Perspective, vol. 4., 2012.

Corps souffrants dans les littératures de la Chine et du Japon au XXe siècle. Extrême-Orient, Extrême-Orient, 39:2015, $\mathrm{P}$. U. de Vincennes.

COUÉGNAS Daniel, Introduction à la paralittérature, Seuil, 1992.

COUNIHAN Carole M, "Introduction - Food and Gender: Identity and Power", in Food and Gender - Identity and Power, COUNIHAND Carole M., KAPLAN Steven L. (eds.), London, Taylor \& Francis, 1998.

CREED Barbara, “Kristeva, Femininity, Abjection”, in The Horror Reader, GELDER Ken (ed.), London, Routledge, 2000 .

CUI Jianjun 催建军, CUI Yunwei 催云伟, [«Sur le sens expressionniste de la caricature dans l'œuvre de Lu Xun »] / 论鲁旬作品中的表现主义漫画感 / Lun Lu Xun zuopin zhongde biaoxian zhuyi manhuagan, 西安石油大 学学报 / Xi'an shiyou daxue xuebao 3:2005, p. 67-71.

CUI Yunwei 催云伟, [《 Le sens pictural expressionniste dans les œuvres de Lu Xun »] / 论鲁式作品中的表现 主义油画感 / Lun Lu Xun zuopin zhongde biaoxian zhuyi youhuagan, 山东示范大学学报 / Shandong shifan daxue xuebao 51.2, 2006, p. 47-51.

CUI Yunwei 催云伟, [« Le sens graphique des gravures expressionnistes dans les œuvres de Lu Xun »] / 论鲁 式作品中的表现主义版画 (木刻) 感 / Lun Lu Xun zuopin zhongde biaoxian zhuyi banhua(muke)gan ", 沈阳示 范大学学报 Shenyang shifan daxue xuebao 28.4, 2004, p. 59-63. 
DAI Jinhua 戴锦华, [Comme en un miroir] / 犹在镜中 / Youzai jingzhong, Zhishi chubanshe, 1999.

DANEREK Stefan, Tjerita and Novel. Literary Discourse in Post New Order Indonesia, Lund, Centre for Languages and Literature, Lund University, 2006.

DANTE Alighieri, La divine comédie, L'enfer/Inferno, RISSET Jacqueline (trad.), Paris, Garnier Flammarion, 2004.

DASCAL Marcelo, "Colonizing and Decolonizing Minds", in Papers of the 2007 World Philosophy Day, KUÇURADI Ioanna (ed.), Ankara, Philosophical Society of Turkey, 2009, p. 308-332.

DAVIDSON Drew, Cross-Media Communications: an Introduction to the Art of Creating Integrated Media Experiences, Pittsburgh, PA, Carnegie Mellon University, ETC Press, 2011. Préface, p. X.

DAVIES Gloria, Lu Xun's Revolution, Writing in a Time of Violence, Cambridge, Harvard U. P., 2013.

DE MAN Paul, Romanticism and Contemporary Criticism, Baltimore, The Johns Hopkins U. P., 1996.

DELEUZE Gilles, Francis Bacon. Logique de la sensation, Seuil, 2002.

DELEUZE Gilles, GUATTARI Félix, Kafka: Toward a Minor Literature, POLAN Dana (trans.), Minneapolis, Minnesota U. P., 1986.

DENG Li 邓莉, [“Understanding and Rewriting Memory: on Han Dong's Novel Banished!'] / 记忆的理解与重构 一读韩东的长篇小说《扎根》/ Jiyi de lijie yu chonggou-du Han Dong de changpian xiaoshuo 'Zha gen', 当代文 坛 Dangdai wentan, 2:2005, p. 62-63.

DENTON Kirk A, “Lu Ling's Literary Art: Myth and Symbol in 'Hungry Guo Su'e”, Modern Chinese Literature 2, 2:1986, p. 197-209.

DIKÖTTER, Frank, The Cultural Revolution: A People’s History, 1962-1976, Londres, Bloomsbury, 2016.

DING Laixian 丁来先, [《La littérature féminine et autres »] / 女性文学与其他 / Nüxing wenxue yu qita, 中华读 书报 / Zhonghua dushu bao, 20/12/1995.

DING Ling 丁玲, [Les Chefs-d'œuvre de la fiction moderne chinoise (๕euvres de DING Ling)] / 中国现代小说精 品 (丁玲卷) / Zhongguo xiandaixiaoshuo jingpin (Dingling juan), Xi'an, Jiangxi renmin chubanshe, 1995.

Đỗ Hoàng Diệu, [Cauchemars] / Bóng đè, Đà Nẵng, Nhà xuất bản Đà Nẵng, 2005.

DOAN Cam Thi, «Quand elles écrivent leur corps », in La Colonisation des corps. De l'Indochine au Vietnam, GUILLEMOT François et LARCHER-Goscha Agathe (dirs.), Éd. Vendémiaire, 2014, p. 246-263.

DOGANIS Basile, Pensées du corps. La philosophie à l'épreuve des arts gestuels japonais (danse, théâtre, arts martiaux), Les Belles Lettres, 2013.

DOWER John W., Embracing Defeat: Japan in the Wake of World War II, New York, Norton, 1999.

DRISColl Mark, Absolute Erotic, Absolute Grotesque - The Living, Dead and Undead in Japan's Imperialism, 1895-1945, Durham, Duke U. P., 2010.

DURAND Gilbert, Les structures anthropologiques de l'imaginaire, Dunod, 1992 [1969].

DURAND-DASTÈs Vincent, LAUREILLARD Marie (éds.), Fantômes dans l'Extrême-Orient d'hier et d'aujourd'hui, Presses de l'INALCO, 2017, 2 vol., DOI : https://doi.org/10.4000/books.pressesinalco.1274.

EIZAWA Kōji 栄沢幸二, [La Pensée de la «Sphère de coprospérité » de la Grande Asie Orientale] / 「大東业共 栄圏」の思想 / «Daitōakyōeiken » no shisō, Kōdansha, coll. « Kōdansha Gendai shisō », 1995.

EL SHIRAZY Habiburrahman, [Les Versets de l'amour] / Ayat-Ayat Cinta, Jakarta, Penerbit Republika, 2004.

ELLSTRÖM Lars, "Guo Moruo, The Goddesses" / 女神 / Nüshen 1921, in A Selective Guide to Chinese Literature 1900-1949, vol. III: The Poem, HAFT Lloyd (ed.), Leiden-New York-København-Köln, Brill, 1989, p. 108-114.

ESKILDSEN Robert, "Of Civilization and Savages: The Mimetic Imperialism of Japan's 1874 Expedition to Taïwan”, The American Historical Review, vol. 107, nº 2, 2002, p. 388-418.

FENG JINTANG 冯金堂, [Les Eaux jaunes] / 黄水传 / Huangshui zhuan, Henan renmin chubanshe, 1961. 
FENWICK Chris, “Irreducible Significance: David Wellbery on Literature” The Point, 06/11/2017, https:// thepointmag.com/dialogue/irreducible-significance-david-wellbery-literature-goethe-cavell/, consulted on $20 / 07 / 2021$.

FERRO Marc, Cinéma et Histoire, Gallimard, 1993 [1977].

FOREST Philippe, Ōe Kenzaburō - Légendes d'un romancier japonais, Nantes, Pleins Feux, 2001.

FouCAult Michel, Le Corps utopique, suivi de Les Hétérotopies, Nouvelles Éditions Lignes, 2009.

FOULCHER Keith and DAY Tony (eds.), Clearing a space. Postcolonial readings of modern Indonesian literature, Leiden, KITLV Press, 2002, p. 109-143.

FREUD Sigmund, Introduction à la psychanalyse, JANKÉLÉVITCH Samuel (trad.), Payot, 1998 [1917].

FREUD Sigmund, Pour introduire le narcissisme, MANNONI Olivier (trad.), Payot, 2012 [1914].

FU XUANCONG 傅拻檫 (éd.), [Poèmes complets des Song] / 全束詩 / Quan song shi, Beijing daxue chubanshe, 1998.

FUCHS Thomas, "The Phenomenology of Body Memory", in Body Memory, Metaphor and Movement, косн Sabine C., FUCHS Thomas, sUMma Michela and MÜLLER Cornelia (eds.), Amsterdam, Philadelphie, 2012, p. 9-22.

FUJII Shōzō 藤井省三, [Le Paysage du “pays natal”] / 魯迅「故郷」の風景 / Rojin : «Kokyō » no fūkei, Heibonsha, 1986.

FUJITA Rina 藤田梨那, TENGTIAN Lina 藤田梨那, [“Medicine, Literature, Body: The Case of Guo Moruo”] / 醫 學・文學・身體一以郭沫若為例 / Yixue - wenxue - shenti - Yi Guo Moruo weili, 中國現代文學研究論叢 / Zhongguo xiandai wenxue yanjiu luncong, 10(1):2015, p. 1-7.

FUJITA Rina 藤田梨那, [“On Guo Moruo’s “The Heavenly Hound””] / 郭沫若の「天狗」論 / Kakumatsujaku no 'Tengu' ron, 国士館大学文学部人文学会紀要 / Kokushikan daigaku bungaku-bu jinbun gakkai kiyō, 36:2003, p. 129-141.

FUSE Hideto 布施英利, [“The Body Sense of ZOO”] / 『ZOO』の身体感覚 / ZOO no shintai kankaku, WAVE, vol. 29, 1991.

GAFFRIC Gwennaël (éd.), « La science-fiction en Asie de l’Est », ReS Futurae, 9:2017, https://doi.org/10.40000/ resf.759.

GÁLIK Marián, “Kuo Mo-jo's The Goddesses: Creative Confrontation with Tagore, Whitman and Goethe”, in Milestones in Sino-Western Literary Confrontation (1898-1979), GÁLIK Marián (ed.), Wiesbaden, Otto Harrassowitz, 1986, p. 43-72.

GANG Yue, The Mouth that Begs: Hunger, Cannibalism, and the Politics of Eating in Modern China, Durham, Duke U. P., 1999.

GAO Hanning 高寒凝, [ "La web-littérature “du lectorat féminin" et la " génération des filles uniques grandies dans le web-environnement »] / 女性向”网络文学与“网络独生女一代 / Nüxingxiang wangluo yu wangluo dushengnü yidai, in [Recherches sur la littérature chinoise moderne] / 中国现代文学研究丛刊 / Zhongguo xiandai wenxue congkan, 8:2016.

GE Hongbing 葛红兵, [《 La littérature individuelle et les écrivains du corps »] / 个体性文学与身体型作家 / Geti xing wenxue yu shenti xing zuojia, [Fleur de montagne] / 山花 / Shanhua, 1997:03, p. 75-78.

GENETTE Gérard, Figures I, II, III, Seuil, 1966, 1969, 1972.

GENETTE Gérard, Narrative Discourse. An Essay in Method, LEWIN Jane E. (trans.), Ithaca, Cornell U. P., 1980. GENETTE Gérard, Nouveau Discours du récit, Seuil, 1983.

GERTH Hans and MILLS C. Wright, "Introduction", in From Max Weber. Essays in Sociology, GERTH Hans and MILLS C. Wright (eds.), Oxford, Oxford U. P., 1958, p. 51.

GEZILI DE YEWAN 格子里的夜晚, [Une vie numérique] / 数字生命 / Shuzi shengming, 起点 / Qidian, http:// book.qidian.com/info/30017, consulté le 25/04/2018. 
GIBRAN Khalil, Les Ailes brisées, colIN Joël (trad.), Babel, 2001.

GLADZIEJEWSKI Paweó, “Shared Representations, Perceptual Symbols, and the Vehicles of Mental Concepts”, Journal of Consciousness Studies 20, 3-4, 2013, p. 102-24.

GODDARD Jean-Christophe et LABRUNE Monique (dir.), Le corps, Vrin, 1992.

GODLEY Michael R., "The end of the queue: hair as symbol in Chinese history”, East Asian History, 8:1994,

p. 53-72.

GOGOL Nicolas, Nouvelles de Pétersbourg / [ПЕТЕРБУРГСКИЕ ПОВЕСТИ (1835-1843)] AUCOUTURIER Gustave, LUNEAU Sylvie et MONGAUlT Henri (trad.), Paris, Gallimard, 1979.

GoldBlatT Howard, Xiao Hong, Boston, Twayne Publishers, 1976.

GONG Ailing 宫爱玲, [““Unbounded Happiness’ at the Time of Distress: On Han Dong’s Banished!”] / 苦难年代 里的”其乐融融”一评韩东《扎根》/ Kunan niandai li de 'qile rongrong'- ping Han Dong 'Zha gen', 长春工业大 学 / Changchun gongye daxue 18, 1:2006, p. 86-88.

GRANT Colin, Negro with a Hat: The Rise and Fall of Marcus Garvey and His Dream of Mother Africa, Oxford, Oxford U. P., 2008.

GRIFFIN Christine, SZMIGIN Isabelle, BENGRY-HOWELL Andrew, HACKLEY Chris, MISTRAL Willm, "Inhabiting the contradictions: Hypersexual femininity and the culture of intoxication among young women in the UK", Feminism \& Psychology, 2012:2, p. 184-206.

GU Dayong 古大勇, [« Lancer des cris de folle souffrance : sous le règne implicite de l'expressionnisme comparaison entre le peintre Munch et l'écrivain Lu Xun»] / 狂痛呐喊 : 在表现主义的潜法则下一画家蒙 克与作家鲁式比较论 / Kuangtong nahan : zai biaoxian zhuyide qianzexia Huajia Mengke yu zuojia Lu Xun bijiaolun, 伊梨示范学院学报 / Yili shifan xueyuan xuebao 2:2004, p. 64-70.

GU Dayong 古大勇, [《Les “appels” de Lu Xun et le “cri” de Munch »] / 鲁式的呐喊与蒙克的呼嚎 / Lu Xunde Nahan yu Mengkede Huhao », 兰州大学学报 / Lanzhou daxue xuebao 29.5, 2001, p. 11-15.

GU Haiyang 古海阳, [“Reconstructing Individual Historical Experience: On Han Dong's Banished! and 'A Small-Town Hero Strides Forth"'] / 个体历史经验的重构一韩东《扎根》、《小城好汉之英特迈往》/Geti lishi jingyan de chonggou-Han Dong 'Zha gen,' 'Xiaocheng haohan zhi yingte maiwang' helun, Xinyu xueyuan xuebao 5, 6:2010, p. 28-30.

GUILLAUD Lauric, « Les oubliés du temps », in Les mondes perdus, GoIMARD Jacques (éd.), Presses de la Cité, 1993, p. III-XXIII.

GULL William Whitney, “Anorexia Nervosa (Apepsia Hysterica, Anorexia Hysterica)”, Clinical Society’s Transactions, vol. 7, 22:1873.

GUILLEMOT François et LARCHER-GOSCHA Agathe, « Le Vietnam à fleur de peau », in La Colonisation des corps. De l'Indochine au Vietnam, GUILLEMOT François et LARCHER-GOSCHA Agathe (éds.), Éd. Vendémiaire, coll. «Chroniques », 2014, p. 13.

GUo Moruo 郭沫若, [Complete Works of Guo Moruo] / 郭沫若全集 / Guo Moruo quanji, 人民文學出版社 Renmin wenxue chubanshe, vol. 1, 1982.

GUO Moruo 郭沫若, The Goddesses, LESTER Jong and BARNES A. C. (trans.), Foreign Languages Press, 2001.

GUo Zhantao 郭战涛, [“ Le taoïsme et le Zi Zhi Tong Jian (Miroir général pour aider au gouvernement) -

réinterpréter l'image du cannibalisme dans “Le journal d'un fou” de Lu Xun »] / 道教与《资治通鉴》-《狂 人日记》吃人意象再解读 / Daojiao yu Zizhi Tongjian - “Kuangren riji” chiren yixiang zai jiedu, [Mensuel des études sur Lu Xun] / 鲁迅研究月刊 / Lu Xun yanjiu yuekan, 2:2015, p. 43-47.

Gutai shiryōshū: Dokyumento Gutai 1954-1972 / 具体資料集ドキュメント具体1954-1972, Ashiya: Ashiya shi bunka shinkō zaidan, 1993, n.p.

HAMON Philippe, Introduction à l'analyse du descriptif, Hachette, 1981. 
HAMON Philippe, Le Descriptif, Hachette supérieur, 1993.

HAN Dong 韩东, Banished! / 扎根 / Zha Gen, Guangzhou, Huacheng chubanshe, 2010.

HAN Dong 韩东, Banished! A Novel, HARMAN Nicky (trans.), Hawai'i U. P., 2009.

HAN Kang et al., [2005, 29e prix Yi Sang] / 2005년도 제29회 이상문학상 작품집 / Ich'ônonyôndo cheishipkuhoe isangmunhaksang chakp'umjip, Séoul, 문학사상사 Munhaksasangsa, 2005.

HAN Kang, « Interview sur Channel Yes », 10/06/2014, http://ch.yes24.com/Article/View/25422, consulté le $21 / 02 / 2018$.

HAN Kang, 채식주의 자 / Ch'aeshikchuûija, P’aju, Ch'angbi, 2007 ; La Végétarienne, JEONG Eun Jin 정 은진 et BAтіLLIOT Jacques (trad.), Paris, Serpent à plumes, 2015 ; The Vegetarian, sмiтн Deborah (trad.), London, Portobello Books, 2015.

HAN Kang, 희 랍어 시간 / Hirabô shigan, P’aju, 문학동네 / Munhakdongne, 2011 ; Leçons de grec, JEONG Eun Jin et Jacques BATILLIOT (trad.), Serpent à plumes, 2017.

HAN Kang, 소년 이 온다 / Sonyôni onda, P'aju, Ch'angbi, 2014 ; Celui qui revient, JEONG Eun Jin et BATILLIOT Jacques (trad.), Paris, Serpent à plumes, 2016 ; Human Acts, sмiтH Deborah (trad.), London, Portobello Books, 2016.

HAN Kang, [Le Fruit de ma femme] / 내 여자의 열 매 / Nae yôjaûi yôlmae, 파주 P'aju, 창비 Ch'angbi, 2000.

HAN Kang, KANG Su Mi 강숨]ᅵ, sHIN Hyông Ch'ôl 신형 철, [« Les strates esthétiques des romans de Han Kang »] / 한강 소설의 미학적 층위 / Han'gang sosôrûi mihakchôk ch'ûngwi, [Village littéraire] / 문학동네 / Munhakdongne, 88:2016, p. 14-61.

HAN Song 韩松, [“The Guide to the Hunting for Women”] / 美女狩猎指南 / Meinü shoulie zhinan, in [The Tomb of Universe] / 宇宙墓碑 / Yuzhou mubei, Shanghai, Shanghai renmin chubanshe, 2014, p. 275-373. HANAN Patrick, «The Technique of Lu Hsün's Fiction », Harvard Journal of Asian Studies 34:1974, p. 53-96. HANSON Clare, “The Maternal Body”, in The Cambridge Companion to The Body in Literature, HILLMAN David and MAUDE Ulrika (eds.), New York, Cambridge U. P., 2015, p. 87-100.

HAROOTUNIAN Harry, “Japan's Postwar and After, 1945-1989: An Overview”, in From Postwar to Postmodern: Art in Japan 1945-1989, CHONG Doryun et al. (eds.), New York, The Museum of Modern Art, 2012, p. 17-21.

HATTORI Sachiko 服部 祥子 (dir.), [Disabled Children and Sex: A Real Image of Puberty] / 障害児と性一思春 期の実像 / Shōgaiji to sei: shishunki no jitsuzō, Nihon Bunkakagakusha, 1989.

HAY John, “The Body Invisible in Chinese Art?", in Body, Subject, and Power in China, BARLow Tani and zITO Angela (eds.) Chicago, University of Chicago Press, 1994, p. 42-77.

HE Xiangyang 何向阳 (éd.), [Anthologie de 60 ans de critique littéraire] / 60 年文学评论精选 / 60 nian wenxue pinglun jingxuan, Wuhan, Changjiang wenyi chubanshe, 2009.

HEINRICH Ari Larissa, The Afterlife of Images: Translating the Pathological Body between China and the West, Durham, Duke U. P., 2008.

HENNINGER Aline, La socialisation de genre à l'école élémentaire dans le Japon contemporain / [Gender Socialization in Primary School in Contemporary Japan], PhD dissertation, INALCO, 2016.

HILLMANN David and ULRIKA Maude (eds.), The Cambridge Companion to the Body in Literature, New York, Cambridge U. P., 2015.

HIROSE Kōjirō 広瀬浩二郎, [A Religious Anthropology of Disabled People] / Shōgaisha no shūkyō minzokugaku, Akashi Library, 1997.

[Histoire des mines au Japon] /日本の炭鉱の歴史 / Nihon no tankō no rekishi, jcoal.or.jp/worldheritage/ 03/05/, consulté le 01/06/2021.

HIRSCH Marianne, The Mother/Daughter Plot: Narrative, Psychoanalysis, Feminism, New Haven, Indiana U. P., 1989. 
HоскX Michel, Questions of Style, Literary Societies and Literary Journals in Modern China, 1911-1937, Leiden, Brill, 2003.

HOMÈRE, L'Odyssée, JAcCoTTET Philippe (trad.), La Découverte, 2004.

HON Lai-Chu (Han Lizhu 韩丽珠), [Body Sewing] / 缝身 / Fengshen, Taibei, Lianhe wenxue chubanshe, 2010.

HON Lai-Chu, [The Kite Family] / 凤笔家族 / Fengzheng jiazu, Taibei, Lianhe wenxue chubanshe, 2008.

HON Lai-Chu, The Kite Family, LINGENFELTER Andrea (trans.), Hong Kong, Hong Kong U. P., 2015

HONG Guo-juin, Taiwan Cinema: A Contested Nation on Screen, New York, Palgrave Macmillan, 2011.

HONG Jianlun 洪健倫, [“Locality, searching for Know-how, making ghost movies with Taiwan characteristics: interview with The Tag-Along director Cheng Wei-hao”] / 接地氣、找Know-how，打造鬼片的「台灣特有

種」專訪《紅衣小女孩》導演程偉豪 / Jiediqi, zhao Know-how, dazao guipian de 'Taiwan teyouzhong' zhuanfang “Hongyi xiaonühai” daoyan Cheng Wei-hao, 放映周刊 / Fangying zhoukan, 534:2015, http:// www.funscreen.com.tw/headline.asp?H_No=592, consulted on 14/06/2021.

HONG Ying 虹影, Une fille de la faim, LOUISGRAND Nathalie (trad.), Seuil, 2000.

HONG Ying 虹影, [Une fille de la faim] / 饥饿的女儿/Ji'e de nüer, Anhui renmin chubanshe, 2013.

HSIA Chih-tsing, A history of modern Chinese fiction, Bloomington, Indiana U. P., 1999.

HSIA T. A., The Gate of Darkness, Studies in the Leftist Literary Movement in China, Seattle, Washington U. P., 1968.

HSU Kai-yu (ed.), Twentieth Century Chinese Poetry: An Anthology, New York, Anchor Books, 1963.

HUANG Wenxiong 黃文雄, [Histoire du cannibalisme en Chine] / 中國食人史 / Zhongguo shiren shi, Taipei, Qianwei chubanshe, 2005.

HUANG Yanfang 黄衍方, [The Tag-Along 2 behind the scene: scriptwriter Jian Shigeng discuss the transforming nature of cultural symbols] / “談《紅衣小女孩 $2 》$ 幕後 編劇簡士耕 : 文化符號是會一直轉 變的” / Tan "Hongyi xiaonühai 2" muhou bianju Jian Shigeng: wenhua fuhao shi hui yizhi zhuanbiande, upmedia.mg/news_info.php?SerialNo=25372, consulted on 22/02/2018.

HUNT Pamela, “The Significance of Scatological Humour: A Case Study of Zhu Wen's 'What Is Garbage, What Is Love' and Han Dong's Banished!", 2010, https://media.paper-republic.org/files/10/06/

P_Hunt_essay_on_scat.pdf, consulted on 28/02/2018.

HUNTER Thomas, « Indo as other. Identity, anxiety and ambiguity in 'Salah Asoehan' », in Clearing a space. Postcolonial readings of modern Indonesian literature, FOULCHER Keith and DAY Tony (eds.), Leiden, KITLV Press, 2002, p. 109-143.

HWANG Ik-koo 黄 益九, [“The Occupation Meets the Flesh Secretly: Postwar Japan in Nikutai no Mon”] / 占領 と肉体の密会『肉体の門』が物語る戦後/ Senryō to nikutai no mikkai: Nikutai no mon ga monogataru sengo, 文学研究論集 / Bungaku kenkyū ronshū, 24:2006, p. 71-88.

HWANG Sok-yong 황석 영, [Shim Chong, le chemin du Lotus] / 심청, 연 꽃의 길 / Sim Cheong, Yeonkkotui gil, Séoul, Munhakdongne, 2007 [2003].

HWANG Sok-yong 황석 영, [ « Le Romancier Hwang Sok-yong : “Je suis un jeune auteur” ”, entretien de Hwang Sok-yong avec intervieweur anonyme »] / 나는 청 년작가이다, 소설가 황석 영 / Naneun cheongnyeon jakgaida, soseolga Hwang Seokyeong, 채 널 예스 / Channel Yes [en ligne], 1:2003, http://ch.yes24.com/Article/ View/12831, consulté le 01/08/2018.

ISHIKAWA Chūichi 石川忠一, [Précis de police de Taïwan] / 臺灣警察要論 / Taïwan keisatsu yōron, Taipei, Shinkōdō Shoten, 1915.

ICHIKAWA Hiroshi 市川浩, 身の構造 / Mi no kōzō, Kōdansha, 1993 [1975].

IGARASHI Yoshikuni, Bodies of Memory: Narratives of War in Postwar Japanese Culture, 1945-1970, Princeton, Princeton U. P., 2000. 
IM Gyeong-bin, [« Un arbre qui rêve du village vert »] / 푸른 마을을 꿈꾸는 나무 / Pureun maeu-reul kkumkku-neun namu, Jungang-ilbo, Séoul, 11/08/1998.

INGOLD Tim, Marcher avec les dragons, MADELIN Pierre (trad.), Bruxelles, Zones sensibles, 2013.

ISHIBASHI Masataka 石橋正孝, [“A Consideration of the Possibilities and Limits of the Body's Representation in Literature”] / 文学における身体表象の可能性と限界についての考察 / Bungaku ni okeru shintai.hyōshō no kanōsei to genkai ni tsuite no kōsatsu, in [Collection of articles from Teikokudaigaku's education center] / 帝 国大学総合教育センタ一論集 / Teikyō Daigaku sōgō kyōiku sentā ronshū, vol. 1, 2010, p. 71-85.

ISHIZUKI Mayuko 石月 麻由子, [“Sakaguchi Ango’s ‘Idiot’ Analyzed through Corporeal Expressions: From the Standpoint of Interconnection between the Flesh and Mind"] / 身体表現から考察する坂口安吾『白痴』肉 体と精神の<連絡>と言う視座に立って / Shintai hyōgen kara kōsatsu suru Sakaguchi Ango, 'Hakuchi': Nikutai to seishin no <renraku> toiu shiza ni tatte, 国文学研究 / Kokubungaku kenkyū 139:2003, p. 121-32.

Iтō Hiromi 伊藤比呂美, [Hand, Leg, Meat, Arm: Hiromi 1955] / 手 · 足 ·肉 ·体 Hiromi 1955 / Te, Ashi, Niku, Karada: Hiromi 1955, Chikumashobō, 1995.

Iтō Hiromi 伊藤比吕美, [On Territory ] / テリトリー論 / Territorii.ron, Shinchōsha, 1985.

Iтō Hiromi 伊藤比呂美, [The Sky of Plants] / 草木の空 / Sōmoku no sora, Daiichishishyūatoriesyuppankikaku, 1978.

Іто̄ Hiromi 伊藤比吕美, [Underripe Plums] / 青梅 / Ao.ume, Shinchōsha, 1982.

ITŌ Hiromi 伊藤比吕美, UENo Chizuko 上野千鶴子, [Noro and Saniwa] / のろとさにわ / Noro to Saniwa, Heibonsha, 1991.

Iтō Hiromi 伊藤比吕美, SAITō Manabu 斎藤学, [Cheerfully Bulimic] / 明るく拒食、元気に拒食 / Akaruku kyoshoku, genki ni kashoku, Heibonsha, 1992.

JAY Martin, Downcast Eyes. The Denigration of Vision in Twentieth-Century French Thought, Berkeley, California U. P., 1993.

JIANG Tao 姜濤, [“Imagining Character in the Dissecting Room - A Comprehensive Investigation of the Image of the Poet in Early Guo Moruo”] / 解剖室中的人格想象一對郭沫若早期詩人形象的擴展性考察 / Jiepoushi zhong de renge xiangxiang - Dui Guo Moruo zaoqi shiren xingxiang de kuozhanxing kaocha, in [Institute of New Poetry of Peking University] / 北京大學新詩研究所 / Beijing daxue xinshi yanjiusuo, 首都師範大學詩歌研 究中心 / Shoudu shifan daxue shige yanjiu zhongxin (ed.), [Proceedings of the Scholarly Conference on New Poetry and Romanticism] / 新詩與浪漫主義學術研討會論文集 / Xinshi yu langmanzhuyi xueshu yantaohui lunwenji, Beijing, 中國詩歌研究中心 / Zhongguo shige yanjiu zhongxin, 2011, p. 116-133.

JIN Siyan, L'écriture féminine chinoise $\mathrm{du} \mathrm{XX}^{\mathrm{e}}$ siècle à nos jours. Trame des souvenirs et de l'imaginaire, You Feng, 2008.

Jo Hoe-gyeong, [Étude sur le roman de Kim Dong-ri] / 김동리 소설 연구 / Kim Dong-ri soseol yeongu, Séoul, Gukhak jaryowon, 1999.

JONAS Hans, La religion gnostique, Flammarion, 1978.

JONES Ann Rosalind, « Writing the Body: Toward an Understanding of "L'Écriture Féminine" », Feminist Studies, vol. 7, 2:1981, p. 247-263.

JORTAY Coraline, Pronominal Politics: (Un)Gendering Narrative and Framing Ambiguity in Chinese Literature, 1917-1937, thèse, ULB, 2020.

JUHUA Sanli 杼花散里, [La Générale en haut et moi en bas] / 将军在上我在下 / Jiangjun zai shang, wo zai xia, Taipei, Sancai wenhua shiye chuban youxian gongsi, 2013.

JUHUA Sanli 杼花散里, CHANGYU 尝谕, [Je suis vraiment une super star] / 我真是大明星 / Wo zhen shida mingxing, https://www.piaotian.com/bookinfo/6/6180.html, consulté le 03/02/2021.

JULLIEN François, De l'essence ou du nu, Seuil, 2000.

JULLIEN François, Ecriture et révolution, Presses de l'ENS, 1979. 
KAKUTA Reizō 角田礼三, [Realities of Sex Education for Disabled Children] / 障害のある子どもへの性教育 の実際 / Shōgai no aru kodomo e no seikyōiku no jissai, Meiji Tosho, 1995, p. 61-63.

KALDIS Nicholas, The Chinese Prose Poem. A Study of Lu Xun's Wild Grass (Yecao), Amherst, Cambria Press, 2014. KALE Sirin, "Why Eating Disorders Are on the Rise in Japan”, Broadly, broadly.vice.com/en_us/article/ 4xkjm3/why-eating-disorders-are-on-the-rise-in-japan, 2016, consulted on 01/06/2021.

KANDINSKY Vassily, Du Spirituel dans l'art, et dans la peinture en particulier, Folio / Denoël, 1994 [1910].

KANEHARA Hitomi, AMEBIC / Amïbikku, Shūeisha, 2005.

KANG Xiaofei, The Cult of the Fox: Power, Gender, and Popular Religion in Late Imperial and Modern China, New York, Columbia U. P., 2005.

KANT Emmanuel, Critique de la faculté de juger, PHILONENKo Alexis (trad.), Vrin, 1965.

KAPROW Allan, Assemblage, Environment, and Happenings, New York, Abrams, 1966.

KAPROW Allan, Essays on the Blurring of Art and Life, KELLEY Jeff (ed.), Berkeley and Los Angeles, California U. P., 2003.

KATŌ Madoka 加藤まどか, [“Questioning the expansion of the family factors theory related to anorexia and bulimia"] / 家族要因説の広がりを問う-拒食症・過食症を手がかりとして / Kazoku.yōin.setsu no hirogari wo tou - kyoshokushō $\cdot$ kashokushōo tegakari to shite, in [Analysis of Contemporary Society: System/Body/ Narrative] / 分析 - 現代社会一制度／身体／物語 / Bunseki Gendai Shakai Seido/Shintai/Monogatari, ŌTA Shôichi 太田省一 (ed.), Yachiyo Shuppan, 1997.

КАШАмото Saburō, [《Vers les tours thérapeutiques : un folklore de la terreur »] / 『治療塔へ』一恐怖の フォークロア」, [Littérature nationale] / 国文学, juillet 1990, p. 12.

KAWAMURA Kunimitsu 川村邦光, [The Maiden's Modern Body and Sexuality] / 乙女の身体・女の近代のセク シュアリテイー / Otome no shintai: onna no kindai to sekushuariti, Kinokuniya Shoten, 1994.

KAWANA Sari, Murder Most Modern - Detective Fiction and Japanese Culture, Minneapolis, London, Minnesota U. P., 2008.

KAYAMA Rika 香山リカ, [Evening Story for Manga 2] / 漫画夜話 2 / [Manga Yawa 2], Kinemajunpōsha, 1998. KELTNER Stacey, Kristeva, New York, Miley, 2013.

KIM Dong-ri, [«La Vallée du lœess »] / 황토기 / Hwangtogi, in [๕uvres de Kim Dong-ri] / 김동리 전 집 / Kim Dong-ri jeonjip, Séoul, Mineumsa, t. 1, 1995.

KIM Hyesoon 김혜순, Autobiographie de la mort, Koo Moduk et MURCIA Claude (trad.), Circé, 2018.

KIM Hyesoon 김혜순, Dentifricetristesse crèmemiroir, KOO Moduk et MURCIA Claude (trad.), Circé, 2016.

KIM Hyesoon 김혜순, [Histoire du roman coréen contemporain] / 한국현대소설사 / Hanguk hyeondae soseolsa, Séoul, Hongseongsa, 1979.

KIM Ye Rim 김예림, [ «À propos de la souffrance ou de la beauté du “devenir-végétal” »] / 식물-되 기’의 고통 혹은 아름다움에 관하여 / Shingmul-toegiûi kot'ong hokûn arûmdaume kwanhayô, [Création et critique], 36 (1): 2008, p. 349-352.

KIMURA Atsushi, WADA Yuji, GoTo Sho-Ichi, TSUZUKI Daisuke, CAI Dongsheng, оKA Takashi, IPPEITA Dan, "Implicit Gender-based Food Stereotypes. Semantic Priming Experiments on Young Japanese", Appetite 52:2009, p. 521-24.

KINKLEY Jeffrey C., Visions of Dystopia in China's New Historical Novel, New York, Columbia U. P., 2015.

KITO Aya 木藤垔也, [One Liter of Tears. A Young Girl's Fight for Life (Aya’s Diary)] / 1リットルの涙 / Ichi rittoru no namida, FA Shuppan, 1986. 
KLEINMAN Arthur and Joan, "How Bodies Remember: Social Memory and Bodily Experience of Criticism, Resistance, and Delegitimation Following China's Cultural Revolution”, New Literary History 25, 3:1994, p. 707-723.

коBAYASHI Fukuko 小林富久子, “New Waves in Japanese Women’s Writing during the1990s: Resident Korean Women Writers' Fiction and the Fiction of 'Eating Disorders”, in 文化論集 / Bunka ronshū, 2002:1, p. 1-13.

косн Sabine C., FUCHS Thomas, sUmma Michela, MÜLLER Cornelia (ed.), “Introduction”, in Body Memory, Metaphor and Movement, косн S. C. et al. (eds.), Amsterdam, John Benjamins, 2012, p. 1-6.

KOIKARI Mire, “Gender, Power, and U.S. Imperialism: The Occupation of Japan, 1945-1952", in Bodies in Contact: Rethinking Colonial Encounters in World History, BALLANTYNE Tony and Antoinette BURTON, Durham, Duke U. P., 2005, p. 342-62.

KOMMER H., « Tjerita Nji Paina », in [Le Bon-vieux temps. Anthologie de littérature pré-indonésienne] / Tempo Doeloe. Antologi Sastra Pra-Indonesia, TOER Pramoedya Ananta (éd.), Jakarta, Lentera Dipantara, 2003,

p. 375-391.

KRISTEVA Julia, Powers of Horror: An Essay on Abjection, RoudiEZ Leon S. (trans.), New York, Columbia U. P., 1982. KURNIAWAN Eka, «Graffiti in the Toilet », ANDERSON Benedict (trad.), Indonesia 86, 2008, p. 55-61.

KURNIAWAN Eka, Beauty is a Wound, TUCKER Annie (trad.), London, Pushkin Press, 2015.

KURNIAWAN Eka, Cantik Itu Luka, Jakarta, Gramedia, 2002.

KURNIAWAN Eka, Cash, NAVEAU Étienne (trad.), Sabine Wespieser, 2019.

KURNIAWAN Eka, L’homme-tigre, NAVEAU Étienne (trad.), Sabine Wespieser, Gallimard, 2017 [2015].

KURNIAWAN Eka, Les Belles de Halimunda, NAVEAU Étienne (trad.), Sabine Wespieser, 2017.

KURNIAWAN Eka, O, Jakarta, Gramedia, 2016.

KURNIAWAN Eka, [Pramoedya Ananta Toer et la littérature réaliste indonésienne] / Pramoedya Ananta Toer dan Sastra Realisme Indonesia, Yogyakarta, Jendela, 1999.

KURNIAWAN Eka, Seperti Dendam, Rindu harus Dibayar Tuntas, Jakarta, Gramedia, 2014.

KUWABARA Jitsuzō 桑原䧞蔵, [《Les coutumes cannibales en Chine»] / 支那人間に於ける食人肉の風習 / Shina jinkan ni okeru shoku jinniku no fūshū », in [Æuvres complètes de Kuwabara Jitsuzô] / 桑原鵱蔵全集 / Kuwabara Jitsuzō zensh̄̄, Iwanami shoten, vol. 2, 1968, p. 153-205.

La Bible de Jérusalem, École biblique de Jérusalem (trad.), Desclée de Brouwer, 2000.

LAIRESSE Gérard de, [Great Book of Painting] / Het groot schilderboeck, Amsterdam, Henri Desbordes, 1707, 2nd printing, in Dutch, 1712.

LAPLANTINE François et NOUSS Alexis, Métissages de Arcimboldo à Zombi, Pauvert, 2001.

LASCH Christopher, La culture du narcissisme, Flammarion, 2006.

LASÈGUE Charles, « De l’anorexie hystérique », Archives Générales de Médecine, 1873, p. 385-403.

LATOUR Bruno, Où atterrir, Comment s'orienter en politique, La Découverte, 2017.

Lawson's official website: 売上高/店舗数 | ローソン公式サイト (lawson.co.jp).

LE BRETON David, Anthropologie de la douleur, Métailié, 1995.

LE FUSTEC Claude, « Le réalisme magique : vers un nouvel imaginaire de l'autre ? Regard sur la littérature africaine américaine ", Amerika, 2:2010, journals.openedition.org/amerika/1164.

LEAVELL James Berry, The Development of the Modern Japanese Police System: Transition from Tokugawa to Meiji, London, University Microfilms International, 1979.

LEE Chulwoo, "Modernity, Legality, and Power in Korea under Japanese Rule", in Colonial Modernity in Korea, SHIN Gi-Wook and ROBINSON Michael (eds.), Cambridge, Harvard University Asia Center, 2000, p. 21-51. 
LEE Jong-moon, [Étude du chamanisme dans l'œuvre romanesque de Hwang Sok-yong] / 이종문, 황석 영 소 설의 샤머니즘 관련성 연구 / Hwang Seokyeong soseolui syameonijeum gwallyeonseong yeongu, Thèse de doctorat, Université de Dongguk, 2015, p. 90-110.

LEE Ju-Ling, "Clothing the Body, Dressing the Identity: The Case of Japanese in Taïwan during the Colonial Period", Journal of Japanese Studies, vol. 43, n 1, 2017, p. 31-64.

LEE Leo Ou-fan, "Kuo Mo-jo", in The Romantic Generation of Modern Chinese Writers, LEE Leo Ou-fan (ed.), Cambridge, MA, Harvard U. P., 1973, p. 177-200.

LEJEUNE Philippe, « Auto-genèse. L'étude génétique des textes autobiographiques » / [Self-genesis. The Genetic Study of Autobiographical Texts]", Genesis, 1:1992, p. 73-87.

LEJEUNE Philippe, Le pacte autobiographique, Seuil, 1975.

LENSON David, The Birth of Tragedy. A Commentary, Boston, Twayne's Masterwork Studies, 1987.

LEPLÂTRE Florine, « Usages du futurisme médical en Chine pré-républicaine : craniotomie et régénération dans deux récits de science-fiction (1904-1905) ", ReS Futurae, 9:2017, http://journals.openedition.org/resf/ 998, consulté le 28/09/2020.

LEVINAS Emmanuel, Éthique et Infini. Dialogue avec Philippe Nemo, Livre de poche, 1992 [1982].

LEW René et SAUVAGNAT François (dir.), La voix, Lysimaque, 1989.

LI Rong 李荣, [The Body Explanation of Modern Chinese Literature] / 中国现代文学的身体阐释 / Zhongguo xiandai wenxue de shenti chanshi, Zhongguo Shehui kexue chubanshe, 2009.

LI Ruei-Teng, Cent trésors de la littérature taïwanaise, Tainan, Guoli Taiwan wenxueguan, 2012.

LI Shi-yung 李時䔨, [ L La langue elle-même est une raison - Lecture lente de Wang Wen-hsing »] / 語言本身 就是一個理由 / Yuyan benshen jiu shi yige liyou, in [L'œil du ciel s'ouvre sur un monde de poussière] / 偶開天 眼覰紅塵慢讀王文興 / Oukai tianyan qu hongchen Mandu Wang Wen-hsing, HUANG Shu-ning 黄恕寧 (éd.), Taipei, Guoli Taiwan daxue chuban zhongxin, vol. 5, 2013.

LI Yifei 李奕霏, [“Writing Zero-Degree Emotions: On Han Dong’s Narrations of Educated Youths”] / 零度情 感'写作一论韩东的知青题材小说 / Lingdu qinggan' xiezuo-lun Han Dong de zhiqing ticai xiaoshuo, 当代文学研 究/ Dangdai wenxue yanjiu, 33:2012, p. 82-85.

LI zHUN 李准, [Le Fleuve jaune s'écoule à l'est] / 黄河东流去 / Huanghe dongliu qu, Beijing chubanshe, 1979.

Liji Yijie 禮記譯解, [Traduction et annotation des Rites], wANG Wenjin 王文錦 (trad.), Zhonghuashuju, 2001.

LIN Bai 林白, [“The Time of Cat's Passion”] / 激情的猫时代 / Jiqing de Mao shidai, in [The Time of Cat's Passion] / 激情的猫时代 / Jiqing de mao shidai, Tianjin, Zhongguo wenlian chubanshe, 2001, p. 237-41. LIN Bai 林白, “The Time of Cat’s Passion”, тUғт Bryna (trans.), Renditions 79:2013, p. 104-7.

LIN Bai 林白, [Guerre solitaire] / 一个人的战争 / Yigeren de zhanzheng, Zuojia chubanshe, 2009.

LIN Bai 林白, “Ceux qui s'aiment ne se séparent jamais », in La Chaise dans le corridor, CHEVALEYRE Véronique (trad.), Bleu de Chine, 2006, p. 55-102.

LIN Julia, Modern Chinese Poetry. An Introduction, Washington, Washington U. P., 1972.

LIN Ruiming 林瑞明, [La Littérature de Taïwan et l'esprit du temps] / 臺灣文學與時代精神 / Taiwan wenxue yu shidai jingshen, Taipei, Yunchen wenhua, 1993.

LIN Shuming 林树明, [《Le corps féminin dans la culture de consommation»] / 消费文化中的女性身体 / Xiaofei wenhua zhong de nüxing shenti, 洛阴师范学院学报 / Luoyang shifan xueyuan xuebao, 6:2005, p. 9-12.

LING Chen 凌晨, [“A Story of Titan”] / 泰坦的故事 / “Taidan de gushi”, 科幻世界 / Kehuan shijie, 7:2007, p. 15-21.

LING Chen 凌晨, “A Story of Titan”, MARTINSEN Joel (trans.), Pathlight, 2013, p. 323-63.

[Littérature nationale] / 『国文学』/Kokubungaku, numéro de juillet 1990. 
LIU Li 刘莉, [Les Femmes chinoises dans La porte des roses] / 玫瑰门中的中国女人 - 铁凝与当代女性作家的 性别认同 / Meiguimen zhong de zhongguo nüren - tiening yu dangdai nüxing zuojia de xingbie rentong, Beijing Shifan daxue chubanshe, 2012.

LIU Lydia, "The Female Body and Nationalist Discourse: Manchuria in Xiao Hong's Field of life and Death", in Body, Subject, and Power in China, zITo Angela and BaRLow Tani (eds.), Chicago, Chicago U. P., 1994, p. 157-178.

LIU Na'ou 刘呐鸥, [“Etiquette and Hygiene”] / 礼仪和卫生 / Liyi he weisheng, in [Urban Scenic Horizon] / 都 市风景线 / Dushi fengjing xian, Shanghai, Shuimo shudian, 1930, p. 107-142.

LIU Zhenyun 刘震云, [๔uvres choisies de Liu Zhenyun] / 刘震云精品文集 / Liu Zhenyun jingpin wenji, Yanbian renmin chubanshe, 1997.

LIU Zhenyun 刘震云, IMBOT-BICHET Geneviève, Se souvenir de 1942, Gallimard, 2013.

LOMBARD Denys, Le Carrefour javanais, t. I, Les limites de l'occidentalisation, EHESS, 1990.

LOMBARD Laurent, « De la représentation (rare) du transgenre dans la littérature italienne contemporaine », Cahiers d'études italiennes, 16:2013, http://cei.revues.org/1255, consulté le 30/09/2016.

LOUVEL Liliane, L'Æil du texte. Texte et image dans la littérature de langue anglaise, Toulouse, P. U. du Mirail, 1998, p. 71-72.

LOZERAND Emmanuel, «Corps, maladie, écriture chez trois auteurs japonais du début du $\mathrm{xx}^{\mathrm{e}}$ siècle : Nakae Chômin, Masaoka Shiki, Natsume Sôseki », Extrême-Orient Extrême-Occident, 39:2015, p. 21-45.

LOZERAND Emmanuel, « Gouttes d'encre. Masaoka Shiki et l'écriture du corps malade », postface à Masaoka Shiki, Un lit de malade six pieds de long, LOZERAND Emmanuel (trad.), Les Belles Lettres, « Collection Japon », 2016, p. 221-254.

LOZERAND Emmanuel, « Santé et maladie chez quelques écrivains japonais modernes (1898-1911) », in Santé et bien-être à l'épreuve de la littérature, DE JESUS CABRAL Maria et DOMINGUES DE ALMEDIA José (dirs.), Limoges,

Lambert-Lucas, 2017, p. 147-155.

LU Ling 路悀, [“Hungry Guo Su'e"] / 饥饿的郭素娥 / Ji'e de Guo Su'e, in [Selected Works] / 路悀文集 / Lu Ling wenji, Lu Ling (ed.), Hefei, Anhui wenyi chubanshe, vol. 4, 1995, p. 1-112.

Lu Tonglin, Misoginy, Cultural Nihilism and Oppositional Politics: Contemporary Chinese Experimental Fiction, Stanford U. P., 1995.

Lu Xun 魯迅, [đuvres complètes] / 魯迅全 / Lu Xun Quanji, Renmin wenxue chubanshe, 1981.

Lu Xun 魯讯, Cris, Bellassen Joël, feng Hanjin, JoIn Jean et LoI Michelle (trad.), Albin Michel, 1995.

LU Xun 魯式, La Mauvaise herbe, RYCKMANS Pierre (trad.), UGE, 1975.

LU Xun 魯迅, [La Mauvaise herbe] / 野草 / Yecao, Renmin wenxue chubanshe, 2006.

LU Xun 魯迅, Toujours sous le dais fleuri, MEUNIER Jacques et FANG Liu (trad.), You Feng, 2014.

Lu Xun 魯讯, [Traductions complètes de Lu Xun] / 鲁迅译文全集 / Lu Xun yiwen quanji, Fuzhou, Fujian jiaoyu chubanshe, 2008, 8 vol.

LUo Guanzhong 羅貫中, [Les Trois Royaumes] / 三國演義 / Sanguo yanyi, HK, Zhonghua shuju, 1987.

MA Jian 马建, [Land of Flesh] / 肉之土 / Rou zhi tu, Taipei, Taichen wenhua, 2010.

MA Jian 马建, The Dark Road / 阴之道 / Yin zhi dao, Taipei, Taichen wenhua, 2012.

MA Jian 马建, The Dark Road, DREW Flora (trans.), London, Vintage Books, 2014.

MACKIE Vera, Feminism in Modern Japan: Citizenship, Embodiment and Sexuality, Cambridge, Cambridge University Press, 2003. 
MAHAYANA Maman S., [« Le déluge de Cantik itu luka »] / Air Bah Cantik itu Luka, in [Le Caractère extrinsèque de la littérature indonésienne] / Ekstrinsikalitas Sastra Indonesia, Jakarta, PT Rajagrafindo Persada, 2007, p. 352-360.

MALIK Kriti, “Why Men Should Eat Spicy Food”, NDTV, food.ndtv.com/food-drinks/why-men-should-eatspicy-food-713829, 2014, consulted on 01/06/2021.

MAN Eva Kit Wah, Bodies in China. Philosophy, Aesthetics, and Politics, Hong Kong, The Chinese U. P., 2016.

MARIN Louis, De la représentation, EHESS/Seuil/Gallimard, 1994.

MARTIN-JUCHAT Fabienne, Le corps et les médias : La chair éprouvée par les médias et les espaces sociaux, Bruxelles, De Boeck, 2008.

MARUO Tsuneki 丸尾常喜, [Recherches sur La mauvaise herbe de Lu Xun] / 魯旬「野草」の研究 / Rojin Yasō no Kenkyū, Tokyo, Kyūko Shoin, 1997.

MARUYAMA Masao, [“From Carnal Literature to Carnal Politics”] / 肉体文学から肉体政治まで / Nikutai bungaku kara nikutai seiji made, in Thought and Behavior in Modern Japanese Politics, MORRIS Ivan (ed.), tr. from the Japanese [新装版現代政治の思想と構造 / Shinsō ban Gendai seiji no shisō to kōdō, Tokyo, Miraisha, 2006 [1964], p. 375-394] by RUCH Barbara, Oxford U. P., 1969, p. 245-67.

MARUYAMA Ōkyo 円山応挙, [The True Manner of Depicting the Human Body] / 人物正写惣本 / Jinbutsu seisha sōhon, Tenri, Tenri Library, 3 vols, 1770.

MASAOKA Shiki 正岡子規, [Une goutte d'eau d'encre] / 墨汁一商 / Bokujū itteki, Iwanami, 1999 [1901].

MASAOKA Shiki 正岡子規, [Un Lit de malade de six pieds de long] / 病床六尺 / Byōshō rokushaku, Iwanami, 2000 [1902].

MASAOKA Shiki 正岡子規, [Chronique drôle de la personne alitée] / 仰臥漫録 / Gyōga manroku, Iwanami bunko, 1999.

MASAOKA Shiki 正岡子規, [๕uvres complètes de Masaoka Shiki] / 正岡子規全集 / Masaoka Shiki zenshû, Kōdansha, vol. 19, 1975.

MASAOKA Shiki 正岡子規, Un lit de malade six pieds de long, LOZERAND Emmanuel (trad.), Les Belles Lettres, 2016. MATSUDA Kyōko, “Inō Kanori’s 'history' of Taïwan: colonial ethnology, the civilizing mission and struggles for survival in east asia", History and Anthropology, vol. 14, 2003, p. 179-196.

матsuмото Michiyo松本充代, [What I lack is] / 私にたりないもの / Watashi ni tarinai mono, Kawadeshobō, 1992.

мATSUмото Michiyo松本充代, [Hidden Voices, A Suicide Note in the Mirror and Other Short Stories] / 潜む 声、鏡に中の遺書、その他の短編 / Hisomu koe, kagami no naka no isho, sono ta no tanpen, Asky, 1999.

MAтsuмото Yūko 松本 侑子, [Dawn Never Comes to an Anorexic] / 巨食症の明けない夜明け / [Kyoshoku.shō no akenai yoake], Shyūeisha, 1988.

MATTEINI Michele, "What the master did not discuss: Self-mutilation, the female body, and filial expectations in early modern China", Asiatica venetiana 8/9, 2003/2004, p. 37-65.

MCDOUGALL Bonnie S., LOUIE Kam Hung, The literature of China in the twentieth century: Ershi-shiji-Zhongguowenxue, London, Hurst, 1997.

McGRath Jason, Post-socialist Modernity. Chinese Cinema, Literature and Criticism in the Market Age, Stanford U. P., 2008.

MENG Gang 孟岗, [L'Utopie du corps à l'ère de consommation] / 消费时代的身体乌托邦 / Xiaofei shidai de shenti wutuobang, thèse, Zhejiang daxue, 2004.

MENG Yue孟悦 et DAI Jinhua 戴锦华, [Émerger de l'horizon de l'histoire] / 浮出历史地表, Maison d'édition du Peuple de Henan / 河南人民出版社, 1989.

MERLEAU-PONTY Maurice, Phénoménologie de la perception, Gallimard, 1945. 
MERLEAU-PONTY Maurice, Phenomenology of Perception, New York, Routledge, 2012.

MERLEAU-PONTY Maurice, The Visible and the Invisible, LINGIS Alphonso (trans.), Evanston, Northwestern U. P., 1968.

MERLEAU-PONTY Maurice, The World of Perception, DAVIS Oliver (trans.), London, New York, Routledge, 2008 [2004].

MI Jiayan [Mi Jialu 米家路], ["The Insolent and Creating Body: Self-Fashioning and Chinese Modernity. A Reinterpretation of Guo Moruo’s Poem “The Heavenly Hound”’] / 張狂與造化的身體 : 自我模塑與中國現代 性一郭沫若詩歌《天狗》再解讀 / Zhangkuang yu zaohua de shenti: ziwo mosu yu Zhongguo xiandaixing - Guo Moruo shige ‘Tiangou’ zaijiedu, ZHAO Fan 趙凡 (trad.), 江南學術 / Jiangnan xueshu, 35(1):2016, p. 13-21.

MI Jiayan, Self-Fashioning and Reflexive Modernity in Modern Chinese Poetry, 1919-1949, Lewiston, NY, Edwin Mellen, 2004.

MINAMI Yūta 南雄太, [Recherche sur les œuvres de Murakami Ryū. La carte du monde de Murakami Ryū] / 村 上龍作家作品研究一村上龍の世界地困 / Murakami Ryū sakka sakuhin kenkyū. Murakami Ryū no sekai chizu, 専 修大学出版局 / Senshūdaigakushuppankyoku, 2007.

MISHIMA Yukio, Sun \& Steel, BESTER John (trans.), Kōdansha International, 1970.

MIZUKAMI Tsutomu 水上勉, [Impression] / 印象 / Inshō, inすばる / Subaru, vol. 9-12, 1987.

MO Yan, Beaux seins, belles fesses, DUTRAIT Noël et Liliane (trad.), Seuil, 2012.

MoI Toril, "Patriarchal Thought and the Drive for Knowledge", in New Directions in Psychoanalysis and Feminism, BRENNAN Teresa (ed.), London and New York, Routledge, 1989, p. 189-205.

MOMOSE Hibiki 百瀬響, [Civilisation et progrès : les coutumes perdues] / 文明開化: 失われた風俗 / Bunmei kaika : ushinawareta füzoku, Yoshikawa Kōbunkan, 2008.

MONCOND'HUY Dominique et SCEPI Henri (éds.), Les Genres de travers. Littérature et transgénéricité, Rennes, P. U. de Rennes, 2008.

MOORE Lisa Jean and CASPER Monica J., The Body. Social and Cultural Dissections, Routledge, 2015.

MORISHIMA Chūryō 森島中良, [Redhead Miscellanea] / 䊼毛雑話 / Kōmo zatsuwa, Edo, 1787.

MU Shiying 穆时英, [“The Man Who Was Treated as a Plaything”] / 被当作消遣品的男人 / Bei dangzuo xiaoqianpin de nanren, in [Tomb] / 公墓 / Gongmu, Tianjin, Bai hua wenyi chubanshe, 2005 [1931].

MU Shiying 穆时英, [“The Platinum Statue”] / 白金女体塑像 / Baijin de nüti suxiang, in [Holy Virgin’s Love] / 生处女的感情 / Shengchunü de ganqing, Hangzhou, Zhejiang wenyi chubanshe, 2004 [1934], p. 72-84.

MU Shiying 穆时英, “Craven A”, in 生处女的感情 / Shengchunü de ganqing, Hangzhou, Zhejiang wenyi chubanshe, 2004 [1933], p. 21-42.

MURAKAMI Ryū 村上龍, Coin Locker Babies 2 $2^{\mathrm{e}}$ partie / コインロッカー・ベイビーズ, 下, Kōdansha, 1984 [1980].

MURAKAMI Ryū 村上龍, Coin Locker Babies / コインロッカー・ベイビーズ, Kōdansha, 2017 [1980].

MURAKAMI Ryū 村上龍, Les bébés de la consigne automatique, ATLAN Corinne (trad.), Arles, Picquier, 1998.

MURAKAMI Ryū 村上龍, IKEZAWA Natsuki 池澤夏樹, [《Aller à l'extrémité de “notre temps”»] / 対談.「“現 代のエッジを行く」/Taidan. Gendai no edji o iku, [Littérature nationale : interprétation et recherches des textes : numéro spécial Murakami Ryū : aller à l'extrémité de “notre temps”] / 国文学 鑑賞と解釈の教材村 上龍特集 “現代のエッジを行く” / Kokubungaku kaishaku to kanshō no kyōzai Murakami Ryū tokusyū Gendai no edji o $i k u$, vol. 46, 9:2001.

MUTA Kazue 牟田和恵, [“The Image of Family of in General Magazines in the Meiji Era”] / 明治期総合雑誌に みる家族像:「家庭」の登場とそのパラドックス / Meiji ki sōgō zasshi ni miru kazoku zō, Shakaigaku hyōron, $41,1: 1990$, p. $12-25$. 
NAGAO Ken 長尾建, [« La dérobade de Ryū, Les bébés de la consigne automatique, Destruction et Vie »] / 龍の脱 出『コインロッカー・ベイビーズ』ー破壊と生, [Littérature nationale : interprétation et recherches des textes : numéro spécial Murakami Ryū : aller à l'extrémité de “notre temps”] / 国文学鑑賞と解釈の教材村 上龍特集 “現代のエッジを行く”/ Kokubungaku kaishaku to kanshō no kyōzai Murakami Ryū tokusyū Gendai no edji o iku, vol. 46, 9:2001.

NAHYANA MAMAN S., [« Le déluge de Cantik itu luka »] / Air Bah Cantik itu Luka, in [Le Caractère extrinsèque de la littérature indonésienne] / Ekstrinsikalitas Sastra Indonesia, Jakarta PT Rajagrafindo Persada, 2007, p. 352-360.

NAKAMURA Masaru 中村勝, [La Création de marchés : marché traditionnel et public au Japon moderne et en Asie de l'Est] / 創られた市場: 近代日本・東アジアの在来市場と公設市場 / Tsukurareta ichiba: kindai Nihon Higashiajia no zairai ichiba to kosetsu ichiba, Nishitokyo, Habesutosha, 2002.

NAKANO Akira 中野明, [À partir de quand la nudité fit-elle honte? Les Japonais et la honte] / 裸はいつから 恥ずかしくなったか: :日本人の羞恥心 / Hadaka wa itsu kara hazukashiku natta ka: Nihonjin no shūchishin, Tokyo, Shinchōsha, 2010.

NANANAN Kiriko 魚喃キリコ, Strawberry Shortcakes, Shōdensha, 2002.

NANCY Jean-Luc, “Exscription”, LYDON Katherine (trans.), Yale French Studies 78, On Bataille, 1990, p. 47-65.

NANTA Arnaud, « Savoirs et colonies : l'archéologie et l'anthropologie japonaises en Corée », in La société japonaise devant la montée du militarisme - Culture populaire et contrôle social dans les années 30, TSCHUDIN JeanJacques et HAMON Claude (éds.), Arles, Picquier, 2007, p. 21-31.

NATSUME Sōseki, [Choses dont je me souviens] / 思い出す事など / Omoidasu koto nado, Iwanami, 2013 [1910-1911].

NAVEAU Étienne, «La littérature indonésienne : une introduction », in Indonésie contemporaine, MADINIER Rémy (dir.), Les Indes savantes, 2016, p. 363-376.

NAVEAU Étienne, «Les Souvenirs de Buru de Pramoedya Ananta Toer (1925-2006). Mémoires et reconnaissances d'un prisonnier politique ", in Communication interculturelle et littérature. Mémoire, littérature et identité, t. $1:$ Les récits de vie : mémoire, histoire et fiction identitaire, CRIHANA Alina et al. (dir.), GalaŢi, Universitate « Dunărea de Jos », Insititul European, 2013, p. 84-102.

NERI Corrado, «Tsai Ming-liang and the Lost Emotions of the Flesh », Positions, Duke U. P., 2008, p. 289-407.

NERI Corrado, Rétro Taiwan : Le temps retrouvé dans le cinéma sinophone contemporain, L'asiathèque, 2016.

NERI Corrado, « Past Masters, New Waves: Tsai Ming-liang / François Truffaut », Transtext(e)s Transcultures / 跨文本跨文化, 1:2006, http://journals.openedition.org/transtexts/183, consulté le 01/03/2018.

o SE Kon 오세곤, [« Les soixante-dix ans depuis la Libération vus à travers le théâtre »] / 연극을 통해서 보는 광복 70년 / Yôn'gûkûl t'onghaesô ponûn kwangbok 70nyôn, [L'Esprit du temps] / 시대정 신 / Shidaejôngshin, 67:2015, https://blog.naver.com/ngmin/220963253431, consulté le 21/02/2018.

ōé Kenzaburō, [Solennel funambule] / 厳肃な綱渡り / Genshuku na tsunawatari, Tokyo, Bungeishunjū, 1965.

ōé Kenzaburō, Notes de Hiroshima / ヒロシマ・ノート / Hiroshima nôto, Bungeishunjū, 1996, Gallimard, [1965].

ōé Kenzaburō, [CEuvres complètes d'Ōe Kenzaburō] (période 1) / 大江健三郎全作品 第一期 / Ōe Kenzaburō zensakuhin dai.ikki, Tokyo, Shinchōsha 新潮社, 6 volumes, 1966-1967.

ōé Kenzaburō, [L'Imagination à l'âge atomique] / 核時代の想像力 / Kaku jidai no sôzôryoku, Tokyo, Shinchōsha, 1970.

ōé Kenzaburō, [L’Homme, être fragile] / 壊れものとしての人間 / Kowaremono toshite no ningen, Tokyo, Kōdansha bungei bunko, 1993 [1970].

ōé Kenzaburō, [«Thématique de Shiki »] / 子規のテマティック / Shiki no tematikku, in [Æuvres complètes de Shiki] / 子規全集 / Shiki zenshū, Kōdansha, vol. 11, 1975. 
ōé Kenzaburō, [đuvres complètes d'Ōe Kenzaburō] (période 2) / 大江健三郎全作品』第二期 / Ōe Kenzaburô zensakuhin dainiki, Tokyo, Shinchōsha, 6 volumes, 1977-1978.

ōé Kenzaburō, Une existence tranquille / 静かな生活 / Shizukana seikatsu, Tokyo, Kōdansha, 1990 (Gallimard, 1995).

ŌÉ Kenzaburō, [Les Tours thérapeutiques] / 治療塔 / Chiryôtô, Kōdansha, 1990.

ōé Kenzaburō, [La Planète des tours thérapeutiques] / 治療塔惑星 / Chiryōtō wakusei, Tokyo, Kōdansha, 1991 ōé Kenzaburō, [L'Arbre vert flamboyant] / 燃えあがる緑の木 / Moeagaru midori no ki, Tokyo, Shinchōsha, 1993-1995.

ōé Kenzaburō, Ōé Kenzaburō, l'écrivain par lui-même / 大江健三郎一作家自身を語る / Ōe Kenzaburō sakka jishin o kataru, Tokyo, Shinchōsha, 2007 (Picquier, 2014).

ōé Kenzaburō, « Je suis né du côté des handicapés », entretien, Le Figaro littéraire, 8 décembre 2005, p. 3.

ōé Kenzaburō, Ōé Kenzaburō, đEuvres, Paris, Gallimard, coll. « Quarto », 2016.

ōé Kenzaburō, « La thématique de Shiki », LOzERAND Emmanuel (trad.), Critique, n 839 : « Et le Japon devint moderne... », avril 2017, p. 276-289.

OGAWA Yōko 小川洋子, [Sugar Time] / Shugā Taimu, Chūō Kōronsha, 1991.

OGINO Anna 荻野アンナ, [Eating Woman] / 食べる女 / Taberu Onna, Bungei Shunjū, 1994.

OGURI Mushitarō 小栗虫太郎, [《Un meurtre parfait »] / 完全犯罪 [1933] / Kanzen hanzai, in [Les Termites] / 白蟻 / Shiroari, Shakai shisō-sha, coll. « Gendai kyōyō bunko », 1997 [1976], p. 5-78.

OGURI Mushitarō 小栗虫太郎, [Les Étranges mondes perdus] (1939-1941) / 人外魔境 / Jingai makyō, Tokyo, Kadokawa shoten, coll. « Kadokawa horā bunko », 1995 [1978].

OGURI Mushitarō 小栗虫太郎, [“Courte biographie d'Oguri Mushitarō»] / 「小伝・小栗虫太郎」/Shōden Oguri Mushitarō, in [Le Monde fantastique d'Oguri Mushitarō] / 『小栗虫太郎ワンダーランド』/Oguri Mushitarō Wandārando, KIDA Jun.ichirō (éd.), Chūseki-sha, 1990 [1979], p. 36-48.

окАмото Makiko 岡本真希子, [ « Intégration et élimination de agents de police auxiliaires de Taïwan : traitement et apaisement des âmes durant le mandat du premier gouverneur militaire 》] 台湾巡查補をめ ぐる統合と排除：前期武官総督期に於ける待遇と慰霊 / Taiwanjin junsaho o meguru tōgō to haijo: zenki bukan sōtokuki ni okeru taigū to irei, [Sciences sociales] / 社会科学 / Shakai kagaku, vol. 41, nº 1, 2011, p. 49-90. OKAZAKI Kyōko 岡崎京子, [Dear Chiwawa] / チワワちゃん / Chihuahua-Chan, Kadokawashoten, 1996. OKAZAKI Kyōko 岡崎京子, [I am Your Toy] / 私はあなたのおもちゃなの / Watashi wa anata no omocha na no, Shōdensha, 1995.

OKAZAKI Kyōko 岡崎京子, [Shotgun from the Lips] / くちびるから散弹銃 1, 2 / Kuchibiru kara sandanjū, Tokyo, Kōdansha, 1989, 1990.

OKAZAKI Kyōko 岡崎京子, [Pink and River’s Edge] / リバーズ・エッジ, Takarajimasha, 2001.

OKAZAKI Kyōko 岡崎京子, [Pink], Magazine House, 2010.

OLIVER Michael, Social Work with Disabled People, London, Macmillan, 1983.

oLson Greta, Reading Eating Disorders - Writings on Bulimia and Anorexia as Confessions of American Culture, Frankfurt, Peter Lang, 2003.

отомо Rio, "A girl with her writing machine", in Girl reading Girl in Japan, AOYAMA Tomoko and HARTLEY Barbara (eds.), Routledge, 2010, p. 130-141.

отомо Rio, "A Girl with the Amoebic Body and her Writing Machine" academia.edu/3344703/ A_Girl_with_the_Amoebic_Body_and_her_Writing_Machine1, 2006 consulted on 01/06/2021.

ou Hong, "Pantheistic Ideas in Guo Moruo's The Goddesses and Whitman's Leaves of Grass", in Whitman East \& West. New Contexts for Reading Walt Whitman, FoLsom Ed (ed.), Iowa U. P., 2002, p. 187-196. 
OUYANG Xiu 欧阴修, [Le nouveau Livre des Tang] / 新唐书 / Xin Tangshu, Beijing, Zhonghua shuju, 1975. PARSIPOUR Shahrnoush, Femmes sans hommes, BALAŸ Christophe (trad.), Lettres persanes, 2006 [1990]. PHAN Hồn Nhiên, Cheval d'acier, DOAN Cam Thi (trad.), Riveneuve, collection « Littérature vietnamienne contemporaine », 2015.

PIKE Kathleen and BOROVOY Amy, "The Rise of Eating Disorders in Japan: Issues of Culture and Limitations of the Model of 'Westernization'", Culture, Medicine and Psychiatry 2004:28, p. 493-531.

PINO Angel et RABUT Isabelle (éds.), Le Petit Bourg aux papayers, You Feng, 2016.

PIRAzzolI Melinda, "Breaking Up From What? The Corporeal Politics of Values in the Duanlie yundong

(Rupture Movement)”, Annali Ca' Foscari. Serie Orientale 55:2019, p. 363-93.

PLANA Muriel, Roman, théâtre, cinéma. Adaptations, hybridations et dialogue des arts, Rosny-sous-Bois, Bréal, 2004.

PLATINI Vincent, Lire, s'évader, résister - essai sur la culture de masse sous le III ${ }^{e}$ Reich, La Découverte, 2014.

PотTS Alex, Flesh and the Ideal. Winckelmann and the Origins of Art History, New Haven, Yale U. P., 2000.

PRŮŠEK Jaroslav, « 'Huai chiu': A Precursor of Modern Chinese Literature », Harvard Journal of Asian Studies 29:1969, p. 169-176.

PRŮŠEK Jaroslav, The Lyrical and the Epic, Studies of Modern Chinese Literature, LEE Leo Ou-fan (éd.), Bloomington, Indiana U. P., 1980.

QIAN Liqun 钱理群, WEN Rumin 温儒敏, WU Fuhui 吴福辉, [Trente années de littérature chinoise moderne] / 中国现代文学三十年 / Zhongguo xiandai wenxue sanshinian, Beijing daxue chubanshe, 1998.

QIDAOJUN 祈袏君, [Mulan n'a pas de frère aîné] / 木兰无长兄 / Mulan wu zhangxiong, Jiangsu Fenghuang chubanshe, 2017.

QIU Jiayi 邱家宜, [ Génération perdue: Wu Zhuoliu comme exemple de journaliste taïwanais dans les premières années d'après-guerre »] / 失落的世代 : 以吳濁流為例看戰後初期的臺灣本土報人 / Shiluo de shidai : yi Wu Zhuoliu wei li kan zhanhou chuqi de Taïwan bentu baoren, Zhonghua chuanbo xuekan 中華傳播學刊, vol. 23, p. 229-266.

QIU Sha 装沙, [Quelques mots sur les idées de Lu Xun sur l'éducation esthétique] / 鲁迅美术教育思想浅谈 / Lu Xun meishu jiaoyu sixiang qiantan », 北方美术 / Beifang meishu 3:2000, p. 56-57.

RABUT Isabelle et PINO Angel (éd.), Pékin - Shanghai. Tradition et modernité dans la littérature chinoise des années trente, Bleu de Chine, 2000.

RICHARD Jean-Pierre, Littérature et sensation, Seuil, 1954.

RICœUR Paul, La Mémoire, l'Histoire, l'Oubli, Seuil, 2000.

RIEFENSTAHL Leni (réar), Schönheit im Olympischen Kampf, Berlin, Deutscher Verlag, 1937.

RIEFENSTAHL Leni (réar), Olympia 2. Teil - Fest der Schönheit, Allemagne, Olympia-Film, 1938.

RITTER Gabriel, "Between Action and the Unknown: The Art of Kazuo Shiraga and Sadamasa Motonaga", Exhibition organized by the Dallas Museum of Art and the Japan Foundation, 2015, wochikochi.jp/english/ special/2015/04/between-action-and-the-unknown-the-art-of-kazuo-shiraga-and-sadamasa-motonaga.php. RITTER Gabriel, Enchanting a disenchanted world: continuity and change in the cathedrals of Consumption, Pine Forge Press, 2010, p. 58.

ROBERT Marthe, Roman des origines et origines du roman, Gallimard, 2002.

ROBERTSON Jennifer, « Blood talks: Eugenic modernity and the creation of new Japanese », History and Anthropology, vol. 13, n³, 2002, p. 191-216.

ROJAS Carlos, Homesickness. Culture, Contagion, and National Transformation in modern China, Boston, Harvard U. P., 2015. 
ROU Shi 柔石, [“A Mother Slave”] / 为奴隶的母亲 / Wei nuli de muqin, in [February] / 二月 / Eryue, Hangzhou, Zhejiang wenyi chubanshe, 2005 [1930], p. 271-98.

ROUSSET Jean, Forme et signification, José Corti, 1995.

ROY olivier, La Sainte ignorance. Le temps de la religion sans culture, Seuil, 2008.

RUARK, Jennifer K., “A Place at the Table”, Chronicle of Higher Education, July 9, 1999, A17.

RUTANGA Murindwa, Nyabingi Movement: People’s Anti-colonial Struggles in Kigezi 1910-1930, Working paper n 18 , 1991, http://murindwa-rutanga.com/pub/Nyabingi\%20Movement.pdf, consulté le 26/04/2018.

RYoo Bo Sun 류보선, “The Body in Contemporary Korean Literature”, Korean Literature Now, 32:2016, p. 21-23.

SAITO Hikaru 斎藤光 (dir.), [An Analysis of Love and Chastity in the Machine Era - Sex and Ideology] / 近代 時代の恋愛一貞操の分析一性のイデオロギー / Kikai jidai no ren.ai-teisō no bunseki - sei to ideorogii, Yumani Shobo, 2006.

SAKAGUCHI Ango, "Discourse on Decadence", LIPPIT Seiji (trans.), Review of Japanese Culture and Society 1:1, 1986, p. 1-5.

SAKAGUCHI Ango, [“The Flesh Itself Meditates”] / 肉体自体が思考する / Nikutai jitai ga shikō suru, in [The Complete Works of Sakaguchi Ango] / 坂口安吾全集 / Sakaguchi Ango Zenshū, Chikuma Shobō, 1998, vol. 4, p. 268-69.

SANG Tze-lan D., "The Modern Girl in Modern Chinese Literature”, in A Companion to Modern Chinese Literature, ZHANG Yingjin (ed.), Malden, John Wiley \& Sons, 2016, p. 411-423.

SARTRE Jean-Paul, “Intimacy”, in Intimacy and Other Stories, LLOYD Alexander (trans.), London and New York, Peter Nevill, 1949, p. 1-45.

SARTRE Jean-Paul, Being and Nothingness: An Essay on Phenomenological Ontology, BARNES Hazel E. (trans.), London, Routledge, 2003 [1957].

SAS Miryam, Experimental Arts in Postwar Japan: Moments of Encounter, Engagement, and Imagined Return, Harvard Asia Center, 2011.

SCHNEIDER Mark A., Culture and Enchantment, Chicago, Chicago U. P., 1993.

Sciences \& vie : Guerres \& histoire, 38:2017.

SCREECH Michael, Rabelais, KISCH Marie-Anne de (trad.), Gallimard, 1992.

SEIZELET Eric, « Les implications politiques de l'introduction du droit français au Japon », Revue internationale de droit comparé, vol. 43, n² 2, 1991, p. 367-388.

SEMANov Vladimir, Lu Hsün and his Predecessors, ALBER Charles J. (trans.), New York, M. E. Sharpe, 1980.

SEO Young-chae, [La Prostituée Shim Chong et les trois cérémonies pour les morts : lecture de Shim Chong de Hwang Sok-yong] / 서영채, 창녀 심청과 세 개의 진혼제 : 황석영의 ‘심청' 읽기 / Changnyeo Sim Cheonggwa se gaeui jinhonje: Hwang Seokyeongui ‘Sim Cheong’ ikgi, Séoul, Munhakdongne, 38:2004, p. 280-298.

SETTIS Salvatore, [Futuro del classico] / The Future of the "Classical", CAMERON Allan (trans.), Cambridge, Polity Press, 2006 [2004].

SHAKESPEARE Tom, “The Social Model of Disability”, in The Disability Studies Reader, DAvIS Lennard (ed.), Routledge, 2013, p. 214-221.

SHARP Jasper, Behind the Pink Curtain: The Complete History of Japanese Sex Cinema, London, FAB press, 2008.

SHARP Lesley A, “The Commodification of the Body and Its Parts", Annual Review of Anthropology 9, 2000, p. 287-328.

SHI Nai'an 施耐庵, LUO Guanzhong, Au bord de l'eau, DARS Jacques (trad.), Gallimard, vol. 1, 2005.

SHI Shu 施淑, [Recueil de littérature des deux rives] / 兩岸文學論集 / Liang'an wenxuelunji, Taipei, Xindi chubanshe, 1997. 
SHIBATA Shōji, [Ōe Kenzaburō - La terre et l'au-delà] / 大江健三郎論一地上と彼岸 / Ōe Kenzaburō - chijô to higan, Yūseidō, 1992.

SHIH Shu-Mei, The Lure of the Modern. Writing Modernism in Semicolonial China 1917-1937, Berkeley, California U. P., 2001.

Sнімамото Shōzō 嶋本 昭三, [“Mambo and Painting”] / Manbo to kaiga, Gutai, 3 / 具体, 1955, reprinted in Gutai shiryōshū: Dokyumento Gutai, p. 274-75.

SHIN Saet Pyôl, [«L'identité végétale et l'imagination communautaire »] / 신샛별, 식물적 주체성과 공동체적 상상력 / Shingmulchôk chuch'esônggwa kongdongch'ejôk sangsangnyôk, [Création et critique] / 창작과 비평 / Ch'angjakkwa pip'yông, 44 (2): 2016, p. 355-373.

SHIRAGA Kazuo 白髮一雄, [“On Distinctive Qualities”] / 資質について / Shishitsu ni tsuite, Gutai 5 / 具体 5, 1956, reprinted in Dokyumento Gutai, p. 287-288.

SHIRAGA Kazuo 白髮一雄, [“Simply Action”] / 行為こそ / Kōi koso, Gutai, 3 / 具体 3 , 1955, reprinted in Dokyumento Gutai, p. 274.

SHIRAGA Kazuo 白䯭一雄, [“The Formation of the Individual Body”] / 個体の確立 / Kotai no kakuritsu, Gutai 4 / 具体 4 , 1956, p. 6-7, reprinted in Dokyumento Gutai, p. 277-78.

SILK Michael, STERN Joseph, Nietzsche on Tragedy, Cambridge U. P., 2016.

SILVERBERg Miriam, Erotic, Grotesque, Nonsense - The Mass Culture of Japanese Modern Times, Berkeley, California U. P., 2006.

SLATER Don, Consumer Culture and Modernity, Cambridge, Polity Press, 1997.

SLAYMAKER Douglas, "Sartre's Fiction in Postwar Japan", in Confluences: Postwar Japan and France, SLAYMAKER Douglas (ed.), Ann Arbor, Center for Japanese Studies, The University of Michigan, 2002, p. 86-109.

SLAYMAKER Douglas, The Body in Postwar Japanese Fiction, London, Routledge/Curzon, 2004.

SONE Hiroyuki, [“Interpretation: Prostitute-like Naked Eyes”] / 解説娼婦的肉眼 / Kaisetsu: Shōfuteki nikugan, in [The Gate of Flesh] / 肉体の門 / Nikutai no mon, TAMURA Taijirō 田村泰次郎, Chikuma shobō, 1988 [1947], p. 239-243.

SONG Zhixin 宋志新, [1942 : La grande famine du Henan] / 1942 河南大饥荒 / 1942 : Henan da jihuang, 湖北人民出 版社, 2005.

SONTAG Susan, La maladie comme métaphore / Le sida et ses métaphores, Christian Bourgois, Collection « Titres » (réédition poche), 2009.

SOUYRI Pierre-François, Moderne sans être occidental : aux origines du Japon d'aujourd'hui, Gallimard, 2016.

SPITZER Leo, « Rabelais et les “rabelaisants” », Études de style, KAUFHOLz Éliane et al. (trad.), Gallimard, 1970, p. 134-165.

STRAFella G., "Roots and Prospects of Xi’s Cultural Policy - China Policy", 2016, news.xinhuanet.com/ politics/2015-10/14/c_1116825558.html.

SUGITA Genpaku 杉田立白 et al., [A Modern Anatomy] / 解体新書 / Kaitai shinsho, Edo, 1774.

SUGITA Hiroko, [Sōseki's Cat and Nietzsche. Modern Japanese Intellectuals Upset by the Modern Philosopher] / 漱石の『猫』とニーチェ一稀代の哲学者に震感した近代日本の知性たち/ Sōseki no neko to nīche - kindai no tetsugakusha ni shinkanshita kindai.nihon no chiseitachi, Hakusuisha, 2010.

sun Mei 孙玫, [ « L'opéra chinois dans le théâtre mondial au xx siècle»] /二十世纪世界戏剧中的中国戏 曲 / Ershi shiji shijie zhongde Zhongguo xiqu, 二十一世纪双月刊 / Ershiyi shiji shuangyue kan 45:1998, p. 103-112.

SUN Yu 孙邰, [Catalogue des peintures collectionnées par Lu Xun] / 鲁式藏画录 / Lu Xun canghua lu, Guangzhou, Huacheng chubanshe, 2008. 


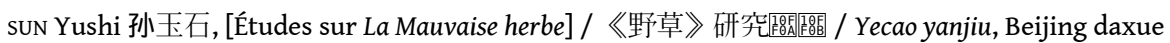
chubanshe, 2007 [1980], p. 222-261.

TAMURA Taijirō 田村泰次郎, [“Body Crash Art”] / 体当たり芸術 / Tai.atari Geijutsu, in サン写真新聞 / San shashin shimbun, October 20, 1955.

TAMURA Taijirō 田村泰次郎, [“The Flesh is the Human”] / 肉体が人間である / Nikutai ga ningen dearu, in [The Gate of the Flesh] / 内体の門, Chikuma shobō, 1988 [1947], p. 219-225.

TAMURA Taijirō 田村泰次郎, [Mon adolescence dans le cénacle littéraire] / 我が文壇青春期 / Waga bundan seishunki, Shinchōsha, 1963.

TANAKA Mitsu 田中美津, [To My Spiritual Sisters] / Inochi no on.na-tachi e, Pandora, 2004 [1972].

TANG Guizhang 唐圭璋 (éd.), [Poèmes à chanter complets des Song] / 全㷊詞 / Quan Song ci, Zhonghua shuju, 1965.

TANG Xiaobing, Origins of the Chinese Avant-Garde. The Modern Woodcut Movement, Berkeley, California U. P., 2008.

TANIZAKI Jun.ichirō 谷崎潤一郎, [Captain Shigemoto's Mother] / 少将滋幹の母 / Shōshō Shigemoto no Haha, Mainichishinbun 毎日新聞, 1950.

TANIZAKI Jun.ichirō 谷崎潤一郎, [The Gourmet Club] / 美食俱楽部 / Bishoku kurabu in [Women are Sacred] / 女人神聖 / Nyonin Shinsei, Tokyo, Shunyōdō, 1920.

TANIZAKI Jun.ichirō 谷崎潤一郎, In Praise of Shadows / In'ei raisan, HARPER Thomas and SEIDENSTICKER Edward (trans.), Sedgwick, Leete's Island Books, (1933-1934) 1977.

TANSMAN Alan (ed.), The Culture of Japanese Fascism, Durham, Duke U. P., 2009.

TAO Jiajie 陶佳洁, [《 Le courant féministe et l'écriture féminine de la Nouvelle période en Chine »] / 中国新 时期女性主义思潮与女性写作 / Zhongguo xin shiqi nüxing zhuyi sichao yu nüxing xiezuo, 当代文坛 / Dangdai wentan, 6:2017, p. 46-50.

TEZUKA Osamu 手塚治虫, MIZUKI Shigeru 水木しげる, YoKoYAMA Mitsuteru 横山光輝, MATSUMOTO Reiji 松本零 士, KUWATA Jirō 桑田次郎, [Chefs-d'œuvre de la bande dessinée d'horreur Jingai makyô (1969-1975)] / ホ ラーコミック保作選 人外魔境 / Horā komikku kesssakusen Jingai makyō, Tokyo, Kadokawa Shoten, coll. « Kadokawa horā bunko », vol. 4, 1999.

TIAMPO Ming, “Gutai Chain: The Collective Spirit of Individualism”, Positions: East Asia Cultures Critique 21:2, 2013, p. 383-415.

TIAMPO Ming, Gutai: Decentering Modernism, Chicago and London, Chicago U. P., 2011.

TIE Ning 铁凝, [La Porte des roses] / 玫瑰门 / Meiguimen, Beijing, Zuojia chubanshe, 2003.

TOER Pramoedya Ananta, [Monologues d'un muet] / Nyanyi Sunyi Seorang Bisu, Jakarta, Hasta Mitra, 2000 [1995].

TOER Pramoedya Ananta, The Mute's Soliloquy. A memoir, SAMUELS Willem (trans.), New York, Penguin Books, 1999.

TOURGUÉNIEV Ivan ИВAH ТУРГЕНЕВ, Gedichte in Prosa, LANGE Wilhelm (trad.), Leipzig, Reclam, 1883.

TOURGUÉNIEV Ivan ИВAH тУРГЕНЕВ, Poèmes en prose, SALOMON Charles et ZVIGUILSKY Alexandre (trads.), Orphée / La Différence, 1990.

TOURGUÉNIEV Ivan ИВAH тУРГЕНЕB, [Poèmes en prose] / 屠格涅夫散文诗集, Xu Weinan 徐蔚南 et WANG Weike 王维克, Shanghai, Xin wenhua shushe, 1923.

TRETIAKOv Serge M., Hurle, Chine ! et autres pièces : Écrits sur le théâtre, AMIARD-CHEVREL Claudine et al. (trad.), Lausanne, L'Âge d'Homme, 1982. 
TS'AI Hui-Yu Caroline, Taiwan in Japan's Empire Building: an institutional approach to colonial engineering, Abingdon, Oxon, Routledge, 2011.

TSU Jing, "Perversions of Masculinity: The Masochistic Male Subject in Yu Dafu, Guo Moruo, and Freud", Positions, 2000:8(2), p. 269-316.

TSUKADA Yukihiro, [“Sex and Bondage: GHQ, Censorship, Tamura Taijiro's “Gate of Flesh”'] / 性を「縛る」G $\mathrm{H}$ Q検閲、田村泰次郎『肉体の門』/ “'Sei’ o <shibaru>: GHQ, ken'etsu, Tamura Taijiro, 'Nikutai no mon', [Annual Review of the Institute for Advanced Social Research], 関西学院大学先端社会研究所紀要 / Kansei gakuin daigaku sentan shakai kenkyūsho kiȳo, vol. 11, 2014, p. 47-60.

TSURUMI Shunsuke, A Cultural History of Postwar Japan: 1945-1980, London, Routledge, 2011 [1987].

TWAIN Mark and BlounT Roy, Cannibalism in the Cars: The Best of Twain's Humorous Sketches, Prion, coll. « Prion humour classics », 2000.

UEHARA Toru, « Past, Current and Future of Anorexia Nevrosa in Japan », Acta Psychopathologica, 2015: 3, p. 1-7.

UKITA Yōzō, « Documentary on the Second Edition of 'Gutai' », Gutai 2, 1955, reprinted in Dokyumento Gutai, p. 271-72.

ULBRICHT, H., Wayang Purwa. Shadows of the Past, Kuala Lumpur \& Singapore, Oxford U. P., 1970.

UMBELINO Luís António, « Memory of the Body, Temptation of Space », The European Legacy 20, 8:2015, p. 844-51.

UTAMI Ayu, Saman, Jakarta, Gramedia, 1998.

UTAMI Ayu, Saman, INANDIAK Elisabeth (trad.), Flammarion, 2008.

VEG Sebastian (trad. et commentaire), Lu Xun Nouvelles et poèmes en prose, Rue d'Ulm, 2015.

VINCENT Keith, “Better than Sex? Masaoka Shiki's Poems on Food”, in Devouring Japan. Global Perspectives on Japanese Culinary Identity, STALKER Nancy K. (ed.), Oxford, Oxford U. P., 2018.

VISSER Robin, Cities Surround the Countryside. Urban Aesthetics in Post-Socialist China, Durham, Duke U. P., 2010. VISSER Robin, “Mal Communication”, in Gutai: Dipingere con il Tempo e lo Spazio - Painting with Time and Space, KANAYAMA Akira et al. (ed.), Milano, Silvana Editoriale, 2011, p. 15-23.

VOISSET Georges, Les lèvres du monde. Littératures comparées de la Caraỉbe à l'Archipel malais, Bécherel, Les Perséides, 2008.

vouilloux Bernard, « Langage et arts visuels. Réflexions intempestives à propos d'un champ de recherches ", Lieux littéraires, 1:2000, p. 203-223.

vouilloux Bernard, La Peinture dans le texte. XVIII ${ }^{e}-X^{e}$ siècles, CNRS Editions, 2005 [1994].

vouiLloux Bernard, Langages de l'art et relations transesthétiques, Éditions de l'Éclat, 1997.

VUILLEUMIER Victor [alias Yu Lewen 宇樂文], [“The Representation of the Body, Medicine, and Dissection: An Ambiguous Stance in Guo Moruo’s Fiction of the 1920s and 1930s”] / 身體的再現、醫學與解剖：20-30 年代 郭沫若小說裡的一種含混的態度 / Shenti de zaixian, yixue yu jiepou: 20-30 niandai Guo Moruo xiaoshuo li de yi zhong hanhu de taidu, 中國現代文學研究論叢 / Zhongguo xiandai wenxue yanjiu luncong, 10(1):2015, p. 8-16.

VUILLEUMIER Victor, “Body, Soul, and Revolution: the Paradoxical Transfiguration of the Body in Modern Chinese Poetry", in Chinese Revolution and Chinese Literature, TAO Dongfeng, YANG Xiaobin et ROSEMARY Roberts (ed.), Newcastle upon Tyne, Cambridge Scholars Publishing, 2009, p. 45-68.

VUILLEUMIER Victor, “Xu Zhimo’s Encounter with 'A Jewish Nightmare’ - How a Chinese Symbolist Viewed an Expressionist Yiddish Play", Studia Orientalia Slovaca 9.2, 2010, p. 145-155.

VUILLEUMIER Victor, « Ai Qing's poetry and Dayanhe, My Nurse », in Routledge Handbook of Modern Chinese Literature, GU Ming Dong (ed.), London, Routledge, 2018. 
VUILLEUMIER Victor, « Le corps souffrant chez Lu Xun : allégorie muette de l'obstacle et appropriation de la modernité », Extrême-Orient Extrême Occident 39:2015, p. 47-84.

WADELL Elizabeth, “Vibrator by Mari Akasaka”, The Quarterly Conversation 10, 2008, https://web.archive.org/ web/20191022042203/http://quarterlyconversation.com/vibrator-by-mari-akasaka-review, consulted on $01 / 06 / 2021$.

WANG David Der-wei, « Chinese Literature from 1841 to 1937 », in The Cambridge History of Chinese Literature, SUN CHANG Kang-I (ed.), New York, Cambridge U. P., vol. 2, 2010, p. 413-564.

WANG Gang 王刚, [English] / 英格力士 / Yinggelishi, Beijing, Renmin wenxue chubanshe, 2005.

WANG Gang 王刚, English, MERZ Martin and WEIZHEN PAN Jane (trans.), New York, Penguin Group, 2009.

WANG Ling 王凌, [“Cold Tone for a Lively Book: The Analysis of Language in Han Dong's Banished!”] / 冷色笔 调, 淡书热闹一韩东小说《扎根》语言分析 / Lengse bidiao, danshu renao-Han Dong xiaoshuo 'Zha gen' yuyan fenxi, 当代文坛 / Dangdai wentan, 4:2004, p. 70-72.

WANG Taisheng 王泰升, [ “"Violence légale” à Taïwan sous domination coloniale japonaise : une évaluation historique »] / 日本殖民統治下臺灣的「法律暴力」及其歷史評價 / Riben zhimin tongzhi xia Taiwan de 'falu baoli' ji qi lishi pingjia, 國立政治大學歷史學報 / Guoli zhengzhi daxue lishi xuebao, 25:2006, p. 1-36.

WANG Wen-hsing, Un homme dos à la mer / 背海的人 / Beihaide ren, Taipei, Hongfan shudian, 1999 ; Un homme dos à la mer, LOIVIER Camille (trad.), Montmorillon, Vagabonde, 2022.

WANG Xin 王新, [« La lutte éternelle entre la terre et le ciel : lecture de La Mauvaise herbe à partir du sens du mouvement de Munch »] / 大地与天空的永恒争执一从蒙克的波荡意象解读鲁团的野草 / Dadi yu tiankongde yongheng zhengzhi Cong Mengkede bolanxiang jiedu Lu Xun de Yecao, 名作欣赏 / Mingzuo xinshang 4:2006, p. 31-34.

WANG Xirong 王锡荣, [Lu Xun dessinateur] / 鲁式画者 / Lu Xun huazhe, Shanghai, Shanghai wenhua chubanshe, 2006.

WANG Yao 王耀, [Æuvres complètes de Wang Yao] / 王耀全集 / Wang Yao Quanji, Shijiazhuang, Hebei jiaoyu chubanshe, 1999, 8 vol.

WANG Yuehua 汪跃华, [“A Duplicated Book: On Han Dong's Banished!”] / 复写之书:韩东《扎根》论 / Fuxie zhi shu: Han Dong 'Zha gen' lun, 文学评论 / Wenxue pinglun, 3:2004, p. 39-46.

WANG Zhiliang 王智量, [Village de montagne affamé] / 饥饿的山村 / Ji'e de shancun, Lijiang chubanshe, 1994.

WANG Zhipeng 王志鹏, [ Particularité de la forme et profondeur de l'expression : brève discussion du réalisme et du modernisme dans l'écriture de fiction de Lu Xun »] / 格式的特别与表现的深切一浅论鲁式小 说创作中的现实主义与现代主义 / Geshide tebie yu biaoxiande shenqie Qianlun Lu Xun xiaoshuo chuangzuo zhongde xianshi zhuyi yu xiandai zhuyi, 滨州职业学院学报 / Binzhou zhiye xueyuann xuebao 2.1:2005, p. 20-22.

WANG-LE Min Sook, « Jeûner pour une offrande corporelle dans le Deungsinbul (Mi-Bouddha, mi-homme) de Kim Dong-ri (1913-1995) », Transtext(e)s Transcultures 跨文本跨文化, 10/2015, https://

journals.openedition.org/transtexts/596, consulté le 10/11/2016.

WEI Hongshan 魏紅珊, [“The Desiring Body and the Fractured Self: The Case of Guo Moruo’s Fiction”] / 欲望 的身體與分裂的自我一以郭沫若小說為例 / Yuwang de shenti yu fenlie de ziwo - Yi Guo Moruo xiaoshuo weili, 郭 沫若學刊 / Guo Moruo xuekan, 2009:2, p. 45-49.

White Paper on Japanese Lifestyle (The) / 国民生活白書 / Kokumin.seikatsu hakusho.

WHITE Theodore H., JАсову Annalee, Thunder out of China, New York, William Sloane Associates, 1946.

WHITE Theodore H., In search of history: a personal adventure, New York, Harper \& Row, 1978.

WHORF Benjamin Lee (CARROLL, John B. ed.), Language, Thought, and Reality - Selected Writings of Benjamin Lee Whorf. Cambridge (Mass.), The MIT Press, 1956.

WILSON George. M. Patriots and Redeemers in Japan: Motives in the Meiji Restoration, Chicago, Chicago U. P., 1992. 
wooD James, How Fiction Works, New York, Farrar, Straus and Giroux, 2008.

Woolf Virginia, A Room of One's Own (repr. 1981), New York, Harcourt, 1929.

WU Chen 吳辰, [“On the Aesthetic Features of Guo Moruo's The Goddesses”] / 論郭沫若《女神》的審美特色 /

Lun Guo Moruo ‘Nüshen’ de shenmei tese, 山東師範大學,學報 / Shandong shifan daxue xuebao, 61(4):2016,

p. 14-30.

wU Congcong 吴聪聪, LI Renjie 李仁杰, [“Filtered Memory: On Han Dong's Literary Creation in Banished!”] /

被过滤的记忆一由《扎根》谈韩东文学创作 / Bei guolü de jiyi-you 'Zha Gen' tan Han Dong wenxue chuangzuo,

作家杂志 Zuojia zazhi, 13:2013, p. 6-7.

WU Tonghu 吴铜虎, [《Les couleurs de Munch et les lignes de Van Gogh »] / 蒙克的色彩与梵高的线条 /

Mengke de secai yu Fan Gao de xiantiao, 贵州示范大学学报 / Guizhou shifan daxue xuebao 5:2009, p. 89-92.

WU Tonghu 吴铜虎, [« Les qualités picturales de Munch et Van Gogh dans la composition textuelle de Lu

Xun »] / 鲁迅文字构图中的蒙克与㷊高的绘画特质 / Lu Xun wenzi goutu zhongde Mengke yu Fan Gao de huihua tezhi, 名作欣赏 Mingzuo xinshang 8:2009, p. 70-74.

WU Wenxing 吳文星, [La Classe dirigeante dans la société taïwanaise pendant la période de l'administration japonaise] / 日治時期臺灣的社會領導階層 / Rizhi shiqi Taiwan de shehui lingdao jieceng, Taipei, Wunan tushu, 2008.

WU Yingjing 吳盈靜, [« La lumière de l'aube sous les fleurs de margousier dans L'orphelin de l'Asie de Wu Zhuoliu»] / 苦楝花下的曙光-論吴溘流『亞細亞的孤兒』中的土地認同 / Kulianhua xia de shuguang-lun Wu Zhuoliu 'Yaxiya de guer' zhong de tudi rentong, 臺灣歷史學會會訊 / Taiwan lishixuehui huixun, vol. 12, 2001, p. 13-20.

WU Yiqin 吴义勤, [“Walking Along with Poetry: Discussing Han Dong’s Fiction”] / 与诗同行一韩东小说论 / Yu shi tong xing-Han Dong xiaoshuo lun, Dangdai zuojia pinglun, 5:1996, p. 73-80.

WU Yu 吳鸕, [《 Le cannibalisme et les rites »] / 喫人與禮教 / Chi ren yu lijiao, in [Recueil des textes de WU $\mathrm{Yu}$ ] / 呉㲊集 / Wu Yu ji, Beijing, Zhonghua shuju, 2013.

WU Zhongjie 吴中杰 (éd.), [Poèmes et proses de Lu Xun commentées par Wu Zhongjie] / 吴中杰评点鲁旬诗 歌散文 / Wu Zhongjie pingdian Lu Xun shige sanwen, Shanghai, Fudan daxue chubanshe, 2006.

XI Jinping 习近平, [“Causeries sur la littérature et l'art »] / 在文艺工作座谈会上的讲话 / Zai wenyi gongzuo zuotanhui shang de jianghua, http://www.xinhuanet.com//politics/2015-10/14/c_1116825558.htm, consulté le 23/04/2018.

WU Zhuoliu 吳濁流, [Vent et pluie devant la fenêtre] / 風雨窗前 / Fengyu chuangqian, Miaoli, Wenxian shuju, 1958.

WU Zhuoliu 吳濁流, [Recueil de Wu Zhuoliu] / 吳濁流集 / Wu Zhuoliu ji, PENG Ruijin 彭瑞金 (éd.), coll.

[« Recueil complet des courtes nouvelles d'écrivains taïwanais de la première génération d'après-guerre »] / 臺灣作家全集 短篇小說選 戰後第一代 / Taiwan zuojia quanji. Duanpian xiaoshuo xuan. Zhanhou diyidai, Taipei, Qianwei chubanshe, 2010 [1991].

WU Zhuoliu 吳濁流, [Lien vers Taïwan] / 臺灣連堯狗 / Taïwan lianciao, ZHONG Zhaozheng 鍾肇政 (trad.), Taipei, Qianwei chubanshe, 1989.

XIANCHENG 鲜橙, [La Promotion de la princesse héritière] / 太子妃升职记 / Taizifei shengzhi ji, site littéraire 《晋江文学城 / Jinjiang wenxuecheng, www.jjwxc.net/oneauthor.php?authorid=137281, 2010 ; site 木子書屋 / Muzi shuwu , https://www.mzsw.org/html/52286/, consulté le 3 février 2021 ; éd. papier, 万卷出版公司 / Wangjuan chuban gongsi, Liaoning, 2012.

XIAO Hong 萧红, [“The Abandoned Child”] / 忘儿 / Qi'er, in [The Complete Works of Xiao Hong] / 萧红全集 / Xiao Hong quanji, Ha'erbin, Heilongjiang daxue chubanshe, vol. 4, 2011 [1933], p. 133-46.

XIAO Hong 萧红, [“The Field of Life and Death”] / 生死场 / Shengsi chang, in [The Complete Works of Xiao Hong] / 萧红全集 / Xiao Hong quanji, Ha'erbin, Heilongjiang daxue chubanshe, vol. 1, 2011 [1935], p. 39-134. 
XIN Yang, “Configuring Female Sickness and Recovering: Chen Ran and Annie Baobei”, Modern Chinese Literature and Culture 23, 1, 2011, p. 169-96.

Xu Guozhang 徐國章, [« Étude préliminaire du système des crimes sommaires à Taïwan durant l'occupation japonaise : l'évolution du système et son efficacité »] / 臺灣日治時期犯罪即決制度初探: 制度的演變及其成 效探討 / Taiwan rizhishiqi fanzui jijue zhidu chutan: Zhidu de yanbian ji chengxiao tantao ", in [Actes du colloque sur les archives de la gouvernance de Taïwan] / 臺灣總督府檔案學術研討會論文集 / Taiwan zongdufu dangan xueshu yantaohui lunwenji, Archives du Musée national d'histoire de Taïwan (éd.) / 國史館臺灣文獻 館 / Guoshiguan Taiwan wenxianguan, Nantou, Guoshiguan Taiwan wenxianguan, 2013, p. 179-228.

Xu Junya 許俊雅, [Recherches sur le roman à Taïwan durant l'occupation japonaise] / 日據時代臺灣小說研 究 / Riju shidai Taiwan xiaoshuo yanjiu, Taipei, Wenshizhe chubanshe, 1995.

Xu Kun 徐坤, [Bateau dans la nuit à deux tons : l'écriture féminine des années 90] / 双调夜行船一九十年代的 女性写作 / Shuangdiao yexing chuan: 90 niandai de nüxing xiezuo, Taiyuan, Shanxi jiaoyu chubanshe, 1999.

xu Shuang, « La web-littérature en Chine : une nouvelle configuration de littérature », Impressions d'ExtrêmeOrient (IDEO), dossier : « Nouvelles tendances littéraires d'Asie », 8:2018, https://journals.openedition.org/ ideo/572, consulté le 30/12/2020.

XU Shuang, « La web littérature en Chine. Création, réception et canonisation : une nouvelle configuration littéraire ", Impressions d'Extrême-Orient, 8:2018, https://doi.org/10.4000/ideo.752.

XUWEI Juquan 须尾俱全, [Le Paradis de la fin du monde] / 末日乐园 / Mori leyuan, 2014, https:// book.qidian.com/info/3312802, consulté le 25/04/2018.

xu Wenying, Eating Identities: Reading Food in Asia American Literature, Honolulu, Hawai'i U. P., 2007.

YAMASHIKI Kazuo 山敷和男, [Akutagawa Ryōnosuke’s Theories on Art] / 芥川龍之介の芸術論 / Akutagawa Ryūnosuke no geijutsuron, Gendai Shichō Shinsha, 2000.

YAMATO Hiroyuki 山登敬之, [Anorexia and Bulimia: Confusion of Alices] / Kyoshokushō to kashokushō: Konwaku suru Arisutachi / 拒食症と過食症:困惑するアリスたち, Kōdansha, 1998.

YANG Jisheng 杨继绳, [Stèle : La vérité de la grande famine des années 60 en Chine] / Mubei : Zhongguo liushi niandai dajihuang jishi / 墓碑: 中國六十年代大鲤荒紀, Tiandi tushu, 2008.

YANG Jisheng, VINCENOLLES Louis, GENTIL Sylvie, Stèles : la grande famine en Chine, 1958-1961, Seuil, 2014.

YANG Xianhui 杨显惠, [Récit de Jiabiangou] / 夹边沟记事 / Jiabiangou jishi, Huacheng chubanshe, 2008.

YANG Yi 杨义, [Histoire de la fiction chinoise moderne] / 中国现代小说史 / Zhongguo xiandai xiaoshuo shi, Renmin chubanshe, 1998, 3 vol.

YAO Xipei 姚锡珮, [“Ce que la bibliothèque de Lu Xun nous apprend sur ses relations littéraires à Tourguénief (1 $1^{\text {ère }}$ partie) »] / 从藏书看鲁迅与屠格涅夫的文学洣源 (上) / Cong cangshu kan Lu Xun yu Tugeniefude wenxue yuanyuan (shang), 鲁迅研究月刊 / Lu Xun yanjiu yuekan 3:1990, p. 56-61.

YATŌ Tamotsu, Young Samurai: Bodybuilders of Japan, Tokyo, Weatherhill, 1966.

YE Rongzhong 葉榮鐘, [《La police japonaise à Taïwan durant l'occupation du Japon »] / 日據時代臺灣的日 本警察 / Riju shidai Taiwan de Riben jingcha, in [La Grande collection de huttes] / 小屋大車集 / Xiaowu dache ji, YE Rongzhong 葉榮鐘, Taipei, Zhongyang shuju, 1967, p. 165-173.

YE Rongzhong 葉榮鐘, [《 Ma jeunesse »] / 我的青少年生活 / Wo de qingshaonian shenghuo, in [Recueil de Ye Rongzhong. Volume Littérature] / 葉榮鐘選集 文學巻 / Ye Rongzhong xua ji, YE Rongzhong 葉榮鐘, Wenxue juan, Taipei, Renjian, 2015.

YE Shengtao 叶圣陶, [“This is a Human Being Too"] / 这也是一个人 / Zhe ye shi yi ge ren, in [The Collected Works of Ye Shengtao] / 叶圣陶集 / Ye Shengtao ji, Suzhou, Jiangsu jiaoyu chubanshe, vol. 1, 1987, p. 99-102.

YE Zhiliang 叶志良, CHEN Siyuan 陈思远, [ "Sur la nature autobiographique de Guerre solitaire de Lin Bai »] / 论 林白《一个人的战争》的自传性 / Lun Lin Bai “Yigeren de zhanzheng” de zizhuan xing, 金华职业技术学院学 报 / Jinhua zhiye jishu xueyuan xuebao, 4:2009, p. 48-52. 
YOKOZUKA Kōichi 横塚光一, [Mum! Don’t Kill Me!] / 母よ ! 殺すな ! / Haha yo! Korosu na!, Seikatsu Shoin, 2007 [1975].

YōRō Takeshi 養老孟司, [Histoire littéraire du corps] / 身体の文学史 / Shintai no bungakushi, Shinchōsha, 2010.

YoSHIDA Akimi 吉田秋生, [The Cherry Orchard] / 櫻の園 / Sakura no Sono, Hakusensha, 2013.

YOSHIHARA Jirō, “Gutai Art Manifesto”, томII Reiko (trans.), web.guggenheim.org/exhibitions/gutai/data/ manifesto.html.

yоSнімото Banana 吉本ばなな, Kitchen / キッチン, Fukutakeshoten, 1988.

YOUQIAN 尤前, [Mon apprentie est encore morte] / 我家徒弟又挂了 / Wo jia tudi you guan le, 起点 / Qidian, book.qidian.com/info/3378072,consulté le 25/04/2018.

YUE Mingbao, "Gendering the Origins of Modern Chinese Fiction", in Gender and Sexuality in Twentieth-Century Chinese Literature and Society, LIU Tonglin (ed.), Albany, State University of New York Press, 1993, p. 47-66.

YUEH-YU Emily, HEI-TUNG NG Neda, « Magic, Medicine, Cannibalism: The China Demaon in Hong Kong Horror », in Horror to the Extreme: Changing Boundaries in Asian Cinema, CHOI Jinhee and WADA-MARCIANO Mitsuyo (eds.), Hong Kong U. P., 2009, p. 156-157.

ZAINI-LAJOUBERT Monique, « Histoire du roman indonésien », in Indonésie contemporaine, MADINIER Rémy (dir.), Les Indes savantes, 2016, p. 407-414.

ZHANG Ailing 张爱玲, [Little Reunion] / 小团圆 / Xiao tuanyuan, Zuojia chubanshe 2009.

ZHANG Ailing 张爱玲, [Un amour à renverser les cités] / 傾城之戀 / Qingcheng zhi lian, Taibei, Huangguan wenhua chubanshe, 1991.

ZHANG Jiang 张江, [L'Auteur peut-il mourir : discussions sur la critique littéraire occidentale moderne] / 作 者能不能死: 当代西方文论考辨 / Zuozhe nengbuneng si : dangdai xifang wenlun kaobian, Beijing, Zhongguo shehui kexue chubanshe, 2017.

ZHANG Lianze 張良澤, [ « La conscience sociale chez Wu Zhuoliu: étude de ses romans avant la libération de Taïwan »] / 吳濁流的社會意識 : 就其描寫臺灣光復以前的小說探討之 / Wu Zhuoliu de shehui yishi: jiu qi miaoxie Taiwan guangfu yi qian de xiaoshuo tantao zhi, in 吳濁流 / Wu Zhuoliu, zHANG Henghao 張恆豪 (éd.), Tainan, Guoli Taiwan wenxueguan, collection « [Recueil d'études des écrivains taïwanais modernes et contemporains 2] / 臺灣現當代作家研究資料彙編 2 / Taiwan xiandangdai zuojia yanjiu ziliao huibian 2 », 2011, p. 185-205.

ZHANG Xinxin 张辛欣, [“On the Same Horizon”] / 在同一地平线上 / Zai tongyi dipingxian shang, in [Representative works] / 张辛欣代表作/ Zhang Xinxin daibiao zuo, Zhengzhou, Huanghe wenyi chubanshe, 1988.

ZHANG Yinde, "The Fiction of Living Beings. Man and Animal in the Work of Mo Yan", in China Perspectives 3:2010, p. 124-32.

ZHANG Yinde, «L'École de Shanghai : le modernisme en question », in Littérature comparée et perspectives chinoises, L'Harmattan, 2008.

ZHANG Yinde, Le roman chinois moderne : 1918-1949, PUF, 1992, p. 151-169.

ZHoNG Xueping, Masculinity Besieged: Issues of Modernity and Male Subjectivity in Chinese Literature of the Late Twentieth Century, Durham, Duke U. P., 2000.

zHu Huizu 朱惠足, [Pouvoir impérial et intimité: les relations raciales dans le roman colonial à Taiwan] / 帝 國下的權力與親密: 殖民地臺灣小說中的種族關係 / Diguo xia de quanli yu qinmi: zhimindi Taïwan xiaoshuo zhong de zhongzu guanxi, Taipei, Maitian chuban, 2017.

zHU Ping, Gender and Subjectivities in Early Twentieth-Century Chinese Literature and Culture, New York, Palgrave MacMillan, 2015.

ZIMMER Heinrich, Philosophies of India, New York, Meridian Books, 1957. 
ZITO Angela and BARLOW Tani (ed.), Body, Subject and Power in China, Chicago, Chicago U. P., 1994. 


\section{Index des noms d'auteur(e)s et de figures historiques}

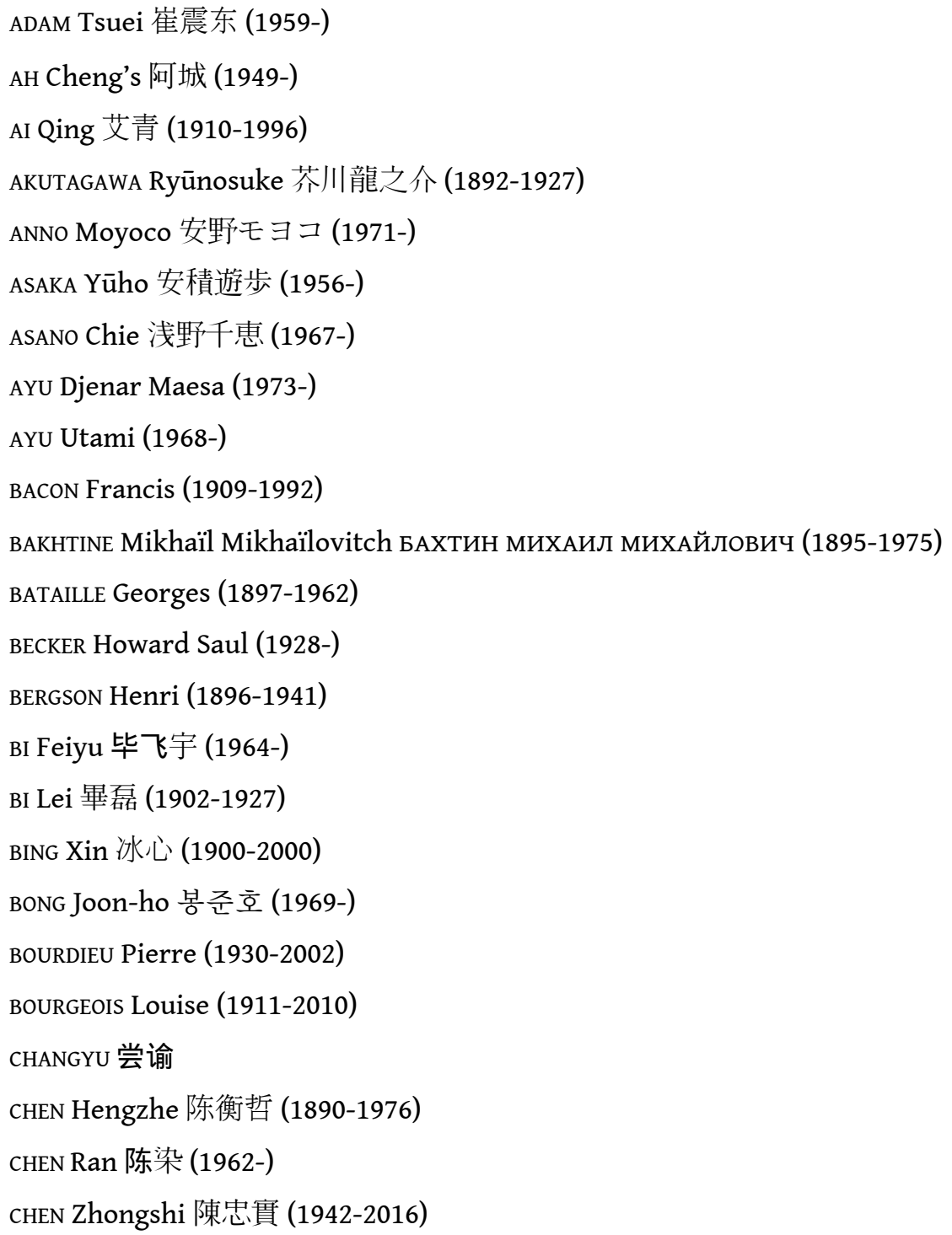


CHENG Wei-hao 程伟豪 (1984-)

CHONG Key Ray (1933-)

CIxous Hélène (1937-)

DAI Jinhua (1959-)

DANTE Alighieri (1265-1321)

DAZAI Osamu 太宰治 (1909-1948)

DING Ling 丁玲 (1904-1986)

Đỗ Hoàng Diệu (1976-)

DUAN Qirui 段祺瑞 (1865-1936)

EDOGAWA Ranpo (1894-1965)

EL SHIRAZY Habiburrahman (1975-)

FREUD Sigmund (1856-1939)

FUJITA Tsuguharu 藤田㖪治 (1886-1968)

GARCÍA MÁRQUEZ Gabriel (1927-2014)

GEZILI DE YEWAN 格子里的夜晚 (1980-2019)

GOGoL Nicolaï Vassilievitch НИКОЛАЙ ВАСИЛЬЕВИЧ ГОГОЛЬ (1809-1852)

GREENAWAY Peter (1942-)

GUNTUR Soehardjonto (1976-)

GUO Moruo 郭沫若 (1892-1978)

HAN Dong 韩东 (1961-)

HANUNG Bramantyo (1975-)

HONG Ying 虹影 (1962-)

ICHIKAWA Hiroshi 市川浩 (1931-2002)

IRIGARAY Luce (1930-)

ITAGAKI Takaho 板垣鷹穗 (1894-1966)

ITō Hiromi 伊藤比呂美 (1955-)

JIN Shengtan (1610-1661)

JUHUA Sanli 杼花散里

KAMEI Hideo 亀井秀雄 (1937-2016)

KAPROW Allan (1927-2006)

KATŌ Madoka 加藤まどか (1965-)

КIM Dong-ri (1913-1995)

KIM Hyesoon 김혜순 (1955)

кITō Aya 木藤亜也 (1962-1988)

KRISTEVA Julia (1941-)

KURIYAGAWA Hakuson 廚川白村 (1880-1923)

KURNIAWAN Eka (1975-)

LACAN Jacques (1901-1981) 
LAIRESSE Gérard de (1641-1711)

LEE Kang-sheng 李康生 (1968-)

LIN Bai 林白 (1962-)

LING Shuhua 凌淑华 (1900-1990)

LIU Heng 刘恒 (1955-)

LIU Hezhen 劉和珍 (1904-1926)

LU Ling 路悀 (1923-1994)

LU Xun 魯迅 (1881-1936)

LU Yin 庐隐 (1899-1934)

MARUO Tsuneki 丸尾常喜 (1937-2008)

MARUYAMA Masao 丸山真男 (1914-1996)

MASAOKA Shiki 正岡子規 (1867-1902)

MATSUMOTO Michiyo 松本充代 (1962-)

MATSUMOTO Yūko 松本侑子 (1963-)

MERLEAU-PONTY Maurice (1908-1961)

MO Yan (1955-)

MORI Ōgai 森鴎外 (1862-1922)

MORISHIMA Chūryō 森島中良 (1756-1810)

мотоNAGA Sadamasa 元永定正 (1922-2011)

MU Shiying 穆时英 (1912-1940)

NANANAN Kiriko 魚喃キリコ (1972-)

NANCY Jean-Luc (1940-2021)

NATSUME Sōseki 夏目漱石 (1867-1916)

NH. Dini (1936-2018)

NIETZSCHE Friedrich (1844-1900)

NOMA Hiroshi 野間宏 (1915-1991)

o'KEEFFE Georgia (1887-1986)

ŌE Kenzaburō (1935-)

OKAZAKI Kyōko 岡崎京子 (1963-)

ŌSHIMA Yumiko 大島弓子 (1947-)

OUYANG Yuqian 歐陽予倩 (1889-1962)

PARK Chan-wook 박찬욱 (1963-)

PARK Cheol-su (1948-2013)

PHAN Hồn Nhiên (1972-)

PRAMOEDYA Ananta Toer (1925-2006)

QIDAOJUN 祈裙君

RABELAIS François (1483 ou 1494-1553)

RADEN Adjeng Kartini (1879-1904) 
RIC@EUR Paul (1913-2005)

SAITo Manabu 斎藤学 (1941-)

SAKAGUCHI Ango 坂口安吾 (1906-1955)

SARTRE Jean-Paul (1905-1980)

SEGAL George (1924-2000)

SHI Zhecun 施蛰存 (1905-2003)

SHIGA Naoya (1883-1971)

SHIмAмото Shōzō 嶋本昭三 (1928-2013)

SHIN Shengtan (1610-1661)

SHIRAGA Kazuo (1924-2008)

SHōNO Yoriko 笙野頼子 (1956-)

SHU Ting 舒婷 (1952-)

SOEHARTO (1921-2008)

SONTAG Susan (1933-2004)

TAMURA Taijirō 田村泰次郎 (1911-1983)

TANAKA Atsuko 田中敦子 (1932-2005)

TAPIÉ Michel (1909-1987)

TIE Ning 铁凝 (1957-)

TOMIOKA Taeko 富岡多恵子 (1935-)

TOURGUÉNIEV Ivan ИВАН СЕРГЕЕВИЧ ТУРГЕНЕВ (1818-1883)

TSUSHIMA Yūko 津島佑子 (1947-2016)

TWAIN Mark (1835-1910)

UKITA Yōzō 浮田要三 (1924-2013)

WANG Gang 王剛 (1960-)

WANG Meng 王蒙 (1935-)

WANG Wen-hsing 背海的人 (1939-)

WANG Zhiliang 王智量 (1928-)

WEI HUI 卫慧 (1973-), v. ZHOU

WEN Tianxiang 文天祥 (1236-1283)

WHITE Theodore H. (1915-1986)

WINCKELMANN Johann (1717-1768)

wu Yu 吳虞 (1872-1949)

XI Jinping 习近平 (1953-)

XIANCHENG 鲜橙 (1980-)

XIAO Hong 萧红 (1911-1942)

XU Guangping (1898-1968)

XU Shoushang 许寿裳 (1883-1948)

XU Xiaobin 徐小斌 (1953-) 
XU Zhimo 徐志摩 (1896-1931)

XUWEI Juquan 须尾俱全

YAM Simon (1955-)

YAMATO Hiroyuki 山登敬之 (1957-)

YAMAZAKI Tsuruko 山崎つる子 (1925-2019)

YAN Lianke 阎连科 (1958-)

YANG Jisheng (1940-)

YE Lingfeng 葉靈鳳 (1905-1975)

YOSHIDA Akimi 吉田秋生 (1956-)

үознімото Banana よしもとばなな (1964-)

YOUQIAN 尤前

Yu Dafu 骫达夫 (1896-1945)

YU Hua 余华 (1960-)

YUAN Shikai 袁世凱 (1859-1916)

YUE Fei 岳飞 (1103-1142)

ZANG Hong 藏洪 (709-757)

ZHANG Ailing 張愛玲 (1920-1995)

ZHANG Jie 张洁 (1937-)

ZHANG Xun 张巡 (709-757)

ZHOU wei Hui 周卫慧 (1973-) 


\section{Index des œuvres}

$301,302(1995)$

[«Ceux qui s'aiment ne se séparent jamais»]/同心爱者不能分手 / Tong xinaizhe buneng fenshou (1989)

[Anemia] / 貧血 / Hinketsu (1978)

Belles de Halimunda (Les) (2017) / Cantik itu luka (2002)

Black Snow / 黑的雪 / Heide xue (1988)

[Bright Anorexia (A), Cheerfully Bulimic]/ あかるく拒食ゲンキに過食/ Akaruku Kyosyoku, Genki ni Kasyoku (1992)

«Ça va, je suis un cochon » / 돼지라서 괜찮아 / Dwaejilaseo gwaenchanha (2012)

Captain Shigemoto's Mother / 少将滋幹の母 / Shōshō Shigemoto no Haha (1950)

Cash / Seperti Dendam, Rindu Harus Dibayar Tuntas (2014)

[Cauchemar des Juifs (Le)] / 猶太人的怖夢 / Youtairende bumeng (1925)

[Cauchemars] / Bóng đè (2006)

«Célébration » / 祝福 / Zhufu (1924)

Challenging Mud / 泥に挑む / Doro ni idomu (1955)

[« Chamane du col Dang (La)»] / 당고개무당 / Danggogae mudang

[«Chamane Eul-hwa (La)»] / 을화 / Eul-hwa (1978)

[Cherry Orchard (The)] / 桜の園 / Sakura no Sono (1986)

Cheval d'acier / Ngựa thép (2014)

[Chihuahua-Chan] / チワワちゃん (1996)

[Choses dont je me souviens] / 思い出すことなど / Omoidasu koto nado (1910)

Cook, the Thief, His Wife and Her Lover (The) (1989)

[Courants dans l'histoire de l'art moderne] / 近代美術史潮論 / Jindai meishushi chao lun (1927)

[Dawn never comes to an anorexic] / 巨食症の明けない夜明け / Kyoshoku-shō no akenai yoake (1987)

[Devil of the Flesh (The)] / 肉体の覀魔 / Nikutai no Akuma (1946) 
[Discours aux Causeries sur la littérature et l'art] / 在文艺工作座谈会上的讲话 / Zai wenyi gongzuo zuotanhui shang de jianghua (2015)

English / 英格力士 / Ying-ge-li-shi (2005)

Fille de la faim (Une) / 饥饿的女儿/Ji'e de nüer (2013)

[Flesh is the Human (The)] / 肉体が人間である / Nikutai ga ningen de aru (1947)

[Flesh Itself Meditates (The)] / 肉体自体が思考する / Nikutai jitai ga shikō suru (1946)

Gate of the Flesh (The) / 肉体の門 / Nikutai no mon (1947)

Geburt der Tragödie aus dem Geiste der Musik (Die) / The birth of tragedy (The) (1872)

[Générale en haut et moi en bas (La)] / 将军在上我在下 / Jiangjun zai shang, wo zai xia (2013)

[Gourmet Club (The)] / 美食俱楽部 / Bishoku-Kurabu (1919)

[Goutte d'encre (Une)] / 墨汁一商 / Bokujū itteki (1901)

Guerre solitaire / 一个人的战争 / Yigeren de zhanzheng (2009)

[Hand, Leg, Meat, Arm] / 手 · 足 ·肉 ·体 / Te, ashi, niku, karada (1995)

[Hidden Voices, A Suicide Note in the Mirror and Other Short Stories] / 潜む声 鏡の

中の遺書 その他の短編 / Hisomu koe kagami no naka no isho sono ta no tanpen (1999)

[Holy Virgin's Love] / 圣处女的感情 / Shengchunü de ganqing (2007)

Homme dos à la mer (Un) / 背海的人 / Bei hai de ren (1999)

[“Hungry Guo Su'e"] / 饥饿的郭素娥 / Ji'e de Guo Su'e (1942)

[In Clothes Called Fat] / 脂肪という名の服を着て / Shibō to iu na no fuku wo kite (2002)

In Praise of Shadows / 陰疑禮讚 / In'ei raisan (1939)

Je suis un cyborg / 싸이보그지 만 괜 찮아 / Saibogeujiman gwaenchanha (2006)

[Je suis vraiment une super star] / 我真是大明星 / Wo zhen shida mingxing (2017)

[Journal de mademoiselle Sophie (Le)] / 莎菲女士日记 / Shafei nüshi riji (1928)

Kitchen /キッチン / Kitchen (1988)

Kōmo zatsuwa / 紅毛雑話 / Painting manual (1787)

Lance Bearer (The)

Lit de malade six pieds de long (Un) / 病牀六尺 / Byōshō rokushaku (1902)

[Literary Too Literary] / 文芸的な、余りに文芸的な / Bungeitekina, amarini bungeitekina (1927)

“Madman's Diary (A)” / 狂人日记 / Kuangren Riji (1918)

Maladie comme métaphore (La) / Illness as Metaphor (1978)

Mauvaise herbe (La) / 野草 / Yecao (1927)

[Mon apprentie est encore morte] / 我家徒弟又挂了 / Wo jia tudi you guan le (2018)

[Mulan n'a pas de frère aîné] / 木兰无长兄 / Mulan wu zhangxiong (2017)

[Noro and Saniwa] / のろとさにわ / Noro to Saniwa (1991)

[Notes éparses d'un homme couché] / 仰臥漫録 / Gyōga manroku (1901)

[On Territory] / テリトリー論 / Territory-ron (1985)

[One Liter of Tears. A Young Girl's Fight for Life (Aya's Diary)] / ーリットルの淚 / Ichi rittoru no namida (1986) 
[Paradis de la fin du monde (Le)] / 末日乐园 / Mori leyuan (2014)

Pink (1989)

[Planète des tours thérapeutiques (La)] / 治療塔惑星 / Chiryōtō wakusei (1991)

[Pork Cutlet Princess] / かつどんひめさま / Katsudon hime sama (1993)

[Porte des roses (La)] / 玫瑰门 / Meigui men

[Pour sortir de la tour d'ivoire] / 出了象牙之塔 / Chule xiangya zhi ta (1924-1925)

[Princess] / 姫 / Hime (1979)

[Promotion de la princesse héritière (La)] / 太子妃升职记 / Taizifei shengzhi ji (2010, 2012)

River's Edge / リバーズ・エッジ (1944)

[Rivière de fleurs] / Manjianghong / 满江红 (1133 ?)

Saman (1998 / 2008)

Shanghai Baby / 上海宝贝 / Shanghai baobei (1999)

[Shotgun from the Lips] / くちびるから散弾銃 / Kuchibiru kara Sandanjū (1996)

[“Sinking”] / 沉沦 / Chenlun (1921)

[Sky of Plants (The)] / 草木の空 / Sōmoku no Sora (1978)

[“Small sun in the water (A)”] / 水の中の小さな太陽 / Mizu no naka no chiisana taiyō (1994)

[“So as not to become warped”] / 歪ませないように / Yugamase nai yōni (1979)

[Symbole de la mélancolie (Le]] / ch. 苦悶的象徵 Kumende xiangzheng / jp. 苦悶の象徵 Kumon no shôchô (1924)

Tag-Along (The) / 紅衣小女孩 / Hongyi xiaonühai (2015)

Tale of Genji / 源氏物語 / Genji monogatari (1008)

Tale of Genji Scroll / 源氏物語絵巻 / Genji monogatari emaki (11??)

Tenants Downstairs (The) / 樓下的訪客 / Louxia de fangke (2016)

[Therapeutic Sexy Trip (A)] / 癒しのセクシー・トリップ / Iyashi no sekushii torippu (1993)

[Tours thérapeutiques (Les)] / 治療塔 / (1990)

[“Trash”] / トラッシュ / Trash (2010-17)

[Tremblement sur la ligne de décadence] / 頽敗線的顫動 / Tuibaixiande chandong (1925)

[Underripe Plums] / 青梅 / Ao-ume (1982)

Une fille de la faim / 饥饿的女儿 / Ji'e de nüer (2013)

[Vallée du lœss (La)] / 황토기 / Hwangtogi (1995)

Versets de l'amour (Les) / Ayat-ayat Cinta (2004)

[Vie numérique (Une)] / 数字生命 / Shuzi shengming (2018)

[“Vinegar and Oil”] / 䣷油 / Su.abura (1985)

Vita sexualis / +夕・セクスアリス / Vita sexualis (1909)

[What I'm lacking] / 私に足りないもの / Watashi ni tarinai mono (1992)

[White Paper on Japanese Lifestyle (The)] / 国民生活白書 / Kokumin-seikatsu Hakusho (s. d.) 


\section{Index des themes}

1980s family (the)

absurde / nonsensu

act / 行為 / $k \bar{o} i$

allégorique

ancienne société cannibale / 吃人的旧社会 / chiren de jiu shehui

art of hurling oneself / 体当たり芸術 / taiatari geijutsu

art of the flesh (the) / 肉体の芸術 / nikutai no geijutsu

$b a /$ 把

bag of skin / 皮囊 / pinang

baihua / 白话, 白話

beauty / 美 / bi

body / 身体 / shintai

bulimia

cannibalism / cannibalisme

cannibalisme

chaman(e)

cochon

commerce d'escorte / 援助交際 / enjo.kōsai

corps / mình, thân, cơ thể

crise

cruelty

Cultural Revolution (1966-1976)

death

dépression, mélancolie, angoisse / 苦悶 / ch. kumen, jp. kumon

disability narrative

Disability Rights Movement

discours indirect 
disease of giant consumption / 拒食症 / kyoshoku-shō

disease of overeating / 過食症 / kashoku-shō

dispositif géographique / 형국론, 形局論 / hyeongguk-ron

douleur

dragon

easy-to-eat store culture

eat store culture

eating disorders

École de Shanghai / 海派 / Haipai (1930-40)

Écrivains de la Nouvelle Génération / 新生代作家 / Xinsheng dai zuojia

embodied

embodiedness

énergie, souffle / 기, 氣 / gi

engourdissement des sens / 麻木 / mamu

fallness / 痽落 / daraku

family

Family Mart

famine de 1942

female identity

femme nouvelle / 新女性 / xin nüxing

forme des veines de la montagne / 산혈형체, 山穴形體 / sanhyeol hyeongche

forme, corps / 상, 像 / sang

gender / genre

GHQ

goodness / 善 / zen

Grande famine

Green Grass Association / 青い芝の会 / Aoi shiba no kai

grotesque / guro

grotesque-ism

Guomindang

Gutai

Gutai Art Association / 具体美術協会 / Gutai Bijutsu Kyōkai

High culture fever / 文化热 / Wenhuare_movement (1979-1989)

homme nouveau / con người mới

humorous pictures or "caricatures" / 笑絵 / warai-e

Incident du 18 mars 1926

intelligence kinésique

karma

karmic retribution

kitchen 
literature of the flesh / 肉体文学

littérature féministe / Sastra Wangi

littérature islamiste / Sastra Islami

lutte

manga / マンガ

manger-homme / 吃人 / chiren

martial heroes' film / 武俠片 / wǔxiápiàn

masturbation

méditation de l'impur / 不浄観 / fujō-kan

mémoire

memory / mémoire

métaphore

mondialisation

monologue introspectif

montagne

Mouvement du 4 Mai

New historical novels / 新历史小说 / xin lishi xiaoshuo

New sensation school / 新感觉派 / xin ganjue pai

Nouvelle littérature / 新文学 / Xin wenxue

nude

Nüwa / 女媧

obscenity

observation des dragons / 간룡법, 看龍法 / ganryong-beop

patriarchal system

phallocentrism

polity / 国体 / kokutai

pork cutlet on rice / カツ井 / katsudon

préservation du relief / 보국형세, 保局形勢 / boguk hyeongse

prostitute / ホテトル嬢 / hotetorujo

prostitution

pulp / カストリ / kasutori culture

pur sentir / pure sensation

raison, principe / 이, 理 / ch. li, cor. yi, jp. li

Renouveau / Đổi Mới

Révolution culturelle (1966-1976)

sauvage

search for the flesh

sexy / ero

slenderism

souffrance 
stereotypical heterosexism

stereotypical theme

superstition

symbolique

tabou

traversée / 穿越 / chuanyue

vengeance

zero-degree narration / 零度写作 / lingdu xiezuo 


\section{Index des xénismes}

$b a /$ 把

baihua / 白话, 白話

bi / 美 / beauty

boguk hyeongse / 보국형세, 保局形勢 / préservation du relief

chiren / 吃人 / manger-homme

chuanyue / 穿越 / traversée

enjo.kōsai / 援助交際 / prostitution

ero / エロ / sexy

ero-guro / エログロ

fujō-kan / 不浄観

ganryong-beop / 간룡법, 看龍法 / observation des dragons

gi / 기, 氣 / énergie, souffle

guro / グロ / grotesque

hotetorujō / ホテトル寋 / prostitute

kashokushō / 過食症 / disease of overeating

katsudon / カツ井 / pork cutlet on rice

$k \bar{o} i$ / 行為 / act

kyoshoku-shō / 拒食症 / disease of giant consumption

lingdu xiezuo / 零度写作 / zero-degree narration

manga / マンガ

$m i /$ 身

nikutai / 肉体 / flesh

nonsensu / absurde

onna-poi / 女っぽい / feminine

otoko-rashii / 男らしい / masculine

pinang / 皮囊 / bag of skin

sang / 상, 像 / corps, forme 
sanhyeol hyeongche / 산혈형체, 山穴形體 / forme des veines de la montagne shintai / 身体 / body

shunga / 春画

ukiyo-e / 浮世絵 / estampe du monde flottant

warai-e / 笑絵 / humorous pictures or "caricatures"

xin ganjue pai / 新感觉派 / New sensation school

xin lishi xiaoshuo / 新历史小说 / New historical novels

xin nüxing / 新女性 / femme nouvelle

Xin wenxue / 新文学 / nouvelle littérature

$y i$ / 이, 理 / raison, principe 